In cooperation with the Texas Department of Transportation

\title{
Atlas of Interoccurrence Intervals for Selected Thresholds of Daily Precipitation in Texas
}

Water-Resources Investigations Report 03-4281

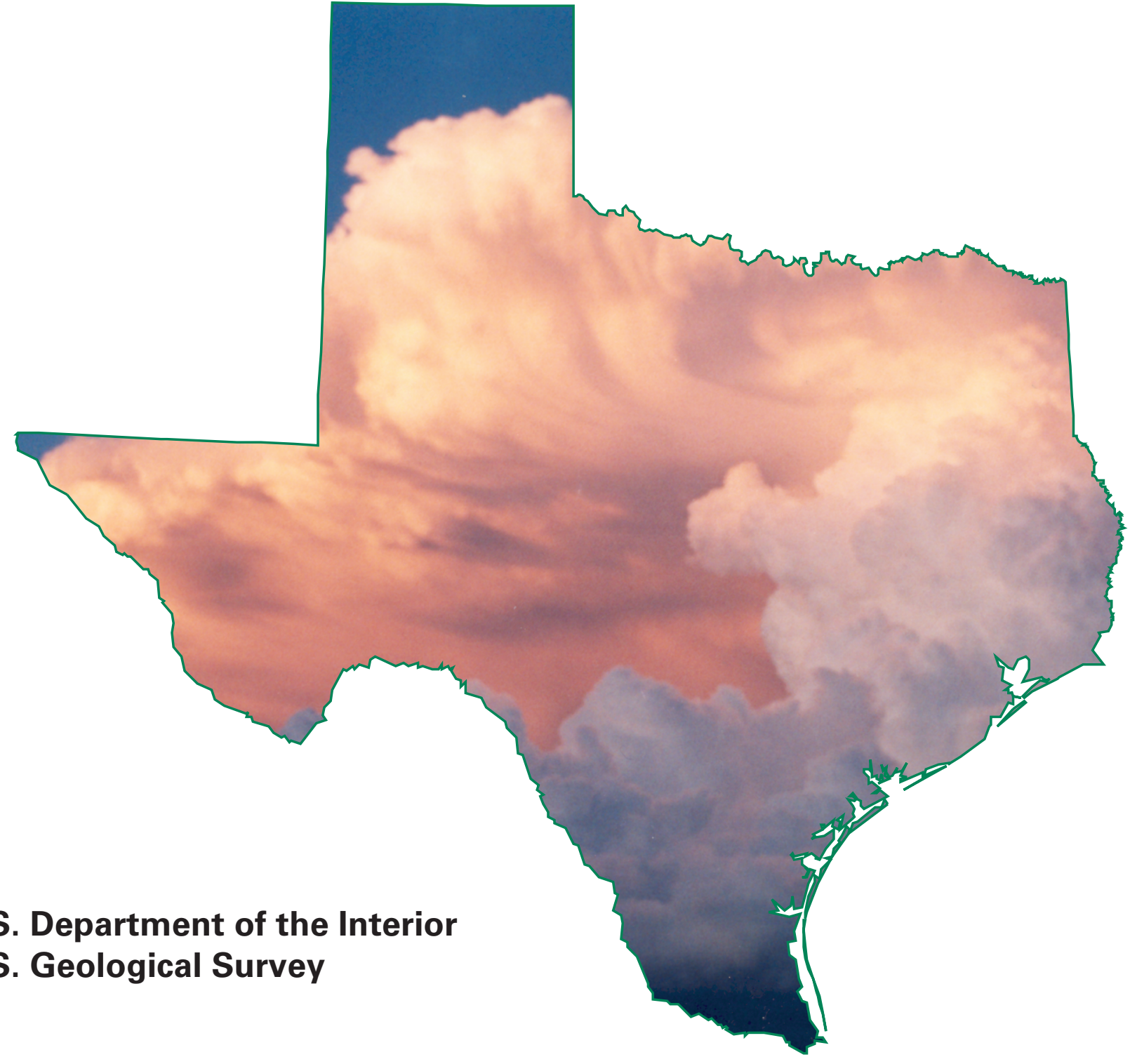




\begin{tabular}{|c|c|c|c|c|}
\hline $\begin{array}{l}\text { 1. Report No. } \\
0-4193-5\end{array}$ & \multicolumn{2}{|c|}{ 2. Government Accession No. } & \multicolumn{2}{|c|}{ 3. Recipient's Catalog No. } \\
\hline \multicolumn{3}{|c|}{ 4. Title and Subtitle } & \multicolumn{2}{|l|}{ 5. Report Date } \\
\hline \multicolumn{3}{|c|}{$\begin{array}{l}\text { ATLAS OF INTEROCCURRENCE INTERVALS FOR } \\
\text { SELECTED THRESHOLDS OF DAILY PRECIPITATION IN } \\
\text { TEXAS }\end{array}$} & \multicolumn{2}{|c|}{ 6. Performing Organization Code } \\
\hline \multicolumn{3}{|c|}{$\begin{array}{l}\text { 7. Author(s) } \\
\text { William H. Asquith and Meghan C. Roussel }\end{array}$} & \multicolumn{2}{|c|}{$\begin{array}{l}\text { 8. Performing Organization Report No. } \\
\text { WRIR 03-4281 }\end{array}$} \\
\hline \multicolumn{3}{|c|}{$\begin{array}{l}\text { 9. Performing Organization Name and Address } \\
\text { U.S. Geological Survey } \\
\text { Water Resources Division } \\
8027 \text { Exchange Drive } \\
\text { Austin, Texas } 78754\end{array}$} & \multicolumn{2}{|c|}{$\begin{array}{l}\text { 11. Contract or Grant No. } \\
\text { Project } 0-4193\end{array}$} \\
\hline \multicolumn{3}{|c|}{$\begin{array}{l}\text { 12. Sponsoring Agency Name and Address } \\
\text { Texas Department of Transportation } \\
\text { Research and Technology Implementation Office } \\
4000 \text { Jackson Ave., Bldg. } 1 \\
\text { P.O. Box } 5080 \\
\text { Austin, TX } 78731\end{array}$} & \multicolumn{2}{|c|}{ 14. Sponsoring Agency Code } \\
\hline \multicolumn{5}{|c|}{$\begin{array}{l}\text { 15. Supplementary Notes } \\
\text { Project conducted in cooperation with the Federal Highway Administration. }\end{array}$} \\
\hline \multicolumn{5}{|c|}{$\begin{array}{l}\text { 16. Abstract } \\
\text { The U.S. Geological Survey, in cooperation with the Texas Department of Transportation, has produced maps depicting } \\
\text { the magnitude and spatial variation of interoccurrence intervals in days of daily precipitation exceeding selected } \\
\text { thresholds in Texas. In the report, a Poisson model of precipitation interoccurrence intervals is described. A } \\
\text { precipitation interoccurrence interval is the time period between two successive rainfall events. Events are defined as } \\
\text { daily precipitation equaling or exceeding a specified depth threshold. Ten precipitation thresholds are considered: } 0.05 \text {, } \\
0.10,0.25,0.50,0.75,1.0,1.5,2.0,2.5 \text {, and } 3.0 \text { inches. Site-specific mean interoccurrence interval and ancillary } \\
\text { statistics are presented for each threshold and for each of } 1,306 \text { National Weather Service daily precipitation gages. } \\
\text { Maps depicting the spatial variation across Texas of the mean interoccurrence interval for each threshold are presented. } \\
\text { Influence of elevation in west Texas and intra-annual variations or month-to-month differences in interoccurrence } \\
\text { interval are documented. }\end{array}$} \\
\hline \multicolumn{2}{|c|}{$\begin{array}{l}\text { 17. Key Words } \\
\text { Precipitation, Interoccurrence interval, Poisson process, } \\
\text { Poisson distribution, Exponential distribution, National } \\
\text { Weather Service rain gage, Texas }\end{array}$} & \multicolumn{3}{|c|}{$\begin{array}{l}\text { 18. Distribution Statement } \\
\text { No restrictions. This document is available to the public } \\
\text { through the U.S. Geological Survey, Information } \\
\text { Services, Box 25286, Denver CO 80225-0286. }\end{array}$} \\
\hline $\begin{array}{l}\text { 19. Security Classif. (of report) } \\
\text { Unclassified }\end{array}$ & $\begin{array}{l}\text { 20. Security Classif. ( } \\
\text { Unclassified }\end{array}$ & his page) & $\begin{array}{c}\text { 21. No. of pages } \\
204\end{array}$ & $\begin{array}{l}\text { 22. Price } \\
\$ 4.00\end{array}$ \\
\hline
\end{tabular}

Form DOT F 1700.7 (8-72) Reproduction of completed page authorized

Cover: Summer thunderstorm viewed from southeast Austin facing north, mid-1990s (photograph by Lynne Fahlquist, U.S. Geological Survey). 
U.S. Department of the Interior

U.S. Geological Survey

\section{Atlas of Interoccurrence Intervals for Selected Thresholds of Daily Precipitation in Texas}

By William H. Asquith and Meghan C. Roussel

\section{U.S. GEOLOGICAL SURVEY}

Water-Resources Investigations 03-4281

In cooperation with the Texas Department of Transportation Austin, Texas 2003 


\section{U.S. DEPARTMENT OF THE INTERIOR}

Gale A. Norton, Secretary

\section{U.S. GEOLOGICAL SURVEY}

Charles G. Groat, Director

Any use of trade, product, or firm names is for descriptive purposes only and does not imply endorsement by the U.S. Government.

For additional information write to

\section{District Chief}

U.S. Geological Survey

8027 Exchange Dr.

Austin, TX 78754-4733

E-mail: dc_tx@usgs.gov

Copies of this report can be purchased from

U.S. Geological Survey

Information Services

Box 25286

Denver, CO 80225-0286

E-mail: infoservices@usgs.gov 


\section{CONTENTS}

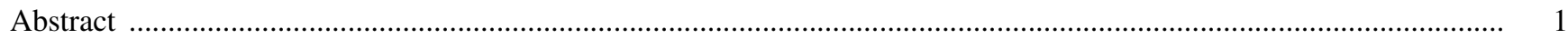

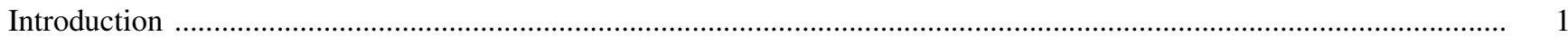

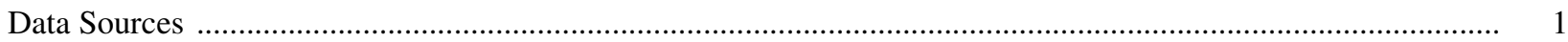

Previous Studies

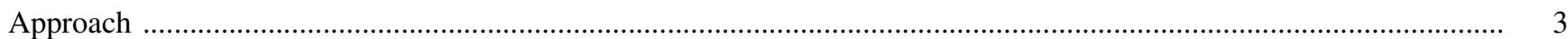

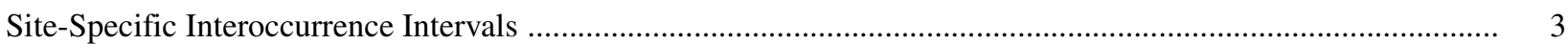

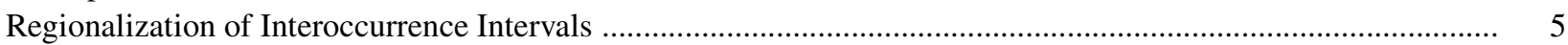

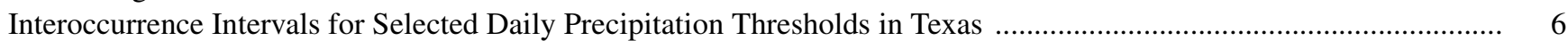

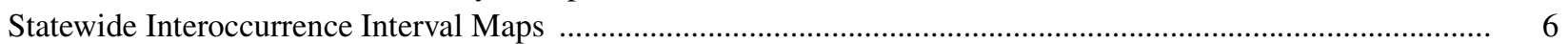

Influence of Elevation on Interoccurrence Intervals West of the Pecos River .....................................................

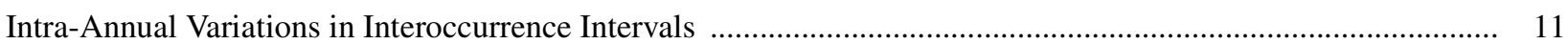

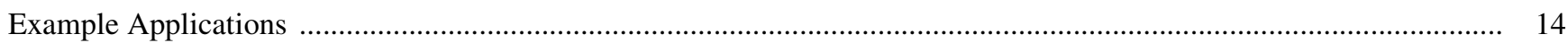

Site-Specific Median 6-Month Daily Precipitation Threshold ................................................................... 15

Site-Specific Median Number of Occurrences ................................................................................... 29

Intra-Annual Variations in Interoccurrence Intervals .........................................................................

Regional Estimation of Mean Interoccurrence Interval ......................................................................... 31

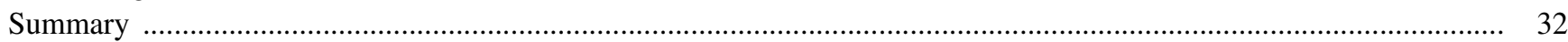

Selected References

\section{FIGURES}

1. Map showing locations of 1,306 National Weather Service daily precipitation stations in Texas ..................... 2

2. Example of abbreviated time series of daily precipitation for station 428 Austin Camp Mabry ...................... 3

3. Example interoccurrence interval computations for a hypothetical data set .................................................

4-13. Maps showing interoccurrence interval for a:

4. 0.05-inch and greater daily precipitation threshold for Texas

5. 0.10-inch and greater daily precipitation threshold for Texas

6. 0.25-inch and greater daily precipitation threshold for Texas

7. 0.50 -inch and greater daily precipitation threshold for Texas

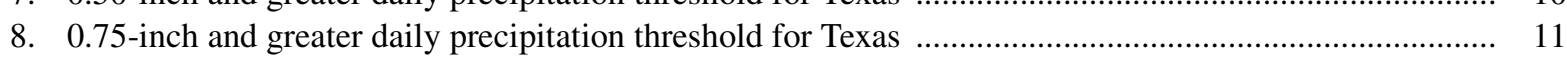

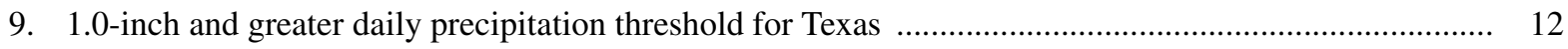

10. 1.5-inch and greater daily precipitation threshold for Texas ............................................................. 13

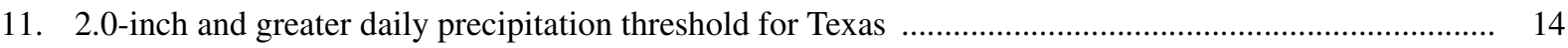

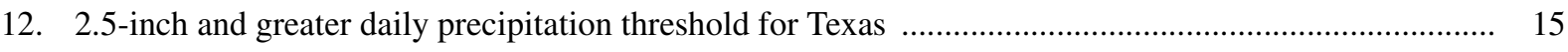

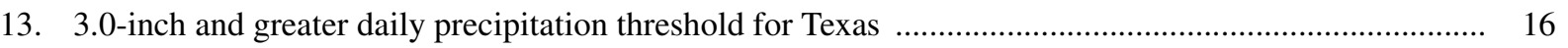

14a-j. Graphs showing relation between interoccurrence interval and elevation west of the Pecos River, west

Texas

15-24. Boxplots showing distribution of ratio of interoccurrence interval for month to mean interoccurrence interval for period of record at station for a:

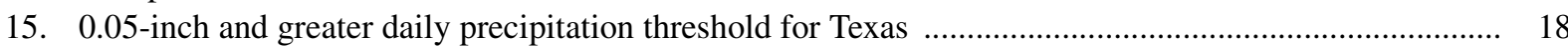

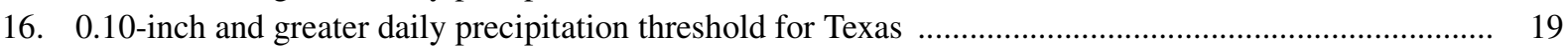

17. 0.25 -inch and greater daily precipitation threshold for Texas .......................................................... 20

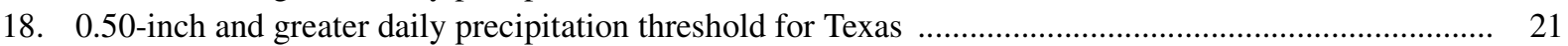

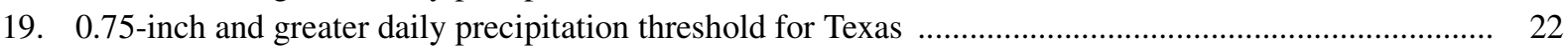

20. 1.0-inch and greater daily precipitation threshold for Texas ............................................................. 23

21. 1.5-inch and greater daily precipitation threshold for Texas ........................................................... 24

22. 2.0-inch and greater daily precipitation threshold for Texas ............................................................ 25

23. 2.5-inch and greater daily precipitation threshold for Texas ........................................................... 26

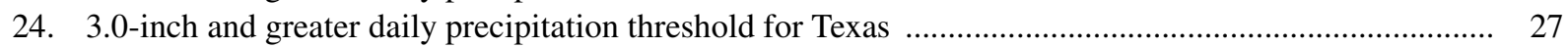


25-28. Graphs showing:

25. Relation between adjusted mean and median interoccurrence ratios and month for 0.05 - and 0.10-inch daily precipitation thresholds for Texas

26. Relation between adjusted mean interoccurrence ratio and month for 0.25-, 0.50-, 0.75-, 1.0-, 1.5-, 2.0-, 2.5-, and 3.0-inch daily precipitation thresholds for Texas

27. Relation between adjusted median interoccurrence ratio and month for 0.25-, 0.50-, 0.75-, 1.0-, 1.5-, 2.0-, 2.5-, and 3.0-inch daily precipitation thresholds for Texas

28. Relation between site-specific daily precipitation threshold and mean interoccurrence interval for station 428 Austin Camp Mabry and estimated median 6-month precipitation

\section{TABLES}

1. Summary of National Weather Service daily precipitation stations in Texas with known names, latitudes, and longitudes

2. National Weather Service daily precipitation stations in Texas, by county

3. Summary of interoccurrence intervals for daily precipitation thresholds of 0.05 and 0.10 inch

4. Summary of interoccurrence intervals for daily precipitation thresholds of 0.25 and 0.50 inch

5. Summary of interoccurrence intervals for daily precipitation thresholds of 0.75 and 1.0 inch 116

6. Summary of interoccurrence intervals for daily precipitation thresholds of 1.5 and 2.0 inches 142

7. Summary of interoccurrence intervals for daily precipitation thresholds of 2.5 and 3.0 inches 170

8. Example computation steps of interoccurrence intervals for a Poisson process described in text 198

9. Summary of statewide interoccurrence interval statistics and diagnostic statistics of interoccurrence interval maps

10. Summary of interoccurrence ratios by month for 0.05 -inch and greater daily precipitation threshold 199

11. Summary of interoccurrence ratios by month for 0.10 -inch and greater daily precipitation threshold 199

12. Summary of interoccurrence ratios by month for 0.25 -inch and greater daily precipitation threshold

13. Summary of interoccurrence ratios by month for 0.50 -inch and greater daily precipitation threshold 200

14. Summary of interoccurrence ratios by month for 0.75 -inch and greater daily precipitation threshold 201

15. Summary of interoccurrence ratios by month for 1.0-inch and greater daily precipitation threshold

16. Summary of interoccurrence ratios by month for 1.5 -inch and greater daily precipitation threshold 202

17. Summary of interoccurrence ratios by month for 2.0-inch and greater daily precipitation threshold

18. Summary of interoccurrence ratios by month for 2.5 -inch and greater daily precipitation threshold

19. Summary of interoccurrence ratios by month for 3.0-inch and greater daily precipitation threshold 203

20. Regional estimates of mean interoccurrence interval of precipitation for Austin, Texas 204

\section{CONVERSION FACTORS AND VERTICAL DATUM}

\begin{tabular}{rcl}
\hline Multiply & By & To obtain \\
\hline & & \\
foot (ft) & 0.3048 & meter $(\mathrm{m})$ \\
inch (in.) & 25.40 & millimeter $(\mathrm{mm})$ \\
mile (mi) & 1.609 & kilometer $(\mathrm{km})$ \\
\hline
\end{tabular}

Vertical coordinate information is referenced to the National Geodetic Vertical Datum of 1929 (NGVD 29). 


\title{
Atlas of Interoccurrence Intervals for Selected Thresholds of Daily Precipitation in Texas
}

\author{
By William H. Asquith and Meghan C. Roussel
}

\section{Abstract}

A Poisson process model is used to define the distribution of interoccurrence intervals of daily precipitation in Texas. A precipitation interoccurrence interval is the time period between two successive rainfall events. Rainfall events are defined as daily precipitation equaling or exceeding a specified depth threshold. Ten precipitation thresholds are considered: $0.05,0.10,0.25,0.50,0.75,1.0$, $1.5,2.0,2.5$, and 3.0 inches. Site-specific mean interoccurrence interval and ancillary statistics are presented for each threshold and for each of 1,306 National Weather Service daily precipitation gages. Maps depicting the spatial variation across Texas of the mean interoccurrence interval for each threshold are presented. The percent change from the statewide standard deviation of the interoccurrence intervals to the root-mean-square error ranges from a magnitude minimum of (negative) -24 to a magnitude maximum of -60 percent for the 0.05- and 2.0-inch thresholds, respectively. Because of the substantial negative percent change, the maps are considered more reliable estimators of the mean interoccurrence interval for most locations in Texas than the statewide mean values.

\section{INTRODUCTION}

A precipitation interoccurrence interval is the time period between two successive rainfall events. Rainfall events are defined as daily precipitation equaling or exceeding a depth threshold. Estimation of the interoccurrence interval for selected depth thresholds in Texas can be useful in developing hydrologic models, in enhancing guidelines for hydraulic structures design, and in assessing "best-management practices" for small watersheds. An interoccurrence interval is the expected or mean interval of time between precipitation "events," in which an event occurs when the daily precipitation depth equals or exceeds a specified threshold (for example, 1 inch). Another application for interoccurrence intervals is for estimation of typical antecedent moisture conditions for a watershed. Antecedent moisture-that is, soil moisture prior to a runoff event-is semi-dependent on how often precipitation occurs. Reliable estimates of typical antecedent moisture conditions across the State could enhance the estimation of rainfall-runoff relations.

Beginning in 2002, the U.S. Geological Survey, in cooperation with the Texas Department of Transportation, began a study of the interoccurrence intervals for selected thresholds of daily precipitation in Texas. The purposes of this report are to provide (1) site-specific estimates of interoccurrence intervals, (2) maps showing the statewide distribution of the expected (average or mean) interoccurrence intervals for selected event thresholds, (3) adjustment factors to account for the calendar variation of precipitation potential, and (4) example applications of interoccurrence intervals. The thresholds selected for this report are $0.05,0.10$, $0.25,0.50,0.75,1.0,1.5,2.0,2.5$, and 3.0 inches.

\section{Data Sources}

A database of daily precipitation in Texas was aggregated using National Weather Service (NWS) precipitation data. The time series of daily precipitation database was assembled for this report using Hydrosphere (2002). Specifically, the Hydrosphere (2002) data are derived from the data archives of the National Climatic Data Center. For this report, the daily NWS precipitation stations considered included only those stations in Texas for which a station name, latitude, and longitude were available. In total 1,306 stations were considered. The total number of days of record represented by the 1,306 stations is in excess of 15.1 million. The earliest year of record is 1895 and the latest year of record is 2001. The average period of record for the stations is about 34 years, and about 660 stations were active as of 2001. The stations are listed in table 1 (at 


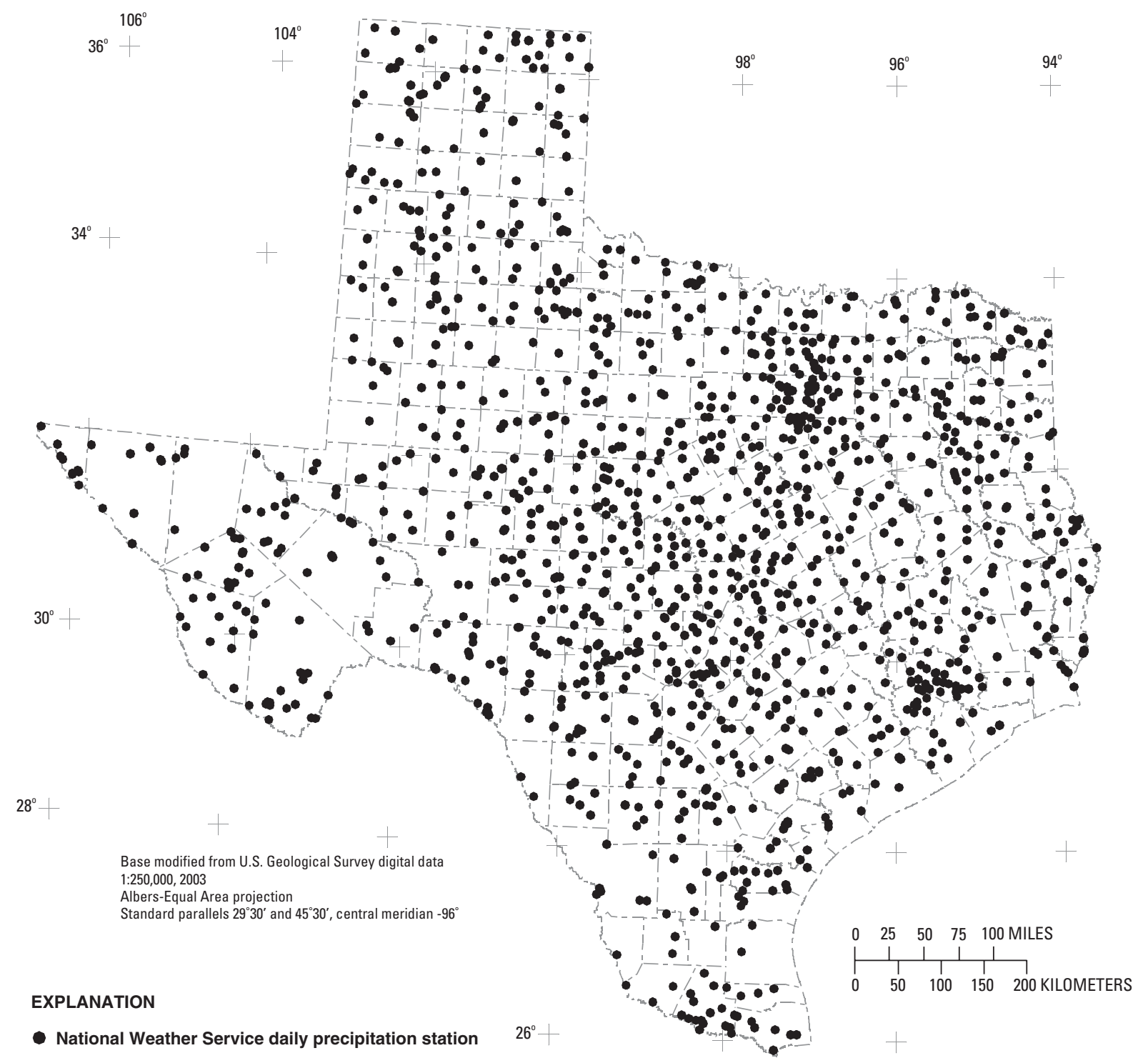

Figure 1. Locations of 1,306 National Weather Service daily precipitation stations in Texas.

end of report); the table contains entries for NWS station name, NWS station number, latitude, longitude, and ancillary data. The stations are listed in alphabetical order and not numerical order of station number. To assist readers in referencing particular stations, an alphabetical ordering of Texas county names and the corresponding sequence numbers of stations within each county is listed in table 2 (at end of report). Finally, a map showing the locations of the 1,306 stations is shown in figure 1 , and an example time series of a subset of the data for the Austin Camp Mabry station is shown in figure 2.

\section{Previous Studies}

Recent studies of precipitation characteristics in Texas pertinent to hydrologic design include Asquith (1998, 1999) and Asquith and Famiglietti (2000). Asquith (1998) presents procedures to estimate the depth-duration frequency of precipitation in Texas; for example, depth of the 6-hour 25-year precipitation. The procedures of Asquith (1998) are based on annual precipitation maxima and therefore are applicable only for the precipitation recurrence intervals of at least 365 days ( 1 year). The procedures described in this report can be used for intervals of less than 1 year such as for 


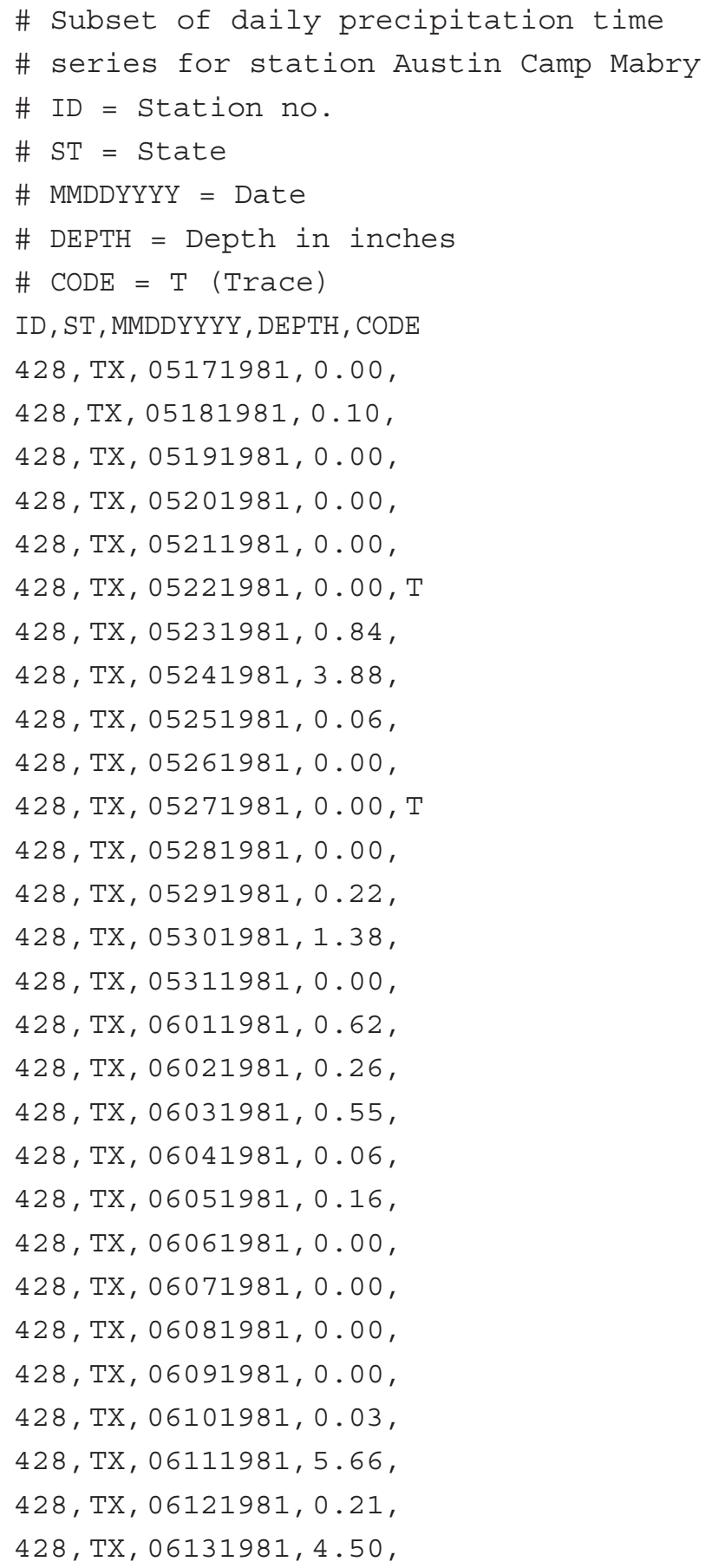

Figure 2. Example of abbreviated time series of daily precipitation for station 428 Austin Camp Mabry.

the estimation of the 6-month depth of daily precipitation. This report compliments Asquith (1998). Asquith (1999) and Asquith and Famiglietti (2000) present precipitation areal-reduction factors associated with the 1-day design storm for areas surrounding the cities of Austin, Dallas, Fort Worth, and Houston.

Bomar (1995, p. 60-63, figs. 30-33) provides the average number of days during each of the four seasons (winter, spring, summer, and fall) for daily precipitation thresholds of 0.01, 0.1, 0.5, and 1.0 inches. Although little content relevant to interoccurrence intervals is provided, Bomar (1995) contains much general information regarding precipitation characteristics and other meteorological topics for Texas.

\section{APPROACH}

A Poisson process model is used in this report to define the distribution of interoccurence intervals for selected thresholds of daily precipitation. According to Clarke (1998, p. 111), "The Poisson process forms the starting-point for many applications in the water sciences [including the study of precipitation] in which probabilistic models are used to describe events occurring irregularly in time or space." When an assumption is made that precipitation events are random variables with characteristics of a Poisson process, then straightforward statistical methods can be used to model the occurrence of precipitation events defined by a given threshold. A major component of a Poisson process is the mean interoccurrence interval-if the mean interoccurrence interval for a given location is known, then the Poisson process is specified. Further information regarding Poisson processes and related statistical topics are discussed in Ross (1994, p. 157-167), Wilks (1995, p. 71-73), Clarke (1998), and Bhat and Miller (2002).

Two steps are used in this report for characterizing and regionalizing interoccurrence intervals in Texas. The first step is to develop estimates of the interoccurrence intervals for each station. Such estimates are referred to as site-specific interoccurrence intervals. The second step is to regionalize the sitespecific estimates by mapping through spatial and geostatistical analysis so that reliable predictions of the interoccurrence intervals for selected precipitation thresholds can be made for any location in Texas.

\section{Site-Specific Interoccurrence Intervals}

The interoccurrence intervals of daily precipitation events that equal or exceed a given threshold for a given station are site-specific estimates and are determined directly from the daily precipitation database. 
For a Poisson process, the random variables $I_{1}, I_{2}, \ldots$ represent the interoccurrence intervals of the events, and the interoccurrence intervals are independent and identically distributed. The exponential distribution (Ross, 1994, p. 223-230; Evans and others, 2000, p. 77-81; Bhat and Miller, 2002, p. 203) describes the cumulative distribution of the interoccurrence intervals of a Poisson process. The distribution in the context of interoccurrence intervals is given by

$F\left(I_{n} \leq x\right)=1-e^{-\lambda x}$ for $x \geq 0$ and $n=1,2, \ldots$

where $F$ is the cumulative or nonexceedance probability for the $I_{n}$ observation that is less than or equal to some interoccurrence interval $x$. The parameter $\lambda$ is known as the arrival rate (of an event, in units of days $^{-1}$ ), the arrival parameter, or the Poisson parameter. The value $1 / \lambda$ is the expected or mean interoccurrence interval $(\Lambda)$ with units of days (per event). In this report, interoccurrence interval is preferred over arrival rate, as it has units of days rather than the abstract units of days ${ }^{-1}$. The variance of the distribution is $1 / \lambda^{2}$. For a homogeneous Poisson process, $\lambda$ is constant. If $\lambda$ varies, then the Poisson process is said to be nonhomogeneous.

The mean interoccurrence interval is computed as the mean interval between successive daily precipitation events that equal or exceed a given threshold. Thus, it follows that

$$
\lambda=\frac{n}{T}=\frac{\text { no. of events above threshold }}{\text { total time observed in days }}=\frac{1}{\Lambda} .
$$

To indicate whether the daily occurrence of precipitation that equals or exceeds a given threshold likely follows a homogeneous Poisson process, hypothesis testing is required. For hypothesis testing Bhat and Miller (2002, p. 254-255) conclude that the standard normal variate, $Z$, for a Poisson process is

$$
\begin{gathered}
Z=\frac{S-\frac{n T}{2}}{\sqrt{\frac{n T^{2}}{12}}} \text {, and } \\
S=\sum_{i=1}^{n} t_{i} \text { for } 0<t_{1}<t_{2}<\ldots<t_{n}<T,
\end{gathered}
$$

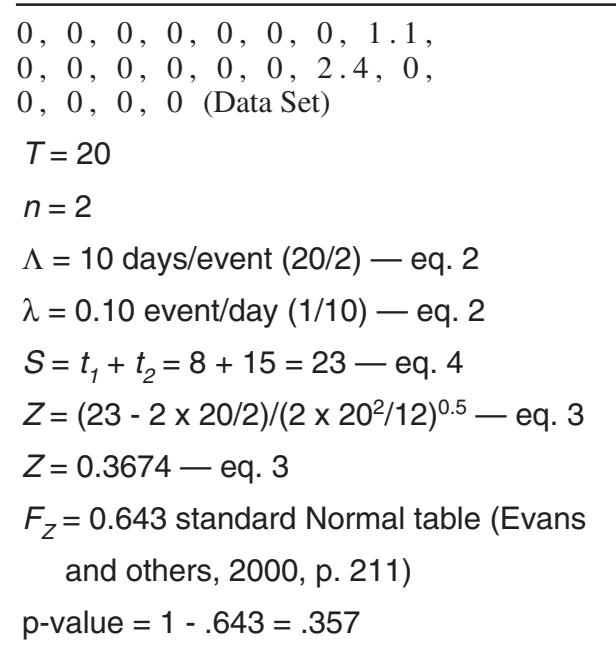

Figure 3. Example interoccurrence interval computations for a hypothetical data set.

where $t_{i}$ is the cumulative time, in days, for each event during the interval $[0, T]$. The statistic $S$ is hereinafter referred to as "sigma S."

The cumulative probability $F_{Z}$ of $Z$ is used to estimate the p-value (Helsel and Hirsch, 1992, p. 97-116) or attained significance level of the data $\left(1-F_{z}=P\right)$. The null hypothesis is that the interoccurrence intervals follow a homogeneous Poisson process. Large absolute values of $Z$ indicate that the process probably is not a (homogeneous) Poisson process with a constant interoccurrence interval. The nonhomogeneous Poisson process for the daily precipitation occurrences is a possible alternative. Bhat and Miller (2002) do not discuss the "power" of a hypothesis test based on equation 3; hence, the potential for the so-called type II error (the Poisson model is incorrect but the model is accepted) of the test on the analysis in this report is unknown.

To demonstrate how the above equations are applied, consider the data set shown at the top of figure 3 . The data set of daily precipitation comprises 20 days $(T=20)$. For a threshold of $1 \mathrm{inch}$, there are two events $(n=2)$. Therefore, the mean interoccurrence interval is 10 days (20/2), the arrival rate is $0.10(2 / 20)$, and the p-value is .357 or 35.7 percent. This p-value is not small-it is greater than a few percent or so-which indicates little statistical evidence that the data should not be modeled as a homogeneous Poisson process. Thus a reasonable conclusion is that the interoccurrence intervals of the example data follow a homogeneous Poisson process. For this report p-values greater than 
.01 provide sufficient evidence to conclude that the data follow a homogeneous Poisson process.

Similar computations to those illustrated in figure 3 for each of the selected depth thresholds $(0.05,0.10$, $0.25,0.50,0.75,1.0,1.5,2.0,2.5$, and 3.0 inches) and for each of the 1,306 daily precipitation stations were done. The computations provide the basis for subsequent analyses presented here. The computational results for each depth threshold are listed in tables 3-7 (at end of report).

The tables identify each station by the sequence number shown in table 1 . For each station, the mean interoccurrence interval is reported along with the total number of events for the indicated threshold within the entire period of record for the station and the total number of days available. The sigma $\mathrm{S}$, standard normal variate, and nonexceedance probability of the standard normal variate provide the basis for the testing of a homogeneous Poisson process. Finally, the p-value corresponding to the nonexceedance probability is reported.

Another important feature of a Poisson process is the distribution of the number of events, $n$, occurring during a given interval $[0, T]$. The probability density function $f_{n}(T)$ of the Poisson process (Ross, 1994, p. 157; Bhat and Miller, 2002, p. 202) is

$$
f_{n}(T)=e^{-\lambda T(\lambda T)^{n}} \frac{e^{-T / \Lambda} \frac{(T / \Lambda)^{n}}{n !}}{n !}
$$

The cumulative distribution function $F_{n}(T)$ of the Poisson process is

$$
F_{n}(T)=e^{-\lambda T} \sum_{i=0}^{n} \frac{(\lambda T)^{i}}{i !}=e^{-T / \Lambda} \sum_{i=0}^{n} \frac{(T / \Lambda)^{i}}{i !}
$$

For example, suppose the mean interoccurrence interval is $\Lambda=10$ days $(\lambda=0.1)$. To the closest integer, what is the median number of occurrences ( $F=0.5$, or a 50 -percent probability) in a $T=60$ day period? From equations 5 and 6 , the probabilities of the Poisson process are computed, and the computations are summarized in table 8 (at end of report). The median number of occurrences for the example is five.

\section{Regionalization of Interoccurrence Intervals}

Regionalization in this report is a two-step process. First, a spatial analysis or "neighborhood smoothing" of the interoccurrence intervals is done for each selected daily precipitation threshold for all stations. Second, geostatistical analysis is done on the smoothed interoccurrence intervals to produce a continuously varying map of interoccurrence intervals for each selected daily precipitation threshold. The maps can be used to estimate the interoccurrence interval for any location in Texas.

Neighborhood smoothing is the process by which the interoccurrence intervals for a particular station and those surrounding it are combined or "pooled" together to develop a more reliable estimate of the interoccurrence interval for the location of the station than can be derived from the data for the station alone. In other words, the neighborhood of $m$ stations surrounding a particular station contains more information-more days of record-about the characteristics of precipitation occurrence in that area than is available at the particular station.

The neighborhood smoothing for each station $(i)$ consisted of computing the "smoothed" mean interoccurrence interval through a weighted average of the station interval and the four nearest station intervals. Record length, as measured by the total number of days, provided the weights. Stations for which the $\mathrm{p}$-value of the mean interoccurrence interval was less than or equal to .01 were not used in the weightedaverage computations. For example, if p-values for two of the five stations (four neighbors plus the $i$ th station) were less than or equal to .01, then only three stations were used in the averaging. The benefit of using stations with p-values greater than .01 is that stations for which interoccurrence intervals do not (with 99-percent or greater probability) follow a homogenous Poisson process are objectively removed from analysis. The critical p-value of .01 was arbitrarily chosen. This significance level eliminates about 3 to 14 percent of the stations for depth thresholds of 0.25 inch and larger. Considering the large database used, eliminating these stations enhances the reliability of interoccurrence interval estimates for a homogenous Poisson model.

The regionalization of interoccurrence intervals included geostatistically based mapping of the smoothed values using the method of kriging (Isaaks and Srivastava, 1989). The kriging was performed using a Gaussian model of the semivariogram with a range of $1,212,900$ meters and a nugget that ranged from 1.59 days squared to 792,490 days squared for the 0.05 - and 3.0-inch thresholds, respectively. The neighborhood for the kriging operation used a minimum of two stations 
and a maximum of 13 stations in conjunction with a circular search method.

Initial interoccurrence interval maps were computer generated, and the resulting lines of equal interval overlain on the mapped values for the smoothed intervals. The computer-generated lines of equal interval were then manually smoothed and variable intervals retained to assist in interpolation near the boundaries of Texas. The smoothed lines of equal interval subsequently were overlain on the mapped site-specific interoccurrence intervals (point values), and visual confirmation of the lines was made. Finally, diagnostic statistics of the maps, which are presented in the section "Statewide Interoccurrence Interval Maps," were computed.

\section{INTEROCCURRENCE INTERVALS FOR SELECTED DAILY PRECIPITATION THRESHOLDS IN TEXAS}

\section{Statewide Interoccurrence Interval Maps}

Maps depicting the spatial variability of the interoccurrence interval for the 10 selected daily precipitation thresholds in Texas are shown in figures 4-13. Specific geographic locations are referred to in this report; the locations are identified only in figure 4. Tick marks of latitude and longitude in increments of 2 degrees are provided to facilitate adapting the maps for digital representation. On all the maps, a general pattern of increasing interoccurrence interval toward the western parts of the State is observed. The contours in the eastern one-half of the State have a pronounced north-south direction. These two characteristics of the maps are consistent with the patterns of mean annual precipitation in Texas. Mean annual precipitation in Texas decreases east to west and is relatively constant with north-south direction (Bomar, 1995).

The accuracy of the maps west of the Pecos River is less relative to the accuracy east of the Pecos River for reasons discussed in the section "Influence of Elevation on Interoccurrence Intervals West of the Pecos River."

Other observations are applicable to all the maps (figs. 4-13). One observation is that interoccurrence intervals in the Hill Country (fig. 4) generally are smaller than intervals in the same longitude range in the rest of the State. Another observation is that the lines of equal interval are approximately perpendicular to the coastline of the Gulf of Mexico. For each of these two observations the line patterns are similar to those of Asquith (1998, figs. 10-23). Details and observations about selected maps follow.

On the map depicting the interoccurrence interval for a 0.05 -inch and greater daily precipitation threshold (fig. 4), interoccurrence interval decreases substantially in the Davis Mountains. Reliable intervals for the Guadalupe and Chisos Mountains are problematic. Precipitation potential generally increases (smaller interoccurrence intervals) over large mountains because of the orographic effect.

On the map depicting the interoccurrence interval for a 0.10 -inch and greater daily precipitation threshold (fig. 5), interoccurrence interval decreases substantially in the Chisos, Davis, and Guadalupe Mountains. Reliable intervals for all three mountain ranges are difficult to develop. Decreases in interoccurrence intervals associated with the mountains also are characteristic of other daily precipitation thresholds.

For the map depicting the interoccurrence interval for a 0.25 -inch and greater daily precipitation threshold (fig. 6), the 7.5-day line of equal interval just outside the northeastern corner of Texas was added to constrain interpolation.

For the map depicting the interoccurrence interval for a 0.50 -inch and greater daily precipitation threshold (fig. 7), the 33-day line of equal interval in the northwestern corner of Texas was added to constrain interpolation.

For the map depicting the interoccurrence interval for a 0.75 -inch and greater daily precipitation threshold (fig. 8), the 58-day line of equal interval in the northwestern corner of Texas was added to constrain interpolation.

A summary of statewide interoccurrence interval statistics and diagnostic statistics of the maps in figures 4-13 is listed in table 9 (at end of report). The statistics in columns 2 and 3 were computed using the interoccurrence interval for all available stations.

As the threshold increases, mean interoccurrence interval increases (table 9). The standard deviation of the interoccurrence intervals increases much more rapidly than the mean; this indicates a larger variation, change, or range in the interoccurrence interval across the State as the threshold increases.

A total of 1,306 stations are considered in this report; not all of these stations are available for the production of each threshold map because of removal of stations based on the p-values described in the "Approach" section. Also, the statewide number of 


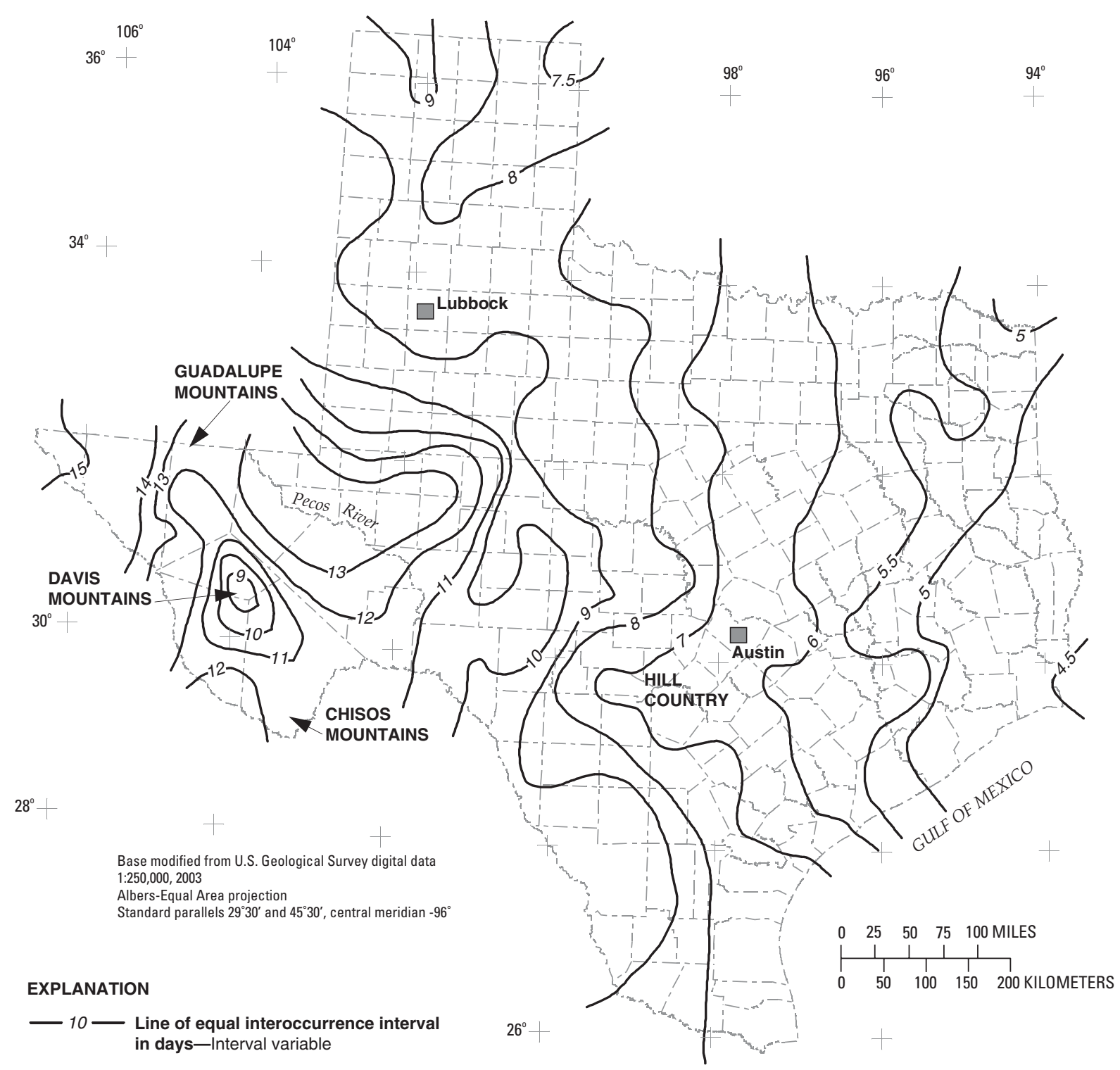

Figure 4. Interoccurrence interval for a 0.05 -inch and greater daily precipitation threshold for Texas.

stations with events for a given threshold decreases with increasing threshold. For example, there are only seven stations with no daily rainfall values greater than 0.05 inch $(1,306-1,299=7)$, but there are 163 stations with no daily rainfall greater than 3.0 inches $(1,306-1,143=163)$.

The number of $\mathrm{p}$-values less than or equal to .01 decreases substantially as the threshold increases. This indicates that as rainfall events are increasingly less frequent, a homogeneous Poisson model of the rainfall occurrence is increasingly more appropriate. This observation is expected because of the natural tendency for a rainy day to be followed by another rainy day-as the threshold becomes progressively smaller, progressively more events are likely to occur on consecutive days. The number of p-values less than or equal to .01 for the 0.05- and 0.10-inch thresholds are large enough that general applicability of a homogeneous Poisson model for these thresholds is questionable. For comparison, about 270 stations have p-values less than or equal to .001 for the 0.05 -inch threshold, compared to 406 stations with p-values less than or equal to .01 (table 9).

Two simple diagnostic statistics of the interoccurrence interval maps are reported in table 9 . The 


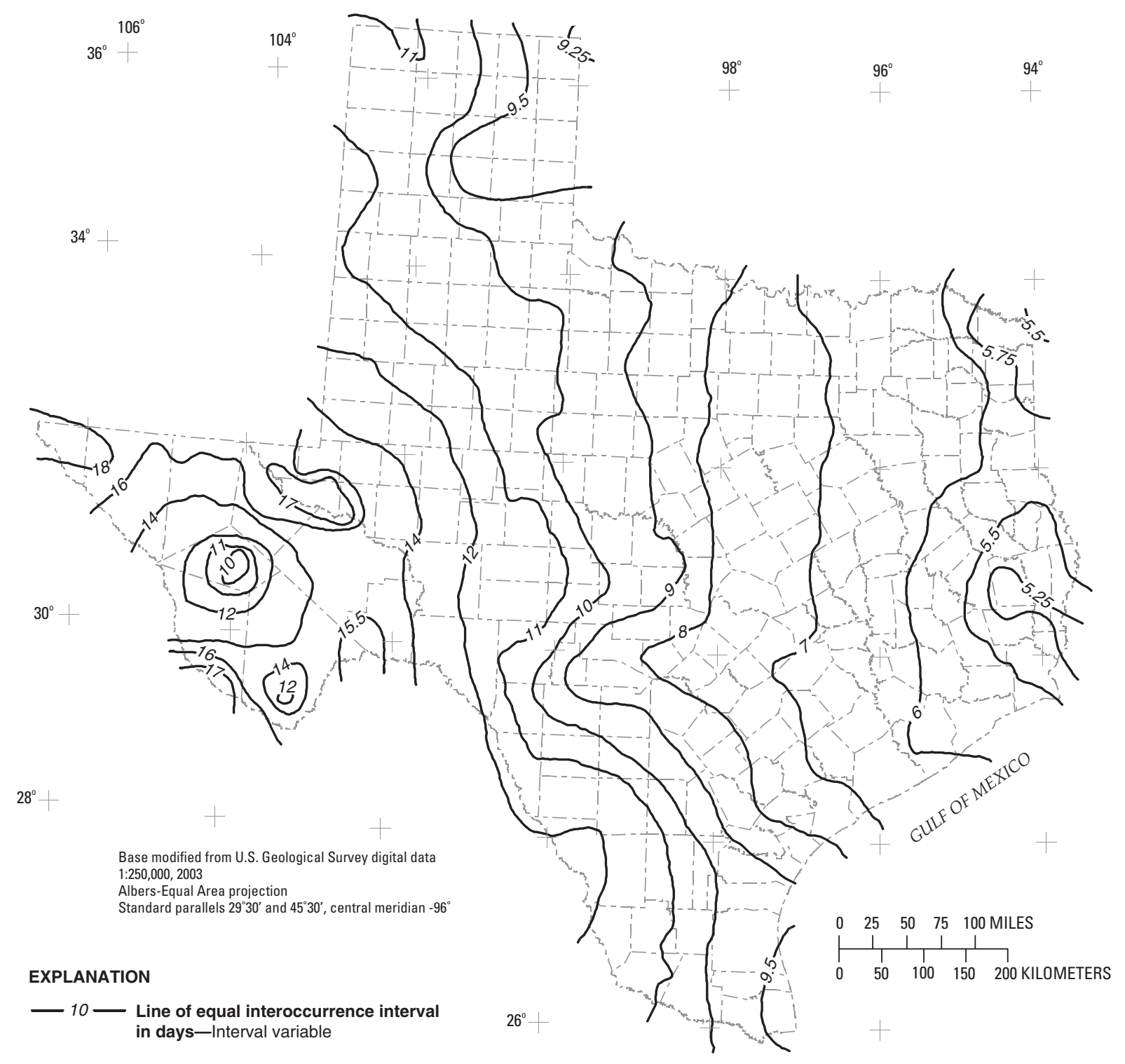

Figure 5. Interoccurrence interval for a 0.10 -inch and greater daily precipitation threshold for Texas.

statistics are the mean bias and the root-mean-square error (RMSE) of the interoccurrence interval map. The bias at the $i$ th station, "station bias," is computed by subtracting the site-specific interoccurrence interval at the station from the interval extracted from the map. The mean bias is the arithmetic average of the station biases for each threshold. The mean bias should be about zero for each map. The mean bias is a simple check on whether the geostatistical analysis and contouring functioned appropriately.

The RMSE error of a map is a more important diagnostic. The RMSE for each threshold is defined as

$$
R M S E=\sqrt{\frac{1}{n} \sum_{i=1}^{n}\left(I_{i}-M_{i}\right)^{2}},
$$

where

$I_{i}$ is the site-specific interoccurrence interval, in days;

$M_{i}$ is the mapped interoccurrence interval, in days; and

$n$ is the number of stations with site-specific interoccurrence intervals for a given threshold. 


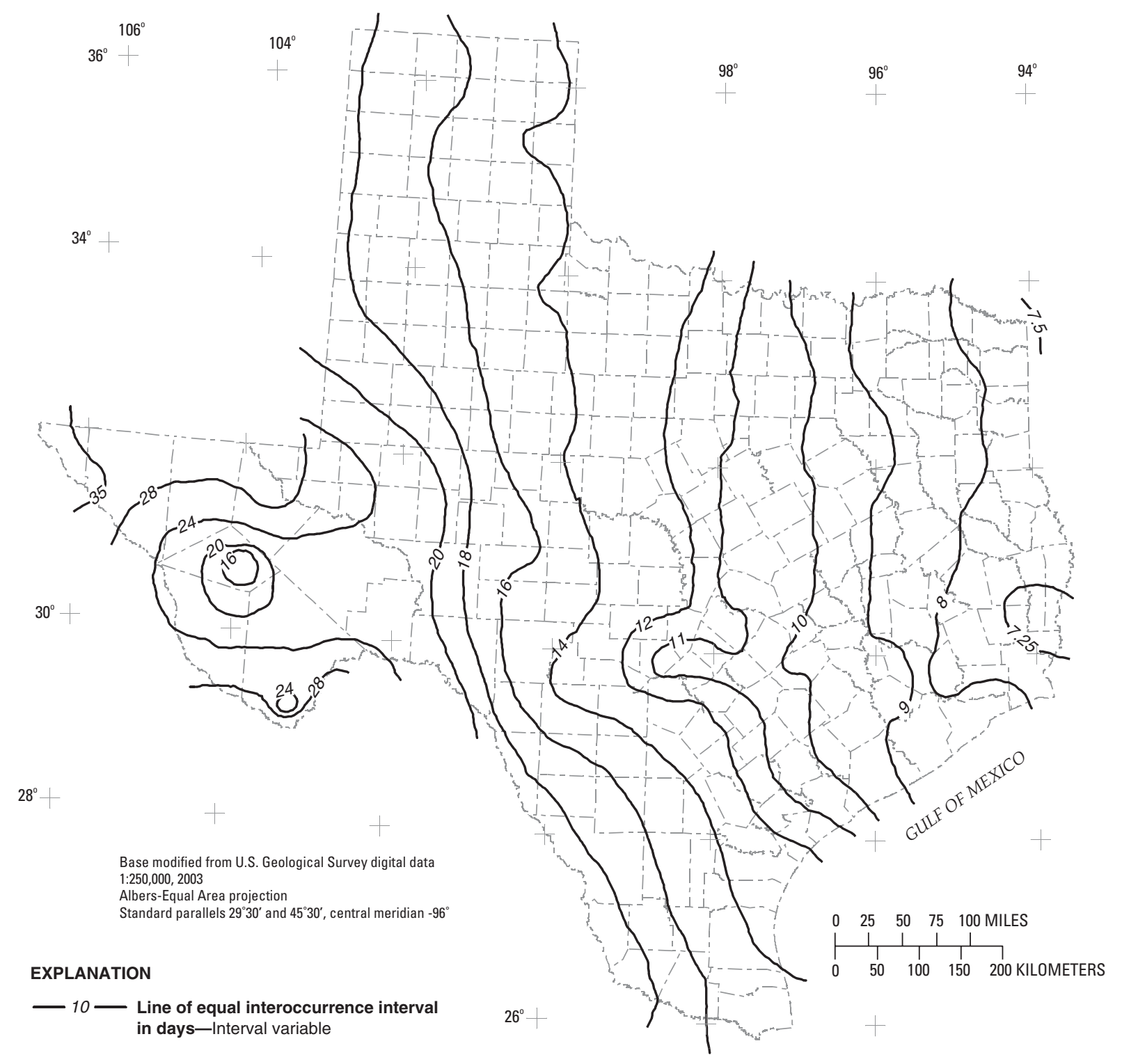

Figure 6. Interoccurrence interval for a 0.25 -inch and greater daily precipitation threshold for Texas.

The number of stations with events and hence an available site-specific interoccurrence interval differs for each threshold. The RMSE reflects how well the lines of equal interval match the site-specific intervals. The percent change from the statewide standard deviation of the interoccurrence intervals to the RMSE ranges from a magnitude minimum of (negative) -24 to a magnitude maximum of - 60 percent for the 0.05 - and 2.0inch thresholds, respectively. Because of the substantial negative percent change, the maps are considered more reliable estimators of the mean interoccurrence interval for most locations in Texas than the statewide mean value.

\section{Influence of Elevation on Interoccurrence Intervals West of the Pecos River}

Because of the orographic influence of elevation on precipitation potential in mountainous regions, an assessment of the relation between interoccurrence interval and elevation west of the Pecos River (fig. 4) is informative. For each of the stations west of the Pecos River, the site-specific mean interoccurrence interval for each threshold is graphed with the corresponding elevation of the station in figure 14 . All stations are represented on the figure without regard to $\mathrm{p}$-value. The interoccurrence interval decreases with elevation up 


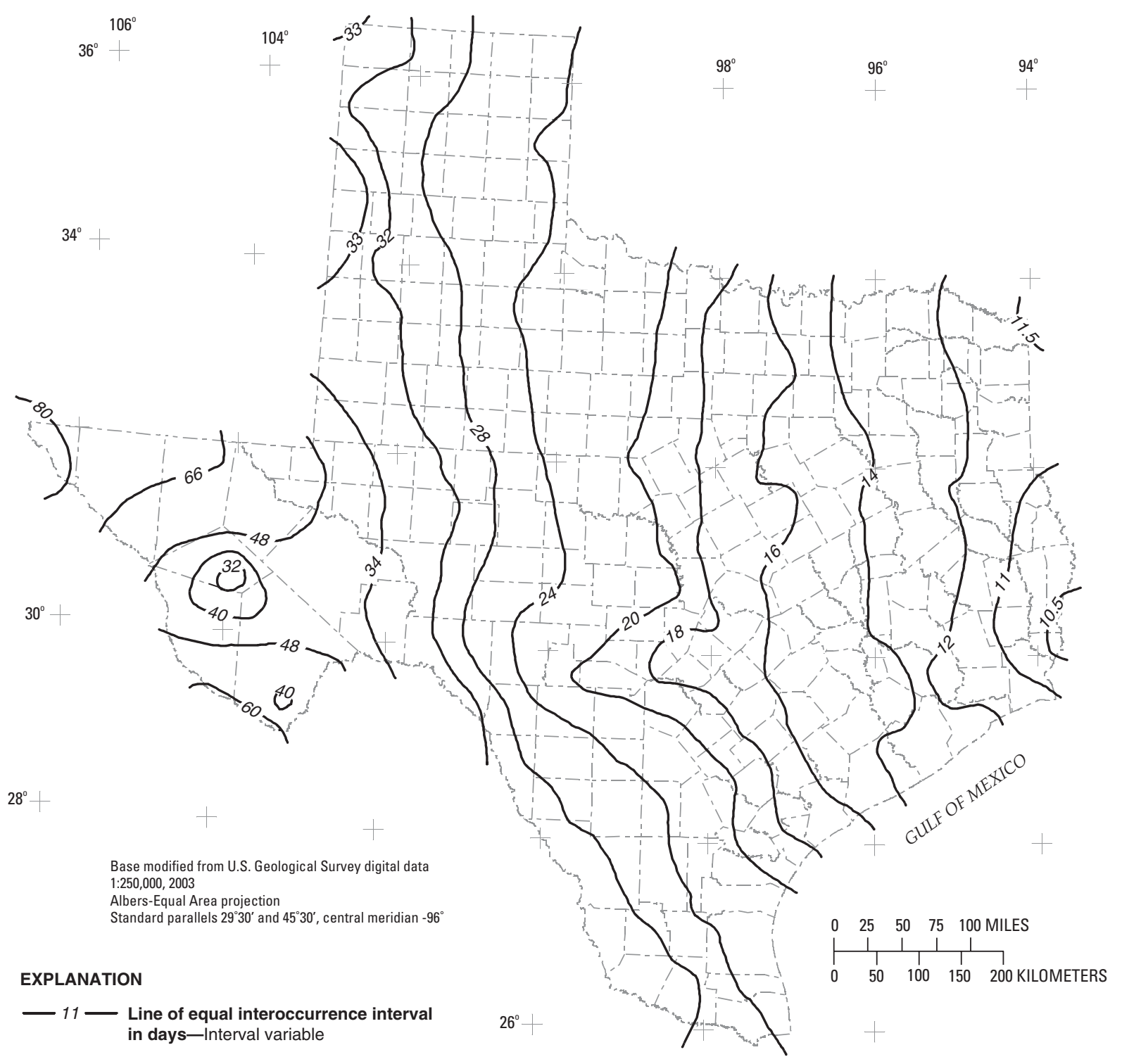

Figure 7. Interoccurrence interval for a 0.50 -inch and greater daily precipitation threshold for Texas.

to about the 1.0-inch threshold. For thresholds larger than 1.0 inch, little or no relation between the interoccurrence interval and elevation is evident for reasons that are not apparent to the authors. However, it is probable and expected that the interoccurrence intervals are relatively smaller for the highest stations (those above 5,000 feet). Because of the influence of elevation, the contours of interoccurrence intervals west of the Pecos River-specifically in and west of the Chisos, Guadalupe, and Davis Mountains (fig. 4) —are less reliable. The reliability of the lines of equal interval diminishes also with increasing threshold because the interoccurrence intervals become very large relative to the typical record lengths at the stations west of the Pecos River. Furthermore, many of those stations have no recorded events for the largest thresholds. This is evident in the fewer number of points plotted in figure $14 \mathrm{j}$ compared to figure $14 \mathrm{a}-\mathrm{f}$. Concerns over the reliability of interoccurrence interval contours in far west Texas is a partial motivation for including the sitespecific interoccurrence intervals in tables 3-7. Readers can choose to investigate specific locations in greater detail. 


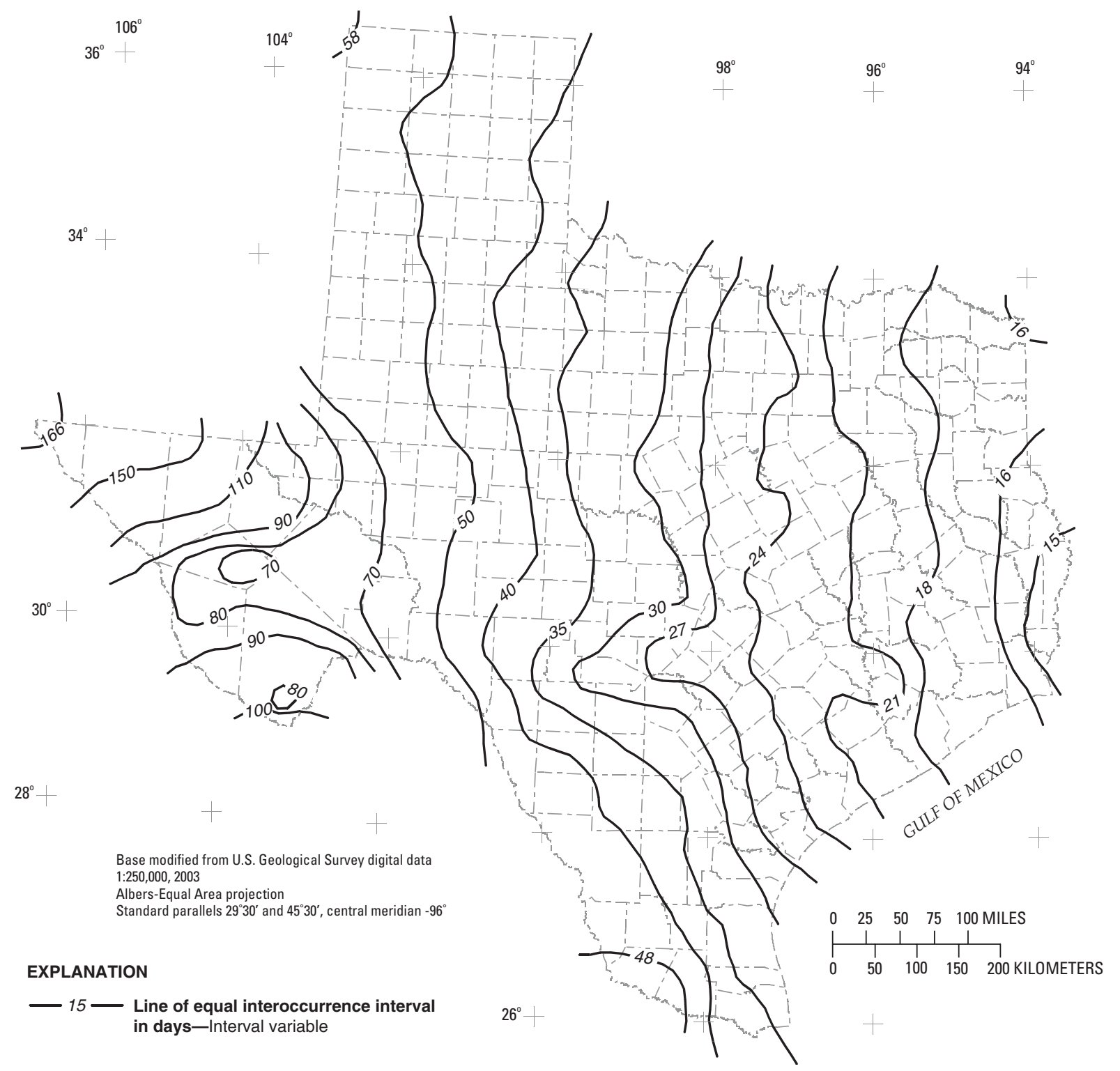

Figure 8. Interoccurrence interval for a 0.75 -inch and greater daily precipitation threshold for Texas.

\section{Intra-Annual Variations in Interoccurrence Intervals}

The interoccurrence interval maps presented in the previous section generally are reliable estimators of the parameter for a homogeneous Poisson model of daily precipitation for a given threshold. However, precipitation characteristics in Texas vary seasonally (see Bomar, 1995). The intra-annual variation documented in this section is derived by grouping the data for the entire State. Geographic or spatial differences in intraannual variations of interoccurrence intervals are not represented, although differences likely exist in a region as large as Texas.

Summaries of the intra-annual variability of precipitation occurrence for each of the 10 thresholds are listed in tables 10-19 (at end of report). Complementing the tables are boxplots showing the distribution of interoccurrence ratios for each month in figures 15-24. The intra-annual variability is reflected in a mean "interoccurrence ratio" for each month. The individual monthly ratios are computed by dividing each interoccurrence interval by the mean interoccurrence interval for the station. Sample sizes are shown in figures 15-24; 


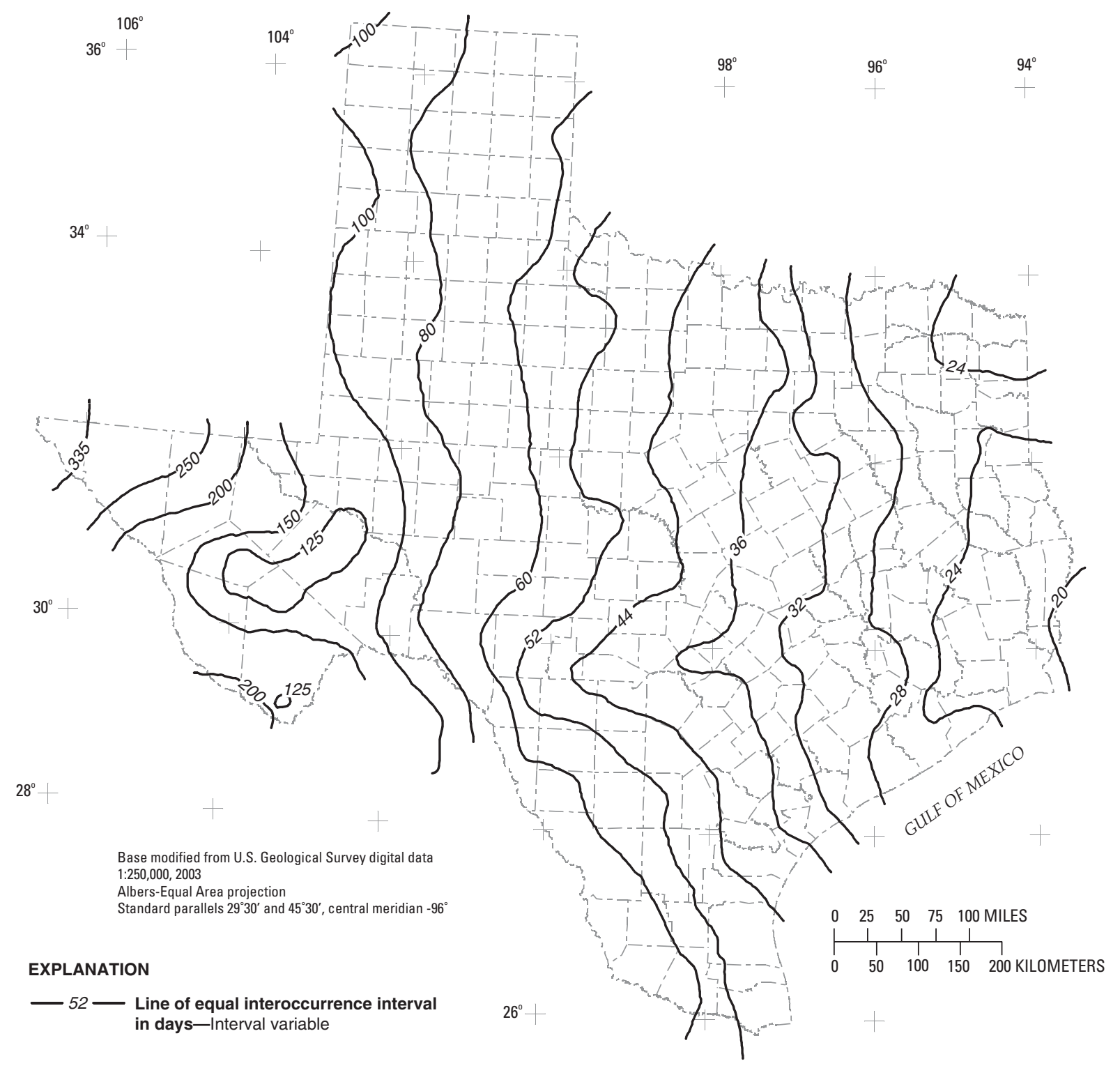

Figure 9. Interoccurrence interval for a 1.0-inch and greater daily precipitation threshold for Texas.

however, the sample sizes do not match the "Statewide no. of stations with events" column in table 9 because not all stations have an event in all months. The mean monthly interoccurrence ratio (column 2) is computed as the average of all the individual monthly ratios for all the stations; likewise, the median monthly ratio (column 3 ) is the median of all the monthly ratios for all the stations.

Interpretation of the ratios is as follows: When the ratio is greater than unity for a given month, the events occurring in that month are less frequent (less likely, longer interval) than the mean interoccurrence interval.
Likewise, when the ratio is less than unity, the events are more frequent (more likely, shorter interval) than the mean interoccurrence interval.

Finally, for each sequence of mean and median monthly ratios an "adjusted ratio" (columns 4 and 5) was computed with an adjustment or offset to ensure that the arithmetic average of all 12 ratios is unity. The adjusted ratios are preferable because the arithmetic average of each of the adjusted ratios multiplied by a specific mean interoccurrence interval recovers the mean interoccurrence interval. Bias is mitigated. 


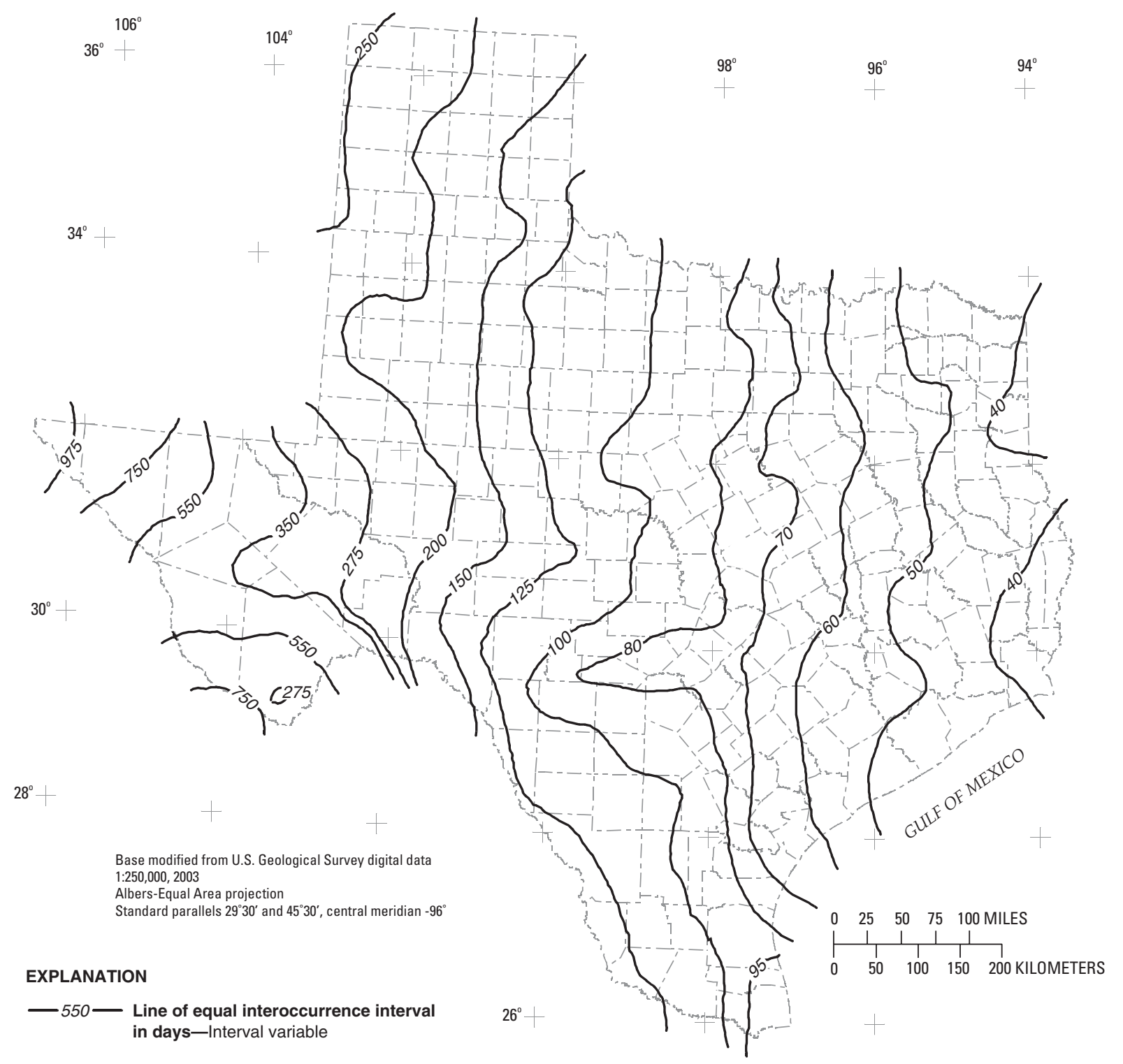

Figure 10. Interoccurrence interval for a 1.5-inch and greater daily precipitation threshold for Texas.

It is informative to graphically illustrate the adjusted interoccurrence ratios listed in tables 10-19. The relation between month and the mean and median adjusted interoccurrence ratios from tables 10 and 11 for the 0.05 - and 0.10 -inch thresholds are graphed in figure 25. The graph shows two distinct troughs in the mean and median ratios. The first trough occurs in May and June and the second in September. The troughs indicate smaller interoccurrence intervals between rainfall events defined by the threshold-months in the rainy seasons. The change in the mean monthly interoc- currence ratio from April to May (from about 1.07 to about 0.79 ) is substantial. To clarify, the April interoccurrence interval is about 107 percent of the mean interoccurrence interval for a location; whereas the May interoccurrence interval is about 79 percent of the mean interoccurrence interval for a location.

The relations between month and adjusted mean interoccurrence ratio for the 0.25-, 0.50-, 0.75-, 1.0-, 1.5-, 2.0-, 2.5-, and 3.0-inch thresholds (tables 12-19) are shown in figure 26 , and the relations between month and adjusted median interoccurrence ratio are 


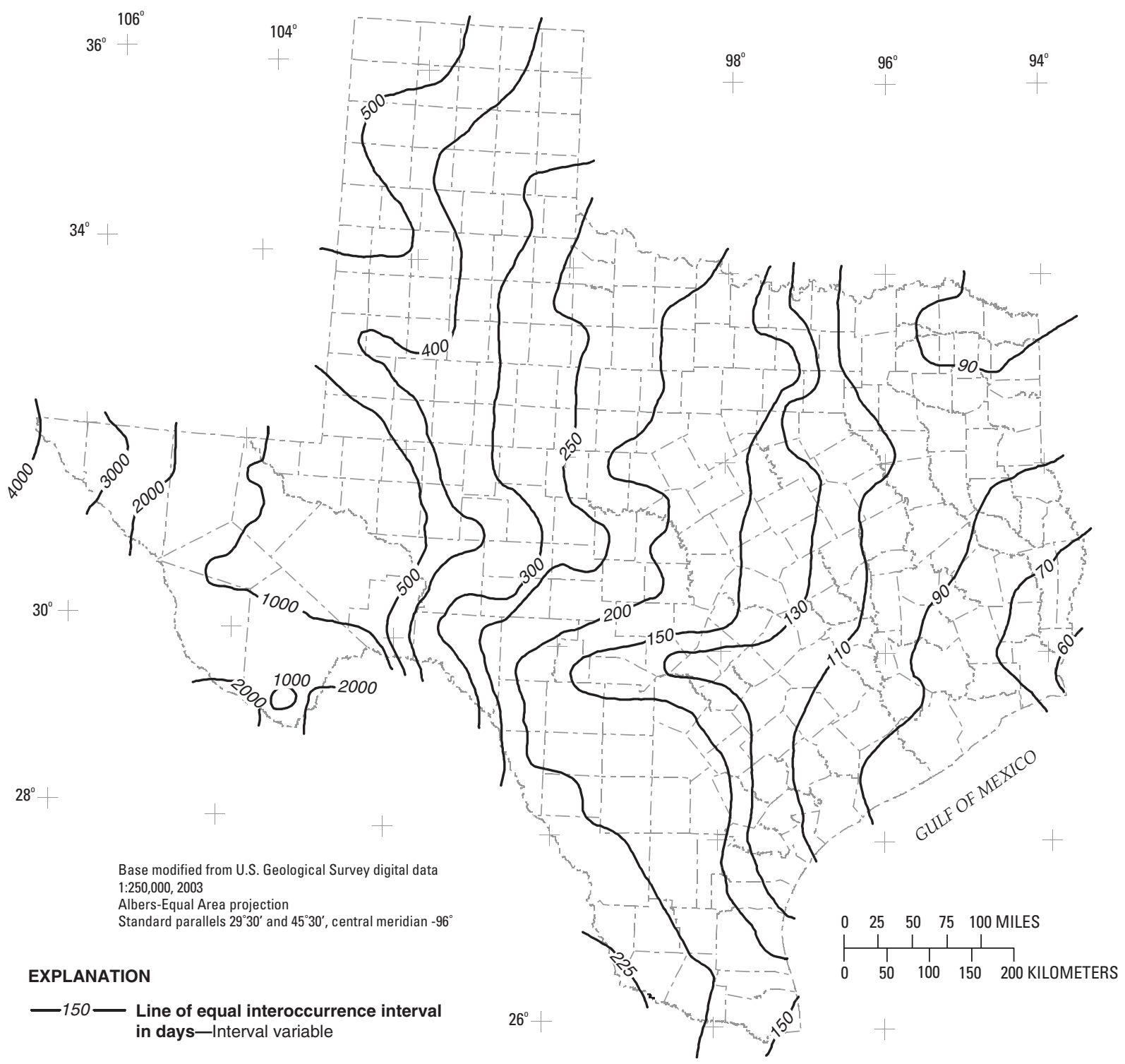

Figure 11. Interoccurrence interval for a 2.0-inch and greater daily precipitation threshold for Texas.

shown in figure 27 . The ratios in each figure mimic the trends established in figure 25 for the 0.05 - and 0.10 -inch thresholds. However, some distinctive differences are visible. One trough is visible between about May-July, and another trough is visible in September and October. For thresholds less than about 1.0 inch the May-July trough is deepest; for larger thresholds the ratios are closer to unity. The ratios peak in January-March for the 1.0-inch and less thresholds, but peak only in March for the larger thresholds. The graphs indicate that considerable and systematic intra-annual variations in the interoccurrence ratios exist.

\section{EXAMPLE APPLICATIONS}

The following examples illustrate the application of the site-specific and regional estimates of the interoccurrence intervals in this report. The underlying assumption for the applications illustrated in this section is that the occurrence of precipitation follows a Poisson process. 


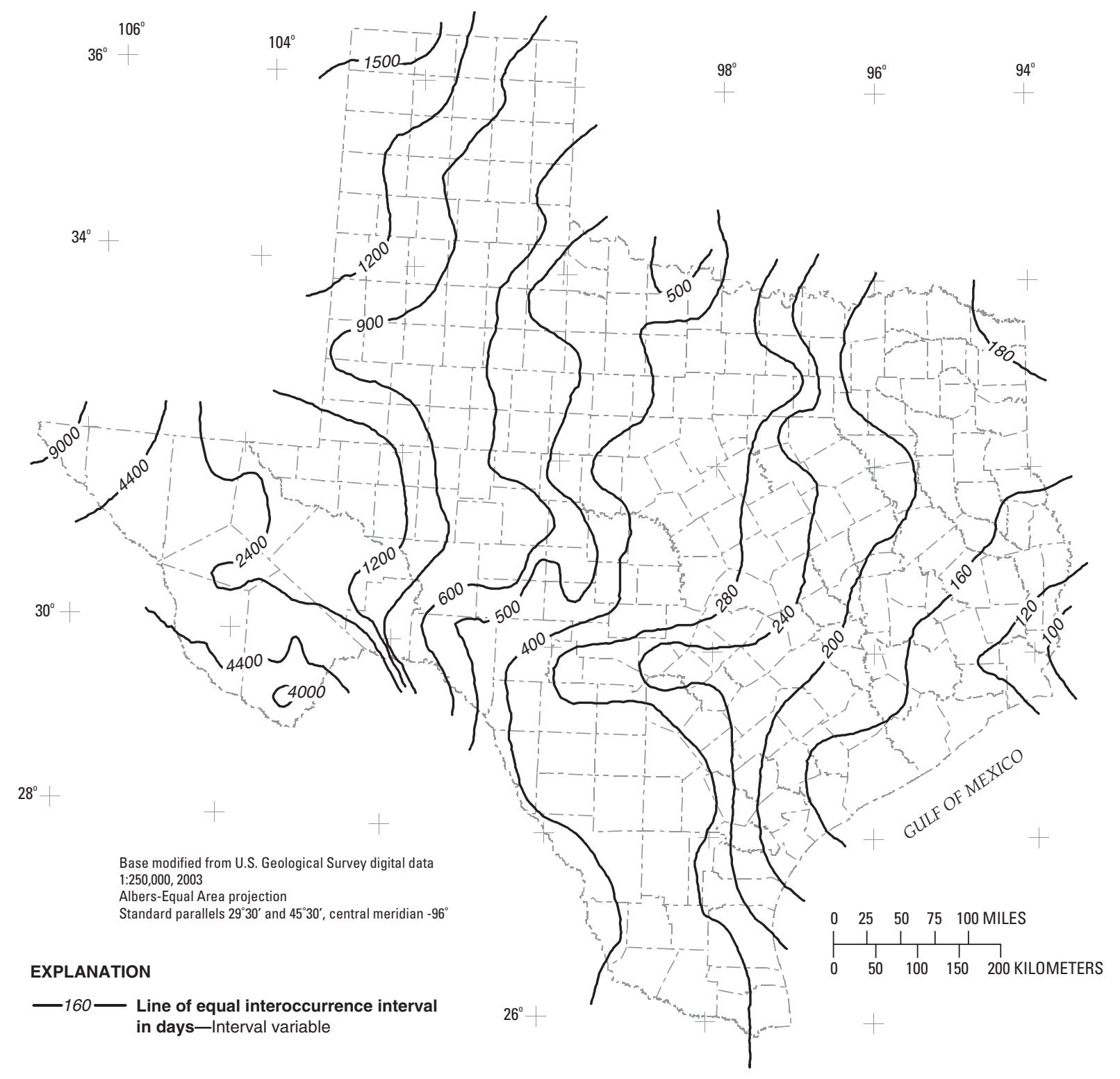

Figure 12. Interoccurrence interval for a 2.5 -inch and greater daily precipitation threshold for Texas.

\section{Site-Specific Median 6-Month Daily Precipitation Threshold}

In hydrologic design practice, it is sometimes useful to set design criteria for occurrences of precipitation that happen at subannual intervals. For example, the median 6-month storm might be required for a design. The 6-month storm can be defined in terms of an interoccurrence interval. Specifically, consider the interoccurrence interval distribution from equation 1. Six months has a day length ( $x$ ) of 182.5 days (365/2), and the median interoccurrence has $F=0.5$. Substituting these values into equation 1 yields

$$
\begin{gathered}
0.5=1-e^{-182.5 \lambda}, \\
\lambda=0.003798, \text { and } \\
1 / \lambda=\Lambda=263 \text { days. }
\end{gathered}
$$

Hence, the median 6-month daily precipitation has a mean interoccurrence interval of about 263 days. 


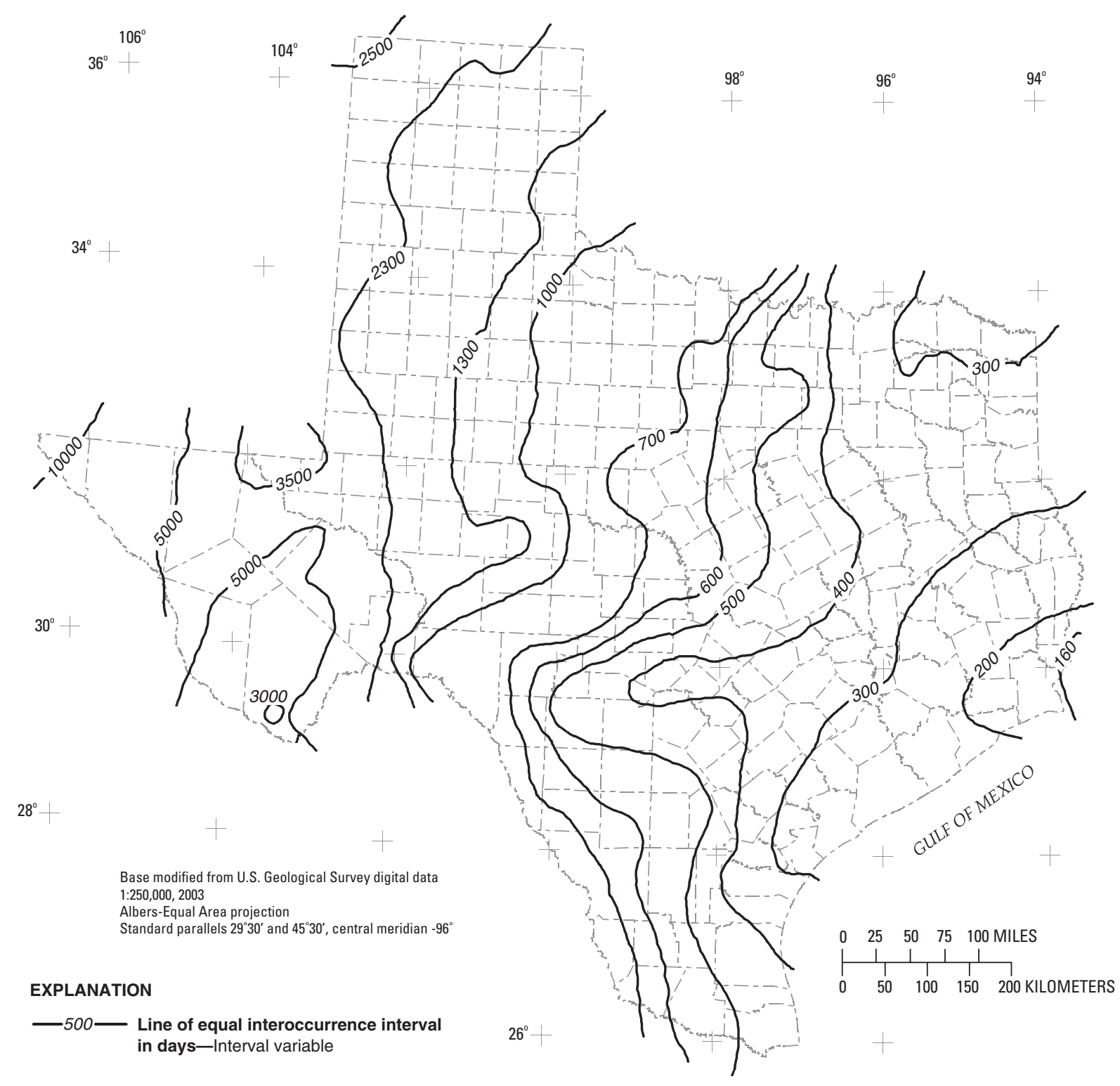

Figure 13. Interoccurrence interval for a 3.0-inch and greater daily precipitation threshold for Texas.

Now for a specific location, it is necessary to estimate the daily precipitation threshold for which the interoccurrence interval is 263 days. This is achieved using either site-specific information or regionalized estimates of the interoccurrence interval for a given location. For this example, site-specific information is used.

Consider the site-specific interoccurrence intervals for station 428 Austin Camp Mabry (sequence no. 46). This is a long-term station with about 72 years of record (table 1). The interoccurrence intervals from tables 4-7 for the selected thresholds are listed in figure 28 . The interoccurrence intervals for daily precipitation thresholds of 0.05 and 0.10 inch (table 3 ) are not used. Also shown in figure 28 is the relation between the daily precipitation threshold and the mean interoccurrence interval; the relation is nearly linear on a semi-log scale. Superimposed on the figure is a dashed horizontal line for the mean interoccurrence interval of 263 days. The dashed line indicates that a precipitation threshold depth with an interoccurrence interval of 263 days is about 2.56 inches. The site-specific median 6-month daily precipitation for Austin Camp Mabry thus is equal to or greater than 2.56 inches. 

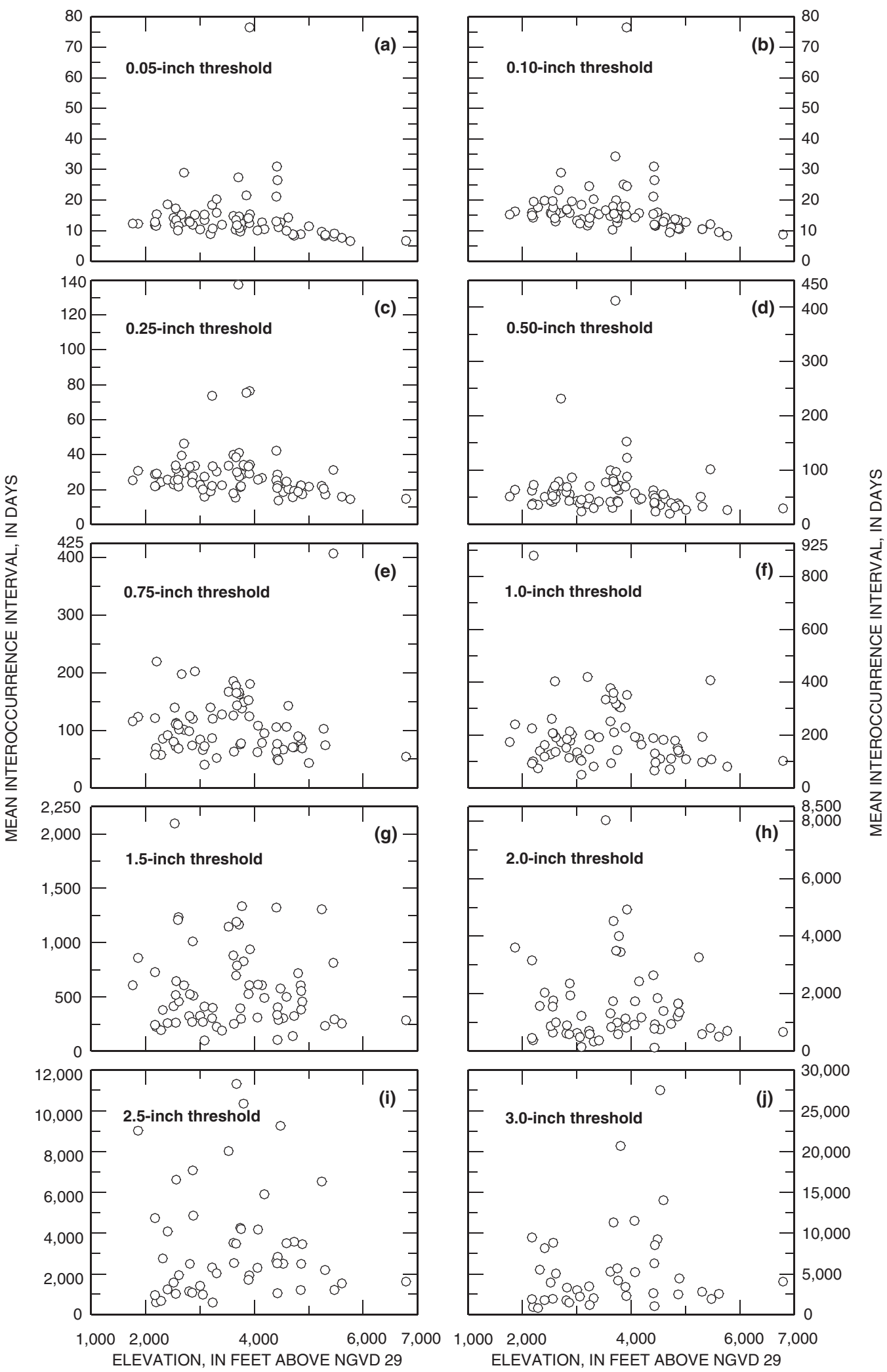

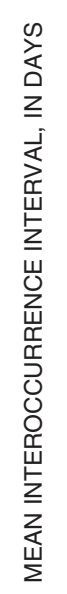

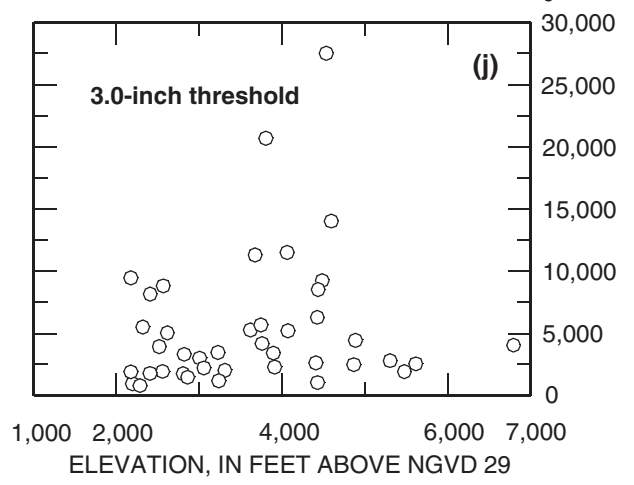

Figure 14. Relation between interoccurrence interval and elevation west of the Pecos River, west Texas. 


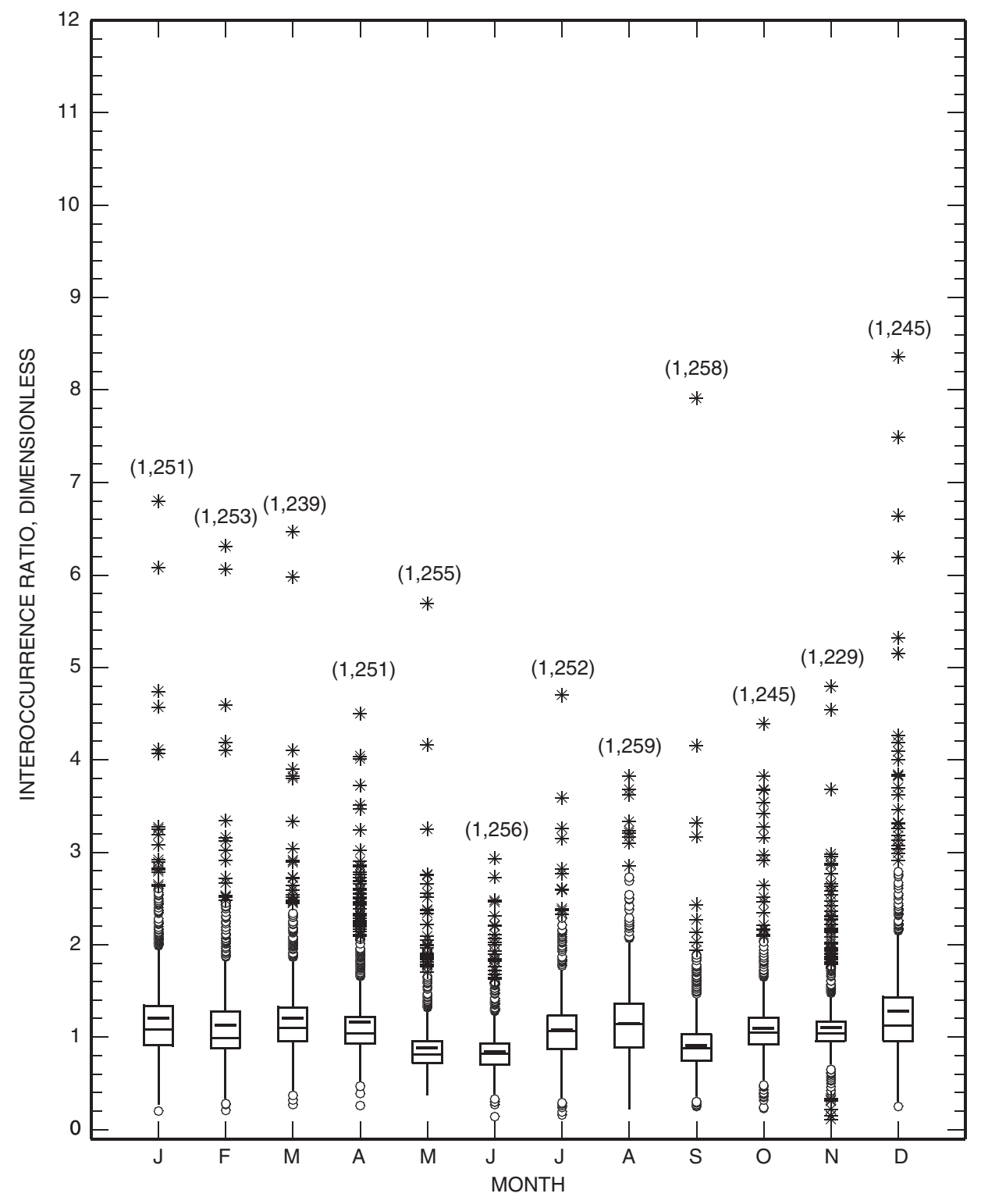

EXPLANATION

$(1,252)$ Sample size

* Data value greater than 3.0 times the IQR outside the box

- Data value 1.5 to 3.0 times the IQR outside the box

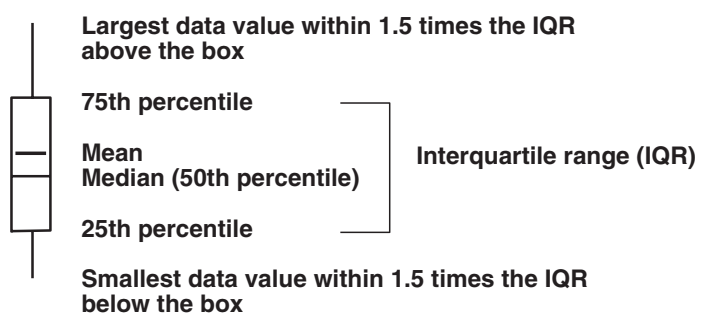

Figure 15. Distribution of ratio of interoccurrence interval for month to mean interoccurrence interval for period of record at station for a 0.05 -inch and greater daily precipitation threshold for Texas. 


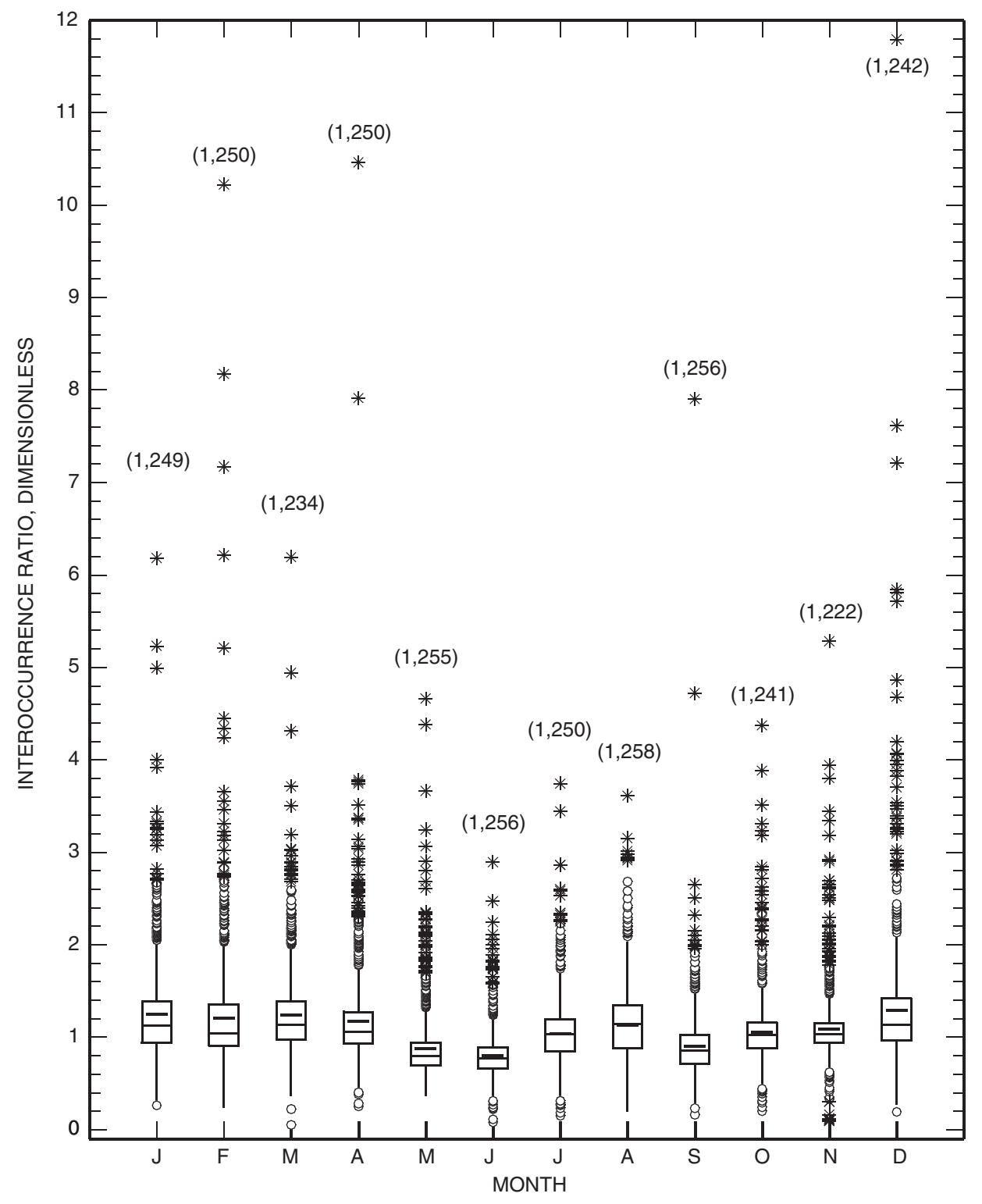

EXPLANATION

$(1,255)$ Sample size

* Data value greater than 3.0 times the IQR outside the box

- Data value 1.5 to 3.0 times the IQR outside the box

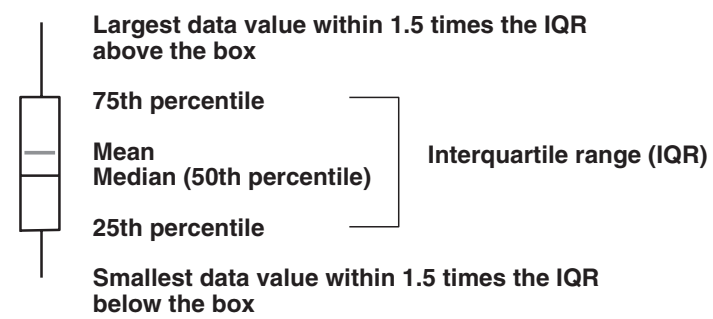

Figure 16. Distribution of ratio of interoccurrence interval for month to mean interoccurrence interval for period of record at station for a 0.10 -inch and greater daily precipitation threshold for Texas. 


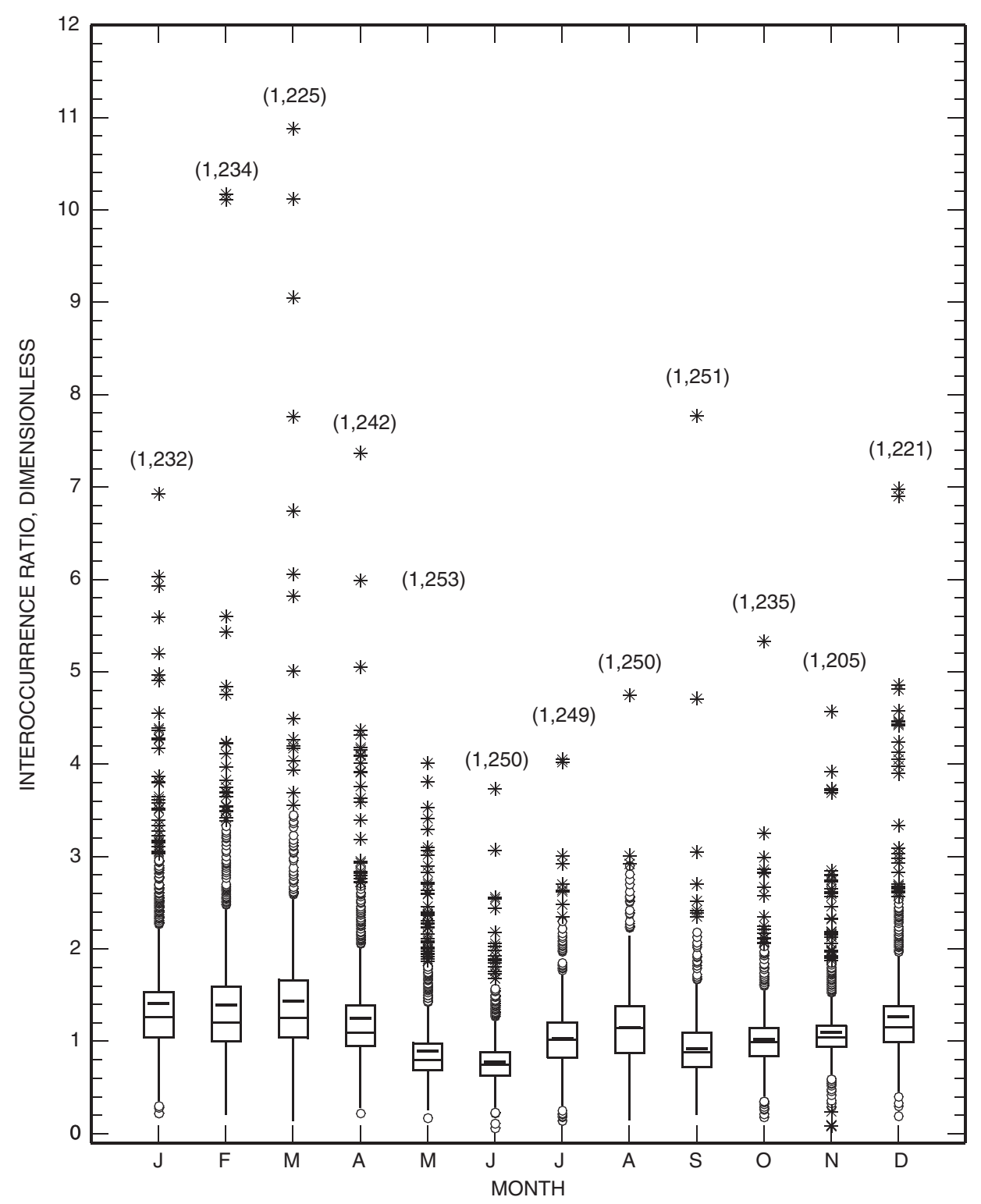

EXPLANATION

$(1,205)$ Sample size

* Data value greater than 3.0 times the IQR outside the box

- Data value 1.5 to 3.0 times the IQR outside the box

Largest data value within 1.5 times the IQR above the box

$\square$

75th percentile

Mean Median (50th percentile)

Interquartile range (IQR)

25th percentile

Smallest data value within 1.5 times the IQR below the box

Figure 17. Distribution of ratio of interoccurrence interval for month to mean interoccurrence interval for period of record at station for a 0.25 -inch and greater daily precipitation threshold for Texas. 


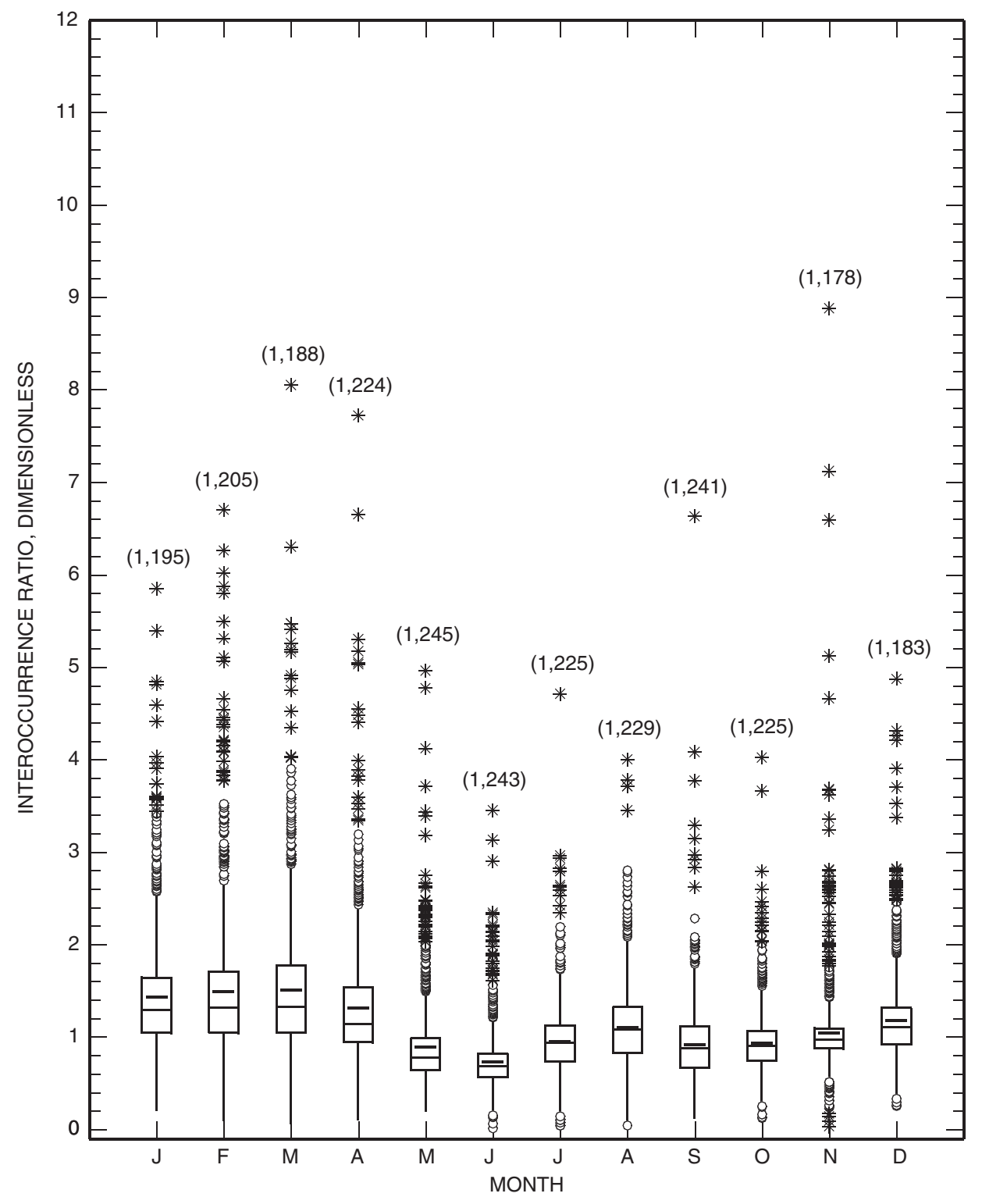

EXPLANATION

$(1,229)$ Sample size

* Data value greater than 3.0 times the IQR outside the box

- Data value 1.5 to 3.0 times the IQR outside the box

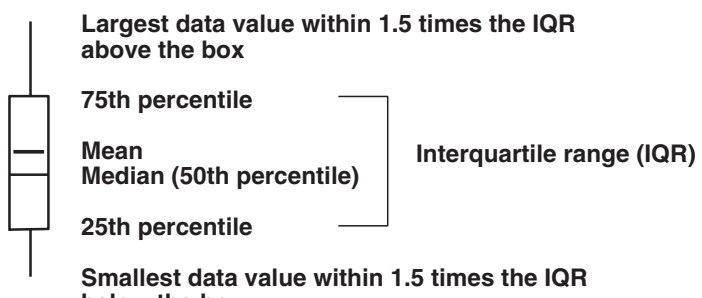

below the box

Figure 18. Distribution of ratio of interoccurrence interval for month to mean interoccurrence interval for period of record at station for a 0.50 -inch and greater daily precipitation threshold for Texas. 


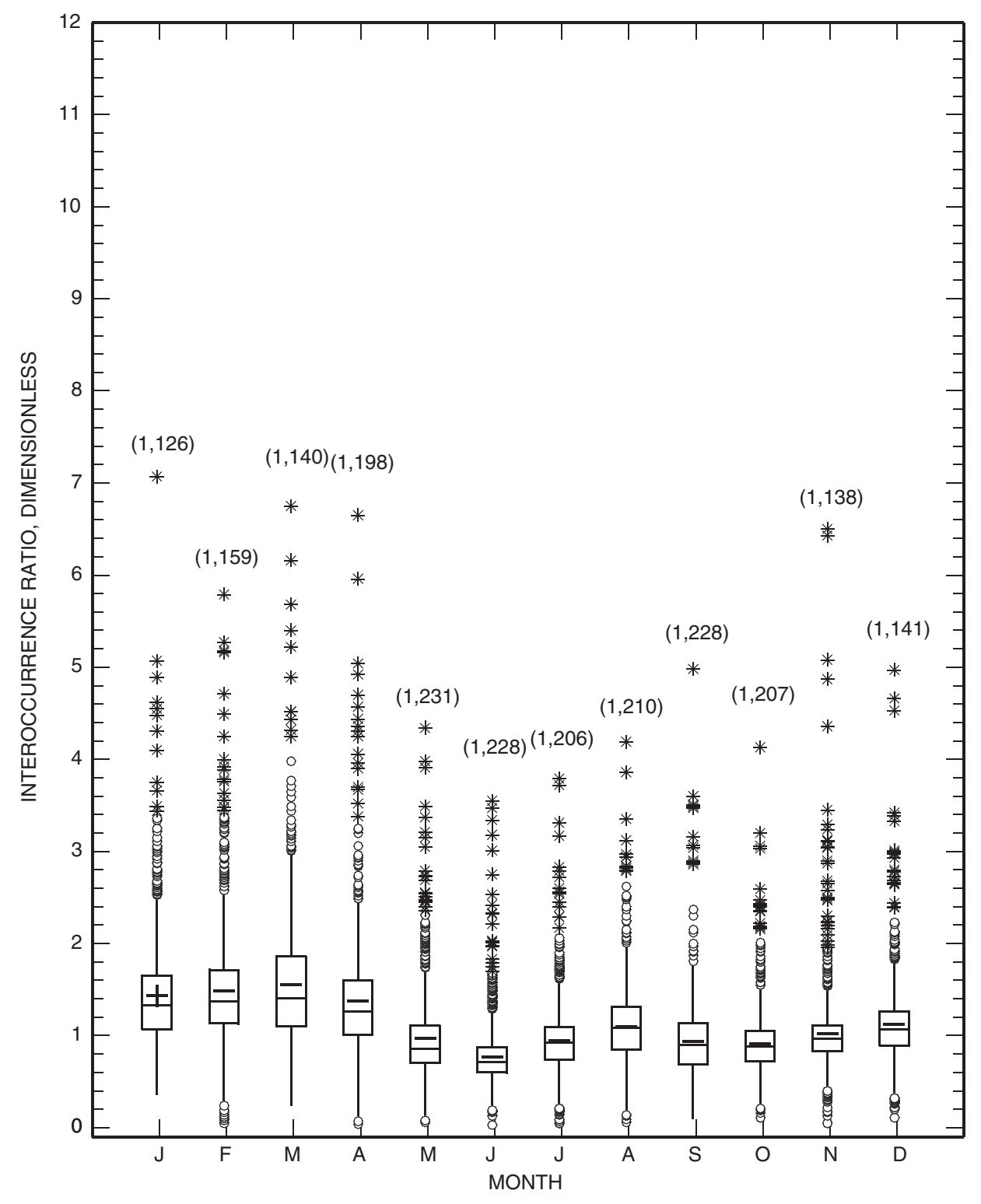

EXPLANATION

$(1,207)$ Sample size

* Data value greater than 3.0 times the IQR outside the box

- Data value 1.5 to 3.0 times the IQR outside the box

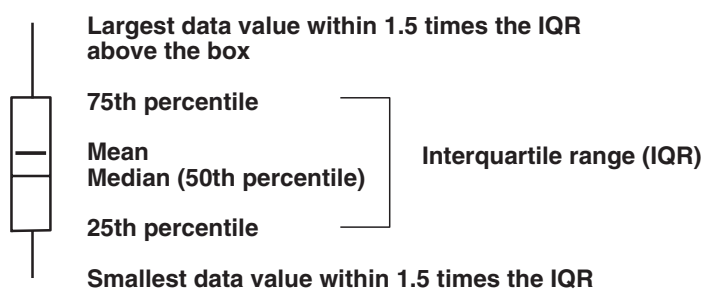

Smallest data value within 1.5 times the IQR below the box

Figure 19. Distribution of ratio of interoccurrence interval for month to mean interoccurrence interval for period of record at station for a 0.75 -inch and greater daily precipitation threshold for Texas. 


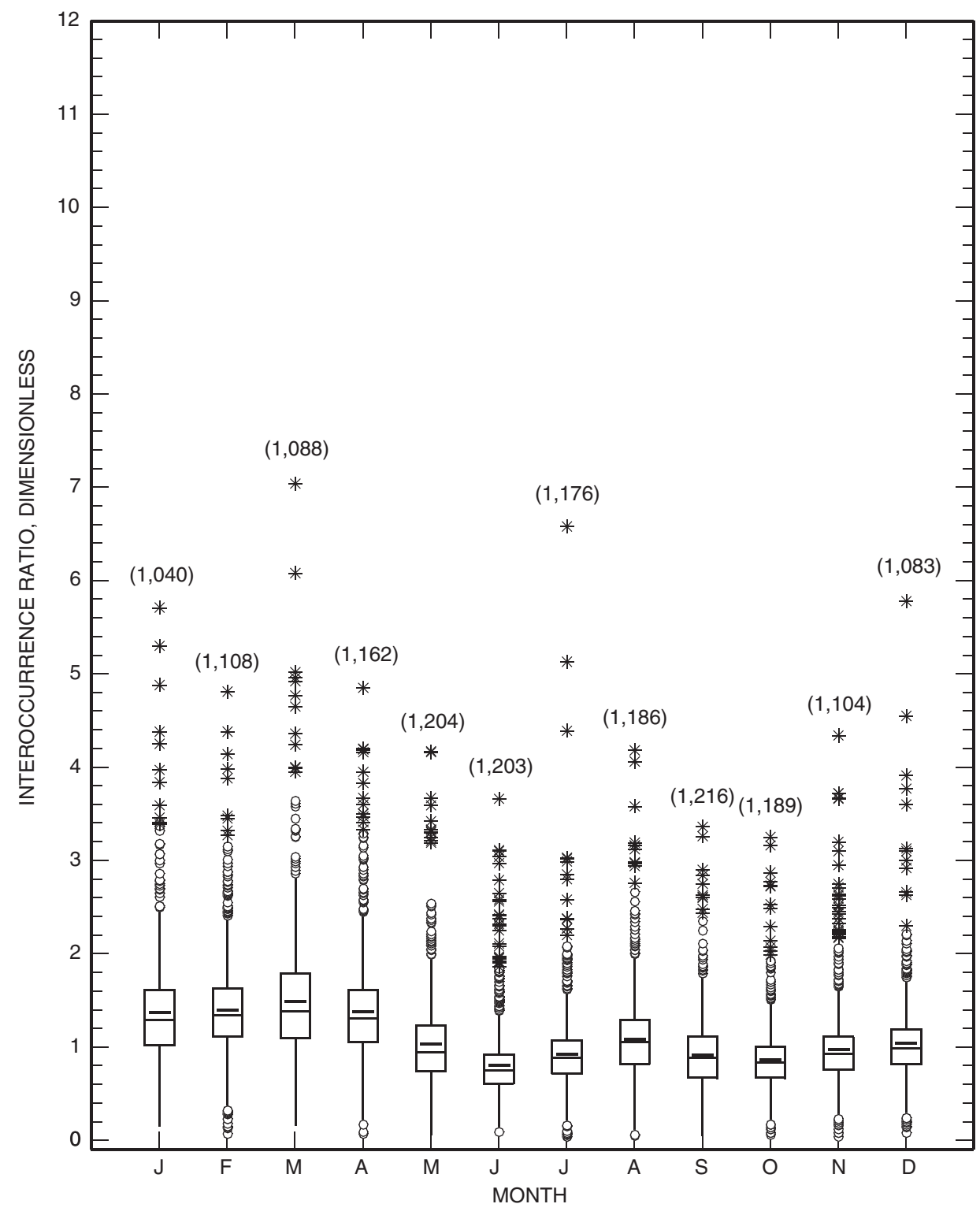

EXPLANATION

$(1,216)$ Sample size

* Data value greater than 3.0 times the IQR outside the box

- Data value 1.5 to 3.0 times the IQR outside the box

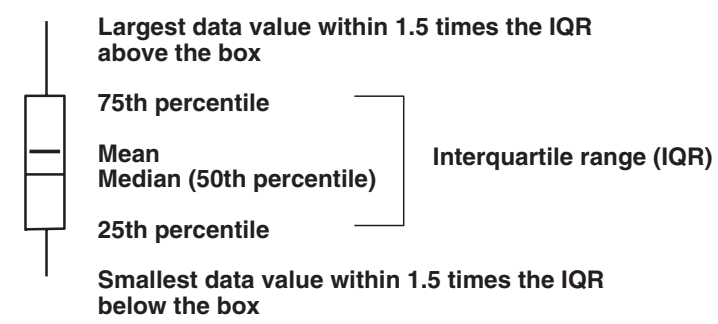

Figure 20. Distribution of ratio of interoccurrence interval for month to mean interoccurrence interval for period of record at station for a 1.0-inch and greater daily precipitation threshold for Texas. 


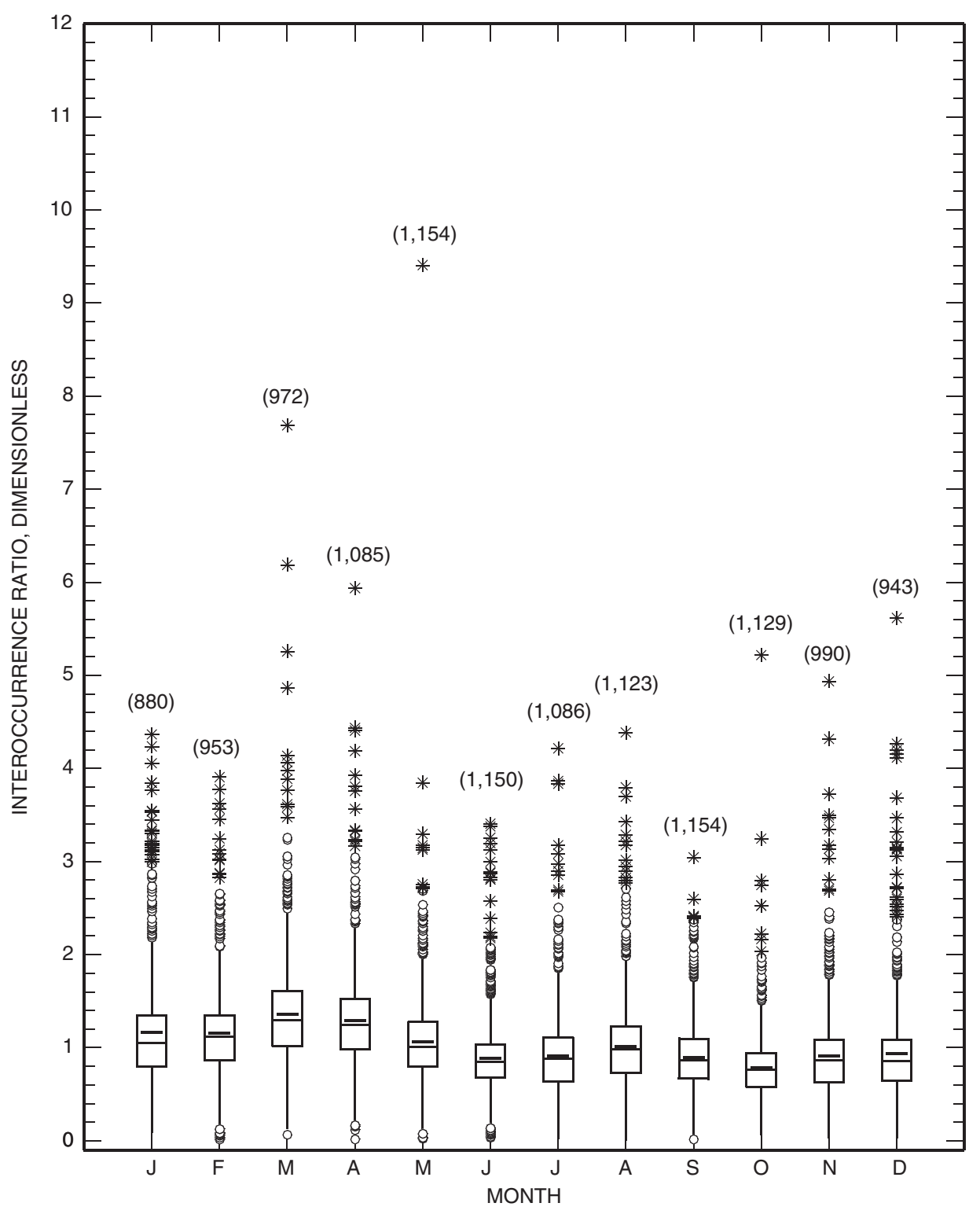

EXPLANATION

\section{$(1,129)$ Sample size}

* Data value greater than 3.0 times the IQR outside the box

- Data value 1.5 to 3.0 times the IQR outside the box

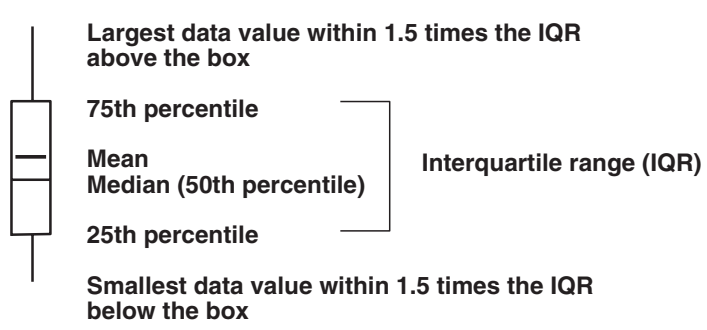

Figure 21. Distribution of ratio of interoccurrence interval for month to mean interoccurrence interval for period of record at station for a 1.5-inch and greater daily precipitation threshold for Texas. 


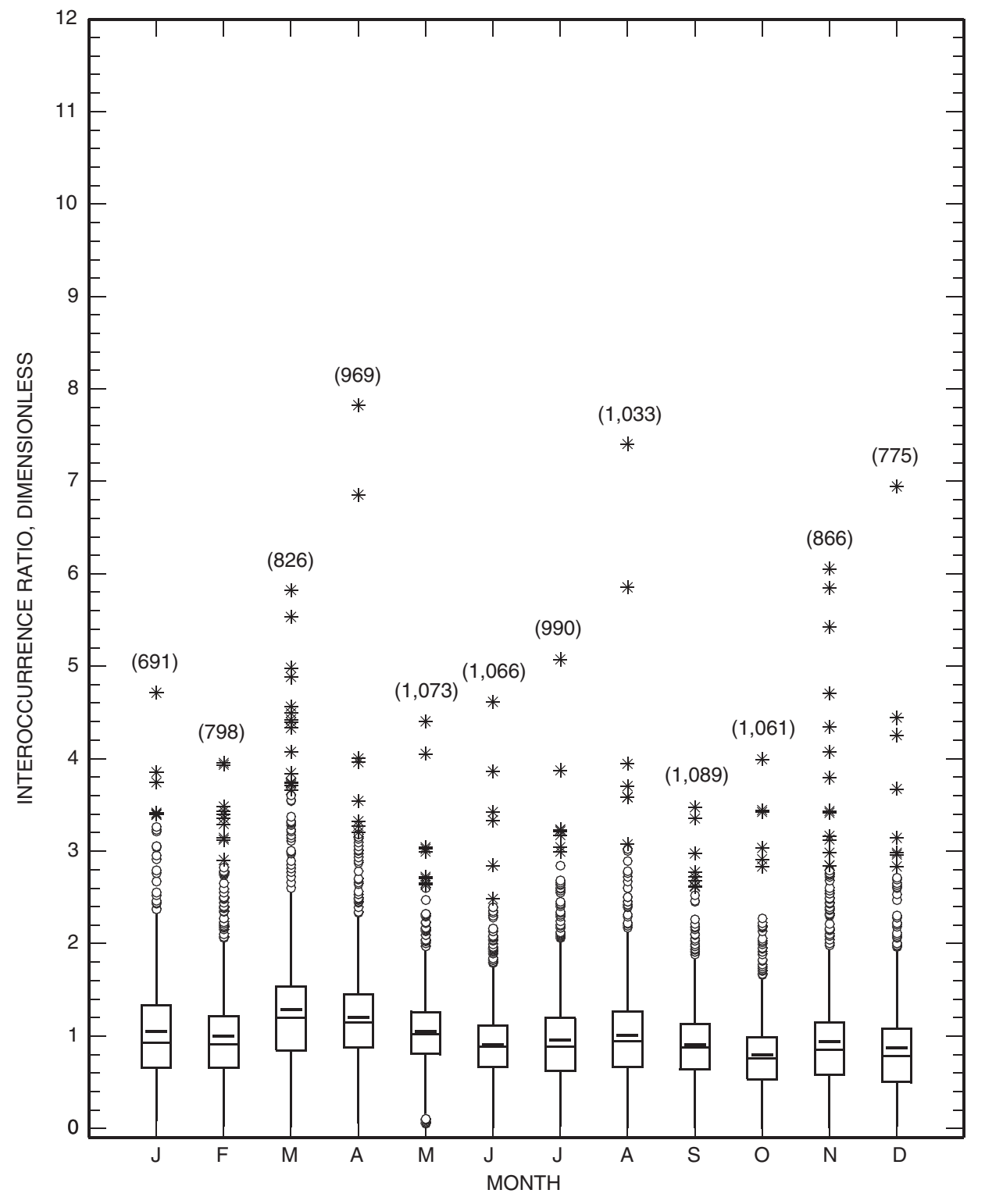

EXPLANATION

$(1,073)$ Sample size

* Data value greater than 3.0 times the IQR outside the box

- Data value 1.5 to 3.0 times the IQR outside the box

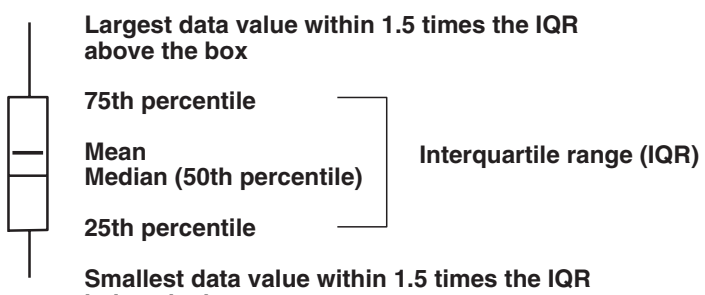

Smallest data value within 1.5 times the IQR below the box

Figure 22. Distribution of ratio of interoccurrence interval for month to mean interoccurrence interval for period of record at station for a 2.0-inch and greater daily precipitation threshold for Texas. 


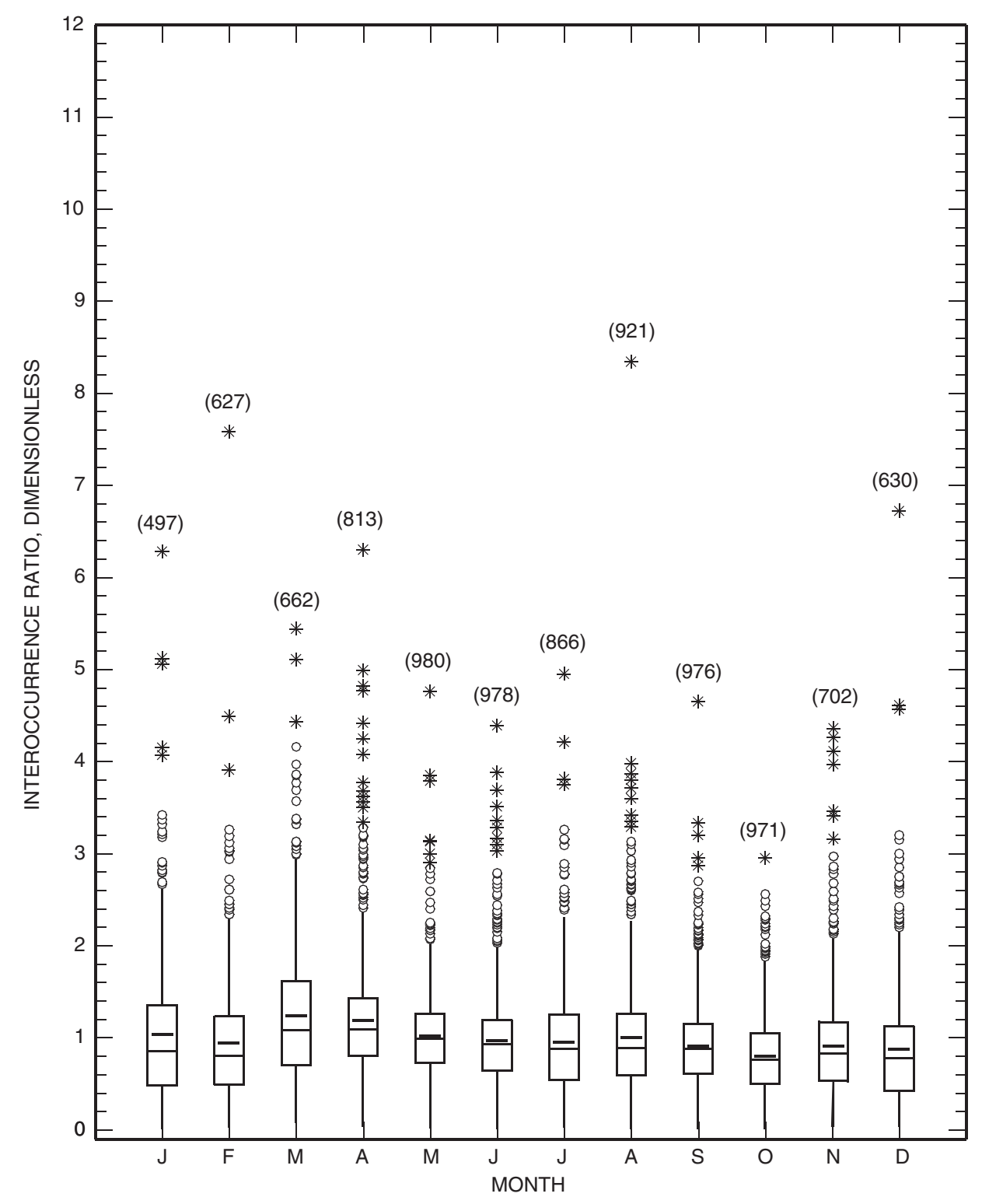

EXPLANATION

(813) Sample size

* Data value greater than 3.0 times the IQR outside the box

- Data value 1.5 to 3.0 times the IQR outside the box

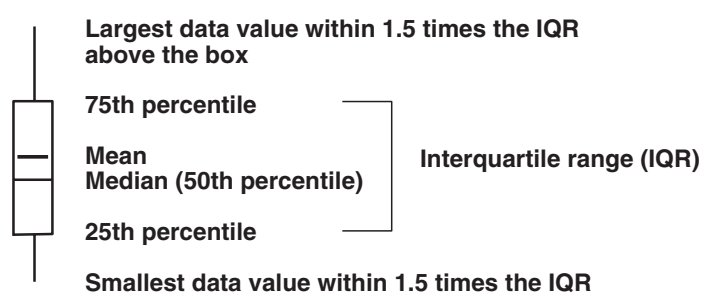

Smallest data value within 1.5 times the IQR below the box

Figure 23. Distribution of ratio of interoccurrence interval for month to mean interoccurrence interval for period of record at station for a 2.5 -inch and greater daily precipitation threshold for Texas. 


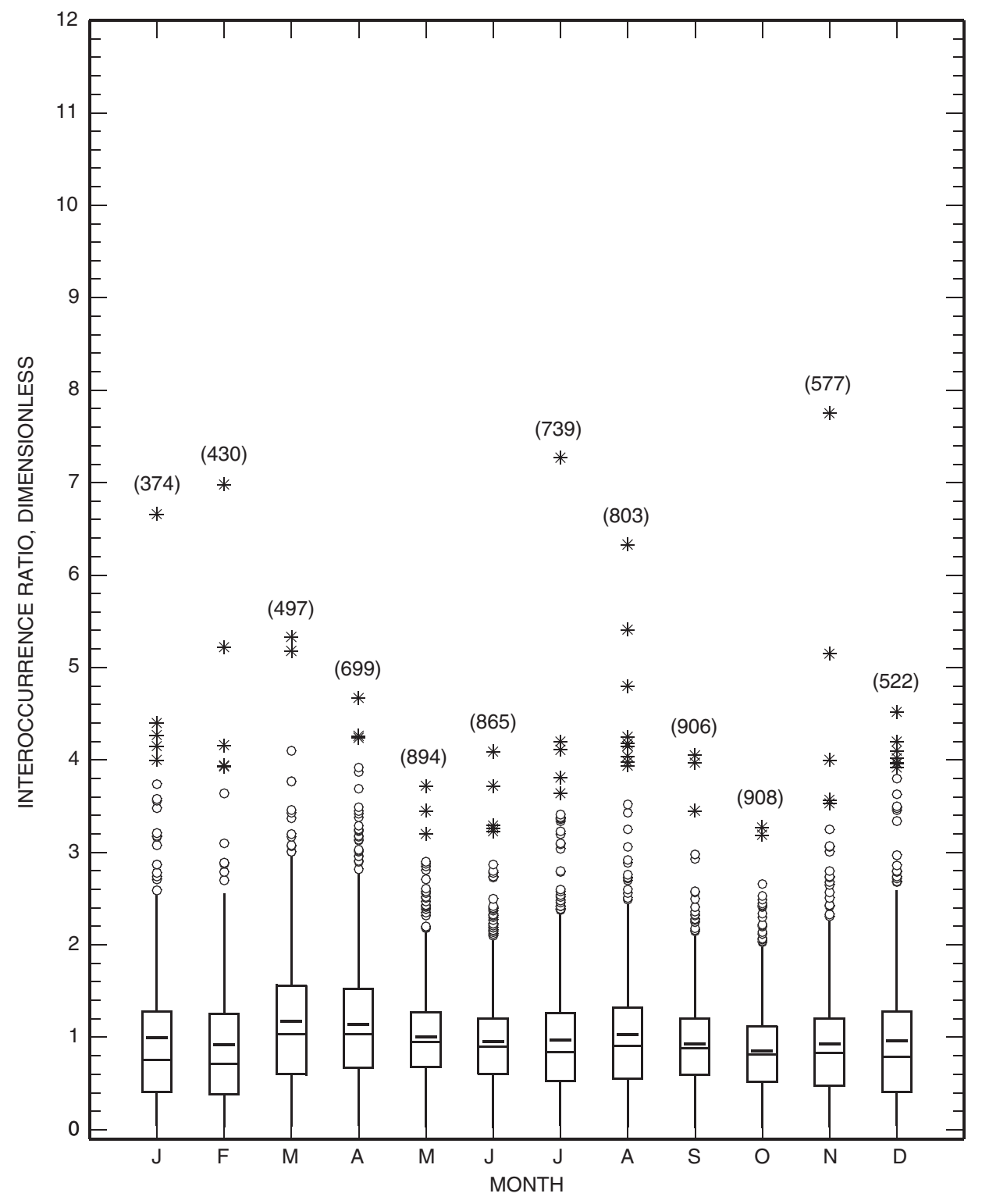

EXPLANATION

(522) Sample size

* Data value greater than 3.0 times the IQR outside the box

$\circ \quad$ Data value 1.5 to 3.0 times the IQR outside the box

Largest data value within 1.5 times the IQR above the box

\begin{tabular}{|l|l|}
\hline & 75th percentile \\
& Mean \\
Median (50th percentile) & Interquartile range (IQR) \\
25 th percentile & Smallest data value within 1.5 times the IQR
\end{tabular}

below the box

Figure 24. Distribution of ratio of interoccurrence interval for month to mean interoccurrence interval for period of record at station for a 3.0-inch and greater daily precipitation threshold for Texas. 


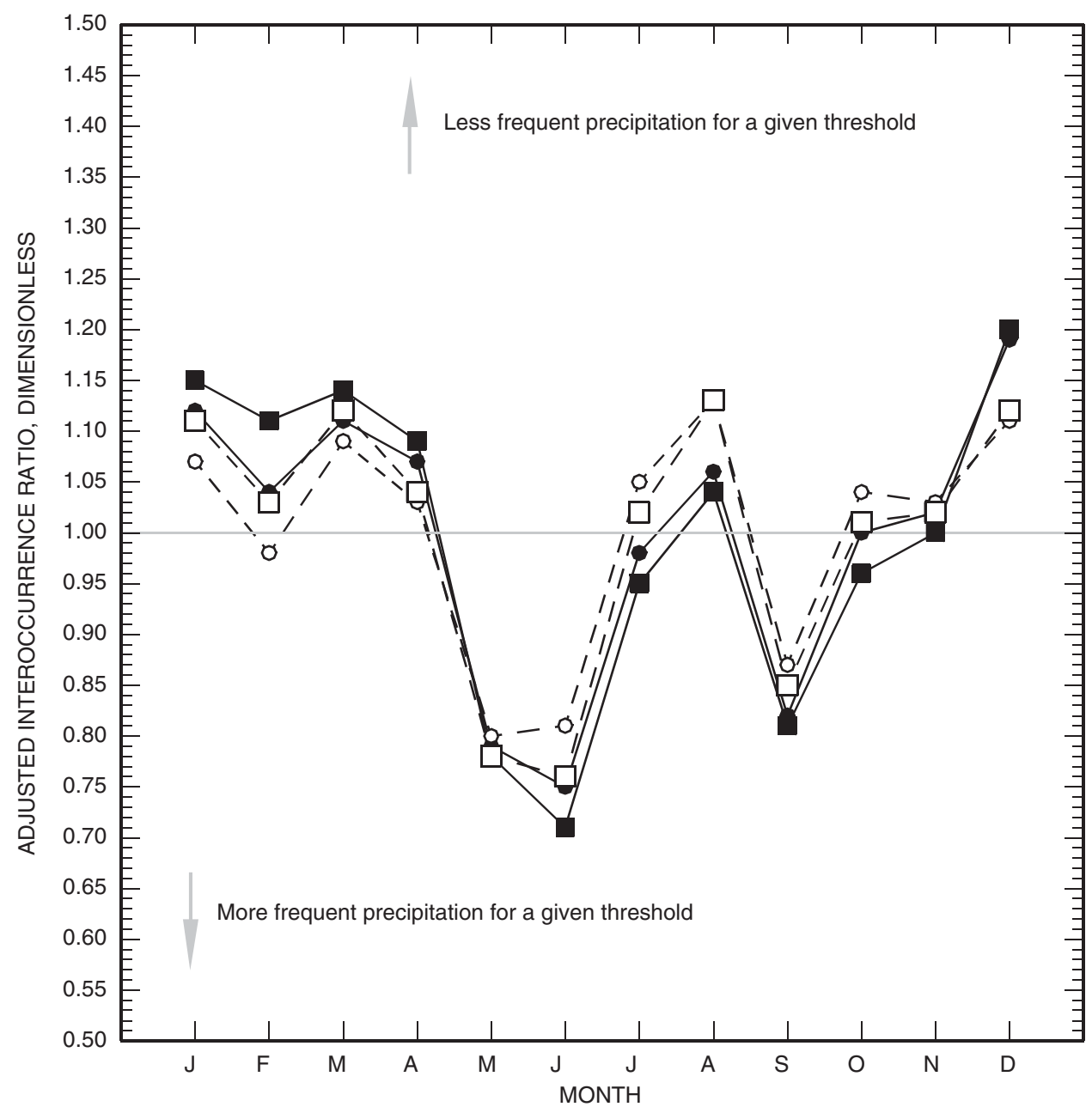

EXPLANATION

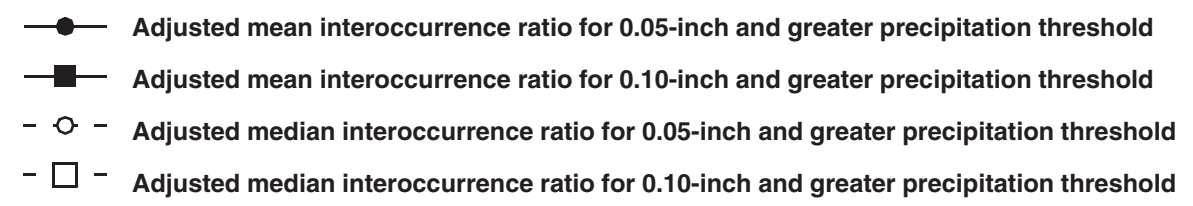

Figure 25. Relation between adjusted mean and median interoccurrence ratios and month for 0.05 - and 0.10 -inch daily precipitation thresholds for Texas.

Asquith and Thompson (2003) and Asquith (2003) concluded that for a 12- to 24-hour duration, an expected dimensionless hyetograph-cumulative temporal representation of storm depth—can be defined by the following equation:

$$
p(F)=F^{0.7830} e^{0.4368(1-F)},
$$

where

$p(F)$ is the fractional percent of the 24-hour storm depth, and
$F$ is the fractional percent of the storm duration.

The equation is appropriate for a duration of 3 hours because the interoccurrence intervals are derived for daily (24-hour) durations. Therefore, it follows that the equation estimates the median 3-hour, 6-month precipitation. For a duration of 3 hours, $F$ is $0.125(3 / 24)$, and $p(F)$ is 0.288 . Hence the site-specific median 3-hour, 6-month precipitation threshold for the Austin Camp Mabry station is 0.74 inch $(0.288 \times 2.56)$. For comparison, the site-specific median 6-hour, 6-month precipitation threshold is 1.20 inches $(0.469 \times 2.56)$. 


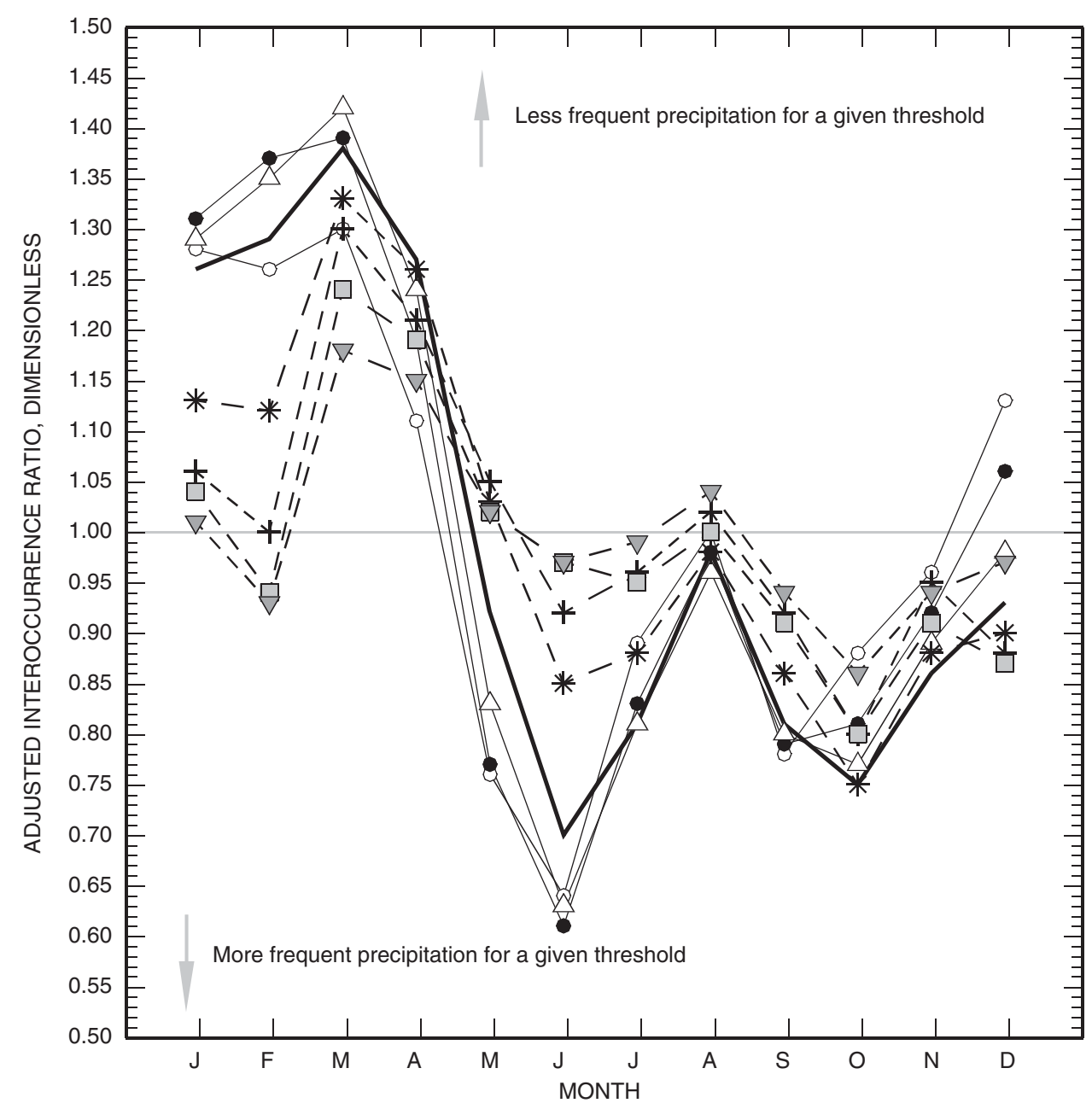

EXPLANATION

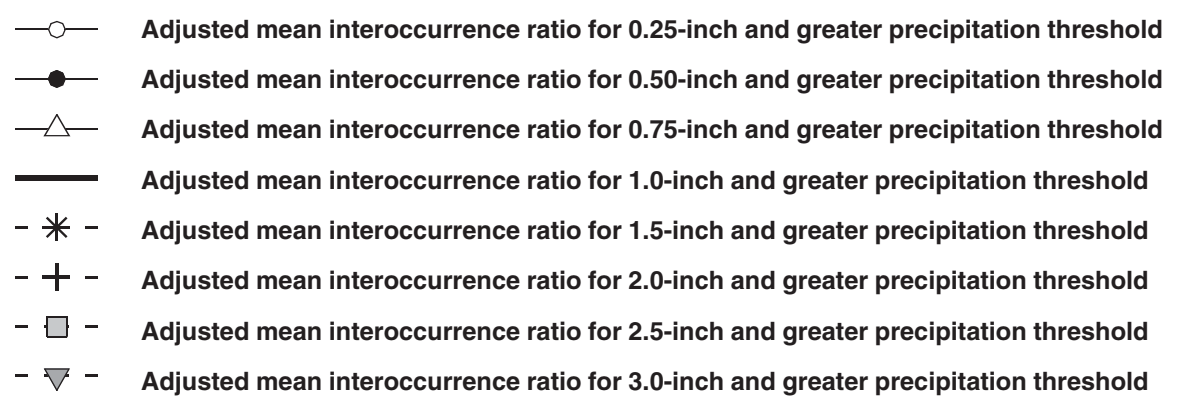

Figure 26. Relation between adjusted mean interoccurrence ratio and month for 0.25-, 0.50-, 0.75-, 1.0-, 1.5-, 2.0-, 2.5-, and 3.0-inch daily precipitation thresholds for Texas.

\section{Site-Specific Median Number of Occurrences}

Suppose that the median number $(n)$ of occurrences in 1 year of daily precipitation with a threshold of 1.0 inch is needed for the Austin Camp Mabry station, which was considered in the previous section. The site-specific mean interoccurrence interval $\Lambda=$ 37.41 days (sequence no. 46, table 5). The total time $T$ is 365 days, and because the median is sought, $F=0.5$. 


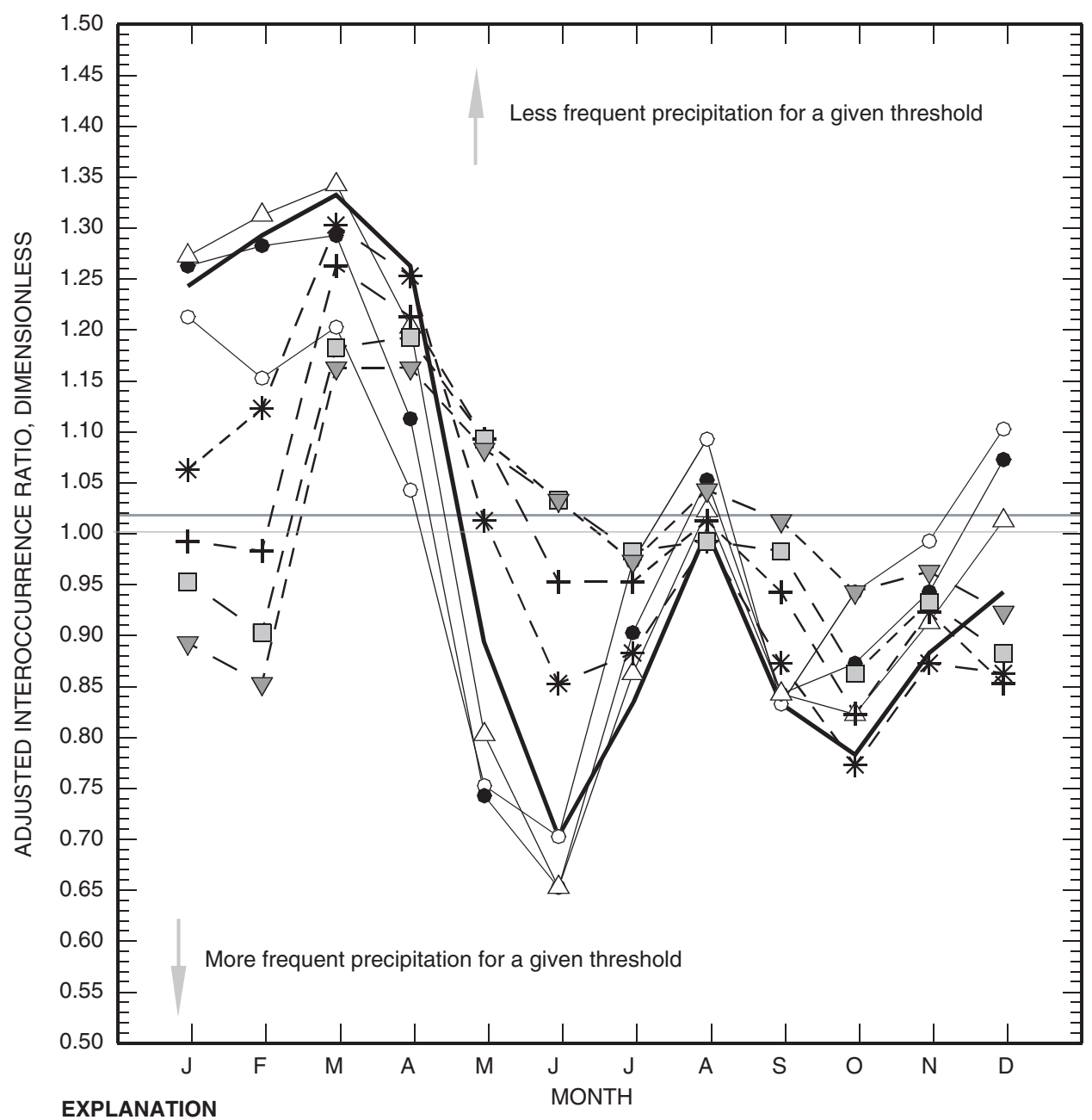

- Adjusted median interoccurrence ratio for 0.25 -inch and greater precipitation threshold

- Adjusted median interoccurrence ratio for 0.50 -inch and greater precipitation threshold

$\triangle \quad$ Adjusted median interoccurrence ratio for 0.75 -inch and greater precipitation threshold

- Adjusted median interoccurrence ratio for 1.0-inch and greater precipitation threshold

- * - Adjusted median interoccurrence ratio for 1.5-inch and greater precipitation threshold

- + - Adjusted median interoccurrence ratio for 2.0-inch and greater precipitation threshold

- $\square$ - Adjusted median interoccurrence ratio for 2.5-inch and greater precipitation threshold

- $\nabla$ - $\quad$ Adjusted median interoccurrence ratio for 3.0-inch and greater precipitation threshold

Figure 27. Relation between adjusted median interoccurrence ratio and month for 0.25-, 0.50-, 0.75-, 1.0-, 1.5-, 2.0-, 2.5-, and 3.0-inch daily precipitation thresholds for Texas.

The estimated number of occurrences per year can be computed from equation 6 :

$$
0.5=e^{-365 /(37.41)} \sum_{i=0}^{n} \frac{(365 / 37.41)^{n}}{n !} \text {, and }
$$

$$
8,635=\sum_{i=0}^{n} \frac{(9.757)^{n}}{n !} .
$$

The value for $n$ can be estimated using a spreadsheet or hand calculator. The right-hand side of equation 


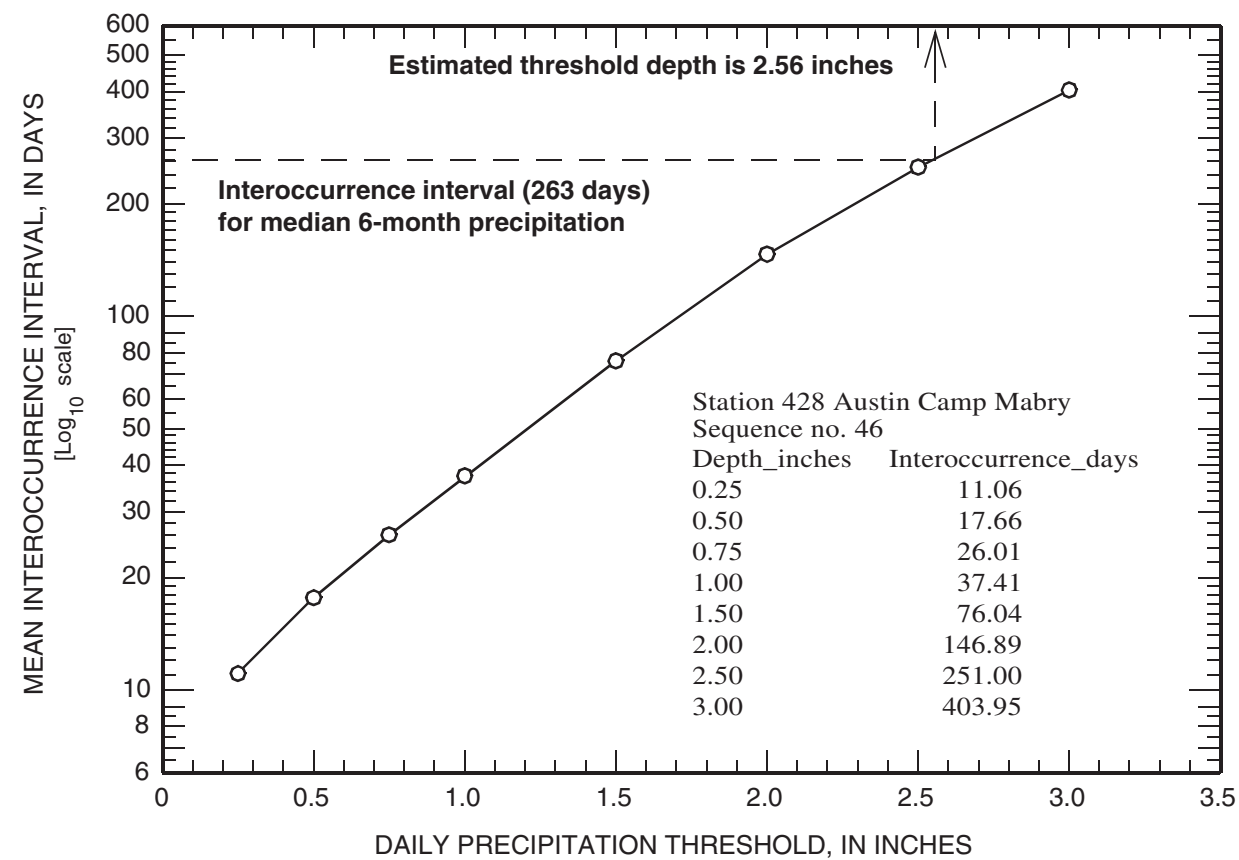

Figure 28. Relation between site-specific daily precipitation threshold and mean interoccurrence interval for station 428 Austin Camp Mabry and estimated median 6-month precipitation.

13 for $n=9$ and $n=10$ is 8,442 and 10,597 , respectively. The value 8,635 is closest to 8,442 ; therefore, the median number of precipitation events per year of 1.0 inch and greater is estimated as 9 .

\section{Intra-Annual Variations in Interoccurrence Intervals}

Suppose that the 75th $(F=0.75)$ and 90 th $(F=$ $0.90)$ percentile of the number of occurrences of June precipitation in a 10-year period of successive months with a threshold of 0.25 inch in Lubbock, Tex. (fig. 4) is needed. The station at the Lubbock International Airport (sequence no. 717, table 1) has the longest period of record in the area (91 years), so the mean interoccurrence interval of 16.92 days is expected to be a reliable site-specific interval. The time $T$ is 10 years multiplied by 30 days in June or 300 days. The expected June interoccurrence interval is 10.83 (0.64 X 16.92) days using the June adjusted mean monthly interoccurrence ratio of 0.64 (table 12). The estimated number of occurrences for the 75 th percentile can be computed from equation 6 as shown in the following equation:

$$
0.75=e^{-300 / 10.83} \sum_{i=0}^{n} \frac{(300 / 10.83)^{n}}{n !} .
$$

The value for $n$ that best approximates the number of June occurrences is 31 for the 75th percentile, and the value for $n$ that best approximates the equality is 34 for the 90th percentile.

\section{Regional Estimation of Mean Interoccurrence Interval}

Suppose that estimates of the mean interoccurrence interval of precipitation are needed for the area surrounding Austin, Tex. The maps depicted in figures 4-13 provide regional estimates that are believed to be generally more reliable than site-specific mean interoccurrence intervals available at any single station. Derived from the maps and manual interpolation, the regional estimates of interoccurrence intervals for each depth threshold for Austin are listed in table 20 (at end of report). For comparison, also listed in the table are the site-specific interoccurrence intervals for a long-term and short-term station in the Austin area (tables 3-7). The intervals in table 20 indicate that the regional estimates are similar to those for both stations; so the applicability of the maps in the Austin area is demonstrated. 


\section{SUMMARY}

A Poisson process model of daily precipitation is used to model the distribution of precipitation interoccurrence intervals, and the mathematics of the model are described in this report. A precipitation interoccurrence interval is the time period between two successive rainfall events. Rainfall events are defined as daily precipitation that equals or exceeds specified depth threshold. Ten precipitation thresholds are considered and include depths of $0.05,0.10,0.25,0.50,0.75,1.0,1.5,2.0,2.5$, and 3.0 inches. For each threshold for each of 1,306 NWS daily precipitation stations, site-specific mean interoccurrence intervals and ancillary statistics are computed, and the results are tabulated. Maps depicting the spatial variation across Texas of the mean interoccurrence interval for each threshold are presented along with diagnostic statistics. The percent change from the statewide standard deviation of the interoccurrence intervals to the RMSE ranges from a magnitude minimum of (negative) -24 to a magnitude maximum of -60 percent for the 0.05 - and 2.0-inch thresholds, respectively. Because of the substantial negative percent change, the maps are considered more reliable estimators of the mean interoccurrence interval for most locations in Texas than the statewide mean values.

The reliability of the maps west of the Pecos River is limited because of reduced station density, influences of elevation, and large interoccurrence intervals relative to the record length at the stations. Analysis of the influences of elevation on the interoccurrence intervals is provided. Interoccurrence interval decreases as station elevation increases, up to rainfall thresholds of about $1 \mathrm{inch}$, and for larger thresholds, little to no relation between interoccurrence interval and elevation is observed. Intra-annual variations or seasonal differences in the interoccurrence interval also are documented. Considerable and systematic seasonal variations reflect the decrease and increase in interoccurrence interval during the course of a year.

Several applications of precipitation interoccurrence intervals are provided through example computations and interpretations. The Poisson model provides a framework to estimate the distribution of the number of event occurrences for specific time periods or the distri- bution of interoccurrence intervals. These distributions can be useful in surface-water hydrology.

\section{SELECTED REFERENCES}

Asquith, W.H., 1998, Depth-duration frequency of precipitation for Texas: U.S. Geological Survey Water-Resources Investigations Report 98-4044, 107 p.

1999, Areal-reduction factors for the precipitation of the 1-day design storm in Texas: U.S. Geological Survey Water-Resources Investigations Report 99-4267, 81 p.

2003, Modeling of runoff-producing rainfall hyetographs in Texas using L-moment statistics: Austin, University of Texas at Austin, Ph.D. dissertation, 386 p.

Asquith, W.H., and Famiglietti, J.S., 2000, Precipitation areal-reduction factor estimation using an annualmaxima centered approach: Journal of Hydrology, v. 230, p. 55-69.

Asquith, W.H., and Thompson, D.B., 2003, Modeling of runoff-producing rainfall hyetographs in Texas using L-moments, in Texas Section, Spring 2003 Meeting, Corpus Christi, Tex., April 2-5, 2003, Proceedings: American Society of Civil Engineers.

Bhat, U.N., and Miller, G.K., 2002, Elements of applied stochastic processes ( $3 \mathrm{~d}$ ed.): Wiley, $461 \mathrm{p}$.

Bomar, G.W., 1995, Texas weather: Austin, University of Texas Press, 275 p.

Clarke, R.T., 1998, Stochastic processes for water scientists-Developments and applications: Wiley, 183 p.

Evans, Merran, Hastings, N.A.J., and Peacock, J.B., 2000, Statistical distributions (3d ed.): Wiley, $221 \mathrm{p}$.

Helsel, D.R., and Hirsch, R.M., 1992, Statistical methods in water resources-Studies in environmental science 49: Amsterdam, Elsevier, 529 p.

Hydrosphere, 2002, NCDC Summary of the day-West 2: Boulder, Colo., Hydrosphere Data Products, Inc., CD-ROM.

Isaaks, E.H., and Srivastava, R.M., 1989, An introduction to applied geostatistics: New York, Oxford University Press, $561 \mathrm{p}$.

Rajagopalan, Balaji, Lall, Upmanu, and Tarboton, D.G., 1996, Nonhomogeneous Markov model for daily precipitation: American Society of Civil Engineers, Journal of Hydrologic Engineering, v. 1, no. 1, p. 33-40.

Ross, Sheldon, 1994, A first course in probability (4th ed.): New York, MacMillan College Publishing Co., 473 p.

Wilks, D.S., 1995, Statistical methods in the atmospheric sciences: San Diego, Academic Press, 467 p. 
Table 1. Summary of National Weather Service daily precipitation stations in Texas with known names, latitudes, and longitudes

[*, county name or elevation provided by authors-entry not available from Hydrosphere (2002). Abbreviations: Seq., sequence; NWS, National Weather Service; Elev., elevation; ft, feet; NGVD 29, National Geodetic Vertical Datum of 1929; mm/yyyy, month and year; CAA, Civilian Aviation Authority; WSO, Weather Service Office; SNA, State Natural Area; LCRA, Lower Colorado River Authority; FAA, Federal Aviation Authority; SCS, Soil Conservation Service; WB; Weather Bureau; WSFO, Weather Service Field Office; NWSO, National Weather Service Office; FCWOS, unknown; ASOS, unknown; CP\&D, unknown; WTU, West Texas Utilities. Directions: SE, southeast; SW, southwest; NNW, north-northwest; NE, northeast; W, west; SSE, south-southeast; NW, northwest; N, north; NNE, north-northeast; ENE, east-northeast; SSW, south-southwest; WNW, west-northwest; S, south; WSW, west-southwest; E, east; ESE, east-southeast]

\begin{tabular}{|c|c|c|c|c|c|c|c|c|c|c|}
\hline $\begin{array}{c}\text { Seq. } \\
\text { no. }\end{array}$ & NWS station name & $\begin{array}{c}\text { NWS } \\
\text { station } \\
\text { no. }\end{array}$ & County & $\begin{array}{c}\text { Elev. } \\
\text { (ft above } \\
\text { NGVD } \\
\text { 29) }\end{array}$ & $\begin{array}{l}\text { Lati- } \\
\text { tude }\end{array}$ & $\begin{array}{l}\text { Longi- } \\
\text { tude }\end{array}$ & $\begin{array}{c}\text { Begining } \\
\text { date } \\
\text { (mm/yyyy) }\end{array}$ & $\begin{array}{c}\text { Ending } \\
\text { date } \\
\text { (mm/yyyy) }\end{array}$ & $\begin{array}{c}\text { Per- } \\
\text { cent } \\
\text { cov- } \\
\text { erage }\end{array}$ & $\begin{array}{c}\text { Years } \\
\text { of } \\
\text { record }\end{array}$ \\
\hline 1 & Abernathy & 12 & Hale & 3,360 & $33^{\circ} 50^{\prime}$ & $101^{\circ} 51^{\prime}$ & $01 / 1948$ & $12 / 2001$ & 99 & 54 \\
\hline 2 & Abilene 2 & 13 & Taylor & 1,865 & $32^{\circ} 12^{\prime}$ & $99^{\circ} 27^{\prime}$ & $05 / 2001$ & $12 / 2001$ & 66 & 1 \\
\hline 3 & Abilene Regional Airport & 16 & Taylor & 1,790 & $32^{\circ} 24^{\prime}$ & $99^{\circ} 40^{\prime}$ & $01 / 1948$ & $12 / 2001$ & 100 & 54 \\
\hline 4 & Acker Ranch & 25 & McMullen & 435 & $28^{\circ} 07^{\prime}$ & $98^{\circ} 32^{\prime}$ & $09 / 1978$ & $12 / 2001$ & 96 & 24 \\
\hline 5 & Ackerly 4 SE & 34 & Howard & 2,655 & $32^{\circ} 30^{\prime}$ & $101^{\circ} 40^{\prime}$ & $01 / 1948$ & $11 / 2001$ & 96 & 54 \\
\hline 6 & Acton Ranch & 40 & Crockett & 2,010 & $30^{\circ} 21^{\prime}$ & $101^{\circ} 15^{\prime}$ & 03/1992 & $12 / 2001$ & 96 & 10 \\
\hline 7 & Adamsville & 50 & Lampasas & 1,030 & $31^{\circ} 17^{\prime}$ & $98^{\circ} 09^{\prime}$ & $09 / 1963$ & $12 / 1987$ & 90 & 25 \\
\hline 8 & Adobe Walls Scout Camp & 70 & Wheeler & 2,582 & $35^{\circ} 31^{\prime}$ & $100^{\circ} 22^{\prime}$ & $04 / 1974$ & $03 / 1978$ & 80 & 5 \\
\hline 9 & Adrian & 68 & Oldham* & 4,042 & $35^{\circ} 17^{\prime}$ & $102^{\circ} 40^{\prime}$ & $05 / 1938$ & $08 / 1948$ & 72 & 11 \\
\hline 10 & Agua Dulce & 73 & Nueces & 85 & $27^{\circ} 47^{\prime}$ & $97^{\circ} 54^{\prime}$ & $03 / 1987$ & 09/1987 & 59 & 1 \\
\hline 11 & Agua Nueva & 81 & Jim Hogg & 459 & $26^{\circ} 54^{\prime}$ & $98^{\circ} 36^{\prime}$ & $01 / 1948$ & $12 / 1964$ & 99 & 17 \\
\hline 12 & Albany & 120 & Shackelford & 1,400 & $32^{\circ} 43^{\prime}$ & $99^{\circ} 18^{\prime}$ & $11 / 1901$ & $12 / 2001$ & 96 & 101 \\
\hline 13 & Aledo $4 \mathrm{SE}$ & 129 & Parker & 790 & $32^{\circ} 39^{\prime}$ & $97^{\circ} 34^{\prime}$ & $09 / 1960$ & $12 / 2001$ & 98 & 42 \\
\hline 14 & Alice & 144 & Jim Wells & 201 & $27^{\circ} 43^{\prime}$ & $98^{\circ} 04^{\prime}$ & $04 / 1911$ & $12 / 2001$ & 96 & 85 \\
\hline 15 & Alice CAA Airport & 145 & Jim Wells & 173 & $27^{\circ} 44^{\prime}$ & $98^{\circ} 02^{\prime}$ & $01 / 1948$ & 09/1952 & 94 & 5 \\
\hline 16 & Allison & 157 & Wheeler & 2,600 & $35^{\circ} 36^{\prime}$ & $100^{\circ} 03^{\prime}$ & $08 / 2001$ & $12 / 2001$ & 34 & 1 \\
\hline 17 & Allison Ranch & 160 & Edwards & 2,280 & $30^{\circ} 05^{\prime}$ & $100^{\circ} 23^{\prime}$ & $10 / 1964$ & $04 / 1965$ & 17 & 2 \\
\hline 18 & Alpine & 174 & Brewster & 4,530 & $30^{\circ} 21^{\prime}$ & $103^{\circ} 39^{\prime}$ & $03 / 1900$ & $12 / 2001$ & 94 & 80 \\
\hline 19 & Alto $5 \mathrm{SW}$ & 190 & Cherokee & 280 & $31^{\circ} 36^{\prime}$ & $95^{\circ} 08^{\prime}$ & $01 / 1948$ & $12 / 2001$ & 98 & 54 \\
\hline 20 & Alvarado & 201 & Johnson & 689 & $32^{\circ} 25^{\prime}$ & $97^{\circ} 13^{\prime}$ & $01 / 1948$ & $10 / 1964$ & 99 & 17 \\
\hline 21 & Alvarado $2 \mathrm{NNW}$ & 202 & Johnson & 835 & $32^{\circ} 27^{\prime}$ & $97^{\circ} 13^{\prime}$ & $04 / 1987$ & $12 / 2001$ & 96 & 15 \\
\hline 22 & Alvin & 204 & Brazoria & 28 & $29^{\circ} 22^{\prime}$ & $95^{\circ} 14^{\prime}$ & $06 / 1898$ & $12 / 2001$ & 93 & 102 \\
\hline 23 & Alvord $3 \mathrm{NE}$ & 206 & Wise & 1,010 & $33^{\circ} 23^{\prime}$ & $97^{\circ} 39^{\prime}$ & $06 / 1948$ & $09 / 1951$ & 81 & 4 \\
\hline 24 & Amarillo WSO Airport & 211 & Potter & 3,586 & $35^{\circ} 13^{\prime}$ & $101^{\circ} 42^{\prime}$ & $01 / 1948$ & $12 / 2001$ & 100 & 54 \\
\hline 25 & Amistad Dam & 225 & Val Verde & 1,157 & $29^{\circ} 27^{\prime}$ & $101^{\circ} 01^{\prime}$ & $05 / 1964$ & $12 / 2001$ & 99 & 38 \\
\hline 26 & Anahuac & 235 & Chambers & 24 & $29^{\circ} 47^{\prime}$ & $94^{\circ} 40^{\prime}$ & 06/1909 & $12 / 2001$ & 96 & 89 \\
\hline 27 & Anderson & 244 & Grimes & 351 & $30^{\circ} 29^{\prime}$ & $95^{\circ} 59^{\prime}$ & $05 / 1914$ & $09 / 1972$ & 96 & 59 \\
\hline 28 & Andice $2 \mathrm{SW}$ & 246 & Williamson & 1,060 & $30^{\circ} 45^{\prime}$ & $97^{\circ} 51^{\prime}$ & $02 / 1968$ & $12 / 2001$ & 97 & 34 \\
\hline 29 & Andrews & 248 & Andrews & 3,192 & $32^{\circ} 20^{\prime}$ & $102^{\circ} 33^{\prime}$ & $01 / 1914$ & $12 / 2001$ & 95 & 46 \\
\hline 30 & Angleton $2 \mathrm{~W}$ & 257 & Brazoria & 27 & $29^{\circ} 09^{\prime}$ & $95^{\circ} 27^{\prime}$ & $06 / 1913$ & $12 / 2001$ & 97 & 89 \\
\hline 31 & Anna & 262 & Collin & 680 & $33^{\circ} 21^{\prime}$ & $96^{\circ} 31^{\prime}$ & $06 / 1898$ & $11 / 1995$ & 95 & 52 \\
\hline 32 & Anson & 268 & Jones & 1,710 & $32^{\circ} 46^{\prime}$ & $99^{\circ} 53^{\prime}$ & $01 / 1898$ & $12 / 2001$ & 86 & 53 \\
\hline 33 & Antelope & 271 & Jack & 1,040 & $33^{\circ} 26^{\prime}$ & $98^{\circ} 22^{\prime}$ & 09/1910 & $12 / 2001$ & 98 & 65 \\
\hline 34 & Aquilla $1 \mathrm{SSE}$ & 297 & Hill & 520 & $31^{\circ} 50^{\prime}$ & $97^{\circ} 13^{\prime}$ & $01 / 1992$ & $12 / 2001$ & 99 & 10 \\
\hline 35 & Aransas Pass 2 & 302 & Aransas & 20 & $27^{\circ} 55^{\prime}$ & $97^{\circ} 08^{\prime}$ & $06 / 1897$ & $07 / 1971$ & 91 & 31 \\
\hline 36 & Aransas Wildlife Refuge & 305 & Aransas & 15 & $28^{\circ} 18^{\prime}$ & $96^{\circ} 48^{\prime}$ & $01 / 1971$ & $12 / 2001$ & 98 & 31 \\
\hline 37 & Aransas Wildlife Refuge & 437 & Aransas & 20 & $28^{\circ} 16^{\prime}$ & $96^{\circ} 48^{\prime}$ & $06 / 1940$ & $12 / 1970$ & 96 & 31 \\
\hline 38 & Archer City & 313 & Archer & 1,045 & $33^{\circ} 34^{\prime}$ & $98^{\circ} 37^{\prime}$ & $04 / 1910$ & $12 / 2001$ & 96 & 64 \\
\hline 39 & Arlington & 337 & Tarrant & 655 & $32^{\circ} 44^{\prime}$ & $97^{\circ} 07^{\prime}$ & $01 / 1948$ & $12 / 2001$ & 99 & 54 \\
\hline 40 & Armstrong & 342 & Kenedy & 30 & $26^{\circ} 56^{\prime}$ & $97^{\circ} 48^{\prime}$ & $08 / 1942$ & $11 / 1978$ & 84 & 34 \\
\hline 41 & Arthur City & 367 & Lamar* & 430 & $33^{\circ} 52^{\prime}$ & $95^{\circ} 30^{\prime}$ & $01 / 1897$ & 06/1970 & 94 & 72 \\
\hline 42 & Asherton & 380 & Dimmit* & 551 & $28^{\circ} 26^{\prime}$ & $99^{\circ} 45^{\prime}$ & $06 / 1948$ & 09/1951 & 51 & 4 \\
\hline 43 & Aspermont & 394 & Stonewall & 1,670 & $33^{\circ} 09^{\prime}$ & $100^{\circ} 14^{\prime}$ & $07 / 1911$ & $12 / 2001$ & 97 & 91 \\
\hline 44 & Athens & 404 & Henderson & 448 & $32^{\circ} 09^{\prime}$ & $95^{\circ} 49^{\prime}$ & $12 / 1903$ & $12 / 2001$ & 93 & 58 \\
\hline
\end{tabular}


Table 1. Summary of National Weather Service daily precipitation stations in Texas with known names, latitudes, and longitudes-Continued

\begin{tabular}{|c|c|c|c|c|c|c|c|c|c|c|}
\hline $\begin{array}{c}\text { Seq. } \\
\text { no. }\end{array}$ & NWS station name & $\begin{array}{c}\text { NWS } \\
\text { station } \\
\text { no. }\end{array}$ & County & $\begin{array}{c}\text { Elev. } \\
\text { (ft above } \\
\text { NGVD } \\
\text { 29) }\end{array}$ & $\begin{array}{l}\text { Lati- } \\
\text { tude }\end{array}$ & $\begin{array}{l}\text { Longi- } \\
\text { tude }\end{array}$ & $\begin{array}{l}\text { Begining } \\
\text { date } \\
\text { (mm/yyyy) }\end{array}$ & $\begin{array}{c}\text { Ending } \\
\text { date } \\
\text { (mm/yyyy) }\end{array}$ & $\begin{array}{l}\text { Per- } \\
\text { cent } \\
\text { cov- } \\
\text { erage }\end{array}$ & $\begin{array}{l}\text { Years } \\
\text { of } \\
\text { record }\end{array}$ \\
\hline 45 & Atlanta & 408 & Cass & 300 & $33^{\circ} 06^{\prime}$ & $94^{\circ} 10^{\prime}$ & $09 / 1930$ & $05 / 2000$ & 91 & 57 \\
\hline 46 & Austin Camp Mabry & 428 & Travis & 673 & $30^{\circ} 19^{\prime}$ & $97^{\circ} 46^{\prime}$ & $06 / 1930$ & $12 / 2001$ & 98 & 72 \\
\hline 47 & Austin Dam & 430 & Travis & 531 & $30^{\circ} 18^{\prime}$ & $97^{\circ} 47^{\prime}$ & $03 / 1948$ & $12 / 1965$ & 97 & 18 \\
\hline 48 & Austin Montopolis Bridge & 432 & Travis* & 512 & $30^{\circ} 15^{\prime}$ & $97^{\circ} 41^{\prime}$ & $01 / 1948$ & $07 / 1963$ & 97 & 16 \\
\hline 49 & Austin Water Treatment Plant & 427 & Travis & 500 & $30^{\circ} 16^{\prime}$ & $97^{\circ} 39^{\prime}$ & 09/1996 & 07/1997 & 41 & 2 \\
\hline 50 & Austin-Bergstrom International Airport & 429 & Travis & 480 & $30^{\circ} 10^{\prime}$ & $97^{\circ} 40^{\prime}$ & 07/1999 & $12 / 2001$ & 83 & 3 \\
\hline 51 & Austwell & 436 & Refugio & 95 & $28^{\circ} 22^{\prime}$ & $96^{\circ} 50^{\prime}$ & 03/1897 & $02 / 1960$ & 96 & 52 \\
\hline 52 & Avalon & 440 & Ellis & 530 & $32^{\circ} 12^{\prime}$ & $96^{\circ} 47^{\prime}$ & $12 / 1964$ & $12 / 2001$ & 94 & 38 \\
\hline 53 & Avery $5 \mathrm{NNW}$ & 443 & Red River & 390 & $33^{\circ} 37^{\prime}$ & $94^{\circ} 48^{\prime}$ & $03 / 2001$ & $12 / 2001$ & 84 & 1 \\
\hline 54 & Bade Ranch & 463 & Sterling* & $* 2,433$ & $31^{\circ} 50^{\prime}$ & $101^{\circ} 10^{\prime}$ & 06/1948 & $10 / 1948$ & 24 & 1 \\
\hline 55 & Baird & 478 & Callahan & 1,732 & $32^{\circ} 24^{\prime}$ & $99^{\circ} 24^{\prime}$ & 03/1948 & $04 / 1980$ & 82 & 33 \\
\hline 56 & Bakers Crossing & 479 & Val Verde & 1,510 & $29^{\circ} 57^{\prime}$ & $101^{\circ} 09^{\prime}$ & $01 / 1981$ & $12 / 1987$ & 93 & 7 \\
\hline 57 & Bakersfield & 482 & Pecos & 2,553 & $30^{\circ} 53^{\prime}$ & $102^{\circ} 18^{\prime}$ & 01/1948 & $12 / 2001$ & 99 & 54 \\
\hline 58 & Bakersfield 11 SSE & 484 & Pecos & 3,081 & $30^{\circ} 43^{\prime}$ & $102^{\circ} 13^{\prime}$ & $07 / 1980$ & 05/1982 & 55 & 3 \\
\hline 59 & Ballinger 2 NW & 493 & Runnels & 1,755 & $31^{\circ} 44^{\prime}$ & $99^{\circ} 58^{\prime}$ & $01 / 1897$ & $12 / 2001$ & 96 & 103 \\
\hline 60 & Balmorhea & 495 & Reeves* & 3,192 & $30^{\circ} 59^{\prime}$ & $103^{\circ} 44^{\prime}$ & 03/1949 & $06 / 1951$ & 38 & 3 \\
\hline 61 & Balmorhea & 498 & Reeves & 3,220 & $30^{\circ} 59^{\prime}$ & $103^{\circ} 44^{\prime}$ & $09 / 1923$ & $12 / 2001$ & 97 & 79 \\
\hline 62 & Balmorhea Circle H Ranch & 497 & Jeff Davis* & 5,003 & $30^{\circ} 51^{\prime}$ & $103^{\circ} 59^{\prime}$ & $03 / 1953$ & $10 / 1953$ & 60 & 1 \\
\hline 63 & Balmorhea Graef Ranch & 499 & Reeves* & 3,304 & $30^{\circ} 56^{\prime}$ & $103^{\circ} 31^{\prime}$ & $03 / 1953$ & $11 / 1958$ & 93 & 6 \\
\hline 64 & Balmorhea wb pan & 496 & Reeves* & 3,222 & $31^{\circ} 00^{\prime}$ & $103^{\circ} 41^{\prime}$ & $01 / 1948$ & $04 / 1950$ & 27 & 3 \\
\hline 65 & Bankersmith & 509 & Kendall & 1,750 & $30^{\circ} 08^{\prime}$ & $98^{\circ} 49^{\prime}$ & $06 / 1948$ & $12 / 2001$ & 92 & 22 \\
\hline 66 & Banquete & 511 & Nueces & 79 & $27^{\circ} 48^{\prime}$ & $97^{\circ} 48^{\prime}$ & $07 / 1978$ & $10 / 1986$ & 71 & 9 \\
\hline 67 & Bardwell Dam & 518 & Ellis & 461 & $32^{\circ} 15^{\prime}$ & $96^{\circ} 38^{\prime}$ & $04 / 1965$ & $12 / 2001$ & 99 & 37 \\
\hline 68 & Barnhart & 528 & Irion & 2,562 & $31^{\circ} 08^{\prime}$ & $101^{\circ} 10^{\prime}$ & 03/1948 & $04 / 1965$ & 80 & 18 \\
\hline 69 & Barstow & 538 & Ward* & 2,572 & $31^{\circ} 28^{\prime}$ & $103^{\circ} 24^{\prime}$ & $05 / 1906$ & $12 / 1919$ & 96 & 14 \\
\hline 70 & Bastrop & 542 & Bastrop* & 381 & $30^{\circ} 06^{\prime}$ & $97^{\circ} 20^{\prime}$ & $12 / 1900$ & $02 / 1915$ & 47 & 6 \\
\hline 71 & Bateman Ranch & 556 & King & 1,811 & $33^{\circ} 35^{\prime}$ & $100^{\circ} 09^{\prime}$ & 05/1971 & $05 / 1973$ & 69 & 3 \\
\hline 72 & Bateman Ranch 2 & 558 & King & 1,785 & $33^{\circ} 36^{\prime}$ & $100^{\circ} 13^{\prime}$ & $11 / 1974$ & $10 / 1988$ & 85 & 14 \\
\hline 73 & Batesville & 560 & Zavala & 745 & $28^{\circ} 57^{\prime}$ & $99^{\circ} 37^{\prime}$ & $06 / 1965$ & 03/2001 & 92 & 37 \\
\hline 74 & Bay City $2 \mathrm{~N}$ & 572 & Matagorda* & 49 & $29^{\circ} 00^{\prime}$ & $95^{\circ} 58^{\prime}$ & $06 / 1948$ & $09 / 1951$ & 83 & 4 \\
\hline 75 & Bay City Waterworks & 569 & Matagorda & 52 & $28^{\circ} 59^{\prime}$ & $95^{\circ} 57^{\prime}$ & 10/1909 & $12 / 2001$ & 93 & 69 \\
\hline 76 & Baylor Ranch & 580 & La Salle* & 400 & $28^{\circ} 18^{\prime}$ & $98^{\circ} 59^{\prime}$ & 07/1947 & 09/1951 & 63 & 5 \\
\hline 77 & Baytown & 586 & Harris & 34 & $29^{\circ} 50^{\prime}$ & $95^{\circ} 00^{\prime}$ & $06 / 1946$ & $12 / 2001$ & 94 & 46 \\
\hline 78 & Bayview & 576 & Cameron* & 20 & $26^{\circ} 07^{\prime}$ & $97^{\circ} 24^{\prime}$ & $06 / 1948$ & $05 / 1950$ & 66 & 3 \\
\hline 79 & Beasley & 605 & Fort Bend* & 102 & $29^{\circ} 30^{\prime}$ & $95^{\circ} 52^{\prime}$ & $06 / 1948$ & $09 / 1950$ & 53 & 3 \\
\hline 80 & Beaumont City & 611 & Jefferson & 20 & $30^{\circ} 06^{\prime}$ & $94^{\circ} 06^{\prime}$ & $11 / 1901$ & $12 / 2001$ & 96 & 101 \\
\hline 81 & Beaumont Research Center & 613 & Jefferson & 27 & $30^{\circ} 04^{\prime}$ & $94^{\circ} 17^{\prime}$ & $01 / 1948$ & $12 / 2001$ & 94 & 41 \\
\hline 82 & Bedias & 635 & Grimes & 335 & $30^{\circ} 47^{\prime}$ & $95^{\circ} 57^{\prime}$ & 08/1949 & $12 / 1985$ & 94 & 37 \\
\hline 83 & Beeville $5 \mathrm{NE}$ & 639 & Bee & 255 & $28^{\circ} 27^{\prime}$ & $97^{\circ} 42^{\prime}$ & $11 / 1901$ & $12 / 2001$ & 98 & 100 \\
\hline 84 & Bellville $6 \mathrm{NNE}$ & 655 & Austin & 280 & $30^{\circ} 01^{\prime}$ & $96^{\circ} 12^{\prime}$ & $11 / 1978$ & $12 / 2001$ & 93 & 23 \\
\hline 85 & Belmont & 660 & Gonzales & 388 & $29^{\circ} 31^{\prime}$ & $97^{\circ} 41^{\prime}$ & 02/1997 & $12 / 2001$ & 90 & 5 \\
\hline 86 & Belton Dam & 665 & Bell & 664 & $31^{\circ} 06^{\prime}$ & $97^{\circ} 29^{\prime}$ & $08 / 1951$ & $12 / 1992$ & 96 & 41 \\
\hline 87 & Benavides & 689 & Duval & 381 & $27^{\circ} 36^{\prime}$ & $98^{\circ} 25^{\prime}$ & $06 / 1948$ & $09 / 1951$ & 83 & 4 \\
\hline 88 & Benavides 2 & 690 & Duval & 380 & $27^{\circ} 35^{\prime}$ & $98^{\circ} 24^{\prime}$ & $07 / 1962$ & $12 / 2001$ & 90 & 39 \\
\hline 89 & Benbrook Dam & 691 & Tarrant & 790 & $32^{\circ} 38^{\prime}$ & $97^{\circ} 26^{\prime}$ & 06/1949 & $12 / 2001$ & 98 & 53 \\
\hline 90 & Benjamin $15 \mathrm{~W}$ & 708 & King & 1,650 & $33^{\circ} 35^{\prime}$ & $100^{\circ} 02^{\prime}$ & 05/1980 & $10 / 2001$ & 79 & 22 \\
\hline 91 & Benjamin 4 SSE & 704 & Knox & 1,401 & $33^{\circ} 32^{\prime}$ & $99^{\circ} 46^{\prime}$ & $06 / 1940$ & 05/1975 & 94 & 36 \\
\hline 92 & Bertram 3 ENE & 738 & Burnet & 1,139 & $30^{\circ} 45^{\prime}$ & $98^{\circ} 00^{\prime}$ & $02 / 1968$ & $12 / 2001$ & 100 & 34 \\
\hline 93 & Bethany & 715 & Panola & 370 & $32^{\circ} 23^{\prime}$ & $94^{\circ} 03^{\prime}$ & $02 / 1983$ & $12 / 1988$ & 97 & 6 \\
\hline 94 & Big Bend Ranch SNA & 764 & Presidio & 4,140 & $29^{\circ} 26^{\prime}$ & $103^{\circ} 57^{\prime}$ & 02/1995 & $12 / 2001$ & 95 & 7 \\
\hline 95 & Big Lake 13 SSW & 777 & Crockett* & 2,802 & $31^{\circ} 01^{\prime}$ & $101^{\circ} 33^{\prime}$ & $09 / 1941$ & $04 / 1949$ & 31 & 9 \\
\hline 96 & Big Lake 2 & 779 & Reagan & 2,703 & $31^{\circ} 11^{\prime}$ & $101^{\circ} 28^{\prime}$ & $08 / 1963$ & $12 / 2001$ & 97 & 39 \\
\hline
\end{tabular}


Table 1. Summary of National Weather Service daily precipitation stations in Texas with known names, latitudes, and longitudes-Continued

\begin{tabular}{|c|c|c|c|c|c|c|c|c|c|c|}
\hline $\begin{array}{l}\text { Seq. } \\
\text { no. }\end{array}$ & NWS station name & $\begin{array}{l}\text { NWS } \\
\text { station } \\
\text { no. }\end{array}$ & County & $\begin{array}{c}\text { Elev. } \\
\text { (ft above } \\
\text { NGVD } \\
\text { 29) }\end{array}$ & $\begin{array}{l}\text { Lati- } \\
\text { tude }\end{array}$ & $\begin{array}{l}\text { Longi- } \\
\text { tude }\end{array}$ & $\begin{array}{l}\text { Begining } \\
\text { date } \\
\text { (mm/yyyy) }\end{array}$ & $\begin{array}{c}\text { Ending } \\
\text { date } \\
\text { (mm/yyyy) }\end{array}$ & $\begin{array}{l}\text { Per- } \\
\text { cent } \\
\text { cov- } \\
\text { erage }\end{array}$ & $\begin{array}{l}\text { Years } \\
\text { of } \\
\text { record }\end{array}$ \\
\hline 97 & Big Lake LCRA 140 & 776 & Reagan & 2,690 & $31^{\circ} 12^{\prime}$ & $101^{\circ} 28^{\prime}$ & $06 / 1948$ & $09 / 1951$ & 77 & 4 \\
\hline 98 & Big Spring & 786 & Howard & 2,500 & $32^{\circ} 14^{\prime}$ & $101^{\circ} 27^{\prime}$ & $01 / 1948$ & $12 / 2001$ & 97 & 54 \\
\hline 99 & Big Spring $5 \mathrm{NE}$ & 781 & Howard & 2,500 & $32^{\circ} 22^{\prime}$ & $101^{\circ} 25^{\prime}$ & 08/2001 & $11 / 2001$ & 31 & 1 \\
\hline 100 & Big Wells 2 W & 787 & Dimmit & 535 & $28^{\circ} 34^{\prime}$ & $99^{\circ} 35^{\prime}$ & $01 / 1916$ & $12 / 2001$ & 97 & 81 \\
\hline 101 & Bishop & 805 & Nueces & 67 & $27^{\circ} 35^{\prime}$ & $97^{\circ} 48^{\prime}$ & $10 / 1934$ & $12 / 2001$ & 87 & 29 \\
\hline 102 & Blackwell & 826 & Nolan* & 2,152 & $32^{\circ} 06^{\prime}$ & $100^{\circ} 18^{\prime}$ & $08 / 1936$ & $08 / 1942$ & 36 & 6 \\
\hline 103 & Blanco & 832 & Blanco & 1,370 & $30^{\circ} 06^{\prime}$ & $98^{\circ} 25^{\prime}$ & $01 / 1897$ & $12 / 2001$ & 99 & 104 \\
\hline 104 & Blanket & 839 & Brown & 1,601 & $31^{\circ} 49^{\prime}$ & $98^{\circ} 47^{\prime}$ & 03/1948 & $12 / 1965$ & 95 & 18 \\
\hline 105 & Blewett $5 \mathrm{NW}$ & 852 & Uvalde & 971 & $29^{\circ} 14^{\prime}$ & $100^{\circ} 06^{\prime}$ & $05 / 1957$ & $11 / 1985$ & 97 & 29 \\
\hline 106 & Bloys Campground & 861 & Jeff Davis & 5,764 & $30^{\circ} 32^{\prime}$ & $104^{\circ} 08^{\prime}$ & 05/1968 & 03/1978 & 89 & 11 \\
\hline 107 & Blue & 866 & Lee & 459 & $30^{\circ} 24^{\prime}$ & $97^{\circ} 09^{\prime}$ & 03/1963 & $12 / 1984$ & 93 & 22 \\
\hline 108 & Boerne & 902 & Kendall & 1,444 & $29^{\circ} 47^{\prime}$ & $98^{\circ} 44^{\prime}$ & $07 / 1897$ & $12 / 2001$ & 98 & 104 \\
\hline 109 & Bon Wier & 917 & Newton & 89 & $30^{\circ} 44^{\prime}$ & $93^{\circ} 39^{\prime}$ & $01 / 1948$ & 06/1988 & 96 & 41 \\
\hline 110 & Bonham 3 NNE & 923 & Fannin & 600 & $33^{\circ} 38^{\prime}$ & $96^{\circ} 10^{\prime}$ & 01/1903 & $12 / 2001$ & 98 & 97 \\
\hline 111 & Bonita 4 NW & 926 & Montague & 985 & $33^{\circ} 50^{\prime}$ & $97^{\circ} 39^{\prime}$ & $06 / 1948$ & $12 / 2001$ & 69 & 5 \\
\hline 112 & Booker & 944 & Lipscomb & 2,750 & $36^{\circ} 27^{\prime}$ & $100^{\circ} 32^{\prime}$ & 05/1922 & $12 / 2001$ & 90 & 70 \\
\hline 113 & Booker Blau Ranch & 942 & Lipscomb* & 2,802 & $36^{\circ} 22^{\prime}$ & $100^{\circ} 32^{\prime}$ & 01/1948 & $11 / 1955$ & 82 & 8 \\
\hline 114 & Bootleg Corner & 947 & Deaf Smith & 4,114 & $34^{\circ} 50^{\prime}$ & $102^{\circ} 49^{\prime}$ & $12 / 1976$ & $10 / 1982$ & 78 & 7 \\
\hline 115 & Boquillas Ranger Station & 950 & Brewster & 1,857 & $29^{\circ} 11^{\prime}$ & $102^{\circ} 57^{\prime}$ & $07 / 1910$ & $12 / 2001$ & 93 & 53 \\
\hline 116 & Borger & 955 & Hutchinson* & 3,114 & $35^{\circ} 39^{\prime}$ & $101^{\circ} 24^{\prime}$ & $04 / 1944$ & $10 / 1961$ & 93 & 17 \\
\hline 117 & Borger & 958 & Hutchinson & 3,067 & $35^{\circ} 41^{\prime}$ & $101^{\circ} 23^{\prime}$ & $02 / 1949$ & $12 / 2001$ & 99 & 53 \\
\hline 118 & Bovina & 977 & Parmer & 4,104 & $34^{\circ} 31^{\prime}$ & $102^{\circ} 53^{\prime}$ & 08/1917 & $06 / 1920$ & 69 & 4 \\
\hline 119 & Bowie & 984 & Montague & 1,080 & $33^{\circ} 33^{\prime}$ & $97^{\circ} 50^{\prime}$ & $01 / 1897$ & $11 / 2001$ & 98 & 77 \\
\hline 120 & Boxelder 3 NNE & 991 & Red River & 440 & $33^{\circ} 30^{\prime}$ & $94^{\circ} 51^{\prime}$ & 04/1949 & $12 / 2001$ & 99 & 53 \\
\hline 121 & Boyd & 996 & Wise & 730 & $33^{\circ} 04^{\prime}$ & $97^{\circ} 33^{\prime}$ & 01/1948 & 06/1999 & 97 & 52 \\
\hline 122 & Boys Ranch & 1000 & Oldham & 3,191 & $35^{\circ} 31^{\prime}$ & $102^{\circ} 15^{\prime}$ & 07/1964 & $12 / 2001$ & 91 & 26 \\
\hline 123 & Brackettville & 1007 & Kinney & 1,118 & $29^{\circ} 19^{\prime}$ & $100^{\circ} 24^{\prime}$ & $02 / 1900$ & $12 / 2001$ & 95 & 99 \\
\hline 124 & Brackettville $22 \mathrm{~N}$ & 1013 & Kinney & 1,675 & $29^{\circ} 36^{\prime}$ & $100^{\circ} 28^{\prime}$ & 09/1978 & $12 / 2001$ & 86 & 24 \\
\hline 125 & Brady & 1017 & McCulloch & 1,720 & $31^{\circ} 07^{\prime}$ & $99^{\circ} 20^{\prime}$ & $01 / 1897$ & $12 / 2001$ & 94 & 69 \\
\hline 126 & Brandon & 1026 & Hill & 640 & $32^{\circ} 03^{\prime}$ & $96^{\circ} 58^{\prime}$ & $01 / 1992$ & $12 / 2001$ & 99 & 10 \\
\hline 127 & Bravo & 1033 & Hartley & 4,160 & $35^{\circ} 37^{\prime}$ & $103^{\circ} 00^{\prime}$ & $01 / 1948$ & $12 / 2001$ & 98 & 54 \\
\hline 128 & Brazoria & 1034 & Brazoria* & 30 & $29^{\circ} 03^{\prime}$ & $95^{\circ} 34^{\prime}$ & $01 / 1897$ & 09/1929 & 93 & 32 \\
\hline 129 & Brazos & 1035 & Palo Pinto & 840 & $32^{\circ} 38^{\prime}$ & $98^{\circ} 08^{\prime}$ & $09 / 1915$ & $12 / 2001$ & 98 & 55 \\
\hline 130 & Breckenridge & 1042 & Stephens & 1,170 & $32^{\circ} 45^{\prime}$ & $98^{\circ} 54^{\prime}$ & $06 / 1898$ & $12 / 2001$ & 92 & 78 \\
\hline 131 & Breckenridge 2 WNW & 1043 & Stephens & 1,332 & $32^{\circ} 46^{\prime}$ & $98^{\circ} 56^{\prime}$ & 03/1973 & 03/1975 & 69 & 3 \\
\hline 132 & Bremond & 1045 & Robertson & 470 & $31^{\circ} 10^{\prime}$ & $96^{\circ} 41^{\prime}$ & $05 / 1963$ & $12 / 2001$ & 99 & 39 \\
\hline 133 & Brenham & 1048 & Washington & 313 & $30^{\circ} 10^{\prime}$ & $96^{\circ} 24^{\prime}$ & $01 / 1902$ & $12 / 2001$ & 99 & 100 \\
\hline 134 & Brewers Store $5 \mathrm{SW}$ & 1053 & Kimble* & 1,762 & $30^{\circ} 41^{\prime}$ & $99^{\circ} 33^{\prime}$ & $06 / 1948$ & 09/1951 & 82 & 4 \\
\hline 135 & Brice $2 \mathrm{~S}$ & 1057 & Hall & 2,228 & $34^{\circ} 41^{\prime}$ & $100^{\circ} 54^{\prime}$ & $06 / 1948$ & 09/1951 & 83 & 4 \\
\hline 136 & Bridgeport & 1063 & Wise & 745 & $33^{\circ} 12^{\prime}$ & $97^{\circ} 46^{\prime}$ & $09 / 1915$ & $12 / 2001$ & 99 & 86 \\
\hline 137 & Briggs & 1068 & Burnet & 1,090 & $30^{\circ} 53^{\prime}$ & $97^{\circ} 56^{\prime}$ & 06/1948 & 07/1998 & 88 & 11 \\
\hline 138 & Brighton & 1073 & Nueces* & 10 & $27^{\circ} 39^{\prime}$ & $97^{\circ} 18^{\prime}$ & $01 / 1897$ & $01 / 1920$ & 91 & 23 \\
\hline 139 & Brite Ranch & 1080 & Presidio* & 4,623 & $30^{\circ} 20^{\prime}$ & $104^{\circ} 32^{\prime}$ & 02/1949 & 08/1949 & 39 & 1 \\
\hline 140 & Britton & 1081 & Tarrant & 561 & $32^{\circ} 33^{\prime}$ & $97^{\circ} 04^{\prime}$ & 06/1948 & 07/1952 & 71 & 5 \\
\hline 141 & Broaddus & 4523 & San Augustine & 269 & $31^{\circ} 19^{\prime}$ & $94^{\circ} 17^{\prime}$ & $09 / 1947$ & $12 / 1976$ & 97 & 30 \\
\hline 142 & Broaddus $1 \mathrm{NE}$ & 1089 & San Augustine & 270 & $31^{\circ} 20^{\prime}$ & $94^{\circ} 13^{\prime}$ & 01/1977 & $12 / 2001$ & 96 & 23 \\
\hline 143 & Bronson & 1094 & Sabine & 322 & $31^{\circ} 21^{\prime}$ & $94^{\circ} 01^{\prime}$ & $07 / 1924$ & $12 / 1979$ & 95 & 52 \\
\hline 144 & Brownfield & 1127 & Terry* & 3,383 & $33^{\circ} 11^{\prime}$ & $102^{\circ} 16^{\prime}$ & 03/1914 & $10 / 1954$ & 94 & 40 \\
\hline 145 & Brownfield 2 & 1128 & Terry & 3,300 & $33^{\circ} 10^{\prime}$ & $102^{\circ} 15^{\prime}$ & $10 / 1953$ & $12 / 2001$ & 98 & 49 \\
\hline 146 & Brownsville WSO Airport & 1136 & Cameron & 19 & $25^{\circ} 54^{\prime}$ & $97^{\circ} 25^{\prime}$ & $12 / 1898$ & $12 / 2001$ & 96 & 81 \\
\hline 147 & Brownwood & 1138 & Brown & 1,385 & $31^{\circ} 40^{\prime}$ & $98^{\circ} 57^{\prime}$ & $01 / 1947$ & $12 / 2001$ & 97 & 55 \\
\hline 148 & Brunson Ranch & 1150 & Glasscock* & 2,513 & $31^{\circ} 59^{\prime}$ & $101^{\circ} 42^{\prime}$ & $01 / 1948$ & 06/1951 & 87 & 4 \\
\hline
\end{tabular}


Table 1. Summary of National Weather Service daily precipitation stations in Texas with known names, latitudes, and longitudes-Continued

\begin{tabular}{|c|c|c|c|c|c|c|c|c|c|c|}
\hline $\begin{array}{c}\text { Seq. } \\
\text { no. }\end{array}$ & NWS station name & $\begin{array}{c}\text { NWS } \\
\text { station } \\
\text { no. }\end{array}$ & County & $\begin{array}{c}\text { Elev. } \\
\text { (ft above } \\
\text { NGVD } \\
\text { 29) }\end{array}$ & $\begin{array}{l}\text { Lati- } \\
\text { tude }\end{array}$ & $\begin{array}{l}\text { Longi- } \\
\text { tude }\end{array}$ & $\begin{array}{c}\text { Begining } \\
\text { date } \\
\text { (mm/yyyy) }\end{array}$ & $\begin{array}{c}\text { Ending } \\
\text { date } \\
\text { (mm/yyyy) }\end{array}$ & $\begin{array}{l}\text { Per- } \\
\text { cent } \\
\text { cov- } \\
\text { erage }\end{array}$ & $\begin{array}{l}\text { Years } \\
\text { of } \\
\text { record }\end{array}$ \\
\hline 149 & Bryan $17 \mathrm{NE}$ & 1156 & Madison & 92 & $30^{\circ} 52^{\prime}$ & $96^{\circ} 11^{\prime}$ & $05 / 1996$ & $11 / 1996$ & 58 & 1 \\
\hline 150 & Bryan CAA Airport & 1154 & Brazos* & 266 & $30^{\circ} 38^{\prime}$ & $96^{\circ} 28^{\prime}$ & $06 / 1948$ & 07/1951 & 79 & 4 \\
\hline 151 & Buchanan Dam & 1165 & Llano* & 1,020 & $30^{\circ} 45^{\prime}$ & $98^{\circ} 25^{\prime}$ & $07 / 1943$ & $02 / 1964$ & 87 & 22 \\
\hline 152 & Buenavista 2 NNW & 1185 & Pecos* & 2,513 & $31^{\circ} 15^{\prime}$ & $102^{\circ} 40^{\prime}$ & 06/1912 & $09 / 1951$ & 94 & 23 \\
\hline 153 & Buffalo & 1188 & Leon & 358 & $31^{\circ} 28^{\prime}$ & $96^{\circ} 03^{\prime}$ & $01 / 1948$ & $11 / 1988$ & 97 & 41 \\
\hline 154 & Buler 2 NE & 1203 & Ochiltree & 2,972 & $36^{\circ} 11^{\prime}$ & $100^{\circ} 50^{\prime}$ & $01 / 1948$ & 05/1977 & 97 & 30 \\
\hline 155 & Bulverde & 1215 & Comal & 1,100 & $29^{\circ} 44^{\prime}$ & $98^{\circ} 27^{\prime}$ & $02 / 1940$ & $12 / 2001$ & 97 & 62 \\
\hline 156 & Bunker Hill & 1224 & Dallam & 4,348 & $36^{\circ} 09^{\prime}$ & $102^{\circ} 56^{\prime}$ & $01 / 1948$ & 07/1990 & 94 & 43 \\
\hline 157 & Bunton Ranch & 1227 & Presidio* & 4,403 & $29^{\circ} 54^{\prime}$ & $104^{\circ} 17^{\prime}$ & $02 / 1948$ & $04 / 1949$ & 17 & 2 \\
\hline 158 & Burkburnett $2 \mathrm{NE}$ & 1236 & Wichita & 953 & $34^{\circ} 07^{\prime}$ & $98^{\circ} 32^{\prime}$ & 06/1996 & $12 / 2001$ & 93 & 6 \\
\hline 159 & Burkett & 1239 & Coleman & 1,555 & $31^{\circ} 59^{\prime}$ & $99^{\circ} 13^{\prime}$ & $03 / 1948$ & $12 / 2001$ & 89 & 54 \\
\hline 160 & Burleson & 1246 & Johnson & 730 & $32^{\circ} 33^{\prime}$ & $97^{\circ} 19^{\prime}$ & $04 / 1985$ & $12 / 2001$ & 96 & 17 \\
\hline 161 & Burleson 2 SSW & 1245 & Johnson & 771 & $32^{\circ} 31^{\prime}$ & $97^{\circ} 20^{\prime}$ & 01/1948 & $01 / 1985$ & 97 & 38 \\
\hline 162 & Burlington 3 WSW & 1248 & Milam & 410 & $31^{\circ} 00^{\prime}$ & $97^{\circ} 02^{\prime}$ & $01 / 1948$ & $04 / 1975$ & 96 & 28 \\
\hline 163 & Burnet & 1250 & Burnet & 1,275 & $30^{\circ} 44^{\prime}$ & $98^{\circ} 14^{\prime}$ & $12 / 1896$ & $12 / 2001$ & 91 & 103 \\
\hline 164 & Burnet 9 WSW & 1253 & Burnet & 880 & $30^{\circ} 44^{\prime}$ & $98^{\circ} 25^{\prime}$ & $08 / 2000$ & $12 / 2001$ & 71 & 2 \\
\hline 165 & Bushland 1 WSW & 1267 & Potter & 3,819 & $35^{\circ} 11^{\prime}$ & $102^{\circ} 04^{\prime}$ & 06/1948 & 09/1951 & 81 & 4 \\
\hline 166 & Byrds 1 NNE & 1285 & Brown & 1,450 & $31^{\circ} 56^{\prime}$ & $99^{\circ} 02^{\prime}$ & 03/1948 & $12 / 1965$ & 96 & 18 \\
\hline 167 & Cadiz & 1304 & Bee* & 351 & $28^{\circ} 26^{\prime}$ & $97^{\circ} 57^{\prime}$ & $06 / 1948$ & 09/1951 & 83 & 4 \\
\hline 168 & Caldwell & 1314 & Burleson & 365 & $30^{\circ} 32^{\prime}$ & $96^{\circ} 42^{\prime}$ & $03 / 1963$ & $12 / 2001$ & 99 & 39 \\
\hline 169 & Calhoun & 1325 & Colorado* & 161 & $29^{\circ} 32^{\prime}$ & $96^{\circ} 20^{\prime}$ & $06 / 1948$ & 09/1951 & 83 & 4 \\
\hline 170 & Callan & 1334 & Menard & 2,123 & $31^{\circ} 03^{\prime}$ & $99^{\circ} 43^{\prime}$ & 03/1948 & $11 / 1965$ & 93 & 18 \\
\hline 171 & Calliham & 1337 & McMullen & 218 & $28^{\circ} 29^{\prime}$ & $98^{\circ} 23^{\prime}$ & $11 / 1978$ & $12 / 2001$ & 94 & 24 \\
\hline 172 & Cameron & 1348 & Milam & 364 & $30^{\circ} 50^{\prime}$ & $96^{\circ} 58^{\prime}$ & 02/1908 & $12 / 2001$ & 96 & 94 \\
\hline 173 & Cameron Ranch & 1349 & Starr* & 459 & $26^{\circ} 33^{\prime}$ & $98^{\circ} 35^{\prime}$ & $04 / 1941$ & 09/1946 & 90 & 6 \\
\hline 174 & Camp San Saba & 1385 & McCulloch & 1,690 & $31^{\circ} 00^{\prime}$ & $99^{\circ} 16^{\prime}$ & $03 / 1948$ & $12 / 1965$ & 94 & 18 \\
\hline 175 & Camp Verde $3 \mathrm{~W}$ & 1395 & Kerr & 1,800 & $29^{\circ} 53^{\prime}$ & $99^{\circ} 08^{\prime}$ & 02/1997 & $12 / 2001$ & 76 & 5 \\
\hline 176 & Camp Wood & 1398 & Real & 1,470 & $29^{\circ} 40^{\prime}$ & $100^{\circ} 01^{\prime}$ & 03/1944 & $12 / 2001$ & 96 & 57 \\
\hline 177 & Canadian & 1412 & Hemphill & 2,300 & $35^{\circ} 54^{\prime}$ & $100^{\circ} 23^{\prime}$ & 11/1906 & $11 / 2001$ & 93 & 95 \\
\hline 178 & Canadian No. 2 & 1413 & Hemphill & 2,440 & $35^{\circ} 54^{\prime}$ & $100^{\circ} 22^{\prime}$ & 08/1946 & $02 / 1949$ & 23 & 4 \\
\hline 179 & Candelaria & 1416 & Presidio & 2,875 & $30^{\circ} 08^{\prime}$ & $104^{\circ} 40^{\prime}$ & $02 / 1948$ & $12 / 2001$ & 99 & 54 \\
\hline 180 & Canton & 1425 & Van Zandt & 490 & $32^{\circ} 33^{\prime}$ & $95^{\circ} 52^{\prime}$ & $01 / 1948$ & $06 / 2001$ & 91 & 50 \\
\hline 181 & Canyon & 1430 & Randall & 3,590 & $34^{\circ} 58^{\prime}$ & $101^{\circ} 55^{\prime}$ & 04/1923 & $12 / 2001$ & 98 & 79 \\
\hline 182 & Canyon Dam & 1429 & Comal & 1,000 & $29^{\circ} 52^{\prime}$ & $98^{\circ} 11^{\prime}$ & $06 / 1961$ & $12 / 2001$ & 93 & 41 \\
\hline 183 & Canyon Dam No. 1 & 1431 & Comal & 980 & $29^{\circ} 51^{\prime}$ & $98^{\circ} 17^{\prime}$ & 08/1996 & $12 / 2001$ & 88 & 6 \\
\hline 184 & Canyon Dam No. 7 & 1438 & Comal & 978 & $29^{\circ} 55^{\prime}$ & $98^{\circ} 13^{\prime}$ & $10 / 1990$ & $04 / 1993$ & 59 & 4 \\
\hline 185 & Capps & 1437 & Moore* & 3,304 & $36^{\circ} 03^{\prime}$ & $101^{\circ} 38^{\prime}$ & 05/1948 & $12 / 1956$ & 67 & 9 \\
\hline 186 & Capps Ranch & 1439 & Mason* & 1,703 & $30^{\circ} 50^{\prime}$ & $99^{\circ} 07^{\prime}$ & 03/1948 & 05/1959 & 52 & 12 \\
\hline 187 & Carbon & 1451 & Eastland & 1,590 & $32^{\circ} 16^{\prime}$ & $98^{\circ} 50^{\prime}$ & $11 / 2001$ & $12 / 2001$ & 17 & 1 \\
\hline 188 & Carr Ranch & 1481 & Gillespie & 2,001 & $30^{\circ} 10^{\prime}$ & $99^{\circ} 07^{\prime}$ & $07 / 1920$ & $11 / 1961$ & 97 & 42 \\
\hline 189 & Carrizo Springs & 1486 & Dimmit & 613 & $28^{\circ} 29^{\prime}$ & $99^{\circ} 52^{\prime}$ & $04 / 1912$ & $12 / 2001$ & 93 & 78 \\
\hline 190 & Carrollton & 1490 & Dallas & 545 & $32^{\circ} 59^{\prime}$ & $96^{\circ} 55^{\prime}$ & $01 / 1948$ & 03/2001 & 96 & 54 \\
\hline 191 & Carta Valley $4 \mathrm{~W}$ & 1492 & Val Verde & 1,780 & $29^{\circ} 48^{\prime}$ & $100^{\circ} 44^{\prime}$ & $08 / 1963$ & $12 / 2000$ & 92 & 38 \\
\hline 192 & Carthage & 1500 & Panola & 340 & $32^{\circ} 08^{\prime}$ & $94^{\circ} 21^{\prime}$ & 01/1908 & $12 / 2001$ & 96 & 52 \\
\hline 193 & Case Ranch $3 \mathrm{~S}$ & 1511 & Sterling & 2,192 & $31^{\circ} 38^{\prime}$ & $101^{\circ} 02^{\prime}$ & 03/1948 & $12 / 1983$ & 93 & 36 \\
\hline 194 & Castell & 1521 & Llano & 1,211 & $30^{\circ} 42^{\prime}$ & $98^{\circ} 58^{\prime}$ & 03/1948 & $02 / 1980$ & 53 & 33 \\
\hline 195 & Castolon & 1524 & Brewster & 2,169 & $29^{\circ} 08^{\prime}$ & $103^{\circ} 30^{\prime}$ & $02 / 1947$ & $12 / 2001$ & 93 & 28 \\
\hline 196 & Catarina & 1528 & Dimmit & 560 & $28^{\circ} 20^{\prime}$ & $99^{\circ} 37^{\prime}$ & $04 / 1959$ & $10 / 2001$ & 92 & 43 \\
\hline 197 & Cedar Creek 4 SE & 1541 & Bastrop & 470 & $30^{\circ} 02^{\prime}$ & $97^{\circ} 27^{\prime}$ & 04/1978 & $12 / 2001$ & 96 & 23 \\
\hline 198 & Celina & 1573 & Collin & 679 & $33^{\circ} 19^{\prime}$ & $96^{\circ} 48^{\prime}$ & $01 / 1948$ & $02 / 1983$ & 96 & 36 \\
\hline 199 & Center & 1578 & Shelby & 325 & $31^{\circ} 48^{\prime}$ & $94^{\circ} 09^{\prime}$ & $07 / 1922$ & $12 / 2001$ & 95 & 66 \\
\hline 200 & Center City & 1580 & Mills & 1,365 & $31^{\circ} 28^{\prime}$ & $98^{\circ} 25^{\prime}$ & $05 / 1963$ & $12 / 2001$ & 99 & 39 \\
\hline
\end{tabular}


Table 1. Summary of National Weather Service daily precipitation stations in Texas with known names, latitudes, and longitudes-Continued

\begin{tabular}{|c|c|c|c|c|c|c|c|c|c|c|}
\hline $\begin{array}{c}\text { Seq. } \\
\text { no. }\end{array}$ & NWS station name & $\begin{array}{c}\text { NWS } \\
\text { station } \\
\text { no. }\end{array}$ & County & $\begin{array}{c}\text { Elev. } \\
\text { (ft above } \\
\text { NGVD } \\
\text { 29) }\end{array}$ & $\begin{array}{l}\text { Lati- } \\
\text { tude }\end{array}$ & $\begin{array}{l}\text { Longi- } \\
\text { tude }\end{array}$ & $\begin{array}{c}\text { Begining } \\
\text { date } \\
\text { (mm/yyyy) }\end{array}$ & $\begin{array}{c}\text { Ending } \\
\text { date } \\
\text { (mm/yyyy) }\end{array}$ & $\begin{array}{l}\text { Per- } \\
\text { cent } \\
\text { cov- } \\
\text { erage }\end{array}$ & $\begin{array}{l}\text { Years } \\
\text { of } \\
\text { record }\end{array}$ \\
\hline 201 & Centerville & 1596 & Leon & 320 & $31^{\circ} 15^{\prime}$ & $95^{\circ} 58^{\prime}$ & $05 / 1937$ & $12 / 2001$ & 97 & 65 \\
\hline 202 & Chalk & 1622 & Cottle & 1,780 & $33^{\circ} 53^{\prime}$ & $100^{\circ} 13^{\prime}$ & $09 / 1990$ & $12 / 1991$ & 66 & 2 \\
\hline 203 & Chalk Mountain & 1625 & Erath & 1,150 & $32^{\circ} 09^{\prime}$ & $97^{\circ} 56^{\prime}$ & $04 / 1963$ & $12 / 2001$ & 99 & 39 \\
\hline 204 & Chambers Hill Guard Station & 1632 & Sabine* & 351 & $31^{\circ} 28^{\prime}$ & $93^{\circ} 50^{\prime}$ & $01 / 1948$ & $03 / 1952$ & 76 & 5 \\
\hline 205 & Chancellor & 1641 & Pecos* & 3,402 & $30^{\circ} 42^{\prime}$ & $103^{\circ} 11^{\prime}$ & $06 / 1948$ & 09/1951 & 26 & 4 \\
\hline 206 & Channing & 1646 & Hartley & 3,800 & $35^{\circ} 41^{\prime}$ & $102^{\circ} 20^{\prime}$ & 08/1904 & $09 / 1951$ & 73 & 11 \\
\hline 207 & Channing $11 \mathrm{NE}$ & 1650 & Moore & 3,725 & $35^{\circ} 46^{\prime}$ & $102^{\circ} 09^{\prime}$ & 03/1990 & 07/1991 & 71 & 2 \\
\hline 208 & Channing 2 & 1649 & Hartley & 3,790 & $35^{\circ} 41^{\prime}$ & $102^{\circ} 19^{\prime}$ & $05 / 1967$ & $12 / 2001$ & 90 & 25 \\
\hline 209 & Chapman Ranch & 1651 & Nueces & 25 & $27^{\circ} 35^{\prime}$ & $97^{\circ} 27^{\prime}$ & $04 / 1959$ & $12 / 2001$ & 97 & 43 \\
\hline 210 & Chappel & 1655 & San Saba* & 1,240 & $31^{\circ} 04^{\prime}$ & $98^{\circ} 34^{\prime}$ & $03 / 1948$ & $12 / 1959$ & 98 & 12 \\
\hline 211 & Charlie & 1659 & Clay & 950 & $34^{\circ} 06^{\prime}$ & $98^{\circ} 19^{\prime}$ & $06 / 1996$ & $12 / 2001$ & 93 & 6 \\
\hline 212 & Charlotte $5 \mathrm{NNW}$ & 1663 & Atascosa & 441 & $28^{\circ} 55^{\prime}$ & $98^{\circ} 44^{\prime}$ & $07 / 1962$ & $12 / 2001$ & 98 & 40 \\
\hline 213 & Cheapside & 1671 & Gonzales & 310 & $29^{\circ} 18^{\prime}$ & $97^{\circ} 20^{\prime}$ & $06 / 1948$ & $12 / 2001$ & 87 & 10 \\
\hline 214 & Cherokee & 1680 & San Saba & 1,490 & $30^{\circ} 59^{\prime}$ & $98^{\circ} 43^{\prime}$ & $06 / 1948$ & 09/1951 & 80 & 4 \\
\hline 215 & Childress $13 \mathrm{NW}$ & 1697 & Childress & 1,713 & $34^{\circ} 34^{\prime}$ & $100^{\circ} 20^{\prime}$ & $02 / 1975$ & 06/1978 & 54 & 4 \\
\hline 216 & Childress 2 & 1694 & Childress & 1,940 & $34^{\circ} 25^{\prime}$ & $100^{\circ} 12^{\prime}$ & $11 / 1997$ & $12 / 2001$ & 83 & 5 \\
\hline 217 & Childress 3 WSW & 1696 & Childress & 1,972 & $34^{\circ} 26^{\prime}$ & $100^{\circ} 15^{\prime}$ & $01 / 1897$ & $12 / 1946$ & 94 & 44 \\
\hline 218 & Childress Municipal Airport & 1698 & Childress & 1,951 & $34^{\circ} 25^{\prime}$ & $100^{\circ} 17^{\prime}$ & $01 / 1948$ & $12 / 2001$ & 96 & 53 \\
\hline 219 & Childress Ranch & 1699 & Jeff Davis* & $* 4,413$ & $31^{\circ} 00^{\prime}$ & $104^{\circ} 02^{\prime}$ & $01 / 1948$ & $01 / 1948$ & 8 & 1 \\
\hline 220 & Chillicothe $2 \mathrm{NE}$ & 1701 & Hardeman & 1,401 & $34^{\circ} 15^{\prime}$ & $99^{\circ} 31^{\prime}$ & $09 / 1906$ & 03/1975 & 96 & 70 \\
\hline 221 & Chireno & 1711 & Nacogdoches & 330 & $31^{\circ} 30^{\prime}$ & $94^{\circ} 21^{\prime}$ & $02 / 1989$ & $12 / 2001$ & 99 & 13 \\
\hline 222 & Chisos Basin & 1715 & Brewster & 5,300 & $29^{\circ} 16^{\prime}$ & $103^{\circ} 18^{\prime}$ & $02 / 1947$ & $12 / 2001$ & 98 & 55 \\
\hline 223 & Choke Canyon Dam & 1720 & Live Oak & 230 & $28^{\circ} 28^{\prime}$ & $98^{\circ} 15^{\prime}$ & $10 / 1983$ & $12 / 2001$ & 94 & 19 \\
\hline 224 & Christoval & 1735 & Tom Green & 2,031 & $31^{\circ} 12^{\prime}$ & $100^{\circ} 29^{\prime}$ & $03 / 1948$ & $12 / 1965$ & 93 & 18 \\
\hline 225 & Cibolo Creek & 1741 & Karnes & 312 & $29^{\circ} 01^{\prime}$ & $97^{\circ} 56^{\prime}$ & $01 / 1948$ & $10 / 1982$ & 92 & 35 \\
\hline 226 & Clarendon & 1761 & Donley & 2,700 & $34^{\circ} 55^{\prime}$ & $100^{\circ} 53^{\prime}$ & $05 / 1904$ & $12 / 2001$ & 95 & 96 \\
\hline 227 & Clarksville $1 \mathrm{~W}$ & 1773 & Red River & 426 & $33^{\circ} 36^{\prime}$ & $95^{\circ} 01^{\prime}$ & $06 / 1948$ & 09/1951 & 82 & 4 \\
\hline 228 & Clarksville $2 \mathrm{NE}$ & 1772 & Red River & 435 & $33^{\circ} 36^{\prime}$ & $95^{\circ} 04^{\prime}$ & $03 / 1903$ & $12 / 2001$ & 96 & 97 \\
\hline 229 & Classens Ranch & 1777 & Bexar & 1,001 & $29^{\circ} 39^{\prime}$ & $98^{\circ} 22^{\prime}$ & $01 / 1947$ & 05/1972 & 96 & 26 \\
\hline 230 & Claude & 1778 & Armstrong & 3,395 & $35^{\circ} 06^{\prime}$ & $101^{\circ} 21^{\prime}$ & $10 / 1904$ & $12 / 2001$ & 89 & 98 \\
\hline 231 & Cleburne & 1800 & Johnson & 783 & $32^{\circ} 20^{\prime}$ & $97^{\circ} 24^{\prime}$ & $01 / 1907$ & $12 / 2001$ & 96 & 94 \\
\hline 232 & Cleburne 7 SE & 1804 & Johnson & 830 & $32^{\circ} 19^{\prime}$ & $97^{\circ} 18^{\prime}$ & $03 / 1995$ & $12 / 2001$ & 98 & 7 \\
\hline 233 & Cleveland & 1810 & Liberty & 196 & $30^{\circ} 21^{\prime}$ & $95^{\circ} 05^{\prime}$ & $06 / 1954$ & $12 / 2001$ & 99 & 48 \\
\hline 234 & Clifton 2 & 1824 & Bosque & 765 & $31^{\circ} 47^{\prime}$ & $97^{\circ} 35^{\prime}$ & $01 / 1992$ & $10 / 1994$ & 94 & 3 \\
\hline 235 & Clifton $9 \mathrm{E}$ & 1823 & Bosque & 669 & $31^{\circ} 48^{\prime}$ & $97^{\circ} 26^{\prime}$ & $03 / 1911$ & 07/1975 & 98 & 65 \\
\hline 236 & Cline & 1832 & Uvalde & 1,001 & $29^{\circ} 15^{\prime}$ & $100^{\circ} 05^{\prime}$ & $06 / 1940$ & $04 / 1957$ & 87 & 18 \\
\hline 237 & Clodine & 1838 & Fort Bend & 87 & $29^{\circ} 42^{\prime}$ & $95^{\circ} 41^{\prime}$ & $01 / 1943$ & $12 / 2001$ & 91 & 56 \\
\hline 238 & Cloudt Ranch & 1843 & Edwards & 2,251 & $30^{\circ} 11^{\prime}$ & $100^{\circ} 22^{\prime}$ & $01 / 1949$ & 08/1964 & 73 & 16 \\
\hline 239 & Coldspring 5 SSW & 1870 & San Jacinto & 355 & $30^{\circ} 32^{\prime}$ & $95^{\circ} 09^{\prime}$ & $06 / 1954$ & $12 / 2001$ & 99 & 48 \\
\hline 240 & Coldwater & 1874 & Dallam & 4,134 & $36^{\circ} 24^{\prime}$ & $102^{\circ} 34^{\prime}$ & $03 / 1941$ & $10 / 1983$ & 85 & 42 \\
\hline 241 & Coleman & 1875 & Coleman & 1,727 & $31^{\circ} 49^{\prime}$ & $99^{\circ} 25^{\prime}$ & $12 / 1896$ & $12 / 2001$ & 98 & 105 \\
\hline 242 & College Station Easterwood & 1889 & Brazos & 314 & $30^{\circ} 35^{\prime}$ & $96^{\circ} 21^{\prime}$ & $08 / 1951$ & $12 / 2001$ & 98 & 49 \\
\hline 243 & College Station $6 \mathrm{SW}$ & 1888 & Brazos & 175 & $30^{\circ} 32^{\prime}$ & $96^{\circ} 25^{\prime}$ & $10 / 1901$ & 05/1984 & 94 & 60 \\
\hline 244 & College Station Agricultural Experiment Station & 1891 & Burleson & 225 & $30^{\circ} 33^{\prime}$ & $96^{\circ} 26^{\prime}$ & $06 / 1981$ & 08/1981 & 25 & 1 \\
\hline 245 & Colorado City & 1903 & Mitchell & 2,105 & $32^{\circ} 23^{\prime}$ & $100^{\circ} 53^{\prime}$ & $07 / 1898$ & $12 / 2001$ & 93 & 78 \\
\hline 246 & Columbus & 1911 & Colorado & 199 & $29^{\circ} 43^{\prime}$ & $96^{\circ} 32^{\prime}$ & $04 / 1915$ & $12 / 2001$ & 98 & 56 \\
\hline 247 & Comanche & 1914 & Comanche & 1,345 & $31^{\circ} 54^{\prime}$ & $98^{\circ} 35^{\prime}$ & $04 / 1901$ & $12 / 2001$ & 95 & 88 \\
\hline 248 & Comfort 2 & 1920 & Kendall & 1,435 & $29^{\circ} 57^{\prime}$ & $98^{\circ} 53^{\prime}$ & 08/1996 & $12 / 2001$ & 90 & 6 \\
\hline 249 & Commerce $4 \mathrm{SW}$ & 1921 & Hunt & 550 & $33^{\circ} 12^{\prime}$ & $95^{\circ} 56^{\prime}$ & $08 / 1948$ & $12 / 2001$ & 82 & 10 \\
\hline 250 & Comstock & 1925 & Val Verde & 1,584 & $29^{\circ} 41^{\prime}$ & $101^{\circ} 11^{\prime}$ & $11 / 1903$ & 09/1987 & 81 & 25 \\
\hline 251 & Comstock $11 \mathrm{WNW}$ & 1926 & Val Verde & 1,260 & $29^{\circ} 42^{\prime}$ & $101^{\circ} 21^{\prime}$ & 03/1992 & $01 / 1993$ & 33 & 2 \\
\hline 252 & Concepcion $3 \mathrm{~S}$ & 1932 & Duval & 260 & $27^{\circ} 21^{\prime}$ & $98^{\circ} 22^{\prime}$ & 05/1941 & 07/2001 & 79 & 8 \\
\hline
\end{tabular}


Table 1. Summary of National Weather Service daily precipitation stations in Texas with known names, latitudes, and longitudes-Continued

\begin{tabular}{|c|c|c|c|c|c|c|c|c|c|c|}
\hline $\begin{array}{c}\text { Seq. } \\
\text { no. }\end{array}$ & NWS station name & $\begin{array}{c}\text { NWS } \\
\text { station } \\
\text { no. }\end{array}$ & County & $\begin{array}{c}\text { Elev. } \\
\text { (ft above } \\
\text { NGVD } \\
\text { 29) }\end{array}$ & $\begin{array}{l}\text { Lati- } \\
\text { tude }\end{array}$ & $\begin{array}{l}\text { Longi- } \\
\text { tude }\end{array}$ & $\begin{array}{l}\text { Begining } \\
\text { date } \\
\text { (mm/yyyy) }\end{array}$ & $\begin{array}{c}\text { Ending } \\
\text { date } \\
\text { (mm/yyyy) }\end{array}$ & $\begin{array}{l}\text { Per- } \\
\text { cent } \\
\text { cov- } \\
\text { erage }\end{array}$ & $\begin{array}{l}\text { Years } \\
\text { of } \\
\text { record }\end{array}$ \\
\hline 253 & Concho Park Ivie Reservoir & 1934 & Concho & 1,575 & $31^{\circ} 33^{\prime}$ & $99^{\circ} 42^{\prime}$ & $03 / 1998$ & $12 / 2001$ & 96 & 4 \\
\hline 254 & Conlen & 1946 & Dallam & 3,820 & $36^{\circ} 14^{\prime}$ & $102^{\circ} 14^{\prime}$ & $01 / 1948$ & $12 / 2001$ & 99 & 54 \\
\hline 255 & Conroe & 1956 & Montgomery & 245 & $30^{\circ} 20^{\prime}$ & $95^{\circ} 29^{\prime}$ & $01 / 1948$ & $12 / 2001$ & 98 & 54 \\
\hline 256 & Cooper & 1970 & Delta & 480 & $33^{\circ} 22^{\prime}$ & $95^{\circ} 41^{\prime}$ & $01 / 1948$ & $02 / 2001$ & 92 & 54 \\
\hline 257 & Coopers Store & 1972 & Brewster* & 2,904 & $29^{\circ} 35^{\prime}$ & $103^{\circ} 08^{\prime}$ & 02/1948 & $04 / 1951$ & 42 & 4 \\
\hline 258 & Cope Ranch & 1974 & Reagan & 2,480 & $31^{\circ} 31^{\prime}$ & $101^{\circ} 16^{\prime}$ & 03/1948 & $12 / 2001$ & 99 & 54 \\
\hline 259 & Copper Breaks State Park & 1995 & Hardeman & 1,475 & $34^{\circ} 06^{\prime}$ & $99^{\circ} 44^{\prime}$ & 05/1989 & $12 / 2001$ & 95 & 13 \\
\hline 260 & Copperas Cove & 1984 & Coryell & 1,070 & $31^{\circ} 07^{\prime}$ & $97^{\circ} 54^{\prime}$ & 09/1915 & 07/1983 & 92 & 66 \\
\hline 261 & Copperas Cove 2 & 1986 & Coryell & 1,240 & $31^{\circ} 07^{\prime}$ & $97^{\circ} 55^{\prime}$ & $02 / 1966$ & $01 / 1970$ & 80 & 5 \\
\hline 262 & Copperas Cove $5 \mathrm{NW}$ & 1990 & Coryell & 1,230 & $31^{\circ} 10^{\prime}$ & $97^{\circ} 58^{\prime}$ & $08 / 1983$ & $12 / 2001$ & 97 & 19 \\
\hline 263 & Cornudas Service Station & 2012 & Hudspeth & 4,480 & $31^{\circ} 47^{\prime}$ & $105^{\circ} 28^{\prime}$ & 05/1948 & 05/2001 & 94 & 54 \\
\hline 264 & Corpus Christi & 2014 & Nueces & 10 & $27^{\circ} 48^{\prime}$ & $97^{\circ} 24^{\prime}$ & $12 / 1946$ & 08/1980 & 93 & 35 \\
\hline 265 & Corpus Christi WSO Airport & 2015 & Nueces & 41 & $27^{\circ} 46^{\prime}$ & $97^{\circ} 30^{\prime}$ & $01 / 1948$ & $12 / 2001$ & 100 & 54 \\
\hline 266 & Corrigan 1 ENE & 2017 & Polk & 200 & $30^{\circ} 59^{\prime}$ & $94^{\circ} 49^{\prime}$ & $01 / 1992$ & $12 / 2001$ & 91 & 10 \\
\hline 267 & Corsicana & 2019 & Navarro & 413 & $32^{\circ} 06^{\prime}$ & $96^{\circ} 28^{\prime}$ & $01 / 1897$ & $12 / 2001$ & 98 & 102 \\
\hline 268 & Corsicana $8 \mathrm{E}$ & 2020 & Navarro & 376 & $32^{\circ} 07^{\prime}$ & $96^{\circ} 20^{\prime}$ & $01 / 1992$ & $12 / 2001$ & 100 & 10 \\
\hline 269 & Coryell City & 2024 & Coryell & 971 & $31^{\circ} 33^{\prime}$ & $97^{\circ} 37^{\prime}$ & $06 / 1948$ & 09/1951 & 49 & 4 \\
\hline 270 & Cottonwood & 2040 & Gillespie & 2,235 & $30^{\circ} 09^{\prime}$ & $99^{\circ} 08^{\prime}$ & $07 / 1962$ & $12 / 2001$ & 97 & 40 \\
\hline 271 & Cottonwood Dam No. 2 & 2043 & El Paso & $* 3,910$ & $31^{\circ} 32^{\prime}$ & $106^{\circ} 04^{\prime}$ & $06 / 1948$ & $11 / 1948$ & 42 & 1 \\
\hline 272 & Cottonwood Dam 1 & 2042 & El Paso* & 3,852 & $31^{\circ} 33^{\prime}$ & $106^{\circ} 05^{\prime}$ & $09 / 1948$ & 03/1949 & 21 & 2 \\
\hline 273 & Cotulla & 2048 & La Salle & 452 & $28^{\circ} 27^{\prime}$ & $99^{\circ} 13^{\prime}$ & 06/1901 & $12 / 2001$ & 94 & 93 \\
\hline 274 & Cotulla FAA Airport & 2050 & La Salle & 463 & $28^{\circ} 27^{\prime}$ & $99^{\circ} 13^{\prime}$ & $10 / 1949$ & $10 / 1981$ & 94 & 33 \\
\hline 275 & Cotulla Hillje Ranch & 2051 & La Salle & 331 & $28^{\circ} 14^{\prime}$ & $99^{\circ} 04^{\prime}$ & $04 / 1956$ & $10 / 1964$ & 82 & 8 \\
\hline 276 & Cox Ranch & 2066 & Concho & 1,903 & $31^{\circ} 21^{\prime}$ & $99^{\circ} 53^{\prime}$ & 03/1948 & $12 / 1965$ & 98 & 18 \\
\hline 277 & Crabb 2 W & 2073 & Fort Bend* & 112 & $29^{\circ} 32^{\prime}$ & $95^{\circ} 45^{\prime}$ & $06 / 1948$ & 09/1951 & 77 & 4 \\
\hline 278 & Crandall & 2080 & Kaufman & 425 & $32^{\circ} 38^{\prime}$ & $96^{\circ} 27^{\prime}$ & $09 / 1960$ & 01/1994 & 93 & 35 \\
\hline 279 & Crane $10 \mathrm{~N}$ & 2084 & Crane & 2,630 & $31^{\circ} 40^{\prime}$ & $102^{\circ} 20^{\prime}$ & $11 / 1999$ & $12 / 2001$ & 67 & 3 \\
\hline 280 & Crane $2 \mathrm{E}$ & 2082 & Crane & 2,602 & $31^{\circ} 24^{\prime}$ & $102^{\circ} 18^{\prime}$ & $05 / 1928$ & $12 / 2001$ & 94 & 48 \\
\hline 281 & Cranfills Gap & 2086 & Bosque & 975 & $31^{\circ} 46^{\prime}$ & $97^{\circ} 50^{\prime}$ & 07/1948 & $12 / 2001$ & 86 & 14 \\
\hline 282 & Crawford & 2088 & McLennan & 705 & $31^{\circ} 32^{\prime}$ & $97^{\circ} 26^{\prime}$ & $01 / 1992$ & $12 / 2001$ & 73 & 5 \\
\hline 283 & Cresson & 2096 & Hood & 1,050 & $32^{\circ} 32^{\prime}$ & $97^{\circ} 37^{\prime}$ & 06/1948 & $12 / 2001$ & 98 & 54 \\
\hline 284 & Crider Ranch & 2104 & Real & 2,323 & $30^{\circ} 04^{\prime}$ & $99^{\circ} 44^{\prime}$ & $01 / 1948$ & 05/1975 & 93 & 28 \\
\hline 285 & Crockett & 2114 & Houston & 347 & $31^{\circ} 18^{\prime}$ & $95^{\circ} 27^{\prime}$ & 08/1904 & 09/2001 & 97 & 92 \\
\hline 286 & Crosbyton & 2121 & Crosby & 3,010 & $33^{\circ} 35^{\prime}$ & $101^{\circ} 14^{\prime}$ & $01 / 1897$ & $12 / 2001$ & 98 & 104 \\
\hline 287 & Cross & 2125 & McMullen & 285 & $28^{\circ} 35^{\prime}$ & $98^{\circ} 33^{\prime}$ & 01/1997 & $12 / 2001$ & 100 & 5 \\
\hline 288 & Cross Plains 1 & 2128 & Callahan & 1,742 & $32^{\circ} 07^{\prime}$ & $99^{\circ} 10^{\prime}$ & 04/1939 & 09/1976 & 95 & 38 \\
\hline 289 & Cross Plains 2 & 2131 & Callahan & 1,790 & $32^{\circ} 07^{\prime}$ & $99^{\circ} 09^{\prime}$ & 06/1948 & 09/1951 & 78 & 4 \\
\hline 290 & Crowell & 2142 & Foard & 1,455 & $33^{\circ} 59^{\prime}$ & $99^{\circ} 43^{\prime}$ & 08/1916 & $12 / 2001$ & 97 & 86 \\
\hline 291 & Crystal City & 2160 & Zavala & 580 & $28^{\circ} 40^{\prime}$ & $99^{\circ} 49^{\prime}$ & 07/1948 & $12 / 2001$ & 99 & 54 \\
\hline 292 & Cuero & 2170 & DeWitt* & 180 & $29^{\circ} 04^{\prime}$ & $97^{\circ} 17^{\prime}$ & $01 / 1948$ & $12 / 1953$ & 100 & 6 \\
\hline 293 & Cuero & 2173 & DeWitt & 178 & $29^{\circ} 05^{\prime}$ & $97^{\circ} 19^{\prime}$ & $12 / 1901$ & $12 / 2001$ & 97 & 100 \\
\hline 294 & Cypress & 2206 & Harris & 150 & $29^{\circ} 58^{\prime}$ & $95^{\circ} 42^{\prime}$ & $01 / 1948$ & $12 / 2001$ & 97 & 53 \\
\hline 295 & Cypress Mill & 2210 & Blanco & 922 & $30^{\circ} 23^{\prime}$ & $98^{\circ} 15^{\prime}$ & $03 / 1948$ & $09 / 1964$ & 96 & 17 \\
\hline 296 & Dacus & 2218 & Montgomery & 240 & $30^{\circ} 25^{\prime}$ & $95^{\circ} 47^{\prime}$ & $06 / 1954$ & $12 / 2001$ & 98 & 48 \\
\hline 297 & Daingerfield $9 \mathrm{~S}$ & 2225 & Morris & 300 & $32^{\circ} 55^{\prime}$ & $94^{\circ} 43^{\prime}$ & $01 / 1948$ & $12 / 2001$ & 99 & 54 \\
\hline 298 & Dallas-Fort Worth International Airport & 2242 & Tarrant & 560 & $32^{\circ} 53^{\prime}$ & $97^{\circ} 01^{\prime}$ & 01/1974 & $12 / 2001$ & 100 & 28 \\
\hline 299 & Dalhart & 2238 & Dallam* & 4,003 & $36^{\circ} 05^{\prime}$ & $102^{\circ} 29^{\prime}$ & $04 / 1948$ & $12 / 1948$ & 64 & 1 \\
\hline 300 & Dalhart $6 \mathrm{SW}$ & 2235 & Hartley & 4,000 & $36^{\circ} 00^{\prime}$ & $102^{\circ} 36^{\prime}$ & 08/1988 & $12 / 2001$ & 95 & 14 \\
\hline 301 & Dalhart Experiment Station & 2239 & Hartley* & 4,003 & $36^{\circ} 01^{\prime}$ & $102^{\circ} 35^{\prime}$ & $11 / 1905$ & $12 / 1953$ & 94 & 49 \\
\hline 302 & Dalhart FAA Airport & 2240 & Hartley & 3,990 & $36^{\circ} 01^{\prime}$ & $102^{\circ} 32^{\prime}$ & $12 / 1948$ & $12 / 2001$ & 98 & 54 \\
\hline 303 & Dallas Arboretum & 2228 & Dallas & 495 & $32^{\circ} 49^{\prime}$ & $96^{\circ} 43^{\prime}$ & 04/1989 & $07 / 1990$ & 67 & 2 \\
\hline 304 & Dallas Love Field & 2244 & Dallas & 440 & $32^{\circ} 51^{\prime}$ & $96^{\circ} 51^{\prime}$ & $01 / 1897$ & $12 / 2001$ & 97 & 71 \\
\hline
\end{tabular}


Table 1. Summary of National Weather Service daily precipitation stations in Texas with known names, latitudes, and longitudes-Continued

\begin{tabular}{|c|c|c|c|c|c|c|c|c|c|c|}
\hline $\begin{array}{l}\text { Seq. } \\
\text { no. }\end{array}$ & NWS station name & $\begin{array}{l}\text { NWS } \\
\text { station } \\
\text { no. }\end{array}$ & County & $\begin{array}{c}\text { Elev. } \\
\text { (ft above } \\
\text { NGVD } \\
\text { 29) }\end{array}$ & $\begin{array}{l}\text { Lati- } \\
\text { tude }\end{array}$ & $\begin{array}{l}\text { Longi- } \\
\text { tude }\end{array}$ & $\begin{array}{c}\text { Begining } \\
\text { date } \\
\text { (mm/yyyy) }\end{array}$ & $\begin{array}{c}\text { Ending } \\
\text { date } \\
\text { (mm/yyyy) }\end{array}$ & $\begin{array}{l}\text { Per- } \\
\text { cent } \\
\text { cov- } \\
\text { erage }\end{array}$ & $\begin{array}{l}\text { Years } \\
\text { of } \\
\text { record }\end{array}$ \\
\hline 305 & Dallas WFAA & 2247 & Dallas* & 479 & $32^{\circ} 46^{\prime}$ & $96^{\circ} 47^{\prime}$ & $11 / 1941$ & $04 / 1951$ & 53 & 8 \\
\hline 306 & Danevang $1 \mathrm{~W}$ & 2266 & Wharton & 70 & $29^{\circ} 03^{\prime}$ & $96^{\circ} 13^{\prime}$ & $01 / 1897$ & $12 / 2001$ & 98 & 104 \\
\hline 307 & Darrouzett & 2282 & Lipscomb & 2,540 & $36^{\circ} 26^{\prime}$ & $100^{\circ} 19^{\prime}$ & $11 / 1941$ & $12 / 2001$ & 95 & 61 \\
\hline 308 & Davilla $2 \mathrm{~N}$ & 2295 & Milam & 556 & $30^{\circ} 48^{\prime}$ & $97^{\circ} 16^{\prime}$ & $01 / 1948$ & $12 / 2001$ & 90 & 54 \\
\hline 309 & Dawson & 2309 & Navarro* & 479 & $31^{\circ} 54^{\prime}$ & $96^{\circ} 43^{\prime}$ & $06 / 1948$ & 09/1951 & 80 & 4 \\
\hline 310 & De Leon $6 \mathrm{~W}$ & 2350 & Comanche & 1,330 & $32^{\circ} 06^{\prime}$ & $98^{\circ} 39^{\prime}$ & $01 / 1992$ & $12 / 2001$ & 85 & 10 \\
\hline 311 & De Long Ranch & 2317 & Schleicher* & 2,402 & $30^{\circ} 59^{\prime}$ & $100^{\circ} 48^{\prime}$ & $04 / 1948$ & $11 / 1958$ & 95 & 11 \\
\hline 312 & Decatur & 2334 & Wise & 1,025 & $33^{\circ} 13^{\prime}$ & $97^{\circ} 35^{\prime}$ & $10 / 1904$ & $12 / 2001$ & 91 & 56 \\
\hline 313 & Decatur 2 SSW & 2338 & Wise & 922 & $33^{\circ} 12^{\prime}$ & $97^{\circ} 36^{\prime}$ & $01 / 1966$ & 09/1972 & 96 & 7 \\
\hline 314 & Decatur $7 \mathrm{~N}$ & 2336 & Wise* & 902 & $33^{\circ} 20^{\prime}$ & $97^{\circ} 35^{\prime}$ & $06 / 1948$ & 09/1951 & 77 & 4 \\
\hline 315 & Dekalb & 2352 & Bowie & 400 & $33^{\circ} 38^{\prime}$ & $94^{\circ} 38^{\prime}$ & $01 / 1948$ & $12 / 2001$ & 95 & 54 \\
\hline 316 & Del Rio 2 NW & 2361 & Val Verde & 1,080 & $29^{\circ} 25^{\prime}$ & $100^{\circ} 54^{\prime}$ & 08/1996 & $12 / 2001$ & 90 & 6 \\
\hline 317 & Del Rio $3 \mathrm{~S}$ & 2362 & Val Verde* & 879 & $29^{\circ} 20^{\prime}$ & $100^{\circ} 53^{\prime}$ & $01 / 1948$ & $02 / 1954$ & 64 & 7 \\
\hline 318 & Del Rio Airport & 2360 & Val Verde & 999 & $29^{\circ} 22^{\prime}$ & $100^{\circ} 55^{\prime}$ & 05/1951 & $12 / 2001$ & 98 & 46 \\
\hline 319 & Del Rio Webb City & 2357 & Val Verde* & 961 & $29^{\circ} 22^{\prime}$ & $100^{\circ} 54^{\prime}$ & $02 / 1946$ & $02 / 1963$ & 79 & 13 \\
\hline 320 & Dell City 5 SSW & 2354 & Hudspeth & 3,770 & $31^{\circ} 52^{\prime}$ & $105^{\circ} 14^{\prime}$ & 07/1979 & $12 / 2001$ & 95 & 23 \\
\hline 321 & Denison Dam & 2394 & Grayson & 613 & $33^{\circ} 49^{\prime}$ & $96^{\circ} 34^{\prime}$ & $02 / 1940$ & 06/1997 & 98 & 58 \\
\hline 322 & Denison Highway 60 Bridge & 2397 & Grayson* & 551 & $33^{\circ} 49^{\prime}$ & $96^{\circ} 32^{\prime}$ & 05/1909 & 05/1949 & 93 & 37 \\
\hline 323 & Denton & 2403 & Denton & 659 & $33^{\circ} 14^{\prime}$ & $97^{\circ} 08^{\prime}$ & 03/1949 & $01 / 1965$ & 86 & 17 \\
\hline 324 & Denton $2 \mathrm{SE}$ & 2404 & Denton & 630 & $33^{\circ} 11^{\prime}$ & $97^{\circ} 06^{\prime}$ & $06 / 1913$ & $12 / 2001$ & 97 & 89 \\
\hline 325 & Denver City & 2408 & Yoakum & 3,590 & $32^{\circ} 57^{\prime}$ & $102^{\circ} 49^{\prime}$ & 05/1986 & $12 / 2001$ & 78 & 15 \\
\hline 326 & Deport 4 NW & 2415 & Lamar & 436 & $33^{\circ} 33^{\prime}$ & $95^{\circ} 22^{\prime}$ & $06 / 1948$ & $03 / 2001$ & 81 & 22 \\
\hline 327 & Derby $1 \mathrm{~S}$ & 2417 & Frio & 470 & $28^{\circ} 45^{\prime}$ & $99^{\circ} 08^{\prime}$ & $04 / 1978$ & $12 / 2001$ & 93 & 24 \\
\hline 328 & Devine & 2430 & Medina* & 689 & $29^{\circ} 09^{\prime}$ & $98^{\circ} 54^{\prime}$ & 07/1948 & $05 / 1954$ & 38 & 7 \\
\hline 329 & Deweyville $5 \mathrm{~S}$ & 2436 & Orange & 20 & $30^{\circ} 14^{\prime}$ & $93^{\circ} 44^{\prime}$ & 05/1954 & 04/1986 & 96 & 33 \\
\hline 330 & Dialville $2 \mathrm{~W}$ & 2444 & Cherokee & 616 & $31^{\circ} 54^{\prime}$ & $95^{\circ} 15^{\prime}$ & $09 / 1897$ & $12 / 2001$ & 96 & 104 \\
\hline 331 & Dickens & 2448 & Dickens & 2,575 & $33^{\circ} 37^{\prime}$ & $100^{\circ} 50^{\prime}$ & $06 / 1964$ & $10 / 1984$ & 87 & 21 \\
\hline 332 & Dilley & 2458 & Frio & 550 & $28^{\circ} 40^{\prime}$ & $99^{\circ} 10^{\prime}$ & 03/1910 & $12 / 2001$ & 98 & 89 \\
\hline 333 & Dime Box & 2462 & Lee & 335 & $30^{\circ} 21^{\prime}$ & $96^{\circ} 50^{\prime}$ & $10 / 1941$ & $12 / 2001$ & 96 & 61 \\
\hline 334 & Dimmitt $2 \mathrm{~N}$ & 2464 & Castro & 3,850 & $34^{\circ} 35^{\prime}$ & $102^{\circ} 18^{\prime}$ & 05/1959 & $12 / 2001$ & 98 & 43 \\
\hline 335 & Dimmitt $6 \mathrm{E}$ & 2463 & Castro & 3,812 & $34^{\circ} 33^{\prime}$ & $102^{\circ} 13^{\prime}$ & $01 / 1923$ & $06 / 1985$ & 97 & 63 \\
\hline 336 & Divide School & 2478 & Kerr* & 2,552 & $30^{\circ} 07^{\prime}$ & $99^{\circ} 37^{\prime}$ & $12 / 1941$ & $02 / 1948$ & 71 & 8 \\
\hline 337 & Doole 6 NNE & 2527 & McCulloch & 1,450 & $31^{\circ} 29^{\prime}$ & $99^{\circ} 34^{\prime}$ & 04/1948 & 07/1979 & 94 & 32 \\
\hline 338 & Doss & 2541 & Gillespie & 1,801 & $30^{\circ} 27^{\prime}$ & $99^{\circ} 08^{\prime}$ & 03/1948 & $12 / 1965$ & 88 & 18 \\
\hline 339 & Douglass $1 \mathrm{~S}$ & 2558 & Nacogdoches & 385 & $31^{\circ} 38^{\prime}$ & $94^{\circ} 53^{\prime}$ & 08/1983 & $12 / 2001$ & 89 & 15 \\
\hline 340 & Dripping Springs 6 E & 2585 & Hays & 1,120 & $30^{\circ} 12^{\prime}$ & $97^{\circ} 58^{\prime}$ & $12 / 1984$ & $12 / 2001$ & 95 & 18 \\
\hline 341 & Dryden & 2590 & Terrell & 2,190 & $30^{\circ} 03^{\prime}$ & $102^{\circ} 07^{\prime}$ & 06/1966 & $10 / 1994$ & 92 & 29 \\
\hline 342 & Dryden $10 \mathrm{NE}$ & 2593 & Terrell & 2,280 & $30^{\circ} 12^{\prime}$ & $101^{\circ} 50^{\prime}$ & $05 / 1937$ & 08/1993 & 86 & 13 \\
\hline 343 & Dryer $1 \mathrm{NW}$ & 2595 & Gonzales & 302 & $29^{\circ} 23^{\prime}$ & $97^{\circ} 16^{\prime}$ & $02 / 1940$ & $04 / 1975$ & 97 & 36 \\
\hline 344 & Dublin & 2598 & Erath & 1,502 & $32^{\circ} 06^{\prime}$ & $98^{\circ} 20^{\prime}$ & $11 / 1898$ & $12 / 2001$ & 92 & 102 \\
\hline 345 & Dublin near SCS 7 & 2599 & Erath* & 1,470 & $32^{\circ} 06^{\prime}$ & $98^{\circ} 15^{\prime}$ & 08/1941 & 06/1943 & 44 & 3 \\
\hline 346 & Dumas & 2617 & Moore & 3,655 & $35^{\circ} 52^{\prime}$ & $101^{\circ} 58^{\prime}$ & $01 / 1937$ & $12 / 2001$ & 91 & 65 \\
\hline 347 & Dumas $8 \mathrm{NE}$ & 2619 & Moore & 3,553 & $35^{\circ} 57^{\prime}$ & $101^{\circ} 53^{\prime}$ & $06 / 1948$ & 09/1951 & 82 & 4 \\
\hline 348 & Dumont & 2621 & King & 2,010 & $33^{\circ} 48^{\prime}$ & $100^{\circ} 31^{\prime}$ & 05/1971 & $12 / 2001$ & 99 & 31 \\
\hline 349 & Duncan Wilson Ranch & 2630 & Schleicher & 2,120 & $30^{\circ} 48^{\prime}$ & $100^{\circ} 10^{\prime}$ & $02 / 1966$ & $11 / 1996$ & 98 & 31 \\
\hline 350 & Dundee 6 NNW & 2633 & Archer & 1,051 & $33^{\circ} 48^{\prime}$ & $98^{\circ} 55^{\prime}$ & 06/1922 & $12 / 2001$ & 96 & 80 \\
\hline 351 & Dunk Ranch & 2636 & Kimble* & 2,201 & $30^{\circ} 22^{\prime}$ & $99^{\circ} 36^{\prime}$ & 03/1948 & 09/1958 & 96 & 11 \\
\hline 352 & E V Ranch & 3013 & Jeff Davis* & 4,423 & $30^{\circ} 53^{\prime}$ & $104^{\circ} 27^{\prime}$ & $02 / 1919$ & $06 / 1923$ & 73 & 4 \\
\hline 353 & EADS & 2669 & Smith* & 522 & $32^{\circ} 34^{\prime}$ & $95^{\circ} 25^{\prime}$ & 01/1948 & $01 / 1958$ & 81 & 11 \\
\hline 354 & Eagle Mountain Lake & 2677 & Tarrant & 760 & $32^{\circ} 52^{\prime}$ & $97^{\circ} 26^{\prime}$ & 04/1978 & $12 / 2001$ & 86 & 22 \\
\hline 355 & Eagle Mountain Lake Dam & 2678 & Tarrant & 679 & $32^{\circ} 53^{\prime}$ & $97^{\circ} 28^{\prime}$ & 07/1940 & $04 / 1975$ & 90 & 34 \\
\hline 356 & Eagle Pass & 2679 & Maverick & 808 & $28^{\circ} 42^{\prime}$ & $100^{\circ} 28^{\prime}$ & $02 / 1900$ & $12 / 2001$ & 97 & 101 \\
\hline
\end{tabular}


Table 1. Summary of National Weather Service daily precipitation stations in Texas with known names, latitudes, and longitudes-Continued

\begin{tabular}{|c|c|c|c|c|c|c|c|c|c|c|}
\hline $\begin{array}{c}\text { Seq. } \\
\text { no. }\end{array}$ & NWS station name & $\begin{array}{c}\text { NWS } \\
\text { station } \\
\text { no. }\end{array}$ & County & $\begin{array}{c}\text { Elev. } \\
\text { (ft above } \\
\text { NGVD } \\
\text { 29) }\end{array}$ & $\begin{array}{l}\text { Lati- } \\
\text { tude }\end{array}$ & $\begin{array}{l}\text { Longi- } \\
\text { tude }\end{array}$ & $\begin{array}{c}\text { Begining } \\
\text { date } \\
\text { (mm/yyyy) }\end{array}$ & $\begin{array}{c}\text { Ending } \\
\text { date } \\
\text { (mm/yyyy) }\end{array}$ & $\begin{array}{l}\text { Per- } \\
\text { cent } \\
\text { cov- } \\
\text { erage }\end{array}$ & $\begin{array}{l}\text { Years } \\
\text { of } \\
\text { record }\end{array}$ \\
\hline 357 & Eastland & 2715 & Eastland & 1,433 & $32^{\circ} 23^{\prime}$ & $98^{\circ} 49^{\prime}$ & $04 / 1904$ & $12 / 2001$ & 94 & 92 \\
\hline 358 & Eden 1 & 2741 & Concho & 2,064 & $31^{\circ} 13^{\prime}$ & $99^{\circ} 50^{\prime}$ & $04 / 1923$ & $02 / 1983$ & 92 & 56 \\
\hline 359 & Eden 2 & 2744 & Concho & 2,070 & $31^{\circ} 13^{\prime}$ & $99^{\circ} 51^{\prime}$ & $06 / 1948$ & $10 / 1986$ & 76 & 8 \\
\hline 360 & Edinburg & 2758 & Hidalgo & 96 & $26^{\circ} 17^{\prime}$ & $98^{\circ} 09^{\prime}$ & $06 / 1948$ & $05 / 1950$ & 66 & 3 \\
\hline 361 & Edmonson & 2766 & Hale* & 3,481 & $34^{\circ} 17^{\prime}$ & $101^{\circ} 54^{\prime}$ & 02/1947 & 06/1947 & 41 & 1 \\
\hline 362 & Edna $3 \mathrm{SW}$ & 2769 & Jackson & 69 & $28^{\circ} 57^{\prime}$ & $96^{\circ} 41^{\prime}$ & 07/1909 & $02 / 1968$ & 97 & 60 \\
\hline 363 & Edna $7 \mathrm{NW}$ & 2770 & Jackson & 70 & $29^{\circ} 03^{\prime}$ & $96^{\circ} 45^{\prime}$ & $12 / 1995$ & $12 / 2001$ & 86 & 7 \\
\hline 364 & Edna Highway 59 Bridge & 2768 & Jackson & 68 & $28^{\circ} 58^{\prime}$ & $96^{\circ} 41^{\prime}$ & 03/1968 & $11 / 1995$ & 94 & 28 \\
\hline 365 & Edom & 2772 & Van Zandt & 508 & $32^{\circ} 22^{\prime}$ & $95^{\circ} 37^{\prime}$ & $04 / 1940$ & $12 / 2001$ & 98 & 62 \\
\hline 366 & Egypt & 2780 & Wharton* & 131 & $29^{\circ} 24^{\prime}$ & $96^{\circ} 14^{\prime}$ & 05/1921 & 05/1928 & 86 & 8 \\
\hline 367 & El Campo & 2786 & Wharton & 112 & $29^{\circ} 12^{\prime}$ & $96^{\circ} 17^{\prime}$ & $10 / 1941$ & $04 / 1974$ & 93 & 34 \\
\hline 368 & El Indio & 2824 & Maverick & 700 & $28^{\circ} 30^{\prime}$ & $100^{\circ} 18^{\prime}$ & $09 / 1978$ & $12 / 2001$ & 96 & 24 \\
\hline 369 & El Paso No. 2 & 2793 & El Paso & 3,918 & $31^{\circ} 48^{\prime}$ & $106^{\circ} 22^{\prime}$ & 08/2001 & $12 / 2001$ & 34 & 1 \\
\hline 370 & El Paso 32 ENE & 2794 & Hudspeth & 5,240 & $31^{\circ} 50^{\prime}$ & $105^{\circ} 57^{\prime}$ & $11 / 1983$ & $12 / 2001$ & 94 & 19 \\
\hline 371 & El Paso Airport & 2797 & El Paso & 3,918 & $31^{\circ} 48^{\prime}$ & $106^{\circ} 22^{\prime}$ & $01 / 1948$ & $12 / 2001$ & 100 & 54 \\
\hline 372 & Eldorado $11 \mathrm{NW}$ & 2812 & Schleicher & 2,490 & $30^{\circ} 58^{\prime}$ & $100^{\circ} 42^{\prime}$ & $12 / 1958$ & 06/1981 & 93 & 24 \\
\hline 373 & Eldorado $11 \mathrm{SW}$ & 2813 & Schleicher* & 2,431 & $30^{\circ} 47^{\prime}$ & $100^{\circ} 46^{\prime}$ & 02/1949 & 09/1951 & 17 & 3 \\
\hline 374 & Eldorado $12 \mathrm{~N}$ & 2815 & Schleicher & 2,380 & $31^{\circ} 02^{\prime}$ & $100^{\circ} 35^{\prime}$ & 08/1999 & $12 / 2001$ & 80 & 3 \\
\hline 375 & Eldorado $19 \mathrm{SW}$ & 2814 & Schleicher* & 2,362 & $30^{\circ} 44^{\prime}$ & $100^{\circ} 53^{\prime}$ & 09/1941 & 09/1975 & 89 & 33 \\
\hline 376 & Eldorado $2 \mathrm{SE}$ & 2792 & Schleicher & 2,434 & $30^{\circ} 50^{\prime}$ & $100^{\circ} 35^{\prime}$ & 09/1981 & 08/1989 & 89 & 9 \\
\hline 377 & Electra & 2818 & Wichita & 1,216 & $34^{\circ} 02^{\prime}$ & $98^{\circ} 55^{\prime}$ & $01 / 1948$ & $10 / 2001$ & 92 & 54 \\
\hline 378 & Elgin & 2820 & Bastrop & 579 & $30^{\circ} 20^{\prime}$ & $97^{\circ} 22^{\prime}$ & $02 / 1962$ & $12 / 2001$ & 100 & 40 \\
\hline 379 & Elmax Ranch & 2830 & Kendall & 1,524 & $29^{\circ} 53^{\prime}$ & $98^{\circ} 39^{\prime}$ & 01/1986 & 07/1986 & 50 & 1 \\
\hline 380 & Elmendorf & 2850 & Bexar & 702 & $29^{\circ} 25^{\prime}$ & $98^{\circ} 20^{\prime}$ & 05/1916 & $04 / 1920$ & 78 & 5 \\
\hline 381 & Emory & 2902 & Rains & 435 & $32^{\circ} 51^{\prime}$ & $95^{\circ} 46^{\prime}$ & $05 / 1897$ & $12 / 2001$ & 87 & 60 \\
\hline 382 & Emory $8 \mathrm{~S}$ & 2904 & Van Zandt & 370 & $32^{\circ} 46^{\prime}$ & $95^{\circ} 45^{\prime}$ & 08/1992 & 07/1993 & 40 & 2 \\
\hline 383 & Encinal & 2906 & Webb & 590 & $28^{\circ} 02^{\prime}$ & $99^{\circ} 25^{\prime}$ & $11 / 1907$ & $12 / 2001$ & 93 & 95 \\
\hline 384 & Engleman Gardens & 2917 & Hidalgo & 69 & $26^{\circ} 20^{\prime}$ & $98^{\circ} 01^{\prime}$ & $09 / 1946$ & $02 / 1970$ & 89 & 25 \\
\hline 385 & Ennis & 2925 & Ellis & 525 & $32^{\circ} 20^{\prime}$ & $96^{\circ} 38^{\prime}$ & 05/1940 & $12 / 1991$ & 96 & 49 \\
\hline 386 & Eola & 2934 & Concho* & $* 1,791$ & $31^{\circ} 24^{\prime}$ & $100^{\circ} 05^{\prime}$ & 08/1911 & $11 / 1912$ & 33 & 2 \\
\hline 387 & Era & 2941 & Cooke* & 951 & $33^{\circ} 30^{\prime}$ & $97^{\circ} 18^{\prime}$ & $09 / 1946$ & $08 / 1953$ & 71 & 8 \\
\hline 388 & Estelle & 2965 & Dallas* & $* 549$ & $32^{\circ} 52^{\prime}$ & $97^{\circ} 01^{\prime}$ & $01 / 1897$ & $12 / 1904$ & 96 & 7 \\
\hline 389 & Euless & 2983 & Tarrant* & 551 & $32^{\circ} 49^{\prime}$ & $97^{\circ} 06^{\prime}$ & $02 / 1946$ & $05 / 1953$ & 33 & 3 \\
\hline 390 & Evadale & 3000 & Jasper & 33 & $30^{\circ} 20^{\prime}$ & $94^{\circ} 05^{\prime}$ & 09/1946 & $12 / 2001$ & 98 & 49 \\
\hline 391 & Evant $1 \mathrm{SSW}$ & 3005 & Coryell & 1,245 & $31^{\circ} 28^{\prime}$ & $98^{\circ} 10^{\prime}$ & $10 / 1941$ & $12 / 2001$ & 91 & 48 \\
\hline 392 & Exxon Gas Plant & 3025 & Kleberg & 84 & $27^{\circ} 28^{\prime}$ & $97^{\circ} 47^{\prime}$ & 07/1998 & $10 / 2001$ & 71 & 4 \\
\hline 393 & Fabens 1 & 3033 & El Paso & 3,612 & $31^{\circ} 30^{\prime}$ & $106^{\circ} 09^{\prime}$ & $06 / 1948$ & 09/1977 & 97 & 30 \\
\hline 394 & Fabens 2 & 3034 & El Paso* & 3,652 & $31^{\circ} 31^{\prime}$ & $106^{\circ} 09^{\prime}$ & $07 / 1949$ & 07/1949 & 8 & 1 \\
\hline 395 & Fair Oaks Ranch & 3038 & Comal & 1,302 & $29^{\circ} 45^{\prime}$ & $98^{\circ} 38^{\prime}$ & $12 / 1946$ & $01 / 1973$ & 89 & 28 \\
\hline 396 & Fairfield $3 \mathrm{~W}$ & 3047 & Freestone & 432 & $31^{\circ} 43^{\prime}$ & $96^{\circ} 12^{\prime}$ & 03/1941 & $12 / 2001$ & 90 & 60 \\
\hline 397 & Falcon Dam & 3060 & Starr & 320 & $26^{\circ} 33^{\prime}$ & $99^{\circ} 08^{\prime}$ & $07 / 1962$ & $12 / 2001$ & 97 & 40 \\
\hline 398 & Falfurrias & 3063 & Brooks & 120 & $27^{\circ} 12^{\prime}$ & $98^{\circ} 07^{\prime}$ & 03/1907 & $12 / 2001$ & 97 & 95 \\
\hline 399 & Falls City 7 WSW & 3065 & Karnes & 343 & $28^{\circ} 57^{\prime}$ & $98^{\circ} 06^{\prime}$ & $08 / 1946$ & $12 / 2001$ & 96 & 56 \\
\hline 400 & Farmersville & 3080 & Collin & 630 & $33^{\circ} 10^{\prime}$ & $96^{\circ} 22^{\prime}$ & $09 / 1947$ & $12 / 2001$ & 96 & 55 \\
\hline 401 & Farnsworth 3 NNW & 3085 & Ochiltree* & 3,002 & $36^{\circ} 22^{\prime}$ & $100^{\circ} 59^{\prime}$ & 01/1941 & $01 / 1958$ & 88 & 18 \\
\hline 402 & Fawcett Ranch & 3103 & Val Verde* & 1,503 & $29^{\circ} 52^{\prime}$ & $100^{\circ} 54^{\prime}$ & $06 / 1948$ & $02 / 1949$ & 33 & 2 \\
\hline 403 & Fedor & 3112 & Lee & 483 & $30^{\circ} 18^{\prime}$ & $97^{\circ} 03^{\prime}$ & $03 / 1963$ & $12 / 2001$ & 95 & 39 \\
\hline 404 & Ferris & 3133 & Ellis & 470 & $32^{\circ} 31^{\prime}$ & $96^{\circ} 40^{\prime}$ & $01 / 1940$ & $12 / 2001$ & 94 & 60 \\
\hline 405 & Fife & 3142 & McCulloch & 1,391 & $31^{\circ} 23^{\prime}$ & $99^{\circ} 22^{\prime}$ & $08 / 1941$ & $07 / 1975$ & 93 & 35 \\
\hline 406 & Fischers store & 3156 & Comal & 1,160 & $29^{\circ} 58^{\prime}$ & $98^{\circ} 15^{\prime}$ & 04/1941 & $12 / 2001$ & 97 & 61 \\
\hline 407 & Flat & 3171 & Coryell & 835 & $31^{\circ} 19^{\prime}$ & $97^{\circ} 38^{\prime}$ & $08 / 1951$ & $12 / 2001$ & 92 & 11 \\
\hline 408 & Flatonia & 3183 & Fayette & 520 & $29^{\circ} 40^{\prime}$ & $97^{\circ} 06^{\prime}$ & $01 / 1908$ & $12 / 2001$ & 99 & 94 \\
\hline
\end{tabular}


Table 1. Summary of National Weather Service daily precipitation stations in Texas with known names, latitudes, and longitudes-Continued

\begin{tabular}{|c|c|c|c|c|c|c|c|c|c|c|}
\hline $\begin{array}{c}\text { Seq. } \\
\text { no. }\end{array}$ & NWS station name & $\begin{array}{l}\text { NWS } \\
\text { station } \\
\text { no. }\end{array}$ & County & $\begin{array}{c}\text { Elev. } \\
\text { (ft above } \\
\text { NGVD } \\
29 \text { ) }\end{array}$ & $\begin{array}{l}\text { Lati- } \\
\text { tude }\end{array}$ & $\begin{array}{l}\text { Longi- } \\
\text { tude }\end{array}$ & $\begin{array}{c}\text { Begining } \\
\text { date } \\
\text { (mm/yyyy) }\end{array}$ & $\begin{array}{c}\text { Ending } \\
\text { date } \\
\text { (mm/yyyy) }\end{array}$ & $\begin{array}{l}\text { Per- } \\
\text { cent } \\
\text { cov- } \\
\text { erage }\end{array}$ & $\begin{array}{l}\text { Years } \\
\text { of } \\
\text { record }\end{array}$ \\
\hline 409 & Fletcher Ranch & 3189 & Presidio* & 4,852 & $30^{\circ} 10^{\prime}$ & $104^{\circ} 12^{\prime}$ & $06 / 1948$ & $09 / 1951$ & 83 & 4 \\
\hline 410 & Flint & 3192 & Smith* & 479 & $32^{\circ} 12^{\prime}$ & $95^{\circ} 21^{\prime}$ & $02 / 1910$ & $08 / 1949$ & 99 & 40 \\
\hline 411 & Flomot $4 \mathrm{NE}$ & 3196 & Motley & 2,360 & $34^{\circ} 16^{\prime}$ & $100^{\circ} 56^{\prime}$ & $08 / 1946$ & $12 / 2001$ & 94 & 56 \\
\hline 412 & Florence $3 \mathrm{SE}$ & 3199 & Williamson & 970 & $30^{\circ} 48^{\prime}$ & $97^{\circ} 45^{\prime}$ & $03 / 1963$ & $12 / 2001$ & 97 & 39 \\
\hline 413 & Floresville & 3201 & Wilson & 400 & $29^{\circ} 07^{\prime}$ & $98^{\circ} 09^{\prime}$ & $06 / 1916$ & $12 / 2001$ & 96 & 86 \\
\hline 414 & Flour Bluff & 3210 & Nueces & 9 & $27^{\circ} 36^{\prime}$ & $97^{\circ} 17^{\prime}$ & 09/1988 & $07 / 1998$ & 85 & 11 \\
\hline 415 & Floydada & 3214 & Floyd & 3,220 & $33^{\circ} 59^{\prime}$ & $101^{\circ} 20^{\prime}$ & $04 / 1911$ & $12 / 2001$ & 93 & 63 \\
\hline 416 & Floydada $9 \mathrm{SE}$ & 3215 & Floyd & 3,130 & $33^{\circ} 52^{\prime}$ & $101^{\circ} 14^{\prime}$ & $01 / 1947$ & $12 / 2001$ & 96 & 55 \\
\hline 417 & Flying V Ranch & 3218 & San Saba* & 1,631 & $31^{\circ} 05^{\prime}$ & $98^{\circ} 45^{\prime}$ & 06/1948 & $12 / 1959$ & 70 & 12 \\
\hline 418 & Follett & 3225 & Lipscomb & 2,769 & $36^{\circ} 26^{\prime}$ & $100^{\circ} 08^{\prime}$ & $06 / 1930$ & $06 / 2001$ & 95 & 72 \\
\hline 419 & Forestburg $5 \mathrm{~S}$ & 3247 & Montague & 1,110 & $33^{\circ} 27^{\prime}$ & $97^{\circ} 34^{\prime}$ & $01 / 1897$ & $12 / 2001$ & 97 & 64 \\
\hline 420 & Forsan & 3253 & Howard & 2,750 & $32^{\circ} 06^{\prime}$ & $101^{\circ} 21^{\prime}$ & $04 / 1949$ & $12 / 2001$ & 99 & 53 \\
\hline 421 & Fort Davis & 3262 & Jeff Davis & 4,880 & $30^{\circ} 39^{\prime}$ & $103^{\circ} 53^{\prime}$ & $01 / 1902$ & $12 / 2001$ & 95 & 90 \\
\hline 422 & Fort Hancock 8 SSE & 3266 & Hudspeth & 3,905 & $31^{\circ} 11^{\prime}$ & $105^{\circ} 44^{\prime}$ & $07 / 1966$ & $12 / 2001$ & 88 & 36 \\
\hline 423 & Fort McIntosh & 3267 & Webb* & 459 & $27^{\circ} 30^{\prime}$ & $99^{\circ} 31^{\prime}$ & $01 / 1897$ & $12 / 1931$ & 96 & 33 \\
\hline 424 & Fort McKavett $14 \mathrm{NW}$ & 3271 & Schleicher & 2,280 & $30^{\circ} 57^{\prime}$ & $100^{\circ} 16^{\prime}$ & $12 / 1958$ & 03/1962 & 15 & 4 \\
\hline 425 & Fort Stockton & 3280 & Pecos & 3,000 & $30^{\circ} 54^{\prime}$ & $102^{\circ} 54^{\prime}$ & 05/1940 & $12 / 2001$ & 95 & 61 \\
\hline 426 & Fort Stockton 1 & 3277 & Pecos* & 3,051 & $30^{\circ} 53^{\prime}$ & $102^{\circ} 53^{\prime}$ & $01 / 1897$ & $12 / 1948$ & 96 & 51 \\
\hline 427 & Fort Worth Clear Fork & 3287 & Tarrant & 533 & $32^{\circ} 44^{\prime}$ & $97^{\circ} 22^{\prime}$ & 08/1947 & 08/1947 & 8 & 1 \\
\hline 428 & Fort Worth Leonard Boulevard & 3286 & Tarrant & 659 & $32^{\circ} 44^{\prime}$ & $97^{\circ} 20^{\prime}$ & $07 / 1953$ & 08/1966 & 94 & 14 \\
\hline 429 & Fort Worth Meacham Field & 3284 & Tarrant & 687 & $32^{\circ} 49^{\prime}$ & $97^{\circ} 21^{\prime}$ & $09 / 1946$ & $12 / 2001$ & 87 & 17 \\
\hline 430 & Fort Worth WB Airport & 3283 & Tarrant & 574 & $32^{\circ} 50^{\prime}$ & $97^{\circ} 03^{\prime}$ & $01 / 1897$ & $12 / 1973$ & 90 & 27 \\
\hline 431 & Fort Worth WSFO & 3285 & Tarrant & 644 & $32^{\circ} 50^{\prime}$ & $97^{\circ} 18^{\prime}$ & $08 / 1948$ & $12 / 2001$ & 88 & 12 \\
\hline 432 & Four Notch Guard Station & 3298 & Walker & 302 & $30^{\circ} 39^{\prime}$ & $95^{\circ} 25^{\prime}$ & $07 / 1940$ & $12 / 1964$ & 95 & 25 \\
\hline 433 & Fowlerton & 3299 & La Salle & 320 & $28^{\circ} 28^{\prime}$ & $98^{\circ} 49^{\prime}$ & $06 / 1913$ & $12 / 2001$ & 88 & 85 \\
\hline 434 & Franklin & 3321 & Robertson & 469 & $31^{\circ} 02^{\prime}$ & $96^{\circ} 29^{\prime}$ & $04 / 1962$ & $12 / 2001$ & 93 & 40 \\
\hline 435 & Frankston $3 \mathrm{WNW}$ & 3325 & Anderson & 430 & $32^{\circ} 04^{\prime}$ & $95^{\circ} 33^{\prime}$ & $12 / 1963$ & $10 / 1966$ & 71 & 4 \\
\hline 436 & Fredericksburg & 3329 & Gillespie & 1,685 & $30^{\circ} 14^{\prime}$ & $98^{\circ} 54^{\prime}$ & $12 / 1896$ & $12 / 2001$ & 97 & 82 \\
\hline 437 & Freeport $2 \mathrm{NW}$ & 3340 & Brazoria & 8 & $28^{\circ} 59^{\prime}$ & $95^{\circ} 23^{\prime}$ & $01 / 1931$ & $12 / 2001$ & 98 & 71 \\
\hline 438 & Freer & 3341 & Duval & 510 & $27^{\circ} 54^{\prime}$ & $98^{\circ} 37^{\prime}$ & $12 / 1947$ & $12 / 2001$ & 93 & 55 \\
\hline 439 & Freer $18 \mathrm{WNW}$ & 3344 & Webb & 336 & $27^{\circ} 56^{\prime}$ & $98^{\circ} 52^{\prime}$ & $07 / 1978$ & $12 / 2001$ & 93 & 24 \\
\hline 440 & Frio Town & 3366 & Frio & 630 & $29^{\circ} 02^{\prime}$ & $99^{\circ} 19^{\prime}$ & $09 / 1947$ & $11 / 1979$ & 91 & 28 \\
\hline 441 & Friona & 3368 & Parmer & 4,010 & $34^{\circ} 38^{\prime}$ & $102^{\circ} 42^{\prime}$ & $11 / 1927$ & $12 / 2001$ & 91 & 71 \\
\hline 442 & Frisco & 3370 & Collin & 740 & $33^{\circ} 09^{\prime}$ & $96^{\circ} 49^{\prime}$ & $11 / 1966$ & $12 / 2001$ & 97 & 36 \\
\hline 443 & Frost & 3379 & Navarro & 522 & $32^{\circ} 05^{\prime}$ & $96^{\circ} 48^{\prime}$ & $10 / 1946$ & $02 / 1985$ & 89 & 40 \\
\hline 444 & Fort McKavett & 3257 & Menard & 2,310 & $30^{\circ} 49^{\prime}$ & $100^{\circ} 06^{\prime}$ & 08/1997 & $12 / 2001$ & 88 & 5 \\
\hline 445 & Funk Ranch & 3401 & Irion & 2,070 & $31^{\circ} 29^{\prime}$ & $100^{\circ} 48^{\prime}$ & $03 / 1948$ & $12 / 2001$ & 97 & 54 \\
\hline 446 & Gageby 3 WNW & 3410 & Hemphill & 2,800 & $35^{\circ} 37^{\prime}$ & $100^{\circ} 23^{\prime}$ & $06 / 1948$ & 09/1951 & 81 & 4 \\
\hline 447 & Gail & 3411 & Borden & 2,530 & $32^{\circ} 46^{\prime}$ & $101^{\circ} 26^{\prime}$ & $01 / 1897$ & 09/2001 & 88 & 71 \\
\hline 448 & Gainesville & 3415 & Cooke & 780 & $33^{\circ} 38^{\prime}$ & $97^{\circ} 08^{\prime}$ & $01 / 1897$ & $03 / 1987$ & 97 & 89 \\
\hline 449 & Gainesville 5 ENE & 3420 & Cooke & 870 & $33^{\circ} 38^{\prime}$ & $97^{\circ} 03^{\prime}$ & 05/1987 & $12 / 2001$ & 97 & 15 \\
\hline 450 & Galveston & 3430 & Galveston & 10 & $29^{\circ} 20^{\prime}$ & $94^{\circ} 46^{\prime}$ & $01 / 1897$ & 09/2001 & 92 & 61 \\
\hline 451 & Galveston WB Airport & 3431 & Galveston & 5 & $29^{\circ} 16^{\prime}$ & $94^{\circ} 52^{\prime}$ & $07 / 1948$ & $09 / 1963$ & 95 & 16 \\
\hline 452 & Garcia Lake & 3441 & Deaf Smith* & 4,203 & $34^{\circ} 53^{\prime}$ & $103^{\circ} 01^{\prime}$ & $06 / 1948$ & 06/1951 & 28 & 3 \\
\hline 453 & Garcia Lake 12 ENE & 3442 & Deaf Smith & 4,134 & $34^{\circ} 55^{\prime}$ & $102^{\circ} 44^{\prime}$ & $07 / 1965$ & 05/1971 & 82 & 7 \\
\hline 454 & Garden City & 3445 & Glasscock & 2,640 & $31^{\circ} 51^{\prime}$ & $101^{\circ} 28^{\prime}$ & $10 / 1912$ & $12 / 2001$ & 97 & 90 \\
\hline 455 & Garden City $16 \mathrm{E}$ & 3446 & Sterling & 2,461 & $31^{\circ} 50^{\prime}$ & $101^{\circ} 12^{\prime}$ & $04 / 1949$ & 09/1951 & 81 & 3 \\
\hline 456 & Garlington Ranch & 3462 & Sterling* & $* 2,620$ & $31^{\circ} 55^{\prime}$ & $100^{\circ} 53^{\prime}$ & $06 / 1948$ & $08 / 1948$ & 18 & 1 \\
\hline 457 & Garner State Park & 3463 & Uvalde* & 1,401 & $29^{\circ} 35^{\prime}$ & $99^{\circ} 44^{\prime}$ & 07/1948 & 05/1950 & 31 & 3 \\
\hline 458 & Gary & 3472 & Panola* & 289 & $32^{\circ} 02^{\prime}$ & $94^{\circ} 22^{\prime}$ & $06 / 1940$ & $01 / 1958$ & 90 & 19 \\
\hline 459 & Gaston & 3480 & Fort Bend* & 102 & $29^{\circ} 43^{\prime}$ & $95^{\circ} 44^{\prime}$ & 09/1947 & 01/1951 & 61 & 5 \\
\hline 460 & Gatesville 4 SSE & 3485 & Coryell & 760 & $31^{\circ} 23^{\prime}$ & $97^{\circ} 43^{\prime}$ & $02 / 1900$ & $12 / 2001$ & 94 & 95 \\
\hline
\end{tabular}


Table 1. Summary of National Weather Service daily precipitation stations in Texas with known names, latitudes, and longitudes-Continued

\begin{tabular}{|c|c|c|c|c|c|c|c|c|c|c|}
\hline $\begin{array}{l}\text { Seq. } \\
\text { no. }\end{array}$ & NWS station name & $\begin{array}{l}\text { NWS } \\
\text { station } \\
\text { no. }\end{array}$ & County & $\begin{array}{c}\text { Elev. } \\
\text { (ft above } \\
\text { NGVD } \\
\text { 29) }\end{array}$ & $\begin{array}{l}\text { Lati- } \\
\text { tude }\end{array}$ & $\begin{array}{l}\text { Longi- } \\
\text { tude }\end{array}$ & $\begin{array}{l}\text { Begining } \\
\text { date } \\
\text { (mm/yyyy) }\end{array}$ & $\begin{array}{c}\text { Ending } \\
\text { date } \\
\text { (mm/yyyy) }\end{array}$ & $\begin{array}{l}\text { Per- } \\
\text { cent } \\
\text { cov- } \\
\text { erage }\end{array}$ & $\begin{array}{l}\text { Years } \\
\text { of } \\
\text { record }\end{array}$ \\
\hline 461 & George West 2 SSW & 3508 & Live Oak & 262 & $28^{\circ} 18^{\prime}$ & $98^{\circ} 07^{\prime}$ & $01 / 1916$ & $12 / 2001$ & 98 & 86 \\
\hline 462 & Georgetown & 3506 & Williamson & 748 & $30^{\circ} 38^{\prime}$ & $97^{\circ} 41^{\prime}$ & $12 / 1896$ & $01 / 1983$ & 90 & 54 \\
\hline 463 & Georgetown Lake & 3507 & Williamson & 840 & $30^{\circ} 41^{\prime}$ & $97^{\circ} 43^{\prime}$ & 07/1981 & $12 / 2001$ & 97 & 21 \\
\hline 464 & Giddings $5 \mathrm{E}$ & 3525 & Lee & 550 & $30^{\circ} 11^{\prime}$ & $96^{\circ} 51^{\prime}$ & $03 / 1940$ & $12 / 2001$ & 99 & 62 \\
\hline 465 & Gilmer & 3547 & Upshur* & 371 & $32^{\circ} 44^{\prime}$ & $94^{\circ} 57^{\prime}$ & $12 / 1950$ & $09 / 1956$ & 77 & 7 \\
\hline 466 & Gilmer 4 WNW & 3546 & Upshur & 390 & $32^{\circ} 44^{\prime}$ & $95^{\circ} 02^{\prime}$ & 03/1929 & $12 / 2001$ & 98 & 73 \\
\hline 467 & Girvin & 3557 & Pecos & 2,313 & $31^{\circ} 04^{\prime}$ & $102^{\circ} 24^{\prime}$ & 09/1947 & 04/1979 & 92 & 33 \\
\hline 468 & Glade & 3564 & Tarrant* & $* 589$ & $32^{\circ} 53^{\prime}$ & $97^{\circ} 06^{\prime}$ & 06/1948 & $09 / 1951$ & 68 & 4 \\
\hline 469 & Gladewater 3 WSW & 3565 & Gregg & 244 & $32^{\circ} 31^{\prime}$ & $94^{\circ} 57^{\prime}$ & $09 / 1946$ & 05/1976 & 87 & 31 \\
\hline 470 & Glen Cove 2 NE & 3579 & Coleman & 2,090 & $31^{\circ} 52^{\prime}$ & $99^{\circ} 37^{\prime}$ & $10 / 2001$ & $12 / 2001$ & 25 & 1 \\
\hline 471 & Glen Rose 2 W & 3591 & Somervell & 655 & $32^{\circ} 14^{\prime}$ & $97^{\circ} 47^{\prime}$ & $12 / 1963$ & $09 / 2000$ & 96 & 38 \\
\hline 472 & Glenfawn & 3585 & Rusk* & 351 & $31^{\circ} 55^{\prime}$ & $94^{\circ} 51^{\prime}$ & $05 / 1949$ & $08 / 1958$ & 92 & 10 \\
\hline 473 & Gold & 3605 & Gillespie & 1,640 & $30^{\circ} 20^{\prime}$ & $98^{\circ} 41^{\prime}$ & 03/1948 & $12 / 2001$ & 97 & 54 \\
\hline 474 & Goldthwaite $1 \mathrm{WSW}$ & 3614 & Mills & 1,500 & $31^{\circ} 27^{\prime}$ & $98^{\circ} 35^{\prime}$ & $04 / 1923$ & $12 / 2001$ & 97 & 71 \\
\hline 475 & Goliad & 3618 & Goliad & 142 & $28^{\circ} 40^{\prime}$ & $97^{\circ} 24^{\prime}$ & 09/1912 & $12 / 2001$ & 97 & 90 \\
\hline 476 & Goliad $1 \mathrm{SE}$ & 3620 & Goliad & 160 & $28^{\circ} 39^{\prime}$ & $97^{\circ} 23^{\prime}$ & $04 / 1949$ & $12 / 2001$ & 94 & 53 \\
\hline 477 & Goliad 6 NE & 3619 & Goliad* & 151 & $28^{\circ} 43^{\prime}$ & $97^{\circ} 20^{\prime}$ & $07 / 1948$ & $12 / 1959$ & 96 & 12 \\
\hline 478 & Gonzales $1 \mathrm{~N}$ & 3622 & Gonzales & 380 & $29^{\circ} 32^{\prime}$ & $97^{\circ} 27^{\prime}$ & $04 / 1915$ & $12 / 2001$ & 99 & 64 \\
\hline 479 & Gonzales $10 \mathrm{SW}$ & 3624 & Gonzales & 365 & $29^{\circ} 26^{\prime}$ & $97^{\circ} 31^{\prime}$ & $02 / 1997$ & $12 / 2001$ & 98 & 5 \\
\hline 480 & Gonzales 2 & 3623 & Gonzales & 302 & $29^{\circ} 30^{\prime}$ & $97^{\circ} 27^{\prime}$ & $11 / 1948$ & $12 / 1964$ & 91 & 17 \\
\hline 481 & Goose Creek & 3640 & Harris & 20 & $29^{\circ} 44^{\prime}$ & $94^{\circ} 58^{\prime}$ & $10 / 1921$ & 08/1956 & 96 & 36 \\
\hline 482 & Gordon $1 \mathrm{SW}$ & 3639 & Palo Pinto & 1,050 & $32^{\circ} 32^{\prime}$ & $98^{\circ} 23^{\prime}$ & $10 / 1991$ & $12 / 2001$ & 92 & 11 \\
\hline 483 & Gordonville & 3642 & Grayson & 755 & $33^{\circ} 47^{\prime}$ & $96^{\circ} 51^{\prime}$ & 06/1948 & $12 / 2001$ & 87 & 10 \\
\hline 484 & Gorman 2 NNE & 3646 & Eastland & 1,380 & $32^{\circ} 14^{\prime}$ & $98^{\circ} 39^{\prime}$ & $09 / 1951$ & 05/1999 & 83 & 9 \\
\hline 485 & Gouldbusk & 3654 & Coleman* & 1,503 & $31^{\circ} 33^{\prime}$ & $99^{\circ} 29^{\prime}$ & $04 / 1948$ & $02 / 1954$ & 33 & 7 \\
\hline 486 & Graham & 3668 & Young & 1,050 & $33^{\circ} 06^{\prime}$ & $98^{\circ} 35^{\prime}$ & $01 / 1897$ & $12 / 2001$ & 96 & 99 \\
\hline 487 & Granbury 2 ENE & 3673 & Hood & 722 & $32^{\circ} 27^{\prime}$ & $97^{\circ} 45^{\prime}$ & $12 / 1943$ & $09 / 1975$ & 90 & 32 \\
\hline 488 & Grandfalls 3 SSE & 3680 & Ward & 2,425 & $31^{\circ} 18^{\prime}$ & $102^{\circ} 49^{\prime}$ & $02 / 1909$ & $11 / 2001$ & 89 & 87 \\
\hline 489 & Granger & 3685 & Williamson & 584 & $30^{\circ} 43^{\prime}$ & $97^{\circ} 26^{\prime}$ & $02 / 1968$ & $12 / 2001$ & 94 & 34 \\
\hline 490 & Granger Dam & 3686 & Williamson & 565 & $30^{\circ} 42^{\prime}$ & $97^{\circ} 20^{\prime}$ & 07/1980 & $12 / 2001$ & 97 & 22 \\
\hline 491 & Grapeland & 3689 & Houston* & 479 & $31^{\circ} 29^{\prime}$ & $95^{\circ} 29^{\prime}$ & $09 / 1935$ & $04 / 1975$ & 87 & 40 \\
\hline 492 & Grapevine Dam & 3691 & Tarrant & 585 & $32^{\circ} 57^{\prime}$ & $97^{\circ} 03^{\prime}$ & $01 / 1897$ & $12 / 2001$ & 95 & 72 \\
\hline 493 & Greenville KGVL Radio & 3734 & Hunt & 545 & $33^{\circ} 10^{\prime}$ & $96^{\circ} 06^{\prime}$ & 03/1900 & $12 / 2001$ & 97 & 101 \\
\hline 494 & Groesbeck & 3770 & Limestone & 469 & $31^{\circ} 31^{\prime}$ & $96^{\circ} 32^{\prime}$ & $12 / 1963$ & 08/1975 & 90 & 13 \\
\hline 495 & Groveton & 3778 & Trinity & 350 & $31^{\circ} 04^{\prime}$ & $95^{\circ} 08^{\prime}$ & $07 / 1923$ & $12 / 2001$ & 87 & 64 \\
\hline 496 & Gruver & 3787 & Hansford & 3,170 & $36^{\circ} 15^{\prime}$ & $101^{\circ} 24^{\prime}$ & 07/1941 & $12 / 2001$ & 97 & 61 \\
\hline 497 & Guadalupe Pass CAA Airport & 3789 & Culberson & 5,452 & $31^{\circ} 50^{\prime}$ & $104^{\circ} 48^{\prime}$ & 06/1948 & 08/1950 & 74 & 3 \\
\hline 498 & Gunter $5 \mathrm{~S}$ & 3822 & Collin & 735 & $33^{\circ} 22^{\prime}$ & $96^{\circ} 46^{\prime}$ & $02 / 1948$ & $11 / 2000$ & 98 & 53 \\
\hline 499 & Guthrie & 3828 & King & 1,740 & $33^{\circ} 37^{\prime}$ & $100^{\circ} 19^{\prime}$ & $09 / 1947$ & $12 / 2001$ & 92 & 53 \\
\hline 500 & Guyer & 3831 & Wilbarger & 1,161 & $34^{\circ} 08^{\prime}$ & $98^{\circ} 56^{\prime}$ & $06 / 1948$ & $09 / 1951$ & 79 & 4 \\
\hline 501 & Hackberry & 3841 & Cottle & 1,670 & $33^{\circ} 56^{\prime}$ & $100^{\circ} 08^{\prime}$ & 05/1971 & $12 / 1987$ & 82 & 17 \\
\hline 502 & Hagansport & 3846 & Franklin & 360 & $33^{\circ} 20^{\prime}$ & $95^{\circ} 14^{\prime}$ & $12 / 1909$ & $12 / 2001$ & 94 & 89 \\
\hline 503 & Hale Center & 3862 & Hale* & 3,432 & $34^{\circ} 05^{\prime}$ & $101^{\circ} 51^{\prime}$ & $01 / 1897$ & $11 / 1957$ & 88 & 19 \\
\hline 504 & Hale Center 14 WNW & 3864 & Hale* & 3,533 & $34^{\circ} 08^{\prime}$ & $102^{\circ} 03^{\prime}$ & $12 / 1946$ & $05 / 1958$ & 78 & 13 \\
\hline 505 & Hall Ranch & 3871 & Kerr & 2,280 & $30^{\circ} 08^{\prime}$ & $99^{\circ} 36^{\prime}$ & 06/1948 & 09/1951 & 73 & 4 \\
\hline 506 & Hallettsville $2 \mathrm{~N}$ & 3873 & Lavaca & 275 & $29^{\circ} 28^{\prime}$ & $96^{\circ} 56^{\prime}$ & $01 / 1897$ & $12 / 2001$ & 98 & 104 \\
\hline 507 & Hallsville $1 \mathrm{~W}$ & 3877 & Harrison & 250 & $32^{\circ} 30^{\prime}$ & $94^{\circ} 35^{\prime}$ & 03/1998 & $12 / 2001$ & 95 & 4 \\
\hline 508 & Hamilton $2 \mathrm{E}$ & 3884 & Hamilton & 1,260 & $31^{\circ} 42^{\prime}$ & $98^{\circ} 05^{\prime}$ & 08/1915 & $12 / 2001$ & 91 & 48 \\
\hline 509 & Hamlin $1 \mathrm{SW}$ & 3890 & Jones & 1,720 & $32^{\circ} 52^{\prime}$ & $100^{\circ} 07^{\prime}$ & $10 / 1910$ & $12 / 2001$ & 93 & 75 \\
\hline 510 & Happy & 3922 & Swisher* & 3,622 & $34^{\circ} 44^{\prime}$ & $101^{\circ} 51^{\prime}$ & 06/1941 & $11 / 1947$ & 82 & 7 \\
\hline 511 & Harleton & 3941 & Harrison & 345 & $32^{\circ} 41^{\prime}$ & $94^{\circ} 34^{\prime}$ & 05/1949 & $12 / 2001$ & 96 & 53 \\
\hline 512 & Harlingen & 3943 & Cameron & 38 & $26^{\circ} 12^{\prime}$ & $97^{\circ} 40^{\prime}$ & 05/1911 & $12 / 2001$ & 97 & 89 \\
\hline
\end{tabular}


Table 1. Summary of National Weather Service daily precipitation stations in Texas with known names, latitudes, and longitudes-Continued

\begin{tabular}{|c|c|c|c|c|c|c|c|c|c|c|}
\hline $\begin{array}{l}\text { Seq. } \\
\text { no. }\end{array}$ & NWS station name & $\begin{array}{c}\text { NWS } \\
\text { station } \\
\text { no. }\end{array}$ & County & $\begin{array}{c}\text { Elev. } \\
\text { (ft above } \\
\text { NGVD } \\
29 \text { ) }\end{array}$ & $\begin{array}{l}\text { Lati- } \\
\text { tude }\end{array}$ & $\begin{array}{l}\text { Longi- } \\
\text { tude }\end{array}$ & $\begin{array}{c}\text { Begining } \\
\text { date } \\
\text { (mm/yyyy) }\end{array}$ & $\begin{array}{c}\text { Ending } \\
\text { date } \\
\text { (mm/yyyy) }\end{array}$ & $\begin{array}{l}\text { Per- } \\
\text { cent } \\
\text { cov- } \\
\text { erage }\end{array}$ & $\begin{array}{c}\text { Years } \\
\text { of } \\
\text { record }\end{array}$ \\
\hline 513 & Harper & 3954 & Gillespie & 2,080 & $30^{\circ} 18^{\prime}$ & $99^{\circ} 15^{\prime}$ & $10 / 1909$ & $12 / 2001$ & 96 & 61 \\
\hline 514 & Harris Lake & 3963 & Brazoria & $* 43$ & $29^{\circ} 15^{\prime}$ & $95^{\circ} 33^{\prime}$ & $12 / 1948$ & $01 / 1949$ & 5 & 2 \\
\hline 515 & Hart & 3972 & Castro & 3,640 & $34^{\circ} 21^{\prime}$ & $102^{\circ} 05^{\prime}$ & $01 / 1947$ & $12 / 2001$ & 90 & 49 \\
\hline 516 & Hart Jones Ranch & 3976 & Hale* & 1,250 & $34^{\circ} 17^{\prime}$ & $102^{\circ} 04^{\prime}$ & $11 / 1946$ & $07 / 1954$ & 84 & 9 \\
\hline 517 & Hartley 4 ESE & 3981 & Hartley & 3,905 & $35^{\circ} 51^{\prime}$ & $102^{\circ} 19^{\prime}$ & $10 / 1947$ & $12 / 2001$ & 97 & 55 \\
\hline 518 & Haskell & 3992 & Haskell & 1,600 & $33^{\circ} 09^{\prime}$ & $99^{\circ} 44^{\prime}$ & $08 / 1897$ & $12 / 2001$ & 97 & 104 \\
\hline 519 & Haskell 6 NW & 3994 & Haskell* & 1,703 & $33^{\circ} 14^{\prime}$ & $99^{\circ} 48^{\prime}$ & $10 / 1941$ & $07 / 1963$ & 92 & 23 \\
\hline 520 & Haskell SCS & 3995 & Haskell* & 1,552 & $33^{\circ} 04^{\prime}$ & $99^{\circ} 37^{\prime}$ & $09 / 1946$ & $08 / 1948$ & 61 & 3 \\
\hline 521 & Hawkins & 4020 & Wood & 335 & $32^{\circ} 34^{\prime}$ & $95^{\circ} 12^{\prime}$ & $04 / 1924$ & $12 / 2001$ & 90 & 54 \\
\hline 522 & Hawley 3 NE & 4026 & Jones & 1,650 & $32^{\circ} 39^{\prime}$ & $99^{\circ} 44^{\prime}$ & $04 / 1973$ & 09/1994 & 92 & 22 \\
\hline 523 & Haynes Ranch & 4033 & Blanco & 1,371 & $30^{\circ} 28^{\prime}$ & $98^{\circ} 25^{\prime}$ & $03 / 1948$ & $11 / 1958$ & 95 & 11 \\
\hline 524 & Heath Canyon & 4051 & Brewster & 1,760 & $29^{\circ} 26^{\prime}$ & $102^{\circ} 49^{\prime}$ & $04 / 1995$ & $12 / 2001$ & 95 & 7 \\
\hline 525 & Heath Farm & 4055 & Brown* & 1,650 & $31^{\circ} 44^{\prime}$ & $99^{\circ} 11^{\prime}$ & $06 / 1948$ & $06 / 1948$ & 8 & 1 \\
\hline 526 & Hebbronville & 4058 & Jim Hogg & 580 & $27^{\circ} 19^{\prime}$ & $98^{\circ} 41^{\prime}$ & $03 / 1905$ & $12 / 2001$ & 93 & 96 \\
\hline 527 & Hemphill & 4076 & Sabine & 300 & $31^{\circ} 21^{\prime}$ & $93^{\circ} 50^{\prime}$ & $06 / 1967$ & $01 / 1992$ & 91 & 26 \\
\hline 528 & Hemphill 6 NE & 4077 & Sabine & 180 & $31^{\circ} 24^{\prime}$ & $93^{\circ} 47^{\prime}$ & $02 / 1992$ & $12 / 2001$ & 96 & 9 \\
\hline 529 & Hempstead & 4080 & Waller & 249 & $30^{\circ} 06^{\prime}$ & $96^{\circ} 05^{\prime}$ & $09 / 1946$ & 09/1978 & 96 & 33 \\
\hline 530 & Henderson & 4081 & Rusk & 420 & $32^{\circ} 10^{\prime}$ & $94^{\circ} 47^{\prime}$ & $07 / 1908$ & $12 / 2001$ & 99 & 94 \\
\hline 531 & Henly & 4088 & Hays & 1,161 & $30^{\circ} 12^{\prime}$ & $98^{\circ} 13^{\prime}$ & $03 / 1948$ & $11 / 1965$ & 97 & 18 \\
\hline 532 & Henrietta & 4093 & Clay & 930 & $33^{\circ} 48^{\prime}$ & $98^{\circ} 12^{\prime}$ & $06 / 1897$ & $12 / 2001$ & 98 & 101 \\
\hline 533 & Hereford & 4098 & Deaf Smith & 3,820 & $34^{\circ} 49^{\prime}$ & $102^{\circ} 24^{\prime}$ & $01 / 1905$ & $12 / 2001$ & 95 & 74 \\
\hline 534 & Hereford $1 \mathrm{SE}$ & 4100 & Deaf Smith* & 3,822 & $34^{\circ} 49^{\prime}$ & $102^{\circ} 24^{\prime}$ & $06 / 1948$ & $08 / 1951$ & 67 & 4 \\
\hline 535 & Hewitt & 4122 & McLennan & 660 & $31^{\circ} 28^{\prime}$ & $97^{\circ} 12^{\prime}$ & $05 / 1879$ & $12 / 2001$ & 99 & 116 \\
\hline 536 & Hext & 4123 & Menard* & 1,923 & $30^{\circ} 52^{\prime}$ & $99^{\circ} 32^{\prime}$ & $06 / 1948$ & $03 / 1952$ & 14 & 3 \\
\hline 537 & Hico & 4137 & Hamilton & 1,025 & $31^{\circ} 59^{\prime}$ & $98^{\circ} 01^{\prime}$ & $11 / 1910$ & $12 / 2001$ & 95 & 92 \\
\hline 538 & Hidalgo & 4139 & Hidalgo* & 102 & $26^{\circ} 06^{\prime}$ & $98^{\circ} 16^{\prime}$ & $09 / 1946$ & $03 / 1955$ & 70 & 9 \\
\hline 539 & Higgins $1 \mathrm{~N}$ & 4140 & Lipscomb & 2,578 & $36^{\circ} 08^{\prime}$ & $100^{\circ} 01^{\prime}$ & $11 / 1907$ & $11 / 2001$ & 95 & 57 \\
\hline 540 & Highbank & 4150 & Falls* & 351 & $31^{\circ} 10^{\prime}$ & $96^{\circ} 49^{\prime}$ & $05 / 1916$ & $07 / 1949$ & 65 & 7 \\
\hline 541 & Hillsboro & 4182 & Hill & 550 & $32^{\circ} 01^{\prime}$ & $97^{\circ} 07^{\prime}$ & $06 / 1903$ & $12 / 2001$ & 97 & 99 \\
\hline 542 & Hindes & 4191 & Atascosa & 360 & $28^{\circ} 43^{\prime}$ & $98^{\circ} 48^{\prime}$ & $06 / 1948$ & 09/1951 & 81 & 4 \\
\hline 543 & Hitchland 6 SSW & 4196 & Hansford & 3,192 & $36^{\circ} 25^{\prime}$ & $101^{\circ} 21^{\prime}$ & $10 / 1947$ & $10 / 1973$ & 85 & 27 \\
\hline 544 & Hondo & 4254 & Medina & 876 & $29^{\circ} 20^{\prime}$ & $99^{\circ} 08^{\prime}$ & $01 / 1900$ & $12 / 2001$ & 98 & 82 \\
\hline 545 & Hondo Airport & 4256 & Medina & 920 & $29^{\circ} 21^{\prime}$ & $99^{\circ} 10^{\prime}$ & $03 / 1975$ & $12 / 2001$ & 92 & 25 \\
\hline 546 & Honey Grove & 4257 & Fannin & 680 & $33^{\circ} 35^{\prime}$ & $95^{\circ} 54^{\prime}$ & $04 / 1898$ & $12 / 2001$ & 99 & 87 \\
\hline 547 & Hords Creek Dam & 4278 & Coleman & 1,942 & $31^{\circ} 51^{\prime}$ & $99^{\circ} 34^{\prime}$ & $07 / 1953$ & $12 / 2001$ & 99 & 49 \\
\hline 548 & Horger & 4280 & Jasper & 112 & $31^{\circ} 00^{\prime}$ & $94^{\circ} 10^{\prime}$ & $09 / 1946$ & $12 / 1982$ & 97 & 37 \\
\hline 549 & Hot Springs & 4299 & Brewster* & 2,201 & $29^{\circ} 11^{\prime}$ & $103^{\circ} 00^{\prime}$ & $07 / 1948$ & 09/1951 & 60 & 4 \\
\hline 550 & Houston Addicks & 4309 & Harris & 91 & $29^{\circ} 46^{\prime}$ & $95^{\circ} 39^{\prime}$ & $06 / 1948$ & $04 / 1951$ & 57 & 4 \\
\hline 551 & Houston Alief & 4311 & Harris & 71 & $29^{\circ} 43^{\prime}$ & $95^{\circ} 36^{\prime}$ & $06 / 1948$ & $12 / 1964$ & 88 & 17 \\
\hline 552 & Houston Barker & 4313 & Harris & 127 & $29^{\circ} 49^{\prime}$ & $95^{\circ} 44^{\prime}$ & $06 / 1948$ & $12 / 2001$ & 99 & 54 \\
\hline 553 & Houston Barker & 520 & Harris & 131 & $29^{\circ} 49^{\prime}$ & $95^{\circ} 44^{\prime}$ & $01 / 1948$ & $05 / 1948$ & 42 & 1 \\
\hline 554 & Houston Bush International Airport & 4300 & Harris & 95 & $29^{\circ} 59^{\prime}$ & $95^{\circ} 21^{\prime}$ & $06 / 1969$ & $12 / 2001$ & 99 & 33 \\
\hline 555 & Houston Deer Park & 2348 & Harris & $* 26$ & $29^{\circ} 43^{\prime}$ & $95^{\circ} 08^{\prime}$ & $01 / 1948$ & $05 / 1948$ & 40 & 1 \\
\hline 556 & Houston Deer Park & 4315 & Harris & 35 & $29^{\circ} 43^{\prime}$ & $95^{\circ} 08^{\prime}$ & $08 / 1945$ & $12 / 2001$ & 96 & 57 \\
\hline 557 & Houston Fairbanks & 3043 & Harris & 89 & $29^{\circ} 48^{\prime}$ & $95^{\circ} 30^{\prime}$ & $09 / 1947$ & $05 / 1948$ & 37 & 2 \\
\hline 558 & Houston Fairbanks & 4317 & Harris & 89 & $29^{\circ} 48^{\prime}$ & $95^{\circ} 31^{\prime}$ & $06 / 1948$ & $03 / 1954$ & 83 & 7 \\
\hline 559 & Houston Golf Crest & 4319 & Harris & 49 & $29^{\circ} 41^{\prime}$ & $95^{\circ} 17^{\prime}$ & $06 / 1948$ & $05 / 1950$ & 53 & 3 \\
\hline 560 & Houston Heights & 4310 & Harris & 59 & $29^{\circ} 47^{\prime}$ & $95^{\circ} 26^{\prime}$ & $10 / 1947$ & $05 / 1948$ & 33 & 2 \\
\hline 561 & Houston Heights & 4321 & Harris & 65 & $29^{\circ} 47^{\prime}$ & $95^{\circ} 26^{\prime}$ & $06 / 1948$ & $12 / 2001$ & 97 & 50 \\
\hline 562 & Houston Hobby Airport & 4307 & Harris & 44 & $29^{\circ} 38^{\prime}$ & $95^{\circ} 16^{\prime}$ & $11 / 1941$ & $12 / 2001$ & 93 & 61 \\
\hline 563 & Houston Independent Heights & 4323 & Harris & 93 & $29^{\circ} 52^{\prime}$ & $95^{\circ} 25^{\prime}$ & $06 / 1948$ & $06 / 1995$ & 96 & 48 \\
\hline 564 & Houston Jersey Village & 4327 & Harris & 112 & $29^{\circ} 53^{\prime}$ & $95^{\circ} 32^{\prime}$ & $06 / 1948$ & $12 / 2001$ & 96 & 54 \\
\hline
\end{tabular}


Table 1. Summary of National Weather Service daily precipitation stations in Texas with known names, latitudes, and longitudes-Continued

\begin{tabular}{|c|c|c|c|c|c|c|c|c|c|c|}
\hline $\begin{array}{c}\text { Seq. } \\
\text { no. }\end{array}$ & NWS station name & $\begin{array}{c}\text { NWS } \\
\text { station } \\
\text { no. }\end{array}$ & County & $\begin{array}{c}\text { Elev. } \\
\text { (ft above } \\
\text { NGVD } \\
\text { 29) }\end{array}$ & $\begin{array}{l}\text { Lati- } \\
\text { tude }\end{array}$ & $\begin{array}{l}\text { Longi- } \\
\text { tude }\end{array}$ & $\begin{array}{l}\text { Begining } \\
\text { date } \\
\text { (mm/yyyy) }\end{array}$ & $\begin{array}{c}\text { Ending } \\
\text { date } \\
\text { (mm/yyyy) }\end{array}$ & $\begin{array}{l}\text { Per- } \\
\text { cent } \\
\text { cov- } \\
\text { erage }\end{array}$ & $\begin{array}{l}\text { Years } \\
\text { of } \\
\text { record }\end{array}$ \\
\hline 565 & Houston NWSO & 4333 & Galveston & 19 & $29^{\circ} 28^{\prime}$ & $95^{\circ} 05^{\prime}$ & $06 / 1905$ & $12 / 2001$ & 79 & 17 \\
\hline 566 & Houston San Jacinto Dam & 4328 & Harris & 58 & $29^{\circ} 55^{\prime}$ & $95^{\circ} 09^{\prime}$ & $06 / 1954$ & 09/1996 & 95 & 43 \\
\hline 567 & Houston Satsuma & 4329 & Harris & 122 & $29^{\circ} 56^{\prime}$ & $95^{\circ} 38^{\prime}$ & $03 / 1923$ & $12 / 1964$ & 93 & 26 \\
\hline 568 & Houston Spring Branch & 4331 & Harris & 92 & $29^{\circ} 48^{\prime}$ & $95^{\circ} 30^{\prime}$ & 04/1954 & $12 / 2000$ & 96 & 45 \\
\hline 569 & Houston WB City & 4305 & Harris* & 43 & $29^{\circ} 46^{\prime}$ & $95^{\circ} 22^{\prime}$ & $11 / 1941$ & $10 / 1969$ & 87 & 29 \\
\hline 570 & Houston-Port & 4326 & Harris & 19 & $29^{\circ} 45^{\prime}$ & $95^{\circ} 17^{\prime}$ & 02/1991 & $12 / 2001$ & 90 & 11 \\
\hline 571 & Houston-Westbury & 4325 & Harris & 65 & $29^{\circ} 41^{\prime}$ & $95^{\circ} 28^{\prime}$ & 06/1948 & $12 / 2001$ & 97 & 51 \\
\hline 572 & Huckabay & 4343 & Erath & 1,415 & $32^{\circ} 20^{\prime}$ & $98^{\circ} 18^{\prime}$ & $04 / 1963$ & $12 / 2001$ & 94 & 37 \\
\hline 573 & Hudspeth River Ranch & 4348 & Val Verde & 1,630 & $29^{\circ} 59^{\prime}$ & $101^{\circ} 10^{\prime}$ & 03/1988 & $12 / 2001$ & 98 & 14 \\
\hline 574 & Humble & 4362 & Harris & 102 & $30^{\circ} 00^{\prime}$ & $95^{\circ} 15^{\prime}$ & $06 / 1954$ & $05 / 1985$ & 95 & 32 \\
\hline 575 & Humble Pump Station 5 WNW & 4363 & Sutton & 2,200 & $30^{\circ} 21^{\prime}$ & $100^{\circ} 18^{\prime}$ & $03 / 1948$ & $12 / 2001$ & 90 & 53 \\
\hline 576 & Hunt $10 \mathrm{~W}$ & 4375 & Kerr & 2,095 & $30^{\circ} 02^{\prime}$ & $99^{\circ} 31^{\prime}$ & 01/1992 & $12 / 2001$ & 99 & 10 \\
\hline 577 & Hunt $3 \mathrm{SW}$ & 4374 & Kerr & 1,870 & $30^{\circ} 01^{\prime}$ & $99^{\circ} 21^{\prime}$ & 09/1947 & 05/1999 & 96 & 53 \\
\hline 578 & Huntsville & 4382 & Walker & 494 & $30^{\circ} 43^{\prime}$ & $95^{\circ} 33^{\prime}$ & 09/1946 & $12 / 2001$ & 98 & 56 \\
\hline 579 & Hurst Springs & 4390 & Coryell & 1,030 & $31^{\circ} 39^{\prime}$ & $97^{\circ} 43^{\prime}$ & $04 / 1963$ & $12 / 2001$ & 99 & 39 \\
\hline 580 & Hurt & 4392 & Hunt* & 679 & $33^{\circ} 13^{\prime}$ & $95^{\circ} 58^{\prime}$ & 06/1948 & 08/1948 & 20 & 1 \\
\hline 581 & Hyatt & 4397 & Tyler* & 112 & $30^{\circ} 34^{\prime}$ & $94^{\circ} 24^{\prime}$ & 09/1946 & $10 / 1953$ & 81 & 8 \\
\hline 582 & Hye & 4402 & Blanco & 1,456 & $30^{\circ} 15^{\prime}$ & $98^{\circ} 34^{\prime}$ & 03/1948 & $12 / 2001$ & 94 & 54 \\
\hline 583 & Imperial & 4425 & Pecos & 2,400 & $31^{\circ} 16^{\prime}$ & $102^{\circ} 42^{\prime}$ & 09/1947 & 09/1993 & 94 & 47 \\
\hline 584 & Independence Heights & 4430 & Harris & 79 & $29^{\circ} 52^{\prime}$ & $95^{\circ} 25^{\prime}$ & $10 / 1947$ & 05/1948 & 33 & 2 \\
\hline 585 & Indian Gap & 4440 & Hamilton & 1,575 & $31^{\circ} 40^{\prime}$ & $98^{\circ} 25^{\prime}$ & $09 / 1947$ & $10 / 1983$ & 97 & 37 \\
\hline 586 & Indian Hot Springs & 4443 & Hudspeth* & 3,704 & $30^{\circ} 51^{\prime}$ & $105^{\circ} 20^{\prime}$ & 08/1947 & $06 / 1953$ & 28 & 4 \\
\hline 587 & Ingram No. 2 & 4458 & Kerr & 1,703 & $30^{\circ} 04^{\prime}$ & $99^{\circ} 14^{\prime}$ & 01/1992 & $12 / 2001$ & 100 & 10 \\
\hline 588 & Iowa Park Experiment Station & 4471 & Wichita & 981 & $33^{\circ} 55^{\prime}$ & $98^{\circ} 39^{\prime}$ & $07 / 1940$ & $02 / 1964$ & 94 & 25 \\
\hline 589 & Iron Bridge Dam & 4483 & Rains & 450 & $32^{\circ} 49^{\prime}$ & $95^{\circ} 55^{\prime}$ & $05 / 1975$ & $12 / 1993$ & 94 & 19 \\
\hline 590 & Itasca & 4503 & Hill & 712 & $32^{\circ} 10^{\prime}$ & $97^{\circ} 09^{\prime}$ & $10 / 1947$ & 06/1949 & 34 & 3 \\
\hline 591 & Itasca & 4505 & Hill & 710 & $32^{\circ} 10^{\prime}$ & $97^{\circ} 09^{\prime}$ & $01 / 1992$ & $12 / 2001$ & 100 & 10 \\
\hline 592 & Jacksboro & 4517 & Jack & 1,100 & $33^{\circ} 14^{\prime}$ & $98^{\circ} 09^{\prime}$ & 03/1941 & $12 / 2001$ & 99 & 61 \\
\hline 593 & Jacksonville & 4525 & Cherokee & 560 & $31^{\circ} 57^{\prime}$ & $95^{\circ} 16^{\prime}$ & 09/1953 & $12 / 2001$ & 88 & 48 \\
\hline 594 & Jacksonville Experiment Station & 4524 & Cherokee & 659 & $31^{\circ} 59^{\prime}$ & $95^{\circ} 17^{\prime}$ & 09/1947 & $12 / 1963$ & 95 & 17 \\
\hline 595 & James River Ranch & 4538 & Mason & 1,552 & $30^{\circ} 32^{\prime}$ & $99^{\circ} 23^{\prime}$ & 03/1948 & $12 / 1975$ & 90 & 28 \\
\hline 596 & Jarrell & 4556 & Williamson & 895 & $30^{\circ} 49^{\prime}$ & $97^{\circ} 36^{\prime}$ & $08 / 1926$ & $12 / 2001$ & 94 & 75 \\
\hline 597 & Jasper & 4563 & Jasper & 290 & $30^{\circ} 54^{\prime}$ & $94^{\circ} 00^{\prime}$ & $09 / 1878$ & $12 / 2001$ & 91 & 62 \\
\hline 598 & Jasper $2 \mathrm{E}$ & 4564 & Jasper* & 220 & $30^{\circ} 55^{\prime}$ & $93^{\circ} 58^{\prime}$ & $12 / 1948$ & $11 / 1952$ & 57 & 5 \\
\hline 599 & Jayton & 4570 & Kent & 2,010 & $33^{\circ} 15^{\prime}$ & $100^{\circ} 34^{\prime}$ & 06/1910 & $12 / 2001$ & 90 & 54 \\
\hline 600 & Jeddo $3 \mathrm{~S}$ & 4575 & Bastrop & 415 & $29^{\circ} 46^{\prime}$ & $97^{\circ} 19^{\prime}$ & 09/1947 & $12 / 2001$ & 97 & 55 \\
\hline 601 & Jefferson & 4577 & Marion & 199 & $32^{\circ} 46^{\prime}$ & $94^{\circ} 20^{\prime}$ & 11/1903 & 09/2001 & 96 & 92 \\
\hline 602 & Jewett & 4591 & Leon & 510 & $31^{\circ} 21^{\prime}$ & $96^{\circ} 09^{\prime}$ & $01 / 1904$ & $01 / 1991$ & 91 & 46 \\
\hline 603 & Joe Pool Lake & 4597 & Dallas & 591 & $32^{\circ} 38^{\prime}$ & $96^{\circ} 58^{\prime}$ & 03/1984 & $12 / 2001$ & 94 & 18 \\
\hline 604 & Johnson City & 4605 & Blanco & 1,232 & $30^{\circ} 17^{\prime}$ & $98^{\circ} 25^{\prime}$ & $10 / 1964$ & $12 / 2001$ & 93 & 36 \\
\hline 605 & Johnson Ranch & 4608 & McCulloch & 1,850 & $31^{\circ} 14^{\prime}$ & $99^{\circ} 29^{\prime}$ & $04 / 1948$ & $12 / 1965$ & 94 & 18 \\
\hline 606 & Jones CB Ranch & 4627 & Schleicher & 2,090 & $30^{\circ} 49^{\prime}$ & $100^{\circ} 08^{\prime}$ & 05/1948 & 05/1964 & 85 & 17 \\
\hline 607 & Jones MW Ranch & 4630 & Schleicher* & 2,280 & $30^{\circ} 57^{\prime}$ & $100^{\circ} 16^{\prime}$ & $03 / 1948$ & $11 / 1958$ & 97 & 11 \\
\hline 608 & Jourdanton & 4647 & Atascosa & 518 & $28^{\circ} 54^{\prime}$ & $98^{\circ} 32^{\prime}$ & 06/1916 & $12 / 2001$ & 93 & 62 \\
\hline 609 & Judkins & 4661 & Ector* & 2,904 & $31^{\circ} 43^{\prime}$ & $102^{\circ} 38^{\prime}$ & 10/1947 & 09/1955 & 88 & 9 \\
\hline 610 & Junction 4 SSW & 4670 & Kimble & 1,747 & $30^{\circ} 26^{\prime}$ & $99^{\circ} 48^{\prime}$ & $01 / 1897$ & $12 / 2001$ & 91 & 92 \\
\hline 611 & Junction FAA Airport & 4671 & Kimble & $* 1,680$ & $30^{\circ} 30^{\prime}$ & $99^{\circ} 46^{\prime}$ & 09/1948 & $06 / 2001$ & 90 & 25 \\
\hline 612 & Juno & 4672 & Val Verde & 1,801 & $30^{\circ} 09^{\prime}$ & $101^{\circ} 07^{\prime}$ & $10 / 1964$ & 03/1975 & 82 & 12 \\
\hline 613 & Juno $10 \mathrm{NNE}$ & 4674 & Crockett & 2,103 & $30^{\circ} 17^{\prime}$ & $101^{\circ} 05^{\prime}$ & $10 / 1975$ & 04/1978 & 47 & 4 \\
\hline 614 & Juno $4 \mathrm{~S}$ & 4675 & Val Verde & 1,820 & $30^{\circ} 05^{\prime}$ & $101^{\circ} 07^{\prime}$ & 09/1980 & 05/1999 & 76 & 12 \\
\hline 615 & Justin & 4679 & Denton & 640 & $33^{\circ} 04^{\prime}$ & $97^{\circ} 17^{\prime}$ & $11 / 2001$ & $12 / 2001$ & 17 & 1 \\
\hline 616 & Kaffie Ranch & 4682 & Jim Hogg & 479 & $27^{\circ} 05^{\prime}$ & $98^{\circ} 35^{\prime}$ & $03 / 1965$ & 09/1971 & 86 & 6 \\
\hline
\end{tabular}


Table 1. Summary of National Weather Service daily precipitation stations in Texas with known names, latitudes, and longitudes-Continued

\begin{tabular}{|c|c|c|c|c|c|c|c|c|c|c|}
\hline $\begin{array}{l}\text { Seq. } \\
\text { no. }\end{array}$ & NWS station name & $\begin{array}{c}\text { NWS } \\
\text { station } \\
\text { no. }\end{array}$ & County & $\begin{array}{c}\text { Elev. } \\
\text { (ft above } \\
\text { NGVD } \\
\text { 29) }\end{array}$ & $\begin{array}{l}\text { Lati- } \\
\text { tude }\end{array}$ & $\begin{array}{l}\text { Longi- } \\
\text { tude }\end{array}$ & $\begin{array}{c}\text { Begining } \\
\text { date } \\
\text { (mm/yyyy) }\end{array}$ & $\begin{array}{c}\text { Ending } \\
\text { date } \\
\text { (mm/yyyy) }\end{array}$ & $\begin{array}{c}\text { Per- } \\
\text { cent } \\
\text { cov- } \\
\text { erage }\end{array}$ & $\begin{array}{c}\text { Years } \\
\text { of } \\
\text { record }\end{array}$ \\
\hline 617 & Kanawha & 4688 & Red River & 515 & $33^{\circ} 50^{\prime}$ & $95^{\circ} 15^{\prime}$ & $05 / 1996$ & $04 / 2001$ & 80 & 6 \\
\hline 618 & Karnack 6 SW & 4693 & Harrison & 170 & $32^{\circ} 35^{\prime}$ & $94^{\circ} 14^{\prime}$ & $05 / 1942$ & $05 / 2000$ & 96 & 59 \\
\hline 619 & Karnes City $2 \mathrm{~N}$ & 4696 & Karnes & 450 & $28^{\circ} 54^{\prime}$ & $97^{\circ} 52^{\prime}$ & $10 / 1919$ & $12 / 2001$ & 95 & 82 \\
\hline 620 & Katy & 4703 & Harris & 142 & $29^{\circ} 47^{\prime}$ & $95^{\circ} 49^{\prime}$ & $06 / 1948$ & $05 / 1950$ & 64 & 3 \\
\hline 621 & Katy City & 4704 & Harris & 153 & $29^{\circ} 48^{\prime}$ & $95^{\circ} 49^{\prime}$ & $04 / 1952$ & $12 / 2001$ & 96 & 47 \\
\hline 622 & Kaufman 3 SE & 4705 & Kaufman & 420 & $32^{\circ} 33^{\prime}$ & $96^{\circ} 16^{\prime}$ & $10 / 1901$ & $12 / 2001$ & 98 & 101 \\
\hline 623 & Kempner & 4745 & Lampasas & 925 & $31^{\circ} 05^{\prime}$ & $98^{\circ} 00^{\prime}$ & $05 / 1963$ & $01 / 1987$ & 92 & 22 \\
\hline 624 & Kendalia & 4757 & Kendall & 1,360 & $29^{\circ} 57^{\prime}$ & $98^{\circ} 30^{\prime}$ & 08/1996 & $12 / 2001$ & 89 & 6 \\
\hline 625 & Kenedy $1 \mathrm{E}$ & 4752 & Karnes & 279 & $28^{\circ} 49^{\prime}$ & $97^{\circ} 51^{\prime}$ & $08 / 1948$ & 04/1977 & 91 & 30 \\
\hline 626 & Kennedale 6 SSW & 4761 & Tarrant & 741 & $32^{\circ} 33^{\prime}$ & $97^{\circ} 14^{\prime}$ & $12 / 1949$ & $01 / 1981$ & 90 & 33 \\
\hline 627 & Kent $5 \mathrm{E}$ & 4767 & Jeff Davis & 4,183 & $31^{\circ} 04^{\prime}$ & $104^{\circ} 09^{\prime}$ & $04 / 1898$ & 04/1976 & 79 & 41 \\
\hline 628 & Kent 8 SE & 4770 & Jeff Davis & 4,860 & $31^{\circ} 00^{\prime}$ & $104^{\circ} 06^{\prime}$ & $04 / 1988$ & $12 / 2001$ & 98 & 14 \\
\hline 629 & Kerrville & 4780 & Kerr & 1,640 & $30^{\circ} 03^{\prime}$ & $99^{\circ} 09^{\prime}$ & $01 / 1897$ & $07 / 1974$ & 97 & 75 \\
\hline 630 & Kerrville 3 NNE & 4782 & Kerr & 1,782 & $30^{\circ} 04^{\prime}$ & $99^{\circ} 06^{\prime}$ & $10 / 1974$ & $12 / 2001$ & 97 & 28 \\
\hline 631 & Killeen & 4791 & Bell & 801 & $31^{\circ} 07^{\prime}$ & $97^{\circ} 42^{\prime}$ & $01 / 1912$ & $08 / 1978$ & 90 & 33 \\
\hline 632 & Killeen $3 \mathrm{~S}$ & 4792 & Bell & 910 & $31^{\circ} 04^{\prime}$ & $97^{\circ} 44^{\prime}$ & $10 / 1978$ & $12 / 2001$ & 90 & 24 \\
\hline 633 & Kingsbury & 4805 & Guadalupe & 510 & $29^{\circ} 41^{\prime}$ & $97^{\circ} 45^{\prime}$ & 02/1997 & $12 / 2001$ & 93 & 5 \\
\hline 634 & Kingston Ranch & 4809 & Jeff Davis & 4,442 & $30^{\circ} 52^{\prime}$ & $103^{\circ} 59^{\prime}$ & $02 / 1949$ & $01 / 1952$ & 26 & 3 \\
\hline 635 & Kingsville & 4810 & Kleberg & 66 & $27^{\circ} 31^{\prime}$ & $97^{\circ} 51^{\prime}$ & $02 / 1902$ & $12 / 2001$ & 89 & 83 \\
\hline 636 & Kirbyville & 4819 & Jasper & 200 & $30^{\circ} 37^{\prime}$ & $93^{\circ} 55^{\prime}$ & $01 / 1929$ & 02/1999 & 98 & 58 \\
\hline 637 & Knapp 2 SW & 4841 & Scurry & 2,290 & $32^{\circ} 37^{\prime}$ & $101^{\circ} 09^{\prime}$ & 08/1931 & $12 / 2001$ & 95 & 70 \\
\hline 638 & Knox City & 4852 & Knox & 1,532 & $33^{\circ} 25^{\prime}$ & $99^{\circ} 49^{\prime}$ & $06 / 1935$ & $11 / 1965$ & 98 & 31 \\
\hline 639 & Kopperl 5 NNE & 4866 & Hill & 620 & $32^{\circ} 08^{\prime}$ & $97^{\circ} 28^{\prime}$ & $12 / 1900$ & $12 / 2001$ & 79 & 18 \\
\hline 640 & Kountze & 4878 & Hardin & 61 & $30^{\circ} 22^{\prime}$ & $94^{\circ} 17^{\prime}$ & $06 / 1948$ & $12 / 2001$ & 89 & 18 \\
\hline 641 & Kress & 4880 & Swisher & 3,480 & $34^{\circ} 22^{\prime}$ & $101^{\circ} 44^{\prime}$ & $06 / 1948$ & $09 / 1951$ & 82 & 4 \\
\hline 642 & Kyle SCS 9 & 4888 & Hays & 722 & $30^{\circ} 00^{\prime}$ & $97^{\circ} 53^{\prime}$ & 08/1941 & $05 / 1942$ & 40 & 2 \\
\hline 643 & La Grange & 4903 & Fayette & 357 & $29^{\circ} 55^{\prime}$ & $96^{\circ} 52^{\prime}$ & $06 / 1910$ & $12 / 2001$ & 98 & 92 \\
\hline 644 & La Joya & 4911 & Hidalgo & 180 & $26^{\circ} 15^{\prime}$ & $98^{\circ} 29^{\prime}$ & $02 / 1995$ & $12 / 2001$ & 96 & 7 \\
\hline 645 & La Pryor & 4920 & Zavala & 759 & $28^{\circ} 59^{\prime}$ & $99^{\circ} 52^{\prime}$ & $04 / 1915$ & $12 / 2001$ & 90 & 83 \\
\hline 646 & La Tuna $1 \mathrm{~S}$ & 4931 & El Paso & 3,800 & $31^{\circ} 58^{\prime}$ & $106^{\circ} 36^{\prime}$ & $03 / 1943$ & $12 / 2001$ & 96 & 59 \\
\hline 647 & La Vernia & 4934 & Wilson* & 479 & $29^{\circ} 22^{\prime}$ & $98^{\circ} 06^{\prime}$ & $09 / 1947$ & $05 / 1950$ & 62 & 4 \\
\hline 648 & Laguna $3 \mathrm{~N}$ & 4907 & Uvalde & 1,160 & $29^{\circ} 27^{\prime}$ & $100^{\circ} 01^{\prime}$ & $08 / 1996$ & $12 / 2001$ & 87 & 6 \\
\hline 649 & Lajitas & 4950 & Brewster & 2,403 & $29^{\circ} 16^{\prime}$ & $103^{\circ} 45^{\prime}$ & 03/1978 & $11 / 2001$ & 93 & 24 \\
\hline 650 & Lake Abilene & 4960 & Taylor & 1,755 & $32^{\circ} 13^{\prime}$ & $99^{\circ} 53^{\prime}$ & $03 / 1962$ & $12 / 2001$ & 98 & 40 \\
\hline 651 & Lake Alan Henry & 4967 & Garza & 2,280 & $33^{\circ} 03^{\prime}$ & $101^{\circ} 02^{\prime}$ & $05 / 1994$ & $12 / 2001$ & 96 & 8 \\
\hline 652 & Lake Bridgeport Dam & 4972 & Wise & 870 & $33^{\circ} 13^{\prime}$ & $97^{\circ} 49^{\prime}$ & $06 / 1948$ & $12 / 2001$ & 44 & 5 \\
\hline 653 & Lake Coffee Mill & 4973 & Fannin* & 502 & $33^{\circ} 44^{\prime}$ & $96^{\circ} 00^{\prime}$ & $06 / 1948$ & $09 / 1951$ & 74 & 4 \\
\hline 654 & Lake Colorado City & 4974 & Mitchell & 2,100 & $32^{\circ} 20^{\prime}$ & $100^{\circ} 55^{\prime}$ & $11 / 1954$ & 03/1993 & 83 & 34 \\
\hline 655 & Lake Dallas & 4977 & Denton* & 522 & $33^{\circ} 07^{\prime}$ & $97^{\circ} 02^{\prime}$ & $10 / 1947$ & $05 / 1956$ & 78 & 10 \\
\hline 656 & Lake Fork Reservoir & 4976 & Wood & 414 & $32^{\circ} 49^{\prime}$ & $95^{\circ} 31^{\prime}$ & $01 / 1989$ & $12 / 2001$ & 100 & 13 \\
\hline 657 & Lake Kemp & 4982 & Baylor & 1,167 & $33^{\circ} 45^{\prime}$ & $99^{\circ} 08^{\prime}$ & $04 / 1962$ & $12 / 2001$ & 93 & 34 \\
\hline 658 & Lake Kickapoo & 4978 & Archer & 1,060 & $33^{\circ} 40^{\prime}$ & $98^{\circ} 47^{\prime}$ & $11 / 1947$ & $02 / 1964$ & 87 & 18 \\
\hline 659 & Lake Palo Pinto & 4979 & Palo Pinto & 900 & $32^{\circ} 38^{\prime}$ & $98^{\circ} 19^{\prime}$ & $05 / 1994$ & $12 / 2001$ & 95 & 8 \\
\hline 660 & Lake Ray Hubbard & 4914 & Kaufman & 440 & $32^{\circ} 48^{\prime}$ & $96^{\circ} 29^{\prime}$ & $05 / 1978$ & 07/1993 & 82 & 16 \\
\hline 661 & Lake Tawakoni & 4980 & Rains & 450 & $32^{\circ} 49^{\prime}$ & $95^{\circ} 55^{\prime}$ & $02 / 1994$ & $12 / 2001$ & 97 & 8 \\
\hline 662 & Lake Victor $3 \mathrm{~W}$ & 4981 & Burnet & 1,401 & $30^{\circ} 54^{\prime}$ & $98^{\circ} 14^{\prime}$ & $03 / 1948$ & $11 / 1965$ & 83 & 18 \\
\hline 663 & Lamesa 1 SSE & 5013 & Dawson & 2,965 & $32^{\circ} 43^{\prime}$ & $101^{\circ} 56^{\prime}$ & $05 / 1910$ & $12 / 2001$ & 97 & 92 \\
\hline 664 & Lampasas & 5018 & Lampasas & 1,032 & $31^{\circ} 04^{\prime}$ & $98^{\circ} 11^{\prime}$ & $01 / 1897$ & $12 / 2001$ & 98 & 104 \\
\hline 665 & Langtry & 5048 & Val Verde & 1,290 & $29^{\circ} 47^{\prime}$ & $101^{\circ} 33^{\prime}$ & $07 / 1897$ & $12 / 2001$ & 86 & 60 \\
\hline 666 & Langtry 2 & 5049 & Val Verde & 1,342 & $29^{\circ} 48^{\prime}$ & $101^{\circ} 34^{\prime}$ & $10 / 1964$ & 08/1968 & 71 & 5 \\
\hline 667 & Laredo 2 & 5060 & Webb & 430 & $27^{\circ} 34^{\prime}$ & $99^{\circ} 29^{\prime}$ & $09 / 1946$ & $12 / 2001$ & 94 & 40 \\
\hline 668 & Laredo WB Airport & 5057 & Webb & 500 & $27^{\circ} 32^{\prime}$ & $99^{\circ} 28^{\prime}$ & $04 / 1915$ & $10 / 1965$ & 89 & 24 \\
\hline
\end{tabular}


Table 1. Summary of National Weather Service daily precipitation stations in Texas with known names, latitudes, and longitudes-Continued

\begin{tabular}{|c|c|c|c|c|c|c|c|c|c|c|}
\hline $\begin{array}{c}\text { Seq. } \\
\text { no. }\end{array}$ & NWS station name & $\begin{array}{c}\text { NWS } \\
\text { station } \\
\text { no. }\end{array}$ & County & $\begin{array}{c}\text { Elev. } \\
\text { (ft above } \\
\text { NGVD } \\
\text { 29) }\end{array}$ & $\begin{array}{l}\text { Lati- } \\
\text { tude }\end{array}$ & $\begin{array}{l}\text { Longi- } \\
\text { tude }\end{array}$ & $\begin{array}{c}\text { Begining } \\
\text { date } \\
\text { (mm/yyyy) }\end{array}$ & $\begin{array}{c}\text { Ending } \\
\text { date } \\
\text { (mm/yyyy) }\end{array}$ & $\begin{array}{l}\text { Per- } \\
\text { cent } \\
\text { cov- } \\
\text { erage }\end{array}$ & $\begin{array}{l}\text { Years } \\
\text { of } \\
\text { record }\end{array}$ \\
\hline 669 & Latex & 5081 & Panola* & 302 & $32^{\circ} 21^{\prime}$ & $94^{\circ} 06^{\prime}$ & $10 / 1947$ & $05 / 1963$ & 91 & 17 \\
\hline 670 & Latimer Ranch & 5086 & Cottle & 1,950 & $33^{\circ} 53^{\prime}$ & $100^{\circ} 23^{\prime}$ & 04/1971 & 09/1994 & 97 & 24 \\
\hline 671 & Lavon Dam & 5094 & Collin & 510 & $33^{\circ} 02^{\prime}$ & $96^{\circ} 29^{\prime}$ & $07 / 1949$ & $12 / 2001$ & 97 & 53 \\
\hline 672 & Lawn & 5097 & Taylor & 1,950 & $32^{\circ} 08^{\prime}$ & $99^{\circ} 44^{\prime}$ & $03 / 1948$ & $12 / 2001$ & 98 & 54 \\
\hline 673 & Leaday & 5109 & Coleman* & $* 1,539$ & $31^{\circ} 34^{\prime}$ & $99^{\circ} 40^{\prime}$ & 03/1948 & $12 / 1965$ & 83 & 18 \\
\hline 674 & Leakey & 5113 & Real & 1,622 & $29^{\circ} 44^{\prime}$ & $99^{\circ} 45^{\prime}$ & 02/1997 & $12 / 2001$ & 98 & 5 \\
\hline 675 & Leakey 2 & 5114 & Real & 1,601 & $29^{\circ} 42^{\prime}$ & $99^{\circ} 50^{\prime}$ & $11 / 1963$ & 01/1971 & 81 & 9 \\
\hline 676 & Leatherwood Ranch & 5116 & Erath & 1,510 & $32^{\circ} 18^{\prime}$ & $98^{\circ} 24^{\prime}$ & $01 / 1992$ & $12 / 2001$ & 91 & 10 \\
\hline 677 & Lenorah & 5158 & Martin & 2,800 & $32^{\circ} 18^{\prime}$ & $101^{\circ} 53^{\prime}$ & 03/1941 & $11 / 2001$ & 94 & 60 \\
\hline 678 & Leon Junction & 5167 & Coryell* & 679 & $31^{\circ} 21^{\prime}$ & $97^{\circ} 36^{\prime}$ & $09 / 1947$ & 08/1948 & 21 & 2 \\
\hline 679 & Levelland & 5183 & Hockley & 3,550 & $33^{\circ} 35^{\prime}$ & $102^{\circ} 22^{\prime}$ & $02 / 1926$ & $12 / 2001$ & 94 & 66 \\
\hline 680 & Lewisville & 5191 & Denton* & 489 & $33^{\circ} 03^{\prime}$ & $97^{\circ} 00^{\prime}$ & 09/1947 & $12 / 1959$ & 92 & 13 \\
\hline 681 & Lewisville $2 \mathrm{E}$ & 5190 & Denton & 455 & $33^{\circ} 03^{\prime}$ & $96^{\circ} 58^{\prime}$ & 05/1909 & 09/1911 & 77 & 3 \\
\hline 682 & Lewisville Dam & 3476 & Denton & 561 & $33^{\circ} 04^{\prime}$ & $97^{\circ} 01^{\prime}$ & 07/1949 & $12 / 1963$ & 93 & 15 \\
\hline 683 & Lewisville Dam & 5192 & Denton & 556 & $33^{\circ} 04^{\prime}$ & $97^{\circ} 00^{\prime}$ & $02 / 1964$ & $12 / 2001$ & 90 & 37 \\
\hline 684 & Lexington & 5193 & Lee & 465 & $30^{\circ} 24^{\prime}$ & $97^{\circ} 00^{\prime}$ & $06 / 1948$ & $12 / 2001$ & 97 & 44 \\
\hline 685 & Liberty & 5196 & Liberty & 35 & $30^{\circ} 03^{\prime}$ & $94^{\circ} 47^{\prime}$ & 05/1904 & $12 / 2001$ & 98 & 98 \\
\hline 686 & Liberty Hill & 5202 & Williamson & 1,040 & $30^{\circ} 40^{\prime}$ & $97^{\circ} 55^{\prime}$ & 03/1948 & $11 / 1965$ & 89 & 18 \\
\hline 687 & Ligon Ranch & 5211 & Llano* & 1,401 & $30^{\circ} 39^{\prime}$ & $98^{\circ} 34^{\prime}$ & 03/1948 & $01 / 1954$ & 61 & 7 \\
\hline 688 & Lillian & 5216 & Johnson* & 751 & $32^{\circ} 30^{\prime}$ & $97^{\circ} 11^{\prime}$ & $09 / 1947$ & $12 / 1959$ & 94 & 13 \\
\hline 689 & Lillian $3 \mathrm{~W}$ & 5218 & Johnson & 745 & $32^{\circ} 30^{\prime}$ & $97^{\circ} 14^{\prime}$ & 03/1981 & 05/1997 & 95 & 17 \\
\hline 690 & Lindale $5 \mathrm{SE}$ & 5228 & Smith & 551 & $32^{\circ} 27^{\prime}$ & $95^{\circ} 22^{\prime}$ & $02 / 1931$ & $06 / 1965$ & 98 & 35 \\
\hline 691 & Linden & 5229 & Cass & 415 & $33^{\circ} 00^{\prime}$ & $94^{\circ} 22^{\prime}$ & 03/1948 & $12 / 2001$ & 95 & 53 \\
\hline 692 & Lipan & 5243 & Hood & 930 & $32^{\circ} 31^{\prime}$ & $98^{\circ} 03^{\prime}$ & 03/1949 & $12 / 2001$ & 97 & 53 \\
\hline 693 & Lipscomb & 5247 & Lipscomb & 2,450 & $36^{\circ} 14^{\prime}$ & $100^{\circ} 16^{\prime}$ & $06 / 1948$ & $12 / 2001$ & 97 & 54 \\
\hline 694 & Little Elm $1 \mathrm{NNE}$ & 5258 & Denton & 551 & $33^{\circ} 10^{\prime}$ & $96^{\circ} 56^{\prime}$ & $01 / 1946$ & 09/1966 & 95 & 21 \\
\hline 695 & Littlefield & 5263 & Lamb & 3,563 & $33^{\circ} 55^{\prime}$ & $102^{\circ} 20^{\prime}$ & $10 / 1916$ & $01 / 1966$ & 88 & 42 \\
\hline 696 & Littlefield & 5265 & Lamb & 3,505 & $33^{\circ} 54^{\prime}$ & $102^{\circ} 19^{\prime}$ & 07/1966 & $12 / 2001$ & 97 & 36 \\
\hline 697 & Livingston 2 NNE & 5271 & Polk & 178 & $30^{\circ} 44^{\prime}$ & $94^{\circ} 56^{\prime}$ & 03/1937 & $12 / 2001$ & 99 & 65 \\
\hline 698 & Llano & 5272 & Llano & 1,020 & $30^{\circ} 44^{\prime}$ & $98^{\circ} 39^{\prime}$ & $12 / 1896$ & $12 / 2001$ & 98 & 105 \\
\hline 699 & Llano $18 \mathrm{~S}$ & 5276 & Llano & 1,380 & $30^{\circ} 30^{\prime}$ & $98^{\circ} 44^{\prime}$ & 09/1997 & $12 / 2001$ & 87 & 5 \\
\hline 700 & Lockhart & 5284 & Caldwell & 547 & $29^{\circ} 53^{\prime}$ & $97^{\circ} 41^{\prime}$ & 09/1947 & $12 / 2001$ & 98 & 55 \\
\hline 701 & Lockhart 2 SW & 5285 & Caldwell & 490 & $29^{\circ} 51^{\prime}$ & $97^{\circ} 41^{\prime}$ & 02/1997 & $12 / 2001$ & 98 & 5 \\
\hline 702 & Loma Alta & 5303 & Val Verde* & 1,923 & $29^{\circ} 55^{\prime}$ & $100^{\circ} 46^{\prime}$ & $10 / 1947$ & 09/1951 & 66 & 5 \\
\hline 703 & Lometa & 5308 & Lampasas & 1,480 & $31^{\circ} 13^{\prime}$ & $98^{\circ} 24^{\prime}$ & 03/1948 & $12 / 1965$ & 72 & 18 \\
\hline 704 & London & 5312 & Kimble & 1,695 & $30^{\circ} 40^{\prime}$ & $99^{\circ} 34^{\prime}$ & $06 / 1948$ & $12 / 1953$ & 19 & 6 \\
\hline 705 & Long Lake $5 \mathrm{SW}$ & 5327 & Freestone & 312 & $31^{\circ} 37^{\prime}$ & $95^{\circ} 51^{\prime}$ & $04 / 1915$ & $10 / 1980$ & 95 & 37 \\
\hline 706 & Longview & 5341 & Gregg & 330 & $32^{\circ} 27^{\prime}$ & $94^{\circ} 44^{\prime}$ & 01/1902 & $12 / 2001$ & 98 & 100 \\
\hline 707 & Longview $11 \mathrm{SE}$ & 5348 & Rusk & 407 & $32^{\circ} 20^{\prime}$ & $94^{\circ} 39^{\prime}$ & 09/1975 & $12 / 2001$ & 94 & 27 \\
\hline 708 & Loop & 5351 & Gaines & 3,245 & $32^{\circ} 54^{\prime}$ & $102^{\circ} 25^{\prime}$ & 08/1948 & 07/1995 & 94 & 47 \\
\hline 709 & Loop $10 \mathrm{SE}$ & 5354 & Gaines & 3,104 & $32^{\circ} 52^{\prime}$ & $102^{\circ} 22^{\prime}$ & $08 / 2001$ & $11 / 2001$ & 16 & 1 \\
\hline 710 & Loraine & 5358 & Mitchell & 2,270 & $32^{\circ} 25^{\prime}$ & $100^{\circ} 43^{\prime}$ & $06 / 1948$ & 09/1951 & 82 & 4 \\
\hline 711 & Lorenzo & 5363 & Crosby & 3,170 & $33^{\circ} 40^{\prime}$ & $101^{\circ} 32^{\prime}$ & $02 / 1947$ & 08/1995 & 84 & 48 \\
\hline 712 & Los Angeles $4 \mathrm{WSW}$ & 5369 & La Salle & 288 & $28^{\circ} 26^{\prime}$ & $99^{\circ} 03^{\prime}$ & $04 / 1998$ & $12 / 2001$ & 92 & 4 \\
\hline 713 & Lovelady & 5398 & Houston & 302 & $31^{\circ} 08^{\prime}$ & $95^{\circ} 27^{\prime}$ & $06 / 1948$ & $10 / 1986$ & 96 & 29 \\
\hline 714 & Lubbock 2 & 5412 & Lubbock* & 3,222 & $33^{\circ} 35^{\prime}$ & $101^{\circ} 53^{\prime}$ & $06 / 1938$ & 05/1939 & 50 & 2 \\
\hline 715 & Lubbock 6 NNE & 5408 & Lubbock & 3,254 & $33^{\circ} 39^{\prime}$ & $101^{\circ} 48^{\prime}$ & $01 / 1998$ & $12 / 2001$ & 100 & 4 \\
\hline 716 & Lubbock $9 \mathrm{~N}$ & 5410 & Lubbock & 3,245 & $33^{\circ} 41^{\prime}$ & $101^{\circ} 49^{\prime}$ & $09 / 1946$ & $02 / 1964$ & 91 & 19 \\
\hline 717 & Lubbock International Airport & 5411 & Lubbock & 3,254 & $33^{\circ} 39^{\prime}$ & $101^{\circ} 49^{\prime}$ & 03/1911 & $12 / 2001$ & 100 & 91 \\
\hline 718 & Lufkin $11 \mathrm{NW}$ & 5415 & Angelina & 350 & $31^{\circ} 25^{\prime}$ & $94^{\circ} 53^{\prime}$ & $01 / 1983$ & $12 / 2001$ & 99 & 19 \\
\hline 719 & Lufkin 2 & 5427 & Angelina & 320 & $31^{\circ} 22^{\prime}$ & $94^{\circ} 43^{\prime}$ & $01 / 1990$ & $12 / 2001$ & 100 & 12 \\
\hline 720 & Lufkin Angelina County Airport & 5424 & Angelina & 288 & $31^{\circ} 14^{\prime}$ & $94^{\circ} 45^{\prime}$ & $11 / 1906$ & $12 / 2001$ & 96 & 95 \\
\hline
\end{tabular}


Table 1. Summary of National Weather Service daily precipitation stations in Texas with known names, latitudes, and longitudes-Continued

\begin{tabular}{|c|c|c|c|c|c|c|c|c|c|c|}
\hline $\begin{array}{c}\text { Seq. } \\
\text { no. }\end{array}$ & NWS station name & $\begin{array}{l}\text { NWS } \\
\text { station } \\
\text { no. }\end{array}$ & County & $\begin{array}{c}\text { Elev. } \\
\text { (ft above } \\
\text { NGVD } \\
\text { 29) }\end{array}$ & $\begin{array}{l}\text { Lati- } \\
\text { tude }\end{array}$ & $\begin{array}{l}\text { Longi- } \\
\text { tude }\end{array}$ & $\begin{array}{l}\text { Begining } \\
\text { date } \\
\text { (mm/yyyy) }\end{array}$ & $\begin{array}{c}\text { Ending } \\
\text { date } \\
\text { (mm/yyyy) }\end{array}$ & $\begin{array}{l}\text { Per- } \\
\text { cent } \\
\text { cov- } \\
\text { erage }\end{array}$ & $\begin{array}{l}\text { Years } \\
\text { of } \\
\text { record }\end{array}$ \\
\hline 721 & Luling & 5429 & Caldwell & 400 & $29^{\circ} 40^{\prime}$ & $97^{\circ} 39^{\prime}$ & $12 / 1901$ & $12 / 2001$ & 99 & 101 \\
\hline 722 & Luling $12 \mathrm{NE}$ & 5430 & Caldwell & 445 & $29^{\circ} 50^{\prime}$ & $97^{\circ} 34^{\prime}$ & 08/1997 & $12 / 2001$ & 88 & 5 \\
\hline 723 & Lumberton & 5435 & Hardin & 39 & $30^{\circ} 15^{\prime}$ & $94^{\circ} 10^{\prime}$ & $11 / 1995$ & $12 / 2001$ & 88 & 7 \\
\hline 724 & Lynxhaven Ranch & 5449 & Kerr & 2,001 & $29^{\circ} 58^{\prime}$ & $99^{\circ} 27^{\prime}$ & $10 / 1947$ & $02 / 1976$ & 83 & 30 \\
\hline 725 & Lytle $3 \mathrm{~W}$ & 5454 & Medina & 722 & $29^{\circ} 14^{\prime}$ & $98^{\circ} 50^{\prime}$ & $12 / 1976$ & $12 / 2001$ & 96 & 26 \\
\hline 726 & Mabank 4 SW & 5461 & Henderson & 341 & $32^{\circ} 20^{\prime}$ & $96^{\circ} 09^{\prime}$ & $06 / 1948$ & $09 / 1951$ & 80 & 4 \\
\hline 727 & Madisonville & 5477 & Madison & 252 & $30^{\circ} 56^{\prime}$ & $95^{\circ} 54^{\prime}$ & $09 / 1918$ & $11 / 2001$ & 92 & 75 \\
\hline 728 & Magnolia $1 \mathrm{~W}$ & 5496 & Montgomery & 260 & $30^{\circ} 13^{\prime}$ & $95^{\circ} 47^{\prime}$ & $06 / 1954$ & 06/1986 & 91 & 31 \\
\hline 729 & Manchaca & 5538 & Travis & 702 & $30^{\circ} 08^{\prime}$ & $97^{\circ} 50^{\prime}$ & 03/1948 & $12 / 1965$ & 98 & 18 \\
\hline 730 & Manchester & 5540 & Red River* & 400 & $33^{\circ} 51^{\prime}$ & $95^{\circ} 08^{\prime}$ & $11 / 1948$ & $11 / 1948$ & 4 & 1 \\
\hline 731 & Mansfield & 5560 & Tarrant & 581 & $32^{\circ} 34^{\prime}$ & $97^{\circ} 09^{\prime}$ & $09 / 1947$ & $10 / 1964$ & 95 & 18 \\
\hline 732 & Mansfield Dam & 5561 & Travis & 551 & $30^{\circ} 24^{\prime}$ & $97^{\circ} 55^{\prime}$ & $06 / 1944$ & 03/1964 & 89 & 21 \\
\hline 733 & Maple & 5570 & Cochran* & 3,904 & $33^{\circ} 47^{\prime}$ & $102^{\circ} 54^{\prime}$ & $09 / 1947$ & $02 / 1949$ & 31 & 3 \\
\hline 734 & Marathon & 5579 & Brewster & 4,055 & $30^{\circ} 12^{\prime}$ & $103^{\circ} 14^{\prime}$ & $01 / 1897$ & $12 / 2001$ & 93 & 68 \\
\hline 735 & Marble Falls & 5580 & Burnet* & 771 & $30^{\circ} 34^{\prime}$ & $98^{\circ} 17^{\prime}$ & $09 / 1946$ & $01 / 1952$ & 77 & 7 \\
\hline 736 & Marco & 5583 & McCulloch & 1,942 & $31^{\circ} 06^{\prime}$ & $99^{\circ} 34^{\prime}$ & $04 / 1948$ & $12 / 1974$ & 82 & 27 \\
\hline 737 & Marfa $19 \mathrm{~S}$ & 5593 & Presidio & 4,403 & $30^{\circ} 02^{\prime}$ & $104^{\circ} 01^{\prime}$ & $12 / 1950$ & $10 / 1961$ & 66 & 11 \\
\hline 738 & Marfa 2 & 5596 & Presidio & 4,730 & $30^{\circ} 18^{\prime}$ & $104^{\circ} 00^{\prime}$ & $12 / 1958$ & $12 / 2001$ & 91 & 43 \\
\hline 739 & Marfa CAA Airport & 5589 & Presidio* & 4,859 & $30^{\circ} 15^{\prime}$ & $103^{\circ} 53^{\prime}$ & 06/1907 & $12 / 1954$ & 85 & 16 \\
\hline 740 & Marfa Charco MR & 5591 & Jeff Davis* & 5,305 & $30^{\circ} 29^{\prime}$ & $104^{\circ} 07^{\prime}$ & $02 / 1949$ & $09 / 1951$ & 88 & 3 \\
\hline 741 & Marfa Ryan & 5594 & Presidio* & 4,705 & $30^{\circ} 22^{\prime}$ & $104^{\circ} 19^{\prime}$ & $05 / 1951$ & $09 / 1951$ & 39 & 1 \\
\hline 742 & Marlin 3 NE & 5611 & Falls & 388 & $31^{\circ} 20^{\prime}$ & $96^{\circ} 51^{\prime}$ & 08/1902 & $12 / 2001$ & 97 & 75 \\
\hline 743 & Marshall & 5618 & Harrison & 352 & $32^{\circ} 32^{\prime}$ & $94^{\circ} 21^{\prime}$ & $07 / 1908$ & $12 / 2001$ & 97 & 94 \\
\hline 744 & Martin Ranch & 5626 & Mason & 1,332 & $30^{\circ} 38^{\prime}$ & $99^{\circ} 10^{\prime}$ & 03/1948 & $12 / 1965$ & 96 & 18 \\
\hline 745 & Marys creek & 5646 & Tarrant & 850 & $32^{\circ} 44^{\prime}$ & $97^{\circ} 30^{\prime}$ & $10 / 1947$ & $10 / 1973$ & 91 & 27 \\
\hline 746 & Mason & 5650 & Mason & 1,430 & $30^{\circ} 44^{\prime}$ & $99^{\circ} 14^{\prime}$ & $11 / 1941$ & $12 / 2001$ & 91 & 61 \\
\hline 747 & Matador & 5658 & Motley & 2,290 & $34^{\circ} 01^{\prime}$ & $100^{\circ} 49^{\prime}$ & $11 / 1947$ & $11 / 2001$ & 97 & 55 \\
\hline 748 & Matagorda 2 & 5659 & Matagorda & 10 & $28^{\circ} 41^{\prime}$ & $95^{\circ} 58^{\prime}$ & $07 / 1910$ & $12 / 2001$ & 98 & 92 \\
\hline 749 & Mathis 4 SSW & 5661 & Jim Wells & 138 & $28^{\circ} 02^{\prime}$ & $97^{\circ} 52^{\prime}$ & $07 / 1964$ & $12 / 2001$ & 98 & 38 \\
\hline 750 & Maud & 5667 & Bowie & 305 & $33^{\circ} 19^{\prime}$ & $94^{\circ} 20^{\prime}$ & $09 / 1946$ & $12 / 2001$ & 91 & 56 \\
\hline 751 & Maurbro & 5670 & Jackson & 30 & $28^{\circ} 55^{\prime}$ & $96^{\circ} 28^{\prime}$ & $01 / 1944$ & $01 / 1966$ & 91 & 23 \\
\hline 752 & Maverick Ranger Station & 5674 & Brewster & 2,703 & $29^{\circ} 17^{\prime}$ & $103^{\circ} 30^{\prime}$ & $01 / 1962$ & $04 / 1965$ & 83 & 4 \\
\hline 753 & Maypearl & 5695 & Ellis & 530 & $32^{\circ} 19^{\prime}$ & $97^{\circ} 01^{\prime}$ & $06 / 1948$ & 08/1994 & 79 & 7 \\
\hline 754 & McCartney Bridge & 5710 & Cass & 230 & $33^{\circ} 19^{\prime}$ & $94^{\circ} 10^{\prime}$ & $08 / 1947$ & $09 / 1954$ & 88 & 8 \\
\hline 755 & McCook & 5721 & Hidalgo & 220 & $26^{\circ} 29^{\prime}$ & $98^{\circ} 23^{\prime}$ & 08/1941 & $12 / 2001$ & 96 & 61 \\
\hline 756 & McDonald Store & 5738 & Callahan* & 1,752 & $32^{\circ} 12^{\prime}$ & $99^{\circ} 24^{\prime}$ & $09 / 1958$ & $12 / 1958$ & 18 & 1 \\
\hline 757 & McIntosh Ranch & 5761 & Schleicher* & 2,352 & $30^{\circ} 44^{\prime}$ & $100^{\circ} 30^{\prime}$ & $04 / 1948$ & $11 / 1965$ & 89 & 18 \\
\hline 758 & McKinney $3 \mathrm{~S}$ & 5766 & Collin & 595 & $33^{\circ} 10^{\prime}$ & $96^{\circ} 37^{\prime}$ & $04 / 1903$ & $12 / 2001$ & 95 & 93 \\
\hline 759 & McLean & 5770 & Gray & 2,860 & $35^{\circ} 14^{\prime}$ & $100^{\circ} 36^{\prime}$ & $06 / 1948$ & $12 / 2001$ & 96 & 44 \\
\hline 760 & McAllen & 5701 & Hidalgo & 100 & $26^{\circ} 12^{\prime}$ & $98^{\circ} 15^{\prime}$ & $06 / 1941$ & $12 / 2001$ & 96 & 61 \\
\hline 761 & McAllen Miller Airport & 5702 & Hidalgo & 100 & $26^{\circ} 10^{\prime}$ & $98^{\circ} 14^{\prime}$ & $01 / 1961$ & $12 / 2001$ & 95 & 39 \\
\hline 762 & McCamey & 5707 & Upton & 2,450 & $31^{\circ} 08^{\prime}$ & $102^{\circ} 11^{\prime}$ & $02 / 1932$ & $12 / 2001$ & 99 & 70 \\
\hline 763 & McCoy & 5723 & Atascosa & 302 & $28^{\circ} 51^{\prime}$ & $98^{\circ} 20^{\prime}$ & 08/1996 & $11 / 1999$ & 81 & 4 \\
\hline 764 & McGregor & 5757 & McLennan & 723 & $31^{\circ} 26^{\prime}$ & $97^{\circ} 24^{\prime}$ & 03/1910 & $12 / 2001$ & 98 & 92 \\
\hline 765 & Medina & 5742 & Bandera & 1,705 & $29^{\circ} 48^{\prime}$ & $99^{\circ} 15^{\prime}$ & $01 / 1966$ & $02 / 2000$ & 95 & 35 \\
\hline 766 & Medina Lake & 5746 & Medina & 1,169 & $29^{\circ} 30^{\prime}$ & $98^{\circ} 54^{\prime}$ & 05/1991 & $03 / 2001$ & 67 & 11 \\
\hline 767 & Memphis & 5821 & Hall & 2,090 & $34^{\circ} 43^{\prime}$ & $100^{\circ} 32^{\prime}$ & 07/1905 & $12 / 2001$ & 95 & 96 \\
\hline 768 & Menard & 5822 & Menard & 1,951 & $30^{\circ} 55^{\prime}$ & $99^{\circ} 47^{\prime}$ & $01 / 1897$ & $12 / 2001$ & 96 & 97 \\
\hline 769 & Menard $13 \mathrm{WNW}$ & 5825 & Menard & 2,201 & $31^{\circ} 03^{\prime}$ & $99^{\circ} 57^{\prime}$ & $04 / 1959$ & $12 / 1965$ & 83 & 7 \\
\hline 770 & Mentone $2 \mathrm{~S}$ & 5828 & Loving & 2,703 & $31^{\circ} 41^{\prime}$ & $103^{\circ} 36^{\prime}$ & $01 / 1949$ & $01 / 1974$ & 86 & 26 \\
\hline 771 & Menzies Al Ranch & 5832 & Menard & 2,251 & $31^{\circ} 04^{\prime}$ & $99^{\circ} 56^{\prime}$ & 03/1948 & 03/1959 & 85 & 12 \\
\hline 772 & Menzies Bill Ranch & 5833 & Edwards* & $* 2,275$ & $30^{\circ} 08^{\prime}$ & $99^{\circ} 58^{\prime}$ & $04 / 1948$ & $09 / 1948$ & 50 & 1 \\
\hline
\end{tabular}


Table 1. Summary of National Weather Service daily precipitation stations in Texas with known names, latitudes, and longitudes-Continued

\begin{tabular}{|c|c|c|c|c|c|c|c|c|c|c|}
\hline $\begin{array}{c}\text { Seq. } \\
\text { no. }\end{array}$ & NWS station name & $\begin{array}{c}\text { NWS } \\
\text { station } \\
\text { no. }\end{array}$ & County & $\begin{array}{c}\text { Elev. } \\
\text { (ft above } \\
\text { NGVD } \\
\text { 29) }\end{array}$ & $\begin{array}{l}\text { Lati- } \\
\text { tude }\end{array}$ & $\begin{array}{l}\text { Longi- } \\
\text { tude }\end{array}$ & $\begin{array}{l}\text { Begining } \\
\text { date } \\
\text { (mm/yyyy) }\end{array}$ & $\begin{array}{c}\text { Ending } \\
\text { date } \\
\text { (mm/yyyy) }\end{array}$ & $\begin{array}{l}\text { Per- } \\
\text { cent } \\
\text { cov- } \\
\text { erage }\end{array}$ & $\begin{array}{l}\text { Years } \\
\text { of } \\
\text { record }\end{array}$ \\
\hline 773 & Mercedes & 5835 & Hidalgo* & 79 & $26^{\circ} 08^{\prime}$ & $97^{\circ} 55^{\prime}$ & $09 / 1946$ & $03 / 1948$ & 47 & 3 \\
\hline 774 & Mercedes 6 SSE & 5836 & Hidalgo & 75 & $26^{\circ} 04^{\prime}$ & $97^{\circ} 54^{\prime}$ & 03/1914 & $12 / 2001$ & 97 & 87 \\
\hline 775 & Mercury & 5840 & McCulloch & 1,440 & $31^{\circ} 25^{\prime}$ & $99^{\circ} 10^{\prime}$ & 09/1949 & $08 / 1950$ & 42 & 2 \\
\hline 776 & Meridian & 5845 & Bosque & 770 & $31^{\circ} 56^{\prime}$ & $97^{\circ} 40^{\prime}$ & $09 / 1982$ & $12 / 2001$ & 96 & 20 \\
\hline 777 & Meridian State Park & 5847 & Bosque & 1,028 & $31^{\circ} 53^{\prime}$ & $97^{\circ} 42^{\prime}$ & $04 / 1963$ & 08/1982 & 94 & 20 \\
\hline 778 & Merrill Ranch & 5854 & Jeff Davis & 5,472 & $30^{\circ} 32^{\prime}$ & $104^{\circ} 03^{\prime}$ & 01/1939 & $12 / 1967$ & 92 & 29 \\
\hline 779 & Mertzon & 5859 & Irion & 2,229 & $31^{\circ} 16^{\prime}$ & $100^{\circ} 49^{\prime}$ & $01 / 1941$ & 08/1987 & 93 & 47 \\
\hline 780 & Mexia & 5869 & Limestone & 535 & $31^{\circ} 41^{\prime}$ & $96^{\circ} 29^{\prime}$ & 09/1904 & $12 / 2001$ & 99 & 96 \\
\hline 781 & Miami & 5875 & Roberts & 2,755 & $35^{\circ} 42^{\prime}$ & $100^{\circ} 38^{\prime}$ & $07 / 1905$ & $12 / 2001$ & 98 & 97 \\
\hline 782 & Mid City 4 SE & 5878 & Lamar & 490 & $33^{\circ} 48^{\prime}$ & $95^{\circ} 30^{\prime}$ & $12 / 1970$ & 09/1997 & 91 & 28 \\
\hline 783 & Middlewell & 5887 & Hartley* & 3,802 & $35^{\circ} 45^{\prime}$ & $102^{\circ} 11^{\prime}$ & $10 / 1947$ & $03 / 1953$ & 57 & 7 \\
\hline 784 & Midkiff & 5888 & Upton & 2,700 & $31^{\circ} 38^{\prime}$ & $101^{\circ} 50^{\prime}$ & 08/1981 & 02/1996 & 81 & 16 \\
\hline 785 & Midland 4 ENE & 5891 & Midland & 2,740 & $32^{\circ} 01^{\prime}$ & $102^{\circ} 01^{\prime}$ & 08/1947 & $12 / 2001$ & 95 & 55 \\
\hline 786 & Midland International Airport & 5890 & Midland & 2,862 & $31^{\circ} 56^{\prime}$ & $102^{\circ} 11^{\prime}$ & 06/1948 & $12 / 2001$ & 99 & 54 \\
\hline 787 & Midlothian & 5896 & Ellis* & 751 & $32^{\circ} 29^{\prime}$ & $97^{\circ} 00^{\prime}$ & $09 / 1947$ & $10 / 1964$ & 92 & 18 \\
\hline 788 & Midlothian 2 & 5897 & Ellis & 750 & $32^{\circ} 29^{\prime}$ & $96^{\circ} 59^{\prime}$ & 08/1997 & $12 / 2001$ & 88 & 5 \\
\hline 789 & Midway 4 NE & 5904 & Madison & 235 & $31^{\circ} 04^{\prime}$ & $95^{\circ} 43^{\prime}$ & $04 / 1978$ & $12 / 2001$ & 95 & 24 \\
\hline 790 & Milam 6 ENE & 5915 & Sabine & 200 & $31^{\circ} 28^{\prime}$ & $93^{\circ} 45^{\prime}$ & 03/1948 & 05/1969 & 76 & 8 \\
\hline 791 & Miller Ranch & 5930 & San Saba & 1,991 & $30^{\circ} 58^{\prime}$ & $98^{\circ} 56^{\prime}$ & 03/1948 & $02 / 1966$ & 72 & 18 \\
\hline 792 & Mineola 2 & 5955 & Wood & 410 & $32^{\circ} 40^{\prime}$ & $95^{\circ} 29^{\prime}$ & $06 / 1965$ & 08/1966 & 62 & 2 \\
\hline 793 & Mineola 4 S & 5954 & Smith & 370 & $32^{\circ} 36^{\prime}$ & $95^{\circ} 29^{\prime}$ & $09 / 1946$ & $10 / 2000$ & 95 & 55 \\
\hline 794 & Mineola 8 ENE & 5956 & Wood & 385 & $32^{\circ} 43^{\prime}$ & $95^{\circ} 22^{\prime}$ & 09/1966 & $10 / 2000$ & 94 & 34 \\
\hline 795 & Mineral Wells FCWOS Airport & 5958 & Palo Pinto & 930 & $32^{\circ} 46^{\prime}$ & $98^{\circ} 03^{\prime}$ & 06/1948 & $12 / 2001$ & 95 & 49 \\
\hline 796 & Mirando City & 5971 & Webb & 771 & $27^{\circ} 27^{\prime}$ & $99^{\circ} 00^{\prime}$ & $10 / 1948$ & $11 / 1963$ & 89 & 16 \\
\hline 797 & Mission $4 \mathrm{~W}$ & 5972 & Hidalgo & 133 & $26^{\circ} 13^{\prime}$ & $98^{\circ} 24^{\prime}$ & $09 / 1910$ & $11 / 1994$ & 93 & 84 \\
\hline 798 & Mission Pumping Station & 5973 & Hidalgo* & 131 & $26^{\circ} 12^{\prime}$ & $98^{\circ} 19^{\prime}$ & $06 / 1948$ & $05 / 1950$ & 63 & 3 \\
\hline 799 & Mobeetie & 5987 & Wheeler & 2,680 & $35^{\circ} 32^{\prime}$ & $100^{\circ} 26^{\prime}$ & $09 / 1947$ & 03/1974 & 89 & 28 \\
\hline 800 & Moline & 5996 & Mills & 1,385 & $31^{\circ} 24^{\prime}$ & $98^{\circ} 19^{\prime}$ & $10 / 1947$ & 09/1951 & 63 & 5 \\
\hline 801 & Monahans & 5999 & Ward & 2,585 & $31^{\circ} 32^{\prime}$ & $102^{\circ} 54^{\prime}$ & 06/1959 & $11 / 2001$ & 94 & 43 \\
\hline 802 & Monahans $1 \mathrm{NW}$ & 6000 & Ward* & 2,631 & $31^{\circ} 36^{\prime}$ & $102^{\circ} 54^{\prime}$ & $02 / 1900$ & 05/1953 & 52 & 8 \\
\hline 803 & Montague & 6014 & Montague & 1,079 & $33^{\circ} 40^{\prime}$ & $97^{\circ} 45^{\prime}$ & $06 / 1943$ & $02 / 1964$ & 91 & 22 \\
\hline 804 & Monte Alto & 6017 & Willacy* & 39 & $26^{\circ} 33^{\prime}$ & $97^{\circ} 58^{\prime}$ & 06/1948 & 05/1950 & 62 & 3 \\
\hline 805 & Montell & 6019 & Uvalde* & 1,302 & $29^{\circ} 32^{\prime}$ & $100^{\circ} 01^{\prime}$ & $09 / 1912$ & $02 / 1944$ & 95 & 33 \\
\hline 806 & Montgomery & 6024 & Montgomery & 320 & $30^{\circ} 23^{\prime}$ & $95^{\circ} 41^{\prime}$ & $06 / 1954$ & $12 / 2001$ & 98 & 48 \\
\hline 807 & Morgan 3 WNW & 6058 & Bosque & 740 & $32^{\circ} 00^{\prime}$ & $97^{\circ} 36^{\prime}$ & $05 / 1965$ & $12 / 2001$ & 97 & 37 \\
\hline 808 & Morgan Mill & 6060 & Erath & 1,030 & $32^{\circ} 22^{\prime}$ & $98^{\circ} 10^{\prime}$ & 03/1949 & $12 / 2001$ & 93 & 53 \\
\hline 809 & Morse & 6070 & Hansford & 3,180 & $36^{\circ} 03^{\prime}$ & $101^{\circ} 28^{\prime}$ & 09/1941 & 01/1998 & 89 & 57 \\
\hline 810 & Morton & 6074 & Cochran & 3,760 & $33^{\circ} 43^{\prime}$ & $102^{\circ} 45^{\prime}$ & $05 / 1935$ & $12 / 2001$ & 95 & 59 \\
\hline 811 & Moscow & 6078 & Polk & 249 & $30^{\circ} 55^{\prime}$ & $94^{\circ} 50^{\prime}$ & 09/1947 & 04/1969 & 88 & 23 \\
\hline 812 & Moss Ranch & 6085 & Llano & 1,180 & $30^{\circ} 33^{\prime}$ & $98^{\circ} 42^{\prime}$ & 03/1948 & 07/1997 & 91 & 20 \\
\hline 813 & Mount Enterprise & 6099 & Rusk* & 610 & $31^{\circ} 58^{\prime}$ & $94^{\circ} 40^{\prime}$ & $04 / 1948$ & 07/1948 & 23 & 1 \\
\hline 814 & Mount Locke & 6104 & Jeff Davis & 6,790 & $30^{\circ} 42^{\prime}$ & $104^{\circ} 01^{\prime}$ & $02 / 1935$ & $12 / 2001$ & 100 & 67 \\
\hline 815 & Mount Pleasant & 6108 & Titus & 425 & $33^{\circ} 10^{\prime}$ & $95^{\circ} 00^{\prime}$ & $03 / 1905$ & $12 / 2001$ & 97 & 86 \\
\hline 816 & Mount Vernon & 6119 & Franklin & 480 & $33^{\circ} 11^{\prime}$ & $95^{\circ} 13^{\prime}$ & 05/1966 & $12 / 2001$ & 98 & 36 \\
\hline 817 & Mountain Creek & 6116 & Dallas & 522 & $32^{\circ} 43^{\prime}$ & $96^{\circ} 56^{\prime}$ & 09/1947 & $10 / 1964$ & 95 & 18 \\
\hline 818 & Muenster & 6130 & Cooke & 1,005 & $33^{\circ} 39^{\prime}$ & $97^{\circ} 22^{\prime}$ & 09/1947 & $12 / 2001$ & 99 & 55 \\
\hline 819 & Muleshoe 1 & 6135 & Bailey & 3,830 & $34^{\circ} 14^{\prime}$ & $102^{\circ} 44^{\prime}$ & 08/1921 & $12 / 2001$ & 98 & 81 \\
\hline 820 & Muleshoe 2 & 6136 & Bailey & 3,800 & $34^{\circ} 13^{\prime}$ & $102^{\circ} 44^{\prime}$ & $06 / 1948$ & $09 / 1951$ & 61 & 4 \\
\hline 821 & Muleshoe National Wildlife Refuge & 6137 & Bailey & 3,740 & $33^{\circ} 57^{\prime}$ & $102^{\circ} 46^{\prime}$ & 02/1980 & $12 / 2001$ & 99 & 22 \\
\hline 822 & Mullin & 6140 & Mills & 1,492 & $31^{\circ} 35^{\prime}$ & $98^{\circ} 40^{\prime}$ & 03/1948 & 09/2001 & 97 & 54 \\
\hline 823 & Munday & 6146 & Knox & 1,480 & $33^{\circ} 27^{\prime}$ & $99^{\circ} 36^{\prime}$ & $09 / 1912$ & $12 / 2001$ & 99 & 90 \\
\hline 824 & Munday 5 SSW & 6148 & Haskell* & 1,503 & $33^{\circ} 23^{\prime}$ & $99^{\circ} 39^{\prime}$ & $10 / 1939$ & $10 / 1948$ & 73 & 8 \\
\hline
\end{tabular}


Table 1. Summary of National Weather Service daily precipitation stations in Texas with known names, latitudes, and longitudes-Continued

\begin{tabular}{|c|c|c|c|c|c|c|c|c|c|c|}
\hline $\begin{array}{l}\text { Seq. } \\
\text { no. }\end{array}$ & NWS station name & $\begin{array}{c}\text { NWS } \\
\text { station } \\
\text { no. }\end{array}$ & County & $\begin{array}{c}\text { Elev. } \\
\text { (ft above } \\
\text { NGVD } \\
\text { 29) }\end{array}$ & $\begin{array}{l}\text { Lati- } \\
\text { tude }\end{array}$ & $\begin{array}{l}\text { Longi- } \\
\text { tude }\end{array}$ & $\begin{array}{c}\text { Begining } \\
\text { date } \\
\text { (mm/yyyy) }\end{array}$ & $\begin{array}{c}\text { Ending } \\
\text { date } \\
\text { (mm/yyyy) }\end{array}$ & $\begin{array}{l}\text { Per- } \\
\text { cent } \\
\text { cov- } \\
\text { erage }\end{array}$ & $\begin{array}{c}\text { Years } \\
\text { of } \\
\text { record }\end{array}$ \\
\hline 825 & Murr Ranch & 6158 & Kimble & 2,402 & $30^{\circ} 41^{\prime}$ & $100^{\circ} 05^{\prime}$ & $03 / 1948$ & $12 / 1965$ & 65 & 17 \\
\hline 826 & Nacogdoches & 6176 & Nacogdoches & 312 & $31^{\circ} 36^{\prime}$ & $94^{\circ} 39^{\prime}$ & $04 / 1900$ & 05/1973 & 98 & 74 \\
\hline 827 & Nacogdoches & 6177 & Nacogdoches & 435 & $31^{\circ} 37^{\prime}$ & $94^{\circ} 38^{\prime}$ & $06 / 1948$ & $12 / 2001$ & 95 & 30 \\
\hline 828 & Naples 1 SW & 6190 & Morris & 361 & $33^{\circ} 11^{\prime}$ & $94^{\circ} 41^{\prime}$ & $12 / 1909$ & $11 / 1981$ & 97 & 73 \\
\hline 829 & Naples $5 \mathrm{NE}$ & 6195 & Bowie & 290 & $33^{\circ} 14^{\prime}$ & $94^{\circ} 40^{\prime}$ & $04 / 1982$ & $01 / 1997$ & 92 & 16 \\
\hline 830 & Natalia & 6205 & Medina & 712 & $29^{\circ} 12^{\prime}$ & $98^{\circ} 51^{\prime}$ & $08 / 1909$ & 08/1976 & 87 & 41 \\
\hline 831 & Navarro Mills Dam & 6210 & Navarro & 454 & $31^{\circ} 57^{\prime}$ & $96^{\circ} 42^{\prime}$ & $01 / 1963$ & $12 / 2001$ & 99 & 39 \\
\hline 832 & Navasota & 6211 & Grimes* & 220 & $30^{\circ} 23^{\prime}$ & $96^{\circ} 07^{\prime}$ & $09 / 1946$ & $12 / 1951$ & 80 & 6 \\
\hline 833 & Nazareth & 6215 & Castro* & 3,704 & $34^{\circ} 33^{\prime}$ & $102^{\circ} 06^{\prime}$ & 03/1906 & 03/1911 & 75 & 6 \\
\hline 834 & Neal Ranch & 6219 & McCulloch* & 1,670 & $31^{\circ} 11^{\prime}$ & $99^{\circ} 09^{\prime}$ & $03 / 1948$ & $12 / 1959$ & 94 & 12 \\
\hline 835 & Negley 4 SSW & 6247 & Red River & 405 & $33^{\circ} 42^{\prime}$ & $95^{\circ} 04^{\prime}$ & $11 / 1948$ & $12 / 2001$ & 94 & 54 \\
\hline 836 & Nelson Ranch & 6257 & Kerr & 2,125 & $29^{\circ} 57^{\prime}$ & $99^{\circ} 31^{\prime}$ & $07 / 1962$ & $02 / 1983$ & 94 & 22 \\
\hline 837 & Neuville & 6265 & Shelby & 480 & $31^{\circ} 39^{\prime}$ & $94^{\circ} 09^{\prime}$ & $09 / 1947$ & $12 / 2001$ & 93 & 35 \\
\hline 838 & New Boston & 6270 & Bowie & 345 & $33^{\circ} 27^{\prime}$ & $94^{\circ} 24^{\prime}$ & $04 / 1980$ & $12 / 2001$ & 94 & 22 \\
\hline 839 & New Boston $3 \mathrm{~W}$ & 6271 & Bowie & 371 & $33^{\circ} 28^{\prime}$ & $94^{\circ} 28^{\prime}$ & $11 / 1973$ & $12 / 1979$ & 88 & 7 \\
\hline 840 & New Braunfels & 6276 & Comal & 710 & $29^{\circ} 44^{\prime}$ & $98^{\circ} 07^{\prime}$ & $01 / 1897$ & $12 / 2001$ & 96 & 104 \\
\hline 841 & New Caney 2 E & 6280 & Montgomery & 235 & $30^{\circ} 08^{\prime}$ & $95^{\circ} 11^{\prime}$ & $01 / 1952$ & $12 / 2001$ & 99 & 50 \\
\hline 842 & New Gulf & 6286 & Wharton & 72 & $29^{\circ} 16^{\prime}$ & $95^{\circ} 54^{\prime}$ & $06 / 1946$ & 02/1999 & 95 & 54 \\
\hline 843 & New Summerfield $2 \mathrm{~W}$ & 6335 & Cherokee & 380 & $31^{\circ} 59^{\prime}$ & $95^{\circ} 08^{\prime}$ & $01 / 1992$ & $12 / 2001$ & 98 & 10 \\
\hline 844 & Newport $1 \mathrm{SW}$ & 6331 & Jack & 1,060 & $33^{\circ} 27^{\prime}$ & $98^{\circ} 01^{\prime}$ & $10 / 1947$ & $12 / 2001$ & 89 & 54 \\
\hline 845 & Newton & 6339 & Newton & 190 & $30^{\circ} 51^{\prime}$ & $93^{\circ} 46^{\prime}$ & $01 / 1966$ & 03/1977 & 85 & 12 \\
\hline 846 & Newton & 6341 & Newton & 150 & $30^{\circ} 49^{\prime}$ & $93^{\circ} 44^{\prime}$ & $01 / 1980$ & $12 / 2001$ & 93 & 19 \\
\hline 847 & Nix Store $1 \mathrm{~W}$ & 6367 & Lampasas & 1,360 & $31^{\circ} 07^{\prime}$ & $98^{\circ} 22^{\prime}$ & $03 / 1948$ & $12 / 2001$ & 91 & 54 \\
\hline 848 & Nixon & 6368 & Gonzales & 400 & $29^{\circ} 16^{\prime}$ & $97^{\circ} 45^{\prime}$ & $06 / 1921$ & $12 / 2001$ & 97 & 81 \\
\hline 849 & Nogalus Guard Station & 6382 & Houston & 400 & $31^{\circ} 16^{\prime}$ & $95^{\circ} 08^{\prime}$ & $09 / 1947$ & $12 / 1969$ & 88 & 21 \\
\hline 850 & Nolan & 6385 & Nolan* & 2,533 & $32^{\circ} 18^{\prime}$ & $100^{\circ} 19^{\prime}$ & $05 / 1922$ & 03/1926 & 78 & 5 \\
\hline 851 & North Fork Dam & 6434 & Williamson & 883 & $30^{\circ} 41^{\prime}$ & $97^{\circ} 43^{\prime}$ & $07 / 1980$ & 06/1981 & 50 & 2 \\
\hline 852 & North Galveston & 6435 & Galveston* & $* 4$ & $29^{\circ} 20^{\prime}$ & $94^{\circ} 46^{\prime}$ & $06 / 1904$ & 08/1905 & 63 & 2 \\
\hline 853 & North Houston & 6438 & Harris* & 110 & $29^{\circ} 53^{\prime}$ & $95^{\circ} 31^{\prime}$ & $09 / 1947$ & 05/1948 & 37 & 2 \\
\hline 854 & Northfield & 6433 & Motley & 2,070 & $34^{\circ} 15^{\prime}$ & $100^{\circ} 36^{\prime}$ & $05 / 1948$ & $12 / 2001$ & 96 & 54 \\
\hline 855 & Northington Ranch & 6448 & Kendall & 1,524 & $29^{\circ} 51^{\prime}$ & $98^{\circ} 39^{\prime}$ & $08 / 1986$ & $12 / 2001$ & 95 & 16 \\
\hline 856 & Notla 3 SE & 6477 & Ochiltree & 2,900 & $36^{\circ} 06^{\prime}$ & $100^{\circ} 35^{\prime}$ & $10 / 1947$ & $12 / 2001$ & 95 & 54 \\
\hline 857 & Novice & 6484 & Coleman & 1,982 & $31^{\circ} 59^{\prime}$ & $99^{\circ} 37^{\prime}$ & $03 / 1948$ & 09/1973 & 96 & 26 \\
\hline 858 & Nugent 1 ESE & 6494 & Jones & 1,591 & $32^{\circ} 41^{\prime}$ & $99^{\circ} 40^{\prime}$ & $08 / 1943$ & $11 / 1972$ & 96 & 30 \\
\hline 859 & O.C. Fisher Dam & 6499 & Tom Green & 1,964 & $31^{\circ} 28^{\prime}$ & $100^{\circ} 29^{\prime}$ & $12 / 1975$ & $12 / 2001$ & 95 & 27 \\
\hline 860 & O'Donnell & 6504 & Lynn & 3,046 & $32^{\circ} 58^{\prime}$ & $101^{\circ} 49^{\prime}$ & $06 / 1948$ & 09/1951 & 83 & 4 \\
\hline 861 & Oak Creek Lake & 6495 & Coke & 2,065 & $32^{\circ} 03^{\prime}$ & $100^{\circ} 18^{\prime}$ & $04 / 1962$ & $12 / 2001$ & 99 & 40 \\
\hline 862 & Oakwood 2 NNE & 6496 & Freestone & 305 & $31^{\circ} 36^{\prime}$ & $95^{\circ} 50^{\prime}$ & $12 / 1980$ & $12 / 2001$ & 95 & 22 \\
\hline 863 & Oakwood 7 NW & 6497 & Freestone & 335 & $31^{\circ} 40^{\prime}$ & $95^{\circ} 55^{\prime}$ & $10 / 1980$ & $11 / 1981$ & 58 & 2 \\
\hline 864 & Odessa & 6502 & Ector & 2,910 & $31^{\circ} 52^{\prime}$ & $102^{\circ} 21^{\prime}$ & $09 / 1950$ & $12 / 2001$ & 95 & 52 \\
\hline 865 & Oilton & 6548 & Webb & 801 & $27^{\circ} 27^{\prime}$ & $98^{\circ} 55^{\prime}$ & $11 / 1964$ & 03/1971 & 80 & 8 \\
\hline 866 & Old 8 Camp 6666 & 6615 & King & 1,790 & $33^{\circ} 33^{\prime}$ & $100^{\circ} 11^{\prime}$ & $10 / 1973$ & 09/2000 & 74 & 10 \\
\hline 867 & Olney & 6636 & Young & 1,195 & $33^{\circ} 22^{\prime}$ & $98^{\circ} 45^{\prime}$ & $01 / 1956$ & $12 / 2001$ & 94 & 46 \\
\hline 868 & Olney 5 NNW & 6641 & Archer & 1,184 & $33^{\circ} 26^{\prime}$ & $98^{\circ} 46^{\prime}$ & $05 / 1941$ & $12 / 2001$ & 98 & 61 \\
\hline 869 & Olton & 6644 & Lamb & 3,610 & $34^{\circ} 11^{\prime}$ & $102^{\circ} 08^{\prime}$ & $04 / 1928$ & $12 / 2001$ & 84 & 60 \\
\hline 870 & Omaha $2 \mathrm{~W}$ & 6649 & Morris & 395 & $33^{\circ} 11^{\prime}$ & $94^{\circ} 46^{\prime}$ & 09/1997 & $12 / 2001$ & 86 & 5 \\
\hline 871 & One O One Ranch & 6654 & Brewster* & 3,612 & $30^{\circ} 10^{\prime}$ & $103^{\circ} 46^{\prime}$ & $02 / 1949$ & $10 / 1949$ & 69 & 1 \\
\hline 872 & Oplin near SCS 174 & 6660 & Callahan* & 2,001 & $32^{\circ} 10^{\prime}$ & $99^{\circ} 31^{\prime}$ & $11 / 1926$ & $02 / 1928$ & 44 & 3 \\
\hline 873 & Orange & 6664 & Orange & 10 & $30^{\circ} 05^{\prime}$ & $93^{\circ} 44^{\prime}$ & $01 / 1905$ & $12 / 2001$ & 96 & 67 \\
\hline 874 & Orange $9 \mathrm{~N}$ & 6680 & Orange & 18 & $30^{\circ} 13^{\prime}$ & $93^{\circ} 44^{\prime}$ & $05 / 1986$ & $12 / 2001$ & 98 & 16 \\
\hline 875 & Orange Dupont & 6663 & Orange* & 10 & $30^{\circ} 04^{\prime}$ & $93^{\circ} 45^{\prime}$ & $11 / 1948$ & $10 / 1950$ & 44 & 3 \\
\hline 876 & Orange Riverside & 6667 & Orange* & 10 & $30^{\circ} 06^{\prime}$ & $93^{\circ} 44^{\prime}$ & $11 / 1947$ & $01 / 1948$ & 8 & 2 \\
\hline
\end{tabular}


Table 1. Summary of National Weather Service daily precipitation stations in Texas with known names, latitudes, and longitudes-Continued

\begin{tabular}{|c|c|c|c|c|c|c|c|c|c|c|}
\hline $\begin{array}{c}\text { Seq. } \\
\text { no. }\end{array}$ & NWS station name & $\begin{array}{c}\text { NWS } \\
\text { station } \\
\text { no. }\end{array}$ & County & $\begin{array}{c}\text { Elev. } \\
\text { (ft above } \\
\text { NGVD } \\
\text { 29) }\end{array}$ & $\begin{array}{l}\text { Lati- } \\
\text { tude }\end{array}$ & $\begin{array}{l}\text { Longi- } \\
\text { tude }\end{array}$ & $\begin{array}{l}\text { Begining } \\
\text { date } \\
\text { (mm/yyyy) }\end{array}$ & $\begin{array}{c}\text { Ending } \\
\text { date } \\
\text { (mm/yyyy) }\end{array}$ & $\begin{array}{l}\text { Per- } \\
\text { cent } \\
\text { cov- } \\
\text { erage }\end{array}$ & $\begin{array}{l}\text { Years } \\
\text { of } \\
\text { record }\end{array}$ \\
\hline 877 & Orson Ranch & 6694 & Borden* & 2,651 & $32^{\circ} 45^{\prime}$ & $101^{\circ} 41^{\prime}$ & $09 / 1947$ & $12 / 1948$ & 59 & 2 \\
\hline 878 & Overton & 6722 & Rusk & 500 & $32^{\circ} 16^{\prime}$ & $94^{\circ} 59^{\prime}$ & $12 / 1943$ & $10 / 1987$ & 95 & 45 \\
\hline 879 & Ozona 1 SSW & 6734 & Crockett & 2,340 & $30^{\circ} 41^{\prime}$ & $101^{\circ} 12^{\prime}$ & $06 / 1948$ & $12 / 2001$ & 94 & 54 \\
\hline 880 & Ozona 8 WSW & 6736 & Crockett & 2,550 & $30^{\circ} 41^{\prime}$ & $101^{\circ} 20^{\prime}$ & $02 / 1951$ & 09/1951 & 61 & 1 \\
\hline 881 & Paducah & 6740 & Cottle & 1,900 & $34^{\circ} 00^{\prime}$ & $100^{\circ} 18^{\prime}$ & 09/1946 & 09/2001 & 96 & 53 \\
\hline 882 & Paducah $10 \mathrm{~S}$ & 6745 & Cottle & 1,950 & $33^{\circ} 52^{\prime}$ & $100^{\circ} 23^{\prime}$ & $12 / 1994$ & $12 / 2001$ & 62 & 8 \\
\hline 883 & Paducah $15 \mathrm{~S}$ & 6742 & King & 1,815 & $33^{\circ} 48^{\prime}$ & $100^{\circ} 18^{\prime}$ & 04/1971 & $12 / 2001$ & 96 & 31 \\
\hline 884 & Paducah 2 WNW & 6743 & Cottle* & 1,890 & $34^{\circ} 02^{\prime}$ & $100^{\circ} 19^{\prime}$ & 06/1913 & $12 / 1950$ & 98 & 38 \\
\hline 885 & Paint Rock & 6747 & Concho & 1,625 & $31^{\circ} 30^{\prime}$ & $99^{\circ} 55^{\prime}$ & 06/1918 & 07/2001 & 97 & 84 \\
\hline 886 & Palacios Municipal Airport & 6750 & Matagorda & 12 & $28^{\circ} 43^{\prime}$ & $96^{\circ} 15^{\prime}$ & $02 / 1943$ & $12 / 2001$ & 99 & 59 \\
\hline 887 & Palestine $2 \mathrm{NE}$ & 6757 & Anderson & 465 & $31^{\circ} 46^{\prime}$ & $95^{\circ} 36^{\prime}$ & $01 / 1930$ & $12 / 2001$ & 98 & 70 \\
\hline 888 & Palo Pinto & 6766 & Palo Pinto & 1,040 & $32^{\circ} 45^{\prime}$ & $98^{\circ} 18^{\prime}$ & 03/1949 & $12 / 2001$ & 98 & 53 \\
\hline 889 & Pampa 2 & 6776 & Gray & 3,150 & $35^{\circ} 33^{\prime}$ & $100^{\circ} 58^{\prime}$ & 04/1964 & $12 / 2001$ & 99 & 38 \\
\hline 890 & Pampa WB Airport & 6775 & Gray & 3,232 & $35^{\circ} 32^{\prime}$ & $100^{\circ} 58^{\prime}$ & 04/1908 & $01 / 1964$ & 87 & 41 \\
\hline 891 & Pandale $1 \mathrm{~N}$ & 6780 & Val Verde & 1,689 & $30^{\circ} 10^{\prime}$ & $101^{\circ} 33^{\prime}$ & 08/1909 & $12 / 2001$ & 89 & 56 \\
\hline 892 & Pandale $11 \mathrm{NE}$ & 6781 & Val Verde & 1,665 & $30^{\circ} 16^{\prime}$ & $101^{\circ} 27^{\prime}$ & $12 / 1981$ & $12 / 2001$ & 94 & 21 \\
\hline 893 & Panhandle & 6785 & Carson & 3,532 & $35^{\circ} 24^{\prime}$ & $101^{\circ} 20^{\prime}$ & 08/1911 & $12 / 2001$ & 96 & 77 \\
\hline 894 & Panter & 6790 & Hood* & 1,001 & $32^{\circ} 20^{\prime}$ & $97^{\circ} 52^{\prime}$ & $12 / 1901$ & $10 / 1917$ & 91 & 17 \\
\hline 895 & Panther Junction & 6792 & Brewster & 3,740 & $29^{\circ} 19^{\prime}$ & $103^{\circ} 12^{\prime}$ & $04 / 1955$ & $12 / 2001$ & 99 & 47 \\
\hline 896 & Paris & 6794 & Lamar & 542 & $33^{\circ} 40^{\prime}$ & $95^{\circ} 34^{\prime}$ & $12 / 1896$ & $12 / 2001$ & 97 & 101 \\
\hline 897 & Park Springs & 6801 & Wise* & 961 & $33^{\circ} 24^{\prime}$ & $97^{\circ} 49^{\prime}$ & $09 / 1947$ & 04/1949 & 17 & 3 \\
\hline 898 & Pasadena Houston & 6825 & Harris* & 30 & $29^{\circ} 43^{\prime}$ & $95^{\circ} 13^{\prime}$ & $10 / 1956$ & $01 / 1960$ & 66 & 5 \\
\hline 899 & Patricia & 6836 & Dawson* & 3,002 & $32^{\circ} 33^{\prime}$ & $102^{\circ} 07^{\prime}$ & 08/1949 & $01 / 1953$ & 8 & 2 \\
\hline 900 & Pattison & 6843 & Harris* & 171 & $29^{\circ} 54^{\prime}$ & $95^{\circ} 50^{\prime}$ & 09/1947 & 03/1952 & 70 & 6 \\
\hline 901 & Pearsall & 6879 & Frio & 635 & $28^{\circ} 53^{\prime}$ & $99^{\circ} 05^{\prime}$ & $03 / 1902$ & $12 / 2001$ & 95 & 98 \\
\hline 902 & Pearsall 9 WNW & 6880 & Frio & 610 & $28^{\circ} 58^{\prime}$ & $99^{\circ} 13^{\prime}$ & $01 / 1980$ & $01 / 1983$ & 77 & 4 \\
\hline 903 & Pecos & 6892 & Reeves & 2,610 & $31^{\circ} 25^{\prime}$ & $103^{\circ} 30^{\prime}$ & $01 / 1904$ & $08 / 2000$ & 96 & 72 \\
\hline 904 & $P e \cos 8 \mathrm{~W}$ & 6893 & Reeves & 2,660 & $31^{\circ} 22^{\prime}$ & $103^{\circ} 38^{\prime}$ & $12 / 2000$ & $12 / 2001$ & 54 & 2 \\
\hline 905 & Penwell & 6932 & Ector & 2,940 & $31^{\circ} 44^{\prime}$ & $102^{\circ} 35^{\prime}$ & $10 / 1955$ & $12 / 2001$ & 97 & 47 \\
\hline 906 & Perryton & 6950 & Ochiltree & 2,942 & $36^{\circ} 23^{\prime}$ & $100^{\circ} 49^{\prime}$ & 01/1907 & $12 / 2001$ & 95 & 91 \\
\hline 907 & Perryton 1 WSW & 6955 & Ochiltree & 2,942 & $36^{\circ} 23^{\prime}$ & $100^{\circ} 49^{\prime}$ & 02/1908 & $02 / 1908$ & 0 & 1 \\
\hline 908 & Perryton $11 \mathrm{WNW}$ & 6953 & Ochiltree & 3,010 & $36^{\circ} 26^{\prime}$ & $100^{\circ} 59^{\prime}$ & $10 / 1947$ & $12 / 2001$ & 98 & 55 \\
\hline 909 & Perryton $21 \mathrm{~S}$ & 6952 & Ochiltree & 2,985 & $36^{\circ} 06^{\prime}$ & $100^{\circ} 44^{\prime}$ & 04/1978 & $12 / 2001$ & 96 & 24 \\
\hline 910 & Persimmon Gap & 6959 & Brewster & 2,865 & $29^{\circ} 39^{\prime}$ & $103^{\circ} 10^{\prime}$ & $05 / 1952$ & $12 / 2001$ & 84 & 23 \\
\hline 911 & Persimmon Gap 6 E & 6960 & Brewster & 2,533 & $29^{\circ} 39^{\prime}$ & $103^{\circ} 05^{\prime}$ & 09/1979 & $05 / 1985$ & 82 & 7 \\
\hline 912 & Pettit 4 NE & 6981 & Hockley* & 3,553 & $33^{\circ} 44^{\prime}$ & $102^{\circ} 28^{\prime}$ & $07 / 1948$ & 09/1951 & 21 & 4 \\
\hline 913 & Pflugerville & 6992 & Travis & 679 & $30^{\circ} 26^{\prime}$ & $97^{\circ} 37^{\prime}$ & 03/1948 & $12 / 1965$ & 98 & 18 \\
\hline 914 & Pidcoke & 7017 & Coryell & 800 & $31^{\circ} 17^{\prime}$ & $97^{\circ} 53^{\prime}$ & 01/1992 & $12 / 2001$ & 99 & 10 \\
\hline 915 & Pierce $1 \mathrm{E}$ & 7020 & Wharton & 105 & $29^{\circ} 14^{\prime}$ & $96^{\circ} 11^{\prime}$ & 05/1904 & $12 / 2001$ & 94 & 98 \\
\hline 916 & Pilot Point & 7028 & Denton & 690 & $33^{\circ} 23^{\prime}$ & $96^{\circ} 57^{\prime}$ & 09/1947 & $12 / 2001$ & 95 & 55 \\
\hline 917 & Pine Springs & 7044 & Culberson & 5,609 & $31^{\circ} 53^{\prime}$ & $104^{\circ} 48^{\prime}$ & 01/1939 & $12 / 2001$ & 96 & 22 \\
\hline 918 & Pineland & 7040 & Sabine & 220 & $31^{\circ} 14^{\prime}$ & $93^{\circ} 57^{\prime}$ & $01 / 1965$ & $12 / 2001$ & 97 & 25 \\
\hline 919 & Pitchfork Ranch & 7060 & Dickens & 1,945 & $33^{\circ} 35^{\prime}$ & $100^{\circ} 31^{\prime}$ & 05/1971 & $12 / 2001$ & 86 & 31 \\
\hline 920 & Pittsburg $5 \mathrm{~S}$ & 7066 & Camp & 345 & $32^{\circ} 55^{\prime}$ & $94^{\circ} 56^{\prime}$ & $04 / 1949$ & $12 / 2001$ & 99 & 53 \\
\hline 921 & Placid & 7072 & McCulloch & 1,631 & $31^{\circ} 19^{\prime}$ & $99^{\circ} 11^{\prime}$ & 03/1948 & $11 / 1965$ & 66 & 18 \\
\hline 922 & Plains & 7074 & Yoakum & 3,675 & $33^{\circ} 11^{\prime}$ & $102^{\circ} 49^{\prime}$ & $01 / 1925$ & $12 / 2001$ & 93 & 67 \\
\hline 923 & Plainview & 7079 & Hale & 3,370 & $34^{\circ} 11^{\prime}$ & $101^{\circ} 42^{\prime}$ & 09/1908 & $12 / 2001$ & 97 & 94 \\
\hline 924 & Plainview Radio KKYN & 7081 & Hale & 3,450 & $34^{\circ} 12^{\prime}$ & $101^{\circ} 43^{\prime}$ & $04 / 2000$ & $12 / 2001$ & 87 & 2 \\
\hline 925 & Plata & 7091 & Presidio & 3,753 & $29^{\circ} 51^{\prime}$ & $104^{\circ} 01^{\prime}$ & $10 / 1964$ & 02/1977 & 82 & 14 \\
\hline 926 & Pleak & 7097 & Fort Bend* & 79 & $29^{\circ} 28^{\prime}$ & $95^{\circ} 47^{\prime}$ & 06/1948 & 09/1950 & 51 & 3 \\
\hline 927 & Pleasanton & 7111 & Atascosa & 416 & $28^{\circ} 57^{\prime}$ & $98^{\circ} 29^{\prime}$ & $01 / 1948$ & $12 / 2001$ & 81 & 15 \\
\hline 928 & Plemons & 7116 & Hutchinson* & 2,802 & $35^{\circ} 46^{\prime}$ & $101^{\circ} 20^{\prime}$ & 09/1906 & 08/1951 & 83 & 25 \\
\hline
\end{tabular}


Table 1. Summary of National Weather Service daily precipitation stations in Texas with known names, latitudes, and longitudes-Continued

\begin{tabular}{|c|c|c|c|c|c|c|c|c|c|c|}
\hline $\begin{array}{c}\text { Seq. } \\
\text { no. }\end{array}$ & NWS station name & $\begin{array}{l}\text { NWS } \\
\text { station } \\
\text { no. }\end{array}$ & County & $\begin{array}{c}\text { Elev. } \\
\text { (ft above } \\
\text { NGVD } \\
29 \text { ) }\end{array}$ & $\begin{array}{l}\text { Lati- } \\
\text { tude }\end{array}$ & $\begin{array}{l}\text { Longi- } \\
\text { tude }\end{array}$ & $\begin{array}{c}\text { Begining } \\
\text { date } \\
\text { (mm/yyyy) }\end{array}$ & $\begin{array}{c}\text { Ending } \\
\text { date } \\
\text { (mm/yyyy) }\end{array}$ & $\begin{array}{l}\text { Per- } \\
\text { cent } \\
\text { cov- } \\
\text { erage }\end{array}$ & $\begin{array}{l}\text { Years } \\
\text { of } \\
\text { record }\end{array}$ \\
\hline 929 & Point Comfort & 7140 & Calhoun & 20 & $28^{\circ} 39^{\prime}$ & $96^{\circ} 33^{\prime}$ & $11 / 1957$ & $12 / 2001$ & 96 & 45 \\
\hline 930 & Polar & 7146 & Garza & 2,352 & $33^{\circ} 01^{\prime}$ & $101^{\circ} 04^{\prime}$ & $09 / 1947$ & $06 / 1975$ & 86 & 29 \\
\hline 931 & Pontotoc & 7164 & Mason & 1,562 & $30^{\circ} 55^{\prime}$ & $98^{\circ} 59^{\prime}$ & $04 / 1948$ & $11 / 1965$ & 83 & 16 \\
\hline 932 & Poolville & 7165 & Parker* & 1,230 & $32^{\circ} 58^{\prime}$ & $97^{\circ} 52^{\prime}$ & $11 / 1947$ & $04 / 1957$ & 81 & 11 \\
\hline 933 & Popham Ranch & 7168 & Reeves* & 3,304 & $30^{\circ} 53^{\prime}$ & $103^{\circ} 33^{\prime}$ & $09 / 1947$ & $10 / 1947$ & 17 & 1 \\
\hline 934 & Port Aransas & 7170 & Nueces & 12 & $27^{\circ} 50^{\prime}$ & $97^{\circ} 03^{\prime}$ & 03/1986 & $12 / 2001$ & 95 & 16 \\
\hline 935 & Port Arthur Airport & 7174 & Jefferson & 16 & $29^{\circ} 57^{\prime}$ & $94^{\circ} 01^{\prime}$ & $10 / 1947$ & $12 / 2001$ & 98 & 55 \\
\hline 936 & Port Arthur City & 7172 & Jefferson & 5 & $29^{\circ} 54^{\prime}$ & $93^{\circ} 58^{\prime}$ & $11 / 1975$ & $12 / 2001$ & 96 & 27 \\
\hline 937 & Port Arthur WB City & 7173 & Jefferson & 10 & $29^{\circ} 52^{\prime}$ & $93^{\circ} 56^{\prime}$ & 03/1911 & $12 / 1967$ & 87 & 28 \\
\hline 938 & Port Isabel & 7179 & Cameron & 17 & $26^{\circ} 04^{\prime}$ & $97^{\circ} 13^{\prime}$ & $01 / 1897$ & $12 / 2001$ & 96 & 72 \\
\hline 939 & Port Lavaca 2 & 7182 & Calhoun & 20 & $28^{\circ} 37^{\prime}$ & $96^{\circ} 38^{\prime}$ & $02 / 1901$ & $02 / 1988$ & 86 & 69 \\
\hline 940 & Port Mansfield & 7184 & Willacy & 9 & $26^{\circ} 33^{\prime}$ & $97^{\circ} 25^{\prime}$ & $02 / 1958$ & $12 / 2001$ & 99 & 44 \\
\hline 941 & Port O'Connor & 7186 & Calhoun & 5 & $28^{\circ} 26^{\prime}$ & $96^{\circ} 25^{\prime}$ & $04 / 1948$ & $10 / 2001$ & 87 & 52 \\
\hline 942 & Possum Kingdom Dam & 7205 & Palo Pinto & 902 & $32^{\circ} 52^{\prime}$ & $98^{\circ} 26^{\prime}$ & $01 / 1939$ & $04 / 1975$ & 97 & 37 \\
\hline 943 & Post & 7206 & Garza & 2,620 & $33^{\circ} 11^{\prime}$ & $101^{\circ} 22^{\prime}$ & $05 / 1910$ & $12 / 2001$ & 97 & 92 \\
\hline 944 & Post Oak School & 7213 & Lee & 318 & $30^{\circ} 16^{\prime}$ & $96^{\circ} 43^{\prime}$ & $04 / 1963$ & 05/1981 & 82 & 19 \\
\hline 945 & Poteet & 7215 & Atascosa & 480 & $29^{\circ} 02^{\prime}$ & $98^{\circ} 35^{\prime}$ & $11 / 1941$ & $12 / 2001$ & 96 & 61 \\
\hline 946 & Poynor & 7230 & Henderson & 531 & $32^{\circ} 05^{\prime}$ & $95^{\circ} 35^{\prime}$ & $09 / 1947$ & $10 / 1975$ & 93 & 29 \\
\hline 947 & Prade Ranch & 7232 & Real & 2,052 & $29^{\circ} 54^{\prime}$ & $99^{\circ} 46^{\prime}$ & $10 / 1955$ & $12 / 2001$ & 90 & 42 \\
\hline 948 & Prairie Mountain & 7243 & Llano & 1,448 & $30^{\circ} 35^{\prime}$ & $98^{\circ} 53^{\prime}$ & $06 / 1948$ & 09/1951 & 83 & 4 \\
\hline 949 & Presidio & 7262 & Presidio & 2,560 & $29^{\circ} 33^{\prime}$ & $104^{\circ} 20^{\prime}$ & $10 / 1927$ & $12 / 2001$ & 97 & 75 \\
\hline 950 & Prewit Ranch & 7268 & Reeves* & 2,703 & $31^{\circ} 17^{\prime}$ & $103^{\circ} 30^{\prime}$ & $09 / 1947$ & 04/1948 & 32 & 2 \\
\hline 951 & Price & 7271 & Rusk & 371 & $32^{\circ} 07^{\prime}$ & $94^{\circ} 58^{\prime}$ & $08 / 1941$ & $11 / 1975$ & 97 & 35 \\
\hline 952 & Priddy $1 \mathrm{NE}$ & 7274 & Mills & 1,470 & $31^{\circ} 40^{\prime}$ & $98^{\circ} 29^{\prime}$ & $01 / 1984$ & 09/1997 & 96 & 14 \\
\hline 953 & Proctor Reservoir & 7300 & Comanche & 1,221 & $31^{\circ} 58^{\prime}$ & $98^{\circ} 30^{\prime}$ & $06 / 1963$ & $11 / 2001$ & 98 & 39 \\
\hline 954 & Provident City & 7299 & Colorado & 151 & $29^{\circ} 17^{\prime}$ & $96^{\circ} 38^{\prime}$ & $09 / 1947$ & 08/1966 & 95 & 20 \\
\hline 955 & Putnam & 7327 & Callahan & 1,591 & $32^{\circ} 22^{\prime}$ & $99^{\circ} 11^{\prime}$ & 03/1911 & $12 / 2001$ & 99 & 91 \\
\hline 956 & Quanah 5 SE & 7336 & Hardeman & 1,495 & $34^{\circ} 15^{\prime}$ & $99^{\circ} 41^{\prime}$ & $10 / 1904$ & $12 / 2001$ & 97 & 98 \\
\hline 957 & Quinlan & 7358 & Hunt & 512 & $32^{\circ} 55^{\prime}$ & $96^{\circ} 08^{\prime}$ & $03 / 1962$ & $11 / 1974$ & 90 & 13 \\
\hline 958 & Quitaque $1 \mathrm{E}$ & 7361 & Briscoe & 2,572 & $34^{\circ} 22^{\prime}$ & $101^{\circ} 03^{\prime}$ & $07 / 1934$ & 03/1976 & 82 & 42 \\
\hline 959 & Quitman & 7363 & Wood & 375 & $32^{\circ} 47^{\prime}$ & $95^{\circ} 26^{\prime}$ & $05 / 1948$ & 08/1987 & 95 & 40 \\
\hline 960 & Quitman 2 & 7365 & Wood & 413 & $32^{\circ} 48^{\prime}$ & $95^{\circ} 26^{\prime}$ & $10 / 1999$ & $12 / 2001$ & 71 & 3 \\
\hline 961 & Rainbow & 7388 & Somervell & 648 & $32^{\circ} 15^{\prime}$ & $97^{\circ} 42^{\prime}$ & $09 / 1946$ & $12 / 2001$ & 98 & 56 \\
\hline 962 & Rancho Escondido & 7411 & Brewster* & 4,803 & $30^{\circ} 01^{\prime}$ & $103^{\circ} 46^{\prime}$ & $09 / 1947$ & 08/1949 & 66 & 3 \\
\hline 963 & Randolph Field & 7422 & Bexar & 760 & $29^{\circ} 31^{\prime}$ & $98^{\circ} 16^{\prime}$ & $06 / 1948$ & 09/1951 & 78 & 4 \\
\hline 964 & Ranger & 7425 & Eastland* & 1,430 & $32^{\circ} 28^{\prime}$ & $98^{\circ} 41^{\prime}$ & $09 / 1946$ & 07/1952 & 79 & 7 \\
\hline 965 & Ranger & 7426 & Eastland & 1,542 & $32^{\circ} 28^{\prime}$ & $98^{\circ} 42^{\prime}$ & $09 / 1947$ & $10 / 1975$ & 96 & 29 \\
\hline 966 & Rankin & 7431 & Upton & 2,615 & $31^{\circ} 13^{\prime}$ & $101^{\circ} 56^{\prime}$ & $08 / 1948$ & $10 / 1984$ & 90 & 27 \\
\hline 967 & Ransom Canyon & 7433 & Lubbock & 3,100 & $33^{\circ} 32^{\prime}$ & $101^{\circ} 41^{\prime}$ & $03 / 1988$ & $12 / 2001$ & 99 & 14 \\
\hline 968 & Ratcliff & 7436 & Houston* & $* 400$ & $31^{\circ} 23^{\prime}$ & $95^{\circ} 08^{\prime}$ & $04 / 1915$ & 07/1919 & 87 & 5 \\
\hline 969 & Ray Roberts Lake & 7463 & Denton & 600 & $33^{\circ} 20^{\prime}$ & $97^{\circ} 03^{\prime}$ & $04 / 1984$ & 04/1988 & 66 & 5 \\
\hline 970 & Raymondville & 7458 & Willacy & 31 & $26^{\circ} 28^{\prime}$ & $97^{\circ} 48^{\prime}$ & $10 / 1910$ & $12 / 2001$ & 97 & 92 \\
\hline 971 & Reagan & 7468 & Falls* & 430 & $31^{\circ} 18^{\prime}$ & $96^{\circ} 42^{\prime}$ & $11 / 1927$ & $10 / 1932$ & 79 & 6 \\
\hline 972 & Red Bluff Crossing & 7480 & San Saba & 1,235 & $31^{\circ} 13^{\prime}$ & $98^{\circ} 35^{\prime}$ & $06 / 1948$ & $12 / 2001$ & 94 & 54 \\
\hline 973 & Red Bluff Dam & 7481 & Reeves & 2,800 & $31^{\circ} 54^{\prime}$ & $103^{\circ} 55^{\prime}$ & $10 / 1939$ & $12 / 2000$ & 89 & 60 \\
\hline 974 & Red Oak & 7495 & Ellis & 600 & $32^{\circ} 31^{\prime}$ & $96^{\circ} 48^{\prime}$ & $11 / 1964$ & 06/1996 & 93 & 33 \\
\hline 975 & Red Rock & 7497 & Bastrop & 520 & $29^{\circ} 58^{\prime}$ & $97^{\circ} 27^{\prime}$ & $05 / 1965$ & $12 / 2000$ & 97 & 35 \\
\hline 976 & Red Springs 2 ESE & 7499 & Baylor & 1,370 & $33^{\circ} 36^{\prime}$ & $99^{\circ} 23^{\prime}$ & $06 / 1948$ & 09/1951 & 61 & 4 \\
\hline 977 & Reeves Station & 7528 & Sutton* & 2,201 & $30^{\circ} 28^{\prime}$ & $100^{\circ} 11^{\prime}$ & $01 / 1949$ & 07/1952 & 13 & 2 \\
\hline 978 & Refugio & 7529 & Refugio & 49 & $28^{\circ} 18^{\prime}$ & $97^{\circ} 17^{\prime}$ & $01 / 1948$ & $11 / 1984$ & 99 & 37 \\
\hline 979 & Refugio $2 \mathrm{NW}$ & 7533 & Refugio & 45 & $28^{\circ} 19^{\prime}$ & $97^{\circ} 17^{\prime}$ & $07 / 1985$ & $12 / 2001$ & 92 & 17 \\
\hline 980 & Refugio $3 \mathrm{SW}$ & 7530 & Refugio & 54 & $28^{\circ} 17^{\prime}$ & $97^{\circ} 19^{\prime}$ & 05/1991 & $12 / 2001$ & 88 & 11 \\
\hline
\end{tabular}


Table 1. Summary of National Weather Service daily precipitation stations in Texas with known names, latitudes, and longitudes-Continued

\begin{tabular}{|c|c|c|c|c|c|c|c|c|c|c|}
\hline $\begin{array}{l}\text { Seq. } \\
\text { no. }\end{array}$ & NWS station name & $\begin{array}{c}\text { NWS } \\
\text { station } \\
\text { no. }\end{array}$ & County & $\begin{array}{c}\text { Elev. } \\
\text { (ft above } \\
\text { NGVD } \\
\text { 29) }\end{array}$ & $\begin{array}{l}\text { Lati- } \\
\text { tude }\end{array}$ & $\begin{array}{l}\text { Longi- } \\
\text { tude }\end{array}$ & $\begin{array}{c}\text { Begining } \\
\text { date } \\
\text { (mm/yyyy) }\end{array}$ & $\begin{array}{c}\text { Ending } \\
\text { date } \\
\text { (mm/yyyy) }\end{array}$ & $\begin{array}{l}\text { Per- } \\
\text { cent } \\
\text { cov- } \\
\text { erage }\end{array}$ & $\begin{array}{l}\text { Years } \\
\text { of } \\
\text { record }\end{array}$ \\
\hline 981 & Regency & 7531 & Mills* & $* 1,241$ & $31^{\circ} 26^{\prime}$ & $98^{\circ} 49^{\prime}$ & $06 / 1948$ & $08 / 1948$ & 4 & 1 \\
\hline 982 & Reiley Ranch & 7534 & Sutton* & 2,451 & $30^{\circ} 38^{\prime}$ & $100^{\circ} 15^{\prime}$ & $04 / 1948$ & $07 / 1950$ & 35 & 3 \\
\hline 983 & Reklaw 3 NNE & 7547 & Rusk & 331 & $31^{\circ} 54^{\prime}$ & $94^{\circ} 59^{\prime}$ & $09 / 1958$ & 03/1988 & 92 & 31 \\
\hline 984 & Rendham & 7552 & Baylor* & 1,352 & $33^{\circ} 35^{\prime}$ & $99^{\circ} 09^{\prime}$ & $09 / 1947$ & $01 / 1958$ & 81 & 12 \\
\hline 985 & Reno & 7556 & Parker & 770 & $32^{\circ} 57^{\prime}$ & $97^{\circ} 34^{\prime}$ & 06/1948 & 09/1951 & 83 & 4 \\
\hline 986 & Rhineland & 7572 & Knox* & 1,201 & $33^{\circ} 32^{\prime}$ & $99^{\circ} 39^{\prime}$ & $01 / 1897$ & 09/1907 & 95 & 10 \\
\hline 987 & Ricardo & 7580 & Kleberg & 59 & $27^{\circ} 25^{\prime}$ & $97^{\circ} 49^{\prime}$ & 06/1909 & $01 / 1975$ & 92 & 67 \\
\hline 988 & Rice & 7581 & Navarro* & 440 & $32^{\circ} 16^{\prime}$ & $96^{\circ} 30^{\prime}$ & $01 / 1949$ & $10 / 1956$ & 80 & 8 \\
\hline 989 & Richards & 7586 & Grimes & 315 & $30^{\circ} 32^{\prime}$ & $95^{\circ} 51^{\prime}$ & $06 / 1954$ & $12 / 2001$ & 94 & 47 \\
\hline 990 & Richardson & 7588 & Collin & 675 & $32^{\circ} 59^{\prime}$ & $96^{\circ} 45^{\prime}$ & $02 / 1948$ & $12 / 2001$ & 96 & 53 \\
\hline 991 & Richland Springs & 7593 & San Saba & 1,380 & $31^{\circ} 16^{\prime}$ & $98^{\circ} 56^{\prime}$ & 03/1948 & $12 / 2001$ & 94 & 54 \\
\hline 992 & Richmond & 7594 & Fort Bend & 101 & $29^{\circ} 35^{\prime}$ & $95^{\circ} 45^{\prime}$ & 06/1919 & $12 / 2001$ & 96 & 57 \\
\hline 993 & Riesel & 7608 & McLennan* & 469 & $31^{\circ} 29^{\prime}$ & $96^{\circ} 53^{\prime}$ & $06 / 1948$ & $05 / 1952$ & 75 & 5 \\
\hline 994 & Riley Ben Ranch & 7612 & Gillespie & 1,631 & $30^{\circ} 26^{\prime}$ & $98^{\circ} 49^{\prime}$ & 03/1948 & $12 / 1965$ & 94 & 18 \\
\hline 995 & Ringgold & 7614 & Montague & 895 & $33^{\circ} 49^{\prime}$ & $97^{\circ} 56^{\prime}$ & $11 / 1947$ & 09/1994 & 94 & 48 \\
\hline 996 & Rio Grande City $1 \mathrm{SE}$ & 7622 & Starr & 172 & $26^{\circ} 23^{\prime}$ & $98^{\circ} 49^{\prime}$ & $01 / 1897$ & $12 / 2001$ & 94 & 84 \\
\hline 997 & Rio Medina & 7628 & Medina & 850 & $29^{\circ} 26^{\prime}$ & $98^{\circ} 52^{\prime}$ & $08 / 1922$ & $12 / 2001$ & 94 & 80 \\
\hline 998 & Rising Star $1 \mathrm{~S}$ & 7633 & Eastland & 1,633 & $32^{\circ} 04^{\prime}$ & $98^{\circ} 57^{\prime}$ & $02 / 1942$ & $12 / 2001$ & 97 & 60 \\
\hline 999 & Rising Star 5 NNE & 7637 & Eastland & 1,605 & $32^{\circ} 09^{\prime}$ & $98^{\circ} 55^{\prime}$ & 09/1991 & $12 / 2001$ & 90 & 11 \\
\hline 1000 & Riverside & 7651 & Walker & 240 & $30^{\circ} 51^{\prime}$ & $95^{\circ} 24^{\prime}$ & $04 / 1915$ & $01 / 1970$ & 91 & 27 \\
\hline 1001 & Roanoke & 7659 & Denton & 655 & $33^{\circ} 00^{\prime}$ & $97^{\circ} 13^{\prime}$ & $08 / 1947$ & $12 / 2001$ & 98 & 55 \\
\hline 1002 & Roaring Springs & 7663 & Motley* & 2,503 & $33^{\circ} 54^{\prime}$ & $100^{\circ} 52^{\prime}$ & 09/1949 & 09/1949 & 4 & 1 \\
\hline 1003 & Robert Lee & 7669 & Coke & 1,780 & $31^{\circ} 54^{\prime}$ & $100^{\circ} 29^{\prime}$ & 03/1908 & $12 / 2001$ & 96 & 58 \\
\hline 1004 & Robstown & 7677 & Nueces & 85 & $27^{\circ} 47^{\prime}$ & $97^{\circ} 39^{\prime}$ & $09 / 1922$ & $12 / 2001$ & 90 & 62 \\
\hline 1005 & Roby & 7678 & Fisher & 1,982 & $32^{\circ} 44^{\prime}$ & $100^{\circ} 23^{\prime}$ & $01 / 1897$ & $06 / 1975$ & 90 & 65 \\
\hline 1006 & Rock Island & 7693 & Colorado* & 249 & $29^{\circ} 32^{\prime}$ & $96^{\circ} 35^{\prime}$ & $01 / 1900$ & $10 / 1907$ & 97 & 8 \\
\hline 1007 & Rock Port & 7705 & Aransas* & 10 & $28^{\circ} 01^{\prime}$ & $97^{\circ} 03^{\prime}$ & 07/1901 & $12 / 1958$ & 86 & 27 \\
\hline 1008 & Rockdale & 7685 & Milam & 528 & $30^{\circ} 39^{\prime}$ & $97^{\circ} 02^{\prime}$ & $03 / 1963$ & $12 / 2001$ & 98 & 39 \\
\hline 1009 & Rockland 1 WSW & 7700 & Jasper & 88 & $31^{\circ} 01^{\prime}$ & $94^{\circ} 24^{\prime}$ & 04/1904 & 06/1979 & 90 & 38 \\
\hline 1010 & Rockport & 7704 & Aransas & 9 & $28^{\circ} 01^{\prime}$ & $97^{\circ} 03^{\prime}$ & $01 / 1959$ & $12 / 2001$ & 100 & 43 \\
\hline 1011 & Rocksprings & 7706 & Edwards & 2,400 & $30^{\circ} 00^{\prime}$ & $100^{\circ} 13^{\prime}$ & $04 / 1932$ & $12 / 2001$ & 89 & 63 \\
\hline 1012 & Rocksprings $14 \mathrm{NE}$ & 7714 & Edwards & 2,173 & $30^{\circ} 09^{\prime}$ & $100^{\circ} 03^{\prime}$ & 04/1978 & 05/1989 & 74 & 11 \\
\hline 1013 & Rocksprings $18 \mathrm{SW}$ & 7712 & Edwards & 1,725 & $29^{\circ} 47^{\prime}$ & $100^{\circ} 25^{\prime}$ & $02 / 1963$ & 07/1993 & 96 & 31 \\
\hline 1014 & Rocksprings 26 SSW & 7717 & Edwards & 1,690 & $29^{\circ} 41^{\prime}$ & $100^{\circ} 25^{\prime}$ & 08/1995 & $12 / 2001$ & 92 & 7 \\
\hline 1015 & Rocksprings $5 \mathrm{~W}$ & 7711 & Edwards* & 2,392 & $30^{\circ} 01^{\prime}$ & $100^{\circ} 18^{\prime}$ & 03/1955 & $06 / 1956$ & 58 & 2 \\
\hline 1016 & Rockwall & 7707 & Rockwall & 543 & $32^{\circ} 56^{\prime}$ & $96^{\circ} 28^{\prime}$ & 09/1946 & $12 / 2001$ & 98 & 56 \\
\hline 1017 & Rockwall & 7708 & Rockwall* & 600 & $32^{\circ} 56^{\prime}$ & $96^{\circ} 27^{\prime}$ & 09/1950 & $02 / 1956$ & 73 & 7 \\
\hline 1018 & Romayor $1 \mathrm{WSW}$ & 7729 & Liberty & 60 & $30^{\circ} 26^{\prime}$ & $94^{\circ} 51^{\prime}$ & $10 / 1982$ & 05/1989 & 83 & 8 \\
\hline 1019 & Romero & 7730 & Hartley* & 4,062 & $35^{\circ} 43^{\prime}$ & $102^{\circ} 55^{\prime}$ & $02 / 1910$ & $04 / 1936$ & 85 & 26 \\
\hline 1020 & Roosevelt 2 E & 7735 & Kimble & 1,962 & $30^{\circ} 29^{\prime}$ & $100^{\circ} 02^{\prime}$ & 03/1948 & $12 / 1965$ & 90 & 18 \\
\hline 1021 & Roscoe & 7743 & Nolan & 2,380 & $32^{\circ} 26^{\prime}$ & $100^{\circ} 31^{\prime}$ & $06 / 1935$ & $12 / 2001$ & 99 & 67 \\
\hline 1022 & Rosebud & 7744 & Falls & 405 & $31^{\circ} 04^{\prime}$ & $96^{\circ} 58^{\prime}$ & $07 / 1965$ & $12 / 2001$ & 93 & 29 \\
\hline 1023 & Rosenberg & 7756 & Fort Bend* & 112 & $29^{\circ} 33^{\prime}$ & $95^{\circ} 47^{\prime}$ & $04 / 1915$ & $02 / 1960$ & 84 & 22 \\
\hline 1024 & Ross & 7768 & McLennan & 571 & $31^{\circ} 43^{\prime}$ & $97^{\circ} 07^{\prime}$ & $08 / 1940$ & $10 / 1975$ & 89 & 36 \\
\hline 1025 & Ross Ranch & 7769 & King & 1,660 & $33^{\circ} 35^{\prime}$ & $100^{\circ} 01^{\prime}$ & 04/1971 & 07/1979 & 86 & 9 \\
\hline 1026 & Rosser & 7773 & Kaufman & 340 & $32^{\circ} 28^{\prime}$ & $96^{\circ} 27^{\prime}$ & 08/1947 & $12 / 2001$ & 95 & 55 \\
\hline 1027 & Rossville & 7779 & Atascosa* & 561 & $29^{\circ} 06^{\prime}$ & $98^{\circ} 41^{\prime}$ & 06/1907 & $05 / 1926$ & 93 & 20 \\
\hline 1028 & Rotan & 7782 & Fisher & 1,935 & $32^{\circ} 51^{\prime}$ & $100^{\circ} 27^{\prime}$ & $07 / 1924$ & $12 / 2001$ & 96 & 78 \\
\hline 1029 & Round Mountain $1 \mathrm{NW}$ & 7787 & Blanco & 1,340 & $30^{\circ} 26^{\prime}$ & $98^{\circ} 21^{\prime}$ & $12 / 1958$ & $12 / 2001$ & 97 & 44 \\
\hline 1030 & Round Rock 3 NE & 7791 & Williamson & 722 & $30^{\circ} 32^{\prime}$ & $97^{\circ} 38^{\prime}$ & $02 / 1968$ & $12 / 2001$ & 99 & 34 \\
\hline 1031 & Ruidosa 7 NE & 7813 & Presidio & 3,625 & $30^{\circ} 02^{\prime}$ & $104^{\circ} 35^{\prime}$ & $07 / 1983$ & $06 / 1990$ & 87 & 8 \\
\hline 1032 & Runge & 7836 & Karnes & 315 & $28^{\circ} 53^{\prime}$ & $97^{\circ} 42^{\prime}$ & $01 / 1897$ & $12 / 2001$ & 96 & 104 \\
\hline
\end{tabular}


Table 1. Summary of National Weather Service daily precipitation stations in Texas with known names, latitudes, and longitudes-Continued

\begin{tabular}{|c|c|c|c|c|c|c|c|c|c|c|}
\hline $\begin{array}{l}\text { Seq. } \\
\text { no. }\end{array}$ & NWS station name & $\begin{array}{c}\text { NWS } \\
\text { station } \\
\text { no. }\end{array}$ & County & $\begin{array}{c}\text { Elev. } \\
\text { (ft above } \\
\text { NGVD } \\
29)\end{array}$ & $\begin{array}{l}\text { Lati- } \\
\text { tude }\end{array}$ & $\begin{array}{l}\text { Longi- } \\
\text { tude }\end{array}$ & $\begin{array}{c}\text { Begining } \\
\text { date } \\
\text { (mm/yyyy) }\end{array}$ & $\begin{array}{c}\text { Ending } \\
\text { date } \\
\text { (mm/yyyy) }\end{array}$ & $\begin{array}{l}\text { Per- } \\
\text { cent } \\
\text { cov- } \\
\text { erage }\end{array}$ & $\begin{array}{c}\text { Years } \\
\text { of } \\
\text { record }\end{array}$ \\
\hline 1033 & Rusk & 7841 & Cherokee & 720 & $31^{\circ} 48^{\prime}$ & $95^{\circ} 08^{\prime}$ & $01 / 1942$ & $12 / 2001$ & 98 & 60 \\
\hline 1034 & Rust Circle Bar Ranch & 7852 & Tom Green* & 2,201 & $31^{\circ} 12^{\prime}$ & $100^{\circ} 10^{\prime}$ & $04 / 1948$ & $06 / 1958$ & 59 & 10 \\
\hline 1035 & Sabinal & 7873 & Uvalde & 953 & $29^{\circ} 21^{\prime}$ & $99^{\circ} 29^{\prime}$ & $09 / 1903$ & $12 / 2001$ & 94 & 98 \\
\hline 1036 & Sabine & 7875 & Jefferson* & 20 & $29^{\circ} 43^{\prime}$ & $93^{\circ} 52^{\prime}$ & $12 / 1898$ & $12 / 1903$ & 29 & 5 \\
\hline 1037 & Saginaw & 7887 & Tarrant* & $* 736$ & $32^{\circ} 52^{\prime}$ & $97^{\circ} 22^{\prime}$ & $03 / 1900$ & $04 / 1901$ & 58 & 2 \\
\hline 1038 & Salado & 7903 & Bell* & 650 & $30^{\circ} 57^{\prime}$ & $97^{\circ} 32^{\prime}$ & $05 / 1910$ & $10 / 1919$ & 95 & 10 \\
\hline 1039 & Salt Flat & 7920 & Hudspeth & 3,722 & $31^{\circ} 44^{\prime}$ & $105^{\circ} 04^{\prime}$ & $10 / 1978$ & 08/1998 & 91 & 21 \\
\hline 1040 & Salt Flat 10 ENE & 7921 & Culberson & 3,891 & $31^{\circ} 47^{\prime}$ & $104^{\circ} 54^{\prime}$ & $01 / 1959$ & $12 / 1977$ & 99 & 19 \\
\hline 1041 & Salt Flat CAA Airport & 7922 & Hudspeth* & 3,717 & $31^{\circ} 45^{\prime}$ & $105^{\circ} 05^{\prime}$ & $06 / 1948$ & $12 / 1957$ & 96 & 10 \\
\hline 1042 & Sam Rayburn Dam & 7936 & Jasper & 189 & $31^{\circ} 03^{\prime}$ & $94^{\circ} 06^{\prime}$ & $01 / 1968$ & $12 / 2001$ & 100 & 34 \\
\hline 1043 & San Angelo $4 \mathrm{NE}$ & 7942 & Tom Green* & 1,903 & $31^{\circ} 31^{\prime}$ & $100^{\circ} 23^{\prime}$ & $08 / 1953$ & $03 / 1955$ & 43 & 3 \\
\hline 1044 & San Angelo Dam & 7940 & Tom Green & 1,962 & $31^{\circ} 28^{\prime}$ & $100^{\circ} 29^{\prime}$ & $07 / 1953$ & $11 / 1975$ & 97 & 23 \\
\hline 1045 & San Angelo WSO Airport & 7943 & Tom Green & 1,916 & $31^{\circ} 21^{\prime}$ & $100^{\circ} 29^{\prime}$ & $09 / 1946$ & $12 / 2001$ & 98 & 56 \\
\hline 1046 & San Antonio 8 NNE & 7947 & Bexar & 788 & $29^{\circ} 31^{\prime}$ & $98^{\circ} 27^{\prime}$ & $05 / 1997$ & $12 / 2001$ & 93 & 5 \\
\hline 1047 & San Antonio International Airport & 7945 & Bexar & 809 & $29^{\circ} 31^{\prime}$ & $98^{\circ} 27^{\prime}$ & $09 / 1946$ & $12 / 2001$ & 99 & 56 \\
\hline 1048 & San Antonio Nursery & 7948 & Bexar* & 591 & $29^{\circ} 18^{\prime}$ & $98^{\circ} 28^{\prime}$ & $01 / 1897$ & $09 / 1951$ & 87 & 38 \\
\hline 1049 & San Antonio Seaworld & 8169 & Bexar & 940 & $29^{\circ} 27^{\prime}$ & $98^{\circ} 42^{\prime}$ & $06 / 1988$ & $12 / 2001$ & 96 & 14 \\
\hline 1050 & San Augustine & 7951 & San Augustine & 310 & $31^{\circ} 30^{\prime}$ & $94^{\circ} 06^{\prime}$ & $06 / 1909$ & $12 / 2001$ & 92 & 36 \\
\hline 1051 & San Benito & 7952 & Cameron & 39 & $26^{\circ} 08^{\prime}$ & $97^{\circ} 38^{\prime}$ & $03 / 1920$ & $06 / 1975$ & 92 & 53 \\
\hline 1052 & San Benito Filter Plant & 7953 & Cameron* & 39 & $26^{\circ} 08^{\prime}$ & $97^{\circ} 38^{\prime}$ & $06 / 1948$ & $05 / 1950$ & 66 & 3 \\
\hline 1053 & San Bernard Wildlife Refuge & 7957 & Brazoria & 12 & $28^{\circ} 55^{\prime}$ & $95^{\circ} 35^{\prime}$ & $11 / 1984$ & $12 / 1987$ & 70 & 4 \\
\hline 1054 & San Diego & 7969 & Jim Wells* & 312 & $27^{\circ} 46^{\prime}$ & $98^{\circ} 14^{\prime}$ & $05 / 1911$ & 06/1911 & 17 & 1 \\
\hline 1055 & San Jacinto & 8045 & Walker & 415 & $30^{\circ} 37^{\prime}$ & $95^{\circ} 43^{\prime}$ & $02 / 1977$ & 09/1986 & 91 & 10 \\
\hline 1056 & San Juanito & 7979 & Hidalgo* & $* 142$ & $26^{\circ} 42^{\prime}$ & $98^{\circ} 14^{\prime}$ & $05 / 1909$ & $08 / 1915$ & 82 & 7 \\
\hline 1057 & San Manuel & 7981 & Hidalgo & 75 & $26^{\circ} 34^{\prime}$ & $98^{\circ} 07^{\prime}$ & $06 / 1948$ & 07/1951 & 35 & 4 \\
\hline 1058 & San Marcos & 7983 & Hays & 612 & $29^{\circ} 52^{\prime}$ & $97^{\circ} 55^{\prime}$ & $12 / 1896$ & $12 / 2001$ & 94 & 103 \\
\hline 1059 & San Perlita & 7990 & Willacy* & 20 & $26^{\circ} 30^{\prime}$ & $97^{\circ} 36^{\prime}$ & $06 / 1948$ & $05 / 1950$ & 65 & 3 \\
\hline 1060 & San Saba & 7992 & San Saba & 1,195 & $31^{\circ} 11^{\prime}$ & $98^{\circ} 43^{\prime}$ & $01 / 1901$ & $02 / 2000$ & 95 & 70 \\
\hline 1061 & San Saba 7 NW & 7994 & San Saba & 1,330 & $31^{\circ} 17^{\prime}$ & $98^{\circ} 45^{\prime}$ & $03 / 2000$ & $12 / 2001$ & 90 & 2 \\
\hline 1062 & Sanatorium & 7997 & Tom Green* & 2,031 & $31^{\circ} 37^{\prime}$ & $100^{\circ} 39^{\prime}$ & $09 / 1947$ & $06 / 1953$ & 81 & 7 \\
\hline 1063 & Sanderson & 8022 & Terrell & 2,855 & $30^{\circ} 08^{\prime}$ & $102^{\circ} 23^{\prime}$ & $01 / 1897$ & $12 / 2001$ & 95 & 69 \\
\hline 1064 & Sanderson 5 NNW & 8023 & Terrell & 3,080 & $30^{\circ} 12^{\prime}$ & $102^{\circ} 25^{\prime}$ & $10 / 1947$ & $09 / 1951$ & 68 & 5 \\
\hline 1065 & Sandy 2 S & 8036 & Blanco & 1,240 & $30^{\circ} 20^{\prime}$ & $98^{\circ} 28^{\prime}$ & 02/1949 & $12 / 1965$ & 98 & 17 \\
\hline 1066 & Sanger & 8043 & Denton & 675 & $33^{\circ} 21^{\prime}$ & $97^{\circ} 10^{\prime}$ & $11 / 1947$ & 07/1999 & 87 & 35 \\
\hline 1067 & Santa Anna & 8047 & Coleman & 1,745 & $31^{\circ} 44^{\prime}$ & $99^{\circ} 18^{\prime}$ & $03 / 1913$ & $09 / 1951$ & 50 & 5 \\
\hline 1068 & Santa Rosa & 8060 & Cameron* & 49 & $26^{\circ} 15^{\prime}$ & $97^{\circ} 50^{\prime}$ & $06 / 1948$ & $05 / 1950$ & 65 & 3 \\
\hline 1069 & Santa Rosa 3 WNW & 8059 & Hidalgo & 50 & $26^{\circ} 16^{\prime}$ & $97^{\circ} 52^{\prime}$ & $03 / 1987$ & $12 / 2001$ & 99 & 15 \\
\hline 1070 & Sarita $7 \mathrm{E}$ & 8081 & Kenedy & 38 & $27^{\circ} 13^{\prime}$ & $97^{\circ} 41^{\prime}$ & $01 / 1900$ & $12 / 2001$ & 95 & 102 \\
\hline 1071 & Schulenburg & 8126 & Fayette & 374 & $29^{\circ} 41^{\prime}$ & $96^{\circ} 54^{\prime}$ & $09 / 1947$ & $12 / 2001$ & 98 & 55 \\
\hline 1072 & Scotland & 8139 & Archer & 964 & $33^{\circ} 40^{\prime}$ & $98^{\circ} 28^{\prime}$ & 04/1997 & $12 / 2001$ & 91 & 5 \\
\hline 1073 & Seagraves & 8154 & Gaines & 3,438 & $32^{\circ} 57^{\prime}$ & $102^{\circ} 34^{\prime}$ & 03/1980 & 02/1981 & 29 & 2 \\
\hline 1074 & Sealy & 8160 & Austin & 194 & $29^{\circ} 47^{\prime}$ & $96^{\circ} 08^{\prime}$ & $10 / 1910$ & $12 / 2001$ & 95 & 87 \\
\hline 1075 & Segovia & 8183 & Kimble & 1,860 & $30^{\circ} 25^{\prime}$ & $99^{\circ} 40^{\prime}$ & $10 / 1958$ & $12 / 1965$ & 89 & 8 \\
\hline 1076 & Seguin & 8186 & Guadalupe & 512 & $29^{\circ} 35^{\prime}$ & $97^{\circ} 57^{\prime}$ & $12 / 1922$ & $04 / 1972$ & 97 & 51 \\
\hline 1077 & Seguin $1 \mathrm{SSW}$ & 8187 & Guadalupe & 503 & $29^{\circ} 33^{\prime}$ & $97^{\circ} 58^{\prime}$ & 07/1992 & $12 / 2001$ & 94 & 10 \\
\hline 1078 & Seminole & 8201 & Gaines & 3,340 & $32^{\circ} 42^{\prime}$ & $102^{\circ} 32^{\prime}$ & $08 / 1922$ & $12 / 2001$ & 94 & 80 \\
\hline 1079 & Seymour & 8221 & Baylor & 1,287 & $33^{\circ} 35^{\prime}$ & $99^{\circ} 16^{\prime}$ & $06 / 1905$ & $12 / 2001$ & 96 & 89 \\
\hline 1080 & Shaeffer Ranch & 8231 & Nueces* & 190 & $27^{\circ} 40^{\prime}$ & $97^{\circ} 45^{\prime}$ & 09/1901 & $02 / 1903$ & 44 & 3 \\
\hline 1081 & Shamrock & 8235 & Wheeler & 2,323 & $35^{\circ} 12^{\prime}$ & $100^{\circ} 15^{\prime}$ & $07 / 1929$ & 09/1987 & 99 & 59 \\
\hline 1082 & Shamrock 2 & 8236 & Wheeler & 2,360 & $35^{\circ} 12^{\prime}$ & $100^{\circ} 15^{\prime}$ & $09 / 1962$ & $12 / 2001$ & 94 & 40 \\
\hline 1083 & Sheffield & 8252 & Pecos & 2,170 & $30^{\circ} 41^{\prime}$ & $101^{\circ} 49^{\prime}$ & $10 / 1938$ & $12 / 2001$ & 92 & 46 \\
\hline 1084 & Sheperd 2 SW & 8265 & San Jacinto* & 180 & $30^{\circ} 29^{\prime}$ & $95^{\circ} 00^{\prime}$ & $06 / 1948$ & $09 / 1951$ & 83 & 4 \\
\hline
\end{tabular}


Table 1. Summary of National Weather Service daily precipitation stations in Texas with known names, latitudes, and longitudes-Continued

\begin{tabular}{|c|c|c|c|c|c|c|c|c|c|c|}
\hline $\begin{array}{c}\text { Seq. } \\
\text { no. }\end{array}$ & NWS station name & $\begin{array}{c}\text { NWS } \\
\text { station } \\
\text { no. }\end{array}$ & County & $\begin{array}{c}\text { Elev. } \\
\text { (ft above } \\
\text { NGVD } \\
\text { 29) }\end{array}$ & $\begin{array}{l}\text { Lati- } \\
\text { tude }\end{array}$ & $\begin{array}{l}\text { Longi- } \\
\text { tude }\end{array}$ & $\begin{array}{c}\text { Begining } \\
\text { date } \\
\text { (mm/yyyy) }\end{array}$ & $\begin{array}{c}\text { Ending } \\
\text { date } \\
\text { (mm/yyyy) }\end{array}$ & $\begin{array}{l}\text { Per- } \\
\text { cent } \\
\text { cov- } \\
\text { erage }\end{array}$ & $\begin{array}{l}\text { Years } \\
\text { of } \\
\text { record }\end{array}$ \\
\hline 1085 & Sherman & 8274 & Grayson & 760 & $33^{\circ} 42^{\prime}$ & $96^{\circ} 38^{\prime}$ & $05 / 1897$ & $12 / 2001$ & 98 & 102 \\
\hline 1086 & Sherwood & 8279 & Irion & 2,172 & $31^{\circ} 17^{\prime}$ & $100^{\circ} 48^{\prime}$ & $04 / 1948$ & $12 / 1965$ & 77 & 18 \\
\hline 1087 & Sierra Blanca 2 E & 8305 & Hudspeth & 4,590 & $31^{\circ} 10^{\prime}$ & $105^{\circ} 21^{\prime}$ & $01 / 1897$ & $12 / 2001$ & 89 & 43 \\
\hline 1088 & Silsbee $4 \mathrm{~N}$ & 8311 & Hardin & 180 & $30^{\circ} 14^{\prime}$ & $94^{\circ} 11^{\prime}$ & $11 / 1995$ & $12 / 2001$ & 88 & 7 \\
\hline 1089 & Silver & 8316 & Coke* & 1,903 & $32^{\circ} 02^{\prime}$ & $100^{\circ} 44^{\prime}$ & 03/1948 & $06 / 1952$ & 65 & 5 \\
\hline 1090 & Silver Valley & 8326 & Coleman & 1,960 & $31^{\circ} 58^{\prime}$ & $99^{\circ} 33^{\prime}$ & $11 / 1973$ & $12 / 2001$ & 96 & 29 \\
\hline 1091 & Silverton & 8323 & Briscoe & 3,280 & $34^{\circ} 27^{\prime}$ & $101^{\circ} 18^{\prime}$ & 09/1946 & $12 / 2001$ & 96 & 56 \\
\hline 1092 & Simms $3 \mathrm{~W}$ & 8333 & Bowie & 320 & $33^{\circ} 21^{\prime}$ & $94^{\circ} 33^{\prime}$ & 05/1981 & 08/1981 & 34 & 1 \\
\hline 1093 & Simms 4 WNW & 8335 & Bowie & 322 & $33^{\circ} 22^{\prime}$ & $94^{\circ} 34^{\prime}$ & $06 / 1948$ & $10 / 1973$ & 87 & 15 \\
\hline 1094 & Sinton & 8354 & San Patricio & 51 & $28^{\circ} 02^{\prime}$ & $97^{\circ} 29^{\prime}$ & $09 / 1921$ & $12 / 2001$ & 92 & 77 \\
\hline 1095 & Sisterdale & 8358 & Kendall & 1,400 & $29^{\circ} 58^{\prime}$ & $98^{\circ} 43^{\prime}$ & 08/1996 & $12 / 2001$ & 90 & 6 \\
\hline 1096 & Slaton $5 \mathrm{SE}$ & 8373 & Lynn & 3,050 & $33^{\circ} 22^{\prime}$ & $101^{\circ} 36^{\prime}$ & $05 / 1949$ & $12 / 2001$ & 99 & 53 \\
\hline 1097 & Slidell & 8378 & Wise & 985 & $33^{\circ} 21^{\prime}$ & $97^{\circ} 23^{\prime}$ & 09/1947 & $11 / 2000$ & 96 & 54 \\
\hline 1098 & Sloan & 8382 & San Saba & 1,302 & $31^{\circ} 09^{\prime}$ & $98^{\circ} 55^{\prime}$ & $04 / 1935$ & $10 / 1975$ & 99 & 41 \\
\hline 1099 & Smith Brothers Ranch & 8400 & Jeff Davis* & $* 5,274$ & $30^{\circ} 29^{\prime}$ & $104^{\circ} 06^{\prime}$ & $06 / 1948$ & $12 / 1948$ & 56 & 1 \\
\hline 1100 & Smithsons Valley & 8414 & Comal* & 1,302 & $29^{\circ} 49^{\prime}$ & $98^{\circ} 20^{\prime}$ & $12 / 1946$ & $06 / 1955$ & 77 & 10 \\
\hline 1101 & Smithville & 8415 & Bastrop & 340 & $30^{\circ} 00^{\prime}$ & $97^{\circ} 09^{\prime}$ & 05/1917 & $12 / 2001$ & 95 & 84 \\
\hline 1102 & Snyder & 8433 & Scurry & 2,335 & $32^{\circ} 42^{\prime}$ & $100^{\circ} 54^{\prime}$ & 05/1911 & $12 / 2001$ & 95 & 91 \\
\hline 1103 & Socorro & 8435 & El Paso* & 3,661 & $31^{\circ} 39^{\prime}$ & $106^{\circ} 17^{\prime}$ & $04 / 1918$ & $10 / 1950$ & 90 & 32 \\
\hline 1104 & Somerville & 8445 & Burleson* & 249 & $30^{\circ} 21^{\prime}$ & $96^{\circ} 31^{\prime}$ & $01 / 1908$ & $09 / 1951$ & 86 & 25 \\
\hline 1105 & Somerville Dam & 8446 & Burleson & 263 & $30^{\circ} 20^{\prime}$ & $96^{\circ} 32^{\prime}$ & $04 / 1963$ & $12 / 2001$ & 95 & 38 \\
\hline 1106 & Sonora & 8449 & Sutton & 2,138 & $30^{\circ} 34^{\prime}$ & $100^{\circ} 39^{\prime}$ & $12 / 1902$ & $12 / 2001$ & 90 & 68 \\
\hline 1107 & Sonora Experiment Station & 8450 & Edwards & 2,503 & $30^{\circ} 16^{\prime}$ & $100^{\circ} 34^{\prime}$ & 09/1959 & $02 / 1964$ & 73 & 6 \\
\hline 1108 & Sour Lake 5 SE & 8455 & Hardin & 33 & $30^{\circ} 06^{\prime}$ & $94^{\circ} 20^{\prime}$ & 08/1948 & $08 / 1948$ & 8 & 1 \\
\hline 1109 & South Camp 6666 & 8468 & King & 1,870 & $33^{\circ} 30^{\prime}$ & $100^{\circ} 26^{\prime}$ & 04/1971 & $12 / 2001$ & 80 & 12 \\
\hline 1110 & South Padre Island & 8487 & Cameron & 5 & $26^{\circ} 04^{\prime}$ & $97^{\circ} 09^{\prime}$ & 02/1992 & $12 / 2001$ & 96 & 10 \\
\hline 1111 & Southland & 8477 & Garza* & 3,022 & $33^{\circ} 22^{\prime}$ & $101^{\circ} 33^{\prime}$ & $08 / 1925$ & 09/1931 & 88 & 7 \\
\hline 1112 & Speaks 2 & 8519 & Lavaca & 143 & $29^{\circ} 16^{\prime}$ & $96^{\circ} 41^{\prime}$ & $01 / 1967$ & $12 / 2001$ & 94 & 35 \\
\hline 1113 & Spearman & 8523 & Hansford & 3,095 & $36^{\circ} 11^{\prime}$ & $101^{\circ} 11^{\prime}$ & 08/1920 & $12 / 2001$ & 98 & 82 \\
\hline 1114 & Spicewood & 8531 & Burnet & 850 & $30^{\circ} 29^{\prime}$ & $98^{\circ} 10^{\prime}$ & $02 / 1968$ & $12 / 2001$ & 98 & 34 \\
\hline 1115 & Spinks Ranch & 8536 & Menard & 2,231 & $30^{\circ} 46^{\prime}$ & $99^{\circ} 50^{\prime}$ & 03/1948 & 03/1964 & 94 & 17 \\
\hline 1116 & Splendora & 8538 & Montgomery* & 161 & $30^{\circ} 14^{\prime}$ & $95^{\circ} 10^{\prime}$ & 09/1947 & 08/1951 & 80 & 5 \\
\hline 1117 & Spring Branch 2 SE & 8544 & Comal & 1,119 & $29^{\circ} 51^{\prime}$ & $98^{\circ} 22^{\prime}$ & 03/1956 & $12 / 2001$ & 98 & 44 \\
\hline 1118 & Spring Branch 3 SSW & 8541 & Comal & 1,190 & $29^{\circ} 50^{\prime}$ & $98^{\circ} 26^{\prime}$ & 03/1995 & 05/1997 & 73 & 3 \\
\hline 1119 & Springtown & 8561 & Parker & 869 & $32^{\circ} 58^{\prime}$ & $97^{\circ} 40^{\prime}$ & $05 / 1957$ & 03/1978 & 94 & 22 \\
\hline 1120 & Spur & 8566 & Dickens & 2,297 & $33^{\circ} 28^{\prime}$ & $100^{\circ} 52^{\prime}$ & 05/1911 & $12 / 2001$ & 97 & 69 \\
\hline 1121 & Stamford 1 & 8583 & Jones & 1,640 & $32^{\circ} 56^{\prime}$ & $99^{\circ} 48^{\prime}$ & 02/1911 & $12 / 2001$ & 94 & 86 \\
\hline 1122 & Stamford 2 & 8584 & Jones & 1,601 & $32^{\circ} 57^{\prime}$ & $99^{\circ} 48^{\prime}$ & $06 / 1948$ & $09 / 1951$ & 70 & 4 \\
\hline 1123 & Stephenville $1 \mathrm{~N}$ & 8623 & Erath & 1,309 & $32^{\circ} 14^{\prime}$ & $98^{\circ} 11^{\prime}$ & 05/1918 & $12 / 2001$ & 93 & 67 \\
\hline 1124 & Stephenville 4 NNE & 8624 & Henderson* & 1,421 & $32^{\circ} 17^{\prime}$ & $96^{\circ} 12^{\prime}$ & 03/1969 & $11 / 1972$ & 94 & 4 \\
\hline 1125 & Stephenville 6 SW & 8625 & Erath & 1,450 & $32^{\circ} 10^{\prime}$ & $98^{\circ} 19^{\prime}$ & $06 / 1948$ & $11 / 1968$ & 86 & 9 \\
\hline 1126 & Sterley & 8628 & Floyd* & 3,251 & $34^{\circ} 12^{\prime}$ & $101^{\circ} 24^{\prime}$ & $03 / 1947$ & $02 / 1960$ & 75 & 14 \\
\hline 1127 & Sterling City & 8630 & Sterling & 2,265 & $31^{\circ} 50^{\prime}$ & $100^{\circ} 58^{\prime}$ & $04 / 1926$ & $12 / 2001$ & 96 & 76 \\
\hline 1128 & Sterling City $8 \mathrm{NE}$ & 8631 & Sterling & 2,710 & $31^{\circ} 55^{\prime}$ & $100^{\circ} 52^{\prime}$ & $06 / 1949$ & $12 / 2001$ & 94 & 28 \\
\hline 1129 & Sterling City $9 \mathrm{NW}$ & 8632 & Sterling* & 2,402 & $31^{\circ} 54^{\prime}$ & $101^{\circ} 08^{\prime}$ & 07/1949 & 08/1960 & 82 & 12 \\
\hline 1130 & Stiles & 8640 & Reagan* & 2,904 & $31^{\circ} 24^{\prime}$ & $101^{\circ} 28^{\prime}$ & $04 / 1916$ & 04/1918 & 69 & 3 \\
\hline 1131 & Stillhouse Hollow Dam & 8646 & Bell & 706 & $31^{\circ} 02^{\prime}$ & $97^{\circ} 31^{\prime}$ & $07 / 1963$ & $12 / 2001$ & 97 & 39 \\
\hline 1132 & Stinnett & 8647 & Hutchinson & 3,130 & $35^{\circ} 50^{\prime}$ & $101^{\circ} 27^{\prime}$ & $02 / 1929$ & $02 / 1944$ & 38 & 5 \\
\hline 1133 & Stockdale $4 \mathrm{~N}$ & 8658 & Wilson & 475 & $29^{\circ} 17^{\prime}$ & $97^{\circ} 58^{\prime}$ & $12 / 1940$ & $12 / 2001$ & 92 & 30 \\
\hline 1134 & Stony & 8677 & Denton* & 702 & $33^{\circ} 14^{\prime}$ & $97^{\circ} 20^{\prime}$ & 09/1948 & 09/1951 & 47 & 4 \\
\hline 1135 & Stowell & 8683 & Chambers* & $* 20$ & $29^{\circ} 47^{\prime}$ & $94^{\circ} 23^{\prime}$ & $06 / 1911$ & $07 / 1915$ & 54 & 4 \\
\hline 1136 & Stratford & 8692 & Sherman & 3,693 & $36^{\circ} 20^{\prime}$ & $102^{\circ} 04^{\prime}$ & $07 / 1911$ & $12 / 2001$ & 95 & 80 \\
\hline
\end{tabular}


Table 1. Summary of National Weather Service daily precipitation stations in Texas with known names, latitudes, and longitudes-Continued

\begin{tabular}{|c|c|c|c|c|c|c|c|c|c|c|}
\hline $\begin{array}{l}\text { Seq. } \\
\text { no. }\end{array}$ & NWS station name & $\begin{array}{c}\text { NWS } \\
\text { station } \\
\text { no. }\end{array}$ & County & $\begin{array}{c}\text { Elev. } \\
\text { (ft above } \\
\text { NGVD } \\
29 \text { ) }\end{array}$ & $\begin{array}{l}\text { Lati- } \\
\text { tude }\end{array}$ & $\begin{array}{l}\text { Longi- } \\
\text { tude }\end{array}$ & $\begin{array}{c}\text { Begining } \\
\text { date } \\
\text { (mm/yyyy) }\end{array}$ & $\begin{array}{c}\text { Ending } \\
\text { date } \\
\text { (mm/yyyy) }\end{array}$ & $\begin{array}{l}\text { Per- } \\
\text { cent } \\
\text { cov- } \\
\text { erage }\end{array}$ & $\begin{array}{c}\text { Years } \\
\text { of } \\
\text { record }\end{array}$ \\
\hline 1137 & Strawn 8 NNE & 8696 & Palo Pinto & 1,180 & $32^{\circ} 39^{\prime}$ & $98^{\circ} 28^{\prime}$ & $03 / 1949$ & $12 / 2001$ & 99 & 53 \\
\hline 1138 & Study Butte & 8713 & Brewster* & 2,602 & $29^{\circ} 19^{\prime}$ & $103^{\circ} 32^{\prime}$ & $03 / 1923$ & $12 / 1929$ & 97 & 7 \\
\hline 1139 & Study Butte & 8714 & Brewster & 2,550 & $29^{\circ} 19^{\prime}$ & $103^{\circ} 30^{\prime}$ & $05 / 1993$ & $12 / 2001$ & 95 & 9 \\
\hline 1140 & Substation 14 & 8721 & Edwards & 2,602 & $30^{\circ} 16^{\prime}$ & $100^{\circ} 35^{\prime}$ & $05 / 1922$ & $12 / 1952$ & 98 & 31 \\
\hline 1141 & Sugar Land & 8728 & Fort Bend & 82 & $29^{\circ} 37^{\prime}$ & $95^{\circ} 38^{\prime}$ & 09/1946 & $12 / 2001$ & 98 & 56 \\
\hline 1142 & Sulphur Springs & 8743 & Hopkins & 495 & $33^{\circ} 09^{\prime}$ & $95^{\circ} 38^{\prime}$ & $01 / 1897$ & $12 / 2001$ & 96 & 93 \\
\hline 1143 & Sunray 4 SW & 8761 & Moore & 3,543 & $35^{\circ} 58^{\prime}$ & $101^{\circ} 52^{\prime}$ & $11 / 1932$ & 08/1984 & 88 & 28 \\
\hline 1144 & Sutherland Springs & 8773 & Wilson* & $* 416$ & $29^{\circ} 17^{\prime}$ & $98^{\circ} 03^{\prime}$ & $06 / 1910$ & $07 / 1917$ & 82 & 8 \\
\hline 1145 & Sweetwater Avenger & 8789 & Nolan* & 2,392 & $32^{\circ} 28^{\prime}$ & $100^{\circ} 28^{\prime}$ & $11 / 1916$ & $10 / 1928$ & 65 & 6 \\
\hline 1146 & Tahoka & 8818 & Lynn & 3,120 & $33^{\circ} 10^{\prime}$ & $101^{\circ} 47^{\prime}$ & $03 / 1913$ & $12 / 2001$ & 95 & 77 \\
\hline 1147 & Talpa & 8824 & Coleman & 1,932 & $31^{\circ} 46^{\prime}$ & $99^{\circ} 42^{\prime}$ & $03 / 1948$ & $05 / 1965$ & 94 & 18 \\
\hline 1148 & Tampico & 8833 & Hall & 2,251 & $34^{\circ} 28^{\prime}$ & $100^{\circ} 49^{\prime}$ & $08 / 1947$ & $11 / 1984$ & 94 & 38 \\
\hline 1149 & Tarpley & 8845 & Bandera & 1,404 & $29^{\circ} 40^{\prime}$ & $99^{\circ} 17^{\prime}$ & $10 / 1937$ & $12 / 2001$ & 94 & 35 \\
\hline 1150 & Tascosa & 8852 & Oldham & 3,412 & $35^{\circ} 34^{\prime}$ & $102^{\circ} 18^{\prime}$ & $09 / 1947$ & $07 / 1984$ & 97 & 38 \\
\hline 1151 & Tatum & 8859 & Rusk & 269 & $32^{\circ} 18^{\prime}$ & $94^{\circ} 31^{\prime}$ & $06 / 1948$ & 09/1951 & 78 & 4 \\
\hline 1152 & Taylor & 8861 & Williamson & 565 & $30^{\circ} 34^{\prime}$ & $97^{\circ} 24^{\prime}$ & $01 / 1929$ & $08 / 2001$ & 99 & 73 \\
\hline 1153 & Taylor $1 \mathrm{NW}$ & 8862 & Williamson & 571 & $30^{\circ} 35^{\prime}$ & $97^{\circ} 24^{\prime}$ & $09 / 2000$ & $12 / 2001$ & 67 & 2 \\
\hline 1154 & Taylor Ranch & 8863 & San Saba & 2,003 & $30^{\circ} 57^{\prime}$ & $98^{\circ} 56^{\prime}$ & 03/1966 & $12 / 2001$ & 99 & 36 \\
\hline 1155 & Teague & 8874 & Freestone* & $* 512$ & $31^{\circ} 38^{\prime}$ & $96^{\circ} 17^{\prime}$ & $10 / 1913$ & $06 / 1917$ & 75 & 5 \\
\hline 1156 & Teague Ranch & 8877 & Gillespie & 1,630 & $30^{\circ} 25^{\prime}$ & $98^{\circ} 48^{\prime}$ & $03 / 1966$ & $12 / 2001$ & 99 & 36 \\
\hline 1157 & Telegraph & 8897 & Kimble & 1,721 & $30^{\circ} 19^{\prime}$ & $99^{\circ} 54^{\prime}$ & $03 / 1948$ & $12 / 2001$ & 93 & 53 \\
\hline 1158 & Telephone & 8898 & Fannin & 541 & $33^{\circ} 47^{\prime}$ & $96^{\circ} 01^{\prime}$ & $09 / 1947$ & $01 / 1958$ & 81 & 12 \\
\hline 1159 & Temple & 8910 & Bell & 635 & $31^{\circ} 05^{\prime}$ & $97^{\circ} 19^{\prime}$ & $01 / 1897$ & $12 / 2001$ & 97 & 104 \\
\hline 1160 & Temple $3 \mathrm{SE}$ & 8911 & Bell* & 650 & $31^{\circ} 03^{\prime}$ & $97^{\circ} 21^{\prime}$ & $06 / 1948$ & $10 / 1952$ & 77 & 5 \\
\hline 1161 & Tennyson & 8920 & Coke & 1,962 & $31^{\circ} 44^{\prime}$ & $100^{\circ} 18^{\prime}$ & $03 / 1948$ & $12 / 1965$ & 87 & 18 \\
\hline 1162 & Terlingua & 8924 & Brewster* & 2,592 & $29^{\circ} 18^{\prime}$ & $103^{\circ} 33^{\prime}$ & $06 / 1948$ & 09/1951 & 83 & 4 \\
\hline 1163 & Terlingua Ranch & 8926 & Brewster & 3,680 & $29^{\circ} 27^{\prime}$ & $103^{\circ} 23^{\prime}$ & $05 / 1993$ & $12 / 2001$ & 96 & 9 \\
\hline 1164 & Terrell & 8929 & Kaufman & 515 & $32^{\circ} 46^{\prime}$ & $96^{\circ} 17^{\prime}$ & $10 / 1947$ & $12 / 2001$ & 95 & 55 \\
\hline 1165 & Tesco & 8939 & Nolan & 2,001 & $32^{\circ} 30^{\prime}$ & $100^{\circ} 15^{\prime}$ & $09 / 1944$ & $12 / 1971$ & 97 & 28 \\
\hline 1166 & Texarkana & 8942 & Bowie & 390 & $33^{\circ} 25^{\prime}$ & $94^{\circ} 05^{\prime}$ & $10 / 1968$ & $12 / 2001$ & 97 & 34 \\
\hline 1167 & Texarkana Dam & 8944 & Cass & 282 & $33^{\circ} 18^{\prime}$ & $94^{\circ} 10^{\prime}$ & $12 / 1955$ & $07 / 1974$ & 92 & 20 \\
\hline 1168 & Texline & 8964 & Dallam* & $* 4,419$ & $36^{\circ} 25^{\prime}$ & $102^{\circ} 50^{\prime}$ & 08/1904 & 08/1912 & 76 & 9 \\
\hline 1169 & Theodore & 8979 & Winkler* & $* 2,856$ & $31^{\circ} 51^{\prime}$ & $103^{\circ} 10^{\prime}$ & $05 / 1910$ & 09/1913 & 85 & 4 \\
\hline 1170 & Thompsons 3 WSW & 8996 & Fort Bend & 72 & $29^{\circ} 29^{\prime}$ & $95^{\circ} 38^{\prime}$ & $02 / 1942$ & $12 / 2001$ & 92 & 49 \\
\hline 1171 & Thorndale & 9001 & Milam & 475 & $30^{\circ} 37^{\prime}$ & $97^{\circ} 12^{\prime}$ & $02 / 1968$ & $12 / 2001$ & 99 & 34 \\
\hline 1172 & Thornton 1 SSE & 9004 & Limestone & 475 & $31^{\circ} 23^{\prime}$ & $96^{\circ} 34^{\prime}$ & $09 / 1947$ & $12 / 2001$ & 96 & 55 \\
\hline 1173 & Three Rivers & 9009 & Live Oak & 151 & $28^{\circ} 28^{\prime}$ & $98^{\circ} 11^{\prime}$ & $05 / 1922$ & $12 / 1987$ & 99 & 66 \\
\hline 1174 & Three Rivers 8 NE & 9007 & Live Oak & 250 & $28^{\circ} 34^{\prime}$ & $98^{\circ} 08^{\prime}$ & $01 / 1988$ & $12 / 2001$ & 99 & 14 \\
\hline 1175 & Throckmorton & 9014 & Throckmorton & 1,370 & $33^{\circ} 10^{\prime}$ & $99^{\circ} 11^{\prime}$ & $01 / 1924$ & 09/1999 & 98 & 76 \\
\hline 1176 & Throckmorton $7 \mathrm{NE}$ & 9016 & Throckmorton & 1,230 & $33^{\circ} 17^{\prime}$ & $99^{\circ} 06^{\prime}$ & $03 / 2000$ & $12 / 2001$ & 89 & 2 \\
\hline 1177 & Throckmorton 8 NNW & 9013 & Throckmorton & $* 1,393$ & $33^{\circ} 18^{\prime}$ & $99^{\circ} 13^{\prime}$ & $01 / 1960$ & $02 / 1964$ & 78 & 5 \\
\hline 1178 & Thurber $5 \mathrm{NE}$ & 9015 & Palo Pinto & 964 & $32^{\circ} 32^{\prime}$ & $98^{\circ} 20^{\prime}$ & $07 / 1910$ & 02/1991 & 90 & 54 \\
\hline 1179 & Tilden 4 SSE & 9031 & McMullen & 345 & $28^{\circ} 24^{\prime}$ & $98^{\circ} 31^{\prime}$ & $11 / 1903$ & $12 / 2001$ & 89 & 54 \\
\hline 1180 & Tilden $9 \mathrm{~S}$ & 9030 & McMullen & 246 & $28^{\circ} 19^{\prime}$ & $98^{\circ} 34^{\prime}$ & 06/1997 & $12 / 2001$ & 92 & 5 \\
\hline 1181 & Tinnin Ranch & 9037 & Reeves* & 3,232 & $31^{\circ} 19^{\prime}$ & $103^{\circ} 59^{\prime}$ & $06 / 1948$ & 09/1951 & 82 & 4 \\
\hline 1182 & Toledo Bend Dam & 9068 & Newton & 190 & $31^{\circ} 10^{\prime}$ & $93^{\circ} 33^{\prime}$ & $07 / 1975$ & $12 / 2001$ & 98 & 27 \\
\hline 1183 & Tomball & 9076 & Harris & 210 & $30^{\circ} 06^{\prime}$ & $95^{\circ} 37^{\prime}$ & $09 / 1946$ & $12 / 2001$ & 96 & 56 \\
\hline 1184 & Tornillo 2 SSE & 9088 & El Paso & 3,525 & $31^{\circ} 24^{\prime}$ & $106^{\circ} 03^{\prime}$ & $09 / 1946$ & $12 / 2001$ & 92 & 24 \\
\hline 1185 & Tow $3 \mathrm{SE}$ & 9099 & Llano & 1,050 & $30^{\circ} 51^{\prime}$ & $98^{\circ} 26^{\prime}$ & $04 / 1978$ & $12 / 2001$ & 98 & 24 \\
\hline 1186 & Town Bluff Dam & 8568 & Tyler & 210 & $30^{\circ} 48^{\prime}$ & $94^{\circ} 11^{\prime}$ & $08 / 1953$ & $01 / 1970$ & 92 & 18 \\
\hline 1187 & Town Bluff Dam & 9101 & Tyler & 214 & $30^{\circ} 48^{\prime}$ & $94^{\circ} 11^{\prime}$ & $02 / 1970$ & $12 / 2001$ & 98 & 32 \\
\hline 1188 & Toyah & 9106 & Reeves & 2,812 & $31^{\circ} 18^{\prime}$ & $103^{\circ} 48^{\prime}$ & $09 / 1947$ & $02 / 1977$ & 91 & 30 \\
\hline
\end{tabular}


Table 1. Summary of National Weather Service daily precipitation stations in Texas with known names, latitudes, and longitudes-Continued

\begin{tabular}{|c|c|c|c|c|c|c|c|c|c|c|}
\hline $\begin{array}{c}\text { Seq. } \\
\text { no. }\end{array}$ & NWS station name & $\begin{array}{c}\text { NWS } \\
\text { station } \\
\text { no. }\end{array}$ & County & $\begin{array}{c}\text { Elev. } \\
\text { (ft above } \\
\text { NGVD } \\
\text { 29) }\end{array}$ & $\begin{array}{l}\text { Lati- } \\
\text { tude }\end{array}$ & $\begin{array}{l}\text { Longi- } \\
\text { tude }\end{array}$ & $\begin{array}{c}\text { Begining } \\
\text { date } \\
\text { (mm/yyyy) }\end{array}$ & $\begin{array}{c}\text { Ending } \\
\text { date } \\
\text { (mm/yyyy) }\end{array}$ & $\begin{array}{l}\text { Per- } \\
\text { cent } \\
\text { cov- } \\
\text { erage }\end{array}$ & $\begin{array}{l}\text { Years } \\
\text { of } \\
\text { record }\end{array}$ \\
\hline 1189 & Trent 2 SSW & 9122 & Taylor & 1,925 & $32^{\circ} 27^{\prime}$ & $100^{\circ} 07^{\prime}$ & $01 / 1948$ & $12 / 2001$ & 97 & 54 \\
\hline 1190 & Trenton & 9125 & Fannin & 760 & $33^{\circ} 23^{\prime}$ & $96^{\circ} 20^{\prime}$ & $10 / 1947$ & $12 / 2001$ & 95 & 55 \\
\hline 1191 & Tri City Airport & 9129 & Hidalgo* & 200 & $26^{\circ} 24^{\prime}$ & $98^{\circ} 21^{\prime}$ & 06/1948 & $05 / 1950$ & 66 & 3 \\
\hline 1192 & Trickham & 9132 & Coleman & 1,401 & $31^{\circ} 35^{\prime}$ & $99^{\circ} 13^{\prime}$ & $03 / 1948$ & $12 / 1965$ & 99 & 18 \\
\hline 1193 & Trinidad 1 SW & 9136 & Henderson & 239 & $32^{\circ} 08^{\prime}$ & $96^{\circ} 06^{\prime}$ & $01 / 1915$ & 04/1966 & 91 & 23 \\
\hline 1194 & Trinidad Power Plant & 9137 & Henderson & 292 & $32^{\circ} 08^{\prime}$ & $96^{\circ} 06^{\prime}$ & 05/1966 & 03/1990 & 91 & 25 \\
\hline 1195 & Trinity & 9139 & Trinity* & 230 & $30^{\circ} 57^{\prime}$ & $95^{\circ} 23^{\prime}$ & $05 / 1900$ & 05/1907 & 76 & 8 \\
\hline 1196 & Troup & 9147 & Smith* & 469 & $32^{\circ} 09^{\prime}$ & $95^{\circ} 07^{\prime}$ & $07 / 1913$ & 01/1931 & 93 & 19 \\
\hline 1197 & Troy & 9153 & Bell & 700 & $31^{\circ} 12^{\prime}$ & $97^{\circ} 17^{\prime}$ & 09/1947 & $12 / 2001$ & 99 & 55 \\
\hline 1198 & Truby 3 ESE & 9154 & Jones & 1,713 & $32^{\circ} 38^{\prime}$ & $99^{\circ} 53^{\prime}$ & $09 / 1947$ & 08/1972 & 95 & 26 \\
\hline 1199 & Trunk & 9162 & Martin* & 2,533 & $32^{\circ} 13^{\prime}$ & $101^{\circ} 43^{\prime}$ & $01 / 1948$ & $01 / 1949$ & 49 & 2 \\
\hline 1200 & Truscott $3 \mathrm{~W}$ & 9163 & Knox & 1,571 & $33^{\circ} 45^{\prime}$ & $99^{\circ} 51^{\prime}$ & $09 / 1948$ & $12 / 2001$ & 92 & 43 \\
\hline 1201 & Tuco Station & 9169 & Hale* & 3,353 & $33^{\circ} 53^{\prime}$ & $101^{\circ} 51^{\prime}$ & $12 / 1946$ & $10 / 1952$ & 75 & 7 \\
\hline 1202 & Tulia & 9175 & Swisher & 3,470 & $34^{\circ} 31^{\prime}$ & $101^{\circ} 45^{\prime}$ & 05/1947 & $12 / 2001$ & 97 & 55 \\
\hline 1203 & Tulia 6 NE & 9176 & Swisher* & 3,504 & $34^{\circ} 36^{\prime}$ & $101^{\circ} 42^{\prime}$ & $01 / 1897$ & $11 / 1952$ & 84 & 51 \\
\hline 1204 & Turkey & 9191 & Hall & 2,330 & $34^{\circ} 23^{\prime}$ & $100^{\circ} 53^{\prime}$ & $10 / 1947$ & $12 / 2001$ & 90 & 47 \\
\hline 1205 & Tyler & 9207 & Smith & 550 & $32^{\circ} 18^{\prime}$ & $95^{\circ} 17^{\prime}$ & 03/1984 & $12 / 2001$ & 99 & 18 \\
\hline 1206 & Tyler 5 NE & 9214 & Smith & 489 & $32^{\circ} 24^{\prime}$ & $95^{\circ} 16^{\prime}$ & $01 / 1955$ & $01 / 1984$ & 97 & 30 \\
\hline 1207 & Tyler Pounds Field & 9213 & Smith & 544 & $32^{\circ} 21^{\prime}$ & $95^{\circ} 24^{\prime}$ & $07 / 1898$ & $11 / 1954$ & 84 & 20 \\
\hline 1208 & Umbarger & 9224 & Randall & 3,746 & $34^{\circ} 57^{\prime}$ & $102^{\circ} 06^{\prime}$ & $10 / 1947$ & $12 / 2001$ & 97 & 55 \\
\hline 1209 & Upland & 9248 & Upton* & 2,602 & $31^{\circ} 23^{\prime}$ & $102^{\circ} 00^{\prime}$ & 08/1948 & 08/1948 & 7 & 1 \\
\hline 1210 & Utopia & 9260 & Uvalde & 1,338 & $29^{\circ} 35^{\prime}$ & $99^{\circ} 31^{\prime}$ & 02/1991 & $12 / 2001$ & 92 & 11 \\
\hline 1211 & Uvalde & 9265 & Uvalde & 912 & $29^{\circ} 13^{\prime}$ & $99^{\circ} 46^{\prime}$ & 03/1905 & $05 / 1985$ & 95 & 81 \\
\hline 1212 & Uvalde & 9267 & Uvalde* & 932 & $29^{\circ} 14^{\prime}$ & $99^{\circ} 48^{\prime}$ & $03 / 1920$ & $12 / 1946$ & 98 & 27 \\
\hline 1213 & Uvalde $3 \mathrm{SW}$ & 9268 & Uvalde & 920 & $29^{\circ} 11^{\prime}$ & $99^{\circ} 49^{\prime}$ & $08 / 1985$ & $12 / 2001$ & 88 & 17 \\
\hline 1214 & Uvalde $9 \mathrm{SW}$ & 9264 & Uvalde & 828 & $29^{\circ} 08^{\prime}$ & $99^{\circ} 54^{\prime}$ & 08/1996 & 08/1998 & 66 & 3 \\
\hline 1215 & Valentine & 9270 & Jeff Davis & 4,430 & $30^{\circ} 35^{\prime}$ & $104^{\circ} 30^{\prime}$ & 06/1978 & $12 / 2001$ & 97 & 24 \\
\hline 1216 & Valentine $10 \mathrm{WSW}$ & 9275 & Presidio & 4,420 & $30^{\circ} 33^{\prime}$ & $104^{\circ} 38^{\prime}$ & 03/1897 & $12 / 2001$ & 96 & 72 \\
\hline 1217 & Valley Junction & 9280 & Robertson & 269 & $30^{\circ} 50^{\prime}$ & $96^{\circ} 38^{\prime}$ & 04/1902 & $12 / 1977$ & 92 & 39 \\
\hline 1218 & Valley Mills & 9282 & Bosque & 630 & $31^{\circ} 39^{\prime}$ & $97^{\circ} 28^{\prime}$ & 01/1992 & $12 / 2001$ & 89 & 10 \\
\hline 1219 & Valley View & 9286 & Cooke & 725 & $33^{\circ} 29^{\prime}$ & $97^{\circ} 09^{\prime}$ & 09/1947 & $12 / 2001$ & 98 & 55 \\
\hline 1220 & Van Horn & 9295 & Culberson & 4,065 & $31^{\circ} 02^{\prime}$ & $104^{\circ} 50^{\prime}$ & $01 / 1939$ & $12 / 2001$ & 91 & 63 \\
\hline 1221 & Vancourt & 9304 & Tom Green & 1,903 & $31^{\circ} 21^{\prime}$ & $100^{\circ} 11^{\prime}$ & $04 / 1948$ & $12 / 1965$ & 89 & 18 \\
\hline 1222 & Vancourt $5 \mathrm{SW}$ & 9307 & Tom Green* & 1,903 & $31^{\circ} 21^{\prime}$ & $100^{\circ} 14^{\prime}$ & 06/1948 & $01 / 1949$ & 31 & 2 \\
\hline 1223 & Vanderpool & 9311 & Bandera & 1,601 & $29^{\circ} 45^{\prime}$ & $99^{\circ} 34^{\prime}$ & 08/1978 & 09/1993 & 94 & 16 \\
\hline 1224 & Vanderpool $10 \mathrm{~N}$ & 9312 & Bandera & 2,265 & $29^{\circ} 48^{\prime}$ & $99^{\circ} 34^{\prime}$ & 08/1996 & $12 / 2001$ & 90 & 6 \\
\hline 1225 & Vanderpool $4 \mathrm{~N}$ & 9313 & Bandera & 1,825 & $29^{\circ} 48^{\prime}$ & $99^{\circ} 34^{\prime}$ & 10/1994 & $12 / 2001$ & 91 & 8 \\
\hline 1226 & Vega & 9330 & Oldham & 4,012 & $35^{\circ} 15^{\prime}$ & $102^{\circ} 25^{\prime}$ & $03 / 1923$ & 04/1983 & 98 & 61 \\
\hline 1227 & Venus & 9337 & Johnson & 659 & $32^{\circ} 26^{\prime}$ & $97^{\circ} 06^{\prime}$ & 09/1947 & $10 / 1964$ & 95 & 18 \\
\hline 1228 & Vernon & 9346 & Wilbarger & 1,227 & $34^{\circ} 09^{\prime}$ & $99^{\circ} 19^{\prime}$ & 03/1904 & $12 / 2001$ & 95 & 72 \\
\hline 1229 & Victoria ASOS & 9364 & Victoria & 115 & $28^{\circ} 51^{\prime}$ & $96^{\circ} 55^{\prime}$ & $07 / 1961$ & $12 / 2001$ & 99 & 41 \\
\hline 1230 & Victoria CP\&D & 9365 & Victoria & 61 & $28^{\circ} 47^{\prime}$ & $97^{\circ} 00^{\prime}$ & $01 / 1922$ & $12 / 2001$ & 94 & 42 \\
\hline 1231 & Victoria Fire Station No. 5 & 9361 & Victoria & 101 & $28^{\circ} 51^{\prime}$ & $97^{\circ} 01^{\prime}$ & $11 / 1999$ & $12 / 2001$ & 71 & 3 \\
\hline 1232 & Victoria Regional Airport & 9367 & Victoria & 104 & $28^{\circ} 50^{\prime}$ & $96^{\circ} 55^{\prime}$ & 04/1999 & $12 / 2001$ & 89 & 3 \\
\hline 1233 & Victoria WB Airport & 9363 & Victoria* & 115 & $28^{\circ} 47^{\prime}$ & $97^{\circ} 05^{\prime}$ & $12 / 1946$ & 06/1961 & 91 & 16 \\
\hline 1234 & Voss $1 \mathrm{WSW}$ & 9410 & Coleman & 1,650 & $31^{\circ} 37^{\prime}$ & $99^{\circ} 35^{\prime}$ & $01 / 1949$ & 03/1981 & 93 & 33 \\
\hline 1235 & Waco & 9421 & McLennan* & 381 & $31^{\circ} 32^{\prime}$ & $97^{\circ} 04^{\prime}$ & 09/1946 & $12 / 1957$ & 94 & 12 \\
\hline 1236 & Waco & 9415 & McLennan & 627 & $31^{\circ} 33^{\prime}$ & $97^{\circ} 12^{\prime}$ & $11 / 1995$ & $12 / 2001$ & 87 & 7 \\
\hline 1237 & Waco Dam & 9417 & McLennan & 495 & $31^{\circ} 36^{\prime}$ & $97^{\circ} 13^{\prime}$ & $03 / 1965$ & $12 / 2001$ & 96 & 37 \\
\hline 1238 & Waco Regional Airport & 9419 & McLennan & 500 & $31^{\circ} 36^{\prime}$ & $97^{\circ} 13^{\prime}$ & 01/1930 & $12 / 2001$ & 100 & 70 \\
\hline 1239 & Waelder $7 \mathrm{~S}$ & 9424 & Gonzales & 342 & $29^{\circ} 36^{\prime}$ & $97^{\circ} 19^{\prime}$ & $09 / 1947$ & $02 / 1993$ & 90 & 46 \\
\hline 1240 & Walcott & 9435 & Deaf Smith & 4,114 & $34^{\circ} 56^{\prime}$ & $102^{\circ} 59^{\prime}$ & 06/1971 & 08/1976 & 86 & 6 \\
\hline
\end{tabular}


Table 1. Summary of National Weather Service daily precipitation stations in Texas with known names, latitudes, and longitudes-Continued

\begin{tabular}{|c|c|c|c|c|c|c|c|c|c|c|}
\hline $\begin{array}{c}\text { Seq. } \\
\text { no. }\end{array}$ & NWS station name & $\begin{array}{c}\text { NWS } \\
\text { station } \\
\text { no. }\end{array}$ & County & $\begin{array}{c}\text { Elev. } \\
\text { (ft above } \\
\text { NGVD } \\
29 \text { ) }\end{array}$ & $\begin{array}{l}\text { Lati- } \\
\text { tude }\end{array}$ & $\begin{array}{l}\text { Longi- } \\
\text { tude }\end{array}$ & $\begin{array}{c}\text { Begining } \\
\text { date } \\
\text { (mm/yyyy) }\end{array}$ & $\begin{array}{c}\text { Ending } \\
\text { date } \\
\text { (mm/yyyy) }\end{array}$ & $\begin{array}{l}\text { Per- } \\
\text { cent } \\
\text { cov- } \\
\text { erage }\end{array}$ & $\begin{array}{c}\text { Years } \\
\text { of } \\
\text { record }\end{array}$ \\
\hline 1241 & Waller & 9448 & Waller & 143 & $30^{\circ} 03^{\prime}$ & $95^{\circ} 56^{\prime}$ & $09 / 1947$ & $08 / 1998$ & 95 & 52 \\
\hline 1242 & Walnut Springs 4 ESE & 9456 & Bosque & 860 & $32^{\circ} 02^{\prime}$ & $97^{\circ} 42^{\prime}$ & $05 / 1963$ & $05 / 1965$ & 68 & 3 \\
\hline 1243 & Warren $2 \mathrm{~S}$ & 9480 & Tyler & 110 & $30^{\circ} 35^{\prime}$ & $94^{\circ} 24^{\prime}$ & $05 / 1935$ & 06/1992 & 94 & 51 \\
\hline 1244 & Washington State Park & 9491 & Washington & 215 & $30^{\circ} 20^{\prime}$ & $96^{\circ} 09^{\prime}$ & $04 / 1915$ & $12 / 2001$ & 92 & 57 \\
\hline 1245 & Water Valley & 9499 & Tom Green & 2,120 & $31^{\circ} 40^{\prime}$ & $100^{\circ} 43^{\prime}$ & $07 / 1898$ & $12 / 2001$ & 94 & 55 \\
\hline 1246 & Water Valley 11 ENE & 9500 & Coke* & 2,142 & $31^{\circ} 42^{\prime}$ & $100^{\circ} 32^{\prime}$ & $04 / 1949$ & $07 / 1958$ & 63 & 10 \\
\hline 1247 & Water Valley $11 \mathrm{NNE}$ & 9501 & Coke & 2,455 & $31^{\circ} 48^{\prime}$ & $100^{\circ} 37^{\prime}$ & $02 / 1959$ & $12 / 2001$ & 97 & 43 \\
\hline 1248 & Waters Bluff & 9503 & Smith & 504 & $32^{\circ} 31^{\prime}$ & $95^{\circ} 07^{\prime}$ & $11 / 2001$ & $12 / 2001$ & 17 & 1 \\
\hline 1249 & Watson & 9504 & Burnet & 1,005 & $30^{\circ} 55^{\prime}$ & $98^{\circ} 01^{\prime}$ & $02 / 1968$ & $12 / 2001$ & 99 & 34 \\
\hline 1250 & Waxahachie & 9522 & Ellis & 630 & $32^{\circ} 25^{\prime}$ & $96^{\circ} 51^{\prime}$ & $05 / 1897$ & $12 / 2001$ & 97 & 104 \\
\hline 1251 & Wayside & 9527 & Armstrong & 3,400 & $34^{\circ} 47^{\prime}$ & $101^{\circ} 32^{\prime}$ & $06 / 1948$ & 09/1951 & 76 & 4 \\
\hline 1252 & Weatherford & 9532 & Parker & 955 & $32^{\circ} 44^{\prime}$ & $97^{\circ} 46^{\prime}$ & $01 / 1902$ & $12 / 2001$ & 97 & 100 \\
\hline 1253 & Webb & 9538 & Tarrant* & 650 & $32^{\circ} 42^{\prime}$ & $97^{\circ} 04^{\prime}$ & $09 / 1947$ & $12 / 1959$ & 94 & 13 \\
\hline 1254 & Weimar & 9549 & Colorado* & $* 416$ & $29^{\circ} 42^{\prime}$ & $96^{\circ} 47^{\prime}$ & $02 / 1902$ & $09 / 1903$ & 62 & 2 \\
\hline 1255 & Welder Wildlife Foundation & 9559 & San Patricio & 50 & $28^{\circ} 06^{\prime}$ & $97^{\circ} 25^{\prime}$ & $10 / 1964$ & $12 / 2001$ & 97 & 38 \\
\hline 1256 & Wellington & 9565 & Collingsworth & 2,040 & $34^{\circ} 50^{\prime}$ & $100^{\circ} 12^{\prime}$ & $04 / 1912$ & $12 / 2001$ & 90 & 62 \\
\hline 1257 & Weslaco $2 \mathrm{E}$ & 9588 & Hidalgo & 75 & $26^{\circ} 09^{\prime}$ & $97^{\circ} 58^{\prime}$ & $09 / 1947$ & $12 / 2001$ & 97 & 55 \\
\hline 1258 & West & 9593 & McLennan & 642 & $31^{\circ} 48^{\prime}$ & $97^{\circ} 06^{\prime}$ & $05 / 1952$ & $12 / 2001$ & 90 & 11 \\
\hline 1259 & Westway & 9646 & Deaf Smith* & $* 3,933$ & $34^{\circ} 49^{\prime}$ & $102^{\circ} 34^{\prime}$ & $09 / 1947$ & $08 / 1948$ & 31 & 2 \\
\hline 1260 & Wharton & 9655 & Wharton & 111 & $29^{\circ} 18^{\prime}$ & $96^{\circ} 05^{\prime}$ & $05 / 1902$ & $12 / 2001$ & 90 & 66 \\
\hline 1261 & Wheeler & 9662 & Wheeler & 2,495 & $35^{\circ} 26^{\prime}$ & $100^{\circ} 16^{\prime}$ & $04 / 1979$ & $12 / 2001$ & 97 & 23 \\
\hline 1262 & Wheelock & 9665 & Robertson & 420 & $30^{\circ} 54^{\prime}$ & $96^{\circ} 23^{\prime}$ & $06 / 1948$ & 09/1951 & 79 & 4 \\
\hline 1263 & White Oak 1 WSW & 9709 & Gregg & 315 & $32^{\circ} 30^{\prime}$ & $94^{\circ} 53^{\prime}$ & $08 / 2000$ & $12 / 2001$ & 71 & 2 \\
\hline 1264 & White River Reservoir & 9711 & Crosby & 2,412 & $33^{\circ} 27^{\prime}$ & $101^{\circ} 05^{\prime}$ & $10 / 1997$ & $12 / 2001$ & 85 & 5 \\
\hline 1265 & Whitehouse $2 \mathrm{SE}$ & 9708 & Smith & 515 & $32^{\circ} 12^{\prime}$ & $95^{\circ} 12^{\prime}$ & $01 / 1992$ & $12 / 2001$ & 94 & 10 \\
\hline 1266 & Whitney Dam & 9715 & Bosque & 574 & $31^{\circ} 51^{\prime}$ & $97^{\circ} 22^{\prime}$ & $10 / 1949$ & $12 / 2001$ & 98 & 53 \\
\hline 1267 & Whitsett & 9717 & Live Oak & 258 & $28^{\circ} 39^{\prime}$ & $98^{\circ} 15^{\prime}$ & $10 / 1964$ & $12 / 2001$ & 97 & 38 \\
\hline 1268 & Whitsett 3 SW & 9716 & Live Oak & 210 & $28^{\circ} 38^{\prime}$ & $98^{\circ} 16^{\prime}$ & $05 / 1914$ & 09/1964 & 96 & 49 \\
\hline 1269 & Wichita Falls 10th Street & 9727 & Wichita & 924 & $33^{\circ} 55^{\prime}$ & $98^{\circ} 32^{\prime}$ & $07 / 1940$ & $07 / 1940$ & 1 & 1 \\
\hline 1270 & Wichita Falls 5 SW Airport & 9723 & Wichita & 988 & $33^{\circ} 54^{\prime}$ & $98^{\circ} 33^{\prime}$ & $01 / 1996$ & $12 / 2001$ & 100 & 6 \\
\hline 1271 & Wichita Falls WSO Airport & 9729 & Wichita & 1,030 & $33^{\circ} 58^{\prime}$ & $98^{\circ} 29^{\prime}$ & $01 / 1897$ & $12 / 2001$ & 95 & 101 \\
\hline 1272 & Wichita Valley 55 & 9732 & Wichita* & 991 & $33^{\circ} 56^{\prime}$ & $98^{\circ} 37^{\prime}$ & $05 / 1939$ & $03 / 1954$ & 68 & 16 \\
\hline 1273 & Wichita Valley Farm 29 & 9730 & Wichita & 961 & $33^{\circ} 56^{\prime}$ & $98^{\circ} 35^{\prime}$ & $04 / 1939$ & $08 / 1972$ & 90 & 29 \\
\hline 1274 & Wiergate & 9734 & Newton* & 180 & $31^{\circ} 01^{\prime}$ & $93^{\circ} 43^{\prime}$ & $08 / 1925$ & $08 / 1943$ & 89 & 19 \\
\hline 1275 & Wildwood & 9754 & Tyler & 200 & $30^{\circ} 31^{\prime}$ & $94^{\circ} 26^{\prime}$ & $02 / 1992$ & $12 / 2001$ & 99 & 10 \\
\hline 1276 & William Harris Reservoir & 9772 & Brazoria & 39 & $29^{\circ} 15^{\prime}$ & $95^{\circ} 33^{\prime}$ & $01 / 1949$ & $04 / 1964$ & 94 & 16 \\
\hline 1277 & Willis & 9780 & Montgomery* & $* 348$ & $30^{\circ} 26^{\prime}$ & $95^{\circ} 29^{\prime}$ & $01 / 1907$ & $01 / 1928$ & 64 & 11 \\
\hline 1278 & Wills Point & 9800 & Van Zandt & 522 & $32^{\circ} 42^{\prime}$ & $96^{\circ} 01^{\prime}$ & $04 / 1905$ & $12 / 2001$ & 97 & 82 \\
\hline 1279 & Wilson & 9809 & Lynn* & 3,202 & $33^{\circ} 20^{\prime}$ & $101^{\circ} 42^{\prime}$ & $07 / 1942$ & $08 / 1946$ & 82 & 5 \\
\hline 1280 & Wilson Ranch & 9813 & Bandera* & 2,280 & $29^{\circ} 54^{\prime}$ & $99^{\circ} 36^{\prime}$ & $09 / 1947$ & $06 / 1962$ & 92 & 16 \\
\hline 1281 & Wimberley $1 \mathrm{NW}$ & 9815 & Hays & 830 & $30^{\circ} 00^{\prime}$ & $98^{\circ} 03^{\prime}$ & $11 / 1984$ & $12 / 2001$ & 94 & 18 \\
\hline 1282 & Wimberley 2 & 9814 & Hays & 1,112 & $29^{\circ} 58^{\prime}$ & $98^{\circ} 04^{\prime}$ & 03/1984 & $10 / 1984$ & 67 & 1 \\
\hline 1283 & Winchell 1 WNW & 9816 & Brown & 1,381 & $31^{\circ} 29^{\prime}$ & $99^{\circ} 11^{\prime}$ & $03 / 1948$ & $01 / 1965$ & 80 & 18 \\
\hline 1284 & Winfield & 9826 & Franklin* & 479 & $33^{\circ} 09^{\prime}$ & $95^{\circ} 08^{\prime}$ & $01 / 1910$ & $10 / 1936$ & 99 & 27 \\
\hline 1285 & Wink FAA Airport & 9830 & Winkler & 2,807 & $31^{\circ} 46^{\prime}$ & $103^{\circ} 12^{\prime}$ & $06 / 1938$ & $12 / 2001$ & 96 & 60 \\
\hline 1286 & Winnsboro $6 \mathrm{SW}$ & 9836 & Wood & 429 & $32^{\circ} 53^{\prime}$ & $95^{\circ} 20^{\prime}$ & $10 / 1947$ & $12 / 2001$ & 91 & 55 \\
\hline 1287 & Winter Haven Experiment & 9842 & Dimmit & 600 & $28^{\circ} 38^{\prime}$ & $99^{\circ} 52^{\prime}$ & $09 / 1947$ & $02 / 1964$ & 91 & 18 \\
\hline 1288 & Winters $1 \mathrm{NNE}$ & 9847 & Runnels & 1,862 & $31^{\circ} 58^{\prime}$ & $99^{\circ} 57^{\prime}$ & $08 / 1968$ & $12 / 2001$ & 97 & 34 \\
\hline 1289 & Winters 9 NNE & 9845 & Taylor & 1,972 & $32^{\circ} 06^{\prime}$ & $99^{\circ} 54^{\prime}$ & 07/1911 & 06/1968 & 86 & 44 \\
\hline 1290 & Witt Ranch & 9853 & Nolan & 2,090 & $32^{\circ} 29^{\prime}$ & $100^{\circ} 18^{\prime}$ & $01 / 1972$ & $11 / 1975$ & 98 & 4 \\
\hline 1291 & Wolf Creek Dam & 9858 & Ochiltree & 2,703 & $36^{\circ} 14^{\prime}$ & $100^{\circ} 40^{\prime}$ & $06 / 1948$ & 09/1951 & 83 & 4 \\
\hline 1292 & Wolfe City & 9859 & Hunt & 660 & $33^{\circ} 21^{\prime}$ & $96^{\circ} 04^{\prime}$ & $09 / 1947$ & $12 / 2001$ & 98 & 55 \\
\hline
\end{tabular}


Table 1. Summary of National Weather Service daily precipitation stations in Texas with known names, latitudes, and longitudes-Continued

\begin{tabular}{|c|c|c|c|c|c|c|c|c|c|c|}
\hline $\begin{array}{l}\text { Seq. } \\
\text { no. }\end{array}$ & NWS station name & $\begin{array}{c}\text { NWS } \\
\text { station } \\
\text { no. }\end{array}$ & County & $\begin{array}{c}\text { Elev. } \\
\text { (ft above } \\
\text { NGVD } \\
29 \text { ) }\end{array}$ & $\begin{array}{l}\text { Lati- } \\
\text { tude }\end{array}$ & $\begin{array}{l}\text { Longi- } \\
\text { tude }\end{array}$ & $\begin{array}{l}\text { Begining } \\
\text { date } \\
\text { (mm/yyyy) }\end{array}$ & $\begin{array}{c}\text { Ending } \\
\text { date } \\
\text { (mm/yyyy) }\end{array}$ & $\begin{array}{l}\text { Per- } \\
\text { cent } \\
\text { cov- } \\
\text { erage }\end{array}$ & $\begin{array}{c}\text { Years } \\
\text { of } \\
\text { record }\end{array}$ \\
\hline 1293 & Woodsboro & 9892 & Refugio* & 49 & $28^{\circ} 14^{\prime}$ & $97^{\circ} 20^{\prime}$ & $08 / 1916$ & $07 / 1964$ & 95 & 49 \\
\hline 1294 & Woodson & 9893 & Throckmorton & 1,263 & $33^{\circ} 01^{\prime}$ & $99^{\circ} 03^{\prime}$ & $06 / 1948$ & $12 / 2001$ & 87 & 16 \\
\hline 1295 & Woodville & 9896 & Tyler* & 230 & $30^{\circ} 47^{\prime}$ & $94^{\circ} 26^{\prime}$ & $04 / 1917$ & 02/1919 & 58 & 3 \\
\hline 1296 & Woodville & 9898 & Tyler & 283 & $30^{\circ} 46^{\prime}$ & $94^{\circ} 24^{\prime}$ & $09 / 1988$ & $12 / 2001$ & 83 & 14 \\
\hline 1297 & Worlds End Ranch & 9904 & Kerr & 1,923 & $29^{\circ} 58^{\prime}$ & $99^{\circ} 26^{\prime}$ & 04/1976 & $08 / 1983$ & 93 & 8 \\
\hline 1298 & Wortham & 9912 & Freestone & 495 & $31^{\circ} 47^{\prime}$ & $96^{\circ} 27^{\prime}$ & $01 / 1992$ & $01 / 1996$ & 81 & 5 \\
\hline 1299 & Wright Patman Dam and Lake & 9916 & Cass & 282 & $33^{\circ} 18^{\prime}$ & $94^{\circ} 10^{\prime}$ & $08 / 1974$ & $12 / 2001$ & 95 & 28 \\
\hline 1300 & WTU Lake Pauline & 9935 & Hardeman & 1,503 & $34^{\circ} 15^{\prime}$ & $99^{\circ} 41^{\prime}$ & $12 / 1978$ & 05/1979 & 25 & 2 \\
\hline 1301 & Yoakum & 9952 & Lavaca & 325 & $29^{\circ} 16^{\prime}$ & $97^{\circ} 07^{\prime}$ & $07 / 1917$ & $12 / 2001$ & 95 & 80 \\
\hline 1302 & Yorktown & 9953 & DeWitt & 260 & $28^{\circ} 58^{\prime}$ & $97^{\circ} 31^{\prime}$ & $10 / 1947$ & $12 / 2001$ & 93 & 55 \\
\hline 1303 & Young & 9959 & Freestone* & $* 348$ & $31^{\circ} 51^{\prime}$ & $96^{\circ} 05^{\prime}$ & $09 / 1947$ & $08 / 1948$ & 40 & 2 \\
\hline 1304 & Youngsport & 9962 & Bell & 735 & $30^{\circ} 57^{\prime}$ & $97^{\circ} 43^{\prime}$ & 01/1992 & $12 / 2001$ & 100 & 10 \\
\hline 1305 & Ysleta & 9966 & El Paso & 3,670 & $31^{\circ} 41^{\prime}$ & $106^{\circ} 19^{\prime}$ & $02 / 1939$ & $12 / 2001$ & 98 & 63 \\
\hline 1306 & Zapata 3 SW & 9976 & Zapata & 320 & $26^{\circ} 52^{\prime}$ & $99^{\circ} 15^{\prime}$ & 01/1909 & $12 / 2001$ & 87 & 47 \\
\hline
\end{tabular}


Table 2. National Weather Service daily precipitation stations in Texas, by county

\begin{tabular}{|c|c|c|c|c|c|}
\hline $\begin{array}{l}\text { County } \\
\text { no. }\end{array}$ & $\begin{array}{l}\text { County } \\
\text { name }\end{array}$ & $\begin{array}{l}\text { Station sequence no. } \\
\text { (table 1) }\end{array}$ & $\begin{array}{l}\text { County } \\
\text { no. }\end{array}$ & $\begin{array}{l}\text { County } \\
\text { name }\end{array}$ & $\begin{array}{l}\text { Station sequence no. } \\
\text { (table 1) }\end{array}$ \\
\hline 1 & Anderson & 435,887 & 49 & Cooke & $387,448,449,818,1219$ \\
\hline 2 & Andrews & 29 & 50 & Coryell & $\begin{array}{l}260,261,262,269,391,407,460,579,678 \\
\quad 914\end{array}$ \\
\hline 3 & Angelina & $718,719,720$ & 51 & Cottle & $202,501,670,881,882,884$ \\
\hline 4 & Aransas & $35,36,37,1007,1010$ & 52 & Crane & 279,280 \\
\hline 5 & Archer & $38,350,658,868,1072$ & 53 & Crockett & $6,95,613,879,880$ \\
\hline 6 & Armstrong & 230,1251 & 54 & Crosby & $286,711,1264$ \\
\hline 7 & Atascosa & $212,542,608,763,927,945,1027$ & 55 & Culberson & $497,917,1040,1220$ \\
\hline 8 & Austin & 84,1074 & 56 & Dallam & $156,240,254,299,1168$ \\
\hline 9 & Bailey & $819,820,821$ & 57 & Dallas & $190,303,304,305,388,603,817$ \\
\hline 10 & Bandera & $765,1149,1223,1224,1225,1280$ & 58 & Dawson & 663,899 \\
\hline 11 & Bastrop & $70,197,378,600,975,1101$ & 59 & DeWitt & $292,293,1302$ \\
\hline 12 & Baylor & $657,976,984,1079$ & 60 & Deaf Smith & $114,452,453,533,534,1240,1259$ \\
\hline 13 & Bee & 83,167 & 61 & Delta & 256 \\
\hline 14 & Bell & $\begin{array}{l}86,631,632,1038,1131,1159,1160,1197, \\
\quad 1304\end{array}$ & 62 & Denton & $\begin{array}{l}323,324,615,655,680,681,682,683,694 \\
\quad 916,969,1001,1066,1134\end{array}$ \\
\hline 15 & Bexar & $229,380,963,1046,1047,1048,1049$ & 63 & Dickens & $331,919,1120$ \\
\hline 16 & Blanco & $103,295,523,582,604,1029,1065$ & 64 & Dimmit & $42,100,189,196,1287$ \\
\hline 17 & Borden & 447,877 & 65 & Donley & 226 \\
\hline 18 & Bosque & $234,235,281,776,777,807,1218,1242,1266$ & 66 & Duval & $87,88,252,438$ \\
\hline 19 & Bowie & $315,750,829,838,839,1092,1093,1166$ & 67 & Eastland & $187,357,484,964,965,998,999$ \\
\hline 20 & Brazoria & $22,30,128,437,514,1053,1276$ & 68 & Ector & $609,864,905$ \\
\hline 21 & Brazos & $150,242,243$ & 69 & Edwards & $\begin{array}{l}17,238,772,1011,1012,1013,1014,1015 \\
\quad 1107,1140\end{array}$ \\
\hline 22 & Brewster & $\begin{array}{l}18,115,195,222,257,524,549,649,734 \\
752,871,895,910,911,962,1138,1139 \\
1162,1163\end{array}$ & 70 & El Paso & $\begin{array}{l}271,272,369,371,393,394,646,1103,1184 \text {, } \\
\quad 1305\end{array}$ \\
\hline 23 & Briscoe & 958,1091 & 71 & Ellis & $52,67,385,404,753,787,788,974,1250$ \\
\hline 24 & Brooks & 398 & 72 & Erath & $203,344,345,572,676,808,1123,1125$ \\
\hline 25 & Brown & $104,147,166,525,1283$ & 73 & Falls & $540,742,971,1022$ \\
\hline 26 & Burleson & $168,244,1104,1105$ & 74 & Fannin & $110,546,653,1158,1190$ \\
\hline 27 & Burnet & $92,137,163,164,662,735,1114,1249$ & 75 & Fayette & $408,643,1071$ \\
\hline 28 & Caldwell & $700,701,721,722$ & 76 & Fisher & 1005,1028 \\
\hline 29 & Calhoun & $929,939,941$ & 77 & Floyd & $415,416,1126$ \\
\hline 30 & Callahan & $55,288,289,756,872,955$ & 78 & Foard & 290 \\
\hline 31 & Cameron & $78,146,512,938,1051,1052,1068,1110$ & 79 & Fort Bend & $79,237,277,459,926,992,1023,1141,1170$ \\
\hline 32 & Camp & 920 & 80 & Franklin & $502,816,1284$ \\
\hline 33 & Carson & 893 & 81 & Freestone & $396,705,862,863,1155,1298,1303$ \\
\hline 34 & Cass & $45,691,754,1167,1299$ & 82 & Frio & $327,332,440,901,902$ \\
\hline 35 & Castro & $334,335,515,833$ & 83 & Gaines & $708,709,1073,1078$ \\
\hline 36 & Chambers & 26,1135 & 84 & Galveston & $450,451,565,852$ \\
\hline 37 & Cherokee & $19,330,593,594,843,1033$ & 85 & Garza & $651,930,943,1111$ \\
\hline 38 & Childress & $215,216,217,218$ & 86 & Gillespie & $188,270,338,436,473,513,994,1156$ \\
\hline 39 & Clay & 211,532 & 87 & Glasscock & 148,454 \\
\hline 40 & Cochran & 733,810 & 88 & Goliad & $475,476,477$ \\
\hline 41 & Coke & $861,1003,1089,1161,1246,1247$ & 89 & Gonzales & $85,213,343,478,479,480,848,1239$ \\
\hline 42 & Coleman & $\begin{array}{l}159,241,470,485,547,673,857,1067,1090 \\
\quad 1147,1192,1234\end{array}$ & 90 & Gray & $759,889,890$ \\
\hline 43 & Collin & $31,198,400,442,498,671,758,990$ & 91 & Grayson & $321,322,483,1085$ \\
\hline 44 & Collingsworth & 1256 & 92 & Gregg & $469,706,1263$ \\
\hline 45 & Colorado & $169,246,954,1006,1254$ & 93 & Grimes & $27,82,832,989$ \\
\hline 46 & Comal & $\begin{array}{l}155,182,183,184,395,406,840,1100,1117 \\
\quad 1118\end{array}$ & 94 & Guadalupe & $633,1076,1077$ \\
\hline 47 & Comanche & $247,310,953$ & 95 & Hale & $1,361,503,504,516,923,924,1201$ \\
\hline 48 & Concho & $253,276,358,359,386,885$ & 96 & Hall & $135,767,1148,1204$ \\
\hline
\end{tabular}


Table 2. National Weather Service daily precipitation stations in Texas, by county-Continued

\begin{tabular}{|c|c|c|c|c|c|}
\hline $\begin{array}{l}\text { County } \\
\text { no. }\end{array}$ & $\begin{array}{l}\text { County } \\
\text { name }\end{array}$ & $\begin{array}{c}\text { Station sequence no. } \\
\text { (table 1) }\end{array}$ & $\begin{array}{l}\text { County } \\
\text { no. }\end{array}$ & $\begin{array}{l}\text { County } \\
\text { name }\end{array}$ & $\begin{array}{l}\text { Station sequence no. } \\
\text { (table 1) }\end{array}$ \\
\hline 97 & Hamilton & $508,537,585$ & 141 & Lamb & $695,696,869$ \\
\hline 98 & Hansford & $496,543,809,1113$ & 142 & Lampasas & $7,623,664,703,847$ \\
\hline 99 & Hardeman & $220,259,956,1300$ & 143 & Lavaca & $506,1112,1301$ \\
\hline 100 & Hardin & $640,723,1088,1108$ & 144 & Lee & $107,333,403,464,684,944$ \\
\hline 101 & Harris & $\begin{array}{l}77,294,481,550,551,552,553,554,555, \\
556,557,558,559,560,561,562,563,564 \\
566,567,568,569,570,571,574,584,620 \\
621,853,898,900,1183\end{array}$ & 145 & Leon & $153,201,602$ \\
\hline 102 & Harrison & $507,511,618,743$ & 146 & Liberty & $233,685,1018$ \\
\hline 103 & Hartley & $127,206,208,300,301,302,517,783,1019$ & 147 & Limestone & $494,780,1172$ \\
\hline 104 & Haskell & $518,519,520,824$ & 148 & Lipscomb & $112,113,307,418,539,693$ \\
\hline 105 & Hays & $340,531,642,1058,1281,1282$ & 149 & Live Oak & $223,461,1173,1174,1267,1268$ \\
\hline 106 & Hemphill & $177,178,446$ & 150 & Llano & $151,194,687,698,699,812,948,1185$ \\
\hline 107 & Henderson & $44,726,946,1124,1193,1194$ & 151 & Loving & 770 \\
\hline 108 & Hidalgo & $\begin{array}{c}360,384,538,644,755,760,761,773,774 \\
797,798,1056,1057,1069,1191,1257\end{array}$ & 152 & Lubbock & $714,715,716,717,967$ \\
\hline 109 & Hill & $34,126,541,590,591,639$ & 153 & Lynn & $860,1096,1146,1279$ \\
\hline 110 & Hockley & 679,912 & 154 & Madison & $149,727,789$ \\
\hline 111 & Hood & $283,487,692,894$ & 155 & Marion & 601 \\
\hline 112 & Hopkins & 1142 & 156 & Martin & 677,1199 \\
\hline 113 & Houston & $285,491,713,849,968$ & 157 & Mason & $186,595,744,746,931$ \\
\hline 114 & Howard & $5,98,99,420$ & 158 & Matagorda & $74,75,748,886$ \\
\hline 115 & Hudspeth & $263,320,370,422,586,1039,1041,1087$ & 159 & Maverick & 356,368 \\
\hline 116 & Hunt & $249,493,580,957,1292$ & 160 & McCulloch & $125,174,337,405,605,736,775,834,921$ \\
\hline 117 & Hutchinson & $116,117,928,1132$ & 161 & McLennan & $\begin{array}{l}282,535,764,993,1024,1235,1236,1237 \\
\quad 1238,1258\end{array}$ \\
\hline 118 & Irion & $68,445,779,1086$ & 162 & McMullen & $4,171,287,1179,1180$ \\
\hline 119 & Jack & $33,592,844$ & 163 & Medina & $328,544,545,725,766,830,997$ \\
\hline 120 & Jackson & $362,363,364,751$ & 164 & Menard & $170,444,536,768,769,771,1115$ \\
\hline 121 & Jasper & $390,548,597,598,636,1009,1042$ & 165 & Midland & 785,786 \\
\hline 122 & Jeff Davis & $\begin{array}{l}62,106,219,352,421,627,628,634,740 \\
\quad 778,814,1099,1215\end{array}$ & 166 & Milam & $162,172,308,1008,1171$ \\
\hline 123 & Jefferson & $80,81,935,936,937,1036$ & 167 & Mills & $200,474,800,822,952,981$ \\
\hline 124 & Jim Hogg & $11,526,616$ & 168 & Mitchell & $245,654,710$ \\
\hline 125 & Jim Wells & $14,15,749,1054$ & 169 & Montague & $111,119,419,803,995$ \\
\hline 126 & Johnson & $20,21,160,161,231,232,688,689,1227$ & 170 & Montgomery & $255,296,728,806,841,1116,1277$ \\
\hline 127 & Jones & $32,509,522,858,1121,1122,1198$ & 171 & Moore & $185,207,346,347,1143$ \\
\hline 128 & Karnes & $225,399,619,625,1032$ & 172 & Morris & $297,828,870$ \\
\hline 129 & Kaufman & $278,622,660,1026,1164$ & 173 & Motley & $411,747,854,1002$ \\
\hline 130 & Kendall & $65,108,248,379,624,855,1095$ & 174 & Nacogdoches & $221,339,826,827$ \\
\hline 131 & Kenedy & 40,1070 & 175 & Navarro & $267,268,309,443,831,988$ \\
\hline 132 & Kent & 599 & 176 & Newton & $109,845,846,1182,1274$ \\
\hline 133 & Kerr & $\begin{array}{l}175,336,505,576,577,587,629,630,724 \\
\quad 836,1297\end{array}$ & 177 & Nolan & $102,850,1021,1145,1165,1290$ \\
\hline 134 & Kimble & $134,351,610,611,704,825,1020,1075,1157$ & 178 & Nueces & $\begin{array}{l}10,66,101,138,209,264,265,414,934,1004 \\
\quad 1080\end{array}$ \\
\hline 135 & King & $71,72,90,348,499,866,883,1025,1109$ & 179 & Ochiltree & $154,401,856,906,907,908,909,1291$ \\
\hline 136 & Kinney & 123,124 & 180 & Oldham & $9,122,1150,1226$ \\
\hline 137 & Kleberg & $392,635,987$ & 181 & Orange & $329,873,874,875,876$ \\
\hline 138 & Knox & $91,638,823,986,1200$ & 182 & Palo Pinto & $129,482,659,795,888,942,1137,1178$ \\
\hline 139 & La Salle & $76,273,274,275,433,712$ & 183 & Panola & $93,192,458,669$ \\
\hline 140 & Lamar & $41,326,782,896$ & 184 & Parker & $13,932,985,1119,1252$ \\
\hline
\end{tabular}


Table 2. National Weather Service daily precipitation stations in Texas, by county-Continued

\begin{tabular}{|c|c|c|c|c|c|}
\hline $\begin{array}{l}\text { County } \\
\text { no. }\end{array}$ & $\begin{array}{l}\text { County } \\
\text { name }\end{array}$ & $\begin{array}{c}\text { Station sequence no. } \\
\text { (table 1) }\end{array}$ & $\begin{array}{c}\text { County } \\
\text { no. }\end{array}$ & $\begin{array}{l}\text { County } \\
\text { name }\end{array}$ & $\begin{array}{l}\text { Station sequence no. } \\
\text { (table 1) }\end{array}$ \\
\hline 185 & Parmer & 118,441 & 220 & Tarrant & $\begin{array}{l}39,89,140,298,354,355,389,427,428,429, \\
430,431,468,492,626,731,745,1037, \\
1253\end{array}$ \\
\hline 186 & Pecos & $57,58,152,205,425,426,467,583,1083$ & 221 & Taylor & $2,3,650,672,1189,1289$ \\
\hline 187 & Polk & $266,697,811$ & 222 & Terrell & $341,342,1063,1064$ \\
\hline 188 & Potter & 24,165 & 223 & Terry & 144,145 \\
\hline 189 & Presidio & $\begin{array}{l}94,139,157,179,409,737,738,739,741 \\
\quad 925,949,1031,1216\end{array}$ & 224 & Throckmorton & $1175,1176,1177,1294$ \\
\hline 190 & Rains & $381,589,661$ & 225 & Titus & 815 \\
\hline 191 & Randall & 181,1208 & 226 & Tom Green & $\begin{array}{l}224,859,1034,1043,1044,1045,1062,1221, \\
\quad 1222,1245\end{array}$ \\
\hline 192 & Reagan & $96,97,258,1130$ & 227 & Travis & $46,47,48,49,50,729,732,913$ \\
\hline 193 & Real & $176,284,674,675,947$ & 228 & Trinity & 495,1195 \\
\hline 194 & Red River & $53,120,227,228,617,730,835$ & 229 & Tyler & $581,1186,1187,1243,1275,1295,1296$ \\
\hline 195 & Reeves & $\begin{array}{l}60,61,63,64,903,904,933,950,973,1181 \\
\quad 1188\end{array}$ & 230 & Upshur & 465,466 \\
\hline 196 & Refugio & $51,978,979,980,1293$ & 231 & Upton & $762,784,966,1209$ \\
\hline 197 & Roberts & 781 & 232 & Uvalde & $\begin{array}{l}105,236,457,648,805,1035,1210,1211 \\
\quad 1212,1213,1214\end{array}$ \\
\hline 198 & Robertson & $132,434,1217,1262$ & 233 & Val Verde & $\begin{array}{l}25,56,191,250,251,316,317,318,319,402 \\
\quad 573,612,614,665,666,702,891,892\end{array}$ \\
\hline 199 & Rockwall & 1016,1017 & 234 & Van Zandt & $180,365,382,1278$ \\
\hline 200 & Runnels & 59,1288 & 235 & Victoria & $1229,1230,1231,1232,1233$ \\
\hline 201 & Rusk & $472,530,707,813,878,951,983,1151$ & 236 & Walker & $432,578,1000,1055$ \\
\hline 202 & Sabine & $143,204,527,528,790,918$ & 237 & Waller & 529,1241 \\
\hline 203 & San Augustine & $141,142,1050$ & 238 & Ward & $69,488,801,802$ \\
\hline 204 & San Jacinto & 239,1084 & 239 & Washington & 133,1244 \\
\hline 205 & San Patricio & 1094,1255 & 240 & Webb & $383,423,439,667,668,796,865$ \\
\hline 206 & San Saba & $\begin{array}{l}210,214,417,791,972,991,1060,1061 \\
\quad 1098,1154\end{array}$ & 241 & Wharton & $306,366,367,842,915,1260$ \\
\hline 207 & Schleicher & $\begin{array}{l}311,349,372,373,374,375,376,424,606 \\
\quad 607,757\end{array}$ & 242 & Wheeler & $8,16,799,1081,1082,1261$ \\
\hline 208 & Scurry & 637,1102 & 243 & Wichita & $158,377,588,1269,1270,1271,1272,1273$ \\
\hline 209 & Shackelford & 12 & 244 & Wilbarger & 500,1228 \\
\hline 210 & Shelby & 199,837 & 245 & Willacy & $804,940,970,1059$ \\
\hline 211 & Sherman & 1136 & 246 & Williamson & $\begin{array}{l}28,412,462,463,489,490,596,686,851, \\
\quad 1030,1152,1153\end{array}$ \\
\hline 212 & Smith & $\begin{array}{l}353,410,690,793,1196,1205,1206,1207 \\
\quad 1248,1265\end{array}$ & 247 & Wilson & $413,647,1133,1144$ \\
\hline 213 & Somervell & 471,961 & 248 & Winkler & 1169,1285 \\
\hline 214 & Starr & $173,397,996$ & 249 & Wise & $23,121,136,312,313,314,652,897,1097$ \\
\hline 215 & Stephens & 130,131 & 250 & Wood & $521,656,792,794,959,960,1286$ \\
\hline 216 & Sterling & $54,193,455,456,1127,1128,1129$ & 251 & Yoakum & 325,922 \\
\hline 217 & Stonewall & 43 & 252 & Young & 486,867 \\
\hline 218 & Sutton & $575,977,982,1106$ & 253 & Zapata & 1306 \\
\hline 219 & Swisher & $510,641,1202,1203$ & 254 & Zavala & $73,291,645$ \\
\hline
\end{tabular}


Table 3. Summary of interoccurrence intervals for daily precipitation thresholds of 0.05 and 0.10 inch

[--, not available, no events that equal or exceed the threshold were observed]

\begin{tabular}{|c|c|c|c|c|c|c|c|c|c|c|c|c|c|c|}
\hline \multirow[b]{2}{*}{$\begin{array}{c}\text { Seq. } \\
\text { no. }\end{array}$} & \multicolumn{7}{|c|}{ Daily precipitation threshold of 0.05 inch and greater } & \multicolumn{7}{|c|}{ Daily precipitation threshold of 0.10 inch and greater } \\
\hline & $\begin{array}{c}\text { Mean } \\
\text { inter- } \\
\text { occur- } \\
\text { rence } \\
\text { interval } \\
\text { (days) }\end{array}$ & $\begin{array}{c}\text { Total } \\
\text { no. of } \\
\text { events }\end{array}$ & $\begin{array}{c}\text { Total } \\
\text { no. of } \\
\text { days } \\
\text { avail- } \\
\text { able }\end{array}$ & $\begin{array}{l}\text { Sigma } \\
\mathrm{S}\end{array}$ & $\begin{array}{c}\text { Stan- } \\
\text { dard } \\
\text { normal } \\
\text { variate }\end{array}$ & $\begin{array}{l}\text { Non- } \\
\text { ex- } \\
\text { ceed- } \\
\text { ance } \\
\text { prob- } \\
\text { ability }\end{array}$ & $\begin{array}{c}\text { p- } \\
\text { value }\end{array}$ & $\begin{array}{l}\text { Mean } \\
\text { inter- } \\
\text { occur- } \\
\text { rence } \\
\text { interval } \\
\text { (days) }\end{array}$ & $\begin{array}{c}\text { Total } \\
\text { no. of } \\
\text { events }\end{array}$ & $\begin{array}{c}\text { Total } \\
\text { no. of } \\
\text { days } \\
\text { avail- } \\
\text { able }\end{array}$ & $\begin{array}{l}\text { Sigma } \\
\mathrm{S}\end{array}$ & $\begin{array}{l}\text { Stan- } \\
\text { dard } \\
\text { normal } \\
\text { variate }\end{array}$ & $\begin{array}{l}\text { Non- } \\
\text { ex- } \\
\text { ceed- } \\
\text { ance } \\
\text { prob- } \\
\text { ability }\end{array}$ & $\begin{array}{c}p- \\
\text { value }\end{array}$ \\
\hline 1 & 7.93 & 2,461 & 19,514 & 24624508 & 2.192 & .986 & .014 & 10.07 & 1,937 & 19,514 & 19604211 & 2.843 & .998 & .002 \\
\hline 2 & 7.27 & 33 & 240 & 3818 & -.357 & .361 & .361 & 9.23 & 26 & 240 & 2995 & -.354 & .362 & .362 \\
\hline 3 & 7.65 & 2,577 & 19,720 & 25885412 & 1.648 & .950 & .050 & 9.40 & 2,099 & 19,720 & 21095151 & 1.530 & .937 & .063 \\
\hline 4 & 8.96 & 939 & 8,416 & 3969846 & .249 & .598 & .402 & 10.51 & 801 & 8,416 & 3367935 & -.039 & .484 & .484 \\
\hline 5 & 10.82 & 1,755 & 18,993 & 16569638 & -.421 & .337 & .337 & 12.23 & 1,553 & 18,993 & 14861685 & .526 & .700 & .300 \\
\hline 6 & 10.48 & 336 & 3,522 & 605347 & .733 & .768 & .232 & 12.36 & 285 & 3,522 & 512981 & .647 & .741 & .259 \\
\hline 7 & 7.49 & 1,103 & 8,264 & 4766073 & 2.631 & .996 & .004 & 8.67 & 953 & 8,264 & 4064079 & 1.715 & .957 & .043 \\
\hline 8 & 8.84 & 165 & 1,458 & 107617 & -2.343 & .010 & .010 & 9.92 & 147 & 1,458 & 94206 & -2.539 & .006 & .006 \\
\hline 9 & 7.67 & 377 & 2,893 & 519681 & -1.582 & .057 & .057 & 9.30 & 311 & 2,893 & 433373 & -1.120 & .132 & .132 \\
\hline 10 & 6.69 & 32 & 214 & 3809 & 1.102 & .865 & .135 & 7.13 & 30 & 214 & 3637 & 1.262 & .896 & .104 \\
\hline 11 & 12.54 & 488 & 6,120 & 1537352 & 1.129 & .871 & .129 & 13.30 & 460 & 6,120 & 1470385 & 1.657 & .951 & .049 \\
\hline 12 & 7.42 & 4,798 & 35,581 & 87180863 & 2.561 & .995 & .005 & 8.75 & 4,066 & 35,581 & 72910815 & .877 & .810 & .190 \\
\hline 13 & 5.99 & 2,507 & 15,005 & 18824756 & .074 & .529 & .471 & 7.05 & 2,128 & 15,005 & 16068381 & .516 & .697 & .303 \\
\hline 14 & 7.13 & 4,204 & 29,966 & 60175440 & -5.016 & 0 & 0 & 8.95 & 3,347 & 29,966 & 48747253 & -2.799 & .003 & .003 \\
\hline 15 & 8.14 & 210 & 1,709 & 168503 & -1.531 & .063 & .063 & 10.89 & 157 & 1,709 & 123164 & -1.778 & .038 & .038 \\
\hline 16 & 12.30 & 10 & 123 & 351 & -2.351 & .009 & .009 & 17.57 & 7 & 123 & 228 & -2.156 & .016 & .016 \\
\hline 17 & 13.67 & 9 & 123 & 485 & -.643 & .260 & .260 & 15.38 & 8 & 123 & 402 & -.896 & .185 & .185 \\
\hline 18 & 8.57 & 3,214 & 27,539 & 46058927 & 4.002 & 1.000 & 0 & 10.98 & 2,509 & 27,539 & 35481609 & 2.345 & .991 & .010 \\
\hline 19 & 5.21 & 3,694 & 19,240 & 37460618 & 5.701 & 0 & 0 & 5.99 & 3,212 & 19,240 & 32259190 & 4.320 & 1.000 & 0 \\
\hline 20 & 7.70 & 795 & 6,122 & 2486368 & 1.061 & .856 & .144 & 7.78 & 787 & 6,122 & 2439636 & .618 & .732 & .268 \\
\hline 21 & 6.12 & 863 & 5,279 & 2221163 & -1.267 & .103 & .103 & 6.99 & 755 & 5,279 & 1965508 & -.652 & .257 & .257 \\
\hline 22 & 5.34 & 6,469 & 34,541 & 117468023 & 7.164 & 0 & 0 & 6.12 & 5,646 & 34,541 & 101424478 & 5.226 & 0 & 0 \\
\hline 23 & 7.24 & 164 & 1,187 & 94883 & -.559 & .288 & .288 & 8.36 & 142 & 1,187 & 82893 & -.339 & .367 & .367 \\
\hline 24 & 7.55 & 2,607 & 19,693 & 26021080 & 1.210 & .887 & .113 & 9.59 & 2,053 & 19,693 & 20460777 & .955 & .830 & .170 \\
\hline 25 & 9.32 & 1,476 & 13,756 & 10157913 & .039 & .516 & .484 & 11.91 & 1,155 & 13,756 & 7932570 & -.085 & .466 & .466 \\
\hline 26 & 4.79 & 6,494 & 31,099 & 102905232 & 2.663 & .996 & .004 & 5.54 & 5,612 & 31,099 & 88186068 & 1.371 & .915 & .085 \\
\hline 27 & 5.66 & 3,675 & 20,787 & 38229396 & .092 & .536 & .464 & 6.53 & 3,183 & 20,787 & 32944918 & -.406 & .342 & .342 \\
\hline 28 & 6.42 & 1,874 & 12,022 & 11451285 & 1.243 & .893 & .107 & 7.34 & 1,639 & 12,022 & 9947026 & .676 & .750 & .250 \\
\hline 29 & 10.39 & 1,529 & 15,884 & 12076351 & -.374 & .354 & .354 & 12.37 & 1,284 & 15,884 & 10180781 & -.102 & .459 & .459 \\
\hline 30 & 4.75 & 6,662 & 31,631 & 103919613 & -1.937 & .026 & .026 & 5.55 & 5,701 & 31,631 & 92565250 & 3.483 & 1.000 & 0 \\
\hline 31 & 5.78 & 3,136 & 18,129 & 28744197 & 1.085 & .861 & .139 & 6.71 & 2,702 & 18,129 & 24718610 & .832 & .797 & .203 \\
\hline 32 & 7.99 & 2,085 & 16,648 & 18406056 & 4.787 & 1.000 & 0 & 9.16 & 1,817 & 16,648 & 15669485 & 2.659 & .996 & .004 \\
\hline 33 & 7.10 & 3,266 & 23,183 & 39449795 & 4.162 & 1.000 & 0 & 8.25 & 2,810 & 23,183 & 33776561 & 3.395 & 1.000 & 0 \\
\hline 34 & 5.70 & 636 & 3,622 & 1112562 & -1.488 & .068 & .068 & 6.80 & 533 & 3,622 & 936091 & -1.209 & .113 & .113 \\
\hline 35 & 6.92 & 1,487 & 10,292 & 7872696 & 1.925 & .973 & .027 & 8.24 & 1,249 & 10,292 & 6703624 & 2.631 & .996 & .004 \\
\hline 36 & 6.11 & 1,814 & 11,081 & 9991839 & -.430 & .334 & .334 & 7.39 & 1,499 & 11,081 & 8297856 & -.059 & .476 & .476 \\
\hline 37 & 6.45 & 1,693 & 10,911 & 9157563 & -.607 & .272 & .272 & 7.86 & 1,389 & 10,911 & 7514678 & -.537 & .296 & .296 \\
\hline 38 & 7.14 & 3,141 & 22,410 & 35977500 & 2.159 & .985 & .015 & 8.39 & 2,672 & 22,410 & 30695035 & 2.259 & .988 & .012 \\
\hline 39 & 6.15 & 3,177 & 19,535 & 31243041 & .666 & .747 & .253 & 7.24 & 2,697 & 19,535 & 26704580 & 1.235 & .892 & .109 \\
\hline 40 & 7.88 & 1,318 & 10,379 & 7361988 & 4.801 & 1.000 & 0 & 8.93 & 1,162 & 10,379 & 6448159 & 4.092 & 1.000 & 0 \\
\hline 41 & 5.72 & 4,335 & 24,787 & 55746924 & 4.290 & 1.000 & 0 & 6.34 & 3,912 & 24,787 & 49991076 & 3.369 & 1.000 & 0 \\
\hline 42 & 8.65 & 86 & 744 & 29522 & -1.240 & .108 & .108 & 10.78 & 69 & 744 & 24496 & -.657 & .256 & .256 \\
\hline 43 & 8.75 & 3,666 & 32,082 & 61513214 & 4.827 & 1.000 & 0 & 10.11 & 3,172 & 32,082 & 52400880 & 2.912 & .998 & .002 \\
\hline 44 & 5.97 & 3,290 & 19,656 & 32857724 & 1.609 & .946 & .054 & 6.89 & 2,855 & 19,656 & 28673716 & 2.028 & .979 & .021 \\
\hline 45 & 5.09 & 3,724 & 18,947 & 34223115 & -3.164 & .001 & .001 & 5.78 & 3,278 & 18,947 & 30529406 & -1.676 & .047 & .047 \\
\hline 46 & 5.96 & 4,338 & 25,853 & 56518846 & .903 & .817 & .183 & 7.37 & 3,510 & 25,853 & 45649991 & .629 & .735 & .265 \\
\hline 47 & 6.55 & 971 & 6,363 & 3145239 & .978 & .836 & .164 & 8.03 & 792 & 6,363 & 2615098 & 1.845 & .967 & .033 \\
\hline 48 & 6.34 & 891 & 5,648 & 2477823 & -.788 & .215 & .215 & 7.96 & 710 & 5,648 & 1985970 & -.439 & .330 & .330 \\
\hline
\end{tabular}


Table 3. Summary of interoccurrence intervals for daily precipitation thresholds of 0.05 and 0.10 inch-Continued

\begin{tabular}{|c|c|c|c|c|c|c|c|c|c|c|c|c|c|c|}
\hline \multirow[b]{2}{*}{$\begin{array}{l}\text { Seq. } \\
\text { no. }\end{array}$} & \multicolumn{7}{|c|}{ Daily precipitation threshold of 0.05 inch and greater } & \multicolumn{7}{|c|}{ Daily precipitation threshold of 0.10 inch and greater } \\
\hline & $\begin{array}{c}\text { Mean } \\
\text { inter- } \\
\text { occur- } \\
\text { rence } \\
\text { interval } \\
\text { (days) }\end{array}$ & $\begin{array}{l}\text { Total } \\
\text { no. of } \\
\text { events }\end{array}$ & $\begin{array}{c}\text { Total } \\
\text { no. of } \\
\text { days } \\
\text { avail- } \\
\text { able }\end{array}$ & $\begin{array}{l}\text { Sigma } \\
\mathrm{S}\end{array}$ & $\begin{array}{c}\text { Stan- } \\
\text { dard } \\
\text { normal } \\
\text { variate }\end{array}$ & $\begin{array}{l}\text { Non- } \\
\text { ex- } \\
\text { ceed- } \\
\text { ance } \\
\text { prob- } \\
\text { ability }\end{array}$ & $\begin{array}{c}\mathrm{p}- \\
\text { value }\end{array}$ & $\begin{array}{l}\text { Mean } \\
\text { inter- } \\
\text { occur- } \\
\text { rence } \\
\text { interval } \\
\text { (days) }\end{array}$ & $\begin{array}{l}\text { Total } \\
\text { no. of } \\
\text { events }\end{array}$ & $\begin{array}{l}\text { Total } \\
\text { no. of } \\
\text { days } \\
\text { avail- } \\
\text { able }\end{array}$ & $\begin{array}{l}\text { Sigma } \\
\mathrm{S}\end{array}$ & $\begin{array}{c}\text { Stan- } \\
\text { dard } \\
\text { normal } \\
\text { variate }\end{array}$ & $\begin{array}{l}\text { Non- } \\
\text { ex- } \\
\text { ceed- } \\
\text { ance } \\
\text { prob- } \\
\text { ability }\end{array}$ & $\begin{array}{c}\mathrm{p}- \\
\text { value }\end{array}$ \\
\hline 49 & 3.87 & 78 & 302 & 12428 & 0.844 & .801 & .199 & 4.79 & 63 & 302 & 10332 & 1.184 & .882 & .118 \\
\hline 50 & 6.58 & 139 & 915 & 68916 & 1.710 & .956 & .044 & 7.69 & 119 & 915 & 58621 & 1.450 & .926 & .074 \\
\hline 51 & 6.65 & 2,741 & 18,223 & 25954431 & 3.558 & 1.000 & 0 & 7.69 & 2,370 & 18,223 & 22071710 & 1.864 & .969 & .031 \\
\hline 52 & 6.29 & 2,084 & 13,109 & 13296456 & -2.102 & .018 & .018 & 6.92 & 1,895 & 13,109 & 12405016 & -.096 & .462 & .462 \\
\hline 53 & 4.31 & 71 & 306 & 10724 & -.187 & .426 & .426 & 5.02 & 61 & 306 & 8878 & -.660 & .255 & .255 \\
\hline 54 & 21.75 & 4 & 87 & 152 & -.438 & .331 & .331 & 21.75 & 4 & 87 & 152 & -.438 & .331 & .331 \\
\hline 55 & 10.00 & 987 & 9,865 & 5306619 & 4.898 & 1.000 & 0 & 10.78 & 915 & 9,865 & 4819279 & 3.553 & 1.000 & 0 \\
\hline 56 & 9.50 & 251 & 2,385 & 319682 & 1.867 & .969 & .031 & 11.52 & 207 & 2,385 & 260043 & 1.332 & .909 & .091 \\
\hline 57 & 12.01 & 1,630 & 19,579 & 17132779 & 5.153 & 0 & 0 & 14.58 & 1,343 & 19,579 & 13811232 & 3.205 & .999 & .001 \\
\hline 58 & 13.51 & 45 & 608 & 14119 & .373 & .645 & .355 & 13.82 & 44 & 608 & 13514 & .119 & .547 & .453 \\
\hline 59 & 8.17 & 4,432 & 36,226 & 82007431 & 2.486 & .994 & .006 & 9.62 & 3,767 & 36,226 & 68352938 & .189 & .575 & .425 \\
\hline 60 & 8.94 & 47 & 420 & 10065 & .235 & .593 & .407 & 11.67 & 36 & 420 & 8155 & .818 & .793 & .207 \\
\hline 61 & 9.75 & 2,859 & 27,865 & 38069113 & -4.101 & 0 & 0 & 12.55 & 2,220 & 27,865 & 30339933 & -1.557 & .060 & .060 \\
\hline 62 & 11.47 & 19 & 218 & 2590 & 1.892 & .971 & .029 & 12.82 & 17 & 218 & 2344 & 1.892 & .971 & .029 \\
\hline 63 & 15.93 & 128 & 2,039 & 143852 & 2.006 & .978 & .022 & 16.18 & 126 & 2,039 & 141684 & 2.002 & .977 & .023 \\
\hline 64 & 18.44 & 16 & 295 & 2049 & -.913 & .181 & .181 & 24.58 & 12 & 295 & 1610 & -.542 & .294 & .294 \\
\hline 65 & 6.59 & 1,126 & 7,422 & 3932579 & -3.422 & 0 & 0 & 8.09 & 918 & 7,422 & 3268796 & -2.124 & .017 & .017 \\
\hline 66 & 7.43 & 314 & 2,334 & 330773 & -2.987 & .001 & .001 & 8.91 & 262 & 2,334 & 280944 & -2.275 & .011 & .011 \\
\hline 67 & 5.62 & 2,373 & 13,340 & 16172284 & 1.836 & .967 & .033 & 6.80 & 1,961 & 13,340 & 13333499 & 1.487 & .932 & .068 \\
\hline 68 & 10.82 & 484 & 5,237 & 1370231 & 3.093 & .999 & .001 & 12.77 & 410 & 5,237 & 1133652 & 1.962 & .975 & .025 \\
\hline 69 & 15.81 & 311 & 4,917 & 781886 & .691 & .755 & .245 & 16.90 & 291 & 4,917 & 733819 & .760 & .776 & .224 \\
\hline 70 & 8.56 & 121 & 1,036 & 73260 & 3.217 & .999 & .001 & 9.09 & 114 & 1,036 & 70380 & 3.548 & 1.000 & 0 \\
\hline 71 & 7.33 & 104 & 762 & 39327 & -.132 & .447 & .447 & 8.02 & 95 & 762 & 35140 & -.492 & .311 & .311 \\
\hline 72 & 8.46 & 516 & 4,366 & 1085310 & -1.436 & .076 & .076 & 9.75 & 448 & 4,366 & 952435 & -.958 & .169 & .169 \\
\hline 73 & 10.19 & 1,227 & 12,503 & 7718573 & .380 & .648 & .352 & 11.04 & 1,133 & 12,503 & 7120291 & .307 & .621 & .379 \\
\hline 74 & 5.42 & 223 & 1,208 & 129674 & -.964 & .168 & .168 & 6.33 & 191 & 1,208 & 113374 & -.413 & .340 & .340 \\
\hline 75 & 5.05 & 4,638 & 23,435 & 56422912 & 4.509 & 1.000 & 0 & 6.13 & 3,822 & 23,435 & 46148513 & 3.262 & .999 & .001 \\
\hline 76 & 10.04 & 115 & 1,154 & 53059 & -3.722 & 0 & 0 & 13.42 & 86 & 1,154 & 39665 & -3.223 & .001 & .001 \\
\hline 77 & 4.89 & 3,238 & 15,838 & 26845916 & 4.629 & 1.000 & 0 & 5.64 & 2,807 & 15,838 & 22872661 & 2.659 & .996 & .004 \\
\hline 78 & 7.96 & 91 & 724 & 34017 & .539 & .705 & .295 & 10.20 & 71 & 724 & 26762 & .602 & .726 & .274 \\
\hline 79 & 6.63 & 88 & 583 & 27487 & 1.162 & .877 & .123 & 7.11 & 82 & 583 & 25880 & 1.297 & .903 & .097 \\
\hline 80 & 4.47 & 7,967 & 35,599 & 146609580 & 5.234 & 0 & 0 & 5.25 & 6,784 & 35,599 & 124298752 & 4.191 & 1.000 & 0 \\
\hline 81 & 4.42 & 3,193 & 14,126 & 23030041 & 2.074 & .981 & .019 & 5.25 & 2,691 & 14,126 & 19571412 & 2.670 & .996 & .004 \\
\hline 82 & 6.58 & 1,926 & 12,671 & 13407260 & 7.507 & 0 & 0 & 6.83 & 1,856 & 12,671 & 12908453 & 7.296 & 0 & 0 \\
\hline 83 & 6.08 & 5,860 & 35,650 & 104726727 & .346 & .635 & .365 & 7.69 & 4,634 & 35,650 & 83340279 & 1.055 & .854 & .146 \\
\hline 84 & 5.27 & 1,483 & 7,812 & 5806865 & .164 & .565 & .435 & 6.09 & 1,283 & 7,812 & 4956622 & -.678 & .249 & .249 \\
\hline 85 & 9.14 & 181 & 1,655 & 133693 & -2.502 & .006 & .006 & 9.30 & 178 & 1,655 & 130778 & -2.591 & .005 & .005 \\
\hline 86 & 5.95 & 2,430 & 14,452 & 18119690 & 2.726 & .997 & .003 & 7.27 & 1,987 & 14,452 & 14976702 & 3.327 & 1.000 & 0 \\
\hline 87 & 9.48 & 128 & 1,213 & 68395 & -2.332 & .010 & .010 & 11.23 & 108 & 1,213 & 57679 & -2.150 & .016 & .016 \\
\hline 88 & 10.33 & 1,244 & 12,853 & 7520134 & -3.625 & 0 & 0 & 11.49 & 1,119 & 12,853 & 6907420 & -2.287 & .011 & .011 \\
\hline 89 & 6.13 & 3,112 & 19,069 & 30547234 & 2.852 & .998 & .002 & 7.36 & 2,591 & 19,069 & 25530702 & 2.951 & .998 & .002 \\
\hline 90 & 9.47 & 675 & 6,392 & 1962075 & -4.072 & 0 & 0 & 10.07 & 635 & 6,392 & 1872162 & -3.383 & 0 & 0 \\
\hline 91 & 8.45 & 1,468 & 12,411 & 9335951 & 1.648 & .950 & .050 & 9.61 & 1,292 & 12,411 & 7985028 & -.252 & .400 & .400 \\
\hline 92 & 6.35 & 1,950 & 12,385 & 12032197 & -.274 & .392 & .392 & 7.31 & 1,695 & 12,385 & 10446036 & -.341 & .366 & .366 \\
\hline 93 & 4.83 & 440 & 2,124 & 463507 & -.293 & .385 & .385 & 5.46 & 389 & 2,124 & 410401 & -.225 & .411 & .411 \\
\hline 94 & 12.75 & 191 & 2,436 & 229562 & -.317 & .376 & .376 & 15.72 & 155 & 2,436 & 183334 & -.623 & .267 & .267 \\
\hline 95 & 12.93 & 80 & 1,034 & 39394 & -.736 & .231 & .231 & 13.97 & 74 & 1,034 & 38226 & -.013 & .495 & .495 \\
\hline 96 & 8.70 & 1,587 & 13,801 & 10515736 & -2.743 & .003 & .003 & 10.85 & 1,272 & 13,801 & 8426730 & -2.468 & .007 & .007 \\
\hline
\end{tabular}


Table 3. Summary of interoccurrence intervals for daily precipitation thresholds of 0.05 and 0.10 inch-Continued

\begin{tabular}{|c|c|c|c|c|c|c|c|c|c|c|c|c|c|c|}
\hline \multirow[b]{2}{*}{$\begin{array}{l}\text { Seq. } \\
\text { no. }\end{array}$} & \multicolumn{7}{|c|}{ Daily precipitation threshold of 0.05 inch and greater } & \multicolumn{7}{|c|}{ Daily precipitation threshold of 0.10 inch and greater } \\
\hline & $\begin{array}{c}\text { Mean } \\
\text { inter- } \\
\text { occur- } \\
\text { rence } \\
\text { interval } \\
\text { (days) }\end{array}$ & $\begin{array}{c}\text { Total } \\
\text { no. of } \\
\text { events }\end{array}$ & $\begin{array}{l}\text { Total } \\
\text { no. of } \\
\text { days } \\
\text { avail- } \\
\text { able }\end{array}$ & $\begin{array}{l}\text { Sigma } \\
\mathrm{S}\end{array}$ & $\begin{array}{c}\text { Stan- } \\
\text { dard } \\
\text { normal } \\
\text { variate }\end{array}$ & $\begin{array}{l}\text { Non- } \\
\text { ex- } \\
\text { ceed- } \\
\text { ance } \\
\text { prob- } \\
\text { ability }\end{array}$ & $\begin{array}{c}\text { p- } \\
\text { value }\end{array}$ & $\begin{array}{c}\text { Mean } \\
\text { inter- } \\
\text { occur- } \\
\text { rence } \\
\text { interval } \\
\text { (days) }\end{array}$ & $\begin{array}{c}\text { Total } \\
\text { no. of } \\
\text { events }\end{array}$ & $\begin{array}{c}\text { Total } \\
\text { no. of } \\
\text { days } \\
\text { avail- } \\
\text { able }\end{array}$ & $\begin{array}{c}\text { Sigma } \\
\mathrm{S}\end{array}$ & $\begin{array}{c}\text { Stan- } \\
\text { dard } \\
\text { normal } \\
\text { variate }\end{array}$ & $\begin{array}{l}\text { Non- } \\
\text { ex- } \\
\text { ceed- } \\
\text { ance } \\
\text { prob- } \\
\text { ability }\end{array}$ & $\begin{array}{c}\mathrm{p}- \\
\text { value }\end{array}$ \\
\hline 97 & 11.40 & 99 & 1,129 & 52226 & -1.129 & .130 & .130 & 14.86 & 76 & 1,129 & 42391 & -0.180 & .429 & .429 \\
\hline 98 & 9.47 & 2,012 & 19,044 & 19274152 & .470 & .681 & .319 & 11.41 & 1,669 & 19,044 & 16129172 & 1.055 & .854 & .146 \\
\hline 99 & 5.18 & 22 & 114 & 1083 & -1.108 & .134 & .134 & 7.13 & 16 & 114 & 660 & -1.914 & .028 & .028 \\
\hline 100 & 10.32 & 2,770 & 28,580 & 37988537 & -3.673 & 0 & 0 & 12.16 & 2,350 & 28,580 & 32501205 & -2.701 & .003 & .003 \\
\hline 101 & 7.50 & 1,228 & 9,211 & 5476688 & -1.920 & .027 & .027 & 9.14 & 1,008 & 9,211 & 4607841 & -.409 & .341 & .341 \\
\hline 102 & 8.99 & 88 & 791 & 36775 & .920 & .821 & .179 & 9.42 & 84 & 791 & 36057 & 1.355 & .912 & .088 \\
\hline 103 & 6.14 & 6,101 & 37,448 & 119345206 & 6.052 & 0 & 0 & 7.28 & 5,143 & 37,448 & 98223961 & 2.485 & .994 & .006 \\
\hline 104 & 8.27 & 759 & 6,276 & 2419955 & .766 & .778 & .222 & 9.61 & 653 & 6,276 & 2078642 & .638 & .738 & .262 \\
\hline 105 & 9.04 & 1,134 & 10,246 & 5737139 & -.726 & .234 & .234 & 10.66 & 961 & 10,246 & 5028055 & 1.144 & .874 & .126 \\
\hline 106 & 6.61 & 539 & 3,562 & 937751 & -.930 & .176 & .176 & 8.34 & 427 & 3,562 & 755638 & -.228 & .410 & .410 \\
\hline 107 & 6.51 & 1,151 & 7,487 & 4040092 & -3.664 & 0 & 0 & 7.35 & 1,019 & 7,487 & 3623938 & -2.764 & .003 & .003 \\
\hline 108 & 5.91 & 6,323 & 37,342 & 121549544 & 4.075 & 1.000 & 0 & 7.39 & 5,054 & 37,342 & 96741117 & 3.103 & .999 & .001 \\
\hline 109 & 4.89 & 2,949 & 14,412 & 21666071 & 1.839 & .967 & .033 & 5.41 & 2,665 & 14,412 & 19538112 & 1.556 & .940 & .060 \\
\hline 110 & 5.53 & 6,278 & 34,710 & 110395377 & 1.815 & .965 & .035 & 6.32 & 5,493 & 34,710 & 96304695 & 1.311 & .905 & .095 \\
\hline 111 & 6.82 & 184 & 1,254 & 121884 & 1.327 & .908 & .092 & 8.09 & 155 & 1,254 & 101492 & .956 & .830 & .170 \\
\hline 112 & 7.99 & 2,872 & 22,955 & 34209769 & 3.510 & 1.000 & 0 & 9.40 & 2,442 & 22,955 & 28472541 & 1.357 & .913 & .087 \\
\hline 113 & 8.88 & 271 & 2,405 & 278287 & -4.164 & 0 & 0 & 10.19 & 236 & 2,405 & 244529 & -3.681 & 0 & 0 \\
\hline 114 & 11.40 & 176 & 2,006 & 177988 & .190 & .575 & .425 & 11.87 & 169 & 2,006 & 168739 & -.102 & .459 & .459 \\
\hline 115 & 12.30 & 1,469 & 18,062 & 13268082 & .008 & .503 & .497 & 16.33 & 1,106 & 18,062 & 9859080 & -.745 & .228 & .228 \\
\hline 116 & 7.95 & 730 & 5,805 & 2064334 & -1.204 & .114 & .114 & 10.20 & 569 & 5,805 & 1606345 & -1.130 & .129 & .129 \\
\hline 117 & 7.30 & 2,632 & 19,214 & 25735968 & 1.583 & .943 & .057 & 9.21 & 2,086 & 19,214 & 20339143 & 1.180 & .881 & .119 \\
\hline 118 & 11.76 & 86 & 1,011 & 48636 & 1.908 & .972 & .028 & 11.89 & 85 & 1,011 & 48092 & 1.905 & .972 & .028 \\
\hline 119 & 6.48 & 4,257 & 27,586 & 59482272 & 1.473 & .930 & .070 & 7.52 & 3,671 & 27,586 & 51532805 & 1.863 & .969 & .031 \\
\hline 120 & 4.84 & 3,960 & 19,175 & 38617056 & 1.868 & .969 & .031 & 5.59 & 3,429 & 19,175 & 33443992 & 1.754 & .960 & .040 \\
\hline 121 & 6.96 & 2,637 & 18,359 & 22825965 & -5.072 & 0 & 0 & 8.02 & 2,289 & 18,359 & 20439994 & -2.255 & .012 & .012 \\
\hline 122 & 9.28 & 931 & 8,643 & 4279015 & 3.359 & 1.000 & 0 & 11.10 & 779 & 8,643 & 3557387 & 2.742 & .997 & .003 \\
\hline 123 & 9.65 & 3,576 & 34,510 & 62219012 & .865 & .806 & .194 & 11.12 & 3,103 & 34,510 & 53839397 & .535 & .704 & .296 \\
\hline 124 & 10.88 & 694 & 7,550 & 3169720 & 9.577 & 0 & 0 & 12.65 & 597 & 7,550 & 2691866 & 8.229 & 0 & 0 \\
\hline 125 & 7.29 & 3,256 & 23,727 & 39748690 & 2.869 & .998 & .002 & 8.88 & 2,673 & 23,727 & 32723399 & 2.859 & .998 & .002 \\
\hline 126 & 5.87 & 617 & 3,619 & 1068792 & -1.837 & .033 & .033 & 6.73 & 538 & 3,619 & 945995 & -1.136 & .128 & .128 \\
\hline 127 & 9.54 & 2,032 & 19,385 & 21135243 & 5.709 & 0 & 0 & 10.92 & 1,775 & 19,385 & 18035640 & 3.527 & 1.000 & 0 \\
\hline 128 & 4.68 & 2,324 & 10,885 & 12781824 & .881 & .811 & .189 & 5.75 & 1,892 & 10,885 & 10366112 & .504 & .693 & .307 \\
\hline 129 & 7.65 & 2,566 & 19,634 & 26133305 & 3.284 & .999 & .001 & 8.51 & 2,306 & 19,634 & 23247355 & 2.239 & .987 & .013 \\
\hline 130 & 7.45 & 3,535 & 26,350 & 47786843 & 2.683 & .996 & .004 & 8.80 & 2,993 & 26,350 & 40030294 & 1.436 & .924 & .076 \\
\hline 131 & 7.18 & 106 & 761 & 43470 & 1.387 & .917 & .083 & 8.55 & 89 & 761 & 35712 & .891 & .814 & .186 \\
\hline 132 & 5.88 & 2,390 & 14,041 & 17474274 & 3.509 & 1.000 & 0 & 6.85 & 2,050 & 14,041 & 14909144 & 2.818 & .998 & .002 \\
\hline 133 & 4.94 & 7,355 & 36,302 & 134289828 & .878 & .810 & .190 & 6.18 & 5,877 & 36,302 & 107249897 & .718 & .763 & .237 \\
\hline 134 & 7.56 & 159 & 1,202 & 80655 & -3.406 & 0 & 0 & 9.18 & 131 & 1,202 & 66308 & -3.128 & .001 & .001 \\
\hline 135 & 6.83 & 178 & 1,216 & 104594 & -.775 & .219 & .219 & 7.70 & 158 & 1,216 & 92885 & -.721 & .236 & .236 \\
\hline 136 & 6.41 & 4,839 & 31,019 & 76445773 & 2.240 & .987 & .013 & 7.55 & 4,111 & 31,019 & 64568218 & 1.409 & .920 & .080 \\
\hline 137 & 6.08 & 581 & 3,532 & 1021677 & -.178 & .429 & .429 & 7.52 & 470 & 3,532 & 843558 & .613 & .730 & .270 \\
\hline 138 & 8.55 & 894 & 7,647 & 3095950 & -4.882 & 0 & 0 & 10.22 & 748 & 7,647 & 2738524 & -2.012 & .022 & .022 \\
\hline 139 & 14.30 & 10 & 143 & 1014 & 2.291 & .989 & .011 & 14.30 & 10 & 143 & 1014 & 2.291 & .989 & .011 \\
\hline 140 & 6.55 & 197 & 1,290 & 123059 & -.766 & .222 & .222 & 7.59 & 170 & 1,290 & 112428 & .572 & .716 & .284 \\
\hline 141 & 5.09 & 2,095 & 10,670 & 11831750 & 4.645 & 1.000 & 0 & 5.67 & 1,882 & 10,670 & 10436481 & 2.964 & .998 & .002 \\
\hline 142 & 4.62 & 1,739 & 8,028 & 6945195 & -.364 & .358 & .358 & 5.46 & 1,470 & 8,028 & 5922560 & .247 & .598 & .402 \\
\hline 143 & 5.11 & 3,515 & 17,968 & 32930171 & 4.395 & 1.000 & 0 & 5.78 & 3,107 & 17,968 & 28708648 & 2.751 & .997 & .003 \\
\hline 144 & 9.91 & 1,390 & 13,768 & 9093555 & -3.207 & .001 & .001 & 11.38 & 1,210 & 13,768 & 7849760 & -3.471 & 0 & 0 \\
\hline
\end{tabular}


Table 3. Summary of interoccurrence intervals for daily precipitation thresholds of 0.05 and 0.10 inch-Continued

\begin{tabular}{|c|c|c|c|c|c|c|c|c|c|c|c|c|c|c|}
\hline \multirow[b]{2}{*}{$\begin{array}{c}\text { Seq. } \\
\text { no. }\end{array}$} & \multicolumn{7}{|c|}{ Daily precipitation threshold of 0.05 inch and greater } & \multicolumn{7}{|c|}{ Daily precipitation threshold of 0.10 inch and greater } \\
\hline & $\begin{array}{c}\text { Mean } \\
\text { inter- } \\
\text { occur- } \\
\text { rence } \\
\text { interval } \\
\text { (days) }\end{array}$ & $\begin{array}{l}\text { Total } \\
\text { no. of } \\
\text { events }\end{array}$ & $\begin{array}{c}\text { Total } \\
\text { no. of } \\
\text { days } \\
\text { avail- } \\
\text { able }\end{array}$ & $\begin{array}{l}\text { Sigma } \\
\mathrm{S}\end{array}$ & $\begin{array}{c}\text { Stan- } \\
\text { dard } \\
\text { normal } \\
\text { variate }\end{array}$ & $\begin{array}{l}\text { Non- } \\
\text { ex- } \\
\text { ceed- } \\
\text { ance } \\
\text { prob- } \\
\text { ability }\end{array}$ & $\begin{array}{c}\mathrm{p}- \\
\text { value }\end{array}$ & $\begin{array}{l}\text { Mean } \\
\text { inter- } \\
\text { occur- } \\
\text { rence } \\
\text { interval } \\
\text { (days) }\end{array}$ & $\begin{array}{l}\text { Total } \\
\text { no. of } \\
\text { events }\end{array}$ & $\begin{array}{l}\text { Total } \\
\text { no. of } \\
\text { days } \\
\text { avail- } \\
\text { able }\end{array}$ & $\begin{array}{l}\text { Sigma } \\
\mathrm{S}\end{array}$ & $\begin{array}{c}\text { Stan- } \\
\text { dard } \\
\text { normal } \\
\text { variate }\end{array}$ & $\begin{array}{l}\text { Non- } \\
\text { ex- } \\
\text { ceed- } \\
\text { ance } \\
\text { prob- } \\
\text { ability }\end{array}$ & $\begin{array}{c}\mathrm{p}- \\
\text { value }\end{array}$ \\
\hline 145 & 8.95 & 1,951 & 17,470 & 17380454 & 1.520 & .936 & .064 & 11.13 & 1,569 & 17,470 & 13939386 & 1.172 & .879 & .121 \\
\hline 146 & 7.56 & 3,748 & 28,335 & 53915703 & 1.629 & .948 & .052 & 9.62 & 2,945 & 28,335 & 42317463 & 1.339 & .910 & .090 \\
\hline 147 & 7.43 & 2,615 & 19,432 & 25394257 & -.046 & .482 & .482 & 8.83 & 2,200 & 19,432 & 21757690 & 1.454 & .927 & .073 \\
\hline 148 & 14.91 & 85 & 1,267 & 46014 & -2.323 & .010 & .010 & 16.04 & 79 & 1,267 & 43517 & -2.009 & .022 & .022 \\
\hline 149 & 10.19 & 21 & 214 & 2513 & .940 & .826 & .174 & 10.70 & 20 & 214 & 2410 & .977 & .836 & .164 \\
\hline 150 & 5.87 & 197 & 1,156 & 110290 & -.764 & .223 & .223 & 7.27 & 159 & 1,156 & 89161 & -.651 & .257 & .257 \\
\hline 151 & 7.26 & 966 & 7,017 & 3269573 & -1.900 & .029 & .029 & 8.75 & 802 & 7,017 & 2699385 & -1.995 & .023 & .023 \\
\hline 152 & 14.25 & 555 & 7,906 & 2194680 & .014 & .506 & .494 & 15.81 & 500 & 7,906 & 1917227 & -1.162 & .123 & .123 \\
\hline 153 & 6.00 & 2,415 & 14,486 & 17084111 & -1.984 & .024 & .024 & 6.70 & 2,161 & 14,486 & 15566766 & -.439 & .330 & .330 \\
\hline 154 & 8.06 & 1,325 & 10,685 & 7218681 & 1.246 & .894 & .106 & 9.82 & 1,088 & 10,685 & 5842688 & .295 & .616 & .384 \\
\hline 155 & 6.53 & 3,376 & 22,051 & 36946092 & -.746 & .228 & .228 & 7.59 & 2,907 & 22,051 & 32070760 & .057 & .523 & .477 \\
\hline 156 & 10.70 & 1,375 & 14,709 & 10100173 & -.078 & .469 & .469 & 11.94 & 1,232 & 14,709 & 9025592 & -.236 & .407 & .407 \\
\hline 157 & 21.17 & 6 & 127 & 445 & .713 & .762 & .238 & 21.17 & 6 & 127 & 445 & .713 & .762 & .238 \\
\hline 158 & 6.39 & 319 & 2,037 & 301499 & -2.228 & .013 & .013 & 7.75 & 263 & 2,037 & 251944 & -1.670 & .048 & .048 \\
\hline 159 & 8.26 & 2,130 & 17,595 & 20011589 & 5.430 & 0 & 0 & 9.12 & 1,929 & 1,7595 & 17817262 & 3.796 & 1.000 & 0 \\
\hline 160 & 5.74 & 1,042 & 5,981 & 3022774 & -1.675 & .047 & .047 & 6.91 & 866 & 5,981 & 2527901 & -1.218 & .112 & .112 \\
\hline 161 & 6.30 & 2,140 & 13,484 & 14892449 & 2.580 & .995 & .005 & 7.50 & 1,798 & 13,484 & 12595159 & 2.866 & .998 & .002 \\
\hline 162 & 6.47 & 1,518 & 9,819 & 7973765 & 4.719 & 1.000 & 0 & 7.60 & 1,292 & 9,819 & 6721427 & 3.714 & 1.000 & 0 \\
\hline 163 & 6.58 & 5,232 & 34,426 & 91749387 & 2.352 & .991 & .009 & 7.81 & 4,409 & 34,426 & 77684132 & 2.716 & .997 & .003 \\
\hline 164 & 5.13 & 101 & 518 & 25630 & -.352 & .362 & .362 & 6.40 & 81 & 518 & 20666 & -.233 & .408 & .408 \\
\hline 165 & 7.65 & 155 & 1,185 & 87241 & -1.079 & .140 & .140 & 9.19 & 129 & 1,185 & 72822 & -.929 & .176 & .176 \\
\hline 166 & 9.34 & 678 & 6,331 & 2423428 & 5.825 & 0 & 0 & 10.28 & 616 & 6,331 & 2156358 & 4.551 & 1.000 & 0 \\
\hline 167 & 7.74 & 156 & 1,207 & 91620 & -.580 & .281 & .281 & 9.73 & 124 & 1,207 & 71055 & -.974 & .165 & .165 \\
\hline 168 & 5.40 & 2,611 & 14,097 & 18420381 & .081 & .532 & .468 & 6.36 & 2,218 & 1,4097 & 15653644 & .105 & .542 & .458 \\
\hline 169 & 5.82 & 209 & 1,216 & 123314 & -.741 & .230 & .230 & 6.72 & 181 & 1,216 & 108377 & -.354 & .362 & .362 \\
\hline 170 & 7.39 & 823 & 6,084 & 2480639 & -.455 & .325 & .325 & 9.30 & 654 & 6,084 & 2010443 & .467 & .680 & .320 \\
\hline 171 & 8.49 & 970 & 8,233 & 3994700 & .023 & .509 & .491 & 9.98 & 825 & 8,233 & 3332516 & -.932 & .176 & .176 \\
\hline 172 & 5.95 & 5,523 & 32,834 & 91894256 & 1.737 & .959 & .041 & 7.06 & 4,648 & 3,2834 & 77315520 & 1.562 & .941 & .059 \\
\hline 173 & 10.36 & 191 & 1,978 & 172020 & -2.139 & .016 & .016 & 11.64 & 170 & 1,978 & 152903 & -2.045 & .020 & .020 \\
\hline 174 & 9.00 & 687 & 6,180 & 2167594 & .957 & .831 & .169 & 9.90 & 624 & 6,180 & 1953956 & .579 & .719 & .281 \\
\hline 175 & 7.05 & 197 & 1,389 & 118369 & -3.278 & .001 & .001 & 8.08 & 172 & 1,389 & 111120 & -1.585 & .057 & .057 \\
\hline 176 & 7.37 & 2,720 & 20,049 & 28195689 & 3.078 & .999 & .001 & 9.12 & 2,198 & 20,049 & 22681040 & 2.385 & .991 & .009 \\
\hline 177 & 7.98 & 4,027 & 32,125 & 65328568 & 1.096 & .863 & .137 & 9.50 & 3,382 & 32,125 & 54708665 & .714 & .762 & .238 \\
\hline 178 & 8.71 & 38 & 331 & 6247 & -.071 & .472 & .472 & 9.46 & 35 & 331 & 5616 & -.312 & .377 & .377 \\
\hline 179 & 10.69 & 1,821 & 19,460 & 18351747 & 2.642 & .996 & .004 & 14.32 & 1,359 & 19,460 & 13891374 & 3.227 & .999 & .001 \\
\hline 180 & 5.46 & 3,051 & 16,672 & 26399727 & 3.636 & 1.000 & 0 & 6.28 & 2,654 & 16,672 & 22910142 & 3.172 & .999 & .001 \\
\hline 181 & 8.29 & 3,408 & 28,238 & 47461771 & -1.378 & .084 & .084 & 10.04 & 2,814 & 28,238 & 38549888 & -2.731 & .003 & .003 \\
\hline 182 & 5.46 & 2,553 & 13,932 & 18020285 & 1.162 & .877 & .123 & 6.88 & 2,025 & 13,932 & 14328708 & 1.230 & .891 & .109 \\
\hline 183 & 6.49 & 298 & 1,934 & 252517 & -3.699 & 0 & 0 & 7.41 & 261 & 1,934 & 228866 & -2.608 & .005 & .005 \\
\hline 184 & 6.81 & 127 & 865 & 56399 & .523 & .699 & .301 & 7.15 & 121 & 865 & 55013 & .976 & .835 & .165 \\
\hline 185 & 11.66 & 189 & 2,204 & 189774 & -2.116 & .017 & .017 & 12.97 & 170 & 2,204 & 172302 & -1.813 & .035 & .035 \\
\hline 186 & 13.68 & 167 & 2,285 & 195844 & .592 & .723 & .277 & 13.93 & 164 & 2,285 & 191102 & .442 & .671 & .329 \\
\hline 187 & 10.17 & 6 & 61 & 149 & -.788 & .215 & .215 & 10.17 & 6 & 61 & 149 & -.788 & .215 & .215 \\
\hline 188 & 6.80 & 2,181 & 1,420 & 15850767 & -1.554 & .060 & .060 & 8.23 & 1,801 & 14,820 & 13421885 & .421 & .663 & .337 \\
\hline 189 & 8.72 & 3,029 & 26,415 & 39990439 & -.036 & .486 & .486 & 11.46 & 2,305 & 26,415 & 30059782 & -1.048 & .147 & .147 \\
\hline 190 & 6.20 & 3,050 & 18,911 & 29037583 & .658 & .745 & .255 & 7.10 & 2,665 & 18,911 & 25767019 & 2.016 & .978 & .022 \\
\hline 191 & 9.37 & 1,368 & 12,819 & 8552565 & -1.575 & .058 & .058 & 10.70 & 1,198 & 12,819 & 7595699 & -.647 & .259 & .259 \\
\hline 192 & 4.83 & 3,765 & 18,176 & 34589636 & 1.160 & .877 & .123 & 5.58 & 3,259 & 18,176 & 29969988 & 1.176 & .880 & .120 \\
\hline
\end{tabular}


Table 3. Summary of interoccurrence intervals for daily precipitation thresholds of 0.05 and 0.10 inch-Continued

\begin{tabular}{|c|c|c|c|c|c|c|c|c|c|c|c|c|c|c|}
\hline \multirow[b]{2}{*}{$\begin{array}{l}\text { Seq. } \\
\text { no. }\end{array}$} & \multicolumn{7}{|c|}{ Daily precipitation threshold of 0.05 inch and greater } & \multicolumn{7}{|c|}{ Daily precipitation threshold of 0.10 inch and greater } \\
\hline & $\begin{array}{c}\text { Mean } \\
\text { inter- } \\
\text { occur- } \\
\text { rence } \\
\text { interval } \\
\text { (days) }\end{array}$ & $\begin{array}{c}\text { Total } \\
\text { no. of } \\
\text { events }\end{array}$ & $\begin{array}{l}\text { Total } \\
\text { no. of } \\
\text { days } \\
\text { avail- } \\
\text { able }\end{array}$ & $\begin{array}{l}\text { Sigma } \\
\mathrm{S}\end{array}$ & $\begin{array}{l}\text { Stan- } \\
\text { dard } \\
\text { normal } \\
\text { variate }\end{array}$ & $\begin{array}{l}\text { Non- } \\
\text { ex- } \\
\text { ceed- } \\
\text { ance } \\
\text { prob- } \\
\text { ability }\end{array}$ & $\begin{array}{c}\text { p- } \\
\text { value }\end{array}$ & $\begin{array}{c}\text { Mean } \\
\text { inter- } \\
\text { occur- } \\
\text { rence } \\
\text { interval } \\
\text { (days) }\end{array}$ & $\begin{array}{l}\text { Total } \\
\text { no. of } \\
\text { events }\end{array}$ & $\begin{array}{l}\text { Total } \\
\text { no. of } \\
\text { days } \\
\text { avail- } \\
\text { able }\end{array}$ & $\begin{array}{c}\text { Sigma } \\
\mathrm{S}\end{array}$ & $\begin{array}{c}\text { Stan- } \\
\text { dard } \\
\text { normal } \\
\text { variate }\end{array}$ & $\begin{array}{l}\text { Non- } \\
\text { ex- } \\
\text { ceed- } \\
\text { ance } \\
\text { prob- } \\
\text { ability }\end{array}$ & $\begin{array}{c}\mathrm{p}- \\
\text { value }\end{array}$ \\
\hline 193 & 11.70 & 1,040 & 12,164 & 6320115 & -0.046 & .482 & .482 & 12.78 & 952 & 12,164 & 5893799 & 0.958 & .831 & .169 \\
\hline 194 & 9.92 & 648 & 6,429 & 2042404 & -.859 & .195 & .195 & 10.25 & 627 & 6,429 & 1987162 & -.610 & .271 & .271 \\
\hline 195 & 11.83 & 802 & 9,486 & 3677676 & -1.628 & .052 & .052 & 15.89 & 597 & 9,486 & 2801401 & -.451 & .326 & .326 \\
\hline 196 & 10.97 & 1,313 & 14,400 & 9196614 & -1.706 & .044 & .044 & 12.96 & 1,111 & 14,400 & 7878150 & -.874 & .191 & .191 \\
\hline 197 & 5.71 & 1,417 & 8,095 & 5822057 & .986 & .838 & .162 & 6.71 & 1,206 & 8,095 & 4997784 & 1.436 & .924 & .076 \\
\hline 198 & 6.60 & 1,919 & 12,665 & 11480135 & -4.195 & 0 & 0 & 7.26 & 1,745 & 12,665 & 10621743 & -2.806 & .003 & .003 \\
\hline 199 & 4.68 & 4,881 & 22,827 & 58060610 & 5.107 & 0 & 0 & 5.35 & 4,267 & 22,827 & 50060086 & 3.156 & .999 & .001 \\
\hline 200 & 6.66 & 2,119 & 14,121 & 15341909 & 2.029 & .979 & .021 & 7.76 & 1,819 & 14,121 & 13088726 & 1.413 & .921 & .079 \\
\hline 201 & 5.20 & 4,408 & 22,939 & 50707231 & .340 & .633 & .367 & 6.26 & 3,664 & 22,939 & 42608866 & 1.459 & .928 & .072 \\
\hline 202 & 7.84 & 62 & 486 & 18148 & 2.790 & .997 & .003 & 9.17 & 53 & 486 & 15484 & 2.551 & .995 & .005 \\
\hline 203 & 6.22 & 2,267 & 14,090 & 15471182 & -2.581 & .005 & .005 & 7.15 & 1,971 & 14,090 & 13777429 & -.600 & .274 & .274 \\
\hline 204 & 5.01 & 277 & 1,388 & 182572 & -1.450 & .074 & .074 & 5.28 & 263 & 1,388 & 172575 & -1.531 & .063 & .063 \\
\hline 205 & 12.00 & 32 & 384 & 6555 & .655 & .744 & .256 & 15.36 & 25 & 384 & 5548 & 1.350 & .911 & .089 \\
\hline 206 & 9.14 & 320 & 2,925 & 480618 & .835 & .798 & .202 & 10.45 & 280 & 2,925 & 414178 & .331 & .630 & .370 \\
\hline 207 & 9.21 & 56 & 516 & 14362 & -.077 & .469 & .469 & 10.53 & 49 & 516 & 12439 & -.195 & .423 & .423 \\
\hline 208 & 8.59 & 959 & 8,239 & 3957175 & .089 & .536 & .464 & 10.11 & 815 & 8,239 & 3389059 & .466 & .680 & .320 \\
\hline 209 & 7.42 & 2,061 & 15,291 & 15097522 & -3.293 & .001 & .001 & 8.63 & 1,772 & 15,291 & 13367600 & -.970 & .166 & .166 \\
\hline 210 & 9.07 & 473 & 4,292 & 1042461 & 1.017 & .845 & .155 & 9.76 & 440 & 4,292 & 955122 & .419 & .662 & .338 \\
\hline 211 & 5.95 & 343 & 2,040 & 336523 & -1.223 & .111 & .111 & 6.99 & 292 & 2,040 & 280097 & -1.763 & .039 & .039 \\
\hline 212 & 7.05 & 2,032 & 14,334 & 14381521 & -.975 & .165 & .165 & 9.11 & 1,574 & 14,334 & 11120104 & -.979 & .164 & .164 \\
\hline 213 & 6.48 & 490 & 3,173 & 773574 & -.188 & .425 & .425 & 7.84 & 405 & 3,173 & 647270 & .257 & .601 & .399 \\
\hline 214 & 8.18 & 142 & 1,162 & 79241 & -.816 & .207 & .207 & 10.56 & 110 & 1,162 & 61739 & -.617 & .269 & .269 \\
\hline 215 & 8.61 & 93 & 801 & 39842 & 1.164 & .878 & .122 & 10.01 & 80 & 801 & 33094 & .510 & .695 & .305 \\
\hline 216 & 7.86 & 193 & 1,517 & 147556 & .192 & .576 & .424 & 9.79 & 155 & 1,517 & 119226 & .304 & .620 & .380 \\
\hline 217 & 9.42 & 1,609 & 15,148 & 12875893 & 3.930 & 1.000 & 0 & 10.27 & 1,475 & 15,148 & 11607275 & 2.594 & .995 & .005 \\
\hline 218 & 7.62 & 2,428 & 18,497 & 23039413 & 2.220 & .987 & .013 & 9.27 & 1,995 & 18,497 & 18909837 & 1.925 & .973 & .027 \\
\hline 219 & 31.00 & 1 & 31 & 24 & .950 & .829 & .171 & 31.00 & 1 & 31 & 24 & .950 & .829 & .171 \\
\hline 220 & 7.37 & 3,326 & 24,501 & 40220830 & -1.285 & .099 & .099 & 8.95 & 2,737 & 24,501 & 33189158 & -.920 & .179 & .179 \\
\hline 221 & 4.50 & 1,048 & 4,717 & 2443369 & -.643 & .260 & .260 & 5.20 & 907 & 4,717 & 2120704 & -.450 & .326 & .326 \\
\hline 222 & 8.29 & 2,386 & 19,773 & 23859518 & .970 & .834 & .166 & 10.55 & 1,874 & 19,773 & 18831674 & 1.232 & .891 & .109 \\
\hline 223 & 6.98 & 933 & 6,509 & 3015930 & -.358 & .360 & .360 & 8.94 & 728 & 6,509 & 2361118 & -.161 & .436 & .436 \\
\hline 224 & 10.71 & 571 & 6,117 & 1821669 & 1.784 & .963 & .037 & 11.79 & 519 & 6,117 & 1653848 & 1.653 & .951 & .049 \\
\hline 225 & 11.37 & 1,041 & 11,834 & 6723888 & 5.120 & 0 & 0 & 11.81 & 1,002 & 11,834 & 6396733 & 4.327 & 1.000 & 0 \\
\hline 226 & 7.50 & 4,459 & 33,447 & 74875876 & .474 & .682 & .318 & 9.00 & 3,718 & 33,447 & 61238799 & -1.595 & .055 & .055 \\
\hline 227 & 4.72 & 254 & 1,200 & 152278 & -.022 & .491 & .491 & 5.61 & 214 & 1,200 & 125152 & -.641 & .261 & .261 \\
\hline 228 & 5.36 & 6,348 & 34,037 & 110807569 & 3.544 & 1.000 & 0 & 6.03 & 5,645 & 34,037 & 97475810 & 1.905 & .972 & .028 \\
\hline 229 & 6.57 & 1,386 & 9,110 & 6401136 & .898 & .815 & .185 & 8.04 & 1,133 & 9,110 & 5272103 & 1.257 & .896 & .104 \\
\hline 230 & 9.02 & 3,549 & 32,007 & 61323009 & 8.224 & 0 & 0 & 10.22 & 3,131 & 32,007 & 52876924 & 5.358 & 0 & 0 \\
\hline 231 & 6.31 & 5,245 & 33,090 & 88809095 & 2.935 & .998 & .002 & 7.37 & 4,490 & 33,090 & 75173826 & 1.385 & .917 & .083 \\
\hline 232 & 6.39 & 391 & 2,497 & 485978 & -.153 & .439 & .439 & 7.28 & 343 & 2,497 & 438073 & .737 & .769 & .231 \\
\hline 233 & 4.55 & 3,799 & 17,281 & 33380308 & 1.805 & .964 & .036 & 5.37 & 3,221 & 17,281 & 28372080 & 1.911 & .972 & .028 \\
\hline 234 & 5.45 & 190 & 1,035 & 92533 & -1.406 & .080 & .080 & 6.43 & 161 & 1,035 & 79202 & -1.086 & .139 & .139 \\
\hline 235 & 6.03 & 3,875 & 23,374 & 44922901 & -.867 & .193 & .193 & 7.22 & 3,238 & 23,374 & 37608793 & -.609 & .271 & .271 \\
\hline 236 & 10.88 & 528 & 5,743 & 1312632 & -5.343 & 0 & 0 & 12.12 & 474 & 5,743 & 1226256 & -3.736 & 0 & 0 \\
\hline 237 & 5.06 & 3,675 & 18,579 & 36696513 & 7.866 & 0 & 0 & 5.93 & 3,132 & 18,579 & 31179914 & 6.947 & 0 & 0 \\
\hline 238 & 11.61 & 367 & 4,259 & 733878 & -2.023 & .022 & .022 & 12.27 & 347 & 4,259 & 706894 & -1.399 & .081 & .081 \\
\hline 239 & 4.49 & 3,863 & 17,330 & 33485658 & .041 & .516 & .484 & 5.39 & 3,214 & 17,330 & 28130428 & .991 & .839 & .161 \\
\hline 240 & 10.25 & 1,273 & 13,047 & 7463924 & -6.255 & 0 & 0 & 11.64 & 1,121 & 13,047 & 6656851 & -5.202 & 0 & 0 \\
\hline
\end{tabular}


Table 3. Summary of interoccurrence intervals for daily precipitation thresholds of 0.05 and 0.10 inch-Continued

\begin{tabular}{|c|c|c|c|c|c|c|c|c|c|c|c|c|c|c|}
\hline \multirow[b]{2}{*}{$\begin{array}{c}\text { Seq. } \\
\text { no. }\end{array}$} & \multicolumn{7}{|c|}{ Daily precipitation threshold of 0.05 inch and greater } & \multicolumn{7}{|c|}{ Daily precipitation threshold of 0.10 inch and greater } \\
\hline & $\begin{array}{c}\text { Mean } \\
\text { inter- } \\
\text { occur- } \\
\text { rence } \\
\text { interval } \\
\text { (days) }\end{array}$ & $\begin{array}{l}\text { Total } \\
\text { no. of } \\
\text { events }\end{array}$ & $\begin{array}{c}\text { Total } \\
\text { no. of } \\
\text { days } \\
\text { avail- } \\
\text { able }\end{array}$ & $\underset{\mathrm{S}}{\text { Sigma }}$ & $\begin{array}{c}\text { Stan- } \\
\text { dard } \\
\text { normal } \\
\text { variate }\end{array}$ & $\begin{array}{l}\text { Non- } \\
\text { ex- } \\
\text { ceed- } \\
\text { ance } \\
\text { prob- } \\
\text { ability }\end{array}$ & $\begin{array}{c}\mathbf{p}- \\
\text { value }\end{array}$ & $\begin{array}{c}\text { Mean } \\
\text { inter- } \\
\text { occur- } \\
\text { rence } \\
\text { interval } \\
\text { (days) }\end{array}$ & $\begin{array}{c}\text { Total } \\
\text { no. of } \\
\text { events }\end{array}$ & $\begin{array}{l}\text { Total } \\
\text { no. of } \\
\text { days } \\
\text { avail- } \\
\text { able }\end{array}$ & $\begin{array}{l}\text { Sigma } \\
\mathrm{S}\end{array}$ & $\begin{array}{l}\text { Stan- } \\
\text { dard } \\
\text { normal } \\
\text { variate }\end{array}$ & $\begin{array}{l}\text { Non- } \\
\text { ex- } \\
\text { ceed- } \\
\text { ance } \\
\text { prob- } \\
\text { ability }\end{array}$ & $\begin{array}{c}\mathrm{p}- \\
\text { value }\end{array}$ \\
\hline 241 & 7.32 & 5,111 & 37,406 & 98119424 & 3.275 & .999 & .001 & 8.58 & 4,360 & 37,406 & 82163986 & 0.868 & .807 & .193 \\
\hline 242 & 5.42 & 3,235 & 17,517 & 28410422 & .267 & .605 & .395 & 6.52 & 2,686 & 17,517 & 23642239 & .446 & .672 & .328 \\
\hline 243 & 5.51 & 3,751 & 20,679 & 38683152 & -.274 & .392 & .392 & 6.68 & 3,094 & 20,679 & 31915373 & -.226 & .411 & .411 \\
\hline 244 & 5.11 & 18 & 92 & 615 & -1.890 & .029 & .029 & 6.57 & 14 & 92 & 456 & -1.892 & .029 & .029 \\
\hline 245 & 8.82 & 2,993 & 26,383 & 39643879 & .388 & .651 & .349 & 10.62 & 2,485 & 26,383 & 32995663 & .566 & .714 & .286 \\
\hline 246 & 5.01 & 4,002 & 20,030 & 42546162 & 6.742 & 0 & 0 & 6.04 & 3,319 & 20,030 & 34417435 & 3.535 & 1.000 & 0 \\
\hline 247 & 7.01 & 4,353 & 30,526 & 67427562 & 1.699 & .955 & .045 & 8.22 & 3,715 & 30,526 & 57596118 & 1.665 & .952 & .048 \\
\hline 248 & 7.62 & 259 & 1,974 & 231904 & -2.588 & .005 & .005 & 8.47 & 233 & 1,974 & 206590 & -2.688 & .004 & .004 \\
\hline 249 & 6.08 & 496 & 3,015 & 738935 & -.453 & .325 & .325 & 6.88 & 438 & 3,015 & 666805 & .358 & .640 & .360 \\
\hline 250 & 10.49 & 708 & 7,429 & 2514959 & -2.014 & .022 & .022 & 13.06 & 569 & 7,429 & 2038119 & -1.475 & .070 & .070 \\
\hline 251 & 12.05 & 20 & 241 & 2213 & -.633 & .263 & .263 & 15.06 & 16 & 241 & 1612 & -1.136 & .128 & .128 \\
\hline 252 & 9.80 & 237 & 2,323 & 278242 & .287 & .613 & .387 & 12.42 & 187 & 2,323 & 214620 & -.281 & .389 & .389 \\
\hline 253 & 8.70 & 161 & 1,400 & 119345 & 1.296 & .902 & .098 & 9.79 & 143 & 1,400 & 104303 & .870 & .808 & .192 \\
\hline 254 & 8.71 & 2,236 & 19,485 & 21360543 & -1.593 & .056 & .056 & 10.53 & 1,850 & 19,485 & 17931352 & -.381 & .351 & .351 \\
\hline 255 & 5.01 & 3,861 & 19,333 & 36795567 & -1.519 & .064 & .064 & 5.75 & 3,363 & 19,333 & 32634934 & .391 & .652 & .348 \\
\hline 256 & 6.70 & 2,720 & 18,213 & 24180757 & -2.148 & .016 & .016 & 7.02 & 2,593 & 18,213 & 23238866 & -1.398 & .081 & .081 \\
\hline 257 & 15.20 & 40 & 608 & 13096 & .843 & .800 & .200 & 19.61 & 31 & 608 & 9508 & .086 & .534 & .466 \\
\hline 258 & 10.34 & 1,883 & 19,465 & 18842362 & 2.117 & .983 & .017 & 12.15 & 1,602 & 19,465 & 15942038 & 1.559 & .940 & .060 \\
\hline 259 & 7.81 & 579 & 4,519 & 1307087 & -.037 & .485 & .485 & 8.95 & 505 & 4,519 & 1121632 & -.662 & .254 & .254 \\
\hline 260 & 7.63 & 2,892 & 22,062 & 33912100 & 5.870 & 0 & 0 & 8.33 & 2,650 & 22,062 & 30165114 & 2.846 & .998 & .002 \\
\hline 261 & 5.92 & 247 & 1,461 & 190859 & 1.573 & .942 & .058 & 6.99 & 209 & 1,461 & 156941 & .700 & .758 & .242 \\
\hline 262 & 7.04 & 954 & 6,712 & 3172907 & -.480 & .316 & .316 & 7.46 & 900 & 6,712 & 2998625 & -.375 & .354 & .354 \\
\hline 263 & 12.76 & 1,453 & 18,538 & 13840890 & 1.829 & .966 & .034 & 16.05 & 1,155 & 18,538 & 11055612 & 1.924 & .973 & .027 \\
\hline 264 & 7.90 & 1,507 & 11,901 & 9101258 & 1.004 & .842 & .158 & 9.58 & 1,242 & 11,901 & 7561348 & 1.411 & .921 & .079 \\
\hline 265 & 6.95 & 2,837 & 19,723 & 29222782 & 4.108 & 1.000 & 0 & 8.76 & 2,251 & 19,723 & 23102988 & 3.349 & 1.000 & 0 \\
\hline 266 & 4.32 & 767 & 3,310 & 1234424 & -1.321 & .093 & .093 & 5.02 & 660 & 3,310 & 1084983 & -.298 & .383 & .383 \\
\hline 267 & 5.79 & 6,292 & 36,415 & 117128880 & 3.079 & .999 & .001 & 6.69 & 5,447 & 36,415 & 100661895 & 1.915 & .972 & .028 \\
\hline 268 & 5.21 & 701 & 3,653 & 1256704 & -.848 & .198 & .198 & 6.24 & 585 & 3,653 & 1045461 & -.903 & .183 & .183 \\
\hline 269 & 5.72 & 124 & 709 & 45549 & .698 & .757 & .243 & 6.33 & 112 & 709 & 41061 & .627 & .734 & .266 \\
\hline 270 & 8.74 & 1,619 & 14,154 & 11558776 & .615 & .731 & .269 & 9.17 & 1,543 & 14,154 & 11022504 & .640 & .739 & .261 \\
\hline 271 & 76.50 & 2 & 153 & 91 & -.993 & .161 & .161 & 76.50 & 2 & 153 & 91 & -.993 & .161 & .161 \\
\hline 272 & 21.57 & 7 & 151 & 615 & .750 & .773 & .227 & 25.17 & 6 & 151 & 541 & .824 & .795 & .205 \\
\hline 273 & 9.46 & 3,357 & 31,770 & 55739003 & 4.541 & 1.000 & 0 & 11.05 & 2,876 & 31,770 & 46877082 & 2.423 & .992 & .008 \\
\hline 274 & 9.17 & 1,241 & 11,383 & 7423502 & 3.113 & .999 & .001 & 11.19 & 1,017 & 11,383 & 6243605 & 4.345 & 1.000 & 0 \\
\hline 275 & 9.73 & 247 & 2,403 & 292787 & -.365 & .357 & .357 & 10.68 & 225 & 2,403 & 265507 & -.464 & .321 & .321 \\
\hline 276 & 8.85 & 730 & 6,457 & 2502378 & 2.891 & .998 & .002 & 10.69 & 604 & 6,457 & 2030139 & 1.749 & .960 & .040 \\
\hline 277 & 5.61 & 200 & 1,121 & 109178 & -.639 & .262 & .262 & 6.75 & 166 & 1,121 & 91194 & -.444 & .329 & .329 \\
\hline 278 & 5.62 & 2,108 & 11,840 & 12477273 & -.013 & .495 & .495 & 6.56 & 1,805 & 11,840 & 10786728 & .696 & .757 & .243 \\
\hline 279 & 14.06 & 52 & 731 & 21304 & 1.510 & .934 & .066 & 18.74 & 39 & 731 & 15419 & .884 & .812 & .189 \\
\hline 280 & 11.13 & 1,481 & 16,485 & 12247989 & .223 & .588 & .412 & 13.75 & 1,199 & 16,485 & 10054183 & 1.040 & .851 & .149 \\
\hline 281 & 6.79 & 650 & 4,415 & 1505852 & 2.184 & .986 & .014 & 8.04 & 549 & 4,415 & 1261142 & 1.648 & .950 & .050 \\
\hline 282 & 5.32 & 252 & 1,341 & 166688 & -.371 & .355 & .355 & 6.07 & 221 & 1,341 & 145131 & -.530 & .298 & .298 \\
\hline 283 & 6.48 & 2,997 & 19,415 & 30771392 & 5.469 & 0 & 0 & 7.53 & 2,577 & 19,415 & 26131424 & 3.920 & 1.000 & 0 \\
\hline 284 & 10.81 & 882 & 9,535 & 4042541 & -1.987 & .023 & .023 & 11.82 & 807 & 9,535 & 3729110 & -1.512 & .065 & .065 \\
\hline 285 & 5.44 & 5,980 & 32,536 & 100690248 & 4.692 & 1.000 & 0 & 6.21 & 5,240 & 32,536 & 87886658 & 3.886 & 1.000 & 0 \\
\hline 286 & 8.05 & 4,645 & 37,369 & 91752044 & 6.750 & 0 & 0 & 9.73 & 3,839 & 37,369 & 74769142 & 4.547 & 1.000 & 0 \\
\hline 287 & 8.01 & 228 & 1,826 & 196372 & -1.482 & .069 & .069 & 9.98 & 183 & 1,826 & 162189 & -.686 & .246 & .246 \\
\hline 288 & 8.75 & 1,507 & 13,182 & 10106541 & 1.177 & .880 & .120 & 9.76 & 1,350 & 13,182 & 8913004 & .108 & .543 & .457 \\
\hline
\end{tabular}


Table 3. Summary of interoccurrence intervals for daily precipitation thresholds of 0.05 and 0.10 inch-Continued

\begin{tabular}{|c|c|c|c|c|c|c|c|c|c|c|c|c|c|c|}
\hline \multirow[b]{2}{*}{$\begin{array}{l}\text { Seq. } \\
\text { no. }\end{array}$} & \multicolumn{7}{|c|}{ Daily precipitation threshold of 0.05 inch and greater } & \multicolumn{7}{|c|}{ Daily precipitation threshold of 0.10 inch and greater } \\
\hline & $\begin{array}{c}\text { Mean } \\
\text { inter- } \\
\text { occur- } \\
\text { rence } \\
\text { interval } \\
\text { (days) }\end{array}$ & $\begin{array}{c}\text { Total } \\
\text { no. of } \\
\text { events }\end{array}$ & $\begin{array}{l}\text { Total } \\
\text { no. of } \\
\text { days } \\
\text { avail- } \\
\text { able }\end{array}$ & $\begin{array}{l}\text { Sigma } \\
\mathrm{S}\end{array}$ & $\begin{array}{l}\text { Stan- } \\
\text { dard } \\
\text { normal } \\
\text { variate }\end{array}$ & $\begin{array}{l}\text { Non- } \\
\text { ex- } \\
\text { ceed- } \\
\text { ance } \\
\text { prob- } \\
\text { ability }\end{array}$ & $\begin{array}{c}\text { p- } \\
\text { value }\end{array}$ & $\begin{array}{c}\text { Mean } \\
\text { inter- } \\
\text { occur- } \\
\text { rence } \\
\text { interval } \\
\text { (days) }\end{array}$ & $\begin{array}{l}\text { Total } \\
\text { no. of } \\
\text { events }\end{array}$ & $\begin{array}{l}\text { Total } \\
\text { no. of } \\
\text { days } \\
\text { avail- } \\
\text { able }\end{array}$ & $\begin{array}{c}\text { Sigma } \\
\mathrm{S}\end{array}$ & $\begin{array}{c}\text { Stan- } \\
\text { dard } \\
\text { normal } \\
\text { variate }\end{array}$ & $\begin{array}{l}\text { Non- } \\
\text { ex- } \\
\text { ceed- } \\
\text { ance } \\
\text { prob- } \\
\text { ability }\end{array}$ & $\begin{array}{c}\text { p- } \\
\text { value }\end{array}$ \\
\hline 289 & 8.53 & 133 & 1,134 & 68029 & -1.955 & .025 & .025 & 10.90 & 104 & 1,134 & 52280 & -2.003 & .023 & .023 \\
\hline 290 & 9.98 & 3,058 & 30,511 & 49042149 & 4.909 & 1.000 & 0 & 10.70 & 2,851 & 30,511 & 45419354 & 4.095 & 1.000 & 0 \\
\hline 291 & 8.65 & 2,255 & 19,511 & 22427038 & 1.602 & .945 & .055 & 11.36 & 1,718 & 19,511 & 17121989 & 1.551 & .940 & .060 \\
\hline 292 & 5.99 & 366 & 2,191 & 378603 & -1.847 & .032 & .032 & 7.53 & 291 & 2,191 & 304308 & -1.342 & .090 & .090 \\
\hline 293 & 6.05 & 5,842 & 35,323 & 98959919 & -5.413 & 0 & 0 & 7.33 & 4,817 & 35,323 & 82871239 & -3.115 & .001 & .001 \\
\hline 294 & 5.93 & 3,175 & 18,834 & 31060924 & 3.793 & 1.000 & 0 & 6.48 & 2,907 & 18,834 & 27930148 & 1.893 & .971 & .029 \\
\hline 295 & 8.34 & 712 & 5,935 & 2089845 & -.503 & .307 & .307 & 9.01 & 659 & 5,935 & 1996611 & .933 & .825 & .176 \\
\hline 296 & 5.18 & 3,320 & 17,191 & 29430402 & 3.124 & .999 & .001 & 5.91 & 2,911 & 17,191 & 25719446 & 2.607 & .995 & .005 \\
\hline 297 & 5.06 & 3,864 & 19,546 & 38536434 & 2.206 & .986 & .014 & 5.85 & 3,340 & 19,546 & 33200722 & 1.714 & .957 & .043 \\
\hline 298 & 5.91 & 1,730 & 10,227 & 8955171 & .886 & .812 & .188 & 6.97 & 1,468 & 10,227 & 7715763 & 1.849 & .968 & .032 \\
\hline 299 & 10.13 & 23 & 233 & 2163 & -1.601 & .055 & .055 & 14.56 & 16 & 233 & 1418 & -1.658 & .049 & .049 \\
\hline 300 & 9.01 & 539 & 4,857 & 1246307 & -1.925 & .027 & .027 & 10.72 & 453 & 4,857 & 1054689 & -1.522 & .064 & .064 \\
\hline 301 & 8.34 & 2,023 & 16,871 & 17409101 & 1.571 & .942 & .058 & 10.64 & 1,585 & 16,871 & 13406257 & .186 & .574 & .426 \\
\hline 302 & 7.93 & 2,436 & 19,309 & 23732608 & .779 & .782 & .218 & 10.35 & 1,865 & 19,309 & 18195317 & .788 & .785 & .215 \\
\hline 303 & 5.35 & 91 & 487 & 23245 & .810 & .791 & .209 & 6.67 & 73 & 487 & 19043 & 1.055 & .854 & .146 \\
\hline 304 & 6.06 & 4,131 & 25,051 & 52619054 & 1.885 & .970 & .030 & 7.16 & 3,498 & 25,051 & 44390356 & 1.347 & .911 & .089 \\
\hline 305 & 7.73 & 201 & 1,554 & 150478 & -.896 & .185 & .185 & 8.49 & 183 & 1,554 & 132022 & -1.676 & .047 & .047 \\
\hline 306 & 5.36 & 6,947 & 37,205 & 133918130 & 5.235 & 0 & 0 & 6.39 & 5,819 & 37,205 & 110549742 & 2.810 & .998 & .002 \\
\hline 307 & 6.77 & 3,122 & 21,126 & 33397384 & 1.232 & .891 & .109 & 8.46 & 2,498 & 21,126 & 26897070 & 1.676 & .953 & .047 \\
\hline 308 & 8.84 & 2,010 & 17,772 & 19454133 & 6.927 & 0 & 0 & 9.35 & 1,900 & 17,772 & 18012197 & 5.048 & 0 & 0 \\
\hline 309 & 6.75 & 173 & 1,167 & 100184 & -.172 & .432 & .432 & 7.39 & 158 & 1,167 & 91368 & -.195 & .423 & .423 \\
\hline 310 & 6.82 & 455 & 3,103 & 637066 & -3.604 & 0 & 0 & 8.17 & 380 & 3,103 & 539457 & -2.870 & .002 & .002 \\
\hline 311 & 10.78 & 353 & 3,804 & 718452 & 2.280 & .989 & .011 & 12.35 & 308 & 3,804 & 627049 & 2.140 & .984 & .016 \\
\hline 312 & 6.55 & 2,829 & 18,527 & 28212261 & 7.051 & 0 & 0 & 7.36 & 2,518 & 18,527 & 24983500 & 6.178 & 0 & 0 \\
\hline 313 & 6.45 & 382 & 2,465 & 464356 & -.464 & .321 & .321 & 7.59 & 325 & 2,465 & 387294 & -1.034 & .151 & .151 \\
\hline 314 & 7.34 & 154 & 1,131 & 91153 & 1.004 & .842 & .158 & 8.50 & 133 & 1,131 & 81113 & 1.567 & .941 & .059 \\
\hline 315 & 4.80 & 3,893 & 18,684 & 36013562 & -1.054 & .146 & .146 & 5.42 & 3,446 & 18,684 & 32524682 & 1.049 & .853 & .147 \\
\hline 316 & 9.12 & 217 & 1,979 & 211687 & -.361 & .359 & .359 & 12.61 & 157 & 1,979 & 149534 & -.813 & .208 & .208 \\
\hline 317 & 13.45 & 121 & 1,627 & 86731 & -2.265 & .012 & .012 & 18.08 & 90 & 1,627 & 64478 & -1.961 & .025 & .025 \\
\hline 318 & 9.99 & 1,652 & 16,498 & 14293106 & 3.439 & 1.000 & 0 & 12.91 & 1,278 & 16,498 & 10987989 & 2.618 & .996 & .004 \\
\hline 319 & 9.66 & 387 & 3,738 & 705956 & -.817 & .207 & .207 & 11.94 & 313 & 3,738 & 582644 & -.123 & .451 & .451 \\
\hline 320 & 11.31 & 709 & 8,016 & 2815670 & -.422 & .337 & .337 & 15.18 & 528 & 8,016 & 2126052 & .185 & .573 & .427 \\
\hline 321 & 5.50 & 3,773 & 20,733 & 39629782 & 1.406 & .920 & .080 & 6.46 & 3,208 & 20,733 & 34022889 & 2.263 & .988 & .012 \\
\hline 322 & 6.84 & 1,833 & 12,538 & 11183917 & -1.982 & .024 & .024 & 7.90 & 1,588 & 12,538 & 9584171 & -2.572 & .005 & .005 \\
\hline 323 & 6.53 & 821 & 5,364 & 2204925 & .068 & .527 & .473 & 7.67 & 699 & 5,364 & 1874283 & -.011 & .496 & .496 \\
\hline 324 & 6.05 & 5,226 & 31,602 & 82908229 & .504 & .693 & .307 & 7.19 & 4,393 & 31,602 & 70358120 & 1.562 & .941 & .059 \\
\hline 325 & 9.53 & 447 & 4,259 & 945995 & -.227 & .410 & .410 & 11.15 & 382 & 4,259 & 804583 & -.370 & .356 & .356 \\
\hline 326 & 5.51 & 1,179 & 6,492 & 3868685 & .647 & .741 & .259 & 5.98 & 1,085 & 6,492 & 3563024 & .666 & .747 & .253 \\
\hline 327 & 8.89 & 914 & 8,126 & 3557929 & -2.195 & .014 & .014 & 9.81 & 828 & 8,126 & 3283260 & -1.199 & .115 & .115 \\
\hline 328 & 9.85 & 99 & 975 & 43506 & -1.699 & .045 & .045 & 12.34 & 79 & 975 & 35549 & -1.185 & .118 & .118 \\
\hline 329 & 4.78 & 2,421 & 11,566 & 15042131 & 6.340 & 0 & 0 & 5.41 & 2,138 & 11,566 & 12978722 & 3.982 & 1.000 & 0 \\
\hline 330 & 5.04 & 7,242 & 36,526 & 133708538 & 1.614 & .947 & .053 & 5.85 & 6,242 & 36,526 & 114801268 & .965 & .833 & .167 \\
\hline 331 & 9.47 & 707 & 6,698 & 2487898 & 2.337 & .990 & .010 & 10.70 & 626 & 6,698 & 2195518 & 2.047 & .980 & .020 \\
\hline 332 & 7.87 & 4,049 & 31,881 & 67543589 & 5.124 & 0 & 0 & 9.98 & 3,195 & 31,881 & 53029781 & 4.037 & 1.000 & 0 \\
\hline 333 & 6.31 & 3,398 & 21,436 & 38562449 & 5.940 & 0 & 0 & 6.94 & 3,089 & 21,436 & 34436437 & 3.863 & 1.000 & 0 \\
\hline 334 & 7.87 & 1,963 & 15,442 & 14908371 & -1.255 & .105 & .105 & 9.92 & 1,557 & 15,442 & 11939347 & -.468 & .320 & .320 \\
\hline 335 & 8.63 & 2,574 & 22,219 & 29054886 & 1.411 & .921 & .079 & 10.20 & 2,179 & 22,219 & 24632741 & 1.420 & .922 & .078 \\
\hline 336 & 6.43 & 322 & 2,070 & 366526 & 3.101 & .999 & .001 & 8.59 & 241 & 2,070 & 271691 & 2.399 & .992 & .008 \\
\hline
\end{tabular}


Table 3. Summary of interoccurrence intervals for daily precipitation thresholds of 0.05 and 0.10 inch-Continued

\begin{tabular}{|c|c|c|c|c|c|c|c|c|c|c|c|c|c|c|}
\hline \multirow[b]{2}{*}{$\begin{array}{c}\text { Seq. } \\
\text { no. }\end{array}$} & \multicolumn{7}{|c|}{ Daily precipitation threshold of 0.05 inch and greater } & \multicolumn{7}{|c|}{ Daily precipitation threshold of 0.10 inch and greater } \\
\hline & $\begin{array}{c}\text { Mean } \\
\text { inter- } \\
\text { occur- } \\
\text { rence } \\
\text { interval } \\
\text { (days) }\end{array}$ & $\begin{array}{c}\text { Total } \\
\text { no. of } \\
\text { events }\end{array}$ & $\begin{array}{l}\text { Total } \\
\text { no. of } \\
\text { days } \\
\text { avail- } \\
\text { able }\end{array}$ & $\begin{array}{l}\text { Sigma } \\
\mathrm{S}\end{array}$ & $\begin{array}{c}\text { Stan- } \\
\text { dard } \\
\text { normal } \\
\text { variate }\end{array}$ & $\begin{array}{l}\text { Non- } \\
\text { ex- } \\
\text { ceed- } \\
\text { ance } \\
\text { prob- } \\
\text { ability }\end{array}$ & $\begin{array}{c}\mathrm{p}- \\
\text { value }\end{array}$ & $\begin{array}{l}\text { Mean } \\
\text { inter- } \\
\text { occur- } \\
\text { rence } \\
\text { interval } \\
\text { (days) }\end{array}$ & $\begin{array}{l}\text { Total } \\
\text { no. of } \\
\text { events }\end{array}$ & $\begin{array}{l}\text { Total } \\
\text { no. of } \\
\text { days } \\
\text { avail- } \\
\text { able }\end{array}$ & $\begin{array}{l}\text { Sigma } \\
\mathrm{S}\end{array}$ & $\begin{array}{c}\text { Stan- } \\
\text { dard } \\
\text { normal } \\
\text { variate }\end{array}$ & $\begin{array}{l}\text { Non- } \\
\text { ex- } \\
\text { ceed- } \\
\text { ance } \\
\text { prob- } \\
\text { ability }\end{array}$ & $\begin{array}{c}\mathrm{p}- \\
\text { value }\end{array}$ \\
\hline 337 & 8.20 & 1,343 & 11,010 & 7821622 & 3.678 & 1.000 & 0 & 9.52 & 1,157 & 11,010 & 6568862 & 1.846 & .968 & .032 \\
\hline 338 & 9.68 & 597 & 5,777 & 1732927 & .208 & .583 & .417 & 10.24 & 564 & 5,777 & 1662481 & .843 & .800 & .200 \\
\hline 339 & 4.85 & 1,002 & 4,859 & 2401168 & -.748 & .227 & .227 & 5.48 & 887 & 4,859 & 2148892 & -.145 & .442 & .442 \\
\hline 340 & 5.70 & 1,094 & 6,240 & 3269078 & -2.420 & .008 & .008 & 7.01 & 890 & 6,240 & 2717310 & -1.107 & .134 & .134 \\
\hline 341 & 11.63 & 837 & 9,737 & 4133253 & .717 & .763 & .237 & 14.28 & 682 & 9,737 & 3334791 & .197 & .578 & .422 \\
\hline 342 & 15.71 & 261 & 4,100 & 483218 & -2.711 & .003 & .003 & 17.67 & 232 & 4,100 & 449743 & -1.434 & .076 & .076 \\
\hline 343 & 6.21 & 2,044 & 12,702 & 13108308 & .765 & .778 & .222 & 7.45 & 1,705 & 12,702 & 10959430 & .865 & .806 & .194 \\
\hline 344 & 6.14 & 5,608 & 34,428 & 99496558 & 3.978 & 1.000 & 0 & 7.36 & 4,679 & 34,428 & 83353313 & 4.132 & 1.000 & 0 \\
\hline 345 & 7.35 & 66 & 485 & 15754 & -.221 & .413 & .413 & 8.08 & 60 & 485 & 14017 & -.492 & .312 & .312 \\
\hline 346 & 7.87 & 2,734 & 21,528 & 28552038 & -2.698 & .003 & .003 & 9.88 & 2,180 & 21,528 & 22741716 & -2.495 & .006 & .006 \\
\hline 347 & 7.74 & 154 & 1,192 & 88942 & -.666 & .253 & .253 & 8.83 & 135 & 1,192 & 74359 & -1.526 & .064 & .064 \\
\hline 348 & 7.71 & 1,449 & 11,166 & 7884827 & -1.670 & .047 & .047 & 8.97 & 1,245 & 11,166 & 6789591 & -1.418 & .078 & .078 \\
\hline 349 & 9.24 & 1,208 & 11,161 & 6666977 & -.663 & .254 & .254 & 10.33 & 1,080 & 11,161 & 5974216 & -.498 & .309 & .309 \\
\hline 350 & 8.49 & 3,321 & 28,183 & 48181272 & 2.951 & .998 & .002 & 9.23 & 3,055 & 28,183 & 44161717 & 2.473 & .993 & .007 \\
\hline 351 & 8.63 & 448 & 3,865 & 919259 & 2.265 & .988 & .012 & 9.74 & 397 & 3,865 & 804178 & 1.663 & .952 & .048 \\
\hline 352 & 26.55 & 40 & 1,062 & 17490 & -1.934 & .027 & .027 & 26.55 & 40 & 1,062 & 17490 & -1.934 & .027 & .027 \\
\hline 353 & 6.80 & 479 & 3,257 & 794005 & .678 & .751 & .249 & 7.16 & 455 & 3,257 & 754487 & .674 & .750 & .250 \\
\hline 354 & 5.73 & 1,201 & 6,876 & 4183513 & .792 & .786 & .214 & 6.89 & 998 & 6,876 & 3507086 & 1.211 & .887 & .113 \\
\hline 355 & 6.30 & 1,772 & 11,170 & 10093865 & 1.453 & .927 & .073 & 7.53 & 1,484 & 11,170 & 8456194 & 1.353 & .912 & .088 \\
\hline 356 & 9.10 & 3,934 & 35,793 & 71815173 & 2.176 & .985 & .015 & 11.63 & 3,078 & 35,793 & 55372956 & .502 & .692 & .308 \\
\hline 357 & 7.18 & 4,398 & 31,587 & 70919601 & 2.414 & .992 & .008 & 8.44 & 3,742 & 31,587 & 60008639 & 1.630 & .948 & .052 \\
\hline 358 & 7.86 & 2,402 & 18,884 & 23399851 & 2.696 & .996 & .004 & 9.53 & 1,981 & 18,884 & 18899407 & .803 & .789 & .211 \\
\hline 359 & 8.32 & 267 & 2,220 & 301823 & .521 & .699 & .301 & 9.82 & 226 & 2,220 & 257409 & .680 & .752 & .248 \\
\hline 360 & 8.32 & 87 & 724 & 30375 & -.574 & .283 & .283 & 12.93 & 56 & 724 & 18708 & -1.000 & .159 & .159 \\
\hline 361 & 7.90 & 19 & 150 & 1821 & 2.098 & .982 & .018 & 9.38 & 16 & 150 & 1589 & 2.246 & .988 & .012 \\
\hline 362 & 6.76 & 3,139 & 21,229 & 36219356 & 8.448 & 0 & 0 & 7.58 & 2,800 & 21,229 & 30688157 & 2.984 & .999 & .001 \\
\hline 363 & 5.61 & 390 & 2,188 & 436566 & .794 & .786 & .214 & 6.45 & 339 & 2,188 & 376893 & .518 & .698 & .302 \\
\hline 364 & 5.56 & 1,724 & 9,588 & 7937535 & -2.848 & .002 & .002 & 6.45 & 1,486 & 9,588 & 6984916 & -1.303 & .096 & .096 \\
\hline 365 & 5.64 & 3,954 & 22,280 & 41909221 & -5.287 & 0 & 0 & 6.40 & 3,482 & 22,280 & 38063220 & -1.914 & .028 & .028 \\
\hline 366 & 8.33 & 303 & 2,525 & 380234 & -.182 & .428 & .428 & 8.59 & 294 & 2,525 & 375816 & .371 & .645 & .355 \\
\hline 367 & 5.57 & 2,072 & 11,543 & 12095035 & .900 & .816 & .184 & 6.56 & 1,760 & 11,543 & 10287152 & .925 & .822 & .178 \\
\hline 368 & 9.50 & 887 & 8,426 & 3538742 & -2.736 & .003 & .003 & 11.48 & 734 & 8,426 & 2975146 & -1.778 & .038 & .038 \\
\hline 369 & 15.38 & 8 & 123 & 394 & -.976 & .165 & .165 & 24.60 & 5 & 123 & 183 & -1.568 & .058 & .058 \\
\hline 370 & 9.71 & 673 & 6,537 & 2088248 & -2.277 & .011 & .011 & 12.08 & 541 & 6,537 & 1636186 & -3.009 & .001 & .001 \\
\hline 371 & 12.02 & 1,640 & 19,719 & 16985834 & 3.541 & 1.000 & 0 & 16.90 & 1,167 & 19,719 & 12144243 & 3.282 & .999 & .001 \\
\hline 372 & 8.92 & 914 & 8,156 & 3917278 & 2.669 & .996 & .004 & 10.29 & 793 & 8,156 & 3334811 & 1.523 & .936 & .064 \\
\hline 373 & 7.15 & 26 & 186 & 2259 & -.581 & .281 & .281 & 8.46 & 22 & 186 & 1821 & -.893 & .186 & .186 \\
\hline 374 & 10.41 & 84 & 874 & 41371 & 2.017 & .978 & .022 & 11.97 & 73 & 874 & 35500 & 1.670 & .952 & .048 \\
\hline 375 & 10.62 & 1,010 & 10,721 & 5421803 & .078 & .531 & .469 & 11.58 & 926 & 10,721 & 5043199 & .843 & .800 & .200 \\
\hline 376 & 9.18 & 317 & 2,910 & 461103 & -.009 & .496 & .496 & 10.51 & 277 & 2,910 & 410350 & .523 & .700 & .300 \\
\hline 377 & 7.01 & 2,590 & 18,150 & 24169990 & 2.497 & .994 & .006 & 8.07 & 2,248 & 18,150 & 21098962 & 2.811 & .998 & .002 \\
\hline 378 & 6.50 & 2,236 & 14,540 & 16544334 & 1.454 & .927 & .073 & 7.31 & 1,990 & 14,540 & 14619347 & .812 & .792 & .208 \\
\hline 379 & 7.24 & 25 & 181 & 2256 & -.025 & .490 & .490 & 8.62 & 21 & 181 & 1845 & -.232 & .408 & .408 \\
\hline 380 & 6.32 & 226 & 1,428 & 179967 & 3.002 & .999 & .001 & 7.48 & 191 & 1,428 & 155136 & 3.293 & 1.000 & 0 \\
\hline 381 & 5.85 & 3,250 & 18,998 & 31134458 & .840 & .800 & .200 & 6.62 & 2,868 & 18,998 & 27546026 & 1.031 & .849 & .151 \\
\hline 382 & 5.59 & 53 & 296 & 7865 & .034 & .514 & .487 & 6.30 & 47 & 296 & 7059 & .176 & .570 & .430 \\
\hline 383 & 9.52 & 3,409 & 32,450 & 51961671 & -6.124 & 0 & 0 & 11.58 & 2,803 & 32,450 & 43901647 & -3.180 & .001 & .001 \\
\hline 384 & 8.34 & 980 & 8,168 & 4398390 & 5.366 & 0 & 0 & 10.06 & 812 & 8,168 & 3545129 & 3.407 & 1.000 & 0 \\
\hline
\end{tabular}


Table 3. Summary of interoccurrence intervals for daily precipitation thresholds of 0.05 and 0.10 inch-Continued

\begin{tabular}{|c|c|c|c|c|c|c|c|c|c|c|c|c|c|c|}
\hline \multirow[b]{2}{*}{$\begin{array}{l}\text { Seq. } \\
\text { no. }\end{array}$} & \multicolumn{7}{|c|}{ Daily precipitation threshold of 0.05 inch and greater } & \multicolumn{7}{|c|}{ Daily precipitation threshold of 0.10 inch and greater } \\
\hline & $\begin{array}{c}\text { Mean } \\
\text { inter- } \\
\text { occur- } \\
\text { rence } \\
\text { interval } \\
\text { (days) }\end{array}$ & $\begin{array}{c}\text { Total } \\
\text { no. of } \\
\text { events }\end{array}$ & $\begin{array}{l}\text { Total } \\
\text { no. of } \\
\text { days } \\
\text { avail- } \\
\text { able }\end{array}$ & $\begin{array}{l}\text { Sigma } \\
\mathrm{S}\end{array}$ & $\begin{array}{l}\text { Stan- } \\
\text { dard } \\
\text { normal } \\
\text { variate }\end{array}$ & $\begin{array}{l}\text { Non- } \\
\text { ex- } \\
\text { ceed- } \\
\text { ance } \\
\text { prob- } \\
\text { ability }\end{array}$ & $\begin{array}{c}\text { p- } \\
\text { value }\end{array}$ & $\begin{array}{c}\text { Mean } \\
\text { inter- } \\
\text { occur- } \\
\text { rence } \\
\text { interval } \\
\text { (days) }\end{array}$ & $\begin{array}{c}\text { Total } \\
\text { no. of } \\
\text { events }\end{array}$ & $\begin{array}{l}\text { Total } \\
\text { no. of } \\
\text { days } \\
\text { avail- } \\
\text { able }\end{array}$ & $\begin{array}{c}\text { Sigma } \\
\mathrm{S}\end{array}$ & $\begin{array}{c}\text { Stan- } \\
\text { dard } \\
\text { normal } \\
\text { variate }\end{array}$ & $\begin{array}{l}\text { Non- } \\
\text { ex- } \\
\text { ceed- } \\
\text { ance } \\
\text { prob- } \\
\text { ability }\end{array}$ & $\begin{array}{c}\text { p- } \\
\text { value }\end{array}$ \\
\hline 385 & 5.92 & 2,911 & 17,222 & 25934335 & 3.235 & .999 & .001 & 6.74 & 2,556 & 17,222 & 22349606 & 1.352 & .912 & .088 \\
\hline 386 & 30.50 & 8 & 244 & 872 & -.522 & .301 & .301 & 30.50 & 8 & 244 & 872 & -.522 & .301 & .301 \\
\hline 387 & 6.85 & 304 & 2,081 & 295890 & -1.950 & .026 & .026 & 7.91 & 263 & 2,081 & 262473 & -1.147 & .126 & .126 \\
\hline 388 & 5.98 & 412 & 2,464 & 481568 & -1.802 & .036 & .036 & 7.31 & 337 & 2,464 & 390689 & -1.876 & .030 & .030 \\
\hline 389 & 7.56 & 48 & 363 & 10048 & 1.840 & .967 & .033 & 9.31 & 39 & 363 & 8683 & 2.452 & .993 & .007 \\
\hline 390 & 4.64 & 3,770 & 17,476 & 33801214 & 2.773 & .997 & .003 & 5.27 & 3,318 & 17,476 & 29624965 & 2.176 & .985 & .015 \\
\hline 391 & 7.26 & 2,200 & 15,972 & 17929177 & 1.665 & .952 & .048 & 8.13 & 1,964 & 15,972 & 16173342 & 2.392 & .992 & .008 \\
\hline 392 & 16.16 & 64 & 1,034 & 21549 & -4.832 & 0 & 0 & 16.95 & 61 & 1,034 & 20938 & -4.546 & 0 & 0 \\
\hline 393 & 14.37 & 737 & 10,594 & 4142698 & 2.876 & .998 & .002 & 19.44 & 545 & 10,594 & 3084394 & 2.767 & .997 & .003 \\
\hline 394 & 10.33 & 3 & 31 & 57 & .677 & .751 & .249 & 10.33 & 3 & 31 & 57 & .677 & .751 & .249 \\
\hline 395 & 8.41 & 1,084 & 9,115 & 5128513 & 2.172 & .985 & .015 & 9.16 & 995 & 9,115 & 4671060 & 1.643 & .950 & .050 \\
\hline 396 & 5.79 & 3,404 & 19,696 & 35121644 & 4.820 & 1.000 & 0 & 6.64 & 2,965 & 19,696 & 30142683 & 3.047 & .999 & .001 \\
\hline 397 & 8.27 & 1,723 & 14,241 & 12053165 & -1.263 & .103 & .103 & 10.81 & 1,318 & 14,241 & 9164896 & -1.474 & .070 & .070 \\
\hline 398 & 7.83 & 4,320 & 33,819 & 73720577 & 1.047 & .852 & .148 & 9.69 & 3,490 & 33,819 & 59062162 & .083 & .533 & .467 \\
\hline 399 & 8.42 & 2,329 & 19,618 & 22345905 & -1.827 & .034 & .034 & 9.20 & 2,132 & 19,618 & 20585838 & -1.250 & .106 & .106 \\
\hline 400 & 6.24 & 3,091 & 19,271 & 29692201 & -.295 & .384 & .384 & 6.90 & 2,792 & 19,271 & 26820201 & -.279 & .390 & .390 \\
\hline 401 & 8.88 & 651 & 5,779 & 1693507 & -4.406 & 0 & 0 & 10.07 & 574 & 5,779 & 1516933 & -3.544 & 0 & 0 \\
\hline 402 & 17.36 & 14 & 243 & 2079 & 1.440 & .925 & .075 & 18.69 & 13 & 243 & 1935 & 1.406 & .920 & .080 \\
\hline 403 & 9.74 & 1,389 & 13,535 & 9536779 & .939 & .826 & .174 & 9.90 & 1,367 & 13,535 & 9381743 & .904 & .817 & .183 \\
\hline 404 & 6.09 & 3,385 & 20,610 & 35618279 & 2.126 & .983 & .017 & 7.03 & 2,932 & 20,610 & 30848521 & 1.969 & .976 & .024 \\
\hline 405 & 7.98 & 1,490 & 11,884 & 9202875 & 2.638 & .996 & .004 & 9.65 & 1,231 & 11,884 & 7673318 & 2.980 & .999 & .001 \\
\hline 406 & 6.79 & 3,194 & 21,685 & 35943593 & 3.710 & 1.000 & 0 & 7.41 & 2,927 & 21,685 & 32662902 & 2.737 & .997 & .003 \\
\hline 407 & 6.11 & 607 & 3,708 & 1103908 & -.814 & .208 & .208 & 7.00 & 530 & 3,708 & 967264 & -.623 & .267 & .267 \\
\hline 408 & 5.63 & 6,061 & 34,142 & 102954806 & -.668 & .252 & .252 & 6.74 & 5,065 & 34,142 & 86392209 & -.103 & .459 & .459 \\
\hline 409 & 8.88 & 137 & 1,216 & 78220 & -1.235 & .108 & .108 & 11.69 & 104 & 1,216 & 60234 & -.838 & .201 & .201 \\
\hline 410 & 5.52 & 2,610 & 14,398 & 18938720 & .703 & .759 & .241 & 6.26 & 2,300 & 14,398 & 16629974 & .363 & .642 & .358 \\
\hline 411 & 9.33 & 2,051 & 19,141 & 20425136 & 3.181 & .999 & .001 & 10.25 & 1,868 & 19,141 & 18157829 & 1.173 & .880 & .120 \\
\hline 412 & 6.98 & 1,992 & 13,897 & 12743916 & -6.130 & 0 & 0 & 7.55 & 1,840 & 13,897 & 11907597 & -5.100 & 0 & 0 \\
\hline 413 & 8.11 & 3,720 & 30,162 & 58952845 & 5.370 & 0 & 0 & 9.09 & 3,317 & 30,162 & 51839380 & 3.621 & 1.000 & 0 \\
\hline 414 & 8.11 & 422 & 3,423 & 678878 & -2.137 & .016 & .016 & 8.94 & 383 & 3,423 & 627076 & -1.470 & .071 & .071 \\
\hline 415 & 8.57 & 2,507 & 21,490 & 27363506 & 1.371 & .915 & .085 & 10.00 & 2,149 & 21,490 & 23461919 & 1.290 & .901 & .099 \\
\hline 416 & 8.92 & 2,169 & 19,352 & 21983447 & 3.829 & 1.000 & 0 & 10.54 & 1,837 & 19,352 & 18422659 & 2.706 & .997 & .003 \\
\hline 417 & 10.38 & 296 & 3,072 & 467708 & .856 & .804 & .196 & 10.49 & 293 & 3,072 & 463373 & .878 & .810 & .190 \\
\hline 418 & 7.78 & 3,204 & 24,925 & 40606359 & 1.661 & .952 & .048 & 9.15 & 2,724 & 24,925 & 34995356 & 2.789 & .997 & .003 \\
\hline 419 & 6.20 & 3,654 & 22,658 & 42041703 & 1.633 & .949 & .051 & 7.31 & 3,098 & 22,658 & 35633462 & 1.473 & .930 & .070 \\
\hline 420 & 10.07 & 1,900 & 19,138 & 19063883 & 3.666 & 1.000 & 0 & 11.63 & 1,645 & 19,138 & 16177156 & 1.947 & .974 & .026 \\
\hline 421 & 8.59 & 3,630 & 31,191 & 59044277 & 4.484 & 1.000 & 0 & 10.52 & 2,965 & 31,191 & 46506311 & .542 & .706 & .294 \\
\hline 422 & 12.46 & 930 & 11,589 & 5181008 & -2.038 & .021 & .021 & 15.25 & 760 & 11,589 & 4266480 & -1.489 & .068 & .068 \\
\hline 423 & 10.14 & 1,144 & 11,596 & 6981870 & 3.082 & .999 & .001 & 12.19 & 951 & 11,596 & 5745008 & 2.239 & .987 & .013 \\
\hline 424 & 19.36 & 11 & 213 & 1637 & 2.283 & .989 & .011 & 21.30 & 10 & 213 & 1441 & 1.934 & .973 & .027 \\
\hline 425 & 10.43 & 2,038 & 21,262 & 21829990 & .592 & .723 & .277 & 13.32 & 1,596 & 21,262 & 17095989 & .526 & .700 & .300 \\
\hline 426 & 10.65 & 1,677 & 17,867 & 15581263 & 2.840 & .998 & .002 & 12.37 & 1,445 & 17,867 & 13280828 & 1.897 & .971 & .029 \\
\hline 427 & -- & -- & -- & -- & -- & -- & -- & -- & -- & -- & -- & -- & -- & -- \\
\hline 428 & 6.35 & 756 & 4,798 & 1823386 & .256 & .601 & .399 & 7.85 & 611 & 4,798 & 1481255 & .452 & .674 & .326 \\
\hline 429 & 5.99 & 903 & 5,412 & 2489988 & .990 & .839 & .161 & 7.19 & 753 & 5,412 & 2092659 & 1.284 & .900 & .100 \\
\hline 430 & 6.19 & 1,441 & 8,915 & 6596774 & 1.776 & .962 & .038 & 7.40 & 1,205 & 8,915 & 5559217 & 2.104 & .982 & .018 \\
\hline 431 & 6.10 & 632 & 3,856 & 1261069 & 1.521 & .936 & .064 & 7.28 & 530 & 3,856 & 1068017 & 1.802 & .964 & .036 \\
\hline 432 & 4.95 & 1,755 & 8,688 & 7684641 & .580 & .719 & .281 & 5.87 & 1,481 & 8,688 & 6517539 & .871 & .808 & .192 \\
\hline
\end{tabular}


Table 3. Summary of interoccurrence intervals for daily precipitation thresholds of 0.05 and 0.10 inch-Continued

\begin{tabular}{|c|c|c|c|c|c|c|c|c|c|c|c|c|c|c|}
\hline \multirow[b]{2}{*}{$\begin{array}{c}\text { Seq. } \\
\text { no. }\end{array}$} & \multicolumn{7}{|c|}{ Daily precipitation threshold of 0.05 inch and greater } & \multicolumn{7}{|c|}{ Daily precipitation threshold of 0.10 inch and greater } \\
\hline & $\begin{array}{c}\text { Mean } \\
\text { inter- } \\
\text { occur- } \\
\text { rence } \\
\text { interval } \\
\text { (days) }\end{array}$ & $\begin{array}{l}\text { Total } \\
\text { no. of } \\
\text { events }\end{array}$ & $\begin{array}{c}\text { Total } \\
\text { no. of } \\
\text { days } \\
\text { avail- } \\
\text { able }\end{array}$ & $\begin{array}{l}\text { Sigma } \\
\mathbf{S}\end{array}$ & $\begin{array}{c}\text { Stan- } \\
\text { dard } \\
\text { normal } \\
\text { variate }\end{array}$ & $\begin{array}{l}\text { Non- } \\
\text { ex- } \\
\text { ceed- } \\
\text { ance } \\
\text { prob- } \\
\text { ability }\end{array}$ & $\begin{array}{c}\text { p- } \\
\text { value }\end{array}$ & $\begin{array}{c}\text { Mean } \\
\text { inter- } \\
\text { occur- } \\
\text { rence } \\
\text { interval } \\
\text { (days) }\end{array}$ & $\begin{array}{c}\text { Total } \\
\text { no. of } \\
\text { events }\end{array}$ & $\begin{array}{l}\text { Total } \\
\text { no. of } \\
\text { days } \\
\text { avail- } \\
\text { able }\end{array}$ & $\begin{array}{l}\text { Sigma } \\
\mathrm{S}\end{array}$ & $\begin{array}{l}\text { Stan- } \\
\text { dard } \\
\text { normal } \\
\text { variate }\end{array}$ & $\begin{array}{l}\text { Non- } \\
\text { ex- } \\
\text { ceed- } \\
\text { ance } \\
\text { prob- } \\
\text { ability }\end{array}$ & $\begin{array}{c}\mathrm{p}- \\
\text { value }\end{array}$ \\
\hline 433 & 11.02 & 2,483 & 27,354 & 35035394 & 2.733 & .997 & .003 & 12.22 & 2,239 & 27,354 & 30829285 & 0.553 & .710 & 290 \\
\hline 434 & 5.61 & 2,423 & 13,597 & 16796612 & 1.676 & .953 & .047 & 6.48 & 2,099 & 13,597 & 14591611 & 1.788 & .963 & .037 \\
\hline 435 & 5.97 & 173 & 1,033 & 93934 & 1.168 & .878 & .122 & 6.98 & 148 & 1,033 & 81631 & 1.430 & .924 & .076 \\
\hline 436 & 6.73 & 4,320 & 29,054 & 64772922 & 3.658 & 1.000 & 0 & 8.27 & 3,515 & 29,054 & 52488064 & 2.867 & .998 & .002 \\
\hline 437 & 5.27 & 4,805 & 25,322 & 64203096 & 6.645 & 0 & 0 & 6.15 & 4,118 & 25,322 & 54317885 & 4.647 & 1.000 & 0 \\
\hline 438 & 9.25 & 2,018 & 18,671 & 19520280 & 2.814 & .998 & .002 & 10.69 & 1,746 & 18,671 & 16944966 & 2.865 & .998 & .002 \\
\hline 439 & 11.45 & 713 & 8,164 & 2809997 & -1.597 & .055 & .055 & 12.58 & 649 & 8,164 & 2634055 & -.253 & .400 & .400 \\
\hline 440 & 8.69 & 1,070 & 9,302 & 4856664 & -1.365 & .086 & .086 & 10.62 & 876 & 9,302 & 4115555 & .519 & .698 & .302 \\
\hline 441 & 8.32 & 2,851 & 23,733 & 35269673 & 3.932 & 1.000 & 0 & 10.49 & 2,263 & 23,733 & 27893982 & 3.191 & .999 & .001 \\
\hline 442 & 5.67 & 2,252 & 12,769 & 14615282 & 1.357 & .913 & .087 & 6.69 & 1,908 & 12,769 & 12281691 & .622 & .733 & .267 \\
\hline 443 & 6.67 & 1,948 & 12,987 & 12919924 & 1.635 & .949 & .051 & 7.71 & 1,685 & 12,987 & 11024389 & .538 & .705 & .295 \\
\hline 444 & 8.36 & 193 & 1,613 & 160307 & .719 & .764 & .236 & 10.02 & 161 & 1,613 & 137716 & 1.332 & .909 & .091 \\
\hline 445 & 9.92 & 1,929 & 19,140 & 18080622 & -1.566 & .059 & .059 & 11.52 & 1,662 & 19,140 & 15944196 & .173 & .569 & .431 \\
\hline 446 & 7.31 & 161 & 1,177 & 93220 & -.355 & .361 & .361 & 8.47 & 139 & 1,177 & 75597 & -1.549 & .061 & .061 \\
\hline 447 & 10.51 & 2,183 & 22,938 & 26354449 & 4.259 & 1.000 & 0 & 11.81 & 1,942 & 22,938 & 23330714 & 3.625 & 1.000 & 0 \\
\hline 448 & 6.16 & 5,117 & 31,527 & 81649011 & 1.516 & .935 & .065 & 7.21 & 4,371 & 31,527 & 69264648 & .602 & .726 & .274 \\
\hline 449 & 5.45 & 975 & 5,317 & 2453983 & -2.881 & .002 & .002 & 6.48 & 821 & 5,317 & 2072508 & -2.504 & .006 & .006 \\
\hline 450 & 5.43 & 3,783 & 20,533 & 39278356 & 1.207 & .886 & .114 & 6.55 & 3,137 & 20,533 & 32792318 & 1.766 & .961 & .039 \\
\hline 451 & 5.41 & 1,029 & 5,562 & 2853160 & -.165 & .435 & .435 & 6.69 & 831 & 5,562 & 2308663 & -.051 & .480 & .480 \\
\hline 452 & 9.21 & 33 & 304 & 4696 & -.635 & .263 & .263 & 12.16 & 25 & 304 & 3689 & -.253 & .400 & .400 \\
\hline 453 & 10.57 & 199 & 2,103 & 217097 & .917 & .820 & .180 & 12.30 & 171 & 2,103 & 186433 & .835 & .798 & .202 \\
\hline 454 & 10.27 & 3,094 & 31,784 & 47533589 & -3.206 & .001 & .001 & 11.87 & 2,677 & 31,784 & 41219179 & -2.788 & .003 & .003 \\
\hline 455 & 8.37 & 106 & 887 & 38463 & -3.243 & .001 & .001 & 10.08 & 88 & 887 & 31080 & -3.309 & 0 & 0 \\
\hline 456 & 33.50 & 2 & 67 & 99 & 1.170 & .879 & .121 & 33.50 & 2 & 67 & 99 & 1.170 & .879 & .121 \\
\hline 457 & 6.20 & 55 & 341 & 10632 & 1.718 & .957 & .043 & 7.75 & 44 & 341 & 8745 & 1.904 & .972 & .028 \\
\hline 458 & 5.63 & 1,105 & 6,223 & 3241535 & -3.294 & 0 & 0 & 6.06 & 1,027 & 6,223 & 3085325 & -1.914 & .028 & .028 \\
\hline 459 & 10.68 & 105 & 1,121 & 61435 & .779 & .782 & .218 & 10.78 & 104 & 1,121 & 60725 & .737 & .769 & .231 \\
\hline 460 & 7.02 & 4,660 & 32,732 & 79989628 & 5.774 & 0 & 0 & 7.91 & 4,136 & 32,732 & 70496807 & 4.619 & 1.000 & 0 \\
\hline 461 & 7.30 & 4,215 & 30,785 & 66579608 & 2.947 & .998 & .002 & 8.87 & 3,472 & 30,785 & 53823516 & .727 & .766 & .234 \\
\hline 462 & 6.02 & 2,947 & 17,740 & 27063505 & 3.322 & 1.000 & 0 & 7.31 & 2,426 & 17,740 & 22307132 & 3.126 & .999 & .001 \\
\hline 463 & 5.49 & 1,363 & 7,477 & 5149739 & .680 & .752 & .248 & 6.74 & 1,109 & 7,477 & 4234013 & 1.225 & .890 & .110 \\
\hline 464 & 5.53 & 4,067 & 22,500 & 45975778 & .536 & .704 & .296 & 6.66 & 3,379 & 22,500 & 38457493 & 1.175 & .880 & .120 \\
\hline 465 & 7.99 & 246 & 1,966 & 233164 & -.972 & .166 & .166 & 8.82 & 223 & 1,966 & 208411 & -1.274 & .101 & .101 \\
\hline 466 & 5.12 & 5,123 & 26,233 & 68884919 & 3.116 & .999 & .001 & 5.97 & 4,391 & 26,233 & 58958580 & 2.718 & .997 & .003 \\
\hline 467 & 11.48 & 964 & 11,063 & 5890540 & 5.629 & 0 & 0 & 14.04 & 788 & 11,063 & 5014684 & 7.316 & 0 & 0 \\
\hline 468 & 7.64 & 130 & 993 & 49616 & -4.568 & 0 & 0 & 8.14 & 122 & 993 & 47990 & -3.974 & 0 & 0 \\
\hline 469 & 5.61 & 1,751 & 9,821 & 9026413 & 3.609 & 1.000 & 0 & 6.13 & 1,603 & 9,821 & 8256584 & 3.392 & 1.000 & 0 \\
\hline 470 & 7.00 & 13 & 91 & 540 & -.544 & .293 & .293 & 7.58 & 12 & 91 & 505 & -.451 & .326 & .326 \\
\hline 471 & 5.99 & 2,236 & 13,388 & 15126801 & .870 & .808 & .192 & 7.16 & 1,871 & 13,388 & 12747968 & 1.337 & .909 & .091 \\
\hline 472 & 6.47 & 521 & 3,372 & 887894 & .427 & .665 & .335 & 6.85 & 492 & 3,372 & 842035 & .580 & .719 & .281 \\
\hline 473 & 7.55 & 2,548 & 19,226 & 24073941 & -1.499 & .067 & .067 & 8.37 & 2,298 & 19,226 & 22067888 & -.086 & .466 & .466 \\
\hline 474 & 7.06 & 3,565 & 25,176 & 45470501 & 1.370 & .915 & .085 & 8.36 & 3,010 & 25,176 & 38465351 & 1.443 & .926 & .074 \\
\hline 475 & 6.02 & 5,305 & 31,959 & 86022332 & 1.862 & .969 & .031 & 7.36 & 4,345 & 31,959 & 70159701 & 1.198 & .885 & .115 \\
\hline 476 & 9.36 & 1,948 & 18,237 & 17759145 & -.016 & .494 & .494 & 9.40 & 1,941 & 18,237 & 17699577 & .003 & .501 & .499 \\
\hline 477 & 5.78 & 727 & 4,201 & 1531522 & .136 & .554 & .446 & 7.82 & 537 & 4,201 & 1137125 & .326 & .628 & .372 \\
\hline 478 & 5.97 & 3,864 & 23,063 & 45047131 & 1.183 & .881 & .119 & 7.41 & 3,113 & 23,063 & 36369499 & 1.271 & .898 & .102 \\
\hline 479 & 6.87 & 261 & 1,792 & 230921 & -.351 & .363 & .363 & 7.59 & 236 & 1,792 & 203318 & -1.024 & .153 & .153 \\
\hline 480 & 7.29 & 780 & 5,684 & 2125702 & -1.987 & .023 & .023 & 8.67 & 656 & 5,684 & 1806988 & -1.365 & .086 & .086 \\
\hline
\end{tabular}


Table 3. Summary of interoccurrence intervals for daily precipitation thresholds of 0.05 and 0.10 inch-Continued

\begin{tabular}{|c|c|c|c|c|c|c|c|c|c|c|c|c|c|c|}
\hline \multirow[b]{2}{*}{$\begin{array}{l}\text { Seq. } \\
\text { no. }\end{array}$} & \multicolumn{7}{|c|}{ Daily precipitation threshold of 0.05 inch and greater } & \multicolumn{7}{|c|}{ Daily precipitation threshold of 0.10 inch and greater } \\
\hline & $\begin{array}{c}\text { Mean } \\
\text { inter- } \\
\text { occur- } \\
\text { rence } \\
\text { interval } \\
\text { (days) }\end{array}$ & $\begin{array}{c}\text { Total } \\
\text { no. of } \\
\text { events }\end{array}$ & $\begin{array}{l}\text { Total } \\
\text { no. of } \\
\text { days } \\
\text { avail- } \\
\text { able }\end{array}$ & $\begin{array}{l}\text { Sigma } \\
\mathrm{S}\end{array}$ & $\begin{array}{l}\text { Stan- } \\
\text { dard } \\
\text { normal } \\
\text { variate }\end{array}$ & $\begin{array}{l}\text { Non- } \\
\text { ex- } \\
\text { ceed- } \\
\text { ance } \\
\text { prob- } \\
\text { ability }\end{array}$ & $\begin{array}{c}\text { p- } \\
\text { value }\end{array}$ & $\begin{array}{c}\text { Mean } \\
\text { inter- } \\
\text { occur- } \\
\text { rence } \\
\text { interval } \\
\text { (days) }\end{array}$ & $\begin{array}{c}\text { Total } \\
\text { no. of } \\
\text { events }\end{array}$ & $\begin{array}{l}\text { Total } \\
\text { no. of } \\
\text { days } \\
\text { avail- } \\
\text { able }\end{array}$ & $\begin{array}{c}\text { Sigma } \\
\mathrm{S}\end{array}$ & $\begin{array}{c}\text { Stan- } \\
\text { dard } \\
\text { normal } \\
\text { variate }\end{array}$ & $\begin{array}{l}\text { Non- } \\
\text { ex- } \\
\text { ceed- } \\
\text { ance } \\
\text { prob- } \\
\text { ability }\end{array}$ & $\begin{array}{c}\text { p- } \\
\text { value }\end{array}$ \\
\hline 481 & 5.28 & 2,395 & 12,650 & 14287808 & -4.815 & 0 & 0 & 6.03 & 2,097 & 12,650 & 12739801 & -3.132 & .001 & .001 \\
\hline 482 & 6.65 & 558 & 3,709 & 1038839 & .159 & .563 & .437 & 7.71 & 481 & 3,709 & 871324 & -.881 & .189 & .189 \\
\hline 483 & 6.30 & 504 & 3,174 & 806846 & .340 & 633 & .367 & 7.18 & 442 & 3,174 & 718477 & .884 & .812 & .189 \\
\hline 484 & 7.30 & 375 & 2,736 & 494289 & -1.223 & .111 & .111 & 8.02 & 341 & 2,736 & 454807 & -.801 & .212 & .212 \\
\hline 485 & 10.73 & 79 & 848 & 32456 & -.478 & .316 & .316 & 10.87 & 78 & 848 & 31987 & -.502 & .308 & .308 \\
\hline 486 & 7.24 & 4,805 & 34,779 & 87400256 & 5.523 & 0 & 0 & 8.27 & 4,207 & 34,779 & 75213655 & 3.157 & .999 & .001 \\
\hline 487 & 6.43 & 1,631 & 10,484 & 8666470 & .955 & .830 & .170 & 7.82 & 1,340 & 10,484 & 7035699 & .103 & .541 & .459 \\
\hline 488 & 13.29 & 2,117 & 28,138 & 32177361 & 6.404 & 0 & 0 & 15.85 & 1,775 & 28,138 & 26190483 & 3.559 & 1.000 & 0 \\
\hline 489 & 6.00 & 1,939 & 11,627 & 10969306 & -2.051 & .020 & .020 & 7.19 & 1,618 & 11,627 & 9209384 & -1.458 & .072 & .072 \\
\hline 490 & 5.82 & 1,341 & 7,802 & 5242592 & .138 & .555 & .445 & 7.17 & 1,088 & 7,802 & 4260400 & .217 & .586 & .414 \\
\hline 491 & 5.89 & 2,168 & 12,770 & 14762067 & 5.356 & 0 & 0 & 6.37 & 2,004 & 12,770 & 13622912 & 5.014 & 0 & 0 \\
\hline 492 & 6.27 & 3,999 & 25,088 & 52908423 & 5.994 & 0 & 0 & 7.41 & 3,385 & 25,088 & 44032540 & 3.729 & 1.000 & 0 \\
\hline 493 & 5.57 & 6,401 & 35,661 & 117976991 & 4.667 & 1.000 & 0 & 6.43 & 5,549 & 35,661 & 101310569 & 3.089 & .999 & .001 \\
\hline 494 & 5.31 & 803 & 4,263 & 1831505 & 3.439 & 1.000 & 0 & 6.45 & 661 & 4,263 & 1499354 & 2.858 & .998 & .002 \\
\hline 495 & 5.20 & 3,914 & 20,344 & 42540308 & 7.422 & 0 & 0 & 5.68 & 3,582 & 20,344 & 38906851 & 7.029 & 0 & 0 \\
\hline 496 & 7.99 & 2,698 & 21,557 & 29750654 & 2.074 & .981 & .019 & 9.76 & 2,208 & 21,557 & 24260922 & 1.580 & .943 & .057 \\
\hline 497 & 8.15 & 100 & 815 & 41703 & .405 & .657 & .343 & 12.16 & 67 & 815 & 29834 & 1.315 & .906 & .094 \\
\hline 498 & 5.91 & 3,227 & 19,069 & 30788296 & .065 & .526 & .474 & 6.93 & 2,750 & 19,069 & 26673646 & 1.572 & .942 & .058 \\
\hline 499 & 8.50 & 2,088 & 17,748 & 19833245 & 5.571 & 0 & 0 & 9.86 & 1,801 & 17,748 & 16821663 & 3.862 & 1.000 & 0 \\
\hline 500 & 7.18 & 161 & 1,156 & 94426 & .323 & .627 & .373 & 8.50 & 136 & 1,156 & 78567 & -.011 & .496 & .496 \\
\hline 501 & 11.19 & 457 & 5,112 & 1210178 & 1.334 & .909 & .091 & 11.73 & 436 & 5,112 & 1146169 & 1.031 & .849 & .151 \\
\hline 502 & 5.67 & 5,393 & 30,570 & 82435112 & .005 & .502 & .498 & 6.36 & 4,805 & 30,570 & 73663606 & .358 & .640 & .360 \\
\hline 503 & 10.95 & 559 & 6,120 & 1692714 & -.427 & .335 & .335 & 12.22 & 501 & 6,120 & 1508864 & -.612 & .270 & .270 \\
\hline 504 & 12.83 & 287 & 3,683 & 481599 & -2.605 & .005 & .005 & 13.95 & 264 & 3,683 & 446129 & -2.317 & .010 & .010 \\
\hline 505 & 7.76 & 138 & 1,071 & 62103 & -3.248 & .001 & .001 & 9.40 & 114 & 1,071 & 53040 & -2.426 & .008 & .008 \\
\hline 506 & 5.94 & 6,292 & 37,401 & 121673241 & 4.682 & 1.000 & 0 & 6.84 & 5,468 & 37,401 & 103974024 & 2.154 & .984 & .016 \\
\hline 507 & 4.81 & 290 & 1,396 & 212376 & 1.451 & .927 & .073 & 5.79 & 241 & 1,396 & 181392 & 2.106 & .982 & .018 \\
\hline 508 & 7.25 & 2,191 & 15,882 & 18174434 & 3.615 & 1.000 & 0 & 8.48 & 1,874 & 15,882 & 15221268 & 1.712 & .957 & .043 \\
\hline 509 & 8.35 & 3,057 & 25,519 & 41526727 & 6.189 & 0 & 0 & 10.12 & 2,522 & 25,519 & 33373519 & 3.228 & .999 & .001 \\
\hline 510 & 9.08 & 231 & 2,098 & 219770 & -2.450 & .007 & .007 & 10.49 & 200 & 2,098 & 202304 & -.875 & .191 & .191 \\
\hline 511 & 5.59 & 3,327 & 18,591 & 29696250 & -3.973 & 0 & 0 & 6.10 & 3,048 & 18,591 & 27456009 & -2.959 & .002 & .002 \\
\hline 512 & 7.14 & 4,418 & 31,532 & 70995558 & 2.217 & .987 & .013 & 8.89 & 3,546 & 31,532 & 55772266 & -.247 & .402 & .402 \\
\hline 513 & 8.03 & 2,653 & 21,306 & 30081213 & 5.741 & 0 & 0 & 9.20 & 2,315 & 21,306 & 25813209 & 3.891 & 1.000 & 0 \\
\hline 514 & 6.33 & 6 & 38 & 123 & .335 & .631 & .369 & 9.50 & 4 & 38 & 100 & 1.094 & .863 & .137 \\
\hline 515 & 9.49 & 1,695 & 16,084 & 14612138 & 5.132 & 0 & 0 & 11.19 & 1,437 & 16,084 & 11944875 & 2.207 & .986 & .014 \\
\hline 516 & 10.70 & 257 & 2,751 & 314392 & -3.072 & .001 & .001 & 13.16 & 209 & 2,751 & 262679 & -2.160 & .015 & .015 \\
\hline 517 & 8.62 & 2,269 & 19,560 & 21798092 & -1.460 & .072 & .072 & 10.42 & 1,878 & 19,560 & 18053331 & -1.281 & .100 & .100 \\
\hline 518 & 7.67 & 4,785 & 36,688 & 90590977 & 3.842 & 1.000 & 0 & 9.07 & 4,047 & 36,688 & 75400166 & 1.725 & .958 & .042 \\
\hline 519 & 8.00 & 964 & 7,716 & 3725421 & .091 & .536 & .464 & 9.91 & 779 & 7,716 & 3002316 & -.049 & .480 & .480 \\
\hline 520 & 6.91 & 97 & 670 & 32837 & .180 & .571 & .429 & 9.05 & 74 & 670 & 25511 & .433 & .668 & .332 \\
\hline 521 & 6.54 & 2,726 & 17,822 & 24290440 & -.004 & .499 & .499 & 7.15 & 2,494 & 17,822 & 22310965 & .338 & .632 & .368 \\
\hline 522 & 9.37 & 790 & 7,402 & 2908071 & -.262 & .397 & .397 & 10.57 & 700 & 7,402 & 2536526 & -.958 & .169 & .169 \\
\hline 523 & 9.35 & 407 & 3,804 & 848821 & 3.372 & 1.000 & 0 & 10.20 & 373 & 3,804 & 764670 & 2.604 & .995 & .005 \\
\hline 524 & 12.36 & 197 & 2,435 & 223244 & -1.683 & .046 & .046 & 15.31 & 159 & 2,435 & 183267 & -1.164 & .122 & .122 \\
\hline 525 & -- & -- & -- & -- & -- & -- & -- & -- & -- & -- & -- & -- & -- & -- \\
\hline 526 & 9.18 & 3,547 & 32,561 & 62440897 & 8.385 & 0 & 0 & 10.92 & 2,983 & 32,561 & 51015510 & 4.774 & 1.000 & 0 \\
\hline 527 & 4.69 & 1,852 & 8,692 & 8171566 & 1.137 & .872 & .128 & 5.41 & 1,607 & 8,692 & 7039442 & .551 & .709 & .291 \\
\hline 528 & 4.70 & 672 & 3,157 & 1037024 & -1.004 & .158 & .158 & 5.32 & 593 & 3,157 & 934966 & -.049 & .480 & .480 \\
\hline
\end{tabular}


Table 3. Summary of interoccurrence intervals for daily precipitation thresholds of 0.05 and 0.10 inch-Continued

\begin{tabular}{|c|c|c|c|c|c|c|c|c|c|c|c|c|c|c|}
\hline \multirow[b]{2}{*}{$\begin{array}{l}\text { Seq. } \\
\text { no. }\end{array}$} & \multicolumn{7}{|c|}{ Daily precipitation threshold of 0.05 inch and greater } & \multicolumn{7}{|c|}{ Daily precipitation threshold of 0.10 inch and greater } \\
\hline & $\begin{array}{c}\text { Mean } \\
\text { inter- } \\
\text { occur- } \\
\text { rence } \\
\text { interval } \\
\text { (days) }\end{array}$ & $\begin{array}{c}\text { Total } \\
\text { no. of } \\
\text { events }\end{array}$ & $\begin{array}{l}\text { Total } \\
\text { no. of } \\
\text { days } \\
\text { avail- } \\
\text { able }\end{array}$ & $\begin{array}{l}\text { Sigma } \\
\mathrm{S}\end{array}$ & $\begin{array}{c}\text { Stan- } \\
\text { dard } \\
\text { normal } \\
\text { variate }\end{array}$ & $\begin{array}{l}\text { Non- } \\
\text { ex- } \\
\text { ceed- } \\
\text { ance } \\
\text { prob- } \\
\text { ability }\end{array}$ & $\begin{array}{c}\mathrm{p}- \\
\text { value }\end{array}$ & $\begin{array}{l}\text { Mean } \\
\text { inter- } \\
\text { occur- } \\
\text { rence } \\
\text { interval } \\
\text { (days) }\end{array}$ & $\begin{array}{l}\text { Total } \\
\text { no. of } \\
\text { events }\end{array}$ & $\begin{array}{l}\text { Total } \\
\text { no. of } \\
\text { days } \\
\text { avail- } \\
\text { able }\end{array}$ & $\begin{array}{l}\text { Sigma } \\
\text { S }\end{array}$ & $\begin{array}{c}\text { Stan- } \\
\text { dard } \\
\text { normal } \\
\text { variate }\end{array}$ & $\begin{array}{l}\text { Non- } \\
\text { ex- } \\
\text { ceed- } \\
\text { ance } \\
\text { prob- } \\
\text { ability }\end{array}$ & $\begin{array}{c}\text { p- } \\
\text { value }\end{array}$ \\
\hline 529 & 5.79 & 2,000 & 11,583 & 12025927 & 2.962 & .998 & .002 & 6.71 & 1,727 & 1,1583 & 10331470 & 2.372 & .991 & .009 \\
\hline 530 & 5.22 & 6,542 & 34,124 & 114122703 & 3.142 & .999 & .001 & 6.01 & 5,680 & 34,124 & 99659956 & 3.701 & 1.000 & 0 \\
\hline 531 & 10.41 & 611 & 6,363 & 1883719 & -1.325 & .093 & .093 & 10.48 & 607 & 6,363 & 1876869 & -1.200 & .115 & .115 \\
\hline 532 & 6.73 & 5,356 & 36,056 & 100075132 & 4.617 & 1.000 & 0 & 7.96 & 4,531 & 36,056 & 82972372 & 1.838 & .967 & .033 \\
\hline 533 & 8.53 & 3,018 & 25,757 & 40443197 & 3.858 & 1.000 & 0 & 10.60 & 2,431 & 25,757 & 31875316 & 1.549 & .939 & .061 \\
\hline 534 & 7.59 & 130 & 986 & 58643 & -1.678 & .047 & .047 & 9.57 & 103 & 986 & 47536 & -1.123 & .131 & .131 \\
\hline 535 & 6.50 & 6,459 & 41,954 & 140816640 & 5.472 & 0 & 0 & 7.63 & 5,496 & 41,954 & 119517359 & 4.709 & 1.000 & 0 \\
\hline 536 & 10.07 & 15 & 151 & 1189 & .335 & .631 & .369 & 10.07 & 15 & 151 & 1189 & .335 & .631 & .369 \\
\hline 537 & 6.48 & 4,918 & 31,864 & 82542081 & 6.493 & 0 & 0 & 7.71 & 4,134 & 31,864 & 67678948 & 3.071 & .999 & .001 \\
\hline 538 & 10.70 & 214 & 2,290 & 232468 & -1.299 & .097 & .097 & 12.87 & 178 & 2,290 & 200071 & -.424 & .336 & .336 \\
\hline 539 & 7.68 & 2,569 & 19,726 & 25990843 & 2.262 & .988 & .012 & 8.94 & 2,206 & 19,726 & 22380292 & 2.328 & .990 & .010 \\
\hline 540 & 7.79 & 214 & 1,666 & 179557 & .184 & .573 & .427 & 8.46 & 197 & 1,666 & 171808 & 1.142 & .873 & .127 \\
\hline 541 & 6.05 & 5,790 & 35,035 & 103810184 & 3.098 & .999 & .001 & 7.09 & 4,940 & 35,035 & 88413582 & 2.641 & .996 & .004 \\
\hline 542 & 8.71 & 136 & 1,184 & 67218 & -3.335 & 0 & 0 & 11.72 & 101 & 1184 & 51183 & -2.506 & .006 & .006 \\
\hline 543 & 9.69 & 864 & 8,373 & 3645829 & .404 & .657 & .343 & 11.13 & 752 & 8373 & 3130913 & -.262 & .397 & .397 \\
\hline 544 & 6.58 & 4,461 & 29,330 & 67663272 & 3.966 & 1.000 & 0 & 8.24 & 3,561 & 29,330 & 53041648 & 1.622 & .948 & .052 \\
\hline 545 & 6.83 & 1,230 & 8,397 & 4974119 & -2.235 & .013 & .013 & 8.48 & 990 & 8,397 & 4014609 & -1.861 & .031 & .031 \\
\hline 546 & 5.51 & 5,701 & 31,403 & 90540761 & 1.500 & .933 & .067 & 6.06 & 5,182 & 31,403 & 82075337 & 1.088 & .862 & .138 \\
\hline 547 & 7.32 & 2,411 & 17,650 & 21272853 & -.017 & .493 & .493 & 8.94 & 1,974 & 17,650 & 17518892 & .434 & .668 & .332 \\
\hline 548 & 4.74 & 2,766 & 13,112 & 17739848 & -1.980 & .024 & .024 & 5.56 & 2,357 & 13,112 & 15325187 & -.693 & .244 & .244 \\
\hline 549 & 15.42 & 57 & 879 & 26349 & .677 & .751 & .249 & 19.53 & 45 & 879 & 20043 & .156 & .562 & .438 \\
\hline 550 & 5.62 & 149 & 838 & 59591 & -.962 & .168 & .168 & 7.29 & 115 & 838 & 45532 & -1.023 & .153 & .153 \\
\hline 551 & 5.78 & 950 & 5,486 & 2682809 & 1.577 & .943 & .057 & 6.96 & 788 & 5,486 & 2230157 & 1.545 & .939 & .061 \\
\hline 552 & 5.39 & 3,631 & 19,567 & 37175303 & 4.852 & 1.000 & 0 & 6.30 & 3,107 & 19,567 & 31641222 & 3.951 & 1.000 & 0 \\
\hline 553 & 3.90 & 39 & 152 & 2529 & -1.588 & .056 & .056 & 5.07 & 30 & 152 & 2010 & -1.123 & .131 & .131 \\
\hline 554 & 4.66 & 2,552 & 11,901 & 15118744 & -.386 & .350 & .350 & 5.63 & 2,114 & 11,901 & 12551407 & -.177 & .430 & .430 \\
\hline 555 & 4.32 & 34 & 147 & 1953 & -2.207 & .014 & .014 & 5.25 & 28 & 147 & 1830 & -1.015 & .155 & .155 \\
\hline 556 & 4.61 & 4,337 & 19,994 & 44291741 & 2.459 & .993 & .007 & 5.41 & 3,696 & 19,994 & 38124332 & 3.350 & 1.000 & 0 \\
\hline 557 & 7.41 & 37 & 274 & 5026 & -.089 & .464 & .464 & 7.41 & 37 & 274 & 5026 & -.089 & .464 & .464 \\
\hline 558 & 8.59 & 248 & 2,129 & 250944 & -1.349 & .089 & .089 & 8.83 & 241 & 2,129 & 245290 & -1.180 & .119 & .119 \\
\hline 559 & 4.75 & 123 & 584 & 36188 & .146 & .558 & .442 & 5.78 & 101 & 584 & 29249 & -.143 & .443 & .443 \\
\hline 560 & 4.44 & 55 & 244 & 6631 & -.151 & .440 & .440 & 5.08 & 48 & 244 & 6011 & .318 & .625 & .375 \\
\hline 561 & 4.73 & 3,763 & 17,792 & 33622959 & .468 & .680 & .320 & 5.42 & 3,283 & 17,792 & 29400805 & .663 & .746 & .254 \\
\hline 562 & 4.78 & 4,328 & 20,691 & 45135744 & .917 & .820 & .180 & 5.64 & 3,668 & 20,691 & 38514719 & 1.569 & .942 & .058 \\
\hline 563 & 5.30 & 3,190 & 16,893 & 26854026 & -.328 & .371 & .371 & 5.87 & 2,879 & 16,893 & 24302906 & -.056 & .478 & .478 \\
\hline 564 & 4.90 & 3,871 & 18,979 & 37963366 & 3.607 & 1.000 & 0 & 5.83 & 3,255 & 18,979 & 32106503 & 3.897 & 1.000 & 0 \\
\hline 565 & 4.89 & 1,001 & 4,897 & 2542650 & 2.050 & .980 & .020 & 5.69 & 861 & 4,897 & 2176138 & 1.639 & .949 & .051 \\
\hline 566 & 4.63 & 3,233 & 14,981 & 24845157 & 2.555 & .995 & .005 & 5.60 & 2,677 & 14,981 & 20428808 & 1.684 & .954 & .046 \\
\hline 567 & 6.79 & 1,295 & 8,792 & 6385569 & 7.585 & 0 & 0 & 7.96 & 1,105 & 8,792 & 5420278 & 6.670 & 0 & 0 \\
\hline 568 & 4.58 & 3,455 & 15,815 & 27945778 & 2.330 & .990 & .010 & 5.46 & 2,899 & 15,815 & 23415530 & 2.000 & .977 & .023 \\
\hline 569 & 4.95 & 1,861 & 9,218 & 8476843 & -.876 & .191 & .191 & 6.08 & 1,517 & 9,218 & 6900217 & -.884 & .188 & .188 \\
\hline 570 & 4.84 & 752 & 3,637 & 1336581 & -1.074 & .141 & .141 & 5.48 & 664 & 3,637 & 1183777 & -.876 & .191 & .191 \\
\hline 571 & 4.85 & 3,738 & 18,133 & 34724450 & 2.606 & .995 & .005 & 5.86 & 3,097 & 18,133 & 28938052 & 2.949 & .998 & .002 \\
\hline 572 & 7.36 & 1,736 & 12,769 & 11806536 & 4.708 & 1.000 & 0 & 8.24 & 1,550 & 12,769 & 10276351 & 2.621 & .996 & .004 \\
\hline 573 & 10.27 & 491 & 5,041 & 1248230 & .331 & .630 & .370 & 12.39 & 407 & 5,041 & 1043534 & .603 & .727 & .273 \\
\hline 574 & 4.82 & 2,316 & 11,163 & 13078969 & .982 & .837 & .163 & 5.82 & 1,918 & 11,163 & 10660549 & -.317 & .376 & .376 \\
\hline 575 & 11.18 & 1,566 & 17,501 & 12832301 & -4.357 & 0 & 0 & 11.81 & 1,482 & 17,501 & 12271536 & -3.582 & 0 & 0 \\
\hline 576 & 7.01 & 516 & 3,617 & 917177 & -.675 & .250 & .250 & 8.26 & 438 & 3,617 & 778170 & -.639 & .262 & .262 \\
\hline
\end{tabular}


Table 3. Summary of interoccurrence intervals for daily precipitation thresholds of 0.05 and 0.10 inch-Continued

\begin{tabular}{|c|c|c|c|c|c|c|c|c|c|c|c|c|c|c|}
\hline \multirow[b]{2}{*}{$\begin{array}{l}\text { Seq. } \\
\text { no. }\end{array}$} & \multicolumn{7}{|c|}{ Daily precipitation threshold of 0.05 inch and greater } & \multicolumn{7}{|c|}{ Daily precipitation threshold of 0.10 inch and greater } \\
\hline & $\begin{array}{c}\text { Mean } \\
\text { inter- } \\
\text { occur- } \\
\text { rence } \\
\text { interval } \\
\text { (days) }\end{array}$ & $\begin{array}{c}\text { Total } \\
\text { no. of } \\
\text { events }\end{array}$ & $\begin{array}{c}\text { Total } \\
\text { no. of } \\
\text { days } \\
\text { avail- } \\
\text { able }\end{array}$ & $\begin{array}{l}\text { Sigma } \\
\mathrm{S}\end{array}$ & $\begin{array}{l}\text { Stan- } \\
\text { dard } \\
\text { normal } \\
\text { variate }\end{array}$ & $\begin{array}{l}\text { Non- } \\
\text { ex- } \\
\text { ceed- } \\
\text { ance } \\
\text { prob- } \\
\text { ability }\end{array}$ & $\begin{array}{c}\text { p- } \\
\text { value }\end{array}$ & $\begin{array}{c}\text { Mean } \\
\text { inter- } \\
\text { occur- } \\
\text { rence } \\
\text { interval } \\
\text { (days) }\end{array}$ & $\begin{array}{c}\text { Total } \\
\text { no. of } \\
\text { events }\end{array}$ & $\begin{array}{c}\text { Total } \\
\text { no. of } \\
\text { days } \\
\text { avail- } \\
\text { able }\end{array}$ & $\begin{array}{c}\text { Sigma } \\
\mathrm{S}\end{array}$ & $\begin{array}{c}\text { Stan- } \\
\text { dard } \\
\text { normal } \\
\text { variate }\end{array}$ & $\begin{array}{l}\text { Non- } \\
\text { ex- } \\
\text { ceed- } \\
\text { ance } \\
\text { prob- } \\
\text { ability }\end{array}$ & $\begin{array}{c}\text { p- } \\
\text { value }\end{array}$ \\
\hline 577 & 8.11 & 2,291 & 18,568 & 22369794 & 4.288 & 1.000 & 0 & 9.07 & 2,047 & 18,568 & 19740937 & 3.037 & .999 & .001 \\
\hline 578 & 4.67 & 4,316 & 20,145 & 43673566 & .525 & .700 & .300 & 5.66 & 3,560 & 20,145 & 36192633 & .964 & .832 & .168 \\
\hline 579 & 6.12 & 2,304 & 14,090 & 16553757 & 1.650 & .950 & .050 & 7.11 & 1,981 & 14,090 & 14308716 & 1.948 & .974 & .026 \\
\hline 580 & 6.08 & 12 & 73 & 431 & -.096 & .462 & .462 & 6.64 & 11 & 73 & 425 & .336 & .632 & .368 \\
\hline 581 & 4.15 & 570 & 2,368 & 668593 & -.385 & .350 & .350 & 4.80 & 493 & 2368 & 570481 & -.872 & .192 & .192 \\
\hline 582 & 8.07 & 2,291 & 18,495 & 22465943 & 5.009 & 0 & 0 & 9.03 & 2,048 & 18,495 & 19806131 & 3.589 & 1.000 & 0 \\
\hline 583 & 18.64 & 868 & 16,182 & 7226285 & 1.477 & .930 & .070 & 19.88 & 814 & 16,182 & 6873872 & 2.159 & .985 & .015 \\
\hline 584 & 4.21 & 58 & 244 & 6886 & -.354 & .362 & .362 & 4.88 & 50 & 244 & 6109 & .018 & .507 & .493 \\
\hline 585 & 7.27 & 1,807 & 13,130 & 12671531 & 5.018 & 0 & 0 & 8.57 & 1,533 & 13,130 & 10431632 & 2.476 & .993 & .007 \\
\hline 586 & 27.47 & 15 & 412 & 2245 & -1.834 & .033 & .033 & 34.33 & 12 & 412 & 1747 & -1.760 & .039 & .039 \\
\hline 587 & 7.77 & 470 & 3,651 & 846503 & -.503 & .308 & .308 & 8.61 & 424 & 3,651 & 761997 & -.554 & .290 & .290 \\
\hline 588 & 7.20 & 1,188 & 8,550 & 4914022 & -1.936 & .026 & .026 & 8.53 & 1,002 & 8,550 & 4118850 & -2.108 & .018 & .018 \\
\hline 589 & 5.26 & 1,246 & 6,553 & 4241848 & 2.386 & .991 & .009 & 6.42 & 1,021 & 6,553 & 3495247 & 2.481 & .993 & .007 \\
\hline 590 & 7.71 & 48 & 370 & 10258 & 1.862 & .969 & .031 & 7.71 & 48 & 370 & 10258 & 1.862 & .969 & .031 \\
\hline 591 & 5.52 & 662 & 3,653 & 1125843 & -3.070 & .001 & .001 & 6.50 & 562 & 3,653 & 980452 & -1.842 & .033 & .033 \\
\hline 592 & 6.77 & 3,263 & 22,076 & 36445404 & 1.177 & .880 & .120 & 7.92 & 2,787 & 22,076 & 31000267 & .706 & .760 & .240 \\
\hline 593 & 5.45 & 2,849 & 15,514 & 23173951 & 4.494 & 1.000 & 0 & 6.20 & 2,503 & 15,514 & 20520400 & 4.930 & 1.000 & 0 \\
\hline 594 & 4.96 & 1,184 & 5,874 & 3553176 & 1.299 & .903 & .097 & 6.01 & 977 & 5,874 & 2918104 & .918 & .821 & .179 \\
\hline 595 & 9.07 & 1,017 & 9,226 & 4729962 & .454 & .675 & .325 & 9.82 & 940 & 9,226 & 4329183 & -.086 & .466 & .466 \\
\hline 596 & 6.12 & 4,202 & 25,710 & 54810738 & 1.650 & .951 & .049 & 7.09 & 3,624 & 25,710 & 47037944 & 1.010 & .844 & .156 \\
\hline 597 & 4.87 & 4,226 & 20,570 & 45469168 & 5.193 & 0 & 0 & 5.53 & 3,720 & 20,570 & 39581091 & 3.647 & 1.000 & 0 \\
\hline 598 & 5.03 & 208 & 1,047 & 92073 & -3.858 & 0 & 0 & 5.75 & 182 & 1,047 & 79120 & -3.963 & 0 & 0 \\
\hline 599 & 9.71 & 1,822 & 17,699 & 16268078 & .662 & .746 & .254 & 10.17 & 1,741 & 17,699 & 15763885 & 1.674 & .953 & .047 \\
\hline 600 & 6.17 & 3,166 & 19,532 & 32996124 & 6.547 & 0 & 0 & 7.39 & 2,643 & 19,532 & 27051244 & 4.277 & 1.000 & 0 \\
\hline 601 & 5.02 & 6,440 & 32,319 & 106588024 & 3.367 & 1.000 & 0 & 5.66 & 5,709 & 32,319 & 94093221 & 2.608 & .995 & .005 \\
\hline 602 & 6.13 & 2,508 & 15,379 & 20120283 & 3.756 & 1.000 & 0 & 6.84 & 2,250 & 15,379 & 17862728 & 2.666 & .996 & .004 \\
\hline 603 & 5.31 & 1,173 & 6,226 & 3729436 & 1.265 & .897 & .103 & 6.33 & 983 & 6,226 & 3107968 & .850 & .802 & .198 \\
\hline 604 & 6.24 & 1,967 & 12,277 & 12169249 & .603 & .727 & .273 & 7.41 & 1,656 & 12,277 & 10243761 & .544 & .707 & .293 \\
\hline 605 & 8.10 & 767 & 6,210 & 2439304 & 1.164 & .878 & .122 & 9.57 & 649 & 6,210 & 2096660 & 1.785 & .963 & .037 \\
\hline 606 & 10.03 & 525 & 5,266 & 1404962 & .650 & .742 & .258 & 11.47 & 459 & 5,266 & 1222867 & .440 & .670 & .330 \\
\hline 607 & 9.48 & 411 & 3,895 & 808196 & .341 & .633 & .367 & 11.03 & 353 & 3,895 & 694360 & .326 & .628 & .372 \\
\hline 608 & 8.12 & 2,605 & 21,162 & 29312477 & 5.609 & 0 & 0 & 9.71 & 2,180 & 21,162 & 24009611 & 3.306 & 1.000 & 0 \\
\hline 609 & 14.26 & 203 & 2,895 & 262526 & -2.630 & .004 & .004 & 17.23 & 168 & 2,895 & 216010 & -2.508 & .006 & .006 \\
\hline 610 & 8.35 & 3,653 & 30,487 & 54999369 & -1.288 & .099 & .099 & 9.50 & 3,210 & 30,487 & 47559552 & -2.752 & .003 & .003 \\
\hline 611 & 7.86 & 1,046 & 8,219 & 4478117 & 2.340 & .990 & .010 & 10.10 & 814 & 8,219 & 3440202 & 1.404 & .920 & .080 \\
\hline 612 & 9.12 & 396 & 3,610 & 684079 & -1.480 & .069 & .069 & 11.01 & 328 & 3,610 & 581711 & -.547 & .292 & .292 \\
\hline 613 & 10.44 & 66 & 689 & 21817 & -.569 & .285 & .285 & 11.48 & 60 & 689 & 19635 & -.672 & .251 & .251 \\
\hline 614 & 9.69 & 346 & 3,354 & 578314 & -.107 & .457 & .457 & 11.73 & 286 & 3,354 & 471356 & -.505 & .307 & .307 \\
\hline 615 & 7.63 & 8 & 61 & 236 & -.161 & .436 & .436 & 8.71 & 7 & 61 & 223 & .204 & .581 & .419 \\
\hline 616 & 9.94 & 189 & 1,878 & 165890 & -1.554 & .060 & .060 & 11.11 & 169 & 1,878 & 149166 & -1.352 & .088 & .088 \\
\hline 617 & 6.69 & 262 & 1,753 & 201961 & -3.380 & 0 & 0 & 6.93 & 253 & 1,753 & 194012 & -3.447 & 0 & 0 \\
\hline 618 & 4.99 & 4,133 & 20,642 & 42947125 & .758 & .776 & .224 & 5.77 & 3,575 & 20,642 & 37338693 & 1.238 & .892 & .108 \\
\hline 619 & 7.29 & 3,903 & 28,443 & 55973136 & .910 & .818 & .182 & 8.34 & 3,412 & 28,443 & 47988867 & -1.115 & .132 & .132 \\
\hline 620 & 6.51 & 107 & 697 & 37658 & .177 & .570 & .430 & 7.74 & 90 & 697 & 31765 & .210 & .583 & .417 \\
\hline 621 & 5.33 & 3,095 & 16,498 & 26117002 & 2.213 & .987 & .013 & 6.28 & 2,628 & 16,498 & 22409196 & 2.993 & .999 & .001 \\
\hline 622 & 5.84 & 6,194 & 36,185 & 110456864 & -1.956 & .025 & .025 & 6.74 & 5,371 & 36,185 & 95539437 & -2.136 & .016 & .016 \\
\hline 623 & 6.81 & 1,081 & 7,364 & 4057369 & 1.104 & .865 & .135 & 8.00 & 921 & 7,364 & 3437883 & .725 & .766 & .234 \\
\hline 624 & 7.34 & 265 & 1,944 & 252111 & -.599 & .275 & .275 & 7.87 & 247 & 1,944 & 228750 & -1.285 & .099 & .099 \\
\hline
\end{tabular}


Table 3. Summary of interoccurrence intervals for daily precipitation thresholds of 0.05 and 0.10 inch-Continued

\begin{tabular}{|c|c|c|c|c|c|c|c|c|c|c|c|c|c|c|}
\hline \multirow[b]{2}{*}{$\begin{array}{c}\text { Seq. } \\
\text { no. }\end{array}$} & \multicolumn{7}{|c|}{ Daily precipitation threshold of 0.05 inch and greater } & \multicolumn{7}{|c|}{ Daily precipitation threshold of 0.10 inch and greater } \\
\hline & $\begin{array}{c}\text { Mean } \\
\text { inter- } \\
\text { occur- } \\
\text { rence } \\
\text { interval } \\
\text { (days) }\end{array}$ & $\begin{array}{c}\text { Total } \\
\text { no. of } \\
\text { events }\end{array}$ & $\begin{array}{c}\text { Total } \\
\text { no. of } \\
\text { days } \\
\text { avail- } \\
\text { able }\end{array}$ & $\begin{array}{l}\text { Sigma } \\
\mathbf{S}\end{array}$ & $\begin{array}{c}\text { Stan- } \\
\text { dard } \\
\text { normal } \\
\text { variate }\end{array}$ & $\begin{array}{l}\text { Non- } \\
\text { ex- } \\
\text { ceed- } \\
\text { ance } \\
\text { prob- } \\
\text { ability }\end{array}$ & $\begin{array}{c}\mathbf{p}- \\
\text { value }\end{array}$ & $\begin{array}{c}\text { Mean } \\
\text { inter- } \\
\text { occur- } \\
\text { rence } \\
\text { interval } \\
\text { (days) }\end{array}$ & $\begin{array}{c}\text { Total } \\
\text { no. of } \\
\text { events }\end{array}$ & $\begin{array}{l}\text { Total } \\
\text { no. of } \\
\text { days } \\
\text { avail- } \\
\text { able }\end{array}$ & $\begin{array}{l}\text { Sigma } \\
\text { S }\end{array}$ & $\begin{array}{l}\text { Stan- } \\
\text { dard } \\
\text { normal } \\
\text { variate }\end{array}$ & $\begin{array}{l}\text { Non- } \\
\text { ex- } \\
\text { ceed- } \\
\text { ance } \\
\text { prob- } \\
\text { ability }\end{array}$ & $\begin{array}{c}\mathrm{p}- \\
\text { value }\end{array}$ \\
\hline 625 & 6.60 & 1,510 & 9,960 & 7884995 & 3.269 & .999 & .001 & 8.26 & 1,206 & 9,960 & 6246594 & 2.411 & .992 & .008 \\
\hline 626 & 7.24 & 1,495 & 10,827 & 8073364 & -.164 & .435 & .435 & 8.13 & 1,332 & 10,827 & 7185174 & -.225 & .411 & .411 \\
\hline 627 & 10.53 & 1,123 & 11,821 & 6851917 & 1.875 & .970 & .030 & 12.54 & 943 & 11,821 & 5940725 & 3.503 & 1.000 & 0 \\
\hline 628 & 8.60 & 582 & 5,003 & 1370446 & -2.452 & .007 & .007 & 10.78 & 464 & 5,003 & 1091438 & -2.226 & .013 & .013 \\
\hline 629 & 5.87 & 4,507 & 26,452 & 61517362 & 3.722 & 1.000 & 0 & 7.51 & 3,523 & 26,452 & 47339847 & 1.643 & .950 & .050 \\
\hline 630 & 5.94 & 1,670 & 9,923 & 8172530 & -.967 & .167 & .167 & 7.50 & 1,323 & 9,923 & 6480972 & -.798 & .213 & .213 \\
\hline 631 & 6.79 & 1,604 & 10,894 & 8969681 & 1.848 & .968 & .032 & 7.88 & 1,382 & 10,894 & 7732516 & 1.752 & .960 & .040 \\
\hline 632 & 5.77 & 1,380 & 7,960 & 5551979 & .698 & .757 & .243 & 6.89 & 1,155 & 7,960 & 4640067 & .553 & .710 & .290 \\
\hline 633 & 6.38 & 267 & 1,702 & 209905 & -2.156 & .016 & .016 & 7.06 & 241 & 1702 & 197091 & -1.049 & .147 & .147 \\
\hline 634 & 11.15 & 26 & 290 & 3556 & -.501 & .308 & .308 & 11.60 & 25 & 290 & 3326 & -.714 & .238 & .238 \\
\hline 635 & 8.69 & 3,089 & 26,842 & 45266631 & 8.845 & 0 & 0 & 10.23 & 2,625 & 26,842 & 37534052 & 5.803 & 0 & 0 \\
\hline 636 & 4.45 & 4,660 & 20,742 & 46871130 & -3.566 & 0 & 0 & 5.17 & 4,015 & 20,742 & 40533774 & -2.915 & .002 & .002 \\
\hline 637 & 10.33 & 2,363 & 24,410 & 29136034 & .863 & .806 & .194 & 11.91 & 2,049 & 24,410 & 25565614 & 1.748 & .960 & .040 \\
\hline 638 & 8.07 & 1,377 & 11,115 & 7841056 & 1.582 & .943 & .057 & 9.52 & 1,167 & 11,115 & 6645593 & 1.460 & .928 & .072 \\
\hline 639 & 6.36 & 821 & 5,224 & 2233257 & 2.055 & .980 & .020 & 7.39 & 707 & 5,224 & 1884622 & .946 & .828 & .172 \\
\hline 640 & 4.35 & 1,348 & 5,865 & 4107494 & 2.485 & .994 & .006 & 4.97 & 1,181 & 5,865 & 3627940 & 2.830 & .998 & .002 \\
\hline 641 & 7.04 & 170 & 1,196 & 101108 & -.123 & .451 & .451 & 8.79 & 136 & 1,196 & 78441 & -.717 & .237 & .237 \\
\hline 642 & 5.96 & 49 & 292 & 8161 & 1.707 & .956 & .044 & 7.68 & 38 & 292 & 5936 & .747 & .772 & .228 \\
\hline 643 & 5.57 & 5,894 & 32,853 & 96258687 & -.768 & .221 & .221 & 6.66 & 4,933 & 32,853 & 82382726 & 2.028 & .979 & .021 \\
\hline 644 & 10.35 & 239 & 2,473 & 311846 & 1.479 & .930 & .070 & 13.37 & 185 & 2,473 & 242329 & 1.398 & .919 & .081 \\
\hline 645 & 8.84 & 3,103 & 27,442 & 41784993 & -1.793 & .036 & .036 & 11.04 & 2,485 & 27,442 & 34045296 & -.130 & .448 & .448 \\
\hline 646 & 13.59 & 1,524 & 20,715 & 15447951 & -1.443 & .075 & .075 & 17.94 & 1,155 & 20,715 & 11964485 & .008 & .503 & .497 \\
\hline 647 & 7.32 & 124 & 907 & 62628 & 2.193 & .986 & .014 & 8.48 & 107 & 907 & 54683 & 2.274 & .989 & .011 \\
\hline 648 & 12.34 & 155 & 1,913 & 124845 & -3.405 & 0 & 0 & 12.34 & 155 & 1,913 & 124845 & -3.405 & 0 & 0 \\
\hline 649 & 12.59 & 649 & 8,170 & 2509365 & -2.360 & .009 & .009 & 15.99 & 511 & 8,170 & 1923587 & -3.073 & .001 & .001 \\
\hline 650 & 7.56 & 1,903 & 14,391 & 13923930 & 1.274 & .899 & .101 & 8.97 & 1,605 & 14,391 & 11604868 & .337 & .632 & .368 \\
\hline 651 & 8.73 & 321 & 2,802 & 452378 & .183 & .573 & .427 & 11.08 & 253 & 2,802 & 349962 & -.349 & .364 & .364 \\
\hline 652 & 6.94 & 115 & 798 & 47791 & .772 & .780 & .220 & 9.07 & 88 & 798 & 36852 & .805 & .790 & .210 \\
\hline 653 & 6.15 & 176 & 1,083 & 99418 & .992 & .839 & .161 & 7.27 & 149 & 1,083 & 84491 & .998 & .841 & .159 \\
\hline 654 & 9.13 & 1,134 & 10,351 & 6092101 & 2.217 & .987 & .013 & 10.88 & 951 & 10,351 & 5099434 & 1.927 & .973 & .027 \\
\hline 655 & 7.69 & 372 & 2,861 & 498435 & -2.116 & .017 & .017 & 8.80 & 325 & 2,861 & 444301 & -1.384 & .083 & .083 \\
\hline 656 & 4.95 & 959 & 4,743 & 2247306 & -.636 & .262 & .262 & 6.01 & 789 & 4,743 & 1862818 & -.216 & .415 & .415 \\
\hline 657 & 7.50 & 1,547 & 11,595 & 9036042 & .511 & .695 & .305 & 8.76 & 1,324 & 11,595 & 7720889 & .370 & .644 & .356 \\
\hline 658 & 7.88 & 727 & 5,729 & 2138127 & 1.248 & .894 & .106 & 9.30 & 616 & 5,729 & 1809837 & 1.104 & .865 & .135 \\
\hline 659 & 6.59 & 421 & 2,776 & 581895 & -.149 & .441 & .441 & 7.80 & 356 & 2,776 & 484084 & -.664 & .253 & .253 \\
\hline 660 & 5.20 & 924 & 4,805 & 2250051 & .715 & .763 & .237 & 6.04 & 795 & 4,805 & 1938316 & .724 & .765 & .235 \\
\hline 661 & 5.13 & 552 & 2,832 & 791513 & .514 & .696 & .304 & 6.25 & 453 & 2,832 & 643472 & .116 & .546 & .454 \\
\hline 662 & 9.62 & 571 & 5,490 & 1626886 & 1.571 & .942 & .058 & 10.06 & 546 & 5,490 & 1549071 & 1.358 & .913 & .087 \\
\hline 663 & 9.55 & 3,417 & 32,622 & 56317146 & 1.058 & .855 & .145 & 11.45 & 2,850 & 32,622 & 46231575 & -.507 & .306 & .306 \\
\hline 664 & 6.40 & 5,794 & 37,084 & 109984292 & 3.132 & .999 & .001 & 7.76 & 4,779 & 37,084 & 90422418 & 2.446 & .993 & .007 \\
\hline 665 & 11.86 & 1,587 & 18,826 & 15190727 & 1.165 & .878 & .122 & 14.54 & 1,295 & 18,826 & 12114610 & -.385 & .350 & .350 \\
\hline 666 & 12.03 & 108 & 1,299 & 77533 & 1.896 & .971 & .029 & 15.46 & 84 & 1,299 & 58488 & 1.144 & .874 & .126 \\
\hline 667 & 9.08 & 1,507 & 13,689 & 10217158 & -.636 & .263 & .263 & 11.51 & 1,189 & 13,689 & 7983827 & -1.132 & .129 & .129 \\
\hline 668 & 10.12 & 767 & 7,761 & 3102169 & 2.028 & .979 & .021 & 13.71 & 566 & 7,761 & 2281365 & 1.595 & .945 & .055 \\
\hline 669 & 5.07 & 1,120 & 5,676 & 3173774 & -.087 & .465 & .465 & 5.93 & 957 & 5,676 & 2704616 & -.224 & .411 & .411 \\
\hline 670 & 7.60 & 1,116 & 8,485 & 4668136 & -.813 & .208 & .208 & 9.16 & 926 & 8,485 & 3836478 & -1.235 & .108 & .108 \\
\hline 671 & 5.47 & 3,435 & 18,797 & 33302341 & 3.203 & .999 & .001 & 6.52 & 2,882 & 18,797 & 27961386 & 3.003 & .999 & .001 \\
\hline 672 & 8.88 & 2,178 & 19,344 & 21412987 & 1.333 & .909 & .091 & 10.04 & 1,927 & 19,344 & 18852798 & .877 & .810 & .190 \\
\hline
\end{tabular}


Table 3. Summary of interoccurrence intervals for daily precipitation thresholds of 0.05 and 0.10 inch-Continued

\begin{tabular}{|c|c|c|c|c|c|c|c|c|c|c|c|c|c|c|}
\hline \multirow[b]{2}{*}{$\begin{array}{l}\text { Seq. } \\
\text { no. }\end{array}$} & \multicolumn{7}{|c|}{ Daily precipitation threshold of 0.05 inch and greater } & \multicolumn{7}{|c|}{ Daily precipitation threshold of 0.10 inch and greater } \\
\hline & $\begin{array}{c}\text { Mean } \\
\text { inter- } \\
\text { occur- } \\
\text { rence } \\
\text { interval } \\
\text { (days) }\end{array}$ & $\begin{array}{c}\text { Total } \\
\text { no. of } \\
\text { events }\end{array}$ & $\begin{array}{l}\text { Total } \\
\text { no. of } \\
\text { days } \\
\text { avail- } \\
\text { able }\end{array}$ & $\begin{array}{l}\text { Sigma } \\
\mathrm{S}\end{array}$ & $\begin{array}{l}\text { Stan- } \\
\text { dard } \\
\text { normal } \\
\text { variate }\end{array}$ & $\begin{array}{l}\text { Non- } \\
\text { ex- } \\
\text { ceed- } \\
\text { ance } \\
\text { prob- } \\
\text { ability }\end{array}$ & $\begin{array}{c}\text { p- } \\
\text { value }\end{array}$ & $\begin{array}{c}\text { Mean } \\
\text { inter- } \\
\text { occur- } \\
\text { rence } \\
\text { interval } \\
\text { (days) }\end{array}$ & $\begin{array}{l}\text { Total } \\
\text { no. of } \\
\text { events }\end{array}$ & $\begin{array}{l}\text { Total } \\
\text { no. of } \\
\text { days } \\
\text { avail- } \\
\text { able }\end{array}$ & $\begin{array}{c}\text { Sigma } \\
\mathrm{S}\end{array}$ & $\begin{array}{c}\text { Stan- } \\
\text { dard } \\
\text { normal } \\
\text { variate }\end{array}$ & $\begin{array}{l}\text { Non- } \\
\text { ex- } \\
\text { ceed- } \\
\text { ance } \\
\text { prob- } \\
\text { ability }\end{array}$ & $\begin{array}{c}p- \\
\text { value }\end{array}$ \\
\hline 673 & 10.56 & 519 & 5,481 & 1425639 & 0.092 & .537 & .463 & 11.01 & 498 & 5,481 & 1383702 & 0.536 & .704 & .296 \\
\hline 674 & 11.04 & 162 & 1,788 & 145512 & .104 & .542 & .459 & 11.18 & 160 & 1,788 & 142917 & -.019 & .492 & .492 \\
\hline 675 & 6.86 & 386 & 2,649 & 513624 & .158 & .563 & .437 & 8.92 & 297 & 2,649 & 394801 & .108 & .543 & .457 \\
\hline 676 & 8.77 & 379 & 3,324 & 586696 & -2.313 & .010 & .010 & 9.18 & 362 & 3,324 & 556960 & -2.448 & .007 & .007 \\
\hline 677 & 10.37 & 1,982 & 20,545 & 21033954 & 2.552 & .995 & .005 & 12.19 & 1,685 & 20,545 & 17518651 & .861 & .805 & .195 \\
\hline 678 & 9.24 & 17 & 157 & 1566 & 1.239 & .892 & .108 & 9.24 & 17 & 157 & 1566 & 1.239 & .892 & .108 \\
\hline 679 & 8.20 & 2,759 & 22,630 & 31902151 & 1.994 & .977 & .023 & 10.23 & 2,212 & 22,630 & 25090678 & .202 & .580 & .420 \\
\hline 680 & 8.00 & 545 & 4,360 & 1265897 & 2.648 & .996 & .004 & 8.70 & 501 & 4,360 & 1124556 & 1.149 & .875 & .125 \\
\hline 681 & 7.48 & 112 & 838 & 44917 & -.786 & .216 & .216 & 7.98 & 105 & 838 & 42359 & -.660 & .255 & .255 \\
\hline 682 & 6.79 & 750 & 5,092 & 1897222 & -.305 & .380 & .380 & 8.13 & 626 & 5,092 & 1618083 & .660 & .745 & .255 \\
\hline 683 & 5.92 & 2,061 & 12,199 & 12627838 & .355 & .639 & .361 & 6.91 & 1,765 & 12,199 & 10788558 & .155 & .562 & .438 \\
\hline 684 & 5.98 & 2,598 & 15,522 & 20214328 & .224 & .589 & .411 & 7.19 & 2,160 & 15,522 & 17045965 & 1.355 & .912 & .088 \\
\hline 685 & 4.56 & 7,723 & 35,218 & 141791766 & 6.489 & 0 & 0 & 5.26 & 6,692 & 35,218 & 121193839 & 4.033 & 1.000 & 0 \\
\hline 686 & 7.93 & 737 & 5,847 & 2147473 & -.156 & .438 & .438 & 8.47 & 690 & 5,847 & 2015429 & -.040 & .484 & .484 \\
\hline 687 & 11.06 & 140 & 1,548 & 104990 & -.637 & .262 & .262 & 11.64 & 133 & 1,548 & 101399 & -.299 & .382 & .382 \\
\hline 688 & 9.43 & 476 & 4,487 & 1092225 & .861 & .805 & .195 & 9.45 & 475 & 4,487 & 1089633 & .849 & .802 & .198 \\
\hline 689 & 6.96 & 848 & 5,899 & 2436681 & -1.301 & .097 & .097 & 7.46 & 791 & 5,899 & 2292282 & -.851 & .197 & .197 \\
\hline 690 & 4.97 & 2,520 & 12,527 & 15476876 & -1.692 & .045 & .045 & 5.88 & 2,131 & 12,527 & 13071916 & -1.651 & .049 & .049 \\
\hline 691 & 5.24 & 3,493 & 18,319 & 32372061 & 1.209 & .887 & .113 & 5.88 & 3,118 & 18,319 & 29059299 & 1.693 & .955 & .045 \\
\hline 692 & 7.60 & 2,473 & 18,792 & 21205039 & -7.530 & 0 & 0 & 8.88 & 2,116 & 18,792 & 18518171 & -5.465 & 0 & 0 \\
\hline 693 & 7.62 & 2,516 & 19,163 & 24761379 & 2.358 & .991 & .009 & 9.18 & 2,087 & 19,163 & 20698114 & 2.776 & .997 & .003 \\
\hline 694 & 7.29 & 1,001 & 7,301 & 3717190 & .945 & .828 & .172 & 7.98 & 915 & 7,301 & 3358148 & .281 & .611 & .389 \\
\hline 695 & 9.46 & 1,430 & 13,531 & 9499593 & -1.185 & .118 & .118 & 11.35 & 1,192 & 13,531 & 7897529 & -1.238 & .108 & .108 \\
\hline 696 & 8.14 & 1,575 & 12,822 & 9920130 & -1.206 & .114 & .114 & 10.23 & 1,254 & 12,822 & 7946824 & -.706 & .240 & .240 \\
\hline 697 & 4.68 & 5,042 & 23,585 & 59208287 & -.516 & .303 & .303 & 5.49 & 4,298 & 23,585 & 51073270 & .872 & .808 & .192 \\
\hline 698 & 7.24 & 5,183 & 37,526 & 101467418 & 5.410 & 0 & 0 & 8.77 & 4,279 & 37,526 & 83436632 & 4.445 & 1.000 & 0 \\
\hline 699 & 6.26 & 253 & 1,583 & 213455 & 1.817 & .965 & .035 & 7.72 & 205 & 1,583 & 172203 & 1.520 & .936 & .064 \\
\hline 700 & 6.06 & 3,244 & 19,662 & 33650754 & 5.441 & 0 & 0 & 7.51 & 2,620 & 19,662 & 27041782 & 4.422 & 1.000 & 0 \\
\hline 701 & 7.63 & 235 & 1,793 & 212993 & .292 & .615 & .385 & 8.08 & 222 & 1,793 & 199589 & .073 & .529 & .471 \\
\hline 702 & 8.91 & 136 & 1,212 & 72548 & -2.419 & .008 & .008 & 11.02 & 110 & 1,212 & 56686 & -2.718 & .003 & .003 \\
\hline 703 & 9.98 & 473 & 4,722 & 1161089 & 1.496 & .933 & .067 & 10.27 & 460 & 4,722 & 1114868 & .985 & .838 & .162 \\
\hline 704 & 9.64 & 44 & 424 & 9296 & -.039 & .484 & .484 & 11.46 & 37 & 424 & 7425 & -.563 & .287 & .287 \\
\hline 705 & 5.99 & 2,145 & 12,843 & 14150307 & 2.191 & .986 & .014 & 6.59 & 1,949 & 12,843 & 12989894 & 2.898 & .998 & .002 \\
\hline 706 & 5.03 & 7,137 & 35,879 & 130770518 & 3.127 & .999 & .001 & 5.74 & 6,250 & 35,879 & 113894304 & 2.165 & .985 & .015 \\
\hline 707 & 4.77 & 1,951 & 9,308 & 9223112 & 1.206 & .886 & .114 & 5.58 & 1,668 & 9308 & 7965217 & 1.844 & .967 & .033 \\
\hline 708 & 11.53 & 1,402 & 16,163 & 11763375 & 2.479 & .993 & .007 & 13.04 & 1,240 & 16,163 & 10379867 & 2.184 & .986 & .014 \\
\hline 709 & 7.38 & 8 & 59 & 273 & .768 & .779 & .221 & 7.38 & 8 & 59 & 273 & .768 & .779 & .221 \\
\hline 710 & 7.88 & 152 & 1,198 & 88077 & -.697 & .243 & .243 & 9.43 & 127 & 1,198 & 72096 & -1.020 & .154 & .154 \\
\hline 711 & 11.45 & 1,294 & 14,820 & 9629743 & .268 & .606 & .394 & 12.19 & 1,216 & 14,820 & 9118694 & .725 & .766 & .234 \\
\hline 712 & 11.56 & 116 & 1,341 & 80076 & .551 & .709 & .291 & 13.28 & 101 & 1,341 & 68907 & .305 & .620 & .380 \\
\hline 713 & 5.38 & 1,889 & 10,153 & 9420026 & -1.331 & .092 & .092 & 5.97 & 1,702 & 10,153 & 8702879 & .518 & .698 & .302 \\
\hline 714 & 9.13 & 40 & 365 & 5778 & -2.284 & .011 & .011 & 12.59 & 29 & 365 & 3480 & -3.194 & .001 & .001 \\
\hline 715 & 9.61 & 152 & 1,460 & 110321 & -.123 & .451 & .451 & 11.68 & 125 & 1,460 & 90913 & -.072 & .471 & .471 \\
\hline 716 & 9.40 & 672 & 6,316 & 2156964 & .736 & .769 & .231 & 11.61 & 544 & 6,316 & 1784470 & 1.564 & .941 & .059 \\
\hline 717 & 8.24 & 4,025 & 33,144 & 67320307 & 1.018 & .846 & .154 & 10.38 & 3,194 & 33,144 & 52787571 & -.265 & .395 & .395 \\
\hline 718 & 4.72 & 1,458 & 6,885 & 5000041 & -.252 & .400 & .400 & 5.51 & 1,250 & 6,885 & 4305727 & .037 & .515 & .485 \\
\hline 719 & 4.89 & 892 & 4,364 & 1871226 & -1.997 & .023 & .023 & 5.52 & 790 & 4,364 & 1652127 & -2.024 & .022 & .022 \\
\hline 720 & 4.93 & 6,743 & 33,230 & 114956102 & 3.708 & 1.000 & 0 & 5.81 & 5,720 & 33,230 & 96890105 & 2.553 & .995 & .005 \\
\hline
\end{tabular}


Table 3. Summary of interoccurrence intervals for daily precipitation thresholds of 0.05 and 0.10 inch-Continued

\begin{tabular}{|c|c|c|c|c|c|c|c|c|c|c|c|c|c|c|}
\hline \multirow[b]{2}{*}{$\begin{array}{c}\text { Seq. } \\
\text { no. }\end{array}$} & \multicolumn{7}{|c|}{ Daily precipitation threshold of 0.05 inch and greater } & \multicolumn{7}{|c|}{ Daily precipitation threshold of 0.10 inch and greater } \\
\hline & $\begin{array}{c}\text { Mean } \\
\text { inter- } \\
\text { occur- } \\
\text { rence } \\
\text { interval } \\
\text { (days) }\end{array}$ & $\begin{array}{c}\text { Total } \\
\text { no. of } \\
\text { events }\end{array}$ & $\begin{array}{l}\text { Total } \\
\text { no. of } \\
\text { days } \\
\text { avail- } \\
\text { able }\end{array}$ & $\begin{array}{l}\text { Sigma } \\
\mathrm{S}\end{array}$ & $\begin{array}{c}\text { Stan- } \\
\text { dard } \\
\text { normal } \\
\text { variate }\end{array}$ & $\begin{array}{l}\text { Non- } \\
\text { ex- } \\
\text { ceed- } \\
\text { ance } \\
\text { prob- } \\
\text { ability }\end{array}$ & $\begin{array}{c}\text { p- } \\
\text { value }\end{array}$ & $\begin{array}{l}\text { Mean } \\
\text { inter- } \\
\text { occur- } \\
\text { rence } \\
\text { interval } \\
\text { (days) }\end{array}$ & $\begin{array}{c}\text { Total } \\
\text { no. of } \\
\text { events }\end{array}$ & $\begin{array}{l}\text { Total } \\
\text { no. of } \\
\text { days } \\
\text { avail- } \\
\text { able }\end{array}$ & $\begin{array}{l}\text { Sigma } \\
\mathrm{S}\end{array}$ & $\begin{array}{c}\text { Stan- } \\
\text { dard } \\
\text { normal } \\
\text { variate }\end{array}$ & $\begin{array}{l}\text { Non- } \\
\text { ex- } \\
\text { ceed- } \\
\text { ance } \\
\text { prob- } \\
\text { ability }\end{array}$ & $\begin{array}{c}\mathrm{p}- \\
\text { value }\end{array}$ \\
\hline 721 & 5.59 & 6,527 & 36,455 & 118286971 & -0.804 & .211 & .211 & 6.98 & 5,223 & 36,455 & 95013161 & -0.249 & .402 & .402 \\
\hline 722 & 6.70 & 241 & 1,614 & 200493 & .830 & .797 & .203 & 7.84 & 206 & 1,614 & 174339 & 1.211 & .887 & .113 \\
\hline 723 & 4.27 & 528 & 2,252 & 595103 & .039 & .515 & .485 & 4.97 & 453 & 2,252 & 510231 & .011 & .504 & .496 \\
\hline 724 & 8.05 & 1,137 & 9,151 & 5438884 & 2.656 & .996 & .004 & 8.99 & 1,018 & 9,151 & 4830018 & 2.043 & .979 & .021 \\
\hline 725 & 6.87 & 1,334 & 9,162 & 6068627 & -.439 & .330 & .330 & 8.54 & 1,073 & 9,162 & 4911956 & -.040 & .484 & .484 \\
\hline 726 & 6.13 & 190 & 1,165 & 115012 & .936 & .825 & .175 & 7.19 & 162 & 1,165 & 97900 & .826 & .795 & .205 \\
\hline 727 & 5.45 & 4,636 & 25,281 & 59652251 & 2.115 & .983 & .017 & 6.24 & 4,050 & 25,281 & 52062450 & 1.870 & .969 & .031 \\
\hline 728 & 5.34 & 1,929 & 10,305 & 10488813 & 4.207 & 1.000 & 0 & 6.04 & 1,707 & 10,305 & 9302696 & 4.128 & 1.000 & 0 \\
\hline 729 & 7.25 & 890 & 6,453 & 2883602 & .216 & .586 & .414 & 8.28 & 779 & 6,453 & 2537688 & .466 & .679 & .321 \\
\hline 730 & 6.50 & 2 & 13 & 9 & -.754 & .226 & .226 & 6.50 & 2 & 13 & 9 & -.754 & .226 & .226 \\
\hline 731 & 8.40 & 744 & 6,252 & 2315484 & -.208 & .417 & .417 & 8.40 & 744 & 6,252 & 2315484 & -.208 & .417 & .417 \\
\hline 732 & 6.75 & 1,010 & 6,819 & 3334745 & -1.740 & .041 & .041 & 8.39 & 813 & 6,819 & 2699492 & -1.291 & .098 & .098 \\
\hline 733 & 14.96 & 23 & 344 & 4693 & 1.548 & .939 & .061 & 17.20 & 20 & 344 & 3943 & 1.133 & .871 & .129 \\
\hline 734 & 10.09 & 2,286 & 23,057 & 25752201 & -1.892 & .029 & .029 & 12.73 & 1,812 & 23,057 & 20112729 & -2.742 & .003 & .003 \\
\hline 735 & 7.04 & 281 & 1,979 & 259653 & -1.921 & .027 & .027 & 8.96 & 221 & 1,979 & 202014 & -1.962 & .025 & .025 \\
\hline 736 & 8.79 & 921 & 8,096 & 3824605 & 1.359 & .913 & .087 & 9.78 & 828 & 80,96 & 3441357 & 1.333 & .909 & .091 \\
\hline 737 & 13.04 & 203 & 2,647 & 284710 & 1.473 & .930 & .070 & 15.48 & 171 & 2,647 & 239442 & 1.313 & .905 & .095 \\
\hline 738 & 8.35 & 1,716 & 14,333 & 12346452 & .284 & .612 & .388 & 11.12 & 1,289 & 14,333 & 9249558 & .080 & .532 & .468 \\
\hline 739 & 11.65 & 428 & 4,987 & 1137833 & 2.371 & .991 & .009 & 13.55 & 368 & 4,987 & 925176 & .274 & .608 & .392 \\
\hline 740 & 8.72 & 111 & 968 & 52427 & -.441 & .330 & .330 & 10.52 & 92 & 968 & 44677 & .056 & .522 & .478 \\
\hline 741 & 8.88 & 16 & 142 & 953 & -1.116 & .132 & .132 & 9.47 & 15 & 142 & 861 & -1.285 & .099 & .099 \\
\hline 742 & 5.58 & 4,747 & 26,466 & 63172979 & .676 & .750 & .250 & 6.77 & 3,908 & 26,466 & 51883438 & .354 & .638 & .362 \\
\hline 743 & 4.93 & 6,780 & 33,395 & 115464929 & 2.842 & .998 & .002 & 5.68 & 5,875 & 33,395 & 99505179 & 1.905 & .972 & .028 \\
\hline 744 & 8.04 & 788 & 6,333 & 2581519 & 1.682 & .954 & .046 & 9.61 & 659 & 6,333 & 2120816 & .726 & .766 & .234 \\
\hline 745 & 6.64 & 1,348 & 8,954 & 6020973 & -.148 & .441 & .441 & 7.90 & 1,133 & 8,954 & 5102879 & .350 & .637 & .363 \\
\hline 746 & 7.49 & 2,745 & 20,552 & 29723174 & 4.876 & 1.000 & 0 & 8.89 & 2,311 & 20,552 & 24911878 & 4.081 & 1.000 & 0 \\
\hline 747 & 7.84 & 2,496 & 19,569 & 25028954 & 2.150 & .984 & .016 & 9.44 & 2,072 & 19,569 & 20739583 & 1.813 & .965 & .035 \\
\hline 748 & 6.24 & 5,278 & 32,946 & 92812855 & 8.493 & 0 & 0 & 7.34 & 4,490 & 32,946 & 78353778 & 6.889 & 0 & 0 \\
\hline 749 & 6.13 & 2,215 & 13,573 & 14320146 & -3.861 & 0 & 0 & 7.73 & 1,756 & 13,573 & 11390849 & -3.205 & .001 & .001 \\
\hline 750 & 5.37 & 3,459 & 18,579 & 31420978 & -2.255 & .012 & .012 & 5.89 & 3,152 & 18,579 & 28719037 & -1.865 & .031 & .031 \\
\hline 751 & 5.85 & 1,303 & 7,628 & 4692204 & -3.490 & 0 & 0 & 7.04 & 1,083 & 7,628 & 4001391 & -1.783 & .037 & .037 \\
\hline 752 & 12.94 & 94 & 1,216 & 55075 & -.610 & .271 & .271 & 15.79 & 77 & 1,216 & 44014 & -.910 & .182 & .182 \\
\hline 753 & 7.13 & 285 & 2,033 & 281016 & -.877 & .190 & .190 & 8.04 & 253 & 2,033 & 258296 & .120 & .548 & .452 \\
\hline 754 & 4.72 & 546 & 2,575 & 664383 & -2.222 & .013 & .013 & 5.56 & 463 & 2,575 & 551420 & -2.794 & .003 & .003 \\
\hline 755 & 7.64 & 2,815 & 21,499 & 29672321 & -1.784 & .037 & .037 & 10.15 & 2,119 & 21,499 & 22565483 & -.745 & .228 & .228 \\
\hline 756 & 32.00 & 2 & 64 & 37 & -1.033 & .151 & .151 & 32.00 & 2 & 64 & 37 & -1.033 & .151 & .151 \\
\hline 757 & 11.57 & 505 & 5,844 & 1565470 & 2.370 & .991 & .009 & 12.23 & 478 & 5,844 & 1464973 & 1.851 & .968 & .032 \\
\hline 758 & 6.08 & 5,322 & 32,344 & 88482876 & 3.546 & 1.000 & 0 & 6.95 & 4,655 & 32,344 & 76515823 & 1.939 & .974 & .026 \\
\hline 759 & 7.16 & 2,149 & 15,392 & 16298873 & -1.164 & .122 & .122 & 8.39 & 1,834 & 15,392 & 14156566 & .221 & .588 & .412 \\
\hline 760 & 8.46 & 2,522 & 21,345 & 27037603 & .393 & .653 & .347 & 10.67 & 2,000 & 21,345 & 21566558 & .804 & .789 & .211 \\
\hline 761 & 8.28 & 1,634 & 13,531 & 11015307 & -.250 & .401 & .401 & 10.50 & 1,289 & 13,531 & 8696495 & -.173 & .431 & .431 \\
\hline 762 & 10.99 & 2,301 & 25,291 & 29134628 & .107 & .542 & .458 & 14.19 & 1,783 & 25,291 & 22577064 & .098 & .539 & .461 \\
\hline 763 & 10.48 & 113 & 1,184 & 56510 & -2.859 & .002 & .002 & 10.48 & 113 & 1,184 & 56510 & -2.859 & .002 & .002 \\
\hline 764 & 6.51 & 5,040 & 32,783 & 87030134 & 6.574 & 0 & 0 & 7.53 & 4,353 & 32,783 & 73765429 & 3.865 & 1.000 & 0 \\
\hline 765 & 6.06 & 2,013 & 12,195 & 12071544 & -1.284 & .100 & .100 & 7.32 & 1,666 & 12,195 & 10137454 & -.146 & .442 & .442 \\
\hline 766 & 6.48 & 413 & 2,674 & 490020 & -3.963 & 0 & 0 & 7.58 & 353 & 2,674 & 419549 & -3.614 & 0 & 0 \\
\hline 767 & 8.96 & 3,705 & 33,202 & 61889219 & .656 & .744 & .256 & 9.92 & 3,348 & 33,202 & 55362015 & -.393 & .347 & .347 \\
\hline 768 & 8.33 & 4,073 & 33,925 & 73247300 & 6.654 & 0 & 0 & 9.98 & 3,398 & 33,925 & 59680213 & 3.576 & 1.000 & 0 \\
\hline
\end{tabular}


Table 3. Summary of interoccurrence intervals for daily precipitation thresholds of 0.05 and 0.10 inch-Continued

\begin{tabular}{|c|c|c|c|c|c|c|c|c|c|c|c|c|c|c|}
\hline \multirow[b]{2}{*}{$\begin{array}{l}\text { Seq. } \\
\text { no. }\end{array}$} & \multicolumn{7}{|c|}{ Daily precipitation threshold of 0.05 inch and greater } & \multicolumn{7}{|c|}{ Daily precipitation threshold of 0.10 inch and greater } \\
\hline & $\begin{array}{c}\text { Mean } \\
\text { inter- } \\
\text { occur- } \\
\text { rence } \\
\text { interval } \\
\text { (days) }\end{array}$ & $\begin{array}{c}\text { Total } \\
\text { no. of } \\
\text { events }\end{array}$ & $\begin{array}{l}\text { Total } \\
\text { no. of } \\
\text { days } \\
\text { avail- } \\
\text { able }\end{array}$ & $\begin{array}{l}\text { Sigma } \\
\mathrm{S}\end{array}$ & $\begin{array}{l}\text { Stan- } \\
\text { dard } \\
\text { normal } \\
\text { variate }\end{array}$ & $\begin{array}{l}\text { Non- } \\
\text { ex- } \\
\text { ceed- } \\
\text { ance } \\
\text { prob- } \\
\text { ability }\end{array}$ & $\begin{array}{c}\text { p- } \\
\text { value }\end{array}$ & $\begin{array}{c}\text { Mean } \\
\text { inter- } \\
\text { occur- } \\
\text { rence } \\
\text { interval } \\
\text { (days) }\end{array}$ & $\begin{array}{l}\text { Total } \\
\text { no. of } \\
\text { events }\end{array}$ & $\begin{array}{l}\text { Total } \\
\text { no. of } \\
\text { days } \\
\text { avail- } \\
\text { able }\end{array}$ & $\begin{array}{c}\text { Sigma } \\
\mathrm{S}\end{array}$ & $\begin{array}{c}\text { Stan- } \\
\text { dard } \\
\text { normal } \\
\text { variate }\end{array}$ & $\begin{array}{l}\text { Non- } \\
\text { ex- } \\
\text { ceed- } \\
\text { ance } \\
\text { prob- } \\
\text { ability }\end{array}$ & $\begin{array}{c}p- \\
\text { value }\end{array}$ \\
\hline 769 & 10.28 & 206 & 2,118 & 201720 & -1.873 & .031 & .031 & 11.90 & 178 & 2,118 & 172915 & -1.911 & .028 & .028 \\
\hline 770 & 15.47 & 526 & 8,136 & 2190574 & .943 & .827 & .173 & 19.61 & 415 & 8,136 & 1775401 & 1.822 & .966 & .034 \\
\hline 771 & 10.12 & 367 & 3,714 & 717410 & 1.747 & .960 & .040 & 11.12 & 334 & 3,714 & 651889 & 1.615 & .947 & .053 \\
\hline 772 & 12.20 & 15 & 183 & 1179 & -.946 & .172 & .172 & 13.07 & 14 & 183 & 1156 & -.632 & .264 & .264 \\
\hline 773 & 8.76 & 59 & 517 & 13041 & -1.928 & .027 & .027 & 11.49 & 45 & 517 & 10063 & -1.568 & .058 & .058 \\
\hline 774 & 7.80 & 3,956 & 30,870 & 60735151 & -.581 & .281 & .281 & 9.64 & 3,204 & 30,870 & 49011551 & -.877 & .190 & .190 \\
\hline 775 & 11.69 & 26 & 304 & 4026 & .165 & .566 & .434 & 12.16 & 25 & 304 & 3871 & .162 & .564 & .436 \\
\hline 776 & 7.12 & 986 & 7,015 & 3449908 & -.134 & .447 & .447 & 7.73 & 907 & 7,015 & 3188652 & .121 & .548 & .452 \\
\hline 777 & 8.59 & 798 & 6,852 & 2433653 & -5.374 & 0 & 0 & 9.10 & 753 & 6,852 & 2331951 & -4.566 & 0 & 0 \\
\hline 778 & 9.09 & 1,072 & 9,749 & 5093470 & -1.433 & .076 & .076 & 10.22 & 954 & 9,749 & 4395628 & -2.930 & .002 & .002 \\
\hline 779 & 9.36 & 1,710 & 16,007 & 13585757 & -.525 & .300 & .300 & 10.97 & 1,459 & 16,007 & 11904492 & 1.288 & .901 & .099 \\
\hline 780 & 5.49 & 6,296 & 34,558 & 109646660 & 1.084 & .861 & .139 & 6.61 & 5,230 & 34,558 & 91771734 & 1.944 & .974 & .026 \\
\hline 781 & 7.24 & 4,784 & 34,614 & 82457373 & -.491 & .312 & .312 & 8.73 & 3,966 & 34,614 & 68052835 & -.932 & .176 & .176 \\
\hline 782 & 6.05 & 1,537 & 9,303 & 7282368 & 1.263 & .897 & .103 & 6.32 & 1,472 & 9,303 & 7029255 & 1.769 & .962 & .038 \\
\hline 783 & 11.32 & 129 & 1,460 & 73427 & -4.333 & 0 & 0 & 12.17 & 120 & 1,460 & 70598 & -3.683 & 0 & 0 \\
\hline 784 & 12.36 & 382 & 4,721 & 764479 & -5.152 & 0 & 0 & 14.26 & 331 & 4,721 & 689993 & -3.684 & 0 & 0 \\
\hline 785 & 12.55 & 1,520 & 19,079 & 14243479 & -1.195 & .116 & .116 & 14.65 & 1,302 & 19,079 & 12198598 & -1.116 & .132 & .132 \\
\hline 786 & 10.50 & 1,861 & 19,541 & 18359848 & .727 & .766 & .234 & 13.62 & 1,435 & 19,541 & 14168265 & .691 & .755 & .245 \\
\hline 787 & 9.22 & 657 & 6,055 & 1973464 & -.348 & .364 & .364 & 9.24 & 655 & 6,055 & 1973129 & -.221 & .413 & .413 \\
\hline 788 & 6.06 & 266 & 1,612 & 226559 & 1.603 & .945 & .055 & 7.23 & 223 & 1,612 & 189817 & 1.450 & .927 & .074 \\
\hline 789 & 5.18 & 1,611 & 8,344 & 6794754 & .762 & .777 & .223 & 5.79 & 1,441 & 8,344 & 6066389 & .597 & .725 & .275 \\
\hline 790 & 6.59 & 337 & 2,219 & 349650 & -2.062 & .020 & .020 & 6.85 & 324 & 2,219 & 337847 & -1.876 & .030 & .030 \\
\hline 791 & 10.62 & 444 & 4,714 & 1048353 & .064 & .526 & .474 & 11.20 & 421 & 4,714 & 997287 & .179 & .571 & .429 \\
\hline 792 & 6.43 & 70 & 450 & 18101 & 2.163 & .985 & .015 & 7.63 & 59 & 450 & 15630 & 2.360 & .991 & .009 \\
\hline 793 & 6.55 & 2,927 & 19,181 & 28996789 & 3.089 & .999 & .001 & 7.06 & 2,716 & 19,181 & 26751416 & 2.438 & .993 & .007 \\
\hline 794 & 4.97 & 2,349 & 11,685 & 13742433 & .113 & .545 & .455 & 5.82 & 2,007 & 11,685 & 11626908 & -.655 & .256 & .256 \\
\hline 795 & 6.41 & 2,661 & 17,047 & 23167884 & 1.918 & .972 & .028 & 7.65 & 2,229 & 17,047 & 19480315 & 2.072 & .981 & .019 \\
\hline 796 & 10.73 & 487 & 5,225 & 1364523 & 2.771 & .997 & .003 & 13.16 & 397 & 5,225 & 1143298 & 3.532 & 1.000 & 0 \\
\hline 797 & 8.47 & 3,362 & 28,459 & 48796044 & 2.008 & .978 & .022 & 10.60 & 2,685 & 28,459 & 39063772 & 2.015 & .978 & .022 \\
\hline 798 & 9.91 & 70 & 694 & 21673 & -1.561 & .059 & .059 & 14.46 & 48 & 694 & 14852 & -1.300 & .097 & .097 \\
\hline 799 & 8.82 & 1,038 & 9,155 & 4384384 & -4.311 & 0 & 0 & 9.14 & 1,002 & 9,155 & 4316190 & -3.233 & .001 & .001 \\
\hline 800 & 7.97 & 144 & 1,148 & 82022 & -.159 & .437 & .437 & 9.81 & 117 & 1,148 & 65697 & -.408 & .342 & .342 \\
\hline 801 & 11.60 & 1,276 & 14,804 & 9452073 & .047 & .519 & .481 & 15.14 & 978 & 1,4804 & 7302270 & .472 & .682 & .318 \\
\hline 802 & 15.59 & 97 & 1,512 & 64159 & -2.134 & .016 & .016 & 19.90 & 76 & 1512 & 47792 & -2.540 & .006 & .006 \\
\hline 803 & 6.69 & 1,091 & 7,299 & 4021909 & .579 & .719 & .281 & 7.93 & 920 & 7,299 & 3336394 & -.331 & .370 & .370 \\
\hline 804 & 8.51 & 80 & 681 & 27084 & -.089 & .465 & .465 & 10.81 & 63 & 681 & 21756 & .195 & .577 & .423 \\
\hline 805 & 7.29 & 1,576 & 11,493 & 9106657 & .381 & .648 & .352 & 8.95 & 1,284 & 11,493 & 7376182 & -.020 & .492 & .492 \\
\hline 806 & 4.87 & 3,525 & 17,161 & 30388007 & .482 & .685 & .315 & 5.77 & 2,974 & 17,161 & 26024909 & 1.875 & .970 & .030 \\
\hline 807 & 6.27 & 2,094 & 13,131 & 13634389 & -.656 & .256 & .256 & 7.12 & 1,845 & 13,131 & 12054753 & -.360 & .359 & .359 \\
\hline 808 & 7.98 & 2,258 & 18,012 & 22152347 & 7.353 & 0 & 0 & 8.67 & 2,078 & 18,012 & 20043962 & 5.609 & 0 & 0 \\
\hline 809 & 9.59 & 1,924 & 18,442 & 19075667 & 5.715 & 0 & 0 & 11.10 & 1,662 & 18,442 & 15914540 & 2.715 & .997 & .003 \\
\hline 810 & 8.99 & 2,289 & 20,566 & 24720058 & 4.162 & 1.000 & 0 & 10.97 & 1,874 & 20,566 & 20200876 & 3.621 & 1.000 & 0 \\
\hline 811 & 5.35 & 1,379 & 7,376 & 4982517 & -1.306 & .096 & .096 & 6.01 & 1,228 & 7,376 & 4405946 & -1.647 & .050 & .050 \\
\hline 812 & 10.04 & 666 & 6,684 & 2303311 & 1.557 & .940 & .060 & 10.59 & 631 & 6,684 & 2184190 & 1.555 & .940 & .060 \\
\hline 813 & 4.88 & 17 & 83 & 657 & -.491 & .312 & .312 & 5.93 & 14 & 83 & 506 & -.837 & .201 & .201 \\
\hline 814 & 6.72 & 3,634 & 24,404 & 44243812 & -.231 & .408 & .408 & 8.78 & 2,780 & 24,404 & 34049986 & .346 & .635 & .365 \\
\hline 815 & 5.38 & 5,645 & 30,377 & 86442940 & 1.068 & .857 & .143 & 6.03 & 5,034 & 30,377 & 76150935 & -.495 & .310 & .310 \\
\hline 816 & 4.88 & 2,648 & 12,922 & 16893238 & -1.123 & .131 & .131 & 5.76 & 2,244 & 12,922 & 14305890 & -1.090 & .138 & .138 \\
\hline
\end{tabular}


Table 3. Summary of interoccurrence intervals for daily precipitation thresholds of 0.05 and 0.10 inch-Continued

\begin{tabular}{|c|c|c|c|c|c|c|c|c|c|c|c|c|c|c|}
\hline \multirow[b]{2}{*}{$\begin{array}{c}\text { Seq. } \\
\text { no. }\end{array}$} & \multicolumn{7}{|c|}{ Daily precipitation threshold of 0.05 inch and greater } & \multicolumn{7}{|c|}{ Daily precipitation threshold of 0.10 inch and greater } \\
\hline & $\begin{array}{c}\text { Mean } \\
\text { inter- } \\
\text { occur- } \\
\text { rence } \\
\text { interval } \\
\text { (days) }\end{array}$ & $\begin{array}{c}\text { Total } \\
\text { no. of } \\
\text { events }\end{array}$ & $\begin{array}{c}\text { Total } \\
\text { no. of } \\
\text { days } \\
\text { avail- } \\
\text { able }\end{array}$ & $\begin{array}{l}\text { Sigma } \\
\mathrm{S}\end{array}$ & $\begin{array}{c}\text { Stan- } \\
\text { dard } \\
\text { normal } \\
\text { variate }\end{array}$ & $\begin{array}{l}\text { Non- } \\
\text { ex- } \\
\text { ceed- } \\
\text { ance } \\
\text { prob- } \\
\text { ability }\end{array}$ & $\begin{array}{c}\mathrm{p}- \\
\text { value }\end{array}$ & $\begin{array}{l}\text { Mean } \\
\text { inter- } \\
\text { occur- } \\
\text { rence } \\
\text { interval } \\
\text { (days) }\end{array}$ & $\begin{array}{l}\text { Total } \\
\text { no. of } \\
\text { events }\end{array}$ & $\begin{array}{l}\text { Total } \\
\text { no. of } \\
\text { days } \\
\text { avail- } \\
\text { able }\end{array}$ & $\begin{array}{l}\text { Sigma } \\
\mathrm{S}\end{array}$ & $\begin{array}{c}\text { Stan- } \\
\text { dard } \\
\text { normal } \\
\text { variate }\end{array}$ & $\begin{array}{l}\text { Non- } \\
\text { ex- } \\
\text { ceed- } \\
\text { ance } \\
\text { prob- } \\
\text { ability }\end{array}$ & $\begin{array}{c}\mathrm{p}- \\
\text { value }\end{array}$ \\
\hline 817 & 7.62 & 816 & 6,220 & 2550892 & 0.256 & .601 & .399 & 7.74 & 804 & 6,220 & 2497915 & -0.050 & .480 & .480 \\
\hline 818 & 5.80 & 3,421 & 19,833 & 34600881 & 2.020 & .978 & .022 & 6.94 & 2,857 & 19,833 & 29076799 & 2.436 & .993 & .007 \\
\hline 819 & 8.37 & 3,447 & 28,862 & 50068754 & .665 & .747 & .253 & 10.44 & 2,764 & 28,862 & 40521059 & 1.447 & .926 & .074 \\
\hline 820 & 7.07 & 126 & 891 & 50480 & -1.958 & .025 & .025 & 9.58 & 93 & 891 & 35308 & -2.469 & .007 & .007 \\
\hline 821 & 8.76 & 909 & 7,961 & 3452785 & -2.388 & .008 & .008 & 11.03 & 722 & 7,961 & 2756693 & -1.898 & .029 & .029 \\
\hline 822 & 7.49 & 2,544 & 19,050 & 25287550 & 3.807 & 1.000 & 0 & 8.51 & 2,238 & 19,050 & 22222932 & 3.483 & 1.000 & 0 \\
\hline 823 & 7.41 & 4,394 & 32,540 & 72234060 & 1.194 & .884 & .116 & 8.96 & 3,631 & 32,540 & 59877723 & 1.416 & .922 & .078 \\
\hline 824 & 7.58 & 280 & 2,122 & 287627 & -.922 & .178 & .178 & 8.73 & 243 & 2,122 & 247701 & -1.060 & .145 & .145 \\
\hline 825 & 18.22 & 222 & 4,045 & 455906 & .397 & .654 & .346 & 18.22 & 222 & 4,045 & 455906 & .397 & .654 & .346 \\
\hline 826 & 4.87 & 5,439 & 26,503 & 73083059 & 1.787 & .963 & .037 & 5.68 & 4,667 & 26,503 & 62261988 & .798 & .788 & .212 \\
\hline 827 & 4.81 & 2,170 & 10,437 & 11539885 & 1.537 & .938 & .062 & 5.59 & 1,866 & 10,437 & 9784280 & .358 & .640 & .360 \\
\hline 828 & 5.88 & 4,394 & 25,827 & 60568979 & 7.744 & 0 & 0 & 6.34 & 4,077 & 25,827 & 55146765 & 5.248 & 0 & 0 \\
\hline 829 & 5.13 & 1,052 & 5,401 & 2716936 & -2.452 & .007 & .007 & 5.61 & 962 & 5,401 & 2515989 & -1.693 & .045 & .045 \\
\hline 830 & 7.30 & 1,794 & 13,102 & 12356078 & 3.768 & 1.000 & 0 & 8.75 & 1,497 & 13,102 & 10162056 & 2.427 & .992 & .008 \\
\hline 831 & 5.64 & 2,514 & 14,176 & 18035850 & 1.056 & .854 & .146 & 6.83 & 2,077 & 14,176 & 15092351 & 1.987 & .977 & .023 \\
\hline 832 & 6.71 & 261 & 1,752 & 210037 & -2.276 & .011 & .011 & 7.62 & 230 & 1,752 & 187945 & -1.765 & .039 & .039 \\
\hline 833 & 8.42 & 195 & 1,641 & 145926 & -2.127 & .017 & .017 & 10.26 & 160 & 1,641 & 117937 & -2.227 & .013 & .013 \\
\hline 834 & 8.89 & 462 & 4,106 & 954054 & .219 & .587 & .413 & 10.21 & 402 & 4,106 & 857554 & 1.357 & .913 & .087 \\
\hline 835 & 5.69 & 3,261 & 18,549 & 29572684 & -2.196 & .014 & .014 & 6.22 & 2,980 & 18,549 & 26993481 & -2.205 & .014 & .014 \\
\hline 836 & 7.02 & 1,075 & 7,548 & 4190875 & 1.873 & .969 & .031 & 8.28 & 912 & 7,548 & 3575917 & 2.037 & .979 & .021 \\
\hline 837 & 5.01 & 2,384 & 11,942 & 14589198 & 2.105 & .982 & .018 & 5.57 & 2,144 & 11,942 & 13014802 & 1.334 & .909 & .091 \\
\hline 838 & 5.23 & 1,457 & 7,618 & 5368788 & -2.155 & .016 & .016 & 5.92 & 1,286 & 7,618 & 4741672 & -1.987 & .023 & .023 \\
\hline 839 & 4.71 & 478 & 2,252 & 528928 & -.654 & .257 & .257 & 5.52 & 408 & 2,252 & 458317 & -.083 & .467 & .467 \\
\hline 840 & 6.42 & 5,675 & 36,458 & 105611504 & 2.727 & .997 & .003 & 7.57 & 4,814 & 36,458 & 88968654 & 1.663 & .952 & .048 \\
\hline 841 & 4.93 & 3,664 & 18,077 & 33047258 & -.221 & .413 & .413 & 5.74 & 3,147 & 18,077 & 28619530 & .599 & .725 & .275 \\
\hline 842 & 5.09 & 3,680 & 18,731 & 34134364 & -1.008 & .157 & .157 & 6.23 & 3,006 & 18,731 & 28009556 & -.483 & .315 & .315 \\
\hline 843 & 6.04 & 595 & 3,594 & 1004681 & -2.550 & .005 & .005 & 6.57 & 547 & 3,594 & 932080 & -2.097 & .018 & .018 \\
\hline 844 & 7.79 & 2,255 & 17,555 & 20597164 & 3.341 & 1.000 & 0 & 8.36 & 2,101 & 17,555 & 19051914 & 2.628 & .996 & .004 \\
\hline 845 & 4.69 & 797 & 3,740 & 1465730 & -.809 & .209 & .209 & 5.22 & 716 & 3,740 & 1349276 & .359 & .640 & .360 \\
\hline 846 & 4.78 & 1,355 & 6,477 & 4213352 & -2.540 & .006 & .006 & 5.41 & 1,197 & 6,477 & 3728098 & -2.294 & .011 & .011 \\
\hline 847 & 8.12 & 2,210 & 17,934 & 21774066 & 8.041 & 0 & 0 & 8.95 & 2,004 & 17,934 & 19452778 & 6.399 & 0 & 0 \\
\hline 848 & 6.11 & 4,711 & 28,763 & 67953258 & .355 & .639 & .361 & 7.60 & 3,787 & 28,763 & 54963067 & .979 & .836 & .164 \\
\hline 849 & 5.20 & 1,304 & 6,785 & 4506677 & 1.172 & .879 & .121 & 6.11 & 1,111 & 6,785 & 3844199 & 1.151 & .875 & .125 \\
\hline 850 & 10.76 & 133 & 1,431 & 89369 & -1.216 & .112 & .112 & 10.76 & 133 & 1,431 & 89369 & -1.216 & .112 & .112 \\
\hline 851 & 5.70 & 64 & 365 & 14197 & 2.986 & .999 & .001 & 6.64 & 55 & 365 & 12335 & 2.940 & .998 & .002 \\
\hline 852 & 5.79 & 79 & 457 & 18638 & .500 & .692 & .309 & 6.18 & 74 & 457 & 17341 & .381 & .648 & .352 \\
\hline 853 & 4.64 & 59 & 274 & 8197 & .188 & .574 & .426 & 5.96 & 46 & 274 & 6285 & -.032 & .487 & .487 \\
\hline 854 & 8.52 & 2,219 & 18,899 & 20923548 & -.175 & .431 & .431 & 10.06 & 1,878 & 18,899 & 17894692 & .628 & .735 & .265 \\
\hline 855 & 5.91 & 943 & 5,570 & 2575394 & -1.030 & .152 & .152 & 7.15 & 779 & 5,570 & 2152990 & -.368 & .356 & .356 \\
\hline 856 & 7.79 & 2,403 & 18,709 & 22519849 & .155 & .562 & .438 & 9.52 & 1,965 & 18,709 & 18643308 & 1.093 & .863 & .137 \\
\hline 857 & 8.43 & 1,079 & 9,100 & 5144663 & 2.726 & .997 & .003 & 9.51 & 957 & 9,100 & 4618130 & 3.246 & .999 & .001 \\
\hline 858 & 8.18 & 1,294 & 10,580 & 6952267 & .974 & .835 & .165 & 9.40 & 1,125 & 10,580 & 6058352 & 1.046 & .852 & .148 \\
\hline 859 & 7.78 & 1,204 & 9,367 & 5721319 & .878 & .810 & .190 & 9.79 & 957 & 9,367 & 4498028 & .190 & .576 & .424 \\
\hline 860 & 9.43 & 129 & 1,217 & 73773 & -1.184 & .118 & .118 & 11.70 & 104 & 1,217 & 56098 & -2.006 & .022 & .022 \\
\hline 861 & 8.07 & 1,791 & 14,445 & 12788652 & -.832 & .203 & .203 & 9.61 & 1,503 & 14,445 & 10797210 & -.360 & .359 & .359 \\
\hline 862 & 6.04 & 1,264 & 7,632 & 4857089 & .430 & .666 & .334 & 6.27 & 1,217 & 7,632 & 4670595 & .345 & .635 & .365 \\
\hline 863 & 5.68 & 75 & 426 & 16343 & .346 & .635 & .365 & 6.27 & 68 & 426 & 14841 & .352 & .638 & .362 \\
\hline 864 & 11.97 & 1,510 & 18,069 & 13858888 & 1.070 & .858 & .142 & 14.68 & 1231 & 18,069 & 11260140 & .758 & .776 & .224 \\
\hline
\end{tabular}


Table 3. Summary of interoccurrence intervals for daily precipitation thresholds of 0.05 and 0.10 inch-Continued

\begin{tabular}{|c|c|c|c|c|c|c|c|c|c|c|c|c|c|c|}
\hline \multirow[b]{2}{*}{$\begin{array}{l}\text { Seq. } \\
\text { no. }\end{array}$} & \multicolumn{7}{|c|}{ Daily precipitation threshold of 0.05 inch and greater } & \multicolumn{7}{|c|}{ Daily precipitation threshold of 0.10 inch and greater } \\
\hline & $\begin{array}{c}\text { Mean } \\
\text { inter- } \\
\text { occur- } \\
\text { rence } \\
\text { interval } \\
\text { (days) }\end{array}$ & $\begin{array}{c}\text { Total } \\
\text { no. of } \\
\text { events }\end{array}$ & $\begin{array}{l}\text { Total } \\
\text { no. of } \\
\text { days } \\
\text { avail- } \\
\text { able }\end{array}$ & $\begin{array}{l}\text { Sigma } \\
\mathrm{S}\end{array}$ & $\begin{array}{l}\text { Stan- } \\
\text { dard } \\
\text { normal } \\
\text { variate }\end{array}$ & $\begin{array}{l}\text { Non- } \\
\text { ex- } \\
\text { ceed- } \\
\text { ance } \\
\text { prob- } \\
\text { ability }\end{array}$ & $\begin{array}{c}\text { p- } \\
\text { value }\end{array}$ & $\begin{array}{c}\text { Mean } \\
\text { inter- } \\
\text { occur- } \\
\text { rence } \\
\text { interval } \\
\text { (days) }\end{array}$ & $\begin{array}{c}\text { Total } \\
\text { no. of } \\
\text { events }\end{array}$ & $\begin{array}{l}\text { Total } \\
\text { no. of } \\
\text { days } \\
\text { avail- } \\
\text { able }\end{array}$ & $\begin{array}{c}\text { Sigma } \\
\mathrm{S}\end{array}$ & $\begin{array}{c}\text { Stan- } \\
\text { dard } \\
\text { normal } \\
\text { variate }\end{array}$ & $\begin{array}{l}\text { Non- } \\
\text { ex- } \\
\text { ceed- } \\
\text { ance } \\
\text { prob- } \\
\text { ability }\end{array}$ & $\begin{array}{c}p- \\
\text { value }\end{array}$ \\
\hline 865 & 9.29 & 252 & 2,342 & 282011 & -1.219 & .112 & .112 & 11.31 & 207 & 2,342 & 230874 & -1.185 & .118 & .118 \\
\hline 866 & 10.97 & 248 & 2,721 & 370217 & 2.653 & .996 & .004 & 12.84 & 212 & 2,721 & 301859 & 1.175 & .880 & .120 \\
\hline 867 & 6.95 & 2,289 & 15,899 & 18409627 & .971 & .834 & .166 & 8.29 & 1,918 & 15,899 & 15544083 & 1.477 & .930 & .070 \\
\hline 868 & 8.25 & 2,636 & 21,746 & 28674364 & .041 & .516 & .484 & 9.00 & 2,416 & 21,746 & 26378012 & .353 & .638 & .362 \\
\hline 869 & 8.67 & 2,127 & 18,440 & 20017944 & 1.658 & .951 & .049 & 10.59 & 1,741 & 18,440 & 16210441 & .713 & .762 & .238 \\
\hline 870 & 5.03 & 313 & 1,575 & 264309 & 2.216 & .987 & .013 & 5.69 & 277 & 1,575 & 232460 & 1.893 & .971 & .029 \\
\hline 871 & 14.82 & 17 & 252 & 2651 & 1.697 & .955 & .045 & 14.82 & 17 & 252 & 2651 & 1.697 & .955 & .045 \\
\hline 872 & 9.35 & 52 & 486 & 12227 & -.404 & .343 & .343 & 9.92 & 49 & 486 & 11426 & -.490 & .312 & .312 \\
\hline 873 & 4.41 & 5,324 & 23,470 & 62751849 & .556 & .711 & .289 & 5.21 & 4,503 & 23,470 & 53397884 & 1.221 & .889 & .111 \\
\hline 874 & 4.00 & 1,430 & 5,724 & 3975056 & -1.882 & .030 & .030 & 4.79 & 1,195 & 5,724 & 3342607 & -1.357 & .088 & .088 \\
\hline 875 & 4.18 & 116 & 485 & 26025 & -1.396 & .081 & .081 & 4.71 & 103 & 485 & 22870 & -1.483 & .069 & .069 \\
\hline 876 & -- & -- & -- & -- & -- & -- & -- & -- & -- & -- & -- & -- & -- & -- \\
\hline 877 & 10.49 & 41 & 430 & 8698 & -.147 & .441 & .441 & 13.44 & 32 & 430 & 7394 & .732 & .768 & .232 \\
\hline 878 & 5.41 & 2,898 & 15,666 & 22414578 & -1.173 & .121 & .121 & 6.00 & 2,613 & 15,666 & 20273171 & -.841 & .200 & .200 \\
\hline 879 & 9.72 & 1,903 & 18,493 & 17191540 & -1.737 & .041 & .041 & 11.65 & 1,587 & 18,493 & 14480983 & -.909 & .182 & .182 \\
\hline 880 & 8.58 & 26 & 223 & 2869 & -.091 & .464 & .464 & 11.15 & 20 & 223 & 2107 & -.427 & .335 & .335 \\
\hline 881 & 7.99 & 2,328 & 18,595 & 22605692 & 3.711 & 1.000 & 0 & 9.62 & 1,933 & 18,595 & 18639920 & 2.830 & .998 & .002 \\
\hline 882 & 9.41 & 194 & 1,826 & 172126 & -.681 & .248 & .248 & 10.14 & 180 & 1,826 & 156056 & -1.171 & .121 & .121 \\
\hline 883 & 7.60 & 1,434 & 10,901 & 7695752 & -1.009 & .157 & .157 & 8.83 & 1,235 & 10,901 & 6620980 & -.998 & .159 & .159 \\
\hline 884 & 7.85 & 1,737 & 13,631 & 11799548 & -.238 & .406 & .406 & 9.51 & 1,433 & 13,631 & 9627362 & -.935 & .175 & .175 \\
\hline 885 & 8.13 & 3,653 & 29,698 & 55129006 & 1.709 & .956 & .044 & 9.48 & 3,133 & 29,698 & 47455866 & 1.946 & .974 & .026 \\
\hline 886 & 5.50 & 3,884 & 21,366 & 41522266 & .077 & .531 & .469 & 6.72 & 3,178 & 21,366 & 34017197 & .192 & .576 & .424 \\
\hline 887 & 5.08 & 4,941 & 25,079 & 63401202 & 2.837 & .998 & .002 & 6.07 & 4,135 & 25,079 & 53109986 & 2.705 & .997 & .003 \\
\hline 888 & 6.86 & 2,759 & 18,929 & 27153257 & 3.626 & 1.000 & 0 & 7.70 & 2,460 & 18,929 & 24440771 & 4.273 & 1.000 & 0 \\
\hline 889 & 7.00 & 1,961 & 13,726 & 13765943 & 1.753 & .960 & .040 & 8.63 & 1,591 & 13,726 & 11175284 & 1.621 & .948 & .052 \\
\hline 890 & 7.77 & 1,685 & 13,085 & 10961684 & -.403 & .344 & .344 & 9.30 & 1,407 & 13,085 & 9057233 & -1.045 & .148 & .148 \\
\hline 891 & 11.95 & 1,518 & 18,143 & 14912483 & 5.596 & 0 & 0 & 14.32 & 1,267 & 1,8143 & 12233193 & 3.967 & 1.000 & 0 \\
\hline 892 & 11.03 & 655 & 7,226 & 2320685 & -.859 & .195 & .195 & 13.07 & 553 & 7,226 & 1968800 & -.595 & .276 & .276 \\
\hline 893 & 7.57 & 3,560 & 26,939 & 49859054 & 4.111 & 1.000 & 0 & 9.36 & 2,878 & 26,939 & 39869556 & 2.647 & .996 & .004 \\
\hline 894 & 6.70 & 840 & 5,631 & 2329896 & -.746 & .228 & .228 & 8.06 & 699 & 5,631 & 1928266 & -.925 & .177 & .177 \\
\hline 895 & 9.74 & 1,753 & 17,071 & 14904134 & -.284 & .388 & .388 & 12.70 & 1,344 & 17,071 & 11548285 & .424 & .664 & .336 \\
\hline 896 & 5.09 & 7,026 & 35,755 & 128275131 & 3.084 & .999 & .001 & 5.97 & 5,987 & 35,755 & 108835494 & 2.258 & .988 & .012 \\
\hline 897 & 10.22 & 18 & 184 & 1674 & .080 & .532 & .468 & 10.22 & 18 & 184 & 1674 & .080 & .532 & .468 \\
\hline 898 & 5.63 & 215 & 1,211 & 129413 & -.150 & .440 & .440 & 5.74 & 211 & 1,211 & 127892 & .026 & .510 & .490 \\
\hline 899 & 9.67 & 6 & 58 & 104 & -1.707 & .044 & .044 & 9.67 & 6 & 58 & 104 & -1.707 & .044 & .044 \\
\hline 900 & 7.58 & 202 & 1,531 & 163162 & 1.358 & .913 & .087 & 8.28 & 185 & 1,531 & 145443 & .636 & .738 & .262 \\
\hline 901 & 8.58 & 3,970 & 34,050 & 68126251 & .867 & .807 & .193 & 10.35 & 3,290 & 34,050 & 56378525 & .650 & .742 & .258 \\
\hline 902 & 12.49 & 90 & 1,124 & 52546 & .639 & .738 & .262 & 12.77 & 88 & 1,124 & 50662 & .396 & .654 & .346 \\
\hline 903 & 12.51 & 2,020 & 25,276 & 26390062 & 2.626 & .996 & .004 & 16.21 & 1,559 & 25,276 & 20168747 & 1.618 & .947 & .053 \\
\hline 904 & 15.23 & 26 & 396 & 4462 & -1.177 & .120 & .120 & 23.29 & 17 & 396 & 3422 & .119 & .547 & .453 \\
\hline 905 & 12.27 & 1,355 & 16,626 & 11259178 & -.028 & .489 & .489 & 14.95 & 1,112 & 16,626 & 9320235 & .476 & .683 & .317 \\
\hline 906 & 8.27 & 3,826 & 31,631 & 62399609 & 3.345 & 1.000 & 0 & 9.82 & 3,220 & 31,631 & 51754874 & 1.600 & .945 & .055 \\
\hline 907 & -- & -- & -- & -- & -- & -- & -- & -- & -- & -- & -- & -- & -- & -- \\
\hline 908 & 7.74 & 2,556 & 19,782 & 24980541 & -1.042 & .149 & .149 & 8.94 & 2213 & 19,782 & 22346266 & 1.703 & .956 & .044 \\
\hline 909 & 8.70 & 965 & 8,392 & 3957273 & -1.221 & .111 & .111 & 9.64 & 871 & 8,392 & 3581863 & -1.019 & .154 & .154 \\
\hline 910 & 11.95 & 593 & 7,085 & 2041759 & -1.184 & .118 & .118 & 15.82 & 448 & 7,085 & 1551187 & -.828 & .204 & .204 \\
\hline 911 & 12.12 & 173 & 2,097 & 186727 & .670 & .749 & .251 & 15.53 & 135 & 2,097 & 144421 & .409 & .659 & .341 \\
\hline 912 & 8.16 & 37 & 302 & 3466 & -4.000 & 0 & 0 & 8.63 & 35 & 302 & 3388 & -3.678 & 0 & 0 \\
\hline
\end{tabular}


Table 3. Summary of interoccurrence intervals for daily precipitation thresholds of 0.05 and 0.10 inch-Continued

\begin{tabular}{|c|c|c|c|c|c|c|c|c|c|c|c|c|c|c|}
\hline \multirow[b]{2}{*}{$\begin{array}{c}\text { Seq. } \\
\text { no. }\end{array}$} & \multicolumn{7}{|c|}{ Daily precipitation threshold of 0.05 inch and greater } & \multicolumn{7}{|c|}{ Daily precipitation threshold of 0.10 inch and greater } \\
\hline & $\begin{array}{c}\text { Mean } \\
\text { inter- } \\
\text { occur- } \\
\text { rence } \\
\text { interval } \\
\text { (days) }\end{array}$ & $\begin{array}{c}\text { Total } \\
\text { no. of } \\
\text { events }\end{array}$ & $\begin{array}{l}\text { Total } \\
\text { no. of } \\
\text { days } \\
\text { avail- } \\
\text { able }\end{array}$ & $\begin{array}{l}\text { Sigma } \\
\text { S }\end{array}$ & $\begin{array}{c}\text { Stan- } \\
\text { dard } \\
\text { normal } \\
\text { variate }\end{array}$ & $\begin{array}{l}\text { Non- } \\
\text { ex- } \\
\text { ceed- } \\
\text { ance } \\
\text { prob- } \\
\text { ability }\end{array}$ & $\begin{array}{c}\text { p- } \\
\text { value }\end{array}$ & $\begin{array}{c}\text { Mean } \\
\text { inter- } \\
\text { occur- } \\
\text { rence } \\
\text { interval } \\
\text { (days) }\end{array}$ & $\begin{array}{c}\text { Total } \\
\text { no. of } \\
\text { events }\end{array}$ & $\begin{array}{c}\text { Total } \\
\text { no. of } \\
\text { days } \\
\text { avail- } \\
\text { able }\end{array}$ & $\begin{array}{l}\text { Sigma } \\
\mathrm{S}\end{array}$ & $\begin{array}{l}\text { Stan- } \\
\text { dard } \\
\text { normal } \\
\text { variate }\end{array}$ & $\begin{array}{l}\text { Non- } \\
\text { ex- } \\
\text { ceed- } \\
\text { ance } \\
\text { prob- } \\
\text { ability }\end{array}$ & $\begin{array}{c}\mathrm{p}- \\
\text { value }\end{array}$ \\
\hline 913 & 7.27 & 884 & 6,422 & 2891414 & 0.960 & .831 & .169 & 8.16 & 787 & 6,422 & 2623246 & 1.850 & .968 & .032 \\
\hline 914 & 5.64 & 642 & 3,622 & 1130385 & -1.218 & .112 & .112 & 6.71 & 540 & 3,622 & 966483 & -.472 & .319 & .319 \\
\hline 915 & 5.12 & 6,567 & 33,649 & 113654315 & 4.024 & 1.000 & 0 & 5.95 & 5,659 & 33,649 & 99637122 & 6.059 & 0 & 0 \\
\hline 916 & 5.63 & 3,398 & 19,131 & 33354443 & 2.643 & .996 & .004 & 6.11 & 3,130 & 19,131 & 31542673 & 5.187 & 0 & 0 \\
\hline 917 & 7.68 & 1,004 & 7,709 & 3939855 & .992 & .839 & .161 & 9.55 & 807 & 7,709 & 3026482 & -1.330 & .092 & .092 \\
\hline 918 & 4.73 & 1,874 & 8,856 & 8090601 & -1.875 & .030 & .030 & 5.43 & 1,630 & 8,856 & 7143185 & -.721 & .235 & .235 \\
\hline 919 & 11.81 & 830 & 9,801 & 4023099 & -.544 & .293 & .293 & 12.16 & 806 & 9,801 & 3876506 & -.913 & .181 & .181 \\
\hline 920 & 5.96 & 3,207 & 19,104 & 30103571 & -1.696 & .045 & .045 & 6.49 & 2,946 & 19,104 & 28041393 & -.330 & .371 & .371 \\
\hline 921 & 8.86 & 493 & 4,368 & 1075744 & -.035 & .486 & .486 & 9.14 & 478 & 4,368 & 1044590 & .023 & .509 & .491 \\
\hline 922 & 10.08 & 2,256 & 22,744 & 26096059 & 1.414 & .921 & .079 & 12.14 & 1,873 & 22,744 & 22079481 & 2.744 & .997 & .003 \\
\hline 923 & 7.94 & 4,209 & 33,427 & 70205000 & -.227 & .410 & .410 & 9.75 & 3,429 & 33,427 & 56749476 & -.993 & .160 & .160 \\
\hline 924 & 9.98 & 64 & 639 & 19557 & -.604 & .273 & .273 & 12.06 & 53 & 639 & 15817 & -.831 & .203 & .203 \\
\hline 925 & 12.40 & 339 & 4,205 & 705640 & -.318 & .375 & .375 & 14.06 & 299 & 4,205 & 639475 & .516 & .697 & .303 \\
\hline 926 & 5.98 & 93 & 556 & 25543 & -.201 & .420 & .420 & 6.78 & 82 & 556 & 22396 & -.275 & .392 & .392 \\
\hline 927 & 6.65 & 667 & 4,433 & 1420726 & -1.745 & .040 & .040 & 8.00 & 554 & 4,433 & 1186691 & -1.370 & .085 & .085 \\
\hline 928 & 7.67 & 994 & 7,623 & 4026714 & 3.432 & 1.000 & 0 & 9.17 & 831 & 7,623 & 3369287 & 3.183 & .999 & .001 \\
\hline 929 & 5.51 & 2,850 & 15,716 & 22600020 & .845 & .801 & .199 & 6.61 & 2,378 & 15,716 & 19004888 & 1.440 & .925 & .075 \\
\hline 930 & 9.22 & 988 & 9,108 & 4616654 & 1.419 & .922 & .078 & 11.00 & 828 & 9,108 & 3871936 & 1.338 & .910 & .091 \\
\hline 931 & 9.80 & 494 & 4,843 & 1172449 & -.765 & .222 & .222 & 10.33 & 469 & 4,843 & 1134440 & -.041 & .484 & .484 \\
\hline 932 & 10.81 & 301 & 3,255 & 475950 & -.854 & .197 & .197 & 11.15 & 292 & 3,255 & 457013 & -1.135 & .128 & .128 \\
\hline 933 & 20.33 & 3 & 61 & 58 & -1.098 & .136 & .136 & 20.33 & 3 & 61 & 58 & -1.098 & .136 & .136 \\
\hline 934 & 6.61 & 839 & 5,547 & 2337033 & .217 & .586 & .414 & 7.90 & 702 & 5,547 & 1929509 & -.412 & .340 & .340 \\
\hline 935 & 4.49 & 4,390 & 19,694 & 43890974 & 1.759 & .961 & .039 & 5.33 & 3,694 & 19,694 & 37063391 & 1.993 & .977 & .023 \\
\hline 936 & 4.69 & 2,026 & 9,510 & 9520901 & -.912 & .181 & .181 & 5.36 & 1,773 & 9,510 & 8301502 & -1.117 & .132 & .132 \\
\hline 937 & 4.86 & 1,836 & 8,920 & 8296647 & .980 & .836 & .164 & 5.69 & 1,569 & 8,920 & 7011535 & .135 & .554 & .446 \\
\hline 938 & 7.49 & 3,379 & 25,311 & 42629455 & -.314 & .377 & .377 & 9.27 & 2,730 & 25,311 & 34832413 & .741 & .771 & .229 \\
\hline 939 & 6.36 & 3,396 & 21,605 & 39977253 & 9.058 & 0 & 0 & 7.39 & 2,923 & 21,605 & 33465827 & 5.606 & 0 & 0 \\
\hline 940 & 7.81 & 2,036 & 15,898 & 15870780 & -1.513 & .065 & .065 & 9.61 & 1,654 & 15,898 & 12921521 & -1.212 & .113 & .113 \\
\hline 941 & 6.38 & 2,579 & 16,452 & 19160054 & -8.520 & 0 & 0 & 7.85 & 2,097 & 16,452 & 15968551 & -5.892 & 0 & 0 \\
\hline 942 & 7.07 & 1,858 & 13,131 & 12296563 & .599 & .725 & .275 & 8.42 & 1,559 & 13,131 & 10346810 & .743 & .771 & .229 \\
\hline 943 & 9.22 & 3,541 & 32,647 & 59220296 & 2.530 & .994 & .006 & 10.75 & 3,036 & 32,647 & 49822790 & .510 & .695 & .305 \\
\hline 944 & 6.17 & 931 & 5,740 & 2827911 & 3.084 & .999 & .001 & 6.78 & 847 & 5,740 & 2513396 & 1.711 & .956 & .044 \\
\hline 945 & 7.10 & 3,008 & 21,362 & 31310055 & -2.420 & .008 & .008 & 8.73 & 2,448 & 21,362 & 25645540 & -1.644 & .050 & .050 \\
\hline 946 & 5.50 & 1,787 & 9,820 & 9062529 & 2.406 & .992 & .008 & 6.41 & 1,531 & 9,820 & 7705028 & 1.693 & .955 & .045 \\
\hline 947 & 7.32 & 1,882 & 13,784 & 12942634 & -.163 & .435 & .435 & 8.80 & 1,566 & 13,784 & 10996124 & 1.291 & .902 & .098 \\
\hline 948 & 6.79 & 179 & 1,216 & 96857 & -2.550 & .005 & .005 & 9.01 & 135 & 1,216 & 71284 & -2.647 & .004 & .004 \\
\hline 949 & 13.48 & 1,967 & 26,509 & 26575843 & 1.486 & .931 & .069 & 17.63 & 1,504 & 26,509 & 20077221 & .480 & .684 & .316 \\
\hline 950 & 29.00 & 8 & 232 & 1135 & 1.093 & .863 & .137 & 29.00 & 8 & 232 & 1135 & 1.093 & .863 & .137 \\
\hline 951 & 5.99 & 2,071 & 12,405 & 13510230 & 4.080 & 1.000 & 0 & 6.63 & 1,871 & 12,405 & 11907837 & 1.956 & .975 & .025 \\
\hline 952 & 7.75 & 636 & 4,929 & 1574227 & .190 & .575 & .425 & 8.05 & 612 & 4,929 & 1513291 & .143 & .557 & .443 \\
\hline 953 & 6.59 & 2,127 & 14,026 & 14887607 & -.156 & .438 & .438 & 7.89 & 1,777 & 14,026 & 12560969 & .579 & .719 & .281 \\
\hline 954 & 7.59 & 912 & 6,922 & 2939951 & -3.587 & 0 & 0 & 8.28 & 836 & 6,922 & 2724466 & -2.924 & .002 & .002 \\
\hline 955 & 8.29 & 3,986 & 33,055 & 69650502 & 6.261 & 0 & 0 & 9.41 & 3,512 & 33,055 & 60017303 & 3.489 & 1.000 & 0 \\
\hline 956 & 7.90 & 4,397 & 34,736 & 80186413 & 5.744 & 0 & 0 & 9.35 & 3,714 & 34,736 & 66141611 & 2.679 & .996 & .004 \\
\hline 957 & 6.01 & 713 & 4,283 & 1505704 & -.642 & .261 & .261 & 6.81 & 629 & 4,283 & 1318564 & -.917 & .180 & .180 \\
\hline 958 & 9.49 & 1,322 & 12,543 & 8258763 & -.244 & .403 & .403 & 10.66 & 1,177 & 12,543 & 7401535 & .161 & .564 & .436 \\
\hline 959 & 5.75 & 2,404 & 13,823 & 16989609 & 1.913 & .972 & .028 & 6.46 & 2,141 & 13,823 & 15083503 & 1.549 & .939 & .061 \\
\hline 960 & 5.79 & 135 & 781 & 60686 & 3.042 & .999 & .001 & 6.56 & 119 & 781 & 53166 & 2.723 & .997 & .003 \\
\hline
\end{tabular}


Table 3. Summary of interoccurrence intervals for daily precipitation thresholds of 0.05 and 0.10 inch-Continued

\begin{tabular}{|c|c|c|c|c|c|c|c|c|c|c|c|c|c|c|}
\hline \multirow[b]{2}{*}{$\begin{array}{l}\text { Seq. } \\
\text { no. }\end{array}$} & \multicolumn{7}{|c|}{ Daily precipitation threshold of 0.05 inch and greater } & \multicolumn{7}{|c|}{ Daily precipitation threshold of 0.10 inch and greater } \\
\hline & $\begin{array}{l}\text { Mean } \\
\text { inter- } \\
\text { occur- } \\
\text { rence } \\
\text { interval } \\
\text { (days) }\end{array}$ & $\begin{array}{c}\text { Total } \\
\text { no. of } \\
\text { events }\end{array}$ & $\begin{array}{l}\text { Total } \\
\text { no. of } \\
\text { days } \\
\text { avail- } \\
\text { able }\end{array}$ & $\begin{array}{l}\text { Sigma } \\
\mathrm{S}\end{array}$ & $\begin{array}{l}\text { Stan- } \\
\text { dard } \\
\text { normal } \\
\text { variate }\end{array}$ & $\begin{array}{l}\text { Non- } \\
\text { ex- } \\
\text { ceed- } \\
\text { ance } \\
\text { prob- } \\
\text { ability }\end{array}$ & $\begin{array}{c}\text { p- } \\
\text { value }\end{array}$ & $\begin{array}{c}\text { Mean } \\
\text { inter- } \\
\text { occur- } \\
\text { rence } \\
\text { interval } \\
\text { (days) }\end{array}$ & $\begin{array}{c}\text { Total } \\
\text { no. of } \\
\text { events }\end{array}$ & $\begin{array}{l}\text { Total } \\
\text { no. of } \\
\text { days } \\
\text { avail- } \\
\text { able }\end{array}$ & $\begin{array}{c}\text { Sigma } \\
\mathrm{S}\end{array}$ & $\begin{array}{l}\text { Stan- } \\
\text { dard } \\
\text { normal } \\
\text { variate }\end{array}$ & $\begin{array}{l}\text { Non- } \\
\text { ex- } \\
\text { ceed- } \\
\text { ance } \\
\text { prob- } \\
\text { ability }\end{array}$ & $\begin{array}{c}p- \\
\text { value }\end{array}$ \\
\hline 961 & 7.17 & 2,803 & 20,090 & 27864195 & -0.951 & .171 & .171 & 8.10 & 2,479 & 20,090 & 25202218 & 1.041 & .851 & .149 \\
\hline 962 & 13.07 & 55 & 719 & 23856 & 2.653 & .996 & .004 & 13.83 & 52 & 719 & 22055 & 2.246 & .988 & .012 \\
\hline 963 & 8.45 & 135 & 1,141 & 65848 & -2.919 & .002 & .002 & 10.66 & 107 & 1,141 & 53268 & -2.282 & .011 & .011 \\
\hline 964 & 8.33 & 242 & 2,015 & 229624 & -1.568 & .058 & .058 & 10.08 & 200 & 2,015 & 195471 & -.733 & .232 & .232 \\
\hline 965 & 7.44 & 1,371 & 10,194 & 7763599 & 7.118 & 0 & 0 & 8.68 & 1,175 & 10,194 & 6522811 & 5.292 & 0 & 0 \\
\hline 966 & 10.31 & 862 & 8,889 & 3912318 & 1.077 & .859 & .141 & 12.06 & 737 & 8,889 & 3329971 & .781 & .782 & .218 \\
\hline 967 & 8.68 & 582 & 5,054 & 1452183 & -.527 & .299 & .299 & 10.94 & 462 & 5,054 & 1152618 & -.474 & .318 & .318 \\
\hline 968 & 5.76 & 275 & 1,583 & 235394 & 2.340 & .990 & .010 & 7.04 & 225 & 1,583 & 194691 & 2.422 & .992 & .008 \\
\hline 969 & 5.53 & 220 & 1,217 & 139876 & 1.153 & .875 & .125 & 6.58 & 185 & 1,217 & 121175 & 1.800 & .964 & .036 \\
\hline 970 & 6.94 & 4,716 & 32,743 & 76520915 & -1.059 & .145 & .145 & 8.84 & 3,704 & 32,743 & 60379977 & -.452 & .326 & .326 \\
\hline 971 & 6.55 & 263 & 1,722 & 233482 & .873 & .809 & .191 & 7.46 & 231 & 1,722 & 202738 & .509 & .695 & .305 \\
\hline 972 & 8.21 & 2,256 & 18,526 & 21631638 & 2.891 & .998 & .002 & 9.06 & 2,044 & 18,526 & 19630747 & 2.883 & .998 & .002 \\
\hline 973 & 13.17 & 1,484 & 19,541 & 14753232 & 1.168 & .879 & .121 & 16.89 & 1,157 & 19,541 & 11743642 & 2.289 & .989 & .011 \\
\hline 974 & 5.56 & 2,006 & 11,161 & 11284335 & .623 & .733 & .267 & 6.41 & 1,742 & 11,161 & 9798918 & .578 & .718 & .282 \\
\hline 975 & 6.15 & 2,018 & 12,404 & 12699753 & 1.145 & .874 & .126 & 6.67 & 1,860 & 12,404 & 11271774 & -1.709 & .044 & .044 \\
\hline 976 & 7.59 & 117 & 888 & 55328 & 1.219 & .889 & .111 & 8.46 & 105 & 888 & 51633 & 1.908 & .972 & .028 \\
\hline 977 & 7.15 & 13 & 93 & 736 & 1.359 & .913 & .087 & 7.15 & 13 & 93 & 736 & 1.359 & .913 & .087 \\
\hline 978 & 5.85 & 2,284 & 13,354 & 15905475 & 3.556 & 1.000 & 0 & 7.37 & 1,811 & 13,354 & 12532888 & 2.687 & .996 & .004 \\
\hline 979 & 5.68 & 1,006 & 5,715 & 2831400 & -.826 & .204 & .204 & 6.97 & 820 & 5,715 & 2306029 & -.786 & .216 & .216 \\
\hline 980 & 7.08 & 505 & 3,573 & 853634 & -2.095 & .018 & .018 & 8.21 & 435 & 3,573 & 751861 & -1.175 & .120 & .120 \\
\hline 981 & 15.00 & 1 & 15 & 15 & 1.732 & .958 & .042 & 15.00 & 1 & 15 & 15 & 1.732 & .958 & .042 \\
\hline 982 & 8.39 & 46 & 386 & 9288 & .543 & .706 & .294 & 9.42 & 41 & 386 & 8751 & 1.175 & .880 & .120 \\
\hline 983 & 5.61 & 1,864 & 10,455 & 9527377 & -1.663 & .048 & .048 & 6.17 & 1,696 & 10,455 & 8687533 & -1.435 & .076 & .076 \\
\hline 984 & 12.58 & 281 & 3,534 & 465104 & -1.838 & .033 & .033 & 13.14 & 269 & 3,534 & 454158 & -1.265 & .103 & .103 \\
\hline 985 & 7.44 & 163 & 1,212 & 100705 & .431 & .667 & .333 & 8.36 & 145 & 1,212 & 90894 & .718 & .763 & .237 \\
\hline 986 & 9.05 & 383 & 3,465 & 575721 & -4.487 & 0 & 0 & 10.13 & 342 & 3,465 & 546671 & -2.478 & .007 & .007 \\
\hline 987 & 9.73 & 2,309 & 22,473 & 24854223 & -3.499 & 0 & 0 & 10.75 & 2,091 & 2,2473 & 23053896 & -1.489 & .068 & .068 \\
\hline 988 & 9.69 & 242 & 2,346 & 241452 & -4.026 & 0 & 0 & 9.78 & 240 & 2,346 & 240946 & -3.867 & 0 & 0 \\
\hline 989 & 5.27 & 3,067 & 16,159 & 23963828 & -3.159 & .001 & .001 & 5.96 & 2,713 & 16,159 & 21351618 & -2.338 & .010 & .010 \\
\hline 990 & 6.02 & 3,094 & 18,639 & 30430815 & 5.334 & 0 & 0 & 6.84 & 2,724 & 18,639 & 26791740 & 5.005 & 0 & 0 \\
\hline 991 & 8.69 & 2,137 & 18,574 & 19896150 & .201 & .580 & .420 & 9.23 & 2,012 & 18,574 & 19052152 & 1.525 & .936 & .064 \\
\hline 992 & 5.03 & 3,978 & 19,989 & 40518733 & 2.090 & .982 & .018 & 6.02 & 3,320 & 19,989 & 33902188 & 2.167 & .985 & .015 \\
\hline 993 & 6.14 & 223 & 1,369 & 152709 & .011 & .504 & .496 & 7.32 & 187 & 1,369 & 129072 & .198 & .579 & .421 \\
\hline 994 & 7.50 & 824 & 6,177 & 2574701 & .582 & .720 & .280 & 8.84 & 699 & 6,177 & 2208685 & 1.057 & .855 & .145 \\
\hline 995 & 7.77 & 2,120 & 16,470 & 18108458 & 2.970 & .999 & .001 & 8.57 & 1,921 & 16,470 & 16270459 & 2.164 & .985 & .015 \\
\hline 996 & 9.37 & 3,079 & 28,850 & 45593368 & 2.551 & .995 & .005 & 11.94 & 2,417 & 28,850 & 35473600 & 1.486 & .931 & .069 \\
\hline 997 & 9.27 & 2,973 & 27,555 & 42286021 & 3.056 & .999 & .001 & 10.00 & 2,756 & 27,555 & 38803527 & 1.994 & .977 & .023 \\
\hline 998 & 7.49 & 2,849 & 21,324 & 31316699 & 2.863 & .998 & .002 & 8.59 & 2,484 & 21,324 & 27373493 & 2.898 & .998 & .002 \\
\hline 999 & 6.89 & 530 & 3,649 & 892774 & -3.060 & .001 & .001 & 8.39 & 435 & 3,649 & 745312 & -2.201 & .014 & .014 \\
\hline 1000 & 5.51 & 1,634 & 9,008 & 7040469 & -3.035 & .001 & .001 & 6.37 & 1,415 & 9,008 & 6244410 & -1.316 & .094 & .094 \\
\hline 1001 & 6.20 & 3,180 & 19,714 & 32086460 & 2.310 & .990 & .010 & 7.22 & 2,732 & 19,714 & 27822505 & 3.003 & .999 & .001 \\
\hline 1002 & 3.50 & 4 & 14 & 38 & 1.237 & .892 & .108 & 4.67 & 3 & 14 & 31 & 1.429 & .923 & .077 \\
\hline 1003 & 9.03 & 2,255 & 20,356 & 24237862 & 4.610 & 1.000 & 0 & 10.37 & 1,963 & 20,356 & 21162644 & 4.545 & 1.000 & 0 \\
\hline 1004 & 6.99 & 2,916 & 20,384 & 31367486 & 5.185 & 0 & 0 & 8.56 & 2,382 & 20,384 & 25245002 & 3.369 & 1.000 & 0 \\
\hline 1005 & 9.78 & 2,182 & 21,346 & 23451150 & .565 & .714 & .286 & 10.86 & 1,966 & 21,346 & 21020141 & .136 & .554 & .446 \\
\hline 1006 & 6.10 & 464 & 2,830 & 629444 & -1.541 & .062 & .062 & 6.55 & 432 & 2,830 & 584339 & -1.587 & .056 & .056 \\
\hline 1007 & 8.51 & 994 & 8,461 & 4266818 & .801 & .788 & .212 & 9.94 & 851 & 8,461 & 3565129 & -.492 & .312 & .312 \\
\hline 1008 & 5.72 & 2,428 & 13,894 & 16916135 & .247 & .598 & .402 & 6.87 & 2,024 & 13,894 & 14009663 & -.283 & .389 & .389 \\
\hline
\end{tabular}


Table 3. Summary of interoccurrence intervals for daily precipitation thresholds of 0.05 and 0.10 inch-Continued

\begin{tabular}{|c|c|c|c|c|c|c|c|c|c|c|c|c|c|c|}
\hline \multirow[b]{2}{*}{$\begin{array}{l}\text { Seq. } \\
\text { no. }\end{array}$} & \multicolumn{7}{|c|}{ Daily precipitation threshold of 0.05 inch and greater } & \multicolumn{7}{|c|}{ Daily precipitation threshold of 0.10 inch and greater } \\
\hline & $\begin{array}{c}\text { Mean } \\
\text { inter- } \\
\text { occur- } \\
\text { rence } \\
\text { interval } \\
\text { (days) }\end{array}$ & $\begin{array}{l}\text { Total } \\
\text { no. of } \\
\text { events }\end{array}$ & $\begin{array}{c}\text { Total } \\
\text { no. of } \\
\text { days } \\
\text { avail- } \\
\text { able }\end{array}$ & $\begin{array}{l}\text { Sigma } \\
\mathrm{S}\end{array}$ & $\begin{array}{c}\text { Stan- } \\
\text { dard } \\
\text { normal } \\
\text { variate }\end{array}$ & $\begin{array}{l}\text { Non- } \\
\text { ex- } \\
\text { ceed- } \\
\text { ance } \\
\text { prob- } \\
\text { ability }\end{array}$ & $\begin{array}{c}\mathrm{p}- \\
\text { value }\end{array}$ & $\begin{array}{l}\text { Mean } \\
\text { inter- } \\
\text { occur- } \\
\text { rence } \\
\text { interval } \\
\text { (days) }\end{array}$ & $\begin{array}{l}\text { Total } \\
\text { no. of } \\
\text { events }\end{array}$ & $\begin{array}{l}\text { Total } \\
\text { no. of } \\
\text { days } \\
\text { avail- } \\
\text { able }\end{array}$ & $\begin{array}{l}\text { Sigma } \\
\mathrm{S}\end{array}$ & $\begin{array}{c}\text { Stan- } \\
\text { dard } \\
\text { normal } \\
\text { variate }\end{array}$ & $\begin{array}{l}\text { Non- } \\
\text { ex- } \\
\text { ceed- } \\
\text { ance } \\
\text { prob- } \\
\text { ability }\end{array}$ & $\begin{array}{c}\mathrm{p}- \\
\text { value }\end{array}$ \\
\hline 1009 & 4.68 & 2,676 & 12,527 & 17110451 & 1.867 & .969 & .031 & 5.49 & 2,281 & 12,527 & 14523707 & 1.370 & .915 & .085 \\
\hline 1010 & 6.09 & 2,571 & 15,655 & 20057145 & -.294 & .384 & .384 & 7.61 & 2,057 & 15,655 & 16111109 & .049 & .519 & .481 \\
\hline 1011 & 8.94 & 2,283 & 20,404 & 23969292 & 2.410 & .992 & .008 & 10.40 & 1,962 & 20,404 & 20489465 & 1.814 & .965 & .035 \\
\hline 1012 & 8.27 & 362 & 2,993 & 546049 & .263 & .604 & .396 & 10.01 & 299 & 2,993 & 434144 & -.891 & .187 & .187 \\
\hline 1013 & 7.66 & 1,427 & 10,925 & 8097384 & 2.538 & .994 & .006 & 9.22 & 1,185 & 10,925 & 6634868 & 1.490 & .932 & .068 \\
\hline 1014 & 10.60 & 221 & 2,342 & 269306 & 1.046 & .852 & .148 & 10.60 & 221 & 2,342 & 269306 & 1.046 & .852 & .148 \\
\hline 1015 & 17.08 & 25 & 427 & 4670 & -1.083 & .139 & .139 & 17.08 & 25 & 427 & 4670 & -1.083 & .139 & .139 \\
\hline 1016 & 6.21 & 3,226 & 20,037 & 34469537 & 6.544 & 0 & 0 & 6.95 & 2,885 & 20,037 & 30168616 & 4.073 & 1.000 & 0 \\
\hline 1017 & 6.67 & 280 & 1,867 & 256788 & -.509 & .305 & .305 & 7.95 & 235 & 1,867 & 222739 & .408 & .658 & .342 \\
\hline 1018 & 6.54 & 370 & 2,421 & 445648 & -.166 & .434 & .434 & 6.67 & 363 & 2,421 & 436878 & -.190 & .424 & .424 \\
\hline 1019 & 8.73 & 924 & 8,062 & 3504693 & -3.109 & .001 & .001 & 9.87 & 817 & 8,062 & 3163558 & -1.951 & .026 & .026 \\
\hline 1020 & 9.80 & 601 & 5,888 & 1782337 & .312 & .622 & .378 & 10.80 & 545 & 5,888 & 1658374 & 1.358 & .913 & .087 \\
\hline 1021 & 8.29 & 2,925 & 24,253 & 34975455 & -1.306 & .096 & .096 & 9.64 & 2,517 & 24,253 & 30555427 & .094 & .537 & .463 \\
\hline 1022 & 6.41 & 1,541 & 9,875 & 7271280 & -3.015 & .001 & .001 & 7.12 & 1,388 & 9,875 & 6657854 & -1.840 & .033 & .033 \\
\hline 1023 & 5.27 & 1,280 & 6,740 & 4244486 & -.993 & .160 & .160 & 6.33 & 1,065 & 6,740 & 3485405 & -1.632 & .051 & .051 \\
\hline 1024 & 6.47 & 1,814 & 11,727 & 10340204 & -2.054 & .020 & .020 & 7.33 & 1,600 & 11,727 & 9367879 & -.101 & .460 & .460 \\
\hline 1025 & 9.74 & 290 & 2,824 & 388275 & -1.527 & .063 & .063 & 9.98 & 283 & 2,824 & 373168 & -1.927 & .027 & .027 \\
\hline 1026 & 6.28 & 3,045 & 19,114 & 31250963 & 7.061 & 0 & 0 & 6.97 & 2,743 & 19,114 & 27692905 & 5.115 & 0 & 0 \\
\hline 1027 & 7.04 & 960 & 6,758 & 3333940 & 1.491 & .932 & .068 & 8.87 & 762 & 6,758 & 2672342 & 1.811 & .965 & .035 \\
\hline 1028 & 8.80 & 3,112 & 27,372 & 43395375 & 1.825 & .966 & .034 & 10.09 & 2,712 & 27,372 & 37787345 & 1.630 & .948 & .052 \\
\hline 1029 & 7.99 & 1,946 & 15,548 & 15211274 & .420 & .663 & .337 & 8.49 & 1,831 & 15,548 & 14296392 & .324 & .627 & .373 \\
\hline 1030 & 6.01 & 2,037 & 12,246 & 11882151 & -3.700 & 0 & 0 & 7.03 & 1,743 & 12,246 & 10436377 & -1.599 & .055 & .055 \\
\hline 1031 & 8.97 & 283 & 2,538 & 324818 & -2.784 & .003 & .003 & 11.28 & 225 & 2,538 & 250160 & -3.218 & .001 & .001 \\
\hline 1032 & 7.05 & 5,166 & 36,443 & 98197689 & 5.377 & 0 & 0 & 8.34 & 4,371 & 36,443 & 81346587 & 2.445 & .993 & .007 \\
\hline 1033 & 5.23 & 4,102 & 21,434 & 44662749 & 1.771 & .962 & .038 & 5.69 & 3,767 & 21,434 & 41064425 & 1.826 & .966 & .034 \\
\hline 1034 & 13.28 & 163 & 2,164 & 186337 & 1.250 & .894 & .106 & 13.61 & 159 & 2,164 & 180346 & 1.055 & .854 & .146 \\
\hline 1035 & 7.77 & 4,349 & 33,805 & 73976820 & .727 & .766 & .234 & 9.42 & 3,588 & 33,805 & 60188674 & -.783 & .217 & .217 \\
\hline 1036 & 4.16 & 129 & 536 & 31582 & -1.701 & .044 & .044 & 5.36 & 100 & 536 & 26350 & -.291 & .386 & .386 \\
\hline 1037 & 7.89 & 54 & 426 & 11556 & .060 & .524 & .476 & 8.35 & 51 & 426 & 10959 & .109 & .544 & .456 \\
\hline 1038 & 8.51 & 408 & 3,471 & 727781 & .973 & .835 & .165 & 8.95 & 388 & 3,471 & 696584 & 1.176 & .880 & .120 \\
\hline 1039 & 10.86 & 645 & 7,002 & 2168180 & -1.753 & .040 & .040 & 14.32 & 489 & 7,002 & 1664868 & -1.054 & .146 & .146 \\
\hline 1040 & 14.14 & 486 & 6,871 & 1694158 & .560 & .712 & .288 & 17.99 & 382 & 6,871 & 1383634 & 1.839 & .967 & .033 \\
\hline 1041 & 14.71 & 238 & 3,500 & 386568 & -1.920 & .027 & .027 & 20.00 & 175 & 3,500 & 278448 & -2.080 & .019 & .019 \\
\hline 1042 & 4.24 & 2,925 & 12,414 & 18050973 & -.539 & .295 & .295 & 4.96 & 2,505 & 12,414 & 15396707 & -.847 & .199 & .199 \\
\hline 1043 & 16.82 & 28 & 471 & 6376 & -.303 & .381 & .381 & 17.44 & 27 & 471 & 5980 & -.536 & .296 & .296 \\
\hline 1044 & 8.75 & 936 & 8,188 & 4019438 & 2.592 & .995 & .005 & 11.07 & 740 & 8,188 & 3216754 & 2.911 & .998 & .002 \\
\hline 1045 & 8.71 & 2,295 & 19,999 & 23808719 & 3.109 & .999 & .001 & 10.92 & 1,831 & 19,999 & 18924421 & 2.491 & .994 & .006 \\
\hline 1046 & 6.25 & 273 & 1,706 & 234928 & .253 & .600 & .400 & 7.72 & 221 & 1,706 & 188580 & .009 & .504 & .496 \\
\hline 1047 & 6.48 & 3,117 & 20,211 & 32381403 & 2.709 & .997 & .003 & 8.17 & 2,475 & 20,211 & 25998497 & 3.402 & 1.000 & 0 \\
\hline 1048 & 6.92 & 1,736 & 12,010 & 10645097 & 1.526 & .936 & .064 & 8.82 & 1,361 & 12,010 & 8291649 & .929 & .824 & .176 \\
\hline 1049 & 6.43 & 762 & 4,897 & 1789007 & -1.967 & .025 & .025 & 7.98 & 614 & 4,897 & 1460945 & -1.211 & .113 & .113 \\
\hline 1050 & 4.74 & 2,539 & 12,043 & 15686750 & 2.273 & .988 & .012 & 5.37 & 2,242 & 12,043 & 13776118 & 1.676 & .953 & .047 \\
\hline 1051 & 7.89 & 2,265 & 17,865 & 19832655 & -1.628 & .052 & .052 & 9.53 & 1,874 & 17,865 & 16524862 & -.961 & .168 & .168 \\
\hline 1052 & 7.24 & 100 & 724 & 33643 & -1.223 & .111 & .111 & 9.17 & 79 & 724 & 27296 & -.701 & .242 & .242 \\
\hline 1053 & 4.93 & 212 & 1,044 & 112677 & .459 & .677 & .323 & 5.38 & 194 & 1,044 & 100710 & -.133 & .447 & .447 \\
\hline 1054 & 12.20 & 5 & 61 & 112 & -1.029 & .152 & .152 & 15.25 & 4 & 61 & 93 & -.823 & .205 & .205 \\
\hline 1055 & 6.31 & 530 & 3,344 & 854586 & -1.421 & .078 & .078 & 6.95 & 481 & 3,344 & 787368 & -.797 & .213 & .213 \\
\hline 1056 & 7.08 & 297 & 2,102 & 357963 & 4.381 & 1.000 & 0 & 9.60 & 219 & 2,102 & 264650 & 3.840 & 1.000 & 0 \\
\hline
\end{tabular}


Table 3. Summary of interoccurrence intervals for daily precipitation thresholds of 0.05 and 0.10 inch-Continued

\begin{tabular}{|c|c|c|c|c|c|c|c|c|c|c|c|c|c|c|}
\hline \multirow[b]{2}{*}{$\begin{array}{l}\text { Seq. } \\
\text { no. }\end{array}$} & \multicolumn{7}{|c|}{ Daily precipitation threshold of 0.05 inch and greater } & \multicolumn{7}{|c|}{ Daily precipitation threshold of 0.10 inch and greater } \\
\hline & $\begin{array}{c}\text { Mean } \\
\text { inter- } \\
\text { occur- } \\
\text { rence } \\
\text { interval } \\
\text { (days) }\end{array}$ & $\begin{array}{c}\text { Total } \\
\text { no. of } \\
\text { events }\end{array}$ & $\begin{array}{l}\text { Total } \\
\text { no. of } \\
\text { days } \\
\text { avail- } \\
\text { able }\end{array}$ & $\begin{array}{l}\text { Sigma } \\
\mathrm{S}\end{array}$ & $\begin{array}{c}\text { Stan- } \\
\text { dard } \\
\text { normal } \\
\text { variate }\end{array}$ & $\begin{array}{l}\text { Non- } \\
\text { ex- } \\
\text { ceed- } \\
\text { ance } \\
\text { prob- } \\
\text { ability }\end{array}$ & $\begin{array}{c}\text { p- } \\
\text { value }\end{array}$ & $\begin{array}{c}\text { Mean } \\
\text { inter- } \\
\text { occur- } \\
\text { rence } \\
\text { interval } \\
\text { (days) }\end{array}$ & $\begin{array}{c}\text { Total } \\
\text { no. of } \\
\text { events }\end{array}$ & $\begin{array}{c}\text { Total } \\
\text { no. of } \\
\text { days } \\
\text { avail- } \\
\text { able }\end{array}$ & $\begin{array}{c}\text { Sigma } \\
\mathrm{S}\end{array}$ & $\begin{array}{c}\text { Stan- } \\
\text { dard } \\
\text { normal } \\
\text { variate }\end{array}$ & $\begin{array}{l}\text { Non- } \\
\text { ex- } \\
\text { ceed- } \\
\text { ance } \\
\text { prob- } \\
\text { ability }\end{array}$ & $\begin{array}{c}\mathrm{p}- \\
\text { value }\end{array}$ \\
\hline 1057 & 11.29 & 45 & 508 & 11386 & -0.045 & .482 & .482 & 14.51 & 35 & 508 & 8744 & -0.168 & .433 & .433 \\
\hline 1058 & 6.23 & 5,658 & 35,247 & 104409758 & 6.136 & 0 & 0 & 7.40 & 4,762 & 35,247 & 86426063 & 3.565 & 1.000 & 0 \\
\hline 1059 & 7.69 & 93 & 715 & 33200 & -.024 & .490 & .490 & 9.93 & 72 & 715 & 25882 & .081 & .532 & .468 \\
\hline 1060 & 7.16 & 3,383 & 24,233 & 41601227 & 1.502 & .933 & .067 & 8.61 & 2,816 & 24,233 & 34557635 & 1.179 & .881 & .119 \\
\hline 1061 & 5.74 & 115 & 660 & 38367 & .204 & .581 & .419 & 7.42 & 89 & 660 & 30619 & .695 & .756 & .244 \\
\hline 1062 & 11.01 & 189 & 2,080 & 179527 & -2.063 & .020 & .020 & 12.38 & 168 & 2,080 & 151846 & -2.939 & .002 & .002 \\
\hline 1063 & 12.00 & 1,989 & 23,869 & 25351574 & 5.252 & 0 & 0 & 14.89 & 1,603 & 23,869 & 19909747 & 2.823 & .998 & .002 \\
\hline 1064 & 15.30 & 81 & 1,239 & 45573 & -1.431 & .076 & .076 & 18.49 & 67 & 1,239 & 39761 & -.596 & .276 & .276 \\
\hline 1065 & 8.26 & 735 & 6,070 & 2272440 & .878 & .810 & .190 & 9.18 & 661 & 6,070 & 2042782 & .814 & .792 & .208 \\
\hline 1066 & 6.53 & 1,701 & 11,113 & 9793676 & 2.585 & .995 & .005 & 7.38 & 1,506 & 11,113 & 8619687 & 2.021 & .978 & .022 \\
\hline 1067 & 8.19 & 112 & 917 & 55833 & 1.600 & .945 & .055 & 9.26 & 99 & 917 & 49779 & 1.666 & .952 & .048 \\
\hline 1068 & 7.38 & 97 & 716 & 33684 & -.512 & .304 & .304 & 9.55 & 75 & 716 & 26902 & .029 & .512 & .488 \\
\hline 1069 & 7.59 & 714 & 5,418 & 1856957 & -1.849 & .032 & .032 & 10.02 & 541 & 5,418 & 1424628 & -1.125 & .130 & .130 \\
\hline 1070 & 7.60 & 4,635 & 35,225 & 85479402 & 5.555 & 0 & 0 & 9.14 & 3,853 & 35,225 & 68897906 & 1.643 & .950 & .050 \\
\hline 1071 & 5.25 & 3,742 & 19,631 & 35385361 & -3.878 & 0 & 0 & 6.33 & 3,103 & 19,631 & 30638520 & .573 & .717 & .283 \\
\hline 1072 & 8.92 & 187 & 1,668 & 153334 & -.399 & .345 & .345 & 9.64 & 173 & 1,668 & 138743 & -.875 & .191 & .191 \\
\hline 1073 & 30.29 & 7 & 212 & 911 & 1.044 & .852 & .148 & 35.33 & 6 & 212 & 699 & .420 & .663 & .337 \\
\hline 1074 & 5.40 & 5,577 & 30,105 & 82160970 & -2.753 & .003 & .003 & 6.46 & 4,657 & 30,105 & 69558636 & -.912 & .181 & .181 \\
\hline 1075 & 9.48 & 273 & 2,589 & 351952 & -.117 & .453 & .453 & 10.15 & 255 & 2,589 & 331761 & .139 & .555 & .445 \\
\hline 1076 & 6.49 & 2,781 & 18,047 & 24957862 & -.497 & .310 & .310 & 8.05 & 2,241 & 18,047 & 20037437 & -.747 & .228 & .228 \\
\hline 1077 & 7.00 & 491 & 3,438 & 842329 & -.077 & .469 & .469 & 8.25 & 417 & 3,438 & 724940 & .401 & .656 & .344 \\
\hline 1078 & 9.06 & 3,036 & 27,513 & 42993626 & 2.808 & .998 & .002 & 11.44 & 2,405 & 27,513 & 33612160 & 1.355 & .912 & .088 \\
\hline 1079 & 7.33 & 4,268 & 31,290 & 69686749 & 4.938 & 1.000 & 0 & 8.67 & 3,611 & 31,290 & 58382046 & 3.478 & 1.000 & 0 \\
\hline 1080 & 7.68 & 63 & 484 & 16965 & 1.550 & .939 & .061 & 8.80 & 55 & 484 & 14354 & 1.008 & .843 & .157 \\
\hline 1081 & 7.48 & 2,839 & 21,232 & 30598738 & 1.408 & .920 & .080 & 9.04 & 2,350 & 21,232 & 25214480 & .898 & .815 & .185 \\
\hline 1082 & 7.46 & 1,836 & 13,695 & 12934267 & 2.139 & .984 & .016 & 9.11 & 1,503 & 13,695 & 10692133 & 2.612 & .996 & .005 \\
\hline 1083 & 12.88 & 1,198 & 15,430 & 8941425 & -1.953 & .025 & .025 & 15.02 & 1,027 & 15,430 & 7783815 & -.977 & .164 & .164 \\
\hline 1084 & 4.91 & 248 & 1,217 & 154880 & .718 & .764 & .236 & 5.74 & 212 & 1217 & 128379 & -.122 & .451 & .451 \\
\hline 1085 & 5.59 & 6,495 & 36,335 & 122536191 & 5.369 & 0 & 0 & 6.50 & 5,589 & 36,335 & 103885107 & 2.993 & .999 & .001 \\
\hline 1086 & 10.65 & 474 & 5,049 & 1363947 & 5.273 & 0 & 0 & 12.82 & 394 & 5,049 & 1089543 & 3.280 & .999 & .001 \\
\hline 1087 & 10.02 & 1,402 & 14,050 & 10014586 & 1.090 & .862 & .138 & 12.97 & 1,083 & 14,050 & 7827689 & 1.645 & .950 & .050 \\
\hline 1088 & 5.40 & 417 & 2,252 & 470189 & .049 & .519 & .481 & 6.09 & 370 & 2,252 & 417924 & .104 & .542 & .458 \\
\hline 1089 & 13.63 & 87 & 1,186 & 47898 & -1.156 & .124 & .124 & 14.46 & 82 & 1,186 & 45817 & -.906 & .183 & .183 \\
\hline 1090 & 7.53 & 1,352 & 10,180 & 7088091 & 1.910 & .972 & .028 & 8.92 & 1,141 & 10,180 & 5962291 & 1.557 & .940 & .060 \\
\hline 1091 & 7.09 & 2,779 & 19,700 & 28516563 & 3.814 & 1.000 & 0 & 8.78 & 2,243 & 19,700 & 22685020 & 2.196 & .986 & .014 \\
\hline 1092 & 3.97 & 31 & 123 & 1258 & -3.280 & .001 & .001 & 4.39 & 28 & 123 & 1228 & -2.629 & .004 & .004 \\
\hline 1093 & 5.07 & 939 & 4,757 & 2221549 & -.282 & .389 & .389 & 5.68 & 838 & 4,757 & 2027415 & .861 & .805 & .195 \\
\hline 1094 & 6.87 & 3,755 & 25,807 & 49265443 & 1.781 & .963 & .038 & 8.19 & 3,152 & 25,807 & 41038466 & .877 & .810 & .190 \\
\hline 1095 & 6.62 & 299 & 1,978 & 286550 & -.928 & .177 & .177 & 7.19 & 275 & 1978 & 261280 & -1.130 & .129 & .129 \\
\hline 1096 & 8.34 & 2,290 & 19,104 & 22581611 & 2.681 & .996 & .004 & 10.31 & 1,853 & 19,104 & 17933376 & .984 & .837 & .163 \\
\hline 1097 & 6.44 & 2,954 & 19,028 & 28595807 & 1.646 & .950 & .050 & 7.16 & 2,657 & 19,028 & 26330474 & 3.715 & 1.000 & 0 \\
\hline 1098 & 7.15 & 2,067 & 14,774 & 15369182 & .517 & .697 & .303 & 8.45 & 1748 & 14,774 & 12879238 & -.186 & .426 & .426 \\
\hline 1099 & 12.12 & 17 & 206 & 1043 & -2.888 & .002 & .002 & 15.85 & 13 & 206 & 788 & -2.570 & .005 & .005 \\
\hline 1100 & 7.07 & 399 & 2,820 & 499565 & -3.876 & 0 & 0 & 8.22 & 343 & 2,820 & 431332 & -3.469 & 0 & 0 \\
\hline 1101 & 5.62 & 5,199 & 29,198 & 75605451 & -.485 & .314 & .314 & 6.73 & 4,341 & 29,198 & 62818437 & -1.001 & .159 & .159 \\
\hline 1102 & 8.75 & 3,606 & 31,561 & 57159434 & .466 & .679 & .321 & 10.23 & 3,084 & 31,561 & 48190881 & -.941 & .173 & .173 \\
\hline 1103 & 13.51 & 775 & 10,466 & 3923209 & -1.574 & .058 & .058 & 18.01 & 581 & 10,466 & 2884781 & -2.137 & .016 & .016 \\
\hline 1104 & 8.02 & 974 & 7,812 & 4223439 & 5.953 & 0 & 0 & 8.56 & 913 & 7,812 & 3887049 & 4.709 & 1.000 & 0 \\
\hline
\end{tabular}


Table 3. Summary of interoccurrence intervals for daily precipitation thresholds of 0.05 and 0.10 inch-Continued

\begin{tabular}{|c|c|c|c|c|c|c|c|c|c|c|c|c|c|c|}
\hline \multirow[b]{2}{*}{$\begin{array}{c}\text { Seq. } \\
\text { no. }\end{array}$} & \multicolumn{7}{|c|}{ Daily precipitation threshold of 0.05 inch and greater } & \multicolumn{7}{|c|}{ Daily precipitation threshold of 0.10 inch and greater } \\
\hline & $\begin{array}{c}\text { Mean } \\
\text { inter- } \\
\text { occur- } \\
\text { rence } \\
\text { interval } \\
\text { (days) }\end{array}$ & $\begin{array}{c}\text { Total } \\
\text { no. of } \\
\text { events }\end{array}$ & $\begin{array}{l}\text { Total } \\
\text { no. of } \\
\text { days } \\
\text { avail- } \\
\text { able }\end{array}$ & $\begin{array}{l}\text { Sigma } \\
\text { S }\end{array}$ & $\begin{array}{l}\text { Stan- } \\
\text { dard } \\
\text { normal } \\
\text { variate }\end{array}$ & $\begin{array}{l}\text { Non- } \\
\text { ex- } \\
\text { ceed- } \\
\text { ance } \\
\text { prob- } \\
\text { ability }\end{array}$ & $\begin{array}{c}\mathbf{p}- \\
\text { value }\end{array}$ & $\begin{array}{l}\text { Mean } \\
\text { inter- } \\
\text { occur- } \\
\text { rence } \\
\text { interval } \\
\text { (days) }\end{array}$ & $\begin{array}{c}\text { Total } \\
\text { no. of } \\
\text { events }\end{array}$ & $\begin{array}{c}\text { Total } \\
\text { no. of } \\
\text { days } \\
\text { avail- } \\
\text { able }\end{array}$ & $\begin{array}{c}\text { Sigma } \\
\mathrm{S}\end{array}$ & $\begin{array}{c}\text { Stan- } \\
\text { dard } \\
\text { normal } \\
\text { variate }\end{array}$ & $\begin{array}{l}\text { Non- } \\
\text { ex- } \\
\text { ceed- } \\
\text { ance } \\
\text { prob- } \\
\text { ability }\end{array}$ & $\begin{array}{c}\mathrm{p}- \\
\text { value }\end{array}$ \\
\hline 1105 & 5.16 & 2,568 & 13,243 & 17316304 & 1.612 & .947 & .053 & 6.28 & 2,108 & 13,243 & 14140596 & 1.040 & .851 & .149 \\
\hline 1106 & 8.61 & 2,611 & 22,491 & 29713126 & 1.058 & .855 & .145 & 10.43 & 2,156 & 22,491 & 24594738 & 1.159 & .877 & .123 \\
\hline 1107 & 10.26 & 157 & 1,610 & 123705 & -.460 & .323 & .323 & 11.58 & 139 & 1,610 & 111852 & -.008 & .497 & .497 \\
\hline 1108 & -- & -- & -- & -- & -- & -- & -- & -- & -- & -- & -- & -- & -- & -- \\
\hline 1109 & 8.67 & 403 & 3,493 & 680852 & -1.136 & .128 & .128 & 9.68 & 361 & 3,493 & 601339 & -1.521 & .064 & .064 \\
\hline 1110 & 9.02 & 389 & 3,510 & 652583 & -1.507 & .066 & .066 & 10.64 & 330 & 3,510 & 541506 & -2.045 & .020 & .020 \\
\hline 1111 & 7.74 & 291 & 2,252 & 322881 & -.432 & .333 & .333 & 8.80 & 256 & 2,252 & 280320 & -.763 & .223 & .223 \\
\hline 1112 & 5.75 & 2,091 & 12,016 & 13210526 & 4.084 & 1.000 & 0 & 6.56 & 1,832 & 12,016 & 11391328 & 2.591 & .995 & .005 \\
\hline 1113 & 7.31 & 4,033 & 29,498 & 59737878 & .472 & .681 & .319 & 9.13 & 3,230 & 29,498 & 47212451 & -.882 & .189 & .189 \\
\hline 1114 & 6.25 & 1,939 & 12,124 & 11937611 & 1.190 & .883 & .117 & 7.39 & 1,640 & 12,124 & 10043049 & .715 & .763 & .237 \\
\hline 1115 & 9.00 & 646 & 5,813 & 1929108 & 1.208 & .886 & .114 & 10.16 & 572 & 5,813 & 1739928 & 1.929 & .973 & .027 \\
\hline 1116 & 6.35 & 229 & 1,453 & 140773 & -4.033 & 0 & 0 & 6.99 & 208 & 1,453 & 130865 & -3.347 & 0 & 0 \\
\hline 1117 & 6.04 & 2,605 & 15,727 & 20377142 & -.463 & .322 & .322 & 7.15 & 2,199 & 15,727 & 17396140 & .490 & .688 & .312 \\
\hline 1118 & 6.78 & 118 & 800 & 48711 & .602 & .726 & .274 & 7.92 & 101 & 800 & 42150 & .754 & .775 & .225 \\
\hline 1119 & 6.21 & 1,221 & 7,586 & 4713580 & 1.076 & .859 & .141 & 7.25 & 1,046 & 7,586 & 4047051 & 1.124 & .869 & .131 \\
\hline 1120 & 8.12 & 3,011 & 24,462 & 36161380 & -1.719 & .043 & .043 & 9.97 & 2,453 & 24,462 & 29703903 & -.854 & .197 & .197 \\
\hline 1121 & 8.39 & 3,539 & 29,675 & 55261746 & 5.400 & 0 & 0 & 9.57 & 3,101 & 29,675 & 47682613 & 3.504 & 1.000 & 0 \\
\hline 1122 & 10.53 & 97 & 1,021 & 50718 & .413 & .660 & .340 & 12.30 & 83 & 1,021 & 42042 & -.123 & .451 & .451 \\
\hline 1123 & 6.86 & 3,324 & 22,804 & 38954362 & 2.777 & .997 & .003 & 8.01 & 2,846 & 22,804 & 32949762 & 1.423 & .923 & .077 \\
\hline 1124 & 6.04 & 227 & 1,371 & 151227 & -.735 & .231 & .231 & 7.00 & 196 & 1371 & 131342 & -.544 & .293 & .293 \\
\hline 1125 & 6.93 & 407 & 2,820 & 600720 & 1.635 & .949 & .051 & 8.39 & 336 & 2,820 & 488979 & 1.020 & .846 & .154 \\
\hline 1126 & 18.66 & 207 & 3,863 & 349081 & -3.163 & .001 & .001 & 19.41 & 199 & 3,863 & 338562 & -2.912 & .002 & .002 \\
\hline 1127 & 9.99 & 2,672 & 26,696 & 37468553 & 4.525 & 1.000 & 0 & 11.32 & 2,358 & 26,696 & 32192931 & 1.920 & .973 & .027 \\
\hline 1128 & 10.43 & 924 & 9,641 & 4360328 & -1.109 & .134 & .134 & 11.29 & 854 & 9,641 & 4086661 & -.369 & .356 & .356 \\
\hline 1129 & 11.64 & 309 & 3,598 & 635278 & 4.348 & 1.000 & 0 & 13.79 & 261 & 3,598 & 519192 & 2.959 & .998 & .002 \\
\hline 1130 & 25.17 & 30 & 755 & 8646 & -2.244 & .012 & .012 & 25.17 & 30 & 755 & 8646 & -2.244 & .012 & .012 \\
\hline 1131 & 5.58 & 2,483 & 13,853 & 17738174 & 2.708 & .997 & .003 & 6.71 & 2,066 & 13,853 & 14652210 & 1.882 & .970 & .030 \\
\hline 1132 & 11.23 & 62 & 696 & 23102 & .965 & .833 & .167 & 13.39 & 52 & 696 & 20083 & 1.371 & .915 & .085 \\
\hline 1133 & 6.18 & 1,635 & 10,098 & 8153403 & -.863 & .194 & .194 & 7.67 & 1,316 & 10,098 & 6517221 & -1.204 & .114 & .114 \\
\hline 1134 & 8.06 & 85 & 685 & 29909 & .437 & .669 & .331 & 9.01 & 76 & 685 & 26280 & .145 & .558 & .442 \\
\hline 1135 & 5.84 & 135 & 789 & 53791 & .202 & .580 & .420 & 6.21 & 127 & 789 & 49459 & -.250 & .401 & .401 \\
\hline 1136 & 8.76 & 3,182 & 27,875 & 43161453 & -2.617 & .004 & .004 & 10.66 & 2,616 & 27,875 & 36239669 & -.537 & .296 & .296 \\
\hline 1137 & 6.69 & 2,860 & 19,145 & 27823841 & 1.511 & .935 & .065 & 7.96 & 2,406 & 19,145 & 23383074 & 1.297 & .903 & .097 \\
\hline 1138 & 11.59 & 213 & 2,469 & 250451 & -1.201 & .115 & .115 & 13.00 & 190 & 2,469 & 221815 & -1.297 & .097 & .097 \\
\hline 1139 & 17.32 & 180 & 3,117 & 290092 & .792 & .786 & .214 & 19.73 & 158 & 3,117 & 253981 & .684 & .753 & .247 \\
\hline 1140 & 7.90 & 1,409 & 11,137 & 7256316 & -4.887 & 0 & 0 & 10.00 & 1,114 & 11,137 & 5771371 & -4.025 & 0 & 0 \\
\hline 1141 & 4.84 & 4,129 & 19,965 & 40125734 & -2.949 & .002 & .002 & 5.88 & 3,397 & 19,965 & 33741200 & -.504 & .307 & .307 \\
\hline 1142 & 5.45 & 5,998 & 32,715 & 104715903 & 9.029 & 0 & 0 & 6.27 & 5,221 & 32,715 & 89694814 & 6.290 & 0 & 0 \\
\hline 1143 & 10.37 & 869 & 9,015 & 4072374 & 2.025 & .979 & .021 & 12.37 & 729 & 9,015 & 3359485 & 1.046 & .852 & .148 \\
\hline 1144 & 9.92 & 242 & 2,401 & 281613 & -.826 & .204 & .204 & 11.17 & 215 & 2,401 & 257947 & -.016 & .494 & .494 \\
\hline 1145 & 11.16 & 128 & 1,429 & 96527 & 1.087 & .861 & .139 & 12.54 & 114 & 1,429 & 91323 & 2.241 & .987 & .013 \\
\hline 1146 & 8.20 & 3,246 & 26,623 & 43949855 & 1.692 & .955 & .045 & 10.26 & 2,596 & 26,623 & 34883694 & .835 & .798 & .202 \\
\hline 1147 & 8.47 & 733 & 6,208 & 2399190 & 2.555 & .995 & .005 & 9.92 & 626 & 6,208 & 2049506 & 2.373 & .991 & .009 \\
\hline 1148 & 9.65 & 1,347 & 13,000 & 8903592 & 1.075 & .859 & .141 & 10.65 & 1,221 & 13,000 & 8216948 & 2.139 & .984 & .016 \\
\hline 1149 & 7.52 & 1,606 & 12,084 & 9386795 & -2.265 & .012 & .012 & 8.63 & 1,400 & 12,084 & 8384367 & -.570 & .284 & .284 \\
\hline 1150 & 8.55 & 1,572 & 13,443 & 10745737 & 1.167 & .878 & .122 & 10.75 & 1,250 & 13,443 & 8490768 & .648 & .741 & .259 \\
\hline 1151 & 5.03 & 227 & 1,142 & 130350 & .148 & .559 & .441 & 5.65 & 202 & 1,142 & 113934 & -.301 & .382 & .382 \\
\hline 1152 & 5.69 & 4,651 & 26,466 & 61647056 & .193 & .576 & .424 & 7.10 & 3,727 & 26,466 & 49708244 & .834 & .798 & .202 \\
\hline
\end{tabular}


Table 3. Summary of interoccurrence intervals for daily precipitation thresholds of 0.05 and 0.10 inch-Continued

\begin{tabular}{|c|c|c|c|c|c|c|c|c|c|c|c|c|c|c|}
\hline \multirow[b]{2}{*}{$\begin{array}{l}\text { Seq. } \\
\text { no. }\end{array}$} & \multicolumn{7}{|c|}{ Daily precipitation threshold of 0.05 inch and greater } & \multicolumn{7}{|c|}{ Daily precipitation threshold of 0.10 inch and greater } \\
\hline & $\begin{array}{c}\text { Mean } \\
\text { inter- } \\
\text { occur- } \\
\text { rence } \\
\text { interval } \\
\text { (days) }\end{array}$ & $\begin{array}{c}\text { Total } \\
\text { no. of } \\
\text { events }\end{array}$ & $\begin{array}{l}\text { Total } \\
\text { no. of } \\
\text { days } \\
\text { avail- } \\
\text { able }\end{array}$ & $\begin{array}{l}\text { Sigma } \\
\mathrm{S}\end{array}$ & $\begin{array}{c}\text { Stan- } \\
\text { dard } \\
\text { normal } \\
\text { variate }\end{array}$ & $\begin{array}{l}\text { Non- } \\
\text { ex- } \\
\text { ceed- } \\
\text { ance } \\
\text { prob- } \\
\text { ability }\end{array}$ & $\begin{array}{c}\text { p- } \\
\text { value }\end{array}$ & $\begin{array}{c}\text { Mean } \\
\text { inter- } \\
\text { occur- } \\
\text { rence } \\
\text { interval } \\
\text { (days) }\end{array}$ & $\begin{array}{c}\text { Total } \\
\text { no. of } \\
\text { events }\end{array}$ & $\begin{array}{l}\text { Total } \\
\text { no. of } \\
\text { days } \\
\text { avail- } \\
\text { able }\end{array}$ & $\begin{array}{c}\text { Sigma } \\
\mathrm{S}\end{array}$ & $\begin{array}{c}\text { Stan- } \\
\text { dard } \\
\text { normal } \\
\text { variate }\end{array}$ & $\begin{array}{l}\text { Non- } \\
\text { ex- } \\
\text { ceed- } \\
\text { ance } \\
\text { prob- } \\
\text { ability }\end{array}$ & $\begin{array}{c}\text { p- } \\
\text { value }\end{array}$ \\
\hline 1153 & 4.92 & 99 & 487 & 22847 & -0.900 & .184 & .184 & 5.73 & 85 & 487 & 19608 & -0.841 & .200 & .200 \\
\hline 1154 & 6.66 & 1,959 & 13,041 & 13061990 & 1.730 & .958 & .042 & 8.10 & 1,610 & 13,041 & 10653198 & 1.027 & .848 & .152 \\
\hline 1155 & 8.25 & 166 & 1,369 & 94981 & -3.662 & 0 & 0 & 8.89 & 154 & 1,369 & 92049 & -2.725 & .003 & .003 \\
\hline 1156 & 6.40 & 2,034 & 13,013 & 13414405 & 1.064 & .856 & .144 & 7.76 & 1,678 & 13,013 & 11061409 & .933 & .824 & .176 \\
\hline 1157 & 8.04 & 2,245 & 18,047 & 20853291 & 2.413 & .992 & .008 & 9.23 & 1,956 & 18,047 & 17992514 & 1.487 & .931 & .069 \\
\hline 1158 & 6.28 & 567 & 3,562 & 993501 & -.667 & .253 & .253 & 6.76 & 527 & 3,562 & 913899 & -1.046 & .148 & .148 \\
\hline 1159 & 6.05 & 6,087 & 36,849 & 112154577 & .006 & .502 & .498 & 7.19 & 5,128 & 36,849 & 94484225 & .004 & .502 & .498 \\
\hline 1160 & 6.80 & 206 & 1,401 & 143133 & -.202 & .420 & .420 & 8.44 & 166 & 1,401 & 115887 & -.076 & .470 & .470 \\
\hline 1161 & 11.26 & 508 & 5,720 & 1545133 & 2.479 & .993 & .007 & 12.22 & 468 & 5,720 & 1418915 & 2.252 & .988 & .012 \\
\hline 1162 & 10.08 & 120 & 1,210 & 73004 & .106 & .542 & .458 & 14.07 & 86 & 1,210 & 55293 & 1.007 & .843 & .157 \\
\hline 1163 & 11.84 & 267 & 3,160 & 408034 & -.928 & .177 & .177 & 15.57 & 203 & 3,160 & 301479 & -1.482 & .069 & .069 \\
\hline 1164 & 6.03 & 3,177 & 19,160 & 31954704 & 4.873 & 1.000 & 0 & 6.80 & 2,818 & 19,160 & 28148076 & 3.922 & 1.000 & 0 \\
\hline 1165 & 8.08 & 1,229 & 9,934 & 6314451 & 2.089 & .982 & .018 & 9.65 & 1,030 & 9,934 & 5300719 & 2.007 & .978 & .022 \\
\hline 1166 & 4.51 & 2,659 & 11,989 & 16222617 & 1.587 & .944 & .056 & 5.29 & 2,268 & 11,989 & 13791567 & 1.189 & .883 & .117 \\
\hline 1167 & 4.63 & 1,457 & 6,751 & 4901659 & -.221 & .412 & .412 & 5.39 & 1,252 & 6,751 & 4216298 & -.143 & .443 & .443 \\
\hline 1168 & 14.19 & 176 & 2,498 & 219469 & -.037 & .485 & .485 & 15.91 & 157 & 2,498 & 200567 & .495 & 690 & .310 \\
\hline 1169 & 14.52 & 86 & 1,249 & 57809 & 1.227 & .890 & .110 & 16.88 & 74 & 1,249 & 51304 & 1.641 & .950 & .050 \\
\hline 1170 & 4.60 & 3,596 & 16,537 & 30973352 & 4.331 & 1.000 & 0 & 5.52 & 2,996 & 16,537 & 25916453 & 4.378 & 1.000 & 0 \\
\hline 1171 & 6.22 & 1,970 & 12,247 & 12455632 & 2.500 & .994 & .006 & 7.10 & 1,726 & 12,247 & 10585545 & .112 & .544 & .456 \\
\hline 1172 & 6.81 & 2,843 & 19,365 & 30559983 & 1.174 & 0 & 0 & 7.59 & 2,553 & 19,365 & 26797811 & 7.358 & 0 & 0 \\
\hline 1173 & 7.28 & 3,265 & 23,783 & 38408747 & -1.063 & .144 & .144 & 8.90 & 2,673 & 23,783 & 31512550 & -.770 & .221 & .221 \\
\hline 1174 & 8.27 & 614 & 5,080 & 1535205 & -.670 & .251 & .251 & 9.60 & 529 & 5,080 & 1308995 & -1.028 & .152 & .152 \\
\hline 1175 & 7.35 & 3,689 & 27,125 & 51262238 & 2.587 & .995 & .005 & 8.66 & 3,132 & 27,125 & 43101078 & 1.422 & .923 & .077 \\
\hline 1176 & 6.88 & 95 & 654 & 29470 & -.867 & .193 & .193 & 7.79 & 84 & 654 & 25254 & -1.280 & .100 & .100 \\
\hline 1177 & 8.36 & 170 & 1,421 & 118787 & -.374 & .354 & .354 & 9.87 & 144 & 1,421 & 101293 & -.207 & .418 & .418 \\
\hline 1178 & 8.50 & 2,101 & 17,848 & 19464696 & 3.029 & .999 & .001 & 9.33 & 1,914 & 1,7848 & 17860963 & 3.462 & 1.000 & 0 \\
\hline 1179 & 7.92 & 2,214 & 17,533 & 19586546 & .745 & .772 & .228 & 10.10 & 1,736 & 1,7533 & 15244408 & .122 & .549 & .451 \\
\hline 1180 & 8.82 & 190 & 1,675 & 159715 & .089 & .535 & .465 & 11.47 & 146 & 1,675 & 123931 & .283 & .612 & .388 \\
\hline 1181 & 10.76 & 112 & 1,205 & 59132 & -2.268 & .012 & .012 & 14.18 & 85 & 1,205 & 45506 & -1.779 & .038 & .038 \\
\hline 1182 & 4.26 & 2,275 & 9,681 & 10970993 & -.309 & .379 & .379 & 5.11 & 1,896 & 9,681 & 9253891 & .627 & .735 & .265 \\
\hline 1183 & 5.15 & 3,811 & 19,606 & 37743021 & 1.098 & .864 & .136 & 5.93 & 3,308 & 19,606 & 32712267 & .872 & .808 & .192 \\
\hline 1184 & 12.12 & 663 & 8,033 & 2520689 & -2.382 & .009 & .009 & 16.74 & 480 & 8,033 & 1814991 & -2.223 & .013 & .013 \\
\hline 1185 & 7.45 & 1,148 & 8,556 & 5073020 & 1.934 & .973 & .027 & 8.28 & 1,033 & 8,556 & 4477877 & .740 & .770 & .230 \\
\hline 1186 & 4.55 & 1,325 & 6,027 & 4028825 & .568 & .715 & .285 & 5.45 & 1,106 & 6,027 & 3369924 & .639 & .739 & .261 \\
\hline 1187 & 4.21 & 2,728 & 11,476 & 15650784 & -.014 & .494 & .494 & 5.06 & 2,266 & 1,1476 & 12966438 & -.228 & .410 & .410 \\
\hline 1188 & 12.85 & 779 & 10,006 & 3974730 & .960 & .831 & .169 & 16.99 & 589 & 10,006 & 2986512 & .567 & .715 & .285 \\
\hline 1189 & 9.69 & 1,978 & 19,158 & 17919666 & -4.178 & 0 & 0 & 10.87 & 1,762 & 19,158 & 16322539 & -2.394 & .008 & .008 \\
\hline 1190 & 5.80 & 3,289 & 19,072 & 32256634 & 2.827 & .998 & .002 & 6.36 & 3,001 & 19,072 & 29532824 & 3.035 & .999 & .001 \\
\hline 1191 & 9.40 & 77 & 724 & 27495 & -.207 & .418 & .418 & 14.78 & 49 & 724 & 15806 & -1.321 & .093 & .093 \\
\hline 1192 & 7.88 & 823 & 6,484 & 2743986 & 1.412 & .921 & .079 & 9.82 & 660 & 6,484 & 2205574 & 1.370 & .915 & .085 \\
\hline 1193 & 6.58 & 1,160 & 7,637 & 4812886 & 5.107 & 0 & 0 & 7.52 & 1,015 & 7,637 & 4050545 & 2.488 & .994 & .006 \\
\hline 1194 & 6.01 & 1,386 & 8,332 & 5405846 & -4.112 & 0 & 0 & 6.90 & 1,207 & 8,332 & 4827845 & -2.400 & .008 & .008 \\
\hline 1195 & 4.51 & 493 & 2,224 & 555427 & .506 & .694 & .307 & 5.30 & 420 & 2,224 & 481255 & 1.080 & .860 & .140 \\
\hline 1196 & 5.10 & 1,260 & 6,424 & 4101117 & .820 & .794 & .206 & 6.09 & 1,055 & 6,424 & 3438926 & .835 & .798 & .202 \\
\hline 1197 & 5.97 & 3,318 & 19,815 & 34108976 & 3.751 & 1.000 & 0 & 7.06 & 2,807 & 19,815 & 28615521 & 2.657 & .996 & .004 \\
\hline 1198 & 8.60 & 1,056 & 9,076 & 4971011 & 2.101 & .982 & .018 & 10.29 & 882 & 9,076 & 4172383 & 2.183 & .985 & .015 \\
\hline 1199 & 21.18 & 17 & 360 & 3708 & 1.512 & .935 & .065 & 24.00 & 15 & 360 & 2996 & .735 & .769 & .231 \\
\hline 1200 & 7.70 & 1,869 & 14,391 & 13475945 & .153 & .561 & .439 & 8.97 & 1,604 & 14,391 & 11592688 & .307 & .621 & .379 \\
\hline
\end{tabular}


Table 3. Summary of interoccurrence intervals for daily precipitation thresholds of 0.05 and 0.10 inch-Continued

\begin{tabular}{|c|c|c|c|c|c|c|c|c|c|c|c|c|c|c|}
\hline \multirow[b]{2}{*}{$\begin{array}{c}\text { Seq. } \\
\text { no. }\end{array}$} & \multicolumn{7}{|c|}{ Daily precipitation threshold of 0.05 inch and greater } & \multicolumn{7}{|c|}{ Daily precipitation threshold of 0.10 inch and greater } \\
\hline & $\begin{array}{c}\text { Mean } \\
\text { inter- } \\
\text { occur- } \\
\text { rence } \\
\text { interval } \\
\text { (days) }\end{array}$ & $\begin{array}{c}\text { Total } \\
\text { no. of } \\
\text { events }\end{array}$ & $\begin{array}{l}\text { Total } \\
\text { no. of } \\
\text { days } \\
\text { avail- } \\
\text { able }\end{array}$ & $\begin{array}{l}\text { Sigma } \\
\text { S }\end{array}$ & $\begin{array}{l}\text { Stan- } \\
\text { dard } \\
\text { normal } \\
\text { variate }\end{array}$ & $\begin{array}{l}\text { Non- } \\
\text { ex- } \\
\text { ceed- } \\
\text { ance } \\
\text { prob- } \\
\text { ability }\end{array}$ & $\begin{array}{c}\mathbf{p}- \\
\text { value }\end{array}$ & $\begin{array}{l}\text { Mean } \\
\text { inter- } \\
\text { occur- } \\
\text { rence } \\
\text { interval } \\
\text { (days) }\end{array}$ & $\begin{array}{l}\text { Total } \\
\text { no. of } \\
\text { events }\end{array}$ & $\begin{array}{c}\text { Total } \\
\text { no. of } \\
\text { days } \\
\text { avail- } \\
\text { able }\end{array}$ & $\begin{array}{c}\text { Sigma } \\
\mathrm{S}\end{array}$ & $\begin{array}{c}\text { Stan- } \\
\text { dard } \\
\text { normal } \\
\text { variate }\end{array}$ & $\begin{array}{l}\text { Non- } \\
\text { ex- } \\
\text { ceed- } \\
\text { ance } \\
\text { prob- } \\
\text { ability }\end{array}$ & $\begin{array}{c}\mathrm{p}- \\
\text { value }\end{array}$ \\
\hline 1201 & 9.35 & 205 & 1,917 & 205821 & 1.177 & .880 & .120 & 11.15 & 172 & 1,917 & 166516 & 0.228 & .590 & .410 \\
\hline 1202 & 7.76 & 2,501 & 19,400 & 25257880 & 3.564 & 1.000 & 0 & 9.76 & 1,987 & 19,400 & 20009484 & 2.947 & .998 & .002 \\
\hline 1203 & 8.83 & 1,778 & 15,694 & 13392232 & -2.930 & .002 & .002 & 9.85 & 1,594 & 15,694 & 11943357 & -3.122 & .001 & .001 \\
\hline 1204 & 7.93 & 1,952 & 15,481 & 15575438 & 2.360 & .991 & .009 & 9.45 & 1,639 & 15,481 & 13259403 & 3.166 & .999 & .001 \\
\hline 1205 & 4.75 & 1,366 & 6,485 & 4426426 & -.041 & .484 & .484 & 5.64 & 1,149 & 6,485 & 3714284 & -.179 & .429 & .429 \\
\hline 1206 & 4.90 & 2,160 & 10,592 & 11437784 & -.011 & .496 & .496 & 5.86 & 1,809 & 10,592 & 9576800 & -.028 & .489 & .489 \\
\hline 1207 & 5.44 & 1,123 & 6,104 & 3412483 & -.253 & .400 & .400 & 6.42 & 951 & 6,104 & 2862646 & -.733 & .232 & .232 \\
\hline 1208 & 8.51 & 2,291 & 19,506 & 22057443 & -1.064 & .144 & .144 & 10.44 & 1,869 & 19,506 & 18140326 & -.362 & .359 & .359 \\
\hline 1209 & -- & -- & -- & -- & -- & -- & -- & -- & -- & -- & -- & -- & -- & -- \\
\hline 1210 & 7.30 & 508 & 3,709 & 901421 & -1.685 & .046 & .046 & 8.06 & 460 & 3,709 & 823965 & -1.267 & .103 & .103 \\
\hline 1211 & 7.86 & 3,571 & 28,061 & 51428876 & 2.739 & .997 & .003 & 10.02 & 2,801 & 28,061 & 39345794 & .108 & .543 & .457 \\
\hline 1212 & 7.68 & 1,254 & 9,634 & 6174615 & 1.362 & .913 & .087 & 9.67 & 996 & 9,634 & 4816831 & .218 & .586 & .414 \\
\hline 1213 & 7.17 & 763 & 5,474 & 1929463 & -3.640 & 0 & 0 & 9.02 & 607 & 5,474 & 1586068 & -1.934 & .027 & .027 \\
\hline 1214 & 10.65 & 68 & 724 & 22456 & -1.253 & .105 & .105 & 10.65 & 68 & 724 & 22456 & -1.253 & .105 & .105 \\
\hline 1215 & 9.06 & 942 & 8,537 & 3813266 & -2.746 & .003 & .003 & 11.70 & 730 & 8,537 & 2972952 & -2.148 & .016 & .016 \\
\hline 1216 & 9.94 & 2,539 & 25,234 & 33506668 & 4.011 & 1.000 & 0 & 12.04 & 2,096 & 25,234 & 27091363 & 1.937 & .974 & .026 \\
\hline 1217 & 6.56 & 2,003 & 13,130 & 13018720 & -.772 & .220 & .220 & 7.57 & 1,734 & 13,130 & 11161743 & -1.406 & .080 & .080 \\
\hline 1218 & 8.38 & 390 & 3,269 & 630405 & -.378 & .353 & .353 & 8.69 & 376 & 3,269 & 605791 & -.480 & .316 & .316 \\
\hline 1219 & 6.18 & 3,176 & 19,632 & 30855257 & -1.003 & .158 & .158 & 6.98 & 2,811 & 19,632 & 27708758 & .386 & .650 & .350 \\
\hline 1220 & 10.90 & 1,918 & 20,914 & 20873200 & 3.089 & .999 & .001 & 14.35 & 1,457 & 20,914 & 15756112 & 2.258 & .988 & .012 \\
\hline 1221 & 11.99 & 490 & 5,877 & 1518327 & 2.089 & .982 & .018 & 12.56 & 468 & 5,877 & 1439562 & 1.753 & .960 & .040 \\
\hline 1222 & 9.96 & 23 & 229 & 2416 & -.686 & .246 & .246 & 10.91 & 21 & 229 & 2257 & -.487 & .313 & .313 \\
\hline 1223 & 5.55 & 987 & 5,476 & 2699826 & -.052 & .479 & .479 & 6.88 & 796 & 5,476 & 2180974 & .034 & .514 & .486 \\
\hline 1224 & 7.22 & 274 & 1,978 & 269687 & -.137 & .445 & .445 & 7.67 & 258 & 1,978 & 255324 & .018 & .507 & .493 \\
\hline 1225 & 6.08 & 436 & 2,649 & 590763 & .832 & .797 & .203 & 7.70 & 344 & 2,649 & 471374 & 1.110 & .866 & .134 \\
\hline 1226 & 7.55 & 2,902 & 21,901 & 30920047 & -2.520 & .006 & .006 & 9.41 & 2,328 & 21,901 & 24462941 & -3.376 & 0 & 0 \\
\hline 1227 & 8.31 & 752 & 6,246 & 2440231 & 1.855 & .968 & .032 & 8.31 & 752 & 6,246 & 2440231 & 1.855 & .968 & .032 \\
\hline 1228 & 7.75 & 3,237 & 25,070 & 41667598 & 2.652 & .996 & .004 & 9.08 & 2,761 & 25,070 & 35605063 & 2.619 & .996 & .004 \\
\hline 1229 & 5.58 & 2,647 & 14,762 & 19922369 & 1.755 & .960 & .040 & 6.84 & 2,159 & 14,762 & 16245263 & 1.564 & .941 & .059 \\
\hline 1230 & 5.70 & 2,518 & 14,360 & 18442939 & 1.748 & .960 & .040 & 6.88 & 2,088 & 14,360 & 15033930 & .222 & .588 & .412 \\
\hline 1231 & 5.90 & 132 & 779 & 54377 & 1.147 & .874 & .126 & 6.66 & 117 & 779 & 47521 & .802 & .789 & .211 \\
\hline 1232 & 5.57 & 175 & 975 & 89621 & 1.157 & .876 & .124 & 6.37 & 153 & 975 & 77987 & .977 & .836 & .164 \\
\hline 1233 & 5.84 & 911 & 5,323 & 2483701 & 1.274 & .899 & .101 & 7.71 & 690 & 5,323 & 1864474 & .695 & .756 & .244 \\
\hline 1234 & 8.70 & 1,284 & 11,164 & 7277535 & .955 & .830 & .170 & 9.47 & 1,179 & 11,164 & 6747430 & 1.502 & .933 & .067 \\
\hline 1235 & 6.23 & 665 & 4,140 & 1324900 & -1.676 & .047 & .047 & 7.61 & 544 & 4,140 & 1120021 & -.217 & .414 & .414 \\
\hline 1236 & 8.56 & 259 & 2,216 & 259893 & -2.630 & .004 & .004 & 10.12 & 219 & 2,216 & 223963 & -1.974 & .024 & .024 \\
\hline 1237 & 5.52 & 2,383 & 13,142 & 16221905 & 3.041 & .999 & .001 & 6.70 & 1,962 & 13,142 & 13343706 & 2.686 & .996 & .004 \\
\hline 1238 & 6.06 & 4,202 & 25,474 & 53297285 & -.469 & .320 & .320 & 7.42 & 3,434 & 25,474 & 43993127 & .590 & .722 & .278 \\
\hline 1239 & 7.03 & 2,141 & 15,049 & 18016227 & 9.483 & 0 & 0 & 7.81 & 1,928 & 15,049 & 15739015 & 6.458 & 0 & 0 \\
\hline 1240 & 9.16 & 205 & 1,877 & 186688 & -.735 & .231 & .231 & 10.04 & 187 & 1,877 & 168561 & -.936 & .175 & .175 \\
\hline 1241 & 6.41 & 2,819 & 18,072 & 26273138 & 2.891 & .998 & .002 & 6.78 & 2,666 & 18,072 & 25011556 & 3.421 & 1.000 & 0 \\
\hline 1242 & 7.47 & 100 & 747 & 38782 & .664 & .747 & .253 & 8.49 & 88 & 747 & 33728 & .425 & .665 & .335 \\
\hline 1243 & 4.32 & 4,075 & 17,604 & 36090543 & .686 & .753 & .247 & 5.08 & 3,464 & 17,604 & 30662073 & .575 & .717 & .283 \\
\hline 1244 & 5.42 & 3,537 & 19,152 & 34619275 & 2.278 & .989 & .011 & 6.35 & 3,016 & 19,152 & 29568760 & 2.264 & .988 & .012 \\
\hline 1245 & 9.33 & 2,032 & 18,958 & 20808139 & 6.270 & 0 & 0 & 11.01 & 1,722 & 18,958 & 17371302 & 4.617 & 1.000 & 0 \\
\hline 1246 & 12.11 & 191 & 2,312 & 215734 & -.549 & .292 & .292 & 13.60 & 170 & 2,312 & 188283 & -.947 & .172 & .172 \\
\hline 1247 & 9.09 & 1,671 & 15,182 & 12819983 & .756 & .775 & .225 & 10.09 & 1,504 & 15,182 & 11376462 & -.238 & .406 & .406 \\
\hline 1248 & 3.81 & 16 & 61 & 501 & .185 & .573 & .427 & 4.36 & 14 & 61 & 448 & .319 & .625 & .375 \\
\hline
\end{tabular}


Table 3. Summary of interoccurrence intervals for daily precipitation thresholds of 0.05 and 0.10 inch-Continued

\begin{tabular}{|c|c|c|c|c|c|c|c|c|c|c|c|c|c|c|}
\hline \multirow[b]{2}{*}{$\begin{array}{l}\text { Seq. } \\
\text { no. }\end{array}$} & \multicolumn{7}{|c|}{ Daily precipitation threshold of 0.05 inch and greater } & \multicolumn{7}{|c|}{ Daily precipitation threshold of 0.10 inch and greater } \\
\hline & $\begin{array}{c}\text { Mean } \\
\text { inter- } \\
\text { occur- } \\
\text { rence } \\
\text { interval } \\
\text { (days) }\end{array}$ & $\begin{array}{c}\text { Total } \\
\text { no. of } \\
\text { events }\end{array}$ & $\begin{array}{l}\text { Total } \\
\text { no. of } \\
\text { days } \\
\text { avail- } \\
\text { able }\end{array}$ & $\begin{array}{l}\text { Sigma } \\
\mathrm{S}\end{array}$ & $\begin{array}{l}\text { Stan- } \\
\text { dard } \\
\text { normal } \\
\text { variate }\end{array}$ & $\begin{array}{l}\text { Non- } \\
\text { ex- } \\
\text { ceed- } \\
\text { ance } \\
\text { prob- } \\
\text { ability }\end{array}$ & $\begin{array}{c}\text { p- } \\
\text { value }\end{array}$ & $\begin{array}{c}\text { Mean } \\
\text { inter- } \\
\text { occur- } \\
\text { rence } \\
\text { interval } \\
\text { (days) }\end{array}$ & $\begin{array}{c}\text { Total } \\
\text { no. of } \\
\text { events }\end{array}$ & $\begin{array}{c}\text { Total } \\
\text { no. of } \\
\text { days } \\
\text { avail- } \\
\text { able }\end{array}$ & $\begin{array}{c}\text { Sigma } \\
\mathrm{S}\end{array}$ & $\begin{array}{c}\text { Stan- } \\
\text { dard } \\
\text { normal } \\
\text { variate }\end{array}$ & $\begin{array}{l}\text { Non- } \\
\text { ex- } \\
\text { ceed- } \\
\text { ance } \\
\text { prob- } \\
\text { ability }\end{array}$ & $\begin{array}{c}p- \\
\text { value }\end{array}$ \\
\hline 1249 & 6.73 & 1,833 & 12,327 & 12052215 & 4.953 & 1.000 & 0 & 7.62 & 1,617 & 12,327 & 10218928 & 1.765 & .961 & .039 \\
\hline 1250 & 6.19 & 5,950 & 36,857 & 114409263 & 5.800 & 0 & 0 & 7.01 & 5,260 & 36,857 & 100021270 & 4.001 & 1.000 & 0 \\
\hline 1251 & 6.48 & 171 & 1,108 & 92020 & -.649 & .258 & .258 & 8.27 & 134 & 1,108 & 71364 & -.776 & .219 & .219 \\
\hline 1252 & 6.14 & 5,774 & 35,470 & 102970077 & .730 & .767 & .233 & 7.45 & 4,762 & 35,470 & 85554385 & 1.557 & .940 & .060 \\
\hline 1253 & 8.44 & 529 & 4,465 & 1264979 & 2.833 & .998 & .002 & 8.51 & 525 & 4,465 & 1263113 & 3.083 & .999 & .001 \\
\hline 1254 & 7.73 & 59 & 456 & 14729 & 1.263 & .897 & .103 & 8.00 & 57 & 456 & 13934 & .944 & .827 & .173 \\
\hline 1255 & 6.26 & 2,146 & 13,428 & 14736233 & 1.827 & .966 & .034 & 7.60 & 1,767 & 13,428 & 11868367 & .029 & .512 & .488 \\
\hline 1256 & 9.10 & 2,251 & 20,473 & 22234499 & -2.881 & .002 & .002 & 10.52 & 1,946 & 20,473 & 19122856 & -3.058 & .001 & .001 \\
\hline 1257 & 7.45 & 2,622 & 19,529 & 26447262 & 2.926 & .998 & .002 & 9.70 & 2,013 & 19,529 & 20320816 & 2.629 & .996 & .004 \\
\hline 1258 & 7.31 & 497 & 3,633 & 868075 & -1.485 & .069 & .069 & 7.43 & 489 & 3,633 & 855573 & -1.410 & .079 & .079 \\
\hline 1259 & 12.05 & 19 & 229 & 2205 & .102 & .541 & .459 & 19.08 & 12 & 229 & 1432 & .253 & 600 & .400 \\
\hline 1260 & 5.31 & 4,103 & 21,784 & 46392280 & 4.226 & 1.000 & 0 & 6.41 & 3,399 & 21,784 & 38054634 & 2.817 & .998 & .002 \\
\hline 1261 & 9.25 & 884 & 8,179 & 3487271 & -1.821 & .034 & .034 & 10.60 & 772 & 8,179 & 3051769 & -1.606 & .054 & .054 \\
\hline 1262 & 7.17 & 161 & 1,154 & 90273 & -.621 & .267 & .267 & 8.30 & 139 & 1,154 & 79196 & -.256 & .399 & .399 \\
\hline 1263 & 3.61 & 143 & 516 & 38269 & .772 & .780 & .220 & 4.10 & 126 & 516 & 33856 & .806 & .790 & .210 \\
\hline 1264 & 9.50 & 163 & 1,548 & 128129 & .345 & .635 & .365 & 11.30 & 137 & 1,548 & 104320 & -.329 & .371 & .371 \\
\hline 1265 & 5.27 & 656 & 3,454 & 1063258 & -2.728 & .003 & .003 & 6.01 & 575 & 3,454 & 962668 & -1.270 & .102 & .102 \\
\hline 1266 & 5.96 & 3,193 & 19,044 & 31535334 & 3.643 & 1.000 & 0 & 7.24 & 2,629 & 19,044 & 25845444 & 2.881 & .998 & .002 \\
\hline 1267 & 8.08 & 1,669 & 13,484 & 12312156 & 6.664 & 0 & 0 & 9.31 & 1,448 & 13,484 & 10434938 & 4.540 & 1.000 & 0 \\
\hline 1268 & 9.37 & 1,832 & 17,161 & 17210360 & 7.031 & 0 & 0 & 10.26 & 1,672 & 17,161 & 15391507 & 5.158 & 0 & 0 \\
\hline 1269 & -- & -- & -- & -- & -- & -- & -- & -- & -- & -- & -- & -- & -- & -- \\
\hline 1270 & 7.15 & 306 & 2,187 & 332022 & -.234 & .407 & .407 & 8.28 & 264 & 2,187 & 280991 & -.750 & .227 & .227 \\
\hline 1271 & 7.19 & 4,878 & 35,073 & 88460355 & 4.126 & 1.000 & 0 & 8.49 & 4,130 & 3,5073 & 73632358 & 1.854 & .968 & .032 \\
\hline 1272 & 8.75 & 456 & 3,991 & 840909 & -2.806 & .003 & .003 & 9.57 & 417 & 3,991 & 761498 & -3.002 & .001 & .001 \\
\hline 1273 & 7.19 & 1,325 & 9,529 & 6461610 & 1.485 & .931 & .069 & 8.91 & 1,069 & 9,529 & 5190285 & 1.079 & .860 & .140 \\
\hline 1274 & 6.56 & 938 & 6,153 & 2834703 & -.939 & .174 & .174 & 6.67 & 923 & 6,153 & 2807565 & -.594 & .276 & .276 \\
\hline 1275 & 4.15 & 868 & 3,606 & 1518312 & -1.523 & .064 & .064 & 5.00 & 721 & 3,606 & 1246277 & -1.921 & .027 & .027 \\
\hline 1276 & 4.93 & 1,121 & 5,527 & 3160923 & 1.180 & .881 & .119 & 5.98 & 925 & 5,527 & 2577425 & .437 & .669 & .331 \\
\hline 1277 & 8.62 & 297 & 2,560 & 305805 & -5.838 & 0 & 0 & 9.08 & 282 & 2,560 & 301889 & -4.760 & 0 & 0 \\
\hline 1278 & 5.30 & 5,473 & 28,977 & 82475557 & 5.139 & 0 & 0 & 6.16 & 4,707 & 28,977 & 69839853 & 2.862 & .998 & .002 \\
\hline 1279 & 11.04 & 136 & 1,501 & 97034 & -.996 & .160 & .160 & 13.90 & 108 & 1,501 & 77223 & -.851 & .198 & .198 \\
\hline 1280 & 7.21 & 746 & 5,379 & 2065465 & 1.394 & .918 & .082 & 8.57 & 628 & 5,379 & 1772385 & 2.143 & .984 & .016 \\
\hline 1281 & 5.60 & 1,108 & 6,203 & 3324177 & -1.884 & .030 & .030 & 6.42 & 966 & 6,203 & 2927917 & -1.224 & .110 & .110 \\
\hline 1282 & 6.13 & 40 & 245 & 5710 & 1.811 & .965 & .035 & 8.45 & 29 & 245 & 4550 & 2.619 & .996 & .004 \\
\hline 1283 & 11.13 & 470 & 5,233 & 1230427 & .021 & .508 & .492 & 11.66 & 449 & 5,233 & 1155037 & -.618 & .268 & .268 \\
\hline 1284 & 5.99 & 1,631 & 9,769 & 7983568 & .149 & .559 & .441 & 6.43 & 1,520 & 9,769 & 7469979 & .414 & .661 & .339 \\
\hline 1285 & 11.31 & 1,862 & 21,056 & 19637509 & .131 & .552 & .448 & 14.81 & 1,422 & 21,056 & 14986039 & .066 & .527 & .473 \\
\hline 1286 & 6.10 & 3,001 & 18,295 & 28592610 & 3.944 & 1.000 & 0 & 6.70 & 2,732 & 18,295 & 25842393 & 3.084 & .999 & .001 \\
\hline 1287 & 9.26 & 646 & 5,980 & 1921410 & -.231 & .409 & .409 & 12.48 & 479 & 5,980 & 1419294 & -.342 & .366 & .366 \\
\hline 1288 & 7.87 & 1,531 & 12,048 & 8742941 & -3.526 & 0 & 0 & 9.43 & 1,278 & 12,048 & 7394052 & -2.450 & .007 & .007 \\
\hline 1289 & 9.33 & 1,487 & 13,868 & 11016237 & 4.569 & 1.000 & 0 & 10.40 & 1,333 & 13,868 & 9638311 & 2.704 & .997 & .003 \\
\hline 1290 & 9.60 & 149 & 1,430 & 105232 & -.259 & .398 & .398 & 10.07 & 142 & 1,430 & 101211 & -.065 & .474 & .474 \\
\hline 1291 & 6.79 & 179 & 1,216 & 108509 & -.069 & .473 & .473 & 8.11 & 150 & 1,216 & 88307 & -.673 & .251 & .251 \\
\hline 1292 & 5.62 & 3,499 & 19,665 & 36151173 & 5.203 & 0 & 0 & 6.43 & 3,060 & 19,665 & 31212651 & 3.583 & 1.000 & 0 \\
\hline 1293 & 6.95 & 2,451 & 17,041 & 22022416 & 4.675 & 1.000 & 0 & 7.98 & 2,136 & 17,041 & 18752275 & 2.430 & .992 & .008 \\
\hline 1294 & 7.62 & 664 & 5,058 & 1665393 & -.369 & .356 & .356 & 8.77 & 577 & 5,058 & 1433329 & -.739 & .230 & .230 \\
\hline 1295 & 5.80 & 110 & 638 & 38405 & 1.716 & .957 & .043 & 6.38 & 100 & 638 & 35309 & 1.851 & .968 & .032 \\
\hline 1296 & 4.22 & 1,009 & 4,257 & 2105442 & -1.081 & .140 & .140 & 4.83 & 882 & 4,257 & 1871681 & -.155 & .438 & .438 \\
\hline
\end{tabular}


Table 3. Summary of interoccurrence intervals for daily precipitation thresholds of 0.05 and 0.10 inch-Continued

\begin{tabular}{|c|c|c|c|c|c|c|c|c|c|c|c|c|c|c|}
\hline \multirow[b]{2}{*}{$\begin{array}{l}\text { Seq. } \\
\text { no. }\end{array}$} & \multicolumn{7}{|c|}{ Daily precipitation threshold of 0.05 inch and greater } & \multicolumn{7}{|c|}{ Daily precipitation threshold of 0.10 inch and greater } \\
\hline & $\begin{array}{c}\text { Mean } \\
\text { inter- } \\
\text { occur- } \\
\text { rence } \\
\text { interval } \\
\text { (days) }\end{array}$ & $\begin{array}{c}\text { Total } \\
\text { no. of } \\
\text { events }\end{array}$ & $\begin{array}{c}\text { Total } \\
\text { no. of } \\
\text { days } \\
\text { avail- } \\
\text { able }\end{array}$ & $\begin{array}{l}\text { Sigma } \\
\mathrm{S}\end{array}$ & $\begin{array}{c}\text { Stan- } \\
\text { dard } \\
\text { normal } \\
\text { variate }\end{array}$ & $\begin{array}{l}\text { Non- } \\
\text { ex- } \\
\text { ceed- } \\
\text { ance } \\
\text { prob- } \\
\text { ability }\end{array}$ & $\begin{array}{c}p- \\
\text { value }\end{array}$ & $\begin{array}{l}\text { Mean } \\
\text { inter- } \\
\text { occur- } \\
\text { rence } \\
\text { interval } \\
\text { (days) }\end{array}$ & $\begin{array}{l}\text { Total } \\
\text { no. of } \\
\text { events }\end{array}$ & $\begin{array}{l}\text { Total } \\
\text { no. of } \\
\text { days } \\
\text { avail- } \\
\text { able }\end{array}$ & $\begin{array}{l}\text { Sigma } \\
\mathrm{S}\end{array}$ & $\begin{array}{c}\text { Stan- } \\
\text { dard } \\
\text { normal } \\
\text { variate }\end{array}$ & $\begin{array}{l}\text { Non- } \\
\text { ex- } \\
\text { ceed- } \\
\text { ance } \\
\text { prob- } \\
\text { ability }\end{array}$ & $\begin{array}{c}\text { p- } \\
\text { value }\end{array}$ \\
\hline 1297 & 8.02 & 338 & 2,709 & 398169 & -4.149 & 0 & 0 & 9.92 & 273 & 2,709 & 308664 & -4.730 & 0 & 0 \\
\hline 1298 & 4.97 & 299 & 1,485 & 206933 & -2.034 & .021 & .021 & 6.09 & 244 & 1,485 & 168484 & -1.895 & .029 & .029 \\
\hline 1299 & 4.63 & 2,096 & 9,696 & 10204793 & .339 & .633 & .367 & 5.44 & 1,784 & 9,696 & 8703021 & .458 & .677 & .323 \\
\hline 1300 & 5.87 & 31 & 182 & 3458 & 2.178 & .985 & .015 & 7.00 & 26 & 182 & 2895 & 1.975 & .976 & .024 \\
\hline 1301 & 5.74 & 4,838 & 27,787 & 69474491 & 4.047 & 1.000 & 0 & 6.90 & 4,029 & 27,787 & 57537777 & 3.066 & .999 & .001 \\
\hline 1302 & 6.39 & 2,924 & 18,695 & 28232680 & 3.086 & .999 & .001 & 7.51 & 2,488 & 18,695 & 23868599 & 2.274 & .989 & .011 \\
\hline 1303 & 10.78 & 27 & 291 & 3603 & -.746 & .228 & .228 & 10.78 & 27 & 291 & 3603 & -.746 & .228 & .228 \\
\hline 1304 & 6.80 & 535 & 3,640 & 928215 & -1.872 & .031 & .031 & 7.47 & 487 & 3,640 & 853806 & -1.403 & .080 & .080 \\
\hline 1305 & 12.92 & 1,753 & 22,655 & 21686985 & 6.683 & 0 & 0 & 17.69 & 1,281 & 22,655 & 15700225 & 5.083 & 0 & 0 \\
\hline 1306 & 9.78 & 1,531 & 14,972 & 12092729 & 3.735 & 1.000 & 0 & 12.38 & 1,209 & 14,972 & 9402072 & 2.339 & .990 & .010 \\
\hline
\end{tabular}


Table 4. Summary of interoccurrence intervals for daily precipitation thresholds of 0.25 and 0.50 inch [--, not available, no events that equal or exceed the threshold were observed]

\begin{tabular}{|c|c|c|c|c|c|c|c|c|c|c|c|c|c|c|}
\hline \multirow[b]{2}{*}{$\begin{array}{l}\text { Seq. } \\
\text { no. }\end{array}$} & \multicolumn{7}{|c|}{ Daily precipitation threshold of 0.25 inch and greater } & \multicolumn{7}{|c|}{ Daily precipitation threshold of 0.50 inch and greater } \\
\hline & $\begin{array}{l}\text { Mean } \\
\text { inter- } \\
\text { occur- } \\
\text { rence } \\
\text { interval } \\
\text { (days) }\end{array}$ & $\begin{array}{l}\text { Total } \\
\text { no. of } \\
\text { events }\end{array}$ & $\begin{array}{l}\text { Total } \\
\text { no. of } \\
\text { days } \\
\text { avail- } \\
\text { able }\end{array}$ & $\begin{array}{c}\text { Sigma } \\
\mathrm{S}\end{array}$ & $\begin{array}{c}\text { Stan- } \\
\text { dard } \\
\text { normal } \\
\text { variate }\end{array}$ & $\begin{array}{l}\text { Non- } \\
\text { ex- } \\
\text { ceed- } \\
\text { ance } \\
\text { prob- } \\
\text { ability }\end{array}$ & $\begin{array}{c}\mathrm{p}- \\
\text { value }\end{array}$ & $\begin{array}{l}\text { Mean } \\
\text { inter- } \\
\text { occur- } \\
\text { rence } \\
\text { interval } \\
\text { (days) }\end{array}$ & $\begin{array}{l}\text { Total } \\
\text { no. of } \\
\text { events }\end{array}$ & $\begin{array}{l}\text { Total } \\
\text { no. of } \\
\text { days } \\
\text { avail- } \\
\text { able }\end{array}$ & $\underset{\mathrm{S}}{\text { Sigma }}$ & $\begin{array}{c}\text { Stan- } \\
\text { dard } \\
\text { normal } \\
\text { variate }\end{array}$ & $\begin{array}{l}\text { Non- } \\
\text { ex- } \\
\text { ceed- } \\
\text { ance } \\
\text { prob- } \\
\text { ability }\end{array}$ & $\begin{array}{c}\text { p- } \\
\text { value }\end{array}$ \\
\hline 1 & 16.64 & 1,173 & 19,514 & 11806259 & 1.873 & .969 & .031 & 29.26 & 667 & 19,514 & 6772781 & 1.821 & .966 & .034 \\
\hline 2 & 12.63 & 19 & 240 & 2143 & -.454 & .325 & .325 & 30.00 & 8 & 240 & 753 & -1.056 & .145 & .145 \\
\hline 3 & 14.14 & 1,395 & 19,720 & 14068377 & 1.475 & .930 & .070 & 23.37 & 844 & 19,720 & 8411682 & .543 & .706 & .294 \\
\hline 4 & 15.97 & 527 & 8,416 & 2185242 & -.581 & .281 & .281 & 26.30 & 320 & 8,416 & 1351736 & .119 & .547 & .453 \\
\hline 5 & 18.09 & 1,050 & 18,993 & 10342772 & 2.091 & .982 & .018 & 29.91 & 635 & 18,993 & 6420769 & 2.826 & .998 & .002 \\
\hline 6 & 18.16 & 194 & 3,522 & 341170 & -.033 & .487 & .487 & 29.85 & 118 & 3,522 & 193727 & -1.274 & .101 & .101 \\
\hline 7 & 12.19 & 678 & 8,264 & 2834925 & .538 & .705 & .295 & 18.53 & 446 & 8,264 & 1867625 & .491 & .688 & .312 \\
\hline 8 & 15.19 & 96 & 1,458 & 62185 & -1.891 & .029 & .029 & 23.90 & 61 & 1,458 & 41079 & -1.031 & .151 & .151 \\
\hline 9 & 13.65 & 212 & 2,893 & 291841 & -1.219 & .112 & .112 & 23.33 & 124 & 2,893 & 162202 & -1.846 & .032 & .032 \\
\hline 10 & 9.73 & 22 & 214 & 2446 & .318 & .625 & .375 & 14.27 & 15 & 214 & 1620 & .063 & .525 & .475 \\
\hline 11 & 19.07 & 321 & 6,120 & 1059378 & 2.436 & .993 & .007 & 27.20 & 225 & 6,120 & 755291 & 2.520 & .994 & .006 \\
\hline 12 & 12.75 & 2,791 & 35,581 & 50239187 & 1.080 & .860 & .140 & 20.38 & 1,746 & 35,581 & 31260530 & .462 & .678 & .322 \\
\hline 13 & 10.09 & 1,487 & 15,005 & 11419981 & 1.579 & .943 & .057 & 15.91 & 943 & 15,005 & 7455537 & 2.862 & .998 & .002 \\
\hline 14 & 13.98 & 2,144 & 29,966 & 32027188 & -.241 & .405 & .405 & 22.65 & 1,323 & 29,966 & 20259481 & 1.389 & .918 & .082 \\
\hline 15 & 19.64 & 87 & 1,709 & 69301 & -1.095 & .137 & .137 & 31.65 & 54 & 1,709 & 37274 & -2.446 & .007 & .007 \\
\hline 16 & 20.50 & 6 & 123 & 174 & -2.242 & .012 & .012 & 24.60 & 5 & 123 & 148 & -2.009 & .022 & .022 \\
\hline 17 & 17.57 & 7 & 123 & 376 & -.580 & .281 & .281 & 30.75 & 4 & 123 & 287 & .577 & .718 & .282 \\
\hline 18 & 18.73 & 1,470 & 27,539 & 20451213 & .689 & .755 & .245 & 36.05 & 764 & 27,539 & 10552616 & .149 & .559 & .441 \\
\hline 19 & 8.29 & 2,322 & 19,240 & 23093085 & 2.823 & .998 & .002 & 12.73 & 1,512 & 19,240 & 15192106 & 2.994 & .999 & .001 \\
\hline 20 & 11.13 & 550 & 6,122 & 1746028 & 1.508 & .934 & .066 & 16.24 & 377 & 6,122 & 1205149 & 1.491 & .932 & .068 \\
\hline 21 & 9.50 & 556 & 5,279 & 1460310 & -.202 & .420 & .420 & 14.00 & 377 & 5,279 & 966605 & -.963 & .168 & .168 \\
\hline 22 & 8.27 & 4,175 & 34,541 & 73773233 & 2.590 & .995 & .005 & 12.17 & 2,838 & 34,541 & 49572012 & 1.051 & .853 & .147 \\
\hline 23 & 12.50 & 95 & 1,187 & 56387 & .001 & .501 & .499 & 18.84 & 63 & 1,187 & 39704 & .851 & .802 & .198 \\
\hline 24 & 16.26 & 1,211 & 19,693 & 11997837 & .373 & .645 & .355 & 29.31 & 672 & 19,693 & 6749175 & .898 & .815 & .185 \\
\hline 25 & 19.38 & 710 & 13,756 & 4715386 & -1.588 & .056 & .056 & 33.39 & 412 & 13,756 & 2711255 & -1.520 & .064 & .064 \\
\hline 26 & 7.71 & 4,034 & 31,099 & 63244216 & .908 & .818 & .182 & 11.40 & 2,727 & 31,099 & 42763577 & .768 & .779 & .221 \\
\hline 27 & 9.18 & 2,265 & 20,787 & 23411983 & -.453 & .325 & .325 & 14.06 & 1,478 & 20,787 & 14973810 & -1.681 & .046 & .046 \\
\hline 28 & 10.35 & 1,162 & 12,022 & 7064673 & .675 & .750 & .250 & 16.72 & 719 & 12,022 & 4375563 & .577 & .718 & .282 \\
\hline 29 & 20.16 & 788 & 15,884 & 6245909 & -.096 & .462 & .462 & 36.35 & 437 & 15,884 & 3379366 & -.952 & .171 & .171 \\
\hline 30 & 8.09 & 3,912 & 31,631 & 63422175 & 2.717 & .997 & .003 & 12.06 & 2,622 & 31,631 & 43253940 & 3.819 & 1.000 & 0 \\
\hline 31 & 9.27 & 1,956 & 18,129 & 18118286 & 1.677 & .953 & .047 & 14.16 & 1,280 & 18,129 & 11948176 & 1.846 & .968 & .032 \\
\hline 32 & 13.17 & 1,264 & 16,648 & 10568153 & .273 & .608 & .392 & 20.58 & 809 & 16,648 & 6721474 & -.093 & .463 & .463 \\
\hline 33 & 11.66 & 1,988 & 23,183 & 23703520 & 2.211 & .986 & .014 & 19.00 & 1,220 & 23,183 & 14402811 & 1.117 & .868 & .132 \\
\hline 34 & 10.32 & 351 & 3,622 & 627829 & -.400 & .345 & .345 & 15.68 & 231 & 3,622 & 415810 & -.159 & .437 & .437 \\
\hline 35 & 12.77 & 806 & 10,292 & 4428495 & 3.329 & 1.000 & 0 & 19.27 & 534 & 10,292 & 2959481 & 3.081 & .999 & .001 \\
\hline 36 & 10.53 & 1,052 & 11,081 & 5859005 & .293 & .615 & .385 & 16.25 & 682 & 11,081 & 3677528 & -1.210 & .113 & .113 \\
\hline 37 & 11.37 & 960 & 10,911 & 5257240 & .205 & .581 & .419 & 17.00 & 642 & 10,911 & 3493800 & -.108 & .457 & .457 \\
\hline 38 & 12.17 & 1,842 & 22,410 & 21390559 & 2.705 & .997 & .003 & 19.24 & 1,165 & 22,410 & 13679506 & 2.834 & .998 & .002 \\
\hline 39 & 10.11 & 1,933 & 19,535 & 19504127 & 2.515 & .994 & .006 & 15.12 & 1,292 & 19,535 & 13313065 & 3.421 & 1.000 & 0 \\
\hline 40 & 13.01 & 798 & 10,379 & 4309625 & 1.990 & .977 & .023 & 20.04 & 518 & 10,379 & 2842382 & 2.262 & .988 & .012 \\
\hline 41 & 8.57 & 2,894 & 24,787 & 36818466 & 2.472 & .993 & .007 & 12.94 & 1,916 & 24,787 & 24216102 & 1.501 & .933 & .067 \\
\hline 42 & 15.50 & 48 & 744 & 17628 & -.153 & .439 & .439 & 24.80 & 30 & 744 & 10402 & -.644 & .260 & .260 \\
\hline 43 & 14.50 & 2,213 & 32,082 & 35996658 & 1.143 & .873 & .127 & 23.28 & 1,378 & 32,082 & 22401537 & .864 & .806 & .194 \\
\hline 44 & 9.29 & 2,115 & 19,656 & 21382488 & 2.285 & .989 & .011 & 13.98 & 1,406 & 19,656 & 14416523 & 2.812 & .998 & .002 \\
\hline 45 & 7.82 & 2,423 & 18,947 & 22767753 & -.693 & .244 & .244 & 11.81 & 1,604 & 18,947 & 15458465 & 1.201 & .885 & .115 \\
\hline 46 & 11.06 & 2,337 & 25,853 & 30536963 & .908 & .818 & .182 & 17.66 & 1,464 & 25,853 & 19009646 & .299 & .617 & .383 \\
\hline 47 & 12.60 & 505 & 6363 & 1683340 & 1.858 & .968 & .032 & 20.20 & 315 & 6,363 & 1053327 & 1.569 & .942 & .058 \\
\hline 48 & 12.20 & 463 & 5648 & 1318165 & .304 & .619 & .381 & 19.82 & 285 & 5,648 & 834700 & 1.085 & .861 & .139 \\
\hline 49 & 7.95 & 38 & 302 & 6803 & 1.982 & .976 & .024 & 12.58 & 24 & 302 & 4536 & 2.135 & .984 & .016 \\
\hline 50 & 11.02 & 83 & 915 & 43676 & 2.370 & .991 & .009 & 16.64 & 55 & 915 & 30299 & 2.622 & .996 & .004 \\
\hline 51 & 11.00 & 1,656 & 18,223 & 15353649 & 1.238 & .892 & .108 & 17.81 & 1,023 & 18,223 & 9424557 & .615 & .731 & .269 \\
\hline 52 & 9.38 & 1,398 & 13,109 & 9135011 & -.199 & .421 & .421 & 13.92 & 942 & 13,109 & 6187999 & .118 & .547 & .453 \\
\hline
\end{tabular}


Table 4. Summary of interoccurrence intervals for daily precipitation thresholds of 0.25 and 0.50 inch-Continued

\begin{tabular}{|c|c|c|c|c|c|c|c|c|c|c|c|c|c|c|}
\hline \multirow[b]{2}{*}{$\begin{array}{l}\text { Seq. } \\
\text { no. }\end{array}$} & \multicolumn{7}{|c|}{ Daily precipitation threshold of 0.25 inch and greater } & \multicolumn{7}{|c|}{ Daily precipitation threshold of 0.50 inch and greater } \\
\hline & $\begin{array}{l}\text { Mean } \\
\text { inter- } \\
\text { occur- } \\
\text { rence } \\
\text { interval } \\
\text { (days) }\end{array}$ & $\begin{array}{c}\text { Total } \\
\text { no. of } \\
\text { events }\end{array}$ & $\begin{array}{l}\text { Total } \\
\text { no. of } \\
\text { days } \\
\text { avail- } \\
\text { able }\end{array}$ & $\begin{array}{l}\text { Sigma } \\
\text { S }\end{array}$ & $\begin{array}{c}\text { Stan- } \\
\text { dard } \\
\text { normal } \\
\text { variate }\end{array}$ & $\begin{array}{l}\text { Non- } \\
\text { ex- } \\
\text { ceed- } \\
\text { ance } \\
\text { prob- } \\
\text { ability }\end{array}$ & $\begin{array}{c}p- \\
\text { value }\end{array}$ & $\begin{array}{l}\text { Mean } \\
\text { inter- } \\
\text { occur- } \\
\text { rence } \\
\text { interval } \\
\text { (days) }\end{array}$ & $\begin{array}{c}\text { Total } \\
\text { no. of } \\
\text { events }\end{array}$ & $\begin{array}{l}\text { Total } \\
\text { no. of } \\
\text { days } \\
\text { avail- } \\
\text { able }\end{array}$ & $\begin{array}{l}\text { Sigma } \\
\mathrm{S}\end{array}$ & $\begin{array}{c}\text { Stan- } \\
\text { dard } \\
\text { normal } \\
\text { variate }\end{array}$ & $\begin{array}{l}\text { Non- } \\
\text { ex- } \\
\text { ceed- } \\
\text { ance } \\
\text { prob- } \\
\text { ability }\end{array}$ & $\begin{array}{c}\mathrm{p}- \\
\text { value }\end{array}$ \\
\hline 53 & 6.25 & 49 & 306 & 7473 & -.039 & .485 & .485 & 8.74 & 35 & 306 & 5189 & -.318 & .375 & .375 \\
\hline 54 & 21.75 & 4 & 87 & 152 & -.438 & .331 & .331 & 29.00 & 3 & 87 & 96 & -.793 & .214 & .214 \\
\hline 55 & 13.86 & 712 & 9,865 & 3585487 & .968 & .833 & 167 & 21.83 & 452 & 9,865 & 2201243 & -.467 & .320 & .320 \\
\hline 56 & 17.28 & 138 & 2,385 & 177927 & 1.652 & .951 & .049 & 27.73 & 86 & 2,385 & 107127 & .716 & .763 & .237 \\
\hline 57 & 22.98 & 852 & 19,579 & 8453534 & .684 & .753 & .247 & 41.75 & 469 & 19,579 & 4743693 & 1.245 & .893 & .107 \\
\hline 58 & 16.00 & 38 & 608 & 11213 & -.313 & .377 & .377 & 24.32 & 25 & 608 & 5748 & -2.110 & .017 & .017 \\
\hline 59 & 14.26 & 2,540 & 36,226 & 45885260 & -.231 & .409 & .409 & 23.24 & 1,559 & 36,226 & 27648184 & -1.429 & .077 & .077 \\
\hline 60 & 19.09 & 22 & 420 & 4754 & .236 & .593 & .407 & 38.18 & 11 & 420 & 1955 & -.883 & .189 & .189 \\
\hline 61 & 22.26 & 1,252 & 27,865 & 17451071 & .027 & .511 & .489 & 48.46 & 575 & 27,865 & 8425456 & 2.148 & .984 & .016 \\
\hline 62 & 21.80 & 10 & 218 & 1445 & 1.784 & .963 & .037 & 27.25 & 8 & 218 & 1156 & 1.596 & .945 & .055 \\
\hline 63 & 18.88 & 108 & 2,039 & 125975 & 2.594 & .995 & .005 & 28.72 & 71 & 2,039 & 86292 & 2.804 & .997 & .003 \\
\hline 64 & 73.75 & 4 & 295 & 628 & .223 & .588 & .412 & -- & -- & -- & -- & -- & -- & -- \\
\hline 65 & 12.03 & 617 & 7,422 & 2249965 & -.746 & .228 & .228 & 19.13 & 388 & 7,422 & 1463106 & .551 & .709 & .291 \\
\hline 66 & 13.57 & 172 & 2,334 & 189826 & -1.233 & .109 & .109 & 20.47 & 114 & 2,334 & 121481 & -1.607 & .054 & .054 \\
\hline 67 & 9.61 & 1,388 & 13,340 & 9392184 & .936 & .825 & .175 & 14.81 & 901 & 13,340 & 6176756 & 1.446 & .926 & .074 \\
\hline 68 & 21.38 & 245 & 5,237 & 656241 & .622 & .733 & .267 & 36.12 & 145 & 5,237 & 382412 & .150 & .560 & .440 \\
\hline 69 & 26.72 & 184 & 4,917 & 430401 & -1.141 & .127 & .127 & 46.39 & 106 & 4,917 & 255716 & -.334 & .369 & .369 \\
\hline 70 & 12.48 & 83 & 1,036 & 51296 & 3.047 & .999 & .001 & 16.98 & 61 & 1,036 & 38609 & 3.002 & .999 & .001 \\
\hline 71 & 11.91 & 64 & 762 & 24510 & .072 & .529 & .471 & 18.14 & 42 & 762 & 15037 & -.677 & .249 & .249 \\
\hline 72 & 13.82 & 316 & 4,366 & 676963 & -.574 & .283 & .283 & 22.74 & 192 & 4,366 & 425957 & .391 & .652 & .348 \\
\hline 73 & 15.55 & 804 & 12,503 & 4972848 & -.521 & .301 & .301 & 23.77 & 526 & 12,503 & 3429382 & 1.705 & .956 & .044 \\
\hline 74 & 9.44 & 128 & 1,208 & 78578 & .321 & .626 & .374 & 14.73 & 82 & 1,208 & 52064 & .803 & .789 & .211 \\
\hline 75 & 8.87 & 2,641 & 23,435 & 31848135 & 2.595 & .995 & .005 & 13.66 & 1,716 & 23,435 & 21065521 & 3.420 & 1.000 & 0 \\
\hline 76 & 21.77 & 53 & 1154 & 26477 & -1.692 & .045 & .045 & 37.23 & 31 & 1,154 & 15746 & -1.154 & .124 & .124 \\
\hline 77 & 7.86 & 2,014 & 15,838 & 16380544 & 2.104 & .982 & .018 & 11.36 & 1,394 & 15,838 & 11275282 & 1.384 & .917 & .083 \\
\hline 78 & 14.48 & 50 & 724 & 18643 & .367 & .643 & .357 & 21.29 & 34 & 724 & 12441 & .109 & .543 & .457 \\
\hline 79 & 9.40 & 62 & 583 & 20776 & 2.040 & .979 & .021 & 13.88 & 42 & 583 & 13946 & 1.561 & .941 & .059 \\
\hline 80 & 7.29 & 4,881 & 35,599 & 89162036 & 3.179 & .999 & .001 & 10.97 & 3,244 & 35,599 & 59449459 & 2.918 & .998 & .002 \\
\hline 81 & 7.39 & 1,911 & 14,126 & 14070741 & 3.216 & .999 & .001 & 11.18 & 1,263 & 14,126 & 9360225 & 3.034 & .999 & .001 \\
\hline 82 & 8.98 & 1,411 & 12,671 & 9647209 & 5.152 & 0 & 0 & 12.61 & 1,005 & 12,671 & 6786390 & 3.615 & 1.000 & 0 \\
\hline 83 & 12.06 & 2,955 & 35,650 & 53468681 & 1.423 & .923 & .077 & 19.72 & 1,808 & 35,650 & 32727788 & 1.143 & .873 & .127 \\
\hline 84 & 9.00 & 868 & 7,812 & 3444851 & .819 & .794 & .206 & 13.52 & 578 & 7,812 & 2279660 & .406 & .657 & .343 \\
\hline 85 & 11.18 & 148 & 1,655 & 118935 & -.608 & .272 & .272 & 14.27 & 116 & 1,655 & 93066 & -.568 & .285 & .285 \\
\hline 86 & 10.59 & 1,365 & 14,452 & 10326222 & 3.002 & .999 & .001 & 17.02 & 849 & 14,452 & 6582640 & 3.684 & 1.000 & 0 \\
\hline 87 & 18.95 & 64 & 1,213 & 37493 & -.472 & .318 & .318 & 28.88 & 42 & 1,213 & 24242 & -.543 & .294 & .294 \\
\hline 88 & 15.43 & 833 & 12,853 & 5302457 & -.475 & .318 & .318 & 23.54 & 546 & 12,853 & 3510940 & .024 & .510 & .490 \\
\hline 89 & 10.90 & 1,750 & 19,069 & 17381359 & 3.022 & .999 & .001 & 16.97 & 1,124 & 19,069 & 11213725 & 2.693 & .996 & .004 \\
\hline 90 & 13.18 & 485 & 6,392 & 1493877 & -1.383 & .083 & .083 & 20.69 & 309 & 6,392 & 995523 & .245 & .597 & .403 \\
\hline 91 & 13.88 & 894 & 12,411 & 5479083 & -.641 & .261 & .261 & 23.51 & 528 & 12,411 & 3170343 & -1.290 & .099 & .099 \\
\hline 92 & 10.72 & 1,155 & 12,385 & 7093274 & -.486 & .313 & .313 & 16.54 & 749 & 12,385 & 4585373 & -.540 & .295 & .295 \\
\hline 93 & 7.35 & 289 & 2,124 & 304913 & -.192 & .424 & .424 & 10.78 & 197 & 2,124 & 210678 & .170 & .568 & .432 \\
\hline 94 & 26.77 & 91 & 2,436 & 108361 & -.369 & .356 & .356 & 45.96 & 53 & 2,436 & 59335 & -1.019 & .154 & .154 \\
\hline 95 & 18.46 & 56 & 1,034 & 28629 & -.145 & .442 & .442 & 24.62 & 42 & 1,034 & 20391 & -.684 & .247 & .247 \\
\hline 96 & 17.85 & 773 & 13,801 & 5227829 & -.959 & .169 & .169 & 30.00 & 460 & 13,801 & 3118275 & -.655 & .256 & .256 \\
\hline 97 & 26.26 & 43 & 1,129 & 23438 & -.391 & .348 & .348 & 53.76 & 21 & 1,129 & 10270 & -1.061 & .144 & .144 \\
\hline 98 & 17.85 & 1,067 & 19,044 & 10612246 & 2.519 & .994 & .006 & 30.52 & 624 & 19,044 & 6371276 & 3.128 & .999 & .001 \\
\hline 99 & 11.40 & 10 & 114 & 451 & -1.144 & .126 & .126 & 22.80 & 5 & 114 & 255 & -.408 & .342 & .342 \\
\hline 100 & 17.45 & 1,638 & 28,580 & 23239089 & -.503 & .308 & .308 & 26.79 & 1,067 & 28,580 & 15586439 & 1.258 & .896 & .104 \\
\hline 101 & 14.28 & 645 & 9,211 & 2908953 & -0.912 & .181 & .181 & 22.80 & 404 & 9,211 & 1800720 & -1.121 & .131 & .131 \\
\hline 102 & 11.46 & 69 & 791 & 30080 & 1.471 & .929 & .071 & 17.98 & 44 & 791 & 19378 & 1.305 & .904 & .096 \\
\hline 103 & 10.93 & 3,427 & 37,448 & 66080651 & 3.024 & .999 & .001 & 17.06 & 2,195 & 37,448 & 42067271 & 1.911 & .972 & .028 \\
\hline 104 & 13.79 & 455 & 6,276 & 1468970 & 1.066 & .857 & .143 & 20.99 & 299 & 6,276 & 982929 & 1.426 & .923 & .077 \\
\hline
\end{tabular}


Table 4. Summary of interoccurrence intervals for daily precipitation thresholds of 0.25 and 0.50 inch-Continued

\begin{tabular}{|c|c|c|c|c|c|c|c|c|c|c|c|c|c|c|}
\hline \multirow[b]{2}{*}{$\begin{array}{c}\text { Seq. } \\
\text { no. }\end{array}$} & \multicolumn{7}{|c|}{ Daily precipitation threshold of 0.25 inch and greater } & \multicolumn{7}{|c|}{ Daily precipitation threshold of 0.50 inch and greater } \\
\hline & $\begin{array}{c}\text { Mean } \\
\text { inter- } \\
\text { occur- } \\
\text { rence } \\
\text { interval } \\
\text { (days) }\end{array}$ & $\begin{array}{l}\text { Total } \\
\text { no. of } \\
\text { events }\end{array}$ & $\begin{array}{l}\text { Total } \\
\text { no. of } \\
\text { days } \\
\text { avail- } \\
\text { able }\end{array}$ & $\begin{array}{l}\text { Sigma } \\
\mathrm{S}\end{array}$ & $\begin{array}{l}\text { Stan- } \\
\text { dard } \\
\text { normal } \\
\text { variate }\end{array}$ & $\begin{array}{l}\text { Non- } \\
\text { ex- } \\
\text { ceed- } \\
\text { ance } \\
\text { prob- } \\
\text { ability }\end{array}$ & $\begin{array}{c}p- \\
\text { value }\end{array}$ & $\begin{array}{l}\text { Mean } \\
\text { inter- } \\
\text { occur- } \\
\text { rence } \\
\text { interval } \\
\text { (days) }\end{array}$ & $\begin{array}{l}\text { Total } \\
\text { no. of } \\
\text { events }\end{array}$ & $\begin{array}{l}\text { Total } \\
\text { no. of } \\
\text { days } \\
\text { avail- } \\
\text { able }\end{array}$ & $\begin{array}{l}\text { Sigma } \\
\mathrm{S}\end{array}$ & $\begin{array}{c}\text { Stan- } \\
\text { dard } \\
\text { normal } \\
\text { variate }\end{array}$ & $\begin{array}{l}\text { Non- } \\
\text { ex- } \\
\text { ceed- } \\
\text { ance } \\
\text { prob- } \\
\text { ability }\end{array}$ & $\begin{array}{c}\mathrm{p}- \\
\text { value }\end{array}$ \\
\hline 105 & 15.98 & 641 & 10,246 & 3334314 & .674 & .750 & .250 & 24.99 & 410 & 10,246 & 2115082 & .245 & .597 & .403 \\
\hline 106 & 14.60 & 244 & 3,562 & 425091 & -.590 & .278 & .278 & 26.99 & 132 & 3,562 & 222409 & -1.074 & .142 & .142 \\
\hline 107 & 10.04 & 746 & 7,487 & 2752395 & -.682 & .248 & .248 & 15.06 & 497 & 7,487 & 1897107 & .759 & .776 & .224 \\
\hline 108 & 11.35 & 3,290 & 37,342 & 62971259 & 2.497 & .994 & .006 & 17.62 & 2,119 & 37,342 & 40144767 & 1.171 & .879 & .121 \\
\hline 109 & 7.12 & 2,025 & 14,412 & 14796772 & 1.093 & .863 & .137 & 10.14 & 1,421 & 14,412 & 10617602 & 2.410 & .992 & .008 \\
\hline 110 & 8.68 & 4,000 & 34,710 & 70675099 & 1.981 & .976 & .024 & 13.27 & 2,616 & 34,710 & 46900647 & 2.927 & .998 & .002 \\
\hline 111 & 11.51 & 109 & 1,254 & 71666 & .879 & .810 & .190 & 17.91 & 70 & 1,254 & 44951 & .350 & .637 & .363 \\
\hline 112 & 14.36 & 1,599 & 22,955 & 18159960 & -.727 & .234 & .234 & 25.71 & 893 & 22,955 & 10163197 & -.435 & .332 & .332 \\
\hline 113 & 15.42 & 156 & 2,405 & 159760 & -3.209 & .001 & .001 & 25.32 & 95 & 2,405 & 98207 & -2.369 & .009 & .009 \\
\hline 114 & 19.29 & 104 & 2,006 & 105681 & .232 & .592 & .408 & 35.19 & 57 & 2,006 & 61906 & 1.083 & .861 & 139 \\
\hline 115 & 30.88 & 585 & 18,062 & 5499764 & 1.718 & .957 & .043 & 64.28 & 281 & 18,062 & 2597827 & .688 & .754 & .246 \\
\hline 116 & 15.36 & 378 & 5,805 & 1064144 & -1.013 & .156 & .156 & 26.75 & 217 & 5,805 & 643919 & .570 & .716 & .284 \\
\hline 117 & 14.76 & 1,302 & 19,214 & 12927752 & 2.096 & .982 & .018 & 26.04 & 738 & 19,214 & 7227077 & .910 & .818 & .182 \\
\hline 118 & 15.32 & 66 & 1,011 & 37623 & 1.797 & .964 & .036 & 28.08 & 36 & 1,011 & 19078 & .503 & .692 & .308 \\
\hline 119 & 10.84 & 2,544 & 27,586 & 36306505 & 3.030 & .999 & .001 & 16.96 & 1,627 & 27,586 & 23435388 & 3.095 & .999 & .001 \\
\hline 120 & 7.58 & 2,530 & 19,175 & 24818102 & 2.018 & .978 & .022 & 11.40 & 1,682 & 19,175 & 16643703 & 2.280 & .989 & .011 \\
\hline 121 & 10.97 & 1,673 & 18,359 & 15831860 & 2.189 & .986 & .014 & 16.65 & 1,103 & 18,359 & 10777679 & 3.708 & 1.000 & 0 \\
\hline 122 & 17.08 & 506 & 8,643 & 2290400 & 1.848 & .968 & .032 & 28.25 & 306 & 8,643 & 1375845 & 1.225 & .890 & .110 \\
\hline 123 & 16.84 & 2,049 & 34,510 & 35312060 & -.096 & .462 & .462 & 26.03 & 1,326 & 34,510 & 23062896 & .504 & .693 & .307 \\
\hline 124 & 17.64 & 428 & 7,550 & 1829463 & 4.741 & 1.000 & 0 & 25.34 & 298 & 7,550 & 1235776 & 2.946 & .998 & .002 \\
\hline 125 & 13.27 & 1,788 & 23,727 & 21741718 & 1.829 & .966 & .034 & 21.61 & 1,098 & 23,727 & 13437696 & 1.813 & .965 & .035 \\
\hline 126 & 9.47 & 382 & 3,619 & 681725 & -.466 & .321 & .321 & 13.87 & 261 & 3,619 & 475198 & .173 & .569 & .431 \\
\hline 127 & 17.97 & 1,079 & 19,385 & 10694785 & 1.287 & .901 & .099 & 32.86 & 590 & 19,385 & 5660381 & -.428 & .334 & .334 \\
\hline 128 & 8.29 & 1,313 & 10,885 & 7204759 & .516 & .697 & .303 & 12.44 & 875 & 10,885 & 4771543 & .101 & .540 & .460 \\
\hline 129 & 11.53 & 1,703 & 19,634 & 16944958 & .969 & .834 & .166 & 17.52 & 1,121 & 19,634 & 11098032 & .491 & .688 & .312 \\
\hline 130 & 12.89 & 2,045 & 26,350 & 26930065 & -.037 & .485 & .485 & 21.55 & 1,223 & 26,350 & 16282048 & .635 & .737 & .263 \\
\hline 131 & 11.89 & 64 & 761 & 27211 & 1.627 & .948 & .052 & 19.51 & 39 & 761 & 16349 & 1.100 & .864 & .136 \\
\hline 132 & 9.63 & 1,458 & 14,041 & 10524859 & 1.867 & .969 & .031 & 14.46 & 971 & 14,041 & 6890429 & .582 & .720 & .280 \\
\hline 133 & 9.16 & 3,962 & 36,302 & 73002260 & 1.649 & .950 & .050 & 14.32 & 2,536 & 36,302 & 46848968 & 1.550 & .939 & .061 \\
\hline 134 & 15.41 & 78 & 1,202 & 41260 & -1.833 & .033 & .033 & 25.04 & 48 & 1,202 & 24446 & -1.831 & .034 & .034 \\
\hline 135 & 12.94 & 94 & 1,216 & 52259 & -1.438 & .075 & .075 & 22.52 & 54 & 1,216 & 31064 & -.685 & .247 & .247 \\
\hline 136 & 10.90 & 2,847 & 31,019 & 44801802 & 1.353 & .912 & .088 & 17.15 & 1,809 & 31,019 & 28552016 & 1.301 & .903 & .097 \\
\hline 137 & 11.28 & 313 & 3,532 & 569648 & .936 & .825 & .175 & 16.58 & 213 & 3,532 & 399794 & 1.588 & .944 & .056 \\
\hline 138 & 15.02 & 509 & 7,647 & 1955572 & .189 & .575 & .425 & 22.29 & 343 & 7,647 & 1373221 & 1.511 & .935 & .065 \\
\hline 139 & 20.43 & 7 & 143 & 641 & 1.286 & .901 & .099 & 47.67 & 3 & 143 & 368 & 2.147 & .984 & .016 \\
\hline 140 & 10.24 & 126 & 1,290 & 86690 & 1.297 & .903 & .097 & 14.33 & 90 & 1,290 & 60441 & .677 & .751 & .249 \\
\hline 141 & 7.62 & 1,400 & 10,670 & 7636745 & 1.456 & .927 & .073 & 11.51 & 927 & 10,670 & 5036177 & .966 & .833 & .167 \\
\hline 142 & 7.57 & 1,061 & 8,028 & 4303608 & .593 & .723 & .277 & 11.07 & 725 & 8,028 & 3001616 & 1.466 & .929 & .071 \\
\hline 143 & 7.64 & 2,353 & 17,968 & 21613560 & 1.885 & .970 & .030 & 10.92 & 1,646 & 17,968 & 15005660 & 1.036 & .850 & .150 \\
\hline 144 & 16.69 & 825 & 13,768 & 5325657 & -3.098 & .001 & .001 & 28.86 & 477 & 13,768 & 3060309 & -2.573 & .005 & .005 \\
\hline 145 & 18.18 & 961 & 17,470 & 8570013 & 1.124 & .869 & .131 & 32.11 & 544 & 17,470 & 4780150 & .241 & .595 & .405 \\
\hline 146 & 15.41 & 1,839 & 28,335 & 26631339 & 1.646 & .950 & .050 & 25.64 & 1,105 & 28,335 & 16058831 & 1.485 & .931 & .069 \\
\hline 147 & 13.00 & 1,495 & 19,432 & 14963986 & 2.022 & .978 & .022 & 20.56 & 945 & 19,432 & 9599913 & 2.426 & .992 & .008 \\
\hline 148 & 21.85 & 58 & 1,267 & 32796 & -1.417 & .078 & .078 & 32.49 & 39 & 1,267 & 23875 & -.364 & .358 & .358 \\
\hline 149 & 10.70 & 20 & 214 & 2410 & .977 & .836 & .164 & 16.46 & 13 & 214 & 1734 & 1.540 & .938 & .062 \\
\hline 150 & 11.68 & 99 & 1,156 & 56387 & -.252 & .401 & .401 & 18.65 & 62 & 1,156 & 34432 & -.534 & .297 & .297 \\
\hline 151 & 13.04 & 538 & 7,017 & 1826189 & -1.307 & .096 & .096 & 21.93 & 320 & 7,017 & 1076429 & -1.278 & .101 & .101 \\
\hline 152 & 23.12 & 342 & 7,906 & 1307264 & -1.058 & .145 & .145 & 44.42 & 178 & 7,906 & 692257 & -.374 & .354 & .354 \\
\hline 153 & 8.99 & 1,611 & 14,486 & 12032370 & 2.168 & .985 & .015 & 14.49 & 1,000 & 14,486 & 7374043 & 0.991 & .839 & .161 \\
\hline 154 & 14.62 & 731 & 10,685 & 3857233 & -.577 & .282 & .282 & 26.91 & 397 & 10,685 & 2059966 & -.993 & .160 & .160 \\
\hline 155 & 10.83 & 2,036 & 22,051 & 22807732 & 1.253 & .895 & .105 & 16.87 & 1,307 & 22,051 & 14620732 & .914 & .820 & .180 \\
\hline 156 & 18.32 & 803 & 14,709 & 5874926 & -.256 & .399 & .399 & 36.05 & 408 & 14,709 & 3016415 & .184 & .573 & .427 \\
\hline
\end{tabular}


Table 4. Summary of interoccurrence intervals for daily precipitation thresholds of 0.25 and 0.50 inch-Continued

\begin{tabular}{|c|c|c|c|c|c|c|c|c|c|c|c|c|c|c|}
\hline \multirow[b]{2}{*}{$\begin{array}{l}\text { Seq. } \\
\text { no. }\end{array}$} & \multicolumn{7}{|c|}{ Daily precipitation threshold of 0.25 inch and greater } & \multicolumn{7}{|c|}{ Daily precipitation threshold of 0.50 inch and greater } \\
\hline & $\begin{array}{l}\text { Mean } \\
\text { inter- } \\
\text { occur- } \\
\text { rence } \\
\text { interval } \\
\text { (days) }\end{array}$ & $\begin{array}{c}\text { Total } \\
\text { no. of } \\
\text { events }\end{array}$ & $\begin{array}{l}\text { Total } \\
\text { no. of } \\
\text { days } \\
\text { avail- } \\
\text { able }\end{array}$ & $\begin{array}{l}\text { Sigma } \\
\text { S }\end{array}$ & $\begin{array}{c}\text { Stan- } \\
\text { dard } \\
\text { normal } \\
\text { variate }\end{array}$ & $\begin{array}{l}\text { Non- } \\
\text { ex- } \\
\text { ceed- } \\
\text { ance } \\
\text { prob- } \\
\text { ability }\end{array}$ & $\begin{array}{c}p- \\
\text { value }\end{array}$ & $\begin{array}{l}\text { Mean } \\
\text { inter- } \\
\text { occur- } \\
\text { rence } \\
\text { interval } \\
\text { (days) }\end{array}$ & $\begin{array}{c}\text { Total } \\
\text { no. of } \\
\text { events }\end{array}$ & $\begin{array}{l}\text { Total } \\
\text { no. of } \\
\text { days } \\
\text { avail- } \\
\text { able }\end{array}$ & $\begin{array}{l}\text { Sigma } \\
\mathrm{S}\end{array}$ & $\begin{array}{l}\text { Stan- } \\
\text { dard } \\
\text { normal } \\
\text { variate }\end{array}$ & $\begin{array}{l}\text { Non- } \\
\text { ex- } \\
\text { ceed- } \\
\text { ance } \\
\text { prob- } \\
\text { ability }\end{array}$ & $\begin{array}{c}\mathrm{p}- \\
\text { value }\end{array}$ \\
\hline 157 & 42.33 & 3 & 127 & 180 & -.165 & .434 & .434 & 63.50 & 2 & 127 & 132 & .096 & .538 & .462 \\
\hline 158 & 11.51 & 177 & 2,037 & 167115 & -1.682 & .046 & .046 & 17.71 & 115 & 2,037 & 102739 & -2.282 & .011 & .011 \\
\hline 159 & 12.52 & 1,405 & 17,595 & 12405607 & .237 & .594 & .406 & 19.51 & 902 & 17,595 & 7961709 & .173 & .569 & .431 \\
\hline 160 & 9.65 & 620 & 5,981 & 1809073 & -1.048 & .147 & 147 & 14.17 & 422 & 5,981 & 1229504 & -.916 & .180 & .180 \\
\hline 161 & 11.30 & 1,193 & 13,484 & 8324930 & 2.095 & .982 & .018 & 16.88 & 799 & 13,484 & 5476275 & .813 & .792 & .208 \\
\hline 162 & 11.01 & 892 & 9,819 & 4592341 & 2.517 & .994 & .006 & 18.08 & 543 & 9,819 & 2846195 & 2.730 & .997 & .003 \\
\hline 163 & 11.24 & 3,064 & 34,426 & 54453267 & 3.113 & .999 & .001 & 18.08 & 1,904 & 34,426 & 34292346 & 3.502 & 1.000 & 0 \\
\hline 164 & 9.59 & 54 & 518 & 13425 & -.511 & .305 & .305 & 19.19 & 27 & 518 & 7014 & .027 & .511 & .489 \\
\hline 165 & 14.81 & 80 & 1,185 & 45807 & -.521 & .301 & .301 & 25.21 & 47 & 1,185 & 29129 & .546 & .708 & .292 \\
\hline 166 & 13.82 & 458 & 6,331 & 1525723 & 1.941 & .974 & .026 & 21.68 & 292 & 6,331 & 948133 & .762 & .777 & .223 \\
\hline 167 & 14.04 & 86 & 1,207 & 50238 & -.515 & .303 & .303 & 24.14 & 50 & 1,207 & 28574 & -.650 & .258 & .258 \\
\hline 168 & 9.32 & 1,512 & 14,097 & 10698467 & .260 & .603 & .397 & 14.44 & 976 & 14,097 & 6974313 & .747 & .772 & .228 \\
\hline 169 & 10.76 & 113 & 1,216 & 73633 & 1.321 & .907 & .093 & 17.88 & 68 & 1,216 & 46697 & 1.849 & .968 & .032 \\
\hline 170 & 14.77 & 412 & 6,084 & 1270904 & .494 & .689 & .311 & 25.89 & 235 & 6,084 & 744779 & 1.111 & .867 & .133 \\
\hline 171 & 14.81 & 556 & 8,233 & 2296632 & .140 & .556 & .444 & 23.66 & 348 & 8,233 & 1434433 & .043 & .517 & .483 \\
\hline 172 & 10.37 & 3,167 & 32,834 & 52792879 & 1.500 & .933 & .067 & 16.18 & 2,029 & 32,834 & 33869040 & 1.309 & .905 & .095 \\
\hline 173 & 19.39 & 102 & 1,978 & 99378 & -.260 & .397 & .397 & 29.52 & 67 & 1,978 & 62938 & -.711 & .238 & .238 \\
\hline 174 & 14.11 & 438 & 6,180 & 1406049 & 1.410 & .921 & .079 & 24.05 & 257 & 6,180 & 822452 & .990 & .839 & .161 \\
\hline 175 & 10.69 & 130 & 1,389 & 90061 & -.049 & .480 & .480 & 15.61 & 89 & 1,389 & 63691 & .497 & .690 & .310 \\
\hline 176 & 13.66 & 1,468 & 20,049 & 15080242 & 1.643 & .950 & .050 & 22.30 & 899 & 20,049 & 9166089 & .888 & .813 & .187 \\
\hline 177 & 14.70 & 2,185 & 32,125 & 35476337 & .876 & .809 & .191 & 25.20 & 1,275 & 32,125 & 20504103 & .074 & .529 & .471 \\
\hline 178 & 12.26 & 27 & 331 & 3522 & -1.906 & .028 & .028 & 18.39 & 18 & 331 & 2546 & -1.068 & .143 & .143 \\
\hline 179 & 25.88 & 752 & 19,460 & 7835703 & 3.367 & 1.000 & 0 & 54.36 & 358 & 19,460 & 3862744 & 3.570 & 1.000 & 0 \\
\hline 180 & 8.63 & 1,933 & 16,672 & 16461116 & 1.643 & .950 & .050 & 12.68 & 1,315 & 16,672 & 11418088 & 2.614 & .996 & .004 \\
\hline 181 & 15.78 & 1,790 & 28,238 & 24319273 & -2.765 & .003 & .003 & 27.90 & 1,012 & 28,238 & 13732293 & -2.145 & .016 & .016 \\
\hline 182 & 10.60 & 1,314 & 13,932 & 9280165 & .870 & .808 & .192 & 16.93 & 823 & 13,932 & 5789500 & .490 & .688 & .312 \\
\hline 183 & 10.51 & 184 & 1,934 & 165794 & -1.602 & .055 & .055 & 15.72 & 123 & 1,934 & 120125 & .191 & .576 & .424 \\
\hline 184 & 8.56 & 101 & 865 & 46493 & 1.120 & .869 & .131 & 13.11 & 66 & 865 & 30108 & .771 & .779 & .221 \\
\hline 185 & 22.04 & 100 & 2,204 & 102869 & -1.152 & .125 & .125 & 33.91 & 65 & 2,204 & 67990 & -.710 & .239 & .239 \\
\hline 186 & 16.68 & 137 & 2,285 & 159033 & .325 & .628 & .373 & 25.39 & 90 & 2,285 & 98579 & -.679 & .249 & .249 \\
\hline 187 & 15.25 & 4 & 61 & 122 & 0 & .500 & .500 & 15.25 & 4 & 61 & 122 & 0 & .500 & .500 \\
\hline 188 & 12.11 & 1,224 & 14,820 & 8970883 & -.661 & .254 & .254 & 18.95 & 782 & 14,820 & 5662066 & -1.108 & .134 & .134 \\
\hline 189 & 17.84 & 1,481 & 26,415 & 19058114 & -1.711 & .044 & .044 & 28.34 & 932 & 26,415 & 12012173 & -1.277 & .101 & .101 \\
\hline 190 & 9.98 & 1,895 & 18,911 & 18923845 & 4.232 & 1.000 & 0 & 15.03 & 1,258 & 18,911 & 12636160 & 3.828 & 1.000 & 0 \\
\hline 191 & 15.94 & 804 & 12,819 & 5240643 & .833 & .798 & .202 & 24.84 & 516 & 12,819 & 3383965 & .912 & .819 & .181 \\
\hline 192 & 7.76 & 2,341 & 18,176 & 21754381 & 1.888 & .971 & .030 & 11.53 & 1,576 & 18,176 & 14812319 & 2.351 & .991 & .009 \\
\hline 193 & 17.78 & 684 & 12,164 & 4408850 & 2.709 & .997 & .003 & 29.10 & 418 & 12,164 & 2790783 & 3.462 & 1.000 & 0 \\
\hline 194 & 12.81 & 502 & 6,429 & 1598064 & -.376 & .354 & .354 & 19.08 & 337 & 6,429 & 1096048 & .375 & .646 & .354 \\
\hline 195 & 28.92 & 328 & 9,486 & 1506907 & -.984 & .163 & .163 & 62.82 & 151 & 9,486 & 653837 & -1.853 & .032 & .032 \\
\hline 196 & 19.12 & 753 & 14,400 & 5290995 & -1.145 & .126 & .126 & 29.75 & 484 & 14,400 & 3498916 & .154 & .561 & .439 \\
\hline 197 & 9.98 & 811 & 8,095 & 3353290 & 1.063 & .856 & .144 & 14.96 & 541 & 8,095 & 2249639 & 1.103 & .865 & .135 \\
\hline 198 & 9.86 & 1,284 & 12,665 & 8130584 & -.003 & .499 & .499 & 14.38 & 881 & 12,665 & 5628767 & .459 & .677 & .323 \\
\hline 199 & 7.32 & 3,117 & 22,827 & 36195361 & 1.684 & .954 & .046 & 10.98 & 2,079 & 22,827 & 24036260 & 1.024 & .847 & .153 \\
\hline 200 & 11.37 & 1,242 & 14,121 & 8994755 & 1.571 & .942 & .058 & 18.78 & 752 & 14,121 & 5454295 & 1.295 & .902 & .098 \\
\hline 201 & 8.90 & 2,577 & 22,939 & 30253920 & 2.074 & .981 & .019 & 13.69 & 1,676 & 22,939 & 19675782 & 1.671 & .953 & .047 \\
\hline 202 & 11.85 & 41 & 486 & 11901 & 2.157 & .985 & .015 & 18.00 & 27 & 486 & 7455 & 1.226 & .890 & .110 \\
\hline 203 & 10.35 & 1,361 & 14,090 & 9787515 & 1.328 & .908 & .092 & 17.40 & 810 & 14,090 & 5826369 & 1.036 & .850 & .150 \\
\hline 204 & 6.31 & 220 & 1,388 & 147858 & -.811 & .209 & .209 & 8.26 & 168 & 1,388 & 117121 & .102 & .541 & .459 \\
\hline 205 & 22.59 & 17 & 384 & 3588 & 0.709 & .761 & .239 & 42.67 & 9 & 384 & 2031 & 0.911 & .819 & .181 \\
\hline 206 & 15.56 & 188 & 2,925 & 263215 & -1.014 & .155 & .155 & 26.84 & 109 & 2,925 & 144407 & -1.702 & .044 & .044 \\
\hline 207 & 15.64 & 33 & 516 & 8467 & -.055 & .478 & .478 & 27.16 & 19 & 516 & 5225 & .498 & .691 & .309 \\
\hline 208 & 16.38 & 503 & 8239 & 2134891 & 1.177 & .880 & .120 & 28.71 & 287 & 8,239 & 1247376 & 1.615 & .947 & .053 \\
\hline
\end{tabular}


Table 4. Summary of interoccurrence intervals for daily precipitation thresholds of 0.25 and 0.50 inch-Continued

\begin{tabular}{|c|c|c|c|c|c|c|c|c|c|c|c|c|c|c|}
\hline \multirow[b]{2}{*}{$\begin{array}{l}\text { Seq. } \\
\text { no. }\end{array}$} & \multicolumn{7}{|c|}{ Daily precipitation threshold of 0.25 inch and greater } & \multicolumn{7}{|c|}{ Daily precipitation threshold of 0.50 inch and greater } \\
\hline & $\begin{array}{l}\text { Mean } \\
\text { inter- } \\
\text { occur- } \\
\text { rence } \\
\text { interval } \\
\text { (days) }\end{array}$ & $\begin{array}{c}\text { Total } \\
\text { no. of } \\
\text { events }\end{array}$ & $\begin{array}{c}\text { Total } \\
\text { no. of } \\
\text { days } \\
\text { avail- } \\
\text { able }\end{array}$ & $\underset{\mathrm{S}}{\text { Sigma }}$ & $\begin{array}{c}\text { Stan- } \\
\text { dard } \\
\text { normal } \\
\text { variate }\end{array}$ & $\begin{array}{c}\text { Non- } \\
\text { ex- } \\
\text { ceed- } \\
\text { ance } \\
\text { prob- } \\
\text { ability }\end{array}$ & $\begin{array}{c}p- \\
\text { value }\end{array}$ & $\begin{array}{c}\text { Mean } \\
\text { inter- } \\
\text { occur- } \\
\text { rence } \\
\text { interval } \\
\text { (days) }\end{array}$ & $\begin{array}{c}\text { Total } \\
\text { no. of } \\
\text { events }\end{array}$ & $\begin{array}{c}\text { Total } \\
\text { no. of } \\
\text { days } \\
\text { avail- } \\
\text { able }\end{array}$ & $\underset{\mathrm{S}}{\operatorname{Sigma}}$ & $\begin{array}{c}\text { Stan- } \\
\text { dard } \\
\text { normal } \\
\text { variate }\end{array}$ & $\begin{array}{c}\text { Non- } \\
\text { ex- } \\
\text { ceed- } \\
\text { ance } \\
\text { prob- } \\
\text { ability }\end{array}$ & $\begin{array}{c}\text { p- } \\
\text { value }\end{array}$ \\
\hline 209 & 12.77 & 1197 & 15291 & 9205128 & .350 & .637 & .363 & 19.55 & 782 & 15,291 & 6077305 & .798 & .788 & .212 \\
\hline 210 & 13.80 & 311 & 4292 & 686179 & .859 & .805 & .195 & 21.35 & 201 & 4,292 & 435770 & .252 & .599 & .401 \\
\hline 211 & 10.57 & 193 & 2,040 & 178486 & -2.246 & .012 & .012 & 19.07 & 107 & 2,040 & 100061 & -1.490 & .068 & .068 \\
\hline 212 & 14.09 & 1,017 & 14,334 & 7246578 & -.320 & .374 & .374 & 22.75 & 630 & 14,334 & 4472937 & -.407 & .342 & .342 \\
\hline 213 & 10.83 & 293 & 3,173 & 482886 & 1.151 & .875 & .125 & 17.06 & 186 & 3,173 & 315463 & 1.631 & .949 & .051 \\
\hline 214 & 15.49 & 75 & 1,162 & 39543 & -1.388 & .083 & .083 & 24.72 & 47 & 1,162 & 25056 & -.979 & .164 & .164 \\
\hline 215 & 14.83 & 54 & 801 & 21277 & -.206 & .418 & .418 & 25.84 & 31 & 801 & 12567 & .118 & .547 & .453 \\
\hline 216 & 14.73 & 103 & 1,517 & 75826 & -.517 & .302 & .302 & 24.47 & 62 & 1,517 & 47038 & .003 & .501 & .499 \\
\hline 217 & 13.97 & 1,084 & 15,148 & 8277751 & .469 & .680 & .320 & 22.28 & 680 & 15,148 & 5048432 & -.894 & .186 & .186 \\
\hline 218 & 14.82 & 1,248 & 18,497 & 11915399 & 1.979 & .976 & .024 & 26.46 & 699 & 18,497 & 6766738 & 2.140 & .984 & .016 \\
\hline 219 & -- & -- & -- & -- & -- & -- & -- & -- & -- & -- & -- & -- & -- & -- \\
\hline 220 & 13.58 & 1,804 & 24,501 & 21934941 & -.549 & .292 & .292 & 21.82 & 1,123 & 24,501 & 13590117 & -.705 & .240 & .240 \\
\hline 221 & 7.09 & 665 & 4,717 & 1560966 & -.212 & .416 & .416 & 10.06 & 469 & 4,717 & 1101769 & -.148 & .441 & .441 \\
\hline 222 & 17.02 & 1,162 & 19,773 & 12142088 & 3.361 & 1.000 & 0 & 32.52 & 608 & 19,773 & 6404983 & 2.799 & .997 & .003 \\
\hline 223 & 14.93 & 436 & 6,509 & 1444920 & .662 & .746 & .254 & 24.47 & 266 & 6,509 & 877364 & .381 & .648 & .352 \\
\hline 224 & 16.90 & 362 & 6,117 & 1116405 & .275 & .608 & .392 & 27.93 & 219 & 6,117 & 670841 & .039 & .516 & .484 \\
\hline 225 & 14.65 & 808 & 11,834 & 5127072 & 3.565 & 1.000 & 0 & 19.86 & 596 & 11,834 & 3745485 & 2.625 & .996 & .004 \\
\hline 226 & 13.91 & 2,405 & 33,447 & 39411656 & -1.707 & .044 & .044 & 24.34 & 1,374 & 33,447 & 22385272 & -1.656 & .049 & .049 \\
\hline 227 & 8.11 & 148 & 1,200 & 87756 & -.248 & .402 & .402 & 12.25 & 98 & 1,200 & 61853 & .890 & .813 & .187 \\
\hline 228 & 8.05 & 4,227 & 34,037 & 72418098 & .753 & .774 & .226 & 11.81 & 2,882 & 34,037 & 49344324 & .563 & .713 & .287 \\
\hline 229 & 12.20 & 747 & 9,110 & 3509840 & 1.492 & .932 & .068 & 19.98 & 456 & 9,110 & 2229401 & 2.712 & .997 & .003 \\
\hline 230 & 14.60 & 2,193 & 32,007 & 35638780 & 1.255 & .895 & .105 & 24.38 & 1,313 & 32,007 & 20654293 & -1.070 & .142 & .142 \\
\hline 231 & 10.47 & 3,162 & 33,090 & 52703606 & .723 & .765 & .235 & 16.33 & 2,027 & 33,090 & 33752249 & .501 & .692 & .308 \\
\hline 232 & 9.49 & 263 & 2,497 & 332833 & .383 & .649 & .351 & 14.86 & 168 & 2,497 & 207986 & -.189 & .425 & .425 \\
\hline 233 & 7.62 & 2,268 & 17,281 & 19824378 & .959 & .831 & .169 & 11.56 & 1,495 & 17,281 & 13209053 & 1.511 & .935 & .065 \\
\hline 234 & 8.85 & 117 & 1,035 & 58485 & -.638 & .262 & .262 & 14.38 & 72 & 1,035 & 36066 & -.471 & .319 & .319 \\
\hline 235 & 10.42 & 2,244 & 23,374 & 26064512 & -.504 & .307 & .307 & 16.43 & 1,423 & 23,374 & 16218554 & -1.619 & .053 & .053 \\
\hline 236 & 17.30 & 332 & 5,743 & 880972 & -2.396 & .008 & .008 & 27.88 & 206 & 5,743 & 550440 & -1.727 & .042 & .042 \\
\hline 237 & 8.62 & 2,155 & 18,579 & 21255322 & 4.966 & 1.000 & 0 & 12.83 & 1,448 & 18,579 & 14493870 & 5.109 & 0 & 0 \\
\hline 238 & 15.49 & 275 & 4,259 & 572595 & -.639 & .262 & .262 & 22.90 & 186 & 4,259 & 379456 & -.992 & .161 & .161 \\
\hline 239 & 7.66 & 2,263 & 17,330 & 19995705 & 1.625 & .948 & .052 & 11.99 & 1,446 & 17,330 & 12967709 & 2.303 & .989 & .011 \\
\hline 240 & 18.51 & 705 & 13,047 & 4487572 & -1.115 & .133 & .133 & 34.52 & 378 & 13,047 & 2545358 & 1.085 & .861 & .139 \\
\hline 241 & 12.48 & 2,998 & 37,406 & 56026179 & -.077 & .469 & .469 & 19.94 & 1,876 & 37,406 & 34915315 & -.367 & .357 & .357 \\
\hline 242 & 9.29 & 1,885 & 17,517 & 16697719 & .856 & .804 & .196 & 14.51 & 1,207 & 17,517 & 10891627 & 1.822 & .966 & .034 \\
\hline 243 & 9.73 & 2,126 & 20,679 & 22015707 & .123 & .549 & .451 & 15.39 & 1,344 & 20,679 & 13752995 & -.655 & .256 & .256 \\
\hline 244 & 9.20 & 10 & 92 & 331 & -1.536 & .062 & .062 & 15.33 & 6 & 92 & 187 & -1.368 & .086 & .086 \\
\hline 245 & 15.83 & 1,667 & 26,383 & 21855729 & -.433 & .333 & .333 & 26.68 & 989 & 26,383 & 12914604 & -.550 & .291 & .291 \\
\hline 246 & 9.03 & 2,219 & 20,030 & 22859786 & 2.337 & .990 & .010 & 13.98 & 1,433 & 20,030 & 15064025 & 3.255 & .999 & .001 \\
\hline 247 & 11.86 & 2,573 & 30,526 & 39749886 & 1.070 & .858 & .142 & 18.40 & 1,659 & 30,526 & 25555968 & .654 & .743 & .257 \\
\hline 248 & 11.09 & 178 & 1,974 & 162767 & -1.699 & .045 & .045 & 16.73 & 118 & 1,974 & 109881 & -1.064 & .144 & .144 \\
\hline 249 & 8.87 & 340 & 3,015 & 519494 & .433 & .667 & .333 & 12.31 & 245 & 3,015 & 377854 & .625 & .734 & .266 \\
\hline 250 & 19.30 & 385 & 7,429 & 1378254 & -1.232 & .109 & .109 & 32.44 & 229 & 7,429 & 811045 & -1.220 & .111 & .111 \\
\hline 251 & 34.43 & 7 & 241 & 699 & -.785 & .216 & .216 & 60.25 & 4 & 241 & 249 & -1.675 & .047 & .047 \\
\hline 252 & 19.04 & 122 & 2,323 & 144437 & .369 & .644 & .356 & 31.82 & 73 & 2,323 & 83645 & -.200 & .421 & .421 \\
\hline 253 & 16.09 & 87 & 1,400 & 64974 & 1.081 & .860 & .140 & 25.46 & 55 & 1,400 & 42136 & 1.213 & .887 & .113 \\
\hline 254 & 16.70 & 1,167 & 19,485 & 11506633 & .714 & .762 & .238 & 31.03 & 628 & 19,485 & 6243774 & .890 & .813 & .187 \\
\hline 255 & 7.99 & 2,420 & 19,333 & 23829771 & 1.591 & .944 & .056 & 12.46 & 1,552 & 19,333 & 15634068 & 2.873 & .998 & .002 \\
\hline 256 & 8.62 & 2,113 & 18,213 & 19309818 & .281 & .610 & .390 & 11.94 & 1,526 & 18,213 & 14020577 & .604 & .727 & .273 \\
\hline 257 & 33.78 & 18 & 608 & 5333 & -0.187 & .426 & .426 & 86.86 & 7 & 608 & 1866 & -0.564 & .286 & .286 \\
\hline 258 & 17.83 & 1,092 & 19,465 & 10958534 & 1.781 & .963 & .037 & 29.85 & 652 & 19,465 & 6635530 & 2.021 & .978 & .022 \\
\hline 259 & 12.38 & 365 & 4,519 & 804901 & -.795 & .213 & .213 & 19.73 & 229 & 4,519 & 487798 & -1.501 & .067 & .067 \\
\hline 260 & 10.90 & 2,024 & 22,062 & 22514680 & .656 & .744 & .256 & 16.63 & 1,327 & 22,062 & 14424841 & -.919 & .179 & .179 \\
\hline
\end{tabular}


Table 4. Summary of interoccurrence intervals for daily precipitation thresholds of 0.25 and 0.50 inch-Continued

\begin{tabular}{|c|c|c|c|c|c|c|c|c|c|c|c|c|c|c|}
\hline \multirow[b]{2}{*}{$\begin{array}{l}\text { Seq. } \\
\text { no. }\end{array}$} & \multicolumn{7}{|c|}{ Daily precipitation threshold of 0.25 inch and greater } & \multicolumn{7}{|c|}{ Daily precipitation threshold of 0.50 inch and greater } \\
\hline & $\begin{array}{l}\text { Mean } \\
\text { inter- } \\
\text { occur- } \\
\text { rence } \\
\text { interval } \\
\text { (days) }\end{array}$ & $\begin{array}{l}\text { Total } \\
\text { no. of } \\
\text { events }\end{array}$ & $\begin{array}{l}\text { Total } \\
\text { no. of } \\
\text { days } \\
\text { avail- } \\
\text { able }\end{array}$ & $\begin{array}{l}\text { Sigma } \\
\text { S }\end{array}$ & $\begin{array}{c}\text { Stan- } \\
\text { dard } \\
\text { normal } \\
\text { variate }\end{array}$ & $\begin{array}{l}\text { Non- } \\
\text { ex- } \\
\text { ceed- } \\
\text { ance } \\
\text { prob- } \\
\text { ability }\end{array}$ & $\begin{array}{c}p- \\
\text { value }\end{array}$ & $\begin{array}{l}\text { Mean } \\
\text { inter- } \\
\text { occur- } \\
\text { rence } \\
\text { interval } \\
\text { (days) }\end{array}$ & $\begin{array}{c}\text { Total } \\
\text { no. of } \\
\text { events }\end{array}$ & $\begin{array}{l}\text { Total } \\
\text { no. of } \\
\text { days } \\
\text { avail- } \\
\text { able }\end{array}$ & $\begin{array}{l}\text { Sigma } \\
\mathrm{S}\end{array}$ & $\begin{array}{c}\text { Stan- } \\
\text { dard } \\
\text { normal } \\
\text { variate }\end{array}$ & $\begin{array}{l}\text { Non- } \\
\text { ex- } \\
\text { ceed- } \\
\text { ance } \\
\text { prob- } \\
\text { ability }\end{array}$ & $\begin{array}{c}\mathrm{p}- \\
\text { value }\end{array}$ \\
\hline 261 & 10.66 & 137 & 1,461 & 103603 & .714 & .762 & .238 & 17.39 & 84 & 1,461 & 60290 & -.277 & .391 & .391 \\
\hline 262 & 10.49 & 640 & 6,712 & 2136380 & -.234 & .408 & .408 & 15.12 & 444 & 6,712 & 1478902 & -.273 & .392 & .392 \\
\hline 263 & 30.19 & 614 & 18,538 & 6028718 & 2.546 & .995 & .005 & 62.42 & 297 & 18,538 & 3033956 & 3.048 & .999 & .001 \\
\hline 264 & 14.37 & 828 & 11,901 & 5158521 & 2.342 & .990 & .010 & 21.76 & 547 & 11,901 & 3473464 & 2.720 & .997 & .003 \\
\hline 265 & 13.23 & 1,491 & 19,723 & 15306217 & 2.742 & .997 & .003 & 21.00 & 939 & 19,723 & 9774384 & 2.949 & .998 & .002 \\
\hline 266 & 7.06 & 469 & 3,310 & 779873 & .178 & .571 & .429 & 10.15 & 326 & 3,310 & 548471 & .518 & .698 & .302 \\
\hline 267 & 9.55 & 3,813 & 36,415 & 70444872 & 1.571 & .942 & .058 & 14.49 & 2,514 & 36,415 & 46198016 & .805 & .790 & .210 \\
\hline 268 & 9.49 & 385 & 3,653 & 672905 & -1.464 & .072 & .072 & 14.00 & 261 & 3,653 & 484499 & .457 & .676 & .324 \\
\hline 269 & 9.09 & 78 & 709 & 30293 & 1.462 & .928 & .072 & 14.47 & 49 & 709 & 19403 & 1.419 & .922 & .078 \\
\hline 270 & 11.67 & 1,213 & 14154 & 8697852 & .797 & .787 & .213 & 17.30 & 818 & 14,154 & 5935581 & 1.255 & .895 & .105 \\
\hline 271 & 76.50 & 2 & 153 & 91 & -.993 & .161 & .161 & 153.00 & 1 & 153 & 56 & -.464 & .321 & .321 \\
\hline 272 & 75.50 & 2 & 151 & 190 & .633 & .736 & .264 & -- & -- & -- & -- & -- & -- & -- \\
\hline 273 & 16.28 & 1,952 & 31,770 & 30968826 & -.096 & .462 & .462 & 26.92 & 1,180 & 31,770 & 18352058 & -1.245 & .107 & .107 \\
\hline 274 & 17.20 & 662 & 11,383 & 4045416 & 3.284 & .999 & .001 & 27.70 & 411 & 11,383 & 2492962 & 2.308 & .990 & .011 \\
\hline 275 & 14.14 & 170 & 2,403 & 195087 & -1.014 & .155 & .155 & 23.56 & 102 & 2,403 & 118416 & -.591 & .277 & .277 \\
\hline 276 & 16.06 & 402 & 6,457 & 1346587 & 1.304 & .904 & .096 & 27.83 & 232 & 6,457 & 758672 & .340 & .633 & .367 \\
\hline 277 & 9.58 & 117 & 1,121 & 65306 & -.078 & .469 & .469 & 17.79 & 63 & 1,121 & 36633 & .515 & .697 & .303 \\
\hline 278 & 9.31 & 1,272 & 11,840 & 7591191 & .500 & .691 & .309 & 14.69 & 806 & 11,840 & 4796101 & .253 & .600 & .400 \\
\hline 279 & 34.81 & 21 & 731 & 8153 & .494 & .689 & .311 & 81.22 & 9 & 731 & 2328 & -1.519 & .064 & .064 \\
\hline 280 & 22.25 & 741 & 16,485 & 6115688 & .062 & .525 & .475 & 42.05 & 392 & 16,485 & 3357536 & 1.342 & .910 & .090 \\
\hline 281 & 11.09 & 398 & 4,415 & 913639 & 1.379 & .916 & .084 & 17.59 & 251 & 4,415 & 569567 & .767 & .778 & .222 \\
\hline 282 & 8.08 & 166 & 1,341 & 107735 & -.715 & .237 & .237 & 14.27 & 94 & 1,341 & 60187 & -.757 & .225 & .225 \\
\hline 283 & 10.74 & 1,807 & 19,415 & 18149653 & 2.553 & .995 & .005 & 16.27 & 1,193 & 19,415 & 12000441 & 2.167 & .985 & .015 \\
\hline 284 & 15.84 & 602 & 9,535 & 2908530 & .570 & .716 & .284 & 22.92 & 416 & 9,535 & 2038789 & .989 & .839 & .161 \\
\hline 285 & 8.42 & 3,863 & 32,536 & 64066398 & 2.095 & .982 & .018 & 12.75 & 2,551 & 32,536 & 42203109 & 1.483 & .931 & .069 \\
\hline 286 & 15.15 & 2,467 & 37,369 & 46503520 & .763 & .777 & .223 & 25.77 & 1,450 & 37,369 & 26534571 & -1.358 & .087 & .087 \\
\hline 287 & 14.27 & 128 & 1,826 & 118314 & .243 & .596 & .404 & 25.72 & 71 & 1,826 & 65374 & .124 & .549 & .451 \\
\hline 288 & 12.68 & 1,040 & 13,182 & 6946674 & .750 & .773 & .227 & 19.50 & 676 & 13,182 & 4522276 & .675 & .750 & .250 \\
\hline 289 & 15.32 & 74 & 1,134 & 35034 & -2.459 & .007 & .007 & 25.20 & 45 & 1,134 & 21462 & -1.846 & .032 & .032 \\
\hline 290 & 13.85 & 2,203 & 30,511 & 34592056 & 2.381 & .991 & .009 & 21.00 & 1,453 & 30,511 & 22381522 & .641 & .739 & .261 \\
\hline 291 & 18.02 & 1,083 & 19,511 & 10777060 & 1.143 & .873 & .127 & 28.53 & 684 & 19,511 & 6714568 & .284 & .612 & .388 \\
\hline 292 & 11.84 & 185 & 2,191 & 200440 & -.259 & .398 & .398 & 19.39 & 113 & 2,191 & 121606 & -.325 & .373 & .373 \\
\hline 293 & 11.04 & 3,200 & 35,323 & 56156528 & -.625 & .266 & .266 & 17.36 & 2,035 & 35,323 & 36246043 & .663 & .746 & .254 \\
\hline 294 & 8.51 & 2,214 & 18,834 & 21243119 & 1.540 & .938 & .062 & 12.05 & 1,563 & 18,834 & 14787984 & .322 & .626 & .374 \\
\hline 295 & 12.16 & 488 & 5,935 & 1506371 & 1.539 & .938 & .062 & 19.78 & 300 & 5,935 & 909335 & .643 & .740 & .260 \\
\hline 296 & 8.16 & 2,107 & 17,191 & 18654519 & 2.387 & .992 & .008 & 12.25 & 1,403 & 17,191 & 12501685 & 2.379 & .991 & .009 \\
\hline 297 & 8.11 & 2,409 & 19,546 & 23752009 & .754 & .775 & .225 & 11.91 & 1,641 & 19,546 & 16482400 & 1.947 & .974 & .026 \\
\hline 298 & 9.77 & 1,047 & 10,227 & 5509865 & 1.633 & .949 & .051 & 15.11 & 677 & 10,227 & 3589342 & 1.660 & .952 & .048 \\
\hline 299 & 21.18 & 11 & 233 & 1047 & -1.051 & .147 & .147 & 38.83 & 6 & 233 & 651 & -.291 & .385 & .385 \\
\hline 300 & 15.87 & 306 & 4,857 & 719172 & -.976 & .165 & .165 & 31.54 & 154 & 4,857 & 373323 & -.038 & .485 & .485 \\
\hline 301 & 17.52 & 963 & 16,871 & 8091273 & -.213 & .416 & .416 & 32.44 & 520 & 16,871 & 4232866 & -1.383 & .083 & .083 \\
\hline 302 & 18.43 & 1,048 & 19,309 & 10166903 & .272 & .607 & .393 & 33.64 & 574 & 19,309 & 5693208 & 1.135 & .872 & .128 \\
\hline 303 & 8.86 & 55 & 487 & 13547 & .148 & .559 & .441 & 12.82 & 38 & 487 & 9078 & -.202 & .420 & .420 \\
\hline 304 & 10.08 & 2,486 & 25,051 & 31890909 & 2.087 & .982 & .018 & 15.51 & 1,615 & 25,051 & 20942456 & 2.456 & .993 & .007 \\
\hline 305 & 11.95 & 130 & 1,554 & 88994 & -2.349 & .009 & .009 & 16.36 & 95 & 1,554 & 64054 & -2.232 & .013 & .013 \\
\hline 306 & 9.15 & 4,065 & 37,205 & 75864357 & .358 & .640 & .360 & 14.07 & 2,645 & 37,205 & 49616324 & .747 & .772 & .228 \\
\hline 307 & 13.45 & 1,571 & 21,126 & 17004818 & 1.698 & .955 & .045 & 24.51 & 862 & 21,126 & 9276063 & .954 & .830 & .170 \\
\hline 308 & 11.26 & 1,578 & 17,772 & 14271550 & 1.224 & .889 & .111 & 15.26 & 1,165 & 17,772 & 10370813 & .106 & .542 & .458 \\
\hline 309 & 10.51 & 111 & 1,167 & 66940 & 0.612 & .730 & .270 & 20.12 & 58 & 1,167 & 35821 & 0.771 & .780 & .220 \\
\hline 310 & 11.41 & 272 & 3,103 & 387650 & -2.326 & .010 & .010 & 18.92 & 164 & 3,103 & 227181 & -2.377 & .009 & .009 \\
\hline 311 & 17.21 & 221 & 3,804 & 444356 & 1.471 & .929 & .071 & 31.70 & 120 & 3,804 & 241569 & 1.108 & .866 & .134 \\
\hline 312 & 10.21 & 1,815 & 18,527 & 17904544 & 4.790 & 1.000 & 0 & 15.70 & 1,180 & 18,527 & 11676538 & 4.058 & 1.000 & 0 \\
\hline
\end{tabular}


Table 4. Summary of interoccurrence intervals for daily precipitation thresholds of 0.25 and 0.50 inch-Continued

\begin{tabular}{|c|c|c|c|c|c|c|c|c|c|c|c|c|c|c|}
\hline \multirow[b]{2}{*}{$\begin{array}{l}\text { Seq. } \\
\text { no. }\end{array}$} & \multicolumn{7}{|c|}{ Daily precipitation threshold of 0.25 inch and greater } & \multicolumn{7}{|c|}{ Daily precipitation threshold of 0.50 inch and greater } \\
\hline & $\begin{array}{l}\text { Mean } \\
\text { inter- } \\
\text { occur- } \\
\text { rence } \\
\text { interval } \\
\text { (days) }\end{array}$ & $\begin{array}{l}\text { Total } \\
\text { no. of } \\
\text { events }\end{array}$ & $\begin{array}{l}\text { Total } \\
\text { no. of } \\
\text { days } \\
\text { avail- } \\
\text { able }\end{array}$ & $\begin{array}{l}\text { Sigma } \\
\mathrm{S}\end{array}$ & $\begin{array}{c}\text { Stan- } \\
\text { dard } \\
\text { normal } \\
\text { variate }\end{array}$ & $\begin{array}{l}\text { Non- } \\
\text { ex- } \\
\text { ceed- } \\
\text { ance } \\
\text { prob- } \\
\text { ability }\end{array}$ & $\begin{array}{c}\mathrm{p}- \\
\text { value }\end{array}$ & $\begin{array}{l}\text { Mean } \\
\text { inter- } \\
\text { occur- } \\
\text { rence } \\
\text { interval } \\
\text { (days) }\end{array}$ & $\begin{array}{l}\text { Total } \\
\text { no. of } \\
\text { events }\end{array}$ & $\begin{array}{l}\text { Total } \\
\text { no. of } \\
\text { days } \\
\text { avail- } \\
\text { able }\end{array}$ & $\begin{array}{l}\text { Sigma } \\
\mathrm{S}\end{array}$ & $\begin{array}{c}\text { Stan- } \\
\text { dard } \\
\text { normal } \\
\text { variate }\end{array}$ & $\begin{array}{l}\text { Non- } \\
\text { ex- } \\
\text { ceed- } \\
\text { ance } \\
\text { prob- } \\
\text { ability }\end{array}$ & $\begin{array}{c}\mathrm{p}- \\
\text { value }\end{array}$ \\
\hline 313 & 10.45 & 236 & 2,465 & 280632 & -.937 & .175 & .175 & 17.24 & 143 & 2,465 & 160132 & -1.894 & .029 & .029 \\
\hline 314 & 11.42 & 99 & 1,131 & 57967 & 610 & .729 & 271 & 15.93 & 71 & 1,131 & 41938 & .650 & .742 & .258 \\
\hline 315 & 7.47 & 2,502 & 18,684 & 23890242 & 1.915 & 972 & .028 & 10.96 & 1,705 & 18,684 & 16587543 & 2.961 & .998 & .002 \\
\hline 316 & 18.16 & 109 & 1,979 & 99776 & -1.355 & 088 & 088 & 34.12 & 58 & 1,979 & 52080 & -1.221 & .111 & .111 \\
\hline 317 & 29.58 & 55 & 1,627 & 38200 & -1.878 & .030 & .030 & 54.23 & 30 & 1,627 & 22997 & -.547 & .292 & .292 \\
\hline 318 & 20.57 & 802 & 16,498 & 6794882 & 1.329 & .908 & .092 & 33.47 & 493 & 16,498 & 4272745 & 1.948 & .974 & .026 \\
\hline 319 & 19.37 & 193 & 3,738 & 359344 & -.092 & .463 & .463 & 31.15 & 120 & 3,738 & 227319 & .257 & .601 & .399 \\
\hline 320 & 28.73 & 279 & 8,016 & 1144627 & .683 & .753 & .247 & 63.62 & 126 & 8,016 & 534568 & 1.138 & .872 & .128 \\
\hline 321 & 9.17 & 2,260 & 20,733 & 23835217 & 1.430 & .924 & .076 & 14.20 & 1,460 & 20,733 & 15508273 & 1.632 & .949 & .051 \\
\hline 322 & 11.23 & 1,117 & 12,538 & 7000532 & -.016 & .494 & .494 & 15.79 & 794 & 12,538 & 5005672 & .275 & .609 & 391 \\
\hline 323 & 11.13 & 482 & 5,364 & 1291138 & -.047 & .481 & .481 & 18.06 & 297 & 5,364 & 805645 & .341 & .633 & .367 \\
\hline 324 & 10.24 & 3,086 & 31,602 & 49913855 & 2.273 & .988 & .012 & 15.71 & 2,012 & 31,602 & 32593050 & 1.959 & .975 & .025 \\
\hline 325 & 17.90 & 238 & 4,259 & 463955 & -2.260 & .012 & .012 & 31.78 & 134 & 4,259 & 272048 & -.935 & .175 & .175 \\
\hline 326 & 7.85 & 827 & 6,492 & 2716380 & .593 & .723 & .277 & 11.37 & 571 & 6,492 & 1849086 & -.098 & .461 & .461 \\
\hline 327 & 14.36 & 566 & 8,126 & 2331885 & .578 & .718 & .282 & 21.79 & 373 & 8,126 & 1559090 & .962 & .832 & .168 \\
\hline 328 & 15.98 & 61 & 975 & 28624 & -.507 & .306 & .306 & 19.90 & 49 & 975 & 22877 & -.513 & .304 & .304 \\
\hline 329 & 7.08 & 1,633 & 11,566 & 9790226 & 2.569 & .995 & .005 & 10.62 & 1,089 & 11,566 & 6408367 & 1.005 & .842 & .158 \\
\hline 330 & 8.17 & 4,473 & 36,526 & 81530137 & -.227 & .410 & .410 & 12.18 & 2,998 & 36,526 & 54520210 & -.402 & .344 & .344 \\
\hline 331 & 15.02 & 446 & 6,698 & 1530198 & .895 & .815 & .185 & 25.57 & 262 & 6,698 & 879359 & .061 & .525 & .475 \\
\hline 332 & 15.78 & 2,020 & 31,881 & 33078104 & 2.123 & .983 & .017 & 25.16 & 1,267 & 31,881 & 20459487 & .802 & .789 & .211 \\
\hline 333 & 9.86 & 2,175 & 21,436 & 23827747 & 1.788 & .963 & .037 & 14.49 & 1,479 & 21,436 & 16454041 & 2.530 & .994 & .006 \\
\hline 334 & 15.97 & 967 & 15,442 & 7535360 & .499 & .691 & .309 & 30.76 & 502 & 15,442 & 4004272 & 1.285 & .901 & .099 \\
\hline 335 & 17.14 & 1,296 & 22,219 & 14053461 & -1.492 & .068 & .068 & 31.70 & 701 & 22,219 & 7653079 & -.793 & .214 & .214 \\
\hline 336 & 13.44 & 154 & 2,070 & 169887 & 1.416 & .922 & .078 & 21.34 & 97 & 2,070 & 109373 & 1.526 & .936 & .064 \\
\hline 337 & 13.27 & 830 & 11,010 & 4611456 & .462 & .678 & .322 & 21.38 & 515 & 11,010 & 2783001 & -.722 & .235 & .235 \\
\hline 338 & 13.22 & 437 & 5777 & 1293163 & .886 & .812 & .188 & 20.41 & 283 & 5,777 & 837542 & .716 & .763 & .237 \\
\hline 339 & 7.65 & 635 & 4859 & 1527663 & -.426 & .335 & .335 & 10.70 & 454 & 4,859 & 1104506 & .051 & .520 & .480 \\
\hline 340 & 10.07 & 620 & 6,240 & 1936424 & .045 & .518 & .482 & 15.88 & 393 & 6,240 & 1248021 & .612 & .730 & .270 \\
\hline 341 & 22.33 & 436 & 9,737 & 2150958 & .482 & .685 & .315 & 39.10 & 249 & 9,737 & 1267738 & 1.251 & .894 & .106 \\
\hline 342 & 24.41 & 168 & 4,100 & 343152 & -.081 & .468 & .468 & 36.61 & 112 & 4,100 & 242512 & 1.031 & .849 & .151 \\
\hline 343 & 11.00 & 1,155 & 12,702 & 7296651 & -.311 & .378 & .378 & 17.40 & 730 & 12,702 & 4655674 & .196 & .578 & .422 \\
\hline 344 & 10.90 & 3,159 & 34,428 & 56399287 & 3.617 & 1.000 & 0 & 17.28 & 1,992 & 34,428 & 36023699 & 3.908 & 1.000 & 0 \\
\hline 345 & 8.98 & 54 & 485 & 12699 & -.385 & .350 & .350 & 13.11 & 37 & 485 & 8529 & -.521 & .301 & .301 \\
\hline 346 & 16.89 & 1,275 & 21,528 & 13357158 & -1.654 & .049 & .049 & 31.20 & 690 & 21,528 & 7284134 & -.876 & .191 & .191 \\
\hline 347 & 13.86 & 86 & 1,192 & 47690 & -1.118 & .132 & .132 & 25.36 & 47 & 1,192 & 26228 & -.756 & .225 & .225 \\
\hline 348 & 14.03 & 796 & 11,166 & 4451641 & .083 & .533 & .467 & 23.41 & 477 & 11,166 & 2651007 & -.172 & .432 & .432 \\
\hline 349 & 14.44 & 773 & 11,161 & 4237151 & -.855 & .196 & .196 & 23.60 & 473 & 11,161 & 2514629 & -1.783 & .037 & .037 \\
\hline 350 & 12.86 & 2,191 & 28,183 & 31341778 & 1.227 & .890 & .110 & 20.32 & 1,387 & 28,183 & 19583217 & .126 & .550 & .450 \\
\hline 351 & 14.53 & 266 & 3,865 & 519250 & .286 & 613 & .387 & 25.60 & 151 & 3,865 & 296976 & .377 & .647 & .353 \\
\hline 352 & 28.70 & 37 & 1,062 & 16823 & -1.514 & .065 & .065 & 37.93 & 28 & 1,062 & 14033 & -.515 & .303 & .303 \\
\hline 353 & 9.44 & 345 & 3,257 & 586564 & 1.416 & .922 & .078 & 14.35 & 227 & 3,257 & 381446 & .831 & .797 & .203 \\
\hline 354 & 9.81 & 701 & 6,876 & 2482902 & 1.387 & .917 & .083 & 15.63 & 440 & 6,876 & 1557536 & 1.076 & .859 & .141 \\
\hline 355 & 10.89 & 1,026 & 11,170 & 5739368 & .089 & .535 & .465 & 16.95 & 659 & 11,170 & 3635833 & -.540 & .295 & .295 \\
\hline 356 & 18.58 & 1,926 & 35,793 & 34512162 & .096 & .538 & .462 & 30.18 & 1,186 & 35,793 & 21094432 & -.368 & .357 & .357 \\
\hline 357 & 12.10 & 2,611 & 31,587 & 41456184 & .471 & .681 & .319 & 19.27 & 1,639 & 31,587 & 26028401 & .387 & .651 & .349 \\
\hline 358 & 14.06 & 1,343 & 18,884 & 12765506 & .425 & .665 & .335 & 22.45 & 841 & 18,884 & 8054260 & .718 & .764 & .236 \\
\hline 359 & 14.70 & 151 & 2,220 & 168619 & .128 & .551 & .449 & 21.77 & 102 & 2,220 & 118941 & .884 & .812 & .188 \\
\hline 360 & 21.29 & 34 & 724 & 11069 & -1.017 & .155 & .155 & 40.22 & 18 & 724 & 5854 & -.747 & .228 & .228 \\
\hline 361 & 16.67 & 9 & 150 & 861 & 1.432 & .924 & .076 & 30.00 & 5 & 150 & 481 & 1.095 & .863 & .137 \\
\hline 362 & 10.04 & 2,114 & 21,229 & 22288882 & -.533 & .297 & .297 & 14.71 & 1,443 & 21,229 & 14971118 & -1.485 & .069 & .069 \\
\hline 363 & 9.16 & 239 & 2,188 & 266243 & .489 & .688 & .312 & 13.94 & 157 & 2,188 & 167215 & -.574 & .283 & .283 \\
\hline 364 & 9.12 & 1,051 & 9,588 & 4988820 & -.554 & .290 & .290 & 14.27 & 672 & 9,588 & 3145664 & -1.058 & .145 & .145 \\
\hline
\end{tabular}


Table 4. Summary of interoccurrence intervals for daily precipitation thresholds of 0.25 and 0.50 inch-Continued

\begin{tabular}{|c|c|c|c|c|c|c|c|c|c|c|c|c|c|c|}
\hline \multirow[b]{2}{*}{$\begin{array}{l}\text { Seq. } \\
\text { no. }\end{array}$} & \multicolumn{7}{|c|}{ Daily precipitation threshold of 0.25 inch and greater } & \multicolumn{7}{|c|}{ Daily precipitation threshold of 0.50 inch and greater } \\
\hline & $\begin{array}{l}\text { Mean } \\
\text { inter- } \\
\text { occur- } \\
\text { rence } \\
\text { interval } \\
\text { (days) }\end{array}$ & $\begin{array}{l}\text { Total } \\
\text { no. of } \\
\text { events }\end{array}$ & $\begin{array}{l}\text { Total } \\
\text { no. of } \\
\text { days } \\
\text { avail- } \\
\text { able }\end{array}$ & $\begin{array}{l}\text { Sigma } \\
\mathrm{S}\end{array}$ & $\begin{array}{c}\text { Stan- } \\
\text { dard } \\
\text { normal } \\
\text { variate }\end{array}$ & $\begin{array}{l}\text { Non- } \\
\text { ex- } \\
\text { ceed- } \\
\text { ance } \\
\text { prob- } \\
\text { ability }\end{array}$ & $\begin{array}{c}\mathrm{p}- \\
\text { value }\end{array}$ & $\begin{array}{l}\text { Mean } \\
\text { inter- } \\
\text { occur- } \\
\text { rence } \\
\text { interval } \\
\text { (days) }\end{array}$ & $\begin{array}{c}\text { Total } \\
\text { no. of } \\
\text { events }\end{array}$ & $\begin{array}{l}\text { Total } \\
\text { no. of } \\
\text { days } \\
\text { avail- } \\
\text { able }\end{array}$ & $\begin{array}{l}\text { Sigma } \\
\text { S }\end{array}$ & $\begin{array}{c}\text { Stan- } \\
\text { dard } \\
\text { normal } \\
\text { variate }\end{array}$ & $\begin{array}{l}\text { Non- } \\
\text { ex- } \\
\text { ceed- } \\
\text { ance } \\
\text { prob- } \\
\text { ability }\end{array}$ & $\begin{array}{c}\mathrm{p}- \\
\text { value }\end{array}$ \\
\hline 365 & 8.61 & 2,588 & 22,280 & 28484266 & -1.058 & .145 & .145 & 12.83 & 1,737 & 22,280 & 19229782 & -.449 & .327 & .327 \\
\hline 366 & 10.22 & 247 & 2,525 & 318428 & .575 & .717 & .283 & 12.02 & 210 & 2,525 & 272092 & .660 & .745 & .255 \\
\hline 367 & 9.39 & 1,229 & 11,543 & 7269443 & 1.509 & 934 & .066 & 14.03 & 823 & 11,543 & 4870065 & 1.257 & .896 & 104 \\
\hline 368 & 17.59 & 479 & 8,426 & 1952752 & -1.226 & .110 & .110 & 30.64 & 275 & 8,426 & 1141458 & -.424 & .336 & .336 \\
\hline 369 & 41.00 & 3 & 123 & 35 & -2.431 & .008 & .008 & 123.00 & 1 & 123 & 16 & -1.281 & .100 & .100 \\
\hline 370 & 22.16 & 295 & 6,537 & 919864 & -1.368 & .086 & .086 & 51.47 & 127 & 6,537 & 350418 & -3.042 & .001 & .001 \\
\hline 371 & 34.35 & 574 & 19,719 & 5880126 & 1.619 & .947 & .053 & 88.43 & 223 & 19,719 & 2343437 & 1.703 & .956 & .044 \\
\hline 372 & 15.57 & 524 & 8,156 & 2173902 & .687 & .754 & .246 & 25.57 & 319 & 8,156 & 1315367 & .345 & .635 & .365 \\
\hline 373 & 10.94 & 17 & 186 & 1261 & -1.446 & .074 & .074 & 20.67 & 9 & 186 & 769 & -.422 & .336 & .336 \\
\hline 374 & 17.14 & 51 & 874 & 24361 & 1.151 & .875 & .125 & 33.62 & 26 & 874 & 12955 & 1.238 & .892 & . 108 \\
\hline 375 & 16.00 & 670 & 10,721 & 3778272 & 2.331 & .990 & .010 & 27.21 & 394 & 10,721 & 2261195 & 2.428 & .992 & .008 \\
\hline 376 & 15.56 & 187 & 2,910 & 271790 & -.026 & .490 & .490 & 24.45 & 119 & 2,910 & 179476 & .691 & .755 & .245 \\
\hline 377 & 11.71 & 1,550 & 18,150 & 14566657 & 2.426 & .992 & .008 & 18.32 & 991 & 18,150 & 9378306 & 2.334 & .990 & .010 \\
\hline 378 & 10.14 & 1,434 & 14,540 & 10454094 & .182 & .572 & .428 & 15.50 & 938 & 14,540 & 6813217 & -.047 & .481 & .481 \\
\hline 379 & 12.07 & 15 & 181 & 1416 & .289 & .614 & .386 & 20.11 & 9 & 181 & 997 & 1.164 & .878 & .122 \\
\hline 380 & 11.24 & 127 & 1,428 & 101436 & 2.316 & .990 & .010 & 17.85 & 80 & 1,428 & 63170 & 1.641 & .950 & .050 \\
\hline 381 & 8.88 & 2,140 & 18,998 & 20553700 & .890 & .813 & .187 & 12.98 & 1,464 & 18,998 & 14326320 & 2.001 & .977 & .023 \\
\hline 382 & 8.00 & 37 & 296 & 5671 & .375 & .646 & .354 & 12.87 & 23 & 296 & 3856 & 1.103 & .865 & .135 \\
\hline 383 & 17.11 & 1,897 & 32,450 & 30487091 & -.715 & .237 & .237 & 26.38 & 1,230 & 32,450 & 20361509 & 1.232 & .891 & .109 \\
\hline 384 & 16.30 & 501 & 8,168 & 2158917 & 2.138 & .984 & .016 & 28.76 & 284 & 8,168 & 1228570 & 1.729 & .958 & .042 \\
\hline 385 & 9.39 & 1,835 & 17,222 & 15941324 & .658 & .745 & .255 & 15.00 & 1,148 & 17,222 & 9731783 & -.912 & .181 & .181 \\
\hline 386 & 30.50 & 8 & 244 & 872 & -.522 & .301 & .301 & 34.86 & 7 & 244 & 765 & -.478 & .317 & .317 \\
\hline 387 & 11.63 & 179 & 2,081 & 185381 & -.108 & .457 & .457 & 18.42 & 113 & 2,081 & 120945 & .528 & .701 & .299 \\
\hline 388 & 10.86 & 227 & 2,464 & 270600 & -.846 & .199 & .199 & 16.43 & 150 & 2,464 & 177840 & -.799 & .212 & .212 \\
\hline 389 & 13.44 & 27 & 363 & 5969 & 1.962 & .975 & .025 & 19.11 & 19 & 363 & 4192 & 1.628 & .948 & .052 \\
\hline 390 & 7.05 & 2,479 & 17,476 & 21842703 & .721 & .765 & .235 & 10.20 & 1,713 & 17,476 & 15096863 & .616 & .731 & .269 \\
\hline 391 & 11.17 & 1,430 & 15,972 & 11894180 & 2.720 & .997 & .003 & 17.12 & 933 & 15,972 & 7634932 & 1.307 & .904 & .096 \\
\hline 392 & 21.10 & 49 & 1,034 & 16942 & -4.016 & 0 & 0 & 25.85 & 40 & 1,034 & 13640 & -3.729 & 0 & 0 \\
\hline 393 & 39.98 & 265 & 10,594 & 1486970 & 1.673 & .953 & .047 & 99.94 & 106 & 10,594 & 609899 & 1.538 & .938 & .062 \\
\hline 394 & 15.50 & 2 & 31 & 32 & .079 & .532 & .468 & 31.00 & 1 & 31 & 18 & .279 & .610 & .390 \\
\hline 395 & 12.93 & 705 & 9,115 & 3385753 & 2.472 & .993 & .007 & 18.99 & 480 & 9,115 & 2267227 & 1.381 & .916 & .084 \\
\hline 396 & 9.23 & 2,135 & 19,696 & 21466366 & 1.678 & .953 & .047 & 13.85 & 1,422 & 19,696 & 14450754 & 2.084 & .981 & .019 \\
\hline 397 & 17.87 & 797 & 14,241 & 5346285 & -2.833 & .002 & .002 & 29.55 & 482 & 14,241 & 3295911 & -1.509 & .066 & .066 \\
\hline 398 & 14.93 & 2,266 & 33,819 & 38609764 & .630 & .736 & .264 & 25.03 & 1,351 & 33,819 & 23265907 & 1.174 & .880 & .120 \\
\hline 399 & 12.77 & 1,536 & 19,618 & 15011129 & -.250 & .401 & .401 & 20.14 & 974 & 19,618 & 9643923 & .509 & .695 & .305 \\
\hline 400 & 8.94 & 2,156 & 19,271 & 20985378 & .818 & .793 & .207 & 13.25 & 1,455 & 19,271 & 14186277 & .785 & .784 & .216 \\
\hline 401 & 14.93 & 387 & 5,779 & 1001316 & -3.563 & 0 & 0 & 26.27 & 220 & 5,779 & 574317 & -2.480 & .007 & .007 \\
\hline 402 & 22.09 & 11 & 243 & 1733 & 1.704 & .956 & .044 & 40.50 & 6 & 243 & 844 & .669 & .748 & .252 \\
\hline 403 & 11.39 & 1,188 & 13,535 & 7919555 & -.893 & .186 & .186 & 15.17 & 892 & 13,535 & 5776183 & -2.232 & .013 & .013 \\
\hline 404 & 9.55 & 2,158 & 20,610 & 22808633 & 2.064 & .980 & .020 & 14.38 & 1,433 & 20,610 & 15185945 & 1.860 & .969 & .031 \\
\hline 405 & 14.37 & 827 & 11,884 & 5147927 & 2.371 & .991 & .009 & 24.11 & 493 & 11,884 & 3059698 & 1.711 & .956 & .044 \\
\hline 406 & 10.36 & 2,094 & 21,685 & 23097048 & 1.371 & .915 & .085 & 15.93 & 1,361 & 21,685 & 15125631 & 1.598 & .945 & .055 \\
\hline 407 & 9.76 & 380 & 3,708 & 688879 & -.750 & .227 & .227 & 15.26 & 243 & 3,708 & 444554 & -.358 & .360 & .360 \\
\hline 408 & 10.06 & 3,395 & 34,142 & 58397246 & .768 & .779 & .221 & 15.75 & 2,168 & 34,142 & 37569426 & 1.219 & .889 & .111 \\
\hline 409 & 22.52 & 54 & 1,216 & 32268 & -.219 & .413 & .413 & 39.23 & 31 & 1,216 & 19514 & .341 & .633 & .367 \\
\hline 410 & 8.62 & 1,670 & 14,398 & 12056647 & .202 & .580 & .420 & 13.03 & 1,105 & 14,398 & 7946613 & -.060 & .476 & .476 \\
\hline 411 & 14.36 & 1,333 & 19,141 & 12853297 & .475 & .683 & .317 & 23.87 & 802 & 19,141 & 7741022 & .419 & .662 & .338 \\
\hline 412 & 10.10 & 1,376 & 13,897 & 9236735 & -2.180 & .015 & .015 & 15.37 & 904 & 13,897 & 6340552 & .490 & .688 & .312 \\
\hline 413 & 12.78 & 2,361 & 30,162 & 35588632 & -0.042 & .483 & .483 & 19.77 & 1,526 & 30,162 & 23069517 & 0.164 & .565 & .435 \\
\hline 414 & 12.45 & 275 & 3,423 & 478361 & .470 & .681 & .319 & 19.45 & 176 & 3,423 & 318336 & 1.305 & .904 & .096 \\
\hline 415 & 15.48 & 1,388 & 21,490 & 15184263 & 1.169 & .879 & .121 & 26.96 & 797 & 21,490 & 8566001 & .013 & .505 & .495 \\
\hline 416 & 16.17 & 1,197 & 19,352 & 12027313 & 2.303 & .989 & .011 & 28.46 & 680 & 19,352 & 6816001 & 1.622 & .948 & .052 \\
\hline
\end{tabular}


Table 4. Summary of interoccurrence intervals for daily precipitation thresholds of 0.25 and 0.50 inch-Continued

\begin{tabular}{|c|c|c|c|c|c|c|c|c|c|c|c|c|c|c|}
\hline \multirow[b]{2}{*}{$\begin{array}{c}\text { Seq. } \\
\text { no. }\end{array}$} & \multicolumn{7}{|c|}{ Daily precipitation threshold of 0.25 inch and greater } & \multicolumn{7}{|c|}{ Daily precipitation threshold of 0.50 inch and greater } \\
\hline & $\begin{array}{c}\text { Mean } \\
\text { inter- } \\
\text { occur- } \\
\text { rence } \\
\text { interval } \\
\text { (days) }\end{array}$ & $\begin{array}{c}\text { Total } \\
\text { no. of } \\
\text { events }\end{array}$ & $\begin{array}{l}\text { Total } \\
\text { no. of } \\
\text { days } \\
\text { avail- } \\
\text { able }\end{array}$ & $\begin{array}{l}\text { Sigma } \\
\mathbf{S}\end{array}$ & $\begin{array}{c}\text { Stan- } \\
\text { dard } \\
\text { normal } \\
\text { variate }\end{array}$ & $\begin{array}{l}\text { Non- } \\
\text { ex- } \\
\text { ceed- } \\
\text { ance } \\
\text { prob- } \\
\text { ability }\end{array}$ & $\begin{array}{c}\text { p- } \\
\text { value }\end{array}$ & $\begin{array}{l}\text { Mean } \\
\text { inter- } \\
\text { occur- } \\
\text { rence } \\
\text { interval } \\
\text { (days) }\end{array}$ & $\begin{array}{c}\text { Total } \\
\text { no. of } \\
\text { events }\end{array}$ & $\begin{array}{l}\text { Total } \\
\text { no. of } \\
\text { days } \\
\text { avail- } \\
\text { able }\end{array}$ & $\begin{array}{l}\text { Sigma } \\
\mathbf{S}\end{array}$ & $\begin{array}{l}\text { Stan- } \\
\text { dard } \\
\text { normal } \\
\text { variate }\end{array}$ & $\begin{array}{l}\text { Non- } \\
\text { ex- } \\
\text { ceed- } \\
\text { ance } \\
\text { prob- } \\
\text { ability }\end{array}$ & $\begin{array}{c}\mathrm{p}- \\
\text { value }\end{array}$ \\
\hline 417 & 13.19 & 233 & 3,072 & 375973 & 1.336 & .909 & .091 & 19.82 & 155 & 3,072 & 232518 & -.504 & .307 & .307 \\
\hline 418 & 13.81 & 1,805 & 24,925 & 23841631 & 4.406 & 1.000 & 0 & 23.60 & 1,056 & 24,925 & 14017937 & 3.668 & 1.000 & 0 \\
\hline 419 & 10.50 & 2,159 & 22,658 & 24671563 & .698 & .757 & .243 & 16.25 & 1,394 & 22,658 & 15887321 & .388 & .651 & .349 \\
\hline 420 & 17.29 & 1,107 & 19,138 & 10672993 & .436 & .668 & .332 & 28.95 & 661 & 19,138 & 6222695 & -.721 & .236 & .236 \\
\hline 421 & 17.48 & 1,784 & 31,191 & 27171844 & -1.711 & .044 & .044 & 35.57 & 877 & 31,191 & 13494886 & -.684 & .247 & .247 \\
\hline 422 & 29.27 & 396 & 11,589 & 2175910 & -1.783 & .037 & .037 & 62.64 & 185 & 11,589 & 920297 & -3.334 & 0 & 0 \\
\hline 423 & 18.79 & 617 & 11,596 & 3636802 & .715 & .763 & .237 & 29.89 & 388 & 11,596 & 2280190 & .464 & .679 & .321 \\
\hline 424 & 53.25 & 4 & 213 & 498 & .586 & .721 & .279 & 106.50 & 2 & 213 & 351 & 1.587 & .944 & .056 \\
\hline 425 & 22.81 & 932 & 21,262 & 9890793 & -.092 & .463 & .463 & 43.84 & 485 & 21,262 & 5282135 & .933 & .825 & .176 \\
\hline 426 & 20.14 & 887 & 17,867 & 8120879 & 1.282 & .900 & .100 & 39.53 & 452 & 17,867 & 4039683 & .016 & .506 & .494 \\
\hline 427 & -- & -- & -- & -- & -- & -- & -- & -- & -- & -- & -- & -- & -- & -- \\
\hline 428 & 11.82 & 406 & 4,798 & 999657 & .920 & .821 & .179 & 17.45 & 275 & 4,798 & 681681 & .956 & .830 & .170 \\
\hline 429 & 10.10 & 536 & 5,412 & 1506439 & 1.549 & .939 & .061 & 16.20 & 334 & 5,412 & 952313 & 1.699 & .955 & .045 \\
\hline 430 & 10.48 & 851 & 8,915 & 3929166 & 1.809 & .965 & .035 & 16.70 & 534 & 8,915 & 2404796 & .412 & .660 & .340 \\
\hline 431 & 9.96 & 387 & 3,856 & 770605 & 1.117 & .868 & .132 & 14.95 & 258 & 3,856 & 527759 & 1.697 & .955 & .045 \\
\hline 432 & 8.57 & 1,014 & 8,688 & 4424642 & .248 & .598 & .402 & 12.65 & 687 & 8,688 & 3043215 & .896 & .815 & .185 \\
\hline 433 & 16.20 & 1,689 & 27,354 & 23018610 & -.252 & .400 & .400 & 24.27 & 1,127 & 27,354 & 15500535 & .327 & .628 & .372 \\
\hline 434 & 9.55 & 1,424 & 13,597 & 9826989 & .985 & .838 & .162 & 14.27 & 953 & 13,597 & 6686347 & 1.711 & .956 & .044 \\
\hline 435 & 9.39 & 110 & 1,033 & 61344 & 1.448 & .926 & .074 & 12.60 & 82 & 1,033 & 44218 & .691 & .755 & .245 \\
\hline 436 & 12.48 & 2,329 & 29,054 & 34412914 & 1.432 & .924 & .076 & 19.93 & 1,458 & 29,054 & 21598437 & 1.305 & .904 & .096 \\
\hline 437 & 8.55 & 2,963 & 25,322 & 38503534 & 2.486 & .994 & .006 & 12.54 & 2,020 & 25,322 & 26344411 & 2.341 & .990 & .010 \\
\hline 438 & 16.10 & 1,160 & 18,671 & 11147389 & 1.733 & .958 & .042 & 23.72 & 787 & 18,671 & 7614564 & 1.769 & .962 & .038 \\
\hline 439 & 17.12 & 477 & 8,164 & 1893842 & -1.035 & .150 & .150 & 24.52 & 333 & 8,164 & 1356391 & -.068 & .473 & .473 \\
\hline 440 & 15.53 & 599 & 9,302 & 2911216 & 1.906 & .972 & .028 & 25.21 & 369 & 9,302 & 1778971 & 1.217 & .888 & .112 \\
\hline 441 & 17.44 & 1,361 & 23,733 & 16905797 & 2.989 & .999 & .001 & 33.19 & 715 & 23,733 & 8950746 & 2.545 & .995 & .005 \\
\hline 442 & 9.13 & 1,398 & 12,769 & 9101489 & 1.277 & .899 & .101 & 13.96 & 915 & 12,769 & 6060333 & 1.960 & .975 & .025 \\
\hline 443 & 10.71 & 1,213 & 12,987 & 7973601 & .743 & .771 & .229 & 16.05 & 809 & 12,987 & 5224169 & -.273 & .393 & .393 \\
\hline 444 & 15.08 & 107 & 1,613 & 91053 & .988 & .838 & .162 & 26.44 & 61 & 1,613 & 51566 & .652 & .743 & .257 \\
\hline 445 & 17.56 & 1,090 & 19,140 & 10824708 & 2.157 & .984 & .016 & 29.58 & 647 & 19,140 & 6541581 & 2.489 & .994 & .006 \\
\hline 446 & 12.66 & 93 & 1,177 & 50218 & -1.377 & .084 & .084 & 24.02 & 49 & 1,177 & 26193 & -1.112 & .133 & .133 \\
\hline 447 & 16.87 & 1,360 & 22,938 & 15848681 & 1.027 & .848 & .152 & 28.35 & 809 & 22,938 & 9341907 & .337 & .632 & .368 \\
\hline 448 & 10.30 & 3,060 & 31,527 & 48053495 & -.363 & .358 & .358 & 16.09 & 1,960 & 31,527 & 30777384 & -.296 & .384 & .384 \\
\hline 449 & 8.97 & 593 & 5,317 & 1546958 & -.790 & .215 & .215 & 13.16 & 404 & 5,317 & 1056752 & -.560 & .288 & .288 \\
\hline 450 & 9.38 & 2,190 & 20,533 & 23109920 & 2.258 & .988 & .012 & 14.58 & 1,408 & 20,533 & 15049280 & 2.671 & .996 & .004 \\
\hline 451 & 9.84 & 565 & 5,562 & 1586922 & .410 & .659 & .341 & 15.16 & 367 & 5,562 & 1001204 & -.632 & .264 & .264 \\
\hline 452 & 19.00 & 16 & 304 & 2505 & .208 & .582 & .418 & 27.64 & 11 & 304 & 1994 & 1.106 & .866 & .134 \\
\hline 453 & 20.22 & 104 & 2,103 & 111403 & .331 & .630 & .370 & 45.72 & 46 & 2,103 & 47412 & -.232 & .408 & .408 \\
\hline 454 & 17.67 & 1,799 & 31,784 & 27641700 & -2.436 & .007 & .007 & 30.71 & 1,035 & 31,784 & 15899152 & -1.860 & .031 & .031 \\
\hline 455 & 16.74 & 53 & 887 & 18391 & -2.744 & .003 & .003 & 32.85 & 27 & 887 & 10541 & -1.077 & .141 & .141 \\
\hline 456 & 67.00 & 1 & 67 & 61 & 1.422 & .922 & .078 & 67.00 & 1 & 67 & 61 & 1.422 & .922 & .078 \\
\hline 457 & 11.00 & 31 & 341 & 6693 & 2.568 & .995 & .005 & 16.24 & 21 & 341 & 4719 & 2.524 & .994 & .006 \\
\hline 458 & 7.77 & 801 & 6,223 & 2440748 & -1.014 & .155 & .155 & 11.27 & 552 & 6,223 & 1734169 & .394 & .653 & .347 \\
\hline 459 & 14.19 & 79 & 1,121 & 43296 & -.342 & .366 & .366 & 18.68 & 60 & 1,121 & 32171 & -.582 & .280 & .280 \\
\hline 460 & 11.01 & 2,974 & 32,732 & 50324874 & 3.207 & .999 & .001 & 16.60 & 1,972 & 32,732 & 32682337 & .974 & .835 & .165 \\
\hline 461 & 13.62 & 2,260 & 30,785 & 34915510 & .304 & .619 & .381 & 22.37 & 1,376 & 30,785 & 21254757 & .227 & .590 & .410 \\
\hline 462 & 10.76 & 1,649 & 17,740 & 15018092 & 1.882 & .970 & .030 & 17.22 & 1,030 & 17,740 & 9360977 & 1.368 & .914 & .086 \\
\hline 463 & 9.86 & 758 & 7,477 & 2908149 & 1.251 & .895 & .105 & 15.61 & 479 & 7,477 & 1816422 & .544 & .707 & .293 \\
\hline 464 & 9.68 & 2,324 & 22,500 & 26536141 & 1.249 & .894 & .106 & 15.17 & 1,483 & 22,500 & 17141085 & 1.828 & .966 & .034 \\
\hline 465 & 10.51 & 187 & 1,966 & 168406 & -1.986 & .024 & .024 & 14.78 & 133 & 1,966 & 118440 & -1.879 & .030 & .030 \\
\hline 466 & 8.23 & 3,186 & 26,233 & 42908445 & 2.619 & .996 & .004 & 12.32 & 2,129 & 26,233 & 28449166 & 1.500 & .933 & .067 \\
\hline 467 & 22.30 & 496 & 11,063 & 3191857 & 6.302 & 0 & 0 & 41.43 & 267 & 11,063 & 1733019 & 4.908 & 1.000 & 0 \\
\hline 468 & 10.79 & 92 & 993 & 38814 & -2.497 & .006 & .006 & 14.60 & 68 & 993 & 26980 & -2.869 & .002 & .002 \\
\hline
\end{tabular}


Table 4. Summary of interoccurrence intervals for daily precipitation thresholds of 0.25 and 0.50 inch-Continued

\begin{tabular}{|c|c|c|c|c|c|c|c|c|c|c|c|c|c|c|}
\hline \multirow[b]{2}{*}{$\begin{array}{l}\text { Seq. } \\
\text { no. }\end{array}$} & \multicolumn{7}{|c|}{ Daily precipitation threshold of 0.25 inch and greater } & \multicolumn{7}{|c|}{ Daily precipitation threshold of 0.50 inch and greater } \\
\hline & $\begin{array}{l}\text { Mean } \\
\text { inter- } \\
\text { occur- } \\
\text { rence } \\
\text { interval } \\
\text { (days) }\end{array}$ & $\begin{array}{l}\text { Total } \\
\text { no. of } \\
\text { events }\end{array}$ & $\begin{array}{l}\text { Total } \\
\text { no. of } \\
\text { days } \\
\text { avail- } \\
\text { able }\end{array}$ & $\begin{array}{l}\text { Sigma } \\
\mathrm{S}\end{array}$ & $\begin{array}{c}\text { Stan- } \\
\text { dard } \\
\text { normal } \\
\text { variate }\end{array}$ & $\begin{array}{l}\text { Non- } \\
\text { ex- } \\
\text { ceed- } \\
\text { ance } \\
\text { prob- } \\
\text { ability }\end{array}$ & $\begin{array}{c}\mathrm{p}- \\
\text { value }\end{array}$ & $\begin{array}{l}\text { Mean } \\
\text { inter- } \\
\text { occur- } \\
\text { rence } \\
\text { interval } \\
\text { (days) }\end{array}$ & $\begin{array}{c}\text { Total } \\
\text { no. of } \\
\text { events }\end{array}$ & $\begin{array}{l}\text { Total } \\
\text { no. of } \\
\text { days } \\
\text { avail- } \\
\text { able }\end{array}$ & $\begin{array}{l}\text { Sigma } \\
\text { S }\end{array}$ & $\begin{array}{c}\text { Stan- } \\
\text { dard } \\
\text { normal } \\
\text { variate }\end{array}$ & $\begin{array}{l}\text { Non- } \\
\text { ex- } \\
\text { ceed- } \\
\text { ance } \\
\text { prob- } \\
\text { ability }\end{array}$ & $\begin{array}{c}\mathrm{p}- \\
\text { value }\end{array}$ \\
\hline 469 & 8.04 & 1,221 & 9,821 & 6169438 & 1.754 & .960 & .040 & 12.10 & 812 & 9,821 & 3967303 & -.248 & .402 & .402 \\
\hline 470 & 15.17 & 6 & 91 & 196 & -1.197 & .116 & .116 & 18.20 & 5 & 91 & 147 & -1.370 & .085 & .085 \\
\hline 471 & 10.35 & 1,293 & 13,388 & 8751613 & .693 & .756 & 244 & 16.84 & 795 & 13,388 & 5331388 & .089 & .535 & 465 \\
\hline 472 & 8.85 & 381 & 3,372 & 648835 & .341 & 633 & .367 & 12.09 & 279 & 3,372 & 473126 & . 168 & .567 & .433 \\
\hline 473 & 11.58 & 1,660 & 19,226 & 16341290 & 1.697 & .955 & .045 & 17.59 & 1,093 & 19,226 & 11030325 & 2.852 & .998 & .002 \\
\hline 474 & 12.28 & 2,050 & 25,176 & 26160634 & 1.080 & .860 & .140 & 19.53 & 1,289 & 25,176 & 16385967 & .613 & .730 & .270 \\
\hline 475 & 11.06 & 2,891 & 31,959 & 47377798 & 2.381 & .991 & .009 & 17.68 & 1,808 & 31,959 & 30157112 & 3.228 & .999 & .001 \\
\hline 476 & 11.50 & 1,586 & 18,237 & 14514468 & .251 & .599 & .401 & 16.13 & 1,131 & 18,237 & 10731690 & 2.365 & .991 & .009 \\
\hline 477 & 12.14 & 346 & 4,201 & 743014 & .720 & .764 & .236 & 20.20 & 208 & 4,201 & 457896 & 1.200 & .885 & .115 \\
\hline 478 & 11.08 & 2,082 & 23,063 & 24662052 & 2.151 & .984 & .016 & 17.17 & 1,343 & 23,063 & 16047375 & 2.298 & .989 & .011 \\
\hline 479 & 10.42 & 172 & 1,792 & 146439 & -1.131 & .129 & .129 & 14.57 & 123 & 1,792 & 105732 & -.780 & .218 & .218 \\
\hline 480 & 12.55 & 453 & 5,684 & 1318533 & .891 & .813 & .187 & 19.33 & 294 & 5,684 & 890513 & 1.954 & .975 & .025 \\
\hline 481 & 8.23 & 1,538 & 12,650 & 9637568 & -.630 & .264 & .264 & 12.53 & 1,010 & 12,650 & 6400313 & .104 & .541 & .459 \\
\hline 482 & 10.60 & 350 & 3,709 & 634842 & -.711 & .239 & .239 & 16.34 & 227 & 3,709 & 406600 & -.891 & .187 & .187 \\
\hline 483 & 9.56 & 332 & 3,174 & 551943 & 1.501 & .933 & .067 & 14.49 & 219 & 3,174 & 366970 & 1.432 & .924 & .076 \\
\hline 484 & 10.94 & 250 & 2,736 & 330297 & -.937 & .174 & .174 & 18.87 & 145 & 2,736 & 199000 & .067 & .527 & .473 \\
\hline 485 & 15.42 & 55 & 848 & 21393 & -1.061 & .144 & .144 & 22.92 & 37 & 848 & 12997 & -1.807 & .035 & .035 \\
\hline 486 & 11.59 & 3,001 & 34,779 & 53134982 & 1.726 & .958 & .042 & 18.49 & 1,881 & 34,779 & 33323900 & 1.411 & .921 & .079 \\
\hline 487 & 11.32 & 926 & 10,484 & 4782043 & -.782 & .217 & .217 & 17.62 & 595 & 10,484 & 3015634 & -1.400 & .081 & .081 \\
\hline 488 & 24.40 & 1,153 & 28,138 & 16165351 & -.204 & .419 & .419 & 44.59 & 631 & 28,138 & 8805519 & -.353 & .362 & .362 \\
\hline 489 & 10.25 & 1,134 & 11,627 & 6583550 & -.079 & .468 & .468 & 15.99 & 727 & 11,627 & 4327476 & 1.117 & .868 & .132 \\
\hline 490 & 10.47 & 745 & 7,802 & 3003158 & 1.577 & .943 & .057 & 16.32 & 478 & 7,802 & 1922421 & 1.173 & .879 & .121 \\
\hline 491 & 8.66 & 1,475 & 12,770 & 9913831 & 3.503 & 1.000 & 0 & 13.14 & 972 & 12,770 & 6422892 & 1.885 & .970 & .030 \\
\hline 492 & 10.65 & 2,355 & 25,088 & 30287564 & 2.124 & .983 & .017 & 16.58 & 1,513 & 25,088 & 19786524 & 2.866 & .998 & .002 \\
\hline 493 & 9.06 & 3,938 & 35,661 & 72414439 & 3.402 & 1.000 & 0 & 13.71 & 2,602 & 35,661 & 47765696 & 2.610 & .995 & .005 \\
\hline 494 & 8.99 & 474 & 4,263 & 1067186 & 2.122 & .983 & .017 & 13.80 & 309 & 4,263 & 690973 & 1.495 & .933 & .067 \\
\hline 495 & 7.58 & 2,683 & 20,344 & 29257933 & 6.464 & 0 & 0 & 11.17 & 1,821 & 20,344 & 19880366 & 5.415 & 0 & 0 \\
\hline 496 & 15.70 & 1,373 & 21,557 & 14906539 & .467 & .680 & .320 & 27.92 & 772 & 21,557 & 8333320 & .071 & .528 & .472 \\
\hline 497 & 31.35 & 26 & 815 & 11044 & .374 & .646 & .354 & 101.88 & 8 & 815 & 3938 & 1.019 & .846 & .154 \\
\hline 498 & 9.71 & 1,963 & 19,069 & 19204355 & 2.001 & .977 & .023 & 14.85 & 1,284 & 19,069 & 12802880 & 2.842 & .998 & .002 \\
\hline 499 & 14.35 & 1,237 & 17,748 & 11363175 & 2.142 & .984 & .016 & 23.14 & 767 & 17,748 & 6988378 & 1.283 & .900 & .100 \\
\hline 500 & 11.56 & 100 & 1,156 & 58406 & .182 & .572 & .428 & 20.28 & 57 & 1,156 & 31937 & -.401 & .344 & .344 \\
\hline 501 & 14.48 & 353 & 5,112 & 904377 & .076 & .530 & .470 & 19.44 & 263 & 5,112 & 671451 & -.033 & .487 & .487 \\
\hline 502 & 8.58 & 3,563 & 30,570 & 55416516 & 1.815 & .965 & .035 & 12.64 & 2,418 & 30,570 & 37850618 & 2.054 & .980 & .020 \\
\hline 503 & 17.69 & 346 & 6,120 & 1019994 & -1.180 & .119 & .119 & 29.71 & 206 & 6,120 & 604269 & -1.029 & .152 & .152 \\
\hline 504 & 20.58 & 179 & 3,683 & 303773 & -1.818 & .035 & .035 & 36.47 & 101 & 3,683 & 184115 & -.176 & .430 & .430 \\
\hline 505 & 14.47 & 74 & 1,071 & 35475 & -1.561 & .059 & .059 & 23.28 & 46 & 1,071 & 20699 & -1.876 & .030 & .030 \\
\hline 506 & 9.83 & 3,804 & 37,401 & 71908857 & 1.160 & .877 & .123 & 15.55 & 2,406 & 37,401 & 45846536 & 1.611 & .946 & .054 \\
\hline 507 & 7.89 & 177 & 1,396 & 136691 & 2.452 & .993 & .007 & 10.66 & 131 & 1,396 & 101798 & 2.246 & .988 & .012 \\
\hline 508 & 12.49 & 1,272 & 15,882 & 10179558 & .481 & .685 & .315 & 19.20 & 827 & 15,882 & 6603455 & .275 & .608 & .392 \\
\hline 509 & 14.79 & 1,726 & 25,519 & 22482975 & 1.503 & .934 & .066 & 23.94 & 1,066 & 25,519 & 13714334 & .469 & .680 & .320 \\
\hline 510 & 16.02 & 131 & 2,098 & 137216 & -.029 & .488 & .488 & 25.28 & 83 & 2,098 & 79246 & -1.418 & .078 & .078 \\
\hline 511 & 8.03 & 2,315 & 18,591 & 21426495 & -.359 & .360 & .360 & 12.04 & 1,544 & 18,591 & 14959989 & 2.882 & .998 & .002 \\
\hline 512 & 14.04 & 2,246 & 31,532 & 35114112 & -.687 & .246 & .246 & 23.02 & 1,370 & 31,532 & 21491225 & -.321 & .374 & .374 \\
\hline 513 & 13.14 & 1,622 & 21,306 & 17814535 & 2.161 & .985 & .015 & 21.26 & 1,002 & 21,306 & 11104865 & 2.212 & .987 & .014 \\
\hline 514 & 19.00 & 2 & 38 & 67 & 1.869 & .969 & .031 & -- & -- & -- & -- & -- & -- & -- \\
\hline 515 & 17.22 & 934 & 16,084 & 7465990 & -.319 & .375 & .375 & 30.29 & 531 & 16,084 & 4137639 & -1.240 & .108 & .108 \\
\hline 516 & 20.84 & 132 & 2,751 & 172123 & -1.035 & .150 & .150 & 39.30 & 70 & 2,751 & 92656 & -.546 & .293 & .293 \\
\hline 517 & 16.56 & 1,181 & 19,560 & 11616014 & 0.339 & .633 & .367 & 31.35 & 624 & 19,560 & 6251553 & 1.055 & .854 & .146 \\
\hline 518 & 13.47 & 2,723 & 36,688 & 50120983 & .308 & .621 & .379 & 23.06 & 1,591 & 36,688 & 28647651 & -1.273 & .102 & .102 \\
\hline 519 & 14.75 & 523 & 7,716 & 1982657 & -.689 & .246 & .246 & 23.45 & 329 & 7,716 & 1243286 & -.643 & .260 & .260 \\
\hline 520 & 12.41 & 54 & 670 & 18932 & .592 & .723 & .277 & 19.71 & 34 & 670 & 11836 & .396 & .654 & .346 \\
\hline
\end{tabular}


Table 4. Summary of interoccurrence intervals for daily precipitation thresholds of 0.25 and 0.50 inch-Continued

\begin{tabular}{|c|c|c|c|c|c|c|c|c|c|c|c|c|c|c|}
\hline \multirow[b]{2}{*}{$\begin{array}{c}\text { Seq. } \\
\text { no. }\end{array}$} & \multicolumn{7}{|c|}{ Daily precipitation threshold of 0.25 inch and greater } & \multicolumn{7}{|c|}{ Daily precipitation threshold of 0.50 inch and greater } \\
\hline & $\begin{array}{l}\text { Mean } \\
\text { inter- } \\
\text { occur- } \\
\text { rence } \\
\text { interval } \\
\text { (days) }\end{array}$ & $\begin{array}{c}\text { Total } \\
\text { no. of } \\
\text { events }\end{array}$ & $\begin{array}{l}\text { Total } \\
\text { no. of } \\
\text { days } \\
\text { avail- } \\
\text { able }\end{array}$ & $\begin{array}{c}\text { Sigma } \\
\mathrm{S}\end{array}$ & $\begin{array}{c}\text { Stan- } \\
\text { dard } \\
\text { normal } \\
\text { variate }\end{array}$ & $\begin{array}{l}\text { Non- } \\
\text { ex- } \\
\text { ceed- } \\
\text { ance } \\
\text { prob- } \\
\text { ability }\end{array}$ & $\begin{array}{c}\mathrm{p}- \\
\text { value }\end{array}$ & $\begin{array}{l}\text { Mean } \\
\text { inter- } \\
\text { occur- } \\
\text { rence } \\
\text { interval } \\
\text { (days) }\end{array}$ & $\begin{array}{c}\text { Total } \\
\text { no. of } \\
\text { events }\end{array}$ & $\begin{array}{l}\text { Total } \\
\text { no. of } \\
\text { days } \\
\text { avail- } \\
\text { able }\end{array}$ & $\begin{array}{l}\text { Sigma } \\
\mathrm{S}\end{array}$ & $\begin{array}{c}\text { Stan- } \\
\text { dard } \\
\text { normal } \\
\text { variate }\end{array}$ & $\begin{array}{l}\text { Non- } \\
\text { ex- } \\
\text { ceed- } \\
\text { ance } \\
\text { prob- } \\
\text { ability }\end{array}$ & $\begin{array}{c}\mathrm{p}- \\
\text { value }\end{array}$ \\
\hline 521 & 9.09 & 1,960 & 17,822 & 17705303 & 1.053 & .854 & .146 & 13.06 & 1,365 & 17,822 & 12283966 & .634 & .737 & .263 \\
\hline 522 & 13.86 & 534 & 7,402 & 1983048 & .136 & .554 & 446 & 20.62 & 359 & 7,402 & 1430139 & 2.507 & .994 & .006 \\
\hline 523 & 13.99 & 272 & 3,804 & 556905 & 2.184 & .986 & .014 & 22.38 & 170 & 3,804 & 353442 & 2.102 & .982 & .018 \\
\hline 524 & 25.37 & 96 & 2,435 & 107801 & -1.318 & .094 & .094 & 51.81 & 47 & 2,435 & 60003 & .577 & .718 & .282 \\
\hline 525 & -- & -- & -- & -- & -- & -- & -- & -- & -- & -- & -- & -- & -- & -- \\
\hline 526 & 16.14 & 2,018 & 32,561 & 33480753 & 1.484 & .931 & .069 & 26.93 & 1,209 & 32,561 & 20253162 & 1.744 & .959 & .041 \\
\hline 527 & 7.34 & 1,185 & 8,692 & 5193629 & .505 & .693 & .307 & 10.55 & 824 & 8,692 & 3669381 & 1.226 & .890 & .110 \\
\hline 528 & 7.26 & 435 & 3,157 & 692247 & .295 & .616 & .384 & 9.90 & 319 & 3,157 & 517444 & .854 & .803 & .197 \\
\hline 529 & 9.68 & 1,197 & 11,583 & 7258866 & 2.822 & .998 & .002 & 14.09 & 822 & 11,583 & 5038446 & 2.898 & .998 & .002 \\
\hline 530 & 8.24 & 4,140 & 34,124 & 72401913 & 2.785 & .997 & .003 & 11.82 & 2,886 & 34,124 & 50625973 & 2.617 & .996 & .004 \\
\hline 531 & 12.07 & 527 & 6,363 & 1698358 & .515 & .697 & .303 & 17.11 & 372 & 6,363 & 1237139 & 1.514 & .935 & .065 \\
\hline 532 & 11.38 & 3,168 & 36,056 & 57706945 & 1.014 & .845 & .155 & 17.83 & 2,022 & 36,056 & 37256561 & 1.718 & .957 & .043 \\
\hline 533 & 16.91 & 1,523 & 25,757 & 19523054 & -.313 & .377 & .377 & 31.30 & 823 & 25,757 & 10455840 & -.671 & .251 & .251 \\
\hline 534 & 15.41 & 64 & 986 & 29246 & -1.013 & .156 & .156 & 29.00 & 34 & 986 & 17340 & .348 & .636 & .364 \\
\hline 535 & 10.63 & 3,946 & 41,954 & 84085122 & 1.722 & .957 & .043 & 16.62 & 2,525 & 41,954 & 52551805 & -.682 & .248 & .248 \\
\hline 536 & 11.62 & 13 & 151 & 926 & -.353 & .362 & .362 & 16.78 & 9 & 151 & 481 & -1.518 & .065 & .065 \\
\hline 537 & 10.95 & 2,910 & 31,864 & 47388318 & 2.068 & .981 & .019 & 17.43 & 1,828 & 31,864 & 29614929 & 1.249 & .894 & .106 \\
\hline 538 & 19.57 & 117 & 2,290 & 133900 & -.009 & .496 & .496 & 32.25 & 71 & 2,290 & 79754 & -.277 & .391 & .391 \\
\hline 539 & 13.62 & 1,448 & 19,726 & 14455306 & .802 & .789 & .211 & 22.60 & 873 & 19,726 & 8758190 & .878 & .810 & .190 \\
\hline 540 & 11.49 & 145 & 1,666 & 131448 & 1.841 & .967 & .033 & 17.35 & 96 & 1,666 & 92989 & 2.763 & .997 & .003 \\
\hline 541 & 10.09 & 3,471 & 35,035 & 61969705 & 1.958 & .975 & .025 & 15.45 & 2,267 & 35,035 & 40166817 & .944 & .827 & .173 \\
\hline 542 & 21.53 & 55 & 1,184 & 24949 & -3.003 & .001 & .001 & 29.60 & 40 & 1,184 & 19400 & -1.980 & .024 & .024 \\
\hline 543 & 16.68 & 502 & 8,373 & 2110672 & .167 & .566 & .434 & 28.19 & 297 & 8,373 & 1261587 & .437 & .669 & .331 \\
\hline 544 & 13.04 & 2,250 & 29,330 & 33132548 & .339 & .633 & .367 & 21.13 & 1,388 & 29,330 & 20016145 & -1.074 & .141 & .141 \\
\hline 545 & 13.27 & 633 & 8,397 & 2597309 & -.989 & .161 & .161 & 22.16 & 379 & 8,397 & 1547786 & -.921 & .179 & .179 \\
\hline 546 & 8.26 & 3,802 & 31,403 & 60793115 & 1.961 & .975 & .025 & 12.32 & 2,549 & 31,403 & 41015073 & 2.167 & .985 & .015 \\
\hline 547 & 13.60 & 1,298 & 17,650 & 11483862 & .158 & .563 & .437 & 21.66 & 815 & 17,650 & 7119349 & -.502 & .308 & .308 \\
\hline 548 & 7.59 & 1,727 & 13,112 & 11416490 & .599 & .725 & .275 & 11.54 & 1,136 & 13,112 & 7583955 & 1.069 & .857 & .143 \\
\hline 549 & 29.30 & 30 & 879 & 13096 & -.064 & .474 & .474 & 73.25 & 12 & 879 & 5427 & .174 & .569 & .431 \\
\hline 550 & 10.48 & 80 & 838 & 33012 & -.235 & .407 & .407 & 16.76 & 50 & 838 & 23224 & 1.329 & .908 & .092 \\
\hline 551 & 10.10 & 543 & 5,486 & 1573600 & 2.280 & .989 & .011 & 15.95 & 344 & 5,486 & 982543 & 1.326 & .908 & .092 \\
\hline 552 & 8.73 & 2,242 & 19,567 & 22923883 & 3.699 & 1.000 & 0 & 13.49 & 1,451 & 19,567 & 15039497 & 3.921 & 1.000 & 0 \\
\hline 553 & 10.86 & 14 & 152 & 1143 & .481 & .685 & .315 & 16.89 & 9 & 152 & 763 & .600 & .726 & .274 \\
\hline 554 & 8.19 & 1,454 & 11,901 & 8717772 & .502 & .692 & .308 & 12.33 & 965 & 11,901 & 5789067 & .439 & .670 & .330 \\
\hline 555 & 7.74 & 19 & 147 & 1357 & -.214 & .415 & .415 & 14.70 & 10 & 147 & 748 & .097 & .539 & .461 \\
\hline 556 & 7.87 & 2,541 & 19,994 & 26322771 & 3.164 & .999 & .001 & 11.62 & 1,721 & 19,994 & 17943899 & 3.087 & .999 & .001 \\
\hline 557 & 7.83 & 35 & 274 & 4816 & .045 & .518 & .482 & 10.54 & 26 & 274 & 3722 & .397 & .654 & .346 \\
\hline 558 & 10.44 & 204 & 2,129 & 209291 & -.896 & .185 & .185 & 15.21 & 140 & 2,129 & 149309 & .038 & .515 & .485 \\
\hline 559 & 8.72 & 67 & 584 & 20341 & .563 & .713 & .287 & 14.60 & 40 & 584 & 11669 & -.010 & .496 & .496 \\
\hline 560 & 7.87 & 31 & 244 & 4263 & 1.227 & .890 & .110 & 12.20 & 20 & 244 & 2746 & .971 & .834 & .166 \\
\hline 561 & 7.76 & 2,293 & 17,792 & 20974668 & 2.343 & .990 & .010 & 11.74 & 1,515 & 17,792 & 13898292 & 2.105 & .982 & .018 \\
\hline 562 & 8.04 & 2,574 & 20,691 & 27172240 & 1.792 & .963 & .037 & 12.07 & 1,715 & 20,691 & 18263324 & 2.105 & .982 & .018 \\
\hline 563 & 8.12 & 2,081 & 16,893 & 18259683 & 3.068 & .999 & .001 & 12.33 & 1,370 & 16,893 & 12544699 & 5.391 & 0 & 0 \\
\hline 564 & 8.34 & 2,277 & 18,979 & 22675167 & 4.084 & 1.000 & 0 & 12.70 & 1,494 & 18,979 & 15009491 & 3.930 & 1.000 & 0 \\
\hline 565 & 7.99 & 613 & 4,897 & 1538862 & 1.084 & .861 & .139 & 11.21 & 437 & 4,897 & 1097849 & .943 & .827 & .173 \\
\hline 566 & 7.96 & 1,881 & 14,981 & 14155922 & .353 & .638 & .362 & 12.03 & 1,245 & 14,981 & 9528127 & 1.327 & .908 & .092 \\
\hline 567 & 12.31 & 714 & 8,792 & 3604488 & 6.868 & 0 & 0 & 20.12 & 437 & 8,792 & 2315732 & 7.439 & 0 & 0 \\
\hline 568 & 7.86 & 2,011 & 15,815 & 16356093 & 2.218 & .987 & .013 & 11.87 & 1,332 & 15,815 & 10883214 & 2.103 & .982 & .018 \\
\hline 569 & 8.92 & 1,034 & 9,218 & 4734852 & -0.361 & .359 & .359 & 13.74 & 671 & 9,218 & 3123490 & 0.448 & .673 & .327 \\
\hline 570 & 7.36 & 494 & 3,637 & 896195 & -.092 & .463 & .463 & 10.19 & 357 & 3,637 & 654609 & .272 & .607 & .393 \\
\hline 571 & 8.44 & 2,148 & 18,133 & 20157093 & 2.812 & .998 & .002 & 12.39 & 1,463 & 18,133 & 14068822 & 4.018 & 1.000 & 0 \\
\hline 572 & 11.28 & 1,132 & 12,769 & 7319074 & .740 & .770 & .230 & 17.59 & 726 & 12,769 & 4584988 & -.505 & .307 & .307 \\
\hline
\end{tabular}


Table 4. Summary of interoccurrence intervals for daily precipitation thresholds of 0.25 and 0.50 inch-Continued

\begin{tabular}{|c|c|c|c|c|c|c|c|c|c|c|c|c|c|c|}
\hline \multirow[b]{2}{*}{$\begin{array}{l}\text { Seq. } \\
\text { no. }\end{array}$} & \multicolumn{7}{|c|}{ Daily precipitation threshold of 0.25 inch and greater } & \multicolumn{7}{|c|}{ Daily precipitation threshold of 0.50 inch and greater } \\
\hline & $\begin{array}{l}\text { Mean } \\
\text { inter- } \\
\text { occur- } \\
\text { rence } \\
\text { interval } \\
\text { (days) }\end{array}$ & $\begin{array}{l}\text { Total } \\
\text { no. of } \\
\text { events }\end{array}$ & $\begin{array}{l}\text { Total } \\
\text { no. of } \\
\text { days } \\
\text { avail- } \\
\text { able }\end{array}$ & $\begin{array}{l}\text { Sigma } \\
\text { S }\end{array}$ & $\begin{array}{c}\text { Stan- } \\
\text { dard } \\
\text { normal } \\
\text { variate }\end{array}$ & $\begin{array}{l}\text { Non- } \\
\text { ex- } \\
\text { ceed- } \\
\text { ance } \\
\text { prob- } \\
\text { ability }\end{array}$ & $\begin{array}{c}p- \\
\text { value }\end{array}$ & $\begin{array}{l}\text { Mean } \\
\text { inter- } \\
\text { occur- } \\
\text { rence } \\
\text { interval } \\
\text { (days) }\end{array}$ & $\begin{array}{c}\text { Total } \\
\text { no. of } \\
\text { events }\end{array}$ & $\begin{array}{l}\text { Total } \\
\text { no. of } \\
\text { days } \\
\text { avail- } \\
\text { able }\end{array}$ & $\begin{array}{l}\text { Sigma } \\
\mathrm{S}\end{array}$ & $\begin{array}{l}\text { Stan- } \\
\text { dard } \\
\text { normal } \\
\text { variate }\end{array}$ & $\begin{array}{l}\text { Non- } \\
\text { ex- } \\
\text { ceed- } \\
\text { ance } \\
\text { prob- } \\
\text { ability }\end{array}$ & $\begin{array}{c}\mathrm{p}- \\
\text { value }\end{array}$ \\
\hline 573 & 18.88 & 267 & 5,041 & 680425 & .313 & .623 & .377 & 30.74 & 164 & 5,041 & 412263 & -.059 & .476 & .476 \\
\hline 574 & 7.88 & 1,417 & 11,163 & 7908486 & -.004 & .498 & .498 & 12.20 & 915 & 11,163 & 5122146 & .155 & .561 & .439 \\
\hline 575 & 15.33 & 1,142 & 17,501 & 9644311 & -2.043 & .021 & .021 & 21.88 & 800 & 17,501 & 6745228 & -1.786 & .037 & .037 \\
\hline 576 & 12.10 & 299 & 3,617 & 565895 & 1.393 & .918 & .082 & 18.94 & 191 & 3,617 & 356700 & .781 & .783 & .217 \\
\hline 577 & 12.65 & 1,468 & 18,568 & 14210735 & 2.833 & .998 & .002 & 19.59 & 948 & 18,568 & 9166233 & 2.212 & .987 & .014 \\
\hline 578 & 8.15 & 2,471 & 20,145 & 25480011 & 2.044 & .980 & .020 & 12.38 & 1,627 & 20,145 & 17089852 & 2.992 & .999 & .001 \\
\hline 579 & 10.13 & 1,391 & 14,090 & 10012296 & 1.402 & .920 & .080 & 16.56 & 851 & 14,090 & 6258105 & 2.215 & .987 & .013 \\
\hline 580 & 9.13 & 8 & 73 & 311 & .319 & .625 & .375 & 14.60 & 5 & 73 & 166 & -.350 & .363 & .363 \\
\hline 581 & 7.31 & 324 & 2,368 & 367557 & -1.305 & .096 & .096 & 10.67 & 222 & 2,368 & 254592 & -.811 & .209 & .209 \\
\hline 582 & 12.33 & 1,500 & 18,495 & 14363857 & 2.382 & .991 & .009 & 18.82 & 983 & 18,495 & 9335727 & 1.466 & .929 & .071 \\
\hline 583 & 27.76 & 583 & 16,182 & 5022639 & 2.709 & .997 & .003 & 47.59 & 340 & 16,182 & 3004255 & 2.941 & .998 & .002 \\
\hline 584 & 7.87 & 31 & 244 & 3632 & -.383 & .351 & .351 & 13.56 & 18 & 244 & 2059 & -.458 & .323 & .323 \\
\hline 585 & 12.43 & 1,056 & 13,130 & 7141614 & 1.697 & .955 & .045 & 20.78 & 632 & 13,130 & 4241719 & .972 & .834 & .166 \\
\hline 586 & 137.33 & 3 & 412 & 430 & -.913 & .181 & .181 & 412.00 & 1 & 412 & 134 & -.605 & .273 & .273 \\
\hline 587 & 12.46 & 293 & 3,651 & 526810 & -.447 & .328 & .328 & 18.07 & 202 & 3,651 & 367173 & -.105 & .458 & .458 \\
\hline 588 & 12.50 & 684 & 8,550 & 2822231 & -1.578 & .057 & .057 & 19.30 & 443 & 8,550 & 1811480 & -1.585 & .056 & .056 \\
\hline 589 & 8.94 & 733 & 6,553 & 2494131 & 1.805 & .964 & .036 & 14.12 & 464 & 6,553 & 1597483 & 1.894 & .971 & .029 \\
\hline 590 & 7.87 & 47 & 370 & 10041 & 1.838 & .967 & .033 & 9.49 & 39 & 370 & 8680 & 2.196 & .986 & .014 \\
\hline 591 & 8.80 & 415 & 3,653 & 756512 & -.069 & .472 & .472 & 12.73 & 287 & 3,653 & 530267 & .339 & .633 & .367 \\
\hline 592 & 11.24 & 1,965 & 22,076 & 21991164 & 1.067 & .857 & .143 & 17.93 & 1,231 & 22,076 & 13896575 & 1.381 & .916 & .084 \\
\hline 593 & 8.46 & 1,834 & 15,514 & 14865018 & 3.330 & 1.000 & 0 & 12.22 & 1,270 & 15,514 & 10293157 & 2.768 & .997 & .003 \\
\hline 594 & 8.40 & 699 & 5,874 & 2099416 & 1.036 & .850 & .150 & 12.58 & 467 & 5,874 & 1397359 & .704 & .759 & .241 \\
\hline 595 & 13.35 & 691 & 9,226 & 3278753 & 1.302 & .904 & .096 & 20.83 & 443 & 9,226 & 2121698 & 1.394 & .918 & .082 \\
\hline 596 & 10.36 & 2,481 & 25,710 & 32642538 & 2.027 & .979 & .021 & 15.73 & 1,634 & 25,710 & 21341320 & 1.121 & .869 & .131 \\
\hline 597 & 7.37 & 2,790 & 20,570 & 29279299 & 1.862 & .969 & .031 & 10.62 & 1,937 & 20,570 & 20205043 & 1.083 & .861 & .139 \\
\hline 598 & 7.27 & 144 & 1,047 & 63500 & -3.277 & .001 & .001 & 9.79 & 107 & 1,047 & 47902 & -2.595 & .005 & .005 \\
\hline 599 & 13.96 & 1,268 & 17,699 & 11653646 & 2.377 & .991 & .009 & 23.23 & 762 & 17,699 & 6850632 & .761 & .777 & .223 \\
\hline 600 & 10.69 & 1,828 & 19,532 & 18694523 & 3.494 & 1.000 & 0 & 16.13 & 1,211 & 19,532 & 12258748 & 2.202 & .986 & .014 \\
\hline 601 & 7.69 & 4,201 & 32,319 & 68413511 & .872 & .808 & .192 & 11.74 & 2,754 & 32,319 & 44708109 & .418 & .662 & .338 \\
\hline 602 & 9.51 & 1,617 & 15,379 & 12760182 & 1.828 & .966 & .034 & 14.44 & 1,065 & 15,379 & 8198492 & .063 & .525 & .475 \\
\hline 603 & 8.78 & 709 & 6,226 & 2237301 & .631 & .736 & .264 & 13.00 & 479 & 6,226 & 1545404 & 1.380 & .916 & .084 \\
\hline 604 & 10.59 & 1,159 & 12,277 & 7233532 & .986 & .838 & .162 & 16.39 & 749 & 12,277 & 4719067 & 1.251 & .894 & .106 \\
\hline 605 & 14.38 & 432 & 6,210 & 1390111 & 1.308 & .905 & .095 & 26.09 & 238 & 6,210 & 772566 & 1.214 & .888 & .112 \\
\hline 606 & 16.10 & 327 & 5,266 & 864802 & .139 & .555 & .445 & 28.01 & 188 & 5,266 & 497825 & .135 & .554 & .446 \\
\hline 607 & 15.96 & 244 & 3,895 & 485185 & .569 & .715 & .285 & 26.50 & 147 & 3,895 & 284847 & -.105 & .458 & .458 \\
\hline 608 & 14.28 & 1,482 & 21,162 & 16229354 & 2.332 & .990 & .010 & 22.71 & 932 & 21,162 & 10022899 & .866 & .807 & .193 \\
\hline 609 & 30.16 & 96 & 2,895 & 122987 & -1.951 & .026 & .026 & 53.61 & 54 & 2,895 & 65426 & -2.074 & .019 & .019 \\
\hline 610 & 13.88 & 2,196 & 30,487 & 32201726 & -3.087 & .001 & .001 & 22.09 & 1,380 & 30,487 & 20048750 & -3.020 & .001 & .001 \\
\hline 611 & 15.60 & 527 & 8,219 & 2237260 & 1.314 & .905 & .095 & 26.09 & 315 & 8,219 & 1347095 & 1.249 & .894 & .106 \\
\hline 612 & 16.56 & 218 & 3,610 & 400975 & .487 & .687 & .313 & 25.97 & 139 & 3,610 & 266504 & 1.270 & .898 & .102 \\
\hline 613 & 15.66 & 44 & 689 & 14407 & -.569 & .285 & .285 & 18.62 & 37 & 689 & 11492 & -1.037 & .150 & .150 \\
\hline 614 & 18.13 & 185 & 3,354 & 295403 & -1.127 & .130 & .130 & 27.27 & 123 & 3,354 & 181777 & -2.281 & .011 & .011 \\
\hline 615 & 15.25 & 4 & 61 & 134 & .341 & .633 & .367 & 30.50 & 2 & 61 & 64 & .121 & .548 & .452 \\
\hline 616 & 15.02 & 125 & 1,878 & 115647 & -.285 & .388 & .388 & 21.10 & 89 & 1,878 & 86879 & .647 & .741 & .259 \\
\hline 617 & 8.01 & 219 & 1,753 & 167927 & -3.208 & .001 & .001 & 11.17 & 157 & 1,753 & 119512 & -2.854 & .002 & .002 \\
\hline 618 & 7.78 & 2,655 & 20,642 & 28108673 & 2.301 & .989 & .011 & 11.44 & 1,804 & 20,642 & 19410677 & 3.128 & .999 & .001 \\
\hline 619 & 12.04 & 2,363 & 28,443 & 32780271 & -2.067 & .019 & .019 & 19.10 & 1,489 & 28,443 & 20715344 & -1.453 & .073 & .073 \\
\hline 620 & 13.67 & 51 & 697 & 19798 & 1.409 & .921 & .079 & 19.36 & 36 & 697 & 14817 & 1.881 & .970 & .030 \\
\hline 621 & 9.21 & 1,791 & 16,498 & 15445910 & 3.334 & 1.000 & 0 & 14.65 & 1,126 & 16,498 & 9832430 & 3.404 & 1.000 & 0 \\
\hline 622 & 9.25 & 3,914 & 36,185 & 69576118 & -1.894 & .029 & .029 & 13.73 & 2,635 & 36,185 & 47297473 & -.702 & .242 & .242 \\
\hline 623 & 11.28 & 653 & 7,364 & 2442168 & .696 & .757 & .243 & 18.09 & 407 & 7,364 & 1520463 & .510 & .695 & .305 \\
\hline 624 & 9.97 & 195 & 1,944 & 177131 & -1.584 & .057 & .057 & 14.62 & 133 & 1,944 & 119828 & -1.460 & .072 & .072 \\
\hline
\end{tabular}


Table 4. Summary of interoccurrence intervals for daily precipitation thresholds of 0.25 and 0.50 inch-Continued

\begin{tabular}{|c|c|c|c|c|c|c|c|c|c|c|c|c|c|c|}
\hline \multirow[b]{2}{*}{$\begin{array}{c}\text { Seq. } \\
\text { no. }\end{array}$} & \multicolumn{7}{|c|}{ Daily precipitation threshold of 0.25 inch and greater } & \multicolumn{7}{|c|}{ Daily precipitation threshold of 0.50 inch and greater } \\
\hline & $\begin{array}{l}\text { Mean } \\
\text { inter- } \\
\text { occur- } \\
\text { rence } \\
\text { interval } \\
\text { (days) }\end{array}$ & $\begin{array}{c}\text { Total } \\
\text { no. of } \\
\text { events }\end{array}$ & $\begin{array}{l}\text { Total } \\
\text { no. of } \\
\text { days } \\
\text { avail- } \\
\text { able }\end{array}$ & $\begin{array}{c}\text { Sigma } \\
\mathrm{S}\end{array}$ & $\begin{array}{c}\text { Stan- } \\
\text { dard } \\
\text { normal } \\
\text { variate }\end{array}$ & $\begin{array}{l}\text { Non- } \\
\text { ex- } \\
\text { ceed- } \\
\text { ance } \\
\text { prob- } \\
\text { ability }\end{array}$ & $\begin{array}{c}\mathrm{p}- \\
\text { value }\end{array}$ & $\begin{array}{l}\text { Mean } \\
\text { inter- } \\
\text { occur- } \\
\text { rence } \\
\text { interval } \\
\text { (days) }\end{array}$ & $\begin{array}{c}\text { Total } \\
\text { no. of } \\
\text { events }\end{array}$ & $\begin{array}{l}\text { Total } \\
\text { no. of } \\
\text { days } \\
\text { avail- } \\
\text { able }\end{array}$ & $\begin{array}{l}\text { Sigma } \\
\mathrm{S}\end{array}$ & $\begin{array}{c}\text { Stan- } \\
\text { dard } \\
\text { normal } \\
\text { variate }\end{array}$ & $\begin{array}{l}\text { Non- } \\
\text { ex- } \\
\text { ceed- } \\
\text { ance } \\
\text { prob- } \\
\text { ability }\end{array}$ & $\begin{array}{c}\mathrm{p}- \\
\text { value }\end{array}$ \\
\hline 625 & 12.27 & 812 & 9,960 & 4320862 & 3.382 & 1.000 & 0 & 19.04 & 523 & 9,960 & 2798800 & 2.954 & .998 & .002 \\
\hline 626 & 10.90 & 993 & 10,827 & 5371124 & -.046 & .482 & .482 & 16.14 & 671 & 10,827 & 3610622 & -.270 & .394 & .394 \\
\hline 627 & 22.78 & 519 & 11,821 & 3283419 & 2.777 & .997 & .003 & 48.25 & 245 & 11,821 & 1571694 & 2.314 & .990 & .010 \\
\hline 628 & 19.32 & 259 & 5,003 & 577015 & -3.049 & .001 & .001 & 40.35 & 124 & 5,003 & 244218 & -4.102 & 0 & 0 \\
\hline 629 & 12.24 & 2,162 & 26,452 & 28651776 & .161 & .564 & .436 & 20.36 & 1,299 & 26,452 & 17046237 & -.488 & .313 & .313 \\
\hline 630 & 11.58 & 857 & 9,923 & 4188069 & -.762 & .223 & .223 & 19.05 & 521 & 9,923 & 2607778 & .349 & .637 & .363 \\
\hline 631 & 11.57 & 942 & 10,894 & 5333907 & 2.101 & .982 & .018 & 18.13 & 601 & 10,894 & 3292484 & .244 & .597 & .403 \\
\hline 632 & 10.23 & 778 & 7,960 & 3160297 & .996 & .840 & .160 & 15.98 & 498 & 7,960 & 2013579 & .615 & .731 & .269 \\
\hline 633 & 9.62 & 177 & 1,702 & 145379 & -.803 & .211 & .211 & 14.42 & 118 & 1,702 & 100420 & 0 & .500 & .500 \\
\hline 634 & 13.81 & 21 & 290 & 2960 & -.222 & .412 & .412 & 24.17 & 12 & 290 & 1737 & -.010 & .496 & .496 \\
\hline 635 & 14.20 & 1,891 & 26,842 & 26037914 & 1.955 & .975 & .025 & 21.75 & 1,234 & 26,842 & 16626291 & .238 & .594 & .406 \\
\hline 636 & 7.11 & 2,919 & 20,742 & 30075203 & -.611 & .271 & .271 & 10.78 & 1,924 & 20,742 & 20288204 & 1.273 & .898 & .102 \\
\hline 637 & 17.35 & 1,407 & 24,410 & 17999159 & 3.128 & .999 & .001 & 27.93 & 874 & 24,410 & 11138424 & 2.262 & .988 & .012 \\
\hline 638 & 14.18 & 784 & 11,115 & 4441487 & .940 & .826 & .174 & 23.25 & 478 & 11,115 & 2783608 & 1.812 & .965 & .035 \\
\hline 639 & 10.55 & 495 & 5,224 & 1335987 & 1.283 & .900 & .100 & 16.22 & 322 & 5,224 & 879698 & 1.428 & .923 & .077 \\
\hline 640 & 7.11 & 825 & 5,865 & 2510236 & 1.870 & .969 & .031 & 10.64 & 551 & 5,865 & 1699225 & 2.099 & .982 & .018 \\
\hline 641 & 15.95 & 75 & 1196 & 42419 & -.813 & .208 & .208 & 27.18 & 44 & 1,196 & 25589 & -.316 & .376 & .376 \\
\hline 642 & 12.70 & 23 & 292 & 3488 & .322 & .626 & .374 & 24.33 & 12 & 292 & 2056 & 1.041 & .851 & .149 \\
\hline 643 & 9.72 & 3,381 & 32,853 & 57107517 & 2.846 & .998 & .002 & 15.37 & 2,138 & 32,853 & 36469162 & 3.077 & .999 & .001 \\
\hline 644 & 20.27 & 122 & 2,473 & 157866 & .889 & .813 & .187 & 32.54 & 76 & 2,473 & 102185 & 1.319 & .906 & .094 \\
\hline 645 & 17.03 & 1,611 & 27,442 & 22140552 & .113 & .545 & .455 & 28.26 & 971 & 27,442 & 13636277 & 1.269 & .898 & .102 \\
\hline 646 & 34.18 & 606 & 20,715 & 6381510 & .712 & .762 & .238 & 71.43 & 290 & 20,715 & 3153559 & 1.472 & .929 & .071 \\
\hline 647 & 11.93 & 76 & 907 & 38421 & 1.733 & .958 & .042 & 19.30 & 47 & 907 & 23996 & 1.494 & .932 & .068 \\
\hline 648 & 15.18 & 126 & 1,913 & 106277 & -2.298 & .011 & .011 & 22.24 & 86 & 1,913 & 79537 & -.532 & .298 & .298 \\
\hline 649 & 25.77 & 317 & 8,170 & 1204469 & -2.155 & .016 & .016 & 51.38 & 159 & 8,170 & 592696 & -1.911 & .028 & .028 \\
\hline 650 & 12.99 & 1,108 & 14,391 & 7901356 & -.515 & .303 & .303 & 21.13 & 681 & 14,391 & 4870792 & -.271 & .393 & .393 \\
\hline 651 & 18.08 & 155 & 2,802 & 221681 & .449 & .673 & .327 & 36.39 & 77 & 2,802 & 106419 & -.205 & .419 & .419 \\
\hline 652 & 15.96 & 50 & 798 & 20353 & .247 & .598 & .402 & 21.00 & 38 & 798 & 15800 & .449 & .673 & .327 \\
\hline 653 & 10.72 & 101 & 1,083 & 56501 & .576 & .718 & .282 & 14.84 & 73 & 1,083 & 42423 & 1.083 & .861 & .139 \\
\hline 654 & 16.67 & 621 & 10,351 & 3335884 & 1.637 & .949 & .051 & 27.31 & 379 & 10,351 & 2066760 & 1.809 & .965 & .035 \\
\hline 655 & 12.12 & 236 & 2,861 & 328043 & -.753 & .226 & .226 & 18.58 & 154 & 2,861 & 207730 & -1.226 & .110 & .110 \\
\hline 656 & 8.37 & 567 & 4,743 & 1337837 & -.209 & .417 & .417 & 12.72 & 373 & 4,743 & 881982 & -.098 & .461 & .461 \\
\hline 657 & 13.21 & 878 & 11,595 & 5142292 & .525 & .700 & .300 & 22.56 & 514 & 11,595 & 3053714 & .973 & .835 & .165 \\
\hline 658 & 13.84 & 414 & 5,729 & 1240742 & 1.630 & .948 & .052 & 21.70 & 264 & 5,729 & 765671 & .351 & .637 & .363 \\
\hline 659 & 11.33 & 245 & 2,776 & 325068 & -1.195 & .116 & .116 & 17.91 & 155 & 2,776 & 205365 & -.980 & .164 & .164 \\
\hline 660 & 8.64 & 556 & 4,805 & 1373717 & 1.160 & .877 & .123 & 12.92 & 372 & 4,805 & 932412 & 1.446 & .926 & .074 \\
\hline 661 & 8.48 & 334 & 2,832 & 488256 & 1.025 & .847 & .153 & 12.37 & 229 & 2,832 & 328209 & .319 & .625 & .375 \\
\hline 662 & 12.86 & 427 & 5,490 & 1193062 & .640 & .739 & .261 & 19.96 & 275 & 5,490 & 802324 & 1.805 & .964 & .036 \\
\hline 663 & 17.89 & 1,824 & 32,622 & 29713720 & -.093 & .463 & .463 & 31.25 & 1,044 & 32,622 & 17283379 & .837 & .799 & .201 \\
\hline 664 & 11.59 & 3,199 & 37,084 & 60706657 & 2.297 & .989 & .011 & 18.49 & 2,006 & 37,084 & 37691502 & 1.035 & .850 & .150 \\
\hline 665 & 23.24 & 810 & 18,826 & 7357442 & -1.727 & .042 & .042 & 38.19 & 493 & 18,826 & 4590034 & -.419 & .338 & .338 \\
\hline 666 & 25.98 & 50 & 1,299 & 34859 & .899 & .816 & .184 & 44.79 & 29 & 1,299 & 21270 & 1.206 & .886 & .114 \\
\hline 667 & 18.04 & 759 & 13,689 & 5024482 & -1.566 & .059 & .059 & 27.99 & 489 & 13,689 & 3147495 & -2.283 & .011 & .011 \\
\hline 668 & 23.81 & 326 & 7,761 & 1279152 & .349 & .636 & .364 & 40.42 & 192 & 7,761 & 764813 & .636 & .738 & .262 \\
\hline 669 & 7.93 & 716 & 5,676 & 2054598 & .515 & .697 & .303 & 11.68 & 486 & 5,676 & 1414802 & .984 & .837 & .163 \\
\hline 670 & 13.51 & 628 & 8,485 & 2644110 & -.329 & .371 & .371 & 22.39 & 379 & 8,485 & 1555000 & -1.110 & .134 & .134 \\
\hline 671 & 9.40 & 1,999 & 18,797 & 19574729 & 3.244 & .999 & .001 & 14.37 & 1,308 & 18,797 & 12823168 & 2.700 & .997 & .003 \\
\hline 672 & 13.75 & 1,407 & 19,344 & 13795246 & .892 & .814 & .186 & 21.74 & 890 & 19,344 & 8808878 & 1.205 & .886 & .114 \\
\hline 673 & 14.77 & 371 & 5,481 & 1051344 & 1.136 & .872 & .128 & 23.42 & 234 & 5,481 & 672696 & 1.298 & .903 & .097 \\
\hline 674 & 13.24 & 135 & 1,788 & 120869 & .030 & .512 & .488 & 16.71 & 107 & 1,788 & 91380 & -.801 & .212 & .212 \\
\hline 675 & 13.66 & 194 & 2,649 & 257025 & .007 & .503 & .497 & 22.64 & 117 & 2,649 & 152050 & -.353 & .362 & .362 \\
\hline 676 & 11.87 & 280 & 3,324 & 404840 & -3.769 & 0 & 0 & 17.68 & 188 & 3,324 & 274136 & -2.913 & .002 & .002 \\
\hline
\end{tabular}


Table 4. Summary of interoccurrence intervals for daily precipitation thresholds of 0.25 and 0.50 inch-Continued

\begin{tabular}{|c|c|c|c|c|c|c|c|c|c|c|c|c|c|c|}
\hline \multirow[b]{2}{*}{$\begin{array}{l}\text { Seq. } \\
\text { no. }\end{array}$} & \multicolumn{7}{|c|}{ Daily precipitation threshold of 0.25 inch and greater } & \multicolumn{7}{|c|}{ Daily precipitation threshold of 0.50 inch and greater } \\
\hline & $\begin{array}{l}\text { Mean } \\
\text { inter- } \\
\text { occur- } \\
\text { rence } \\
\text { interval } \\
\text { (days) }\end{array}$ & $\begin{array}{c}\text { Total } \\
\text { no. of } \\
\text { events }\end{array}$ & $\begin{array}{c}\text { Total } \\
\text { no. of } \\
\text { days } \\
\text { avail- } \\
\text { able }\end{array}$ & $\underset{\mathrm{S}}{\text { Sigma }}$ & $\begin{array}{c}\text { Stan- } \\
\text { dard } \\
\text { normal } \\
\text { variate }\end{array}$ & $\begin{array}{l}\text { Non- } \\
\text { ex- } \\
\text { ceed- } \\
\text { ance } \\
\text { prob- } \\
\text { ability }\end{array}$ & $\begin{array}{c}p- \\
\text { value }\end{array}$ & $\begin{array}{l}\text { Mean } \\
\text { inter- } \\
\text { occur- } \\
\text { rence } \\
\text { interval } \\
\text { (days) }\end{array}$ & $\begin{array}{c}\text { Total } \\
\text { no. of } \\
\text { events }\end{array}$ & $\begin{array}{c}\text { Total } \\
\text { no. of } \\
\text { days } \\
\text { avail- } \\
\text { able }\end{array}$ & $\underset{\mathrm{S}}{\text { Sigma }}$ & $\begin{array}{c}\text { Stan- } \\
\text { dard } \\
\text { normal } \\
\text { variate }\end{array}$ & $\begin{array}{c}\text { Non- } \\
\text { ex- } \\
\text { ceed- } \\
\text { ance } \\
\text { prob- } \\
\text { ability }\end{array}$ & $\begin{array}{c}p- \\
\text { value }\end{array}$ \\
\hline 677 & 17.68 & 1,162 & 20,545 & 11890990 & -.226 & .411 & .411 & 30.94 & 664 & 20,545 & 6746562 & -.487 & .313 & .313 \\
\hline 678 & 9.24 & 17 & 157 & 1566 & 1.239 & .892 & .108 & 17.44 & 9 & 157 & 668 & -.283 & .388 & .388 \\
\hline 679 & 16.66 & 1,358 & 22,630 & 15509607 & .598 & .725 & .275 & 30.50 & 742 & 22,630 & 8413495 & .100 & .540 & .460 \\
\hline 680 & 11.75 & 371 & 4,360 & 821875 & .540 & .705 & .295 & 18.24 & 239 & 4,360 & 534728 & .705 & .759 & .241 \\
\hline 681 & 10.22 & 82 & 838 & 34578 & .100 & .540 & .460 & 12.32 & 68 & 838 & 27244 & -.626 & .266 & .266 \\
\hline 682 & 11.57 & 440 & 5,092 & 1114366 & -.191 & .424 & .424 & 18.58 & 274 & 5,092 & 702254 & .191 & .576 & .424 \\
\hline 683 & 9.71 & 1,257 & 12,199 & 7731544 & .516 & .697 & .303 & 14.79 & 825 & 12,199 & 5177722 & 1.440 & .925 & .075 \\
\hline 684 & 10.21 & 1,520 & 15,522 & 12223894 & 2.445 & .993 & .007 & 16.07 & 966 & 15,522 & 7774115 & 1.989 & .977 & .023 \\
\hline 685 & 7.42 & 4,746 & 35,218 & 85682145 & 3.012 & .999 & .001 & 11.27 & 3,126 & 35,218 & 56907194 & 3.275 & .999 & .001 \\
\hline 686 & 11.67 & 501 & 5,847 & 1542610 & 2.063 & .980 & .020 & 17.61 & 332 & 5,847 & 1031623 & 1.984 & .976 & .024 \\
\hline 687 & 14.20 & 109 & 1,548 & 82549 & -.390 & .348 & .348 & 18.88 & 82 & 1,548 & 61498 & -.487 & .313 & .313 \\
\hline 688 & 10.06 & 446 & 4,487 & 1037105 & 1.335 & .909 & .091 & 13.64 & 329 & 4,487 & 740111 & .085 & .534 & .466 \\
\hline 689 & 9.44 & 625 & 5,899 & 1857185 & .323 & .627 & .373 & 14.35 & 411 & 5,899 & 1266709 & 1.578 & .943 & .057 \\
\hline 690 & 8.37 & 1,496 & 12,527 & 9118300 & -1.801 & .036 & .036 & 12.67 & 989 & 12,527 & 6056181 & -1.217 & .112 & .112 \\
\hline 691 & 7.61 & 2,408 & 18,319 & 22469042 & 1.591 & .944 & .056 & 11.02 & 1,663 & 18,319 & 15926971 & 3.222 & .999 & .001 \\
\hline 692 & 11.97 & 1,570 & 18,792 & 14378022 & -1.739 & .041 & .041 & 17.85 & 1,053 & 18,792 & 10097126 & 1.154 & .876 & .124 \\
\hline 693 & 14.41 & 1,330 & 19,163 & 13263315 & 2.577 & .995 & .005 & 26.04 & 736 & 19,163 & 7339138 & 1.913 & .972 & .028 \\
\hline 694 & 10.36 & 705 & 7,301 & 2610731 & .664 & .746 & .254 & 15.67 & 466 & 7,301 & 1765630 & 1.418 & .922 & .078 \\
\hline 695 & 18.04 & 750 & 13,531 & 5028803 & -.424 & .336 & .336 & 32.61 & 415 & 13,531 & 2723525 & -1.058 & .145 & .145 \\
\hline 696 & 16.50 & 777 & 12,822 & 4980127 & -.012 & .495 & .495 & 30.53 & 420 & 12,822 & 2709040 & .217 & .586 & .414 \\
\hline 697 & 7.71 & 3,060 & 23,585 & 36567957 & 1.282 & .900 & .100 & 11.59 & 2,035 & 23,585 & 24813108 & 2.655 & .996 & .004 \\
\hline 698 & 12.90 & 2,908 & 37,526 & 56767236 & 3.774 & 1.000 & 0 & 21.04 & 1,784 & 37,526 & 34744242 & 2.778 & .997 & .003 \\
\hline 699 & 11.47 & 138 & 1,583 & 116511 & 1.357 & .913 & .087 & 18.20 & 87 & 1,583 & 73277 & 1.036 & .850 & .150 \\
\hline 700 & 11.11 & 1,770 & 19,662 & 18163120 & 3.192 & .999 & .001 & 17.13 & 1,148 & 19,662 & 12101346 & 4.240 & 1.000 & 0 \\
\hline 701 & 10.36 & 173 & 1,793 & 148825 & -.921 & .179 & .179 & 14.70 & 122 & 1,793 & 110188 & .143 & .557 & .443 \\
\hline 702 & 17.07 & 71 & 1,212 & 35911 & -2.413 & .008 & .008 & 28.86 & 42 & 1,212 & 20119 & -2.352 & .009 & .009 \\
\hline 703 & 12.11 & 390 & 4,722 & 933701 & .480 & .684 & .316 & 17.75 & 266 & 4,722 & 647835 & .891 & .813 & .187 \\
\hline 704 & 16.96 & 25 & 424 & 4862 & -.716 & .237 & .237 & 28.27 & 15 & 424 & 2692 & -1.029 & .152 & .152 \\
\hline 705 & 8.76 & 1,466 & 12,843 & 9773286 & 2.532 & .994 & .006 & 13.49 & 952 & 12,843 & 6283918 & 1.492 & .932 & .068 \\
\hline 706 & 7.92 & 4,533 & 35,879 & 82786121 & 2.103 & .982 & .018 & 11.63 & 3,085 & 35,879 & 56634010 & 2.244 & .988 & .012 \\
\hline 707 & 7.74 & 1,202 & 9,308 & 5710706 & 1.252 & .895 & .105 & 11.19 & 832 & 9,308 & 4051330 & 2.312 & .990 & .010 \\
\hline 708 & 19.38 & 834 & 16,163 & 7022583 & 2.097 & .982 & .018 & 34.03 & 475 & 16,163 & 3950141 & 1.096 & .863 & .137 \\
\hline 709 & 14.75 & 4 & 59 & 175 & 1.673 & .953 & .047 & -- & -- & -- & -- & -- & -- & -- \\
\hline 710 & 16.64 & 72 & 1,198 & 39297 & -1.306 & .096 & .096 & 29.95 & 40 & 1,198 & 23196 & -.349 & .363 & .363 \\
\hline 711 & 16.49 & 899 & 14,820 & 6860903 & 1.554 & .940 & .060 & 26.37 & 562 & 14,820 & 4391562 & 2.240 & .987 & .013 \\
\hline 712 & 19.16 & 70 & 1,341 & 47606 & .207 & .582 & .418 & 30.48 & 44 & 1,341 & 27044 & -.957 & .169 & .169 \\
\hline 713 & 8.48 & 1,197 & 10,153 & 6306833 & 2.271 & .988 & .012 & 12.55 & 809 & 10,153 & 4277612 & 2.048 & .980 & .020 \\
\hline 714 & 26.07 & 14 & 365 & 1609 & -2.400 & .008 & .008 & 45.63 & 8 & 365 & 690 & -2.584 & .005 & .005 \\
\hline 715 & 17.38 & 84 & 1,460 & 63055 & .449 & .673 & .327 & 29.20 & 50 & 1,460 & 34446 & -.689 & .245 & .245 \\
\hline 716 & 18.80 & 336 & 6,316 & 1098780 & 1.128 & .870 & .130 & 35.29 & 179 & 6,316 & 557320 & -.326 & .372 & .372 \\
\hline 717 & 16.92 & 1,959 & 33,144 & 32161943 & -.715 & .238 & .238 & 31.66 & 1,047 & 33,144 & 17136494 & -.693 & .244 & .244 \\
\hline 718 & 7.62 & 904 & 6,885 & 3089589 & -.375 & .354 & .354 & 10.54 & 653 & 6,885 & 2235437 & -.246 & .403 & .403 \\
\hline 719 & 7.51 & 581 & 4,364 & 1223720 & -1.450 & .074 & .074 & 10.42 & 419 & 4,364 & 871348 & -1.664 & .048 & .048 \\
\hline 720 & 8.10 & 4,105 & 33,230 & 69121389 & 1.492 & .932 & .068 & 12.45 & 2,669 & 33,230 & 43994009 & -.709 & .239 & .239 \\
\hline 721 & 10.74 & 3,395 & 36,455 & 62710938 & 1.351 & .912 & .088 & 17.09 & 2,133 & 36,455 & 39951396 & 2.206 & .986 & .014 \\
\hline 722 & 10.35 & 156 & 1,614 & 139425 & 2.326 & .990 & .010 & 14.67 & 110 & 1,614 & 94089 & 1.089 & .862 & .138 \\
\hline 723 & 6.76 & 333 & 2,252 & 377866 & .245 & .597 & .403 & 9.97 & 226 & 2,252 & 256790 & .237 & .594 & .406 \\
\hline 724 & 12.82 & 714 & 9,151 & 3371494 & 1.482 & .931 & .069 & 20.61 & 444 & 9,151 & 2103332 & 1.290 & .901 & .099 \\
\hline 725 & 13.82 & 663 & 9,162 & 3072791 & 0.523 & .699 & .301 & 21.76 & 421 & 9,162 & 2027135 & 1.816 & .965 & .035 \\
\hline 726 & 10.31 & 113 & 1,165 & 64994 & -.232 & .408 & .408 & 18.20 & 64 & 1,165 & 37508 & .085 & .534 & .466 \\
\hline 727 & 8.58 & 2,945 & 25,281 & 37543483 & .801 & .788 & .212 & 13.15 & 1,923 & 25,281 & 24406359 & .308 & .621 & .379 \\
\hline 728 & 8.30 & 1,242 & 10,305 & 6716997 & 3.029 & .999 & .001 & 12.37 & 833 & 10,305 & 4568859 & 3.224 & .999 & .001 \\
\hline
\end{tabular}


Table 4. Summary of interoccurrence intervals for daily precipitation thresholds of 0.25 and 0.50 inch-Continued

\begin{tabular}{|c|c|c|c|c|c|c|c|c|c|c|c|c|c|c|}
\hline \multirow[b]{2}{*}{$\begin{array}{l}\text { Seq. } \\
\text { no. }\end{array}$} & \multicolumn{7}{|c|}{ Daily precipitation threshold of 0.25 inch and greater } & \multicolumn{7}{|c|}{ Daily precipitation threshold of 0.50 inch and greater } \\
\hline & $\begin{array}{l}\text { Mean } \\
\text { inter- } \\
\text { occur- } \\
\text { rence } \\
\text { interval } \\
\text { (days) }\end{array}$ & $\begin{array}{c}\text { Total } \\
\text { no. of } \\
\text { events }\end{array}$ & $\begin{array}{c}\text { Total } \\
\text { no. of } \\
\text { days } \\
\text { avail- } \\
\text { able }\end{array}$ & $\underset{\mathrm{S}}{\text { Sigma }}$ & $\begin{array}{c}\text { Stan- } \\
\text { dard } \\
\text { normal } \\
\text { variate }\end{array}$ & $\begin{array}{c}\text { Non- } \\
\text { ex- } \\
\text { ceed- } \\
\text { ance } \\
\text { prob- } \\
\text { ability }\end{array}$ & $\begin{array}{c}p- \\
\text { value }\end{array}$ & $\begin{array}{c}\text { Mean } \\
\text { inter- } \\
\text { occur- } \\
\text { rence } \\
\text { interval } \\
\text { (days) }\end{array}$ & $\begin{array}{c}\text { Total } \\
\text { no. of } \\
\text { events }\end{array}$ & $\begin{array}{c}\text { Total } \\
\text { no. of } \\
\text { days } \\
\text { avail- } \\
\text { able }\end{array}$ & $\underset{\mathrm{S}}{\operatorname{Sigma}}$ & $\begin{array}{c}\text { Stan- } \\
\text { dard } \\
\text { normal } \\
\text { variate }\end{array}$ & $\begin{array}{c}\text { Non- } \\
\text { ex- } \\
\text { ceed- } \\
\text { ance } \\
\text { prob- } \\
\text { ability }\end{array}$ & $\begin{array}{c}\text { p- } \\
\text { value }\end{array}$ \\
\hline 729 & 11.88 & 543 & 6,453 & 1780479 & .656 & .744 & .256 & 18.70 & 345 & 6,453 & 1168833 & 1.610 & .946 & .054 \\
\hline 730 & 13.00 & 1 & 13 & 6 & -.133 & .447 & .447 & 13.00 & 1 & 13 & 6 & -.133 & .447 & .447 \\
\hline 731 & 11.35 & 551 & 6,252 & 1736723 & .338 & .632 & .368 & 15.40 & 406 & 6,252 & 1243900 & -.695 & .244 & .244 \\
\hline 732 & 12.82 & 532 & 6,819 & 1731496 & -1.814 & .035 & .035 & 21.51 & 317 & 6,819 & 1045734 & -1.001 & .159 & .159 \\
\hline 733 & 26.46 & 13 & 344 & 2529 & .818 & .793 & .207 & 49.14 & 7 & 344 & 1576 & 1.416 & .922 & .078 \\
\hline 734 & 20.55 & 1,122 & 23,057 & 12166769 & -3.446 & 0 & 0 & 37.13 & 621 & 23,057 & 6699801 & -2.770 & .003 & .003 \\
\hline 735 & 15.11 & 131 & 1,979 & 115843 & -2.108 & .018 & .018 & 29.10 & 68 & 1,979 & 56863 & -2.213 & .013 & .013 \\
\hline 736 & 13.06 & 620 & 8,096 & 2625192 & 1.984 & .976 & .024 & 20.44 & 396 & 8,096 & 1701881 & 2.126 & .983 & .017 \\
\hline 737 & 25.45 & 104 & 2,647 & 147077 & 1.211 & .887 & .113 & 54.02 & 49 & 2,647 & 71429 & 1.230 & .891 & .109 \\
\hline 738 & 19.34 & 741 & 14,333 & 5390606 & .712 & .762 & .238 & 39.93 & 359 & 14,333 & 2699143 & 1.612 & .947 & .054 \\
\hline 739 & 22.46 & 222 & 4,987 & 524243 & -1.367 & .086 & .086 & 44.53 & 112 & 4,987 & 238069 & -2.704 & .003 & .003 \\
\hline 740 & 17.29 & 56 & 968 & 28830 & .825 & .795 & .205 & 33.38 & 29 & 968 & 12768 & -.843 & .200 & .200 \\
\hline 741 & 15.78 & 9 & 142 & 542 & -.789 & .215 & .215 & 20.29 & 7 & 142 & 508 & .101 & .540 & .460 \\
\hline 742 & 9.75 & 2,714 & 26,466 & 36179510 & .666 & .747 & .253 & 15.32 & 1,728 & 26,466 & 23481431 & 1.936 & .974 & .026 \\
\hline 743 & 7.78 & 4,294 & 33,395 & 72672082 & 1.540 & .938 & .062 & 11.39 & 2,933 & 33,395 & 49936576 & 1.844 & .967 & .033 \\
\hline 744 & 14.07 & 450 & 6,333 & 1450179 & .651 & .742 & .258 & 24.74 & 256 & 6,333 & 827032 & .561 & .713 & .287 \\
\hline 745 & 11.64 & 769 & 8,954 & 3531808 & 1.242 & .893 & .107 & 17.66 & 507 & 8,954 & 2334324 & 1.108 & .866 & .134 \\
\hline 746 & 12.93 & 1,590 & 20,552 & 17079797 & 3.132 & .999 & .001 & 20.68 & 994 & 20,552 & 10909280 & 3.715 & 1.000 & 0 \\
\hline 747 & 14.97 & 1,307 & 19,569 & 13256751 & 2.294 & .989 & .011 & 26.59 & 736 & 19,569 & 7517058 & 2.060 & .980 & .020 \\
\hline 748 & 10.38 & 3,173 & 32,946 & 53894649 & 3.035 & .999 & .001 & 15.48 & 2,128 & 32,946 & 35139584 & .194 & .577 & .423 \\
\hline 749 & 11.88 & 1,143 & 13,573 & 7201561 & -4.193 & 0 & 0 & 18.62 & 729 & 13,573 & 4500147 & -4.227 & 0 & 0 \\
\hline 750 & 7.79 & 2,386 & 18,579 & 21988371 & -.673 & .250 & .250 & 11.20 & 1,659 & 18,579 & 15608768 & .904 & .817 & .183 \\
\hline 751 & 10.06 & 758 & 7,628 & 2853514 & -.619 & .268 & .268 & 15.57 & 490 & 7,628 & 1864812 & -.083 & .467 & .467 \\
\hline 752 & 29.66 & 41 & 1,216 & 24753 & -.078 & .469 & .469 & 71.53 & 17 & 1,216 & 10686 & .242 & .596 & .404 \\
\hline 753 & 10.64 & 191 & 2,033 & 203039 & 1.096 & .863 & .137 & 15.17 & 134 & 2,033 & 144632 & 1.240 & .892 & .108 \\
\hline 754 & 7.62 & 338 & 2,575 & 394465 & -2.979 & .001 & .001 & 11.34 & 227 & 2,575 & 272494 & -1.765 & .039 & .039 \\
\hline 755 & 17.67 & 1,217 & 21,499 & 13113325 & .144 & .557 & .443 & 30.03 & 716 & 21,499 & 7708194 & .070 & .528 & .472 \\
\hline 756 & 64.00 & 1 & 64 & 17 & -.812 & .209 & .209 & 64.00 & 1 & 64 & 17 & -.812 & .209 & .209 \\
\hline 757 & 16.65 & 351 & 5,844 & 1073596 & 1.518 & .935 & .065 & 27.06 & 216 & 5,844 & 647874 & .674 & .750 & .250 \\
\hline 758 & 9.56 & 3,383 & 32,344 & 56229822 & 2.799 & .997 & .003 & 14.52 & 2,228 & 32,344 & 36764242 & 1.663 & .952 & .048 \\
\hline 759 & 12.68 & 1,214 & 15,392 & 9587696 & 1.581 & .943 & .057 & 21.90 & 703 & 15,392 & 5686612 & 2.346 & .991 & .010 \\
\hline 760 & 17.20 & 1,241 & 21,345 & 13487352 & 1.119 & .868 & .132 & 29.24 & 730 & 21,345 & 8016377 & 1.354 & .912 & .088 \\
\hline 761 & 16.48 & 821 & 13,531 & 5586561 & .287 & .613 & .387 & 28.43 & 476 & 13,531 & 3188238 & -.377 & .353 & .353 \\
\hline 762 & 23.88 & 1,059 & 25,291 & 13454526 & .265 & .604 & .396 & 42.87 & 590 & 25,291 & 7427365 & -.189 & .425 & .425 \\
\hline 763 & 14.44 & 82 & 1,184 & 40681 & -2.541 & .006 & .006 & 17.94 & 66 & 1,184 & 34204 & -1.753 & .040 & .040 \\
\hline 764 & 10.70 & 3,065 & 32,783 & 51093162 & 1.629 & .948 & .052 & 16.57 & 1,979 & 32,783 & 32938154 & 1.186 & .882 & .118 \\
\hline 765 & 11.16 & 1,093 & 12,195 & 6661742 & -.024 & .490 & .490 & 18.12 & 673 & 12,195 & 4139086 & .388 & .651 & .349 \\
\hline 766 & 10.83 & 247 & 2,674 & 302537 & -2.284 & .011 & .011 & 15.82 & 169 & 2,674 & 196673 & -2.918 & .002 & .002 \\
\hline 767 & 14.39 & 2,307 & 33,202 & 38401907 & .225 & .589 & .411 & 24.43 & 1,359 & 33,202 & 22235774 & -.920 & .179 & .179 \\
\hline 768 & 14.66 & 2,314 & 33,925 & 39465593 & .455 & .675 & .325 & 23.96 & 1,416 & 33,925 & 24112633 & .254 & .600 & .400 \\
\hline 769 & 16.94 & 125 & 2,118 & 116863 & -2.269 & .012 & .012 & 24.92 & 85 & 2,118 & 81269 & -1.552 & .060 & .060 \\
\hline 770 & 34.92 & 233 & 8,136 & 987103 & 1.095 & .863 & .137 & 67.80 & 120 & 8,136 & 518077 & 1.163 & .877 & .123 \\
\hline 771 & 16.51 & 225 & 3,714 & 416763 & -.066 & .474 & .474 & 26.34 & 141 & 3,714 & 261722 & -.009 & .496 & .496 \\
\hline 772 & 14.08 & 13 & 183 & 1038 & -.795 & .213 & .213 & 16.64 & 11 & 183 & 874 & -.756 & .225 & .225 \\
\hline 773 & 18.46 & 28 & 517 & 6506 & -.927 & .177 & .177 & 30.41 & 17 & 517 & 3871 & -.851 & .198 & .198 \\
\hline 774 & 15.37 & 2,008 & 30,870 & 30764239 & -.574 & .283 & .283 & 25.51 & 1,210 & 30,870 & 18744183 & .219 & .587 & .413 \\
\hline 775 & 13.82 & 22 & 304 & 3567 & .542 & .706 & .294 & 19.00 & 16 & 304 & 2752 & .912 & .819 & .181 \\
\hline 776 & 10.71 & 655 & 7,015 & 2306910 & .183 & .573 & .427 & 15.18 & 462 & 7,015 & 1698062 & 1.783 & .963 & .037 \\
\hline 777 & 11.75 & 583 & 6,852 & 1890077 & -2.246 & .012 & .012 & 16.92 & 405 & 6,852 & 1383772 & -0.094 & .462 & .462 \\
\hline 778 & 16.41 & 594 & 9,749 & 2648854 & -3.595 & 0 & 0 & 31.96 & 305 & 9,749 & 1287111 & -4.061 & 0 & 0 \\
\hline 779 & 16.55 & 967 & 16,007 & 8169378 & 2.993 & .999 & .001 & 27.65 & 579 & 16,007 & 5051786 & 3.757 & 1.000 & 0 \\
\hline 780 & 9.63 & 3,588 & 34,558 & 63614594 & 2.707 & .997 & .003 & 14.41 & 2,399 & 34,558 & 43390285 & 3.966 & 1.000 & 0 \\
\hline
\end{tabular}


Table 4. Summary of interoccurrence intervals for daily precipitation thresholds of 0.25 and 0.50 inch-Continued

\begin{tabular}{|c|c|c|c|c|c|c|c|c|c|c|c|c|c|c|}
\hline \multirow[b]{2}{*}{$\begin{array}{l}\text { Seq. } \\
\text { no. }\end{array}$} & \multicolumn{7}{|c|}{ Daily precipitation threshold of 0.25 inch and greater } & \multicolumn{7}{|c|}{ Daily precipitation threshold of 0.50 inch and greater } \\
\hline & $\begin{array}{l}\text { Mean } \\
\text { inter- } \\
\text { occur- } \\
\text { rence } \\
\text { interval } \\
\text { (days) }\end{array}$ & $\begin{array}{l}\text { Total } \\
\text { no. of } \\
\text { events }\end{array}$ & $\begin{array}{l}\text { Total } \\
\text { no. of } \\
\text { days } \\
\text { avail- } \\
\text { able }\end{array}$ & $\begin{array}{l}\text { Sigma } \\
\text { S }\end{array}$ & $\begin{array}{c}\text { Stan- } \\
\text { dard } \\
\text { normal } \\
\text { variate }\end{array}$ & $\begin{array}{l}\text { Non- } \\
\text { ex- } \\
\text { ceed- } \\
\text { ance } \\
\text { prob- } \\
\text { ability }\end{array}$ & $\begin{array}{c}p- \\
\text { value }\end{array}$ & $\begin{array}{l}\text { Mean } \\
\text { inter- } \\
\text { occur- } \\
\text { rence } \\
\text { interval } \\
\text { (days) }\end{array}$ & $\begin{array}{c}\text { Total } \\
\text { no. of } \\
\text { events }\end{array}$ & $\begin{array}{l}\text { Total } \\
\text { no. of } \\
\text { days } \\
\text { avail- } \\
\text { able }\end{array}$ & $\begin{array}{l}\text { Sigma } \\
\mathrm{S}\end{array}$ & $\begin{array}{l}\text { Stan- } \\
\text { dard } \\
\text { normal } \\
\text { variate }\end{array}$ & $\begin{array}{l}\text { Non- } \\
\text { ex- } \\
\text { ceed- } \\
\text { ance } \\
\text { prob- } \\
\text { ability }\end{array}$ & $\begin{array}{c}\mathrm{p}- \\
\text { value }\end{array}$ \\
\hline 781 & 13.70 & 2,526 & 34,614 & 43558220 & -.317 & .376 & .376 & 23.91 & 1,448 & 34,614 & 24587111 & -1.245 & .107 & .107 \\
\hline 782 & 8.01 & 1,161 & 9,303 & 5512121 & 1.221 & .889 & .111 & 11.84 & 786 & 9,303 & 3775776 & 1.590 & .944 & .056 \\
\hline 783 & 18.48 & 79 & 1,460 & 47073 & -2.829 & .002 & .002 & 27.04 & 54 & 1,460 & 30596 & -2.849 & .002 & .002 \\
\hline 784 & 21.17 & 223 & 4,721 & 463590 & -3.086 & .001 & .001 & 33.96 & 139 & 4,721 & 304665 & -1.459 & .072 & .072 \\
\hline 785 & 22.06 & 865 & 19,079 & 8291989 & .249 & .598 & .402 & 38.31 & 498 & 19,079 & 4963990 & 1.736 & .959 & .041 \\
\hline 786 & 22.96 & 851 & 19,541 & 8431539 & .710 & .761 & .239 & 41.84 & 467 & 19,541 & 4616387 & .439 & .670 & .330 \\
\hline 787 & 10.28 & 589 & 6,055 & 1796182 & .306 & .620 & .380 & 15.73 & 385 & 6,055 & 1113046 & -1.532 & .063 & .063 \\
\hline 788 & 9.77 & 165 & 1,612 & 135479 & .416 & .661 & .339 & 13.11 & 123 & 1,612 & 99943 & .156 & .562 & .438 \\
\hline 789 & 7.68 & 1,086 & 8,344 & 4567668 & .465 & .679 & .321 & 15.25 & 547 & 8,344 & 2317346 & .626 & .734 & .266 \\
\hline 790 & 8.34 & 266 & 2,219 & 288738 & -.612 & .270 & .270 & 11.21 & 198 & 2,219 & 219103 & -.064 & .474 & .474 \\
\hline 791 & 13.87 & 340 & 4,714 & 817584 & .646 & .741 & .259 & 19.48 & 242 & 4,714 & 587683 & .817 & .793 & .207 \\
\hline 792 & 9.38 & 48 & 450 & 12908 & 2.342 & .990 & .010 & 14.52 & 31 & 450 & 8805 & 2.530 & .994 & .006 \\
\hline 793 & 9.06 & 2,117 & 19,181 & 20683287 & 1.492 & .932 & .068 & 13.42 & 1,429 & 19,181 & 13771465 & .318 & .625 & .375 \\
\hline 794 & 8.00 & 1,461 & 11,685 & 8385367 & -1.168 & .122 & .122 & 12.06 & 969 & 11,685 & 5496814 & -1.567 & .059 & .059 \\
\hline 795 & 11.27 & 1,513 & 17,047 & 13271699 & 1.963 & .975 & .025 & 17.76 & 960 & 17,047 & 8510813 & 2.153 & .984 & .016 \\
\hline 796 & 21.59 & 242 & 5,225 & 683575 & 2.189 & .986 & .014 & 35.30 & 148 & 5,225 & 410148 & 1.281 & .900 & .100 \\
\hline 797 & 17.15 & 1,659 & 28,459 & 24123107 & 1.543 & .939 & .061 & 29.77 & 956 & 28,459 & 13690375 & .342 & .634 & .366 \\
\hline 798 & 22.39 & 31 & 694 & 9177 & -1.417 & .078 & .078 & 33.05 & 21 & 694 & 5203 & -2.270 & .012 & .012 \\
\hline 799 & 13.21 & 693 & 9,155 & 3098339 & -1.062 & .144 & .144 & 22.77 & 402 & 9,155 & 1861866 & .410 & .659 & .341 \\
\hline 800 & 14.72 & 78 & 1,148 & 43092 & -.574 & .283 & .283 & 24.96 & 46 & 1,148 & 27348 & .420 & .663 & .337 \\
\hline 801 & 24.97 & 593 & 14,804 & 4391299 & .018 & .507 & .493 & 44.46 & 333 & 14,804 & 2547303 & 1.057 & .855 & .145 \\
\hline 802 & 33.60 & 45 & 1,512 & 26597 & -2.535 & .006 & .006 & 63.00 & 24 & 1,512 & 12697 & -2.547 & .005 & .005 \\
\hline 803 & 11.20 & 652 & 7,299 & 2368251 & -.209 & .417 & .417 & 17.67 & 413 & 7,299 & 1527963 & .484 & .686 & .314 \\
\hline 804 & 17.46 & 39 & 681 & 13270 & -.008 & .497 & .497 & 30.96 & 22 & 681 & 6328 & -1.261 & .104 & .104 \\
\hline 805 & 13.83 & 831 & 11,493 & 4682039 & -.976 & .165 & .165 & 23.46 & 490 & 11,493 & 2721514 & -1.284 & .100 & .100 \\
\hline 806 & 8.25 & 2,079 & 17,161 & 18431372 & 2.623 & .996 & .004 & 12.38 & 1,386 & 17,161 & 12426870 & 2.897 & .998 & .002 \\
\hline 807 & 9.82 & 1,337 & 13,131 & 8882903 & .756 & .775 & .225 & 14.87 & 883 & 13,131 & 5978638 & 1.610 & .946 & .054 \\
\hline 808 & 11.63 & 1,549 & 18,012 & 14488661 & 2.631 & .996 & .004 & 17.14 & 1,051 & 18,012 & 9507767 & .252 & .599 & .401 \\
\hline 809 & 16.41 & 1,124 & 18,442 & 10536323 & .963 & .832 & .168 & 28.37 & 650 & 18,442 & 6024707 & .229 & .591 & .409 \\
\hline 810 & 17.65 & 1,165 & 20,566 & 12363645 & 1.895 & .971 & .029 & 33.88 & 607 & 20,566 & 6289618 & .327 & .628 & .372 \\
\hline 811 & 7.85 & 940 & 7,376 & 3421642 & -.691 & .245 & .245 & 11.49 & 642 & 7,376 & 2389328 & .401 & .656 & .344 \\
\hline 812 & 13.64 & 490 & 6,684 & 1697586 & 1.405 & .920 & .080 & 21.42 & 312 & 6,684 & 1119934 & 2.266 & .988 & .012 \\
\hline 813 & 7.55 & 11 & 83 & 363 & -1.177 & .120 & .120 & 10.38 & 8 & 83 & 299 & -.487 & .313 & .313 \\
\hline 814 & 14.86 & 1,642 & 24,404 & 20213620 & .623 & .733 & .267 & 30.02 & 813 & 24,404 & 9981111 & .303 & .619 & .381 \\
\hline 815 & 8.08 & 3,758 & 30,377 & 56887715 & -.355 & .361 & .361 & 11.93 & 2,547 & 30,377 & 39292002 & 1.371 & .915 & .085 \\
\hline 816 & 7.91 & 1,634 & 12,922 & 10490376 & -.444 & .329 & .329 & 11.71 & 1,104 & 12,922 & 7175938 & .347 & .636 & .364 \\
\hline 817 & 11.19 & 556 & 6,220 & 1764796 & .842 & .800 & .200 & 16.33 & 381 & 6,220 & 1188525 & .103 & .541 & .459 \\
\hline 818 & 10.07 & 1,969 & 19,833 & 19987355 & 1.818 & .965 & .035 & 16.02 & 1,238 & 19,833 & 12568946 & 1.451 & .927 & .073 \\
\hline 819 & 17.30 & 1,668 & 28,862 & 24137436 & .196 & .578 & .422 & 32.18 & 897 & 28,862 & 12651426 & -1.175 & .120 & .120 \\
\hline 820 & 14.37 & 62 & 891 & 25869 & -.865 & .194 & .194 & 26.21 & 34 & 891 & 15984 & .558 & .712 & .288 \\
\hline 821 & 18.51 & 430 & 7,961 & 1656600 & -1.154 & .124 & .124 & 32.49 & 245 & 7,961 & 989750 & .404 & .657 & .343 \\
\hline 822 & 12.18 & 1,564 & 19,050 & 15617716 & 3.314 & 1.000 & 0 & 19.54 & 975 & 19,050 & 9802072 & 3.000 & .999 & .001 \\
\hline 823 & 13.33 & 2,442 & 32,540 & 40553747 & 1.772 & .962 & .038 & 22.14 & 1,470 & 32,540 & 23941714 & .069 & .528 & .473 \\
\hline 824 & 12.86 & 165 & 2,122 & 172976 & -.266 & .395 & .395 & 19.83 & 107 & 2,122 & 107286 & -.985 & .162 & .162 \\
\hline 825 & 19.35 & 209 & 4,045 & 436011 & .788 & .785 & .215 & 24.22 & 167 & 4,045 & 366942 & 1.934 & .973 & .027 \\
\hline 826 & 7.85 & 3,375 & 26,503 & 44659745 & -.144 & .443 & .443 & 12.02 & 2,205 & 26,503 & 29063716 & -.434 & .332 & .332 \\
\hline 827 & 7.70 & 1,356 & 10,437 & 7164375 & .794 & .786 & .214 & 11.74 & 889 & 10,437 & 4753343 & 1.270 & .898 & .102 \\
\hline 828 & 8.22 & 3,143 & 25,827 & 41751582 & 2.786 & .997 & .003 & 11.89 & 2,173 & 25,827 & 28870398 & 2.329 & .990 & .010 \\
\hline 829 & 7.46 & 724 & 5,401 & 1934058 & -0.503 & .307 & .307 & 10.89 & 496 & 5,401 & 1320077 & -0.558 & .289 & .289 \\
\hline 830 & 13.38 & 979 & 13,102 & 6557874 & 1.221 & .889 & .111 & 20.83 & 629 & 13,102 & 4211155 & .955 & .830 & .170 \\
\hline 831 & 9.97 & 1,422 & 14,176 & 10321498 & 1.571 & .942 & .058 & 15.28 & 928 & 14,176 & 6828810 & 2.015 & .978 & .022 \\
\hline 832 & 11.02 & 159 & 1,752 & 133388 & -.925 & .178 & .178 & 18.44 & 95 & 1,752 & 76173 & -1.430 & .076 & .076 \\
\hline
\end{tabular}


Table 4. Summary of interoccurrence intervals for daily precipitation thresholds of 0.25 and 0.50 inch-Continued

\begin{tabular}{|c|c|c|c|c|c|c|c|c|c|c|c|c|c|c|}
\hline \multirow[b]{2}{*}{$\begin{array}{c}\text { Seq. } \\
\text { no. }\end{array}$} & \multicolumn{7}{|c|}{ Daily precipitation threshold of 0.25 inch and greater } & \multicolumn{7}{|c|}{ Daily precipitation threshold of 0.50 inch and greater } \\
\hline & $\begin{array}{c}\text { Mean } \\
\text { inter- } \\
\text { occur- } \\
\text { rence } \\
\text { interval } \\
\text { (days) }\end{array}$ & $\begin{array}{c}\text { Total } \\
\text { no. of } \\
\text { events }\end{array}$ & $\begin{array}{l}\text { Total } \\
\text { no. of } \\
\text { days } \\
\text { avail- } \\
\text { able }\end{array}$ & $\begin{array}{l}\text { Sigma } \\
\mathbf{S}\end{array}$ & $\begin{array}{l}\text { Stan- } \\
\text { dard } \\
\text { normal } \\
\text { variate }\end{array}$ & $\begin{array}{l}\text { Non- } \\
\text { ex- } \\
\text { ceed- } \\
\text { ance } \\
\text { prob- } \\
\text { ability }\end{array}$ & $\begin{array}{c}\text { p- } \\
\text { value }\end{array}$ & $\begin{array}{l}\text { Mean } \\
\text { inter- } \\
\text { occur- } \\
\text { rence } \\
\text { interval } \\
\text { (days) }\end{array}$ & $\begin{array}{c}\text { Total } \\
\text { no. of } \\
\text { events }\end{array}$ & $\begin{array}{l}\text { Total } \\
\text { no. of } \\
\text { days } \\
\text { avail- } \\
\text { able }\end{array}$ & $\begin{array}{l}\text { Sigma } \\
\mathbf{S}\end{array}$ & $\begin{array}{l}\text { Stan- } \\
\text { dard } \\
\text { normal } \\
\text { variate }\end{array}$ & $\begin{array}{l}\text { Non- } \\
\text { ex- } \\
\text { ceed- } \\
\text { ance } \\
\text { prob- } \\
\text { ability }\end{array}$ & $\begin{array}{c}p- \\
\text { value }\end{array}$ \\
\hline 833 & 16.25 & 101 & 1,641 & 73756 & -1.915 & .028 & .028 & 28.29 & 58 & 1,641 & 45228 & -.654 & .256 & .256 \\
\hline 834 & 14.88 & 276 & 4,106 & 598385 & 1.613 & .947 & .053 & 25.82 & 159 & 4,106 & 333420 & .468 & .680 & .320 \\
\hline 835 & 8.09 & 2,293 & 18,549 & 20916812 & -1.364 & .086 & .086 & 11.64 & 1,594 & 18,549 & 14690273 & -.436 & .331 & .331 \\
\hline 836 & 12.54 & 602 & 7,548 & 2363795 & 1.718 & .957 & .043 & 21.08 & 358 & 7,548 & 1381553 & .739 & .770 & .230 \\
\hline 837 & 7.41 & 1,612 & 11,942 & 9807974 & 1.320 & .907 & .093 & 11.07 & 1,079 & 11,942 & 6573995 & 1.159 & .877 & .123 \\
\hline 838 & 7.88 & 967 & 7,618 & 3607585 & -1.107 & .134 & .134 & 11.15 & 683 & 7,618 & 2614725 & .229 & .591 & .409 \\
\hline 839 & 7.71 & 292 & 2,252 & 318877 & -.893 & .186 & .186 & 12.11 & 186 & 2,252 & 206319 & -.352 & .363 & .363 \\
\hline 840 & 11.23 & 3,248 & 36,458 & 60493334 & 2.143 & .984 & .016 & 17.49 & 2,084 & 36,458 & 39588209 & 3.328 & 1.000 & 0 \\
\hline 841 & 7.97 & 2,267 & 18,077 & 20703004 & .856 & .804 & .196 & 12.02 & 1,504 & 18,077 & 13927583 & 1.649 & .950 & .050 \\
\hline 842 & 8.91 & 2,102 & 18,731 & 19859384 & .698 & .757 & .243 & 13.55 & 1,382 & 18,731 & 13287530 & 1.713 & .957 & .043 \\
\hline 843 & 8.83 & 407 & 3,594 & 714364 & -.813 & .208 & .208 & 12.93 & 278 & 3,594 & 506798 & .418 & .662 & .338 \\
\hline 844 & 11.16 & 1,573 & 17,555 & 14055769 & 1.238 & .892 & .108 & 15.83 & 1,109 & 17,555 & 9564235 & -1.007 & .157 & .157 \\
\hline 845 & 7.00 & 534 & 3,740 & 1028112 & 1.184 & .882 & .118 & 10.28 & 364 & 3,740 & 714229 & 1.629 & .948 & .052 \\
\hline 846 & 7.29 & 889 & 6,477 & 2865087 & -.250 & .401 & .401 & 10.48 & 618 & 6,477 & 2027481 & .561 & .713 & .287 \\
\hline 847 & 11.50 & 1,560 & 17,934 & 14728493 & 3.619 & 1.000 & 0 & 16.79 & 1,068 & 17,934 & 9787314 & 1.245 & .893 & .107 \\
\hline 848 & 11.33 & 2,538 & 28,763 & 37346894 & 2.024 & .979 & .021 & 17.72 & 1,623 & 28,763 & 24595059 & 3.749 & 1.000 & 0 \\
\hline 849 & 8.69 & 781 & 6,785 & 2683056 & .612 & .730 & .270 & 14.28 & 475 & 6,785 & 1608472 & -.070 & .472 & .472 \\
\hline 850 & 13.13 & 109 & 1,431 & 72872 & -1.187 & .118 & .118 & 21.36 & 67 & 1,431 & 44284 & -1.081 & .140 & .140 \\
\hline 851 & 9.61 & 38 & 365 & 8833 & 2.922 & .998 & .002 & 13.04 & 28 & 365 & 6362 & 2.246 & .988 & .012 \\
\hline 852 & 7.75 & 59 & 457 & 13495 & .013 & .505 & .495 & 9.52 & 48 & 457 & 11207 & .262 & .603 & .397 \\
\hline 853 & 7.83 & 35 & 274 & 4771 & -.051 & .480 & .480 & 11.91 & 23 & 274 & 3105 & -.121 & .452 & .452 \\
\hline 854 & 14.94 & 1,265 & 18,899 & 12231394 & 1.432 & .924 & .076 & 26.92 & 702 & 18,899 & 7003549 & 2.560 & .995 & .005 \\
\hline 855 & 10.30 & 541 & 5,570 & 1529326 & .605 & .727 & .273 & 15.78 & 353 & 5,570 & 1001131 & .597 & .725 & .275 \\
\hline 856 & 14.96 & 1,251 & 18,709 & 11922655 & 1.153 & .875 & .125 & 26.54 & 705 & 18,709 & 6721120 & .880 & .811 & .190 \\
\hline 857 & 13.62 & 668 & 9,100 & 3263071 & 3.294 & 1.000 & 0 & 21.36 & 426 & 9,100 & 2153782 & 3.974 & 1.000 & 0 \\
\hline 858 & 14.05 & 753 & 10,580 & 4034916 & .615 & .731 & .269 & 21.95 & 482 & 10,580 & 2631850 & 1.224 & .889 & .111 \\
\hline 859 & 15.01 & 624 & 9,367 & 2915686 & -.101 & .460 & .460 & 24.59 & 381 & 9,367 & 1785434 & .019 & .508 & .492 \\
\hline 860 & 18.16 & 67 & 1,217 & 34389 & -2.219 & .013 & .013 & 34.77 & 35 & 1,217 & 17885 & -1.642 & .050 & .050 \\
\hline 861 & 14.35 & 1,007 & 14,445 & 7093445 & -1.357 & .087 & .087 & 24.48 & 590 & 14,445 & 4109211 & -1.501 & .067 & .067 \\
\hline 862 & 8.23 & 927 & 7,632 & 3622130 & 1.263 & .897 & .103 & 12.25 & 623 & 7,632 & 2469582 & 1.677 & .953 & .047 \\
\hline 863 & 7.61 & 56 & 426 & 13001 & 1.166 & .878 & .122 & 12.17 & 35 & 426 & 8933 & 2.032 & .979 & .021 \\
\hline 864 & 23.47 & 770 & 18,069 & 6972385 & .109 & .544 & .456 & 39.45 & 458 & 18,069 & 4144913 & .064 & .525 & .475 \\
\hline 865 & 18.59 & 126 & 2,342 & 136594 & -1.443 & .075 & .075 & 30.42 & 77 & 2,342 & 88086 & -.351 & .363 & .363 \\
\hline 866 & 18.26 & 149 & 2,721 & 206874 & .434 & .668 & .332 & 29.90 & 91 & 2,721 & 126808 & .401 & .656 & .344 \\
\hline 867 & 12.24 & 1,299 & 15,899 & 10710520 & 2.322 & .990 & .010 & 20.00 & 795 & 15,899 & 6696488 & 2.910 & .998 & .002 \\
\hline 868 & 12.46 & 1,746 & 21,746 & 19292227 & 1.174 & .880 & .120 & 20.29 & 1,072 & 21,746 & 11932335 & 1.345 & .911 & .089 \\
\hline 869 & 16.87 & 1,093 & 18,440 & 9867273 & -1.194 & .116 & .116 & 30.84 & 598 & 18,440 & 5294697 & -1.681 & .046 & .046 \\
\hline 870 & 7.43 & 212 & 1,575 & 176650 & 1.465 & .929 & .071 & 11.01 & 143 & 1,575 & 119420 & 1.252 & .895 & .105 \\
\hline 871 & 18.00 & 14 & 252 & 2306 & 1.991 & .977 & .023 & 42.00 & 6 & 252 & 922 & .932 & .824 & .176 \\
\hline 872 & 13.50 & 36 & 486 & 8156 & -.703 & .241 & .241 & 19.44 & 25 & 486 & 5592 & -.689 & .246 & .246 \\
\hline 873 & 7.16 & 3,276 & 23,470 & 38508162 & .166 & .566 & .434 & 10.48 & 2,240 & 23,470 & 26287375 & .003 & .501 & .499 \\
\hline 874 & 6.68 & 857 & 5,724 & 2381869 & -1.465 & .071 & .071 & 9.52 & 601 & 5,724 & 1651141 & -1.701 & .044 & .044 \\
\hline 875 & 7.46 & 65 & 485 & 14511 & -1.109 & .134 & .134 & 12.76 & 38 & 485 & 8388 & -.958 & .169 & .169 \\
\hline 876 & -- & -- & -- & -- & -- & -- & -- & -- & -- & -- & -- & -- & -- & -- \\
\hline 877 & 17.92 & 24 & 430 & 5846 & 1.128 & .870 & .130 & 26.88 & 16 & 430 & 4212 & 1.555 & .940 & .060 \\
\hline 878 & 7.98 & 1,963 & 15,666 & 15422098 & .229 & .591 & .409 & 11.85 & 1,322 & 15,666 & 10245044 & -.670 & .251 & .251 \\
\hline 879 & 18.10 & 1,022 & 18,493 & 9575937 & .738 & .770 & .230 & 30.12 & 614 & 18,493 & 5776263 & .748 & .773 & .227 \\
\hline 880 & 24.78 & 9 & 223 & 815 & -.976 & .165 & .165 & 74.33 & 3 & 223 & 205 & -1.161 & .123 & .123 \\
\hline 881 & 14.70 & 1,265 & 18,595 & 12312471 & 2.887 & .998 & .002 & 24.47 & 760 & 18,595 & 7293773 & 1.539 & .938 & .062 \\
\hline 882 & 13.33 & 137 & 1,826 & 115307 & -1.584 & .057 & .057 & 20.75 & 88 & 1,826 & 73956 & -1.292 & .098 & .098 \\
\hline 883 & 13.21 & 825 & 10,901 & 4378824 & -1.304 & .096 & .096 & 21.98 & 496 & 10,901 & 2652567 & -.726 & .234 & .234 \\
\hline 884 & 14.36 & 949 & 13,631 & 6288924 & -1.477 & .070 & .070 & 24.87 & 548 & 13,631 & 3588815 & -1.586 & .056 & .056 \\
\hline
\end{tabular}


Table 4. Summary of interoccurrence intervals for daily precipitation thresholds of 0.25 and 0.50 inch-Continued

\begin{tabular}{|c|c|c|c|c|c|c|c|c|c|c|c|c|c|c|}
\hline \multirow[b]{2}{*}{$\begin{array}{l}\text { Seq. } \\
\text { no. }\end{array}$} & \multicolumn{7}{|c|}{ Daily precipitation threshold of 0.25 inch and greater } & \multicolumn{7}{|c|}{ Daily precipitation threshold of 0.50 inch and greater } \\
\hline & $\begin{array}{l}\text { Mean } \\
\text { inter- } \\
\text { occur- } \\
\text { rence } \\
\text { interval } \\
\text { (days) }\end{array}$ & $\begin{array}{c}\text { Total } \\
\text { no. of } \\
\text { events }\end{array}$ & $\begin{array}{l}\text { Total } \\
\text { no. of } \\
\text { days } \\
\text { avail- } \\
\text { able }\end{array}$ & $\begin{array}{c}\text { Sigma } \\
\mathbf{S}\end{array}$ & $\begin{array}{c}\text { Stan- } \\
\text { dard } \\
\text { normal } \\
\text { variate }\end{array}$ & $\begin{array}{l}\text { Non- } \\
\text { ex- } \\
\text { ceed- } \\
\text { ance } \\
\text { prob- } \\
\text { ability }\end{array}$ & $\begin{array}{c}p- \\
\text { value }\end{array}$ & $\begin{array}{l}\text { Mean } \\
\text { inter- } \\
\text { occur- } \\
\text { rence } \\
\text { interval } \\
\text { (days) }\end{array}$ & $\begin{array}{c}\text { Total } \\
\text { no. of } \\
\text { events }\end{array}$ & $\begin{array}{c}\text { Total } \\
\text { no. of } \\
\text { days } \\
\text { avail- } \\
\text { able }\end{array}$ & $\underset{\mathrm{S}}{\text { Sigma }}$ & $\begin{array}{c}\text { Stan- } \\
\text { dard } \\
\text { normal } \\
\text { variate }\end{array}$ & $\begin{array}{c}\text { Non- } \\
\text { ex- } \\
\text { ceed- } \\
\text { ance } \\
\text { prob- } \\
\text { ability }\end{array}$ & $\begin{array}{c}p- \\
\text { value }\end{array}$ \\
\hline 885 & 14.10 & 2,106 & 29,698 & 31604706 & .846 & .801 & .199 & 22.67 & 1,310 & 29,698 & 19556709 & .337 & .632 & .368 \\
\hline 886 & 9.68 & 2,208 & 21,366 & 23807526 & .757 & .775 & .225 & 15.03 & 1,422 & 21,366 & 15399429 & .895 & .815 & .185 \\
\hline 887 & 8.71 & 2,880 & 25,079 & 37365560 & 3.222 & .999 & .001 & 13.17 & 1,904 & 25,079 & 24686379 & 2.568 & .995 & .005 \\
\hline 888 & 10.86 & 1,743 & 18,929 & 17145077 & 2.843 & .998 & .002 & 16.95 & 1,117 & 18,929 & 11033462 & 2.528 & .994 & .006 \\
\hline 889 & 13.94 & 985 & 13,726 & 6902184 & 1.143 & .873 & .127 & 26.70 & 514 & 13,726 & 3655942 & 1.429 & .923 & .077 \\
\hline 890 & 14.62 & 895 & 13,085 & 5747203 & -.959 & .169 & .169 & 24.97 & 524 & 13,085 & 3288918 & -1.612 & .054 & .054 \\
\hline 891 & 21.60 & 840 & 18,143 & 8049984 & 2.832 & .998 & .002 & 34.10 & 532 & 18,143 & 4887440 & .508 & .694 & .306 \\
\hline 892 & 19.85 & 364 & 7,226 & 1313486 & -.041 & .483 & .483 & 32.70 & 221 & 7,226 & 784469 & -.452 & .326 & .326 \\
\hline 893 & 14.47 & 1,862 & 26,939 & 25415334 & .999 & .841 & .159 & 24.94 & 1,080 & 26,939 & 14237163 & -1.213 & .113 & .113 \\
\hline 894 & 11.42 & 493 & 5,631 & 1356094 & -.885 & .188 & .188 & 17.22 & 327 & 5,631 & 912394 & -.282 & .389 & .389 \\
\hline 895 & 21.53 & 793 & 17,071 & 6853565 & .612 & .730 & .270 & 43.55 & 392 & 17,071 & 3533534 & 1.923 & .973 & .027 \\
\hline 896 & 8.39 & 4,261 & 35,755 & 78040924 & 2.768 & .997 & .003 & 12.59 & 2,840 & 35,755 & 52245719 & 2.679 & .996 & .004 \\
\hline 897 & 14.15 & 13 & 184 & 1192 & -.021 & .492 & .492 & 20.44 & 9 & 184 & 811 & -.107 & .457 & .457 \\
\hline 898 & 6.92 & 175 & 1,211 & 107083 & .242 & .596 & .404 & 9.24 & 131 & 1,211 & 79355 & .009 & .503 & .497 \\
\hline 899 & 19.33 & 3 & 58 & 70 & -.586 & .279 & .279 & 29.00 & 2 & 58 & 44 & -.591 & .277 & .277 \\
\hline 900 & 11.01 & 139 & 1,531 & 106744 & .065 & .526 & .474 & 17.01 & 90 & 1,531 & 68162 & -.175 & .431 & .431 \\
\hline 901 & 15.56 & 2,189 & 34,050 & 37854078 & 1.275 & .899 & .101 & 24.34 & 1,399 & 34,050 & 24451383 & 1.723 & .958 & .042 \\
\hline 902 & 16.78 & 67 & 1,124 & 39728 & .781 & .783 & .218 & 22.04 & 51 & 1,124 & 30605 & .839 & .799 & .201 \\
\hline 903 & 28.72 & 880 & 25,276 & 11203495 & .379 & .648 & .352 & 56.04 & 451 & 25,276 & 5754618 & .354 & .638 & .362 \\
\hline 904 & 39.60 & 10 & 396 & 2049 & .191 & .576 & .424 & 79.20 & 5 & 396 & 1434 & 1.737 & .959 & .041 \\
\hline 905 & 24.52 & 678 & 16,626 & 5825750 & 1.517 & .935 & .065 & 47.37 & 351 & 16,626 & 2962410 & .495 & .690 & .310 \\
\hline 906 & 14.82 & 2,135 & 31,631 & 33644608 & -.288 & .387 & .387 & 25.76 & 1,228 & 31,631 & 19308874 & -.352 & .362 & .362 \\
\hline 907 & -- & -- & -- & -- & -- & -- & -- & -- & -- & -- & -- & -- & -- & -- \\
\hline 908 & 14.38 & 1,376 & 19,782 & 13856245 & 1.162 & .877 & .123 & 27.10 & 730 & 19,782 & 7526596 & 1.984 & .976 & .024 \\
\hline 909 & 13.85 & 606 & 8,392 & 2571356 & .479 & .684 & .316 & 23.91 & 351 & 8,392 & 1463351 & -.208 & .418 & .418 \\
\hline 910 & 27.68 & 256 & 7,085 & 888482 & -.562 & .287 & .287 & 56.23 & 126 & 7,085 & 440254 & -.266 & .395 & .395 \\
\hline 911 & 25.27 & 83 & 2,097 & 88867 & .334 & .631 & .369 & 58.25 & 36 & 2,097 & 39189 & .397 & .654 & .346 \\
\hline 912 & 12.08 & 25 & 302 & 2415 & -3.120 & .001 & .001 & 17.77 & 17 & 302 & 1749 & -2.276 & .011 & .011 \\
\hline 913 & 12.54 & 512 & 6,422 & 1702202 & 1.387 & .917 & .083 & 20.20 & 318 & 6,422 & 1081905 & 1.839 & .967 & .033 \\
\hline 914 & 10.06 & 360 & 3,622 & 629990 & -1.107 & .134 & .134 & 14.97 & 242 & 3,622 & 417816 & -1.257 & .104 & .104 \\
\hline 915 & 8.63 & 3,899 & 33,649 & 69087504 & 5.752 & 0 & 0 & 13.39 & 2,513 & 33,649 & 45211660 & 6.021 & 0 & 0 \\
\hline 916 & 9.12 & 2,098 & 19,131 & 20865701 & 3.152 & .999 & .001 & 13.05 & 1,466 & 19,131 & 14936797 & 4.321 & 1.000 & 0 \\
\hline 917 & 16.03 & 481 & 7,709 & 1754946 & -2.030 & .021 & .021 & 32.80 & 235 & 7,709 & 816437 & -2.620 & .004 & .004 \\
\hline 918 & 7.24 & 1,224 & 8,856 & 5504809 & .950 & .829 & .171 & 10.80 & 820 & 8,856 & 3743759 & 1.541 & .938 & .062 \\
\hline 919 & 15.10 & 649 & 9,801 & 3072851 & -1.493 & .068 & .068 & 22.03 & 445 & 9,801 & 2142157 & -.646 & .259 & .259 \\
\hline 920 & 8.31 & 2,298 & 19,104 & 22395179 & 1.682 & .954 & .046 & 11.70 & 1,633 & 19,104 & 15992079 & 1.766 & .961 & .039 \\
\hline 921 & 11.65 & 375 & 4,368 & 843193 & .991 & .839 & .161 & 18.43 & 237 & 4,368 & 553116 & 1.829 & .966 & .034 \\
\hline 922 & 18.57 & 1,225 & 22,744 & 14289112 & 1.560 & .941 & .059 & 31.59 & 720 & 22,744 & 8571698 & 2.179 & .985 & .015 \\
\hline 923 & 15.71 & 2,128 & 33,427 & 35303565 & -.590 & .278 & .278 & 28.33 & 1,180 & 33,427 & 19233661 & -1.473 & .070 & .070 \\
\hline 924 & 18.26 & 35 & 639 & 10131 & -.964 & .168 & .168 & 39.94 & 16 & 639 & 4490 & -.843 & .200 & .200 \\
\hline 925 & 22.02 & 191 & 4,205 & 426074 & 1.460 & .928 & .072 & 41.63 & 101 & 4,205 & 233116 & 1.702 & .956 & .044 \\
\hline 926 & 12.36 & 45 & 556 & 13217 & .657 & .744 & .256 & 20.59 & 27 & 556 & 8627 & 1.344 & .911 & .089 \\
\hline 927 & 11.70 & 379 & 4,433 & 823278 & -.673 & .250 & .250 & 17.73 & 250 & 4,433 & 537291 & -.832 & .203 & .203 \\
\hline 928 & 14.17 & 538 & 7,623 & 2213194 & 3.186 & .999 & .001 & 24.75 & 308 & 7,623 & 1286938 & 2.926 & .998 & .002 \\
\hline 929 & 9.76 & 1,611 & 15,716 & 12785028 & .691 & .755 & .245 & 15.10 & 1,041 & 15,716 & 8059559 & -.824 & .205 & .205 \\
\hline 930 & 17.19 & 530 & 9,108 & 2534889 & 2.004 & .977 & .023 & 31.19 & 292 & 9,108 & 1421840 & 2.049 & .980 & .020 \\
\hline 931 & 13.72 & 353 & 4,843 & 875809 & .800 & .788 & .212 & 21.72 & 223 & 4,843 & 565655 & 1.229 & .890 & .110 \\
\hline 932 & 13.23 & 246 & 3,255 & 368813 & -2.141 & .016 & .016 & 19.26 & 169 & 3,255 & 262613 & -1.018 & .154 & .154 \\
\hline 933 & 30.50 & 2 & 61 & 50 & -0.442 & .329 & .329 & 30.50 & 2 & 61 & 50 & -0.442 & .329 & .329 \\
\hline 934 & 12.55 & 442 & 5,547 & 1222782 & -.092 & .463 & .463 & 19.00 & 292 & 5,547 & 822028 & .445 & .672 & .328 \\
\hline 935 & 7.37 & 2,674 & 19,694 & 26983438 & 2.220 & .987 & .013 & 10.94 & 1,801 & 19,694 & 18487305 & 3.120 & .999 & .001 \\
\hline 936 & 7.32 & 1,299 & 9,510 & 6004721 & -1.739 & .041 & .041 & 10.36 & 918 & 9,510 & 4342006 & -.278 & .391 & .391 \\
\hline
\end{tabular}


Table 4. Summary of interoccurrence intervals for daily precipitation thresholds of 0.25 and 0.50 inch-Continued

\begin{tabular}{|c|c|c|c|c|c|c|c|c|c|c|c|c|c|c|}
\hline \multirow[b]{2}{*}{$\begin{array}{c}\text { Seq. } \\
\text { no. }\end{array}$} & \multicolumn{7}{|c|}{ Daily precipitation threshold of 0.25 inch and greater } & \multicolumn{7}{|c|}{ Daily precipitation threshold of 0.50 inch and greater } \\
\hline & $\begin{array}{l}\text { Mean } \\
\text { inter- } \\
\text { occur- } \\
\text { rence } \\
\text { interval } \\
\text { (days) }\end{array}$ & $\begin{array}{c}\text { Total } \\
\text { no. of } \\
\text { events }\end{array}$ & $\begin{array}{l}\text { Total } \\
\text { no. of } \\
\text { days } \\
\text { avail- } \\
\text { able }\end{array}$ & $\begin{array}{c}\text { Sigma } \\
\mathrm{S}\end{array}$ & $\begin{array}{c}\text { Stan- } \\
\text { dard } \\
\text { normal } \\
\text { variate }\end{array}$ & $\begin{array}{l}\text { Non- } \\
\text { ex- } \\
\text { ceed- } \\
\text { ance } \\
\text { prob- } \\
\text { ability }\end{array}$ & $\begin{array}{c}\mathrm{p}- \\
\text { value }\end{array}$ & $\begin{array}{l}\text { Mean } \\
\text { inter- } \\
\text { occur- } \\
\text { rence } \\
\text { interval } \\
\text { (days) }\end{array}$ & $\begin{array}{c}\text { Total } \\
\text { no. of } \\
\text { events }\end{array}$ & $\begin{array}{l}\text { Total } \\
\text { no. of } \\
\text { days } \\
\text { avail- } \\
\text { able }\end{array}$ & $\begin{array}{l}\text { Sigma } \\
\mathrm{S}\end{array}$ & $\begin{array}{c}\text { Stan- } \\
\text { dard } \\
\text { normal } \\
\text { variate }\end{array}$ & $\begin{array}{l}\text { Non- } \\
\text { ex- } \\
\text { ceed- } \\
\text { ance } \\
\text { prob- } \\
\text { ability }\end{array}$ & $\begin{array}{c}\mathrm{p}- \\
\text { value }\end{array}$ \\
\hline 937 & 8.03 & 1,111 & 8,920 & 4949829 & -.061 & .476 & .476 & 11.26 & 792 & 8,920 & 3487755 & -.615 & .269 & .269 \\
\hline 938 & 14.56 & 1,739 & 25,311 & 22500761 & 1.618 & 947 & .053 & 24.01 & 1,054 & 25,311 & 13583296 & 1.030 & .849 & .152 \\
\hline 939 & 10.57 & 2,044 & 21,605 & 22541152 & 1.634 & .949 & .051 & 15.81 & 1,367 & 21,605 & 15097836 & 1.435 & .924 & .076 \\
\hline 940 & 14.79 & 1,075 & 15,898 & 8293211 & -1.675 & .047 & .047 & 24.05 & 661 & 15,898 & 5204908 & -.419 & .338 & .338 \\
\hline 941 & 11.28 & 1,458 & 16,452 & 11078624 & -5.045 & 0 & 0 & 17.62 & 934 & 16,452 & 7253189 & -2.962 & .002 & .002 \\
\hline 942 & 12.57 & 1,045 & 13,131 & 7000071 & 1.135 & .872 & .128 & 20.84 & 630 & 13,131 & 4187386 & .537 & .704 & .296 \\
\hline 943 & 15.82 & 2,064 & 32,647 & 33287922 & -.943 & .173 & .173 & 27.12 & 1,204 & 32,647 & 19630039 & -.072 & .471 & .471 \\
\hline 944 & 9.62 & 597 & 5,740 & 1760149 & 1.155 & .876 & .124 & 13.51 & 425 & 5,740 & 1245047 & .741 & .770 & .230 \\
\hline 945 & 13.43 & 1,591 & 21,362 & 17280544 & 1.167 & .878 & .122 & 21.05 & 1,015 & 21,362 & 11026667 & .944 & .827 & .173 \\
\hline 946 & 8.99 & 1,092 & 9,820 & 5498180 & 1.457 & .927 & .073 & 14.25 & 689 & 9,820 & 3426751 & .588 & .722 & .278 \\
\hline 947 & 12.97 & 1,063 & 13,784 & 7594372 & 2.067 & .981 & .019 & 19.92 & 692 & 13,784 & 5088565 & 3.050 & .999 & .001 \\
\hline 948 & 15.79 & 77 & 1,216 & 43094 & -1.208 & .114 & .114 & 26.44 & 46 & 1,216 & 27147 & -.345 & .365 & .365 \\
\hline 949 & 31.94 & 830 & 26,509 & 11160575 & .723 & .765 & .235 & 64.66 & 410 & 26,509 & 5644184 & 1.354 & .912 & .088 \\
\hline 950 & 46.40 & 5 & 232 & 617 & .247 & .598 & .402 & 232.00 & 1 & 232 & 75 & -.612 & .270 & .270 \\
\hline 951 & 8.71 & 1,424 & 12,405 & 8711441 & -.895 & .186 & .186 & 12.79 & 970 & 12,405 & 5880288 & -1.221 & .111 & .111 \\
\hline 952 & 10.08 & 489 & 4,929 & 1239050 & 1.078 & .859 & .141 & 15.21 & 324 & 4,929 & 844183 & 1.784 & .963 & .037 \\
\hline 953 & 11.46 & 1,224 & 14,026 & 8679506 & .675 & .750 & .250 & 18.36 & 764 & 14,026 & 5504459 & 1.309 & .905 & .095 \\
\hline 954 & 11.01 & 629 & 6,922 & 2197379 & .407 & .658 & .342 & 16.25 & 426 & 6,922 & 1595654 & 2.940 & .998 & .002 \\
\hline 955 & 13.34 & 2,478 & 33,055 & 41341548 & .814 & .792 & .208 & 21.04 & 1,571 & 33,055 & 25996493 & .084 & .534 & .466 \\
\hline 956 & 13.66 & 2,543 & 34,736 & 44400437 & .462 & .678 & .322 & 22.13 & 1,570 & 34,736 & 27100627 & -.421 & .337 & .337 \\
\hline 957 & 9.21 & 465 & 4,283 & 982972 & -.481 & .315 & .315 & 13.51 & 317 & 4,283 & 695189 & .742 & .771 & .229 \\
\hline 958 & 15.28 & 821 & 12,543 & 5197414 & .468 & .680 & .320 & 25.65 & 489 & 12,543 & 3119807 & .663 & .746 & .254 \\
\hline 959 & 8.75 & 1,579 & 13,823 & 11062206 & .939 & .826 & .174 & 13.22 & 1,046 & 13,823 & 7178770 & -.393 & .347 & .347 \\
\hline 960 & 9.41 & 83 & 781 & 35927 & 1.712 & .957 & .044 & 13.02 & 60 & 781 & 26210 & 1.592 & .944 & .056 \\
\hline 961 & 11.36 & 1,769 & 20,090 & 18117512 & 1.426 & .923 & .077 & 18.17 & 1,106 & 20,090 & 11205178 & .495 & .690 & .310 \\
\hline 962 & 18.92 & 38 & 719 & 16025 & 1.848 & .968 & .032 & 32.68 & 22 & 719 & 10027 & 2.176 & .985 & .015 \\
\hline 963 & 15.63 & 73 & 1,141 & 38639 & -1.069 & .143 & .143 & 23.77 & 48 & 1,141 & 24890 & -1.093 & .137 & .137 \\
\hline 964 & 15.38 & 131 & 2,015 & 133481 & .225 & .589 & .411 & 24.57 & 82 & 2,015 & 79285 & -.632 & .264 & .264 \\
\hline 965 & 12.25 & 832 & 10,194 & 4416043 & 2.066 & .981 & .019 & 19.31 & 528 & 10,194 & 2698163 & .103 & .541 & .459 \\
\hline 966 & 19.41 & 458 & 8,889 & 2022058 & -.246 & .403 & .403 & 32.09 & 277 & 8,889 & 1239354 & .193 & .576 & .424 \\
\hline 967 & 17.86 & 283 & 5,054 & 680261 & -1.421 & .078 & .078 & 35.34 & 143 & 5,054 & 346976 & -.825 & .205 & .205 \\
\hline 968 & 9.54 & 166 & 1,583 & 140600 & 1.565 & .941 & .059 & 14.52 & 109 & 1,583 & 93584 & 1.532 & .937 & .063 \\
\hline 969 & 9.82 & 124 & 1,217 & 78207 & .704 & .759 & .241 & 14.66 & 83 & 1,217 & 54587 & 1.275 & .899 & .101 \\
\hline 970 & 14.01 & 2,337 & 32,743 & 38043512 & -.474 & .318 & .318 & 22.95 & 1,427 & 32,743 & 23206397 & -.436 & .331 & .331 \\
\hline 971 & 10.31 & 167 & 1,722 & 149474 & .885 & .812 & .188 & 15.11 & 114 & 1,722 & 103482 & 1.004 & .842 & .158 \\
\hline 972 & 12.39 & 1,495 & 18,526 & 14662765 & 3.939 & 1.000 & 0 & 19.86 & 933 & 18,526 & 9176223 & 3.268 & .999 & .001 \\
\hline 973 & 29.34 & 666 & 19,541 & 6874338 & 2.522 & .994 & .006 & 59.94 & 326 & 19,541 & 3391252 & 2.023 & .978 & .022 \\
\hline 974 & 9.13 & 1,222 & 11,161 & 6930202 & .984 & .837 & .163 & 13.87 & 805 & 11,161 & 4587145 & 1.038 & .850 & .150 \\
\hline 975 & 9.57 & 1,296 & 12,404 & 7840411 & -1.531 & .063 & .063 & 15.04 & 825 & 12,404 & 5037551 & -.769 & .221 & .221 \\
\hline 976 & 11.68 & 76 & 888 & 36192 & 1.095 & .863 & .137 & 18.12 & 49 & 888 & 24259 & 1.395 & .918 & .082 \\
\hline 977 & 8.46 & 11 & 93 & 636 & 1.398 & .919 & .081 & 11.63 & 8 & 93 & 464 & 1.212 & .887 & .113 \\
\hline 978 & 10.99 & 1,215 & 13,354 & 8452912 & 2.533 & .994 & .006 & 17.08 & 782 & 13,354 & 5523370 & 2.801 & .997 & .003 \\
\hline 979 & 10.41 & 549 & 5,715 & 1545273 & -.608 & .272 & .272 & 16.47 & 347 & 5,715 & 973127 & -.600 & .274 & .274 \\
\hline 980 & 11.13 & 321 & 3,573 & 544933 & -1.544 & .061 & .061 & 16.70 & 214 & 3,573 & 355380 & -1.785 & .037 & .037 \\
\hline 981 & 15.00 & 1 & 15 & 15 & 1.732 & .958 & .042 & 15.00 & 1 & 15 & 15 & 1.732 & .958 & .042 \\
\hline 982 & 11.70 & 33 & 386 & 7076 & 1.105 & .865 & .135 & 13.79 & 28 & 386 & 5985 & .985 & .838 & .162 \\
\hline 983 & 8.20 & 1,275 & 10,455 & 6649435 & -.145 & .442 & .442 & 11.81 & 885 & 10,455 & 4725162 & 1.101 & .864 & .136 \\
\hline 984 & 17.16 & 206 & 3,534 & 363524 & -.033 & .487 & .487 & 25.42 & 139 & 3,534 & 249335 & .310 & .622 & .378 \\
\hline 985 & 11.43 & 106 & 1,212 & 68662 & 1.229 & .890 & .110 & 19.24 & 63 & 1,212 & 40522 & 0.844 & .801 & .199 \\
\hline 986 & 13.38 & 259 & 3,465 & 455623 & .429 & .666 & .334 & 19.58 & 177 & 3,465 & 334434 & 2.088 & .982 & .018 \\
\hline 987 & 14.90 & 1,508 & 22,473 & 16956770 & .048 & .519 & .481 & 23.19 & 969 & 22,473 & 10797160 & -.451 & .326 & .326 \\
\hline 988 & 11.56 & 203 & 2,346 & 210435 & -2.869 & .002 & .002 & 16.07 & 146 & 2,346 & 162777 & -1.036 & .150 & .150 \\
\hline
\end{tabular}


Table 4. Summary of interoccurrence intervals for daily precipitation thresholds of 0.25 and 0.50 inch-Continued

\begin{tabular}{|c|c|c|c|c|c|c|c|c|c|c|c|c|c|c|}
\hline \multirow[b]{2}{*}{$\begin{array}{l}\text { Seq. } \\
\text { no. }\end{array}$} & \multicolumn{7}{|c|}{ Daily precipitation threshold of 0.25 inch and greater } & \multicolumn{7}{|c|}{ Daily precipitation threshold of 0.50 inch and greater } \\
\hline & $\begin{array}{l}\text { Mean } \\
\text { inter- } \\
\text { occur- } \\
\text { rence } \\
\text { interval } \\
\text { (days) }\end{array}$ & $\begin{array}{c}\text { Total } \\
\text { no. of } \\
\text { events }\end{array}$ & $\begin{array}{l}\text { Total } \\
\text { no. of } \\
\text { days } \\
\text { avail- } \\
\text { able }\end{array}$ & $\begin{array}{l}\text { Sigma } \\
\text { S }\end{array}$ & $\begin{array}{c}\text { Stan- } \\
\text { dard } \\
\text { normal } \\
\text { variate }\end{array}$ & $\begin{array}{l}\text { Non- } \\
\text { ex- } \\
\text { ceed- } \\
\text { ance } \\
\text { prob- } \\
\text { ability }\end{array}$ & $\begin{array}{c}p- \\
\text { value }\end{array}$ & $\begin{array}{l}\text { Mean } \\
\text { inter- } \\
\text { occur- } \\
\text { rence } \\
\text { interval } \\
\text { (days) }\end{array}$ & $\begin{array}{c}\text { Total } \\
\text { no. of } \\
\text { events }\end{array}$ & $\begin{array}{l}\text { Total } \\
\text { no. of } \\
\text { days } \\
\text { avail- } \\
\text { able }\end{array}$ & $\begin{array}{l}\text { Sigma } \\
\mathrm{S}\end{array}$ & $\begin{array}{l}\text { Stan- } \\
\text { dard } \\
\text { normal } \\
\text { variate }\end{array}$ & $\begin{array}{l}\text { Non- } \\
\text { ex- } \\
\text { ceed- } \\
\text { ance } \\
\text { prob- } \\
\text { ability }\end{array}$ & $\begin{array}{c}\mathrm{p}- \\
\text { value }\end{array}$ \\
\hline 989 & 8.22 & 1,967 & 16,159 & 16015761 & .596 & .724 & .276 & 12.49 & 1,294 & 16,159 & 10665760 & 1.257 & .896 & .104 \\
\hline 990 & 9.64 & 1,933 & 18,639 & 19093740 & 4.562 & 1.000 & 0 & 15.14 & 1,231 & 18,639 & 12127475 & 3.471 & 1.000 & 0 \\
\hline 991 & 12.53 & 1,483 & 18,574 & 14192376 & 2.033 & .979 & .021 & 18.93 & 981 & 18,574 & 9592248 & 2.868 & .998 & .002 \\
\hline 992 & 8.48 & 2,356 & 19,989 & 24367254 & 2.929 & .998 & .002 & 12.86 & 1,554 & 19,989 & 16244767 & 3.136 & .999 & .001 \\
\hline 993 & 11.80 & 116 & 1,369 & 86327 & 1.627 & .948 & .052 & 18.25 & 75 & 1,369 & 59002 & 2.239 & .987 & .013 \\
\hline 994 & 13.03 & 474 & 6,177 & 1538239 & 1.914 & .972 & .028 & 20.80 & 297 & 6,177 & 981477 & 2.089 & .982 & .018 \\
\hline 995 & 11.52 & 1,430 & 16,470 & 12076845 & 1.673 & .953 & .047 & 17.77 & 927 & 16,470 & 7832664 & 1.374 & .915 & .085 \\
\hline 996 & 19.56 & 1,475 & 28,850 & 21690733 & 1.294 & .902 & .098 & 32.09 & 899 & 28,850 & 13470342 & 2.011 & .978 & .022 \\
\hline 997 & 13.30 & 2,072 & 27,555 & 28876153 & .909 & .818 & .182 & 19.75 & 1,395 & 27,555 & 19563985 & 1.159 & .877 & .123 \\
\hline 998 & 12.23 & 1,743 & 21,324 & 19117467 & 2.076 & .981 & .019 & 19.09 & 1,117 & 21,324 & 12335214 & 2.070 & .981 & .019 \\
\hline 999 & 12.63 & 289 & 3,649 & 498453 & -1.610 & .054 & .054 & 18.71 & 195 & 3,649 & 333881 & -1.489 & .068 & .068 \\
\hline 1000 & 8.88 & 1,015 & 9,008 & 4572842 & .016 & .506 & .494 & 13.80 & 653 & 9,008 & 2991465 & .758 & .776 & .224 \\
\hline 1001 & 10.00 & 1,972 & 19,714 & 20364699 & 3.667 & 1.000 & 0 & 15.34 & 1,285 & 19,714 & 13461744 & 3.900 & 1.000 & 0 \\
\hline 1002 & 4.67 & 3 & 14 & 31 & 1.429 & .923 & .077 & 4.67 & 3 & 14 & 31 & 1.429 & .923 & .077 \\
\hline 1003 & 15.43 & 1,319 & 20,356 & 14048605 & 2.923 & .998 & .002 & 25.87 & 787 & 20,356 & 8387163 & 2.287 & .989 & .011 \\
\hline 1004 & 13.14 & 1,551 & 20,384 & 16359102 & 2.379 & .991 & .009 & 20.55 & 992 & 20,384 & 10287133 & .953 & .830 & .170 \\
\hline 1005 & 14.87 & 1,436 & 21,346 & 15348745 & .096 & .538 & .462 & 23.61 & 904 & 21,346 & 9685859 & .202 & .580 & .420 \\
\hline 1006 & 9.16 & 309 & 2,830 & 432415 & -.336 & .369 & .369 & 14.01 & 202 & 2,830 & 282867 & -.255 & .399 & .399 \\
\hline 1007 & 13.83 & 612 & 8,461 & 2515018 & -1.226 & .110 & .110 & 19.41 & 436 & 8,461 & 1768363 & -1.493 & .068 & .068 \\
\hline 1008 & 9.83 & 1,414 & 13,894 & 9801188 & -.145 & .442 & .442 & 14.70 & 945 & 13,894 & 6472045 & -.753 & .226 & .226 \\
\hline 1009 & 7.73 & 1,620 & 12,527 & 10183571 & .252 & .600 & .400 & 12.14 & 1,032 & 12,527 & 6439324 & -.212 & .416 & .416 \\
\hline 1010 & 11.39 & 1,375 & 15,655 & 10680232 & -.493 & .311 & .311 & 17.65 & 887 & 15,655 & 6783132 & -1.188 & .118 & .118 \\
\hline 1011 & 15.01 & 1,359 & 20,404 & 13992029 & .587 & .721 & .279 & 23.64 & 863 & 20,404 & 9007648 & 1.175 & .880 & .120 \\
\hline 1012 & 14.60 & 205 & 2,993 & 299823 & -.563 & .287 & .287 & 25.15 & 119 & 2,993 & 185643 & .802 & .789 & .211 \\
\hline 1013 & 14.28 & 765 & 10,925 & 4331853 & 1.755 & .960 & .040 & 23.85 & 458 & 10,925 & 2539935 & .565 & .714 & .286 \\
\hline 1014 & 14.28 & 164 & 2,342 & 199431 & .853 & .803 & .197 & 21.29 & 110 & 2,342 & 137362 & 1.206 & .886 & .114 \\
\hline 1015 & 23.72 & 18 & 427 & 3422 & -.805 & .210 & .210 & 42.70 & 10 & 427 & 1452 & -1.752 & .040 & .040 \\
\hline 1016 & 9.48 & 2,113 & 20,037 & 21528049 & 1.350 & .911 & .089 & 14.46 & 1,386 & 20,037 & 14031593 & .678 & .751 & .249 \\
\hline 1017 & 10.43 & 179 & 1,867 & 182363 & 2.117 & .983 & .017 & 16.38 & 114 & 1,867 & 117267 & 1.885 & .970 & .030 \\
\hline 1018 & 9.68 & 250 & 2,421 & 287567 & -1.363 & .087 & .087 & 11.26 & 215 & 2,421 & 247108 & -1.283 & .100 & .100 \\
\hline 1019 & 15.10 & 534 & 8,062 & 2095664 & -1.058 & .145 & 145 & 27.33 & 295 & 8,062 & 1212751 & .591 & .723 & .277 \\
\hline 1020 & 15.74 & 374 & 5,888 & 1170747 & 2.120 & .983 & .017 & 24.84 & 237 & 5,888 & 731326 & 1.284 & .900 & .100 \\
\hline 1021 & 14.58 & 1,664 & 24,253 & 20449628 & .949 & .829 & .171 & 23.85 & 1,017 & 24,253 & 12359936 & .122 & .549 & .451 \\
\hline 1022 & 9.52 & 1,037 & 9,875 & 5062573 & -.628 & .265 & .265 & 13.83 & 714 & 9,875 & 3540028 & .192 & .576 & .424 \\
\hline 1023 & 9.49 & 710 & 6,740 & 2283249 & -2.111 & .017 & .017 & 14.46 & 466 & 6,740 & 1493655 & -1.828 & .034 & .034 \\
\hline 1024 & 10.30 & 1,139 & 11,727 & 6672767 & -.050 & .480 & .480 & 15.17 & 773 & 11,727 & 4615638 & .884 & .811 & .189 \\
\hline 1025 & 13.20 & 214 & 2,824 & 288493 & -1.147 & .126 & .126 & 19.61 & 144 & 2,824 & 189995 & -1.363 & .086 & .086 \\
\hline 1026 & 9.59 & 1,993 & 19,114 & 19700443 & 2.652 & .996 & .004 & 14.94 & 1,279 & 19,114 & 12594279 & 1.880 & .970 & .030 \\
\hline 1027 & 13.96 & 484 & 6,758 & 1682418 & 1.095 & .863 & .137 & 22.45 & 301 & 6,758 & 1031087 & .414 & .661 & .339 \\
\hline 1028 & 14.62 & 1,872 & 27,372 & 25937652 & .929 & .823 & .177 & 23.99 & 1,141 & 27,372 & 16433883 & 3.065 & .999 & .001 \\
\hline 1029 & 11.11 & 1,400 & 15,548 & 11158936 & 1.640 & .949 & .051 & 17.75 & 876 & 15,548 & 7116915 & 2.310 & .990 & .010 \\
\hline 1030 & 10.06 & 1,217 & 12,246 & 7444940 & -.055 & .478 & .478 & 15.90 & 770 & 12,246 & 4863667 & 1.519 & .936 & .064 \\
\hline 1031 & 19.67 & 129 & 2,538 & 142080 & -2.598 & .005 & .005 & 39.05 & 65 & 2,538 & 68257 & -2.409 & .008 & .008 \\
\hline 1032 & 12.16 & 2,997 & 36,443 & 54626381 & .029 & .511 & .489 & 19.70 & 1,850 & 36,443 & 33535070 & -.386 & .350 & .350 \\
\hline 1033 & 7.80 & 2,749 & 21,434 & 29544646 & .258 & .602 & .398 & 11.55 & 1,856 & 21,434 & 20276426 & 1.447 & .926 & .074 \\
\hline 1034 & 16.91 & 128 & 2,164 & 144174 & .803 & .789 & .211 & 28.10 & 77 & 2,164 & 85459 & .391 & .652 & .348 \\
\hline 1035 & 14.42 & 2,344 & 33,805 & 39603497 & -.034 & .487 & .487 & 23.69 & 1,427 & 33,805 & 24231723 & .303 & .619 & .381 \\
\hline 1036 & 6.54 & 82 & 536 & 22444 & .334 & .631 & .369 & 9.40 & 57 & 536 & 14164 & -.952 & .171 & .171 \\
\hline 1037 & 11.83 & 36 & 426 & 7721 & 0.072 & .529 & .471 & 18.52 & 23 & 426 & 3911 & -1.675 & .047 & .047 \\
\hline 1038 & 11.97 & 290 & 3,471 & 526390 & 1.354 & .912 & .088 & 17.89 & 194 & 3,471 & 351925 & 1.092 & .863 & .138 \\
\hline 1039 & 27.57 & 254 & 7,002 & 883876 & -.167 & .434 & .434 & 68.65 & 102 & 7,002 & 384944 & 1.364 & .914 & .086 \\
\hline 1040 & 33.35 & 206 & 6,871 & 764478 & 1.994 & .977 & .023 & 70.11 & 98 & 6,871 & 375252 & 1.965 & .975 & .025 \\
\hline
\end{tabular}


Table 4. Summary of interoccurrence intervals for daily precipitation thresholds of 0.25 and 0.50 inch-Continued

\begin{tabular}{|c|c|c|c|c|c|c|c|c|c|c|c|c|c|c|}
\hline \multirow[b]{2}{*}{$\begin{array}{c}\text { Seq. } \\
\text { no. }\end{array}$} & \multicolumn{7}{|c|}{ Daily precipitation threshold of 0.25 inch and greater } & \multicolumn{7}{|c|}{ Daily precipitation threshold of 0.50 inch and greater } \\
\hline & $\begin{array}{c}\text { Mean } \\
\text { inter- } \\
\text { occur- } \\
\text { rence } \\
\text { interval } \\
\text { (days) }\end{array}$ & $\begin{array}{c}\text { Total } \\
\text { no. of } \\
\text { events }\end{array}$ & $\begin{array}{l}\text { Total } \\
\text { no. of } \\
\text { days } \\
\text { avail- } \\
\text { able }\end{array}$ & $\begin{array}{l}\text { Sigma } \\
\mathbf{S}\end{array}$ & $\begin{array}{c}\text { Stan- } \\
\text { dard } \\
\text { normal } \\
\text { variate }\end{array}$ & $\begin{array}{l}\text { Non- } \\
\text { ex- } \\
\text { ceed- } \\
\text { ance } \\
\text { prob- } \\
\text { ability }\end{array}$ & $\begin{array}{c}\text { p- } \\
\text { value }\end{array}$ & $\begin{array}{c}\text { Mean } \\
\text { inter- } \\
\text { occur- } \\
\text { rence } \\
\text { interval } \\
\text { (days) }\end{array}$ & $\begin{array}{c}\text { Total } \\
\text { no. of } \\
\text { events }\end{array}$ & $\begin{array}{l}\text { Total } \\
\text { no. of } \\
\text { days } \\
\text { avail- } \\
\text { able }\end{array}$ & $\begin{array}{l}\text { Sigma } \\
\mathbf{S}\end{array}$ & $\begin{array}{l}\text { Stan- } \\
\text { dard } \\
\text { normal } \\
\text { variate }\end{array}$ & $\begin{array}{l}\text { Non- } \\
\text { ex- } \\
\text { ceed- } \\
\text { ance } \\
\text { prob- } \\
\text { ability }\end{array}$ & $\begin{array}{c}p- \\
\text { value }\end{array}$ \\
\hline 1041 & 41.18 & 85 & 3,500 & 143163 & -.600 & .274 & .274 & 97.22 & 36 & 3,500 & 61780 & -.201 & .420 & .420 \\
\hline 1042 & 6.78 & 1,830 & 12,414 & 11291906 & -.436 & .331 & .331 & 9.55 & 1,300 & 12,414 & 8052989 & -.125 & .450 & .450 \\
\hline 1043 & 23.55 & 20 & 471 & 3827 & -1.452 & .073 & .073 & 42.82 & 11 & 471 & 1449 & -2.531 & .006 & .006 \\
\hline 1044 & 17.09 & 479 & 8,188 & 2122627 & 3.124 & .999 & .001 & 29.88 & 274 & 8,188 & 1216279 & 2.416 & .992 & .008 \\
\hline 1045 & 16.96 & 1,179 & 19,999 & 12396330 & 3.062 & .999 & .001 & 29.72 & 673 & 19,999 & 7140937 & 2.746 & .997 & .003 \\
\hline 1046 & 11.30 & 151 & 1,706 & 128909 & .018 & .507 & .493 & 18.15 & 94 & 1,706 & 82404 & .465 & .679 & .321 \\
\hline 1047 & 12.44 & 1,625 & 20,211 & 17278002 & 3.642 & 1.000 & 0 & 19.99 & 1,011 & 20,211 & 10747107 & 2.859 & .998 & .002 \\
\hline 1048 & 13.66 & 879 & 12,010 & 5077204 & -1.957 & .025 & .025 & 20.92 & 574 & 12,010 & 3417667 & -.352 & .363 & .363 \\
\hline 1049 & 12.34 & 397 & 4,897 & 959633 & -.441 & .330 & .330 & 18.69 & 262 & 4,897 & 653756 & .535 & .704 & .296 \\
\hline 1050 & 7.32 & 1,646 & 12,043 & 10261242 & 2.480 & .993 & .007 & 10.69 & 1,127 & 12,043 & 7093797 & 2.635 & .996 & .004 \\
\hline 1051 & 14.45 & 1,236 & 17,865 & 10881243 & -.879 & .190 & .190 & 22.90 & 780 & 17,865 & 6766015 & -1.398 & .081 & .081 \\
\hline 1052 & 14.20 & 51 & 724 & 18159 & -.203 & .420 & .420 & 24.97 & 29 & 724 & 11014 & .459 & .677 & .323 \\
\hline 1053 & 7.40 & 141 & 1,044 & 74185 & .163 & .565 & .435 & 10.34 & 101 & 1,044 & 51409 & -.434 & .332 & .332 \\
\hline 1054 & 20.33 & 3 & 61 & 75 & -.541 & .294 & .294 & 30.50 & 2 & 61 & 24 & -1.486 & .069 & .069 \\
\hline 1055 & 8.89 & 376 & 3,344 & 631239 & .137 & .555 & .445 & 12.48 & 268 & 3,344 & 464975 & 1.068 & .857 & .143 \\
\hline 1056 & 16.42 & 128 & 2,102 & 149940 & 2.245 & .988 & .012 & 29.19 & 72 & 2,102 & 82651 & 1.356 & .912 & .088 \\
\hline 1057 & 18.82 & 27 & 508 & 7068 & .276 & .609 & .391 & 39.08 & 13 & 508 & 3595 & .554 & .710 & .290 \\
\hline 1058 & 10.98 & 3,209 & 35,247 & 57276842 & 1.254 & .895 & .105 & 16.87 & 2,089 & 35,247 & 37311044 & 1.066 & .857 & .143 \\
\hline 1059 & 15.54 & 46 & 715 & 15457 & -.706 & .240 & .240 & 23.07 & 31 & 715 & 9736 & -1.172 & .121 & .121 \\
\hline 1060 & 12.85 & 1,886 & 24,233 & 23119542 & .882 & .811 & .189 & 20.68 & 1,172 & 24,233 & 14510966 & 1.296 & .903 & .097 \\
\hline 1061 & 11.38 & 58 & 660 & 19208 & .047 & .519 & .481 & 22.76 & 29 & 660 & 9144 & -.415 & .339 & .339 \\
\hline 1062 & 20.19 & 103 & 2,080 & 87976 & -3.142 & .001 & .001 & 33.55 & 62 & 2,080 & 54874 & -2.032 & .021 & .021 \\
\hline 1063 & 24.11 & 990 & 23,869 & 12122449 & 1.417 & .922 & .078 & 43.80 & 545 & 23,869 & 6788159 & 1.765 & .961 & .039 \\
\hline 1064 & 27.53 & 45 & 1,239 & 28510 & .264 & .604 & .396 & 45.89 & 27 & 1,239 & 17653 & .499 & .691 & .309 \\
\hline 1065 & 12.97 & 468 & 6,070 & 1418740 & -.043 & .483 & .483 & 20.58 & 295 & 6,070 & 923950 & .951 & .829 & .171 \\
\hline 1066 & 10.33 & 1,076 & 11,113 & 6090819 & 1.065 & .856 & .144 & 16.06 & 692 & 11,113 & 4011757 & 1.975 & .976 & .024 \\
\hline 1067 & 11.91 & 77 & 917 & 40436 & 2.209 & .986 & .014 & 16.09 & 57 & 917 & 29821 & 1.845 & .967 & .033 \\
\hline 1068 & 14.61 & 49 & 716 & 17022 & -.359 & .360 & .360 & 28.64 & 25 & 716 & 8095 & -.827 & .204 & .204 \\
\hline 1069 & 16.52 & 328 & 5,418 & 867495 & -.743 & .229 & .229 & 28.67 & 189 & 5,418 & 492291 & -.917 & .180 & .180 \\
\hline 1070 & 13.77 & 2,559 & 35,225 & 44493411 & -1.122 & .131 & .131 & 22.02 & 1,600 & 35,225 & 27978640 & -.495 & .310 & .310 \\
\hline 1071 & 9.91 & 1,981 & 19,631 & 19707976 & 1.045 & .852 & .148 & 15.62 & 1,257 & 19,631 & 12850638 & 2.551 & .995 & .005 \\
\hline 1072 & 12.00 & 139 & 1,668 & 112439 & -.614 & .270 & .270 & 16.35 & 102 & 1,668 & 84766 & -.062 & .475 & .475 \\
\hline 1073 & 70.67 & 3 & 212 & 357 & .368 & .644 & .356 & 212.00 & 1 & 212 & 130 & .392 & .653 & .347 \\
\hline 1074 & 9.34 & 3,223 & 30,105 & 48967986 & .920 & .821 & .179 & 14.13 & 2,130 & 30,105 & 32662064 & 1.497 & .933 & .067 \\
\hline 1075 & 14.30 & 181 & 2,589 & 237494 & .317 & .624 & .376 & 22.91 & 113 & 2,589 & 147868 & .200 & .579 & .421 \\
\hline 1076 & 12.11 & 1,490 & 18,047 & 13346504 & -.490 & .312 & .312 & 19.26 & 937 & 18,047 & 8545305 & .566 & .714 & .286 \\
\hline 1077 & 11.77 & 292 & 3,438 & 518010 & .947 & .828 & .172 & 17.28 & 199 & 3,438 & 360123 & 1.289 & .901 & .099 \\
\hline 1078 & 19.31 & 1,425 & 27,513 & 19688009 & .284 & .612 & .388 & 35.23 & 781 & 27,513 & 10640997 & -.463 & .322 & .322 \\
\hline 1079 & 12.70 & 2,464 & 31,290 & 39452855 & 2.015 & .978 & .022 & 20.74 & 1,509 & 31,290 & 24030581 & 1.204 & .886 & .114 \\
\hline 1080 & 12.41 & 39 & 484 & 10130 & .793 & .786 & .214 & 20.17 & 24 & 484 & 6549 & 1.083 & .860 & .140 \\
\hline 1081 & 13.69 & 1,551 & 21,232 & 16738975 & 1.133 & .871 & .129 & 23.88 & 889 & 21,232 & 9637910 & 1.096 & .863 & .137 \\
\hline 1082 & 13.72 & 998 & 13,695 & 7169346 & 2.687 & .996 & .004 & 23.37 & 586 & 13,695 & 4243691 & 2.414 & .992 & .008 \\
\hline 1083 & 22.01 & 701 & 15,430 & 5464314 & .476 & .683 & .317 & 36.65 & 421 & 15,430 & 3392734 & 1.584 & .943 & .057 \\
\hline 1084 & 9.36 & 130 & 1,217 & 77637 & -.367 & .357 & .357 & 14.66 & 83 & 1,217 & 49513 & -.310 & .378 & .378 \\
\hline 1085 & 9.03 & 4,026 & 36,335 & 74023922 & 1.325 & .907 & .093 & 13.75 & 2,643 & 36,335 & 48491084 & .880 & .810 & .190 \\
\hline 1086 & 19.20 & 263 & 5,049 & 694129 & 1.277 & .899 & .101 & 30.42 & 166 & 5,049 & 416587 & -.132 & .447 & .447 \\
\hline 1087 & 24.65 & 570 & 14,050 & 4000169 & -.042 & .483 & .483 & 55.98 & 251 & 14,050 & 1731200 & -.499 & .309 & .309 \\
\hline 1088 & 7.71 & 292 & 2,252 & 331126 & .210 & .583 & .417 & 10.88 & 207 & 2,252 & 236362 & .351 & .637 & .363 \\
\hline 1089 & 19.13 & 62 & 1,186 & 33651 & -1.156 & .124 & .124 & 27.58 & 43 & 1,186 & 22985 & -1.120 & .131 & .131 \\
\hline 1090 & 13.33 & 764 & 10,180 & 3938128 & .608 & .728 & .272 & 20.90 & 487 & 10,180 & 2532555 & .828 & .796 & .204 \\
\hline 1091 & 14.41 & 1,367 & 19,700 & 13748064 & 1.347 & .911 & .089 & 26.62 & 740 & 19,700 & 7353021 & .414 & .660 & .340 \\
\hline 1092 & 5.13 & 24 & 123 & 949 & -3.030 & .001 & .001 & 7.24 & 17 & 123 & 717 & -2.244 & .012 & .012 \\
\hline
\end{tabular}


Table 4. Summary of interoccurrence intervals for daily precipitation thresholds of 0.25 and 0.50 inch-Continued

\begin{tabular}{|c|c|c|c|c|c|c|c|c|c|c|c|c|c|c|}
\hline \multirow[b]{2}{*}{$\begin{array}{l}\text { Seq. } \\
\text { no. }\end{array}$} & \multicolumn{7}{|c|}{ Daily precipitation threshold of 0.25 inch and greater } & \multicolumn{7}{|c|}{ Daily precipitation threshold of 0.50 inch and greater } \\
\hline & $\begin{array}{l}\text { Mean } \\
\text { inter- } \\
\text { occur- } \\
\text { rence } \\
\text { interval } \\
\text { (days) }\end{array}$ & $\begin{array}{c}\text { Total } \\
\text { no. of } \\
\text { events }\end{array}$ & $\begin{array}{l}\text { Total } \\
\text { no. of } \\
\text { days } \\
\text { avail- } \\
\text { able }\end{array}$ & $\begin{array}{l}\text { Sigma } \\
\text { S }\end{array}$ & $\begin{array}{c}\text { Stan- } \\
\text { dard } \\
\text { normal } \\
\text { variate }\end{array}$ & $\begin{array}{l}\text { Non- } \\
\text { ex- } \\
\text { ceed- } \\
\text { ance } \\
\text { prob- } \\
\text { ability }\end{array}$ & $\begin{array}{c}p- \\
\text { value }\end{array}$ & $\begin{array}{l}\text { Mean } \\
\text { inter- } \\
\text { occur- } \\
\text { rence } \\
\text { interval } \\
\text { (days) }\end{array}$ & $\begin{array}{c}\text { Total } \\
\text { no. of } \\
\text { events }\end{array}$ & $\begin{array}{l}\text { Total } \\
\text { no. of } \\
\text { days } \\
\text { avail- } \\
\text { able }\end{array}$ & $\begin{array}{l}\text { Sigma } \\
\mathrm{S}\end{array}$ & $\begin{array}{c}\text { Stan- } \\
\text { dard } \\
\text { normal } \\
\text { variate }\end{array}$ & $\begin{array}{l}\text { Non- } \\
\text { ex- } \\
\text { ceed- } \\
\text { ance } \\
\text { prob- } \\
\text { ability }\end{array}$ & $\begin{array}{c}\mathrm{p}- \\
\text { value }\end{array}$ \\
\hline 1093 & 7.58 & 628 & 4,757 & 1525800 & .933 & .824 & .176 & 10.96 & 434 & 4,757 & 1066724 & 1.204 & .886 & .114 \\
\hline 1094 & 12.31 & 2,097 & 25,807 & 27290053 & 678 & .751 & 249 & 18.78 & 1,374 & 25,807 & 18196218 & 1.690 & .955 & .045 \\
\hline 1095 & 9.24 & 214 & 1,978 & 206099 & -.664 & .253 & .253 & 14.76 & 134 & 1,978 & 129617 & -.440 & .330 & .330 \\
\hline 1096 & 16.01 & 1,193 & 19,104 & 11613874 & 1.146 & .874 & .126 & 28.73 & 665 & 19,104 & 6564377 & 1.493 & .932 & .068 \\
\hline 1097 & 9.73 & 1,955 & 19,028 & 19745013 & 4.715 & 1.000 & 0 & 13.94 & 1,365 & 19,028 & 14138045 & 5.674 & 0 & 0 \\
\hline 1098 & 12.16 & 1,215 & 14,774 & 8900839 & -.500 & .309 & .309 & 19.26 & 767 & 14,774 & 5671558 & .049 & .519 & .481 \\
\hline 1099 & 20.60 & 10 & 206 & 666 & -1.936 & .026 & .026 & 51.50 & 4 & 206 & 226 & -1.564 & .059 & .059 \\
\hline 1100 & 12.21 & 231 & 2,820 & 290486 & -2.847 & .002 & .002 & 19.58 & 144 & 2,820 & 185009 & -1.846 & .032 & .032 \\
\hline 1101 & 9.90 & 2,948 & 29,198 & 43002249 & -.078 & .469 & .469 & 15.37 & 1,900 & 29,198 & 27727546 & -.029 & .489 & .489 \\
\hline 1102 & 15.51 & 2,035 & 31,561 & 31656430 & -1.112 & .133 & .133 & 26.06 & 1,211 & 31,561 & 18910572 & -.630 & .265 & .265 \\
\hline 1103 & 38.62 & 271 & 10,466 & 1403531 & -.294 & .384 & .384 & 84.40 & 124 & 10,466 & 654316 & .161 & .564 & .436 \\
\hline 1104 & 10.75 & 727 & 7,812 & 2996753 & 2.584 & .995 & .005 & 16.04 & 487 & 7,812 & 1997383 & 1.912 & .972 & .028 \\
\hline 1105 & 9.15 & 1,448 & 13,243 & 9864724 & 1.903 & .971 & .029 & 14.85 & 892 & 13,243 & 6109634 & 1.780 & .962 & .038 \\
\hline 1106 & 15.68 & 1,434 & 22,491 & 16414557 & 1.174 & .880 & .120 & 25.70 & 875 & 22,491 & 9931057 & .475 & .683 & .317 \\
\hline 1107 & 17.50 & 92 & 1,610 & 71474 & -.580 & .281 & .281 & 29.27 & 55 & 1,610 & 37437 & -1.984 & .024 & .024 \\
\hline 1108 & -- & -- & -- & -- & -- & -- & -- & -- & -- & -- & -- & -- & -- & -- \\
\hline 1109 & 13.23 & 264 & 3,493 & 439988 & -1.287 & .099 & .099 & 20.19 & 173 & 3,493 & 281385 & -1.565 & .059 & .059 \\
\hline 1110 & 16.71 & 210 & 3,510 & 334833 & -2.296 & .011 & .011 & 25.44 & 138 & 3,510 & 212132 & -2.525 & .006 & .006 \\
\hline 1111 & 15.86 & 142 & 2,252 & 152374 & -.971 & .166 & .166 & 28.51 & 79 & 2,252 & 84072 & -.845 & .199 & .199 \\
\hline 1112 & 8.77 & 1,370 & 12,016 & 8300879 & .545 & .707 & .293 & 13.31 & 903 & 12,016 & 5549933 & 1.196 & .884 & .116 \\
\hline 1113 & 15.01 & 1,965 & 29,498 & 28698962 & -.749 & .227 & .227 & 26.87 & 1,098 & 29,498 & 16024037 & -.604 & .273 & .273 \\
\hline 1114 & 10.85 & 1,117 & 12,124 & 6778999 & .066 & .526 & .474 & 17.00 & 713 & 12,124 & 4233279 & -.952 & .171 & .171 \\
\hline 1115 & 14.42 & 403 & 5,813 & 1250228 & 2.342 & .990 & .010 & 24.95 & 233 & 5,813 & 692392 & .593 & .723 & .277 \\
\hline 1116 & 8.81 & 165 & 1,453 & 110458 & -1.747 & .040 & .040 & 11.53 & 126 & 1,453 & 83676 & -1.670 & .047 & .047 \\
\hline 1117 & 10.88 & 1,445 & 15,727 & 11533577 & .990 & .839 & .161 & 17.36 & 906 & 15,727 & 7113805 & -.077 & .469 & .469 \\
\hline 1118 & 11.11 & 72 & 800 & 31584 & 1.421 & .922 & .078 & 18.18 & 44 & 800 & 19672 & 1.353 & .912 & .088 \\
\hline 1119 & 10.79 & 703 & 7,586 & 2645359 & -.364 & .358 & .358 & 17.52 & 433 & 7,586 & 1582730 & -1.309 & .095 & .095 \\
\hline 1120 & 15.29 & 1,600 & 24,462 & 19485050 & -.299 & .382 & .382 & 25.80 & 948 & 24,462 & 11488929 & -.488 & .313 & .313 \\
\hline 1121 & 13.91 & 2,133 & 29,675 & 32140186 & 1.243 & .893 & .107 & 22.21 & 1,336 & 29,675 & 19818501 & -.014 & .494 & .494 \\
\hline 1122 & 17.31 & 59 & 1,021 & 30769 & .287 & .613 & .387 & 24.31 & 42 & 1,021 & 20054 & -.726 & .234 & .234 \\
\hline 1123 & 11.41 & 1,998 & 22,804 & 23092564 & 1.058 & .855 & 145 & 18.89 & 1,207 & 22,804 & 13822865 & .265 & .605 & .395 \\
\hline 1124 & 9.72 & 141 & 1,371 & 90941 & -1.216 & .112 & .112 & 15.23 & 90 & 1,371 & 54260 & -1.980 & .024 & .024 \\
\hline 1125 & 12.59 & 224 & 2,820 & 339615 & 1.951 & .974 & .026 & 18.19 & 155 & 2,820 & 221252 & .267 & .605 & .395 \\
\hline 1126 & 22.59 & 171 & 3,863 & 292259 & -2.608 & .005 & .005 & 32.46 & 119 & 3,863 & 215064 & -1.215 & .112 & .112 \\
\hline 1127 & 16.20 & 1,648 & 26,696 & 21645124 & -1.126 & .130 & .130 & 26.88 & 993 & 26,696 & 12571074 & -2.815 & .002 & .002 \\
\hline 1128 & 16.15 & 597 & 9,641 & 2868482 & -.138 & .445 & .445 & 26.13 & 369 & 9,641 & 1736798 & -.785 & .216 & .216 \\
\hline 1129 & 21.29 & 169 & 3,598 & 322896 & 1.397 & .919 & .081 & 38.28 & 94 & 3,598 & 174209 & .507 & .694 & .306 \\
\hline 1130 & 47.19 & 16 & 755 & 4758 & -1.471 & .071 & .071 & 83.89 & 9 & 755 & 3055 & -.524 & .300 & .300 \\
\hline 1131 & 10.08 & 1,374 & 13,853 & 9725930 & 1.409 & .921 & .079 & 15.83 & 875 & 13,853 & 6315066 & 2.150 & .984 & .016 \\
\hline 1132 & 21.09 & 33 & 696 & 12279 & .689 & .754 & .246 & 31.64 & 22 & 696 & 7936 & .297 & .617 & .383 \\
\hline 1133 & 11.59 & 871 & 10,098 & 4326753 & -.824 & .205 & .205 & 17.50 & 577 & 10,098 & 2906937 & -.091 & .464 & .464 \\
\hline 1134 & 15.93 & 43 & 685 & 15213 & .374 & .646 & .354 & 24.46 & 28 & 685 & 10257 & .638 & .738 & .262 \\
\hline 1135 & 7.74 & 102 & 789 & 39091 & -.499 & .309 & .309 & 9.39 & 84 & 789 & 30492 & -1.268 & .103 & .103 \\
\hline 1136 & 17.08 & 1,632 & 27,875 & 23043573 & .915 & .820 & .180 & 32.19 & 866 & 27,875 & 12401283 & 1.400 & .919 & .081 \\
\hline 1137 & 11.42 & 1,677 & 19,145 & 16312357 & 1.146 & .874 & .126 & 18.18 & 1,053 & 19,145 & 10224690 & .808 & .790 & .210 \\
\hline 1138 & 21.85 & 113 & 2,469 & 135136 & -.576 & .282 & .282 & 47.48 & 52 & 2,469 & 68216 & .783 & .783 & .217 \\
\hline 1139 & 33.88 & 92 & 3,117 & 139526 & -.447 & .328 & .328 & 52.83 & 59 & 3,117 & 81364 & -1.532 & .063 & .063 \\
\hline 1140 & 16.07 & 693 & 11,137 & 3622667 & -2.792 & .003 & .003 & 26.71 & 417 & 11,137 & 2222638 & -1.515 & .065 & .065 \\
\hline 1141 & 8.41 & 2,373 & 19,965 & 24040206 & 1.253 & .895 & .105 & 12.75 & 1,566 & 19,965 & 16208211 & 2.524 & .994 & .006 \\
\hline 1142 & 8.50 & 3,851 & 32,715 & 65385948 & 4.084 & 1.000 & 0 & 12.35 & 2,649 & 32,715 & 44393476 & 2.186 & .986 & .014 \\
\hline 1143 & 19.90 & 453 & 9,015 & 2058911 & .307 & .621 & .379 & 34.54 & 261 & 9,015 & 1190768 & .340 & .633 & .367 \\
\hline 1144 & 15.39 & 156 & 2,401 & 189712 & .281 & .611 & .389 & 21.63 & 111 & 2,401 & 137327 & .558 & .711 & .289 \\
\hline
\end{tabular}


Table 4. Summary of interoccurrence intervals for daily precipitation thresholds of 0.25 and 0.50 inch-Continued

\begin{tabular}{|c|c|c|c|c|c|c|c|c|c|c|c|c|c|c|}
\hline \multirow[b]{2}{*}{$\begin{array}{c}\text { Seq. } \\
\text { no. }\end{array}$} & \multicolumn{7}{|c|}{ Daily precipitation threshold of 0.25 inch and greater } & \multicolumn{7}{|c|}{ Daily precipitation threshold of 0.50 inch and greater } \\
\hline & $\begin{array}{c}\text { Mean } \\
\text { inter- } \\
\text { occur- } \\
\text { rence } \\
\text { interval } \\
\text { (days) }\end{array}$ & $\begin{array}{c}\text { Total } \\
\text { no. of } \\
\text { events }\end{array}$ & $\begin{array}{l}\text { Total } \\
\text { no. of } \\
\text { days } \\
\text { avail- } \\
\text { able }\end{array}$ & $\begin{array}{l}\text { Sigma } \\
\mathbf{S}\end{array}$ & $\begin{array}{c}\text { Stan- } \\
\text { dard } \\
\text { normal } \\
\text { variate }\end{array}$ & $\begin{array}{l}\text { Non- } \\
\text { ex- } \\
\text { ceed- } \\
\text { ance } \\
\text { prob- } \\
\text { ability }\end{array}$ & $\begin{array}{c}\text { p- } \\
\text { value }\end{array}$ & $\begin{array}{l}\text { Mean } \\
\text { inter- } \\
\text { occur- } \\
\text { rence } \\
\text { interval } \\
\text { (days) }\end{array}$ & $\begin{array}{c}\text { Total } \\
\text { no. of } \\
\text { events }\end{array}$ & $\begin{array}{l}\text { Total } \\
\text { no. of } \\
\text { days } \\
\text { avail- } \\
\text { able }\end{array}$ & $\begin{array}{l}\text { Sigma } \\
\mathbf{S}\end{array}$ & $\begin{array}{c}\text { Stan- } \\
\text { dard } \\
\text { normal } \\
\text { variate }\end{array}$ & $\begin{array}{l}\text { Non- } \\
\text { ex- } \\
\text { ceed- } \\
\text { ance } \\
\text { prob- } \\
\text { ability }\end{array}$ & $\begin{array}{c}p- \\
\text { value }\end{array}$ \\
\hline 1145 & 18.32 & 78 & 1,429 & 68641 & 3.544 & 1.000 & 0 & 30.40 & 47 & 1,429 & 41846 & 2.922 & .998 & .002 \\
\hline 1146 & 16.13 & 1,651 & 26,623 & 21842531 & -.432 & .333 & .333 & 29.22 & 911 & 26,623 & 12078881 & -.207 & .418 & .418 \\
\hline 1147 & 15.07 & 412 & 6,208 & 1335545 & 1.559 & .940 & .060 & 26.19 & 237 & 6,208 & 755263 & .711 & .761 & .239 \\
\hline 1148 & 15.28 & 851 & 13,000 & 5771933 & 2.196 & .986 & .014 & 27.08 & 480 & 13,000 & 3187039 & .815 & .793 & .207 \\
\hline 1149 & 12.55 & 963 & 12,084 & 5932563 & 1.054 & .854 & .146 & 20.38 & 593 & 12,084 & 3747954 & 1.943 & .974 & .026 \\
\hline 1150 & 17.39 & 773 & 13,443 & 5221984 & .243 & .596 & .404 & 31.56 & 426 & 13,443 & 2883370 & .250 & .599 & .401 \\
\hline 1151 & 8.16 & 140 & 1,142 & 79867 & -.019 & .493 & .493 & 13.13 & 87 & 1,142 & 48139 & -.500 & .309 & .309 \\
\hline 1152 & 10.51 & 2,518 & 26,466 & 33612692 & .762 & .777 & .223 & 16.51 & 1,603 & 26,466 & 21709645 & 1.625 & .948 & .052 \\
\hline 1153 & 8.12 & 60 & 487 & 15029 & .385 & .650 & .350 & 11.33 & 43 & 487 & 10488 & .019 & .508 & .492 \\
\hline 1154 & 12.18 & 1,071 & 13,041 & 7139884 & 1.270 & .898 & .102 & 20.16 & 647 & 13,041 & 4271122 & .547 & .708 & .292 \\
\hline 1155 & 10.37 & 132 & 1,369 & 76309 & -3.093 & .001 & .001 & 15.21 & 90 & 1,369 & 51985 & -2.566 & .005 & .005 \\
\hline 1156 & 11.61 & 1,121 & 13,013 & 7427614 & 1.064 & .856 & .144 & 18.59 & 700 & 13,013 & 4663898 & 1.100 & .864 & .136 \\
\hline 1157 & 13.88 & 1,300 & 18,047 & 11938863 & 1.109 & .866 & .134 & 21.64 & 834 & 18,047 & 7713833 & 1.251 & .895 & .105 \\
\hline 1158 & 8.97 & 397 & 3,562 & 713471 & .313 & .623 & .377 & 12.91 & 276 & 3,562 & 512816 & 1.245 & .893 & .107 \\
\hline 1159 & 10.37 & 3,552 & 36,849 & 65799398 & .561 & .713 & .287 & 15.82 & 2,330 & 36,849 & 42847201 & -.160 & .437 & .437 \\
\hline 1160 & 13.60 & 103 & 1,401 & 75278 & .762 & .777 & .223 & 21.55 & 65 & 1,401 & 49672 & 1.270 & .898 & .102 \\
\hline 1161 & 16.02 & 357 & 5,720 & 1086964 & 2.114 & .983 & .017 & 24.34 & 235 & 5,720 & 704913 & 1.296 & .903 & .097 \\
\hline 1162 & 25.75 & 47 & 1,210 & 31977 & 1.479 & .930 & .070 & 71.18 & 17 & 1,210 & 10726 & .306 & .620 & .380 \\
\hline 1163 & 30.10 & 105 & 3,160 & 152851 & -1.396 & .081 & .081 & 79.00 & 40 & 3,160 & 55703 & -1.300 & .097 & .097 \\
\hline 1164 & 9.27 & 2,066 & 19,160 & 20575180 & 3.114 & .999 & .001 & 13.94 & 1,375 & 19,160 & 13463232 & 1.418 & .922 & .078 \\
\hline 1165 & 15.12 & 657 & 9,934 & 3387783 & 1.693 & .955 & .045 & 24.77 & 401 & 9,934 & 2059370 & 1.177 & .880 & .120 \\
\hline 1166 & 7.09 & 1,691 & 11,989 & 10352925 & 1.519 & .936 & .064 & 10.60 & 1,131 & 11,989 & 6943396 & 1.406 & .920 & .080 \\
\hline 1167 & 7.67 & 880 & 6,751 & 2965875 & -.079 & .468 & .468 & 11.95 & 565 & 6,751 & 1929947 & .492 & .689 & .311 \\
\hline 1168 & 20.65 & 121 & 2,498 & 161488 & 1.306 & .904 & .096 & 32.44 & 77 & 2,498 & 100338 & .658 & .745 & .255 \\
\hline 1169 & 28.39 & 44 & 1,249 & 28838 & .569 & .715 & .285 & 62.45 & 20 & 1,249 & 13147 & .408 & .658 & .342 \\
\hline 1170 & 7.91 & 2,091 & 16,537 & 18160387 & 3.990 & 1.000 & 0 & 11.98 & 1,380 & 16,537 & 11852163 & 2.490 & .994 & .006 \\
\hline 1171 & 10.11 & 1,211 & 12,247 & 7414870 & -.006 & .498 & .498 & 15.56 & 787 & 12,247 & 4730153 & -.898 & .185 & .185 \\
\hline 1172 & 10.33 & 1,874 & 19,365 & 19012891 & 3.586 & 1.000 & 0 & 14.83 & 1,306 & 19,365 & 13205669 & 2.774 & .997 & .003 \\
\hline 1173 & 13.84 & 1,719 & 23,783 & 20630027 & .662 & .746 & .254 & 22.72 & 1,047 & 23,783 & 12492062 & .188 & .574 & .426 \\
\hline 1174 & 14.68 & 346 & 5,080 & 888596 & .358 & .640 & .360 & 24.08 & 211 & 5,080 & 542295 & .298 & .617 & .383 \\
\hline 1175 & 12.91 & 2,101 & 27,125 & 28845723 & .978 & .836 & .164 & 21.34 & 1,271 & 27,125 & 17458783 & .791 & .785 & .215 \\
\hline 1176 & 12.82 & 51 & 654 & 14104 & -1.908 & .028 & .028 & 20.44 & 32 & 654 & 8888 & -1.476 & .070 & .070 \\
\hline 1177 & 13.53 & 105 & 1,421 & 75325 & .172 & .568 & .432 & 18.46 & 77 & 1,421 & 54051 & -.183 & .427 & .427 \\
\hline 1178 & 12.58 & 1,419 & 17,848 & 13134627 & 2.429 & .992 & .008 & 19.25 & 927 & 17,848 & 8491821 & 1.398 & .919 & .081 \\
\hline 1179 & 15.18 & 1,155 & 17,533 & 10122726 & -.015 & .494 & .494 & 24.73 & 709 & 17,533 & 6314826 & .737 & .769 & .231 \\
\hline 1180 & 16.42 & 102 & 1,675 & 86765 & .274 & .608 & .392 & 27.46 & 61 & 1,675 & 52706 & .429 & .666 & .334 \\
\hline 1181 & 33.47 & 36 & 1,205 & 17402 & -2.055 & .020 & .020 & 70.88 & 17 & 1,205 & 10618 & .262 & .603 & .397 \\
\hline 1182 & 7.27 & 1,332 & 9,681 & 6584043 & 1.338 & .910 & .090 & 10.69 & 906 & 9,681 & 4434929 & .588 & .722 & .278 \\
\hline 1183 & 8.38 & 2,339 & 19,606 & 23536633 & 2.219 & .987 & .013 & 13.03 & 1,505 & 19,606 & 15246996 & 2.248 & .988 & .012 \\
\hline 1184 & 33.75 & 238 & 8033 & 902824 & -1.484 & .069 & .069 & 77.99 & 103 & 8,033 & 381604 & -1.364 & .086 & .086 \\
\hline 1185 & 11.23 & 762 & 8556 & 3231864 & -.410 & .341 & .341 & 19.90 & 430 & 8,556 & 1923115 & 1.632 & .949 & .051 \\
\hline 1186 & 8.00 & 753 & 6027 & 2272209 & .064 & .525 & .475 & 12.33 & 489 & 6,027 & 1508780 & .914 & .820 & .180 \\
\hline 1187 & 7.11 & 1,615 & 11,476 & 9252299 & -.109 & .456 & .456 & 10.55 & 1,088 & 11,476 & 6311464 & .627 & .735 & .265 \\
\hline 1188 & 33.13 & 302 & 10,006 & 1505352 & -.111 & .456 & .456 & 69.49 & 144 & 10,006 & 758005 & 1.084 & .861 & .139 \\
\hline 1189 & 14.67 & 1,306 & 19,158 & 12417469 & -.464 & .321 & .321 & 23.05 & 831 & 19,158 & 8241288 & 1.763 & .961 & .039 \\
\hline 1190 & 8.51 & 2,240 & 19,072 & 21815362 & 1.745 & .960 & .040 & 12.81 & 1,489 & 19,072 & 14673652 & 2.234 & .987 & .013 \\
\hline 1191 & 25.86 & 28 & 724 & 8673 & -1.323 & .093 & .093 & 45.25 & 16 & 724 & 4793 & -1.195 & .116 & .116 \\
\hline 1192 & 14.87 & 436 & 6,484 & 1444309 & .788 & .785 & .215 & 24.38 & 266 & 6,484 & 907068 & 1.464 & .928 & .072 \\
\hline 1193 & 10.32 & 740 & 7,637 & 2881212 & 0.926 & .823 & .177 & 14.72 & 519 & 7,637 & 2026463 & 0.889 & .813 & .187 \\
\hline 1194 & 9.45 & 882 & 8,332 & 3606009 & -.958 & .169 & .169 & 13.82 & 603 & 8,332 & 2559276 & .799 & .788 & .212 \\
\hline 1195 & 7.44 & 299 & 2,224 & 345035 & 1.130 & .871 & .129 & 11.71 & 190 & 2,224 & 214765 & .394 & .653 & .347 \\
\hline 1196 & 8.65 & 743 & 6,424 & 2462931 & 1.512 & .935 & .065 & 12.87 & 499 & 6,424 & 1623796 & .507 & .694 & .306 \\
\hline
\end{tabular}


Table 4. Summary of interoccurrence intervals for daily precipitation thresholds of 0.25 and 0.50 inch-Continued

\begin{tabular}{|c|c|c|c|c|c|c|c|c|c|c|c|c|c|c|}
\hline \multirow[b]{2}{*}{$\begin{array}{l}\text { Seq. } \\
\text { no. }\end{array}$} & \multicolumn{7}{|c|}{ Daily precipitation threshold of 0.25 inch and greater } & \multicolumn{7}{|c|}{ Daily precipitation threshold of 0.50 inch and greater } \\
\hline & $\begin{array}{l}\text { Mean } \\
\text { inter- } \\
\text { occur- } \\
\text { rence } \\
\text { interval } \\
\text { (days) }\end{array}$ & $\begin{array}{c}\text { Total } \\
\text { no. of } \\
\text { events }\end{array}$ & $\begin{array}{l}\text { Total } \\
\text { no. of } \\
\text { days } \\
\text { avail- } \\
\text { able }\end{array}$ & $\begin{array}{l}\text { Sigma } \\
\mathrm{S}\end{array}$ & $\begin{array}{l}\text { Stan- } \\
\text { dard } \\
\text { normal } \\
\text { variate }\end{array}$ & $\begin{array}{l}\text { Non- } \\
\text { ex- } \\
\text { ceed- } \\
\text { ance } \\
\text { prob- } \\
\text { ability }\end{array}$ & $\begin{array}{c}p- \\
\text { value }\end{array}$ & $\begin{array}{l}\text { Mean } \\
\text { inter- } \\
\text { occur- } \\
\text { rence } \\
\text { interval } \\
\text { (days) }\end{array}$ & $\begin{array}{c}\text { Total } \\
\text { no. of } \\
\text { events }\end{array}$ & $\begin{array}{l}\text { Total } \\
\text { no. of } \\
\text { days } \\
\text { avail- } \\
\text { able }\end{array}$ & $\begin{array}{l}\text { Sigma } \\
\mathrm{S}\end{array}$ & $\begin{array}{c}\text { Stan- } \\
\text { dard } \\
\text { normal } \\
\text { variate }\end{array}$ & $\begin{array}{l}\text { Non- } \\
\text { ex- } \\
\text { ceed- } \\
\text { ance } \\
\text { prob- } \\
\text { ability }\end{array}$ & $\begin{array}{c}p- \\
\text { value }\end{array}$ \\
\hline 1197 & 10.33 & 1,918 & 19,815 & 19484985 & 1.926 & .973 & .027 & 16.11 & 1,230 & 19,815 & 12629095 & 2.208 & .986 & .014 \\
\hline 1198 & 14.83 & 612 & 9,076 & 2916124 & 2.143 & 984 & 016 & 24.07 & 377 & 9,076 & 1823768 & 2.220 & .987 & .013 \\
\hline 1199 & 36.00 & 10 & 360 & 1792 & -.024 & 490 & 490 & 51.43 & 7 & 360 & 1417 & .571 & .716 & .284 \\
\hline 1200 & 13.45 & 1,070 & 14,391 & 7822422 & .907 & .818 & 182 & 22.77 & 632 & 14,391 & 4708117 & 1.537 & .938 & .062 \\
\hline 1201 & 17.92 & 107 & 1,917 & 104608 & .358 & .640 & .360 & 33.63 & 57 & 1,917 & 54616 & -.004 & .498 & .498 \\
\hline 1202 & 15.93 & 1,218 & 19,400 & 12417363 & 3.084 & .999 & .001 & 29.57 & 656 & 19,400 & 6758811 & 2.758 & .997 & .003 \\
\hline 1203 & 14.36 & 1,093 & 15,694 & 8181126 & -2.642 & .004 & .004 & 23.32 & 673 & 15,694 & 4952706 & -2.794 & .003 & .003 \\
\hline 1204 & 14.77 & 1,048 & 15,481 & 8589418 & 3.300 & 1.000 & 0 & 25.98 & 596 & 15,481 & 4954175 & 3.124 & .999 & .001 \\
\hline 1205 & 7.90 & 821 & 6,485 & 2628384 & -.628 & .265 & .265 & 11.90 & 545 & 6,485 & 1785157 & .412 & .660 & .340 \\
\hline 1206 & 8.19 & 1,294 & 10,592 & 6920137 & .610 & .729 & .271 & 13.13 & 807 & 10,592 & 4273141 & -.008 & .497 & .497 \\
\hline 1207 & 8.92 & 684 & 6,104 & 2054307 & -.722 & .235 & .235 & 13.60 & 449 & 6,104 & 1357802 & -.336 & .368 & .368 \\
\hline 1208 & 17.04 & 1,145 & 19,506 & 11171525 & .023 & .509 & .491 & 31.26 & 624 & 19,506 & 6134901 & .349 & .636 & .364 \\
\hline 1209 & -- & -- & -- & -- & -- & -- & -- & -- & -- & -- & -- & -- & -- & -- \\
\hline 1210 & 12.00 & 309 & 3,709 & 562416 & -.565 & .286 & .286 & 17.09 & 217 & 3,709 & 389650 & -.810 & .209 & .209 \\
\hline 1211 & 15.44 & 1,817 & 28,061 & 25307987 & -.537 & .296 & .296 & 24.99 & 1,123 & 28,061 & 15751309 & -.018 & .493 & .493 \\
\hline 1212 & 15.41 & 625 & 9,634 & 3020313 & .139 & .555 & .445 & 25.83 & 373 & 9,634 & 1837702 & .763 & .777 & .223 \\
\hline 1213 & 13.69 & 400 & 5,474 & 1045075 & -1.573 & .058 & .058 & 23.29 & 235 & 5,474 & 646187 & .124 & .549 & .451 \\
\hline 1214 & 12.93 & 56 & 724 & 19110 & -.743 & .229 & .229 & 19.05 & 38 & 724 & 12408 & -1.046 & .148 & .148 \\
\hline 1215 & 22.41 & 381 & 8,537 & 1515566 & -2.302 & .011 & .011 & 49.06 & 174 & 8,537 & 677303 & -2.012 & .022 & .022 \\
\hline 1216 & 21.01 & 1,201 & 25,234 & 15142904 & -.040 & .484 & .484 & 40.70 & 620 & 25,234 & 7511778 & -1.713 & .043 & .043 \\
\hline 1217 & 10.45 & 1,257 & 13,130 & 8187847 & -.479 & .316 & .316 & 15.80 & 831 & 13,130 & 5448967 & -.060 & .476 & .476 \\
\hline 1218 & 10.75 & 304 & 3,269 & 495285 & -.097 & .461 & .461 & 14.66 & 223 & 3,269 & 366093 & .114 & .545 & .455 \\
\hline 1219 & 9.95 & 1,974 & 19,632 & 19804405 & 1.698 & .955 & .045 & 15.36 & 1,278 & 19,632 & 12966935 & 2.083 & .981 & .019 \\
\hline 1220 & 25.76 & 812 & 20,914 & 8672032 & 1.052 & .853 & .147 & 57.61 & 363 & 20,914 & 3894682 & .859 & .805 & .195 \\
\hline 1221 & 16.06 & 366 & 5,877 & 1119550 & 1.358 & .913 & .087 & 23.41 & 251 & 5,877 & 764884 & 1.017 & .845 & .155 \\
\hline 1222 & 22.90 & 10 & 229 & 837 & -1.473 & .070 & .070 & 57.25 & 4 & 229 & 427 & -.235 & .407 & .407 \\
\hline 1223 & 11.22 & 488 & 5,476 & 1354716 & .532 & .703 & .297 & 17.17 & 319 & 5,476 & 909725 & 1.286 & .901 & .099 \\
\hline 1224 & 11.05 & 179 & 1,978 & 172034 & -.654 & .257 & .257 & 18.84 & 105 & 1,978 & 103287 & -.095 & .462 & .462 \\
\hline 1225 & 11.47 & 231 & 2,649 & 315626 & .832 & .797 & .203 & 17.78 & 149 & 2,649 & 201974 & .495 & .690 & .310 \\
\hline 1226 & 16.06 & 1,364 & 21,901 & 14297347 & -2.737 & .003 & .003 & 30.25 & 724 & 21,901 & 7466197 & -2.716 & .003 & .003 \\
\hline 1227 & 11.46 & 545 & 6,246 & 1701337 & -.017 & .493 & .493 & 17.95 & 348 & 6,246 & 1056859 & -.890 & .187 & .187 \\
\hline 1228 & 13.24 & 1,893 & 25,070 & 24219294 & 1.558 & .940 & .060 & 20.82 & 1,204 & 25,070 & 14956490 & -.540 & .295 & .295 \\
\hline 1229 & 10.14 & 1,456 & 14,762 & 11037717 & 1.790 & .963 & .037 & 15.81 & 934 & 14,762 & 7040427 & 1.125 & .870 & .130 \\
\hline 1230 & 10.29 & 1,396 & 14,360 & 9947560 & -.489 & .312 & .312 & 16.78 & 856 & 14,360 & 6185592 & .326 & .628 & .372 \\
\hline 1231 & 10.39 & 75 & 779 & 30548 & .686 & .754 & .246 & 16.94 & 46 & 779 & 19068 & .755 & .775 & .225 \\
\hline 1232 & 9.03 & 108 & 975 & 57171 & 1.546 & .939 & .061 & 15.98 & 61 & 975 & 32375 & 1.200 & .885 & .115 \\
\hline 1233 & 11.70 & 455 & 5,323 & 1241481 & .931 & .824 & .176 & 19.86 & 268 & 5,323 & 740086 & 1.066 & .857 & .143 \\
\hline 1234 & 13.09 & 853 & 11,164 & 4967944 & 2.194 & .986 & .014 & 20.34 & 549 & 11,164 & 3254101 & 2.511 & .994 & .006 \\
\hline 1235 & 11.47 & 361 & 4,140 & 772099 & 1.093 & .863 & .137 & 19.53 & 212 & 4,140 & 449572 & .617 & .731 & .269 \\
\hline 1236 & 13.35 & 166 & 2,216 & 172094 & -1.436 & .076 & .076 & 18.31 & 121 & 2,216 & 122571 & -1.634 & .051 & .051 \\
\hline 1237 & 9.74 & 1,349 & 13,142 & 9235706 & 2.666 & .996 & .004 & 15.25 & 862 & 13,142 & 5939802 & 2.474 & .993 & .007 \\
\hline 1238 & 10.80 & 2,359 & 25,474 & 30350053 & .850 & .802 & .198 & 17.12 & 1,488 & 25,474 & 19187193 & .827 & .796 & .204 \\
\hline 1239 & 10.43 & 1,443 & 15,049 & 11134814 & 1.678 & .953 & .047 & 16.30 & 923 & 15,049 & 6886661 & -.443 & .329 & .329 \\
\hline 1240 & 18.22 & 103 & 1,877 & 88482 & -1.488 & .068 & .068 & 29.33 & 64 & 1,877 & 54018 & -1.395 & .082 & .082 \\
\hline 1241 & 9.05 & 1,997 & 18,072 & 18498402 & 1.945 & .974 & .026 & 14.26 & 1,267 & 18,072 & 11577561 & .694 & .756 & .244 \\
\hline 1242 & 10.83 & 69 & 747 & 26812 & .581 & .719 & .281 & 16.60 & 45 & 747 & 18006 & .829 & .796 & .204 \\
\hline 1243 & 7.30 & 2,412 & 17,604 & 21563270 & 1.334 & .909 & .091 & 11.04 & 1,595 & 17,604 & 14356563 & 1.564 & .941 & .059 \\
\hline 1244 & 8.95 & 2,139 & 19,152 & 20935811 & 1.771 & .962 & .038 & 14.31 & 1,338 & 19,152 & 13213402 & 1.981 & .976 & .024 \\
\hline 1245 & 16.25 & 1,167 & 18,958 & 11500746 & 2.347 & .991 & .009 & 26.04 & 728 & 18,958 & 7250053 & 2.366 & .991 & .009 \\
\hline 1246 & 19.27 & 120 & 2,312 & 125697 & -1.781 & .037 & .037 & 32.56 & 71 & 2,312 & 75642 & -1.144 & .126 & .126 \\
\hline 1247 & 15.06 & 1,008 & 15,182 & 7599956 & -.372 & .355 & .355 & 24.25 & 626 & 15,182 & 4636474 & -1.053 & .146 & .146 \\
\hline 1248 & 6.78 & 9 & 61 & 268 & -.123 & .451 & .451 & 7.63 & 8 & 61 & 244 & 0 & .500 & .500 \\
\hline
\end{tabular}


Table 4. Summary of interoccurrence intervals for daily precipitation thresholds of 0.25 and 0.50 inch-Continued

\begin{tabular}{|c|c|c|c|c|c|c|c|c|c|c|c|c|c|c|}
\hline \multirow[b]{2}{*}{$\begin{array}{c}\text { Seq. } \\
\text { no. }\end{array}$} & \multicolumn{7}{|c|}{ Daily precipitation threshold of 0.25 inch and greater } & \multicolumn{7}{|c|}{ Daily precipitation threshold of 0.50 inch and greater } \\
\hline & $\begin{array}{c}\text { Mean } \\
\text { inter- } \\
\text { occur- } \\
\text { rence } \\
\text { interval } \\
\text { (days) }\end{array}$ & $\begin{array}{c}\text { Total } \\
\text { no. of } \\
\text { events }\end{array}$ & $\begin{array}{l}\text { Total } \\
\text { no. of } \\
\text { days } \\
\text { avail- } \\
\text { able }\end{array}$ & $\begin{array}{l}\text { Sigma } \\
\mathbf{S}\end{array}$ & $\begin{array}{c}\text { Stan- } \\
\text { dard } \\
\text { normal } \\
\text { variate }\end{array}$ & $\begin{array}{l}\text { Non- } \\
\text { ex- } \\
\text { ceed- } \\
\text { ance } \\
\text { prob- } \\
\text { ability }\end{array}$ & $\begin{array}{c}\text { p- } \\
\text { value }\end{array}$ & $\begin{array}{c}\text { Mean } \\
\text { inter- } \\
\text { occur- } \\
\text { rence } \\
\text { interval } \\
\text { (days) }\end{array}$ & $\begin{array}{c}\text { Total } \\
\text { no. of } \\
\text { events }\end{array}$ & $\begin{array}{l}\text { Total } \\
\text { no. of } \\
\text { days } \\
\text { avail- } \\
\text { able }\end{array}$ & $\begin{array}{l}\text { Sigma } \\
\mathbf{S}\end{array}$ & $\begin{array}{l}\text { Stan- } \\
\text { dard } \\
\text { normal } \\
\text { variate }\end{array}$ & $\begin{array}{l}\text { Non- } \\
\text { ex- } \\
\text { ceed- } \\
\text { ance } \\
\text { prob- } \\
\text { ability }\end{array}$ & $\begin{array}{c}p- \\
\text { value }\end{array}$ \\
\hline 1249 & 10.82 & 1,139 & 12,327 & 7143647 & 1.028 & .848 & .152 & 17.24 & 715 & 12,327 & 4607504 & 2.108 & .982 & .018 \\
\hline 1250 & 9.79 & 3,764 & 36,857 & 70367661 & 1.536 & .938 & .062 & 15.02 & 2,454 & 36,857 & 46189486 & 1.833 & .967 & .033 \\
\hline 1251 & 14.58 & 76 & 1,108 & 34712 & -2.651 & .004 & .004 & 25.18 & 44 & 1,108 & 19354 & -2.367 & .009 & .009 \\
\hline 1252 & 10.81 & 3,280 & 35,470 & 59282760 & 1.896 & .971 & .029 & 16.96 & 2,092 & 35,470 & 38018075 & 1.957 & .975 & .025 \\
\hline 1253 & 10.58 & 422 & 4,465 & 999139 & 2.154 & .984 & .016 & 14.45 & 309 & 4,465 & 721673 & 1.405 & .920 & .080 \\
\hline 1254 & 10.13 & 45 & 456 & 11284 & 1.160 & .877 & .123 & 14.25 & 32 & 456 & 7626 & .443 & .671 & .329 \\
\hline 1255 & 11.59 & 1,159 & 13,428 & 7681787 & -.756 & .225 & .225 & 17.72 & 758 & 13,428 & 4910502 & -1.675 & .047 & .047 \\
\hline 1256 & 14.86 & 1,378 & 20,473 & 13796578 & -1.410 & .079 & .079 & 23.95 & 855 & 20,473 & 8704901 & -.274 & .392 & .392 \\
\hline 1257 & 15.52 & 1,258 & 19,529 & 12616559 & 1.665 & .952 & .048 & 27.66 & 706 & 19,529 & 7218973 & 2.171 & .985 & .015 \\
\hline 1258 & 9.69 & 375 & 3,633 & 670787 & -.512 & .304 & .304 & 14.36 & 253 & 3,633 & 466938 & .441 & .671 & .329 \\
\hline 1259 & 28.63 & 8 & 229 & 1087 & .915 & .820 & .180 & 57.25 & 4 & 229 & 658 & 1.513 & .935 & .065 \\
\hline 1260 & 9.10 & 2,395 & 21,784 & 26689199 & 1.959 & .975 & .025 & 13.96 & 1,561 & 21,784 & 17363924 & 1.455 & .927 & .073 \\
\hline 1261 & 14.76 & 554 & 8,179 & 2201408 & -1.155 & .124 & .124 & 24.27 & 337 & 8,179 & 1385536 & .170 & .568 & .432 \\
\hline 1262 & 12.41 & 93 & 1,154 & 53341 & -.100 & .460 & .460 & 20.61 & 56 & 1,154 & 30845 & -.589 & .278 & .278 \\
\hline 1263 & 6.88 & 75 & 516 & 19978 & .487 & .687 & .313 & 9.21 & 56 & 516 & 14347 & -.091 & .464 & .464 \\
\hline 1264 & 18.21 & 85 & 1,548 & 67002 & .294 & .616 & .384 & 28.67 & 54 & 1,548 & 47254 & 1.662 & .952 & .048 \\
\hline 1265 & 8.36 & 413 & 3454 & 696464 & -.829 & .204 & .204 & 12.42 & 278 & 3,454 & 491148 & .664 & .747 & .253 \\
\hline 1266 & 10.47 & 1,819 & 19,044 & 17773966 & 1.934 & .973 & .027 & 16.53 & 1,152 & 19,044 & 11306407 & 1.806 & .965 & .035 \\
\hline 1267 & 13.40 & 1,006 & 13,484 & 6737185 & -.367 & .357 & .357 & 20.65 & 653 & 13,484 & 4283253 & -1.199 & .115 & .115 \\
\hline 1268 & 14.04 & 1,222 & 17,161 & 10837138 & 2.031 & .979 & .021 & 21.72 & 790 & 17,161 & 6692184 & -.621 & .267 & .267 \\
\hline 1269 & -- & -- & -- & -- & -- & -- & -- & -- & -- & -- & -- & -- & -- & -- \\
\hline 1270 & 11.76 & 186 & 2,187 & 200636 & -.320 & .374 & .374 & 19.18 & 114 & 2,187 & 123050 & -.239 & .406 & .406 \\
\hline 1271 & 12.31 & 2,849 & 35,073 & 50120614 & .294 & .616 & .384 & 19.55 & 1,794 & 35,073 & 30969360 & -1.145 & .126 & .126 \\
\hline 1272 & 14.10 & 283 & 3,991 & 517565 & -2.433 & .007 & .007 & 21.93 & 182 & 3,991 & 329275 & -2.182 & .015 & .015 \\
\hline 1273 & 12.72 & 749 & 9,529 & 3645000 & 1.015 & .845 & .155 & 19.85 & 480 & 9,529 & 2264787 & -.368 & .356 & .356 \\
\hline 1274 & 7.86 & 783 & 6,153 & 2423725 & .298 & .617 & .383 & 11.27 & 546 & 6,153 & 1707461 & .667 & .748 & .252 \\
\hline 1275 & 7.44 & 485 & 3,606 & 849934 & -1.070 & .142 & .142 & 10.70 & 337 & 3,606 & 605214 & -.125 & .450 & .450 \\
\hline 1276 & 8.92 & 620 & 5,527 & 1750637 & .938 & .826 & .174 & 14.06 & 393 & 5,527 & 1123985 & 1.199 & .885 & .115 \\
\hline 1277 & 10.20 & 251 & 2,560 & 283733 & -3.207 & .001 & .001 & 12.49 & 205 & 2,560 & 250390 & -1.135 & .128 & .128 \\
\hline 1278 & 8.58 & 3,378 & 28,977 & 49543026 & 1.236 & .892 & .108 & 12.69 & 2,283 & 28,977 & 33058287 & -.047 & .481 & .481 \\
\hline 1279 & 19.75 & 76 & 1,501 & 51586 & -1.443 & .074 & .074 & 33.36 & 45 & 1,501 & 28902 & -1.676 & .047 & .047 \\
\hline 1280 & 13.83 & 389 & 5,379 & 1129092 & 2.706 & .997 & .003 & 24.34 & 221 & 5,379 & 650855 & 2.447 & .993 & .007 \\
\hline 1281 & 9.50 & 653 & 6,203 & 2015262 & -.219 & .413 & .413 & 14.98 & 414 & 6,203 & 1267370 & -.457 & .324 & .324 \\
\hline 1282 & 12.90 & 19 & 245 & 3061 & 2.379 & .991 & .009 & 22.27 & 11 & 245 & 1617 & 1.149 & .875 & .125 \\
\hline 1283 & 14.03 & 373 & 5,233 & 979295 & .115 & .546 & .454 & 20.68 & 253 & 5,233 & 715445 & 2.225 & .987 & .013 \\
\hline 1284 & 8.99 & 1,087 & 9,769 & 5286111 & -.251 & .401 & .401 & 12.94 & 755 & 9,769 & 3691382 & .046 & .518 & .482 \\
\hline 1285 & 27.49 & 766 & 21,056 & 8096794 & .192 & .576 & .424 & 51.74 & 407 & 21,056 & 4242807 & -.343 & .366 & .366 \\
\hline 1286 & 8.62 & 2,123 & 18,295 & 19916912 & 2.041 & .979 & .021 & 12.38 & 1,478 & 18,295 & 13725523 & 1.012 & .844 & .156 \\
\hline 1287 & 20.14 & 297 & 5,980 & 870702 & -.583 & .280 & .280 & 32.15 & 186 & 5,980 & 542667 & -.572 & .284 & .284 \\
\hline 1288 & 13.90 & 867 & 12,048 & 4960996 & -2.557 & .005 & .005 & 22.99 & 524 & 12,048 & 2963659 & -2.423 & .008 & .008 \\
\hline 1289 & 15.01 & 924 & 13,868 & 6427615 & .169 & .567 & .433 & 23.08 & 601 & 13,868 & 4112206 & -.562 & .287 & .287 \\
\hline 1290 & 13.24 & 108 & 1,430 & 77830 & .142 & .557 & .443 & 26.00 & 55 & 1,430 & 42225 & .947 & .828 & .172 \\
\hline 1291 & 12.28 & 99 & 1,216 & 62548 & .675 & .750 & .250 & 20.27 & 60 & 1,216 & 37207 & .267 & .605 & .395 \\
\hline 1292 & 8.61 & 2,285 & 19,665 & 23041953 & 2.118 & .983 & .017 & 12.89 & 1,526 & 19,665 & 15289857 & 1.287 & .901 & .099 \\
\hline 1293 & 11.81 & 1,443 & 17,041 & 12371676 & .410 & .659 & .341 & 18.32 & 930 & 17,041 & 8029743 & .704 & .759 & .241 \\
\hline 1294 & 12.94 & 391 & 5,058 & 980505 & -.289 & .386 & .386 & 19.45 & 260 & 5,058 & 638370 & -.814 & .208 & .208 \\
\hline 1295 & 8.51 & 75 & 638 & 27236 & 2.076 & .981 & .019 & 11.39 & 56 & 638 & 22140 & 3.103 & .999 & .001 \\
\hline 1296 & 6.84 & 622 & 4,257 & 1313942 & -.326 & .372 & .372 & 10.04 & 424 & 4,257 & 903377 & .035 & .514 & .486 \\
\hline 1297 & 14.04 & 193 & 2,709 & 215104 & -4.263 & 0 & 0 & 23.35 & 116 & 2,709 & 139889 & -2.046 & .020 & .020 \\
\hline 1298 & 9.71 & 153 & 1,485 & 105802 & -1.471 & .071 & .071 & 14.28 & 104 & 1,485 & 65888 & -2.592 & .005 & .005 \\
\hline 1299 & 7.46 & 1,299 & 9,696 & 6303542 & .059 & .524 & .476 & 11.15 & 870 & 9,696 & 4275821 & .703 & .759 & .241 \\
\hline 1300 & 10.11 & 18 & 182 & 1948 & 1.391 & .918 & .082 & 20.22 & 9 & 182 & 978 & 1.009 & .843 & .157 \\
\hline
\end{tabular}


Table 4. Summary of interoccurrence intervals for daily precipitation thresholds of 0.25 and 0.50 inch-Continued

\begin{tabular}{|c|c|c|c|c|c|c|c|c|c|c|c|c|c|c|}
\hline \multirow[b]{2}{*}{$\begin{array}{l}\text { Seq. } \\
\text { no. }\end{array}$} & \multicolumn{7}{|c|}{ Daily precipitation threshold of 0.25 inch and greater } & \multicolumn{7}{|c|}{ Daily precipitation threshold of 0.50 inch and greater } \\
\hline & $\begin{array}{l}\text { Mean } \\
\text { inter- } \\
\text { occur- } \\
\text { rence } \\
\text { interval } \\
\text { (days) }\end{array}$ & $\begin{array}{l}\text { Total } \\
\text { no. of } \\
\text { events }\end{array}$ & $\begin{array}{l}\text { Total } \\
\text { no. of } \\
\text { days } \\
\text { avail- } \\
\text { able }\end{array}$ & $\begin{array}{l}\text { Sigma } \\
\mathrm{S}\end{array}$ & $\begin{array}{c}\text { Stan- } \\
\text { dard } \\
\text { normal } \\
\text { variate }\end{array}$ & $\begin{array}{l}\text { Non- } \\
\text { ex- } \\
\text { ceed- } \\
\text { ance } \\
\text { prob- } \\
\text { ability }\end{array}$ & $\begin{array}{c}\mathrm{p}- \\
\text { value }\end{array}$ & $\begin{array}{l}\text { Mean } \\
\text { inter- } \\
\text { occur- } \\
\text { rence } \\
\text { interval } \\
\text { (days) }\end{array}$ & $\begin{array}{l}\text { Total } \\
\text { no. of } \\
\text { events }\end{array}$ & $\begin{array}{l}\text { Total } \\
\text { no. of } \\
\text { days } \\
\text { avail- } \\
\text { able }\end{array}$ & $\begin{array}{l}\text { Sigma } \\
\text { S }\end{array}$ & $\begin{array}{c}\text { Stan- } \\
\text { dard } \\
\text { normal } \\
\text { variate }\end{array}$ & $\begin{array}{l}\text { Non- } \\
\text { ex- } \\
\text { ceed- } \\
\text { ance } \\
\text { prob- } \\
\text { ability }\end{array}$ & $\begin{array}{c}\mathrm{p}- \\
\text { value }\end{array}$ \\
\hline 1301 & 10.13 & 2,743 & 27,787 & 38738166 & 1.496 & .933 & .067 & 15.71 & 1,769 & 27,787 & 25040330 & 1.372 & .915 & .085 \\
\hline 1302 & 10.97 & 1,704 & 18,695 & 16438350 & 2.290 & .989 & .011 & 17.14 & 1,091 & 18,695 & 10621370 & 2.374 & .991 & .009 \\
\hline 1303 & 11.64 & 25 & 291 & 3301 & -.801 & .212 & .212 & 15.32 & 19 & 291 & 2343 & -1.151 & .125 & .125 \\
\hline 1304 & 10.49 & 347 & 3,640 & 613074 & -.943 & .173 & .173 & 16.70 & 218 & 3,640 & 391833 & -.318 & .375 & .375 \\
\hline 1305 & 33.97 & 667 & 22,655 & 8004215 & 2.657 & .996 & .004 & 79.77 & 284 & 22,655 & 3352707 & 1.231 & .891 & .109 \\
\hline 1306 & 19.91 & 752 & 14,972 & 5788249 & 1.340 & .910 & .090 & 31.59 & 474 & 14,972 & 3605392 & .606 & .728 & .272 \\
\hline
\end{tabular}


Table 5. Summary of interoccurrence intervals for daily precipitation thresholds of 0.75 and 1.0 inch

[--, not available, no events that equal or exceed the threshold were observed]

\begin{tabular}{|c|c|c|c|c|c|c|c|c|c|c|c|c|c|c|}
\hline \multirow[b]{2}{*}{$\begin{array}{l}\text { Seq. } \\
\text { no. }\end{array}$} & \multicolumn{7}{|c|}{ Daily precipitation threshold of 0.75 inch and greater } & \multicolumn{7}{|c|}{ Daily precipitation threshold of 1.0 inch and greater } \\
\hline & $\begin{array}{l}\text { Mean } \\
\text { inter- } \\
\text { occur- } \\
\text { rence } \\
\text { interval } \\
\text { (days) }\end{array}$ & $\begin{array}{l}\text { Total } \\
\text { no. of } \\
\text { events }\end{array}$ & $\begin{array}{l}\text { Total } \\
\text { no. of } \\
\text { days } \\
\text { avail- } \\
\text { able }\end{array}$ & $\begin{array}{c}\text { Sigma } \\
S\end{array}$ & $\begin{array}{l}\text { Stan- } \\
\text { dard } \\
\text { normal } \\
\text { variate }\end{array}$ & $\begin{array}{l}\text { Non- } \\
\text { ex- } \\
\text { ceed- } \\
\text { ance } \\
\text { prob- } \\
\text { ability }\end{array}$ & $\begin{array}{c}\mathrm{p}- \\
\text { value }\end{array}$ & $\begin{array}{l}\text { Mean } \\
\text { inter- } \\
\text { occur- } \\
\text { rence } \\
\text { interval } \\
\text { (days) }\end{array}$ & $\begin{array}{l}\text { Total } \\
\text { no. of } \\
\text { events }\end{array}$ & $\begin{array}{l}\text { Total } \\
\text { no. of } \\
\text { days } \\
\text { avail- } \\
\text { able }\end{array}$ & $\begin{array}{l}\text { Sigma } \\
\mathbf{S}\end{array}$ & $\begin{array}{c}\text { Stan- } \\
\text { dard } \\
\text { normal } \\
\text { variate }\end{array}$ & $\begin{array}{l}\text { Non- } \\
\text { ex- } \\
\text { ceed- } \\
\text { ance } \\
\text { prob- } \\
\text { ability }\end{array}$ & $\begin{array}{c}\mathrm{p}- \\
\text { value }\end{array}$ \\
\hline 1 & 51.22 & 381 & 19,514 & 3816445 & 0.901 & .816 & .184 & 80.97 & 241 & 19,514 & 2356211 & 0.055 & .522 & .478 \\
\hline 2 & 60.00 & 4 & 240 & 324 & -1.126 & .130 & .130 & 80.00 & 3 & 240 & 202 & -1.317 & .094 & .094 \\
\hline 3 & 36.93 & 534 & 19,720 & 5374840 & .833 & .798 & .202 & 57.66 & 342 & 19,720 & 3313512 & -.557 & .289 & .289 \\
\hline 4 & 39.51 & 213 & 8,416 & 869674 & -.751 & .226 & .226 & 57.25 & 147 & 8,416 & 603669 & -.506 & .306 & .306 \\
\hline 5 & 52.04 & 365 & 18,993 & 3780885 & 3.004 & .999 & .001 & 78.81 & 241 & 18,993 & 2509901 & 2.599 & .995 & .005 \\
\hline 6 & 43.48 & 81 & 3,522 & 130253 & -1.354 & .088 & .088 & 71.88 & 49 & 3,522 & 79066 & -1.015 & .155 & .155 \\
\hline 7 & 29.20 & 283 & 8,264 & 1150974 & -.458 & .324 & .324 & 43.27 & 191 & 8,264 & 752391 & -1.117 & .132 & .132 \\
\hline 8 & 47.03 & 31 & 1,458 & 23820 & .521 & .699 & .301 & 60.75 & 24 & 1,458 & 18210 & .346 & .635 & .365 \\
\hline 9 & 35.28 & 82 & 2,893 & 109801 & -1.165 & .122 & .122 & 53.57 & 54 & 2,893 & 73522 & -.748 & .227 & .227 \\
\hline 10 & 21.40 & 10 & 214 & 934 & -.696 & .243 & .243 & 23.78 & 9 & 214 & 898 & -.351 & .363 & .363 \\
\hline 11 & 38.49 & 159 & 6,120 & 528232 & 1.872 & .969 & .031 & 49.36 & 124 & 6,120 & 414674 & 1.791 & .963 & .037 \\
\hline 12 & 31.71 & 1,122 & 35,581 & 20143403 & .530 & .702 & .298 & 48.54 & 733 & 35,581 & 13456244 & 1.495 & .933 & .067 \\
\hline 13 & 24.24 & 619 & 15,005 & 4914642 & 2.511 & .994 & .006 & 36.51 & 411 & 15,005 & 3222921 & 1.587 & .944 & .056 \\
\hline 14 & 34.56 & 867 & 29,966 & 13233500 & .955 & .830 & .170 & 49.04 & 611 & 29,966 & 9655906 & 2.344 & .990 & .010 \\
\hline 15 & 43.82 & 39 & 1,709 & 29539 & -1.229 & .110 & .110 & 63.30 & 27 & 1,709 & 20357 & -1.059 & .145 & .145 \\
\hline 16 & 41.00 & 3 & 123 & 65 & -1.943 & .026 & .026 & 61.50 & 2 & 123 & 47 & -1.514 & .065 & .065 \\
\hline 17 & 123.00 & 1 & 123 & 65 & .099 & .539 & .461 & -- & -- & -- & -- & -- & -- & -- \\
\hline 18 & 66.84 & 412 & 27,539 & 5702876 & .185 & .573 & .427 & 110.60 & 249 & 27,539 & 3543901 & .919 & .821 & .179 \\
\hline 19 & 18.27 & 1,053 & 19,240 & 10664329 & 2.966 & .998 & .002 & 27.06 & 711 & 19,240 & 7255350 & 2.806 & .997 & .003 \\
\hline 20 & 22.34 & 274 & 6,122 & 843730 & .172 & .568 & .432 & 30.31 & 202 & 6,122 & 593462 & -.990 & .161 & .161 \\
\hline 21 & 21.03 & 251 & 5,279 & 647771 & -.611 & .271 & .271 & 32.99 & 160 & 5,279 & 408608 & -.711 & .239 & .239 \\
\hline 22 & 17.25 & 2,003 & 34,541 & 34900898 & .690 & .755 & .245 & 23.87 & 1,447 & 34,541 & 25309464 & .841 & .800 & .200 \\
\hline 23 & 26.38 & 45 & 1,187 & 28275 & .682 & .752 & .248 & 38.29 & 31 & 1,187 & 17659 & -.388 & .349 & .349 \\
\hline 24 & 49.61 & 397 & 19,693 & 3944886 & .316 & .624 & .376 & 84.16 & 234 & 19,693 & 2278020 & -.300 & .382 & .382 \\
\hline 25 & 50.95 & 270 & 13,756 & 1746381 & -1.696 & .045 & .045 & 72.40 & 190 & 13,756 & 1241939 & -1.185 & .118 & .118 \\
\hline 26 & 16.33 & 1,905 & 31,099 & 29882011 & .664 & .747 & .253 & 22.18 & 1,402 & 31,099 & 21974171 & .517 & .697 & .303 \\
\hline 27 & 19.95 & 1,042 & 20,787 & 10490866 & -1.751 & .040 & .040 & 27.72 & 750 & 20,787 & 7477228 & -1.934 & .027 & .027 \\
\hline 28 & 25.10 & 479 & 12,022 & 3002141 & 1.618 & .947 & .053 & 38.66 & 311 & 12,022 & 2025405 & 2.549 & .995 & .005 \\
\hline 29 & 64.83 & 245 & 15,884 & 1872784 & -1.017 & .155 & .155 & 101.82 & 156 & 15,884 & 1161097 & -1.359 & .087 & .087 \\
\hline 30 & 16.61 & 1,904 & 31,631 & 31437517 & 3.325 & 1.000 & 0 & 22.43 & 1,410 & 31,631 & 23414945 & 3.252 & .999 & .001 \\
\hline 31 & 21.11 & 859 & 18,129 & 8024998 & 1.556 & .940 & .060 & 30.07 & 603 & 18,129 & 5659170 & 1.504 & .934 & .066 \\
\hline 32 & 31.06 & 536 & 16,648 & 4334756 & -1.141 & .127 & .127 & 45.49 & 366 & 16,648 & 2978856 & -.737 & .231 & .231 \\
\hline 33 & 28.10 & 825 & 23,183 & 9673148 & .573 & .717 & .283 & 42.00 & 552 & 23,183 & 6534766 & .867 & .807 & .193 \\
\hline 34 & 21.18 & 171 & 3,622 & 321822 & .888 & .813 & .187 & 35.86 & 101 & 3,622 & 184604 & .161 & .564 & .436 \\
\hline 35 & 28.91 & 356 & 10,292 & 1973949 & 2.533 & .994 & .006 & 39.89 & 258 & 10,292 & 1445751 & 2.474 & .993 & .007 \\
\hline 36 & 23.18 & 478 & 11,081 & 2556998 & -1.306 & .096 & .096 & 30.28 & 366 & 11,081 & 1934485 & -1.525 & .064 & .064 \\
\hline 37 & 23.62 & 462 & 10,911 & 2553236 & .484 & .686 & .314 & 34.31 & 318 & 10,911 & 1764488 & .528 & .701 & .299 \\
\hline 38 & 28.77 & 779 & 22,410 & 9241429 & 2.840 & .998 & .002 & 44.38 & 505 & 22,410 & 5878191 & 1.511 & .935 & .065 \\
\hline 39 & 21.61 & 904 & 19,535 & 9256678 & 2.518 & .994 & .006 & 31.41 & 622 & 19,535 & 6377995 & 2.152 & .984 & .016 \\
\hline 40 & 32.13 & 323 & 10,379 & 1752800 & 1.422 & .923 & .077 & 45.93 & 226 & 10,379 & 1230876 & 1.289 & .901 & .099 \\
\hline 41 & 19.25 & 1,288 & 24,787 & 16300241 & 1.314 & .906 & .094 & 28.20 & 879 & 24,787 & 11203741 & 1.461 & .928 & .072 \\
\hline 42 & 37.20 & 20 & 744 & 6755 & -.713 & .238 & .238 & 53.14 & 14 & 744 & 5243 & .044 & .517 & .483 \\
\hline 43 & 38.06 & 843 & 32,082 & 13449045 & -.273 & .392 & .392 & 57.50 & 558 & 32,082 & 8800541 & -.687 & .246 & .246 \\
\hline 44 & 19.88 & 989 & 19,656 & 10148703 & 2.403 & .992 & .008 & 27.72 & 709 & 19,656 & 7288384 & 2.120 & .983 & .017 \\
\hline 45 & 16.96 & 1,117 & 18,947 & 11075906 & 2.702 & .997 & .003 & 23.33 & 812 & 18,947 & 8245983 & 3.551 & 1.000 & 0 \\
\hline 46 & 26.01 & 994 & 25,853 & 12721692 & -.541 & .294 & .294 & 37.41 & 691 & 25,853 & 8810086 & -.623 & .267 & .267 \\
\hline 47 & 28.41 & 224 & 6,363 & 764381 & 1.882 & .970 & .030 & 42.42 & 150 & 6,363 & 528453 & 2.277 & .989 & .011 \\
\hline 48 & 28.24 & 200 & 5,648 & 601364 & 1.586 & .944 & .056 & 41.23 & 137 & 5,648 & 425808 & 2.039 & .979 & .021 \\
\hline
\end{tabular}


Table 5. Summary of interoccurrence intervals for daily precipitation thresholds of 0.75 and 1.0 inch-Continued

\begin{tabular}{|c|c|c|c|c|c|c|c|c|c|c|c|c|c|c|}
\hline \multirow[b]{2}{*}{$\begin{array}{c}\text { Seq. } \\
\text { no. }\end{array}$} & \multicolumn{7}{|c|}{ Daily precipitation threshold of 0.75 inch and greater } & \multicolumn{7}{|c|}{ Daily precipitation threshold of 1.0 inch and greater } \\
\hline & $\begin{array}{l}\text { Mean } \\
\text { inter- } \\
\text { occur- } \\
\text { rence } \\
\text { interval } \\
\text { (days) }\end{array}$ & $\begin{array}{c}\text { Total } \\
\text { no. of } \\
\text { events }\end{array}$ & $\begin{array}{l}\text { Total } \\
\text { no. of } \\
\text { days } \\
\text { avail- } \\
\text { able }\end{array}$ & $\begin{array}{l}\text { Sigma } \\
\mathrm{S}\end{array}$ & $\begin{array}{l}\text { Stan- } \\
\text { dard } \\
\text { normal } \\
\text { variate }\end{array}$ & $\begin{array}{l}\text { Non- } \\
\text { ex- } \\
\text { ceed- } \\
\text { ance } \\
\text { prob- } \\
\text { ability }\end{array}$ & $\begin{array}{c}\text { p- } \\
\text { value }\end{array}$ & $\begin{array}{c}\text { Mean } \\
\text { inter- } \\
\text { occur- } \\
\text { rence } \\
\text { interval } \\
\text { (days) }\end{array}$ & $\begin{array}{l}\text { Total } \\
\text { no. of } \\
\text { events }\end{array}$ & $\begin{array}{l}\text { Total } \\
\text { no. of } \\
\text { days } \\
\text { avail- } \\
\text { able }\end{array}$ & $\begin{array}{c}\text { Sigma } \\
\mathrm{S}\end{array}$ & $\begin{array}{c}\text { Stan- } \\
\text { dard } \\
\text { normal } \\
\text { variate }\end{array}$ & $\begin{array}{l}\text { Non- } \\
\text { ex- } \\
\text { ceed- } \\
\text { ance } \\
\text { prob- } \\
\text { ability }\end{array}$ & $\begin{array}{c}\text { p- } \\
\text { value }\end{array}$ \\
\hline 49 & 15.10 & 20 & 302 & 3540 & 1.334 & .909 & .091 & 27.46 & 11 & 302 & 1775 & 0.394 & .653 & .347 \\
\hline 50 & 24.73 & 37 & 915 & 19784 & 1.778 & .962 & .038 & 45.75 & 20 & 915 & 11406 & 1.910 & .972 & .028 \\
\hline 51 & 25.96 & 702 & 18,223 & 6380688 & -.112 & .455 & .455 & 35.66 & 511 & 18,223 & 4610113 & -.386 & .350 & .350 \\
\hline 52 & 20.58 & 637 & 13,109 & 4192991 & .186 & .574 & .426 & 30.28 & 433 & 13,109 & 2766926 & -.904 & .183 & .183 \\
\hline 53 & 12.75 & 24 & 306 & 3616 & -.129 & .448 & .448 & 18.00 & 17 & 306 & 2969 & 1.010 & .844 & .156 \\
\hline 54 & 29.00 & 3 & 87 & 96 & -.793 & .214 & .214 & 29.00 & 3 & 87 & 96 & -.793 & .214 & .214 \\
\hline 55 & 32.13 & 307 & 9,865 & 1482908 & -.629 & .265 & .265 & 44.84 & 220 & 9,865 & 1067504 & -.418 & .338 & .338 \\
\hline 56 & 39.75 & 60 & 2,385 & 70580 & -.182 & .428 & .428 & 72.27 & 33 & 2,385 & 34616 & -1.198 & .116 & 116 \\
\hline 57 & 72.25 & 271 & 19,579 & 2777083 & 1.334 & .909 & .091 & 117.95 & 166 & 19,579 & 1800859 & 2.414 & .992 & .008 \\
\hline 58 & 40.53 & 15 & 608 & 3295 & -1.861 & .031 & .031 & 50.67 & 12 & 608 & 2559 & -1.791 & .037 & .037 \\
\hline 59 & 36.74 & 986 & 36,226 & 17905266 & .140 & .556 & .444 & 54.48 & 665 & 36,226 & 11882118 & -.605 & .273 & .273 \\
\hline 60 & 140.00 & 3 & 420 & 527 & -.491 & .312 & .312 & 420.00 & 1 & 420 & 339 & 1.064 & .856 & .144 \\
\hline 61 & 86.81 & 321 & 27,865 & 4661655 & 1.314 & .905 & .095 & 146.66 & 190 & 27,865 & 2743441 & .868 & .807 & .193 \\
\hline 62 & 43.60 & 5 & 218 & 701 & 1.109 & .866 & .134 & 109.00 & 2 & 218 & 304 & .966 & .833 & .167 \\
\hline 63 & 52.28 & 39 & 2,039 & 44242 & 1.219 & .889 & .111 & 81.56 & 25 & 2,039 & 27321 & .623 & .733 & .267 \\
\hline 64 & -- & -- & -- & -- & -- & -- & -- & -- & -- & -- & -- & -- & -- & -- \\
\hline 65 & 27.09 & 274 & 7,422 & 1025613 & .248 & .598 & .402 & 37.87 & 196 & 7,422 & 726917 & -.015 & .494 & .494 \\
\hline 66 & 27.79 & 84 & 2,334 & 88293 & -1.577 & .057 & .057 & 36.47 & 64 & 2,334 & 65242 & -1.753 & .040 & .040 \\
\hline 67 & 21.98 & 607 & 13,340 & 4192949 & 1.521 & .936 & .064 & 32.94 & 405 & 13,340 & 2801767 & 1.296 & .902 & .098 \\
\hline 68 & 63.10 & 83 & 5,237 & 224905 & .550 & .709 & .291 & 104.74 & 50 & 5,237 & 139837 & .834 & .798 & .202 \\
\hline 69 & 86.26 & 57 & 4,917 & 145693 & .519 & .698 & .302 & 144.62 & 34 & 4,917 & 83246 & -.041 & .483 & .483 \\
\hline 70 & 25.27 & 41 & 1,036 & 26910 & 2.962 & .998 & .002 & 34.53 & 30 & 1,036 & 20035 & 2.744 & .997 & .003 \\
\hline 71 & 27.21 & 28 & 762 & 10233 & -.374 & .354 & .354 & 40.11 & 19 & 762 & 6758 & -.502 & .308 & .308 \\
\hline 72 & 34.38 & 127 & 4,366 & 286151 & .627 & .735 & .265 & 50.18 & 87 & 4,366 & 193643 & .317 & .624 & .376 \\
\hline 73 & 34.73 & 360 & 12,503 & 2313199 & .915 & .820 & .180 & 48.65 & 257 & 12,503 & 1707227 & 1.739 & .959 & .041 \\
\hline 74 & 25.17 & 48 & 1,208 & 31087 & .867 & .807 & .193 & 35.53 & 34 & 1,208 & 22527 & .979 & .836 & .164 \\
\hline 75 & 19.24 & 1,218 & 23,435 & 15081105 & 3.427 & 1.000 & 0 & 25.95 & 903 & 23,435 & 11392532 & 3.993 & 1.000 & 0 \\
\hline 76 & 57.70 & 20 & 1,154 & 11509 & -.021 & .492 & .492 & 96.17 & 12 & 1,154 & 7085 & .140 & .556 & .444 \\
\hline 77 & 15.81 & 1,002 & 15,838 & 8066916 & .913 & .819 & .181 & 20.98 & 755 & 15,838 & 6105921 & 1.012 & .844 & .156 \\
\hline 78 & 32.91 & 22 & 724 & 7595 & -.376 & .353 & .353 & 45.25 & 16 & 724 & 5369 & -.506 & .306 & .306 \\
\hline 79 & 20.82 & 28 & 583 & 9834 & 1.878 & .970 & .030 & 30.68 & 19 & 583 & 6881 & 1.830 & .966 & .034 \\
\hline 80 & 15.47 & 2,301 & 35,599 & 42448046 & 3.025 & .999 & .001 & 21.39 & 1,664 & 35,599 & 30579926 & 2.294 & .989 & .011 \\
\hline 81 & 15.51 & 911 & 14,126 & 6698981 & 2.150 & .984 & .016 & 21.57 & 655 & 14,126 & 4856381 & 2.205 & .986 & .014 \\
\hline 82 & 18.34 & 691 & 12,671 & 4448061 & .730 & .767 & .233 & 23.60 & 537 & 12,671 & 3488710 & 1.021 & .846 & .154 \\
\hline 83 & 29.61 & 1,204 & 35,650 & 21613219 & .425 & .665 & .335 & 42.34 & 842 & 35,650 & 15310488 & 1.011 & .844 & .156 \\
\hline 84 & 19.29 & 405 & 7,812 & 1608603 & .588 & .722 & .278 & 26.13 & 299 & 7,812 & 1165131 & -.071 & .472 & .472 \\
\hline 85 & 20.69 & 80 & 1,655 & 66921 & .169 & .567 & .433 & 27.58 & 60 & 1,655 & 51787 & .578 & .718 & .282 \\
\hline 86 & 25.00 & 578 & 14,452 & 4461186 & 2.837 & .998 & .002 & 36.40 & 397 & 14,452 & 3010978 & 1.711 & .956 & .044 \\
\hline 87 & 37.91 & 32 & 1,213 & 18157 & -.632 & .264 & .264 & 50.54 & 24 & 1,213 & 14164 & -.229 & .410 & .410 \\
\hline 88 & 33.65 & 382 & 12,853 & 2467741 & .177 & .570 & .430 & 46.07 & 279 & 12,853 & 1881674 & 1.431 & .924 & .076 \\
\hline 89 & 25.91 & 736 & 19,069 & 7303011 & 1.913 & .972 & .028 & 39.32 & 485 & 19,069 & 4835973 & 1.747 & .960 & .040 \\
\hline 90 & 32.12 & 199 & 6,392 & 618410 & -.676 & .250 & .250 & 50.33 & 127 & 6,392 & 420634 & .709 & .761 & .239 \\
\hline 91 & 36.72 & 338 & 12,411 & 2000528 & -1.472 & .071 & .071 & 57.73 & 215 & 12,411 & 1287022 & -.898 & .185 & .185 \\
\hline 92 & 25.07 & 494 & 12,385 & 3169387 & 1.388 & .917 & .083 & 35.90 & 345 & 12,385 & 2193436 & .859 & .805 & .195 \\
\hline 93 & 15.17 & 140 & 2,124 & 151358 & .369 & .644 & .356 & 19.67 & 108 & 2,124 & 116361 & .261 & .603 & .397 \\
\hline 94 & 78.58 & 31 & 2,436 & 34152 & -.921 & .179 & .179 & 187.39 & 13 & 2,436 & 16300 & .184 & .573 & .427 \\
\hline 95 & 36.93 & 28 & 1,034 & 11619 & -1.809 & .035 & .035 & 54.42 & 19 & 1,034 & 8012 & -1.392 & .082 & .082 \\
\hline 96 & 48.26 & 286 & 13,801 & 1938551 & -.519 & .302 & .302 & 74.20 & 186 & 13,801 & 1260498 & -.423 & .336 & .336 \\
\hline 97 & 75.27 & 15 & 1,129 & 6151 & -1.835 & .033 & .033 & 102.64 & 11 & 1,129 & 4729 & -1.370 & .085 & .085 \\
\hline 98 & 50.52 & 377 & 19,044 & 3845520 & 2.396 & .992 & .008 & 74.39 & 256 & 19,044 & 2617430 & 2.044 & .980 & .020 \\
\hline 99 & 28.50 & 4 & 114 & 156 & -1.094 & .137 & .137 & 38.00 & 3 & 114 & 148 & -.404 & .343 & .343 \\
\hline 100 & 39.26 & 728 & 28,580 & 10944880 & 2.434 & .993 & .007 & 55.28 & 517 & 28,580 & 7903819 & 2.750 & .997 & .003 \\
\hline
\end{tabular}


Table 5. Summary of interoccurrence intervals for daily precipitation thresholds of 0.75 and 1.0 inch-Continued

\begin{tabular}{|c|c|c|c|c|c|c|c|c|c|c|c|c|c|c|}
\hline \multirow[b]{2}{*}{$\begin{array}{c}\text { Seq. } \\
\text { no. }\end{array}$} & \multicolumn{7}{|c|}{ Daily precipitation threshold of 0.75 inch and greater } & \multicolumn{7}{|c|}{ Daily precipitation threshold of 1.0 inch and greater } \\
\hline & $\begin{array}{l}\text { Mean } \\
\text { inter- } \\
\text { occur- } \\
\text { rence } \\
\text { interval } \\
\text { (days) }\end{array}$ & $\begin{array}{c}\text { Total } \\
\text { no. of } \\
\text { events }\end{array}$ & $\begin{array}{c}\text { Total } \\
\text { no. of } \\
\text { days } \\
\text { avail- } \\
\text { able }\end{array}$ & $\begin{array}{l}\text { Sigma } \\
\text { S }\end{array}$ & $\begin{array}{c}\text { Stan- } \\
\text { dard } \\
\text { normal } \\
\text { variate }\end{array}$ & $\begin{array}{l}\text { Non- } \\
\text { ex- } \\
\text { ceed- } \\
\text { ance } \\
\text { prob- } \\
\text { ability }\end{array}$ & $\begin{array}{c}\mathrm{p}- \\
\text { value }\end{array}$ & $\begin{array}{l}\text { Mean } \\
\text { inter- } \\
\text { occur- } \\
\text { rence } \\
\text { interval } \\
\text { (days) }\end{array}$ & $\begin{array}{l}\text { Total } \\
\text { no. of } \\
\text { events }\end{array}$ & $\begin{array}{l}\text { Total } \\
\text { no. of } \\
\text { days } \\
\text { avail- } \\
\text { able }\end{array}$ & $\begin{array}{l}\text { Sigma } \\
\text { S }\end{array}$ & $\begin{array}{c}\text { Stan- } \\
\text { dard } \\
\text { normal } \\
\text { variate }\end{array}$ & $\begin{array}{l}\text { Non- } \\
\text { ex- } \\
\text { ceed- } \\
\text { ance } \\
\text { prob- } \\
\text { ability }\end{array}$ & $\begin{array}{c}\mathrm{p}- \\
\text { value }\end{array}$ \\
\hline 101 & 32.78 & 281 & 9,211 & 1254807 & -0.883 & .189 & .189 & 44.93 & 205 & 9,211 & 883272 & -1.599 & .055 & .055 \\
\hline 102 & 24.72 & 32 & 791 & 14199 & 1.195 & .884 & .116 & 30.42 & 26 & 791 & 10752 & .403 & .656 & .344 \\
\hline 103 & 25.90 & 1,446 & 37,448 & 27896360 & 1.998 & .977 & .023 & 38.25 & 979 & 37,448 & 19349503 & 3.012 & .999 & .001 \\
\hline 104 & 30.32 & 207 & 6,276 & 693950 & 1.703 & .956 & .044 & 45.15 & 139 & 6,276 & 464542 & 1.328 & .908 & .092 \\
\hline 105 & 37.67 & 272 & 10,246 & 1347592 & -.940 & .174 & .174 & 50.72 & 202 & 10,246 & 1023732 & -.264 & .396 & .396 \\
\hline 106 & 47.49 & 75 & 3,562 & 110515 & -2.590 & .005 & .005 & 80.96 & 44 & 3,562 & 67480 & -1.596 & .055 & .055 \\
\hline 107 & 22.42 & 334 & 7,487 & 1281725 & .795 & .787 & .213 & 30.44 & 246 & 7,487 & 946678 & .760 & .776 & .224 \\
\hline 108 & 25.79 & 1,448 & 37,342 & 27643807 & 1.483 & .931 & .069 & 36.83 & 1,014 & 37,342 & 19139044 & .602 & .726 & .274 \\
\hline 109 & 14.59 & 988 & 14,412 & 7329318 & 1.604 & .946 & .054 & 20.44 & 705 & 14,412 & 5227872 & 1.337 & .909 & .091 \\
\hline 110 & 19.39 & 1,790 & 34,710 & 32348723 & 3.027 & .999 & .001 & 27.46 & 1,264 & 34,710 & 22652374 & 2.009 & .978 & .022 \\
\hline 111 & 29.86 & 42 & 1,254 & 28472 & .911 & .819 & .181 & 50.16 & 25 & 1,254 & 15566 & -.060 & .476 & .476 \\
\hline 112 & 43.31 & 530 & 22,955 & 5949267 & -.877 & .190 & .190 & 71.07 & 323 & 22,955 & 3730114 & .192 & .576 & .424 \\
\hline 113 & 40.76 & 59 & 2,405 & 59770 & -2.096 & .018 & .018 & 77.58 & 31 & 2,405 & 36089 & -.308 & .379 & .379 \\
\hline 114 & 54.22 & 37 & 2,006 & 42268 & 1.464 & .928 & .072 & 111.44 & 18 & 2,006 & 19042 & .402 & .656 & .344 \\
\hline 115 & 123.71 & 146 & 18,062 & 1365879 & .752 & .774 & .226 & 240.83 & 75 & 18,062 & 738307 & 1.351 & .912 & .088 \\
\hline 116 & 49.20 & 118 & 5,805 & 354698 & .670 & .749 & .251 & 73.48 & 79 & 5,805 & 241625 & .828 & .796 & .204 \\
\hline 117 & 44.58 & 431 & 19,214 & 4277397 & 1.188 & .883 & .118 & 73.06 & 263 & 19,214 & 2537355 & .119 & .547 & .453 \\
\hline 118 & 45.96 & 22 & 1,011 & 11459 & .247 & .598 & .402 & 59.47 & 17 & 1,011 & 8352 & -.201 & .420 & .420 \\
\hline 119 & 26.32 & 1,048 & 27,586 & 15253953 & 3.099 & .999 & .001 & 38.21 & 722 & 27,586 & 10646424 & 3.215 & .999 & .001 \\
\hline 120 & 16.42 & 1,168 & 19,175 & 11639799 & 2.334 & .990 & .010 & 22.91 & 837 & 19,175 & 8394900 & 2.312 & .990 & .010 \\
\hline 121 & 24.35 & 754 & 18,359 & 7443885 & 3.591 & 1.000 & 0 & 35.86 & 512 & 18,359 & 5186240 & 4.056 & 1.000 & 0 \\
\hline 122 & 46.22 & 187 & 8,643 & 824133 & .469 & .681 & .319 & 68.06 & 127 & 8,643 & 566031 & .612 & .730 & .270 \\
\hline 123 & 38.22 & 903 & 34,510 & 15891790 & 1.037 & .850 & .150 & 53.34 & 647 & 34,510 & 11438954 & 1.085 & .861 & .139 \\
\hline 124 & 35.12 & 215 & 7,550 & 830836 & .601 & .726 & .274 & 45.21 & 167 & 7,550 & 616097 & -.509 & .306 & .306 \\
\hline 125 & 34.19 & 694 & 23,727 & 8529918 & 1.644 & .950 & .050 & 50.38 & 471 & 23,727 & 5743426 & 1.048 & .853 & .147 \\
\hline 126 & 19.46 & 186 & 3,619 & 353174 & 1.166 & .878 & .122 & 27.84 & 130 & 3,619 & 235077 & -.013 & .495 & .495 \\
\hline 127 & 56.35 & 344 & 19,385 & 3265114 & -.666 & .253 & .253 & 91.01 & 213 & 19,385 & 1977936 & -1.060 & .145 & .145 \\
\hline 128 & 17.42 & 625 & 10,885 & 3451819 & .640 & .739 & .261 & 23.06 & 472 & 10,885 & 2589015 & .295 & .616 & .384 \\
\hline 129 & 26.53 & 740 & 19,634 & 7436364 & 1.114 & .867 & .133 & 40.57 & 484 & 19,634 & 4917611 & 1.333 & .909 & .091 \\
\hline 130 & 32.61 & 808 & 26,350 & 10922421 & 1.281 & .900 & .100 & 47.82 & 551 & 26,350 & 7477177 & 1.220 & .889 & .111 \\
\hline 131 & 31.71 & 24 & 761 & 9546 & .385 & .650 & .350 & 42.28 & 18 & 761 & 6779 & -.075 & .470 & .470 \\
\hline 132 & 21.54 & 652 & 14,041 & 4755950 & 1.726 & .958 & .042 & 31.70 & 443 & 14,041 & 3201049 & 1.066 & .857 & .143 \\
\hline 133 & 21.27 & 1,707 & 36,302 & 31674693 & 1.596 & .945 & .055 & 29.39 & 1,235 & 36,302 & 22920540 & 1.369 & .914 & .086 \\
\hline 134 & 32.49 & 37 & 1,202 & 18344 & -1.845 & .033 & .033 & 38.77 & 31 & 1,202 & 15019 & -1.870 & .031 & .031 \\
\hline 135 & 33.78 & 36 & 1,216 & 22352 & .220 & .587 & .413 & 55.27 & 22 & 1,216 & 14318 & .572 & .716 & .284 \\
\hline 136 & 26.42 & 1,174 & 31,019 & 18483631 & .898 & .815 & .185 & 39.67 & 782 & 31,019 & 12767654 & 2.553 & .995 & .005 \\
\hline 137 & 26.56 & 133 & 3,532 & 246419 & .982 & .837 & .163 & 40.14 & 88 & 3,532 & 168710 & 1.391 & .918 & .082 \\
\hline 138 & 32.27 & 237 & 7,647 & 986463 & 2.363 & .991 & .009 & 46.91 & 163 & 7,647 & 678563 & 1.963 & .975 & .025 \\
\hline 139 & 143.00 & 1 & 143 & 105 & .812 & .791 & .209 & -- & -- & -- & -- & -- & -- & -- \\
\hline 140 & 21.50 & 60 & 1,290 & 39077 & .131 & .552 & .448 & 29.32 & 44 & 1,290 & 28815 & .176 & .570 & .430 \\
\hline 141 & 16.39 & 651 & 10,670 & 3519164 & .586 & .721 & .279 & 24.25 & 440 & 10,670 & 2346493 & -.014 & .494 & .494 \\
\hline 142 & 15.09 & 532 & 8,028 & 2204335 & 1.289 & .901 & .099 & 20.53 & 391 & 8,028 & 1630846 & 1.339 & .910 & .090 \\
\hline 143 & 15.68 & 1,146 & 17,968 & 10398759 & .587 & .721 & .279 & 22.38 & 803 & 17,968 & 7275692 & .419 & .662 & .338 \\
\hline 144 & 49.35 & 279 & 13,768 & 1809796 & -1.670 & .048 & .048 & 75.65 & 182 & 13,768 & 1126366 & -2.360 & .009 & .009 \\
\hline 145 & 52.78 & 331 & 17,470 & 2888294 & -.033 & .487 & .487 & 76.29 & 229 & 17,470 & 1988337 & -.157 & .438 & .438 \\
\hline 146 & 38.09 & 744 & 28,335 & 10833897 & 1.315 & .906 & .094 & 53.26 & 532 & 28,335 & 7720978 & .975 & .835 & .165 \\
\hline 147 & 30.22 & 643 & 19,432 & 6630128 & 2.691 & .996 & .004 & 44.88 & 433 & 19,432 & 4599240 & 3.360 & 1.000 & 0 \\
\hline 148 & 52.79 & 24 & 1,267 & 16020 & .455 & .676 & .324 & 97.46 & 13 & 1,267 & 8304 & .052 & .521 & .479 \\
\hline 149 & 23.78 & 9 & 214 & 1127 & .885 & .812 & .188 & 23.78 & 9 & 214 & 1127 & .885 & .812 & .188 \\
\hline 150 & 27.52 & 42 & 1,156 & 24894 & .286 & .613 & .387 & 38.53 & 30 & 1,156 & 17385 & .025 & .510 & .490 \\
\hline 151 & 35.09 & 200 & 7,017 & 683327 & -.641 & .261 & .261 & 50.85 & 138 & 7,017 & 468234 & -.670 & .252 & .252 \\
\hline 152 & 80.67 & 98 & 7,906 & 368358 & -.843 & .200 & .200 & 127.52 & 62 & 7,906 & 235480 & -.535 & .297 & .297 \\
\hline
\end{tabular}


Table 5. Summary of interoccurrence intervals for daily precipitation thresholds of 0.75 and 1.0 inch-Continued

\begin{tabular}{|c|c|c|c|c|c|c|c|c|c|c|c|c|c|c|}
\hline \multirow[b]{2}{*}{$\begin{array}{l}\text { Seq. } \\
\text { no. }\end{array}$} & \multicolumn{7}{|c|}{ Daily precipitation threshold of 0.75 inch and greater } & \multicolumn{7}{|c|}{ Daily precipitation threshold of 1.0 inch and greater } \\
\hline & $\begin{array}{l}\text { Mean } \\
\text { inter- } \\
\text { occur- } \\
\text { rence } \\
\text { interval } \\
\text { (days) }\end{array}$ & $\begin{array}{c}\text { Total } \\
\text { no. of } \\
\text { events }\end{array}$ & $\begin{array}{l}\text { Total } \\
\text { no. of } \\
\text { days } \\
\text { avail- } \\
\text { able }\end{array}$ & $\underset{\mathrm{S}}{\text { Sigma }}$ & $\begin{array}{c}\text { Stan- } \\
\text { dard } \\
\text { normal } \\
\text { variate }\end{array}$ & $\begin{array}{l}\text { Non- } \\
\text { ex- } \\
\text { ceed- } \\
\text { ance } \\
\text { prob- } \\
\text { ability }\end{array}$ & $\begin{array}{c}\mathrm{p}- \\
\text { value }\end{array}$ & $\begin{array}{l}\text { Mean } \\
\text { inter- } \\
\text { occur- } \\
\text { rence } \\
\text { interval } \\
\text { (days) }\end{array}$ & $\begin{array}{l}\text { Total } \\
\text { no. of } \\
\text { events }\end{array}$ & $\begin{array}{l}\text { Total } \\
\text { no. of } \\
\text { days } \\
\text { avail- } \\
\text { able }\end{array}$ & $\begin{array}{c}\text { Sigma } \\
\mathrm{S}\end{array}$ & $\begin{array}{c}\text { Stan- } \\
\text { dard } \\
\text { normal } \\
\text { variate }\end{array}$ & $\begin{array}{l}\text { Non- } \\
\text { ex- } \\
\text { ceed- } \\
\text { ance } \\
\text { prob- } \\
\text { ability }\end{array}$ & $\begin{array}{c}\mathrm{p}- \\
\text { value }\end{array}$ \\
\hline 153 & 19.82 & 731 & 14,486 & 5525248 & 2.040 & .979 & .021 & 27.44 & 528 & 14,486 & 4103452 & 2.905 & .998 & .002 \\
\hline 154 & 45.08 & 237 & 10,685 & 1194343 & -1.513 & .065 & .065 & 72.20 & 148 & 10,685 & 719615 & -1.894 & .029 & .029 \\
\hline 155 & 24.58 & 897 & 22,051 & 9883796 & -.032 & .487 & .487 & 35.51 & 621 & 22,051 & 7026580 & 1.133 & .871 & .129 \\
\hline 156 & 66.26 & 222 & 14,709 & 1638658 & .094 & .538 & .462 & 122.58 & 120 & 14,709 & 836275 & -.995 & .160 & .160 \\
\hline 157 & 63.50 & 2 & 127 & 132 & .096 & .538 & .462 & -- & -- & -- & -- & -- & -- & -- \\
\hline 158 & 30.40 & 67 & 2,037 & 59015 & -1.917 & .028 & .028 & 65.71 & 31 & 2,037 & 25686 & -1.798 & .036 & .036 \\
\hline 159 & 28.80 & 611 & 17,595 & 5439143 & .509 & .694 & .306 & 42.92 & 410 & 17,595 & 3672502 & .637 & .738 & .262 \\
\hline 160 & 22.66 & 264 & 5,981 & 791067 & .056 & .522 & .478 & 34.37 & 174 & 5,981 & 518903 & -.063 & .475 & .475 \\
\hline 161 & 25.88 & 521 & 13,484 & 3506179 & -.072 & .471 & .471 & 38.31 & 352 & 13,484 & 2312386 & -.833 & .203 & .203 \\
\hline 162 & 26.76 & 367 & 9,819 & 1932838 & 2.413 & .992 & .008 & 37.91 & 259 & 9,819 & 1373263 & 2.230 & .987 & .013 \\
\hline 163 & 27.61 & 1,247 & 34,426 & 22708965 & 3.546 & 1.000 & 0 & 39.34 & 875 & 34,426 & 15924216 & 2.935 & .998 & .002 \\
\hline 164 & 27.26 & 19 & 518 & 5137 & .331 & .630 & .370 & 37.00 & 14 & 518 & 3626 & 0 & .500 & .500 \\
\hline 165 & 35.91 & 33 & 1,185 & 18761 & -.403 & .344 & .344 & 53.86 & 22 & 1,185 & 12472 & -.351 & .363 & .363 \\
\hline 166 & 30.01 & 211 & 6,331 & 685374 & .657 & .744 & .256 & 40.07 & 158 & 6,331 & 518275 & .789 & .785 & .215 \\
\hline 167 & 34.49 & 35 & 1,207 & 18556 & -1.245 & .107 & .107 & 54.86 & 22 & 1,207 & 11877 & -.857 & .196 & .196 \\
\hline 168 & 20.43 & 690 & 14,097 & 4899962 & .341 & .634 & .366 & 29.99 & 470 & 14,097 & 3280606 & -.365 & .358 & .358 \\
\hline 169 & 24.82 & 49 & 1,216 & 32279 & 1.012 & .844 & .156 & 36.85 & 33 & 1,216 & 19247 & -.405 & .343 & .343 \\
\hline 170 & 39.77 & 153 & 6,084 & 476839 & .525 & .700 & .300 & 64.04 & 95 & 6,084 & 293760 & .279 & .610 & .390 \\
\hline 171 & 34.74 & 237 & 8,233 & 976559 & .026 & .510 & .490 & 52.44 & 157 & 8,233 & 652731 & .216 & .586 & .414 \\
\hline 172 & 23.97 & 1,370 & 32,834 & 22876958 & 1.099 & .864 & .136 & 35.15 & 934 & 32,834 & 15468144 & .465 & .679 & .321 \\
\hline 173 & 44.96 & 44 & 1,978 & 41009 & -.662 & .254 & .254 & 54.94 & 36 & 1,978 & 33919 & -.492 & .311 & .311 \\
\hline 174 & 35.72 & 173 & 6,180 & 568933 & 1.464 & .928 & .072 & 51.93 & 119 & 6,180 & 377970 & .527 & .701 & .299 \\
\hline 175 & 22.40 & 62 & 1,389 & 47916 & 1.538 & .938 & .062 & 31.57 & 44 & 1,389 & 36025 & 2.056 & .980 & .020 \\
\hline 176 & 33.25 & 603 & 20,049 & 6212491 & 1.180 & .881 & .119 & 47.17 & 425 & 20,049 & 4394766 & 1.126 & .870 & .130 \\
\hline 177 & 40.67 & 790 & 32,125 & 12686451 & -.011 & .496 & .496 & 61.78 & 520 & 32,125 & 8203217 & -.706 & .240 & .240 \\
\hline 178 & 30.09 & 11 & 331 & 1538 & -.891 & .186 & .186 & 41.38 & 8 & 331 & 1202 & -.451 & .326 & .326 \\
\hline 179 & 106.92 & 182 & 19,460 & 1956464 & 2.449 & .993 & .007 & 178.53 & 109 & 19,460 & 1170290 & 1.871 & .969 & .031 \\
\hline 180 & 18.50 & 901 & 16,672 & 7754751 & 1.689 & .954 & .046 & 26.26 & 635 & 16,672 & 5333639 & .332 & .630 & .370 \\
\hline 181 & 47.14 & 599 & 28,238 & 8366593 & -.455 & .325 & .325 & 75.71 & 373 & 28,238 & 5372059 & .671 & .749 & .251 \\
\hline 182 & 25.33 & 550 & 13,932 & 3836822 & .059 & .523 & .477 & 36.57 & 381 & 13,932 & 2623913 & -.384 & .351 & .351 \\
\hline 183 & 23.02 & 84 & 1,934 & 84612 & .661 & .746 & .254 & 36.49 & 53 & 1,934 & 55358 & 1.011 & .844 & .156 \\
\hline 184 & 16.32 & 53 & 865 & 23548 & .344 & .635 & .365 & 19.66 & 44 & 865 & 19480 & .272 & .607 & .393 \\
\hline 185 & 62.97 & 35 & 2,204 & 35475 & -.822 & .206 & .206 & 100.18 & 22 & 2,204 & 22664 & -.529 & .298 & .298 \\
\hline 186 & 33.12 & 69 & 2,285 & 78377 & -.083 & .467 & .467 & 40.80 & 56 & 2,285 & 67341 & .681 & .752 & .248 \\
\hline 187 & 15.25 & 4 & 61 & 122 & 0 & .500 & .500 & 30.50 & 2 & 61 & 63 & .080 & .532 & .468 \\
\hline 188 & 29.06 & 510 & 14,820 & 3744959 & -.353 & .362 & .362 & 42.34 & 350 & 14,820 & 2650953 & .718 & .763 & .237 \\
\hline 189 & 43.02 & 614 & 26,415 & 8060020 & -.261 & .397 & .397 & 61.86 & 427 & 26,415 & 5549472 & -.572 & .284 & .284 \\
\hline 190 & 22.54 & 839 & 18,911 & 8320703 & 2.451 & .993 & .007 & 31.47 & 601 & 18,911 & 5894721 & 1.584 & .943 & .057 \\
\hline 191 & 38.04 & 337 & 12,819 & 2238277 & 1.152 & .875 & .125 & 52.75 & 243 & 12,819 & 1605844 & .838 & .799 & .201 \\
\hline 192 & 16.00 & 1,136 & 18,176 & 10818202 & 2.795 & .997 & .003 & 22.44 & 810 & 18,176 & 7878114 & 3.461 & 1.000 & 0 \\
\hline 193 & 45.90 & 265 & 12,164 & 1851240 & 4.190 & 1.000 & 0 & 78.48 & 155 & 12,164 & 1068225 & 2.871 & .998 & .002 \\
\hline 194 & 28.70 & 224 & 6,429 & 697861 & -.799 & .212 & .212 & 39.93 & 161 & 6,429 & 516710 & -.035 & .486 & .486 \\
\hline 195 & 121.62 & 78 & 9,486 & 352836 & -.708 & .240 & .240 & 225.86 & 42 & 9,486 & 184569 & -.825 & .205 & .205 \\
\hline 196 & 42.48 & 339 & 14,400 & 2476327 & .464 & .679 & .321 & 64.29 & 224 & 14,400 & 1646616 & .544 & .707 & .293 \\
\hline 197 & 21.47 & 377 & 8,095 & 1618569 & 2.042 & .979 & .021 & 29.22 & 277 & 8,095 & 1190239 & 1.776 & .962 & .038 \\
\hline 198 & 21.36 & 593 & 12,665 & 3827926 & .817 & .793 & .207 & 31.20 & 406 & 12,665 & 2720115 & 2.024 & .979 & .021 \\
\hline 199 & 15.79 & 1,446 & 22,827 & 16494786 & -.037 & .485 & .485 & 22.06 & 1,035 & 22,827 & 11975702 & .768 & .779 & .221 \\
\hline 200 & 28.88 & 489 & 14,121 & 3585921 & 1.479 & .930 & .070 & 44.13 & 320 & 14,121 & 2330142 & .971 & .834 & .166 \\
\hline 201 & 20.02 & 1,146 & 22,939 & 13503066 & 1.602 & .945 & .055 & 27.84 & 824 & 22,939 & 9880966 & 2.263 & .988 & .012 \\
\hline 202 & 23.14 & 21 & 486 & 5873 & 1.198 & .884 & .116 & 34.71 & 14 & 486 & 3749 & .661 & .746 & .254 \\
\hline 203 & 25.62 & 550 & 14,090 & 4016226 & 1.483 & .931 & .069 & 38.50 & 366 & 14,090 & 2693176 & 1.474 & .930 & .070 \\
\hline 204 & 13.48 & 103 & 1,388 & 71578 & .024 & .509 & .491 & 18.76 & 74 & 1,388 & 51129 & -.066 & .474 & .474 \\
\hline
\end{tabular}


Table 5. Summary of interoccurrence intervals for daily precipitation thresholds of 0.75 and 1.0 inch-Continued

\begin{tabular}{|c|c|c|c|c|c|c|c|c|c|c|c|c|c|c|}
\hline \multirow[b]{2}{*}{$\begin{array}{c}\text { Seq. } \\
\text { no. }\end{array}$} & \multicolumn{7}{|c|}{ Daily precipitation threshold of 0.75 inch and greater } & \multicolumn{7}{|c|}{ Daily precipitation threshold of 1.0 inch and greater } \\
\hline & $\begin{array}{l}\text { Mean } \\
\text { inter- } \\
\text { occur- } \\
\text { rence } \\
\text { interval } \\
\text { (days) }\end{array}$ & $\begin{array}{c}\text { Total } \\
\text { no. of } \\
\text { events }\end{array}$ & $\begin{array}{c}\text { Total } \\
\text { no. of } \\
\text { days } \\
\text { avail- } \\
\text { able }\end{array}$ & $\begin{array}{l}\text { Sigma } \\
\mathrm{S}\end{array}$ & $\begin{array}{c}\text { Stan- } \\
\text { dard } \\
\text { normal } \\
\text { variate }\end{array}$ & $\begin{array}{l}\text { Non- } \\
\text { ex- } \\
\text { ceed- } \\
\text { ance } \\
\text { prob- } \\
\text { ability }\end{array}$ & $\begin{array}{c}\mathrm{p}- \\
\text { value }\end{array}$ & $\begin{array}{c}\text { Mean } \\
\text { inter- } \\
\text { occur- } \\
\text { rence } \\
\text { interval } \\
\text { (days) }\end{array}$ & $\begin{array}{l}\text { Total } \\
\text { no. of } \\
\text { events }\end{array}$ & $\begin{array}{l}\text { Total } \\
\text { no. of } \\
\text { days } \\
\text { avail- } \\
\text { able }\end{array}$ & $\begin{array}{l}\text { Sigma } \\
\text { S }\end{array}$ & $\begin{array}{l}\text { Stan- } \\
\text { dard } \\
\text { normal } \\
\text { variate }\end{array}$ & $\begin{array}{l}\text { Non- } \\
\text { ex- } \\
\text { ceed- } \\
\text { ance } \\
\text { prob- } \\
\text { ability }\end{array}$ & $\begin{array}{c}\mathrm{p}- \\
\text { value }\end{array}$ \\
\hline 205 & 128.00 & 3 & 384 & 509 & -0.349 & .364 & .364 & 192.00 & 2 & 384 & 333 & -0.325 & .372 & .372 \\
\hline 206 & 44.32 & 66 & 2,925 & 84691 & -1.725 & .042 & .042 & 86.03 & 34 & 2,925 & 42292 & -1.510 & .066 & .066 \\
\hline 207 & 39.69 & 13 & 516 & 3118 & -.439 & .330 & .330 & 73.71 & 7 & 516 & 1692 & -.289 & .386 & .386 \\
\hline 208 & 45.77 & 180 & 8,239 & 807911 & 2.081 & .981 & .019 & 71.64 & 115 & 8,239 & 543104 & 2.720 & .997 & .003 \\
\hline 209 & 27.90 & 548 & 15,291 & 4170015 & -.191 & .424 & .424 & 39.61 & 386 & 15,291 & 3042089 & 1.049 & .853 & .147 \\
\hline 210 & 33.80 & 127 & 4,292 & 289100 & 1.186 & .882 & .118 & 49.91 & 86 & 4,292 & 187637 & .268 & .606 & .394 \\
\hline 211 & 32.90 & 62 & 2,040 & 58585 & -1.004 & .158 & .158 & 51.00 & 40 & 2,040 & 41414 & .165 & .566 & .434 \\
\hline 212 & 34.96 & 410 & 14,334 & 2901243 & -.444 & .328 & .328 & 49.09 & 292 & 14,334 & 2065778 & -.382 & .351 & .351 \\
\hline 213 & 23.33 & 136 & 3,173 & 232555 & 1.572 & .942 & .058 & 32.71 & 97 & 3,173 & 162731 & .980 & .836 & .164 \\
\hline 214 & 41.50 & 28 & 1,162 & 14589 & -.946 & .172 & .172 & 61.16 & 19 & 1,162 & 10772 & -.183 & .428 & .428 \\
\hline 215 & 33.38 & 24 & 801 & 9435 & -.156 & .438 & .438 & 61.62 & 13 & 801 & 5903 & .835 & .798 & .202 \\
\hline 216 & 35.28 & 43 & 1,517 & 30905 & -.596 & .276 & .276 & 54.18 & 28 & 1,517 & 22143 & .391 & .652 & .348 \\
\hline 217 & 35.23 & 430 & 15,148 & 3184004 & -.803 & .211 & .211 & 51.70 & 293 & 15,148 & 2128580 & -1.210 & .113 & .113 \\
\hline 218 & 45.01 & 411 & 18,497 & 4001427 & 1.850 & .968 & .032 & 72.25 & 256 & 18,497 & 2446908 & .928 & .823 & .177 \\
\hline 219 & -- & -- & -- & -- & -- & -- & -- & -- & -- & -- & -- & -- & -- & -- \\
\hline 220 & 35.87 & 683 & 24,501 & 8190214 & -.957 & .169 & .169 & 54.21 & 452 & 24,501 & 5187302 & -2.327 & .010 & .010 \\
\hline 221 & 14.04 & 336 & 4,717 & 801365 & .357 & .639 & .361 & 18.64 & 253 & 4,717 & 628624 & 1.474 & .930 & .070 \\
\hline 222 & 56.01 & 353 & 19,773 & 3763159 & 2.548 & .995 & .005 & 97.40 & 203 & 19,773 & 2124848 & 1.450 & .926 & .074 \\
\hline 223 & 37.84 & 172 & 6,509 & 567978 & .333 & .630 & .370 & 53.35 & 122 & 6,509 & 395660 & -.067 & .473 & .473 \\
\hline 224 & 45.99 & 133 & 6,117 & 403395 & -.166 & .434 & .434 & 81.56 & 75 & 6,117 & 217131 & -.802 & .211 & .211 \\
\hline 225 & 27.91 & 424 & 11,834 & 2682641 & 2.471 & .993 & .007 & 34.70 & 341 & 11,834 & 2090509 & 1.154 & .876 & .124 \\
\hline 226 & 40.69 & 822 & 33,447 & 13558696 & -.679 & .249 & .249 & 62.29 & 537 & 33,447 & 8609652 & -1.658 & .049 & .049 \\
\hline 227 & 17.14 & 70 & 1,200 & 45274 & 1.130 & .871 & .129 & 24.49 & 49 & 1,200 & 32357 & 1.219 & .889 & .111 \\
\hline 228 & 16.97 & 2,006 & 34,037 & 34163435 & .055 & .522 & .478 & 23.60 & 1,442 & 34,037 & 24567134 & .071 & .528 & .472 \\
\hline 229 & 29.48 & 309 & 9,110 & 1506593 & 2.144 & .984 & .016 & 42.37 & 215 & 9,110 & 1067417 & 2.285 & .989 & .011 \\
\hline 230 & 37.79 & 847 & 32,007 & 13389170 & -.617 & .269 & .269 & 57.77 & 554 & 32,007 & 8471987 & -1.812 & .035 & .035 \\
\hline 231 & 24.75 & 1,337 & 33,090 & 22250397 & .371 & .645 & .355 & 35.43 & 934 & 33,090 & 15545690 & .317 & .625 & .375 \\
\hline 232 & 20.81 & 120 & 2,497 & 151862 & .259 & .602 & .398 & 30.45 & 82 & 2,497 & 108770 & .979 & .836 & .164 \\
\hline 233 & 15.88 & 1,088 & 17,281 & 9756868 & 2.164 & .985 & .015 & 21.85 & 791 & 17,281 & 7014677 & 1.283 & .900 & .100 \\
\hline 234 & 21.12 & 49 & 1,035 & 24507 & -.407 & .342 & .342 & 38.33 & 27 & 1,035 & 11576 & -1.544 & .061 & .061 \\
\hline 235 & 24.84 & 941 & 23,374 & 10573000 & -2.051 & .020 & .020 & 36.93 & 633 & 23,374 & 7048168 & -2.060 & .020 & .020 \\
\hline 236 & 38.03 & 151 & 5,743 & 405285 & -1.390 & .082 & .082 & 50.38 & 114 & 5,743 & 311246 & -.910 & .182 & .182 \\
\hline 237 & 17.97 & 1,034 & 18,579 & 10290330 & 3.972 & 1.000 & 0 & 24.45 & 760 & 18,579 & 7329926 & 1.826 & .966 & .034 \\
\hline 238 & 34.91 & 122 & 4,259 & 236171 & -1.740 & .041 & .041 & 52.58 & 81 & 4,259 & 174476 & .180 & .571 & .429 \\
\hline 239 & 17.61 & 984 & 17,330 & 9020415 & 3.148 & .999 & .001 & 24.72 & 701 & 17,330 & 6360491 & 2.162 & .985 & .015 \\
\hline 240 & 60.97 & 214 & 13,047 & 1417960 & .398 & .655 & .345 & 111.51 & 117 & 13,047 & 765577 & .057 & .523 & .477 \\
\hline 241 & 30.59 & 1,223 & 37,406 & 22751455 & -.324 & .373 & .373 & 44.85 & 834 & 37,406 & 15839033 & .772 & .780 & .220 \\
\hline 242 & 20.88 & 839 & 17,517 & 7530962 & 1.247 & .894 & .106 & 30.10 & 582 & 17,517 & 5088157 & -.076 & .470 & .470 \\
\hline 243 & 22.19 & 932 & 20,679 & 9501051 & -.743 & .229 & .229 & 32.46 & 637 & 20,679 & 6394465 & -1.273 & .102 & .102 \\
\hline 244 & 18.40 & 5 & 92 & 154 & -1.280 & .100 & .100 & 18.40 & 5 & 92 & 154 & -1.280 & .100 & .100 \\
\hline 245 & 43.25 & 610 & 26,383 & 7761323 & -1.518 & .065 & .065 & 63.88 & 413 & 26,383 & 5094793 & -2.283 & .011 & .011 \\
\hline 246 & 19.95 & 1,004 & 20,030 & 10705029 & 3.548 & 1.000 & 0 & 27.51 & 728 & 20,030 & 7827685 & 3.441 & 1.000 & 0 \\
\hline 247 & 27.60 & 1,106 & 30,526 & 17294155 & 1.410 & .921 & .079 & 43.30 & 705 & 30,526 & 11139169 & 1.619 & .947 & .053 \\
\hline 248 & 25.97 & 76 & 1,974 & 75667 & .132 & .552 & .448 & 34.63 & 57 & 1,974 & 57416 & .269 & .606 & .394 \\
\hline 249 & 18.50 & 163 & 3,015 & 260636 & 1.342 & .910 & .090 & 23.56 & 128 & 3,015 & 201090 & .826 & .795 & .205 \\
\hline 250 & 54.63 & 136 & 7,429 & 460476 & -1.787 & .037 & .037 & 80.75 & 92 & 7,429 & 320582 & -1.028 & .152 & .152 \\
\hline 251 & 120.50 & 2 & 241 & 165 & -.773 & .220 & .220 & -- & -- & -- & -- & -- & -- & -- \\
\hline 252 & 49.43 & 47 & 2,323 & 50009 & -.997 & .160 & .160 & 70.39 & 33 & 2,323 & 34520 & -.989 & .161 & .161 \\
\hline 253 & 37.84 & 37 & 1,400 & 29497 & 1.463 & .928 & .072 & 50.00 & 28 & 1,400 & 24062 & 2.087 & .982 & .018 \\
\hline 254 & 53.68 & 363 & 19,485 & 3611287 & .698 & .757 & .243 & 89.38 & 218 & 19,485 & 2212720 & 1.070 & .858 & .142 \\
\hline 255 & 18.14 & 1,066 & 19,333 & 10771117 & 2.561 & .995 & .005 & 24.85 & 778 & 19,333 & 7958402 & 2.813 & .998 & .002 \\
\hline 256 & 17.07 & 1,067 & 18,213 & 10198824 & 2.808 & .998 & .002 & 24.32 & 749 & 18,213 & 7262480 & 3.070 & .999 & .001 \\
\hline
\end{tabular}


Table 5. Summary of interoccurrence intervals for daily precipitation thresholds of 0.75 and 1.0 inch-Continued

\begin{tabular}{|c|c|c|c|c|c|c|c|c|c|c|c|c|c|c|}
\hline \multirow[b]{2}{*}{$\begin{array}{c}\text { Seq. } \\
\text { no. }\end{array}$} & \multicolumn{7}{|c|}{ Daily precipitation threshold of 0.75 inch and greater } & \multicolumn{7}{|c|}{ Daily precipitation threshold of 1.0 inch and greater } \\
\hline & $\begin{array}{l}\text { Mean } \\
\text { inter- } \\
\text { occur- } \\
\text { rence } \\
\text { interval } \\
\text { (days) }\end{array}$ & $\begin{array}{c}\text { Total } \\
\text { no. of } \\
\text { events }\end{array}$ & $\begin{array}{l}\text { Total } \\
\text { no. of } \\
\text { days } \\
\text { avail- } \\
\text { able }\end{array}$ & $\begin{array}{l}\text { Sigma } \\
\mathrm{S}\end{array}$ & $\begin{array}{l}\text { Stan- } \\
\text { dard } \\
\text { normal } \\
\text { variate }\end{array}$ & $\begin{array}{l}\text { Non- } \\
\text { ex- } \\
\text { ceed- } \\
\text { ance } \\
\text { prob- } \\
\text { ability }\end{array}$ & $\begin{array}{c}\text { p- } \\
\text { value }\end{array}$ & $\begin{array}{c}\text { Mean } \\
\text { inter- } \\
\text { occur- } \\
\text { rence } \\
\text { interval } \\
\text { (days) }\end{array}$ & $\begin{array}{l}\text { Total } \\
\text { no. of } \\
\text { events }\end{array}$ & $\begin{array}{l}\text { Total } \\
\text { no. of } \\
\text { days } \\
\text { avail- } \\
\text { able }\end{array}$ & $\begin{array}{c}\text { Sigma } \\
\mathrm{S}\end{array}$ & $\begin{array}{c}\text { Stan- } \\
\text { dard } \\
\text { normal } \\
\text { variate }\end{array}$ & $\begin{array}{l}\text { Non- } \\
\text { ex- } \\
\text { ceed- } \\
\text { ance } \\
\text { prob- } \\
\text { ability }\end{array}$ & $\begin{array}{c}p- \\
\text { value }\end{array}$ \\
\hline 257 & 202.67 & 3 & 608 & 803 & -0359 & .360 & .360 & 202.67 & 3 & 608 & 803 & -0.359 & .360 & .360 \\
\hline 258 & 50.56 & 385 & 19,465 & 3869214 & 1.108 & .866 & .134 & 78.49 & 248 & 19,465 & 2610376 & 2.223 & .987 & .013 \\
\hline 259 & 28.07 & 161 & 4,519 & 321096 & -2.579 & .005 & .005 & 44.74 & 101 & 4,519 & 202781 & -1.940 & .026 & .026 \\
\hline 260 & 24.93 & 885 & 22,062 & 9570882 & -1.011 & .156 & .156 & 35.58 & 620 & 22,062 & 6842022 & .018 & .507 & .493 \\
\hline 261 & 28.65 & 51 & 1,461 & 35621 & -.543 & .294 & .294 & 42.97 & 34 & 1,461 & 23146 & -.688 & .246 & .246 \\
\hline 262 & 23.72 & 283 & 6,712 & 929823 & -.611 & .271 & .271 & 31.96 & 210 & 6,712 & 696125 & -.308 & .379 & .379 \\
\hline 263 & 122.77 & 151 & 18,538 & 1559730 & 2.435 & .993 & .007 & 226.07 & 82 & 18,538 & 874095 & 2.353 & .991 & .009 \\
\hline 264 & 32.25 & 369 & 11,901 & 2416640 & 3.347 & 1.000 & 0 & 44.41 & 268 & 11,901 & 1753392 & 2.821 & .998 & .002 \\
\hline 265 & 31.01 & 636 & 19,723 & 6577974 & 2.132 & .983 & .017 & 42.88 & 460 & 19,723 & 4811537 & 2.254 & .988 & .012 \\
\hline 266 & 13.68 & 242 & 3,310 & 399511 & -.067 & .473 & .473 & 18.60 & 178 & 3,310 & 289296 & -.415 & .339 & .339 \\
\hline 267 & 21.96 & 1,658 & 36,415 & 30338883 & .352 & .638 & .362 & 31.39 & 1,160 & 36,415 & 21509566 & 1.086 & .861 & .139 \\
\hline 268 & 20.18 & 181 & 3,653 & 336695 & .430 & .666 & .334 & 29.70 & 123 & 3,653 & 236091 & .977 & .836 & .164 \\
\hline 269 & 23.63 & 30 & 709 & 11934 & 1.159 & .877 & .123 & 35.45 & 20 & 709 & 8674 & 1.731 & .958 & .042 \\
\hline 270 & 27.12 & 522 & 14,154 & 3751926 & .618 & .732 & .268 & 38.36 & 369 & 14,154 & 2663116 & .659 & .745 & .255 \\
\hline 271 & -- & -- & -- & -- & -- & -- & -- & -- & -- & -- & -- & -- & -- & -- \\
\hline 272 & -- & -- & -- & -- & -- & -- & -- & -- & -- & -- & -- & -- & -- & -- \\
\hline 273 & 40.01 & 794 & 31,770 & 12560740 & -.201 & .420 & .420 & 55.93 & 568 & 31,770 & 8982155 & -.185 & .426 & .426 \\
\hline 274 & 43.28 & 263 & 11,383 & 1646467 & 2.807 & .998 & .003 & 57.20 & 199 & 11,383 & 1248542 & 2.501 & .994 & .006 \\
\hline 275 & 36.97 & 65 & 2,403 & 74198 & -.697 & .243 & .243 & 42.91 & 56 & 2,403 & 64077 & -.618 & .268 & .268 \\
\hline 276 & 41.66 & 155 & 6,457 & 492516 & -.341 & .367 & .367 & 67.97 & 95 & 6,457 & 303153 & -.196 & .422 & .422 \\
\hline 277 & 23.85 & 47 & 1,121 & 28457 & .953 & .830 & .170 & 33.97 & 33 & 1,121 & 19449 & .512 & .696 & .304 \\
\hline 278 & 21.49 & 551 & 11,840 & 3292423 & .380 & .648 & .352 & 31.49 & 376 & 11,840 & 2192244 & -.508 & .306 & .306 \\
\hline 279 & 365.50 & 2 & 731 & 449 & -.945 & .172 & .172 & 365.50 & 2 & 731 & 449 & -.945 & .172 & .172 \\
\hline 280 & 68.40 & 241 & 16,485 & 2095962 & 1.483 & .931 & .069 & 111.39 & 148 & 16,485 & 1273652 & .929 & .823 & .177 \\
\hline 281 & 26.44 & 167 & 4,415 & 398495 & 1.812 & .965 & .035 & 38.73 & 114 & 4,415 & 265818 & 1.041 & .851 & .149 \\
\hline 282 & 23.12 & 58 & 1,341 & 38818 & -.024 & .490 & .490 & 34.39 & 39 & 1,341 & 28041 & .782 & .783 & .217 \\
\hline 283 & 25.25 & 769 & 19,415 & 7575233 & .709 & .761 & .239 & 39.79 & 488 & 19,415 & 4772123 & .282 & .611 & .389 \\
\hline 284 & 34.18 & 279 & 9,535 & 1359736 & .644 & .740 & .260 & 50.45 & 189 & 9,535 & 981989 & 2.139 & .984 & .016 \\
\hline 285 & 18.03 & 1,805 & 32,536 & 29195740 & -.421 & .337 & .337 & 24.01 & 1,355 & 32,536 & 21206112 & -2.421 & .008 & .008 \\
\hline 286 & 41.20 & 907 & 37,369 & 16619580 & -1.007 & .157 & .157 & 63.34 & 590 & 37,369 & 10769344 & -.971 & .166 & .166 \\
\hline 287 & 44.54 & 41 & 1,826 & 36508 & -.274 & .392 & .392 & 62.97 & 29 & 1,826 & 25813 & -.234 & .407 & .407 \\
\hline 288 & 28.97 & 455 & 13,182 & 3056487 & .709 & .761 & .239 & 42.25 & 312 & 13,182 & 1987641 & -1.023 & .153 & .153 \\
\hline 289 & 39.10 & 29 & 1,134 & 13393 & -1.730 & .042 & .042 & 59.68 & 19 & 1,134 & 7386 & -2.374 & .009 & .009 \\
\hline 290 & 31.72 & 962 & 30,511 & 14691629 & .058 & .523 & .477 & 46.16 & 661 & 30,511 & 10064198 & -.087 & .465 & .465 \\
\hline 291 & 43.36 & 450 & 19,511 & 4398844 & .074 & .530 & .470 & 65.69 & 297 & 19,511 & 2884179 & -.136 & .446 & .446 \\
\hline 292 & 28.09 & 78 & 2,191 & 85284 & -.030 & .488 & .488 & 38.44 & 57 & 2,191 & 61458 & -.206 & .418 & .418 \\
\hline 293 & 25.21 & 1,401 & 35,323 & 25002283 & .677 & .751 & .249 & 34.77 & 1,016 & 35,323 & 18548346 & 1.859 & .968 & .032 \\
\hline 294 & 17.14 & 1,099 & 18,834 & 10550431 & 1.116 & .868 & .132 & 22.50 & 837 & 18,834 & 8064310 & 1.159 & .877 & .123 \\
\hline 295 & 28.81 & 206 & 5,935 & 636007 & 1.005 & .842 & .158 & 41.80 & 142 & 5,935 & 441014 & .961 & .832 & .168 \\
\hline 296 & 18.02 & 954 & 17,191 & 8485662 & 1.863 & .969 & .031 & 25.10 & 685 & 17,191 & 6028516 & 1.083 & .860 & .140 \\
\hline 297 & 16.94 & 1,154 & 19,546 & 11713827 & 2.274 & .989 & .012 & 23.84 & 820 & 19,546 & 8292648 & 1.725 & .958 & .042 \\
\hline 298 & 22.83 & 448 & 10,227 & 2351736 & .974 & .835 & .165 & 35.63 & 287 & 10,227 & 1508334 & .815 & .792 & .208 \\
\hline 299 & 77.67 & 3 & 233 & 272 & -.665 & .253 & .253 & 116.50 & 2 & 233 & 136 & -1.020 & .154 & .154 \\
\hline 300 & 55.19 & 88 & 4,857 & 213723 & .001 & .500 & .500 & 105.59 & 46 & 4,857 & 118162 & .678 & .751 & .249 \\
\hline 301 & 53.56 & 315 & 16,871 & 2582375 & -.865 & .193 & .193 & 88.33 & 191 & 16,871 & 1556581 & -.811 & .209 & .209 \\
\hline 302 & 56.29 & 343 & 19,309 & 3506228 & 1.886 & .970 & .030 & 91.51 & 211 & 19,309 & 2243834 & 2.553 & .995 & .005 \\
\hline 303 & 14.76 & 33 & 487 & 7629 & -.503 & .307 & .307 & 17.39 & 28 & 487 & 5816 & -1.347 & .089 & .089 \\
\hline 304 & 23.24 & 1,078 & 25,051 & 13847506 & 1.453 & .927 & .073 & 33.49 & 748 & 25,051 & 9749664 & 1.924 & .973 & .027 \\
\hline 305 & 21.00 & 74 & 1,554 & 50437 & -1.830 & .034 & .034 & 28.78 & 54 & 1,554 & 39449 & -.761 & .223 & .223 \\
\hline 306 & 20.44 & 1,820 & 37,205 & 34021584 & .360 & .641 & .359 & 28.21 & 1,319 & 37,205 & 24771550 & .602 & .726 & .274 \\
\hline 307 & 40.71 & 519 & 21,126 & 5827471 & 2.485 & .994 & .006 & 66.43 & 318 & 21,126 & 3636380 & 2.550 & .995 & .005 \\
\hline 308 & 20.57 & 864 & 17,772 & 7711403 & .225 & .589 & .411 & 27.34 & 650 & 17,772 & 5745663 & -.231 & .409 & .409 \\
\hline
\end{tabular}


Table 5. Summary of interoccurrence intervals for daily precipitation thresholds of 0.75 and 1.0 inch-Continued

\begin{tabular}{|c|c|c|c|c|c|c|c|c|c|c|c|c|c|c|}
\hline \multirow[b]{2}{*}{$\begin{array}{l}\text { Seq. } \\
\text { no. }\end{array}$} & \multicolumn{7}{|c|}{ Daily precipitation threshold of 0.75 inch and greater } & \multicolumn{7}{|c|}{ Daily precipitation threshold of 1.0 inch and greater } \\
\hline & $\begin{array}{l}\text { Mean } \\
\text { inter- } \\
\text { occur- } \\
\text { rence } \\
\text { interval } \\
\text { (days) }\end{array}$ & $\begin{array}{c}\text { Total } \\
\text { no. of } \\
\text { events }\end{array}$ & $\begin{array}{c}\text { Total } \\
\text { no. of } \\
\text { days } \\
\text { avail- } \\
\text { able }\end{array}$ & $\begin{array}{l}\text { Sigma } \\
\mathrm{S}\end{array}$ & $\begin{array}{c}\text { Stan- } \\
\text { dard } \\
\text { normal } \\
\text { variate }\end{array}$ & $\begin{array}{l}\text { Non- } \\
\text { ex- } \\
\text { ceed- } \\
\text { ance } \\
\text { prob- } \\
\text { ability }\end{array}$ & $\begin{array}{c}\mathrm{p}- \\
\text { value }\end{array}$ & $\begin{array}{c}\text { Mean } \\
\text { inter- } \\
\text { occur- } \\
\text { rence } \\
\text { interval } \\
\text { (days) }\end{array}$ & $\begin{array}{l}\text { Total } \\
\text { no. of } \\
\text { events }\end{array}$ & $\begin{array}{l}\text { Total } \\
\text { no. of } \\
\text { days } \\
\text { avail- } \\
\text { able }\end{array}$ & $\begin{array}{l}\text { Sigma } \\
\text { S }\end{array}$ & $\begin{array}{l}\text { Stan- } \\
\text { dard } \\
\text { normal } \\
\text { variate }\end{array}$ & $\begin{array}{l}\text { Non- } \\
\text { ex- } \\
\text { ceed- } \\
\text { ance } \\
\text { prob- } \\
\text { ability }\end{array}$ & $\begin{array}{c}\mathrm{p}- \\
\text { value }\end{array}$ \\
\hline 309 & 40.24 & 29 & 1,167 & 19423 & 1.379 & .916 & .084 & 53.05 & 22 & 1,167 & 15105 & 1.435 & .924 & .076 \\
\hline 310 & 26.52 & 117 & 3,103 & 159373 & -2.286 & .011 & .011 & 37.39 & 83 & 3,103 & 114346 & -1.768 & .039 & .039 \\
\hline 311 & 46.39 & 82 & 3,804 & 161205 & .527 & .701 & .299 & 62.36 & 61 & 3,804 & 114167 & -.216 & .414 & .414 \\
\hline 312 & 22.99 & 806 & 18,527 & 8038207 & 3.766 & 1.000 & 0 & 36.05 & 514 & 18,527 & 5182727 & 3.474 & 1.000 & 0 \\
\hline 313 & 28.66 & 86 & 2,465 & 105532 & -.070 & .472 & .472 & 42.50 & 58 & 2,465 & 69642 & -.340 & .367 & .367 \\
\hline 314 & 21.34 & 53 & 1,131 & 31831 & .782 & .783 & .217 & 31.42 & 36 & 1,131 & 22573 & 1.131 & .871 & .129 \\
\hline 315 & 15.74 & 1,187 & 18,684 & 11544875 & 2.454 & .993 & .007 & 22.46 & 832 & 18,684 & 8215903 & 2.850 & .998 & .002 \\
\hline 316 & 52.08 & 38 & 1,979 & 34686 & -.828 & .204 & .204 & 82.46 & 24 & 1,979 & 22782 & -.345 & .365 & .365 \\
\hline 317 & 90.39 & 18 & 1,627 & 13441 & -.603 & .273 & .273 & 125.15 & 13 & 1,627 & 10768 & .114 & .545 & .455 \\
\hline 318 & 52.38 & 315 & 16,498 & 2685993 & 1.036 & .850 & .150 & 76.38 & 216 & 16,498 & 1855739 & 1.057 & .855 & .145 \\
\hline 319 & 43.98 & 85 & 3,738 & 165895 & .707 & .760 & .240 & 69.22 & 54 & 3,738 & 105536 & .581 & .719 & .281 \\
\hline 320 & 138.21 & 58 & 8,016 & 246321 & .786 & .784 & .216 & 308.31 & 26 & 8,016 & 113553 & .792 & .786 & .214 \\
\hline 321 & 21.35 & 971 & 20,733 & 10414057 & 1.867 & .969 & .031 & 30.58 & 678 & 20,733 & 7311360 & 1.815 & .965 & .035 \\
\hline 322 & 22.15 & 566 & 12,538 & 3637858 & 1.041 & .851 & .149 & 28.18 & 445 & 12,538 & 2897912 & 1.417 & .922 & .078 \\
\hline 323 & 26.17 & 205 & 5,364 & 566418 & .749 & .773 & .227 & 36.49 & 147 & 5,364 & 402292 & .428 & .666 & .334 \\
\hline 324 & 24.01 & 1,316 & 31,602 & 21465786 & 2.030 & .979 & .021 & 35.91 & 880 & 31,602 & 14395457 & 1.813 & .965 & .035 \\
\hline 325 & 56.79 & 75 & 4,259 & 142660 & -1.602 & .055 & .055 & 85.18 & 50 & 4,259 & 90809 & -1.802 & .036 & .036 \\
\hline 326 & 15.83 & 410 & 6,492 & 1324655 & -.164 & .435 & .435 & 21.71 & 299 & 6,492 & 974450 & .120 & .548 & .452 \\
\hline 327 & 33.44 & 243 & 8,126 & 982818 & -.123 & .451 & .451 & 45.65 & 178 & 8,126 & 707274 & -.509 & .305 & .305 \\
\hline 328 & 29.55 & 33 & 975 & 15795 & -.181 & .428 & .428 & 36.11 & 27 & 975 & 13398 & .161 & .564 & .436 \\
\hline 329 & 15.10 & 766 & 11,566 & 4545608 & 1.254 & .895 & .105 & 20.88 & 554 & 11,566 & 3267450 & .810 & .791 & .209 \\
\hline 330 & 17.62 & 2,073 & 36,526 & 37723414 & -.283 & .389 & .389 & 25.09 & 1,456 & 36,526 & 26500049 & -.226 & .411 & .411 \\
\hline 331 & 39.87 & 168 & 6,698 & 551821 & -.431 & .333 & .333 & 61.45 & 109 & 6,698 & 375424 & .514 & .696 & .304 \\
\hline 332 & 38.37 & 831 & 31,881 & 13461504 & .810 & .791 & .209 & 56.03 & 569 & 31,881 & 9224376 & .703 & .759 & .241 \\
\hline 333 & 21.33 & 1,005 & 21,436 & 11099993 & 1.674 & .953 & .047 & 30.32 & 707 & 21,436 & 7708389 & .795 & .787 & .213 \\
\hline 334 & 49.65 & 311 & 15,442 & 2604898 & 2.591 & .995 & .005 & 82.58 & 187 & 15,442 & 1582523 & 2.275 & .989 & .011 \\
\hline 335 & 52.65 & 422 & 22,219 & 4562835 & -.952 & .171 & .171 & 83.22 & 267 & 22,219 & 2904196 & -.592 & .277 & .277 \\
\hline 336 & 37.64 & 55 & 2,070 & 57360 & .098 & .539 & .461 & 47.05 & 44 & 2,070 & 44698 & -.212 & .416 & .416 \\
\hline 337 & 32.38 & 340 & 11,010 & 1822342 & -.842 & .200 & .200 & 50.27 & 219 & 11,010 & 1146162 & -1.264 & .103 & .103 \\
\hline 338 & 33.01 & 175 & 5,777 & 515254 & .443 & .671 & .329 & 45.85 & 126 & 5,777 & 368905 & .265 & .604 & .396 \\
\hline 339 & 15.83 & 307 & 4,859 & 751701 & .238 & .594 & .406 & 23.03 & 211 & 4,859 & 535306 & 1.113 & .867 & .133 \\
\hline 340 & 22.77 & 274 & 6,240 & 872649 & .596 & .724 & .276 & 34.29 & 182 & 6,240 & 566867 & -.040 & .484 & .484 \\
\hline 341 & 69.55 & 140 & 9,737 & 725105 & 1.308 & .905 & .095 & 101.43 & 96 & 9,737 & 513008 & 1.657 & .951 & .049 \\
\hline 342 & 57.75 & 71 & 4,100 & 167022 & 2.153 & .984 & .016 & 74.55 & 55 & 4,100 & 126636 & 1.582 & .943 & .057 \\
\hline 343 & 26.14 & 486 & 12,702 & 3083273 & -.041 & .484 & .484 & 36.50 & 348 & 12,702 & 2177717 & -.474 & .318 & .318 \\
\hline 344 & 26.48 & 1,300 & 34,428 & 23568194 & 3.321 & 1.000 & 0 & 38.77 & 888 & 34,428 & 15945979 & 2.228 & .987 & .013 \\
\hline 345 & 19.40 & 25 & 485 & 5354 & -1.012 & .156 & .156 & 24.25 & 20 & 485 & 4213 & -1.017 & .155 & .155 \\
\hline 346 & 51.01 & 422 & 21,528 & 4370954 & -1.343 & .090 & .090 & 85.09 & 253 & 21,528 & 2642717 & -.815 & .208 & .208 \\
\hline 347 & 38.45 & 31 & 1,192 & 16500 & -1.031 & .151 & .151 & 66.22 & 18 & 1,192 & 10097 & -.432 & .333 & .333 \\
\hline 348 & 36.97 & 302 & 11,166 & 1707649 & .385 & .650 & .350 & 53.43 & 209 & 11,166 & 1187060 & .434 & .668 & .332 \\
\hline 349 & 35.77 & 312 & 11,161 & 1682274 & -1.034 & .151 & .151 & 51.43 & 217 & 11,161 & 1201546 & -.199 & .421 & .421 \\
\hline 350 & 32.39 & 870 & 28,183 & 12359662 & .417 & .662 & .338 & 49.27 & 572 & 28,183 & 8118999 & .302 & .619 & .382 \\
\hline 351 & 39.04 & 99 & 3,865 & 203176 & 1.068 & .857 & .143 & 60.39 & 64 & 3,865 & 128755 & .569 & .715 & .285 \\
\hline 352 & 50.57 & 21 & 1,062 & 9930 & -.869 & .192 & .192 & 66.38 & 16 & 1,062 & 8727 & .188 & .575 & .425 \\
\hline 353 & 19.74 & 165 & 3,257 & 283502 & 1.225 & .890 & .110 & 27.14 & 120 & 3,257 & 200846 & .527 & .701 & .299 \\
\hline 354 & 23.00 & 299 & 6,876 & 1055627 & .806 & .790 & .210 & 34.73 & 198 & 6,876 & 670964 & -.349 & .363 & .363 \\
\hline 355 & 25.39 & 440 & 11,170 & 2404983 & -.775 & .219 & .219 & 36.50 & 306 & 11,170 & 1697357 & -.207 & .418 & .418 \\
\hline 356 & 44.74 & 800 & 35,793 & 14206021 & -.380 & .352 & .352 & 62.91 & 569 & 35,793 & 10153443 & -.120 & .452 & .452 \\
\hline 357 & 30.79 & 1,026 & 31,587 & 16437469 & .799 & .788 & .212 & 46.66 & 677 & 31,587 & 10846059 & .649 & .742 & .258 \\
\hline 358 & 35.04 & 539 & 18,884 & 5168984 & .630 & .736 & .264 & 52.17 & 362 & 18,884 & 3433515 & .150 & .559 & .441 \\
\hline 359 & 33.64 & 66 & 2,220 & 78647 & 1.035 & .850 & .150 & 48.26 & 46 & 2,220 & 54404 & .769 & .779 & .221 \\
\hline 360 & 45.25 & 16 & 724 & 5201 & -.707 & .240 & .240 & 48.27 & 15 & 724 & 5110 & -.395 & .346 & .346 \\
\hline
\end{tabular}


Table 5. Summary of interoccurrence intervals for daily precipitation thresholds of 0.75 and 1.0 inch-Continued

\begin{tabular}{|c|c|c|c|c|c|c|c|c|c|c|c|c|c|c|}
\hline \multirow[b]{2}{*}{$\begin{array}{c}\text { Seq. } \\
\text { no. }\end{array}$} & \multicolumn{7}{|c|}{ Daily precipitation threshold of 0.75 inch and greater } & \multicolumn{7}{|c|}{ Daily precipitation threshold of 1.0 inch and greater } \\
\hline & $\begin{array}{l}\text { Mean } \\
\text { inter- } \\
\text { occur- } \\
\text { rence } \\
\text { interval } \\
\text { (days) }\end{array}$ & $\begin{array}{c}\text { Total } \\
\text { no. of } \\
\text { events }\end{array}$ & $\begin{array}{l}\text { Total } \\
\text { no. of } \\
\text { days } \\
\text { avail- } \\
\text { able }\end{array}$ & $\begin{array}{l}\text { Sigma } \\
\mathrm{S}\end{array}$ & $\begin{array}{c}\text { Stan- } \\
\text { dard } \\
\text { normal } \\
\text { variate }\end{array}$ & $\begin{array}{l}\text { Non- } \\
\text { ex- } \\
\text { ceed- } \\
\text { ance } \\
\text { prob- } \\
\text { ability }\end{array}$ & $\begin{array}{c}p- \\
\text { value }\end{array}$ & $\begin{array}{l}\text { Mean } \\
\text { inter- } \\
\text { occur- } \\
\text { rence } \\
\text { interval } \\
\text { (days) }\end{array}$ & $\begin{array}{l}\text { Total } \\
\text { no. of } \\
\text { events }\end{array}$ & $\begin{array}{l}\text { Total } \\
\text { no. of } \\
\text { days } \\
\text { avail- } \\
\text { able }\end{array}$ & $\begin{array}{l}\text { Sigma } \\
\mathrm{S}\end{array}$ & $\begin{array}{c}\text { Stan- } \\
\text { dard } \\
\text { normal } \\
\text { variate }\end{array}$ & $\begin{array}{l}\text { Non- } \\
\text { ex- } \\
\text { ceed- } \\
\text { ance } \\
\text { prob- } \\
\text { ability }\end{array}$ & $\begin{array}{c}\mathrm{p}- \\
\text { value }\end{array}$ \\
\hline 361 & 30.00 & 5 & 150 & 481 & 1.095 & .863 & .137 & 75.00 & 2 & 150 & 194 & 0.719 & .764 & .236 \\
\hline 362 & 21.93 & 968 & 21,229 & 10081708 & -1.013 & 156 & 156 & 29.40 & 722 & 21,229 & 7432594 & -1.403 & .080 & .080 \\
\hline 363 & 20.26 & 108 & 2,188 & 109702 & -1.287 & .099 & .099 & 26.36 & 83 & 2,188 & 84377 & -1.117 & .132 & .132 \\
\hline 364 & 20.23 & 474 & 9,588 & 2272101 & -.004 & .498 & .498 & 27.71 & 346 & 9,588 & 1644860 & -.269 & .394 & .394 \\
\hline 365 & 18.52 & 1,203 & 22,280 & 13451516 & .225 & .589 & .411 & 26.31 & 847 & 22,280 & 9312503 & -.658 & .255 & .255 \\
\hline 366 & 17.18 & 147 & 2,525 & 186727 & .129 & .551 & .449 & 17.66 & 143 & 2,525 & 181138 & .069 & .528 & .473 \\
\hline 367 & 20.01 & 577 & 11,543 & 3407248 & .963 & .832 & .168 & 29.30 & 394 & 11,543 & 2312843 & .588 & .722 & .278 \\
\hline 368 & 47.34 & 178 & 8,426 & 750977 & .033 & .513 & .487 & 62.42 & 135 & 8,426 & 567446 & -.046 & .482 & .482 \\
\hline 369 & -- & -- & -- & -- & -- & -- & -- & -- & -- & -- & -- & -- & -- & -- \\
\hline 370 & 110.80 & 59 & 6,537 & 158574 & -2.364 & .009 & .009 & 217.90 & 30 & 6,537 & 63246 & -3.368 & 0 & 0 \\
\hline 371 & 180.91 & 109 & 19,719 & 1187166 & 1.893 & .971 & .029 & 352.13 & 56 & 19,719 & 595308 & 1.014 & .845 & .155 \\
\hline 372 & 41.83 & 195 & 8,156 & 827027 & .968 & .833 & .167 & 74.83 & 109 & 8,156 & 488433 & 1.787 & .963 & .037 \\
\hline 373 & 26.57 & 7 & 186 & 554 & -.683 & .247 & .247 & 37.20 & 5 & 186 & 491 & .217 & .586 & .414 \\
\hline 374 & 48.56 & 18 & 874 & 8378 & .478 & .684 & .316 & 62.43 & 14 & 874 & 6259 & .149 & .559 & .441 \\
\hline 375 & 46.82 & 229 & 10,721 & 1323633 & 2.052 & .980 & .020 & 73.94 & 145 & 10,721 & 824906 & 1.278 & .899 & .101 \\
\hline 376 & 37.31 & 78 & 2,910 & 126961 & 1.816 & .965 & .035 & 61.92 & 47 & 2,910 & 75852 & 1.297 & .903 & .097 \\
\hline 377 & 28.45 & 638 & 18,150 & 5943010 & 1.157 & .876 & .124 & 43.84 & 414 & 18,150 & 3970471 & 2.002 & .977 & .023 \\
\hline 378 & 22.58 & 644 & 14,540 & 4647809 & -.320 & .375 & .375 & 33.74 & 431 & 14,540 & 3078945 & -.625 & .266 & .266 \\
\hline 379 & 25.86 & 7 & 181 & 744 & .799 & .788 & .212 & 36.20 & 5 & 181 & 507 & .467 & .680 & .320 \\
\hline 380 & 29.75 & 48 & 1,428 & 36680 & .843 & .800 & .200 & 42.00 & 34 & 1,428 & 24629 & .147 & .558 & .442 \\
\hline 381 & 18.79 & 1,011 & 18,998 & 9868378 & 1.519 & .936 & .064 & 26.03 & 730 & 18,998 & 7017370 & .561 & .712 & .288 \\
\hline 382 & 19.73 & 15 & 296 & 2423 & .613 & .730 & .270 & 26.91 & 11 & 296 & 1677 & .173 & .569 & .431 \\
\hline 383 & 39.67 & 818 & 32,450 & 13708556 & 1.629 & .948 & .052 & 53.90 & 602 & 32,450 & 10274098 & 2.204 & .986 & .014 \\
\hline 384 & 43.45 & 188 & 8,168 & 814570 & 1.447 & .926 & .074 & 66.95 & 122 & 8,168 & 532212 & 1.304 & .904 & .096 \\
\hline 385 & 20.70 & 832 & 17,222 & 7064067 & -.699 & .242 & .242 & 28.66 & 601 & 17,222 & 5077978 & -.798 & .213 & .213 \\
\hline 386 & 40.67 & 6 & 244 & 599 & -.771 & .220 & .220 & 48.80 & 5 & 244 & 489 & -.768 & .221 & .221 \\
\hline 387 & 27.03 & 77 & 2,081 & 83155 & .576 & .718 & .282 & 35.88 & 58 & 2,081 & 62599 & .492 & .689 & .311 \\
\hline 388 & 24.16 & 102 & 2,464 & 125194 & -.065 & .474 & .474 & 36.78 & 67 & 2,464 & 88349 & .997 & .841 & .159 \\
\hline 389 & 25.93 & 14 & 363 & 2855 & .801 & .788 & .212 & 33.00 & 11 & 363 & 2015 & .053 & .521 & .479 \\
\hline 390 & 14.92 & 1,171 & 17,476 & 10323661 & .530 & .702 & .298 & 19.88 & 879 & 17,476 & 7680616 & -.001 & .500 & .500 \\
\hline 391 & 25.89 & 617 & 15,972 & 5079375 & 1.327 & .908 & .092 & 38.39 & 416 & 15,972 & 3377441 & .588 & .722 & .278 \\
\hline 392 & 35.66 & 29 & 1,034 & 10085 & -3.053 & .001 & .001 & 51.70 & 20 & 1,034 & 7195 & -2.356 & .009 & .009 \\
\hline 393 & 185.86 & 57 & 10,594 & 337272 & 1.531 & .937 & .063 & 378.36 & 28 & 10,594 & 180195 & 1.970 & .976 & .024 \\
\hline 394 & -- & -- & -- & -- & -- & -- & -- & -- & -- & -- & -- & -- & -- & -- \\
\hline 395 & 27.88 & 327 & 9,115 & 1568762 & 1.649 & .950 & .050 & 39.29 & 232 & 9,115 & 1152754 & 2.381 & .991 & .009 \\
\hline 396 & 20.12 & 979 & 19,696 & 10250394 & 3.424 & 1.000 & 0 & 28.26 & 697 & 19,696 & 7316116 & 3.012 & .999 & .001 \\
\hline 397 & 47.00 & 303 & 14,241 & 2092411 & -.910 & .182 & .182 & 69.81 & 204 & 14,241 & 1449890 & -.046 & .482 & .482 \\
\hline 398 & 37.54 & 901 & 33,819 & 15575633 & 1.161 & .877 & .123 & 54.02 & 626 & 33,819 & 10781164 & .802 & .789 & .211 \\
\hline 399 & 29.82 & 658 & 19,618 & 6505898 & .355 & .639 & .361 & 43.99 & 446 & 19,618 & 4512190 & 1.149 & .875 & .125 \\
\hline 400 & 18.93 & 1,018 & 19,271 & 9875325 & .374 & .646 & .354 & 26.15 & 737 & 19,271 & 7160406 & .391 & .652 & .348 \\
\hline 401 & 42.49 & 136 & 5,779 & 358814 & -1.756 & .040 & .040 & 69.63 & 83 & 5,779 & 219476 & -1.339 & .090 & .090 \\
\hline 402 & 40.50 & 6 & 243 & 844 & .669 & .748 & .252 & 60.75 & 4 & 243 & 537 & .364 & .642 & .358 \\
\hline 403 & 21.35 & 634 & 13,535 & 4111332 & -1.822 & .034 & .034 & 29.68 & 456 & 13,535 & 2932449 & -1.840 & .033 & .033 \\
\hline 404 & 20.63 & 999 & 20,610 & 10697144 & 2.140 & .984 & .016 & 30.04 & 686 & 20,610 & 7318513 & 1.600 & .945 & .055 \\
\hline 405 & 36.34 & 327 & 11,884 & 2030896 & 1.416 & .922 & .078 & 57.14 & 208 & 11,884 & 1335701 & 2.016 & .978 & .022 \\
\hline 406 & 24.01 & 903 & 21,685 & 10124534 & 1.774 & .962 & .038 & 33.21 & 653 & 21,685 & 7269454 & 1.183 & .882 & .118 \\
\hline 407 & 23.03 & 161 & 3,708 & 302294 & .280 & .610 & .390 & 32.24 & 115 & 3,708 & 211529 & -.146 & .442 & .442 \\
\hline 408 & 23.68 & 1,442 & 34,142 & 25230532 & 1.641 & .950 & .050 & 34.18 & 999 & 34,142 & 17433729 & 1.219 & .889 & .111 \\
\hline 409 & 76.00 & 16 & 1,216 & 12556 & 2.014 & .978 & .022 & 152.00 & 8 & 1,216 & 6940 & 2.091 & .982 & .018 \\
\hline 410 & 18.90 & 762 & 14,398 & 5567750 & .716 & .763 & .237 & 26.71 & 539 & 14,398 & 3981862 & 1.053 & .854 & .146 \\
\hline 411 & 40.64 & 471 & 19,141 & 4568878 & .510 & .695 & .305 & 64.02 & 299 & 19,141 & 2893662 & .336 & .632 & .369 \\
\hline 412 & 22.90 & 607 & 13,897 & 4416349 & 2.009 & .978 & .022 & 31.58 & 440 & 13,897 & 3207298 & 1.782 & .963 & .037 \\
\hline
\end{tabular}


Table 5. Summary of interoccurrence intervals for daily precipitation thresholds of 0.75 and 1.0 inch-Continued

\begin{tabular}{|c|c|c|c|c|c|c|c|c|c|c|c|c|c|c|}
\hline \multirow[b]{2}{*}{$\begin{array}{c}\text { Seq. } \\
\text { no. }\end{array}$} & \multicolumn{7}{|c|}{ Daily precipitation threshold of 0.75 inch and greater } & \multicolumn{7}{|c|}{ Daily precipitation threshold of 1.0 inch and greater } \\
\hline & $\begin{array}{l}\text { Mean } \\
\text { inter- } \\
\text { occur- } \\
\text { rence } \\
\text { interval } \\
\text { (days) }\end{array}$ & $\begin{array}{c}\text { Total } \\
\text { no. of } \\
\text { events }\end{array}$ & $\begin{array}{c}\text { Total } \\
\text { no. of } \\
\text { days } \\
\text { avail- } \\
\text { able }\end{array}$ & $\begin{array}{l}\text { Sigma } \\
\text { S }\end{array}$ & $\begin{array}{c}\text { Stan- } \\
\text { dard } \\
\text { normal } \\
\text { variate }\end{array}$ & $\begin{array}{l}\text { Non- } \\
\text { ex- } \\
\text { ceed- } \\
\text { ance } \\
\text { prob- } \\
\text { ability }\end{array}$ & $\begin{array}{c}\mathrm{p}- \\
\text { value }\end{array}$ & $\begin{array}{c}\text { Mean } \\
\text { inter- } \\
\text { occur- } \\
\text { rence } \\
\text { interval } \\
\text { (days) }\end{array}$ & $\begin{array}{l}\text { Total } \\
\text { no. of } \\
\text { events }\end{array}$ & $\begin{array}{l}\text { Total } \\
\text { no. of } \\
\text { days } \\
\text { avail- } \\
\text { able }\end{array}$ & $\begin{array}{l}\text { Sigma } \\
\text { S }\end{array}$ & $\begin{array}{c}\text { Stan- } \\
\text { dard } \\
\text { normal } \\
\text { variate }\end{array}$ & $\begin{array}{l}\text { Non- } \\
\text { ex- } \\
\text { ceed- } \\
\text { ance } \\
\text { prob- } \\
\text { ability }\end{array}$ & $\begin{array}{c}\mathrm{p}- \\
\text { value }\end{array}$ \\
\hline 413 & 29.80 & 1,012 & 30,162 & 15215669 & -0.167 & .434 & .434 & 42.54 & 709 & 30,162 & 10520782 & -0.740 & .230 & .230 \\
\hline 414 & 24.28 & 141 & 3,423 & 255607 & 1.218 & .888 & .112 & 34.23 & 100 & 3,423 & 179666 & .862 & .806 & .194 \\
\hline 415 & 44.13 & 487 & 21,490 & 5175616 & -.418 & .338 & .338 & 70.46 & 305 & 21,490 & 3187189 & -.831 & .203 & .203 \\
\hline 416 & 47.43 & 408 & 19,352 & 4280643 & 2.950 & .998 & .002 & 72.75 & 266 & 19,352 & 2801939 & 2.504 & .994 & .006 \\
\hline 417 & 27.19 & 113 & 3,072 & 165009 & -.908 & .182 & .182 & 37.93 & 81 & 3,072 & 124408 & -.001 & .500 & .500 \\
\hline 418 & 40.59 & 614 & 24,925 & 8082003 & 2.412 & .992 & .008 & 65.25 & 382 & 24,925 & 5181217 & 2.990 & .999 & .001 \\
\hline 419 & 25.18 & 900 & 22,658 & 10171371 & -.126 & .450 & .450 & 36.25 & 625 & 22,658 & 6942843 & -.843 & .200 & .200 \\
\hline 420 & 46.01 & 416 & 19,138 & 4010058 & .261 & .603 & .397 & 70.10 & 273 & 19,138 & 2667521 & .605 & .727 & .273 \\
\hline 421 & 68.85 & 453 & 31,191 & 7098964 & .179 & .571 & .429 & 134.44 & 232 & 31,191 & 3427386 & -1.391 & .082 & .082 \\
\hline 422 & 124.61 & 93 & 11,589 & 474353 & -2.000 & .023 & .023 & 214.61 & 54 & 11,589 & 248152 & -2.634 & .004 & .004 \\
\hline 423 & 45.65 & 254 & 11,596 & 1524613 & .973 & .835 & .165 & 63.37 & 183 & 11,596 & 1082497 & .474 & .682 & .318 \\
\hline 424 & -- & -- & -- & -- & -- & -- & -- & -- & -- & -- & -- & -- & -- & -- \\
\hline 425 & 84.37 & 252 & 21,262 & 2849968 & 1.755 & .960 & .040 & 135.43 & 157 & 21,262 & 1718773 & .646 & .741 & .259 \\
\hline 426 & 66.17 & 270 & 17,867 & 2333817 & -.923 & .178 & .178 & 110.29 & 162 & 17,867 & 1363941 & -1.269 & .102 & .102 \\
\hline 427 & -- & -- & -- & -- & -- & -- & -- & -- & -- & -- & -- & -- & -- & -- \\
\hline 428 & 26.96 & 178 & 4,798 & 451889 & 1.346 & .911 & .089 & 44.84 & 107 & 4,798 & 284470 & 1.939 & .974 & .026 \\
\hline 429 & 25.65 & 211 & 5,412 & 586010 & .663 & .746 & .254 & 40.09 & 135 & 5,412 & 371669 & .350 & .637 & .363 \\
\hline 430 & 26.30 & 339 & 8,915 & 1575939 & 1.369 & .914 & .086 & 39.27 & 227 & 8,915 & 1052874 & 1.058 & .855 & .145 \\
\hline 431 & 21.30 & 181 & 3,856 & 375621 & 1.780 & .962 & .038 & 32.13 & 120 & 3,856 & 237836 & .531 & .702 & .298 \\
\hline 432 & 18.06 & 481 & 8,688 & 2143397 & .981 & .837 & .163 & 24.54 & 354 & 8,688 & 1532867 & -.104 & .459 & .459 \\
\hline 433 & 35.11 & 779 & 27,354 & 10632363 & -.100 & .460 & .460 & 50.10 & 546 & 27,354 & 7406179 & -.333 & .370 & .370 \\
\hline 434 & 21.08 & 645 & 13,597 & 4555275 & 1.708 & .956 & .044 & 30.90 & 440 & 13,597 & 3086577 & 1.157 & .876 & .124 \\
\hline 435 & 22.96 & 45 & 1,033 & 25265 & 1.011 & .844 & .156 & 36.89 & 28 & 1,033 & 15509 & .664 & .746 & .254 \\
\hline 436 & 30.27 & 960 & 29,054 & 14296038 & 1.347 & .911 & .089 & 43.43 & 669 & 29,054 & 10014277 & 1.363 & .914 & .086 \\
\hline 437 & 17.39 & 1,456 & 25,322 & 18763175 & 1.179 & .881 & .119 & 23.71 & 1,068 & 25,322 & 13895493 & 1.564 & .941 & .059 \\
\hline 438 & 34.96 & 534 & 18,671 & 5251899 & 2.142 & .984 & .016 & 48.37 & 386 & 18,671 & 3804214 & 1.895 & .971 & .029 \\
\hline 439 & 36.12 & 226 & 8,164 & 922755 & .006 & .503 & .497 & 53.71 & 152 & 8,164 & 607359 & -.451 & .326 & .326 \\
\hline 440 & 36.91 & 252 & 9,302 & 1206184 & .801 & .788 & .212 & 51.68 & 180 & 9,302 & 877905 & 1.130 & .871 & .129 \\
\hline 441 & 56.24 & 422 & 23,733 & 5312329 & 2.165 & .985 & .015 & 96.48 & 246 & 23,733 & 3243076 & 3.014 & .999 & .001 \\
\hline 442 & 21.14 & 604 & 12,769 & 4086621 & 2.543 & .995 & .005 & 30.92 & 413 & 12,769 & 2711899 & 1.003 & .842 & .158 \\
\hline 443 & 23.07 & 563 & 12,987 & 3642910 & -.145 & .442 & .442 & 32.80 & 396 & 12,987 & 2532992 & -.515 & .303 & .303 \\
\hline 444 & 41.36 & 39 & 1,613 & 35311 & 1.327 & .908 & .092 & 62.04 & 26 & 1,613 & 24245 & 1.380 & .916 & .084 \\
\hline 445 & 48.83 & 392 & 19,140 & 3995111 & 2.228 & .987 & .013 & 69.35 & 276 & 19,140 & 2806325 & 1.798 & .964 & .036 \\
\hline 446 & 39.23 & 30 & 1,177 & 19093 & .773 & .780 & .220 & 51.17 & 23 & 1,177 & 14782 & .765 & .778 & .222 \\
\hline 447 & 45.78 & 501 & 22,938 & 5922464 & 1.191 & .883 & .117 & 70.15 & 327 & 22,938 & 3843378 & .777 & .781 & .219 \\
\hline 448 & 23.87 & 1,321 & 31,527 & 20599431 & -.678 & .249 & .249 & 34.65 & 910 & 31,527 & 14111111 & -.851 & .197 & .197 \\
\hline 449 & 19.41 & 274 & 5,317 & 723233 & -.205 & .419 & .419 & 27.84 & 191 & 5,317 & 494309 & -.635 & .263 & .263 \\
\hline 450 & 21.23 & 967 & 20,533 & 10382970 & 2.470 & .993 & .007 & 29.67 & 692 & 20,533 & 7493748 & 2.497 & .994 & .006 \\
\hline 451 & 22.52 & 247 & 5,562 & 700315 & .531 & .702 & .298 & 31.60 & 176 & 5,562 & 499777 & .485 & .686 & .314 \\
\hline 452 & 38.00 & 8 & 304 & 1248 & .129 & .551 & .449 & 60.80 & 5 & 304 & 940 & .917 & .820 & .180 \\
\hline 453 & 70.10 & 30 & 2,103 & 28370 & -.955 & .170 & .170 & 161.77 & 13 & 2,103 & 9789 & -1.773 & .038 & .038 \\
\hline 454 & 51.94 & 612 & 31,784 & 9480184 & -1.083 & .140 & .140 & 79.86 & 398 & 31,784 & 6140862 & -1.006 & .157 & .157 \\
\hline 455 & 55.44 & 16 & 887 & 6694 & -.393 & .347 & .347 & 88.70 & 10 & 887 & 4904 & .579 & .719 & .281 \\
\hline 456 & 67.00 & 1 & 67 & 61 & 1.422 & .922 & .078 & 67.00 & 1 & 67 & 61 & 1.422 & .922 & .078 \\
\hline 457 & 26.23 & 13 & 341 & 2696 & 1.351 & .912 & .088 & 48.71 & 7 & 341 & 1833 & 2.455 & .993 & .007 \\
\hline 458 & 15.44 & 403 & 6,223 & 1255000 & .030 & .512 & .488 & 20.81 & 299 & 6,223 & 927939 & -.077 & .469 & .469 \\
\hline 459 & 30.30 & 37 & 1,121 & 20733 & -.003 & .499 & .499 & 37.37 & 30 & 1,121 & 16657 & -.089 & .464 & .464 \\
\hline 460 & 25.35 & 1,291 & 32,732 & 21157258 & .085 & .534 & .466 & 35.58 & 920 & 32,732 & 15008070 & -.170 & .433 & .433 \\
\hline 461 & 34.40 & 895 & 30,785 & 13778351 & .008 & .503 & .497 & 47.58 & 647 & 30,785 & 9923648 & -.156 & .438 & .438 \\
\hline 462 & 25.38 & 699 & 17,740 & 6339841 & 1.032 & .849 & .151 & 36.58 & 485 & 17,740 & 4416703 & 1.018 & .845 & .155 \\
\hline 463 & 22.87 & 327 & 7,477 & 1254399 & .818 & .793 & .207 & 32.94 & 227 & 7,477 & 867843 & .591 & .723 & .277 \\
\hline 464 & 21.64 & 1,040 & 22,500 & 11866676 & .796 & .787 & .213 & 29.15 & 772 & 22,500 & 8908288 & 1.237 & .892 & .108 \\
\hline
\end{tabular}


Table 5. Summary of interoccurrence intervals for daily precipitation thresholds of 0.75 and 1.0 inch-Continued

\begin{tabular}{|c|c|c|c|c|c|c|c|c|c|c|c|c|c|c|}
\hline \multirow[b]{2}{*}{$\begin{array}{l}\text { Seq. } \\
\text { no. }\end{array}$} & \multicolumn{7}{|c|}{ Daily precipitation threshold of 0.75 inch and greater } & \multicolumn{7}{|c|}{ Daily precipitation threshold of 1.0 inch and greater } \\
\hline & $\begin{array}{l}\text { Mean } \\
\text { inter- } \\
\text { occur- } \\
\text { rence } \\
\text { interval } \\
\text { (days) }\end{array}$ & $\begin{array}{c}\text { Total } \\
\text { no. of } \\
\text { events }\end{array}$ & $\begin{array}{l}\text { Total } \\
\text { no. of } \\
\text { days } \\
\text { avail- } \\
\text { able }\end{array}$ & $\underset{\mathrm{S}}{\text { Sigma }}$ & $\begin{array}{c}\text { Stan- } \\
\text { dard } \\
\text { normal } \\
\text { variate }\end{array}$ & $\begin{array}{l}\text { Non- } \\
\text { ex- } \\
\text { ceed- } \\
\text { ance } \\
\text { prob- } \\
\text { ability }\end{array}$ & $\begin{array}{c}\mathrm{p}- \\
\text { value }\end{array}$ & $\begin{array}{c}\text { Mean } \\
\text { inter- } \\
\text { occur- } \\
\text { rence } \\
\text { interval } \\
\text { (days) }\end{array}$ & $\begin{array}{l}\text { Total } \\
\text { no. of } \\
\text { events }\end{array}$ & $\begin{array}{l}\text { Total } \\
\text { no. of } \\
\text { days } \\
\text { avail- } \\
\text { able }\end{array}$ & $\underset{\mathrm{S}}{\text { Sigma }}$ & $\begin{array}{c}\text { Stan- } \\
\text { dard } \\
\text { normal } \\
\text { variate }\end{array}$ & $\begin{array}{l}\text { Non- } \\
\text { ex- } \\
\text { ceed- } \\
\text { ance } \\
\text { prob- } \\
\text { ability }\end{array}$ & $\begin{array}{c}\mathrm{p}- \\
\text { value }\end{array}$ \\
\hline 465 & 21.84 & 90 & 1,966 & 86646 & -0.339 & .367 & .367 & 29.79 & 66 & 1,966 & 61646 & -0.701 & .242 & .242 \\
\hline 466 & 17.61 & 1,490 & 26,233 & 20069205 & 1.798 & .964 & .036 & 25.13 & 1,044 & 26,233 & 13938363 & 1.000 & .841 & .159 \\
\hline 467 & 85.76 & 129 & 11,063 & 778473 & 1.790 & .963 & .037 & 140.04 & 79 & 11,063 & 461478 & .863 & .806 & .194 \\
\hline 468 & 19.86 & 50 & 993 & 17694 & -3.518 & 0 & 0 & 23.09 & 43 & 993 & 14390 & -3.702 & 0 & 0 \\
\hline 469 & 17.05 & 576 & 9,821 & 2747067 & -1.196 & .116 & .116 & 23.67 & 415 & 9,821 & 1988485 & -.855 & .196 & .196 \\
\hline 470 & 45.50 & 2 & 91 & 91 & 0 & .500 & .500 & 91.00 & 1 & 91 & 45 & -.019 & .492 & .492 \\
\hline 471 & 25.40 & 527 & 13,388 & 3513667 & -.159 & .437 & .437 & 37.09 & 361 & 13,388 & 2408000 & -.116 & .454 & .454 \\
\hline 472 & 16.21 & 208 & 3,372 & 368882 & 1.296 & .902 & .098 & 26.34 & 128 & 3,372 & 216726 & .083 & .533 & .467 \\
\hline 473 & 26.34 & 730 & 19,226 & 7442434 & 2.834 & .998 & .002 & 36.55 & 526 & 19,226 & 5395254 & 2.662 & .996 & .004 \\
\hline 474 & 29.87 & 843 & 25,176 & 10682541 & .336 & .632 & .369 & 45.61 & 552 & 25,176 & 6938988 & -.056 & .478 & .478 \\
\hline 475 & 25.73 & 1,242 & 31,959 & 20845372 & 3.072 & .999 & .001 & 36.15 & 884 & 31,959 & 14855240 & 2.659 & .996 & .004 \\
\hline 476 & 23.81 & 766 & 18,237 & 7152107 & 1.149 & .875 & .125 & 32.45 & 562 & 18,237 & 5472772 & 2.790 & .997 & .003 \\
\hline 477 & 29.17 & 144 & 4,201 & 336467 & 2.336 & .990 & .010 & 42.01 & 100 & 4,201 & 240258 & 2.491 & .994 & .006 \\
\hline 478 & 25.40 & 908 & 23,063 & 10841512 & 1.849 & .968 & .032 & 35.76 & 645 & 23,063 & 7799784 & 2.141 & .984 & .016 \\
\hline 479 & 19.06 & 94 & 1,792 & 77593 & -1.322 & .093 & .093 & 28.00 & 64 & 1,792 & 48958 & -2.026 & .021 & .021 \\
\hline 480 & 28.85 & 197 & 5,684 & 590817 & 1.344 & .910 & .090 & 38.93 & 146 & 5,684 & 443063 & 1.419 & .922 & .078 \\
\hline 481 & 17.10 & 740 & 12,650 & 4796714 & 1.170 & .879 & .121 & 23.25 & 544 & 12,650 & 3581184 & 1.648 & .950 & .050 \\
\hline 482 & 24.73 & 150 & 3,709 & 270859 & -.558 & .289 & .289 & 36.01 & 103 & 3,709 & 196873 & .539 & .705 & .295 \\
\hline 483 & 20.61 & 154 & 3,174 & 257877 & 1.185 & .882 & .118 & 28.60 & 111 & 3,174 & 188589 & 1.288 & .901 & .099 \\
\hline 484 & 28.50 & 96 & 2,736 & 138645 & .946 & .828 & .172 & 40.24 & 68 & 2,736 & 100102 & 1.087 & .861 & .139 \\
\hline 485 & 32.62 & 26 & 848 & 8887 & -1.712 & .043 & .043 & 53.00 & 16 & 848 & 5254 & -1.563 & .059 & .059 \\
\hline 486 & 28.23 & 1,232 & 34,779 & 21644107 & .625 & .734 & .266 & 40.25 & 864 & 34,779 & 15177097 & .517 & .697 & .303 \\
\hline 487 & 25.02 & 419 & 10,484 & 2124781 & -1.156 & .124 & .124 & 35.54 & 295 & 10,484 & 1471654 & -1.438 & .075 & .075 \\
\hline 488 & 78.38 & 359 & 28,138 & 5086509 & .232 & .592 & .408 & 119.23 & 236 & 28,138 & 3402856 & .662 & .746 & .254 \\
\hline 489 & 23.21 & 501 & 11,627 & 2980156 & .900 & .816 & .184 & 36.11 & 322 & 11,627 & 1913427 & .689 & .754 & .246 \\
\hline 490 & 23.86 & 327 & 7,802 & 1301774 & .642 & .740 & .261 & 35.46 & 220 & 7,802 & 882815 & .736 & .769 & .231 \\
\hline 491 & 19.41 & 658 & 12,770 & 4288945 & .927 & .823 & .177 & 28.57 & 447 & 12,770 & 2910420 & .723 & .765 & .235 \\
\hline 492 & 25.16 & 997 & 25,088 & 12915779 & 1.790 & .963 & .037 & 36.57 & 686 & 25,088 & 8778307 & .913 & .819 & .181 \\
\hline 493 & 19.34 & 1,844 & 35,661 & 34208113 & 3.006 & .999 & .001 & 27.64 & 1,290 & 35,661 & 23578912 & 1.562 & .941 & .059 \\
\hline 494 & 20.01 & 213 & 4,263 & 455447 & .080 & .532 & .468 & 28.23 & 151 & 4,263 & 329438 & .501 & .692 & .308 \\
\hline 495 & 15.99 & 1,272 & 20,344 & 14018491 & 5.155 & 0 & 0 & 22.36 & 910 & 20,344 & 9961439 & 3.979 & 1.000 & 0 \\
\hline 496 & 46.46 & 464 & 21,557 & 4959904 & -.308 & .379 & .379 & 74.85 & 288 & 21,557 & 2971678 & -1.255 & .105 & .105 \\
\hline 497 & 407.50 & 2 & 815 & 1230 & 1.247 & .894 & .106 & 407.50 & 2 & 815 & 1230 & 1.247 & .894 & .106 \\
\hline 498 & 21.55 & 885 & 19,069 & 8826043 & 2.369 & .991 & .009 & 30.91 & 617 & 19,069 & 6145176 & 1.919 & .973 & .027 \\
\hline 499 & 35.93 & 494 & 17,748 & 4660213 & 2.428 & .992 & .008 & 53.95 & 329 & 17,748 & 3031486 & 1.205 & .886 & .114 \\
\hline 500 & 29.64 & 39 & 1,156 & 22813 & .130 & .552 & .448 & 42.82 & 27 & 1,156 & 16336 & .421 & .663 & .337 \\
\hline 501 & 29.90 & 171 & 5,112 & 415260 & -1.131 & .129 & .129 & 39.02 & 131 & 5,112 & 313947 & -1.237 & .108 & .108 \\
\hline 502 & 18.61 & 1,643 & 30,570 & 26010100 & 2.507 & .994 & .006 & 25.78 & 1,186 & 30,570 & 19019612 & 2.934 & .998 & .002 \\
\hline 503 & 44.67 & 137 & 6,120 & 401830 & -.841 & .200 & .200 & 65.11 & 94 & 6,120 & 272165 & -.904 & .183 & .183 \\
\hline 504 & 54.97 & 67 & 3,683 & 126901 & .405 & .657 & .343 & 87.69 & 42 & 3,683 & 73644 & -.537 & .296 & .296 \\
\hline 505 & 34.55 & 31 & 1,071 & 14158 & -1.419 & .078 & .078 & 56.37 & 19 & 1,071 & 7762 & -1.790 & .037 & .037 \\
\hline 506 & 22.95 & 1,630 & 37,401 & 31885358 & 3.220 & .999 & .001 & 32.41 & 1,154 & 37,401 & 22670035 & 2.971 & .999 & .001 \\
\hline 507 & 15.17 & 92 & 1,396 & 71137 & 1.791 & .963 & .037 & 19.94 & 70 & 1,396 & 54840 & 1.774 & .962 & .038 \\
\hline 508 & 30.43 & 522 & 15,882 & 4212481 & .642 & .740 & .260 & 44.49 & 357 & 15,882 & 2872389 & .432 & .667 & .333 \\
\hline 509 & 36.67 & 696 & 25,519 & 9045930 & .851 & .802 & .198 & 58.94 & 433 & 25,519 & 5552637 & .181 & .572 & .428 \\
\hline 510 & 45.61 & 46 & 2,098 & 44706 & -.864 & .194 & .194 & 72.35 & 29 & 2,098 & 30178 & -.075 & .470 & .470 \\
\hline 511 & 16.60 & 1,120 & 18,591 & 10910877 & 2.783 & .997 & .003 & 22.62 & 822 & 18,591 & 8239990 & 3.894 & 1.000 & 0 \\
\hline 512 & 33.33 & 946 & 31,532 & 14754241 & -.573 & .283 & .283 & 47.06 & 670 & 31,532 & 10431522 & -.559 & .288 & .288 \\
\hline 513 & 30.79 & 692 & 21,306 & 7624456 & 1.561 & .941 & .059 & 45.62 & 467 & 21,306 & 5070719 & .721 & .764 & .236 \\
\hline 514 & -- & -- & -- & -- & -- & -- & -- & -- & -- & -- & -- & -- & -- & -- \\
\hline 515 & 49.04 & 328 & 16,084 & 2532199 & -1.256 & .105 & .105 & 80.02 & 201 & 16,084 & 1497259 & -1.811 & .035 & .035 \\
\hline 516 & 58.53 & 47 & 2,751 & 65603 & .175 & .570 & .430 & 137.55 & 20 & 2,751 & 27297 & -.060 & .476 & .476 \\
\hline
\end{tabular}


Table 5. Summary of interoccurrence intervals for daily precipitation thresholds of 0.75 and 1.0 inch-Continued

\begin{tabular}{|c|c|c|c|c|c|c|c|c|c|c|c|c|c|c|}
\hline \multirow[b]{2}{*}{$\begin{array}{l}\text { Seq. } \\
\text { no. }\end{array}$} & \multicolumn{7}{|c|}{ Daily precipitation threshold of 0.75 inch and greater } & \multicolumn{7}{|c|}{ Daily precipitation threshold of 1.0 inch and greater } \\
\hline & $\begin{array}{l}\text { Mean } \\
\text { inter- } \\
\text { occur- } \\
\text { rence } \\
\text { interval } \\
\text { (days) }\end{array}$ & $\begin{array}{c}\text { Total } \\
\text { no. of } \\
\text { events }\end{array}$ & $\begin{array}{c}\text { Total } \\
\text { no. of } \\
\text { days } \\
\text { avail- } \\
\text { able }\end{array}$ & $\begin{array}{l}\text { Sigma } \\
\mathrm{S}\end{array}$ & $\begin{array}{c}\text { Stan- } \\
\text { dard } \\
\text { normal } \\
\text { variate }\end{array}$ & $\begin{array}{l}\text { Non- } \\
\text { ex- } \\
\text { ceed- } \\
\text { ance } \\
\text { prob- } \\
\text { ability }\end{array}$ & $\begin{array}{c}\mathrm{p}- \\
\text { value }\end{array}$ & $\begin{array}{c}\text { Mean } \\
\text { inter- } \\
\text { occur- } \\
\text { rence } \\
\text { interval } \\
\text { (days) }\end{array}$ & $\begin{array}{l}\text { Total } \\
\text { no. of } \\
\text { events }\end{array}$ & $\begin{array}{l}\text { Total } \\
\text { no. of } \\
\text { days } \\
\text { avail- } \\
\text { able }\end{array}$ & $\begin{array}{l}\text { Sigma } \\
\text { S }\end{array}$ & $\begin{array}{l}\text { Stan- } \\
\text { dard } \\
\text { normal } \\
\text { variate }\end{array}$ & $\begin{array}{l}\text { Non- } \\
\text { ex- } \\
\text { ceed- } \\
\text { ance } \\
\text { prob- } \\
\text { ability }\end{array}$ & $\begin{array}{c}\text { p- } \\
\text { value }\end{array}$ \\
\hline 517 & 54.79 & 357 & 19,560 & 3569276 & 0.729 & .767 & .233 & 86.93 & 225 & 19,560 & 2194988 & -0.065 & .474 & .474 \\
\hline 518 & 35.69 & 1,028 & 36,688 & 19219936 & 1.067 & .857 & .143 & 56.71 & 647 & 36,688 & 12042377 & .645 & .741 & .259 \\
\hline 519 & 39.57 & 195 & 7,716 & 775487 & .745 & .772 & .228 & 59.81 & 129 & 7,716 & 514880 & .680 & .752 & .248 \\
\hline 520 & 31.91 & 21 & 670 & 7340 & .344 & .635 & .365 & 51.54 & 13 & 670 & 4261 & -.135 & .446 & .446 \\
\hline 521 & 17.91 & 995 & 17,822 & 8950443 & .518 & .698 & .302 & 24.31 & 733 & 17,822 & 6562488 & .221 & .587 & .413 \\
\hline 522 & 30.59 & 242 & 7,402 & 962270 & 2.004 & .977 & .023 & 46.85 & 158 & 7,402 & 622578 & 1.408 & .920 & .080 \\
\hline 523 & 32.24 & 118 & 3,804 & 239285 & 1.245 & .893 & .107 & 55.94 & 68 & 3,804 & 142103 & 1.410 & .921 & .079 \\
\hline 524 & 115.95 & 21 & 2,435 & 23634 & -.600 & .274 & .274 & 173.93 & 14 & 2,435 & 16698 & -.132 & .447 & .447 \\
\hline 525 & -- & -- & -- & -- & -- & -- & -- & -- & -- & -- & -- & -- & -- & -- \\
\hline 526 & 42.45 & 767 & 32,561 & 12567138 & .307 & .621 & .379 & 61.21 & 532 & 32,561 & 8472080 & -.872 & .192 & .192 \\
\hline 527 & 14.94 & 582 & 8,692 & 2605343 & 1.255 & .895 & .105 & 19.71 & 441 & 8,692 & 2031108 & 2.173 & .985 & .015 \\
\hline 528 & 14.42 & 219 & 3,157 & 340350 & -.396 & .346 & .346 & 19.49 & 162 & 3,157 & 245712 & -.863 & .194 & .194 \\
\hline 529 & 19.90 & 582 & 11,583 & 3584271 & 2.648 & .996 & .004 & 28.05 & 413 & 11,583 & 2645731 & 3.736 & 1.000 & 0 \\
\hline 530 & 17.03 & 2,004 & 34,124 & 35137149 & 2.143 & .984 & .016 & 23.63 & 1,444 & 34,124 & 25483177 & 2.259 & .988 & .012 \\
\hline 531 & 25.66 & 248 & 6,363 & 812833 & .824 & .795 & .205 & 36.99 & 172 & 6,363 & 604996 & 2.398 & .992 & .008 \\
\hline 532 & 27.95 & 1,290 & 36,056 & 23890172 & 1.696 & .955 & .045 & 41.02 & 879 & 36,056 & 16320809 & 1.537 & .938 & .062 \\
\hline 533 & 54.80 & 470 & 25,757 & 5957377 & -.593 & .277 & .277 & 89.75 & 287 & 25,757 & 3582585 & -.901 & .184 & .184 \\
\hline 534 & 58.00 & 17 & 986 & 8993 & .522 & .699 & .301 & 70.43 & 14 & 986 & 6946 & .041 & .517 & .484 \\
\hline 535 & 25.15 & 1,668 & 41,954 & 35068929 & .160 & .564 & .436 & 36.29 & 1,156 & 41,954 & 24525840 & .671 & .749 & .251 \\
\hline 536 & 25.17 & 6 & 151 & 225 & -2.135 & .016 & .016 & 25.17 & 6 & 151 & 225 & -2.135 & .016 & .016 \\
\hline 537 & 26.71 & 1,193 & 31,864 & 19271876 & .834 & .798 & .202 & 38.39 & 830 & 31,864 & 13527023 & 1.145 & .874 & .126 \\
\hline 538 & 45.80 & 50 & 2,290 & 55353 & -.406 & .342 & .342 & 61.89 & 37 & 2,290 & 42513 & .037 & .515 & .485 \\
\hline 539 & 38.16 & 517 & 19,726 & 5159478 & .466 & .679 & .321 & 58.02 & 340 & 19,726 & 3306146 & -.450 & .326 & .326 \\
\hline 540 & 23.47 & 71 & 1,666 & 70400 & 2.778 & .997 & .003 & 28.24 & 59 & 1,666 & 58573 & 2.552 & .995 & .005 \\
\hline 541 & 23.03 & 1,521 & 35,035 & 26832431 & .477 & .683 & .317 & 32.47 & 1,079 & 35,035 & 18598299 & -.912 & .181 & .181 \\
\hline 542 & 47.36 & 25 & 1,184 & 13092 & -.999 & .159 & .159 & 62.32 & 19 & 1,184 & 10924 & -.218 & .414 & .414 \\
\hline 543 & 44.78 & 187 & 8,373 & 789247 & .193 & .576 & .424 & 72.81 & 115 & 8,373 & 457036 & -.942 & .173 & .173 \\
\hline 544 & 31.14 & 942 & 29,330 & 13589725 & -.865 & .194 & .194 & 44.04 & 666 & 29,330 & 9739509 & -.125 & .450 & .450 \\
\hline 545 & 32.80 & 256 & 8,397 & 1040814 & -.877 & .190 & .190 & 49.11 & 171 & 8,397 & 700626 & -.546 & .292 & .292 \\
\hline 546 & 17.59 & 1,785 & 31,403 & 28919494 & 2.330 & .990 & .010 & 25.76 & 1,219 & 31,403 & 19849634 & 2.242 & .988 & .012 \\
\hline 547 & 34.01 & 519 & 17,650 & 4601625 & .185 & .573 & .427 & 50.57 & 349 & 17,650 & 3096855 & .178 & .571 & .429 \\
\hline 548 & 16.96 & 773 & 13,112 & 5168017 & .952 & .829 & .171 & 23.41 & 560 & 13,112 & 3804373 & 1.485 & .931 & .069 \\
\hline 549 & 219.75 & 4 & 879 & 1933 & .345 & .635 & .365 & 879.00 & 1 & 879 & 770 & 1.303 & .904 & .096 \\
\hline 550 & 25.39 & 33 & 838 & 15148 & .951 & .829 & .171 & 32.23 & 26 & 838 & 11717 & .667 & .748 & .252 \\
\hline 551 & 21.60 & 254 & 5,486 & 742754 & 1.824 & .966 & .034 & 30.48 & 180 & 5,486 & 526866 & 1.559 & .941 & .060 \\
\hline 552 & 19.15 & 1,022 & 19,567 & 10725254 & 4.023 & 1.000 & 0 & 27.37 & 715 & 19,567 & 7447130 & 2.992 & .999 & .001 \\
\hline 553 & 25.33 & 6 & 152 & 636 & 1.675 & .953 & .047 & 152.00 & 1 & 152 & 104 & .638 & .738 & .262 \\
\hline 554 & 17.87 & 666 & 11,901 & 4007684 & .504 & .693 & .307 & 24.85 & 479 & 11,901 & 2919549 & .921 & .821 & .179 \\
\hline 555 & 24.50 & 6 & 147 & 411 & -.289 & .386 & .386 & 147.00 & 1 & 147 & 19 & -1.284 & .100 & .100 \\
\hline 556 & 16.59 & 1,205 & 19,994 & 12581419 & 2.670 & .996 & .004 & 22.17 & 902 & 19,994 & 9551562 & 3.082 & .999 & .001 \\
\hline 557 & 21.08 & 13 & 274 & 1851 & .246 & .597 & .403 & 34.25 & 8 & 274 & 924 & -.769 & .221 & .221 \\
\hline 558 & 19.90 & 107 & 2,129 & 118336 & .698 & .757 & .243 & 27.65 & 77 & 2,129 & 86956 & .925 & .823 & .178 \\
\hline 559 & 18.84 & 31 & 584 & 9195 & .152 & .561 & .439 & 27.81 & 21 & 584 & 6131 & -.001 & .499 & .499 \\
\hline 560 & 22.18 & 11 & 244 & 1477 & .578 & .718 & .282 & 30.50 & 8 & 244 & 977 & .005 & .502 & .498 \\
\hline 561 & 16.68 & 1,067 & 17,792 & 9992478 & 2.983 & .999 & .001 & 22.35 & 796 & 17,792 & 7502447 & 2.907 & .998 & .002 \\
\hline 562 & 16.61 & 1,246 & 20,691 & 13569971 & 3.223 & .999 & .001 & 22.59 & 916 & 20,691 & 10024513 & 3.032 & .999 & .001 \\
\hline 563 & 16.93 & 998 & 16,893 & 9153430 & 4.698 & 1.000 & 0 & 22.49 & 751 & 16,893 & 6769725 & 3.191 & .999 & .001 \\
\hline 564 & 17.80 & 1,066 & 18,979 & 10817960 & 3.925 & 1.000 & 0 & 24.52 & 774 & 18,979 & 7838514 & 3.239 & .999 & .001 \\
\hline 565 & 15.35 & 319 & 4,897 & 818599 & 1.486 & .931 & .069 & 21.67 & 226 & 4,897 & 557999 & .218 & .586 & .414 \\
\hline 566 & 16.95 & 884 & 14,981 & 6775450 & 1.197 & .884 & .116 & 23.05 & 650 & 14,981 & 5010518 & 1.285 & .901 & .099 \\
\hline 567 & 32.21 & 273 & 8,792 & 1484463 & 6.781 & 0 & 0 & 47.78 & 184 & 8,792 & 1026251 & 6.314 & 0 & 0 \\
\hline 568 & 17.08 & 926 & 15,815 & 7592335 & 1.943 & .974 & .026 & 22.56 & 701 & 15,815 & 5782022 & 1.976 & .976 & .024 \\
\hline
\end{tabular}


Table 5. Summary of interoccurrence intervals for daily precipitation thresholds of 0.75 and 1.0 inch-Continued

\begin{tabular}{|c|c|c|c|c|c|c|c|c|c|c|c|c|c|c|}
\hline \multirow[b]{2}{*}{$\begin{array}{c}\text { Seq. } \\
\text { no. }\end{array}$} & \multicolumn{7}{|c|}{ Daily precipitation threshold of 0.75 inch and greater } & \multicolumn{7}{|c|}{ Daily precipitation threshold of 1.0 inch and greater } \\
\hline & $\begin{array}{l}\text { Mean } \\
\text { inter- } \\
\text { occur- } \\
\text { rence } \\
\text { interval } \\
\text { (days) }\end{array}$ & $\begin{array}{c}\text { Total } \\
\text { no. of } \\
\text { events }\end{array}$ & $\begin{array}{l}\text { Total } \\
\text { no. of } \\
\text { days } \\
\text { avail- } \\
\text { able }\end{array}$ & $\begin{array}{l}\text { Sigma } \\
\mathrm{S}\end{array}$ & $\begin{array}{l}\text { Stan- } \\
\text { dard } \\
\text { normal } \\
\text { variate }\end{array}$ & $\begin{array}{l}\text { Non- } \\
\text { ex- } \\
\text { ceed- } \\
\text { ance } \\
\text { prob- } \\
\text { ability }\end{array}$ & $\begin{array}{c}p- \\
\text { value }\end{array}$ & $\begin{array}{c}\text { Mean } \\
\text { inter- } \\
\text { occur- } \\
\text { rence } \\
\text { interval } \\
\text { (days) }\end{array}$ & $\begin{array}{l}\text { Total } \\
\text { no. of } \\
\text { events }\end{array}$ & $\begin{array}{l}\text { Total } \\
\text { no. of } \\
\text { days } \\
\text { avail- } \\
\text { able }\end{array}$ & $\begin{array}{c}\text { Sigma } \\
\mathrm{S}\end{array}$ & $\begin{array}{l}\text { Stan- } \\
\text { dard } \\
\text { normal } \\
\text { variate }\end{array}$ & $\begin{array}{l}\text { Non- } \\
\text { ex- } \\
\text { ceed- } \\
\text { ance } \\
\text { prob- } \\
\text { ability }\end{array}$ & $\begin{array}{c}\mathrm{p}- \\
\text { value }\end{array}$ \\
\hline 569 & 20.17 & 457 & 9,218 & 2142156 & 0.630 & .736 & .264 & 29.83 & 309 & 9,218 & 1424851 & 0.014 & .506 & .494 \\
\hline 570 & 13.37 & 272 & 3,637 & 490407 & -.244 & .404 & .404 & 17.40 & 209 & 3,637 & 381198 & .075 & .530 & .470 \\
\hline 571 & 17.85 & 1,016 & 18,133 & 9815677 & 3.621 & 1.000 & 0 & 24.60 & 737 & 18,133 & 7270029 & 4.138 & 1.000 & 0 \\
\hline 572 & 24.99 & 511 & 12,769 & 3185061 & -.929 & .176 & .176 & 32.83 & 389 & 12,769 & 2358756 & -1.717 & .043 & .043 \\
\hline 573 & 45.01 & 112 & 5,041 & 267983 & -.929 & .176 & .176 & 65.47 & 77 & 5,041 & 183327 & -.842 & .200 & .200 \\
\hline 574 & 16.74 & 667 & 11,163 & 3823084 & 1.204 & .886 & .114 & 23.26 & 480 & 11,163 & 2773818 & 1.341 & .910 & 090 \\
\hline 575 & 33.66 & 520 & 17,501 & 4443228 & -.929 & .176 & .176 & 47.82 & 366 & 17,501 & 3127404 & -.779 & .218 & .218 \\
\hline 576 & 29.89 & 121 & 3,617 & 229665 & .944 & .827 & .173 & 45.79 & 79 & 3,617 & 151985 & .982 & .837 & .163 \\
\hline 577 & 29.66 & 626 & 18,568 & 6126778 & 2.349 & .991 & .009 & 42.78 & 434 & 18,568 & 4349990 & 2.872 & .998 & .002 \\
\hline 578 & 17.99 & 1,120 & 20,145 & 11929125 & 3.329 & 1.000 & 0 & 25.06 & 804 & 20,145 & 8373750 & 1.671 & .953 & .047 \\
\hline 579 & 24.04 & 586 & 14,090 & 4304999 & 1.794 & .964 & .036 & 36.79 & 383 & 14,090 & 2762081 & .802 & .789 & .211 \\
\hline 580 & 18.25 & 4 & 73 & 141 & -.119 & .453 & .453 & 36.50 & 2 & 73 & 80 & .235 & .593 & .407 \\
\hline 581 & 15.48 & 153 & 2,368 & 172987 & -.966 & .167 & .167 & 21.33 & 111 & 2,368 & 127192 & -.588 & .278 & .278 \\
\hline 582 & 26.88 & 688 & 18,495 & 6519181 & 1.120 & .869 & .131 & 36.99 & 500 & 18,495 & 4738923 & .965 & .833 & .167 \\
\hline 583 & 81.73 & 198 & 16,182 & 1825579 & 3.401 & 1.000 & 0 & 118.99 & 136 & 16,182 & 1220300 & 2.201 & .986 & .014 \\
\hline 584 & 24.40 & 10 & 244 & 1227 & .031 & .513 & .487 & 27.11 & 9 & 244 & 1092 & -.028 & .489 & .489 \\
\hline 585 & 32.50 & 404 & 13,130 & 2704936 & .691 & .755 & .245 & 46.40 & 283 & 13,130 & 1880462 & .354 & .638 & .362 \\
\hline 586 & -- & -- & -- & -- & -- & -- & -- & -- & -- & -- & -- & -- & -- & -- \\
\hline 587 & 26.65 & 137 & 3,651 & 252503 & .195 & .577 & .423 & 36.88 & 99 & 3,651 & 192659 & 1.138 & .872 & .128 \\
\hline 588 & 28.69 & 298 & 8,550 & 1224029 & -1.172 & .121 & .121 & 43.85 & 195 & 8,550 & 797530 & -1.047 & .148 & .148 \\
\hline 589 & 20.67 & 317 & 6,553 & 1104406 & 1.952 & .975 & .025 & 30.06 & 218 & 6,553 & 750547 & 1.299 & .903 & .097 \\
\hline 590 & 13.70 & 27 & 370 & 5952 & 1.724 & .958 & .042 & 18.50 & 20 & 370 & 4149 & .940 & .826 & .174 \\
\hline 591 & 19.03 & 192 & 3,653 & 352753 & .141 & .556 & .444 & 30.70 & 119 & 3,653 & 225750 & .730 & .767 & .233 \\
\hline 592 & 27.36 & 807 & 22,076 & 9132402 & 1.241 & .893 & .107 & 40.88 & 540 & 22,076 & 6056490 & .648 & .741 & .259 \\
\hline 593 & 17.35 & 894 & 15,514 & 7367654 & 3.233 & .999 & .001 & 23.90 & 649 & 15,514 & 5399566 & 3.202 & .999 & .001 \\
\hline 594 & 18.13 & 324 & 5,874 & 969455 & .585 & .721 & .279 & 25.65 & 229 & 5,874 & 685806 & .516 & .697 & .303 \\
\hline 595 & 31.06 & 297 & 9,226 & 1398384 & .617 & .731 & .269 & 47.80 & 193 & 9,226 & 920554 & .817 & .793 & .207 \\
\hline 596 & 23.39 & 1,099 & 25,710 & 14404574 & 1.126 & .870 & .130 & 32.71 & 786 & 25,710 & 10475678 & 1.786 & .963 & .037 \\
\hline 597 & 14.98 & 1,373 & 20,570 & 14303691 & .829 & .796 & .204 & 20.39 & 1,009 & 20,570 & 10726274 & 1.849 & .968 & .032 \\
\hline 598 & 14.75 & 71 & 1,047 & 29702 & -2.932 & .002 & .002 & 20.14 & 52 & 1,047 & 21663 & -2.551 & .005 & .005 \\
\hline 599 & 37.18 & 476 & 17,699 & 4311266 & .887 & .812 & .188 & 56.73 & 312 & 17,699 & 2799560 & .427 & .665 & .335 \\
\hline 600 & 23.14 & 844 & 19,532 & 8484359 & 1.477 & .930 & .070 & 31.97 & 611 & 19,532 & 6230478 & 1.890 & .971 & .029 \\
\hline 601 & 16.75 & 1,929 & 32,319 & 31555759 & .937 & .826 & .174 & 24.26 & 1,332 & 32,319 & 21308352 & -.635 & .263 & .263 \\
\hline 602 & 20.53 & 749 & 15,379 & 5701552 & -.476 & .317 & .317 & 29.46 & 522 & 15,379 & 3929910 & -.828 & .204 & .204 \\
\hline 603 & 18.42 & 338 & 6,226 & 1147701 & 2.890 & .998 & .002 & 27.92 & 223 & 6,226 & 721872 & 1.031 & .849 & .151 \\
\hline 604 & 24.85 & 494 & 12,277 & 3056242 & .302 & .619 & .381 & 37.20 & 330 & 12,277 & 2026225 & .008 & .503 & .497 \\
\hline 605 & 39.81 & 156 & 6,210 & 508009 & 1.055 & .854 & .146 & 63.37 & 98 & 6,210 & 325325 & 1.185 & .882 & .118 \\
\hline 606 & 48.31 & 109 & 5,266 & 301008 & .883 & .811 & .189 & 75.23 & 70 & 5,266 & 200037 & 1.237 & .892 & .108 \\
\hline 607 & 44.77 & 87 & 3,895 & 172717 & .313 & .623 & .377 & 68.33 & 57 & 3,895 & 124412 & 1.579 & .943 & .057 \\
\hline 608 & 33.07 & 640 & 21,162 & 6841590 & .451 & .674 & .326 & 46.51 & 455 & 21,162 & 4865805 & .395 & .654 & .347 \\
\hline 609 & 107.22 & 27 & 2,895 & 33422 & -1.304 & .096 & .096 & 152.37 & 19 & 2,895 & 24319 & -.874 & .191 & .191 \\
\hline 610 & 33.25 & 917 & 30,487 & 13224424 & -2.829 & .002 & .002 & 48.16 & 633 & 30,487 & 9229872 & -1.894 & .029 & .029 \\
\hline 611 & 42.81 & 192 & 8,219 & 853031 & 1.947 & .974 & .026 & 69.65 & 118 & 8,219 & 532468 & 1.845 & .967 & .033 \\
\hline 612 & 41.49 & 87 & 3,610 & 175926 & 1.944 & .974 & .026 & 63.33 & 57 & 3,610 & 123593 & 2.632 & .996 & .004 \\
\hline 613 & 36.26 & 19 & 689 & 5952 & -.685 & .247 & .247 & 43.06 & 16 & 689 & 4972 & -.679 & .249 & .249 \\
\hline 614 & 41.41 & 81 & 3,354 & 117531 & -2.101 & .018 & .018 & 60.98 & 55 & 3,354 & 81612 & -1.479 & .070 & .070 \\
\hline 615 & 30.50 & 2 & 61 & 64 & .121 & .548 & .452 & 30.50 & 2 & 61 & 64 & .121 & .548 & .452 \\
\hline 616 & 32.38 & 58 & 1,878 & 59805 & 1.294 & .902 & .098 & 49.42 & 38 & 1,878 & 38493 & .841 & .800 & .200 \\
\hline 617 & 14.37 & 122 & 1,753 & 91222 & -2.811 & .002 & .002 & 20.87 & 84 & 1,753 & 62737 & -2.348 & .009 & .009 \\
\hline 618 & 15.67 & 1,317 & 20,642 & 14359098 & 3.544 & 1.000 & 0 & 21.77 & 948 & 20,642 & 10664525 & 4.798 & 1.000 & 0 \\
\hline 619 & 28.59 & 995 & 28,443 & 13982348 & -.649 & .258 & .258 & 40.17 & 708 & 28,443 & 10031828 & -.169 & .433 & .433 \\
\hline 620 & 30.30 & 23 & 697 & 9013 & 1.034 & .849 & .151 & 46.47 & 15 & 697 & 6045 & 1.049 & .853 & .147 \\
\hline
\end{tabular}


Table 5. Summary of interoccurrence intervals for daily precipitation thresholds of 0.75 and 1.0 inch-Continued

\begin{tabular}{|c|c|c|c|c|c|c|c|c|c|c|c|c|c|c|}
\hline \multirow[b]{2}{*}{$\begin{array}{c}\text { Seq. } \\
\text { no. }\end{array}$} & \multicolumn{7}{|c|}{ Daily precipitation threshold of 0.75 inch and greater } & \multicolumn{7}{|c|}{ Daily precipitation threshold of 1.0 inch and greater } \\
\hline & $\begin{array}{l}\text { Mean } \\
\text { inter- } \\
\text { occur- } \\
\text { rence } \\
\text { interval } \\
\text { (days) }\end{array}$ & $\begin{array}{c}\text { Total } \\
\text { no. of } \\
\text { events }\end{array}$ & $\begin{array}{c}\text { Total } \\
\text { no. of } \\
\text { days } \\
\text { avail- } \\
\text { able }\end{array}$ & $\begin{array}{l}\text { Sigma } \\
\mathrm{S}\end{array}$ & $\begin{array}{c}\text { Stan- } \\
\text { dard } \\
\text { normal } \\
\text { variate }\end{array}$ & $\begin{array}{l}\text { Non- } \\
\text { ex- } \\
\text { ceed- } \\
\text { ance } \\
\text { prob- } \\
\text { ability }\end{array}$ & $\begin{array}{c}\mathrm{p}- \\
\text { value }\end{array}$ & $\begin{array}{c}\text { Mean } \\
\text { inter- } \\
\text { occur- } \\
\text { rence } \\
\text { interval } \\
\text { (days) }\end{array}$ & $\begin{array}{l}\text { Total } \\
\text { no. of } \\
\text { events }\end{array}$ & $\begin{array}{l}\text { Total } \\
\text { no. of } \\
\text { days } \\
\text { avail- } \\
\text { able }\end{array}$ & $\begin{array}{l}\text { Sigma } \\
\text { S }\end{array}$ & $\begin{array}{l}\text { Stan- } \\
\text { dard } \\
\text { normal } \\
\text { variate }\end{array}$ & $\begin{array}{l}\text { Non- } \\
\text { ex- } \\
\text { ceed- } \\
\text { ance } \\
\text { prob- } \\
\text { ability }\end{array}$ & $\begin{array}{c}\text { p- } \\
\text { value }\end{array}$ \\
\hline 621 & 21.32 & 774 & 16,498 & 6773828 & 2.937 & .998 & .002 & 29.57 & 558 & 16,498 & 4944376 & 3.035 & .999 & .001 \\
\hline 622 & 19.62 & 1,844 & 36,185 & 32954814 & -.909 & .182 & .182 & 27.99 & 1,293 & 36,185 & 23281472 & -.299 & .383 & .383 \\
\hline 623 & 27.17 & 271 & 7,364 & 1023441 & .732 & .768 & .232 & 41.14 & 179 & 7,364 & 673634 & .512 & .696 & .304 \\
\hline 624 & 20.68 & 94 & 1,944 & 80246 & -2.044 & .020 & .020 & 28.17 & 69 & 1,944 & 59933 & -1.531 & .063 & .063 \\
\hline 625 & 28.54 & 349 & 9,960 & 1873560 & 2.523 & .994 & .006 & 40.32 & 247 & 9,960 & 1312340 & 1.821 & .966 & .034 \\
\hline 626 & 23.64 & 458 & 10,827 & 2426129 & -.796 & .213 & .213 & 32.51 & 333 & 10,827 & 1795035 & -.134 & .447 & .447 \\
\hline 627 & 95.33 & 124 & 11,821 & 766655 & .888 & .813 & .187 & 164.18 & 72 & 11,821 & 446024 & .707 & .760 & .240 \\
\hline 628 & 65.83 & 76 & 5,003 & 145696 & -3.528 & 0 & 0 & 113.71 & 44 & 5,003 & 77813 & -3.367 & 0 & 0 \\
\hline 629 & 31.16 & 849 & 26,452 & 11137099 & -.413 & .340 & .340 & 45.77 & 578 & 26,452 & 7686639 & .229 & .591 & .409 \\
\hline 630 & 28.43 & 349 & 9,923 & 1728145 & -.064 & .474 & .474 & 40.17 & 247 & 9,923 & 1202337 & -.514 & .304 & .304 \\
\hline 631 & 26.19 & 416 & 10,894 & 2266033 & .001 & .501 & .499 & 39.62 & 275 & 10,894 & 1487681 & -.196 & .422 & .422 \\
\hline 632 & 25.03 & 318 & 7,960 & 1305688 & .977 & .836 & .164 & 35.86 & 222 & 7,960 & 906301 & .664 & .747 & .253 \\
\hline 633 & 20.51 & 83 & 1,702 & 74070 & .768 & .779 & .221 & 32.73 & 52 & 1,702 & 47820 & 1.007 & .843 & .157 \\
\hline 634 & 48.33 & 6 & 290 & 488 & -1.863 & .031 & .031 & 96.67 & 3 & 290 & 406 & -.200 & .421 & .421 \\
\hline 635 & 32.30 & 831 & 26,842 & 11118641 & -.153 & .439 & .439 & 45.04 & 596 & 26,842 & 8003446 & .024 & .510 & .490 \\
\hline 636 & 15.03 & 1,380 & 20,742 & 14586641 & 1.235 & .892 & .109 & 20.68 & 1,003 & 20,742 & 10751134 & 1.841 & .967 & .033 \\
\hline 637 & 46.23 & 528 & 24,410 & 6847254 & 2.489 & .994 & .006 & 66.51 & 367 & 24,410 & 4785115 & 2.266 & .988 & .012 \\
\hline 638 & 39.28 & 283 & 11,115 & 1668555 & 1.775 & .962 & .038 & 62.10 & 179 & 11,115 & 1049915 & 1.284 & .900 & .100 \\
\hline 639 & 25.48 & 205 & 5,224 & 555090 & .909 & .818 & .182 & 41.13 & 127 & 5,224 & 335772 & .238 & .594 & .406 \\
\hline 640 & 15.39 & 381 & 5,865 & 1143833 & .803 & .789 & .211 & 19.29 & 304 & 5,865 & 921318 & 1.011 & .844 & .156 \\
\hline 641 & 46.00 & 26 & 1,196 & 15716 & .095 & .538 & .462 & 92.00 & 13 & 1,196 & 7758 & -.013 & .495 & .495 \\
\hline 642 & 41.71 & 7 & 292 & 1149 & .570 & .715 & .285 & 58.40 & 5 & 292 & 714 & -.085 & .466 & .466 \\
\hline 643 & 22.61 & 1,453 & 32,853 & 24460977 & 1.641 & .950 & .050 & 31.05 & 1,058 & 32,853 & 17586977 & .673 & .750 & .250 \\
\hline 644 & 49.46 & 50 & 2,473 & 69466 & 1.514 & .935 & .065 & 65.08 & 38 & 2,473 & 52827 & 1.327 & .908 & .092 \\
\hline 645 & 41.20 & 666 & 27,442 & 9485612 & 1.699 & .955 & .045 & 57.65 & 476 & 27,442 & 6803917 & 1.578 & .943 & .057 \\
\hline 646 & 149.03 & 139 & 20,715 & 1582133 & 2.020 & .978 & .022 & 304.63 & 68 & 20,715 & 770224 & 1.337 & .909 & .091 \\
\hline 647 & 27.49 & 33 & 907 & 16430 & .974 & .835 & .165 & 41.23 & 22 & 907 & 11853 & 1.528 & .937 & .063 \\
\hline 648 & 28.13 & 68 & 1,913 & 64966 & -.017 & .493 & .493 & 36.09 & 53 & 1,913 & 53706 & .749 & .773 & .227 \\
\hline 649 & 91.80 & 89 & 8,170 & 325534 & -1.709 & .044 & .044 & 163.40 & 50 & 8,170 & 180011 & -1.453 & .073 & .073 \\
\hline 650 & 32.20 & 447 & 14,391 & 3139899 & -.871 & .192 & .192 & 44.83 & 321 & 14,391 & 2340812 & .417 & .662 & .338 \\
\hline 651 & 47.49 & 59 & 2,802 & 76917 & -.924 & .178 & .178 & 82.41 & 34 & 2,802 & 47914 & .059 & .524 & .476 \\
\hline 652 & 31.92 & 25 & 798 & 9985 & .009 & .503 & .497 & 44.33 & 18 & 798 & 7092 & -.092 & .463 & .463 \\
\hline 653 & 24.07 & 45 & 1,083 & 25953 & .756 & .775 & .225 & 30.94 & 35 & 1,083 & 20001 & .567 & .715 & .285 \\
\hline 654 & 45.80 & 226 & 10,351 & 1204846 & .783 & .783 & .217 & 70.90 & 146 & 10,351 & 823309 & 1.875 & .970 & .030 \\
\hline 655 & 26.99 & 106 & 2,861 & 138583 & -1.535 & .062 & .062 & 37.16 & 77 & 2,861 & 98429 & -1.617 & .053 & .053 \\
\hline 656 & 18.38 & 258 & 4,743 & 606720 & -.233 & .408 & .408 & 23.25 & 204 & 4,743 & 478781 & -.256 & .399 & .399 \\
\hline 657 & 34.82 & 333 & 11,595 & 1999044 & 1.121 & .869 & .131 & 57.69 & 201 & 11,595 & 1197215 & .673 & .749 & .251 \\
\hline 658 & 32.55 & 176 & 5,729 & 515130 & .500 & .692 & .308 & 49.39 & 116 & 5,729 & 337370 & .286 & .612 & .388 \\
\hline 659 & 26.19 & 106 & 2,776 & 145228 & -.230 & .409 & .409 & 38.03 & 73 & 2,776 & 96691 & -.677 & .249 & .249 \\
\hline 660 & 18.62 & 258 & 4,805 & 640341 & .920 & .821 & .179 & 26.69 & 180 & 4,805 & 435757 & .178 & .571 & .429 \\
\hline 661 & 18.04 & 157 & 2,832 & 229110 & .664 & .746 & .254 & 25.29 & 112 & 2,832 & 155802 & -.323 & .374 & .374 \\
\hline 662 & 29.52 & 186 & 5,490 & 539164 & 1.323 & .907 & .093 & 40.97 & 134 & 5,490 & 391628 & 1.297 & .903 & .097 \\
\hline 663 & 52.70 & 619 & 32,622 & 10162803 & .283 & .611 & .389 & 84.08 & 388 & 32,622 & 6368203 & .213 & .584 & .416 \\
\hline 664 & 29.22 & 1,269 & 37,084 & 23995181 & 1.220 & .889 & .111 & 42.87 & 865 & 37,084 & 16200775 & .514 & .696 & .304 \\
\hline 665 & 62.96 & 299 & 18,826 & 2795485 & -.202 & .420 & .420 & 97.04 & 194 & 18,826 & 1782951 & -.570 & .284 & .284 \\
\hline 666 & 76.41 & 17 & 1,299 & 11960 & .594 & .724 & .276 & 162.38 & 8 & 1,299 & 4907 & -.273 & .393 & .393 \\
\hline 667 & 44.88 & 305 & 13,689 & 1995826 & -1.329 & .092 & .092 & 63.67 & 215 & 13,689 & 1409639 & -1.069 & .143 & .143 \\
\hline 668 & 57.07 & 136 & 7,761 & 516630 & -.426 & .335 & .335 & 88.19 & 88 & 7,761 & 318866 & -1.076 & .141 & .141 \\
\hline 669 & 17.91 & 317 & 5,676 & 938287 & 1.325 & .907 & .093 & 26.77 & 212 & 5,676 & 617299 & .656 & .744 & .256 \\
\hline 670 & 36.57 & 232 & 8,485 & 983160 & -.030 & .488 & .488 & 58.52 & 145 & 8,485 & 603141 & -.408 & .342 & .342 \\
\hline 671 & 21.71 & 866 & 18,797 & 8421206 & 1.767 & .961 & .039 & 31.91 & 589 & 18,797 & 5848056 & 2.372 & .991 & .009 \\
\hline 672 & 33.29 & 581 & 19,344 & 5673688 & .403 & .657 & .343 & 51.72 & 374 & 19,344 & 3566602 & -.470 & .319 & .319 \\
\hline
\end{tabular}


Table 5. Summary of interoccurrence intervals for daily precipitation thresholds of 0.75 and 1.0 inch-Continued

\begin{tabular}{|c|c|c|c|c|c|c|c|c|c|c|c|c|c|c|}
\hline \multirow[b]{2}{*}{$\begin{array}{l}\text { Seq. } \\
\text { no. }\end{array}$} & \multicolumn{7}{|c|}{ Daily precipitation threshold of 0.75 inch and greater } & \multicolumn{7}{|c|}{ Daily precipitation threshold of 1.0 inch and greater } \\
\hline & $\begin{array}{l}\text { Mean } \\
\text { inter- } \\
\text { occur- } \\
\text { rence } \\
\text { interval } \\
\text { (days) }\end{array}$ & $\begin{array}{c}\text { Total } \\
\text { no. of } \\
\text { events }\end{array}$ & $\begin{array}{l}\text { Total } \\
\text { no. of } \\
\text { days } \\
\text { avail- } \\
\text { able }\end{array}$ & $\begin{array}{l}\text { Sigma } \\
\mathrm{S}\end{array}$ & $\begin{array}{l}\text { Stan- } \\
\text { dard } \\
\text { normal } \\
\text { variate }\end{array}$ & $\begin{array}{l}\text { Non- } \\
\text { ex- } \\
\text { ceed- } \\
\text { ance } \\
\text { prob- } \\
\text { ability }\end{array}$ & $\begin{array}{c}\text { p- } \\
\text { value }\end{array}$ & $\begin{array}{c}\text { Mean } \\
\text { inter- } \\
\text { occur- } \\
\text { rence } \\
\text { interval } \\
\text { (days) }\end{array}$ & $\begin{array}{l}\text { Total } \\
\text { no. of } \\
\text { events }\end{array}$ & $\begin{array}{l}\text { Total } \\
\text { no. of } \\
\text { days } \\
\text { avail- } \\
\text { able }\end{array}$ & $\begin{array}{c}\text { Sigma } \\
\mathrm{S}\end{array}$ & $\begin{array}{l}\text { Stan- } \\
\text { dard } \\
\text { normal } \\
\text { variate }\end{array}$ & $\begin{array}{l}\text { Non- } \\
\text { ex- } \\
\text { ceed- } \\
\text { ance } \\
\text { prob- } \\
\text { ability }\end{array}$ & $\begin{array}{c}\text { p- } \\
\text { value }\end{array}$ \\
\hline 673 & 36.06 & 152 & 5,481 & 459375 & 2.195 & .986 & .014 & 54.81 & 100 & 5,481 & 295511 & 1.356 & .912 & .088 \\
\hline 674 & 22.63 & 79 & 1,788 & 66938 & -.804 & .211 & .211 & 28.84 & 62 & 1,788 & 49163 & -1.542 & .062 & .062 \\
\hline 675 & 35.80 & 74 & 2,649 & 90609 & -1.126 & .130 & .130 & 49.98 & 53 & 2,649 & 63926 & -1.127 & .130 & .130 \\
\hline 676 & 24.26 & 137 & 3,324 & 210195 & -1.558 & .060 & .060 & 36.13 & 92 & 3,324 & 144661 & -.896 & .185 & .185 \\
\hline 677 & 52.82 & 389 & 20,545 & 3989584 & -.055 & .478 & .478 & 81.53 & 252 & 20,545 & 2582159 & -.069 & .472 & .472 \\
\hline 678 & 39.25 & 4 & 157 & 197 & -1.291 & .098 & .098 & 39.25 & 4 & 157 & 197 & -1.291 & .098 & .098 \\
\hline 679 & 48.77 & 464 & 22,630 & 5273159 & .163 & .565 & .435 & 77.50 & 292 & 22,630 & 3185604 & -1.060 & .145 & .145 \\
\hline 680 & 25.06 & 174 & 4,360 & 385480 & .371 & .645 & .355 & 36.95 & 118 & 4,360 & 257210 & -.002 & .499 & .499 \\
\hline 681 & 19.49 & 43 & 838 & 17878 & -.088 & .465 & .465 & 22.05 & 38 & 838 & 16379 & .307 & .620 & .380 \\
\hline 682 & 28.29 & 180 & 5,092 & 459299 & .052 & .521 & .479 & 44.28 & 115 & 5,092 & 295417 & .167 & .566 & .434 \\
\hline 683 & 22.38 & 545 & 12,199 & 3422573 & 1.196 & .884 & .116 & 30.73 & 397 & 12,199 & 2517298 & 1.365 & .914 & .086 \\
\hline 684 & 23.41 & 663 & 15,522 & 5270905 & 1.087 & .861 & .139 & 32.96 & 471 & 15,522 & 3678366 & .236 & .593 & .407 \\
\hline 685 & 16.29 & 2,162 & 35,218 & 39712243 & 3.473 & 1.000 & 0 & 22.16 & 1,589 & 35,218 & 29234428 & 3.094 & .999 & .001 \\
\hline 686 & 29.24 & 200 & 5,847 & 629926 & 1.895 & .971 & .029 & 41.18 & 142 & 5,847 & 461715 & 2.316 & .990 & .010 \\
\hline 687 & 24.97 & 62 & 1,548 & 46601 & -.394 & .347 & .347 & 32.94 & 47 & 1,548 & 34526 & -.605 & .273 & .273 \\
\hline 688 & 17.81 & 252 & 4,487 & 554529 & -.527 & .299 & .299 & 24.39 & 184 & 4,487 & 407392 & -.308 & .379 & .379 \\
\hline 689 & 21.07 & 280 & 5,899 & 863914 & 1.336 & .909 & .091 & 33.14 & 178 & 5,899 & 531658 & .293 & .615 & .385 \\
\hline 690 & 18.21 & 688 & 12,527 & 4314440 & .054 & .522 & .478 & 25.41 & 493 & 12,527 & 3127724 & .496 & .690 & .310 \\
\hline 691 & 15.64 & 1,171 & 18,319 & 11219708 & 2.730 & .997 & .003 & 21.81 & 840 & 18,319 & 8281519 & 3.833 & 1.000 & 0 \\
\hline 692 & 27.47 & 684 & 18,792 & 6642245 & 1.518 & .935 & .065 & 39.40 & 477 & 18,792 & 4875030 & 3.318 & 1.000 & 0 \\
\hline 693 & 42.30 & 453 & 19,163 & 4606744 & 2.262 & .988 & .012 & 67.48 & 284 & 19,163 & 2857625 & 1.464 & .928 & .072 \\
\hline 694 & 22.82 & 320 & 7,301 & 1194743 & .705 & .760 & .240 & 32.89 & 222 & 7,301 & 839251 & .918 & .821 & .179 \\
\hline 695 & 51.65 & 262 & 13,531 & 1784489 & .189 & .575 & .425 & 76.45 & 177 & 13,531 & 1190946 & -.126 & .450 & .450 \\
\hline 696 & 50.68 & 253 & 12,822 & 1613736 & -.140 & .444 & .444 & 83.26 & 154 & 12,822 & 967241 & -.437 & .331 & .331 \\
\hline 697 & 16.53 & 1,427 & 23,585 & 17217320 & 1.514 & .935 & .065 & 22.53 & 1,047 & 23,585 & 12770155 & 1.922 & .973 & .027 \\
\hline 698 & 32.72 & 1,147 & 37,526 & 22168642 & 1.765 & .961 & .039 & 48.42 & 775 & 37,526 & 14552960 & .039 & .515 & .485 \\
\hline 699 & 27.29 & 58 & 1,583 & 47494 & .456 & .676 & .324 & 35.18 & 45 & 1,583 & 36328 & .232 & .592 & .408 \\
\hline 700 & 25.21 & 780 & 19,662 & 8051120 & 2.416 & .992 & .008 & 36.82 & 534 & 19,662 & 5473196 & 1.704 & .956 & .044 \\
\hline 701 & 21.60 & 83 & 1,793 & 77863 & .732 & .768 & .232 & 35.16 & 51 & 1,793 & 43985 & -.470 & .319 & .319 \\
\hline 702 & 40.40 & 30 & 1,212 & 15011 & -1.654 & .049 & .049 & 60.60 & 20 & 1,212 & 10290 & -1.170 & .121 & .121 \\
\hline 703 & 24.72 & 191 & 4,722 & 471092 & 1.069 & .857 & .143 & 34.22 & 138 & 4,722 & 347780 & 1.372 & .915 & .085 \\
\hline 704 & 38.55 & 11 & 424 & 1471 & -2.121 & .017 & .017 & 42.40 & 10 & 424 & 1116 & -2.594 & .005 & .005 \\
\hline 705 & 19.97 & 643 & 12,843 & 4281266 & 1.619 & .947 & .053 & 28.86 & 445 & 12,843 & 3006824 & 1.908 & .972 & .028 \\
\hline 706 & 16.85 & 2,129 & 35,879 & 39091429 & 1.880 & .970 & .030 & 23.75 & 1,511 & 35,879 & 27788699 & 1.694 & .955 & .045 \\
\hline 707 & 15.78 & 590 & 9,308 & 2895401 & 2.291 & .989 & .011 & 21.50 & 433 & 9,308 & 2132241 & 2.094 & .982 & .018 \\
\hline 708 & 58.35 & 277 & 16,163 & 2333312 & 1.220 & .889 & .111 & 88.32 & 183 & 16,163 & 1562828 & 1.330 & .908 & .092 \\
\hline 709 & -- & -- & -- & -- & -- & -- & -- & -- & -- & -- & -- & -- & -- & -- \\
\hline 710 & 49.92 & 24 & 1,198 & 14322 & -.032 & .487 & .487 & 70.47 & 17 & 1,198 & 9412 & -.541 & .294 & .294 \\
\hline 711 & 40.83 & 363 & 14,820 & 2871235 & 2.226 & .987 & .013 & 61.75 & 240 & 14,820 & 1947108 & 2.546 & .995 & .005 \\
\hline 712 & 43.26 & 31 & 1,341 & 18449 & -1.084 & .139 & .139 & 63.86 & 21 & 1,341 & 12332 & -.986 & .162 & .162 \\
\hline 713 & 19.27 & 527 & 10,153 & 2823556 & 2.203 & .986 & .014 & 27.89 & 364 & 10,153 & 2010166 & 2.903 & .998 & .002 \\
\hline 714 & 73.00 & 5 & 365 & 503 & -1.738 & .041 & .041 & 121.67 & 3 & 365 & 255 & -1.603 & .055 & .055 \\
\hline 715 & 50.35 & 29 & 1,460 & 20307 & -.380 & .352 & .352 & 76.84 & 19 & 1,460 & 12456 & -.770 & .221 & .221 \\
\hline 716 & 58.48 & 108 & 6,316 & 348440 & .389 & .651 & .349 & 85.35 & 74 & 6,316 & 261154 & 1.751 & .960 & .040 \\
\hline 717 & 52.28 & 634 & 33,144 & 10347522 & -.661 & .255 & .255 & 88.15 & 376 & 33,144 & 5995699 & -1.269 & .102 & .102 \\
\hline 718 & 15.23 & 452 & 6,885 & 1501622 & -1.287 & .099 & .099 & 21.52 & 320 & 6,885 & 1071971 & -.833 & .202 & .202 \\
\hline 719 & 14.69 & 297 & 4,364 & 636354 & -.539 & .295 & .295 & 20.49 & 213 & 4,364 & 460989 & -.205 & .419 & .419 \\
\hline 720 & 18.23 & 1,823 & 33,230 & 30037999 & -.613 & .270 & .270 & 25.80 & 1,288 & 33,230 & 21209630 & -.553 & .290 & .290 \\
\hline 721 & 25.40 & 1,435 & 36,455 & 26796511 & 1.606 & .946 & .054 & 37.39 & 975 & 36,455 & 18362807 & 1.799 & .964 & .036 \\
\hline 722 & 22.42 & 72 & 1,614 & 59850 & .442 & .671 & .329 & 36.68 & 44 & 1,614 & 38638 & 1.013 & .844 & .156 \\
\hline 723 & 13.73 & 164 & 2,252 & 188184 & .423 & .664 & .336 & 19.58 & 115 & 2,252 & 132972 & .500 & .691 & .309 \\
\hline 724 & 31.23 & 293 & 9,151 & 1353760 & .291 & .614 & .386 & 45.30 & 202 & 9,151 & 929792 & .148 & .559 & .441 \\
\hline
\end{tabular}


Table 5. Summary of interoccurrence intervals for daily precipitation thresholds of 0.75 and 1.0 inch-Continued

\begin{tabular}{|c|c|c|c|c|c|c|c|c|c|c|c|c|c|c|}
\hline \multirow[b]{2}{*}{$\begin{array}{c}\text { Seq. } \\
\text { no. }\end{array}$} & \multicolumn{7}{|c|}{ Daily precipitation threshold of 0.75 inch and greater } & \multicolumn{7}{|c|}{ Daily precipitation threshold of 1.0 inch and greater } \\
\hline & $\begin{array}{l}\text { Mean } \\
\text { inter- } \\
\text { occur- } \\
\text { rence } \\
\text { interval } \\
\text { (days) }\end{array}$ & $\begin{array}{c}\text { Total } \\
\text { no. of } \\
\text { events }\end{array}$ & $\begin{array}{c}\text { Total } \\
\text { no. of } \\
\text { days } \\
\text { avail- } \\
\text { able }\end{array}$ & $\begin{array}{l}\text { Sigma } \\
\text { S }\end{array}$ & $\begin{array}{c}\text { Stan- } \\
\text { dard } \\
\text { normal } \\
\text { variate }\end{array}$ & $\begin{array}{l}\text { Non- } \\
\text { ex- } \\
\text { ceed- } \\
\text { ance } \\
\text { prob- } \\
\text { ability }\end{array}$ & $\begin{array}{c}\mathrm{p}- \\
\text { value }\end{array}$ & $\begin{array}{l}\text { Mean } \\
\text { inter- } \\
\text { occur- } \\
\text { rence } \\
\text { interval } \\
\text { (days) }\end{array}$ & $\begin{array}{l}\text { Total } \\
\text { no. of } \\
\text { events }\end{array}$ & $\begin{array}{l}\text { Total } \\
\text { no. of } \\
\text { days } \\
\text { avail- } \\
\text { able }\end{array}$ & $\begin{array}{l}\text { Sigma } \\
\text { S }\end{array}$ & $\begin{array}{c}\text { Stan- } \\
\text { dard } \\
\text { normal } \\
\text { variate }\end{array}$ & $\begin{array}{l}\text { Non- } \\
\text { ex- } \\
\text { ceed- } \\
\text { ance } \\
\text { prob- } \\
\text { ability }\end{array}$ & $\begin{array}{c}\mathrm{p}- \\
\text { value }\end{array}$ \\
\hline 725 & 33.81 & 271 & 9,162 & 1329181 & 2.015 & .978 & .022 & 49.26 & 186 & 9,162 & 903179 & 1.417 & .922 & .078 \\
\hline 726 & 25.89 & 45 & 1,165 & 26965 & .334 & .631 & .369 & 43.15 & 27 & 1,165 & 15139 & -.337 & .368 & .368 \\
\hline 727 & 18.94 & 1,335 & 25,281 & 17025693 & .565 & .714 & .286 & 26.70 & 947 & 25,281 & 12046024 & .336 & .632 & .368 \\
\hline 728 & 17.98 & 573 & 10,305 & 3118581 & 2.334 & .990 & .010 & 25.63 & 402 & 10,305 & 2199639 & 2.152 & .984 & .016 \\
\hline 729 & 29.60 & 218 & 6,453 & 768528 & 2.369 & .991 & .009 & 40.59 & 159 & 6,453 & 568093 & 2.345 & .990 & .010 \\
\hline 730 & -- & -- & -- & -- & -- & -- & -- & -- & -- & -- & -- & -- & -- & -- \\
\hline 731 & 23.86 & 262 & 6,252 & 765970 & -1.816 & .035 & .035 & 30.20 & 207 & 6,252 & 605581 & -1.598 & .055 & .055 \\
\hline 732 & 32.94 & 207 & 6,819 & 719147 & .473 & .682 & .318 & 49.06 & 139 & 6,819 & 477156 & .139 & .555 & .445 \\
\hline 733 & 344.00 & 1 & 344 & 307 & 1.360 & .913 & .087 & 344.00 & 1 & 344 & 307 & 1.360 & .913 & .087 \\
\hline 734 & 62.49 & 369 & 23,057 & 3964230 & -2.267 & .012 & .012 & 95.28 & 242 & 23,057 & 2540814 & -2.406 & .008 & .008 \\
\hline 735 & 43.98 & 45 & 1,979 & 38358 & -1.610 & .054 & .054 & 70.68 & 28 & 1,979 & 23756 & -1.307 & .096 & .096 \\
\hline 736 & 31.14 & 260 & 8,096 & 1077847 & .673 & .749 & .251 & 51.24 & 158 & 8,096 & 625005 & -.496 & .310 & .310 \\
\hline 737 & 105.88 & 25 & 2,647 & 35769 & .702 & .759 & .241 & 189.07 & 14 & 2,647 & 24921 & 2.236 & .987 & .013 \\
\hline 738 & 70.96 & 202 & 14,333 & 1518043 & 1.197 & .884 & .116 & 111.11 & 129 & 14,333 & 977314 & 1.124 & .870 & .131 \\
\hline 739 & 85.98 & 58 & 4,987 & 120116 & -2.235 & .013 & .013 & 142.49 & 35 & 4,987 & 77745 & -1.119 & .132 & .132 \\
\hline 740 & 74.46 & 13 & 968 & 5440 & -.846 & .199 & .199 & 193.60 & 5 & 968 & 2441 & .034 & .513 & .487 \\
\hline 741 & 71.00 & 2 & 142 & 77 & -1.121 & .131 & .131 & 71.00 & 2 & 142 & 77 & -1.121 & .131 & .131 \\
\hline 742 & 23.26 & 1,138 & 26,466 & 15543195 & 1.878 & .970 & .030 & 32.76 & 808 & 26,466 & 11057307 & 1.681 & .954 & .046 \\
\hline 743 & 16.39 & 2,037 & 33,395 & 35483367 & 3.380 & 1.000 & 0 & 23.03 & 1,450 & 33,395 & 25885816 & 4.561 & 1.000 & 0 \\
\hline 744 & 37.92 & 167 & 6,333 & 543941 & .641 & .739 & .261 & 55.55 & 114 & 6,333 & 363113 & .109 & .544 & .456 \\
\hline 745 & 26.57 & 337 & 8,954 & 1571942 & 1.332 & .909 & .092 & 36.40 & 246 & 8,954 & 1179713 & 1.933 & .973 & .027 \\
\hline 746 & 31.28 & 657 & 20,552 & 7160933 & 2.694 & .996 & .004 & 46.08 & 446 & 20,552 & 4845764 & 2.096 & .982 & .018 \\
\hline 747 & 43.39 & 451 & 19,569 & 4543578 & 1.090 & .862 & .138 & 68.42 & 286 & 19,569 & 2993730 & 2.045 & .980 & .020 \\
\hline 748 & 21.55 & 1,529 & 32,946 & 24592845 & -1.598 & .055 & .055 & 28.57 & 1,153 & 32,946 & 18176151 & -2.531 & .006 & .006 \\
\hline 749 & 26.98 & 503 & 13,573 & 3035274 & -4.305 & 0 & 0 & 38.45 & 353 & 13,573 & 2099166 & -4.027 & 0 & 0 \\
\hline 750 & 15.81 & 1,175 & 18,579 & 11374963 & 2.501 & .994 & .006 & 22.07 & 842 & 18,579 & 8267330 & 2.863 & .998 & .002 \\
\hline 751 & 22.70 & 336 & 7,628 & 1286599 & .126 & .550 & .450 & 32.19 & 237 & 7,628 & 945307 & 1.221 & .889 & .111 \\
\hline 752 & 101.33 & 12 & 1,216 & 7871 & .473 & .682 & .318 & 173.71 & 7 & 1,216 & 4153 & -.111 & .456 & .456 \\
\hline 753 & 23.37 & 87 & 2,033 & 93967 & 1.011 & .844 & .156 & 33.88 & 60 & 2,033 & 65983 & 1.098 & .864 & .136 \\
\hline 754 & 17.52 & 147 & 2,575 & 179064 & -1.132 & .129 & .129 & 25.00 & 103 & 2,575 & 113226 & -2.570 & .005 & .005 \\
\hline 755 & 44.98 & 478 & 21,499 & 5184507 & .341 & .633 & .367 & 63.61 & 338 & 21,499 & 3581420 & -.455 & .325 & .325 \\
\hline 756 & -- & -- & -- & -- & -- & -- & -- & -- & -- & -- & -- & -- & -- & -- \\
\hline 757 & 42.04 & 139 & 5,844 & 408363 & .111 & .544 & .456 & 60.88 & 96 & 5,844 & 269274 & -.680 & .248 & .248 \\
\hline 758 & 21.17 & 1,528 & 32,344 & 25217706 & 1.389 & .918 & .082 & 29.65 & 1,091 & 32,344 & 17994027 & 1.136 & .872 & .128 \\
\hline 759 & 35.63 & 432 & 15,392 & 3631537 & 3.323 & 1.000 & 0 & 51.65 & 298 & 15,392 & 2495283 & 2.632 & .996 & .004 \\
\hline 760 & 45.13 & 473 & 21,345 & 5305287 & 1.919 & .973 & .027 & 64.68 & 330 & 21,345 & 3688055 & 1.484 & .931 & .069 \\
\hline 761 & 44.51 & 304 & 13,531 & 2010274 & -.682 & .248 & .248 & 62.07 & 218 & 13,531 & 1422994 & -.900 & .184 & .184 \\
\hline 762 & 73.10 & 346 & 25,291 & 4460405 & .626 & .734 & .266 & 111.91 & 226 & 25,291 & 2794501 & -.578 & .282 & .282 \\
\hline 763 & 28.19 & 42 & 1,184 & 22678 & -.987 & .162 & .162 & 37.00 & 32 & 1,184 & 16902 & -1.056 & .146 & .146 \\
\hline 764 & 24.52 & 1,337 & 32,783 & 22062250 & .424 & .664 & .336 & 36.03 & 910 & 32,783 & 14922871 & .023 & .509 & .491 \\
\hline 765 & 25.84 & 472 & 12,195 & 2939052 & .798 & .787 & .213 & 34.55 & 353 & 12,195 & 2211197 & .889 & .813 & .187 \\
\hline 766 & 22.66 & 118 & 2,674 & 136003 & -2.595 & .005 & .005 & 33.43 & 80 & 2,674 & 92714 & -2.063 & .020 & .020 \\
\hline 767 & 38.65 & 859 & 33,202 & 13821140 & -1.563 & .059 & .059 & 60.04 & 553 & 33,202 & 9031442 & -.661 & .254 & .254 \\
\hline 768 & 36.60 & 927 & 33,925 & 15775787 & .173 & .569 & .431 & 55.52 & 611 & 33,925 & 10293675 & -.291 & .386 & .386 \\
\hline 769 & 40.73 & 52 & 2,118 & 52778 & -.519 & .302 & .302 & 57.24 & 37 & 2,118 & 40846 & .447 & .673 & .327 \\
\hline 770 & 108.48 & 75 & 8,136 & 328837 & 1.167 & .878 & .122 & 184.91 & 44 & 8,136 & 198735 & 1.267 & .897 & .103 \\
\hline 771 & 43.69 & 85 & 3,714 & 158984 & .115 & .546 & .454 & 58.03 & 64 & 3,714 & 124958 & .712 & .762 & .238 \\
\hline 772 & 36.60 & 5 & 183 & 564 & .902 & .816 & .184 & 45.75 & 4 & 183 & 389 & .218 & .586 & .414 \\
\hline 773 & 36.93 & 14 & 517 & 3041 & -1.035 & .150 & .150 & 51.70 & 10 & 517 & 2312 & -.578 & .282 & .282 \\
\hline 774 & 37.83 & 816 & 30,870 & 12703828 & .428 & .666 & .334 & 55.22 & 559 & 30,870 & 8879965 & 1.195 & .884 & .116 \\
\hline 775 & 25.33 & 12 & 304 & 1864 & .132 & .552 & .448 & 43.43 & 7 & 304 & 1007 & -.246 & .403 & .403 \\
\hline 776 & 22.56 & 311 & 7,015 & 1182398 & 2.564 & .995 & .005 & 31.46 & 223 & 7,015 & 866321 & 2.783 & .997 & .003 \\
\hline
\end{tabular}


Table 5. Summary of interoccurrence intervals for daily precipitation thresholds of 0.75 and 1.0 inch-Continued

\begin{tabular}{|c|c|c|c|c|c|c|c|c|c|c|c|c|c|c|}
\hline \multirow[b]{2}{*}{$\begin{array}{l}\text { Seq. } \\
\text { no. }\end{array}$} & \multicolumn{7}{|c|}{ Daily precipitation threshold of 0.75 inch and greater } & \multicolumn{7}{|c|}{ Daily precipitation threshold of 1.0 inch and greater } \\
\hline & $\begin{array}{c}\text { Mean } \\
\text { inter- } \\
\text { occur- } \\
\text { rence } \\
\text { interval } \\
\text { (days) }\end{array}$ & $\begin{array}{l}\text { Total } \\
\text { no. of } \\
\text { events }\end{array}$ & $\begin{array}{l}\text { Total } \\
\text { no. of } \\
\text { days } \\
\text { avail- } \\
\text { able }\end{array}$ & $\begin{array}{l}\text { Sigma } \\
\mathrm{S}\end{array}$ & $\begin{array}{c}\text { Stan- } \\
\text { dard } \\
\text { normal } \\
\text { variate }\end{array}$ & $\begin{array}{l}\text { Non- } \\
\text { ex- } \\
\text { ceed- } \\
\text { ance } \\
\text { prob- } \\
\text { ability }\end{array}$ & $\begin{array}{c}\mathrm{p}- \\
\text { value }\end{array}$ & $\begin{array}{l}\text { Mean } \\
\text { inter- } \\
\text { occur- } \\
\text { rence } \\
\text { interval } \\
\text { (days) }\end{array}$ & $\begin{array}{c}\text { Total } \\
\text { no. of } \\
\text { events }\end{array}$ & $\begin{array}{l}\text { Total } \\
\text { no. of } \\
\text { days } \\
\text { avail- } \\
\text { able }\end{array}$ & $\begin{array}{l}\text { Sigma } \\
\mathrm{S}\end{array}$ & $\begin{array}{c}\text { Stan- } \\
\text { dard } \\
\text { normal } \\
\text { variate }\end{array}$ & $\begin{array}{l}\text { Non- } \\
\text { ex- } \\
\text { ceed- } \\
\text { ance } \\
\text { prob- } \\
\text { ability }\end{array}$ & $\begin{array}{c}\mathrm{p}- \\
\text { value }\end{array}$ \\
\hline 777 & 22.84 & 300 & 6,852 & 1049973 & 0.647 & .741 & .259 & 31.87 & 215 & 6,852 & 768977 & 1.117 & .868 & .132 \\
\hline 778 & 65.87 & 148 & 9,749 & 599823 & -3.552 & 0 & 0 & 108.32 & 90 & 9,749 & 380514 & -2.180 & .015 & .015 \\
\hline 779 & 43.98 & 364 & 16,007 & 3100357 & 2.122 & .983 & .017 & 65.07 & 246 & 16,007 & 2156447 & 2.588 & .995 & .005 \\
\hline 780 & 21.33 & 1,620 & 34,558 & 29538428 & 3.851 & 1.000 & 0 & 30.97 & 1,116 & 34,558 & 20187559 & 2.713 & .997 & .003 \\
\hline 781 & 40.20 & 861 & 34,614 & 14713144 & -.642 & .261 & .261 & 62.26 & 556 & 34,614 & 9621266 & -.006 & .498 & .498 \\
\hline 782 & 15.90 & 585 & 9,303 & 2893196 & 2.649 & .996 & .004 & 21.74 & 428 & 9,303 & 2128056 & 2.470 & .993 & .007 \\
\hline 783 & 47.10 & 31 & 1,460 & 18753 & -1.652 & .049 & .049 & 81.11 & 18 & 1,460 & 11052 & -1.168 & .122 & .122 \\
\hline 784 & 51.32 & 92 & 4,721 & 198502 & -1.428 & .077 & .077 & 80.02 & 59 & 4,721 & 127175 & -1.155 & .124 & .124 \\
\hline 785 & 63.60 & 300 & 19,079 & 3042393 & 1.893 & .971 & .029 & 93.53 & 204 & 19,079 & 2099508 & 1.951 & .974 & .026 \\
\hline 786 & 68.33 & 286 & 19,541 & 2773778 & -.216 & .415 & .415 & 112.31 & 174 & 19,541 & 1681521 & -.249 & .402 & .402 \\
\hline 787 & 21.78 & 278 & 6,055 & 820032 & -.742 & .229 & .229 & 32.21 & 188 & 6,055 & 538280 & -1.289 & .099 & .099 \\
\hline 788 & 20.15 & 80 & 1,612 & 63038 & -.347 & .364 & .364 & 27.79 & 58 & 1,612 & 47402 & .185 & .573 & .427 \\
\hline 789 & 20.16 & 414 & 8,344 & 1721144 & -.124 & .451 & .451 & 27.09 & 308 & 8,344 & 1266590 & -.435 & .332 & .332 \\
\hline 790 & 15.63 & 142 & 2,219 & 152815 & -.620 & .268 & .268 & 22.19 & 100 & 2,219 & 110640 & -.048 & .481 & .481 \\
\hline 791 & 29.46 & 160 & 4,714 & 394253 & .995 & .840 & .160 & 38.64 & 122 & 4,714 & 297633 & .671 & .749 & .251 \\
\hline 792 & 26.47 & 17 & 450 & 4797 & 1.815 & .965 & .035 & 37.50 & 12 & 450 & 3387 & 1.527 & .937 & .063 \\
\hline 793 & 19.14 & 1,002 & 19,181 & 9612653 & .017 & .507 & .493 & 25.89 & 741 & 19,181 & 7116229 & .064 & .526 & .474 \\
\hline 794 & 17.57 & 665 & 11,685 & 3889313 & .047 & .519 & .481 & 25.80 & 453 & 11,685 & 2669205 & .314 & .623 & .377 \\
\hline 795 & 27.99 & 609 & 17,047 & 5332492 & 1.167 & .878 & .122 & 39.92 & 427 & 17,047 & 3877645 & 2.342 & .990 & .010 \\
\hline 796 & 60.06 & 87 & 5,225 & 224497 & -.198 & .421 & .421 & 91.67 & 57 & 5,225 & 152632 & .327 & .628 & .372 \\
\hline 797 & 45.75 & 622 & 28,459 & 9099762 & 1.215 & .888 & .112 & 65.88 & 432 & 28,459 & 6333997 & 1.094 & .863 & .137 \\
\hline 798 & 43.38 & 16 & 694 & 4205 & -1.681 & .046 & .046 & 69.40 & 10 & 694 & 2934 & -.846 & .199 & .199 \\
\hline 799 & 38.47 & 238 & 9,155 & 1118583 & .715 & .763 & .237 & 62.28 & 147 & 9,155 & 672636 & -.008 & .497 & .497 \\
\hline 800 & 42.52 & 27 & 1,148 & 15384 & -.066 & .474 & .474 & 76.53 & 15 & 1,148 & 8990 & .296 & .616 & .384 \\
\hline 801 & 77.10 & 192 & 14,804 & 1483606 & 1.054 & .854 & .146 & 120.36 & 123 & 14,804 & 939310 & .609 & .729 & .271 \\
\hline 802 & 108.00 & 14 & 1,512 & 8461 & -1.300 & .097 & .097 & 137.46 & 11 & 1,512 & 6155 & -1.493 & .068 & .068 \\
\hline 803 & 25.61 & 285 & 7,299 & 1031729 & -.236 & .407 & .407 & 37.24 & 196 & 7,299 & 699188 & -.546 & .292 & .292 \\
\hline 804 & 48.64 & 14 & 681 & 4138 & -.855 & .196 & .196 & 61.91 & 11 & 681 & 2345 & -2.148 & .016 & .016 \\
\hline 805 & 35.58 & 323 & 11,493 & 1799785 & -.945 & .172 & .172 & 53.46 & 215 & 11,493 & 1195002 & -.832 & .203 & .203 \\
\hline 806 & 18.43 & 931 & 17,161 & 8225526 & 1.568 & .942 & .058 & 26.36 & 651 & 17,161 & 5772899 & 1.479 & .930 & .070 \\
\hline 807 & 21.96 & 598 & 13,131 & 4219251 & 3.162 & .999 & .001 & 34.20 & 384 & 13,131 & 2705414 & 2.481 & .993 & .007 \\
\hline 808 & 25.84 & 697 & 18,012 & 6325309 & .351 & .637 & .363 & 38.32 & 470 & 18,012 & 4391670 & 1.409 & .921 & .079 \\
\hline 809 & 47.78 & 386 & 18,442 & 3483727 & -.723 & .235 & .235 & 74.36 & 248 & 18,442 & 2252940 & -.404 & .343 & .343 \\
\hline 810 & 57.77 & 356 & 20,566 & 3797208 & 1.218 & .888 & .112 & 86.78 & 237 & 20,566 & 2519168 & .898 & .815 & .185 \\
\hline 811 & 16.25 & 454 & 7,376 & 1741886 & 1.489 & .932 & .068 & 23.57 & 313 & 7,376 & 1178068 & .630 & .736 & .264 \\
\hline 812 & 31.23 & 214 & 6,684 & 749577 & 1.218 & .888 & .112 & 43.69 & 153 & 6,684 & 518166 & .287 & .613 & .387 \\
\hline 813 & 11.86 & 7 & 83 & 273 & -.276 & .391 & .391 & 11.86 & 7 & 83 & 273 & -.276 & .391 & .391 \\
\hline 814 & 54.60 & 447 & 24,404 & 5532950 & .528 & .701 & .299 & 102.97 & 237 & 24,404 & 2896358 & .041 & .517 & .484 \\
\hline 815 & 17.05 & 1,782 & 30,377 & 27847456 & 2.111 & .983 & .017 & 23.49 & 1,293 & 30,377 & 20091186 & 1.435 & .924 & .076 \\
\hline 816 & 16.59 & 779 & 12,922 & 5184446 & 1.454 & .927 & .073 & 23.93 & 540 & 12,922 & 3696012 & 2.389 & .992 & .008 \\
\hline 817 & 25.29 & 246 & 6,220 & 766142 & .038 & .515 & .485 & 35.34 & 176 & 6,220 & 545525 & -.077 & .469 & .469 \\
\hline 818 & 23.81 & 833 & 19,833 & 8344413 & .508 & .694 & .306 & 34.86 & 569 & 19,833 & 5757434 & .842 & .800 & .200 \\
\hline 819 & 57.61 & 501 & 28,862 & 7037440 & -1.032 & .151 & .151 & 98.17 & 294 & 28,862 & 4037995 & -1.433 & .076 & .076 \\
\hline 820 & 42.43 & 21 & 891 & 10081 & .616 & .731 & .269 & 68.54 & 13 & 891 & 7038 & 1.344 & .911 & .089 \\
\hline 821 & 57.69 & 138 & 7,961 & 561888 & .466 & .679 & .321 & 90.47 & 88 & 7,961 & 344018 & -.291 & .386 & .386 \\
\hline 822 & 30.19 & 631 & 19,050 & 6488999 & 3.466 & 1.000 & 0 & 44.41 & 429 & 19,050 & 4420474 & 2.935 & .998 & .002 \\
\hline 823 & 34.99 & 930 & 32,540 & 15248791 & .411 & .659 & .341 & 54.87 & 593 & 32,540 & 9449170 & -.870 & .192 & .192 \\
\hline 824 & 32.65 & 65 & 2,122 & 63220 & -1.163 & .122 & .122 & 57.35 & 37 & 2,122 & 31977 & -1.954 & .025 & .025 \\
\hline 825 & 34.87 & 116 & 4,045 & 249706 & 1.200 & .885 & .115 & 49.94 & 81 & 4,045 & 170915 & .675 & .750 & .250 \\
\hline 826 & 17.18 & 1,543 & 26,503 & 20303277 & -.478 & .316 & .316 & 24.61 & 1,077 & 26,503 & 14248460 & -.093 & .463 & .463 \\
\hline 827 & 16.94 & 616 & 10,437 & 3293339 & 1.053 & .854 & .146 & 24.67 & 423 & 10,437 & 2279848 & 1.169 & .879 & .121 \\
\hline 828 & 17.25 & 1,497 & 25,827 & 19973109 & 2.224 & .987 & .013 & 24.32 & 1,062 & 25,827 & 13829030 & .473 & .682 & .318 \\
\hline
\end{tabular}


Table 5. Summary of interoccurrence intervals for daily precipitation thresholds of 0.75 and 1.0 inch-Continued

\begin{tabular}{|c|c|c|c|c|c|c|c|c|c|c|c|c|c|c|}
\hline \multirow[b]{2}{*}{$\begin{array}{c}\text { Seq. } \\
\text { no. }\end{array}$} & \multicolumn{7}{|c|}{ Daily precipitation threshold of 0.75 inch and greater } & \multicolumn{7}{|c|}{ Daily precipitation threshold of 1.0 inch and greater } \\
\hline & $\begin{array}{c}\text { Mean } \\
\text { inter- } \\
\text { occur- } \\
\text { rence } \\
\text { interval } \\
\text { (days) }\end{array}$ & $\begin{array}{l}\text { Total } \\
\text { no. of } \\
\text { events }\end{array}$ & $\begin{array}{c}\text { Total } \\
\text { no. of } \\
\text { days } \\
\text { avail- } \\
\text { able }\end{array}$ & $\begin{array}{c}\text { Sigma } \\
\mathrm{S}\end{array}$ & $\begin{array}{c}\text { Stan- } \\
\text { dard } \\
\text { normal } \\
\text { variate }\end{array}$ & $\begin{array}{l}\text { Non- } \\
\text { ex- } \\
\text { ceed- } \\
\text { ance } \\
\text { prob- } \\
\text { ability }\end{array}$ & $\begin{array}{c}\mathbf{p}- \\
\text { value }\end{array}$ & $\begin{array}{c}\text { Mean } \\
\text { inter- } \\
\text { occur- } \\
\text { rence } \\
\text { interval } \\
\text { (days) }\end{array}$ & $\begin{array}{l}\text { Total } \\
\text { no. of } \\
\text { events }\end{array}$ & $\begin{array}{l}\text { Total } \\
\text { no. of } \\
\text { days } \\
\text { avail- } \\
\text { able }\end{array}$ & $\begin{array}{l}\text { Sigma } \\
\mathbf{S}\end{array}$ & $\begin{array}{c}\text { Stan- } \\
\text { dard } \\
\text { normal } \\
\text { variate }\end{array}$ & $\begin{array}{l}\text { Non- } \\
\text { ex- } \\
\text { ceed- } \\
\text { ance } \\
\text { prob- } \\
\text { ability }\end{array}$ & $\begin{array}{c}\mathrm{p}- \\
\text { value }\end{array}$ \\
\hline 829 & 15.61 & 346 & 5,401 & 949465 & 0.520 & .699 & .301 & 21.96 & 246 & 5,401 & 682664 & 0.750 & .773 & .227 \\
\hline 830 & 31.20 & 420 & 13,102 & 2786579 & .454 & .675 & .325 & 43.82 & 299 & 13,102 & 1986685 & .427 & .665 & .335 \\
\hline 831 & 22.43 & 632 & 14,176 & 4611228 & 1.279 & .900 & .100 & 32.82 & 432 & 14,176 & 3149226 & 1.025 & .847 & .153 \\
\hline 832 & 27.81 & 63 & 1,752 & 51946 & -.808 & .210 & .210 & 40.74 & 43 & 1,752 & 35268 & -.724 & .235 & .235 \\
\hline 833 & 46.89 & 35 & 1,641 & 27091 & -.580 & .281 & .281 & 71.35 & 23 & 1,641 & 17140 & -.762 & .223 & .223 \\
\hline 834 & 36.66 & 112 & 4,106 & 235279 & .426 & .665 & .335 & 60.38 & 68 & 4,106 & 149102 & .972 & .834 & .166 \\
\hline 835 & 16.21 & 1,144 & 18,549 & 10842431 & 1.283 & .900 & .100 & 21.47 & 864 & 18,549 & 8347840 & 2.126 & .983 & .017 \\
\hline 836 & 32.82 & 230 & 7,548 & 894623 & .805 & .790 & .210 & 45.20 & 167 & 7,548 & 642318 & .428 & .666 & .334 \\
\hline 837 & 15.59 & 766 & 11,942 & 4724151 & 1.576 & .942 & .058 & 22.28 & 536 & 11,942 & 3345618 & 1.819 & .966 & .034 \\
\hline 838 & 15.12 & 504 & 7,618 & 1970088 & 1.020 & .846 & .154 & 20.87 & 365 & 7,618 & 1465257 & 1.784 & .963 & .037 \\
\hline 839 & 18.16 & 124 & 2,252 & 135823 & -.525 & .300 & .300 & 28.51 & 79 & 2,252 & 85852 & -.537 & .296 & .296 \\
\hline 840 & 25.93 & 1,406 & 36,458 & 26660988 & 2.613 & .996 & .004 & 37.55 & 971 & 36,458 & 18735667 & 3.157 & .999 & .001 \\
\hline 841 & 16.88 & 1,071 & 18,077 & 10002076 & 1.885 & .970 & .030 & 23.69 & 763 & 18,077 & 7253236 & 2.476 & .993 & .007 \\
\hline 842 & 19.00 & 986 & 18,731 & 9562652 & 1.933 & .973 & .027 & 26.35 & 711 & 18,731 & 6903127 & 1.694 & .955 & .045 \\
\hline 843 & 16.87 & 213 & 3,594 & 390561 & .515 & .697 & .303 & 22.60 & 159 & 3,594 & 295855 & .775 & .781 & .219 \\
\hline 844 & 25.44 & 690 & 17,555 & 6007889 & -.365 & .358 & .358 & 35.47 & 495 & 17,555 & 4283431 & -.545 & .293 & .293 \\
\hline 845 & 14.06 & 266 & 3,740 & 520896 & 1.333 & .909 & .091 & 19.38 & 193 & 3,740 & 389709 & 1.920 & .973 & .027 \\
\hline 846 & 13.75 & 471 & 6,477 & 1569010 & 1.076 & .859 & .141 & 18.35 & 353 & 6,477 & 1174662 & .896 & .815 & .185 \\
\hline 847 & 25.15 & 713 & 17,934 & 6465986 & .525 & .700 & .300 & 34.62 & 518 & 17,934 & 4558736 & -.731 & .232 & .232 \\
\hline 848 & 27.87 & 1,032 & 28,763 & 15742070 & 3.376 & 1.000 & 0 & 40.57 & 709 & 28,763 & 10738849 & 2.453 & .993 & .007 \\
\hline 849 & 20.56 & 330 & 6,785 & 1132375 & .361 & .641 & .359 & 27.92 & 243 & 6,785 & 841675 & .567 & .714 & .286 \\
\hline 850 & 39.75 & 36 & 1,431 & 25851 & .038 & .515 & .485 & 57.24 & 25 & 1,431 & 19027 & .552 & .709 & .291 \\
\hline 851 & 22.81 & 16 & 365 & 3941 & 2.423 & .992 & .008 & 28.08 & 13 & 365 & 3202 & 2.183 & .986 & .015 \\
\hline 852 & 14.28 & 32 & 457 & 7883 & .765 & .778 & .222 & 19.04 & 24 & 457 & 5578 & .145 & .558 & .442 \\
\hline 853 & 21.08 & 13 & 274 & 1612 & -.593 & .277 & .277 & 39.14 & 7 & 274 & 636 & -1.544 & .061 & .061 \\
\hline 854 & 43.55 & 434 & 18,899 & 4412777 & 2.742 & .997 & .003 & 62.79 & 301 & 18,899 & 3054137 & 2.217 & .987 & .013 \\
\hline 855 & 22.02 & 253 & 5,570 & 727538 & .897 & .815 & .185 & 31.47 & 177 & 5,570 & 513282 & .951 & .829 & .171 \\
\hline 856 & 45.19 & 414 & 18,709 & 3848100 & -.224 & .411 & .411 & 73.08 & 256 & 18,709 & 2381651 & -.152 & .440 & .440 \\
\hline 857 & 32.62 & 279 & 9,100 & 1404751 & 3.084 & .999 & .001 & 46.67 & 195 & 9,100 & 984016 & 2.638 & .996 & .004 \\
\hline 858 & 35.27 & 300 & 10,580 & 1629225 & .798 & .788 & .212 & 55.68 & 190 & 10,580 & 1077669 & 1.724 & .958 & .042 \\
\hline 859 & 38.23 & 245 & 9,367 & 1170692 & .549 & .708 & .292 & 56.09 & 167 & 9,367 & 798635 & .472 & .681 & .319 \\
\hline 860 & 43.46 & 28 & 1,217 & 13854 & -1.713 & .043 & .043 & 110.64 & 11 & 1,217 & 6158 & -.460 & .323 & .323 \\
\hline 861 & 40.35 & 358 & 14,445 & 2553289 & -.410 & .341 & .341 & 56.87 & 254 & 14,445 & 1791835 & -.642 & .260 & .260 \\
\hline 862 & 18.35 & 416 & 7,632 & 1630372 & .955 & .830 & .170 & 24.70 & 309 & 7,632 & 1227069 & 1.238 & .892 & .108 \\
\hline 863 & 19.36 & 22 & 426 & 5466 & 1.352 & .912 & .088 & 32.77 & 13 & 426 & 3310 & 1.220 & .889 & .111 \\
\hline 864 & 70.04 & 258 & 18,069 & 2331953 & .013 & .505 & .495 & 106.92 & 169 & 18,069 & 1506875 & -.294 & .384 & .384 \\
\hline 865 & 46.84 & 50 & 2,342 & 60089 & .322 & .626 & .374 & 80.76 & 29 & 2,342 & 34482 & .144 & .557 & .443 \\
\hline 866 & 46.12 & 59 & 2,721 & 84382 & .682 & .752 & .248 & 75.58 & 36 & 2,721 & 49391 & .088 & .535 & .465 \\
\hline 867 & 31.55 & 504 & 15,899 & 4238229 & 2.249 & .988 & .012 & 44.29 & 359 & 15,899 & 3021937 & 1.933 & .973 & .027 \\
\hline 868 & 30.98 & 702 & 21,746 & 7976208 & 2.064 & .981 & .019 & 45.98 & 473 & 21,746 & 5473817 & 2.424 & .992 & .008 \\
\hline 869 & 50.11 & 368 & 18,440 & 3204125 & -1.849 & .032 & .032 & 81.59 & 226 & 18,440 & 1919055 & -2.058 & .020 & .020 \\
\hline 870 & 14.58 & 108 & 1,575 & 99334 & 3.023 & .999 & .001 & 21.00 & 75 & 1,575 & 67883 & 2.240 & .987 & .013 \\
\hline 871 & 126.00 & 2 & 252 & 299 & .457 & .676 & .324 & 252.00 & 1 & 252 & 211 & 1.168 & .879 & .121 \\
\hline 872 & 24.30 & 20 & 486 & 4916 & .089 & .536 & .464 & 37.39 & 13 & 486 & 3503 & .680 & .752 & .248 \\
\hline 873 & 14.44 & 1,625 & 23,470 & 19081853 & .046 & .518 & .482 & 19.62 & 1,196 & 23,470 & 13892289 & -.609 & .271 & .271 \\
\hline 874 & 13.41 & 427 & 5,724 & 1164629 & -1.682 & .046 & .046 & 17.89 & 320 & 5,724 & 893461 & -.757 & .225 & .225 \\
\hline 875 & 16.72 & 29 & 485 & 6754 & -.369 & .356 & .356 & 20.21 & 24 & 485 & 5564 & -.373 & .355 & .355 \\
\hline 876 & -- & -- & -- & -- & -- & -- & -- & -- & -- & -- & -- & -- & -- & -- \\
\hline 877 & 43.00 & 10 & 430 & 2633 & 1.231 & .891 & .109 & 86.00 & 5 & 430 & 1174 & .357 & .639 & .361 \\
\hline 878 & 17.29 & 906 & 15,666 & 6915822 & -1.329 & .092 & .092 & 24.21 & 647 & 15,666 & 4831468 & -2.056 & .020 & .020 \\
\hline 879 & 47.91 & 386 & 18,493 & 3639603 & .672 & .749 & .251 & 70.32 & 263 & 18,493 & 2519726 & 1.015 & .845 & .155 \\
\hline 880 & 111.50 & 2 & 223 & 171 & -.571 & .284 & .284 & 111.50 & 2 & 223 & 171 & -.571 & .284 & .284 \\
\hline
\end{tabular}


Table 5. Summary of interoccurrence intervals for daily precipitation thresholds of 0.75 and 1.0 inch-Continued

\begin{tabular}{|c|c|c|c|c|c|c|c|c|c|c|c|c|c|c|}
\hline \multirow[b]{2}{*}{$\begin{array}{l}\text { Seq. } \\
\text { no. }\end{array}$} & \multicolumn{7}{|c|}{ Daily precipitation threshold of 0.75 inch and greater } & \multicolumn{7}{|c|}{ Daily precipitation threshold of 1.0 inch and greater } \\
\hline & $\begin{array}{l}\text { Mean } \\
\text { inter- } \\
\text { occur- } \\
\text { rence } \\
\text { interval } \\
\text { (days) }\end{array}$ & $\begin{array}{l}\text { Total } \\
\text { no. of } \\
\text { events }\end{array}$ & $\begin{array}{l}\text { Total } \\
\text { no. of } \\
\text { days } \\
\text { avail- } \\
\text { able }\end{array}$ & $\begin{array}{c}\text { Sigma } \\
\mathrm{S}\end{array}$ & $\begin{array}{l}\text { Stan- } \\
\text { dard } \\
\text { normal } \\
\text { variate }\end{array}$ & $\begin{array}{l}\text { Non- } \\
\text { ex- } \\
\text { ceed- } \\
\text { ance } \\
\text { prob- } \\
\text { ability }\end{array}$ & $\begin{array}{c}\mathrm{p}- \\
\text { value }\end{array}$ & $\begin{array}{c}\text { Mean } \\
\text { inter- } \\
\text { occur- } \\
\text { rence } \\
\text { interval } \\
\text { (days) }\end{array}$ & $\begin{array}{c}\text { Total } \\
\text { no. of } \\
\text { events }\end{array}$ & $\begin{array}{l}\text { Total } \\
\text { no. of } \\
\text { days } \\
\text { avail- } \\
\text { able }\end{array}$ & $\begin{array}{l}\text { Sigma } \\
\mathrm{S}\end{array}$ & $\begin{array}{c}\text { Stan- } \\
\text { dard } \\
\text { normal } \\
\text { variate }\end{array}$ & $\begin{array}{l}\text { Non- } \\
\text { ex- } \\
\text { ceed- } \\
\text { ance } \\
\text { prob- } \\
\text { ability }\end{array}$ & $\begin{array}{c}\mathrm{p}- \\
\text { value }\end{array}$ \\
\hline 881 & 37.64 & 494 & 18,595 & 4675673 & 0.693 & .756 & .244 & 57.93 & 321 & 18,595 & 3045734 & 0.637 & .738 & .262 \\
\hline 882 & 32.04 & 57 & 1,826 & 46850 & -1.304 & .096 & .096 & 46.82 & 39 & 1,826 & 31290 & -1.311 & .095 & .095 \\
\hline 883 & 33.85 & 322 & 10,901 & 1716686 & -.680 & .248 & .248 & 55.06 & 198 & 10,901 & 1082691 & .079 & .531 & .469 \\
\hline 884 & 40.45 & 337 & 13,631 & 2255954 & -.566 & .286 & .286 & 60.85 & 224 & 13,631 & 1471064 & -.944 & .173 & .173 \\
\hline 885 & 35.74 & 831 & 29,698 & 12568619 & .927 & .823 & .177 & 54.49 & 545 & 29,698 & 8337617 & 1.224 & .889 & .111 \\
\hline 886 & 20.72 & 1,031 & 21,366 & 11097552 & .421 & 663 & .337 & 28.68 & 745 & 21,366 & 7981120 & .132 & .553 & .447 \\
\hline 887 & 19.16 & 1,309 & 25,079 & 17428621 & 3.873 & 1.000 & 0 & 27.03 & 928 & 25,079 & 12358115 & 3.271 & .999 & .001 \\
\hline 888 & 25.41 & 745 & 18,929 & 7372999 & 2.159 & .985 & .015 & 39.27 & 482 & 18,929 & 4904405 & 2.855 & .998 & .002 \\
\hline 889 & 45.91 & 299 & 13,726 & 2239128 & 2.731 & .997 & .003 & 75.42 & 182 & 13,726 & 1345906 & 1.812 & .965 & .035 \\
\hline 890 & 43.33 & 302 & 13,085 & 1920649 & -.841 & .200 & .200 & 69.60 & 188 & 13,085 & 1167269 & -1.211 & .113 & .113 \\
\hline 891 & 51.99 & 349 & 18,143 & 3322902 & 1.604 & .946 & .054 & 71.43 & 254 & 18,143 & 2368376 & .769 & .779 & .221 \\
\hline 892 & 50.53 & 143 & 7,226 & 510900 & -.231 & .409 & .409 & 66.91 & 108 & 7,226 & 387318 & -.133 & .447 & .447 \\
\hline 893 & 42.69 & 631 & 26,939 & 8254493 & -1.253 & .105 & .105 & 66.03 & 408 & 26,939 & 5182512 & -1.993 & .023 & .023 \\
\hline 894 & 25.60 & 220 & 5,631 & 640053 & .856 & .804 & .196 & 36.80 & 153 & 5,631 & 447943 & .854 & .803 & .197 \\
\hline 895 & 75.87 & 225 & 17,071 & 1988496 & .920 & .821 & .179 & 143.45 & 119 & 17,071 & 1059488 & .814 & .792 & .208 \\
\hline 896 & 17.88 & 2,000 & 35,755 & 37085619 & 2.883 & .998 & .002 & 26.08 & 1,371 & 35,755 & 25241555 & 1.914 & .972 & .028 \\
\hline 897 & 23.00 & 8 & 184 & 720 & -.107 & .458 & .458 & 36.80 & 5 & 184 & 506 & .387 & .651 & .349 \\
\hline 898 & 14.42 & 84 & 1,211 & 51662 & .250 & .599 & .401 & 19.85 & 61 & 1,211 & 38609 & .613 & .730 & .270 \\
\hline 899 & 29.00 & 2 & 58 & 44 & -.591 & .277 & .277 & 29.00 & 2 & 58 & 44 & -.591 & .277 & .277 \\
\hline 900 & 22.52 & 68 & 1,531 & 55511 & .949 & .829 & .171 & 33.28 & 46 & 1,531 & 32406 & -.936 & .175 & .175 \\
\hline 901 & 35.80 & 951 & 34,050 & 16766017 & 1.898 & .971 & .029 & 53.37 & 638 & 34,050 & 11318113 & 1.837 & .967 & .033 \\
\hline 902 & 33.06 & 34 & 1,124 & 20776 & .882 & .811 & .189 & 51.09 & 22 & 1,124 & 13192 & .544 & .707 & .293 \\
\hline 903 & 101.92 & 248 & 25,276 & 3230972 & .842 & .800 & .200 & 191.49 & 132 & 25,276 & 1786764 & 1.414 & .921 & .079 \\
\hline 904 & 198.00 & 2 & 396 & 564 & 1.039 & .851 & .149 & -- & -- & -- & -- & -- & -- & -- \\
\hline 905 & 77.33 & 215 & 16,626 & 1785877 & -.020 & .492 & .492 & 116.27 & 143 & 16,626 & 1151767 & -.645 & .260 & .260 \\
\hline 906 & 43.63 & 725 & 31,631 & 11514491 & .196 & .578 & .422 & 72.22 & 438 & 31,631 & 7056694 & .678 & .751 & .249 \\
\hline 907 & -- & -- & -- & -- & -- & -- & -- & -- & -- & -- & -- & -- & -- & -- \\
\hline 908 & 47.55 & 416 & 19,782 & 4306022 & 1.643 & .950 & .050 & 75.50 & 262 & 19,782 & 2670311 & .853 & .803 & .197 \\
\hline 909 & 41.55 & 202 & 8,392 & 864314 & .486 & .686 & .314 & 67.14 & 125 & 8,392 & 585650 & 2.258 & .988 & .012 \\
\hline 910 & 120.09 & 59 & 7,085 & 213717 & .300 & .618 & .382 & 214.70 & 33 & 7,085 & 135024 & 1.542 & .938 & .062 \\
\hline 911 & 139.80 & 15 & 2,097 & 16030 & .129 & .551 & .449 & 262.13 & 8 & 2,097 & 9121 & .428 & .666 & .334 \\
\hline 912 & 20.13 & 15 & 302 & 1635 & -1.866 & .031 & .031 & 21.57 & 14 & 302 & 1522 & -1.815 & .035 & .035 \\
\hline 913 & 28.42 & 226 & 6,422 & 773230 & 1.706 & .956 & .044 & 40.39 & 159 & 6,422 & 559890 & 2.111 & .983 & .017 \\
\hline 914 & 21.95 & 165 & 3,622 & 291705 & -.529 & .298 & .298 & 36.59 & 99 & 3,622 & 179807 & .050 & .520 & .480 \\
\hline 915 & 19.39 & 1,735 & 33,649 & 31506167 & 5.723 & 0 & 0 & 26.75 & 1,258 & 33,649 & 23029175 & 5.410 & 0 & 0 \\
\hline 916 & 19.78 & 967 & 19,131 & 9918218 & 3.892 & 1.000 & 0 & 26.28 & 728 & 19,131 & 7756241 & 5.319 & 0 & 0 \\
\hline 917 & 60.23 & 128 & 7,709 & 405934 & -3.473 & 0 & 0 & 105.60 & 73 & 7,709 & 226941 & -2.863 & .002 & .002 \\
\hline 918 & 14.49 & 611 & 8,856 & 2829840 & 1.968 & .975 & .025 & 19.09 & 464 & 8,856 & 2155596 & 1.834 & .967 & .033 \\
\hline 919 & 32.89 & 298 & 9,801 & 1463782 & .070 & .528 & .472 & 46.67 & 210 & 9,801 & 1087163 & 1.416 & .922 & .078 \\
\hline 920 & 17.26 & 1,107 & 19,104 & 10856603 & 1.540 & .938 & .062 & 24.56 & 778 & 19,104 & 7601365 & 1.105 & .865 & .135 \\
\hline 921 & 26.63 & 164 & 4,368 & 395415 & 2.306 & .989 & .011 & 40.44 & 108 & 4,368 & 270085 & 2.611 & .995 & .005 \\
\hline 922 & 54.67 & 416 & 22,744 & 4884173 & 1.146 & .874 & .126 & 89.54 & 254 & 22,744 & 2932783 & .423 & .664 & .336 \\
\hline 923 & 46.88 & 713 & 33,427 & 11224622 & -2.686 & .004 & .004 & 73.63 & 454 & 33,427 & 7239608 & -1.694 & .045 & .045 \\
\hline 924 & 63.90 & 10 & 639 & 2448 & -1.281 & .100 & .100 & 106.50 & 6 & 639 & 1897 & -.044 & .482 & .482 \\
\hline 925 & 77.87 & 54 & 4,205 & 134035 & 2.298 & .989 & .011 & 113.65 & 37 & 4,205 & 95697 & 2.425 & .992 & .008 \\
\hline 926 & 29.26 & 19 & 556 & 6122 & 1.201 & .885 & .115 & 39.71 & 14 & 556 & 4438 & .909 & .818 & .182 \\
\hline 927 & 23.83 & 186 & 4,433 & 403934 & -.478 & .317 & .317 & 34.63 & 128 & 4,433 & 289978 & .433 & .667 & .333 \\
\hline 928 & 42.59 & 179 & 7,623 & 769917 & 2.977 & .999 & .001 & 71.92 & 106 & 7,623 & 440691 & 1.619 & .947 & .053 \\
\hline 929 & 20.90 & 752 & 15,716 & 5774203 & -1.085 & .139 & .139 & 28.32 & 555 & 15,716 & 4313960 & -.442 & .329 & .329 \\
\hline 930 & 48.97 & 186 & 9,108 & 893586 & 1.298 & .903 & .097 & 69.53 & 131 & 9,108 & 626656 & 1.000 & .841 & .159 \\
\hline 931 & 33.40 & 145 & 4,843 & 358699 & .450 & .674 & .326 & 43.63 & 111 & 4,843 & 282763 & .949 & .829 & .171 \\
\hline 932 & 26.90 & 121 & 3,255 & 191169 & -.557 & .289 & .289 & 50.08 & 65 & 3,255 & 99522 & -.827 & .204 & .204 \\
\hline
\end{tabular}


Table 5. Summary of interoccurrence intervals for daily precipitation thresholds of 0.75 and 1.0 inch-Continued

\begin{tabular}{|c|c|c|c|c|c|c|c|c|c|c|c|c|c|c|}
\hline \multirow[b]{2}{*}{$\begin{array}{c}\text { Seq. } \\
\text { no. }\end{array}$} & \multicolumn{7}{|c|}{ Daily precipitation threshold of 0.75 inch and greater } & \multicolumn{7}{|c|}{ Daily precipitation threshold of 1.0 inch and greater } \\
\hline & $\begin{array}{l}\text { Mean } \\
\text { inter- } \\
\text { occur- } \\
\text { rence } \\
\text { interval } \\
\text { (days) }\end{array}$ & $\begin{array}{c}\text { Total } \\
\text { no. of } \\
\text { events }\end{array}$ & $\begin{array}{c}\text { Total } \\
\text { no. of } \\
\text { days } \\
\text { avail- } \\
\text { able }\end{array}$ & $\begin{array}{l}\text { Sigma } \\
\text { S }\end{array}$ & $\begin{array}{c}\text { Stan- } \\
\text { dard } \\
\text { normal } \\
\text { variate }\end{array}$ & $\begin{array}{l}\text { Non- } \\
\text { ex- } \\
\text { ceed- } \\
\text { ance } \\
\text { prob- } \\
\text { ability }\end{array}$ & $\begin{array}{c}\mathrm{p}- \\
\text { value }\end{array}$ & $\begin{array}{c}\text { Mean } \\
\text { inter- } \\
\text { occur- } \\
\text { rence } \\
\text { interval } \\
\text { (days) }\end{array}$ & $\begin{array}{l}\text { Total } \\
\text { no. of } \\
\text { events }\end{array}$ & $\begin{array}{l}\text { Total } \\
\text { no. of } \\
\text { days } \\
\text { avail- } \\
\text { able }\end{array}$ & $\begin{array}{l}\text { Sigma } \\
\text { S }\end{array}$ & $\begin{array}{c}\text { Stan- } \\
\text { dard } \\
\text { normal } \\
\text { variate }\end{array}$ & $\begin{array}{l}\text { Non- } \\
\text { ex- } \\
\text { ceed- } \\
\text { ance } \\
\text { prob- } \\
\text { ability }\end{array}$ & $\begin{array}{c}\mathrm{p}- \\
\text { value }\end{array}$ \\
\hline 933 & -- & -- & -- & -- & -- & -- & -- & -- & -- & -- & -- & -- & -- & - \\
\hline 934 & 26.29 & 211 & 5,547 & 591162 & 0.256 & .601 & .399 & 35.33 & 157 & 5,547 & 447564 & 0.604 & .727 & .273 \\
\hline 935 & 15.27 & 1,290 & 19,694 & 13288481 & 2.869 & .998 & .002 & 21.04 & 936 & 19,694 & 9625071 & 2.347 & .991 & .009 \\
\hline 936 & 14.48 & 657 & 9,510 & 3094711 & -.417 & .338 & .338 & 20.23 & 470 & 9,510 & 2224195 & -.179 & .429 & .429 \\
\hline 937 & 15.73 & 567 & 8,920 & 2496735 & -.523 & .300 & .300 & 21.14 & 422 & 8,920 & 1813100 & -1.305 & .096 & .096 \\
\hline 938 & 35.01 & 723 & 25,311 & 9510669 & 1.836 & .967 & .033 & 48.30 & 524 & 25,311 & 6868416 & 1.417 & .922 & .078 \\
\hline 939 & 21.98 & 983 & 21,605 & 10835039 & 1.106 & .865 & .135 & 30.22 & 715 & 21,605 & 7971699 & 1.487 & .931 & .069 \\
\hline 940 & 35.81 & 444 & 15,898 & 3593282 & .661 & .746 & .254 & 47.60 & 334 & 15,898 & 2746843 & 1.095 & .863 & .137 \\
\hline 941 & 24.56 & 670 & 16,452 & 5167374 & -2.799 & .003 & .003 & 33.85 & 486 & 16,452 & 3813468 & -1.761 & .039 & .039 \\
\hline 942 & 32.91 & 399 & 13,131 & 2604661 & -.198 & .422 & .422 & 51.90 & 253 & 13,131 & 1617805 & -.718 & .237 & .237 \\
\hline 943 & 43.18 & 756 & 32,647 & 12444161 & .400 & .655 & .345 & 67.59 & 483 & 32,647 & 8085643 & .972 & .834 & .166 \\
\hline 944 & 19.66 & 292 & 5,740 & 856977 & .669 & .748 & .252 & 28.56 & 201 & 5,740 & 562168 & -.626 & .266 & .266 \\
\hline 945 & 32.66 & 654 & 21,362 & 7307457 & 2.042 & .979 & .021 & 45.16 & 473 & 21,362 & 5262263 & 1.567 & .941 & .059 \\
\hline 946 & 21.03 & 467 & 9,820 & 2300671 & .126 & .550 & .450 & 29.85 & 329 & 9,820 & 1601034 & -.279 & .390 & .390 \\
\hline 947 & 30.36 & 454 & 13,784 & 3344086 & 2.537 & .994 & .006 & 44.47 & 310 & 13,784 & 2264557 & 1.828 & .966 & .034 \\
\hline 948 & 36.85 & 33 & 1,216 & 20852 & .391 & .652 & .348 & 64.00 & 19 & 1,216 & 11977 & .278 & .609 & .391 \\
\hline 949 & 113.29 & 234 & 26,509 & 3330847 & 1.959 & .975 & .025 & 207.10 & 128 & 26,509 & 1789450 & 1.073 & .858 & .142 \\
\hline 950 & -- & -- & -- & -- & -- & -- & -- & -- & -- & -- & -- & -- & -- & -- \\
\hline 951 & 18.85 & 658 & 12,405 & 3967543 & -1.238 & .108 & .108 & 26.79 & 463 & 12,405 & 2775577 & -1.248 & .106 & .106 \\
\hline 952 & 22.71 & 217 & 4,929 & 582721 & 2.286 & .989 & .011 & 32.86 & 150 & 4,929 & 403717 & 1.953 & .975 & .025 \\
\hline 953 & 26.62 & 527 & 14,026 & 3774330 & .844 & .801 & .199 & 39.40 & 356 & 14,026 & 2600365 & 1.358 & .913 & .087 \\
\hline 954 & 22.85 & 303 & 6,922 & 1141251 & 2.661 & .996 & .004 & 32.35 & 214 & 6,922 & 800037 & 2.032 & .979 & .021 \\
\hline 955 & 33.29 & 993 & 33,055 & 16464841 & .176 & .570 & .430 & 49.48 & 668 & 33,055 & 10871666 & -.684 & .247 & .247 \\
\hline 956 & 34.26 & 1,014 & 34,736 & 17666559 & .174 & .569 & .431 & 50.05 & 694 & 34,736 & 11976611 & -.291 & .386 & .386 \\
\hline 957 & 20.11 & 213 & 4,283 & 469014 & .714 & .762 & .238 & 27.63 & 155 & 4,283 & 333139 & .078 & .531 & .469 \\
\hline 958 & 42.66 & 294 & 12,543 & 1893510 & .800 & .788 & .212 & 65.33 & 192 & 12,543 & 1299918 & 1.909 & .972 & .028 \\
\hline 959 & 18.46 & 749 & 13,823 & 5080482 & -.881 & .189 & .189 & 26.18 & 528 & 13,823 & 3547582 & -1.109 & .134 & .134 \\
\hline 960 & 16.62 & 47 & 781 & 20352 & 1.293 & .902 & .098 & 22.97 & 34 & 781 & 14807 & 1.164 & .878 & .122 \\
\hline 961 & 27.63 & 727 & 20,090 & 7208711 & -.601 & .274 & .274 & 38.78 & 518 & 20,090 & 5224344 & .159 & .563 & .437 \\
\hline 962 & 89.88 & 8 & 719 & 3427 & .939 & .826 & .174 & 179.75 & 4 & 719 & 1653 & .518 & .698 & .302 \\
\hline 963 & 38.03 & 30 & 1,141 & 15443 & -.927 & .177 & .177 & 63.39 & 18 & 1,141 & 7403 & -2.051 & .020 & .020 \\
\hline 964 & 37.32 & 54 & 2,015 & 51400 & -.703 & .241 & .241 & 54.46 & 37 & 2,015 & 35850 & -.404 & .343 & .343 \\
\hline 965 & 29.04 & 351 & 10,194 & 1798450 & .171 & .568 & .432 & 43.38 & 235 & 10,194 & 1184859 & -.287 & .387 & .387 \\
\hline 966 & 57.35 & 155 & 8,889 & 693737 & .152 & .560 & .440 & 84.66 & 105 & 8,889 & 463132 & -.135 & .446 & .446 \\
\hline 967 & 56.79 & 89 & 5,054 & 206431 & -1.342 & .090 & .090 & 99.10 & 51 & 5,054 & 130301 & .137 & .554 & .446 \\
\hline 968 & 20.04 & 79 & 1,583 & 67179 & 1.145 & .874 & .126 & 28.78 & 55 & 1,583 & 45291 & .519 & .698 & .302 \\
\hline 969 & 21.73 & 56 & 1,217 & 37868 & 1.442 & .925 & .075 & 29.68 & 41 & 1,217 & 27851 & 1.290 & .901 & .099 \\
\hline 970 & 34.14 & 959 & 32,743 & 15761568 & .209 & .583 & .417 & 48.22 & 679 & 32,743 & 11312440 & .797 & .787 & .213 \\
\hline 971 & 20.75 & 83 & 1,722 & 73987 & .557 & .711 & .289 & 30.75 & 56 & 1,722 & 51102 & .776 & .781 & .219 \\
\hline 972 & 31.24 & 593 & 18,526 & 5916306 & 3.251 & .999 & .001 & 45.97 & 403 & 18,526 & 3960421 & 2.118 & .983 & .017 \\
\hline 973 & 98.69 & 198 & 19,541 & 2073829 & 1.755 & .960 & .040 & 152.66 & 128 & 19,541 & 1371487 & 1.894 & .971 & .029 \\
\hline 974 & 20.90 & 534 & 11,161 & 3032709 & .708 & .760 & .240 & 30.50 & 366 & 11,161 & 2092936 & .819 & .794 & .206 \\
\hline 975 & 22.19 & 559 & 12,404 & 3465577 & -.016 & .494 & .494 & 30.18 & 411 & 12,404 & 2585200 & .498 & .691 & .309 \\
\hline 976 & 24.00 & 37 & 888 & 17947 & .974 & .835 & .165 & 29.60 & 30 & 888 & 13696 & .268 & .606 & .394 \\
\hline 977 & 18.60 & 5 & 93 & 251 & .308 & .621 & .379 & 31.00 & 3 & 93 & 135 & -.097 & .461 & .461 \\
\hline 978 & 24.11 & 554 & 13,354 & 3944102 & 2.701 & .997 & .003 & 33.81 & 395 & 13,354 & 2803559 & 2.169 & .985 & .015 \\
\hline 979 & 23.52 & 243 & 5,715 & 695151 & .030 & .512 & .488 & 32.66 & 175 & 5,715 & 510205 & .465 & .679 & .321 \\
\hline 980 & 23.66 & 151 & 3,573 & 265213 & -.359 & .360 & .360 & 30.28 & 118 & 3,573 & 199951 & -.969 & .166 & .166 \\
\hline 981 & 15.00 & 1 & 15 & 15 & 1.732 & .958 & .042 & 15.00 & 1 & 15 & 15 & 1.732 & .958 & .042 \\
\hline 982 & 22.71 & 17 & 386 & 3452 & .372 & .645 & .355 & 27.57 & 14 & 386 & 2718 & .038 & .515 & .485 \\
\hline 983 & 16.81 & 622 & 10,455 & 3340484 & 1.182 & .881 & .119 & 25.82 & 405 & 10,455 & 2191099 & 1.218 & .888 & .112 \\
\hline 984 & 36.43 & 97 & 3,534 & 178979 & .754 & .775 & .225 & 52.75 & 67 & 3,534 & 112348 & -.723 & .235 & .235 \\
\hline
\end{tabular}


Table 5. Summary of interoccurrence intervals for daily precipitation thresholds of 0.75 and 1.0 inch-Continued

\begin{tabular}{|c|c|c|c|c|c|c|c|c|c|c|c|c|c|c|}
\hline \multirow[b]{2}{*}{$\begin{array}{l}\text { Seq. } \\
\text { no. }\end{array}$} & \multicolumn{7}{|c|}{ Daily precipitation threshold of 0.75 inch and greater } & \multicolumn{7}{|c|}{ Daily precipitation threshold of 1.0 inch and greater } \\
\hline & $\begin{array}{l}\text { Mean } \\
\text { inter- } \\
\text { occur- } \\
\text { rence } \\
\text { interval } \\
\text { (days) }\end{array}$ & $\begin{array}{c}\text { Total } \\
\text { no. of } \\
\text { events }\end{array}$ & $\begin{array}{l}\text { Total } \\
\text { no. of } \\
\text { days } \\
\text { avail- } \\
\text { able }\end{array}$ & $\begin{array}{c}\text { Sigma } \\
\mathrm{S}\end{array}$ & $\begin{array}{c}\text { Stan- } \\
\text { dard } \\
\text { normal } \\
\text { variate }\end{array}$ & $\begin{array}{l}\text { Non- } \\
\text { ex- } \\
\text { ceed- } \\
\text { ance } \\
\text { prob- } \\
\text { ability }\end{array}$ & $\begin{array}{c}p- \\
\text { value }\end{array}$ & $\begin{array}{l}\text { Mean } \\
\text { inter- } \\
\text { occur- } \\
\text { rence } \\
\text { interval } \\
\text { (days) }\end{array}$ & $\begin{array}{l}\text { Total } \\
\text { no. of } \\
\text { events }\end{array}$ & $\begin{array}{l}\text { Total } \\
\text { no. of } \\
\text { days } \\
\text { avail- } \\
\text { able }\end{array}$ & $\begin{array}{l}\text { Sigma } \\
\mathrm{S}\end{array}$ & $\begin{array}{c}\text { Stan- } \\
\text { dard } \\
\text { normal } \\
\text { variate }\end{array}$ & $\begin{array}{l}\text { Non- } \\
\text { ex- } \\
\text { ceed- } \\
\text { ance } \\
\text { prob- } \\
\text { ability }\end{array}$ & $\begin{array}{c}\mathrm{p}- \\
\text { value }\end{array}$ \\
\hline 985 & 26.93 & 45 & 1,212 & 26760 & -0.217 & .414 & .414 & 35.65 & 34 & 1,212 & 20131 & -0.232 & .408 & .408 \\
\hline 986 & 27.94 & 124 & 3,465 & 250027 & 3.160 & .999 & .001 & 36.86 & 94 & 3,465 & 202786 & 4.118 & 1.000 & 0 \\
\hline 987 & 34.15 & 658 & 22,473 & 7291417 & -.614 & .270 & .270 & 49.94 & 450 & 22,473 & 4914806 & -1.029 & .152 & .152 \\
\hline 988 & 20.22 & 116 & 2,346 & 133117 & -.405 & .343 & .343 & 27.93 & 84 & 2,346 & 98091 & -.071 & .472 & .472 \\
\hline 989 & 18.06 & 895 & 16,159 & 7424104 & 1.383 & .917 & .083 & 25.94 & 623 & 16,159 & 5145913 & .965 & .833 & .167 \\
\hline 990 & 22.01 & 847 & 18,639 & 8355440 & 2.949 & .998 & .002 & 30.21 & 617 & 18,639 & 6071672 & 2.406 & .992 & .008 \\
\hline 991 & 28.93 & 642 & 18,574 & 6085950 & .911 & .819 & .181 & 42.70 & 435 & 18,574 & 4344220 & 2.722 & .997 & .003 \\
\hline 992 & 18.42 & 1,085 & 19,989 & 11607926 & 4.019 & 1.000 & 0 & 26.34 & 759 & 19,989 & 8127519 & 3.408 & 1.000 & 0 \\
\hline 993 & 36.03 & 38 & 1,369 & 29923 & 1.606 & .946 & .054 & 54.76 & 25 & 1,369 & 21131 & 2.034 & .979 & .021 \\
\hline 994 & 31.68 & 195 & 6,177 & 628241 & 1.044 & .852 & .148 & 43.50 & 142 & 6,177 & 453897 & .722 & .765 & .235 \\
\hline 995 & 26.61 & 619 & 16,470 & 5312674 & 1.819 & .966 & .034 & 37.52 & 439 & 16,470 & 3799635 & 1.852 & .968 & .032 \\
\hline 996 & 48.41 & 596 & 28,850 & 9101362 & 2.479 & .993 & .007 & 67.09 & 430 & 28,850 & 6637811 & 2.519 & .994 & .006 \\
\hline 997 & 28.79 & 957 & 27,555 & 13601234 & 1.691 & .955 & .045 & 40.46 & 681 & 27,555 & 9792562 & 1.976 & .976 & .024 \\
\hline 998 & 30.03 & 710 & 21,324 & 7855829 & 1.743 & .959 & .041 & 43.52 & 490 & 21,324 & 5465064 & 1.766 & .961 & .039 \\
\hline 999 & 28.73 & 127 & 3,649 & 214622 & -1.440 & .075 & .075 & 40.10 & 91 & 3,649 & 149238 & -1.671 & .047 & .047 \\
\hline 1000 & 20.38 & 442 & 9,008 & 2055012 & 1.175 & .880 & .120 & 29.73 & 303 & 9,008 & 1438469 & 1.630 & .948 & .052 \\
\hline 1001 & 22.92 & 860 & 19,714 & 8993437 & 3.094 & .999 & .001 & 32.80 & 601 & 19,714 & 6351730 & 3.065 & .999 & .001 \\
\hline 1002 & 7.00 & 2 & 14 & 18 & .700 & .758 & .242 & 14.00 & 1 & 14 & 6 & -.247 & .402 & .402 \\
\hline 1003 & 40.71 & 500 & 20,356 & 5391799 & 2.305 & .989 & .011 & 63.22 & 322 & 20,356 & 3517026 & 2.273 & .989 & .012 \\
\hline 1004 & 29.46 & 692 & 20,384 & 7303890 & 1.622 & .948 & .052 & 39.05 & 522 & 20,384 & 5447926 & .950 & .829 & .171 \\
\hline 1005 & 35.82 & 596 & 21,346 & 6276111 & -.565 & .286 & .286 & 53.23 & 401 & 21,346 & 4061282 & -1.772 & .038 & .038 \\
\hline 1006 & 21.12 & 134 & 2,830 & 181882 & -.817 & .207 & .207 & 30.76 & 92 & 2,830 & 119844 & -1.319 & .094 & .094 \\
\hline 1007 & 27.47 & 308 & 8,461 & 1234613 & -1.595 & .055 & .055 & 35.70 & 237 & 8,461 & 897867 & -2.786 & .003 & .003 \\
\hline 1008 & 22.56 & 616 & 13,894 & 4232818 & -.468 & .320 & .320 & 33.40 & 416 & 13,894 & 2868437 & -.263 & .396 & .396 \\
\hline 1009 & 17.37 & 721 & 12,527 & 4580215 & .662 & .746 & .254 & 24.66 & 508 & 12,527 & 3276959 & 1.167 & .878 & .122 \\
\hline 1010 & 24.97 & 627 & 15,655 & 4727091 & -1.597 & .055 & .055 & 33.74 & 464 & 15,655 & 3505659 & -1.297 & .097 & .097 \\
\hline 1011 & 36.70 & 556 & 20,404 & 5699440 & .195 & .577 & .423 & 51.92 & 393 & 20,404 & 4080244 & .607 & .728 & .272 \\
\hline 1012 & 37.89 & 79 & 2,993 & 124817 & .859 & .805 & .195 & 59.86 & 50 & 2,993 & 81921 & 1.162 & .877 & .123 \\
\hline 1013 & 35.70 & 306 & 10,925 & 1656303 & -.276 & .391 & .391 & 53.29 & 205 & 10,925 & 1088879 & -.685 & .247 & .247 \\
\hline 1014 & 29.65 & 79 & 2,342 & 95641 & .521 & 699 & .301 & 45.04 & 52 & 2,342 & 62260 & .281 & .611 & .389 \\
\hline 1015 & 61.00 & 7 & 427 & 1054 & -1.351 & .088 & .088 & 85.40 & 5 & 427 & 853 & -.778 & .218 & .218 \\
\hline 1016 & 20.68 & 969 & 20,037 & 9678398 & -.164 & .435 & .435 & 30.27 & 662 & 20,037 & 6577240 & -.370 & .356 & .356 \\
\hline 1017 & 24.57 & 76 & 1,867 & 81895 & 2.330 & .990 & .010 & 36.61 & 51 & 1,867 & 58097 & 2.725 & .997 & .003 \\
\hline 1018 & 15.04 & 161 & 2,421 & 178968 & -1.796 & .036 & .036 & 19.52 & 124 & 2,421 & 137435 & -1.628 & .052 & .052 \\
\hline 1019 & 43.58 & 185 & 8,062 & 793656 & 1.514 & .935 & .065 & 76.06 & 106 & 8,062 & 459382 & 1.340 & .910 & .090 \\
\hline 1020 & 43.29 & 136 & 5,888 & 435180 & 1.755 & .960 & .040 & 66.91 & 88 & 5,888 & 270194 & .698 & .757 & .243 \\
\hline 1021 & 39.56 & 613 & 24,253 & 7511446 & .449 & .673 & .327 & 60.03 & 404 & 24,253 & 4990861 & .652 & .743 & .257 \\
\hline 1022 & 19.59 & 504 & 9,875 & 2484157 & -.068 & .473 & .473 & 28.46 & 347 & 9,875 & 1681763 & -.594 & .276 & .276 \\
\hline 1023 & 21.53 & 313 & 6,740 & 1015292 & -1.148 & .126 & .126 & 29.05 & 232 & 6,740 & 749017 & -1.108 & .134 & .134 \\
\hline 1024 & 22.64 & 518 & 11,727 & 3064586 & .354 & .638 & .362 & 31.27 & 375 & 11,727 & 2294421 & 1.458 & .928 & .072 \\
\hline 1025 & 32.84 & 86 & 2,824 & 110467 & -1.450 & .074 & .074 & 48.69 & 58 & 2,824 & 78057 & -.618 & .268 & .268 \\
\hline 1026 & 21.12 & 905 & 19,114 & 8822182 & 1.043 & .851 & .149 & 30.73 & 622 & 19,114 & 6211280 & 1.939 & .974 & .026 \\
\hline 1027 & 32.65 & 207 & 6,758 & 704173 & .168 & .567 & .433 & 48.27 & 140 & 6,758 & 484445 & .493 & .689 & .311 \\
\hline 1028 & 38.12 & 718 & 27,372 & 10292748 & 2.202 & .986 & .014 & 58.12 & 471 & 27,372 & 6877934 & 2.518 & .994 & .006 \\
\hline 1029 & 25.96 & 599 & 15,548 & 4916063 & 2.362 & .991 & .009 & 38.77 & 401 & 15,548 & 3308429 & 2.126 & .983 & .017 \\
\hline 1030 & 23.11 & 530 & 12,246 & 3312098 & .822 & .794 & .206 & 34.21 & 358 & 12,246 & 2279170 & 1.303 & .904 & .096 \\
\hline 1031 & 63.45 & 40 & 2,538 & 42764 & -1.726 & .042 & .042 & 94.00 & 27 & 2,538 & 30079 & -1.099 & .136 & .136 \\
\hline 1032 & 29.46 & 1,237 & 36,443 & 22488081 & -.140 & .444 & .444 & 41.84 & 871 & 36,443 & 15925233 & .175 & .569 & .431 \\
\hline 1033 & 16.16 & 1,326 & 21,434 & 14621675 & 1.824 & .966 & .034 & 22.63 & 947 & 21,434 & 10550003 & 2.106 & .982 & .018 \\
\hline 1034 & 42.43 & 51 & 2,164 & 53011 & -.487 & .313 & .313 & 56.95 & 38 & 2,164 & 42086 & .252 & .599 & .401 \\
\hline 1035 & 33.84 & 999 & 33,805 & 17111557 & .733 & .768 & .232 & 48.36 & 699 & 33,805 & 12037811 & .864 & .806 & .194 \\
\hline 1036 & 11.91 & 45 & 536 & 12195 & .130 & .552 & .448 & 14.11 & 38 & 536 & 10368 & .193 & .577 & .423 \\
\hline
\end{tabular}


Table 5. Summary of interoccurrence intervals for daily precipitation thresholds of 0.75 and 1.0 inch-Continued

\begin{tabular}{|c|c|c|c|c|c|c|c|c|c|c|c|c|c|c|}
\hline \multirow[b]{2}{*}{$\begin{array}{l}\text { Seq. } \\
\text { no. }\end{array}$} & \multicolumn{7}{|c|}{ Daily precipitation threshold of 0.75 inch and greater } & \multicolumn{7}{|c|}{ Daily precipitation threshold of 1.0 inch and greater } \\
\hline & $\begin{array}{l}\text { Mean } \\
\text { inter- } \\
\text { occur- } \\
\text { rence } \\
\text { interval } \\
\text { (days) }\end{array}$ & $\begin{array}{c}\text { Total } \\
\text { no. of } \\
\text { events }\end{array}$ & $\begin{array}{c}\text { Total } \\
\text { no. of } \\
\text { days } \\
\text { avail- } \\
\text { able }\end{array}$ & $\begin{array}{l}\text { Sigma } \\
\mathrm{S}\end{array}$ & $\begin{array}{c}\text { Stan- } \\
\text { dard } \\
\text { normal } \\
\text { variate }\end{array}$ & $\begin{array}{l}\text { Non- } \\
\text { ex- } \\
\text { ceed- } \\
\text { ance } \\
\text { prob- } \\
\text { ability }\end{array}$ & $\begin{array}{c}\mathrm{p}- \\
\text { value }\end{array}$ & $\begin{array}{c}\text { Mean } \\
\text { inter- } \\
\text { occur- } \\
\text { rence } \\
\text { interval } \\
\text { (days) }\end{array}$ & $\begin{array}{l}\text { Total } \\
\text { no. of } \\
\text { events }\end{array}$ & $\begin{array}{l}\text { Total } \\
\text { no. of } \\
\text { days } \\
\text { avail- } \\
\text { able }\end{array}$ & $\begin{array}{l}\text { Sigma } \\
\text { S }\end{array}$ & $\begin{array}{l}\text { Stan- } \\
\text { dard } \\
\text { normal } \\
\text { variate }\end{array}$ & $\begin{array}{l}\text { Non- } \\
\text { ex- } \\
\text { ceed- } \\
\text { ance } \\
\text { prob- } \\
\text { ability }\end{array}$ & $\begin{array}{c}\mathrm{p}- \\
\text { value }\end{array}$ \\
\hline 1037 & 26.63 & 16 & 426 & 2557 & -1.730 & .042 & .042 & 30.43 & 14 & 426 & 1739 & -2.701 & .003 & .003 \\
\hline 1038 & 25.90 & 134 & 3,471 & 250585 & 1.554 & .940 & .060 & 36.93 & 94 & 3,471 & 169418 & .647 & .741 & .259 \\
\hline 1039 & 162.84 & 43 & 7,002 & 175332 & 1.870 & .969 & .031 & 318.27 & 22 & 7,002 & 93391 & 1.727 & .958 & .042 \\
\hline 1040 & 152.69 & 45 & 6,871 & 150229 & -.328 & .371 & .371 & 229.03 & 30 & 6,871 & 102807 & -.024 & .491 & .491 \\
\hline 1041 & 166.67 & 21 & 3,500 & 35611 & -.246 & .403 & .403 & 318.18 & 11 & 3,500 & 16867 & -.711 & .239 & .239 \\
\hline 1042 & 13.32 & 932 & 12,414 & 5739574 & -.415 & .339 & .339 & 18.31 & 678 & 12,414 & 4235911 & .295 & .616 & .384 \\
\hline 1043 & 67.29 & 7 & 471 & 744 & -2.514 & .006 & .006 & 94.20 & 5 & 471 & 436 & -2.439 & .007 & .007 \\
\hline 1044 & 43.79 & 187 & 8,188 & 843395 & 2.408 & .992 & .008 & 67.12 & 122 & 8,188 & 563061 & 2.436 & .993 & .007 \\
\hline 1045 & 47.84 & 418 & 19,999 & 4478145 & 2.528 & .994 & .006 & 72.46 & 276 & 19,999 & 2960951 & 2.097 & .982 & .018 \\
\hline 1046 & 29.41 & 58 & 1,706 & 51947 & .659 & .745 & .255 & 36.30 & 47 & 1,706 & 45670 & 1.652 & .951 & .049 \\
\hline 1047 & 29.33 & 689 & 20,211 & 7463533 & 3.270 & .999 & .001 & 42.91 & 471 & 20,211 & 5067747 & 2.433 & .993 & .007 \\
\hline 1048 & 32.03 & 375 & 12,010 & 2279094 & .405 & .657 & .343 & 45.67 & 263 & 12,010 & 1587582 & .147 & .558 & .442 \\
\hline 1049 & 27.98 & 175 & 4,897 & 437192 & .466 & .679 & .321 & 39.81 & 123 & 4,897 & 295058 & -.390 & .348 & .348 \\
\hline 1050 & 14.96 & 805 & 12,043 & 5134164 & 2.908 & .998 & .002 & 20.17 & 597 & 12,043 & 3726801 & 1.554 & .940 & .060 \\
\hline 1051 & 33.39 & 535 & 17,865 & 4720704 & -.488 & .313 & .313 & 45.34 & 394 & 17,865 & 3569250 & .487 & .687 & .313 \\
\hline 1052 & 42.59 & 17 & 724 & 5417 & -.855 & .196 & .196 & 65.82 & 11 & 724 & 3328 & -.944 & .173 & .173 \\
\hline 1053 & 14.70 & 71 & 1,044 & 35952 & -.437 & .331 & .331 & 19.70 & 53 & 1,044 & 26586 & -.492 & .311 & .311 \\
\hline 1054 & 30.50 & 2 & 61 & 24 & -1.486 & .069 & .069 & 61.00 & 1 & 61 & 21 & -.540 & .295 & .295 \\
\hline 1055 & 17.06 & 196 & 3,344 & 345115 & 1.288 & .901 & .099 & 26.54 & 126 & 3,344 & 219956 & .857 & .804 & .196 \\
\hline 1056 & 41.22 & 51 & 2,102 & 59375 & 1.332 & .909 & .091 & 55.32 & 38 & 2,102 & 43374 & .919 & .821 & .179 \\
\hline 1057 & 63.50 & 8 & 508 & 1869 & -.393 & .347 & .347 & 84.67 & 6 & 508 & 1449 & -.209 & .417 & .417 \\
\hline 1058 & 25.12 & 1,403 & 35,247 & 25156200 & 1.129 & .871 & .129 & 35.25 & 1,000 & 35,247 & 18085362 & 1.435 & .924 & .076 \\
\hline 1059 & 32.50 & 22 & 715 & 6565 & -1.343 & .090 & .090 & 55.00 & 13 & 715 & 3230 & -1.905 & .028 & .028 \\
\hline 1060 & 32.05 & 756 & 24,233 & 9596743 & 2.270 & .988 & .012 & 47.99 & 505 & 24,233 & 6393207 & 1.745 & .960 & .040 \\
\hline 1061 & 34.74 & 19 & 660 & 6023 & -.297 & .383 & .383 & 41.25 & 16 & 660 & 5238 & -.055 & .478 & .478 \\
\hline 1062 & 56.22 & 37 & 2,080 & 32658 & -1.594 & .055 & .055 & 86.67 & 24 & 2,080 & 18869 & -2.071 & .019 & .019 \\
\hline 1063 & 74.13 & 322 & 23,869 & 3951468 & .878 & .810 & .190 & 114.76 & 208 & 23,869 & 2518225 & .361 & .641 & .359 \\
\hline 1064 & 72.88 & 17 & 1,239 & 11408 & .594 & .724 & .276 & 103.25 & 12 & 1,239 & 8452 & .822 & .794 & .206 \\
\hline 1065 & 32.46 & 187 & 6,070 & 567023 & -.022 & .491 & .491 & 49.75 & 122 & 6,070 & 362071 & -.424 & .336 & .336 \\
\hline 1066 & 24.11 & 461 & 11,113 & 2627238 & .954 & .830 & .170 & 35.28 & 315 & 11,113 & 1778623 & .498 & .691 & .309 \\
\hline 1067 & 27.79 & 33 & 917 & 16661 & 1.007 & .843 & .157 & 36.68 & 25 & 917 & 12757 & .978 & .836 & .164 \\
\hline 1068 & 34.10 & 21 & 716 & 7183 & -.354 & .362 & .362 & 44.75 & 16 & 716 & 5514 & -.259 & .398 & .398 \\
\hline 1069 & 43.34 & 125 & 5,418 & 319033 & -1.120 & .131 & .131 & 53.12 & 102 & 5,418 & 271678 & -.294 & .384 & .384 \\
\hline 1070 & 31.82 & 1,107 & 35,225 & 19343838 & -.453 & .325 & .325 & 45.10 & 781 & 35,225 & 13455605 & -1.055 & .146 & .146 \\
\hline 1071 & 22.72 & 864 & 19,631 & 8784398 & 1.824 & .966 & .034 & 32.88 & 597 & 19,631 & 6086929 & 1.640 & .949 & .051 \\
\hline 1072 & 26.90 & 62 & 1,668 & 49466 & -.591 & .277 & .277 & 37.07 & 45 & 1,668 & 35171 & -.730 & .233 & .233 \\
\hline 1073 & -- & -- & -- & -- & -- & -- & -- & -- & -- & -- & -- & -- & -- & -- \\
\hline 1074 & 20.11 & 1,497 & 30,105 & 22846017 & .929 & .824 & .176 & 28.14 & 1,070 & 30,105 & 16173364 & .236 & .593 & .407 \\
\hline 1075 & 34.07 & 76 & 2,589 & 102592 & .646 & .741 & .259 & 53.94 & 48 & 2,589 & 59474 & -.514 & .304 & .304 \\
\hline 1076 & 28.51 & 633 & 18,047 & 5727330 & .118 & .547 & .453 & 41.78 & 432 & 18,047 & 3859926 & -.353 & .362 & .362 \\
\hline 1077 & 25.85 & 133 & 3,438 & 237781 & .800 & .788 & .212 & 36.19 & 95 & 3,438 & 167558 & .440 & .670 & .330 \\
\hline 1078 & 58.04 & 474 & 27,513 & 6558858 & .221 & .588 & .412 & 90.21 & 305 & 27,513 & 4350930 & 1.119 & .868 & .132 \\
\hline 1079 & 32.26 & 970 & 31,290 & 15362475 & .664 & .747 & .253 & 48.97 & 639 & 31,290 & 10153176 & .683 & .753 & .247 \\
\hline 1080 & 34.57 & 14 & 484 & 3997 & 1.165 & .878 & .122 & 48.40 & 10 & 484 & 2864 & 1.005 & .842 & .158 \\
\hline 1081 & 40.29 & 527 & 21,232 & 5495408 & -.705 & .240 & .240 & 61.54 & 345 & 21,232 & 3717048 & .479 & .684 & .316 \\
\hline 1082 & 41.25 & 332 & 13,695 & 2388340 & 1.596 & .945 & .055 & 60.60 & 226 & 13,695 & 1618577 & 1.195 & .884 & .116 \\
\hline 1083 & 58.23 & 265 & 15,430 & 2084867 & .557 & .711 & .289 & 92.95 & 166 & 15,430 & 1263541 & -.299 & .383 & .383 \\
\hline 1084 & 18.72 & 65 & 1,217 & 39564 & .004 & .502 & .498 & 27.04 & 45 & 1,217 & 27057 & -.138 & .445 & .445 \\
\hline 1085 & 20.22 & 1,797 & 36,335 & 32943675 & .667 & .748 & .252 & 29.52 & 1,231 & 36,335 & 22635761 & .738 & .770 & .230 \\
\hline 1086 & 46.75 & 108 & 5,049 & 251769 & -1.378 & .084 & .084 & 63.91 & 79 & 5,049 & 176767 & -1.750 & .040 & .040 \\
\hline 1087 & 106.44 & 132 & 14,050 & 899796 & -.590 & .278 & .278 & 182.47 & 77 & 14,050 & 513030 & -.784 & .217 & .217 \\
\hline 1088 & 15.01 & 150 & 2,252 & 171107 & .277 & .609 & .391 & 19.75 & 114 & 2,252 & 134013 & .814 & .792 & .208 \\
\hline
\end{tabular}


Table 5. Summary of interoccurrence intervals for daily precipitation thresholds of 0.75 and 1.0 inch-Continued

\begin{tabular}{|c|c|c|c|c|c|c|c|c|c|c|c|c|c|c|}
\hline \multirow[b]{2}{*}{$\begin{array}{l}\text { Seq. } \\
\text { no. }\end{array}$} & \multicolumn{7}{|c|}{ Daily precipitation threshold of 0.75 inch and greater } & \multicolumn{7}{|c|}{ Daily precipitation threshold of 1.0 inch and greater } \\
\hline & $\begin{array}{l}\text { Mean } \\
\text { inter- } \\
\text { occur- } \\
\text { rence } \\
\text { interval } \\
\text { (days) }\end{array}$ & $\begin{array}{c}\text { Total } \\
\text { no. of } \\
\text { events }\end{array}$ & $\begin{array}{l}\text { Total } \\
\text { no. of } \\
\text { days } \\
\text { avail- } \\
\text { able }\end{array}$ & $\underset{\mathrm{S}}{\text { Sigma }}$ & $\begin{array}{c}\text { Stan- } \\
\text { dard } \\
\text { normal } \\
\text { variate }\end{array}$ & $\begin{array}{l}\text { Non- } \\
\text { ex- } \\
\text { ceed- } \\
\text { ance } \\
\text { prob- } \\
\text { ability }\end{array}$ & $\begin{array}{c}\mathrm{p}- \\
\text { value }\end{array}$ & $\begin{array}{l}\text { Mean } \\
\text { inter- } \\
\text { occur- } \\
\text { rence } \\
\text { interval } \\
\text { (days) }\end{array}$ & $\begin{array}{l}\text { Total } \\
\text { no. of } \\
\text { events }\end{array}$ & $\begin{array}{l}\text { Total } \\
\text { no. of } \\
\text { days } \\
\text { avail- } \\
\text { able }\end{array}$ & $\begin{array}{c}\text { Sigma } \\
\mathrm{S}\end{array}$ & $\begin{array}{c}\text { Stan- } \\
\text { dard } \\
\text { normal } \\
\text { variate }\end{array}$ & $\begin{array}{l}\text { Non- } \\
\text { ex- } \\
\text { ceed- } \\
\text { ance } \\
\text { prob- } \\
\text { ability }\end{array}$ & $\begin{array}{c}\mathrm{p}- \\
\text { value }\end{array}$ \\
\hline 1089 & 40.90 & 29 & 1,186 & 15934 & -0.685 & .247 & .247 & 59.30 & 20 & 1,186 & 11345 & -0.336 & .368 & .368 \\
\hline 1090 & 32.32 & 315 & 10,180 & 1564067 & -.753 & .226 & .226 & 49.90 & 204 & 10,180 & 1018058 & -.484 & .314 & .314 \\
\hline 1091 & 43.58 & 452 & 19,700 & 4467686 & .128 & .551 & .449 & 71.64 & 275 & 19,700 & 2697469 & -.120 & .452 & .452 \\
\hline 1092 & 11.18 & 11 & 123 & 379 & -2.526 & .006 & .006 & 12.30 & 10 & 123 & 285 & -2.939 & .002 & .002 \\
\hline 1093 & 16.24 & 293 & 4,757 & 746990 & 2.131 & .983 & .017 & 23.67 & 201 & 4,757 & 511013 & 1.692 & .955 & .045 \\
\hline 1094 & 27.25 & 947 & 25,807 & 12779459 & 2.442 & .993 & .007 & 38.18 & 676 & 25,807 & 9267621 & 2.813 & .998 & .002 \\
\hline 1095 & 20.39 & 97 & 1,978 & 91989 & -.701 & .242 & .242 & 28.67 & 69 & 1,978 & 63661 & -.966 & .167 & .167 \\
\hline 1096 & 48.74 & 392 & 19,104 & 3873492 & 1.182 & .881 & .119 & 75.21 & 254 & 19,104 & 2504542 & .891 & .814 & .186 \\
\hline 1097 & 21.77 & 874 & 19,028 & 9134635 & 5.046 & 0 & 0 & 30.84 & 617 & 19,028 & 6374564 & 3.697 & 1.000 & 0 \\
\hline 1098 & 29.61 & 499 & 14,774 & 3711226 & .264 & .604 & .396 & 43.45 & 340 & 14,774 & 2495189 & -.208 & .417 & .417 \\
\hline 1099 & 103.00 & 2 & 206 & 154 & -.618 & .268 & .268 & -- & -- & -- & -- & -- & -- & -- \\
\hline 1100 & 26.11 & 108 & 2,820 & 142836 & -1.116 & .132 & .132 & 39.72 & 71 & 2,820 & 95796 & -.629 & .265 & .265 \\
\hline 1101 & 22.34 & 1,307 & 29,198 & 19386346 & 1.002 & .842 & .158 & 31.98 & 913 & 29,198 & 13646907 & 1.249 & .894 & .106 \\
\hline 1102 & 40.62 & 777 & 31,561 & 12009573 & -.992 & .161 & .161 & 65.89 & 479 & 31,561 & 7396357 & -.815 & .208 & .208 \\
\hline 1103 & 177.39 & 59 & 10,466 & 301673 & -.305 & .380 & .380 & 337.61 & 31 & 10,466 & 169956 & .460 & .677 & .323 \\
\hline 1104 & 23.53 & 332 & 7,812 & 1357346 & 1.474 & .930 & .070 & 32.28 & 242 & 7,812 & 1013616 & 1.949 & .974 & .026 \\
\hline 1105 & 21.78 & 608 & 13,243 & 4177929 & 1.613 & .947 & .053 & 30.87 & 429 & 13,243 & 2948992 & 1.369 & .914 & .086 \\
\hline 1106 & 40.67 & 553 & 22,491 & 6215129 & -.024 & .490 & .490 & 60.95 & 369 & 22,491 & 4126469 & -.185 & .426 & .426 \\
\hline 1107 & 43.51 & 37 & 1,610 & 25571 & -1.491 & .068 & .068 & 59.63 & 27 & 1,610 & 19714 & -.837 & .201 & .201 \\
\hline 1108 & -- & -- & -- & -- & -- & -- & -- & -- & -- & -- & -- & -- & -- & -- \\
\hline 1109 & 32.95 & 106 & 3,493 & 169607 & -1.495 & .067 & .067 & 51.37 & 68 & 3,493 & 112124 & -.798 & .212 & .212 \\
\hline 1110 & 33.75 & 104 & 3,510 & 161449 & -2.039 & .021 & .021 & 44.43 & 79 & 3,510 & 123161 & -1.719 & .043 & .043 \\
\hline 1111 & 53.62 & 42 & 2,252 & 40915 & -1.514 & .065 & .065 & 86.62 & 26 & 2,252 & 23940 & -1.610 & .054 & .054 \\
\hline 1112 & 18.32 & 656 & 12,016 & 4108993 & 1.888 & .970 & .030 & 25.35 & 474 & 12,016 & 3000079 & 2.017 & .978 & .022 \\
\hline 1113 & 44.76 & 659 & 29,498 & 9527094 & -.881 & .189 & .189 & 72.12 & 409 & 29,498 & 5910921 & -.705 & .240 & .240 \\
\hline 1114 & 25.63 & 473 & 12,124 & 2818241 & -.645 & .260 & .260 & 36.74 & 330 & 12,124 & 1948151 & -.823 & .205 & .205 \\
\hline 1115 & 41.52 & 140 & 5,813 & 422448 & .783 & .783 & .217 & 62.51 & 93 & 5,813 & 282010 & .723 & .765 & .235 \\
\hline 1116 & 18.39 & 79 & 1,453 & 55509 & -.506 & .307 & .307 & 25.05 & 58 & 1,453 & 40708 & -.447 & .327 & .327 \\
\hline 1117 & 26.26 & 599 & 15,727 & 4764742 & .491 & .688 & .312 & 36.07 & 436 & 15,727 & 3420610 & -.083 & .467 & .467 \\
\hline 1118 & 27.59 & 29 & 800 & 12366 & .616 & .731 & .269 & 38.10 & 21 & 800 & 8048 & -.333 & .370 & .370 \\
\hline 1119 & 26.62 & 285 & 7,586 & 1043373 & -1.018 & .154 & .154 & 40.35 & 188 & 7,586 & 679965 & -1.103 & .135 & .135 \\
\hline 1120 & 42.03 & 582 & 24,462 & 7138860 & .120 & .548 & .452 & 69.10 & 354 & 24,462 & 4321767 & -.060 & .476 & .476 \\
\hline 1121 & 32.15 & 923 & 29,675 & 13701865 & .026 & .511 & .489 & 49.21 & 603 & 29,675 & 8768291 & -.850 & .198 & .198 \\
\hline 1122 & 32.94 & 31 & 1,021 & 14056 & -1.078 & .141 & .141 & 44.39 & 23 & 1,021 & 8917 & -1.998 & .023 & .023 \\
\hline 1123 & 28.79 & 792 & 22,804 & 9162882 & .715 & .763 & .237 & 44.19 & 516 & 22,804 & 5746719 & -.914 & .180 & .180 \\
\hline 1124 & 23.24 & 59 & 1,371 & 35017 & -1.785 & .037 & .037 & 42.84 & 32 & 1,371 & 20840 & -.490 & .312 & .312 \\
\hline 1125 & 30.32 & 93 & 2,820 & 134834 & .472 & .681 & .319 & 47.00 & 60 & 2,820 & 87655 & .485 & .686 & .314 \\
\hline 1126 & 47.69 & 81 & 3,863 & 155634 & -.082 & .467 & .467 & 64.38 & 60 & 3,863 & 121229 & .618 & .732 & .268 \\
\hline 1127 & 41.78 & 639 & 26,696 & 7971135 & -2.866 & .002 & .002 & 63.87 & 418 & 26,696 & 5207068 & -2.364 & .009 & .009 \\
\hline 1128 & 43.82 & 220 & 9,641 & 1066090 & .135 & .554 & .446 & 65.59 & 147 & 9,641 & 679062 & -.876 & .191 & .191 \\
\hline 1129 & 63.12 & 57 & 3,598 & 107537 & .637 & .738 & .262 & 94.68 & 38 & 3,598 & 74947 & 1.029 & .848 & .152 \\
\hline 1130 & 94.38 & 8 & 755 & 2586 & -.704 & .241 & .241 & 125.83 & 6 & 755 & 1421 & -1.581 & .057 & .057 \\
\hline 1131 & 23.88 & 580 & 13,853 & 4217690 & 2.080 & .981 & .019 & 34.38 & 403 & 13,853 & 2999915 & 2.598 & .995 & .005 \\
\hline 1132 & 53.54 & 13 & 696 & 3819 & -.973 & .165 & .165 & 99.43 & 7 & 696 & 1641 & -1.496 & .067 & .067 \\
\hline 1133 & 26.30 & 384 & 10,098 & 1921181 & -.309 & .379 & .379 & 36.85 & 274 & 10,098 & 1350842 & -.675 & .250 & .250 \\
\hline 1134 & 52.69 & 13 & 685 & 4558 & .148 & .559 & .441 & 85.63 & 8 & 685 & 2780 & .072 & .529 & .471 \\
\hline 1135 & 14.61 & 54 & 789 & 18501 & -1.674 & .047 & .047 & 18.79 & 42 & 789 & 14689 & -1.274 & .101 & .101 \\
\hline 1136 & 54.23 & 514 & 27,875 & 7303219 & .764 & .777 & .223 & 87.38 & 319 & 27,875 & 4406333 & -.276 & .391 & .391 \\
\hline 1137 & 28.62 & 669 & 19,145 & 6566155 & 1.134 & .872 & .128 & 41.89 & 457 & 19,145 & 4504849 & 1.102 & .865 & .135 \\
\hline 1138 & 68.58 & 36 & 2,469 & 47824 & .791 & .785 & .215 & 137.17 & 18 & 2,469 & 22535 & .104 & .541 & .459 \\
\hline 1139 & 111.32 & 28 & 3,117 & 32712 & -2.295 & .011 & .011 & 207.80 & 15 & 3,117 & 18052 & -1.528 & .063 & .063 \\
\hline 1140 & 41.56 & 268 & 11,137 & 1445480 & -.891 & .187 & .187 & 62.92 & 177 & 11,137 & 944577 & -.960 & .169 & .169 \\
\hline
\end{tabular}


Table 5. Summary of interoccurrence intervals for daily precipitation thresholds of 0.75 and 1.0 inch-Continued

\begin{tabular}{|c|c|c|c|c|c|c|c|c|c|c|c|c|c|c|}
\hline \multirow[b]{2}{*}{$\begin{array}{l}\text { Seq. } \\
\text { no. }\end{array}$} & \multicolumn{7}{|c|}{ Daily precipitation threshold of 0.75 inch and greater } & \multicolumn{7}{|c|}{ Daily precipitation threshold of 1.0 inch and greater } \\
\hline & $\begin{array}{l}\text { Mean } \\
\text { inter- } \\
\text { occur- } \\
\text { rence } \\
\text { interval } \\
\text { (days) }\end{array}$ & $\begin{array}{c}\text { Total } \\
\text { no. of } \\
\text { events }\end{array}$ & $\begin{array}{c}\text { Total } \\
\text { no. of } \\
\text { days } \\
\text { avail- } \\
\text { able }\end{array}$ & $\begin{array}{l}\text { Sigma } \\
\mathrm{S}\end{array}$ & $\begin{array}{c}\text { Stan- } \\
\text { dard } \\
\text { normal } \\
\text { variate }\end{array}$ & $\begin{array}{l}\text { Non- } \\
\text { ex- } \\
\text { ceed- } \\
\text { ance } \\
\text { prob- } \\
\text { ability }\end{array}$ & $\begin{array}{c}\mathrm{p}- \\
\text { value }\end{array}$ & $\begin{array}{c}\text { Mean } \\
\text { inter- } \\
\text { occur- } \\
\text { rence } \\
\text { interval } \\
\text { (days) }\end{array}$ & $\begin{array}{l}\text { Total } \\
\text { no. of } \\
\text { events }\end{array}$ & $\begin{array}{l}\text { Total } \\
\text { no. of } \\
\text { days } \\
\text { avail- } \\
\text { able }\end{array}$ & $\begin{array}{l}\text { Sigma } \\
\mathrm{S}\end{array}$ & $\begin{array}{l}\text { Stan- } \\
\text { dard } \\
\text { normal } \\
\text { variate }\end{array}$ & $\begin{array}{l}\text { Non- } \\
\text { ex- } \\
\text { ceed- } \\
\text { ance } \\
\text { prob- } \\
\text { ability }\end{array}$ & $\begin{array}{c}\mathrm{p}- \\
\text { value }\end{array}$ \\
\hline 1141 & 18.10 & 1,103 & 19,965 & 11502752 & 2.571 & .995 & .005 & 25.53 & 782 & 19,965 & 8354266 & 3.400 & 1.000 & 0 \\
\hline 1142 & 17.61 & 1,858 & 32,715 & 30899306 & 1.246 & .894 & .107 & 25.28 & 1,294 & 32,715 & 21572601 & 1.195 & .884 & .116 \\
\hline 1143 & 59.70 & 151 & 9,015 & 688094 & .233 & .592 & .408 & 96.94 & 93 & 9,015 & 404712 & -.577 & .282 & .282 \\
\hline 1144 & 34.80 & 69 & 2,401 & 87381 & .790 & .785 & .215 & 46.17 & 52 & 2,401 & 64420 & .399 & .655 & .345 \\
\hline 1145 & 62.13 & 23 & 1,429 & 22071 & 2.850 & .998 & .002 & 95.27 & 15 & 1,429 & 13936 & 2.015 & .978 & .022 \\
\hline 1146 & 49.12 & 542 & 26,623 & 7177519 & -.209 & .417 & .417 & 75.21 & 354 & 26,623 & 4693628 & -.129 & .449 & .449 \\
\hline 1147 & 38.09 & 163 & 6,208 & 523334 & .760 & .776 & .224 & 53.98 & 115 & 6,208 & 361393 & .231 & .591 & .409 \\
\hline 1148 & 48.87 & 266 & 13,000 & 1723852 & -.084 & .466 & .466 & 80.75 & 161 & 13,000 & 1032806 & -.288 & .387 & .387 \\
\hline 1149 & 28.98 & 417 & 12,084 & 2629348 & 1.542 & .938 & .062 & 40.96 & 295 & 12,084 & 1797283 & .249 & .598 & .402 \\
\hline 1150 & 52.11 & 258 & 13,443 & 1782189 & .771 & .779 & .221 & 81.97 & 164 & 13,443 & 1120483 & .365 & .643 & .357 \\
\hline 1151 & 23.31 & 49 & 1,142 & 26235 & -.756 & .225 & .225 & 38.07 & 30 & 1,142 & 14592 & -1.406 & .080 & .080 \\
\hline 1152 & 24.28 & 1,090 & 26,466 & 14840253 & 1.650 & .951 & .049 & 35.10 & 754 & 26,466 & 10224402 & 1.176 & .880 & .120 \\
\hline 1153 & 15.71 & 31 & 487 & 7336 & -.272 & .393 & .393 & 21.17 & 23 & 487 & 5429 & -.254 & .400 & .400 \\
\hline 1154 & 29.98 & 435 & 13,041 & 2823204 & -.168 & .433 & .433 & 43.62 & 299 & 13,041 & 1911278 & -.589 & .278 & .278 \\
\hline 1155 & 19.84 & 69 & 1,369 & 40572 & -2.028 & .021 & .021 & 29.13 & 47 & 1,369 & 29412 & -1.019 & .154 & .154 \\
\hline 1156 & 27.87 & 467 & 13,013 & 3120984 & 1.016 & .845 & .155 & 39.55 & 329 & 13,013 & 2209198 & 1.006 & .843 & .157 \\
\hline 1157 & 33.55 & 538 & 18,047 & 5057275 & 1.677 & .953 & .047 & 54.36 & 332 & 18,047 & 3124041 & 1.351 & .912 & .088 \\
\hline 1158 & 18.27 & 195 & 3,562 & 359751 & .868 & .807 & .193 & 25.09 & 142 & 3,562 & 265307 & 1.012 & .844 & .156 \\
\hline 1159 & 23.90 & 1,542 & 36,849 & 28745036 & .801 & .788 & .212 & 34.73 & 1,061 & 36,849 & 19662402 & .329 & .629 & .371 \\
\hline 1160 & 34.17 & 41 & 1,401 & 31990 & 1.263 & .897 & .103 & 48.31 & 29 & 1,401 & 22276 & .901 & .816 & .184 \\
\hline 1161 & 37.39 & 153 & 5,720 & 447142 & .468 & .680 & .320 & 57.78 & 99 & 5,720 & 296052 & .786 & .784 & .216 \\
\hline 1162 & 110.00 & 11 & 1,210 & 6839 & .159 & .563 & .437 & 403.33 & 3 & 1,210 & 2734 & 1.519 & .936 & .064 \\
\hline 1163 & 143.64 & 22 & 3,160 & 31664 & -.724 & .235 & .235 & 210.67 & 15 & 3,160 & 22969 & -.207 & .418 & .418 \\
\hline 1164 & 19.86 & 965 & 19,160 & 9383742 & .809 & .791 & .209 & 27.81 & 689 & 19,160 & 6772023 & 1.181 & .881 & .119 \\
\hline 1165 & 42.09 & 236 & 9,934 & 1234330 & 1.410 & .921 & .079 & 60.21 & 165 & 9,934 & 871861 & 1.420 & .922 & .078 \\
\hline 1166 & 15.57 & 770 & 11,989 & 4825046 & 2.179 & .985 & .015 & 22.28 & 538 & 11,989 & 3384776 & 1.990 & .977 & .023 \\
\hline 1167 & 18.20 & 371 & 6,751 & 1283796 & .839 & .799 & .201 & 25.87 & 261 & 6,751 & 909645 & .910 & .818 & .182 \\
\hline 1168 & 46.26 & 54 & 2,498 & 66050 & -.263 & .396 & .396 & 55.51 & 45 & 2,498 & 57471 & .262 & .603 & .397 \\
\hline 1169 & 104.08 & 12 & 1,249 & 7798 & .243 & .596 & .404 & 156.13 & 8 & 1,249 & 4181 & -.799 & .212 & .212 \\
\hline 1170 & 17.74 & 932 & 16,537 & 8024946 & 2.187 & .986 & .014 & 24.36 & 679 & 16,537 & 5821595 & 1.666 & .952 & .048 \\
\hline 1171 & 23.06 & 531 & 12,247 & 3212568 & -.479 & .316 & .316 & 34.02 & 360 & 12,247 & 2155172 & -.735 & .231 & .231 \\
\hline 1172 & 21.54 & 899 & 19,365 & 8975691 & 1.618 & .947 & .053 & 29.57 & 655 & 19,365 & 6510948 & 1.181 & .881 & .119 \\
\hline 1173 & 33.31 & 714 & 23,783 & 8500988 & .057 & .523 & .477 & 48.94 & 486 & 23,783 & 5885031 & .699 & .758 & .242 \\
\hline 1174 & 36.55 & 139 & 5,080 & 370013 & .981 & .837 & .163 & 54.04 & 94 & 5,080 & 255451 & 1.174 & .880 & .120 \\
\hline 1175 & 33.57 & 808 & 27,125 & 11099654 & .634 & .737 & .263 & 50.23 & 540 & 27,125 & 7517668 & 1.066 & .857 & .143 \\
\hline 1176 & 32.70 & 20 & 654 & 5912 & -.744 & .229 & .229 & 46.71 & 14 & 654 & 3801 & -1.100 & .136 & .136 \\
\hline 1177 & 27.33 & 52 & 1,421 & 36914 & -.011 & .496 & .496 & 36.44 & 39 & 1,421 & 29814 & .822 & .794 & .206 \\
\hline 1178 & 28.51 & 626 & 17,848 & 5669091 & .641 & .739 & .261 & 42.70 & 418 & 17,848 & 3747084 & .160 & .564 & .436 \\
\hline 1179 & 38.20 & 459 & 17,533 & 4111872 & .812 & .792 & .208 & 55.66 & 315 & 17,533 & 2798061 & .408 & .658 & .342 \\
\hline 1180 & 44.08 & 38 & 1,675 & 32906 & .363 & .642 & .358 & 62.04 & 27 & 1,675 & 24029 & .564 & .714 & .286 \\
\hline 1181 & 120.50 & 10 & 1,205 & 6623 & .544 & .707 & .293 & 200.83 & 6 & 1,205 & 3056 & -.656 & .256 & .256 \\
\hline 1182 & 14.87 & 651 & 9,681 & 3272001 & 1.695 & .955 & .045 & 20.30 & 477 & 9,681 & 2431088 & 2.002 & .977 & .023 \\
\hline 1183 & 18.69 & 1,049 & 19,606 & 10855035 & 3.119 & .999 & .001 & 26.07 & 752 & 19,606 & 7907333 & 3.450 & 1.000 & 0 \\
\hline 1184 & 167.35 & 48 & 8,033 & 175792 & -1.058 & .145 & .145 & 334.71 & 24 & 8,033 & 79504 & -1.487 & .069 & .069 \\
\hline 1185 & 29.81 & 287 & 8,556 & 1222825 & -.119 & .453 & .453 & 41.33 & 207 & 8,556 & 888757 & .090 & .536 & .464 \\
\hline 1186 & 17.99 & 335 & 6,027 & 1038258 & .902 & .817 & .183 & 25.98 & 232 & 6,027 & 707935 & .332 & .630 & .370 \\
\hline 1187 & 14.55 & 789 & 11,476 & 4617499 & .970 & .834 & .166 & 20.03 & 573 & 11,476 & 3349278 & .774 & .781 & .219 \\
\hline 1188 & 125.08 & 80 & 10,006 & 420734 & .793 & .786 & .214 & 185.30 & 54 & 10,006 & 284238 & .663 & .746 & .254 \\
\hline 1189 & 34.46 & 556 & 19,158 & 5560050 & 1.795 & .964 & .036 & 51.50 & 372 & 19,158 & 3836609 & 2.561 & .995 & .005 \\
\hline 1190 & 18.96 & 1,006 & 19,072 & 9749032 & .892 & .814 & .186 & 27.44 & 695 & 19,072 & 6700593 & .504 & .693 & .307 \\
\hline 1191 & 65.82 & 11 & 724 & 3152 & -1.197 & .116 & .116 & 120.67 & 6 & 724 & 1244 & -1.813 & .035 & .035 \\
\hline 1192 & 34.31 & 189 & 6,484 & 640714 & 1.087 & .861 & .139 & 58.41 & 111 & 6,484 & 353947 & -.300 & .382 & .382 \\
\hline
\end{tabular}


Table 5. Summary of interoccurrence intervals for daily precipitation thresholds of 0.75 and 1.0 inch-Continued

\begin{tabular}{|c|c|c|c|c|c|c|c|c|c|c|c|c|c|c|}
\hline \multirow[b]{2}{*}{$\begin{array}{c}\text { Seq. } \\
\text { no. }\end{array}$} & \multicolumn{7}{|c|}{ Daily precipitation threshold of 0.75 inch and greater } & \multicolumn{7}{|c|}{ Daily precipitation threshold of 1.0 inch and greater } \\
\hline & $\begin{array}{c}\text { Mean } \\
\text { inter- } \\
\text { occur- } \\
\text { rence } \\
\text { interval } \\
\text { (days) }\end{array}$ & $\begin{array}{c}\text { Total } \\
\text { no. of } \\
\text { events }\end{array}$ & $\begin{array}{l}\text { Total } \\
\text { no. of } \\
\text { days } \\
\text { avail- } \\
\text { able }\end{array}$ & $\begin{array}{l}\text { Sigma } \\
\mathrm{S}\end{array}$ & $\begin{array}{l}\text { Stan- } \\
\text { dard } \\
\text { normal } \\
\text { variate }\end{array}$ & $\begin{array}{l}\text { Non- } \\
\text { ex- } \\
\text { ceed- } \\
\text { ance } \\
\text { prob- } \\
\text { ability }\end{array}$ & $\begin{array}{c}\text { p- } \\
\text { value }\end{array}$ & $\begin{array}{c}\text { Mean } \\
\text { inter- } \\
\text { occur- } \\
\text { rence } \\
\text { interval } \\
\text { (days) }\end{array}$ & $\begin{array}{c}\text { Total } \\
\text { no. of } \\
\text { events }\end{array}$ & $\begin{array}{c}\text { Total } \\
\text { no. of } \\
\text { days } \\
\text { avail- } \\
\text { able }\end{array}$ & $\begin{array}{l}\text { Sigma } \\
\mathrm{S}\end{array}$ & $\begin{array}{c}\text { Stan- } \\
\text { dard } \\
\text { normal } \\
\text { variate }\end{array}$ & $\begin{array}{l}\text { Non- } \\
\text { ex- } \\
\text { ceed- } \\
\text { ance } \\
\text { prob- } \\
\text { ability }\end{array}$ & $\begin{array}{c}\text { p- } \\
\text { value }\end{array}$ \\
\hline 1193 & 21.82 & 350 & 7,637 & 1328263 & -0.199 & .421 & .421 & 33.79 & 226 & 7,637 & 854958 & -0.242 & .404 & .404 \\
\hline 1194 & 20.57 & 405 & 8,332 & 1697858 & .220 & .587 & .413 & 28.83 & 289 & 8,332 & 1265362 & 1.501 & .933 & .067 \\
\hline 1195 & 16.47 & 135 & 2,224 & 154925 & .644 & .740 & .260 & 23.66 & 94 & 2,224 & 100194 & -.696 & .243 & .243 \\
\hline 1196 & 18.05 & 356 & 6,424 & 1154358 & .311 & .622 & .378 & 26.55 & 242 & 6,424 & 777781 & .017 & .507 & .493 \\
\hline 1197 & 23.99 & 826 & 19,815 & 8529806 & 2.106 & .982 & .018 & 35.07 & 565 & 19,815 & 5750694 & 1.125 & .870 & .130 \\
\hline 1198 & 37.35 & 243 & 9,076 & 1207247 & 2.559 & .995 & .005 & 55.68 & 163 & 9,076 & 821213 & 2.437 & .993 & .007 \\
\hline 1199 & 60.00 & 6 & 360 & 1256 & .691 & .755 & .245 & 72.00 & 5 & 360 & 904 & .017 & .507 & .493 \\
\hline 1200 & 34.18 & 421 & 14,391 & 3082424 & .623 & .733 & .267 & 48.13 & 299 & 14,391 & 2218783 & .937 & .826 & .174 \\
\hline 1201 & 58.09 & 33 & 1,917 & 32761 & .356 & .639 & .361 & 91.29 & 21 & 1,917 & 22856 & 1.076 & .859 & .141 \\
\hline 1202 & 51.19 & 379 & 19,400 & 3869230 & 1.770 & .962 & .038 & 84.72 & 229 & 19,400 & 2326329 & 1.239 & .892 & .108 \\
\hline 1203 & 39.43 & 398 & 15,694 & 2822862 & -3.322 & 0 & 0 & 64.85 & 242 & 15,694 & 1687109 & -3.006 & .001 & .001 \\
\hline 1204 & 44.61 & 347 & 15,481 & 2912047 & 2.716 & .997 & .003 & 73.72 & 210 & 15,481 & 1868892 & 3.758 & 1.000 & 0 \\
\hline 1205 & 17.02 & 381 & 6,485 & 1271837 & .997 & .841 & .159 & 23.16 & 280 & 6,485 & 955354 & 1.515 & .935 & .065 \\
\hline 1206 & 19.76 & 536 & 10,592 & 2792980 & -.645 & .259 & .259 & 27.16 & 390 & 10,592 & 2012775 & -.872 & .192 & .192 \\
\hline 1207 & 19.08 & 320 & 6,104 & 948645 & -.888 & .187 & .187 & 27.13 & 225 & 6,104 & 685419 & -.049 & .481 & .481 \\
\hline 1208 & 53.15 & 367 & 19,506 & 3681876 & .950 & .829 & .171 & 84.08 & 232 & 19,506 & 2358161 & 1.113 & .867 & .133 \\
\hline 1209 & -- & -- & -- & -- & -- & -- & -- & -- & -- & -- & -- & -- & -- & -- \\
\hline 1210 & 25.23 & 147 & 3,709 & 256766 & -1.221 & .111 & .111 & 35.66 & 104 & 3,709 & 178994 & -1.271 & .102 & .102 \\
\hline 1211 & 37.72 & 744 & 28,061 & 10571473 & .601 & .726 & .274 & 54.91 & 511 & 28,061 & 7360273 & 1.041 & .851 & .149 \\
\hline 1212 & 40.82 & 236 & 9,634 & 1166503 & .695 & .756 & .244 & 56.01 & 172 & 9,634 & 859471 & .849 & .802 & .198 \\
\hline 1213 & 34.87 & 157 & 5,474 & 423763 & -.300 & .382 & .382 & 48.88 & 112 & 5,474 & 330420 & 1.428 & .923 & .077 \\
\hline 1214 & 32.91 & 22 & 724 & 7574 & -.398 & .345 & .345 & 45.25 & 16 & 724 & 5790 & -.002 & .499 & .499 \\
\hline 1215 & 88.01 & 97 & 8,537 & 342437 & -2.950 & .002 & .002 & 131.34 & 65 & 8,537 & 240703 & -1.850 & .032 & .032 \\
\hline 1216 & 76.93 & 328 & 25,234 & 3935717 & -1.536 & .062 & .062 & 135.67 & 186 & 25,234 & 2097835 & -2.506 & .006 & .006 \\
\hline 1217 & 23.45 & 560 & 13,130 & 3738903 & .697 & .757 & .243 & 32.10 & 409 & 13,130 & 2749923 & .846 & .801 & .199 \\
\hline 1218 & 20.69 & 158 & 3,269 & 256952 & -.110 & .456 & .456 & 26.80 & 122 & 3,269 & 197563 & -.177 & .430 & .430 \\
\hline 1219 & 22.99 & 854 & 19,632 & 8818311 & 2.629 & .996 & .004 & 33.05 & 594 & 19,632 & 6292602 & 3.344 & 1.000 & 0 \\
\hline 1220 & 108.36 & 193 & 20,914 & 2048269 & .359 & .640 & .360 & 193.65 & 108 & 20,914 & 1185527 & .895 & .815 & .185 \\
\hline 1221 & 36.73 & 160 & 5,877 & 474513 & .203 & .580 & .420 & 54.93 & 107 & 5,877 & 307851 & -.374 & .354 & .354 \\
\hline 1222 & 114.50 & 2 & 229 & 39 & -2.032 & .021 & .021 & -- & -- & -- & -- & -- & -- & -- \\
\hline 1223 & 25.59 & 214 & 5,476 & 603556 & .762 & .777 & .223 & 34.88 & 157 & 5,476 & 436595 & .340 & .633 & .367 \\
\hline 1224 & 26.03 & 76 & 1,978 & 73212 & -.392 & .348 & .348 & 36.63 & 54 & 1,978 & 57346 & .939 & .826 & .174 \\
\hline 1225 & 24.99 & 106 & 2,649 & 143314 & .371 & .645 & .356 & 33.11 & 80 & 2,649 & 105352 & -.089 & .465 & .465 \\
\hline 1226 & 53.03 & 413 & 21,901 & 4241707 & -2.186 & .014 & .014 & 83.27 & 263 & 21,901 & 2671935 & -2.029 & .021 & .021 \\
\hline 1227 & 28.52 & 219 & 6,246 & 685982 & .077 & .531 & .469 & 38.80 & 161 & 6,246 & 510304 & .328 & .629 & .371 \\
\hline 1228 & 31.50 & 796 & 25,070 & 9987253 & .046 & .518 & .482 & 45.42 & 552 & 25,070 & 6982856 & .374 & .646 & .354 \\
\hline 1229 & 23.32 & 633 & 14,762 & 4746346 & .692 & .755 & .245 & 32.09 & 460 & 14,762 & 3487761 & 1.012 & .844 & .156 \\
\hline 1230 & 24.09 & 596 & 14,360 & 4310172 & .305 & .620 & .380 & 33.55 & 428 & 14,360 & 3148045 & .875 & .809 & .191 \\
\hline 1231 & 21.64 & 36 & 779 & 15102 & .800 & .788 & .212 & 29.96 & 26 & 779 & 10832 & .615 & .731 & .269 \\
\hline 1232 & 23.21 & 42 & 975 & 23265 & 1.530 & .937 & .063 & 29.55 & 33 & 975 & 18736 & 1.638 & .949 & .051 \\
\hline 1233 & 29.74 & 179 & 5,323 & 496072 & .957 & .831 & .169 & 42.93 & 124 & 5,323 & 364121 & 1.993 & .977 & .023 \\
\hline 1234 & 31.18 & 358 & 11,164 & 2132733 & 2.204 & .986 & .014 & 46.52 & 240 & 11,164 & 1369336 & .594 & .724 & .276 \\
\hline 1235 & 28.55 & 145 & 4,140 & 315745 & 1.084 & .861 & .139 & 40.19 & 103 & 4,140 & 221730 & .702 & .759 & .241 \\
\hline 1236 & 26.07 & 85 & 2,216 & 86253 & -1.344 & .089 & .089 & 36.33 & 61 & 2,216 & 62828 & -.953 & .170 & .170 \\
\hline 1237 & 21.87 & 601 & 13,142 & 4050772 & 1.092 & .863 & .137 & 30.71 & 428 & 13,142 & 2796161 & -.207 & .418 & .418 \\
\hline 1238 & 25.84 & 986 & 25,474 & 12791528 & 1.008 & .843 & .157 & 38.54 & 661 & 25,474 & 8433908 & .078 & .531 & .469 \\
\hline 1239 & 23.40 & 643 & 15,049 & 4873781 & .323 & .626 & .374 & 34.60 & 435 & 15,049 & 3277055 & .043 & .517 & .483 \\
\hline 1240 & 50.73 & 37 & 1,877 & 29119 & -1.701 & .045 & .045 & 93.85 & 20 & 1,877 & 15563 & -1.324 & .093 & .093 \\
\hline 1241 & 20.17 & 896 & 18,072 & 8253585 & 1.008 & .843 & .157 & 28.42 & 636 & 18,072 & 5914330 & 1.273 & .898 & .102 \\
\hline 1242 & 26.68 & 28 & 747 & 11895 & 1.259 & .896 & .104 & 35.57 & 21 & 747 & 8738 & .905 & .817 & .183 \\
\hline 1243 & 15.92 & 1,106 & 17,604 & 10023323 & 1.706 & .956 & .044 & 22.12 & 796 & 17,604 & 7176527 & 1.187 & .882 & .118 \\
\hline 1244 & 20.29 & 944 & 19,152 & 9214290 & 1.028 & .848 & .152 & 28.25 & 678 & 19,152 & 6561616 & .480 & .684 & .316 \\
\hline
\end{tabular}


Table 5. Summary of interoccurrence intervals for daily precipitation thresholds of 0.75 and 1.0 inch-Continued

\begin{tabular}{|c|c|c|c|c|c|c|c|c|c|c|c|c|c|c|}
\hline \multirow[b]{2}{*}{$\begin{array}{l}\text { Seq. } \\
\text { no. }\end{array}$} & \multicolumn{7}{|c|}{ Daily precipitation threshold of 0.75 inch and greater } & \multicolumn{7}{|c|}{ Daily precipitation threshold of 1.0 inch and greater } \\
\hline & $\begin{array}{l}\text { Mean } \\
\text { inter- } \\
\text { occur- } \\
\text { rence } \\
\text { interval } \\
\text { (days) }\end{array}$ & $\begin{array}{c}\text { Total } \\
\text { no. of } \\
\text { events }\end{array}$ & $\begin{array}{c}\text { Total } \\
\text { no. of } \\
\text { days } \\
\text { avail- } \\
\text { able }\end{array}$ & $\begin{array}{l}\text { Sigma } \\
\mathrm{S}\end{array}$ & $\begin{array}{c}\text { Stan- } \\
\text { dard } \\
\text { normal } \\
\text { variate }\end{array}$ & $\begin{array}{l}\text { Non- } \\
\text { ex- } \\
\text { ceed- } \\
\text { ance } \\
\text { prob- } \\
\text { ability }\end{array}$ & $\begin{array}{c}\mathrm{p}- \\
\text { value }\end{array}$ & $\begin{array}{c}\text { Mean } \\
\text { inter- } \\
\text { occur- } \\
\text { rence } \\
\text { interval } \\
\text { (days) }\end{array}$ & $\begin{array}{l}\text { Total } \\
\text { no. of } \\
\text { events }\end{array}$ & $\begin{array}{l}\text { Total } \\
\text { no. of } \\
\text { days } \\
\text { avail- } \\
\text { able }\end{array}$ & $\begin{array}{l}\text { Sigma } \\
\text { S }\end{array}$ & $\begin{array}{l}\text { Stan- } \\
\text { dard } \\
\text { normal } \\
\text { variate }\end{array}$ & $\begin{array}{l}\text { Non- } \\
\text { ex- } \\
\text { ceed- } \\
\text { ance } \\
\text { prob- } \\
\text { ability }\end{array}$ & $\begin{array}{c}\mathrm{p}- \\
\text { value }\end{array}$ \\
\hline 1245 & 40.42 & 469 & 18,958 & 4652097 & 1.742 & .959 & .041 & 61.95 & 306 & 18,958 & 3086632 & 1.944 & .974 & .026 \\
\hline 1246 & 64.22 & 36 & 2,312 & 39364 & -.562 & .287 & .287 & 77.07 & 30 & 2,312 & 32466 & -.606 & .272 & .272 \\
\hline 1247 & 38.34 & 396 & 15,182 & 3055202 & .564 & .713 & .287 & 60.01 & 253 & 15,182 & 1966440 & .659 & .745 & .255 \\
\hline 1248 & 8.71 & 7 & 61 & 206 & -.161 & .436 & .436 & 10.17 & 6 & 61 & 193 & .232 & .592 & .408 \\
\hline 1249 & 27.21 & 453 & 12,327 & 2928168 & 1.797 & .964 & .036 & 42.51 & 290 & 12,327 & 1895133 & 1.778 & .962 & .038 \\
\hline 1250 & 22.19 & 1,661 & 36,857 & 31248556 & 1.473 & .930 & .070 & 32.25 & 1,143 & 36,857 & 21332682 & .748 & .773 & .227 \\
\hline 1251 & 44.32 & 25 & 1,108 & 11675 & -1.360 & .087 & .087 & 61.56 & 18 & 1,108 & 7864 & -1.553 & .060 & .060 \\
\hline 1252 & 25.26 & 1,404 & 35,470 & 25532251 & 1.648 & .950 & .050 & 37.94 & 935 & 35,470 & 17254163 & 2.146 & .984 & .016 \\
\hline 1253 & 20.11 & 222 & 4,465 & 503163 & .393 & .653 & .347 & 26.42 & 169 & 4,465 & 360798 & -.984 & .163 & .163 \\
\hline 1254 & 18.24 & 25 & 456 & 5796 & .146 & .558 & .442 & 19.00 & 24 & 456 & 5757 & .442 & .671 & .329 \\
\hline 1255 & 24.87 & 540 & 13,428 & 3506521 & -1.322 & .093 & .093 & 34.08 & 394 & 13,428 & 2544266 & -1.313 & .095 & .095 \\
\hline 1256 & 37.29 & 549 & 20,473 & 5709544 & .648 & .741 & .259 & 57.83 & 354 & 20,473 & 3904076 & 2.521 & .994 & .006 \\
\hline 1257 & 42.45 & 460 & 19,529 & 4758703 & 2.209 & .986 & .014 & 59.36 & 329 & 19,529 & 3434156 & 2.168 & .985 & .015 \\
\hline 1258 & 21.12 & 172 & 3,633 & 323041 & .771 & .780 & .220 & 30.28 & 120 & 3,633 & 226682 & .757 & .776 & .224 \\
\hline 1259 & 229.00 & 1 & 229 & 228 & 1.717 & .957 & .043 & 229.00 & 1 & 229 & 228 & 1.717 & .957 & .043 \\
\hline 1260 & 20.36 & 1,070 & 21,784 & 12170025 & 2.507 & .994 & .006 & 28.82 & 756 & 21,784 & 8762476 & 3.054 & .999 & .001 \\
\hline 1261 & 38.04 & 215 & 8,179 & 879861 & .018 & .507 & .493 & 56.02 & 146 & 8,179 & 607088 & .351 & .637 & .363 \\
\hline 1262 & 27.48 & 42 & 1,154 & 23923 & -.144 & .443 & .443 & 44.39 & 26 & 1,154 & 13707 & -.762 & .223 & .223 \\
\hline 1263 & 12.29 & 42 & 516 & 10910 & .077 & .531 & .469 & 15.64 & 33 & 516 & 8424 & -.105 & .458 & .458 \\
\hline 1264 & 40.74 & 38 & 1,548 & 30151 & .268 & .606 & .394 & 81.47 & 19 & 1,548 & 14286 & -.216 & .415 & .415 \\
\hline 1265 & 17.36 & 199 & 3,454 & 372397 & 2.042 & .979 & .021 & 23.66 & 146 & 3,454 & 272643 & 1.702 & .956 & .044 \\
\hline 1266 & 24.35 & 782 & 19,044 & 7581193 & .878 & .810 & .190 & 36.14 & 527 & 19,044 & 5072154 & .428 & .666 & .334 \\
\hline 1267 & 30.23 & 446 & 13,484 & 3014479 & .092 & .537 & .463 & 45.25 & 298 & 13,484 & 1981873 & -.405 & .343 & .343 \\
\hline 1268 & 31.72 & 541 & 17,161 & 4566602 & -.655 & .256 & .256 & 43.89 & 391 & 17,161 & 3269748 & -.870 & .192 & .192 \\
\hline 1269 & -- & -- & -- & -- & -- & -- & -- & -- & -- & -- & -- & -- & -- & -- \\
\hline 1270 & 34.17 & 64 & 2,187 & 67773 & -.438 & .331 & .331 & 49.71 & 44 & 2,187 & 48154 & .010 & .504 & .496 \\
\hline 1271 & 30.47 & 1,151 & 35,073 & 19872285 & -.909 & .182 & .182 & 45.14 & 777 & 35,073 & 13161745 & -1.645 & .050 & .050 \\
\hline 1272 & 33.82 & 118 & 3,991 & 232558 & -.233 & .408 & .408 & 49.27 & 81 & 3,991 & 169824 & .790 & .785 & .215 \\
\hline 1273 & 29.41 & 324 & 9,529 & 1563768 & .405 & .657 & .343 & 42.35 & 225 & 9,529 & 1074790 & .067 & .527 & .473 \\
\hline 1274 & 15.90 & 387 & 6,153 & 1227049 & 1.043 & .851 & .149 & 21.98 & 280 & 6,153 & 897155 & 1.202 & .885 & .115 \\
\hline 1275 & 14.84 & 243 & 3,606 & 456806 & 1.151 & .875 & .125 & 19.92 & 181 & 3,606 & 347439 & 1.506 & .934 & .066 \\
\hline 1276 & 20.55 & 269 & 5,527 & 756832 & .514 & .696 & .304 & 27.50 & 201 & 5,527 & 545502 & -.440 & .330 & .330 \\
\hline 1277 & 15.42 & 166 & 2,560 & 214498 & .212 & .584 & .416 & 17.53 & 146 & 2,560 & 199537 & 1.417 & .922 & .078 \\
\hline 1278 & 19.22 & 1,508 & 28,977 & 21626925 & -.683 & .247 & .247 & 27.81 & 1,042 & 28,977 & 15279515 & .676 & .750 & .250 \\
\hline 1279 & 62.54 & 24 & 1,501 & 13056 & -2.335 & .010 & .010 & 75.05 & 20 & 1,501 & 9721 & -2.729 & .003 & .003 \\
\hline 1280 & 38.98 & 138 & 5,379 & 427031 & 3.063 & .999 & .001 & 56.62 & 95 & 5,379 & 281374 & 1.709 & .956 & .044 \\
\hline 1281 & 21.54 & 288 & 6,203 & 884892 & -.274 & .392 & .392 & 30.86 & 201 & 6,203 & 623404 & 0 & .500 & .500 \\
\hline 1282 & 49.00 & 5 & 245 & 891 & 1.761 & .961 & .039 & 122.50 & 2 & 245 & 409 & 1.640 & .949 & .051 \\
\hline 1283 & 30.42 & 172 & 5,233 & 492673 & 2.152 & .984 & .016 & 43.98 & 119 & 5,233 & 373399 & 3.765 & 1.000 & 0 \\
\hline 1284 & 16.53 & 591 & 9,769 & 2983915 & 1.417 & .922 & .078 & 21.86 & 447 & 9,769 & 2282486 & 1.662 & .952 & .048 \\
\hline 1285 & 88.10 & 239 & 21,056 & 2446490 & -.742 & .229 & .229 & 135.85 & 155 & 21,056 & 1569786 & -.820 & .206 & .206 \\
\hline 1286 & 17.68 & 1,035 & 18,295 & 9529412 & .363 & .642 & .358 & 24.33 & 752 & 18,295 & 6803569 & -.520 & .301 & .301 \\
\hline 1287 & 46.72 & 128 & 5,980 & 364138 & -.951 & .171 & .171 & 62.29 & 96 & 5,980 & 273455 & -.803 & .211 & .211 \\
\hline 1288 & 35.23 & 342 & 12,048 & 1936994 & -1.916 & .028 & .028 & 52.84 & 228 & 12,048 & 1363878 & -.183 & .427 & .427 \\
\hline 1289 & 35.47 & 391 & 13,868 & 2744909 & .426 & .665 & .335 & 50.80 & 273 & 13,868 & 1975475 & 1.247 & .894 & .106 \\
\hline 1290 & 39.72 & 36 & 1,430 & 27977 & .903 & .817 & .183 & 59.58 & 24 & 1,430 & 19900 & 1.355 & .912 & .088 \\
\hline 1291 & 29.66 & 41 & 1,216 & 25170 & .108 & .543 & .457 & 43.43 & 28 & 1,216 & 16778 & -.132 & .447 & .447 \\
\hline 1292 & 18.80 & 1,046 & 19,665 & 10597381 & 1.703 & .956 & .044 & 27.01 & 728 & 19,665 & 7292171 & .876 & .809 & .191 \\
\hline 1293 & 27.71 & 615 & 17,041 & 5256567 & .135 & .554 & .446 & 39.82 & 428 & 17,041 & 3662560 & .155 & .562 & .438 \\
\hline 1294 & 32.01 & 158 & 5,058 & 378815 & -1.132 & .129 & .129 & 45.16 & 112 & 5,058 & 265261 & -1.164 & .122 & .122 \\
\hline 1295 & 19.33 & 33 & 638 & 13818 & 3.111 & .999 & .001 & 27.74 & 23 & 638 & 9895 & 2.896 & .998 & .002 \\
\hline 1296 & 14.05 & 303 & 4,257 & 648959 & .188 & .575 & .425 & 19.18 & 222 & 4,257 & 478106 & .305 & .620 & .380 \\
\hline
\end{tabular}


Table 5. Summary of interoccurrence intervals for daily precipitation thresholds of 0.75 and 1.0 inch-Continued

\begin{tabular}{|c|c|c|c|c|c|c|c|c|c|c|c|c|c|c|}
\hline \multirow[b]{2}{*}{$\begin{array}{l}\text { Seq. } \\
\text { no. }\end{array}$} & \multicolumn{7}{|c|}{ Daily precipitation threshold of 0.75 inch and greater } & \multicolumn{7}{|c|}{ Daily precipitation threshold of 1.0 inch and greater } \\
\hline & $\begin{array}{l}\text { Mean } \\
\text { inter- } \\
\text { occur- } \\
\text { rence } \\
\text { interval } \\
\text { (days) }\end{array}$ & $\begin{array}{l}\text { Total } \\
\text { no. of } \\
\text { events }\end{array}$ & $\begin{array}{l}\text { Total } \\
\text { no. of } \\
\text { days } \\
\text { avail- } \\
\text { able }\end{array}$ & $\begin{array}{l}\text { Sigma } \\
\mathrm{S}\end{array}$ & $\begin{array}{l}\text { Stan- } \\
\text { dard } \\
\text { normal } \\
\text { variate }\end{array}$ & $\begin{array}{l}\text { Non- } \\
\text { ex- } \\
\text { ceed- } \\
\text { ance } \\
\text { prob- } \\
\text { ability }\end{array}$ & $\begin{array}{c}\mathrm{p}- \\
\text { value }\end{array}$ & $\begin{array}{l}\text { Mean } \\
\text { inter- } \\
\text { occur- } \\
\text { rence } \\
\text { interval } \\
\text { (days) }\end{array}$ & $\begin{array}{l}\text { Total } \\
\text { no. of } \\
\text { events }\end{array}$ & $\begin{array}{l}\text { Total } \\
\text { no. of } \\
\text { days } \\
\text { avail- } \\
\text { able }\end{array}$ & $\begin{array}{l}\text { Sigma } \\
\text { S }\end{array}$ & $\begin{array}{c}\text { Stan- } \\
\text { dard } \\
\text { normal } \\
\text { variate }\end{array}$ & $\begin{array}{l}\text { Non- } \\
\text { ex- } \\
\text { ceed- } \\
\text { ance } \\
\text { prob- } \\
\text { ability }\end{array}$ & $\begin{array}{c}\mathrm{p}- \\
\text { value }\end{array}$ \\
\hline 1297 & 32.25 & 84 & 2,709 & 103760 & -1.398 & .081 & .081 & 41.68 & 65 & 2,709 & 81499 & -1.038 & .150 & .150 \\
\hline 1298 & 23.57 & 63 & 1,485 & 43281 & -1.028 & .152 & .152 & 33.75 & 44 & 1,485 & 30691 & -.696 & .243 & .243 \\
\hline 1299 & 15.82 & 613 & 9,696 & 3090327 & 1.710 & .956 & .044 & 23.03 & 421 & 9,696 & 2160608 & 2.083 & .981 & .019 \\
\hline 1300 & 26.00 & 7 & 182 & 897 & 1.870 & .969 & .031 & 60.67 & 3 & 182 & 477 & 2.242 & .988 & .012 \\
\hline 1301 & 23.18 & 1,199 & 27,787 & 17035794 & 1.359 & .913 & .087 & 31.72 & 876 & 27,787 & 12594492 & 1.785 & .963 & .037 \\
\hline 1302 & 26.07 & 717 & 18,695 & 6938528 & 1.636 & .949 & .051 & 37.09 & 504 & 18,695 & 4800181 & .735 & .769 & .231 \\
\hline 1303 & 17.12 & 17 & 291 & 2289 & -.533 & .297 & .297 & 20.79 & 14 & 291 & 1806 & -.735 & .231 & .231 \\
\hline 1304 & 24.11 & 151 & 3,640 & 278253 & .266 & .605 & .395 & 35.00 & 104 & 3,640 & 198098 & .823 & .795 & .205 \\
\hline 1305 & 165.37 & 137 & 22,655 & 1624674 & .951 & .829 & .171 & 359.60 & 63 & 22,655 & 776214 & 1.206 & .886 & .114 \\
\hline 1306 & 46.21 & 324 & 14,972 & 2416316 & -.118 & .453 & .453 & 66.54 & 225 & 14,972 & 1668647 & -.242 & .404 & .404 \\
\hline
\end{tabular}


Table 6. Summary of interoccurrence intervals for daily precipitation thresholds of 1.5 and 2.0 inches

[--, not available, no events that equal or exceed the threshold were observed]

\begin{tabular}{|c|c|c|c|c|c|c|c|c|c|c|c|c|c|c|}
\hline \multirow[b]{2}{*}{$\begin{array}{l}\text { Seq. } \\
\text { no. }\end{array}$} & \multicolumn{7}{|c|}{ Daily precipitation threshold of 1.5 inches and greater } & \multicolumn{7}{|c|}{ Daily precipitation threshold of 2.0 inches and greater } \\
\hline & $\begin{array}{l}\text { Mean } \\
\text { inter- } \\
\text { occur- } \\
\text { rence } \\
\text { interval } \\
\text { (days) }\end{array}$ & $\begin{array}{c}\text { Total } \\
\text { no. of } \\
\text { events }\end{array}$ & $\begin{array}{l}\text { Total } \\
\text { no. of } \\
\text { days } \\
\text { avail- } \\
\text { able }\end{array}$ & $\begin{array}{l}\text { Sigma } \\
\mathrm{S}\end{array}$ & $\begin{array}{c}\text { Stan- } \\
\text { dard } \\
\text { normal } \\
\text { variate }\end{array}$ & $\begin{array}{l}\text { Non- } \\
\text { ex- } \\
\text { ceed- } \\
\text { ance } \\
\text { prob- } \\
\text { ability }\end{array}$ & $\begin{array}{c}\mathrm{p}- \\
\text { value }\end{array}$ & $\begin{array}{l}\text { Mean } \\
\text { inter- } \\
\text { occur- } \\
\text { rence } \\
\text { interval } \\
\text { (days) }\end{array}$ & $\begin{array}{c}\text { Total } \\
\text { no. of } \\
\text { events }\end{array}$ & $\begin{array}{l}\text { Total } \\
\text { no. of } \\
\text { days } \\
\text { avail- } \\
\text { able }\end{array}$ & $\begin{array}{l}\text { Sigma } \\
\mathrm{S}\end{array}$ & $\begin{array}{c}\text { Stan- } \\
\text { dard } \\
\text { normal } \\
\text { variate }\end{array}$ & $\begin{array}{l}\text { Non- } \\
\text { ex- } \\
\text { ceed- } \\
\text { ance } \\
\text { prob- } \\
\text { ability }\end{array}$ & $\begin{array}{c}\mathrm{p}- \\
\text { value }\end{array}$ \\
\hline 1 & 216.82 & 90 & 19,514 & 792213 & -1.608 & .054 & .054 & 464.62 & 42 & 19,514 & 424095 & 0.392 & .652 & .348 \\
\hline 2 & 120.00 & 2 & 240 & 197 & -.439 & .330 & .330 & -- & -- & -- & -- & -- & -- & -- \\
\hline 3 & 135.07 & 146 & 19,720 & 1429563 & -.145 & .442 & .442 & 318.07 & 62 & 19,720 & 585942 & -.566 & .286 & .286 \\
\hline 4 & 115.29 & 73 & 8,416 & 266028 & -1.983 & .024 & .024 & 179.06 & 47 & 8,416 & 183840 & -.837 & .201 & .201 \\
\hline 5 & 191.85 & 99 & 18,993 & 1010585 & 1.291 & .902 & 098 & 345.33 & 55 & 18,993 & 574560 & 1.285 & .901 & .099 \\
\hline 6 & 135.46 & 26 & 3,522 & 44689 & -.212 & .416 & .416 & 220.13 & 16 & 3,522 & 32131 & .973 & .835 & .165 \\
\hline 7 & 81.82 & 101 & 8,264 & 375112 & -1.761 & .039 & .039 & 165.28 & 50 & 8,264 & 165644 & -2.428 & .008 & .008 \\
\hline 8 & 208.29 & 7 & 1,458 & 5869 & .688 & .754 & .246 & 729.00 & 2 & 1,458 & 1566 & .181 & .572 & .428 \\
\hline 9 & 144.65 & 20 & 2,893 & 26812 & -.567 & .285 & .285 & 289.30 & 10 & 2,893 & 13466 & -.378 & .353 & .353 \\
\hline 10 & 214.00 & 1 & 214 & 92 & -.243 & .404 & .404 & 214.00 & 1 & 214 & 92 & -.243 & .404 & .404 \\
\hline 11 & 91.34 & 67 & 6,120 & 197715 & -.505 & .307 & .307 & 136.00 & 45 & 6,120 & 135097 & -.220 & .413 & .413 \\
\hline 12 & 99.39 & 358 & 35,581 & 6726653 & 1.840 & .967 & .033 & 193.38 & 184 & 35,581 & 3349281 & .544 & .707 & .293 \\
\hline 13 & 84.77 & 177 & 15,005 & 1365025 & .644 & .740 & .260 & 180.78 & 83 & 15,005 & 637951 & .386 & .650 & .350 \\
\hline 14 & 97.61 & 307 & 29,966 & 4626092 & .174 & .569 & .431 & 161.98 & 185 & 29,966 & 2824996 & .452 & .674 & .326 \\
\hline 15 & 142.42 & 12 & 1,709 & 11744 & .872 & .808 & .192 & 189.89 & 9 & 1,709 & 9399 & 1.154 & .876 & .124 \\
\hline 16 & -- & -- & -- & -- & -- & -- & -- & -- & -- & -- & -- & -- & -- & -- \\
\hline 17 & -- & -- & -- & -- & -- & -- & -- & -- & -- & -- & -- & -- & -- & -- \\
\hline 18 & 305.99 & 90 & 27,539 & 1286438 & .626 & .734 & .266 & 764.97 & 36 & 27,539 & 576286 & 1.689 & .954 & .046 \\
\hline 19 & 52.43 & 367 & 19,240 & 3819007 & 2.711 & .997 & .003 & 95.25 & 202 & 19,240 & 2033011 & 1.137 & .872 & .128 \\
\hline 20 & 57.76 & 106 & 6,122 & 309431 & -.826 & .204 & .204 & 103.76 & 59 & 6,122 & 179499 & -.081 & .468 & .468 \\
\hline 21 & 71.34 & 74 & 5,279 & 179532 & -1.205 & .114 & .114 & 128.76 & 41 & 5,279 & 98177 & -1.029 & .152 & .152 \\
\hline 22 & 46.18 & 748 & 34,541 & 12991495 & .268 & .606 & .394 & 75.75 & 456 & 34,541 & 7716839 & -.744 & .228 & .228 \\
\hline 23 & 59.35 & 20 & 1,187 & 11844 & -.017 & .493 & .493 & 148.38 & 8 & 1,187 & 4958 & .217 & .586 & .414 \\
\hline 24 & 194.98 & 101 & 19,693 & 884153 & -1.931 & .027 & .027 & 468.88 & 42 & 19,693 & 387482 & -.708 & .240 & .240 \\
\hline 25 & 143.29 & 96 & 13,756 & 570877 & -2.298 & .011 & .011 & 264.54 & 52 & 13,756 & 300194 & -2.007 & .022 & .022 \\
\hline 26 & 39.62 & 785 & 31,099 & 12296383 & .358 & .640 & .360 & 66.17 & 470 & 31,099 & 7474794 & .856 & .804 & .196 \\
\hline 27 & 49.61 & 419 & 20,787 & 4244664 & -.897 & .185 & .185 & 88.08 & 236 & 20,787 & 2301509 & -1.642 & .050 & .050 \\
\hline 28 & 86.49 & 139 & 12,022 & 859757 & .592 & .723 & .277 & 166.97 & 72 & 12,022 & 446181 & .455 & .675 & .325 \\
\hline 29 & 233.59 & 68 & 15,884 & 492487 & -1.258 & .104 & .104 & 512.39 & 31 & 15,884 & 182711 & -2.487 & .006 & .006 \\
\hline 30 & 39.10 & 809 & 31,631 & 13831485 & 3.992 & 1.000 & 0 & 65.49 & 483 & 31,631 & 8470977 & 4.146 & 1.000 & 0 \\
\hline 31 & 60.84 & 298 & 18,129 & 2903316 & 2.237 & .987 & .013 & 107.91 & 168 & 18,129 & 1643022 & 1.772 & .962 & .038 \\
\hline 32 & 104.05 & 160 & 16,648 & 1293183 & -.636 & .262 & .262 & 198.19 & 84 & 16,648 & 677086 & -.502 & .308 & .308 \\
\hline 33 & 87.15 & 266 & 23,183 & 3217226 & 1.227 & .890 & .110 & 183.99 & 126 & 23,183 & 1511678 & .681 & .752 & .248 \\
\hline 34 & 78.74 & 46 & 3,622 & 77235 & -.856 & .196 & .196 & 164.64 & 22 & 3,622 & 42501 & .542 & .706 & .294 \\
\hline 35 & 68.61 & 150 & 10,292 & 840037 & 1.873 & .969 & .031 & 113.10 & 91 & 10,292 & 505010 & 1.296 & .902 & .098 \\
\hline 36 & 51.07 & 217 & 11,081 & 1146377 & -1.187 & .118 & .118 & 81.48 & 136 & 11,081 & 755338 & .049 & .520 & .480 \\
\hline 37 & 65.34 & 167 & 10,911 & 944793 & .829 & .796 & .204 & 106.97 & 102 & 10,911 & 584847 & .892 & .814 & .186 \\
\hline 38 & 91.84 & 244 & 22,410 & 2762838 & .285 & .612 & .388 & 176.46 & 127 & 22,410 & 1416904 & -.084 & .466 & .466 \\
\hline 39 & 61.43 & 318 & 19,535 & 3245017 & 1.382 & .916 & .084 & 135.66 & 144 & 19,535 & 1582865 & 2.606 & .995 & .005 \\
\hline 40 & 81.09 & 128 & 10,379 & 697898 & .993 & .839 & .161 & 152.63 & 68 & 10,379 & 357175 & .174 & .569 & .431 \\
\hline 41 & 54.36 & 456 & 24,787 & 5752843 & .664 & .747 & .254 & 105.93 & 234 & 24,787 & 2996780 & .884 & .811 & .189 \\
\hline 42 & 67.64 & 11 & 744 & 3770 & -.452 & .326 & .326 & 93.00 & 8 & 744 & 2434 & -.892 & .186 & .186 \\
\hline 43 & 127.82 & 251 & 32,082 & 3902049 & -.847 & .199 & .199 & 286.45 & 112 & 32,082 & 1724842 & -.732 & .232 & .232 \\
\hline 44 & 52.98 & 371 & 19,656 & 3807606 & 1.477 & .930 & .070 & 102.38 & 192 & 19,656 & 1972998 & 1.094 & .863 & .137 \\
\hline 45 & 47.37 & 400 & 18,947 & 4075934 & 2.619 & .996 & .004 & 83.84 & 226 & 18,947 & 2282998 & 1.727 & .958 & .042 \\
\hline 46 & 76.04 & 340 & 25,853 & 4437882 & .312 & .622 & .378 & 146.89 & 176 & 25,853 & 2187903 & -.880 & .189 & .189 \\
\hline 47 & 79.54 & 80 & 6,363 & 291554 & 2.254 & .988 & .012 & 159.08 & 40 & 6,363 & 147952 & 1.781 & .963 & .037 \\
\hline 48 & 72.41 & 78 & 5,648 & 251106 & 2.141 & .984 & .016 & 131.35 & 43 & 5,648 & 134130 & 1.188 & .882 & .118 \\
\hline
\end{tabular}


Table 6. Summary of interoccurrence intervals for daily precipitation thresholds of 1.5 and 2.0 inches-Continued

\begin{tabular}{|c|c|c|c|c|c|c|c|c|c|c|c|c|c|c|}
\hline \multirow[b]{2}{*}{$\begin{array}{l}\text { Seq. } \\
\text { no. }\end{array}$} & \multicolumn{7}{|c|}{ Daily precipitation threshold of 1.5 inches and greater } & \multicolumn{7}{|c|}{ Daily precipitation threshold of 2.0 inches and greater } \\
\hline & $\begin{array}{l}\text { Mean } \\
\text { inter- } \\
\text { occur- } \\
\text { rence } \\
\text { interval } \\
\text { (days) }\end{array}$ & $\begin{array}{c}\text { Total } \\
\text { no. of } \\
\text { events }\end{array}$ & $\begin{array}{l}\text { Total } \\
\text { no. of } \\
\text { days } \\
\text { avail- } \\
\text { able }\end{array}$ & $\begin{array}{l}\text { Sigma } \\
\mathrm{S}\end{array}$ & $\begin{array}{c}\text { Stan- } \\
\text { dard } \\
\text { normal } \\
\text { variate }\end{array}$ & $\begin{array}{l}\text { Non- } \\
\text { ex- } \\
\text { ceed- } \\
\text { ance } \\
\text { prob- } \\
\text { ability }\end{array}$ & $\begin{array}{c}\mathrm{p}- \\
\text { value }\end{array}$ & $\begin{array}{l}\text { Mean } \\
\text { inter- } \\
\text { occur- } \\
\text { rence } \\
\text { interval } \\
\text { (days) }\end{array}$ & $\begin{array}{c}\text { Total } \\
\text { no. of } \\
\text { events }\end{array}$ & $\begin{array}{l}\text { Total } \\
\text { no. of } \\
\text { days } \\
\text { avail- } \\
\text { able }\end{array}$ & $\begin{array}{c}\text { Sigma } \\
\mathrm{S}\end{array}$ & $\begin{array}{l}\text { Stan- } \\
\text { dard } \\
\text { normal } \\
\text { variate }\end{array}$ & $\begin{array}{l}\text { Non- } \\
\text { ex- } \\
\text { ceed- } \\
\text { ance } \\
\text { prob- } \\
\text { ability }\end{array}$ & $\begin{array}{c}\mathrm{p}- \\
\text { value }\end{array}$ \\
\hline 49 & 43.14 & 7 & 302 & 1187 & .564 & .713 & .287 & 100.67 & 3 & 302 & 770 & 2.099 & .982 & .018 \\
\hline 50 & 101.67 & 9 & 915 & 4872 & .952 & .829 & .171 & 130.71 & 7 & 915 & 3358 & .223 & .588 & .412 \\
\hline 51 & 69.03 & 264 & 18,223 & 2453903 & .567 & .715 & .285 & 127.43 & 143 & 18,223 & 1315188 & .195 & .577 & .423 \\
\hline 52 & 61.26 & 214 & 13,109 & 1359432 & -.781 & .218 & .218 & 116.01 & 113 & 13,109 & 730693 & -.248 & .402 & .402 \\
\hline 53 & 38.25 & 8 & 306 & 1448 & .897 & .815 & .185 & 61.20 & 5 & 306 & 1166 & 2.030 & .979 & .021 \\
\hline 54 & 29.00 & 3 & 87 & 96 & -.793 & .214 & .214 & 43.50 & 2 & 87 & 61 & -.732 & .232 & .232 \\
\hline 55 & 87.30 & 113 & 9,865 & 582592 & .833 & .798 & .202 & 170.09 & 58 & 9,865 & 308843 & 1.049 & .853 & .147 \\
\hline 56 & 149.06 & 16 & 2,385 & 14434 & -1.687 & .046 & .046 & 298.13 & 8 & 2,385 & 5168 & -2.245 & .012 & .012 \\
\hline 57 & 264.58 & 74 & 19,579 & 831124 & 2.195 & .986 & .014 & 652.63 & 30 & 19,579 & 304705 & .356 & .639 & .361 \\
\hline 58 & 101.33 & 6 & 608 & 978 & -1.968 & .025 & .025 & 152.00 & 4 & 608 & 483 & -2.088 & .018 & .018 \\
\hline 59 & 130.78 & 277 & 36,226 & 5043182 & .149 & .559 & .441 & 283.02 & 128 & 36,226 & 2445721 & 1.076 & .859 & .141 \\
\hline 60 & -- & -- & -- & -- & -- & -- & -- & -- & -- & -- & -- & -- & -- & -- \\
\hline 61 & 306.21 & 91 & 27,865 & 1283805 & .208 & .582 & .418 & 714.49 & 39 & 27,865 & 605650 & 1.240 & .892 & .108 \\
\hline 62 & -- & -- & -- & -- & -- & -- & -- & -- & -- & -- & -- & -- & -- & -- \\
\hline 63 & 226.56 & 9 & 2,039 & 9024 & -.086 & .466 & .466 & 339.83 & 6 & 2,039 & 4789 & -.921 & .179 & .179 \\
\hline 64 & -- & -- & -- & -- & -- & -- & -- & -- & -- & -- & -- & -- & -- & -- \\
\hline 65 & 73.49 & 101 & 7,422 & 391097 & .756 & .775 & .225 & 134.95 & 55 & 7,422 & 223773 & 1.238 & .892 & .108 \\
\hline 66 & 63.08 & 37 & 2,334 & 40624 & -.623 & .267 & .267 & 106.09 & 22 & 2,334 & 21496 & -1.322 & .093 & .093 \\
\hline 67 & 66.04 & 202 & 13,340 & 1359022 & .213 & .585 & .415 & 123.52 & 108 & 13,340 & 754510 & .853 & .803 & .197 \\
\hline 68 & 201.42 & 26 & 5,237 & 69366 & .167 & .566 & .434 & 654.63 & 8 & 5,237 & 21574 & .146 & .558 & .442 \\
\hline 69 & 351.21 & 14 & 4,917 & 35508 & .205 & .581 & .419 & 1639.00 & 3 & 4,917 & 8379 & .408 & .658 & .342 \\
\hline 70 & 69.07 & 15 & 1,036 & 11295 & 3.043 & .999 & .001 & 148.00 & 7 & 1,036 & 5263 & 2.069 & .981 & .019 \\
\hline 71 & 95.25 & 8 & 762 & 4072 & 1.646 & .950 & .050 & 381.00 & 2 & 762 & 1012 & .804 & .789 & .211 \\
\hline 72 & 111.95 & 39 & 4,366 & 89271 & .525 & .700 & .300 & 242.56 & 18 & 4,366 & 34656 & -.867 & .193 & .193 \\
\hline 73 & 84.48 & 148 & 12,503 & 951567 & .600 & .726 & .274 & 148.85 & 84 & 12,503 & 568960 & 1.325 & .907 & .093 \\
\hline 74 & 63.58 & 19 & 1,208 & 13860 & 1.568 & .942 & .058 & 80.53 & 15 & 1,208 & 10262 & .890 & .813 & .187 \\
\hline 75 & 46.41 & 505 & 23,435 & 6447518 & 3.487 & 1.000 & 0 & 78.64 & 298 & 23,435 & 3907084 & 3.556 & 1.000 & 0 \\
\hline 76 & 128.22 & 9 & 1,154 & 5488 & .295 & .616 & .384 & 230.80 & 5 & 1,154 & 3600 & .960 & .831 & .169 \\
\hline 77 & 38.63 & 410 & 15,838 & 3359253 & 1.215 & .888 & .112 & 68.56 & 231 & 15,838 & 1936535 & 1.543 & .939 & .061 \\
\hline 78 & 90.50 & 8 & 724 & 2383 & -.868 & .193 & .193 & 120.67 & 6 & 724 & 1352 & -1.602 & .055 & .055 \\
\hline 79 & 83.29 & 7 & 583 & 2755 & 1.605 & .946 & .054 & 116.60 & 5 & 583 & 2023 & 1.503 & .934 & .066 \\
\hline 80 & 39.34 & 905 & 35,599 & 16377478 & .870 & .808 & .192 & 70.91 & 502 & 35,599 & 9010886 & .328 & .629 & .371 \\
\hline 81 & 40.48 & 349 & 14,126 & 2638406 & 2.276 & .989 & .011 & 70.99 & 199 & 14,126 & 1514357 & 1.892 & .971 & .029 \\
\hline 82 & 45.58 & 278 & 12,671 & 1814451 & .872 & .808 & .192 & 79.69 & 159 & 12,671 & 1030440 & .501 & .692 & .308 \\
\hline 83 & 79.58 & 448 & 35,650 & 8424492 & 2.015 & .978 & .022 & 143.75 & 248 & 35,650 & 4553909 & .823 & .795 & .205 \\
\hline 84 & 53.51 & 146 & 7,812 & 543873 & -.969 & .166 & .166 & 95.27 & 82 & 7,812 & 300943 & -.948 & .172 & .172 \\
\hline 85 & 55.17 & 30 & 1,655 & 27894 & 1.173 & .880 & .120 & 91.94 & 18 & 1,655 & 14930 & .017 & .507 & .493 \\
\hline 86 & 76.47 & 189 & 14,452 & 1411273 & .794 & .786 & .214 & 145.98 & 99 & 14,452 & 775461 & 1.448 & .926 & .074 \\
\hline 87 & 101.08 & 12 & 1,213 & 6996 & -.233 & .408 & .408 & 134.78 & 9 & 1,213 & 5623 & .157 & .562 & .438 \\
\hline 88 & 82.92 & 155 & 12,853 & 1044511 & 1.048 & .853 & .147 & 158.68 & 81 & 12,853 & 548622 & .841 & .800 & .200 \\
\hline 89 & 81.49 & 234 & 19,069 & 2384582 & 1.823 & .966 & .034 & 168.75 & 113 & 19,069 & 1149124 & 1.226 & .890 & .110 \\
\hline 90 & 103.10 & 62 & 6,392 & 214051 & 1.094 & .863 & .137 & 188.00 & 34 & 6,392 & 112756 & .380 & .648 & .352 \\
\hline 91 & 130.64 & 95 & 12,411 & 573741 & -.452 & .326 & .326 & 253.29 & 49 & 12,411 & 255429 & -1.940 & .026 & .026 \\
\hline 92 & 75.06 & 165 & 12,385 & 1026611 & .106 & .542 & .458 & 137.61 & 90 & 12,385 & 540775 & -.488 & .313 & .313 \\
\hline 93 & 34.26 & 62 & 2,124 & 67578 & 0.359 & .640 & .360 & 60.69 & 35 & 2,124 & 35433 & -0.479 & .316 & .316 \\
\hline 94 & 609.00 & 4 & 2,436 & 3981 & -.634 & .263 & .263 & 2436.00 & 1 & 2,436 & 478 & -1.052 & .146 & .146 \\
\hline 95 & 94.00 & 11 & 1,034 & 4879 & -.816 & .207 & .207 & 258.50 & 4 & 1,034 & 1316 & -1.260 & .104 & .104 \\
\hline 96 & 155.07 & 89 & 13,801 & 571154 & -1.144 & .126 & .126 & 373.00 & 37 & 13,801 & 256011 & .029 & .511 & .489 \\
\hline
\end{tabular}


Table 6. Summary of interoccurrence intervals for daily precipitation thresholds of 1.5 and 2.0 inches—Continued

\begin{tabular}{|c|c|c|c|c|c|c|c|c|c|c|c|c|c|c|}
\hline \multirow[b]{2}{*}{$\begin{array}{c}\text { Seq. } \\
\text { no. }\end{array}$} & \multicolumn{7}{|c|}{ Daily precipitation threshold of 1.5 inches and greater } & \multicolumn{7}{|c|}{ Daily precipitation threshold of 2.0 inches and greater } \\
\hline & $\begin{array}{l}\text { Mean } \\
\text { inter- } \\
\text { occur- } \\
\text { rence } \\
\text { interval } \\
\text { (days) }\end{array}$ & $\begin{array}{c}\text { Total } \\
\text { no. of } \\
\text { events }\end{array}$ & $\begin{array}{l}\text { Total } \\
\text { no. of } \\
\text { days } \\
\text { avail- } \\
\text { able }\end{array}$ & $\begin{array}{l}\text { Sigma } \\
\mathrm{S}\end{array}$ & $\begin{array}{l}\text { Stan- } \\
\text { dard } \\
\text { normal } \\
\text { variate }\end{array}$ & $\begin{array}{l}\text { Non- } \\
\text { ex- } \\
\text { ceed- } \\
\text { ance } \\
\text { prob- } \\
\text { ability }\end{array}$ & $\begin{array}{c}\text { p- } \\
\text { value }\end{array}$ & $\begin{array}{l}\text { Mean } \\
\text { inter- } \\
\text { occur- } \\
\text { rence } \\
\text { interval } \\
\text { (days) }\end{array}$ & $\begin{array}{c}\text { Total } \\
\text { no. of } \\
\text { events }\end{array}$ & $\begin{array}{l}\text { Total } \\
\text { no. of } \\
\text { days } \\
\text { avail- } \\
\text { able }\end{array}$ & $\begin{array}{c}\text { Sigma } \\
\mathrm{S}\end{array}$ & $\begin{array}{c}\text { Stan- } \\
\text { dard } \\
\text { normal } \\
\text { variate }\end{array}$ & $\begin{array}{l}\text { Non- } \\
\text { ex- } \\
\text { ceed- } \\
\text { ance } \\
\text { prob- } \\
\text { ability }\end{array}$ & $\begin{array}{c}\mathrm{p}- \\
\text { value }\end{array}$ \\
\hline 97 & 376.33 & 3 & 1,129 & 1299 & -.699 & .242 & .242 & 564.50 & 2 & 1,129 & 952 & -.384 & .350 & .350 \\
\hline 98 & 149.95 & 127 & 19,044 & 1294319 & 1.372 & .915 & .085 & 317.40 & 60 & 19,044 & 625279 & 1.267 & .897 & .103 \\
\hline 99 & 114.00 & 1 & 114 & 98 & 1.246 & .894 & .106 & 114.00 & 1 & 114 & 98 & 1.246 & .894 & .106 \\
\hline 100 & 110.78 & 258 & 28,580 & 4065934 & 2.861 & .998 & .002 & 194.42 & 147 & 28,580 & 2287340 & 1.867 & .969 & .031 \\
\hline 101 & 92.11 & 100 & 9,211 & 437782 & -.856 & .196 & .196 & 187.98 & 49 & 9,211 & 228392 & .146 & .558 & .442 \\
\hline 102 & 49.44 & 16 & 791 & 7242 & 1.001 & .841 & .159 & 79.10 & 10 & 791 & 4554 & .830 & .797 & .203 \\
\hline 103 & 79.17 & 473 & 37,448 & 9364361 & 2.160 & .985 & .015 & 158.68 & 236 & 37,448 & 4872472 & 2.731 & .997 & .003 \\
\hline 104 & 96.55 & 65 & 6,276 & 232255 & 1.937 & .974 & .026 & 224.14 & 28 & 6,276 & 103605 & 1.642 & .950 & .050 \\
\hline 105 & 89.10 & 115 & 10,246 & 575078 & -.444 & .329 & .329 & 150.68 & 68 & 10,246 & 354177 & .238 & .594 & .406 \\
\hline 106 & 274.00 & 13 & 3,562 & 14491 & -2.336 & .010 & .010 & 712.40 & 5 & 3,562 & 5975 & -1.274 & .101 & .101 \\
\hline 107 & 63.99 & 117 & 7,487 & 460420 & .960 & .831 & .169 & 133.70 & 56 & 7,487 & 195378 & -.882 & .189 & .189 \\
\hline 108 & 68.90 & 542 & 37,342 & 10176170 & .225 & .589 & .411 & 124.47 & 300 & 37,342 & 5546614 & -.293 & .385 & .385 \\
\hline 109 & 36.21 & 398 & 14,412 & 2957601 & 1.080 & .860 & .140 & 67.66 & 213 & 14,412 & 1534407 & -.008 & .497 & .497 \\
\hline 110 & 53.24 & 652 & 34,710 & 12065743 & 2.933 & .998 & .002 & 102.69 & 338 & 34710 & 6155040 & 1.569 & .942 & .058 \\
\hline 111 & 73.77 & 17 & 1,254 & 9801 & -.575 & .283 & .283 & 125.40 & 10 & 1,254 & 4530 & -1.520 & .064 & .064 \\
\hline 112 & 192.90 & 119 & 22,955 & 1346782 & -.263 & .396 & .396 & 425.09 & 54 & 22,955 & 625039 & .108 & .543 & .457 \\
\hline 113 & 218.64 & 11 & 2,405 & 10070 & -1.371 & .085 & .085 & 267.22 & 9 & 2,405 & 7511 & -1.590 & .056 & .056 \\
\hline 114 & 154.31 & 13 & 2,006 & 13873 & .399 & .655 & .345 & 334.33 & 6 & 2,006 & 7881 & 1.313 & .905 & .095 \\
\hline 115 & 860.10 & 21 & 18,062 & 208999 & .810 & .791 & .209 & 3612.40 & 5 & 18,062 & 35245 & -.850 & .198 & .198 \\
\hline 116 & 207.32 & 28 & 5,805 & 86243 & .561 & .712 & .288 & 580.50 & 10 & 5,805 & 25446 & -.675 & .250 & .250 \\
\hline 117 & 198.08 & 97 & 19,214 & 941644 & .179 & .571 & .429 & 457.48 & 42 & 19,214 & 402568 & -.026 & .490 & .490 \\
\hline 118 & 144.43 & 7 & 1,011 & 3811 & .353 & .638 & .362 & 505.50 & 2 & 1,011 & 1133 & .296 & .616 & .384 \\
\hline 119 & 83.85 & 329 & 27,586 & 4662511 & .863 & .806 & .194 & 160.38 & 172 & 27,586 & 2403847 & .301 & .618 & .382 \\
\hline 120 & 44.70 & 429 & 19,175 & 4214370 & .884 & .812 & .188 & 84.47 & 227 & 19,175 & 2219862 & .522 & .699 & .301 \\
\hline 121 & 71.16 & 258 & 18,359 & 2766617 & 4.679 & 1.000 & 0 & 130.21 & 141 & 18,359 & 1530989 & 3.761 & 1.000 & 0 \\
\hline 122 & 157.15 & 55 & 8,643 & 248741 & .598 & .725 & .275 & 308.68 & 28 & 8,643 & 129569 & .649 & .742 & .258 \\
\hline 123 & 105.21 & 328 & 34,510 & 5719266 & .331 & .630 & .371 & 176.97 & 195 & 34,510 & 3475913 & .799 & .788 & .212 \\
\hline 124 & 87.79 & 86 & 7,550 & 321709 & -.146 & .442 & .442 & 125.83 & 60 & 7,550 & 220208 & -.373 & .355 & .355 \\
\hline 125 & 121.06 & 196 & 23,727 & 2460249 & 1.408 & .920 & .080 & 244.61 & 97 & 23,727 & 1244998 & 1.397 & .919 & .081 \\
\hline 126 & 57.44 & 63 & 3,619 & 105090 & -1.074 & .141 & .141 & 109.67 & 33 & 3,619 & 65655 & .990 & .839 & .161 \\
\hline 127 & 210.71 & 92 & 19,385 & 880485 & -.209 & .417 & .417 & 523.92 & 37 & 19,385 & 347693 & -.321 & .374 & .374 \\
\hline 128 & 44.07 & 247 & 10,885 & 1316941 & -.554 & .290 & .290 & 77.20 & 141 & 10,885 & 782592 & .407 & .658 & .342 \\
\hline 129 & 89.25 & 220 & 19,634 & 2242216 & .981 & .837 & .163 & 173.75 & 113 & 19,634 & 1097961 & -.189 & .425 & .425 \\
\hline 130 & 104.56 & 252 & 26,350 & 3402515 & .683 & .752 & .248 & 215.98 & 122 & 26,350 & 1652488 & .537 & .704 & .296 \\
\hline 131 & 95.13 & 8 & 761 & 2369 & -1.086 & .139 & .139 & 152.20 & 5 & 761 & 1579 & -.659 & .255 & .255 \\
\hline 132 & 66.23 & 212 & 14,041 & 1526447 & .646 & .741 & .259 & 126.50 & 111 & 14,041 & 790702 & .268 & .606 & .394 \\
\hline 133 & 56.02 & 648 & 36,302 & 11829598 & .254 & .600 & .400 & 106.46 & 341 & 36,302 & 6441003 & 1.300 & .903 & .097 \\
\hline 134 & 109.27 & 11 & 1,202 & 3323 & -2.857 & .002 & .002 & 300.50 & 4 & 1,202 & 1103 & -1.875 & .030 & .030 \\
\hline 135 & 121.60 & 10 & 1,216 & 6372 & .263 & .604 & .396 & 304.00 & 4 & 1,216 & 2302 & -.185 & .426 & .426 \\
\hline 136 & 83.16 & 373 & 31,019 & 6051385 & 1.540 & .938 & .062 & 159.07 & 195 & 31,019 & 3076307 & .416 & .661 & .339 \\
\hline 137 & 75.15 & 47 & 3,532 & 90765 & 1.111 & .867 & .133 & 235.47 & 15 & 3,532 & 27701 & .307 & .620 & .380 \\
\hline 138 & 84.03 & 91 & 7,647 & 412793 & 3.080 & .999 & .001 & 129.61 & 59 & 7,647 & 268256 & 2.517 & .994 & .006 \\
\hline 139 & -- & -- & -- & -- & -- & -- & -- & -- & -- & -- & -- & -- & -- & -- \\
\hline 140 & 64.50 & 20 & 1,290 & 13472 & .344 & .634 & .366 & 143.33 & 9 & 1,290 & 6197 & .351 & .637 & .363 \\
\hline 141 & 47.85 & 223 & 10,670 & 1141068 & -1.057 & .145 & .145 & 95.27 & 112 & 10,670 & 552467 & -1.382 & .084 & .084 \\
\hline 142 & 39.74 & 202 & 8,028 & 861541 & 1.540 & .938 & .062 & 75.03 & 107 & 8,028 & 462483 & 1.376 & .916 & .084 \\
\hline 143 & 43.72 & 411 & 17,968 & 3681383 & -.105 & .458 & .458 & 84.36 & 213 & 17,968 & 1840908 & -.960 & .169 & .169 \\
\hline 144 & 167.90 & 82 & 13,768 & 531733 & -.910 & .181 & .181 & 312.91 & 44 & 13,768 & 286814 & -.610 & .271 & .271 \\
\hline
\end{tabular}


Table 6. Summary of interoccurrence intervals for daily precipitation thresholds of 1.5 and 2.0 inches-Continued

\begin{tabular}{|c|c|c|c|c|c|c|c|c|c|c|c|c|c|c|}
\hline \multirow[b]{2}{*}{$\begin{array}{l}\text { Seq. } \\
\text { no. }\end{array}$} & \multicolumn{7}{|c|}{ Daily precipitation threshold of 1.5 inches and greater } & \multicolumn{7}{|c|}{ Daily precipitation threshold of 2.0 inches and greater } \\
\hline & $\begin{array}{c}\text { Mean } \\
\text { inter- } \\
\text { occur- } \\
\text { rence } \\
\text { interval } \\
\text { (days) }\end{array}$ & $\begin{array}{c}\text { Total } \\
\text { no. of } \\
\text { events }\end{array}$ & $\begin{array}{l}\text { Total } \\
\text { no. of } \\
\text { days } \\
\text { avail- } \\
\text { able }\end{array}$ & $\begin{array}{l}\text { Sigma } \\
\mathrm{S}\end{array}$ & $\begin{array}{c}\text { Stan- } \\
\text { dard } \\
\text { normal } \\
\text { variate }\end{array}$ & $\begin{array}{l}\text { Non- } \\
\text { ex- } \\
\text { ceed- } \\
\text { ance } \\
\text { prob- } \\
\text { ability }\end{array}$ & $\begin{array}{c}\text { p- } \\
\text { value }\end{array}$ & $\begin{array}{l}\text { Mean } \\
\text { inter- } \\
\text { occur- } \\
\text { rence } \\
\text { interval } \\
\text { (days) }\end{array}$ & $\begin{array}{c}\text { Total } \\
\text { no. of } \\
\text { events }\end{array}$ & $\begin{array}{c}\text { Total } \\
\text { no. of } \\
\text { days } \\
\text { avail- } \\
\text { able }\end{array}$ & $\begin{array}{c}\text { Sigma } \\
\mathrm{S}\end{array}$ & $\begin{array}{l}\text { Stan- } \\
\text { dard } \\
\text { normal } \\
\text { variate }\end{array}$ & $\begin{array}{l}\text { Non- } \\
\text { ex- } \\
\text { ceed- } \\
\text { ance } \\
\text { prob- } \\
\text { ability }\end{array}$ & $\begin{array}{c}p- \\
\text { value }\end{array}$ \\
\hline 145 & 172.97 & 101 & 17,470 & 884271 & .040 & .516 & .484 & 356.53 & 49 & 17,470 & 446322 & .519 & .698 & .302 \\
\hline 146 & 101.92 & 278 & 28,335 & 4033791 & .698 & .757 & .243 & 168.66 & 168 & 28,335 & 2566251 & 1.755 & .960 & .040 \\
\hline 147 & 93.42 & 208 & 19,432 & 2306508 & 3.530 & 1.000 & 0 & 168.97 & 115 & 19,432 & 1244734 & 2.118 & .983 & .017 \\
\hline 148 & 253.40 & 5 & 1,267 & 3291 & .151 & .560 & .440 & 316.75 & 4 & 1,267 & 2764 & .314 & .623 & .377 \\
\hline 149 & 35.67 & 6 & 214 & 832 & 1.256 & .895 & .105 & 71.33 & 3 & 214 & 498 & 1.654 & .951 & .049 \\
\hline 150 & 96.33 & 12 & 1,156 & 6196 & -.640 & .261 & .261 & 192.67 & 6 & 1,156 & 3459 & -.011 & .496 & .496 \\
\hline 151 & 104.73 & 67 & 7,017 & 221196 & -.837 & .201 & .201 & 212.64 & 33 & 7,017 & 117640 & .160 & .564 & .436 \\
\hline 152 & 416.11 & 19 & 7,906 & 74062 & -.105 & .458 & .458 & 878.44 & 9 & 7,906 & 30212 & -.784 & .217 & .217 \\
\hline 153 & 53.45 & 271 & 14,486 & 2115933 & 2.224 & .987 & .013 & 94.07 & 154 & 14,486 & 1226952 & 2.149 & .984 & .016 \\
\hline 154 & 209.51 & 51 & 10,685 & 230446 & -1.908 & .028 & .028 & 508.81 & 21 & 10,685 & 75291 & -2.611 & .005 & .005 \\
\hline 155 & 69.78 & 316 & 22,051 & 3611690 & 1.128 & .870 & .130 & 118.55 & 186 & 22,051 & 2194560 & 1.657 & .951 & .049 \\
\hline 156 & 358.76 & 41 & 14,709 & 266258 & -1.298 & .097 & .097 & 668.59 & 22 & 14,709 & 140790 & -1.055 & .146 & .146 \\
\hline 157 & -- & -- & -- & -- & -- & -- & -- & -- & -- & -- & -- & -- & -- & -- \\
\hline 158 & 107.21 & 19 & 2,037 & 16367 & -1.164 & .122 & .122 & 226.33 & 9 & 2,037 & 9122 & -.025 & .490 & .490 \\
\hline 159 & 83.79 & 210 & 17,595 & 1860284 & .174 & .569 & .431 & 162.92 & 108 & 17,595 & 937839 & -.233 & .408 & .408 \\
\hline 160 & 74.76 & 80 & 5,981 & 243381 & .268 & .606 & .394 & 115.02 & 52 & 5,981 & 149917 & -.449 & .327 & .327 \\
\hline 161 & 82.72 & 163 & 13,484 & 1134662 & .719 & .764 & .236 & 175.12 & 77 & 13,484 & 516833 & -.067 & .473 & .473 \\
\hline 162 & 79.19 & 124 & 9,819 & 655636 & 1.485 & .931 & .069 & 138.30 & 71 & 9,819 & 397825 & 2.062 & .980 & .020 \\
\hline 163 & 85.42 & 403 & 34,426 & 7216573 & 1.402 & .920 & .080 & 162.39 & 212 & 34,426 & 3639893 & -.064 & .474 & .474 \\
\hline 164 & 86.33 & 6 & 518 & 1846 & .797 & .787 & .213 & 172.67 & 3 & 518 & 1149 & 1.436 & .925 & .075 \\
\hline 165 & 237.00 & 5 & 1,185 & 1939 & -1.338 & .090 & .090 & 592.50 & 2 & 1,185 & 1388 & .420 & .663 & .337 \\
\hline 166 & 84.41 & 75 & 6,331 & 245640 & .520 & .698 & .302 & 143.89 & 44 & 6,331 & 138682 & -.050 & .480 & .480 \\
\hline 167 & 172.43 & 7 & 1,207 & 3248 & -1.059 & .145 & .145 & 603.50 & 2 & 1,207 & 1329 & .248 & .598 & .402 \\
\hline 168 & 60.24 & 234 & 14,097 & 1631326 & -.290 & .386 & .386 & 120.49 & 117 & 14,097 & 824113 & -.013 & .495 & .495 \\
\hline 169 & 50.67 & 24 & 1,216 & 14081 & -.297 & .383 & .383 & 93.54 & 13 & 1,216 & 8938 & .817 & .793 & .207 \\
\hline 170 & 132.26 & 46 & 6,084 & 151774 & .994 & .840 & .160 & 304.20 & 20 & 6,084 & 70566 & 1.238 & .892 & .108 \\
\hline 171 & 111.26 & 74 & 8,233 & 299377 & -.257 & .399 & .399 & 216.66 & 38 & 8,233 & 148426 & -.546 & .293 & .293 \\
\hline 172 & 70.01 & 469 & 32,834 & 7681410 & -.089 & .465 & .465 & 152.72 & 215 & 32,834 & 3561249 & .227 & .590 & .410 \\
\hline 173 & 94.19 & 21 & 1,978 & 22151 & .528 & .701 & .299 & 164.83 & 12 & 1,978 & 14069 & 1.113 & .867 & .133 \\
\hline 174 & 137.33 & 45 & 6,180 & 148485 & .788 & .785 & .215 & 280.91 & 22 & 6,180 & 76544 & 1.024 & .847 & .153 \\
\hline 175 & 57.88 & 24 & 1,389 & 19166 & 1.272 & .898 & .102 & 77.17 & 18 & 1,389 & 13519 & .598 & .725 & .275 \\
\hline 176 & 89.50 & 224 & 20,049 & 2381594 & 1.571 & .942 & .058 & 151.89 & 132 & 20,049 & 1433382 & 1.657 & .951 & .049 \\
\hline 177 & 153.71 & 209 & 32,125 & 3233006 & -.925 & .177 & .177 & 356.94 & 90 & 32,125 & 1409876 & -.406 & .342 & .342 \\
\hline 178 & 82.75 & 4 & 331 & 604 & -.304 & .381 & .381 & 331.00 & 1 & 331 & 214 & .508 & .694 & .306 \\
\hline 179 & 512.11 & 38 & 19,460 & 441776 & 2.080 & .981 & .019 & 1946.00 & 10 & 19,460 & 130513 & 1.870 & .969 & .031 \\
\hline 180 & 49.92 & 334 & 16,672 & 2861321 & .877 & .810 & .190 & 91.10 & 183 & 16,672 & 1653730 & 1.970 & .976 & .024 \\
\hline 181 & 196.10 & 144 & 28,238 & 2012657 & -.209 & .417 & .417 & 397.72 & 71 & 28,238 & 989506 & -.188 & .425 & .425 \\
\hline 182 & 72.56 & 192 & 13,932 & 1336052 & -.026 & .490 & .490 & 131.43 & 106 & 13,932 & 726243 & -.294 & .385 & .385 \\
\hline 183 & 66.69 & 29 & 1,934 & 34301 & 2.082 & .981 & .019 & 107.44 & 18 & 1,934 & 18201 & .336 & .631 & .369 \\
\hline 184 & 39.32 & 22 & 865 & 10283 & .656 & .744 & .256 & 54.06 & 16 & 865 & 6280 & -.641 & .261 & .261 \\
\hline 185 & 314.86 & 7 & 2,204 & 7057 & -.390 & .348 & .348 & 1102.00 & 2 & 2,204 & 2092 & -.125 & .450 & .450 \\
\hline 186 & 78.79 & 29 & 2,285 & 30996 & -.602 & .274 & .274 & 142.81 & 16 & 2,285 & 19678 & .530 & .702 & .298 \\
\hline 187 & -- & -- & -- & -- & -- & -- & -- & -- & -- & -- & -- & -- & -- & -- \\
\hline 188 & 99.46 & 149 & 14,820 & 1151185 & .902 & .816 & .184 & 208.73 & 71 & 14,820 & 571104 & 1.248 & .894 & .106 \\
\hline 189 & 119.53 & 221 & 26,415 & 2811276 & -0.949 & .171 & .171 & 220.13 & 120 & 26,415 & 1406427 & -2.137 & .016 & .016 \\
\hline 190 & 62.41 & 303 & 18,911 & 2998027 & 1.400 & .919 & .081 & 116.02 & 163 & 18,911 & 1675817 & 1.931 & .973 & .027 \\
\hline 191 & 107.72 & 119 & 12,819 & 816950 & 1.343 & .910 & .090 & 210.15 & 61 & 12,819 & 378287 & -.439 & .330 & .330 \\
\hline 192 & 46.02 & 395 & 18,176 & 3977036 & 3.714 & 1.000 & 0 & 83.38 & 218 & 18,176 & 2182206 & 2.595 & .995 & .005 \\
\hline
\end{tabular}


Table 6. Summary of interoccurrence intervals for daily precipitation thresholds of 1.5 and 2.0 inches—Continued

\begin{tabular}{|c|c|c|c|c|c|c|c|c|c|c|c|c|c|c|}
\hline \multirow[b]{2}{*}{$\begin{array}{l}\text { Seq. } \\
\text { no. }\end{array}$} & \multicolumn{7}{|c|}{ Daily precipitation threshold of 1.5 inches and greater } & \multicolumn{7}{|c|}{ Daily precipitation threshold of 2.0 inches and greater } \\
\hline & $\begin{array}{l}\text { Mean } \\
\text { inter- } \\
\text { occur- } \\
\text { rence } \\
\text { interval } \\
\text { (days) }\end{array}$ & $\begin{array}{c}\text { Total } \\
\text { no. of } \\
\text { events }\end{array}$ & $\begin{array}{l}\text { Total } \\
\text { no. of } \\
\text { days } \\
\text { avail- } \\
\text { able }\end{array}$ & $\begin{array}{l}\text { Sigma } \\
\mathrm{S}\end{array}$ & $\begin{array}{c}\text { Stan- } \\
\text { dard } \\
\text { normal } \\
\text { variate }\end{array}$ & $\begin{array}{l}\text { Non- } \\
\text { ex- } \\
\text { ceed- } \\
\text { ance } \\
\text { prob- } \\
\text { ability }\end{array}$ & $\begin{array}{c}\mathrm{p}- \\
\text { value }\end{array}$ & $\begin{array}{l}\text { Mean } \\
\text { inter- } \\
\text { occur- } \\
\text { rence } \\
\text { interval } \\
\text { (days) }\end{array}$ & $\begin{array}{l}\text { Total } \\
\text { no. of } \\
\text { events }\end{array}$ & $\begin{array}{l}\text { Total } \\
\text { no. of } \\
\text { days } \\
\text { avail- } \\
\text { able }\end{array}$ & $\begin{array}{l}\text { Sigma } \\
\mathrm{S}\end{array}$ & $\begin{array}{c}\text { Stan- } \\
\text { dard } \\
\text { normal } \\
\text { variate }\end{array}$ & $\begin{array}{l}\text { Non- } \\
\text { ex- } \\
\text { ceed- } \\
\text { ance } \\
\text { prob- } \\
\text { ability }\end{array}$ & $\begin{array}{c}\mathrm{p}- \\
\text { value }\end{array}$ \\
\hline 193 & 196.19 & 62 & 12,164 & 460696 & 3.024 & .999 & .001 & 380.13 & 32 & 12,164 & 250645 & 2.820 & .998 & .002 \\
\hline 194 & 88.07 & 73 & 6,429 & 227554 & -.448 & .327 & .327 & 149.51 & 43 & 6,429 & 132158 & -.498 & .309 & .309 \\
\hline 195 & 729.69 & 13 & 9,486 & 43982 & -1.790 & .037 & .037 & 3162.00 & 3 & 9,486 & 6958 & -1.533 & .063 & .063 \\
\hline 196 & 112.50 & 128 & 14,400 & 924235 & .056 & .522 & .478 & 202.82 & 71 & 14,400 & 526458 & .436 & .668 & .332 \\
\hline 197 & 68.60 & 118 & 8,095 & 521134 & 1.715 & .957 & .043 & 132.71 & 61 & 8,095 & 278016 & 1.705 & .956 & .044 \\
\hline 198 & 63.97 & 198 & 12,665 & 1334641 & 1.571 & .942 & .058 & 118.36 & 107 & 12,665 & 725172 & 1.259 & .896 & .104 \\
\hline 199 & 41.81 & 546 & 22,827 & 6309060 & .502 & .692 & .308 & 76.86 & 297 & 22,827 & 3423393 & .296 & .616 & .384 \\
\hline 200 & 93.52 & 151 & 14,121 & 1036128 & -.599 & .275 & .275 & 172.21 & 82 & 14,121 & 540614 & -1.039 & .150 & .150 \\
\hline 201 & 55.54 & 413 & 22,939 & 4793769 & .423 & .664 & .336 & 114.70 & 200 & 22,939 & 2381189 & .932 & .824 & .176 \\
\hline 202 & 97.20 & 5 & 486 & 1753 & 1.715 & .957 & .043 & 486.00 & 1 & 486 & 383 & .998 & .841 & .159 \\
\hline 203 & 82.88 & 170 & 14,090 & 1242751 & .850 & .802 & .198 & 151.51 & 93 & 14,090 & 689784 & .882 & .811 & .189 \\
\hline 204 & 34.70 & 40 & 1,388 & 26644 & -.440 & .330 & .330 & 51.41 & 27 & 1,388 & 16387 & -1.129 & .129 & .129 \\
\hline 205 & 192.00 & 2 & 384 & 333 & -.325 & .372 & .372 & 384.00 & 1 & 384 & 170 & -.199 & .421 & .421 \\
\hline 206 & 243.75 & 12 & 2,925 & 16602 & -.324 & .373 & .373 & 585.00 & 5 & 2,925 & 3731 & -1.897 & .029 & .029 \\
\hline 207 & 103.20 & 5 & 516 & 1054 & -.709 & .239 & .239 & 516.00 & 1 & 516 & 446 & 1.262 & .897 & .104 \\
\hline 208 & 161.55 & 51 & 8,239 & 263064 & 3.119 & .999 & .001 & 411.95 & 20 & 8,239 & 113836 & 2.956 & .998 & .002 \\
\hline 209 & 69.51 & 220 & 15,291 & 1733555 & .787 & .784 & .216 & 119.46 & 128 & 15,291 & 1023950 & .908 & .818 & .182 \\
\hline 210 & 116.00 & 37 & 4,292 & 93154 & 1.825 & .966 & .034 & 252.47 & 17 & 4,292 & 51140 & 2.869 & .998 & .002 \\
\hline 211 & 113.33 & 18 & 2,040 & 16342 & -.808 & .210 & .210 & 291.43 & 7 & 2,040 & 6231 & -.583 & .280 & .280 \\
\hline 212 & 94.93 & 151 & 14,334 & 1092873 & .210 & .583 & .417 & 174.81 & 82 & 14,334 & 567247 & -.546 & .293 & .293 \\
\hline 213 & 58.76 & 54 & 3,173 & 91861 & .920 & .821 & .179 & 105.77 & 30 & 3,173 & 49067 & .293 & .615 & .385 \\
\hline 214 & 105.64 & 11 & 1,162 & 5146 & -1.119 & .132 & .132 & 581.00 & 2 & 1,162 & 1294 & .278 & .610 & .390 \\
\hline 215 & 133.50 & 6 & 801 & 2557 & .272 & .607 & .393 & 200.25 & 4 & 801 & 1444 & -.342 & .366 & .366 \\
\hline 216 & 168.56 & 9 & 1,517 & 8134 & .995 & .840 & .160 & 379.25 & 4 & 1,517 & 3399 & .417 & .662 & .338 \\
\hline 217 & 114.76 & 132 & 15,148 & 930418 & -1.380 & .084 & .084 & 201.97 & 75 & 15,148 & 545722 & -.590 & .278 & .278 \\
\hline 218 & 179.58 & 103 & 18,497 & 1010286 & 1.065 & .856 & .144 & 330.30 & 56 & 18,497 & 575818 & 1.449 & .926 & .074 \\
\hline 219 & -- & -- & -- & -- & -- & -- & -- & -- & -- & -- & -- & -- & -- & -- \\
\hline 220 & 119.52 & 205 & 24,501 & 2325259 & -1.838 & .033 & .033 & 237.87 & 103 & 24,501 & 1149374 & -1.566 & .059 & .059 \\
\hline 221 & 32.76 & 144 & 4,717 & 357775 & 1.111 & .867 & .133 & 56.83 & 83 & 4,717 & 193573 & -.176 & .430 & .430 \\
\hline 222 & 235.39 & 84 & 19,773 & 904969 & 1.424 & .923 & .077 & 599.18 & 33 & 19,773 & 376914 & 1.545 & .939 & .061 \\
\hline 223 & 110.32 & 59 & 6,509 & 196801 & .332 & .630 & .370 & 241.07 & 27 & 6,509 & 89039 & .120 & .548 & .452 \\
\hline 224 & 174.77 & 35 & 6,117 & 96752 & -.986 & .162 & .162 & 339.83 & 18 & 6,117 & 52218 & -.378 & .353 & .353 \\
\hline 225 & 62.95 & 188 & 11,834 & 1126206 & .295 & .616 & .384 & 103.81 & 114 & 11,834 & 622628 & -1.423 & .077 & .077 \\
\hline 226 & 135.96 & 246 & 33,447 & 4067333 & -.308 & .379 & .379 & 274.16 & 122 & 33,447 & 1980127 & -.564 & .286 & .286 \\
\hline 227 & 52.17 & 23 & 1,200 & 15839 & 1.227 & .890 & .110 & 200.00 & 6 & 1,200 & 4026 & .502 & .692 & .308 \\
\hline 228 & 46.95 & 725 & 34,037 & 12570821 & .879 & .810 & .190 & 95.34 & 357 & 34,037 & 6385917 & 1.672 & .953 & .047 \\
\hline 229 & 82.07 & 111 & 9,110 & 571949 & 2.395 & .992 & .008 & 149.34 & 61 & 9,110 & 309699 & 1.550 & .939 & .061 \\
\hline 230 & 140.38 & 228 & 32,007 & 3653463 & .033 & .513 & .487 & 313.79 & 102 & 32,007 & 1642097 & .104 & .542 & .458 \\
\hline 231 & 68.94 & 480 & 33,090 & 8003597 & .296 & .616 & .384 & 140.81 & 235 & 33,090 & 4042118 & 1.052 & .854 & .146 \\
\hline 232 & 64.03 & 39 & 2,497 & 47875 & -.181 & .428 & .428 & 104.04 & 24 & 2,497 & 31688 & .488 & .687 & .313 \\
\hline 233 & 40.00 & 432 & 17,281 & 3772048 & .380 & .648 & .352 & 69.68 & 248 & 17,281 & 2234656 & 1.169 & .879 & .121 \\
\hline 234 & 115.00 & 9 & 1,035 & 2358 & -2.565 & .005 & .005 & 258.75 & 4 & 1,035 & 1215 & -1.431 & .076 & .076 \\
\hline 235 & 73.50 & 318 & 23,374 & 3457580 & -2.152 & .016 & .016 & 130.58 & 179 & 23,374 & 1999733 & -1.022 & .154 & .154 \\
\hline 236 & 100.75 & 57 & 5,743 & 153958 & -.776 & .219 & .219 & 147.26 & 39 & 5,743 & 104008 & -.771 & .220 & .220 \\
\hline 237 & 46.22 & 402 & 18,579 & 3790615 & 0.523 & .699 & .301 & 88.47 & 210 & 18,579 & 1983693 & 0.423 & .664 & .336 \\
\hline 238 & 137.39 & 31 & 4,259 & 72102 & .889 & .813 & .187 & 250.53 & 17 & 4,259 & 40618 & .871 & .808 & .192 \\
\hline 239 & 45.73 & 379 & 17,330 & 3450746 & 1.712 & .957 & .043 & 84.13 & 206 & 17,330 & 1894765 & 1.529 & .937 & .063 \\
\hline 240 & 362.42 & 36 & 13,047 & 259898 & 1.109 & .866 & .134 & 931.93 & 14 & 13,047 & 105299 & .991 & .839 & .161 \\
\hline
\end{tabular}


Table 6. Summary of interoccurrence intervals for daily precipitation thresholds of 1.5 and 2.0 inches-Continued

\begin{tabular}{|c|c|c|c|c|c|c|c|c|c|c|c|c|c|c|}
\hline \multirow[b]{2}{*}{$\begin{array}{l}\text { Seq. } \\
\text { no. }\end{array}$} & \multicolumn{7}{|c|}{ Daily precipitation threshold of 1.5 inches and greater } & \multicolumn{7}{|c|}{ Daily precipitation threshold of 2.0 inches and greater } \\
\hline & $\begin{array}{l}\text { Mean } \\
\text { inter- } \\
\text { occur- } \\
\text { rence } \\
\text { interval } \\
\text { (days) }\end{array}$ & $\begin{array}{c}\text { Total } \\
\text { no. of } \\
\text { events }\end{array}$ & $\begin{array}{l}\text { Total } \\
\text { no. of } \\
\text { days } \\
\text { avail- } \\
\text { able }\end{array}$ & $\begin{array}{l}\text { Sigma } \\
\mathrm{S}\end{array}$ & $\begin{array}{c}\text { Stan- } \\
\text { dard } \\
\text { normal } \\
\text { variate }\end{array}$ & $\begin{array}{l}\text { Non- } \\
\text { ex- } \\
\text { ceed- } \\
\text { ance } \\
\text { prob- } \\
\text { ability }\end{array}$ & $\begin{array}{c}\mathrm{p}- \\
\text { value }\end{array}$ & $\begin{array}{l}\text { Mean } \\
\text { inter- } \\
\text { occur- } \\
\text { rence } \\
\text { interval } \\
\text { (days) }\end{array}$ & $\begin{array}{c}\text { Total } \\
\text { no. of } \\
\text { events }\end{array}$ & $\begin{array}{c}\text { Total } \\
\text { no. of } \\
\text { days } \\
\text { avail- } \\
\text { able }\end{array}$ & $\begin{array}{l}\text { Sigma } \\
\mathbf{S}\end{array}$ & $\begin{array}{l}\text { Stan- } \\
\text { dard } \\
\text { normal } \\
\text { variate }\end{array}$ & $\begin{array}{l}\text { Non- } \\
\text { ex- } \\
\text { ceed- } \\
\text { ance } \\
\text { prob- } \\
\text { ability }\end{array}$ & $\begin{array}{c}p- \\
\text { value }\end{array}$ \\
\hline 241 & 94.22 & 397 & 37,406 & 7654302 & 1.065 & .857 & .143 & 173.98 & 215 & 37,406 & 4164637 & .906 & .818 & .182 \\
\hline 242 & 59.38 & 295 & 17,517 & 2515618 & -.785 & .216 & .216 & 114.49 & 153 & 17,517 & 1304529 & -.568 & .285 & .285 \\
\hline 243 & 63.05 & 328 & 20,679 & 3290229 & -.935 & .175 & .175 & 109.41 & 189 & 20,679 & 1975008 & .254 & .600 & .400 \\
\hline 244 & 30.67 & 3 & 92 & 103 & -.761 & .223 & .223 & 46.00 & 2 & 92 & 99 & .186 & .574 & .426 \\
\hline 245 & 139.59 & 189 & 26,383 & 2243801 & -2.382 & .009 & .009 & 263.83 & 100 & 26,383 & 1128154 & -2.508 & .006 & .006 \\
\hline 246 & 51.36 & 390 & 20,030 & 4160527 & 2.230 & .987 & .013 & 95.84 & 209 & 20,030 & 2203750 & 1.323 & .907 & .093 \\
\hline 247 & 93.07 & 328 & 30,526 & 5136876 & .818 & .793 & .207 & 195.68 & 156 & 30,526 & 2644854 & 2.397 & .992 & .008 \\
\hline 248 & 82.25 & 24 & 1,974 & 23549 & -.050 & .480 & .480 & 131.60 & 15 & 1,974 & 15299 & .224 & .589 & .411 \\
\hline 249 & 47.11 & 64 & 3,015 & 102400 & .850 & .802 & .198 & 79.34 & 38 & 3,015 & 64925 & 1.424 & .923 & .077 \\
\hline 250 & 181.20 & 41 & 7,429 & 140610 & -.851 & .197 & .197 & 323.00 & 23 & 7,429 & 76102 & -.907 & .182 & .182 \\
\hline 251 & -- & -- & -- & -- & -- & -- & -- & -- & -- & -- & -- & -- & -- & -- \\
\hline 252 & 145.19 & 16 & 2,323 & 20037 & .542 & .706 & .294 & 178.69 & 13 & 2,323 & 14378 & -.298 & .383 & .383 \\
\hline 253 & 107.69 & 13 & 1,400 & 10177 & .739 & .770 & .230 & 233.33 & 6 & 1,400 & 5298 & 1.109 & .866 & .134 \\
\hline 254 & 237.62 & 82 & 19,485 & 770239 & -.562 & .287 & .287 & 573.09 & 34 & 19,485 & 323841 & -.226 & .411 & .411 \\
\hline 255 & 47.85 & 404 & 19,333 & 4213333 & 2.746 & .997 & .003 & 93.40 & 207 & 19,333 & 2228445 & 2.833 & .998 & .002 \\
\hline 256 & 48.31 & 377 & 18,213 & 3735119 & 2.958 & .998 & .002 & 92.45 & 197 & 18,213 & 1935059 & 1.912 & .972 & .028 \\
\hline 257 & -- & -- & -- & -- & -- & -- & -- & -- & -- & -- & -- & -- & -- & -- \\
\hline 258 & 190.83 & 102 & 19,465 & 1142955 & 2.647 & .996 & .004 & 405.52 & 48 & 19,465 & 582661 & 2.967 & .999 & .002 \\
\hline 259 & 86.90 & 52 & 4,519 & 101349 & -1.716 & .043 & .043 & 205.41 & 22 & 4,519 & 40331 & -1.533 & .063 & .063 \\
\hline 260 & 69.60 & 317 & 22,062 & 3474463 & -.197 & .422 & .422 & 135.35 & 163 & 22,062 & 1794264 & -.047 & .481 & .481 \\
\hline 261 & 112.39 & 13 & 1,461 & 10084 & .386 & .650 & .350 & 146.10 & 10 & 1,461 & 7138 & -.125 & .450 & .450 \\
\hline 262 & 65.17 & 103 & 6,712 & 342087 & -.182 & .428 & .428 & 156.09 & 43 & 6,712 & 141413 & -.228 & .410 & .410 \\
\hline 263 & 579.31 & 32 & 18,538 & 347466 & 1.680 & .954 & .046 & 1853.80 & 10 & 18,538 & 121165 & 1.683 & .954 & .046 \\
\hline 264 & 86.87 & 137 & 11,901 & 914976 & 2.481 & .993 & .007 & 135.24 & 88 & 11,901 & 585043 & 1.905 & .972 & .028 \\
\hline 265 & 76.15 & 259 & 19,723 & 2699990 & 1.592 & .944 & .056 & 118.81 & 166 & 19,723 & 1726704 & 1.223 & .889 & .111 \\
\hline 266 & 30.37 & 109 & 3,310 & 182170 & .178 & .571 & .429 & 47.97 & 69 & 3,310 & 122696 & 1.071 & .858 & .142 \\
\hline 267 & 63.00 & 578 & 36,415 & 10589408 & .259 & .602 & .398 & 124.71 & 292 & 36,415 & 5428619 & .624 & .734 & .266 \\
\hline 268 & 54.52 & 67 & 3,653 & 123346 & .112 & .545 & .455 & 91.33 & 40 & 3,653 & 80312 & 1.087 & .861 & .139 \\
\hline 269 & 177.25 & 4 & 709 & 1986 & 1.388 & .917 & .083 & 709.00 & 1 & 709 & 556 & .985 & .838 & .163 \\
\hline 270 & 74.50 & 190 & 14,154 & 1356631 & .213 & .584 & .416 & 138.77 & 102 & 14,154 & 718772 & -.075 & .470 & .470 \\
\hline 271 & -- & -- & -- & -- & -- & -- & -- & -- & -- & -- & -- & -- & -- & -- \\
\hline 272 & -- & -- & -- & -- & -- & -- & -- & -- & -- & -- & -- & -- & -- & -- \\
\hline 273 & 111.08 & 286 & 31,770 & 4558269 & .098 & .539 & .461 & 190.24 & 167 & 31,770 & 2670299 & .148 & .559 & .441 \\
\hline 274 & 117.35 & 97 & 11,383 & 654439 & 3.163 & .999 & .001 & 223.20 & 51 & 11,383 & 350619 & 2.572 & .995 & .005 \\
\hline 275 & 80.10 & 30 & 2,403 & 34232 & -.477 & .317 & .317 & 184.85 & 13 & 2,403 & 11431 & -1.675 & .047 & .047 \\
\hline 276 & 146.75 & 44 & 6,457 & 143329 & .103 & .541 & .459 & 379.82 & 17 & 6,457 & 48775 & -.795 & .213 & .213 \\
\hline 277 & 70.06 & 16 & 1,121 & 9677 & .548 & .708 & .292 & 124.56 & 9 & 1,121 & 5400 & .366 & .643 & .357 \\
\hline 278 & 66.15 & 179 & 11,840 & 1088955 & .640 & .739 & .261 & 122.06 & 97 & 11,840 & 615175 & 1.216 & .888 & .112 \\
\hline 279 & 731.00 & 1 & 731 & 297 & -.325 & .373 & .373 & -- & -- & -- & -- & -- & -- & -- \\
\hline 280 & 246.05 & 67 & 16,485 & 557749 & .141 & .556 & .444 & 433.82 & 38 & 16,485 & 350093 & 1.257 & .896 & .104 \\
\hline 281 & 98.11 & 45 & 4,415 & 106802 & .873 & .809 & .191 & 191.96 & 23 & 4,415 & 49946 & -.135 & .446 & .446 \\
\hline 282 & 60.96 & 22 & 1,341 & 16632 & 1.036 & .850 & .150 & 149.00 & 9 & 1,341 & 6487 & .390 & .652 & .348 \\
\hline 283 & 83.33 & 233 & 19,415 & 2234035 & -.325 & .373 & .373 & 176.50 & 110 & 19,415 & 1029971 & -.644 & .260 & .260 \\
\hline 284 & 107.14 & 89 & 9,535 & 489805 & 2.522 & .994 & .006 & 179.91 & 53 & 9,535 & 286762 & 1.701 & .956 & .044 \\
\hline 285 & 50.76 & 641 & 32,536 & 10037785 & -1.640 & .051 & .051 & 91.65 & 355 & 32,536 & 5544008 & -1.306 & .096 & .096 \\
\hline 286 & 148.29 & 252 & 37,369 & 4483974 & -1.311 & .095 & .095 & 278.87 & 134 & 37,369 & 2378181 & -1.005 & .157 & .157 \\
\hline 287 & 130.43 & 14 & 1,826 & 12025 & -.384 & .351 & .351 & 182.60 & 10 & 1,826 & 8214 & -.550 & .291 & .291 \\
\hline 288 & 89.07 & 148 & 13,182 & 936382 & -.844 & .199 & .199 & 183.08 & 72 & 13,182 & 453364 & -.656 & .256 & .256 \\
\hline
\end{tabular}


Table 6. Summary of interoccurrence intervals for daily precipitation thresholds of 1.5 and 2.0 inches—Continued

\begin{tabular}{|c|c|c|c|c|c|c|c|c|c|c|c|c|c|c|}
\hline \multirow[b]{2}{*}{$\begin{array}{l}\text { Seq. } \\
\text { no. }\end{array}$} & \multicolumn{7}{|c|}{ Daily precipitation threshold of 1.5 inches and greater } & \multicolumn{7}{|c|}{ Daily precipitation threshold of 2.0 inches and greater } \\
\hline & $\begin{array}{l}\text { Mean } \\
\text { inter- } \\
\text { occur- } \\
\text { rence } \\
\text { interval } \\
\text { (days) }\end{array}$ & $\begin{array}{c}\text { Total } \\
\text { no. of } \\
\text { events }\end{array}$ & $\begin{array}{l}\text { Total } \\
\text { no. of } \\
\text { days } \\
\text { avail- } \\
\text { able }\end{array}$ & $\begin{array}{l}\text { Sigma } \\
\mathbf{S}\end{array}$ & $\begin{array}{l}\text { Stan- } \\
\text { dard } \\
\text { normal } \\
\text { variate }\end{array}$ & $\begin{array}{l}\text { Non- } \\
\text { ex- } \\
\text { ceed- } \\
\text { ance } \\
\text { prob- } \\
\text { ability }\end{array}$ & $\begin{array}{c}\text { p- } \\
\text { value }\end{array}$ & $\begin{array}{l}\text { Mean } \\
\text { inter- } \\
\text { occur- } \\
\text { rence } \\
\text { interval } \\
\text { (days) }\end{array}$ & $\begin{array}{c}\text { Total } \\
\text { no. of } \\
\text { events }\end{array}$ & $\begin{array}{l}\text { Total } \\
\text { no. of } \\
\text { days } \\
\text { avail- } \\
\text { able }\end{array}$ & $\begin{array}{c}\text { Sigma } \\
\mathrm{S}\end{array}$ & $\begin{array}{c}\text { Stan- } \\
\text { dard } \\
\text { normal } \\
\text { variate }\end{array}$ & $\begin{array}{l}\text { Non- } \\
\text { ex- } \\
\text { ceed- } \\
\text { ance } \\
\text { prob- } \\
\text { ability }\end{array}$ & $\begin{array}{c}\mathrm{p}- \\
\text { value }\end{array}$ \\
\hline 289 & 162.00 & 7 & 1,134 & 2459 & -1.743 & .041 & .041 & 378.00 & 3 & 1,134 & 1366 & $\begin{array}{l}-.591 \\
\end{array}$ & .277 & .277 \\
\hline 290 & 90.27 & 338 & 30,511 & 5169003 & .078 & .531 & .469 & 186.04 & 164 & 30,511 & 2604859 & .913 & .819 & .181 \\
\hline 291 & 130.07 & 150 & 19,511 & 1406778 & -.820 & .206 & .206 & 219.23 & 89 & 19,511 & 785504 & -1.557 & .060 & .060 \\
\hline 292 & 99.59 & 22 & 2,191 & 24303 & .068 & .527 & .473 & 182.58 & 12 & 2,191 & 15353 & 1.007 & .843 & .157 \\
\hline 293 & 68.99 & 512 & 35,323 & 9403766 & 1.565 & .941 & .059 & 113.22 & 312 & 35,323 & 5620063 & .609 & .729 & .271 \\
\hline 294 & 44.01 & 428 & 18,834 & 4212250 & 1.616 & .947 & .053 & 88.01 & 214 & 18,834 & 2140775 & 1.578 & .943 & .057 \\
\hline 295 & 89.92 & 66 & 5,935 & 197473 & .116 & .546 & .454 & 169.57 & 35 & 5,935 & 105887 & .200 & .579 & .421 \\
\hline 296 & 50.41 & 341 & 17,191 & 3104632 & 1.894 & .971 & .029 & 103.56 & 166 & 17,191 & 1522919 & 1.503 & .934 & .067 \\
\hline 297 & 49.48 & 395 & 19,546 & 4086016 & 2.013 & .978 & .022 & 93.97 & 208 & 19,546 & 2103267 & .866 & .807 & .193 \\
\hline 298 & 76.90 & 133 & 10,227 & 701473 & .628 & .735 & .265 & 189.39 & 54 & 10,227 & 284651 & .393 & .653 & .347 \\
\hline 299 & -- & -- & -- & -- & -- & -- & -- & -- & -- & -- & -- & -- & -- & -- \\
\hline 300 & 323.80 & 15 & 4,857 & 39638 & .591 & .723 & .277 & 607.13 & 8 & 4,857 & 18362 & -.269 & .394 & .394 \\
\hline 301 & 208.28 & 81 & 16,871 & 677145 & -.140 & .444 & .444 & 468.64 & 36 & 16,871 & 299615 & -.139 & .445 & .445 \\
\hline 302 & 257.45 & 75 & 19,309 & 828262 & 2.158 & .985 & .015 & 715.15 & 27 & 19,309 & 292468 & 1.098 & .864 & .136 \\
\hline 303 & 28.65 & 17 & 487 & 3395 & -1.284 & .100 & .100 & 48.70 & 10 & 487 & 1995 & -.990 & .161 & .161 \\
\hline 304 & 66.80 & 375 & 25,051 & 4615472 & -.583 & .280 & .280 & 132.55 & 189 & 25,051 & 2310189 & -.575 & .283 & .283 \\
\hline 305 & 53.59 & 29 & 1,554 & 21526 & -.417 & .338 & .338 & 86.33 & 18 & 1,554 & 13137 & -.446 & .328 & .328 \\
\hline 306 & 54.47 & 683 & 37,205 & 12926597 & .788 & .784 & .216 & 91.64 & 406 & 37,205 & 7704052 & .700 & .758 & .242 \\
\hline 307 & 167.67 & 126 & 21,126 & 1460581 & 1.894 & .971 & .029 & 346.33 & 61 & 21,126 & 735308 & 1.910 & .972 & .028 \\
\hline 308 & 55.71 & 319 & 17,772 & 2836392 & .019 & .508 & .492 & 102.14 & 174 & 17,772 & 1579995 & .500 & .691 & .309 \\
\hline 309 & 129.67 & 9 & 1,167 & 5787 & .530 & .702 & .298 & 194.50 & 6 & 1,167 & 4379 & 1.064 & .856 & .144 \\
\hline 310 & 70.52 & 44 & 3,103 & 56565 & -1.969 & .024 & .024 & 155.15 & 20 & 3,103 & 28144 & -.720 & .236 & .236 \\
\hline 311 & 126.80 & 30 & 3,804 & 57320 & .043 & .517 & .483 & 951.00 & 4 & 3,804 & 8036 & .195 & .577 & .423 \\
\hline 312 & 73.52 & 252 & 18,527 & 2577817 & 2.867 & .998 & .002 & 147.04 & 126 & 18,527 & 1322168 & 2.581 & .995 & .005 \\
\hline 313 & 91.30 & 27 & 2,465 & 28991 & -1.159 & .123 & .123 & 176.07 & 14 & 2,465 & 13469 & -1.422 & .078 & .078 \\
\hline 314 & 62.83 & 18 & 1,131 & 10149 & -.022 & .491 & .491 & 161.57 & 7 & 1,131 & 3770 & -.218 & .414 & .414 \\
\hline 315 & 43.05 & 434 & 18,684 & 4429540 & 3.338 & 1.000 & 0 & 80.53 & 232 & 18,684 & 2474209 & 3.735 & 1.000 & 0 \\
\hline 316 & 152.23 & 13 & 1,979 & 11345 & -.737 & .231 & .231 & 329.83 & 6 & 1,979 & 7379 & 1.031 & .849 & .151 \\
\hline 317 & 162.70 & 10 & 1,627 & 8533 & .268 & .606 & .394 & 271.17 & 6 & 1,627 & 3544 & -1.162 & .123 & .123 \\
\hline 318 & 170.08 & 97 & 16,498 & 766236 & -.723 & .235 & .235 & 358.65 & 46 & 16,498 & 363966 & -.480 & .316 & .316 \\
\hline 319 & 124.60 & 30 & 3,738 & 54640 & -.242 & .404 & .404 & 219.88 & 17 & 3,738 & 26800 & -1.118 & .132 & .132 \\
\hline 320 & 1336.00 & 6 & 8,016 & 18095 & -1.050 & .147 & .147 & 4008.00 & 2 & 8,016 & 3290 & -1.444 & .074 & .074 \\
\hline 321 & 62.64 & 331 & 20,733 & 3506866 & .694 & .756 & .244 & 120.54 & 172 & 20,733 & 1819906 & .470 & .681 & .319 \\
\hline 322 & 63.97 & 196 & 12,538 & 1283637 & 1.084 & .861 & .139 & 100.30 & 125 & 12,538 & 869459 & 2.121 & .983 & .017 \\
\hline 323 & 69.66 & 77 & 5,364 & 208264 & .129 & .551 & .449 & 178.80 & 30 & 5,364 & 78675 & -.211 & .417 & .417 \\
\hline 324 & 74.36 & 425 & 31,602 & 6964578 & 1.325 & .907 & .093 & 151.21 & 209 & 31,602 & 3685380 & 2.904 & .998 & .002 \\
\hline 325 & 224.16 & 19 & 4,259 & 30667 & -1.827 & .034 & .034 & 425.90 & 10 & 4,259 & 20746 & -.141 & .444 & .444 \\
\hline 326 & 42.16 & 154 & 6,492 & 497485 & -.103 & .459 & .459 & 82.18 & 79 & 6,492 & 273644 & 1.033 & .849 & .151 \\
\hline 327 & 97.90 & 83 & 8,126 & 329378 & -.367 & .357 & .357 & 198.20 & 41 & 8,126 & 142278 & -1.618 & .053 & .053 \\
\hline 328 & 121.88 & 8 & 975 & 4257 & .448 & .673 & .327 & 487.50 & 2 & 975 & 1122 & .369 & .644 & .356 \\
\hline 329 & 35.70 & 324 & 11,566 & 1869716 & -.066 & .474 & .474 & 60.24 & 192 & 11,566 & 1044374 & -1.426 & .077 & .077 \\
\hline 330 & 48.25 & 757 & 36,526 & 14126929 & 1.040 & .851 & .149 & 94.87 & 385 & 36526 & 7131873 & .486 & .687 & .313 \\
\hline 331 & 133.96 & 50 & 6,698 & 179571 & .887 & .812 & .188 & 279.08 & 24 & 6,698 & 88325 & .839 & .799 & .201 \\
\hline 332 & 113.46 & 281 & 31,881 & 4501781 & .146 & .558 & .442 & 192.05 & 166 & 31,881 & 2809226 & 1.376 & .915 & .085 \\
\hline 333 & 60.73 & 353 & 21,436 & 3831266 & 0.411 & .660 & .340 & 117.78 & 182 & 21,436 & 1968974 & 0.219 & .587 & .413 \\
\hline 334 & 195.47 & 79 & 15,442 & 693338 & 2.104 & .982 & .018 & 514.73 & 30 & 15,442 & 270027 & 1.573 & .942 & .058 \\
\hline 335 & 224.43 & 99 & 22,219 & 1016485 & -1.306 & .096 & .096 & 435.67 & 51 & 22,219 & 475911 & -1.980 & .024 & .024 \\
\hline 336 & 86.25 & 24 & 2,070 & 24827 & -.004 & .498 & .498 & 188.18 & 11 & 2,070 & 7935 & -1.741 & .041 & .041 \\
\hline
\end{tabular}


Table 6. Summary of interoccurrence intervals for daily precipitation thresholds of 1.5 and 2.0 inches-Continued

\begin{tabular}{|c|c|c|c|c|c|c|c|c|c|c|c|c|c|c|}
\hline \multirow[b]{2}{*}{$\begin{array}{l}\text { Seq. } \\
\text { no. }\end{array}$} & \multicolumn{7}{|c|}{ Daily precipitation threshold of 1.5 inches and greater } & \multicolumn{7}{|c|}{ Daily precipitation threshold of 2.0 inches and greater } \\
\hline & $\begin{array}{l}\text { Mean } \\
\text { inter- } \\
\text { occur- } \\
\text { rence } \\
\text { interval } \\
\text { (days) }\end{array}$ & $\begin{array}{l}\text { Total } \\
\text { no. of } \\
\text { events }\end{array}$ & $\begin{array}{l}\text { Total } \\
\text { no. of } \\
\text { days } \\
\text { avail- } \\
\text { able }\end{array}$ & $\begin{array}{l}\text { Sigma } \\
\mathrm{S}\end{array}$ & $\begin{array}{c}\text { Stan- } \\
\text { dard } \\
\text { normal } \\
\text { variate }\end{array}$ & $\begin{array}{l}\text { Non- } \\
\text { ex- } \\
\text { ceed- } \\
\text { ance } \\
\text { prob- } \\
\text { ability }\end{array}$ & $\begin{array}{c}\mathrm{p}- \\
\text { value }\end{array}$ & $\begin{array}{l}\text { Mean } \\
\text { inter- } \\
\text { occur- } \\
\text { rence } \\
\text { interval } \\
\text { (days) }\end{array}$ & $\begin{array}{c}\text { Total } \\
\text { no. of } \\
\text { events }\end{array}$ & $\begin{array}{l}\text { Total } \\
\text { no. of } \\
\text { days } \\
\text { avail- } \\
\text { able }\end{array}$ & $\begin{array}{l}\text { Sigma } \\
\mathrm{S}\end{array}$ & $\begin{array}{c}\text { Stan- } \\
\text { dard } \\
\text { normal } \\
\text { variate }\end{array}$ & $\begin{array}{l}\text { Non- } \\
\text { ex- } \\
\text { ceed- } \\
\text { ance } \\
\text { prob- } \\
\text { ability }\end{array}$ & $\begin{array}{c}p- \\
\text { value }\end{array}$ \\
\hline 337 & 91.75 & 120 & 11,010 & 608871 & -1.486 & .069 & .069 & 220.20 & 50 & 11,010 & 273751 & -.067 & .473 & .473 \\
\hline 338 & 94.71 & 61 & 5,777 & 175788 & -.032 & .487 & .487 & 231.08 & 25 & 5,777 & 77831 & .674 & .750 & .250 \\
\hline 339 & 42.25 & 115 & 4,859 & 308399 & 1.928 & .973 & .027 & 79.66 & 61 & 4,859 & 151644 & .314 & .623 & .377 \\
\hline 340 & 65.00 & 96 & 6,240 & 306610 & .402 & .656 & .344 & 127.35 & 49 & 6,240 & 151039 & -.146 & .442 & .442 \\
\hline 341 & 226.44 & 43 & 9,737 & 236400 & 1.468 & .929 & .071 & 389.48 & 25 & 9,737 & 149054 & 1.945 & .974 & .026 \\
\hline 342 & 195.24 & 21 & 4,100 & 54229 & 2.061 & .980 & .020 & 410.00 & 10 & 4,100 & 32206 & 3.128 & .999 & .001 \\
\hline 343 & 67.56 & 188 & 12,702 & 1197007 & .060 & .524 & .476 & 125.76 & 101 & 12,702 & 679530 & 1.033 & .849 & .151 \\
\hline 344 & 77.54 & 444 & 34,428 & 8089324 & 2.131 & .983 & .017 & 140.52 & 245 & 34,428 & 4427337 & 1.349 & .911 & .089 \\
\hline 345 & 48.50 & 10 & 485 & 2281 & -.325 & .373 & .373 & 121.25 & 4 & 485 & 988 & .064 & .526 & .474 \\
\hline 346 & 207.00 & 104 & 21,528 & 1032715 & -1.369 & .086 & .086 & 422.12 & 51 & 21,528 & 519469 & -.665 & .253 & .253 \\
\hline 347 & 198.67 & 6 & 1,192 & 3861 & .338 & .632 & .368 & 397.33 & 3 & 1,192 & 1918 & .218 & .586 & .414 \\
\hline 348 & 122.70 & 91 & 11,166 & 529736 & .705 & .760 & .240 & 248.13 & 45 & 11,166 & 266282 & .696 & .757 & .243 \\
\hline 349 & 116.26 & 96 & 11,161 & 539908 & .132 & .553 & .447 & 223.22 & 50 & 11,161 & 298633 & .861 & .805 & .195 \\
\hline 350 & 104.38 & 270 & 28,183 & 3891903 & .652 & .743 & .257 & 242.96 & 116 & 28,183 & 1677162 & .486 & .686 & .314 \\
\hline 351 & 120.78 & 32 & 3,865 & 65460 & .574 & .717 & .283 & 227.35 & 17 & 3,865 & 31382 & -.320 & .375 & .375 \\
\hline 352 & 106.20 & 10 & 1,062 & 5195 & -.119 & .453 & .453 & 132.75 & 8 & 1,062 & 3922 & -.376 & .353 & .353 \\
\hline 353 & 48.61 & 67 & 3,257 & 113461 & .565 & .714 & .286 & 125.27 & 26 & 3,257 & 41421 & -.192 & .424 & .424 \\
\hline 354 & 75.56 & 91 & 6,876 & 324413 & .610 & .729 & .271 & 156.27 & 44 & 6,876 & 158846 & .575 & .717 & .283 \\
\hline 355 & 76.51 & 146 & 11,170 & 801966 & -.345 & .365 & .365 & 134.58 & 83 & 11,170 & 449905 & -.465 & .321 & .321 \\
\hline 356 & 118.52 & 302 & 35,793 & 5385287 & -.108 & .457 & .457 & 192.44 & 186 & 35,793 & 3186521 & -1.009 & .156 & .156 \\
\hline 357 & 100.60 & 314 & 31,587 & 4865988 & -.577 & .282 & .282 & 214.88 & 147 & 31,587 & 2197786 & -1.120 & .131 & .131 \\
\hline 358 & 116.57 & 162 & 18,884 & 1524585 & -.072 & .471 & .471 & 222.17 & 85 & 18,884 & 794926 & -.152 & .440 & .440 \\
\hline 359 & 148.00 & 15 & 2,220 & 20756 & 1.654 & .951 & .049 & 277.50 & 8 & 2,220 & 13174 & 2.369 & .991 & .009 \\
\hline 360 & 72.40 & 10 & 724 & 2913 & -1.070 & .142 & .142 & 181.00 & 4 & 724 & 1256 & -.459 & .323 & .323 \\
\hline 361 & 150.00 & 1 & 150 & 99 & .554 & .710 & .290 & -- & -- & -- & -- & -- & -- & -- \\
\hline 362 & 56.61 & 375 & 21,229 & 3894798 & -.722 & .235 & .235 & 103.05 & 206 & 21,229 & 2215254 & .326 & .628 & .372 \\
\hline 363 & 44.65 & 49 & 2,188 & 51158 & -.554 & .290 & .290 & 84.15 & 26 & 2,188 & 26628 & -.564 & .286 & .286 \\
\hline 364 & 49.17 & 195 & 9,588 & 966864 & .829 & .796 & .204 & 78.59 & 122 & 9,588 & 583711 & -.038 & .485 & .485 \\
\hline 365 & 49.84 & 447 & 22,280 & 4960014 & -.144 & .443 & .443 & 95.21 & 234 & 22,280 & 2588776 & -.183 & .427 & .427 \\
\hline 366 & 51.53 & 49 & 2,525 & 63712 & .363 & .642 & .358 & 52.60 & 48 & 2,525 & 62638 & .404 & .657 & .343 \\
\hline 367 & 54.45 & 212 & 11,543 & 1246887 & .481 & .685 & .315 & 103.99 & 111 & 11,543 & 643405 & .079 & .531 & .469 \\
\hline 368 & 123.91 & 68 & 8,426 & 274666 & -.589 & .278 & .278 & 210.65 & 40 & 8,426 & 151435 & -1.111 & .133 & .133 \\
\hline 369 & -- & -- & -- & -- & -- & -- & -- & -- & -- & -- & -- & -- & -- & -- \\
\hline 370 & 1307.40 & 5 & 6,537 & 10121 & -1.474 & .070 & .070 & 3268.50 & 2 & 6,537 & 3833 & -1.013 & .156 & .156 \\
\hline 371 & 939.00 & 21 & 19,719 & 169542 & -1.438 & .075 & .075 & 4929.75 & 4 & 19,719 & 38667 & -.068 & .473 & .473 \\
\hline 372 & 156.85 & 52 & 8,156 & 235042 & 1.354 & .912 & .088 & 429.26 & 19 & 8,156 & 95112 & 1.718 & .957 & .043 \\
\hline 373 & 93.00 & 2 & 186 & 235 & .645 & .741 & .259 & 93.00 & 2 & 186 & 235 & .645 & .741 & .259 \\
\hline 374 & 174.80 & 5 & 874 & 2870 & 1.214 & .888 & .112 & 291.33 & 3 & 874 & 2097 & 1.799 & .964 & .036 \\
\hline 375 & 164.94 & 65 & 10,721 & 372105 & .949 & .829 & .171 & 345.84 & 31 & 10,721 & 164365 & -.105 & .458 & .458 \\
\hline 376 & 171.18 & 17 & 2,910 & 30705 & 1.724 & .958 & .042 & 485.00 & 6 & 2,910 & 10235 & .731 & .768 & .232 \\
\hline 377 & 95.53 & 190 & 18,150 & 1819494 & 1.319 & .906 & .094 & 187.11 & 97 & 18,150 & 940776 & 1.172 & .879 & .121 \\
\hline 378 & 76.13 & 191 & 14,540 & 1425014 & .628 & .735 & .265 & 132.18 & 110 & 14,540 & 810775 & .252 & .599 & .401 \\
\hline 379 & 36.20 & 5 & 181 & 507 & .467 & .680 & .320 & 181.00 & 1 & 181 & 138 & .909 & .818 & .182 \\
\hline 380 & 119.00 & 12 & 1,428 & 9270 & .492 & .688 & .312 & 142.80 & 10 & 1,428 & 7387 & .190 & .575 & .425 \\
\hline 381 & 48.34 & 393 & 18,998 & 3857071 & 1.140 & .873 & .127 & 86.75 & 219 & 18,998 & 2237040 & 1.932 & .973 & .027 \\
\hline 382 & 74.00 & 4 & 296 & 507 & -.497 & .309 & .309 & 74.00 & 4 & 296 & 507 & -.497 & .309 & .309 \\
\hline 383 & 111.51 & 291 & 32,450 & 5155415 & 2.716 & .997 & .003 & 178.30 & 182 & 32,450 & 3149701 & 1.557 & .940 & .060 \\
\hline 384 & 115.04 & 71 & 8,168 & 281314 & -.435 & .332 & .332 & 204.20 & 40 & 8,168 & 164531 & .079 & .531 & .469 \\
\hline
\end{tabular}


Table 6. Summary of interoccurrence intervals for daily precipitation thresholds of 1.5 and 2.0 inches—Continued

\begin{tabular}{|c|c|c|c|c|c|c|c|c|c|c|c|c|c|c|}
\hline \multirow[b]{2}{*}{$\begin{array}{l}\text { Seq. } \\
\text { no. }\end{array}$} & \multicolumn{7}{|c|}{ Daily precipitation threshold of 1.5 inches and greater } & \multicolumn{7}{|c|}{ Daily precipitation threshold of 2.0 inches and greater } \\
\hline & $\begin{array}{l}\text { Mean } \\
\text { inter- } \\
\text { occur- } \\
\text { rence } \\
\text { interval } \\
\text { (days) }\end{array}$ & $\begin{array}{c}\text { Total } \\
\text { no. of } \\
\text { events }\end{array}$ & $\begin{array}{l}\text { Total } \\
\text { no. of } \\
\text { days } \\
\text { avail- } \\
\text { able }\end{array}$ & $\begin{array}{l}\text { Sigma } \\
\mathrm{S}\end{array}$ & $\begin{array}{c}\text { Stan- } \\
\text { dard } \\
\text { normal } \\
\text { variate }\end{array}$ & $\begin{array}{l}\text { Non- } \\
\text { ex- } \\
\text { ceed- } \\
\text { ance } \\
\text { prob- } \\
\text { ability }\end{array}$ & $\begin{array}{c}\mathrm{p}- \\
\text { value }\end{array}$ & $\begin{array}{l}\text { Mean } \\
\text { inter- } \\
\text { occur- } \\
\text { rence } \\
\text { interval } \\
\text { (days) }\end{array}$ & $\begin{array}{l}\text { Total } \\
\text { no. of } \\
\text { events }\end{array}$ & $\begin{array}{l}\text { Total } \\
\text { no. of } \\
\text { days } \\
\text { avail- } \\
\text { able }\end{array}$ & $\begin{array}{l}\text { Sigma } \\
\mathrm{S}\end{array}$ & $\begin{array}{c}\text { Stan- } \\
\text { dard } \\
\text { normal } \\
\text { variate }\end{array}$ & $\begin{array}{l}\text { Non- } \\
\text { ex- } \\
\text { ceed- } \\
\text { ance } \\
\text { prob- } \\
\text { ability }\end{array}$ & $\begin{array}{c}\mathrm{p}- \\
\text { value }\end{array}$ \\
\hline 385 & 60.43 & 285 & 17,222 & 2406362 & -.569 & .285 & .285 & 112.56 & 153 & 17,222 & 1323393 & .096 & .538 & .462 \\
\hline 386 & 61.00 & 4 & 244 & 390 & -.696 & .243 & .243 & 244.00 & 1 & 244 & 236 & 1.619 & .947 & .053 \\
\hline 387 & 83.24 & 25 & 2,081 & 25807 & -.068 & .473 & .473 & 189.18 & 11 & 2,081 & 9913 & -.769 & .221 & .221 \\
\hline 388 & 66.60 & 37 & 2,464 & 49021 & .794 & .786 & .214 & 136.89 & 18 & 2,464 & 22695 & .172 & .568 & .432 \\
\hline 389 & 60.50 & 6 & 363 & 1156 & .261 & .603 & .397 & 90.75 & 4 & 363 & 1030 & 1.451 & .927 & .073 \\
\hline 390 & 36.87 & 474 & 17,476 & 4239364 & .888 & .813 & .187 & 69.35 & 252 & 17,476 & 2222616 & .258 & .602 & .398 \\
\hline 391 & 84.51 & 189 & 15,972 & 1518345 & .142 & .556 & .444 & 164.66 & 97 & 15,972 & 777028 & .053 & .521 & .479 \\
\hline 392 & 79.54 & 13 & 1,034 & 5010 & -1.590 & .056 & .056 & 94.00 & 11 & 1,034 & 3682 & -2.025 & .021 & .021 \\
\hline 393 & 882.83 & 12 & 10,594 & 79136 & 1.470 & .929 & .071 & 1324.25 & 8 & 10,594 & 44093 & .199 & .579 & .421 \\
\hline 394 & -- & -- & -- & -- & -- & -- & -- & -- & -- & -- & -- & -- & -- & -- \\
\hline 395 & 80.66 & 113 & 9,115 & 572006 & 2.038 & .979 & .021 & 128.38 & 71 & 9,115 & 351349 & 1.252 & .895 & .105 \\
\hline 396 & 53.38 & 369 & 19,696 & 3763569 & 1.187 & .882 & .118 & 106.47 & 185 & 19,696 & 1782911 & -.504 & .307 & .307 \\
\hline 397 & 146.81 & 97 & 14,241 & 655769 & -.862 & .194 & .194 & 249.84 & 57 & 14,241 & 377261 & -.922 & .178 & .178 \\
\hline 398 & 102.17 & 331 & 33,819 & 5811676 & 1.208 & .887 & .113 & 183.80 & 184 & 33,819 & 3260653 & 1.127 & .870 & .130 \\
\hline 399 & 82.43 & 238 & 19,618 & 2356859 & .255 & .601 & .399 & 153.27 & 128 & 19,618 & 1208789 & -.730 & .233 & .233 \\
\hline 400 & 48.79 & 395 & 19,271 & 3846362 & .365 & .642 & .358 & 89.63 & 215 & 19,271 & 2123773 & .639 & .739 & .261 \\
\hline 401 & 160.53 & 36 & 5,779 & 86302 & -1.770 & .038 & .038 & 275.19 & 21 & 5,779 & 44363 & -2.134 & .016 & .016 \\
\hline 402 & 81.00 & 3 & 243 & 503 & 1.140 & .873 & .127 & 121.50 & 2 & 243 & 263 & .202 & .580 & .420 \\
\hline 403 & 63.84 & 212 & 13,535 & 1368183 & -1.169 & .121 & .121 & 123.05 & 110 & 13,535 & 775829 & .766 & .778 & .222 \\
\hline 404 & 62.64 & 329 & 20,610 & 3502365 & 1.038 & .850 & .150 & 127.22 & 162 & 20,610 & 1721999 & .695 & .756 & .244 \\
\hline 405 & 126.43 & 94 & 11,884 & 621661 & 1.898 & .971 & .029 & 237.68 & 50 & 11,884 & 336400 & 1.620 & .947 & .053 \\
\hline 406 & 71.57 & 303 & 21,685 & 3498207 & 1.954 & .975 & .025 & 134.69 & 161 & 21,685 & 1930907 & 2.332 & .990 & .010 \\
\hline 407 & 65.05 & 57 & 3,708 & 94293 & -1.409 & .079 & .079 & 168.55 & 22 & 3,708 & 42175 & .276 & .609 & .391 \\
\hline 408 & 63.70 & 536 & 34,142 & 9573712 & 1.857 & .968 & .032 & 121.94 & 280 & 34,142 & 5071652 & 1.769 & .962 & .038 \\
\hline 409 & 608.00 & 2 & 1,216 & 1263 & .095 & .538 & .462 & 1216.00 & 1 & 1,216 & 423 & -.527 & .299 & .299 \\
\hline 410 & 54.13 & 266 & 14,398 & 1979834 & .957 & .831 & .169 & 105.87 & 136 & 14,398 & 1010121 & .641 & .739 & .261 \\
\hline 411 & 137.71 & 139 & 19,141 & 1382074 & .795 & .787 & .213 & 290.02 & 66 & 19,141 & 699169 & 1.504 & .934 & .066 \\
\hline 412 & 63.17 & 220 & 13,897 & 1678240 & 2.514 & .994 & .006 & 122.98 & 113 & 13,897 & 922857 & 3.228 & .999 & .001 \\
\hline 413 & 84.96 & 355 & 30,162 & 5316140 & -.229 & .409 & .409 & 157.92 & 191 & 30,162 & 2806641 & -.614 & .270 & .270 \\
\hline 414 & 55.21 & 62 & 3,423 & 114868 & 1.125 & .870 & .130 & 106.97 & 32 & 3,423 & 58059 & .589 & .722 & .278 \\
\hline 415 & 167.89 & 128 & 21,490 & 1335196 & -.572 & .284 & .284 & 383.75 & 56 & 21,490 & 591260 & -.225 & .411 & .411 \\
\hline 416 & 161.27 & 120 & 19,352 & 1165926 & .079 & .531 & .469 & 365.13 & 53 & 19,352 & 450823 & -1.525 & .064 & .064 \\
\hline 417 & 76.80 & 40 & 3,072 & 69933 & 1.514 & .935 & .065 & 219.43 & 14 & 3,072 & 30731 & 2.781 & .997 & .003 \\
\hline 418 & 169.56 & 147 & 24,925 & 2029638 & 2.266 & .988 & .012 & 361.23 & 69 & 24,925 & 959655 & 1.669 & .952 & .048 \\
\hline 419 & 73.33 & 309 & 22,658 & 3256270 & -2.126 & .017 & .017 & 137.32 & 165 & 22,658 & 1704349 & -1.963 & .025 & .025 \\
\hline 420 & 156.87 & 122 & 19,138 & 1223307 & .916 & .820 & .180 & 324.37 & 59 & 19,138 & 634310 & 1.643 & .950 & .050 \\
\hline 421 & 458.69 & 68 & 31,191 & 1066494 & .081 & .532 & .468 & 1356.13 & 23 & 31,191 & 374814 & .373 & .646 & .355 \\
\hline 422 & 609.95 & 19 & 11,589 & 78496 & -2.167 & .015 & .015 & 827.79 & 14 & 11,589 & 54848 & -2.099 & .018 & .018 \\
\hline 423 & 126.04 & 92 & 11,596 & 507837 & -.797 & .213 & .213 & 218.79 & 53 & 11,596 & 305590 & -.070 & .472 & .472 \\
\hline 424 & -- & -- & -- & -- & -- & -- & -- & -- & -- & -- & -- & -- & -- & -- \\
\hline 425 & 327.11 & 65 & 21,262 & 697772 & .137 & .554 & .446 & 644.30 & 33 & 21,262 & 325711 & -.712 & .238 & .238 \\
\hline 426 & 270.71 & 66 & 17,867 & 543110 & -1.110 & .134 & .134 & 496.31 & 36 & 17,867 & 269152 & -1.695 & .045 & .045 \\
\hline 427 & -- & -- & -- & -- & -- & -- & -- & -- & -- & -- & -- & -- & -- & -- \\
\hline 428 & 94.08 & 51 & 4,798 & 137603 & 1.542 & .938 & .062 & 137.09 & 35 & 4,798 & 97984 & 1.711 & .956 & .044 \\
\hline 429 & 79.59 & 68 & 5,412 & 189348 & 0.415 & .661 & .339 & 174.58 & 31 & 5,412 & 80472 & -0.393 & .347 & .347 \\
\hline 430 & 84.10 & 106 & 8,915 & 500952 & 1.074 & .859 & .141 & 151.10 & 59 & 8,915 & 310930 & 2.425 & .992 & .008 \\
\hline 431 & 68.86 & 56 & 3,856 & 109102 & .136 & .554 & .446 & 167.65 & 23 & 3,856 & 37593 & -1.265 & .103 & .103 \\
\hline 432 & 44.33 & 196 & 8,688 & 797648 & -1.532 & .063 & .063 & 79.71 & 109 & 8,688 & 454199 & -.737 & .231 & .231 \\
\hline
\end{tabular}


Table 6. Summary of interoccurrence intervals for daily precipitation thresholds of 1.5 and 2.0 inches-Continued

\begin{tabular}{|c|c|c|c|c|c|c|c|c|c|c|c|c|c|c|}
\hline \multirow[b]{2}{*}{$\begin{array}{l}\text { Seq. } \\
\text { no. }\end{array}$} & \multicolumn{7}{|c|}{ Daily precipitation threshold of 1.5 inches and greater } & \multicolumn{7}{|c|}{ Daily precipitation threshold of 2.0 inches and greater } \\
\hline & $\begin{array}{l}\text { Mean } \\
\text { inter- } \\
\text { occur- } \\
\text { rence } \\
\text { interval } \\
\text { (days) }\end{array}$ & $\begin{array}{c}\text { Total } \\
\text { no. of } \\
\text { events }\end{array}$ & $\begin{array}{l}\text { Total } \\
\text { no. of } \\
\text { days } \\
\text { avail- } \\
\text { able }\end{array}$ & $\begin{array}{l}\text { Sigma } \\
\mathbf{S}\end{array}$ & $\begin{array}{c}\text { Stan- } \\
\text { dard } \\
\text { normal } \\
\text { variate }\end{array}$ & $\begin{array}{l}\text { Non- } \\
\text { ex- } \\
\text { ceed- } \\
\text { ance } \\
\text { prob- } \\
\text { ability }\end{array}$ & $\begin{array}{c}\mathrm{p}- \\
\text { value }\end{array}$ & $\begin{array}{l}\text { Mean } \\
\text { inter- } \\
\text { occur- } \\
\text { rence } \\
\text { interval } \\
\text { (days) }\end{array}$ & $\begin{array}{c}\text { Total } \\
\text { no. of } \\
\text { events }\end{array}$ & $\begin{array}{l}\text { Total } \\
\text { no. of } \\
\text { days } \\
\text { avail- } \\
\text { able }\end{array}$ & $\begin{array}{l}\text { Sigma } \\
\mathrm{S}\end{array}$ & $\begin{array}{l}\text { Stan- } \\
\text { dard } \\
\text { normal } \\
\text { variate }\end{array}$ & $\begin{array}{l}\text { Non- } \\
\text { ex- } \\
\text { ceed- } \\
\text { ance } \\
\text { prob- } \\
\text { ability }\end{array}$ & $\begin{array}{c}\mathrm{p}- \\
\text { value }\end{array}$ \\
\hline 433 & 97.35 & 281 & 27,354 & 3821694 & -.163 & .435 & .435 & 182.36 & 150 & 27,354 & 2077289 & .266 & .605 & .395 \\
\hline 434 & 62.37 & 218 & 13,597 & 1509906 & .480 & .684 & .316 & 123.61 & 110 & 13,597 & 787311 & .959 & .831 & .169 \\
\hline 435 & 73.79 & 14 & 1,033 & 8899 & 1.495 & .933 & .067 & 114.78 & 9 & 1,033 & 6778 & 2.380 & .991 & .009 \\
\hline 436 & 90.51 & 321 & 29,054 & 4986936 & 2.155 & .984 & .016 & 168.92 & 172 & 29,054 & 2584073 & .777 & .781 & .219 \\
\hline 437 & 40.07 & 632 & 25,322 & 8194629 & 1.050 & .853 & .147 & 65.60 & 386 & 25,322 & 5000521 & .789 & .785 & .215 \\
\hline 438 & 81.89 & 228 & 18,671 & 2239279 & 1.361 & .913 & .087 & 142.53 & 131 & 18,671 & 1297048 & 1.201 & .885 & .115 \\
\hline 439 & 97.19 & 84 & 8,164 & 321410 & -.994 & .160 & .160 & 189.86 & 43 & 8,164 & 170220 & -.343 & .366 & .366 \\
\hline 440 & 95.90 & 97 & 9,302 & 473618 & .850 & .802 & .198 & 157.66 & 59 & 9,302 & 295141 & 1.005 & .843 & .157 \\
\hline 441 & 263.70 & 90 & 23,733 & 1109547 & .640 & .739 & .261 & 641.43 & 37 & 23,733 & 391904 & -1.132 & .129 & .129 \\
\hline 442 & 61.99 & 206 & 12,769 & 1416144 & 1.908 & .972 & .028 & 115.04 & 111 & 12,769 & 780582 & 1.852 & .968 & .032 \\
\hline 443 & 69.08 & 188 & 12,987 & 1215450 & -.104 & .459 & .459 & 154.61 & 84 & 12,987 & 535384 & -.293 & .385 & .385 \\
\hline 444 & 115.21 & 14 & 1,613 & 12005 & .410 & .659 & .341 & 322.60 & 5 & 1,613 & 5225 & 1.145 & .874 & .126 \\
\hline 445 & 153.12 & 125 & 19,140 & 1251821 & .900 & .816 & .184 & 348.00 & 55 & 19,140 & 525392 & -.023 & .491 & .491 \\
\hline 446 & 90.54 & 13 & 1,177 & 7865 & .175 & .570 & .430 & 235.40 & 5 & 1,177 & 3333 & .514 & .696 & .304 \\
\hline 447 & 158.19 & 145 & 22,938 & 1563190 & -1.252 & .105 & .105 & 358.41 & 64 & 22,938 & 647710 & -1.629 & .052 & .052 \\
\hline 448 & 69.29 & 455 & 31,527 & 6999417 & -.891 & .187 & .187 & 135.31 & 233 & 31,527 & 3447453 & -1.623 & .052 & .052 \\
\hline 449 & 50.64 & 105 & 5,317 & 262547 & -1.055 & .146 & .146 & 91.67 & 58 & 5,317 & 151292 & -.248 & .402 & .402 \\
\hline 450 & 58.17 & 353 & 20,533 & 3908194 & 2.551 & .995 & .005 & 102.15 & 201 & 20,533 & 2179221 & 1.376 & .916 & .084 \\
\hline 451 & 63.93 & 87 & 5,562 & 245579 & .243 & .596 & .404 & 111.24 & 50 & 5,562 & 146492 & .656 & .744 & .256 \\
\hline 452 & 101.33 & 3 & 304 & 371 & -.559 & .288 & .288 & 152.00 & 2 & 304 & 287 & -.137 & .445 & .445 \\
\hline 453 & 262.88 & 8 & 2,103 & 4986 & -1.995 & .023 & .023 & 701.00 & 3 & 2,103 & 3069 & -.081 & .468 & .468 \\
\hline 454 & 194.99 & 163 & 31,784 & 2652250 & .528 & .701 & .299 & 392.40 & 81 & 31,784 & 1244206 & -.521 & .301 & .301 \\
\hline 455 & 221.75 & 4 & 887 & 1916 & .277 & .609 & .391 & 443.50 & 2 & 887 & 1029 & .392 & .653 & .348 \\
\hline 456 & -- & -- & -- & -- & -- & -- & -- & -- & -- & -- & -- & -- & -- & -- \\
\hline 457 & 68.20 & 5 & 341 & 1242 & 1.770 & .962 & .038 & 113.67 & 3 & 341 & 640 & .754 & .774 & .226 \\
\hline 458 & 41.21 & 151 & 6,223 & 473007 & .144 & .557 & .443 & 73.21 & 85 & 6,223 & 270962 & .392 & .652 & .348 \\
\hline 459 & 62.28 & 18 & 1,121 & 10489 & .291 & .615 & .385 & 93.42 & 12 & 1,121 & 6805 & .071 & .528 & .472 \\
\hline 460 & 69.94 & 468 & 32,732 & 7361236 & -1.458 & .072 & .072 & 126.87 & 258 & 32,732 & 3919545 & -1.996 & .023 & .023 \\
\hline 461 & 89.49 & 344 & 30,785 & 5418453 & .749 & .773 & .227 & 165.51 & 186 & 30,785 & 2747502 & -.953 & .170 & .170 \\
\hline 462 & 72.41 & 245 & 17,740 & 2226419 & .665 & .747 & .253 & 133.38 & 133 & 17,740 & 1168366 & -.192 & .424 & .424 \\
\hline 463 & 64.46 & 116 & 7,477 & 490026 & 2.424 & .992 & .008 & 124.62 & 60 & 7,477 & 230475 & .369 & .644 & .356 \\
\hline 464 & 59.37 & 379 & 22,500 & 4228074 & -.282 & .389 & .389 & 109.22 & 206 & 22,500 & 2272394 & -.484 & .314 & .314 \\
\hline 465 & 61.44 & 32 & 1,966 & 34350 & .901 & .816 & .184 & 109.22 & 18 & 1,966 & 19858 & .899 & .816 & .184 \\
\hline 466 & 52.26 & 502 & 26,233 & 6643780 & .350 & .637 & .363 & 95.05 & 276 & 26,233 & 3778116 & 1.256 & .895 & .105 \\
\hline 467 & 381.48 & 29 & 11,063 & 167402 & .406 & .658 & .342 & 1580.43 & 7 & 11,063 & 41365 & .313 & .623 & .377 \\
\hline 468 & 33.10 & 30 & 993 & 10735 & -2.650 & .004 & .004 & 58.41 & 17 & 993 & 5594 & -2.408 & .008 & .008 \\
\hline 469 & 50.62 & 194 & 9,821 & 913009 & -1.004 & .158 & .158 & 99.20 & 99 & 9,821 & 469536 & -.589 & .278 & .278 \\
\hline 470 & 91.00 & 1 & 91 & 45 & -.019 & .492 & .492 & -- & -- & -- & -- & -- & -- & -- \\
\hline 471 & 71.59 & 187 & 13,388 & 1299324 & .900 & .816 & .184 & 143.96 & 93 & 13,388 & 628076 & .149 & .559 & .441 \\
\hline 472 & 46.83 & 72 & 3,372 & 124820 & .415 & .661 & .339 & 80.29 & 42 & 3,372 & 78090 & 1.154 & .876 & .124 \\
\hline 473 & 73.95 & 260 & 19,226 & 2788580 & 3.232 & .999 & .001 & 141.37 & 136 & 19,226 & 1388330 & 1.251 & .894 & .106 \\
\hline 474 & 96.46 & 261 & 25,176 & 3441019 & 1.325 & .907 & .093 & 215.18 & 117 & 25,176 & 1509061 & .461 & .678 & .322 \\
\hline 475 & 69.93 & 457 & 31,959 & 7769221 & 2.366 & .991 & .009 & 119.25 & 268 & 31,959 & 4612940 & 2.188 & .986 & .014 \\
\hline 476 & 57.90 & 315 & 18,237 & 3007785 & 1.450 & .926 & .074 & 94.01 & 194 & 18,237 & 1761852 & -.097 & .461 & .461 \\
\hline 477 & 75.02 & 56 & 4,201 & 138656 & 2.317 & .990 & .010 & 150.04 & 28 & 4,201 & 75056 & 2.531 & .994 & .006 \\
\hline 478 & 72.07 & 320 & 23,063 & 3791807 & .854 & .803 & .197 & 133.31 & 173 & 23,063 & 2059532 & .738 & .770 & .230 \\
\hline 479 & 44.80 & 40 & 1,792 & 29901 & -1.815 & .035 & .035 & 81.46 & 22 & 1,792 & 15241 & -1.843 & .033 & .033 \\
\hline 480 & 64.59 & 88 & 5,684 & 258124 & .522 & 699 & .301 & 107.25 & 53 & 5,684 & 154465 & .321 & .626 & .374 \\
\hline
\end{tabular}


Table 6. Summary of interoccurrence intervals for daily precipitation thresholds of 1.5 and 2.0 inches—Continued

\begin{tabular}{|c|c|c|c|c|c|c|c|c|c|c|c|c|c|c|}
\hline \multirow[b]{2}{*}{$\begin{array}{l}\text { Seq. } \\
\text { no. }\end{array}$} & \multicolumn{7}{|c|}{ Daily precipitation threshold of 1.5 inches and greater } & \multicolumn{7}{|c|}{ Daily precipitation threshold of 2.0 inches and greater } \\
\hline & $\begin{array}{l}\text { Mean } \\
\text { inter- } \\
\text { occur- } \\
\text { rence } \\
\text { interval } \\
\text { (days) }\end{array}$ & $\begin{array}{c}\text { Total } \\
\text { no. of } \\
\text { events }\end{array}$ & $\begin{array}{l}\text { Total } \\
\text { no. of } \\
\text { days } \\
\text { avail- } \\
\text { able }\end{array}$ & $\begin{array}{l}\text { Sigma } \\
\mathrm{S}\end{array}$ & $\begin{array}{c}\text { Stan- } \\
\text { dard } \\
\text { normal } \\
\text { variate }\end{array}$ & $\begin{array}{l}\text { Non- } \\
\text { ex- } \\
\text { ceed- } \\
\text { ance } \\
\text { prob- } \\
\text { ability }\end{array}$ & $\begin{array}{c}\mathrm{p}- \\
\text { value }\end{array}$ & $\begin{array}{l}\text { Mean } \\
\text { inter- } \\
\text { occur- } \\
\text { rence } \\
\text { interval } \\
\text { (days) }\end{array}$ & $\begin{array}{l}\text { Total } \\
\text { no. of } \\
\text { events }\end{array}$ & $\begin{array}{l}\text { Total } \\
\text { no. of } \\
\text { days } \\
\text { avail- } \\
\text { able }\end{array}$ & $\begin{array}{l}\text { Sigma } \\
\mathrm{S}\end{array}$ & $\begin{array}{c}\text { Stan- } \\
\text { dard } \\
\text { normal } \\
\text { variate }\end{array}$ & $\begin{array}{l}\text { Non- } \\
\text { ex- } \\
\text { ceed- } \\
\text { ance } \\
\text { prob- } \\
\text { ability }\end{array}$ & $\begin{array}{c}p- \\
\text { value }\end{array}$ \\
\hline 481 & 43.17 & 293 & 12,650 & 1979310 & 2.017 & .978 & .022 & 75.30 & 168 & 12,650 & 1181073 & 2.503 & .994 & .006 \\
\hline 482 & 77.27 & 48 & 3,709 & 86146 & -.387 & .349 & .349 & 137.37 & 27 & 3,709 & 46938 & -.563 & .287 & .287 \\
\hline 483 & 70.53 & 45 & 3,174 & 77409 & .975 & .835 & .165 & 144.27 & 22 & 3,174 & 36221 & .304 & .619 & .381 \\
\hline 484 & 76.00 & 36 & 2,736 & 48753 & -.105 & .458 & .458 & 109.44 & 25 & 2,736 & 32591 & -.407 & .342 & .342 \\
\hline 485 & 84.80 & 10 & 848 & 2912 & -1.716 & .043 & .043 & 212.00 & 4 & 848 & 656 & -2.124 & .017 & .017 \\
\hline 486 & 88.50 & 393 & 34,779 & 6959733 & .631 & .736 & .264 & 177.44 & 196 & 34,779 & 3605822 & 1.405 & .920 & .080 \\
\hline 487 & 81.91 & 128 & 10,484 & 672203 & .036 & .514 & .486 & 156.48 & 67 & 10,484 & 374060 & .922 & .822 & .178 \\
\hline 488 & 287.12 & 98 & 28,138 & 1427256 & .603 & .727 & .273 & 639.50 & 44 & 28,138 & 576943 & -.781 & .217 & .217 \\
\hline 489 & 74.06 & 157 & 11,627 & 967254 & 1.297 & .903 & .097 & 149.06 & 78 & 11,627 & 484660 & 1.053 & .854 & .146 \\
\hline 490 & 71.58 & 109 & 7,802 & 445758 & .874 & .809 & .191 & 152.98 & 51 & 7,802 & 237513 & 2.398 & .992 & .008 \\
\hline 491 & 53.66 & 238 & 12,770 & 1605934 & 1.518 & .935 & .065 & 107.31 & 119 & 12,770 & 785927 & .649 & .742 & .258 \\
\hline 492 & 72.30 & 347 & 25,088 & 4212568 & -1.039 & .149 & .149 & 140.94 & 178 & 25,088 & 2223798 & -.094 & .463 & .463 \\
\hline 493 & 53.71 & 664 & 35,661 & 12251265 & 1.552 & .940 & .060 & 94.84 & 376 & 35,661 & 7013373 & 1.549 & .939 & .061 \\
\hline 494 & 49.57 & 86 & 4,263 & 187503 & .368 & .643 & .357 & 85.26 & 50 & 4,263 & 109564 & .344 & .634 & .366 \\
\hline 495 & 42.65 & 477 & 20,344 & 5293694 & 3.443 & 1.000 & 0 & 79.78 & 255 & 20,344 & 2886619 & 3.122 & .999 & .001 \\
\hline 496 & 184.25 & 117 & 21,557 & 1167391 & -1.392 & .082 & .082 & 365.37 & 59 & 21,557 & 463890 & -3.599 & 0 & 0 \\
\hline 497 & 815.00 & 1 & 815 & 465 & .244 & .597 & .403 & 815.00 & 1 & 815 & 465 & .244 & .597 & .403 \\
\hline 498 & 61.32 & 311 & 19,069 & 3122212 & 1.617 & .947 & .053 & 123.03 & 155 & 19,069 & 1566826 & 1.298 & .903 & .097 \\
\hline 499 & 111.62 & 159 & 17,748 & 1451606 & .629 & .735 & .265 & 253.54 & 70 & 17,748 & 621840 & .015 & .506 & .494 \\
\hline 500 & 88.92 & 13 & 1,156 & 10756 & 2.695 & .996 & .004 & 231.20 & 5 & 1,156 & 4075 & 1.588 & .944 & .056 \\
\hline 501 & 76.30 & 67 & 5,112 & 162934 & -.689 & .246 & .246 & 146.06 & 35 & 5,112 & 85929 & -.404 & .343 & .343 \\
\hline 502 & 50.70 & 603 & 30,570 & 9701944 & 2.239 & .987 & .013 & 95.23 & 321 & 30,570 & 5320771 & 2.620 & .996 & .004 \\
\hline 503 & 145.71 & 42 & 6,120 & 122252 & -.547 & .292 & .292 & 278.18 & 22 & 6,120 & 57147 & -1.228 & .110 & .110 \\
\hline 504 & 230.19 & 16 & 3,683 & 30571 & .260 & .603 & .397 & 334.82 & 11 & 3,683 & 19432 & -.234 & .408 & .408 \\
\hline 505 & 153.00 & 7 & 1,071 & 3408 & -.416 & .339 & .339 & 535.50 & 2 & 1,071 & 386 & -1.567 & .059 & .059 \\
\hline 506 & 63.07 & 593 & 37,401 & 11882537 & 3.017 & .999 & .001 & 111.98 & 334 & 37,401 & 6796651 & 2.791 & .997 & .003 \\
\hline 507 & 33.24 & 42 & 1,396 & 33420 & 1.571 & .942 & .058 & 60.70 & 23 & 1,396 & 16634 & .300 & .618 & .382 \\
\hline 508 & 86.79 & 183 & 15,882 & 1463501 & .166 & .566 & .434 & 206.26 & 77 & 15,882 & 594791 & -.414 & .339 & .339 \\
\hline 509 & 118.14 & 216 & 25,519 & 2633674 & -1.130 & .129 & .129 & 274.40 & 93 & 25,519 & 1107088 & -1.120 & .131 & .131 \\
\hline 510 & 233.11 & 9 & 2,098 & 11505 & 1.136 & .872 & .128 & 299.71 & 7 & 2,098 & 8286 & .589 & .722 & .278 \\
\hline 511 & 43.95 & 423 & 18,591 & 4411782 & 4.347 & 1.000 & 0 & 83.37 & 223 & 18,591 & 2429865 & 4.454 & 1.000 & 0 \\
\hline 512 & 97.32 & 324 & 31,532 & 5073560 & -.211 & .416 & .416 & 166.84 & 189 & 31,532 & 2934804 & -.359 & .360 & .360 \\
\hline 513 & 96.85 & 220 & 21,306 & 2314913 & -.315 & .376 & .376 & 191.95 & 111 & 21,306 & 1260821 & 1.209 & .887 & .113 \\
\hline 514 & -- & -- & -- & -- & -- & -- & -- & -- & -- & -- & -- & -- & -- & -- \\
\hline 515 & 223.39 & 72 & 16,084 & 550659 & -.720 & .236 & .236 & 554.62 & 29 & 16,084 & 247196 & .559 & .712 & .288 \\
\hline 516 & 343.88 & 8 & 2,751 & 13821 & 1.254 & .895 & .105 & 1375.50 & 2 & 2,751 & 2924 & .154 & .561 & .439 \\
\hline 517 & 219.78 & 89 & 19,560 & 882444 & .226 & .589 & .411 & 489.00 & 40 & 19,560 & 435456 & 1.239 & .892 & .108 \\
\hline 518 & 123.53 & 297 & 36,688 & 5598504 & .824 & .795 & .205 & 286.63 & 128 & 36,688 & 2310916 & -.310 & .378 & .378 \\
\hline 519 & 130.78 & 59 & 7,716 & 251389 & 1.389 & .918 & .082 & 308.64 & 25 & 7,716 & 104564 & .729 & .767 & .233 \\
\hline 520 & 167.50 & 4 & 670 & 1005 & -.866 & .193 & .193 & 670.00 & 1 & 670 & 641 & 1.582 & .943 & .057 \\
\hline 521 & 45.93 & 388 & 17,822 & 3479285 & .215 & .585 & .415 & 90.47 & 197 & 17,822 & 1834622 & 1.096 & .863 & .137 \\
\hline 522 & 86.07 & 86 & 7,402 & 370391 & 2.630 & .996 & .004 & 189.80 & 39 & 7,402 & 155320 & .823 & .795 & .205 \\
\hline 523 & 140.89 & 27 & 3,804 & 60708 & 1.639 & .949 & .051 & 237.75 & 16 & 3,804 & 36191 & 1.311 & .905 & .095 \\
\hline 524 & 608.75 & 4 & 2,435 & 4812 & -.041 & .484 & .484 & -- & -- & -- & -- & -- & -- & -- \\
\hline 525 & -- & -- & -- & -- & -- & -- & -- & -- & -- & -- & -- & -- & -- & -- \\
\hline 526 & 115.06 & 283 & 32,561 & 4606957 & -0.003 & .499 & .499 & 206.08 & 158 & 32,561 & 2538262 & -0.288 & .387 & .387 \\
\hline 527 & 36.37 & 239 & 8,692 & 1144122 & 2.718 & .997 & .003 & 67.38 & 129 & 8,692 & 617864 & 2.008 & .978 & .022 \\
\hline 528 & 36.29 & 87 & 3,157 & 130251 & -.833 & .203 & .203 & 63.14 & 50 & 3,157 & 78968 & .007 & .503 & .497 \\
\hline
\end{tabular}


Table 6. Summary of interoccurrence intervals for daily precipitation thresholds of 1.5 and 2.0 inches-Continued

\begin{tabular}{|c|c|c|c|c|c|c|c|c|c|c|c|c|c|c|}
\hline \multirow[b]{2}{*}{$\begin{array}{l}\text { Seq. } \\
\text { no. }\end{array}$} & \multicolumn{7}{|c|}{ Daily precipitation threshold of 1.5 inches and greater } & \multicolumn{7}{|c|}{ Daily precipitation threshold of 2.0 inches and greater } \\
\hline & $\begin{array}{l}\text { Mean } \\
\text { inter- } \\
\text { occur- } \\
\text { rence } \\
\text { interval } \\
\text { (days) }\end{array}$ & $\begin{array}{l}\text { Total } \\
\text { no. of } \\
\text { events }\end{array}$ & $\begin{array}{l}\text { Total } \\
\text { no. of } \\
\text { days } \\
\text { avail- } \\
\text { able }\end{array}$ & $\begin{array}{l}\text { Sigma } \\
\mathrm{S}\end{array}$ & $\begin{array}{c}\text { Stan- } \\
\text { dard } \\
\text { normal } \\
\text { variate }\end{array}$ & $\begin{array}{l}\text { Non- } \\
\text { ex- } \\
\text { ceed- } \\
\text { ance } \\
\text { prob- } \\
\text { ability }\end{array}$ & $\begin{array}{c}\mathrm{p}- \\
\text { value }\end{array}$ & $\begin{array}{l}\text { Mean } \\
\text { inter- } \\
\text { occur- } \\
\text { rence } \\
\text { interval } \\
\text { (days) }\end{array}$ & $\begin{array}{c}\text { Total } \\
\text { no. of } \\
\text { events }\end{array}$ & $\begin{array}{l}\text { Total } \\
\text { no. of } \\
\text { days } \\
\text { avail- } \\
\text { able }\end{array}$ & $\begin{array}{l}\text { Sigma } \\
\mathrm{S}\end{array}$ & $\begin{array}{l}\text { Stan- } \\
\text { dard } \\
\text { normal } \\
\text { variate }\end{array}$ & $\begin{array}{l}\text { Non- } \\
\text { ex- } \\
\text { ceed- } \\
\text { ance } \\
\text { prob- } \\
\text { ability }\end{array}$ & $\begin{array}{c}\mathrm{p}- \\
\text { value }\end{array}$ \\
\hline 529 & 57.92 & 200 & 11,583 & 1276963 & 2.509 & .994 & .006 & 104.35 & 111 & 11,583 & 710558 & 1.922 & .973 & .027 \\
\hline 530 & 47.66 & 716 & 34,124 & 12739084 & 1.983 & .976 & .024 & 92.48 & 369 & 34,124 & 6559215 & 1.392 & .918 & .082 \\
\hline 531 & 63.00 & 101 & 6,363 & 341049 & 1.068 & .857 & .143 & 109.71 & 58 & 6,363 & 199405 & 1.064 & .856 & .144 \\
\hline 532 & 87.30 & 413 & 36,056 & 7627113 & .858 & .805 & .195 & 183.03 & 197 & 36,056 & 3610461 & .404 & .657 & .343 \\
\hline 533 & 220.15 & 117 & 25,757 & 1394414 & -1.397 & .081 & .081 & 415.44 & 62 & 25,757 & 764298 & -.584 & .280 & .280 \\
\hline 534 & 98.60 & 10 & 986 & 4410 & -.578 & .282 & .282 & 328.67 & 3 & 986 & 1926 & .907 & .818 & .182 \\
\hline 535 & 72.84 & 576 & 41,954 & 11975006 & -.371 & .355 & .355 & 140.31 & 299 & 41,954 & 6101876 & -.813 & .208 & .208 \\
\hline 536 & 75.50 & 2 & 151 & 48 & -1.671 & .047 & .047 & -- & -- & -- & -- & -- & -- & -- \\
\hline 537 & 81.29 & 392 & 31,864 & 6532502 & 1.577 & .943 & .057 & 168.59 & 189 & 31,864 & 3081298 & .555 & .710 & .290 \\
\hline 538 & 120.53 & 19 & 2,290 & 18431 & -1.154 & .124 & .124 & 190.83 & 12 & 2,290 & 10566 & -1.386 & .083 & .083 \\
\hline 539 & 132.39 & 149 & 19,726 & 1361803 & -1.551 & .061 & .061 & 308.22 & 64 & 19,726 & 612100 & -.420 & .337 & .337 \\
\hline 540 & 83.30 & 20 & 1,666 & 20308 & 1.696 & .955 & .045 & 151.46 & 11 & 1,666 & 10183 & .640 & .739 & .261 \\
\hline 541 & 65.98 & 531 & 35,035 & 8950532 & -1.507 & .066 & .066 & 122.07 & 287 & 35,035 & 4790591 & -1.383 & .083 & .083 \\
\hline 542 & 74.00 & 16 & 1,184 & 9132 & -.249 & .402 & .402 & 118.40 & 10 & 1,184 & 6149 & .212 & .584 & .416 \\
\hline 543 & 270.10 & 31 & 8,373 & 129092 & -.051 & .480 & .480 & 523.31 & 16 & 8,373 & 82680 & 1.623 & .948 & .052 \\
\hline 544 & 82.85 & 354 & 29,330 & 5247177 & .350 & .637 & .363 & 143.78 & 204 & 29,330 & 2995261 & .030 & .512 & .488 \\
\hline 545 & 113.47 & 74 & 8,397 & 314760 & .195 & .577 & .423 & 209.93 & 40 & 8,397 & 145772 & -1.446 & .074 & .074 \\
\hline 546 & 49.22 & 638 & 31,403 & 10165564 & .646 & .741 & .259 & 85.33 & 368 & 31,403 & 5975493 & 1.135 & .872 & .128 \\
\hline 547 & 104.44 & 169 & 17,650 & 1412539 & -1.191 & .117 & .117 & 191.85 & 92 & 17,650 & 770160 & -.854 & .197 & .197 \\
\hline 548 & 47.51 & 276 & 13,112 & 1875409 & 1.049 & .853 & .147 & 85.14 & 154 & 13,112 & 1013172 & .076 & .530 & .470 \\
\hline 549 & -- & -- & -- & -- & -- & -- & -- & -- & -- & -- & -- & -- & -- & -- \\
\hline 550 & 41.90 & 20 & 838 & 8944 & .521 & .699 & .301 & 93.11 & 9 & 838 & 4115 & .474 & .682 & .318 \\
\hline 551 & 53.78 & 102 & 5,486 & 302053 & 1.392 & .918 & .082 & 94.59 & 58 & 5,486 & 169673 & .877 & .810 & .190 \\
\hline 552 & 50.17 & 390 & 19,567 & 4085051 & 2.416 & .992 & .008 & 93.62 & 209 & 19,567 & 2290779 & 3.013 & .999 & .001 \\
\hline 553 & -- & -- & -- & -- & -- & -- & -- & -- & -- & -- & -- & -- & -- & -- \\
\hline 554 & 44.57 & 267 & 11,901 & 1603368 & .260 & .603 & .397 & 82.65 & 144 & 11,901 & 863549 & .162 & .564 & .436 \\
\hline 555 & -- & -- & -- & -- & -- & -- & -- & -- & -- & -- & -- & -- & -- & -- \\
\hline 556 & 39.83 & 502 & 19,994 & 5376936 & 2.772 & .997 & .003 & 70.15 & 285 & 19,994 & 3075740 & 2.326 & .990 & .010 \\
\hline 557 & 54.80 & 5 & 274 & 635 & -.283 & .389 & .389 & 91.33 & 3 & 274 & 396 & -.110 & .456 & .456 \\
\hline 558 & 40.94 & 52 & 2,129 & 59040 & .832 & .797 & .203 & 78.85 & 27 & 2,129 & 29186 & .139 & .555 & .445 \\
\hline 559 & 97.33 & 6 & 584 & 1374 & -.915 & .180 & .180 & 146.00 & 4 & 584 & 1112 & -.166 & .434 & .434 \\
\hline 560 & 244.00 & 1 & 244 & 54 & -.965 & .167 & .167 & -- & -- & -- & -- & -- & -- & -- \\
\hline 561 & 40.71 & 437 & 17,792 & 4154022 & 2.482 & .993 & .007 & 77.36 & 230 & 17,792 & 2298751 & 3.244 & .999 & .001 \\
\hline 562 & 40.97 & 505 & 20,691 & 5535609 & 2.318 & .990 & .010 & 80.20 & 258 & 20,691 & 2800173 & 1.366 & .914 & .086 \\
\hline 563 & 44.11 & 383 & 16,893 & 3423390 & 1.974 & .976 & .024 & 72.19 & 234 & 16,893 & 1951135 & -.340 & .367 & .367 \\
\hline 564 & 46.07 & 412 & 18,979 & 4150241 & 2.163 & .985 & .015 & 93.03 & 204 & 18,979 & 2127756 & 2.452 & .993 & .007 \\
\hline 565 & 39.49 & 124 & 4,897 & 324865 & 1.350 & .911 & .089 & 63.60 & 77 & 4,897 & 211576 & 1.858 & .968 & .032 \\
\hline 566 & 42.44 & 353 & 14,981 & 2830420 & 2.293 & .989 & .011 & 76.05 & 197 & 14,981 & 1522338 & .770 & .779 & .221 \\
\hline 567 & 86.20 & 102 & 8,792 & 583656 & 5.277 & 0 & 0 & 151.59 & 58 & 8,792 & 331088 & 3.938 & 1.000 & 0 \\
\hline 568 & 41.95 & 377 & 15,815 & 3193436 & 2.395 & .992 & .008 & 85.03 & 186 & 15,815 & 1585085 & 1.836 & .967 & .033 \\
\hline 569 & 55.53 & 166 & 9,218 & 728435 & -1.069 & .143 & .143 & 97.03 & 95 & 9,218 & 413382 & -.944 & .173 & .173 \\
\hline 570 & 29.10 & 125 & 3,637 & 239424 & 1.032 & .849 & .151 & 46.63 & 78 & 3,637 & 150781 & .964 & .832 & .168 \\
\hline 571 & 45.79 & 396 & 18,133 & 3984926 & 3.788 & 1.000 & 0 & 90.67 & 200 & 18,133 & 2036025 & 3.009 & .999 & .001 \\
\hline 572 & 69.02 & 185 & 12,769 & 1127909 & -1.062 & .144 & .144 & 138.79 & 92 & 12769 & 599324 & .338 & .632 & .368 \\
\hline 573 & 129.26 & 39 & 5,041 & 96976 & -0.146 & .442 & .442 & 265.32 & 19 & 5,041 & 47640 & -0.039 & .484 & .484 \\
\hline 574 & 46.90 & 238 & 11,163 & 1419545 & 1.833 & .967 & .033 & 74.92 & 149 & 11,163 & 948182 & 2.963 & .998 & .002 \\
\hline 575 & 101.75 & 172 & 17,501 & 1521810 & .252 & .600 & .400 & 203.50 & 86 & 17501 & 800845 & 1.031 & .849 & .151 \\
\hline 576 & 75.35 & 48 & 3,617 & 84973 & -.254 & .400 & .400 & 109.61 & 33 & 3,617 & 56946 & -.456 & .324 & .324 \\
\hline
\end{tabular}


Table 6. Summary of interoccurrence intervals for daily precipitation thresholds of 1.5 and 2.0 inches—Continued

\begin{tabular}{|c|c|c|c|c|c|c|c|c|c|c|c|c|c|c|}
\hline \multirow[b]{2}{*}{$\begin{array}{l}\text { Seq. } \\
\text { no. }\end{array}$} & \multicolumn{7}{|c|}{ Daily precipitation threshold of 1.5 inches and greater } & \multicolumn{7}{|c|}{ Daily precipitation threshold of 2.0 inches and greater } \\
\hline & $\begin{array}{l}\text { Mean } \\
\text { inter- } \\
\text { occur- } \\
\text { rence } \\
\text { interval } \\
\text { (days) }\end{array}$ & $\begin{array}{c}\text { Total } \\
\text { no. of } \\
\text { events }\end{array}$ & $\begin{array}{l}\text { Total } \\
\text { no. of } \\
\text { days } \\
\text { avail- } \\
\text { able }\end{array}$ & $\begin{array}{l}\text { Sigma } \\
\mathrm{S}\end{array}$ & $\begin{array}{c}\text { Stan- } \\
\text { dard } \\
\text { normal } \\
\text { variate }\end{array}$ & $\begin{array}{l}\text { Non- } \\
\text { ex- } \\
\text { ceed- } \\
\text { ance } \\
\text { prob- } \\
\text { ability }\end{array}$ & $\begin{array}{c}\mathrm{p}- \\
\text { value }\end{array}$ & $\begin{array}{l}\text { Mean } \\
\text { inter- } \\
\text { occur- } \\
\text { rence } \\
\text { interval } \\
\text { (days) }\end{array}$ & $\begin{array}{l}\text { Total } \\
\text { no. of } \\
\text { events }\end{array}$ & $\begin{array}{l}\text { Total } \\
\text { no. of } \\
\text { days } \\
\text { avail- } \\
\text { able }\end{array}$ & $\begin{array}{l}\text { Sigma } \\
\mathrm{S}\end{array}$ & $\begin{array}{c}\text { Stan- } \\
\text { dard } \\
\text { normal } \\
\text { variate }\end{array}$ & $\begin{array}{l}\text { Non- } \\
\text { ex- } \\
\text { ceed- } \\
\text { ance } \\
\text { prob- } \\
\text { ability }\end{array}$ & $\begin{array}{c}\mathrm{p}- \\
\text { value }\end{array}$ \\
\hline 577 & 80.73 & 230 & 18,568 & 2367091 & 2.851 & .998 & .002 & 141.74 & 131 & 18,568 & 1374639 & 2.583 & .995 & .005 \\
\hline 578 & 47.74 & 422 & 20,145 & 4293089 & .356 & .639 & .361 & 95.47 & 211 & 20,145 & 2237755 & 1.331 & .908 & .092 \\
\hline 579 & 81.92 & 172 & 14,090 & 1198770 & -.243 & .404 & .404 & 156.56 & 90 & 14,090 & 587509 & -1.206 & .114 & .114 \\
\hline 580 & 73.00 & 1 & 73 & 57 & .973 & .835 & .165 & -- & -- & -- & -- & -- & -- & -- \\
\hline 581 & 33.83 & 70 & 2,368 & 82222 & -.115 & .454 & .454 & 64.00 & 37 & 2,368 & 43995 & .045 & .518 & .482 \\
\hline 582 & 73.10 & 253 & 18,495 & 2452698 & 1.332 & .908 & .092 & 141.18 & 131 & 18,495 & 1366602 & 2.539 & .994 & .006 \\
\hline 583 & 261.00 & 62 & 16,182 & 575087 & 1.997 & .977 & .023 & 522.00 & 31 & 16,182 & 317733 & 2.573 & .995 & .005 \\
\hline 584 & 61.00 & 4 & 244 & 542 & .383 & .649 & .351 & -- & -- & -- & -- & -- & -- & -- \\
\hline 585 & 115.18 & 114 & 13,130 & 702580 & -1.133 & .129 & .129 & 205.16 & 64 & 13,130 & 382910 & -1.229 & .110 & .110 \\
\hline 586 & -- & -- & -- & -- & -- & -- & -- & -- & -- & -- & -- & -- & -- & -- \\
\hline 587 & 70.21 & 52 & 3,651 & 102509 & .998 & .841 & .159 & 135.22 & 27 & 3,651 & 53098 & .696 & .757 & .243 \\
\hline 588 & 92.94 & 92 & 8,550 & 362292 & -1.310 & .095 & .095 & 198.84 & 43 & 8,550 & 169854 & -.863 & .194 & .194 \\
\hline 589 & 58.51 & 112 & 6,553 & 398684 & 1.584 & .943 & .057 & 96.37 & 68 & 6,553 & 243602 & 1.333 & .909 & .091 \\
\hline 590 & 52.86 & 7 & 370 & 1866 & 2.021 & .978 & .022 & 123.33 & 3 & 370 & 827 & 1.470 & .929 & .071 \\
\hline 591 & 57.08 & 64 & 3,653 & 119785 & .343 & .634 & .366 & 117.84 & 31 & 3,653 & 59732 & .530 & .702 & .298 \\
\hline 592 & 83.94 & 263 & 22,076 & 2917305 & .139 & .555 & .445 & 159.97 & 138 & 22,076 & 1472222 & -.682 & .248 & .248 \\
\hline 593 & 48.63 & 319 & 15,514 & 2685192 & 2.634 & .996 & .004 & 100.09 & 155 & 15,514 & 1294899 & 1.660 & .952 & .048 \\
\hline 594 & 48.55 & 121 & 5,874 & 361635 & .336 & .631 & .369 & 96.30 & 61 & 5,874 & 186915 & .586 & .721 & .279 \\
\hline 595 & 99.20 & 93 & 9,226 & 438001 & .350 & .637 & .363 & 205.02 & 45 & 9,226 & 178935 & -1.604 & .054 & .054 \\
\hline 596 & 65.92 & 390 & 25,710 & 5123330 & .750 & .773 & .227 & 147.76 & 174 & 25,710 & 2388432 & 1.549 & .939 & .061 \\
\hline 597 & 37.61 & 547 & 20,570 & 6005927 & 2.736 & .997 & .003 & 65.93 & 312 & 20,570 & 3412787 & 1.944 & .974 & .026 \\
\hline 598 & 37.39 & 28 & 1,047 & 13388 & -.794 & .214 & .214 & 74.79 & 14 & 1,047 & 5881 & -1.280 & .100 & .100 \\
\hline 599 & 135.11 & 131 & 17,699 & 1280131 & 2.067 & .981 & .019 & 256.51 & 69 & 17,699 & 661420 & 1.197 & .884 & .116 \\
\hline 600 & 63.42 & 308 & 19,532 & 3101272 & .943 & .827 & .173 & 111.61 & 175 & 19,532 & 1713560 & .061 & .524 & .476 \\
\hline 601 & 47.46 & 681 & 32,319 & 10889829 & -.472 & .319 & .319 & 95.62 & 338 & 32,319 & 5290585 & -.999 & .159 & .159 \\
\hline 602 & 57.38 & 268 & 15,379 & 2042435 & -.253 & .400 & .400 & 99.86 & 154 & 15,379 & 1177071 & -.129 & .449 & .449 \\
\hline 603 & 63.53 & 98 & 6,226 & 339015 & 1.908 & .972 & .028 & 97.28 & 64 & 6,226 & 215987 & 1.165 & .878 & .122 \\
\hline 604 & 71.38 & 172 & 12,277 & 1101794 & .989 & .839 & .161 & 133.45 & 92 & 12,277 & 591391 & .784 & .783 & .217 \\
\hline 605 & 177.43 & 35 & 6,210 & 115787 & .671 & .749 & .251 & 326.84 & 19 & 6,210 & 71221 & 1.565 & .941 & .059 \\
\hline 606 & 169.87 & 31 & 5,266 & 95983 & 1.697 & .955 & .045 & 239.36 & 22 & 5,266 & 69110 & 1.569 & .942 & .058 \\
\hline 607 & 155.80 & 25 & 3,895 & 58426 & 1.732 & .958 & .042 & 432.78 & 9 & 3,895 & 20122 & .769 & .779 & .221 \\
\hline 608 & 89.29 & 237 & 21,162 & 2468317 & -.419 & .338 & .338 & 162.79 & 130 & 21,162 & 1399777 & .348 & .636 & .364 \\
\hline 609 & 263.18 & 11 & 2,895 & 16205 & .102 & .541 & .459 & 579.00 & 5 & 2,895 & 6899 & -.181 & .428 & .428 \\
\hline 610 & 100.62 & 303 & 30,487 & 4386732 & -1.515 & .065 & .065 & 194.19 & 157 & 30,487 & 2200967 & -1.744 & .041 & .041 \\
\hline 611 & 164.38 & 50 & 8,219 & 218218 & .760 & .776 & .224 & 342.46 & 24 & 8,219 & 110069 & .984 & .837 & .163 \\
\hline 612 & 150.42 & 24 & 3,610 & 56350 & 2.552 & .995 & .005 & 277.69 & 13 & 3,610 & 29443 & 1.591 & .944 & .056 \\
\hline 613 & 68.90 & 10 & 689 & 2348 & -1.744 & .041 & .041 & 98.43 & 7 & 689 & 1457 & -1.814 & .035 & .035 \\
\hline 614 & 145.83 & 23 & 3,354 & 36057 & -.541 & .294 & .294 & 258.00 & 13 & 3,354 & 20383 & -.406 & .342 & .342 \\
\hline 615 & 61.00 & 1 & 61 & 47 & .937 & .826 & .174 & 61.00 & 1 & 61 & 47 & .937 & .826 & .174 \\
\hline 616 & 85.36 & 22 & 1,878 & 19209 & -.570 & .284 & .284 & 104.33 & 18 & 1,878 & 15105 & -.781 & .217 & .217 \\
\hline 617 & 38.96 & 45 & 1,753 & 27472 & -3.526 & 0 & 0 & 67.42 & 26 & 1,753 & 15287 & -2.907 & .002 & .002 \\
\hline 618 & 43.73 & 472 & 20,642 & 5334421 & 3.576 & 1.000 & 0 & 86.37 & 239 & 20,642 & 2707437 & 2.613 & .996 & .004 \\
\hline 619 & 84.15 & 338 & 28,443 & 4640461 & -1.102 & .135 & .135 & 153.75 & 185 & 28,443 & 2508320 & -1.098 & .136 & .136 \\
\hline 620 & 77.44 & 9 & 697 & 3676 & .894 & .814 & .186 & -- & -- & -- & -- & -- & -- & -- \\
\hline 621 & 52.71 & 313 & 16,498 & 2799898 & 2.587 & .995 & .005 & 94.82 & 174 & 16,498 & 1478794 & 0.692 & .755 & .245 \\
\hline 622 & 59.71 & 606 & 36,185 & 11041040 & .299 & .618 & .382 & 113.43 & 319 & 36,185 & 5825553 & .290 & .614 & .386 \\
\hline 623 & 92.05 & 80 & 7,364 & 291267 & -.173 & .431 & .431 & 179.61 & 41 & 7,364 & 143238 & -.567 & .285 & .285 \\
\hline 624 & 48.60 & 40 & 1,944 & 36151 & -.769 & .221 & .221 & 77.76 & 25 & 1,944 & 24962 & .236 & .593 & .407 \\
\hline
\end{tabular}


Table 6. Summary of interoccurrence intervals for daily precipitation thresholds of 1.5 and 2.0 inches-Continued

\begin{tabular}{|c|c|c|c|c|c|c|c|c|c|c|c|c|c|c|}
\hline \multirow[b]{2}{*}{$\begin{array}{l}\text { Seq. } \\
\text { no. }\end{array}$} & \multicolumn{7}{|c|}{ Daily precipitation threshold of 1.5 inches and greater } & \multicolumn{7}{|c|}{ Daily precipitation threshold of 2.0 inches and greater } \\
\hline & $\begin{array}{l}\text { Mean } \\
\text { inter- } \\
\text { occur- } \\
\text { rence } \\
\text { interval } \\
\text { (days) }\end{array}$ & $\begin{array}{l}\text { Total } \\
\text { no. of } \\
\text { events }\end{array}$ & $\begin{array}{l}\text { Total } \\
\text { no. of } \\
\text { days } \\
\text { avail- } \\
\text { able }\end{array}$ & $\begin{array}{c}\text { Sigma } \\
\mathrm{S}\end{array}$ & $\begin{array}{c}\text { Stan- } \\
\text { dard } \\
\text { normal } \\
\text { variate }\end{array}$ & $\begin{array}{l}\text { Non- } \\
\text { ex- } \\
\text { ceed- } \\
\text { ance } \\
\text { prob- } \\
\text { ability }\end{array}$ & $\begin{array}{c}\text { p- } \\
\text { value }\end{array}$ & $\begin{array}{l}\text { Mean } \\
\text { inter- } \\
\text { occur- } \\
\text { rence } \\
\text { interval } \\
\text { (days) }\end{array}$ & $\begin{array}{c}\text { Total } \\
\text { no. of } \\
\text { events }\end{array}$ & $\begin{array}{l}\text { Total } \\
\text { no. of } \\
\text { days } \\
\text { avail- } \\
\text { able }\end{array}$ & $\begin{array}{l}\text { Sigma } \\
\mathrm{S}\end{array}$ & $\begin{array}{l}\text { Stan- } \\
\text { dard } \\
\text { normal } \\
\text { variate }\end{array}$ & $\begin{array}{l}\text { Non- } \\
\text { ex- } \\
\text { ceed- } \\
\text { ance } \\
\text { prob- } \\
\text { ability }\end{array}$ & $\begin{array}{c}p- \\
\text { value }\end{array}$ \\
\hline 625 & 73.24 & 136 & 9,960 & 726421 & 1.466 & .929 & .071 & 121.46 & 82 & 9,960 & 440936 & 1.251 & .895 & .105 \\
\hline 626 & 63.69 & 170 & 10,827 & 888638 & -.777 & .219 & .219 & 130.45 & 83 & 10,827 & 452714 & .119 & .547 & .453 \\
\hline 627 & 492.54 & 24 & 11,821 & 130297 & -.691 & .245 & .245 & 1182.10 & 10 & 11,821 & 65350 & .579 & .719 & .281 \\
\hline 628 & 555.89 & 9 & 5,003 & 17400 & -1.180 & .119 & .119 & 1667.67 & 3 & 5,003 & 2808 & -1.878 & .030 & .030 \\
\hline 629 & 91.85 & 288 & 26,452 & 3824250 & .117 & .547 & .453 & 164.30 & 161 & 26,452 & 2191702 & .643 & .740 & .260 \\
\hline 630 & 79.38 & 125 & 9,923 & 631030 & .339 & .633 & .367 & 141.76 & 70 & 9,923 & 333133 & -.591 & .277 & .277 \\
\hline 631 & 83.16 & 131 & 10,894 & 729641 & .447 & .672 & .328 & 162.60 & 67 & 10,894 & 368927 & .155 & .561 & .439 \\
\hline 632 & 70.44 & 113 & 7,960 & 467633 & .733 & .768 & .232 & 139.65 & 57 & 7,960 & 252643 & 1.486 & .931 & .069 \\
\hline 633 & 63.04 & 27 & 1,702 & 23556 & .227 & .590 & .410 & 141.83 & 12 & 1,702 & 9390 & -.483 & .315 & .315 \\
\hline 634 & 290.00 & 1 & 290 & 150 & .060 & .524 & .476 & -- & -- & -- & -- & -- & -- & -- \\
\hline 635 & 80.85 & 332 & 26,842 & 4673067 & 1.539 & .938 & .062 & 133.54 & 201 & 26,842 & 2810705 & 1.029 & .848 & .152 \\
\hline 636 & 39.06 & 531 & 20,742 & 5736844 & 1.666 & .952 & .048 & 67.34 & 308 & 20,742 & 3226372 & .306 & .620 & .380 \\
\hline 637 & 143.59 & 170 & 24,410 & 2166865 & 1.002 & .842 & .158 & 317.01 & 77 & 24,410 & 1022631 & 1.340 & .910 & .090 \\
\hline 638 & 129.24 & 86 & 11,115 & 518344 & 1.358 & .913 & .087 & 277.88 & 40 & 11,115 & 237098 & .729 & .767 & .233 \\
\hline 639 & 87.07 & 60 & 5,224 & 157008 & .025 & .510 & .490 & 163.25 & 32 & 5,224 & 84264 & .080 & .532 & .468 \\
\hline 640 & 34.30 & 171 & 5,865 & 544065 & 1.925 & .973 & .027 & 56.39 & 104 & 5,865 & 321306 & .946 & .828 & .172 \\
\hline 641 & 170.86 & 7 & 1,196 & 4470 & .311 & .622 & .378 & 398.67 & 3 & 1,196 & 2184 & .652 & .743 & .257 \\
\hline 642 & -- & -- & -- & -- & -- & -- & -- & -- & -- & -- & -- & -- & -- & -- \\
\hline 643 & 58.56 & 561 & 32,853 & 9314895 & .444 & .671 & .329 & 107.36 & 306 & 32,853 & 5238906 & 1.280 & .900 & .100 \\
\hline 644 & 117.76 & 21 & 2,473 & 29578 & 1.104 & .865 & .135 & 247.30 & 10 & 2,473 & 13921 & .689 & .755 & .245 \\
\hline 645 & 117.27 & 234 & 27,442 & 3312271 & .838 & .799 & .201 & 194.62 & 141 & 27,442 & 1905208 & -.313 & .377 & .377 \\
\hline 646 & 828.60 & 25 & 20,715 & 291263 & 1.081 & .860 & .140 & 3452.50 & 6 & 20,715 & 68275 & .419 & .662 & .338 \\
\hline 647 & 75.58 & 12 & 907 & 6738 & 1.429 & .923 & .077 & 113.38 & 8 & 907 & 4486 & 1.159 & .877 & .123 \\
\hline 648 & 57.97 & 33 & 1,913 & 34900 & 1.051 & .853 & .147 & 119.56 & 16 & 1,913 & 16692 & .628 & .735 & .265 \\
\hline 649 & 510.63 & 16 & 8,170 & 42421 & -2.432 & .008 & .008 & 2042.50 & 4 & 8,170 & 10834 & -1.167 & .122 & .122 \\
\hline 650 & 99.25 & 145 & 14,391 & 1077660 & .686 & .754 & .246 & 208.57 & 69 & 14,391 & 502732 & .181 & .572 & .428 \\
\hline 651 & 254.73 & 11 & 2,802 & 17372 & .731 & .768 & .232 & 560.40 & 5 & 2,802 & 9361 & 1.303 & .904 & .096 \\
\hline 652 & 99.75 & 8 & 798 & 3092 & -.154 & .439 & .439 & 199.50 & 4 & 798 & 1853 & .558 & .711 & .289 \\
\hline 653 & 77.36 & 14 & 1,083 & 7120 & -.394 & .347 & .347 & 120.33 & 9 & 1,083 & 4195 & -.723 & .235 & .235 \\
\hline 654 & 152.22 & 68 & 10,351 & 397091 & 1.833 & .967 & .033 & 333.90 & 31 & 10,351 & 185104 & 1.483 & .931 & .069 \\
\hline 655 & 79.47 & 36 & 2,861 & 47427 & -.822 & .206 & .206 & 178.81 & 16 & 2,861 & 18443 & -1.346 & .089 & .089 \\
\hline 656 & 43.92 & 108 & 4,743 & 253451 & -.188 & .426 & .426 & 84.70 & 56 & 4,743 & 127565 & -.511 & .305 & .305 \\
\hline 657 & 124.68 & 93 & 11,595 & 551703 & .388 & .651 & .349 & 241.56 & 48 & 11,595 & 297840 & .844 & .800 & .200 \\
\hline 658 & 98.78 & 58 & 5,729 & 160227 & -.470 & .319 & .319 & 184.81 & 31 & 5,729 & 79238 & -1.038 & .150 & .150 \\
\hline 659 & 81.65 & 34 & 2,776 & 45173 & -.432 & .333 & .333 & 173.50 & 16 & 2,776 & 21354 & -.266 & .395 & .395 \\
\hline 660 & 57.89 & 83 & 4,805 & 200992 & .125 & .550 & .450 & 94.22 & 51 & 4,805 & 125723 & .323 & .627 & .373 \\
\hline 661 & 51.49 & 55 & 2,832 & 75271 & -.430 & .334 & .334 & 83.29 & 34 & 2,832 & 48034 & -.023 & .491 & .491 \\
\hline 662 & 78.43 & 70 & 5,490 & 209983 & 1.345 & .911 & .089 & 171.56 & 32 & 5,490 & 90847 & .335 & .631 & .369 \\
\hline 663 & 183.27 & 178 & 32,622 & 3034039 & 1.040 & .851 & .149 & 418.23 & 78 & 32,622 & 1263437 & -.106 & .458 & .458 \\
\hline 664 & 90.23 & 411 & 37,084 & 7476123 & -.667 & .253 & .253 & 188.24 & 197 & 37,084 & 3565217 & -.583 & .280 & .280 \\
\hline 665 & 206.88 & 91 & 18,826 & 872066 & .299 & .617 & .383 & 470.65 & 40 & 18,826 & 337707 & -1.129 & .129 & .129 \\
\hline 666 & 324.75 & 4 & 1,299 & 2591 & -.009 & .496 & .496 & 433.00 & 3 & 1,299 & 2362 & .637 & .738 & .262 \\
\hline 667 & 113.13 & 121 & 13,689 & 754033 & -1.706 & .044 & .044 & 220.79 & 62 & 13,689 & 348840 & -2.427 & .008 & .008 \\
\hline 668 & 172.47 & 45 & 7,761 & 151455 & -1.542 & .062 & .062 & 298.50 & 26 & 7,761 & 81805 & -1.671 & .047 & .047 \\
\hline 669 & 67.57 & 84 & 5,676 & 241379 & 0.199 & .579 & .421 & 172.00 & 33 & 5,676 & 103026 & 0.996 & .840 & .160 \\
\hline 670 & 132.58 & 64 & 8,485 & 259813 & -.597 & .275 & .275 & 249.56 & 34 & 8,485 & 135608 & -.605 & .273 & .273 \\
\hline 671 & 61.03 & 308 & 18,797 & 3134779 & 2.521 & .994 & .006 & 114.62 & 164 & 18,797 & 1658790 & 1.690 & .954 & .046 \\
\hline 672 & 113.79 & 170 & 19,344 & 1666502 & .306 & .620 & .380 & 233.06 & 83 & 19,344 & 788638 & -.278 & .391 & .391 \\
\hline
\end{tabular}


Table 6. Summary of interoccurrence intervals for daily precipitation thresholds of 1.5 and 2.0 inches—Continued

\begin{tabular}{|c|c|c|c|c|c|c|c|c|c|c|c|c|c|c|}
\hline \multirow[b]{2}{*}{$\begin{array}{l}\text { Seq. } \\
\text { no. }\end{array}$} & \multicolumn{7}{|c|}{ Daily precipitation threshold of 1.5 inches and greater } & \multicolumn{7}{|c|}{ Daily precipitation threshold of 2.0 inches and greater } \\
\hline & $\begin{array}{c}\text { Mean } \\
\text { inter- } \\
\text { occur- } \\
\text { rence } \\
\text { interval } \\
\text { (days) }\end{array}$ & $\begin{array}{c}\text { Total } \\
\text { no. of } \\
\text { events }\end{array}$ & $\begin{array}{c}\text { Total } \\
\text { no. of } \\
\text { days } \\
\text { avail- } \\
\text { able }\end{array}$ & $\begin{array}{c}\text { Sigma } \\
\mathrm{S}\end{array}$ & $\begin{array}{l}\text { Stan- } \\
\text { dard } \\
\text { normal } \\
\text { variate }\end{array}$ & $\begin{array}{l}\text { Non- } \\
\text { ex- } \\
\text { ceed- } \\
\text { ance } \\
\text { prob- } \\
\text { ability }\end{array}$ & $\begin{array}{c}\mathrm{p}- \\
\text { value }\end{array}$ & $\begin{array}{l}\text { Mean } \\
\text { inter- } \\
\text { occur- } \\
\text { rence } \\
\text { interval } \\
\text { (days) }\end{array}$ & $\begin{array}{c}\text { Total } \\
\text { no. of } \\
\text { events }\end{array}$ & $\begin{array}{l}\text { Total } \\
\text { no. of } \\
\text { days } \\
\text { avail- } \\
\text { able }\end{array}$ & $\begin{array}{c}\text { Sigma } \\
\mathrm{S}\end{array}$ & $\begin{array}{l}\text { Stan- } \\
\text { dard } \\
\text { normal } \\
\text { variate }\end{array}$ & $\begin{array}{l}\text { Non- } \\
\text { ex- } \\
\text { ceed- } \\
\text { ance } \\
\text { prob- } \\
\text { ability }\end{array}$ & $\begin{array}{c}p- \\
\text { value }\end{array}$ \\
\hline 673 & 119.15 & 46 & 5,481 & 127088 & .096 & .538 & .462 & 249.14 & 22 & 5,481 & 61519 & .166 & .566 & .434 \\
\hline 674 & 74.50 & 24 & 1,788 & 19765 & -.669 & .252 & .252 & 111.75 & 16 & 1,788 & 13526 & -.377 & .353 & .353 \\
\hline 675 & 85.45 & 31 & 2,649 & 38215 & -.668 & .252 & .252 & 139.42 & 19 & 2,649 & 22912 & -.676 & .250 & .250 \\
\hline 676 & 63.92 & 52 & 3,324 & 77514 & -1.288 & .099 & .099 & 138.50 & 24 & 3,324 & 32933 & -1.480 & .070 & .070 \\
\hline 677 & 190.23 & 108 & 20,545 & 1116512 & .115 & .546 & .454 & 321.02 & 64 & 20,545 & 731139 & 1.553 & .940 & .060 \\
\hline 678 & 157.00 & 1 & 157 & 51 & -.607 & .272 & .272 & -- & -- & -- & -- & -- & -- & -- \\
\hline 679 & 202.05 & 112 & 22,630 & 1107434 & -2.312 & .010 & .010 & 411.46 & 55 & 22,630 & 557542 & -1.337 & .091 & .091 \\
\hline 680 & 75.17 & 58 & 4,360 & 134624 & .854 & .803 & .197 & 145.33 & 30 & 4,360 & 68243 & .412 & .660 & .340 \\
\hline 681 & 52.38 & 16 & 838 & 6065 & -.660 & .255 & .255 & 93.11 & 9 & 838 & 3116 & -.903 & .183 & .183 \\
\hline 682 & 89.33 & 57 & 5,092 & 144870 & -.023 & .491 & .491 & 181.86 & 28 & 5,092 & 64208 & -.910 & .181 & .181 \\
\hline 683 & 60.09 & 203 & 12,199 & 1273135 & .696 & .757 & .243 & 115.09 & 106 & 12,199 & 668884 & .616 & .731 & .269 \\
\hline 684 & 67.78 & 229 & 15,522 & 1864105 & 1.281 & .900 & .100 & 136.16 & 114 & 15,522 & 884905 & .003 & .501 & .499 \\
\hline 685 & 39.98 & 881 & 35,218 & 15843391 & 1.093 & .863 & .137 & 66.45 & 530 & 35,218 & 9584355 & 1.075 & .859 & .141 \\
\hline 686 & 88.59 & 66 & 5,847 & 230424 & 2.733 & .997 & .003 & 167.06 & 35 & 5,847 & 112615 & 1.031 & .849 & .151 \\
\hline 687 & 73.71 & 21 & 1,548 & 14516 & -.849 & .198 & .198 & 309.60 & 5 & 1,548 & 4108 & .238 & .594 & .406 \\
\hline 688 & 46.74 & 96 & 4,487 & 215636 & .021 & .508 & .492 & 77.36 & 58 & 4,487 & 128301 & -.185 & .427 & .427 \\
\hline 689 & 67.03 & 88 & 5,899 & 279037 & 1.220 & .889 & .111 & 111.30 & 53 & 5,899 & 166004 & .781 & .783 & .218 \\
\hline 690 & 49.71 & 252 & 12,527 & 1591511 & .228 & .590 & .410 & 92.11 & 136 & 12,527 & 849535 & -.055 & .478 & .478 \\
\hline 691 & 43.00 & 426 & 18,319 & 4250130 & 3.190 & .999 & .001 & 76.65 & 239 & 18,319 & 2309268 & 1.470 & .929 & .071 \\
\hline 692 & 73.98 & 254 & 18,792 & 2749215 & 4.194 & 1.000 & 0 & 147.97 & 127 & 18,792 & 1401842 & 3.411 & 1.000 & 0 \\
\hline 693 & 158.37 & 121 & 19,163 & 1229790 & 1.157 & .876 & .124 & 407.72 & 47 & 19,163 & 463399 & .345 & .635 & .365 \\
\hline 694 & 66.98 & 109 & 7,301 & 384560 & -.607 & .272 & .272 & 135.20 & 54 & 7,301 & 174130 & -1.485 & .069 & .069 \\
\hline 695 & 201.96 & 67 & 13,531 & 441384 & -.372 & .355 & .355 & 563.79 & 24 & 13,531 & 178693 & .853 & .803 & .197 \\
\hline 696 & 194.27 & 66 & 12,822 & 370823 & -1.739 & .041 & .041 & 457.93 & 28 & 12,822 & 128974 & -2.580 & .005 & .005 \\
\hline 697 & 47.08 & 501 & 23,585 & 6077525 & 1.112 & .867 & .133 & 88.00 & 268 & 23,585 & 3301155 & 1.263 & .897 & .103 \\
\hline 698 & 104.53 & 359 & 37,526 & 6867341 & .640 & .739 & .261 & 207.33 & 181 & 37,526 & 3279140 & -.803 & .211 & .211 \\
\hline 699 & 93.12 & 17 & 1,583 & 13505 & .026 & .511 & .489 & 175.89 & 9 & 1,583 & 7610 & .355 & .639 & .361 \\
\hline 700 & 70.98 & 277 & 19,662 & 2821583 & 1.042 & .851 & .149 & 125.24 & 157 & 19,662 & 1502617 & -.574 & .283 & .283 \\
\hline 701 & 61.83 & 29 & 1,793 & 27253 & .450 & .674 & .326 & 94.37 & 19 & 1,793 & 19174 & .949 & .829 & .171 \\
\hline 702 & 134.67 & 9 & 1,212 & 4472 & -.936 & .175 & .175 & 242.40 & 5 & 1,212 & 2052 & -1.250 & .106 & .106 \\
\hline 703 & 71.55 & 66 & 4,722 & 174448 & 1.682 & .954 & .046 & 131.17 & 36 & 4,722 & 100280 & 1.869 & .969 & .031 \\
\hline 704 & 141.33 & 3 & 424 & 381 & -1.203 & .115 & .115 & 424.00 & 1 & 424 & 24 & -1.536 & .062 & .062 \\
\hline 705 & 59.74 & 215 & 12,843 & 1449848 & 1.273 & .899 & .101 & 112.66 & 114 & 12,843 & 791284 & 1.496 & .933 & .067 \\
\hline 706 & 49.63 & 723 & 35,879 & 13672157 & 2.520 & .994 & .006 & 101.07 & 355 & 35,879 & 6759781 & 2.005 & .978 & .022 \\
\hline 707 & 41.19 & 226 & 9,308 & 1169208 & 2.907 & .998 & .002 & 87.81 & 106 & 9,308 & 530592 & 1.347 & .911 & .089 \\
\hline 708 & 183.67 & 88 & 16,163 & 764712 & 1.223 & .889 & .111 & 384.83 & 42 & 16,163 & 348974 & .316 & .624 & .376 \\
\hline 709 & -- & -- & -- & -- & -- & -- & -- & -- & -- & -- & -- & -- & -- & -- \\
\hline 710 & 149.75 & 8 & 1,198 & 3193 & -1.635 & .051 & .051 & 199.67 & 6 & 1,198 & 2460 & -1.339 & .090 & .090 \\
\hline 711 & 134.73 & 110 & 14,820 & 878173 & 1.406 & .920 & .080 & 269.46 & 55 & 14,820 & 454662 & 1.485 & .931 & .069 \\
\hline 712 & 89.40 & 15 & 1,341 & 8369 & -1.126 & .130 & .130 & 268.20 & 5 & 1,341 & 2226 & -1.301 & .097 & .097 \\
\hline 713 & 57.69 & 176 & 10,153 & 938271 & 1.152 & .875 & .125 & 125.35 & 81 & 10,153 & 433268 & .837 & .799 & .201 \\
\hline 714 & 365.00 & 1 & 365 & 17 & -1.571 & .058 & .058 & -- & -- & -- & -- & -- & -- & -- \\
\hline 715 & 146.00 & 10 & 1,460 & 7551 & .188 & .575 & .425 & 730.00 & 2 & 1,460 & 1412 & -.081 & .468 & .468 \\
\hline 716 & 263.17 & 24 & 6,316 & 78646 & .320 & .625 & .375 & 574.18 & 11 & 6,316 & 41358 & 1.095 & .863 & .137 \\
\hline 717 & 211.11 & 157 & 33,144 & 2523987 & -0.649 & .258 & .258 & 466.82 & 71 & 33,144 & 1109051 & -0.838 & 0.201 & .201 \\
\hline 718 & 38.46 & 179 & 6,885 & 580798 & -1.332 & .092 & .092 & 79.14 & 87 & 6,885 & 292995 & -.351 & .363 & .363 \\
\hline 719 & 38.62 & 113 & 4,364 & 244036 & -.189 & .425 & .425 & 68.19 & 64 & 4,364 & 135068 & -.454 & .325 & .325 \\
\hline 720 & 49.08 & 677 & 33,230 & 10770761 & -1.914 & .028 & .028 & 92.31 & 360 & 33,230 & 5694619 & -1.576 & .058 & .058 \\
\hline
\end{tabular}


Table 6. Summary of interoccurrence intervals for daily precipitation thresholds of 1.5 and 2.0 inches-Continued

\begin{tabular}{|c|c|c|c|c|c|c|c|c|c|c|c|c|c|c|}
\hline \multirow[b]{2}{*}{$\begin{array}{l}\text { Seq. } \\
\text { no. }\end{array}$} & \multicolumn{7}{|c|}{ Daily precipitation threshold of 1.5 inches and greater } & \multicolumn{7}{|c|}{ Daily precipitation threshold of 2.0 inches and greater } \\
\hline & $\begin{array}{l}\text { Mean } \\
\text { inter- } \\
\text { occur- } \\
\text { rence } \\
\text { interval } \\
\text { (days) }\end{array}$ & $\begin{array}{c}\text { Total } \\
\text { no. of } \\
\text { events }\end{array}$ & $\begin{array}{l}\text { Total } \\
\text { no. of } \\
\text { days } \\
\text { avail- } \\
\text { able }\end{array}$ & $\begin{array}{l}\text { Sigma } \\
\mathrm{S}\end{array}$ & $\begin{array}{c}\text { Stan- } \\
\text { dard } \\
\text { normal } \\
\text { variate }\end{array}$ & $\begin{array}{l}\text { Non- } \\
\text { ex- } \\
\text { ceed- } \\
\text { ance } \\
\text { prob- } \\
\text { ability }\end{array}$ & $\begin{array}{c}\text { p- } \\
\text { value }\end{array}$ & $\begin{array}{l}\text { Mean } \\
\text { inter- } \\
\text { occur- } \\
\text { rence } \\
\text { interval } \\
\text { (days) }\end{array}$ & $\begin{array}{c}\text { Total } \\
\text { no. of } \\
\text { events }\end{array}$ & $\begin{array}{c}\text { Total } \\
\text { no. of } \\
\text { days } \\
\text { avail- } \\
\text { able }\end{array}$ & $\begin{array}{c}\text { Sigma } \\
\mathrm{S}\end{array}$ & $\begin{array}{l}\text { Stan- } \\
\text { dard } \\
\text { normal } \\
\text { variate }\end{array}$ & $\begin{array}{l}\text { Non- } \\
\text { ex- } \\
\text { ceed- } \\
\text { ance } \\
\text { prob- } \\
\text { ability }\end{array}$ & $\begin{array}{c}\mathrm{p}- \\
\text { value }\end{array}$ \\
\hline 721 & 74.40 & 490 & 36,455 & 8784663 & -.630 & .264 & .264 & 135.52 & 269 & 36,455 & 4972061 & .399 & .655 & .345 \\
\hline 722 & 73.36 & 22 & 1,614 & 17058 & -.319 & .375 & .375 & 134.50 & 12 & 1,614 & 8561 & -.696 & .243 & .243 \\
\hline 723 & 35.19 & 64 & 2,252 & 79111 & 1.355 & .912 & .088 & 56.30 & 40 & 2,252 & 49468 & 1.077 & .859 & .141 \\
\hline 724 & 90.60 & 101 & 9,151 & 478078 & .601 & .726 & .274 & 166.38 & 55 & 9,151 & 256813 & .263 & .604 & .396 \\
\hline 725 & 91.62 & 100 & 9,162 & 450745 & -.278 & .390 & .390 & 190.88 & 48 & 9,162 & 225725 & .319 & .625 & .375 \\
\hline 726 & 77.67 & 15 & 1,165 & 7842 & -.688 & .246 & .246 & 116.50 & 10 & 1,165 & 5417 & -.384 & .351 & .351 \\
\hline 727 & 50.77 & 498 & 25,281 & 6213964 & -.497 & .309 & .309 & 96.13 & 263 & 25,281 & 3163833 & -1.357 & .087 & .087 \\
\hline 728 & 44.04 & 234 & 10,305 & 1328494 & 2.699 & .997 & .003 & 82.44 & 125 & 10,305 & 701150 & 1.716 & .957 & .043 \\
\hline 729 & 64.53 & 100 & 6,453 & 340622 & .965 & .833 & .167 & 115.23 & 56 & 6,453 & 201737 & 1.510 & .935 & .066 \\
\hline 730 & -- & -- & -- & -- & -- & -- & -- & -- & -- & -- & -- & -- & -- & -- \\
\hline 731 & 62.52 & 100 & 6,252 & 286853 & -1.427 & .077 & .077 & 109.68 & 57 & 6,252 & 162232 & -1.171 & .121 & .121 \\
\hline 732 & 119.63 & 57 & 6,819 & 200724 & .430 & .666 & .334 & 235.14 & 29 & 6,819 & 108631 & .920 & .821 & .179 \\
\hline 733 & 344.00 & 1 & 344 & 307 & 1.360 & .913 & .087 & -- & -- & -- & -- & -- & -- & -- \\
\hline 734 & 311.58 & 74 & 23,057 & 886033 & .575 & .717 & .283 & 922.28 & 25 & 23,057 & 286857 & -.041 & .484 & .484 \\
\hline 735 & 197.90 & 10 & 1,979 & 8769 & -.623 & .267 & .267 & 659.67 & 3 & 1,979 & 4269 & 1.314 & .906 & .094 \\
\hline 736 & 103.80 & 78 & 8,096 & 334065 & .888 & .813 & .187 & 238.12 & 34 & 8,096 & 171691 & 2.499 & .994 & .006 \\
\hline 737 & 1323.50 & 2 & 2,647 & 3632 & .912 & .819 & .181 & 2647.00 & 1 & 2,647 & 2018 & .909 & .818 & .182 \\
\hline 738 & 325.75 & 44 & 14,333 & 322063 & .246 & .597 & .403 & 955.53 & 15 & 14,333 & 136573 & 1.814 & .965 & .035 \\
\hline 739 & 383.62 & 13 & 4,987 & 29935 & -.478 & .316 & .316 & 1662.33 & 3 & 4,987 & 4988 & -1.000 & .159 & .159 \\
\hline 740 & -- & -- & -- & -- & -- & -- & -- & -- & -- & -- & -- & -- & -- & -- \\
\hline 741 & 142.00 & 1 & 142 & 33 & -.927 & .177 & .177 & -- & -- & -- & -- & -- & -- & -- \\
\hline 742 & 65.19 & 406 & 26,466 & 5471680 & .644 & .740 & .260 & 117.11 & 226 & 26,466 & 3065312 & .650 & .742 & .258 \\
\hline 743 & 47.24 & 707 & 33,395 & 12543690 & 2.881 & .998 & .002 & 92.76 & 360 & 33,395 & 6245793 & 1.283 & .900 & .100 \\
\hline 744 & 126.66 & 50 & 6,333 & 146779 & -.893 & .186 & .186 & 226.18 & 28 & 6,333 & 75037 & -1.408 & .080 & .080 \\
\hline 745 & 78.54 & 114 & 8,954 & 541905 & 1.142 & .873 & .127 & 172.19 & 52 & 8,954 & 266313 & 1.798 & .964 & .036 \\
\hline 746 & 100.75 & 204 & 20,552 & 2184463 & 1.040 & .851 & .149 & 228.36 & 90 & 20,552 & 1000908 & 1.352 & .912 & .088 \\
\hline 747 & 147.14 & 133 & 19,569 & 1342364 & .630 & .736 & .265 & 305.77 & 64 & 19,569 & 664013 & .837 & .798 & .202 \\
\hline 748 & 49.03 & 672 & 32,946 & 10484068 & -2.376 & .009 & .009 & 79.39 & 415 & 32,946 & 6314049 & -2.696 & .004 & .004 \\
\hline 749 & 71.44 & 190 & 13,573 & 1129738 & -2.957 & .002 & .002 & 122.28 & 111 & 13,573 & 626590 & -3.070 & .001 & .001 \\
\hline 750 & 43.61 & 426 & 18,579 & 4351067 & 3.557 & 1.000 & 0 & 84.07 & 221 & 18,579 & 2338096 & 3.576 & 1.000 & 0 \\
\hline 751 & 56.09 & 136 & 7,628 & 517121 & -.062 & .475 & .475 & 97.80 & 78 & 7,628 & 272603 & -1.280 & .100 & .100 \\
\hline 752 & 608.00 & 2 & 1,216 & 1498 & .568 & .715 & .285 & -- & -- & -- & -- & -- & -- & -- \\
\hline 753 & 67.77 & 30 & 2,033 & 35264 & 1.484 & .931 & .069 & 184.82 & 11 & 2,033 & 13473 & 1.177 & .880 & .120 \\
\hline 754 & 47.69 & 54 & 2,575 & 65859 & -.671 & .251 & .251 & 85.83 & 30 & 2,575 & 32470 & -1.512 & .065 & .065 \\
\hline 755 & 123.56 & 174 & 21,499 & 1786737 & -1.022 & .153 & .153 & 223.95 & 96 & 21,499 & 1000530 & -.517 & .303 & .303 \\
\hline 756 & -- & -- & -- & -- & -- & -- & -- & -- & -- & -- & -- & -- & -- & -- \\
\hline 757 & 135.91 & 43 & 5,844 & 132012 & .576 & .717 & .283 & 365.25 & 16 & 5,844 & 56557 & 1.453 & .927 & .073 \\
\hline 758 & 57.55 & 562 & 32,344 & 9222459 & .605 & .727 & .273 & 100.45 & 322 & 32,344 & 5219309 & .071 & .528 & .472 \\
\hline 759 & 112.35 & 137 & 15,392 & 1149207 & 1.824 & .966 & .034 & 236.80 & 65 & 15,392 & 541140 & 1.142 & .873 & .127 \\
\hline 760 & 124.10 & 172 & 21,345 & 1968656 & 1.646 & .950 & .050 & 215.61 & 99 & 21,345 & 1100753 & .721 & .764 & .236 \\
\hline 761 & 119.74 & 113 & 13,531 & 698511 & -1.589 & .056 & .056 & 198.99 & 68 & 13,531 & 430944 & -.904 & .183 & .183 \\
\hline 762 & 258.07 & 98 & 25,291 & 1202722 & -.506 & .307 & .307 & 683.54 & 37 & 25,291 & 465646 & -.050 & .480 & .480 \\
\hline 763 & 84.57 & 14 & 1,184 & 8591 & .237 & .594 & .406 & 98.67 & 12 & 1,184 & 7293 & .160 & .563 & .437 \\
\hline 764 & 68.30 & 480 & 32,783 & 7751277 & -.563 & .287 & .287 & 142.54 & 230 & 32,783 & 3645865 & -.865 & .194 & .194 \\
\hline 765 & 62.22 & 196 & 12,195 & 1202791 & 0.156 & .562 & .438 & 110.86 & 110 & 12,195 & 719558 & 1.323 & .907 & .093 \\
\hline 766 & 74.28 & 36 & 2,674 & 40683 & -1.608 & .054 & .054 & 116.26 & 23 & 2,674 & 25063 & -1.537 & .062 & .062 \\
\hline 767 & 134.97 & 246 & 33,202 & 4013892 & -.465 & .321 & .321 & 313.23 & 106 & 33,202 & 1949854 & 1.927 & .973 & .027 \\
\hline 768 & 117.80 & 288 & 33,925 & 4862636 & -.136 & .446 & .446 & 224.67 & 151 & 33,925 & 2412530 & -1.237 & .108 & .108 \\
\hline
\end{tabular}


Table 6. Summary of interoccurrence intervals for daily precipitation thresholds of 1.5 and 2.0 inches—Continued

\begin{tabular}{|c|c|c|c|c|c|c|c|c|c|c|c|c|c|c|}
\hline \multirow[b]{2}{*}{$\begin{array}{l}\text { Seq. } \\
\text { no. }\end{array}$} & \multicolumn{7}{|c|}{ Daily precipitation threshold of 1.5 inches and greater } & \multicolumn{7}{|c|}{ Daily precipitation threshold of 2.0 inches and greater } \\
\hline & $\begin{array}{c}\text { Mean } \\
\text { inter- } \\
\text { occur- } \\
\text { rence } \\
\text { interval } \\
\text { (days) }\end{array}$ & $\begin{array}{c}\text { Total } \\
\text { no. of } \\
\text { events }\end{array}$ & $\begin{array}{l}\text { Total } \\
\text { no. of } \\
\text { days } \\
\text { avail- } \\
\text { able }\end{array}$ & $\begin{array}{l}\text { Sigma } \\
\mathrm{S}\end{array}$ & $\begin{array}{l}\text { Stan- } \\
\text { dard } \\
\text { normal } \\
\text { variate }\end{array}$ & $\begin{array}{l}\text { Non- } \\
\text { ex- } \\
\text { ceed- } \\
\text { ance } \\
\text { prob- } \\
\text { ability }\end{array}$ & $\begin{array}{c}\text { p- } \\
\text { value }\end{array}$ & $\begin{array}{l}\text { Mean } \\
\text { inter- } \\
\text { occur- } \\
\text { rence } \\
\text { interval } \\
\text { (days) }\end{array}$ & $\begin{array}{c}\text { Total } \\
\text { no. of } \\
\text { events }\end{array}$ & $\begin{array}{l}\text { Total } \\
\text { no. of } \\
\text { days } \\
\text { avail- } \\
\text { able }\end{array}$ & $\begin{array}{c}\text { Sigma } \\
\mathrm{S}\end{array}$ & $\begin{array}{l}\text { Stan- } \\
\text { dard } \\
\text { normal } \\
\text { variate }\end{array}$ & $\begin{array}{l}\text { Non- } \\
\text { ex- } \\
\text { ceed- } \\
\text { ance } \\
\text { prob- } \\
\text { ability }\end{array}$ & $\begin{array}{c}p- \\
\text { value }\end{array}$ \\
\hline 769 & 105.90 & 20 & 2,118 & 24050 & 1.050 & .853 & .147 & 192.55 & 11 & 2,118 & 13299 & .814 & .792 & .208 \\
\hline 770 & 406.80 & 20 & 8,136 & 103060 & 2.066 & .981 & .019 & 625.85 & 13 & 8,136 & 64272 & 1.345 & .911 & .089 \\
\hline 771 & 206.33 & 18 & 3,714 & 38179 & 1.045 & .852 & .148 & 619.00 & 6 & 3,714 & 11618 & .181 & .572 & .428 \\
\hline 772 & 61.00 & 3 & 183 & 333 & .639 & .739 & .261 & 61.00 & 3 & 183 & 333 & .639 & .739 & .261 \\
\hline 773 & 86.17 & 6 & 517 & 1641 & .246 & .597 & .403 & 129.25 & 4 & 517 & 852 & -.610 & .271 & .271 \\
\hline 774 & 100.55 & 307 & 30,870 & 4862391 & .793 & .786 & .214 & 179.48 & 172 & 30,870 & 2751020 & .823 & .795 & .205 \\
\hline 775 & 76.00 & 4 & 304 & 669 & .348 & .636 & .364 & 152.00 & 2 & 304 & 390 & .693 & .756 & .244 \\
\hline 776 & 65.56 & 107 & 7,015 & 397786 & 1.073 & .858 & .142 & 134.90 & 52 & 7,015 & 200556 & 1.244 & .893 & .107 \\
\hline 777 & 60.11 & 114 & 6,852 & 404820 & .675 & .750 & .250 & 116.14 & 59 & 6,852 & 211631 & .625 & .734 & .266 \\
\hline 778 & 295.42 & 33 & 9,749 & 129201 & -1.958 & .025 & .025 & 609.31 & 16 & 9,749 & 50747 & -2.420 & .008 & .008 \\
\hline 779 & 132.29 & 121 & 16,007 & 1113508 & 2.854 & .998 & .002 & 275.98 & 58 & 16,007 & 498902 & .986 & .838 & .162 \\
\hline 780 & 59.48 & 581 & 34,558 & 10395427 & 1.482 & .931 & .069 & 111.48 & 310 & 34,558 & 5525010 & .959 & .831 & .169 \\
\hline 781 & 151.82 & 228 & 34,614 & 4001617 & .369 & .644 & .356 & 323.50 & 107 & 34,614 & 1900104 & .467 & .680 & .320 \\
\hline 782 & 38.60 & 241 & 9,303 & 1190907 & 1.677 & .953 & .047 & 69.95 & 133 & 9,303 & 666436 & 1.543 & .939 & .061 \\
\hline 783 & 162.22 & 9 & 1,460 & 7228 & .520 & 699 & .301 & 365.00 & 4 & 1,460 & 3288 & .437 & .669 & .331 \\
\hline 784 & 196.71 & 24 & 4,721 & 52436 & -.632 & .264 & .264 & 472.10 & 10 & 4,721 & 24329 & .168 & .567 & .433 \\
\hline 785 & 244.60 & 78 & 19,079 & 795829 & 1.064 & .856 & .144 & 545.11 & 35 & 19,079 & 310784 & -.709 & .239 & .239 \\
\hline 786 & 267.69 & 73 & 19,541 & 719600 & .132 & .552 & .448 & 610.66 & 32 & 19,541 & 356893 & 1.386 & .917 & .083 \\
\hline 787 & 62.42 & 97 & 6,055 & 272415 & -1.235 & .109 & .109 & 85.28 & 71 & 6,055 & 197962 & -1.154 & .124 & .124 \\
\hline 788 & 47.41 & 34 & 1,612 & 28819 & .522 & .699 & .301 & 100.75 & 16 & 1,612 & 13121 & .121 & .548 & .452 \\
\hline 789 & 50.27 & 166 & 8,344 & 680283 & -.395 & .346 & .346 & 93.75 & 89 & 8,344 & 390822 & .859 & .805 & .195 \\
\hline 790 & 40.35 & 55 & 2,219 & 65613 & .966 & .833 & .167 & 76.52 & 29 & 2,219 & 31771 & -.117 & .453 & .453 \\
\hline 791 & 73.66 & 64 & 4,714 & 168235 & 1.597 & .945 & .055 & 124.05 & 38 & 4,714 & 103544 & 1.666 & .952 & .048 \\
\hline 792 & 64.29 & 7 & 450 & 1842 & .777 & .781 & .219 & 75.00 & 6 & 450 & 1514 & .515 & .697 & .303 \\
\hline 793 & 49.44 & 388 & 19,181 & 3692616 & -.261 & .397 & .397 & 104.25 & 184 & 19,181 & 1774690 & .134 & .553 & .447 \\
\hline 794 & 51.93 & 225 & 11,685 & 1367067 & 1.038 & .850 & .150 & 108.19 & 108 & 11,685 & 648468 & .499 & .691 & .309 \\
\hline 795 & 84.39 & 202 & 17,047 & 1895726 & 2.488 & .994 & .006 & 168.78 & 101 & 17,047 & 942311 & 1.647 & .950 & .050 \\
\hline 796 & 168.55 & 31 & 5,225 & 82412 & .170 & .567 & .433 & 307.35 & 17 & 5,225 & 37858 & -1.054 & .146 & .146 \\
\hline 797 & 127.05 & 224 & 28,459 & 3317838 & 1.061 & .856 & .144 & 210.81 & 135 & 28,459 & 2039020 & 1.237 & .892 & .108 \\
\hline 798 & 115.67 & 6 & 694 & 1973 & -.222 & .412 & .412 & 694.00 & 1 & 694 & 486 & .694 & .756 & .244 \\
\hline 799 & 130.79 & 70 & 9,155 & 307449 & -.587 & .279 & .279 & 416.14 & 22 & 9,155 & 79744 & -1.691 & .045 & .045 \\
\hline 800 & 229.60 & 5 & 1,148 & 3488 & .834 & .798 & .202 & 1148.00 & 1 & 1,148 & 1038 & 1.400 & .919 & .081 \\
\hline 801 & 250.92 & 59 & 14,804 & 462808 & .795 & .787 & .213 & 779.16 & 19 & 14,804 & 147994 & .395 & .654 & .346 \\
\hline 802 & 302.40 & 5 & 1,512 & 2675 & -1.132 & .129 & .129 & 504.00 & 3 & 1,512 & 1238 & -1.362 & .087 & .087 \\
\hline 803 & 72.99 & 100 & 7,299 & 362071 & -.137 & .446 & .446 & 165.89 & 44 & 7,299 & 162523 & .139 & .555 & .445 \\
\hline 804 & 136.20 & 5 & 681 & 673 & -2.342 & .010 & .010 & 340.50 & 2 & 681 & 224 & -1.644 & .050 & .050 \\
\hline 805 & 102.62 & 112 & 11,493 & 649993 & .182 & .572 & .428 & 191.55 & 60 & 11,493 & 298240 & -1.811 & .035 & .035 \\
\hline 806 & 49.60 & 346 & 17,161 & 3072696 & 1.127 & .870 & .130 & 104.01 & 165 & 17,161 & 1442881 & .426 & .665 & .335 \\
\hline 807 & 67.69 & 194 & 13,131 & 1365634 & 1.741 & .959 & .041 & 139.69 & 94 & 13,131 & 638737 & .587 & .721 & .279 \\
\hline 808 & 78.31 & 230 & 18,012 & 2059922 & -.145 & .442 & .442 & 159.40 & 113 & 18,012 & 1057366 & .718 & .764 & .236 \\
\hline 809 & 196.19 & 94 & 18,442 & 809784 & -1.104 & .135 & .135 & 409.82 & 45 & 18,442 & 423840 & .249 & .598 & .402 \\
\hline 810 & 201.63 & 102 & 20,566 & 1053792 & .082 & .533 & .467 & 373.93 & 55 & 20,566 & 526880 & -.879 & .190 & .190 \\
\hline 811 & 48.53 & 152 & 7,376 & 593009 & 1.236 & .892 & .108 & 83.82 & 88 & 7,376 & 324754 & .011 & .504 & .496 \\
\hline 812 & 113.29 & 59 & 6,684 & 221678 & 1.653 & .951 & .049 & 257.08 & 26 & 6,684 & 109515 & 2.299 & .989 & .011 \\
\hline 813 & 20.75 & 4 & 83 & 207 & 0.856 & .804 & .196 & 83.00 & 1 & 83 & 66 & 1.023 & .847 & .153 \\
\hline 814 & 287.11 & 85 & 24,404 & 1072648 & .546 & .707 & .293 & 677.89 & 36 & 24,404 & 459049 & .468 & .680 & .320 \\
\hline 815 & 47.10 & 645 & 30,377 & 10416012 & 2.781 & .997 & .003 & 98.95 & 307 & 30,377 & 5094391 & 2.809 & .998 & .002 \\
\hline 816 & 47.86 & 270 & 12,922 & 1918599 & 2.841 & .998 & .002 & 91.65 & 141 & 12,922 & 1050139 & 3.141 & .999 & .001 \\
\hline
\end{tabular}


Table 6. Summary of interoccurrence intervals for daily precipitation thresholds of 1.5 and 2.0 inches-Continued

\begin{tabular}{|c|c|c|c|c|c|c|c|c|c|c|c|c|c|c|}
\hline \multirow[b]{2}{*}{$\begin{array}{l}\text { Seq. } \\
\text { no. }\end{array}$} & \multicolumn{7}{|c|}{ Daily precipitation threshold of 1.5 inches and greater } & \multicolumn{7}{|c|}{ Daily precipitation threshold of 2.0 inches and greater } \\
\hline & $\begin{array}{l}\text { Mean } \\
\text { inter- } \\
\text { occur- } \\
\text { rence } \\
\text { interval } \\
\text { (days) }\end{array}$ & $\begin{array}{c}\text { Total } \\
\text { no. of } \\
\text { events }\end{array}$ & $\begin{array}{l}\text { Total } \\
\text { no. of } \\
\text { days } \\
\text { avail- } \\
\text { able }\end{array}$ & $\begin{array}{l}\text { Sigma } \\
\mathrm{S}\end{array}$ & $\begin{array}{c}\text { Stan- } \\
\text { dard } \\
\text { normal } \\
\text { variate }\end{array}$ & $\begin{array}{l}\text { Non- } \\
\text { ex- } \\
\text { ceed- } \\
\text { ance } \\
\text { prob- } \\
\text { ability }\end{array}$ & $\begin{array}{c}\mathrm{p}- \\
\text { value }\end{array}$ & $\begin{array}{l}\text { Mean } \\
\text { inter- } \\
\text { occur- } \\
\text { rence } \\
\text { interval } \\
\text { (days) }\end{array}$ & $\begin{array}{c}\text { Total } \\
\text { no. of } \\
\text { events }\end{array}$ & $\begin{array}{c}\text { Total } \\
\text { no. of } \\
\text { days } \\
\text { avail- } \\
\text { able }\end{array}$ & $\begin{array}{l}\text { Sigma } \\
\mathbf{S}\end{array}$ & $\begin{array}{l}\text { Stan- } \\
\text { dard } \\
\text { normal } \\
\text { variate }\end{array}$ & $\begin{array}{l}\text { Non- } \\
\text { ex- } \\
\text { ceed- } \\
\text { ance } \\
\text { prob- } \\
\text { ability }\end{array}$ & $\begin{array}{c}p- \\
\text { value }\end{array}$ \\
\hline 817 & 76.79 & 81 & 6,220 & 235949 & -.988 & .162 & .162 & 163.68 & 38 & 6,220 & 111713 & -.584 & .280 & .280 \\
\hline 818 & 69.59 & 285 & 19,833 & 2953122 & 1.313 & .905 & .095 & 135.84 & 146 & 19,833 & 1541242 & 1.351 & .912 & .088 \\
\hline 819 & 246.68 & 117 & 28,862 & 1630463 & -.643 & .260 & .260 & 627.44 & 46 & 28,862 & 630103 & -.597 & .275 & .275 \\
\hline 820 & 99.00 & 9 & 891 & 5452 & 1.869 & .969 & .031 & 148.50 & 6 & 891 & 3650 & 1.551 & .940 & .061 \\
\hline 821 & 215.16 & 37 & 7,961 & 143382 & -.279 & .390 & .390 & 468.29 & 17 & 7,961 & 62702 & -.524 & .300 & .300 \\
\hline 822 & 100.26 & 190 & 19,050 & 1849253 & .521 & .699 & .301 & 216.48 & 88 & 19,050 & 909179 & 1.376 & .916 & .084 \\
\hline 823 & 122.33 & 266 & 32,540 & 4338506 & .070 & .528 & .472 & 225.97 & 144 & 32,540 & 2435791 & .824 & .795 & .205 \\
\hline 824 & 117.89 & 18 & 2,122 & 17527 & -.605 & .273 & .273 & 303.14 & 7 & 2,122 & 6916 & -.315 & .376 & .376 \\
\hline 825 & 96.31 & 42 & 4,045 & 91746 & .899 & .816 & .184 & 161.80 & 25 & 4,045 & 49918 & -.110 & .456 & .456 \\
\hline 826 & 48.10 & 551 & 26,503 & 7310775 & .051 & .520 & .480 & 95.34 & 278 & 26,503 & 3716231 & .253 & .600 & .400 \\
\hline 827 & 48.10 & 217 & 10,437 & 1196663 & 1.448 & .926 & .074 & 89.21 & 117 & 10,437 & 664882 & 1.667 & .952 & .048 \\
\hline 828 & 49.19 & 525 & 25,827 & 7088266 & 1.807 & .965 & .035 & 92.57 & 279 & 25,827 & 3614313 & .092 & .537 & .463 \\
\hline 829 & 37.51 & 144 & 5,401 & 386685 & -.117 & .453 & .453 & 62.08 & 87 & 5,401 & 219391 & -1.069 & .143 & .143 \\
\hline 830 & 79.89 & 164 & 13,102 & 1105045 & .633 & .737 & .263 & 140.88 & 93 & 13,102 & 620843 & .318 & .625 & .375 \\
\hline 831 & 65.94 & 215 & 14,176 & 1617842 & 1.565 & .941 & .059 & 119.13 & 119 & 14,176 & 939363 & 2.148 & .984 & .016 \\
\hline 832 & 97.33 & 18 & 1,752 & 13966 & -.840 & .201 & .201 & 159.27 & 11 & 1,752 & 8459 & -.702 & .242 & .242 \\
\hline 833 & 126.23 & 13 & 1,641 & 9830 & -.490 & .312 & .312 & 410.25 & 4 & 1,641 & 1999 & -1.354 & .088 & .088 \\
\hline 834 & 164.24 & 25 & 4,106 & 57622 & 1.063 & .856 & .144 & 342.17 & 12 & 4,106 & 32997 & 2.036 & .979 & .021 \\
\hline 835 & 40.86 & 454 & 18,549 & 4368436 & 1.383 & .917 & .083 & 70.00 & 265 & 18,549 & 2568675 & 1.273 & .898 & .102 \\
\hline 836 & 95.54 & 79 & 7,548 & 315975 & .921 & .821 & .179 & 132.42 & 57 & 7,548 & 223588 & .515 & .697 & .303 \\
\hline 837 & 42.20 & 283 & 11,942 & 1835641 & 2.515 & .994 & .006 & 76.06 & 157 & 11,942 & 984319 & 1.085 & .861 & .139 \\
\hline 838 & 37.53 & 203 & 7,618 & 814192 & 1.307 & .904 & .096 & 69.89 & 109 & 7,618 & 444034 & 1.257 & .896 & .104 \\
\hline 839 & 53.62 & 42 & 2,252 & 47150 & -.034 & .487 & .487 & 102.36 & 22 & 2,252 & 22026 & -.901 & .184 & .184 \\
\hline 840 & 74.71 & 488 & 36,458 & 9590737 & 2.989 & .999 & .001 & 128.83 & 283 & 36,458 & 5503945 & 1.949 & .974 & .026 \\
\hline 841 & 41.27 & 438 & 18,077 & 4281776 & 2.957 & .998 & .002 & 76.27 & 237 & 18,077 & 2386210 & 3.038 & .999 & .001 \\
\hline 842 & 49.16 & 381 & 18,731 & 3780686 & 2.013 & .978 & .022 & 92.27 & 203 & 18,731 & 2061177 & 2.077 & .981 & .019 \\
\hline 843 & 40.38 & 89 & 3,594 & 174573 & 1.496 & .933 & .067 & 102.69 & 35 & 3,594 & 71715 & 1.437 & .925 & .075 \\
\hline 844 & 79.43 & 221 & 17,555 & 1913206 & -.353 & .362 & .362 & 156.74 & 112 & 17,555 & 906360 & -1.431 & .076 & .076 \\
\hline 845 & 41.10 & 91 & 3,740 & 188858 & 1.815 & .965 & .035 & 69.26 & 54 & 3,740 & 115252 & 1.799 & .964 & .036 \\
\hline 846 & 33.22 & 195 & 6,477 & 639047 & .289 & .614 & .386 & 64.13 & 101 & 6,477 & 332835 & .306 & .620 & .380 \\
\hline 847 & 66.18 & 271 & 17,934 & 2332124 & -1.149 & .125 & .125 & 129.02 & 139 & 17,934 & 1114146 & -2.167 & .015 & .015 \\
\hline 848 & 76.91 & 374 & 28,763 & 5762070 & 2.388 & .992 & .008 & 139.63 & 206 & 28,763 & 3297190 & 2.808 & .998 & .002 \\
\hline 849 & 53.01 & 128 & 6,785 & 441696 & .337 & .632 & .368 & 84.81 & 80 & 6,785 & 291297 & 1.136 & .872 & .128 \\
\hline 850 & 159.00 & 9 & 1,431 & 6819 & .306 & .620 & .380 & 477.00 & 3 & 1,431 & 2773 & .876 & .809 & .191 \\
\hline 851 & 91.25 & 4 & 365 & 1128 & 1.889 & .971 & .029 & 182.50 & 2 & 365 & 448 & .557 & .711 & .289 \\
\hline 852 & 45.70 & 10 & 457 & 2732 & 1.072 & .858 & .142 & 76.17 & 6 & 457 & 1459 & .272 & .607 & .393 \\
\hline 853 & 91.33 & 3 & 274 & 260 & -1.102 & .135 & .135 & 137.00 & 2 & 274 & 156 & -1.055 & .146 & .146 \\
\hline 854 & 143.17 & 132 & 18,899 & 1312350 & 1.037 & .850 & .150 & 299.98 & 63 & 18,899 & 665388 & 1.618 & .947 & .053 \\
\hline 855 & 62.58 & 89 & 5,570 & 272820 & 1.645 & .950 & .050 & 121.09 & 46 & 5,570 & 135231 & .653 & .743 & .257 \\
\hline 856 & 194.89 & 96 & 18,709 & 923491 & .481 & .685 & .315 & 479.72 & 39 & 18,709 & 354343 & -.311 & .378 & .378 \\
\hline 857 & 100.00 & 91 & 9,100 & 436563 & .898 & .815 & .185 & 206.82 & 44 & 9,100 & 222673 & 1.290 & .901 & .099 \\
\hline 858 & 127.47 & 83 & 10,580 & 502101 & 2.265 & .988 & .012 & 293.89 & 36 & 10,580 & 204660 & .776 & .781 & .219 \\
\hline 859 & 130.10 & 72 & 9,367 & 343926 & .293 & .615 & .385 & 323.00 & 29 & 9,367 & 136557 & .051 & .520 & .480 \\
\hline 860 & 304.25 & 4 & 1,217 & 2776 & .487 & .687 & .313 & 1217.00 & 1 & 1,217 & 1177 & 1.618 & .947 & .053 \\
\hline 861 & 111.12 & 130 & 14,445 & 891977 & -0.988 & .162 & .162 & 225.70 & 64 & 14,445 & 382230 & -2.398 & .008 & .008 \\
\hline 862 & 48.61 & 157 & 7,632 & 596983 & -.077 & .469 & .469 & 88.74 & 86 & 7,632 & 316044 & -.594 & .276 & .276 \\
\hline 863 & 71.00 & 6 & 426 & 1337 & .196 & .578 & .422 & 213.00 & 2 & 426 & 579 & .880 & .810 & .190 \\
\hline 864 & 269.69 & 67 & 18,069 & 633405 & .658 & .745 & .255 & 645.32 & 28 & 18,069 & 264812 & .429 & .666 & .334 \\
\hline
\end{tabular}


Table 6. Summary of interoccurrence intervals for daily precipitation thresholds of 1.5 and 2.0 inches—Continued

\begin{tabular}{|c|c|c|c|c|c|c|c|c|c|c|c|c|c|c|}
\hline \multirow[b]{2}{*}{$\begin{array}{l}\text { Seq. } \\
\text { no. }\end{array}$} & \multicolumn{7}{|c|}{ Daily precipitation threshold of 1.5 inches and greater } & \multicolumn{7}{|c|}{ Daily precipitation threshold of 2.0 inches and greater } \\
\hline & $\begin{array}{l}\text { Mean } \\
\text { inter- } \\
\text { occur- } \\
\text { rence } \\
\text { interval } \\
\text { (days) }\end{array}$ & $\begin{array}{c}\text { Total } \\
\text { no. of } \\
\text { events }\end{array}$ & $\begin{array}{l}\text { Total } \\
\text { no. of } \\
\text { days } \\
\text { avail- } \\
\text { able }\end{array}$ & $\begin{array}{l}\text { Sigma } \\
\mathrm{S}\end{array}$ & $\begin{array}{c}\text { Stan- } \\
\text { dard } \\
\text { normal } \\
\text { variate }\end{array}$ & $\begin{array}{l}\text { Non- } \\
\text { ex- } \\
\text { ceed- } \\
\text { ance } \\
\text { prob- } \\
\text { ability }\end{array}$ & $\begin{array}{c}\mathrm{p}- \\
\text { value }\end{array}$ & $\begin{array}{l}\text { Mean } \\
\text { inter- } \\
\text { occur- } \\
\text { rence } \\
\text { interval } \\
\text { (days) }\end{array}$ & $\begin{array}{l}\text { Total } \\
\text { no. of } \\
\text { events }\end{array}$ & $\begin{array}{l}\text { Total } \\
\text { no. of } \\
\text { days } \\
\text { avail- } \\
\text { able }\end{array}$ & $\begin{array}{l}\text { Sigma } \\
\mathrm{S}\end{array}$ & $\begin{array}{c}\text { Stan- } \\
\text { dard } \\
\text { normal } \\
\text { variate }\end{array}$ & $\begin{array}{l}\text { Non- } \\
\text { ex- } \\
\text { ceed- } \\
\text { ance } \\
\text { prob- } \\
\text { ability }\end{array}$ & $\begin{array}{c}\mathrm{p}- \\
\text { value }\end{array}$ \\
\hline 865 & 156.13 & 15 & 2,342 & 15976 & -.607 & .272 & .272 & 390.33 & 6 & 2,342 & 6119 & -.548 & .292 & .292 \\
\hline 866 & 209.31 & 13 & 2,721 & 17132 & -.196 & .422 & .422 & 340.13 & 8 & 2,721 & 8469 & -1.087 & .139 & .139 \\
\hline 867 & 89.83 & 177 & 15,899 & 1504875 & 1.602 & .945 & .055 & 160.60 & 99 & 15,899 & 886763 & 2.185 & .986 & .014 \\
\hline 868 & 98.85 & 220 & 21,746 & 2515283 & 1.323 & .907 & .093 & 178.25 & 122 & 21,746 & 1398843 & 1.043 & .852 & .148 \\
\hline 869 & 200.44 & 92 & 18,440 & 830606 & -.345 & .365 & .365 & 439.05 & 42 & 18,440 & 348126 & -1.134 & .128 & .128 \\
\hline 870 & 43.75 & 36 & 1,575 & 31028 & .982 & .837 & .163 & 105.00 & 15 & 1,575 & 14059 & 1.276 & .899 & .101 \\
\hline 871 & -- & -- & -- & -- & -- & -- & -- & -- & -- & -- & -- & -- & -- & -- \\
\hline 872 & 81.00 & 6 & 486 & 1100 & -1.042 & .149 & .149 & 486.00 & 1 & 486 & 36 & -1.476 & .070 & .070 \\
\hline 873 & 34.77 & 675 & 23,470 & 8050994 & .738 & .770 & .230 & 57.38 & 409 & 23,470 & 4805740 & .045 & .518 & .482 \\
\hline 874 & 29.97 & 191 & 5,724 & 548147 & .066 & .526 & .474 & 47.70 & 120 & 5,724 & 350745 & .404 & .657 & .343 \\
\hline 875 & 44.09 & 11 & 485 & 2708 & .087 & .535 & .465 & 97.00 & 5 & 485 & 1349 & .436 & .669 & .331 \\
\hline 876 & -- & -- & -- & -- & -- & -- & -- & -- & -- & -- & -- & -- & -- & -- \\
\hline 877 & 143.33 & 3 & 430 & 861 & 1.005 & .842 & .158 & 430.00 & 1 & 430 & 237 & .177 & .570 & .430 \\
\hline 878 & 48.65 & 322 & 15,666 & 2474150 & -.592 & .277 & .277 & 97.30 & 161 & 15,666 & 1191248 & -1.218 & .112 & .112 \\
\hline 879 & 139.05 & 133 & 18,493 & 1222741 & -.114 & .454 & .454 & 271.96 & 68 & 18,493 & 640897 & .276 & .609 & .391 \\
\hline 880 & -- & -- & -- & -- & -- & -- & -- & -- & -- & -- & -- & -- & -- & -- \\
\hline 881 & 135.73 & 137 & 18,595 & 1343072 & 1.103 & .865 & .135 & 261.90 & 71 & 18,595 & 700790 & .899 & .816 & .184 \\
\hline 882 & 79.39 & 23 & 1,826 & 17828 & -1.254 & .105 & .105 & 228.25 & 8 & 1,826 & 7702 & .267 & .605 & .395 \\
\hline 883 & 109.01 & 100 & 10,901 & 553167 & .258 & .602 & .398 & 259.55 & 42 & 10,901 & 220358 & -.420 & .337 & .337 \\
\hline 884 & 148.16 & 92 & 13,631 & 626617 & -.011 & .496 & .496 & 309.80 & 44 & 13,631 & 305369 & .210 & .583 & .417 \\
\hline 885 & 121.71 & 244 & 29,698 & 3922393 & 2.235 & .987 & .013 & 247.48 & 120 & 29,698 & 1938265 & 1.665 & .952 & .048 \\
\hline 886 & 48.78 & 438 & 21,366 & 4816798 & 1.066 & .857 & .143 & 80.63 & 265 & 21,366 & 2997938 & 1.663 & .952 & .048 \\
\hline 887 & 56.36 & 445 & 25,079 & 6019512 & 2.877 & .998 & .002 & 115.04 & 218 & 25,079 & 2978791 & 2.294 & .989 & .011 \\
\hline 888 & 78.87 & 240 & 18,929 & 2403373 & 1.558 & .940 & .060 & 153.89 & 123 & 18,929 & 1254593 & 1.493 & .932 & .068 \\
\hline 889 & 165.37 & 83 & 13,726 & 636979 & 1.866 & .969 & .031 & 415.94 & 33 & 13,726 & 254610 & 1.236 & .892 & .108 \\
\hline 890 & 172.17 & 76 & 13,085 & 455008 & -1.282 & .100 & .100 & 436.17 & 30 & 13,085 & 201836 & .269 & .606 & .394 \\
\hline 891 & 151.19 & 120 & 18,143 & 1063785 & -.432 & .333 & .333 & 266.81 & 68 & 18,143 & 636593 & .457 & .676 & .324 \\
\hline 892 & 129.04 & 56 & 7,226 & 200242 & -.134 & .447 & .447 & 225.81 & 32 & 7,226 & 121530 & .501 & .692 & .308 \\
\hline 893 & 159.40 & 169 & 26,939 & 2034365 & -2.394 & .008 & .008 & 324.57 & 83 & 26,939 & 997351 & -1.703 & .044 & .044 \\
\hline 894 & 89.38 & 63 & 5,631 & 173772 & -.279 & .390 & .390 & 187.70 & 30 & 5,631 & 80546 & -.440 & .330 & .330 \\
\hline 895 & 397.00 & 43 & 17,071 & 414046 & 1.455 & .927 & .073 & 1004.18 & 17 & 17,071 & 184787 & 1.953 & .975 & .025 \\
\hline 896 & 50.86 & 703 & 35,755 & 13131934 & 2.061 & .980 & .020 & 92.87 & 385 & 35,755 & 7393520 & 2.522 & .994 & .006 \\
\hline 897 & 61.33 & 3 & 184 & 379 & 1.120 & .869 & .132 & 92.00 & 2 & 184 & 323 & 1.850 & .968 & .032 \\
\hline 898 & 34.60 & 35 & 1,211 & 24532 & 1.615 & .947 & .053 & 67.28 & 18 & 1,211 & 14155 & 2.195 & .986 & .014 \\
\hline 899 & 58.00 & 1 & 58 & 20 & -.538 & .296 & .296 & 58.00 & 1 & 58 & 20 & -.538 & .296 & .296 \\
\hline 900 & 66.57 & 23 & 1,531 & 15153 & -1.158 & .124 & .124 & 127.58 & 12 & 1,531 & 10345 & .757 & .775 & .225 \\
\hline 901 & 100.15 & 340 & 34,050 & 6177297 & 2.145 & .984 & .016 & 183.07 & 186 & 34,050 & 3337238 & 1.273 & .898 & .102 \\
\hline 902 & 93.67 & 12 & 1,124 & 7316 & .509 & .695 & .305 & 187.33 & 6 & 1,124 & 2393 & -1.232 & .109 & .109 \\
\hline 903 & 459.56 & 55 & 25,276 & 795453 & 1.855 & .968 & .032 & 1011.04 & 25 & 25,276 & 362446 & 1.275 & .899 & .101 \\
\hline 904 & -- & -- & -- & -- & -- & -- & -- & -- & -- & -- & -- & -- & -- & -- \\
\hline 905 & 302.29 & 55 & 16,626 & 476401 & .539 & .705 & .295 & 722.87 & 23 & 16,626 & 219952 & 1.249 & .894 & .106 \\
\hline 906 & 169.15 & 187 & 31,631 & 2913617 & -.351 & .363 & .363 & 385.74 & 82 & 31,631 & 1318690 & .264 & .604 & .396 \\
\hline 907 & -- & -- & -- & -- & -- & -- & -- & -- & -- & -- & -- & -- & -- & -- \\
\hline 908 & 195.86 & 101 & 19,782 & 1068168 & 1.205 & .886 & .114 & 460.05 & 43 & 19,782 & 420467 & -.129 & .448 & .448 \\
\hline 909 & 182.44 & 46 & 8,392 & 218950 & 1.578 & .943 & .057 & 466.22 & 18 & 8,392 & 92697 & 1.671 & .953 & .047 \\
\hline 910 & 1012.14 & 7 & 7,085 & 29373 & .846 & .801 & .199 & 2361.67 & 3 & 7,085 & 9902 & -.205 & .419 & .419 \\
\hline 911 & 2097.00 & 1 & 2,097 & 698 & -.579 & .281 & .281 & -- & -- & -- & -- & -- & -- & -- \\
\hline 912 & 37.75 & 8 & 302 & 1092 & -.470 & .319 & .319 & 75.50 & 4 & 302 & 590 & -.080 & .468 & .468 \\
\hline
\end{tabular}


Table 6. Summary of interoccurrence intervals for daily precipitation thresholds of 1.5 and 2.0 inches-Continued

\begin{tabular}{|c|c|c|c|c|c|c|c|c|c|c|c|c|c|c|}
\hline \multirow[b]{2}{*}{$\begin{array}{l}\text { Seq. } \\
\text { no. }\end{array}$} & \multicolumn{7}{|c|}{ Daily precipitation threshold of 1.5 inches and greater } & \multicolumn{7}{|c|}{ Daily precipitation threshold of 2.0 inches and greater } \\
\hline & $\begin{array}{l}\text { Mean } \\
\text { inter- } \\
\text { occur- } \\
\text { rence } \\
\text { interval } \\
\text { (days) }\end{array}$ & $\begin{array}{c}\text { Total } \\
\text { no. of } \\
\text { events }\end{array}$ & $\begin{array}{l}\text { Total } \\
\text { no. of } \\
\text { days } \\
\text { avail- } \\
\text { able }\end{array}$ & $\begin{array}{l}\text { Sigma } \\
\mathrm{S}\end{array}$ & $\begin{array}{c}\text { Stan- } \\
\text { dard } \\
\text { normal } \\
\text { variate }\end{array}$ & $\begin{array}{l}\text { Non- } \\
\text { ex- } \\
\text { ceed- } \\
\text { ance } \\
\text { prob- } \\
\text { ability }\end{array}$ & $\begin{array}{c}\text { p- } \\
\text { value }\end{array}$ & $\begin{array}{c}\text { Mean } \\
\text { inter- } \\
\text { occur- } \\
\text { rence } \\
\text { interval } \\
\text { (days) }\end{array}$ & $\begin{array}{c}\text { Total } \\
\text { no. of } \\
\text { events }\end{array}$ & $\begin{array}{c}\text { Total } \\
\text { no. of } \\
\text { days } \\
\text { avail- } \\
\text { able }\end{array}$ & $\begin{array}{c}\text { Sigma } \\
\mathrm{S}\end{array}$ & $\begin{array}{l}\text { Stan- } \\
\text { dard } \\
\text { normal } \\
\text { variate }\end{array}$ & $\begin{array}{l}\text { Non- } \\
\text { ex- } \\
\text { ceed- } \\
\text { ance } \\
\text { prob- } \\
\text { ability }\end{array}$ & $\begin{array}{c}\mathrm{p}- \\
\text { value }\end{array}$ \\
\hline 913 & 80.28 & 80 & 6,422 & 294049 & 2.242 & .988 & .012 & 133.79 & 48 & 6,422 & 180045 & 2.018 & .978 & .022 \\
\hline 914 & 78.74 & 46 & 3,622 & 89572 & .884 & .811 & .189 & 190.63 & 19 & 3,622 & 34582 & .038 & .515 & .485 \\
\hline 915 & 52.17 & 645 & 33,649 & 11457251 & 2.454 & .993 & .007 & 96.97 & 347 & 33,649 & 6278364 & 2.433 & .993 & .007 \\
\hline 916 & 52.85 & 362 & 19,131 & 3934158 & 4.487 & 1.000 & 0 & 101.76 & 188 & 19,131 & 2067897 & 3.560 & 1.000 & 0 \\
\hline 917 & 256.97 & 30 & 7,709 & 93192 & -1.841 & .033 & .033 & 513.93 & 15 & 7,709 & 40661 & -1.991 & .023 & .023 \\
\hline 918 & 37.21 & 238 & 8,856 & 1117683 & 1.618 & .947 & .053 & 68.12 & 130 & 8,856 & 618581 & 1.473 & .930 & .070 \\
\hline 919 & 100.01 & 98 & 9,801 & 470645 & -.343 & .366 & .366 & 227.93 & 43 & 9,801 & 225409 & .792 & .786 & .214 \\
\hline 920 & 49.24 & 388 & 19,104 & 3846467 & 1.292 & .902 & .098 & 93.65 & 204 & 19,104 & 2064758 & 1.475 & .930 & .070 \\
\hline 921 & 85.65 & 51 & 4,368 & 132303 & 2.323 & .990 & .010 & 208.00 & 21 & 4,368 & 53539 & 1.328 & .908 & .092 \\
\hline 922 & 187.97 & 121 & 22,744 & 1374733 & -.018 & .493 & .493 & 406.14 & 56 & 22,744 & 626580 & -.209 & .417 & .417 \\
\hline 923 & 173.20 & 193 & 33,427 & 3123693 & -.761 & .223 & .223 & 464.26 & 72 & 33,427 & 1085257 & -1.443 & .075 & .075 \\
\hline 924 & 639.00 & 1 & 639 & 399 & .431 & .667 & .333 & 639.00 & 1 & 639 & 399 & .431 & .667 & .333 \\
\hline 925 & 300.36 & 14 & 4,205 & 36390 & 1.531 & .937 & .063 & 600.71 & 7 & 4,205 & 16886 & .675 & .750 & .250 \\
\hline 926 & 69.50 & 8 & 556 & 2953 & 1.606 & .946 & .054 & 111.20 & 5 & 556 & 1864 & 1.321 & .907 & .093 \\
\hline 927 & 68.20 & 65 & 4,433 & 140194 & -.376 & .354 & .354 & 105.55 & 42 & 4,433 & 88857 & -.511 & .305 & .305 \\
\hline 928 & 195.46 & 39 & 7,623 & 157177 & .621 & .733 & .267 & 476.44 & 16 & 7,623 & 62705 & .196 & .578 & .422 \\
\hline 929 & 48.66 & 323 & 15,716 & 2483045 & -.676 & .250 & .250 & 75.56 & 208 & 15,716 & 1594695 & -.608 & .272 & .272 \\
\hline 930 & 162.64 & 56 & 9,108 & 276150 & 1.074 & .858 & .142 & 350.31 & 26 & 9,108 & 128193 & .730 & .767 & .233 \\
\hline 931 & 105.28 & 46 & 4,843 & 131644 & 2.136 & .984 & .016 & 193.72 & 25 & 4,843 & 85385 & 3.555 & 1.000 & 0 \\
\hline 932 & 95.74 & 34 & 3,255 & 56135 & .146 & .558 & .442 & 203.44 & 16 & 3,255 & 27145 & .294 & .616 & .384 \\
\hline 933 & -- & -- & -- & -- & -- & -- & -- & -- & -- & -- & -- & -- & -- & -- \\
\hline 934 & 62.33 & 89 & 5,547 & 255617 & .581 & .719 & .281 & 95.64 & 58 & 5,547 & 168448 & .622 & .733 & .267 \\
\hline 935 & 37.66 & 523 & 19,694 & 5502216 & 2.709 & .997 & .003 & 66.09 & 298 & 19,694 & 3252145 & 3.238 & .999 & .001 \\
\hline 936 & 35.62 & 267 & 9,510 & 1276702 & .159 & .563 & .437 & 58.70 & 162 & 9,510 & 767861 & -.070 & .472 & .472 \\
\hline 937 & 37.48 & 238 & 8,920 & 1001195 & -1.518 & .065 & .065 & 61.10 & 146 & 8,920 & 635068 & -.517 & .303 & .303 \\
\hline 938 & 94.09 & 269 & 25,311 & 3626810 & 1.857 & .968 & .032 & 164.36 & 154 & 25,311 & 2083847 & 1.488 & .932 & .068 \\
\hline 939 & 54.28 & 398 & 21,605 & 4436266 & 1.100 & .864 & .136 & 89.65 & 241 & 21,605 & 2823056 & 2.269 & .988 & .012 \\
\hline 940 & 98.14 & 162 & 15,898 & 1344085 & .965 & .833 & .167 & 165.60 & 96 & 15,898 & 728394 & -.772 & .220 & .220 \\
\hline 941 & 62.08 & 265 & 16,452 & 2049099 & -1.692 & .045 & .045 & 104.13 & 158 & 16,452 & 1219767 & -1.339 & .090 & .090 \\
\hline 942 & 116.20 & 113 & 13,131 & 720357 & -.535 & .296 & .296 & 226.40 & 58 & 13,131 & 357573 & -.805 & .211 & .211 \\
\hline 943 & 151.14 & 216 & 32,647 & 3478218 & -.344 & .365 & .365 & 302.29 & 108 & 32,647 & 1726998 & -.367 & .357 & .357 \\
\hline 944 & 52.66 & 109 & 5,740 & 311764 & -.062 & .475 & .475 & 104.36 & 55 & 5,740 & 128469 & -2.391 & .008 & .008 \\
\hline 945 & 89.76 & 238 & 21,362 & 2525291 & -.177 & .430 & .430 & 169.54 & 126 & 21,362 & 1366356 & .297 & .617 & .383 \\
\hline 946 & 58.45 & 168 & 9,820 & 849772 & .678 & .751 & .249 & 116.91 & 84 & 9,820 & 468890 & 2.173 & .985 & .015 \\
\hline 947 & 83.04 & 166 & 13,784 & 1255926 & 2.182 & .985 & .015 & 149.83 & 92 & 13,784 & 694569 & 1.585 & .944 & .056 \\
\hline 948 & 135.11 & 9 & 1,216 & 6404 & .885 & .812 & .188 & 608.00 & 2 & 1,216 & 1646 & .866 & .807 & .193 \\
\hline 949 & 646.56 & 41 & 26,509 & 631572 & 1.799 & .964 & .036 & 1767.27 & 15 & 26,509 & 216947 & .612 & .730 & .270 \\
\hline 950 & -- & -- & -- & -- & -- & -- & -- & -- & -- & -- & -- & -- & -- & -- \\
\hline 951 & 52.79 & 235 & 12,405 & 1460535 & .054 & .521 & .479 & 106.94 & 116 & 12,405 & 724486 & .130 & .552 & .448 \\
\hline 952 & 61.61 & 80 & 4,929 & 211349 & 1.115 & .868 & .133 & 140.83 & 35 & 4,929 & 103745 & 2.077 & .981 & .019 \\
\hline 953 & 87.66 & 160 & 14,026 & 1118686 & -.066 & .474 & .474 & 157.60 & 89 & 14,026 & 647887 & .621 & .733 & .267 \\
\hline 954 & 63.51 & 109 & 6,922 & 408170 & 1.482 & .931 & .069 & 115.37 & 60 & 6,922 & 218426 & .696 & .757 & .243 \\
\hline 955 & 109.09 & 303 & 33,055 & 5061479 & .323 & .627 & .373 & 254.27 & 130 & 33,055 & 2066218 & -.757 & .225 & .225 \\
\hline 956 & 104.31 & 333 & 34,736 & 5605167 & -.975 & .165 & .165 & 221.25 & 157 & 34,736 & 2792503 & .523 & .700 & .300 \\
\hline 957 & 49.80 & 86 & 4,283 & 177427 & -0.588 & .278 & .278 & 79.32 & 54 & 4,283 & 111903 & -0.411 & .340 & .340 \\
\hline 958 & 169.50 & 74 & 12,543 & 460895 & -.103 & .459 & .459 & 404.61 & 31 & 12,543 & 172127 & -1.106 & .135 & .135 \\
\hline 959 & 52.96 & 261 & 13,823 & 1716364 & -1.358 & .087 & .087 & 99.45 & 139 & 13,823 & 966690 & .127 & .551 & .449 \\
\hline 960 & 43.39 & 18 & 781 & 8428 & 1.463 & .928 & .072 & 111.57 & 7 & 781 & 3558 & 1.382 & .917 & .083 \\
\hline
\end{tabular}


Table 6. Summary of interoccurrence intervals for daily precipitation thresholds of 1.5 and 2.0 inches—Continued

\begin{tabular}{|c|c|c|c|c|c|c|c|c|c|c|c|c|c|c|}
\hline \multirow[b]{2}{*}{$\begin{array}{l}\text { Seq. } \\
\text { no. }\end{array}$} & \multicolumn{7}{|c|}{ Daily precipitation threshold of 1.5 inches and greater } & \multicolumn{7}{|c|}{ Daily precipitation threshold of 2.0 inches and greater } \\
\hline & $\begin{array}{l}\text { Mean } \\
\text { inter- } \\
\text { occur- } \\
\text { rence } \\
\text { interval } \\
\text { (days) }\end{array}$ & $\begin{array}{l}\text { Total } \\
\text { no. of } \\
\text { events }\end{array}$ & $\begin{array}{c}\text { Total } \\
\text { no. of } \\
\text { days } \\
\text { avail- } \\
\text { able }\end{array}$ & $\begin{array}{c}\text { Sigma } \\
\mathrm{S}\end{array}$ & $\begin{array}{l}\text { Stan- } \\
\text { dard } \\
\text { normal } \\
\text { variate }\end{array}$ & $\begin{array}{l}\text { Non- } \\
\text { ex- } \\
\text { ceed- } \\
\text { ance } \\
\text { prob- } \\
\text { ability }\end{array}$ & $\begin{array}{c}\mathrm{p}- \\
\text { value }\end{array}$ & $\begin{array}{l}\text { Mean } \\
\text { inter- } \\
\text { occur- } \\
\text { rence } \\
\text { interval } \\
\text { (days) }\end{array}$ & $\begin{array}{c}\text { Total } \\
\text { no. of } \\
\text { events }\end{array}$ & $\begin{array}{l}\text { Total } \\
\text { no. of } \\
\text { days } \\
\text { avail- } \\
\text { able }\end{array}$ & $\begin{array}{l}\text { Sigma } \\
\mathrm{S}\end{array}$ & $\begin{array}{l}\text { Stan- } \\
\text { dard } \\
\text { normal } \\
\text { variate }\end{array}$ & $\begin{array}{l}\text { Non- } \\
\text { ex- } \\
\text { ceed- } \\
\text { ance } \\
\text { prob- } \\
\text { ability }\end{array}$ & $\begin{array}{c}p- \\
\text { value }\end{array}$ \\
\hline 961 & 81.34 & 247 & 20,090 & 2487625 & .071 & .529 & .472 & 158.19 & 127 & 20,090 & 1308513 & .502 & .692 & .308 \\
\hline 962 & 719.00 & 1 & 719 & 320 & -.190 & .424 & .424 & -- & -- & -- & -- & -- & -- & -- \\
\hline 963 & 126.78 & 9 & 1,141 & 4182 & -.964 & .168 & .168 & 190.17 & 6 & 1,141 & 2771 & -.808 & .210 & .210 \\
\hline 964 & 100.75 & 20 & 2,015 & 20884 & .282 & .611 & .389 & 201.50 & 10 & 2,015 & 10268 & .105 & .542 & .458 \\
\hline 965 & 99.94 & 102 & 10,194 & 475533 & -1.493 & .068 & .068 & 208.04 & 49 & 10,194 & 219338 & -1.477 & .070 & .070 \\
\hline 966 & 240.24 & 37 & 8,889 & 171664 & .462 & .678 & .322 & 444.45 & 20 & 8,889 & 88316 & -.050 & .480 & .480 \\
\hline 967 & 202.16 & 25 & 5,054 & 63001 & -.024 & .490 & .490 & 722.00 & 7 & 5,054 & 16036 & -.428 & .334 & .334 \\
\hline 968 & 56.54 & 28 & 1,583 & 23050 & .367 & .643 & .357 & 121.77 & 13 & 1,583 & 9389 & -.547 & .292 & .292 \\
\hline 969 & 71.59 & 17 & 1,217 & 12253 & 1.318 & .906 & .094 & 173.86 & 7 & 1,217 & 5263 & 1.080 & .860 & .140 \\
\hline 970 & 92.23 & 355 & 32,743 & 6050733 & 1.341 & .910 & .090 & 170.54 & 192 & 32,743 & 3342256 & 1.519 & .936 & .064 \\
\hline 971 & 57.40 & 30 & 1,722 & 26704 & .321 & .626 & .374 & 107.63 & 16 & 1,722 & 13818 & .021 & .508 & .492 \\
\hline 972 & 84.98 & 218 & 18,526 & 2160333 & 1.786 & .963 & .037 & 187.13 & 99 & 18,526 & 942048 & .470 & .681 & .319 \\
\hline 973 & 325.68 & 60 & 19,541 & 599352 & .300 & .618 & .382 & 630.36 & 31 & 19,541 & 331191 & .901 & .816 & .184 \\
\hline 974 & 67.64 & 165 & 11,161 & 922315 & .037 & .515 & .485 & 139.51 & 80 & 11,161 & 436565 & -.343 & .366 & .366 \\
\hline 975 & 59.07 & 210 & 12,404 & 1264092 & -.739 & .230 & .230 & 121.61 & 102 & 12,404 & 580818 & -1.432 & .076 & .076 \\
\hline 976 & 63.43 & 14 & 888 & 6814 & .624 & .733 & .267 & 111.00 & 8 & 888 & 4575 & 1.411 & .921 & .079 \\
\hline 977 & -- & -- & -- & -- & -- & -- & -- & -- & -- & -- & -- & -- & -- & -- \\
\hline 978 & 59.88 & 223 & 13,354 & 1632530 & 2.494 & .994 & .006 & 103.52 & 129 & 13,354 & 970769 & 2.500 & .994 & .006 \\
\hline 979 & 60.16 & 95 & 5,715 & 290180 & 1.164 & .878 & .122 & 112.06 & 51 & 5,715 & 162077 & 1.387 & .917 & .083 \\
\hline 980 & 51.04 & 70 & 3,573 & 123631 & -.165 & .434 & .434 & 87.15 & 41 & 3,573 & 72876 & -.056 & .478 & .478 \\
\hline 981 & -- & -- & -- & -- & -- & -- & -- & -- & -- & -- & -- & -- & -- & -- \\
\hline 982 & 77.20 & 5 & 386 & 670 & -1.184 & .118 & .118 & 128.67 & 3 & 386 & 585 & .031 & .512 & .488 \\
\hline 983 & 52.28 & 200 & 10,455 & 1092960 & 1.112 & .867 & .133 & 111.22 & 94 & 10,455 & 482489 & -.304 & .381 & .381 \\
\hline 984 & 90.62 & 39 & 3,534 & 69010 & .015 & .506 & .494 & 271.85 & 13 & 3,534 & 23683 & .194 & .577 & .423 \\
\hline 985 & 80.80 & 15 & 1,212 & 7866 & -.903 & .183 & .183 & 173.14 & 7 & 1,212 & 5180 & 1.013 & .844 & .156 \\
\hline 986 & 86.63 & 40 & 3,465 & 88433 & 3.024 & .999 & .001 & 123.75 & 28 & 3,465 & 60211 & 2.211 & .986 & .014 \\
\hline 987 & 93.64 & 240 & 22,473 & 2532442 & -1.635 & .051 & .051 & 161.68 & 139 & 22,473 & 1468250 & -1.224 & .111 & .111 \\
\hline 988 & 65.17 & 36 & 2,346 & 49470 & 1.782 & .963 & .037 & 156.40 & 15 & 2,346 & 21240 & 1.390 & .918 & .082 \\
\hline 989 & 47.67 & 339 & 16,159 & 2788752 & .580 & .719 & .281 & 95.05 & 170 & 16,159 & 1428994 & .912 & .819 & .181 \\
\hline 990 & 55.97 & 333 & 18,639 & 3356862 & 2.582 & .995 & .005 & 109.00 & 171 & 18,639 & 1722772 & 1.835 & .967 & .033 \\
\hline 991 & 86.39 & 215 & 18,574 & 2190643 & 2.467 & .993 & .007 & 182.10 & 102 & 18,574 & 1096633 & 2.758 & .997 & .003 \\
\hline 992 & 49.36 & 405 & 19,989 & 4244976 & 1.698 & .955 & .045 & 100.45 & 199 & 19,989 & 2102553 & 1.396 & .919 & .081 \\
\hline 993 & 152.11 & 9 & 1,369 & 5715 & -.376 & .354 & .354 & 342.25 & 4 & 1,369 & 3138 & .506 & .694 & .306 \\
\hline 994 & 98.05 & 63 & 6,177 & 227113 & 2.299 & .989 & .011 & 199.26 & 31 & 6,177 & 112233 & 1.661 & .952 & .048 \\
\hline 995 & 72.24 & 228 & 16,470 & 1893429 & .221 & .587 & .413 & 143.22 & 115 & 16,470 & 956332 & .183 & .572 & .428 \\
\hline 996 & 131.74 & 219 & 28,850 & 3472742 & 2.545 & .995 & .005 & 216.92 & 133 & 28,850 & 2108687 & 1.980 & .976 & .024 \\
\hline 997 & 75.91 & 363 & 27,555 & 5430150 & 2.830 & .998 & .002 & 135.74 & 203 & 27,555 & 3109454 & 2.758 & .997 & .003 \\
\hline 998 & 87.75 & 243 & 21,324 & 2772917 & 1.897 & .971 & .029 & 148.08 & 144 & 21324 & 1630983 & 1.295 & .902 & .098 \\
\hline 999 & 68.85 & 53 & 3,649 & 80355 & -2.131 & .017 & .017 & 125.83 & 29 & 3,649 & 38958 & -2.460 & .007 & .007 \\
\hline 1000 & 54.59 & 165 & 9,008 & 760690 & .525 & .700 & .300 & 100.09 & 90 & 9,008 & 422775 & .706 & .760 & .240 \\
\hline 1001 & 65.71 & 300 & 19,714 & 3187246 & 2.335 & .990 & .010 & 118.05 & 167 & 19,714 & 1821586 & 2.386 & .991 & .009 \\
\hline 1002 & -- & -- & -- & -- & -- & -- & -- & -- & -- & -- & -- & -- & -- & -- \\
\hline 1003 & 141.36 & 144 & 20,356 & 1536538 & 1.006 & .843 & .157 & 278.85 & 73 & 20,356 & 791763 & .971 & .834 & .166 \\
\hline 1004 & 69.33 & 294 & 20,384 & 2960151 & -.360 & .360 & .360 & 122.80 & 166 & 20,384 & 1606779 & -1.122 & .131 & .131 \\
\hline 1005 & 111.76 & 191 & 21,346 & 1858496 & -2.114 & .017 & .017 & 260.32 & 82 & 21,346 & 819299 & -1.002 & .158 & .158 \\
\hline 1006 & 57.76 & 49 & 2,830 & 57919 & -1.996 & .023 & .023 & 113.20 & 25 & 2,830 & 29704 & -1.388 & .083 & .083 \\
\hline 1007 & 66.10 & 128 & 8,461 & 510275 & -1.130 & .129 & .129 & 119.17 & 71 & 8,461 & 284670 & -.763 & .223 & .223 \\
\hline 1008 & 67.45 & 206 & 13,894 & 1363847 & -1.168 & .121 & .121 & 115.78 & 120 & 13,894 & 825678 & -.181 & .428 & .428 \\
\hline
\end{tabular}


Table 6. Summary of interoccurrence intervals for daily precipitation thresholds of 1.5 and 2.0 inches-Continued

\begin{tabular}{|c|c|c|c|c|c|c|c|c|c|c|c|c|c|c|}
\hline \multirow[b]{2}{*}{$\begin{array}{l}\text { Seq. } \\
\text { no. }\end{array}$} & \multicolumn{7}{|c|}{ Daily precipitation threshold of 1.5 inches and greater } & \multicolumn{7}{|c|}{ Daily precipitation threshold of 2.0 inches and greater } \\
\hline & $\begin{array}{l}\text { Mean } \\
\text { inter- } \\
\text { occur- } \\
\text { rence } \\
\text { interval } \\
\text { (days) }\end{array}$ & $\begin{array}{c}\text { Total } \\
\text { no. of } \\
\text { events }\end{array}$ & $\begin{array}{l}\text { Total } \\
\text { no. of } \\
\text { days } \\
\text { avail- } \\
\text { able }\end{array}$ & $\begin{array}{l}\text { Sigma } \\
\mathbf{S}\end{array}$ & $\begin{array}{l}\text { Stan- } \\
\text { dard } \\
\text { normal } \\
\text { variate }\end{array}$ & $\begin{array}{l}\text { Non- } \\
\text { ex- } \\
\text { ceed- } \\
\text { ance } \\
\text { prob- } \\
\text { ability }\end{array}$ & $\begin{array}{c}\mathrm{p}- \\
\text { value }\end{array}$ & $\begin{array}{l}\text { Mean } \\
\text { inter- } \\
\text { occur- } \\
\text { rence } \\
\text { interval } \\
\text { (days) }\end{array}$ & $\begin{array}{c}\text { Total } \\
\text { no. of } \\
\text { events }\end{array}$ & $\begin{array}{l}\text { Total } \\
\text { no. of } \\
\text { days } \\
\text { avail- } \\
\text { able }\end{array}$ & $\begin{array}{l}\text { Sigma } \\
\mathrm{S}\end{array}$ & $\begin{array}{l}\text { Stan- } \\
\text { dard } \\
\text { normal } \\
\text { variate }\end{array}$ & $\begin{array}{l}\text { Non- } \\
\text { ex- } \\
\text { ceed- } \\
\text { ance } \\
\text { prob- } \\
\text { ability }\end{array}$ & $\begin{array}{c}\mathrm{p}- \\
\text { value }\end{array}$ \\
\hline 1009 & 49.71 & 252 & 12,527 & 1531234 & -.822 & .206 & .206 & 86.39 & 145 & 12,527 & 875090 & -.761 & .224 & .224 \\
\hline 1010 & 59.53 & 263 & 15,655 & 1961212 & -1.329 & .092 & .092 & 101.00 & 155 & 15,655 & 1128494 & -1.507 & .066 & .066 \\
\hline 1011 & 105.72 & 193 & 20,404 & 2137592 & 2.061 & .980 & .020 & 175.90 & 116 & 20,404 & 1285622 & 1.611 & .946 & .054 \\
\hline 1012 & 130.13 & 23 & 2,993 & 41098 & 1.612 & .946 & .054 & 299.30 & 10 & 2,993 & 15737 & .283 & .611 & .389 \\
\hline 1013 & 121.39 & 90 & 10,925 & 427310 & -2.150 & .016 & .016 & 206.13 & 53 & 10,925 & 257946 & -1.375 & .085 & .085 \\
\hline 1014 & 63.30 & 37 & 2,342 & 43996 & .163 & .565 & .435 & 123.26 & 19 & 2,342 & 22965 & .243 & .596 & .404 \\
\hline 1015 & 427.00 & 1 & 427 & 208 & -.045 & .482 & .482 & 427.00 & 1 & 427 & 208 & -.045 & .482 & .482 \\
\hline 1016 & 58.59 & 342 & 20,037 & 3368155 & -.544 & .293 & .293 & 103.82 & 193 & 20,037 & 1919615 & -.174 & .431 & .431 \\
\hline 1017 & 69.15 & 27 & 1,867 & 28961 & 1.341 & .910 & .090 & 124.47 & 15 & 1,867 & 14559 & .267 & .605 & .395 \\
\hline 1018 & 35.09 & 69 & 2,421 & 81191 & -.402 & .344 & .344 & 71.21 & 34 & 2,421 & 36150 & -1.229 & .110 & .110 \\
\hline 1019 & 149.30 & 54 & 8,062 & 225279 & .445 & .672 & .328 & 298.59 & 27 & 8,062 & 115545 & .555 & .710 & .290 \\
\hline 1020 & 147.20 & 40 & 5,888 & 128845 & 1.031 & .849 & .151 & 392.53 & 15 & 5,888 & 61599 & 2.649 & .996 & .004 \\
\hline 1021 & 122.49 & 198 & 24,253 & 2450383 & .501 & .692 & .308 & 247.48 & 98 & 24,253 & 1192801 & .064 & .525 & .475 \\
\hline 1022 & 54.86 & 180 & 9,875 & 890715 & .051 & .521 & .479 & 101.80 & 97 & 9,875 & 503479 & .874 & .809 & .191 \\
\hline 1023 & 54.80 & 123 & 6,740 & 387020 & -1.274 & .101 & .101 & 140.42 & 48 & 6,740 & 124305 & -2.779 & .003 & .003 \\
\hline 1024 & 63.39 & 185 & 11,727 & 1095860 & .241 & .595 & .405 & 113.85 & 103 & 11,727 & 642501 & 1.122 & .869 & .131 \\
\hline 1025 & 108.62 & 26 & 2,824 & 33535 & -.764 & .222 & .222 & 256.73 & 11 & 2,824 & 18277 & 1.015 & .845 & .155 \\
\hline 1026 & 63.71 & 300 & 19,114 & 2997039 & 1.360 & .913 & .087 & 141.59 & 135 & 19,114 & 1392144 & 1.590 & .944 & .056 \\
\hline 1027 & 92.58 & 73 & 6,758 & 244241 & -.146 & .442 & .442 & 160.91 & 42 & 6,758 & 137257 & -.369 & .356 & .356 \\
\hline 1028 & 128.51 & 213 & 27,372 & 3192877 & 2.409 & .992 & .008 & 248.84 & 110 & 27,372 & 1621715 & 1.403 & .920 & .080 \\
\hline 1029 & 80.98 & 192 & 15,548 & 1536898 & .712 & .762 & .238 & 153.94 & 101 & 15,548 & 846084 & 1.350 & .912 & .088 \\
\hline 1030 & 68.03 & 180 & 12,246 & 1179896 & 1.639 & .949 & .051 & 122.46 & 100 & 12,246 & 703888 & 2.591 & .995 & .005 \\
\hline 1031 & 253.80 & 10 & 2,538 & 12295 & -.171 & .432 & .432 & 846.00 & 3 & 2,538 & 3633 & -.137 & .445 & .445 \\
\hline 1032 & 79.40 & 459 & 36,443 & 8562445 & .882 & .811 & .189 & 148.75 & 245 & 36,443 & 4574738 & .671 & .749 & .251 \\
\hline 1033 & 43.30 & 495 & 21,434 & 5543561 & 1.734 & .959 & .042 & 90.06 & 238 & 21,434 & 2685782 & 1.416 & .922 & .078 \\
\hline 1034 & 127.29 & 17 & 2,164 & 18099 & -.115 & .454 & .454 & 240.44 & 9 & 2,164 & 10059 & .171 & .568 & .432 \\
\hline 1035 & 94.96 & 356 & 33,805 & 6040171 & .124 & .550 & .450 & 185.74 & 182 & 33,805 & 2947364 & -.979 & .164 & .164 \\
\hline 1036 & 20.62 & 26 & 536 & 7314 & .439 & .669 & .331 & 31.53 & 17 & 536 & 4631 & .118 & .547 & .453 \\
\hline 1037 & 38.73 & 11 & 426 & 1480 & -2.116 & .017 & .017 & 60.86 & 7 & 426 & 876 & -1.890 & .029 & .029 \\
\hline 1038 & 68.06 & 51 & 3,471 & 85237 & -.458 & .324 & .324 & 115.70 & 30 & 3,471 & 49104 & -.540 & .295 & .295 \\
\hline 1039 & 1750.50 & 4 & 7,002 & 23680 & 2.394 & .992 & .008 & 3501.00 & 2 & 7,002 & 13319 & 2.210 & .986 & .014 \\
\hline 1040 & 528.54 & 13 & 6,871 & 50756 & .852 & .803 & .197 & 1145.17 & 6 & 6,871 & 29947 & 1.921 & .973 & .027 \\
\hline 1041 & 1166.67 & 3 & 3,500 & 6106 & .489 & .688 & .312 & 3500.00 & 1 & 3,500 & 2269 & .514 & .696 & .304 \\
\hline 1042 & 32.84 & 378 & 12,414 & 2416697 & 1.011 & .844 & .156 & 64.32 & 193 & 12,414 & 1191552 & -.129 & .449 & .449 \\
\hline 1043 & 157.00 & 3 & 471 & 148 & -2.372 & .009 & .009 & -- & -- & -- & -- & -- & -- & -- \\
\hline 1044 & 132.07 & 62 & 8,188 & 251891 & -.104 & .459 & .459 & 292.43 & 28 & 8,188 & 111931 & -.216 & .414 & .414 \\
\hline 1045 & 176.98 & 113 & 19,999 & 1254300 & 2.026 & .979 & .021 & 363.62 & 55 & 19,999 & 568911 & .442 & .671 & .329 \\
\hline 1046 & 89.79 & 19 & 1,706 & 18873 & 1.242 & .893 & .107 & 121.86 & 14 & 1,706 & 14941 & 1.628 & .948 & .052 \\
\hline 1047 & 84.57 & 239 & 20,211 & 2580551 & 1.833 & .967 & .033 & 144.36 & 140 & 20,211 & 1502649 & 1.273 & .898 & .102 \\
\hline 1048 & 96.08 & 125 & 12,010 & 736790 & -.357 & .361 & .361 & 190.64 & 63 & 12,010 & 373534 & -.174 & .431 & .431 \\
\hline 1049 & 73.09 & 67 & 4,897 & 163513 & -.046 & .481 & .481 & 144.03 & 34 & 4,897 & 82007 & -.151 & .440 & .440 \\
\hline 1050 & 38.23 & 315 & 12,043 & 2033703 & 2.219 & .987 & .013 & 72.99 & 165 & 12,043 & 1066098 & 1.625 & .948 & .052 \\
\hline 1051 & 85.89 & 208 & 17,865 & 1867861 & .133 & .553 & .447 & 147.65 & 121 & 17,865 & 1074304 & -.115 & .454 & .454 \\
\hline 1052 & 80.44 & 9 & 724 & 2712 & -.871 & .192 & .192 & 144.80 & 5 & 724 & 1286 & -1.121 & .131 & .131 \\
\hline 1053 & 31.64 & 33 & 1,044 & 17373 & 0.085 & .534 & .466 & 61.41 & 17 & 1,044 & 7576 & -1.045 & .148 & .148 \\
\hline 1054 & 61.00 & 1 & 61 & 21 & -.540 & .295 & .295 & -- & -- & -- & -- & -- & -- & -- \\
\hline 1055 & 42.87 & 78 & 3,344 & 134190 & .443 & .671 & .329 & 85.74 & 39 & 3,344 & 68115 & .482 & .685 & .315 \\
\hline 1056 & 140.13 & 15 & 2,102 & 17216 & .617 & .731 & .269 & 191.09 & 11 & 2,102 & 11880 & .159 & .563 & .437 \\
\hline
\end{tabular}


Table 6. Summary of interoccurrence intervals for daily precipitation thresholds of 1.5 and 2.0 inches—Continued

\begin{tabular}{|c|c|c|c|c|c|c|c|c|c|c|c|c|c|c|}
\hline \multirow[b]{2}{*}{$\begin{array}{l}\text { Seq. } \\
\text { no. }\end{array}$} & \multicolumn{7}{|c|}{ Daily precipitation threshold of 1.5 inches and greater } & \multicolumn{7}{|c|}{ Daily precipitation threshold of 2.0 inches and greater } \\
\hline & $\begin{array}{l}\text { Mean } \\
\text { inter- } \\
\text { occur- } \\
\text { rence } \\
\text { interval } \\
\text { (days) }\end{array}$ & $\begin{array}{l}\text { Total } \\
\text { no. of } \\
\text { events }\end{array}$ & $\begin{array}{c}\text { Total } \\
\text { no. of } \\
\text { days } \\
\text { avail- } \\
\text { able }\end{array}$ & $\begin{array}{c}\text { Sigma } \\
\mathrm{S}\end{array}$ & $\begin{array}{l}\text { Stan- } \\
\text { dard } \\
\text { normal } \\
\text { variate }\end{array}$ & $\begin{array}{l}\text { Non- } \\
\text { ex- } \\
\text { ceed- } \\
\text { ance } \\
\text { prob- } \\
\text { ability }\end{array}$ & $\begin{array}{c}\mathrm{p}- \\
\text { value }\end{array}$ & $\begin{array}{l}\text { Mean } \\
\text { inter- } \\
\text { occur- } \\
\text { rence } \\
\text { interval } \\
\text { (days) }\end{array}$ & $\begin{array}{c}\text { Total } \\
\text { no. of } \\
\text { events }\end{array}$ & $\begin{array}{l}\text { Total } \\
\text { no. of } \\
\text { days } \\
\text { avail- } \\
\text { able }\end{array}$ & $\begin{array}{l}\text { Sigma } \\
\mathrm{S}\end{array}$ & $\begin{array}{l}\text { Stan- } \\
\text { dard } \\
\text { normal } \\
\text { variate }\end{array}$ & $\begin{array}{l}\text { Non- } \\
\text { ex- } \\
\text { ceed- } \\
\text { ance } \\
\text { prob- } \\
\text { ability }\end{array}$ & $\begin{array}{c}p- \\
\text { value }\end{array}$ \\
\hline 1057 & 127.00 & 4 & 508 & 970 & -.157 & .438 & .438 & 508.00 & 1 & 508 & 29 & -1.534 & .063 & .063 \\
\hline 1058 & 70.78 & 498 & 35,247 & 9001749 & .992 & .839 & .161 & 133.01 & 265 & 35,247 & 4676164 & .036 & .514 & .486 \\
\hline 1059 & 119.17 & 6 & 715 & 782 & -2.696 & .004 & .004 & 143.00 & 5 & 715 & 753 & -2.242 & .013 & .013 \\
\hline 1060 & 95.41 & 254 & 24,233 & 3236740 & 1.428 & .923 & .077 & 193.86 & 125 & 24,233 & 1553247 & .495 & .690 & .310 \\
\hline 1061 & 82.50 & 8 & 660 & 2378 & -.486 & .313 & .313 & 220.00 & 3 & 660 & 873 & -.355 & .361 & .361 \\
\hline 1062 & 115.56 & 18 & 2,080 & 15544 & -1.247 & .106 & .106 & 346.67 & 6 & 2,080 & 4452 & -1.216 & .112 & .112 \\
\hline 1063 & 271.24 & 88 & 23,869 & 1135563 & 1.320 & .907 & .093 & 596.73 & 40 & 23,869 & 532251 & 1.259 & .896 & .104 \\
\hline 1064 & 413.00 & 3 & 1,239 & 1752 & -.172 & .432 & .432 & 1239.00 & 1 & 1,239 & 469 & -.421 & .337 & .337 \\
\hline 1065 & 86.71 & 70 & 6,070 & 216451 & .273 & .608 & .392 & 195.81 & 31 & 6,070 & 93547 & -.055 & .478 & .478 \\
\hline 1066 & 78.82 & 141 & 11,113 & 788616 & .135 & .554 & .446 & 148.17 & 75 & 11,113 & 479448 & 2.257 & .988 & .012 \\
\hline 1067 & 61.13 & 15 & 917 & 8299 & 1.387 & .917 & .083 & 131.00 & 7 & 917 & 3822 & .875 & .809 & .191 \\
\hline 1068 & 79.56 & 9 & 716 & 2791 & -.695 & .244 & .244 & 238.67 & 3 & 716 & 459 & -1.718 & .043 & .043 \\
\hline 1069 & 106.24 & 51 & 5,418 & 148077 & .888 & .813 & .187 & 216.72 & 25 & 5,418 & 76952 & 1.180 & .881 & .119 \\
\hline 1070 & 82.49 & 427 & 35,225 & 7241354 & -1.329 & .092 & .092 & 142.61 & 247 & 35,225 & 4180575 & -1.062 & .144 & .144 \\
\hline 1071 & 60.78 & 323 & 19,631 & 3409304 & 2.346 & .991 & .010 & 116.16 & 169 & 19,631 & 1731489 & .986 & .838 & .162 \\
\hline 1072 & 75.82 & 22 & 1,668 & 16205 & -.949 & .171 & .171 & 151.64 & 11 & 1,668 & 8240 & -.585 & .279 & .279 \\
\hline 1073 & -- & -- & -- & -- & -- & -- & -- & -- & -- & -- & -- & -- & -- & -- \\
\hline 1074 & 51.82 & 581 & 30,105 & 9081448 & 1.604 & .946 & .054 & 88.54 & 340 & 30,105 & 5206102 & .551 & .709 & .291 \\
\hline 1075 & 103.56 & 25 & 2,589 & 30828 & -.411 & .341 & .341 & 258.90 & 10 & 2,589 & 14598 & 699 & .758 & .242 \\
\hline 1076 & 86.76 & 208 & 18,047 & 1979475 & 1.365 & .914 & .086 & 155.58 & 116 & 18,047 & 1079778 & .589 & .722 & .278 \\
\hline 1077 & 78.14 & 44 & 3,438 & 74735 & -.137 & .446 & .446 & 137.52 & 25 & 3,438 & 41089 & -.380 & .352 & .352 \\
\hline 1078 & 205.32 & 134 & 27,513 & 1934838 & .995 & .840 & .160 & 410.64 & 67 & 27,513 & 971878 & .772 & .780 & .220 \\
\hline 1079 & 107.53 & 291 & 31,290 & 4759680 & 1.343 & .910 & .090 & 211.42 & 148 & 31,290 & 2353586 & .347 & .636 & .364 \\
\hline 1080 & 69.14 & 7 & 484 & 2318 & 1.688 & .954 & .046 & 121.00 & 4 & 484 & 1146 & .637 & .738 & .262 \\
\hline 1081 & 136.10 & 156 & 21,232 & 1668294 & .159 & .563 & .437 & 268.76 & 79 & 21,232 & 843101 & .081 & .532 & .468 \\
\hline 1082 & 131.68 & 104 & 13,695 & 760743 & 1.206 & .886 & .114 & 291.38 & 47 & 13,695 & 369920 & 1.774 & .962 & .038 \\
\hline 1083 & 244.92 & 63 & 15,430 & 493460 & .210 & .583 & .417 & 467.58 & 33 & 15,430 & 276450 & .854 & .803 & .197 \\
\hline 1084 & 48.68 & 25 & 1,217 & 15567 & .202 & .580 & .420 & 71.59 & 17 & 1,217 & 9458 & -.612 & .270 & .270 \\
\hline 1085 & 59.57 & 610 & 36,335 & 11268122 & .718 & .763 & .237 & 108.79 & 334 & 36,335 & 6014412 & -.279 & .390 & .390 \\
\hline 1086 & 120.21 & 42 & 5,049 & 92553 & -1.427 & .077 & .077 & 280.50 & 18 & 5,049 & 41768 & -.594 & .276 & .276 \\
\hline 1087 & 501.79 & 28 & 14,050 & 192994 & -.173 & .431 & .431 & 1405.00 & 10 & 14,050 & 62157 & -.631 & .264 & .264 \\
\hline 1088 & 34.65 & 65 & 2,252 & 79273 & 1.161 & .877 & .123 & 52.37 & 43 & 2,252 & 55300 & 1.614 & .947 & .053 \\
\hline 1089 & 118.60 & 10 & 1,186 & 5808 & -.113 & .455 & .455 & 296.50 & 4 & 1,186 & 1918 & -.663 & .254 & .254 \\
\hline 1090 & 104.95 & 97 & 10,180 & 508553 & .512 & .696 & .304 & 248.29 & 41 & 10,180 & 224963 & .865 & .806 & .194 \\
\hline 1091 & 169.83 & 116 & 19,700 & 1191423 & .797 & .787 & .213 & 419.15 & 47 & 19,700 & 493082 & .773 & .780 & .220 \\
\hline 1092 & 24.60 & 5 & 123 & 135 & -2.173 & .015 & .015 & -- & -- & -- & -- & -- & -- & -- \\
\hline 1093 & 46.18 & 103 & 4,757 & 239521 & -.392 & .348 & .348 & 97.08 & 49 & 4,757 & 100290 & -1.691 & .045 & .045 \\
\hline 1094 & 64.84 & 398 & 25,807 & 5544066 & 2.748 & .997 & .003 & 107.08 & 241 & 25,807 & 3408715 & 2.585 & .995 & .005 \\
\hline 1095 & 61.81 & 32 & 1,978 & 28691 & -.916 & .180 & .180 & 109.89 & 18 & 1,978 & 18839 & .428 & .666 & .334 \\
\hline 1096 & 156.59 & 122 & 19,104 & 1252153 & 1.425 & .923 & .077 & 382.08 & 50 & 19,104 & 517136 & 1.014 & .845 & .155 \\
\hline 1097 & 63.85 & 298 & 19,028 & 3117552 & 2.978 & .999 & .001 & 136.89 & 139 & 19,028 & 1448153 & 1.941 & .974 & .026 \\
\hline 1098 & 89.54 & 165 & 14,774 & 1172629 & -.844 & .199 & .199 & 173.81 & 85 & 14,774 & 579272 & -1.237 & .108 & .108 \\
\hline 1099 & -- & -- & -- & -- & -- & -- & -- & -- & -- & -- & -- & -- & -- & -- \\
\hline 1100 & 94.00 & 30 & 2,820 & 40616 & -.378 & .353 & .353 & 216.92 & 13 & 2,820 & 21094 & .942 & .827 & .173 \\
\hline 1101 & 62.39 & 468 & 29,198 & 7206874 & 2.054 & .980 & .020 & 121.15 & 241 & 29,198 & 3531100 & 0.097 & .539 & .461 \\
\hline 1102 & 143.46 & 220 & 31,561 & 3561258 & .663 & .746 & .254 & 281.80 & 112 & 31,561 & 1811742 & .460 & .677 & .323 \\
\hline 1103 & 697.73 & 15 & 10,466 & 84462 & .510 & .695 & .305 & 1744.33 & 6 & 10,466 & 27987 & -.461 & .322 & .322 \\
\hline 1104 & 67.35 & 116 & 7,812 & 462838 & .401 & .656 & .344 & 130.20 & 60 & 7,812 & 247199 & .735 & .769 & .231 \\
\hline
\end{tabular}


Table 6. Summary of interoccurrence intervals for daily precipitation thresholds of 1.5 and 2.0 inches-Continued

\begin{tabular}{|c|c|c|c|c|c|c|c|c|c|c|c|c|c|c|}
\hline \multirow[b]{2}{*}{$\begin{array}{l}\text { Seq. } \\
\text { no. }\end{array}$} & \multicolumn{7}{|c|}{ Daily precipitation threshold of 1.5 inches and greater } & \multicolumn{7}{|c|}{ Daily precipitation threshold of 2.0 inches and greater } \\
\hline & $\begin{array}{l}\text { Mean } \\
\text { inter- } \\
\text { occur- } \\
\text { rence } \\
\text { interval } \\
\text { (days) }\end{array}$ & $\begin{array}{l}\text { Total } \\
\text { no. of } \\
\text { events }\end{array}$ & $\begin{array}{l}\text { Total } \\
\text { no. of } \\
\text { days } \\
\text { avail- } \\
\text { able }\end{array}$ & $\begin{array}{l}\text { Sigma } \\
\mathrm{S}\end{array}$ & $\begin{array}{c}\text { Stan- } \\
\text { dard } \\
\text { normal } \\
\text { variate }\end{array}$ & $\begin{array}{l}\text { Non- } \\
\text { ex- } \\
\text { ceed- } \\
\text { ance } \\
\text { prob- } \\
\text { ability }\end{array}$ & $\begin{array}{c}\mathrm{p}- \\
\text { value }\end{array}$ & $\begin{array}{l}\text { Mean } \\
\text { inter- } \\
\text { occur- } \\
\text { rence } \\
\text { interval } \\
\text { (days) }\end{array}$ & $\begin{array}{c}\text { Total } \\
\text { no. of } \\
\text { events }\end{array}$ & $\begin{array}{l}\text { Total } \\
\text { no. of } \\
\text { days } \\
\text { avail- } \\
\text { able }\end{array}$ & $\begin{array}{l}\text { Sigma } \\
\mathrm{S}\end{array}$ & $\begin{array}{l}\text { Stan- } \\
\text { dard } \\
\text { normal } \\
\text { variate }\end{array}$ & $\begin{array}{l}\text { Non- } \\
\text { ex- } \\
\text { ceed- } \\
\text { ance } \\
\text { prob- } \\
\text { ability }\end{array}$ & $\begin{array}{c}\mathrm{p}- \\
\text { value }\end{array}$ \\
\hline 1105 & 65.56 & 202 & 13,243 & 1333156 & -.081 & .468 & .468 & 128.57 & 103 & 13,243 & 663544 & -.476 & .317 & .317 \\
\hline 1106 & 123.58 & 182 & 22,491 & 2039179 & -.086 & .466 & .466 & 234.28 & 96 & 22,491 & 1132891 & .838 & .799 & .201 \\
\hline 1107 & 123.85 & 13 & 1,610 & 7847 & -1.562 & .059 & .059 & 402.50 & 4 & 1,610 & 2348 & -.938 & .174 & .174 \\
\hline 1108 & -- & -- & -- & -- & -- & -- & -- & -- & -- & -- & -- & -- & -- & -- \\
\hline 1109 & 112.68 & 31 & 3,493 & 53956 & -.033 & .487 & .487 & 232.87 & 15 & 3,493 & 21872 & -1.108 & .134 & .134 \\
\hline 1110 & 71.63 & 49 & 3,510 & 79582 & -.904 & .183 & .183 & 117.00 & 30 & 3,510 & 49646 & -.541 & .294 & .294 \\
\hline 1111 & 160.86 & 14 & 2,252 & 9832 & -2.439 & .007 & .007 & 563.00 & 4 & 2,252 & 2601 & -1.464 & .072 & .072 \\
\hline 1112 & 44.84 & 268 & 12,016 & 1701706 & 1.612 & .947 & .053 & 84.03 & 143 & 12,016 & 910721 & 1.243 & .893 & .107 \\
\hline 1113 & 187.89 & 157 & 29,498 & 2235229 & -.753 & .226 & .226 & 404.08 & 73 & 29,498 & 1000734 & -1.044 & .148 & .148 \\
\hline 1114 & 87.22 & 139 & 12,124 & 830060 & -.304 & .380 & .380 & 151.55 & 80 & 12,124 & 496832 & .379 & .648 & .352 \\
\hline 1115 & 157.11 & 37 & 5,813 & 114369 & .669 & .748 & .252 & 363.31 & 16 & 5,813 & 37527 & -1.337 & .091 & .091 \\
\hline 1116 & 48.43 & 30 & 1,453 & 21242 & -.241 & .405 & .405 & 90.81 & 16 & 1,453 & 11405 & -.131 & .448 & .448 \\
\hline 1117 & 65.53 & 240 & 15,727 & 1832354 & -.780 & .218 & .218 & 125.82 & 125 & 15,727 & 969428 & -.266 & .395 & .395 \\
\hline 1118 & 80.00 & 10 & 800 & 3818 & -.249 & .402 & .402 & 160.00 & 5 & 800 & 1688 & -.604 & .273 & .273 \\
\hline 1119 & 80.70 & 94 & 7,586 & 336595 & -.940 & .174 & .174 & 140.48 & 54 & 7,586 & 211374 & .407 & .658 & .342 \\
\hline 1120 & 154.82 & 158 & 24,462 & 1911338 & -.238 & .406 & .406 & 321.87 & 76 & 24,462 & 861023 & -1.113 & .133 & .133 \\
\hline 1121 & 109.91 & 270 & 29,675 & 3734837 & -1.927 & .027 & .027 & 211.96 & 140 & 29,675 & 1934999 & -1.403 & .080 & .080 \\
\hline 1122 & 92.82 & 11 & 1,021 & 4911 & -.721 & .236 & .236 & 170.17 & 6 & 1,021 & 3225 & .224 & .589 & .411 \\
\hline 1123 & 96.22 & 237 & 22,804 & 2696196 & -.060 & .476 & .476 & 190.03 & 120 & 22,804 & 1369054 & .011 & .505 & .495 \\
\hline 1124 & 97.93 & 14 & 1,371 & 8220 & -.930 & .176 & .176 & 171.38 & 8 & 1,371 & 4427 & -.944 & .173 & .173 \\
\hline 1125 & 85.46 & 33 & 2,820 & 45952 & -.124 & .451 & .451 & 165.88 & 17 & 2,820 & 20660 & -.986 & .162 & .162 \\
\hline 1126 & 120.72 & 32 & 3,863 & 60945 & -.137 & .446 & .446 & 227.24 & 17 & 3,863 & 31299 & -.334 & .369 & .369 \\
\hline 1127 & 142.00 & 188 & 26,696 & 2344011 & -1.565 & .059 & .059 & 287.05 & 93 & 26,696 & 1082879 & -2.133 & .016 & .016 \\
\hline 1128 & 132.07 & 73 & 9,641 & 334600 & -.727 & .234 & .234 & 292.15 & 33 & 9,641 & 154939 & -.259 & .398 & .398 \\
\hline 1129 & 257.00 & 14 & 3,598 & 24978 & -.054 & .479 & .479 & 449.75 & 8 & 3,598 & 15105 & .243 & .596 & .404 \\
\hline 1130 & 755.00 & 1 & 755 & 192 & -.851 & .197 & .197 & -- & -- & -- & -- & -- & -- & -- \\
\hline 1131 & 73.69 & 188 & 13,853 & 1374273 & 1.315 & .906 & .094 & 138.53 & 100 & 13,853 & 763348 & 1.768 & .961 & .039 \\
\hline 1132 & 348.00 & 2 & 696 & 453 & -.855 & .196 & .196 & 696.00 & 1 & 696 & 227 & -.602 & .274 & .274 \\
\hline 1133 & 69.64 & 145 & 10,098 & 681514 & -1.441 & .075 & .075 & 138.33 & 73 & 10,098 & 349664 & -.759 & .224 & .224 \\
\hline 1134 & 228.33 & 3 & 685 & 987 & -.118 & .453 & .453 & 342.50 & 2 & 685 & 392 & -1.048 & .147 & .147 \\
\hline 1135 & 35.86 & 22 & 789 & 6540 & -2.002 & .023 & .023 & 60.69 & 13 & 789 & 4217 & -1.110 & .134 & .134 \\
\hline 1136 & 228.48 & 122 & 27,875 & 1682413 & -.202 & .420 & .420 & 536.06 & 52 & 27,875 & 696395 & -.489 & .313 & .313 \\
\hline 1137 & 92.94 & 206 & 19,145 & 1978185 & .079 & .531 & .469 & 187.70 & 102 & 19,145 & 1020262 & .786 & .784 & .216 \\
\hline 1138 & 1234.50 & 2 & 2,469 & 3625 & 1.147 & .874 & .126 & -- & -- & -- & -- & -- & -- & -- \\
\hline 1139 & 519.50 & 6 & 3,117 & 7634 & -.779 & .218 & .218 & 1558.50 & 2 & 3,117 & 2825 & -.230 & .409 & .409 \\
\hline 1140 & 128.01 & 87 & 11,137 & 440260 & -1.474 & .070 & .070 & 227.29 & 49 & 11,137 & 265712 & -.318 & .375 & .375 \\
\hline 1141 & 47.09 & 424 & 19,965 & 4578374 & 2.914 & .998 & .002 & 84.24 & 237 & 19,965 & 2706806 & 3.843 & 1.000 & 0 \\
\hline 1142 & 49.20 & 665 & 32,715 & 10976721 & .406 & .658 & .342 & 88.42 & 370 & 32,715 & 6265283 & 1.173 & .879 & .121 \\
\hline 1143 & 231.15 & 39 & 9,015 & 164377 & -.702 & .241 & .241 & 751.25 & 12 & 9,015 & 43397 & -1.186 & .118 & .118 \\
\hline 1144 & 77.45 & 31 & 2,401 & 37794 & .150 & .560 & .440 & 141.24 & 17 & 2,401 & 20669 & .091 & .536 & .464 \\
\hline 1145 & 238.17 & 6 & 1,429 & 5302 & 1.005 & .842 & .158 & 476.33 & 3 & 1,429 & 2335 & .268 & .606 & .394 \\
\hline 1146 & 184.88 & 144 & 26,623 & 1925543 & .094 & .538 & .462 & 451.24 & 59 & 26,623 & 791940 & .111 & .544 & .456 \\
\hline 1147 & 105.22 & 59 & 6,208 & 202021 & 1.372 & .915 & .085 & 206.93 & 30 & 6,208 & 99617 & .662 & .746 & .254 \\
\hline 1148 & 185.71 & 70 & 13,000 & 466484 & .366 & .643 & .357 & 325.00 & 40 & 13,000 & 251436 & -.361 & .359 & .359 \\
\hline 1149 & 82.77 & 146 & 12,084 & 887598 & 0.130 & .552 & .448 & 132.79 & 91 & 12,084 & 594306 & 1.337 & .909 & .091 \\
\hline 1150 & 210.05 & 64 & 13,443 & 454448 & .782 & .783 & .217 & 463.55 & 29 & 13,443 & 212659 & .849 & .802 & .198 \\
\hline 1151 & 95.17 & 12 & 1,142 & 6325 & -.462 & .322 & .322 & 228.40 & 5 & 1,142 & 2767 & -.119 & .452 & .452 \\
\hline 1152 & 70.77 & 374 & 26,466 & 5005695 & .383 & .649 & .351 & 144.62 & 183 & 26,466 & 2527223 & 1.022 & .846 & .154 \\
\hline
\end{tabular}


Table 6. Summary of interoccurrence intervals for daily precipitation thresholds of 1.5 and 2.0 inches—Continued

\begin{tabular}{|c|c|c|c|c|c|c|c|c|c|c|c|c|c|c|}
\hline \multirow[b]{2}{*}{$\begin{array}{l}\text { Seq. } \\
\text { no. }\end{array}$} & \multicolumn{7}{|c|}{ Daily precipitation threshold of 1.5 inches and greater } & \multicolumn{7}{|c|}{ Daily precipitation threshold of 2.0 inches and greater } \\
\hline & $\begin{array}{l}\text { Mean } \\
\text { inter- } \\
\text { occur- } \\
\text { rence } \\
\text { interval } \\
\text { (days) }\end{array}$ & $\begin{array}{c}\text { Total } \\
\text { no. of } \\
\text { events }\end{array}$ & $\begin{array}{l}\text { Total } \\
\text { no. of } \\
\text { days } \\
\text { avail- } \\
\text { able }\end{array}$ & $\begin{array}{l}\text { Sigma } \\
\mathrm{S}\end{array}$ & $\begin{array}{c}\text { Stan- } \\
\text { dard } \\
\text { normal } \\
\text { variate }\end{array}$ & $\begin{array}{l}\text { Non- } \\
\text { ex- } \\
\text { ceed- } \\
\text { ance } \\
\text { prob- } \\
\text { ability }\end{array}$ & $\begin{array}{c}\mathrm{p}- \\
\text { value }\end{array}$ & $\begin{array}{l}\text { Mean } \\
\text { inter- } \\
\text { occur- } \\
\text { rence } \\
\text { interval } \\
\text { (days) }\end{array}$ & $\begin{array}{l}\text { Total } \\
\text { no. of } \\
\text { events }\end{array}$ & $\begin{array}{l}\text { Total } \\
\text { no. of } \\
\text { days } \\
\text { avail- } \\
\text { able }\end{array}$ & $\begin{array}{l}\text { Sigma } \\
\mathrm{S}\end{array}$ & $\begin{array}{c}\text { Stan- } \\
\text { dard } \\
\text { normal } \\
\text { variate }\end{array}$ & $\begin{array}{l}\text { Non- } \\
\text { ex- } \\
\text { ceed- } \\
\text { ance } \\
\text { prob- } \\
\text { ability }\end{array}$ & $\begin{array}{c}\mathrm{p}- \\
\text { value }\end{array}$ \\
\hline 1153 & 34.79 & 14 & 487 & 3376 & -.063 & .475 & .475 & 48.70 & 10 & 487 & 2350 & -.191 & .424 & .424 \\
\hline 1154 & 92.49 & 141 & 13,041 & 905434 & -.312 & .377 & .377 & 207.00 & 63 & 13,041 & 409885 & -.030 & .488 & .488 \\
\hline 1155 & 52.65 & 26 & 1,369 & 16023 & -.880 & .189 & .189 & 85.56 & 16 & 1,369 & 7989 & -1.874 & .030 & .030 \\
\hline 1156 & 82.89 & 157 & 13,013 & 1046698 & .535 & .704 & .296 & 153.09 & 85 & 13,013 & 538228 & -.428 & .334 & .334 \\
\hline 1157 & 116.43 & 155 & 18,047 & 1552037 & 2.365 & .991 & .009 & 265.40 & 68 & 18,047 & 678758 & 1.517 & .935 & .065 \\
\hline 1158 & 50.89 & 70 & 3,562 & 126709 & .237 & .594 & .406 & 107.94 & 33 & 3,562 & 65587 & 1.154 & .876 & .124 \\
\hline 1159 & 68.62 & 537 & 36,849 & 10150087 & 1.039 & .851 & .149 & 124.07 & 297 & 36,849 & 5522074 & .273 & .607 & .393 \\
\hline 1160 & 107.77 & 13 & 1,401 & 9637 & .364 & .642 & .358 & 280.20 & 5 & 1,401 & 1931 & -1.738 & .041 & .041 \\
\hline 1161 & 154.60 & 37 & 5,720 & 104316 & -.150 & .440 & .440 & 336.47 & 17 & 5,720 & 45733 & -.424 & .336 & .336 \\
\hline 1162 & 1210.00 & 1 & 1,210 & 1122 & 1.480 & .931 & .069 & -- & -- & -- & -- & -- & -- & -- \\
\hline 1163 & 790.00 & 4 & 3,160 & 4035 & -1.252 & .105 & .105 & -- & -- & -- & -- & -- & -- & -- \\
\hline 1164 & 54.74 & 350 & 19,160 & 3603650 & 2.422 & .992 & .008 & 101.38 & 189 & 19,160 & 1981519 & 2.248 & .988 & .012 \\
\hline 1165 & 125.75 & 79 & 9,934 & 415115 & .892 & .814 & .186 & 283.83 & 35 & 9,934 & 166269 & -.447 & .328 & .328 \\
\hline 1166 & 43.60 & 275 & 11,989 & 1751554 & 1.796 & .964 & .036 & 85.03 & 141 & 11,989 & 920716 & 1.837 & .967 & .033 \\
\hline 1167 & 55.79 & 121 & 6,751 & 417553 & .425 & .665 & .335 & 112.52 & 60 & 6,751 & 196033 & -.430 & .333 & .333 \\
\hline 1168 & 146.94 & 17 & 2,498 & 25616 & 1.474 & .930 & .070 & 416.33 & 6 & 2,498 & 6826 & -.378 & .353 & .353 \\
\hline 1169 & 624.50 & 2 & 1,249 & 1033 & -.424 & .336 & .336 & -- & -- & -- & -- & -- & -- & -- \\
\hline 1170 & 45.43 & 364 & 16,537 & 3230145 & 2.420 & .992 & .008 & 95.59 & 173 & 16,537 & 1536130 & 1.683 & .954 & .046 \\
\hline 1171 & 74.68 & 164 & 12,247 & 1013600 & .206 & .582 & .418 & 157.01 & 78 & 12,247 & 475085 & -.082 & .467 & .467 \\
\hline 1172 & 59.95 & 323 & 19,365 & 3161625 & .340 & .633 & .367 & 111.29 & 174 & 19,365 & 1733915 & .667 & .747 & .253 \\
\hline 1173 & 94.75 & 251 & 23,783 & 2865573 & -1.096 & .137 & .137 & 171.10 & 139 & 23,783 & 1592328 & -.749 & .227 & .227 \\
\hline 1174 & 103.67 & 49 & 5,080 & 140326 & 1.546 & .939 & .061 & 175.17 & 29 & 5,080 & 84778 & 1.408 & .920 & .080 \\
\hline 1175 & 113.02 & 240 & 27,125 & 3329847 & .617 & .731 & .269 & 248.85 & 109 & 27,125 & 1509606 & .383 & .649 & .351 \\
\hline 1176 & 327.00 & 2 & 654 & 596 & -.217 & .414 & .414 & 654.00 & 1 & 654 & 257 & -.371 & .355 & .355 \\
\hline 1177 & 56.84 & 25 & 1,421 & 21156 & 1.655 & .951 & .049 & 129.18 & 11 & 1,421 & 7314 & -.369 & .356 & .356 \\
\hline 1178 & 88.80 & 201 & 17,848 & 1863693 & .958 & .831 & .169 & 174.98 & 102 & 17,848 & 911754 & .029 & .512 & .488 \\
\hline 1179 & 116.11 & 151 & 17,533 & 1297811 & -.417 & .338 & .338 & 240.18 & 73 & 17,533 & 633221 & -.156 & .438 & .438 \\
\hline 1180 & 104.69 & 16 & 1,675 & 12670 & -.377 & .353 & .353 & 152.27 & 11 & 1,675 & 8808 & -.252 & .400 & .400 \\
\hline 1181 & 401.67 & 3 & 1,205 & 2040 & .386 & .650 & .350 & 602.50 & 2 & 1,205 & 886 & -.649 & .258 & .258 \\
\hline 1182 & 37.24 & 260 & 9,681 & 1320151 & 1.368 & .914 & .086 & 69.15 & 140 & 9,681 & 702401 & .748 & .773 & .227 \\
\hline 1183 & 48.65 & 403 & 19,606 & 4316784 & 3.223 & .999 & .001 & 92.48 & 212 & 19,606 & 2286293 & 2.525 & .994 & .006 \\
\hline 1184 & 1147.57 & 7 & 8,033 & 28835 & .117 & .547 & .453 & 8033.00 & 1 & 8,033 & 3174 & -.363 & .358 & .358 \\
\hline 1185 & 90.06 & 95 & 8,556 & 401948 & -.185 & .426 & .426 & 225.16 & 38 & 8,556 & 171127 & .562 & .713 & .287 \\
\hline 1186 & 51.51 & 117 & 6,027 & 359586 & .372 & .645 & .355 & 92.72 & 65 & 6,027 & 210942 & 1.074 & .859 & .141 \\
\hline 1187 & 37.14 & 309 & 11,476 & 1892738 & 2.055 & .980 & .020 & 65.95 & 174 & 11,476 & 1083073 & 1.937 & .974 & .026 \\
\hline 1188 & 526.63 & 19 & 10,006 & 104361 & .739 & .770 & .230 & 909.64 & 11 & 10,006 & 63698 & .905 & .817 & .183 \\
\hline 1189 & 110.10 & 174 & 19,158 & 1799440 & 1.819 & .966 & .034 & 258.89 & 74 & 19,158 & 749628 & .857 & .804 & .196 \\
\hline 1190 & 57.62 & 331 & 19,072 & 3327483 & 1.708 & .956 & .044 & 105.96 & 180 & 19,072 & 1899021 & 2.471 & .993 & .007 \\
\hline 1191 & 181.00 & 4 & 724 & 867 & -1.390 & .082 & .082 & 241.33 & 3 & 724 & 728 & -.989 & .161 & .161 \\
\hline 1192 & 129.68 & 50 & 6,484 & 165442 & .253 & .600 & .400 & 294.73 & 22 & 6,484 & 66805 & -.515 & .303 & .303 \\
\hline 1193 & 68.19 & 112 & 7,637 & 434858 & .308 & .621 & .379 & 117.49 & 65 & 7,637 & 235879 & -.693 & .244 & .244 \\
\hline 1194 & 54.46 & 153 & 8,332 & 693949 & 1.901 & .971 & .029 & 105.47 & 79 & 8,332 & 386661 & 2.692 & .996 & .004 \\
\hline 1195 & 47.32 & 47 & 2,224 & 54817 & .580 & .719 & .281 & 105.91 & 21 & 2,224 & 23830 & .163 & .565 & .435 \\
\hline 1196 & 54.44 & 118 & 6,424 & 371285 & -.384 & .351 & .351 & 112.70 & 57 & 6,424 & 179161 & -.280 & .390 & .390 \\
\hline 1197 & 68.56 & 289 & 19,815 & 3040271 & 1.820 & .966 & .034 & 140.53 & 141 & 19,815 & 1487191 & 1.329 & .908 & .092 \\
\hline 1198 & 114.89 & 79 & 9,076 & 398461 & 1.716 & .957 & .043 & 245.30 & 37 & 9,076 & 187226 & 1.212 & .887 & .113 \\
\hline 1199 & 120.00 & 3 & 360 & 485 & -.306 & .380 & .380 & 180.00 & 2 & 360 & 270 & -.612 & .270 & .270 \\
\hline 1200 & 102.06 & 141 & 14,391 & 1080010 & 1.327 & .908 & .092 & 194.47 & 74 & 14,391 & 567467 & .979 & .836 & .164 \\
\hline
\end{tabular}


Table 6. Summary of interoccurrence intervals for daily precipitation thresholds of 1.5 and 2.0 inches-Continued

\begin{tabular}{|c|c|c|c|c|c|c|c|c|c|c|c|c|c|c|}
\hline \multirow[b]{2}{*}{$\begin{array}{l}\text { Seq. } \\
\text { no. }\end{array}$} & \multicolumn{7}{|c|}{ Daily precipitation threshold of 1.5 inches and greater } & \multicolumn{7}{|c|}{ Daily precipitation threshold of 2.0 inches and greater } \\
\hline & $\begin{array}{l}\text { Mean } \\
\text { inter- } \\
\text { occur- } \\
\text { rence } \\
\text { interval } \\
\text { (days) }\end{array}$ & $\begin{array}{c}\text { Total } \\
\text { no. of } \\
\text { events }\end{array}$ & $\begin{array}{l}\text { Total } \\
\text { no. of } \\
\text { days } \\
\text { avail- } \\
\text { able }\end{array}$ & $\begin{array}{l}\text { Sigma } \\
\mathrm{S}\end{array}$ & $\begin{array}{c}\text { Stan- } \\
\text { dard } \\
\text { normal } \\
\text { variate }\end{array}$ & $\begin{array}{l}\text { Non- } \\
\text { ex- } \\
\text { ceed- } \\
\text { ance } \\
\text { prob- } \\
\text { ability }\end{array}$ & $\begin{array}{c}\text { p- } \\
\text { value }\end{array}$ & $\begin{array}{l}\text { Mean } \\
\text { inter- } \\
\text { occur- } \\
\text { rence } \\
\text { interval } \\
\text { (days) }\end{array}$ & $\begin{array}{c}\text { Total } \\
\text { no. of } \\
\text { events }\end{array}$ & $\begin{array}{l}\text { Total } \\
\text { no. of } \\
\text { days } \\
\text { avail- } \\
\text { able }\end{array}$ & $\begin{array}{l}\text { Sigma } \\
\mathrm{S}\end{array}$ & $\begin{array}{l}\text { Stan- } \\
\text { dard } \\
\text { normal } \\
\text { variate }\end{array}$ & $\begin{array}{l}\text { Non- } \\
\text { ex- } \\
\text { ceed- } \\
\text { ance } \\
\text { prob- } \\
\text { ability }\end{array}$ & $\begin{array}{c}p- \\
\text { value }\end{array}$ \\
\hline 1201 & 191.70 & 10 & 1,917 & 10029 & .254 & .600 & .400 & 273.86 & 7 & 1,917 & 7124 & .283 & .611 & .389 \\
\hline 1202 & 213.19 & 91 & 19,400 & 946895 & 1.202 & .885 & .115 & 451.16 & 43 & 19,400 & 510077 & 2.532 & .994 & .006 \\
\hline 1203 & 149.47 & 105 & 15,694 & 681239 & -3.074 & .001 & .001 & 290.63 & 54 & 15,694 & 361415 & -1.872 & .031 & .031 \\
\hline 1204 & 188.79 & 82 & 15,481 & 723291 & 2.189 & .986 & .014 & 322.52 & 48 & 15,481 & 426426 & 1.773 & .962 & .038 \\
\hline 1205 & 44.12 & 147 & 6,485 & 511435 & 1.533 & .937 & .063 & 93.99 & 69 & 6,485 & 244449 & 1.332 & .909 & .091 \\
\hline 1206 & 56.04 & 189 & 10,592 & 977250 & -.564 & .287 & .287 & 109.20 & 97 & 10,592 & 451910 & -2.052 & .020 & .020 \\
\hline 1207 & 54.50 & 112 & 6,104 & 348903 & .380 & .648 & .352 & 105.24 & 58 & 6,104 & 174196 & -.210 & .417 & .417 \\
\hline 1208 & 214.35 & 91 & 19,506 & 907317 & .369 & .644 & .356 & 527.19 & 37 & 19,506 & 345993 & -.434 & .332 & .332 \\
\hline 1209 & -- & -- & -- & -- & -- & -- & -- & -- & -- & -- & -- & -- & -- & -- \\
\hline 1210 & 62.86 & 59 & 3,709 & 95745 & -1.662 & .048 & .048 & 90.46 & 41 & 3,709 & 73143 & -.422 & .337 & .337 \\
\hline 1211 & 109.19 & 257 & 28,061 & 3577743 & -.216 & .414 & .414 & 179.88 & 156 & 28,061 & 2105669 & -.821 & .206 & .206 \\
\hline 1212 & 108.25 & 89 & 9,634 & 459152 & 1.160 & .877 & .123 & 181.77 & 53 & 9,634 & 288865 & 1.658 & .951 & .049 \\
\hline 1213 & 107.33 & 51 & 5,474 & 131581 & -.709 & .239 & .239 & 248.82 & 22 & 5,474 & 49634 & -1.427 & .077 & .077 \\
\hline 1214 & 72.40 & 10 & 724 & 4432 & 1.229 & .890 & .110 & 103.43 & 7 & 724 & 3099 & 1.022 & .847 & .154 \\
\hline 1215 & 406.52 & 21 & 8,537 & 81080 & -.758 & .224 & .224 & 948.56 & 9 & 8,537 & 22639 & -2.134 & .016 & .016 \\
\hline 1216 & 336.45 & 75 & 25,234 & 812815 & -2.116 & .017 & .017 & 788.56 & 32 & 25,234 & 384771 & -.460 & .323 & .323 \\
\hline 1217 & 62.82 & 209 & 13,130 & 1360704 & -.208 & .418 & .418 & 110.34 & 119 & 13,130 & 724689 & -1.368 & .086 & .086 \\
\hline 1218 & 52.73 & 62 & 3,269 & 99556 & -.240 & .405 & .405 & 108.97 & 30 & 3,269 & 50177 & .221 & .587 & .413 \\
\hline 1219 & 65.88 & 298 & 19,632 & 3210557 & 2.917 & .998 & .002 & 121.94 & 161 & 19,632 & 1708818 & 1.786 & .963 & .037 \\
\hline 1220 & 615.12 & 34 & 20,914 & 374244 & .531 & .702 & .298 & 1742.83 & 12 & 20,914 & 135299 & .469 & .681 & .319 \\
\hline 1221 & 117.54 & 50 & 5,877 & 144443 & -.207 & .418 & .418 & 293.85 & 20 & 5,877 & 56915 & -.245 & .403 & .403 \\
\hline 1222 & -- & -- & -- & -- & -- & -- & -- & -- & -- & -- & -- & -- & -- & -- \\
\hline 1223 & 63.67 & 86 & 5,476 & 258425 & 1.566 & .941 & .059 & 111.76 & 49 & 5,476 & 144845 & .965 & .833 & .167 \\
\hline 1224 & 70.64 & 28 & 1,978 & 32394 & 1.556 & .940 & .060 & 141.29 & 14 & 1,978 & 14835 & .463 & .678 & .322 \\
\hline 1225 & 63.07 & 42 & 2,649 & 54554 & -.217 & .414 & .414 & 120.41 & 22 & 2,649 & 26322 & -.785 & .216 & .216 \\
\hline 1226 & 238.05 & 92 & 21,901 & 915777 & -1.512 & .065 & .065 & 576.34 & 38 & 21,901 & 379807 & -.932 & .176 & .176 \\
\hline 1227 & 81.12 & 77 & 6,246 & 244193 & .235 & .593 & .407 & 178.46 & 35 & 6,246 & 126227 & 1.586 & .944 & .056 \\
\hline 1228 & 97.17 & 258 & 25,070 & 3291462 & .494 & .689 & .311 & 181.67 & 138 & 25,070 & 1753871 & .283 & .611 & .389 \\
\hline 1229 & 61.51 & 240 & 14,762 & 1862289 & 1.376 & .916 & .084 & 103.23 & 143 & 14,762 & 1114698 & 1.162 & .877 & .123 \\
\hline 1230 & 61.90 & 232 & 14,360 & 1811664 & 2.311 & .990 & .010 & 101.84 & 141 & 14,360 & 1145307 & 2.701 & .997 & .003 \\
\hline 1231 & 48.69 & 16 & 779 & 6383 & .168 & .567 & .433 & 86.56 & 9 & 779 & 3901 & .586 & .721 & .279 \\
\hline 1232 & 57.35 & 17 & 975 & 10618 & 2.008 & .978 & .022 & 121.88 & 8 & 975 & 5901 & 2.514 & .994 & .006 \\
\hline 1233 & 81.89 & 65 & 5,323 & 199740 & 2.159 & .985 & .015 & 120.98 & 44 & 5,323 & 135922 & 1.846 & .968 & .032 \\
\hline 1234 & 105.32 & 106 & 11,164 & 606457 & .445 & .672 & .328 & 192.48 & 58 & 11,164 & 333149 & .383 & .649 & .351 \\
\hline 1235 & 86.25 & 48 & 4,140 & 109279 & 1.198 & .884 & .116 & 153.33 & 27 & 4,140 & 59643 & .604 & .727 & .273 \\
\hline 1236 & 65.18 & 34 & 2,216 & 33282 & -1.177 & .120 & .120 & 123.11 & 18 & 2,216 & 16739 & -1.181 & .119 & .119 \\
\hline 1237 & 62.88 & 209 & 13,142 & 1455336 & 1.495 & .933 & .067 & 126.37 & 104 & 13,142 & 734264 & 1.315 & .906 & .094 \\
\hline 1238 & 79.36 & 321 & 25,474 & 4060181 & -.216 & .415 & .415 & 175.68 & 145 & 25,474 & 1936417 & 1.011 & .844 & .156 \\
\hline 1239 & 65.72 & 229 & 15,049 & 1659534 & -.967 & .167 & .167 & 133.18 & 113 & 15,049 & 861746 & .249 & .598 & .402 \\
\hline 1240 & 268.14 & 7 & 1,877 & 5980 & -.411 & .340 & .340 & 469.25 & 4 & 1,877 & 3500 & -.234 & .407 & .407 \\
\hline 1241 & 52.38 & 345 & 18,072 & 3192693 & .777 & .781 & .219 & 105.68 & 171 & 18,072 & 1637315 & 1.351 & .912 & .088 \\
\hline 1242 & 62.25 & 12 & 747 & 6316 & 2.455 & .993 & .007 & 106.71 & 7 & 747 & 4019 & 2.462 & .993 & .007 \\
\hline 1243 & 41.32 & 426 & 17,604 & 3847659 & .934 & .825 & .175 & 68.23 & 258 & 17,604 & 2324703 & .659 & .745 & .255 \\
\hline 1244 & 57.69 & 332 & 19,152 & 3196340 & .170 & .567 & .433 & 114.68 & 167 & 19,152 & 1602897 & .052 & .521 & .479 \\
\hline 1245 & 141.48 & 134 & 18,958 & 1327389 & 0.903 & .817 & .183 & 296.22 & 64 & 18,958 & 713745 & 2.446 & .993 & .007 \\
\hline 1246 & 144.50 & 16 & 2,312 & 20358 & .698 & .757 & .243 & 289.00 & 8 & 2,312 & 11212 & 1.040 & .851 & .149 \\
\hline 1247 & 125.47 & 121 & 15,182 & 883773 & -.721 & .236 & .236 & 230.03 & 66 & 15,182 & 499960 & -.029 & .488 & .488 \\
\hline 1248 & 20.33 & 3 & 61 & 135 & 1.426 & .923 & .077 & -- & -- & -- & -- & -- & -- & -- \\
\hline
\end{tabular}


Table 6. Summary of interoccurrence intervals for daily precipitation thresholds of 1.5 and 2.0 inches—Continued

\begin{tabular}{|c|c|c|c|c|c|c|c|c|c|c|c|c|c|c|}
\hline \multirow[b]{2}{*}{$\begin{array}{l}\text { Seq. } \\
\text { no. }\end{array}$} & \multicolumn{7}{|c|}{ Daily precipitation threshold of 1.5 inches and greater } & \multicolumn{7}{|c|}{ Daily precipitation threshold of 2.0 inches and greater } \\
\hline & $\begin{array}{l}\text { Mean } \\
\text { inter- } \\
\text { occur- } \\
\text { rence } \\
\text { interval } \\
\text { (days) }\end{array}$ & $\begin{array}{c}\text { Total } \\
\text { no. of } \\
\text { events }\end{array}$ & $\begin{array}{l}\text { Total } \\
\text { no. of } \\
\text { days } \\
\text { avail- } \\
\text { able }\end{array}$ & $\begin{array}{l}\text { Sigma } \\
\mathrm{S}\end{array}$ & $\begin{array}{c}\text { Stan- } \\
\text { dard } \\
\text { normal } \\
\text { variate }\end{array}$ & $\begin{array}{l}\text { Non- } \\
\text { ex- } \\
\text { ceed- } \\
\text { ance } \\
\text { prob- } \\
\text { ability }\end{array}$ & $\begin{array}{c}\mathrm{p}- \\
\text { value }\end{array}$ & $\begin{array}{l}\text { Mean } \\
\text { inter- } \\
\text { occur- } \\
\text { rence } \\
\text { interval } \\
\text { (days) }\end{array}$ & $\begin{array}{l}\text { Total } \\
\text { no. of } \\
\text { events }\end{array}$ & $\begin{array}{l}\text { Total } \\
\text { no. of } \\
\text { days } \\
\text { avail- } \\
\text { able }\end{array}$ & $\begin{array}{l}\text { Sigma } \\
\mathrm{S}\end{array}$ & $\begin{array}{c}\text { Stan- } \\
\text { dard } \\
\text { normal } \\
\text { variate }\end{array}$ & $\begin{array}{l}\text { Non- } \\
\text { ex- } \\
\text { ceed- } \\
\text { ance } \\
\text { prob- } \\
\text { ability }\end{array}$ & $\begin{array}{c}p- \\
\text { value }\end{array}$ \\
\hline 1249 & 95.56 & 129 & 12,327 & 828694 & .831 & .797 & 203 & 192.61 & 64 & 12,327 & 388224 & -.219 & .413 & .413 \\
\hline 1250 & 66.29 & 556 & 36,857 & 10230399 & -.063 & .475 & .475 & 130.70 & 282 & 36,857 & 5236363 & .221 & .588 & .412 \\
\hline 1251 & 221.60 & 5 & 1,108 & 1406 & -1.907 & .028 & .028 & 554.00 & 2 & 1,108 & 376 & -1.618 & .053 & .053 \\
\hline 1252 & 76.61 & 463 & 35,470 & 8683065 & 2.141 & .984 & .016 & 164.21 & 216 & 35,470 & 4125784 & 1.961 & .975 & .025 \\
\hline 1253 & 47.50 & 94 & 4,465 & 205360 & -.360 & .360 & .360 & 91.12 & 49 & 4,465 & 106163 & -.358 & .360 & .360 \\
\hline 1254 & 30.40 & 15 & 456 & 3176 & -.479 & .316 & .316 & 41.46 & 11 & 456 & 2552 & .101 & .540 & .460 \\
\hline 1255 & 58.90 & 228 & 13,428 & 1480272 & -.863 & .194 & .194 & 104.91 & 128 & 13,428 & 855056 & -.099 & .461 & .461 \\
\hline 1256 & 116.99 & 175 & 20,473 & 1975168 & 2.351 & .991 & .009 & 227.48 & 90 & 20,473 & 1027540 & 1.895 & .971 & .029 \\
\hline 1257 & 103.33 & 189 & 19,529 & 1947139 & 1.312 & .905 & .095 & 189.60 & 103 & 19,529 & 1051526 & .800 & .788 & .212 \\
\hline 1258 & 58.60 & 62 & 3,633 & 110345 & -.276 & .391 & .391 & 103.80 & 35 & 3,633 & 62296 & -.207 & .418 & .418 \\
\hline 1259 & -- & -- & -- & -- & -- & -- & -- & -- & -- & -- & -- & -- & -- & -- \\
\hline 1260 & 54.87 & 397 & 21,784 & 4700163 & 3.001 & .999 & .001 & 96.39 & 226 & 21,784 & 2625210 & 1.731 & .958 & .042 \\
\hline 1261 & 115.20 & 71 & 8,179 & 327398 & 1.862 & .969 & .031 & 247.85 & 33 & 8,179 & 162706 & 2.046 & .980 & .020 \\
\hline 1262 & 72.13 & 16 & 1,154 & 7150 & -1.562 & .059 & .059 & 128.22 & 9 & 1,154 & 4010 & -1.184 & .118 & .118 \\
\hline 1263 & 86.00 & 6 & 516 & 1536 & -.033 & .487 & .487 & 172.00 & 3 & 516 & 726 & -.186 & .426 & .426 \\
\hline 1264 & 172.00 & 9 & 1,548 & 8249 & .957 & .831 & .169 & 309.60 & 5 & 1,548 & 4254 & .384 & .650 & .350 \\
\hline 1265 & 41.61 & 83 & 3,454 & 149564 & .685 & .753 & .247 & 80.33 & 43 & 3,454 & 93061 & 2.875 & .998 & .002 \\
\hline 1266 & 79.68 & 239 & 19,044 & 2226666 & -.578 & .282 & .282 & 160.03 & 119 & 19,044 & 1198261 & 1.086 & .861 & .139 \\
\hline 1267 & 97.71 & 138 & 13,484 & 953977 & .516 & .697 & .303 & 175.12 & 77 & 13,484 & 556622 & 1.098 & .864 & .136 \\
\hline 1268 & 90.80 & 189 & 17,161 & 1628591 & .101 & .540 & .460 & 157.44 & 109 & 17,161 & 989206 & 1.043 & .851 & .149 \\
\hline 1269 & -- & -- & -- & -- & -- & -- & -- & -- & -- & -- & -- & -- & -- & -- \\
\hline 1270 & 145.80 & 15 & 2,187 & 15052 & -.552 & .290 & .290 & 364.50 & 6 & 2,187 & 5678 & -.571 & .284 & .284 \\
\hline 1271 & 95.83 & 366 & 35,073 & 5908720 & -2.631 & .004 & .004 & 193.77 & 181 & 35,073 & 2729065 & -3.267 & .001 & .001 \\
\hline 1272 & 107.87 & 37 & 3,991 & 67718 & -.873 & .192 & .192 & 307.00 & 13 & 3,991 & 21325 & -1.111 & .133 & .133 \\
\hline 1273 & 89.90 & 106 & 9,529 & 484486 & -.726 & .234 & .234 & 186.84 & 51 & 9,529 & 260421 & .887 & .812 & .188 \\
\hline 1274 & 37.75 & 163 & 6,153 & 522355 & .921 & .821 & .179 & 65.46 & 94 & 6,153 & 295484 & .365 & .643 & .357 \\
\hline 1275 & 33.39 & 108 & 3,606 & 203821 & .841 & .800 & .200 & 54.64 & 66 & 3,606 & 120769 & .209 & .583 & .417 \\
\hline 1276 & 53.14 & 104 & 5,527 & 272624 & -.908 & .182 & .182 & 83.74 & 66 & 5,527 & 170645 & -.906 & .182 & .182 \\
\hline 1277 & 25.60 & 100 & 2,560 & 137205 & 1.246 & .894 & .107 & 39.39 & 65 & 2,560 & 93669 & 1.757 & .961 & .039 \\
\hline 1278 & 56.60 & 512 & 28,977 & 7646493 & 1.207 & .886 & .114 & 103.12 & 281 & 28,977 & 4263621 & 1.372 & .915 & .085 \\
\hline 1279 & 250.17 & 6 & 1,501 & 3184 & -1.243 & .107 & .107 & 500.33 & 3 & 1,501 & 2286 & .046 & .518 & .482 \\
\hline 1280 & 94.37 & 57 & 5,379 & 178105 & 2.116 & .983 & .017 & 168.09 & 32 & 5,379 & 100932 & 1.693 & .955 & .045 \\
\hline 1281 & 63.95 & 97 & 6,203 & 283715 & -.971 & .166 & .166 & 126.59 & 49 & 6,203 & 145979 & -.478 & .316 & .316 \\
\hline 1282 & 245.00 & 1 & 245 & 221 & 1.393 & .918 & .082 & -- & -- & -- & -- & -- & -- & -- \\
\hline 1283 & 106.80 & 49 & 5,233 & 146266 & 1.708 & .956 & .044 & 249.19 & 21 & 5,233 & 61636 & .966 & .833 & .167 \\
\hline 1284 & 36.86 & 265 & 9,769 & 1373204 & 1.717 & .957 & .043 & 70.28 & 139 & 9,769 & 746474 & 2.031 & .979 & .021 \\
\hline 1285 & 309.65 & 68 & 21,056 & 739611 & .473 & .682 & .318 & 726.07 & 29 & 21,056 & 343945 & 1.180 & .881 & .119 \\
\hline 1286 & 49.72 & 368 & 18,295 & 3430289 & .632 & .736 & .264 & 97.83 & 187 & 18,295 & 1750470 & .552 & .710 & .290 \\
\hline 1287 & 127.23 & 47 & 5,980 & 152766 & 1.034 & .849 & .151 & 206.21 & 29 & 5,980 & 95641 & .961 & .832 & .168 \\
\hline 1288 & 107.57 & 112 & 12,048 & 656808 & -.486 & .314 & .314 & 236.24 & 51 & 12,048 & 264079 & -1.737 & .041 & .041 \\
\hline 1289 & 120.59 & 115 & 13,868 & 842370 & 1.047 & .852 & .148 & 277.36 & 50 & 13,868 & 363456 & .592 & .723 & .277 \\
\hline 1290 & 110.00 & 13 & 1,430 & 12071 & 1.865 & .969 & .031 & 238.33 & 6 & 1,430 & 6588 & 2.273 & .988 & .012 \\
\hline 1291 & 135.11 & 9 & 1,216 & 6907 & 1.363 & .913 & .087 & 243.20 & 5 & 1,216 & 4125 & 1.382 & .917 & .083 \\
\hline 1292 & 53.29 & 369 & 19,665 & 3822435 & 1.781 & .963 & .037 & 100.33 & 196 & 19,665 & 2018817 & 1.153 & .876 & .124 \\
\hline 1293 & 73.45 & 232 & 17,041 & 1958471 & -0.244 & .404 & .404 & 139.68 & 122 & 17,041 & 1019696 & -0.365 & .358 & .358 \\
\hline 1294 & 91.96 & 55 & 5,058 & 129780 & -.860 & .195 & .195 & 174.41 & 29 & 5,058 & 60243 & -1.666 & .048 & .048 \\
\hline 1295 & 45.57 & 14 & 638 & 6213 & 2.535 & .994 & .006 & 91.14 & 7 & 638 & 2746 & 1.053 & .854 & .146 \\
\hline 1296 & 33.26 & 128 & 4,257 & 272239 & -.015 & .494 & .494 & 61.70 & 69 & 4,257 & 154327 & .731 & .768 & .232 \\
\hline
\end{tabular}


Table 6. Summary of interoccurrence intervals for daily precipitation thresholds of 1.5 and 2.0 inches-Continued

\begin{tabular}{|c|c|c|c|c|c|c|c|c|c|c|c|c|c|c|}
\hline \multirow[b]{2}{*}{$\begin{array}{l}\text { Seq. } \\
\text { no. }\end{array}$} & \multicolumn{7}{|c|}{ Daily precipitation threshold of 1.5 inches and greater } & \multicolumn{7}{|c|}{ Daily precipitation threshold of 2.0 inches and greater } \\
\hline & $\begin{array}{c}\text { Mean } \\
\text { inter- } \\
\text { occur- } \\
\text { rence } \\
\text { interval } \\
\text { (days) }\end{array}$ & $\begin{array}{c}\text { Total } \\
\text { no. of } \\
\text { events }\end{array}$ & $\begin{array}{c}\text { Total } \\
\text { no. of } \\
\text { days } \\
\text { avail- } \\
\text { able }\end{array}$ & $\underset{\mathbf{S}}{\operatorname{Sigma}}$ & $\begin{array}{c}\text { Stan- } \\
\text { dard } \\
\text { normal } \\
\text { variate }\end{array}$ & $\begin{array}{c}\text { Non- } \\
\text { ex- } \\
\text { ceed- } \\
\text { ance } \\
\text { prob- } \\
\text { ability }\end{array}$ & $\begin{array}{c}\mathrm{p}- \\
\text { value }\end{array}$ & $\begin{array}{l}\text { Mean } \\
\text { inter- } \\
\text { occur- } \\
\text { rence } \\
\text { interval } \\
\text { (days) }\end{array}$ & $\begin{array}{c}\text { Total } \\
\text { no. of } \\
\text { events }\end{array}$ & $\begin{array}{c}\text { Total } \\
\text { no. of } \\
\text { days } \\
\text { avail- } \\
\text { able }\end{array}$ & $\underset{\mathbf{S}}{\text { Sigma }}$ & $\begin{array}{c}\text { Stan- } \\
\text { dard } \\
\text { normal } \\
\text { variate }\end{array}$ & $\begin{array}{l}\text { Non- } \\
\text { ex- } \\
\text { ceed- } \\
\text { ance } \\
\text { prob- } \\
\text { ability }\end{array}$ & $\begin{array}{c}\text { p- } \\
\text { value }\end{array}$ \\
\hline 1297 & 73.22 & 37 & 2,709 & 48301 & -.382 & .351 & .351 & 129.00 & 21 & 2,709 & 24573 & -1.080 & .140 & .140 \\
\hline 1298 & 67.50 & 22 & 1,485 & 16752 & .207 & .582 & .418 & 114.23 & 13 & 1,485 & 10055 & .260 & .603 & .397 \\
\hline 1299 & 49.47 & 196 & 9,696 & 1046312 & 2.453 & .993 & .007 & 93.23 & 104 & 9,696 & 566117 & 2.169 & .985 & .015 \\
\hline 1300 & -- & -- & -- & -- & -- & -- & -- & -- & -- & -- & -- & -- & -- & -- \\
\hline 1301 & 59.63 & 466 & 27,787 & 6704412 & 1.329 & .908 & .092 & 105.25 & 264 & 27,787 & 3763077 & .730 & .767 & .233 \\
\hline 1302 & 69.24 & 270 & 18,695 & 2685836 & 1.827 & .966 & .034 & 127.18 & 147 & 18,695 & 1462240 & 1.347 & .911 & .089 \\
\hline 1303 & 32.33 & 9 & 291 & 1071 & -.946 & .172 & .172 & 97.00 & 3 & 291 & 360 & -.526 & .300 & .300 \\
\hline 1304 & 80.89 & 45 & 3,640 & 91438 & 1.353 & .912 & .088 & 165.46 & 22 & 3,640 & 44321 & .869 & .807 & .193 \\
\hline 1305 & 1192.37 & 19 & 22,655 & 227193 & .420 & .663 & .337 & 4531.00 & 5 & 22,655 & 67950 & .774 & .780 & .220 \\
\hline 1306 & 125.82 & 119 & 14,972 & 853457 & -.793 & .214 & .214 & 216.99 & 69 & 14,972 & 456871 & -1.662 & .048 & .048 \\
\hline
\end{tabular}


Table 7. Summary of interoccurrence intervals for daily precipitation thresholds of 2.5 and 3.0 inches

[--, not available, no events that equal or exceed the threshold were observed]

\begin{tabular}{|c|c|c|c|c|c|c|c|c|c|c|c|c|c|c|}
\hline \multirow[b]{2}{*}{$\begin{array}{l}\text { Seq. } \\
\text { no. }\end{array}$} & \multicolumn{7}{|c|}{ Daily precipitation threshold of 2.5 inches and greater } & \multicolumn{7}{|c|}{ Daily precipitation threshold of 3.0 inches and greater } \\
\hline & $\begin{array}{l}\text { Mean } \\
\text { inter- } \\
\text { occur- } \\
\text { rence } \\
\text { interval } \\
\text { (days) }\end{array}$ & $\begin{array}{c}\text { Total } \\
\text { no. of } \\
\text { events }\end{array}$ & $\begin{array}{l}\text { Total } \\
\text { no. of } \\
\text { days } \\
\text { avail- } \\
\text { able }\end{array}$ & $\begin{array}{l}\text { Sigma } \\
\mathrm{S}\end{array}$ & $\begin{array}{c}\text { Stan- } \\
\text { dard } \\
\text { normal } \\
\text { variate }\end{array}$ & $\begin{array}{l}\text { Non- } \\
\text { ex- } \\
\text { ceed- } \\
\text { ance } \\
\text { prob- } \\
\text { ability }\end{array}$ & $\begin{array}{c}\mathrm{p}- \\
\text { value }\end{array}$ & $\begin{array}{l}\text { Mean } \\
\text { inter- } \\
\text { occur- } \\
\text { rence } \\
\text { interval } \\
\text { (days) }\end{array}$ & $\begin{array}{l}\text { Total } \\
\text { no. of } \\
\text { events }\end{array}$ & $\begin{array}{l}\text { Total } \\
\text { no. of } \\
\text { days } \\
\text { avail- } \\
\text { able }\end{array}$ & $\begin{array}{l}\text { Sigma } \\
\mathrm{S}\end{array}$ & $\begin{array}{c}\text { Stan- } \\
\text { dard } \\
\text { normal } \\
\text { variate }\end{array}$ & $\begin{array}{l}\text { Non- } \\
\text { ex- } \\
\text { ceed- } \\
\text { ance } \\
\text { prob- } \\
\text { ability }\end{array}$ & $\begin{array}{c}\text { p- } \\
\text { value }\end{array}$ \\
\hline 1 & 780.56 & 25 & 19,514 & 212846 & -1.103 & .135 & .135 & 1393.86 & 14 & 19,514 & 132246 & -0.207 & .418 & .418 \\
\hline 2 & -- & -- & -- & -- & -- & -- & -- & -- & -- & -- & -- & -- & -- & -- \\
\hline 3 & 616.25 & 32 & 19,720 & 291421 & -.748 & .227 & .227 & 1232.50 & 16 & 19,720 & 171243 & .592 & .723 & .277 \\
\hline 4 & 336.64 & 25 & 8,416 & 102472 & -.225 & .411 & .411 & 601.14 & 14 & 8,416 & 55451 & -.381 & .352 & .352 \\
\hline 5 & 542.66 & 35 & 18,993 & 409143 & 2.367 & .991 & .009 & 791.38 & 24 & 18,993 & 277041 & 1.829 & .966 & .034 \\
\hline 6 & 440.25 & 8 & 3,522 & 17211 & 1.086 & .861 & .139 & 587.00 & 6 & 3,522 & 13216 & 1.064 & .856 & .144 \\
\hline 7 & 344.33 & 24 & 8,264 & 66044 & -2.834 & .002 & .002 & 918.22 & 9 & 8,264 & 28172 & -1.260 & .104 & .104 \\
\hline 8 & 1458.00 & 1 & 1,458 & 1144 & .986 & .838 & .162 & -- & -- & -- & -- & -- & -- & -- \\
\hline 9 & 964.33 & 3 & 2,893 & 3144 & -.827 & .204 & .204 & 964.33 & 3 & 2,893 & 3144 & -.827 & .204 & .204 \\
\hline 10 & -- & -- & -- & -- & -- & -- & -- & -- & -- & -- & -- & -- & -- & -- \\
\hline 11 & 235.39 & 26 & 6,120 & 79568 & .001 & .500 & .500 & 470.77 & 13 & 6,120 & 27153 & -1.982 & .024 & .024 \\
\hline 12 & 408.98 & 87 & 35,581 & 1591886 & .460 & .677 & .323 & 741.27 & 48 & 35,581 & 893570 & .557 & .711 & .289 \\
\hline 13 & 341.02 & 44 & 15,005 & 332762 & .092 & .537 & .463 & 468.91 & 32 & 15,005 & 257755 & .721 & .765 & .235 \\
\hline 14 & 285.39 & 105 & 29,966 & 1666498 & 1.052 & .854 & .146 & 416.19 & 72 & 29,966 & 1160118 & 1.108 & .866 & .134 \\
\hline 15 & 427.25 & 4 & 1,709 & 4739 & 1.339 & .910 & .090 & 854.50 & 2 & 1,709 & 1996 & .411 & .660 & .340 \\
\hline 16 & -- & -- & -- & -- & -- & -- & -- & -- & -- & -- & -- & -- & -- & -- \\
\hline 17 & -- & -- & -- & -- & -- & -- & -- & -- & -- & -- & -- & -- & -- & -- \\
\hline 18 & 2503.55 & 11 & 27,539 & 201407 & 1.894 & .971 & .029 & 27539.00 & 1 & 27,539 & 15717 & .245 & .597 & .403 \\
\hline 19 & 179.81 & 107 & 19,240 & 1035566 & .108 & .543 & .457 & 305.40 & 63 & 19,240 & 636376 & .688 & .754 & .246 \\
\hline 20 & 156.97 & 39 & 6,122 & 116962 & -.219 & .413 & .413 & 211.10 & 29 & 6,122 & 76831 & -1.254 & .105 & .105 \\
\hline 21 & 229.52 & 23 & 5,279 & 58716 & -.273 & .393 & .393 & 310.53 & 17 & 5,279 & 45520 & .103 & .541 & .459 \\
\hline 22 & 122.92 & 281 & 34,541 & 4861710 & .052 & .521 & .479 & 200.82 & 172 & 34,541 & 2907358 & -.483 & .315 & .315 \\
\hline 23 & 395.67 & 3 & 1,187 & 2496 & 1.206 & .886 & .114 & 1187.00 & 1 & 1,187 & 729 & .395 & .654 & .346 \\
\hline 24 & 1094.06 & 18 & 19,693 & 148728 & -1.182 & .119 & .119 & 1790.27 & 11 & 19,693 & 83292 & -1.327 & .092 & .092 \\
\hline 25 & 474.35 & 29 & 13,756 & 152515 & -2.195 & .014 & .014 & 724.00 & 19 & 13,756 & 94945 & -2.065 & .019 & .019 \\
\hline 26 & 108.74 & 286 & 31,099 & 4821907 & 2.468 & .993 & .007 & 172.77 & 180 & 31,099 & 3010805 & 1.759 & .961 & .039 \\
\hline 27 & 159.90 & 130 & 20,787 & 1294939 & -.822 & .206 & .206 & 296.96 & 70 & 20,787 & 647462 & -1.595 & .055 & .055 \\
\hline 28 & 364.30 & 33 & 12,022 & 201547 & .160 & .563 & .437 & 667.89 & 18 & 12,022 & 106342 & -.126 & .450 & .450 \\
\hline 29 & 1221.85 & 13 & 15,884 & 73639 & -1.791 & .037 & .037 & 1764.89 & 9 & 15,884 & 48731 & -1.654 & .049 & .049 \\
\hline 30 & 115.44 & 274 & 31,631 & 4721196 & 2.565 & .995 & .005 & 158.95 & 199 & 31,631 & 3442734 & 2.294 & .989 & .011 \\
\hline 31 & 226.61 & 80 & 18,129 & 752069 & .575 & .717 & .283 & 412.02 & 44 & 18,129 & 413102 & .411 & .659 & .341 \\
\hline 32 & 369.96 & 45 & 16,648 & 351288 & -.723 & .235 & .235 & 594.57 & 28 & 16,648 & 190766 & -1.664 & .048 & .048 \\
\hline 33 & 463.66 & 50 & 23,183 & 689340 & 2.320 & .990 & .010 & 858.63 & 27 & 23,183 & 377145 & 1.845 & .968 & .032 \\
\hline 34 & 329.27 & 11 & 3,622 & 24206 & 1.236 & .892 & .108 & 452.75 & 8 & 3,622 & 18207 & 1.258 & .896 & .104 \\
\hline 35 & 183.79 & 56 & 10,292 & 320280 & 1.444 & .926 & .074 & 233.91 & 44 & 10,292 & 262012 & 1.806 & .965 & .035 \\
\hline 36 & 124.51 & 89 & 11,081 & 473113 & -.663 & .254 & .254 & 167.89 & 66 & 11,081 & 360413 & -.202 & .420 & .420 \\
\hline 37 & 145.48 & 75 & 10,911 & 429388 & .742 & .771 & .229 & 222.67 & 49 & 10,911 & 273715 & .290 & .614 & .386 \\
\hline 38 & 306.99 & 73 & 22,410 & 816760 & -.022 & .491 & .491 & 589.74 & 38 & 22,410 & 449418 & .593 & .723 & .277 \\
\hline 39 & 257.04 & 76 & 19,535 & 850611 & 2.203 & .986 & .014 & 527.97 & 37 & 19,535 & 425041 & 1.855 & .968 & .032 \\
\hline 40 & 280.51 & 37 & 10,379 & 197938 & .325 & .628 & .373 & 494.24 & 21 & 10,379 & 115027 & .441 & .670 & .330 \\
\hline 41 & 204.85 & 121 & 24,787 & 1654907 & 1.973 & .976 & .024 & 387.30 & 64 & 24,787 & 936493 & 2.504 & .994 & .006 \\
\hline 42 & 148.80 & 5 & 744 & 1210 & -1.354 & .088 & .088 & 248.00 & 3 & 744 & 639 & -1.282 & .100 & .100 \\
\hline 43 & 534.70 & 60 & 32,082 & 969104 & .093 & .537 & .463 & 972.18 & 33 & 32,082 & 476370 & -.996 & .160 & .160 \\
\hline 44 & 183.70 & 107 & 19,656 & 1106708 & .939 & .826 & .174 & 344.84 & 57 & 19,656 & 609769 & 1.157 & .876 & .124 \\
\hline 45 & 164.76 & 115 & 18,947 & 1126293 & 0.628 & .735 & .265 & 332.40 & 57 & 18,947 & 604100 & 1.553 & .940 & .060 \\
\hline 46 & 251.00 & 103 & 25,853 & 1312353 & -.252 & .401 & .401 & 403.95 & 64 & 25,853 & 857743 & .510 & .695 & .305 \\
\hline 47 & 254.52 & 25 & 6,363 & 98877 & 2.106 & .982 & .018 & 454.50 & 14 & 6,363 & 54830 & 1.497 & .933 & .067 \\
\hline 48 & 225.92 & 25 & 5,648 & 83885 & 1.630 & .948 & .052 & 353.00 & 16 & 5,648 & 58021 & 1.968 & .975 & .025 \\
\hline
\end{tabular}


Table 7. Summary of interoccurrence intervals for daily precipitation thresholds of 2.5 and 3.0 inches-Continued

\begin{tabular}{|c|c|c|c|c|c|c|c|c|c|c|c|c|c|c|}
\hline \multirow[b]{2}{*}{$\begin{array}{l}\text { Seq. } \\
\text { no. }\end{array}$} & \multicolumn{7}{|c|}{ Daily precipitation threshold of 2.5 inches and greater } & \multicolumn{7}{|c|}{ Daily precipitation threshold of 3.0 inches and greater } \\
\hline & $\begin{array}{l}\text { Mean } \\
\text { inter- } \\
\text { occur- } \\
\text { rence } \\
\text { interval } \\
\text { (days) }\end{array}$ & $\begin{array}{c}\text { Total } \\
\text { no. of } \\
\text { events }\end{array}$ & $\begin{array}{l}\text { Total } \\
\text { no. of } \\
\text { days } \\
\text { avail- } \\
\text { able }\end{array}$ & $\begin{array}{l}\text { Sigma } \\
\mathrm{S}\end{array}$ & $\begin{array}{c}\text { Stan- } \\
\text { dard } \\
\text { normal } \\
\text { variate }\end{array}$ & $\begin{array}{l}\text { Non- } \\
\text { ex- } \\
\text { ceed- } \\
\text { ance } \\
\text { prob- } \\
\text { ability }\end{array}$ & $\begin{array}{c}\text { p- } \\
\text { value }\end{array}$ & $\begin{array}{l}\text { Mean } \\
\text { inter- } \\
\text { occur- } \\
\text { rence } \\
\text { interval } \\
\text { (days) }\end{array}$ & $\begin{array}{c}\text { Total } \\
\text { no. of } \\
\text { events }\end{array}$ & $\begin{array}{l}\text { Total } \\
\text { no. of } \\
\text { days } \\
\text { avail- } \\
\text { able }\end{array}$ & $\begin{array}{l}\text { Sigma } \\
\mathrm{S}\end{array}$ & $\begin{array}{l}\text { Stan- } \\
\text { dard } \\
\text { normal } \\
\text { variate }\end{array}$ & $\begin{array}{l}\text { Non- } \\
\text { ex- } \\
\text { ceed- } \\
\text { ance } \\
\text { prob- } \\
\text { ability }\end{array}$ & $\begin{array}{c}p- \\
\text { value }\end{array}$ \\
\hline 49 & 302.00 & 1 & 302 & 250 & 1.136 & .872 & .128 & -- & -- & -- & -- & -- & -- & -- \\
\hline 50 & 305.00 & 3 & 915 & 1990 & 1.350 & .911 & .089 & 457.50 & 2 & 915 & 1175 & 696 & .757 & .243 \\
\hline 51 & 207.08 & 88 & 18,223 & 810265 & .171 & .568 & .432 & 303.72 & 60 & 18,223 & 534836 & -.291 & .386 & .386 \\
\hline 52 & 189.99 & 69 & 13,109 & 435408 & -.536 & .296 & .296 & 267.53 & 49 & 13,109 & 306640 & -.549 & .292 & .292 \\
\hline 53 & 61.20 & 5 & 306 & 1166 & 2.030 & .979 & .021 & 76.50 & 4 & 306 & 874 & 1.483 & .931 & .069 \\
\hline 54 & 43.50 & 2 & 87 & 61 & -.732 & .232 & .232 & 87.00 & 1 & 87 & 36 & -.299 & .383 & .383 \\
\hline 55 & 290.15 & 34 & 9,865 & 179514 & .711 & .761 & .239 & 448.41 & 22 & 9,865 & 128322 & 1.483 & .931 & .069 \\
\hline 56 & 1192.50 & 2 & 2,385 & 2248 & -.141 & .444 & .444 & 1192.50 & 2 & 2,385 & 2248 & -.141 & .444 & .444 \\
\hline 57 & 1030.47 & 19 & 19,579 & 198078 & .490 & .688 & .312 & 1957.90 & 10 & 19,579 & 96866 & -.058 & .477 & .477 \\
\hline 58 & -- & -- & -- & -- & -- & -- & -- & -- & -- & -- & -- & -- & -- & -- \\
\hline 59 & 510.23 & 71 & 36,226 & 1338771 & .599 & .725 & .275 & 754.71 & 48 & 36,226 & 815088 & -.750 & .227 & .227 \\
\hline 60 & -- & -- & -- & -- & -- & -- & -- & -- & -- & -- & -- & -- & -- & -- \\
\hline 61 & 2322.08 & 12 & 27,865 & 146967 & -.726 & .234 & .234 & 3483.13 & 8 & 27,865 & 103239 & -.361 & .359 & .359 \\
\hline 62 & -- & -- & -- & -- & -- & -- & -- & -- & -- & -- & -- & -- & -- & -- \\
\hline 63 & 2039.00 & 1 & 2,039 & 878 & -.240 & .405 & .405 & 2039.00 & 1 & 2,039 & 878 & -.240 & .405 & .405 \\
\hline 64 & -- & -- & -- & -- & -- & -- & -- & -- & -- & -- & -- & -- & -- & -- \\
\hline 65 & 247.40 & 30 & 7,422 & 108434 & -.247 & .403 & .403 & 436.59 & 17 & 7,422 & 64978 & .214 & .585 & .415 \\
\hline 66 & 259.33 & 9 & 2,334 & 8773 & -.856 & .196 & .196 & 333.43 & 7 & 2,334 & 7259 & -.511 & .305 & .305 \\
\hline 67 & 238.21 & 56 & 13,340 & 395815 & .774 & .780 & .220 & 392.35 & 34 & 13,340 & 236244 & .422 & .663 & .337 \\
\hline 68 & 1745.67 & 3 & 5,237 & 8650 & .303 & .619 & .381 & -- & -- & -- & -- & -- & -- & -- \\
\hline 69 & 2458.50 & 2 & 4,917 & 7857 & 1.465 & .928 & .072 & 4917.00 & 1 & 4,917 & 3035 & .406 & .658 & .342 \\
\hline 70 & 207.20 & 5 & 1,036 & 3477 & 1.326 & .908 & .092 & 259.00 & 4 & 1,036 & 2786 & 1.194 & .884 & .116 \\
\hline 71 & 762.00 & 1 & 762 & 472 & .414 & .660 & .340 & 762.00 & 1 & 762 & 472 & .414 & .660 & .340 \\
\hline 72 & 335.85 & 13 & 4,366 & 26334 & -.450 & .326 & .326 & 873.20 & 5 & 4,366 & 12331 & .502 & .692 & .308 \\
\hline 73 & 271.80 & 46 & 12,503 & 261089 & -1.082 & .140 & .140 & 520.96 & 24 & 12,503 & 117759 & -1.825 & .034 & .034 \\
\hline 74 & 134.22 & 9 & 1,208 & 4712 & -.692 & .245 & .245 & 172.57 & 7 & 1,208 & 3172 & -1.145 & .126 & .126 \\
\hline 75 & 131.66 & 178 & 23,435 & 2317446 & 2.567 & .995 & .005 & 203.78 & 115 & 23,435 & 1544566 & 2.716 & .997 & .003 \\
\hline 76 & 288.50 & 4 & 1,154 & 3461 & 1.731 & .958 & .042 & 577.00 & 2 & 1,154 & 2172 & 2.161 & .985 & .015 \\
\hline 77 & 104.20 & 152 & 15,838 & 1264332 & 1.076 & .859 & .141 & 179.98 & 88 & 15,838 & 711821 & .349 & .636 & .364 \\
\hline 78 & 241.33 & 3 & 724 & 631 & -1.257 & .104 & .104 & 241.33 & 3 & 724 & 631 & -1.257 & .104 & .104 \\
\hline 79 & 194.33 & 3 & 583 & 1150 & .945 & .828 & .172 & -- & -- & -- & -- & -- & -- & -- \\
\hline 80 & 120.27 & 296 & 35,599 & 5290243 & .122 & .549 & .451 & 183.50 & 194 & 35,599 & 3467659 & .102 & .541 & .459 \\
\hline 81 & 114.85 & 123 & 14,126 & 918689 & 1.104 & .865 & .135 & 170.19 & 83 & 14,126 & 630506 & 1.192 & .883 & .117 \\
\hline 82 & 162.45 & 78 & 12,671 & 458369 & -1.108 & .134 & .134 & 248.45 & 51 & 12,671 & 301131 & -.841 & .200 & .200 \\
\hline 83 & 252.84 & 141 & 35,650 & 2662734 & 1.223 & .889 & .111 & 434.76 & 82 & 35,650 & 1497925 & .389 & .651 & .349 \\
\hline 84 & 177.55 & 44 & 7,812 & 163663 & -.548 & .292 & .292 & 325.50 & 24 & 7,812 & 92181 & -.142 & .444 & .444 \\
\hline 85 & 183.89 & 9 & 1,655 & 8082 & .443 & .671 & .329 & 331.00 & 5 & 1,655 & 4158 & .019 & .508 & .492 \\
\hline 86 & 253.54 & 57 & 14,452 & 430537 & .592 & .723 & .277 & 466.19 & 31 & 14,452 & 218102 & -.254 & .400 & .400 \\
\hline 87 & 242.60 & 5 & 1,213 & 4583 & 1.980 & .976 & .024 & 404.33 & 3 & 1,213 & 3418 & 2.636 & .996 & .004 \\
\hline 88 & 279.41 & 46 & 12,853 & 300551 & .196 & .578 & .422 & 357.03 & 36 & 12,853 & 218023 & -.599 & .275 & .275 \\
\hline 89 & 359.79 & 53 & 19,069 & 531418 & .651 & .742 & .258 & 681.04 & 28 & 19,069 & 296662 & 1.020 & .846 & .154 \\
\hline 90 & 304.38 & 21 & 6,392 & 69736 & .310 & .622 & .378 & 581.09 & 11 & 6,392 & 41225 & .992 & .839 & .161 \\
\hline 91 & 459.67 & 27 & 12,411 & 156385 & -.600 & .274 & .274 & 886.50 & 14 & 12,411 & 87025 & .011 & .504 & .496 \\
\hline 92 & 252.76 & 49 & 12,385 & 299125 & -.172 & .432 & .432 & 476.35 & 26 & 12,385 & 153504 & -.412 & .340 & .340 \\
\hline 93 & 141.60 & 15 & 2,124 & 15712 & -0.092 & .463 & .463 & 236.00 & 9 & 2,124 & 9429 & -0.070 & .472 & .472 \\
\hline 94 & -- & -- & -- & -- & -- & -- & -- & -- & -- & -- & -- & -- & -- & -- \\
\hline 95 & 258.50 & 4 & 1,034 & 1316 & -1.260 & .104 & .104 & 344.67 & 3 & 1,034 & 1074 & -.923 & .178 & .178 \\
\hline 96 & 811.82 & 17 & 13,801 & 134009 & 1.017 & .845 & .155 & 1533.44 & 9 & 13,801 & 64895 & .234 & .592 & .408 \\
\hline
\end{tabular}


Table 7. Summary of interoccurrence intervals for daily precipitation thresholds of 2.5 and 3.0 inches—Continued

\begin{tabular}{|c|c|c|c|c|c|c|c|c|c|c|c|c|c|c|}
\hline \multirow[b]{2}{*}{$\begin{array}{l}\text { Seq. } \\
\text { no. }\end{array}$} & \multicolumn{7}{|c|}{ Daily precipitation threshold of 2.5 inches and greater } & \multicolumn{7}{|c|}{ Daily precipitation threshold of 3.0 inches and greater } \\
\hline & $\begin{array}{l}\text { Mean } \\
\text { inter- } \\
\text { occur- } \\
\text { rence } \\
\text { interval } \\
\text { (days) }\end{array}$ & $\begin{array}{l}\text { Total } \\
\text { no. of } \\
\text { events }\end{array}$ & $\begin{array}{l}\text { Total } \\
\text { no. of } \\
\text { days } \\
\text { avail- } \\
\text { able }\end{array}$ & $\begin{array}{l}\text { Sigma } \\
\mathrm{S}\end{array}$ & $\begin{array}{c}\text { Stan- } \\
\text { dard } \\
\text { normal } \\
\text { variate }\end{array}$ & $\begin{array}{l}\text { Non- } \\
\text { ex- } \\
\text { ceed- } \\
\text { ance } \\
\text { prob- } \\
\text { ability }\end{array}$ & $\begin{array}{c}\mathrm{p}- \\
\text { value }\end{array}$ & $\begin{array}{l}\text { Mean } \\
\text { inter- } \\
\text { occur- } \\
\text { rence } \\
\text { interval } \\
\text { (days) }\end{array}$ & $\begin{array}{l}\text { Total } \\
\text { no. of } \\
\text { events }\end{array}$ & $\begin{array}{l}\text { Total } \\
\text { no. of } \\
\text { days } \\
\text { avail- } \\
\text { able }\end{array}$ & $\begin{array}{l}\text { Sigma } \\
\mathrm{S}\end{array}$ & $\begin{array}{c}\text { Stan- } \\
\text { dard } \\
\text { normal } \\
\text { variate }\end{array}$ & $\begin{array}{l}\text { Non- } \\
\text { ex- } \\
\text { ceed- } \\
\text { ance } \\
\text { prob- } \\
\text { ability }\end{array}$ & $\begin{array}{c}\mathrm{p}- \\
\text { value }\end{array}$ \\
\hline 97 & -- & -- & -- & -- & -- & -- & -- & -- & -- & -- & -- & -- & -- & -- \\
\hline 98 & 1002.32 & 19 & 19,044 & 195478 & 608 & .728 & .272 & 1904.40 & 10 & 19,044 & 109987 & .849 & .802 & .198 \\
\hline 99 & 114.00 & 1 & 114 & 98 & 1.246 & .894 & .106 & -- & -- & -- & -- & -- & -- & -- \\
\hline 100 & 381.07 & 75 & 28,580 & 1185372 & 1.590 & .944 & .056 & 595.42 & 48 & 28,580 & 732062 & .807 & .790 & .210 \\
\hline 101 & 354.27 & 26 & 9,211 & 142806 & 1.701 & .956 & .044 & 575.69 & 16 & 9,211 & 84012 & .971 & .834 & .166 \\
\hline 102 & 158.20 & 5 & 791 & 2007 & .058 & .523 & .477 & 263.67 & 3 & 791 & 791 & -1.000 & .159 & .159 \\
\hline 103 & 294.87 & 127 & 37,448 & 2589350 & 1.735 & .959 & .041 & 492.74 & 76 & 37,448 & 1521393 & 1.044 & .852 & .148 \\
\hline 104 & 418.40 & 15 & 6,276 & 42880 & -.597 & .275 & .275 & 784.50 & 8 & 6,276 & 26210 & .216 & .585 & .415 \\
\hline 105 & 209.10 & 49 & 10,246 & 232876 & -.877 & .190 & .190 & 341.53 & 30 & 10,246 & 146141 & -.466 & .321 & .321 \\
\hline 106 & -- & -- & -- & -- & -- & -- & -- & -- & -- & -- & -- & -- & -- & -- \\
\hline 107 & 374.35 & 20 & 7,487 & 87877 & 1.346 & .911 & .089 & 623.92 & 12 & 7,487 & 53075 & 1.089 & .862 & .138 \\
\hline 108 & 208.62 & 179 & 37,342 & 3407032 & .450 & .674 & .326 & 339.47 & 110 & 37,342 & 2084232 & .269 & .606 & .394 \\
\hline 109 & 113.48 & 127 & 14,412 & 879085 & -.770 & .221 & .221 & 189.63 & 76 & 14,412 & 495437 & -1.440 & .075 & .075 \\
\hline 110 & 197.22 & 176 & 34,710 & 3327355 & 2.053 & .980 & .020 & 357.84 & 97 & 34,710 & 1923601 & 2.434 & .993 & .007 \\
\hline 111 & 313.50 & 4 & 1,254 & 2247 & -.361 & .359 & .359 & 627.00 & 2 & 1,254 & 1107 & -.287 & .387 & .387 \\
\hline 112 & 740.48 & 31 & 22,955 & 318355 & -1.015 & .155 & .155 & 1275.28 & 18 & 22,955 & 209701 & .111 & .544 & .456 \\
\hline 113 & 601.25 & 4 & 2,405 & 4123 & -.495 & .310 & .310 & 1202.50 & 2 & 2,405 & 2870 & .474 & .682 & .318 \\
\hline 114 & 1003.00 & 2 & 2,006 & 2525 & .634 & .737 & .263 & 2006.00 & 1 & 2,006 & 1913 & 1.572 & .942 & .058 \\
\hline 115 & 9031.00 & 2 & 18,062 & 6370 & -1.586 & .056 & .056 & -- & -- & -- & -- & -- & -- & -- \\
\hline 116 & 829.29 & 7 & 5,805 & 17441 & -.649 & .258 & .258 & 2902.50 & 2 & 5,805 & 3281 & -1.065 & .144 & .144 \\
\hline 117 & 1280.93 & 15 & 19,214 & 181524 & 1.742 & .959 & .041 & 2401.75 & 8 & 19,214 & 75272 & -.101 & .460 & .460 \\
\hline 118 & 1011.00 & 1 & 1,011 & 355 & -.516 & .303 & .303 & 1011.00 & 1 & 1,011 & 355 & -.516 & .303 & .303 \\
\hline 119 & 336.42 & 82 & 27,586 & 1076588 & -.755 & .225 & .225 & 562.98 & 49 & 27,586 & 631094 & -.803 & .211 & .211 \\
\hline 120 & 147.50 & 130 & 19,175 & 1381499 & 2.141 & .984 & .016 & 245.83 & 78 & 19,175 & 880399 & 2.712 & .997 & .003 \\
\hline 121 & 244.79 & 75 & 18,359 & 796992 & 2.365 & .991 & .009 & 390.62 & 47 & 18,359 & 496744 & 1.797 & .964 & .036 \\
\hline 122 & 617.36 & 14 & 8,643 & 62402 & .204 & .581 & .419 & 1728.60 & 5 & 8,643 & 17010 & -.824 & .205 & .205 \\
\hline 123 & 367.13 & 94 & 34,510 & 1568151 & -.557 & .289 & .289 & 639.07 & 54 & 34,510 & 874522 & -.782 & .217 & .217 \\
\hline 124 & 235.94 & 32 & 7,550 & 119499 & -.106 & .458 & .458 & 290.39 & 26 & 7,550 & 90579 & -.681 & .248 & .248 \\
\hline 125 & 474.54 & 50 & 23,727 & 648400 & 1.140 & .873 & .127 & 719.00 & 33 & 23,727 & 433923 & 1.078 & .859 & .141 \\
\hline 126 & 201.06 & 18 & 3,619 & 33600 & .232 & .592 & .408 & 329.00 & 11 & 3,619 & 24953 & 1.457 & .927 & .073 \\
\hline 127 & 1076.94 & 18 & 19,385 & 179312 & .204 & .581 & .419 & 1762.27 & 11 & 19,385 & 110086 & .187 & .574 & .426 \\
\hline 128 & 149.11 & 73 & 10,885 & 402170 & .181 & .572 & .428 & 222.14 & 49 & 10,885 & 294633 & 1.271 & .898 & .102 \\
\hline 129 & 332.78 & 59 & 19,634 & 566782 & -.285 & .388 & .388 & 613.56 & 32 & 19,634 & 284584 & -.922 & .178 & .178 \\
\hline 130 & 418.25 & 63 & 26,350 & 840489 & .173 & .569 & .431 & 908.62 & 29 & 26,350 & 427952 & 1.120 & .869 & .131 \\
\hline 131 & 380.50 & 2 & 761 & 1011 & .805 & .789 & .211 & 380.50 & 2 & 761 & 1011 & .805 & .789 & .211 \\
\hline 132 & 222.87 & 63 & 14,041 & 440646 & -.051 & .480 & .480 & 484.17 & 29 & 14,041 & 225906 & 1.022 & .847 & .153 \\
\hline 133 & 199.46 & 182 & 36,302 & 3490836 & 1.325 & .907 & .093 & 297.56 & 122 & 36,302 & 2254321 & .345 & .635 & .365 \\
\hline 134 & 1202.00 & 1 & 1,202 & 308 & -.844 & .199 & .199 & 1202.00 & 1 & 1,202 & 308 & -.844 & .199 & .199 \\
\hline 135 & -- & -- & -- & -- & -- & -- & -- & -- & -- & -- & -- & -- & -- & -- \\
\hline 136 & 356.54 & 87 & 31,019 & 1473343 & 1.485 & .931 & .069 & 633.04 & 49 & 31,019 & 862816 & 1.641 & .950 & .050 \\
\hline 137 & 392.44 & 9 & 3,532 & 20349 & 1.457 & .927 & .073 & 706.40 & 5 & 3,532 & 12828 & 1.754 & .960 & .040 \\
\hline 138 & 224.91 & 34 & 7,647 & 154138 & 1.875 & .970 & .030 & 402.47 & 19 & 7,647 & 90641 & 1.870 & .969 & .031 \\
\hline 139 & -- & -- & -- & -- & -- & -- & -- & -- & -- & -- & -- & -- & -- & -- \\
\hline 140 & 645.00 & 2 & 1,290 & 435 & -1.624 & .052 & .052 & 645.00 & 2 & 1,290 & 435 & -1.624 & .052 & .052 \\
\hline 141 & 205.19 & 52 & 10,670 & 263527 & -0.626 & .266 & .266 & 333.44 & 32 & 10,670 & 173708 & 0.172 & .568 & .432 \\
\hline 142 & 131.61 & 61 & 8,028 & 263580 & 1.035 & .850 & .150 & 243.27 & 33 & 8,028 & 151294 & 1.415 & .921 & .079 \\
\hline 143 & 164.84 & 109 & 17,968 & 924451 & -1.012 & .156 & .156 & 299.47 & 60 & 17,968 & 533337 & -.142 & .444 & .444 \\
\hline 144 & 655.62 & 21 & 13,768 & 149917 & .294 & .616 & .384 & 1251.64 & 11 & 13,768 & 85603 & .749 & .773 & .227 \\
\hline
\end{tabular}


Table 7. Summary of interoccurrence intervals for daily precipitation thresholds of 2.5 and 3.0 inches-Continued

\begin{tabular}{|c|c|c|c|c|c|c|c|c|c|c|c|c|c|c|}
\hline \multirow[b]{2}{*}{$\begin{array}{l}\text { Seq. } \\
\text { no. }\end{array}$} & \multicolumn{7}{|c|}{ Daily precipitation threshold of 2.5 inches and greater } & \multicolumn{7}{|c|}{ Daily precipitation threshold of 3.0 inches and greater } \\
\hline & $\begin{array}{l}\text { Mean } \\
\text { inter- } \\
\text { occur- } \\
\text { rence } \\
\text { interval } \\
\text { (days) }\end{array}$ & $\begin{array}{c}\text { Total } \\
\text { no. of } \\
\text { events }\end{array}$ & $\begin{array}{l}\text { Total } \\
\text { no. of } \\
\text { days } \\
\text { avail- } \\
\text { able }\end{array}$ & $\begin{array}{l}\text { Sigma } \\
\mathrm{S}\end{array}$ & $\begin{array}{c}\text { Stan- } \\
\text { dard } \\
\text { normal } \\
\text { variate }\end{array}$ & $\begin{array}{l}\text { Non- } \\
\text { ex- } \\
\text { ceed- } \\
\text { ance } \\
\text { prob- } \\
\text { ability }\end{array}$ & $\begin{array}{c}\text { p- } \\
\text { value }\end{array}$ & $\begin{array}{c}\text { Mean } \\
\text { inter- } \\
\text { occur- } \\
\text { rence } \\
\text { interval } \\
\text { (days) }\end{array}$ & $\begin{array}{c}\text { Total } \\
\text { no. of } \\
\text { events }\end{array}$ & $\begin{array}{l}\text { Total } \\
\text { no. of } \\
\text { days } \\
\text { avail- } \\
\text { able }\end{array}$ & $\begin{array}{l}\text { Sigma } \\
\mathbf{S}\end{array}$ & $\begin{array}{l}\text { Stan- } \\
\text { dard } \\
\text { normal } \\
\text { variate }\end{array}$ & $\begin{array}{l}\text { Non- } \\
\text { ex- } \\
\text { ceed- } \\
\text { ance } \\
\text { prob- } \\
\text { ability }\end{array}$ & $\begin{array}{c}p- \\
\text { value }\end{array}$ \\
\hline 145 & 970.56 & 18 & 17,470 & 169419 & .570 & .716 & .284 & 1941.11 & 9 & 17,470 & 84916 & .417 & .661 & .339 \\
\hline 146 & 277.79 & 102 & 28,335 & 1561901 & 1.414 & .921 & .079 & 449.76 & 63 & 28,335 & 911350 & .290 & .614 & .386 \\
\hline 147 & 347.00 & 56 & 19,432 & 601434 & 1.366 & .914 & .086 & 647.73 & 30 & 19,432 & 297521 & .197 & .578 & .422 \\
\hline 148 & 633.50 & 2 & 1,267 & 1050 & -.420 & .337 & .337 & 633.50 & 2 & 1,267 & 1050 & -.420 & .337 & .337 \\
\hline 149 & -- & -- & -- & -- & -- & -- & -- & -- & -- & -- & -- & -- & -- & -- \\
\hline 150 & 231.20 & 5 & 1,156 & 2774 & -.156 & .438 & .438 & 385.33 & 3 & 1,156 & 1772 & .066 & .526 & .474 \\
\hline 151 & 438.56 & 16 & 7,017 & 49911 & -.768 & .221 & .221 & 779.67 & 9 & 7,017 & 24084 & -1.233 & .109 & .109 \\
\hline 152 & 1581.20 & 5 & 7,906 & 18637 & -.221 & .413 & .413 & 3953.00 & 2 & 7,906 & 4285 & -1.122 & .131 & .131 \\
\hline 153 & 204.03 & 71 & 14,486 & 584167 & 1.984 & .976 & .024 & 344.91 & 42 & 14,486 & 366033 & 2.281 & .989 & .011 \\
\hline 154 & 2671.25 & 4 & 10,685 & 6840 & -2.355 & .009 & .009 & 10685.00 & 1 & 10,685 & 529 & -1.561 & .059 & .059 \\
\hline 155 & 204.18 & 108 & 22,051 & 1319710 & 1.949 & .974 & .026 & 315.01 & 70 & 22,051 & 790596 & .353 & .638 & .362 \\
\hline 156 & 1470.90 & 10 & 14,709 & 63546 & -.745 & .228 & .228 & 2101.29 & 7 & 14,709 & 38893 & -1.121 & .131 & .131 \\
\hline 157 & -- & -- & -- & -- & -- & -- & -- & -- & -- & -- & -- & -- & -- & -- \\
\hline 158 & 679.00 & 3 & 2,037 & 3059 & .003 & .501 & .499 & 1018.50 & 2 & 2,037 & 1453 & -.702 & .241 & .241 \\
\hline 159 & 288.44 & 61 & 17,595 & 576742 & 1.011 & .844 & .156 & 488.75 & 36 & 17,595 & 358561 & 1.373 & .915 & .085 \\
\hline 160 & 221.52 & 27 & 5,981 & 74811 & -.661 & .254 & .254 & 351.82 & 17 & 5,981 & 45088 & -.808 & .210 & .210 \\
\hline 161 & 328.88 & 41 & 13,484 & 268673 & -.311 & .378 & .378 & 499.41 & 27 & 13,484 & 208558 & 1.311 & .905 & .095 \\
\hline 162 & 245.48 & 40 & 9,819 & 214241 & .996 & .840 & .160 & 490.95 & 20 & 9,819 & 99770 & .125 & .550 & .450 \\
\hline 163 & 324.77 & 106 & 34,426 & 1738089 & -.845 & .199 & .199 & 521.61 & 66 & 34,426 & 1142812 & .084 & .533 & .467 \\
\hline 164 & 259.00 & 2 & 518 & 869 & 1.660 & .952 & .048 & 518.00 & 1 & 518 & 396 & .916 & .820 & .180 \\
\hline 165 & 1185.00 & 1 & 1,185 & 1048 & 1.332 & .908 & .092 & 1185.00 & 1 & 1,185 & 1048 & 1.332 & .908 & .092 \\
\hline 166 & 234.48 & 27 & 6,331 & 78383 & -.746 & .228 & .228 & 316.55 & 20 & 6,331 & 66146 & .347 & .636 & .364 \\
\hline 167 & 1207.00 & 1 & 1,207 & 1190 & 1.683 & .954 & .046 & 1207.00 & 1 & 1,207 & 1190 & 1.683 & .954 & .046 \\
\hline 168 & 256.31 & 55 & 14,097 & 416885 & .968 & .833 & .167 & 414.62 & 34 & 14,097 & 249825 & .429 & .666 & .334 \\
\hline 169 & 152.00 & 8 & 1,216 & 5284 & .423 & .664 & .336 & 202.67 & 6 & 1,216 & 4055 & .473 & .682 & .318 \\
\hline 170 & 608.40 & 10 & 6,084 & 34897 & .806 & .790 & .210 & 1014.00 & 6 & 6,084 & 18298 & .011 & .504 & .496 \\
\hline 171 & 433.32 & 19 & 8,233 & 68280 & -.959 & .169 & .169 & 748.46 & 11 & 8,233 & 36880 & -1.066 & .143 & .143 \\
\hline 172 & 288.02 & 114 & 32,834 & 1944254 & .719 & .764 & .236 & 505.14 & 65 & 32,834 & 1172530 & 1.380 & .916 & .084 \\
\hline 173 & 329.67 & 6 & 1,978 & 7590 & 1.184 & .882 & .118 & 659.33 & 3 & 1,978 & 2760 & -.209 & .417 & .417 \\
\hline 174 & 772.50 & 8 & 6,180 & 21459 & -.646 & .259 & .259 & 1030.00 & 6 & 6,180 & 14309 & -.968 & .167 & .167 \\
\hline 175 & 154.33 & 9 & 1,389 & 5545 & -.587 & .279 & .279 & 277.80 & 5 & 1,389 & 2654 & -.913 & .181 & .181 \\
\hline 176 & 267.32 & 75 & 20,049 & 806459 & 1.090 & .862 & .138 & 455.66 & 44 & 20,049 & 497016 & 1.457 & .927 & .073 \\
\hline 177 & 845.40 & 38 & 32,125 & 637384 & .473 & .682 & .318 & 1606.25 & 20 & 32,125 & 337048 & .381 & .648 & .352 \\
\hline 178 & 331.00 & 1 & 331 & 214 & .508 & .694 & .306 & 331.00 & 1 & 331 & 214 & .508 & .694 & .306 \\
\hline 179 & 4865.00 & 4 & 19,460 & 51880 & 1.154 & .876 & .124 & -- & -- & -- & -- & -- & -- & -- \\
\hline 180 & 155.81 & 107 & 16,672 & 947815 & 1.122 & .869 & .131 & 287.45 & 58 & 16,672 & 521433 & 1.035 & .850 & .150 \\
\hline 181 & 830.53 & 34 & 28,238 & 484641 & .097 & .539 & .461 & 1486.21 & 19 & 28,238 & 264187 & -.115 & .454 & .454 \\
\hline 182 & 224.71 & 62 & 13,932 & 434624 & .086 & .534 & .466 & 366.63 & 38 & 13,932 & 283038 & .739 & .770 & .230 \\
\hline 183 & 241.75 & 8 & 1,934 & 7506 & -.146 & .442 & .442 & 322.33 & 6 & 1,934 & 5308 & -.361 & .359 & .359 \\
\hline 184 & 173.00 & 5 & 865 & 2193 & .055 & .522 & .478 & 173.00 & 5 & 865 & 2193 & .055 & .522 & .478 \\
\hline 185 & 2204.00 & 1 & 2,204 & 1219 & .184 & .573 & .427 & 2204.00 & 1 & 2,204 & 1219 & .184 & .573 & .427 \\
\hline 186 & 190.42 & 12 & 2,285 & 14574 & .378 & .647 & .353 & 326.43 & 7 & 2,285 & 10427 & 1.392 & .918 & .082 \\
\hline 187 & -- & -- & -- & -- & -- & -- & -- & -- & -- & -- & -- & -- & -- & -- \\
\hline 188 & 423.43 & 35 & 14,820 & 282007 & .895 & .815 & .185 & 673.64 & 22 & 14,820 & 174830 & .589 & .722 & .278 \\
\hline 189 & 394.25 & 67 & 26,415 & 795777 & -1.428 & .077 & .077 & 574.24 & 46 & 26,415 & 557870 & -0.961 & .168 & .168 \\
\hline 190 & 212.48 & 89 & 18,911 & 893344 & 1.006 & .843 & .157 & 370.80 & 51 & 18,911 & 510179 & .717 & .763 & .237 \\
\hline 191 & 400.59 & 32 & 12,819 & 191236 & -.663 & .254 & .254 & 582.68 & 22 & 12,819 & 123257 & -1.023 & .153 & .153 \\
\hline 192 & 156.69 & 116 & 18,176 & 1203270 & 2.638 & .996 & .004 & 259.66 & 70 & 18,176 & 742700 & 2.427 & .992 & .008 \\
\hline
\end{tabular}


Table 7. Summary of interoccurrence intervals for daily precipitation thresholds of 2.5 and 3.0 inches—Continued

\begin{tabular}{|c|c|c|c|c|c|c|c|c|c|c|c|c|c|c|}
\hline \multirow[b]{2}{*}{$\begin{array}{l}\text { Seq. } \\
\text { no. }\end{array}$} & \multicolumn{7}{|c|}{ Daily precipitation threshold of 2.5 inches and greater } & \multicolumn{7}{|c|}{ Daily precipitation threshold of 3.0 inches and greater } \\
\hline & $\begin{array}{l}\text { Mean } \\
\text { inter- } \\
\text { occur- } \\
\text { rence } \\
\text { interval } \\
\text { (days) }\end{array}$ & $\begin{array}{c}\text { Total } \\
\text { no. of } \\
\text { events }\end{array}$ & $\begin{array}{l}\text { Total } \\
\text { no. of } \\
\text { days } \\
\text { avail- } \\
\text { able }\end{array}$ & $\begin{array}{l}\text { Sigma } \\
\mathrm{S}\end{array}$ & $\begin{array}{c}\text { Stan- } \\
\text { dard } \\
\text { normal } \\
\text { variate }\end{array}$ & $\begin{array}{l}\text { Non- } \\
\text { ex- } \\
\text { ceed- } \\
\text { ance } \\
\text { prob- } \\
\text { ability }\end{array}$ & $\begin{array}{c}\text { p- } \\
\text { value }\end{array}$ & $\begin{array}{l}\text { Mean } \\
\text { inter- } \\
\text { occur- } \\
\text { rence } \\
\text { interval } \\
\text { (days) }\end{array}$ & $\begin{array}{l}\text { Total } \\
\text { no. of } \\
\text { events }\end{array}$ & $\begin{array}{c}\text { Total } \\
\text { no. of } \\
\text { days } \\
\text { avail- } \\
\text { able }\end{array}$ & $\begin{array}{l}\text { Sigma } \\
\mathrm{S}\end{array}$ & $\begin{array}{c}\text { Stan- } \\
\text { dard } \\
\text { normal } \\
\text { variate }\end{array}$ & $\begin{array}{l}\text { Non- } \\
\text { ex- } \\
\text { ceed- } \\
\text { ance } \\
\text { prob- } \\
\text { ability }\end{array}$ & $\begin{array}{c}\text { p- } \\
\text { value }\end{array}$ \\
\hline 193 & 608.20 & 20 & 12,164 & 161903 & 2.564 & .995 & .005 & 1013.67 & 12 & 12,164 & 93260 & 1.667 & .952 & .048 \\
\hline 194 & 306.14 & 21 & 6,429 & 61592 & -.695 & .244 & .244 & 535.75 & 12 & 6,429 & 35064 & -.546 & .293 & .293 \\
\hline 195 & 4743.00 & 2 & 9,486 & 4829 & -1.203 & .115 & .115 & 9486.00 & 1 & 9,486 & 3968 & -.283 & .389 & .389 \\
\hline 196 & 306.38 & 47 & 14,400 & 329959 & -.296 & .384 & .384 & 533.33 & 27 & 14,400 & 186898 & -.347 & .364 & .364 \\
\hline 197 & 269.83 & 30 & 8,095 & 122530 & .086 & .534 & .466 & 449.72 & 18 & 8,095 & 77178 & .436 & .669 & .331 \\
\hline 198 & 214.66 & 59 & 12,665 & 429778 & 2.000 & .977 & .023 & 395.78 & 32 & 12,665 & 252292 & 2.401 & .992 & .008 \\
\hline 199 & 140.91 & 162 & 22,827 & 1902471 & .638 & .738 & .262 & 271.75 & 84 & 22,827 & 1034376 & 1.253 & .895 & .105 \\
\hline 200 & 455.52 & 31 & 14,121 & 168577 & -2.216 & .013 & .013 & 941.40 & 15 & 14,121 & 85509 & -1.292 & .098 & .098 \\
\hline 201 & 216.41 & 106 & 22,939 & 1164595 & -.751 & .227 & .227 & 358.42 & 64 & 22,939 & 734411 & .007 & .503 & .497 \\
\hline 202 & 486.00 & 1 & 486 & 383 & .998 & .841 & .159 & 486.00 & 1 & 486 & 383 & .998 & .841 & .159 \\
\hline 203 & 370.79 & 38 & 14,090 & 297188 & 1.176 & .880 & .120 & 640.46 & 22 & 14,090 & 181011 & 1.364 & .914 & .086 \\
\hline 204 & 86.75 & 16 & 1,388 & 10453 & -.406 & .342 & .342 & 173.50 & 8 & 1,388 & 5632 & .071 & .528 & .472 \\
\hline 205 & -- & -- & -- & -- & -- & -- & -- & -- & -- & -- & -- & -- & -- & -- \\
\hline 206 & 2925.00 & 1 & 2,925 & 398 & -1.261 & .104 & .104 & 2925.00 & 1 & 2,925 & 398 & -1.261 & .104 & .104 \\
\hline 207 & -- & -- & -- & -- & -- & -- & -- & -- & -- & -- & -- & -- & -- & -- \\
\hline 208 & 2059.75 & 4 & 8,239 & 28652 & 2.559 & .995 & .005 & 2746.33 & 3 & 8,239 & 21841 & 2.302 & .989 & .011 \\
\hline 209 & 201.20 & 76 & 15,291 & 633013 & 1.350 & .911 & .089 & 294.06 & 52 & 15,291 & 454271 & 1.782 & .963 & .037 \\
\hline 210 & 390.18 & 11 & 4,292 & 36316 & 3.093 & .999 & .001 & 476.89 & 9 & 4,292 & 28800 & 2.552 & .995 & .005 \\
\hline 211 & 1020.00 & 2 & 2,040 & 1544 & -.596 & .276 & .276 & 2040.00 & 1 & 2,040 & 496 & -.890 & .187 & .187 \\
\hline 212 & 304.98 & 47 & 14,334 & 294032 & -1.509 & .066 & .066 & 511.93 & 28 & 14,334 & 177843 & -1.043 & .149 & .149 \\
\hline 213 & 186.65 & 17 & 3,173 & 24901 & -.548 & .292 & .292 & 317.30 & 10 & 3,173 & 13107 & -.952 & .171 & .171 \\
\hline 214 & 1162.00 & 1 & 1,162 & 279 & -.900 & .184 & .184 & -- & -- & -- & -- & -- & -- & -- \\
\hline 215 & 801.00 & 1 & 801 & 99 & -1.304 & .096 & .096 & -- & -- & -- & -- & -- & -- & -- \\
\hline 216 & 758.50 & 2 & 1,517 & 1446 & -.115 & .454 & .454 & 758.50 & 2 & 1,517 & 1446 & -.115 & .454 & .454 \\
\hline 217 & 459.03 & 33 & 15,148 & 286658 & 1.462 & .928 & .072 & 797.26 & 19 & 15,148 & 172317 & 1.491 & .932 & .068 \\
\hline 218 & 840.77 & 22 & 18,497 & 267252 & 2.547 & .995 & .005 & 2055.22 & 9 & 18,497 & 115292 & 2.001 & .977 & .023 \\
\hline 219 & -- & -- & -- & -- & -- & -- & -- & -- & -- & -- & -- & -- & -- & -- \\
\hline 220 & 429.84 & 57 & 24,501 & 635948 & -1.167 & .122 & .122 & 720.62 & 34 & 24,501 & 365656 & -1.233 & .109 & .109 \\
\hline 221 & 81.33 & 58 & 4,717 & 128771 & -.774 & .220 & .220 & 174.70 & 27 & 4,717 & 64708 & .145 & .558 & .442 \\
\hline 222 & 2197.00 & 9 & 19,773 & 111916 & 1.340 & .910 & .090 & 2824.71 & 7 & 19,773 & 78460 & .613 & .730 & .270 \\
\hline 223 & 464.93 & 14 & 6,509 & 57863 & 1.750 & .960 & .040 & 929.86 & 7 & 6,509 & 26449 & .738 & .770 & .230 \\
\hline 224 & 611.70 & 10 & 6,117 & 25921 & -.835 & .202 & .202 & 1019.50 & 6 & 6,117 & 17386 & -.223 & .412 & .412 \\
\hline 225 & 182.06 & 65 & 11,834 & 334638 & -1.814 & .035 & .035 & 328.72 & 36 & 11,834 & 174613 & -1.873 & .031 & .031 \\
\hline 226 & 530.91 & 63 & 33,447 & 962695 & -1.186 & .118 & .118 & 857.62 & 39 & 33,447 & 635030 & -.285 & .388 & .388 \\
\hline 227 & 600.00 & 2 & 1,200 & 1059 & -.288 & .387 & .387 & 1200.00 & 1 & 1,200 & 821 & .638 & .738 & .262 \\
\hline 228 & 190.15 & 179 & 34,037 & 3217699 & 1.304 & .904 & .096 & 286.03 & 119 & 34,037 & 2218404 & 1.803 & .964 & .036 \\
\hline 229 & 260.29 & 35 & 9,110 & 161576 & .138 & .555 & .445 & 379.58 & 24 & 9,110 & 112747 & .266 & .605 & .395 \\
\hline 230 & 653.20 & 49 & 32,007 & 805361 & .328 & .628 & .372 & 1524.14 & 21 & 32,007 & 290316 & -1.081 & .140 & .140 \\
\hline 231 & 246.94 & 134 & 33,090 & 2250790 & .305 & .620 & .380 & 429.74 & 77 & 33,090 & 1332590 & .699 & .758 & .242 \\
\hline 232 & 156.06 & 16 & 2,497 & 22280 & .799 & .788 & .212 & 499.40 & 5 & 2,497 & 8092 & 1.148 & .874 & .126 \\
\hline 233 & 135.01 & 128 & 17,281 & 1200817 & 1.680 & .954 & .046 & 230.41 & 75 & 17,281 & 682594 & .800 & .788 & .212 \\
\hline 234 & 1035.00 & 1 & 1,035 & 56 & -1.545 & .061 & .061 & -- & -- & -- & -- & -- & -- & -- \\
\hline 235 & 220.51 & 106 & 23,374 & 1227879 & -.158 & .437 & .437 & 477.02 & 49 & 23,374 & 564114 & -.181 & .428 & .428 \\
\hline 236 & 337.82 & 17 & 5,743 & 51500 & .393 & .653 & .347 & 717.88 & 8 & 5,743 & 31004 & 1.713 & .957 & .043 \\
\hline 237 & 157.45 & 118 & 18,579 & 1086052 & -0.174 & .431 & .431 & 229.37 & 81 & 18,579 & 675300 & -1.598 & .055 & .055 \\
\hline 238 & 1064.75 & 4 & 4,259 & 11242 & 1.108 & .866 & .134 & 1419.67 & 3 & 4,259 & 8583 & 1.031 & .849 & .151 \\
\hline 239 & 158.99 & 109 & 17,330 & 1009625 & 1.247 & .894 & .106 & 247.57 & 70 & 17,330 & 690326 & 2.002 & .977 & .023 \\
\hline 240 & 3261.75 & 4 & 13,047 & 32182 & .808 & .790 & .210 & 3261.75 & 4 & 13,047 & 32182 & .808 & .790 & .210 \\
\hline
\end{tabular}


Table 7. Summary of interoccurrence intervals for daily precipitation thresholds of 2.5 and 3.0 inches-Continued

\begin{tabular}{|c|c|c|c|c|c|c|c|c|c|c|c|c|c|c|}
\hline \multirow[b]{2}{*}{$\begin{array}{l}\text { Seq. } \\
\text { no. }\end{array}$} & \multicolumn{7}{|c|}{ Daily precipitation threshold of 2.5 inches and greater } & \multicolumn{7}{|c|}{ Daily precipitation threshold of 3.0 inches and greater } \\
\hline & $\begin{array}{l}\text { Mean } \\
\text { inter- } \\
\text { occur- } \\
\text { rence } \\
\text { interval } \\
\text { (days) }\end{array}$ & $\begin{array}{c}\text { Total } \\
\text { no. of } \\
\text { events }\end{array}$ & $\begin{array}{l}\text { Total } \\
\text { no. of } \\
\text { days } \\
\text { avail- } \\
\text { able }\end{array}$ & $\begin{array}{l}\text { Sigma } \\
\mathrm{S}\end{array}$ & $\begin{array}{c}\text { Stan- } \\
\text { dard } \\
\text { normal } \\
\text { variate }\end{array}$ & $\begin{array}{l}\text { Non- } \\
\text { ex- } \\
\text { ceed- } \\
\text { ance } \\
\text { prob- } \\
\text { ability }\end{array}$ & $\begin{array}{c}\text { p- } \\
\text { value }\end{array}$ & $\begin{array}{l}\text { Mean } \\
\text { inter- } \\
\text { occur- } \\
\text { rence } \\
\text { interval } \\
\text { (days) }\end{array}$ & $\begin{array}{c}\text { Total } \\
\text { no. of } \\
\text { events }\end{array}$ & $\begin{array}{l}\text { Total } \\
\text { no. of } \\
\text { days } \\
\text { avail- } \\
\text { able }\end{array}$ & $\begin{array}{l}\text { Sigma } \\
\mathrm{S}\end{array}$ & $\begin{array}{l}\text { Stan- } \\
\text { dard } \\
\text { normal } \\
\text { variate }\end{array}$ & $\begin{array}{l}\text { Non- } \\
\text { ex- } \\
\text { ceed- } \\
\text { ance } \\
\text { prob- } \\
\text { ability }\end{array}$ & $\begin{array}{c}p- \\
\text { value }\end{array}$ \\
\hline 241 & 317.00 & 118 & 37,406 & 2192019 & -.127 & .449 & .449 & 575.48 & 65 & 37,406 & 1111414 & -1.198 & .116 & .116 \\
\hline 242 & 211.05 & 83 & 17,517 & 694820 & -.698 & .243 & .243 & 350.34 & 50 & 17,517 & 418158 & -.553 & .290 & .290 \\
\hline 243 & 183.00 & 113 & 20,679 & 1156186 & -.192 & .424 & .424 & 375.98 & 55 & 20,679 & 580662 & .271 & .607 & .393 \\
\hline 244 & 92.00 & 1 & 92 & 7 & -1.469 & .071 & .071 & 92.00 & 1 & 92 & 7 & -1.469 & .071 & .071 \\
\hline 245 & 561.34 & 47 & 26,383 & 533547 & -1.656 & .049 & .049 & 909.76 & 29 & 26,383 & 288489 & -2.294 & .011 & .011 \\
\hline 246 & 200.30 & 100 & 20,030 & 996201 & -.092 & .463 & .463 & 317.94 & 63 & 20,030 & 613952 & -.370 & .356 & .356 \\
\hline 247 & 492.36 & 62 & 30,526 & 943632 & -.039 & .485 & .485 & 744.54 & 41 & 30,526 & 623684 & -.037 & .485 & .485 \\
\hline 248 & 219.33 & 9 & 1,974 & 11562 & 1.567 & .941 & .059 & 329.00 & 6 & 1,974 & 6197 & .197 & .578 & .422 \\
\hline 249 & 177.35 & 17 & 3,015 & 28270 & .736 & .769 & .231 & 274.09 & 11 & 3,015 & 18082 & .520 & .698 & .302 \\
\hline 250 & 495.27 & 15 & 7,429 & 55977 & .031 & .512 & .488 & 675.36 & 11 & 7,429 & 35740 & -.720 & .236 & .236 \\
\hline 251 & -- & -- & -- & -- & -- & -- & -- & -- & -- & -- & -- & -- & -- & -- \\
\hline 252 & 290.38 & 8 & 2,323 & 9986 & .366 & .643 & .357 & 387.17 & 6 & 2,323 & 7667 & .425 & .665 & .335 \\
\hline 253 & 466.67 & 3 & 1,400 & 2948 & 1.211 & .887 & .113 & 466.67 & 3 & 1,400 & 2948 & 1.211 & .887 & .113 \\
\hline 254 & 1391.79 & 14 & 19,485 & 112433 & -1.139 & .128 & .128 & 3247.50 & 6 & 19,485 & 68184 & .706 & .760 & .240 \\
\hline 255 & 165.24 & 117 & 19,333 & 1283998 & 2.535 & .994 & .006 & 284.31 & 68 & 19,333 & 701380 & .957 & .831 & .169 \\
\hline 256 & 175.13 & 104 & 18,213 & 998011 & .950 & .829 & .171 & 298.57 & 61 & 18,213 & 587270 & .774 & .780 & .220 \\
\hline 257 & -- & -- & -- & -- & -- & -- & -- & -- & -- & -- & -- & -- & -- & -- \\
\hline 258 & 778.60 & 25 & 19,465 & 314435 & 2.532 & .994 & .006 & 1145.00 & 17 & 19,465 & 207297 & 1.806 & .965 & .035 \\
\hline 259 & 410.82 & 11 & 4,519 & 23635 & -.282 & .389 & .389 & 753.17 & 6 & 4,519 & 15148 & .498 & .691 & .309 \\
\hline 260 & 265.81 & 83 & 22,062 & 863542 & -.897 & .185 & .185 & 565.69 & 39 & 22,062 & 402271 & -.702 & .241 & .241 \\
\hline 261 & 487.00 & 3 & 1,461 & 1583 & -.833 & .202 & .202 & 1461.00 & 1 & 1,461 & 222 & -1.206 & .114 & .114 \\
\hline 262 & 291.83 & 23 & 6,712 & 71100 & -.655 & .256 & .256 & 559.33 & 12 & 6,712 & 33301 & -1.039 & .150 & .150 \\
\hline 263 & 9269.00 & 2 & 18,538 & 20505 & .260 & .603 & .397 & 9269.00 & 2 & 18,538 & 20505 & .260 & .603 & .397 \\
\hline 264 & 216.38 & 55 & 11,901 & 371327 & 1.729 & .958 & .042 & 276.77 & 43 & 11,901 & 301718 & 2.035 & .979 & .021 \\
\hline 265 & 201.26 & 98 & 19,723 & 1009122 & .758 & .776 & .224 & 358.60 & 55 & 19,723 & 585110 & 1.012 & .844 & .156 \\
\hline 266 & 100.30 & 33 & 3,310 & 63218 & 1.567 & .941 & .059 & 174.21 & 19 & 3,310 & 36233 & 1.150 & .875 & .125 \\
\hline 267 & 244.40 & 149 & 36,415 & 3175375 & 3.604 & 1.000 & 0 & 423.43 & 86 & 36,415 & 1810004 & 2.505 & .994 & .006 \\
\hline 268 & 158.83 & 23 & 3,653 & 48935 & 1.369 & .915 & .085 & 260.93 & 14 & 3,653 & 26051 & .122 & .548 & .452 \\
\hline 269 & -- & -- & -- & -- & -- & -- & -- & -- & -- & -- & -- & -- & -- & -- \\
\hline 270 & 232.03 & 61 & 14,154 & 424107 & -.238 & .406 & .406 & 337.00 & 42 & 14,154 & 286378 & -.410 & .341 & .341 \\
\hline 271 & -- & -- & -- & -- & -- & -- & -- & -- & -- & -- & -- & -- & -- & -- \\
\hline 272 & -- & -- & -- & -- & -- & -- & -- & -- & -- & -- & -- & -- & -- & -- \\
\hline 273 & 324.18 & 98 & 31,770 & 1538862 & -.197 & .422 & .422 & 588.33 & 54 & 31,770 & 817089 & -.604 & .273 & .273 \\
\hline 274 & 344.94 & 33 & 11,383 & 226468 & 2.047 & .980 & .020 & 569.15 & 20 & 11,383 & 143371 & 2.010 & .978 & .022 \\
\hline 275 & 300.38 & 8 & 2,403 & 7473 & -1.090 & .138 & .138 & 600.75 & 4 & 2,403 & 4741 & -.047 & .481 & .481 \\
\hline 276 & 1291.40 & 5 & 6,457 & 16273 & .031 & .513 & .487 & 2152.33 & 3 & 6,457 & 7665 & -.626 & .266 & .266 \\
\hline 277 & 224.20 & 5 & 1,121 & 2182 & -.858 & .196 & .196 & 224.20 & 5 & 1,121 & 2182 & -.858 & .196 & .196 \\
\hline 278 & 215.27 & 55 & 11,840 & 324256 & -.053 & .479 & .479 & 394.67 & 30 & 11,840 & 174972 & -.140 & .444 & .444 \\
\hline 279 & -- & -- & -- & -- & -- & -- & -- & -- & -- & -- & -- & -- & -- & -- \\
\hline 280 & 867.63 & 19 & 16,485 & 186864 & 1.459 & .928 & .072 & 1648.50 & 10 & 16,485 & 104238 & 1.450 & .926 & .074 \\
\hline 281 & 441.50 & 10 & 4,415 & 24042 & .488 & .687 & .313 & 735.83 & 6 & 4,415 & 10237 & -.964 & .168 & .168 \\
\hline 282 & 335.25 & 4 & 1,341 & 3631 & 1.226 & .890 & .110 & 1341.00 & 1 & 1,341 & 319 & -.908 & .182 & .182 \\
\hline 283 & 313.15 & 62 & 19,415 & 618455 & .376 & .647 & .354 & 554.71 & 35 & 19,415 & 359933 & .608 & .728 & .272 \\
\hline 284 & 297.97 & 32 & 9,535 & 172613 & 1.288 & .901 & .099 & 501.84 & 19 & 9,535 & 106037 & 1.288 & .901 & .099 \\
\hline 285 & 161.07 & 202 & 32,536 & 3106669 & -1.344 & .089 & .089 & 278.09 & 117 & 32,536 & 1802222 & -0.996 & .160 & .160 \\
\hline 286 & 622.82 & 60 & 37,369 & 1075139 & -.550 & .291 & .291 & 1009.97 & 37 & 37,369 & 640889 & -.769 & .221 & .221 \\
\hline 287 & 456.50 & 4 & 1,826 & 5049 & 1.325 & .907 & .093 & 608.67 & 3 & 1,826 & 4083 & 1.472 & .929 & .071 \\
\hline 288 & 439.40 & 30 & 13,182 & 196574 & -.056 & .478 & .478 & 693.79 & 19 & 13,182 & 110448 & -.891 & .187 & .187 \\
\hline
\end{tabular}


Table 7. Summary of interoccurrence intervals for daily precipitation thresholds of 2.5 and 3.0 inches—Continued

\begin{tabular}{|c|c|c|c|c|c|c|c|c|c|c|c|c|c|c|}
\hline \multirow[b]{2}{*}{$\begin{array}{l}\text { Seq. } \\
\text { no. }\end{array}$} & \multicolumn{7}{|c|}{ Daily precipitation threshold of 2.5 inches and greater } & \multicolumn{7}{|c|}{ Daily precipitation threshold of 3.0 inches and greater } \\
\hline & $\begin{array}{l}\text { Mean } \\
\text { inter- } \\
\text { occur- } \\
\text { rence } \\
\text { interval } \\
\text { (days) }\end{array}$ & $\begin{array}{l}\text { Total } \\
\text { no. of } \\
\text { events }\end{array}$ & $\begin{array}{l}\text { Total } \\
\text { no. of } \\
\text { days } \\
\text { avail- } \\
\text { able }\end{array}$ & $\begin{array}{l}\text { Sigma } \\
\mathrm{S}\end{array}$ & $\begin{array}{c}\text { Stan- } \\
\text { dard } \\
\text { normal } \\
\text { variate }\end{array}$ & $\begin{array}{l}\text { Non- } \\
\text { ex- } \\
\text { ceed- } \\
\text { ance } \\
\text { prob- } \\
\text { ability }\end{array}$ & $\begin{array}{c}\mathrm{p}- \\
\text { value }\end{array}$ & $\begin{array}{l}\text { Mean } \\
\text { inter- } \\
\text { occur- } \\
\text { rence } \\
\text { interval } \\
\text { (days) }\end{array}$ & $\begin{array}{l}\text { Total } \\
\text { no. of } \\
\text { events }\end{array}$ & $\begin{array}{l}\text { Total } \\
\text { no. of } \\
\text { days } \\
\text { avail- } \\
\text { able }\end{array}$ & $\begin{array}{l}\text { Sigma } \\
\mathrm{S}\end{array}$ & $\begin{array}{c}\text { Stan- } \\
\text { dard } \\
\text { normal } \\
\text { variate }\end{array}$ & $\begin{array}{l}\text { Non- } \\
\text { ex- } \\
\text { ceed- } \\
\text { ance } \\
\text { prob- } \\
\text { ability }\end{array}$ & $\begin{array}{c}p- \\
\text { value }\end{array}$ \\
\hline 289 & 1134.00 & 1 & 1,134 & 494 & -.223 & .412 & .412 & -- & -- & -- & -- & -- & -- & -- \\
\hline 290 & 406.81 & 75 & 30,511 & 1260248 & 1.522 & .936 & .064 & 649.17 & 47 & 30,511 & 752241 & .584 & .720 & .280 \\
\hline 291 & 464.55 & 42 & 19,511 & 307747 & -2.794 & .003 & .003 & 929.10 & 21 & 19,511 & 153316 & -1.997 & .023 & .023 \\
\hline 292 & 365.17 & 6 & 2,191 & 8620 & 1.321 & .907 & .093 & 438.20 & 5 & 2,191 & 8197 & 1.923 & .973 & .027 \\
\hline 293 & 215.38 & 164 & 35,323 & 3067213 & 1.307 & .904 & .096 & 349.73 & 101 & 35,323 & 1934583 & 1.471 & .929 & .071 \\
\hline 294 & 171.22 & 110 & 18,834 & 1096118 & 1.057 & .855 & .145 & 269.06 & 70 & 18,834 & 683733 & .540 & .705 & .295 \\
\hline 295 & 312.37 & 19 & 5,935 & 68267 & 1.591 & .944 & .056 & 539.55 & 11 & 5,935 & 36830 & .737 & .769 & .231 \\
\hline 296 & 173.65 & 99 & 17,191 & 877258 & .533 & .703 & .297 & 286.52 & 60 & 17,191 & 485387 & -.789 & .215 & .215 \\
\hline 297 & 182.67 & 107 & 19,546 & 941909 & -1.779 & .038 & .038 & 305.41 & 64 & 19,546 & 548731 & -1.700 & .045 & .045 \\
\hline 298 & 340.90 & 30 & 10,227 & 162381 & .555 & .711 & .289 & 601.59 & 17 & 10,227 & 99680 & 1.048 & .853 & .148 \\
\hline 299 & -- & -- & -- & -- & -- & -- & -- & -- & -- & -- & -- & -- & -- & -- \\
\hline 300 & 1214.25 & 4 & 4,857 & 10918 & .429 & .666 & .334 & 2428.50 & 2 & 4,857 & 5530 & .339 & .633 & .367 \\
\hline 301 & 1124.73 & 15 & 16,871 & 146430 & 1.055 & .854 & .146 & 2410.14 & 7 & 16,871 & 60252 & .093 & .537 & .463 \\
\hline 302 & 1930.90 & 10 & 19,309 & 117397 & 1.183 & .882 & .118 & 3218.17 & 6 & 19,309 & 71623 & 1.003 & .842 & .158 \\
\hline 303 & 97.40 & 5 & 487 & 1443 & .717 & .763 & .237 & 243.50 & 2 & 487 & 341 & -.734 & .231 & .231 \\
\hline 304 & 248.03 & 101 & 25,051 & 1249117 & -.220 & .413 & .413 & 431.91 & 58 & 25,051 & 758235 & .577 & .718 & .282 \\
\hline 305 & 129.50 & 12 & 1,554 & 9045 & -.180 & .429 & .429 & 172.67 & 9 & 1,554 & 8137 & .850 & .802 & .198 \\
\hline 306 & 149.42 & 249 & 37,205 & 4847599 & 1.272 & .898 & .102 & 246.39 & 151 & 37,205 & 3015652 & 1.566 & .941 & .059 \\
\hline 307 & 621.35 & 34 & 21,126 & 403358 & 1.243 & .893 & .107 & 1408.40 & 15 & 21,126 & 204288 & 1.941 & .974 & .026 \\
\hline 308 & 195.30 & 91 & 17,772 & 828420 & .405 & .657 & .343 & 286.65 & 62 & 17,772 & 557044 & .151 & .560 & .440 \\
\hline 309 & 583.50 & 2 & 1,167 & 1542 & .787 & .784 & .216 & 1167.00 & 1 & 1,167 & 1150 & 1.682 & .954 & .046 \\
\hline 310 & 310.30 & 10 & 3,103 & 14367 & -.405 & .343 & .343 & 620.60 & 5 & 3,103 & 5884 & -.935 & .175 & .175 \\
\hline 311 & 1902.00 & 2 & 3,804 & 6837 & 1.953 & .975 & .025 & 3804.00 & 1 & 3,804 & 3603 & 1.549 & .939 & .061 \\
\hline 312 & 268.51 & 69 & 18,527 & 735645 & 2.171 & .985 & .015 & 451.88 & 41 & 18,527 & 400160 & .594 & .724 & .276 \\
\hline 313 & 308.13 & 8 & 2,465 & 9274 & -.291 & .385 & .385 & 616.25 & 4 & 2,465 & 5711 & .549 & .708 & .292 \\
\hline 314 & 377.00 & 3 & 1,131 & 2061 & .645 & .740 & .260 & 565.50 & 2 & 1,131 & 1475 & .745 & .772 & .228 \\
\hline 315 & 140.48 & 133 & 18,684 & 1414525 & 2.766 & .997 & .003 & 252.49 & 74 & 18,684 & 833374 & 3.062 & .999 & .001 \\
\hline 316 & 659.67 & 3 & 1,979 & 2926 & -.043 & .483 & .483 & 659.67 & 3 & 1,979 & 2926 & -.043 & .483 & .483 \\
\hline 317 & 542.33 & 3 & 1,627 & 1559 & -1.084 & .139 & .139 & 813.50 & 2 & 1,627 & 544 & -1.631 & .052 & .052 \\
\hline 318 & 611.04 & 27 & 16,498 & 193587 & -1.177 & .120 & .120 & 1499.82 & 11 & 16,498 & 67244 & -1.487 & .068 & .068 \\
\hline 319 & 311.50 & 12 & 3,738 & 18982 & -.922 & .178 & .178 & 934.50 & 4 & 3,738 & 3968 & -1.626 & .052 & .052 \\
\hline 320 & -- & -- & -- & -- & -- & -- & -- & -- & -- & -- & -- & -- & -- & -- \\
\hline 321 & 227.84 & 91 & 20,733 & 1016513 & 1.281 & .900 & .100 & 431.94 & 48 & 20,733 & 526208 & .690 & .755 & .245 \\
\hline 322 & 227.96 & 55 & 12,538 & 364715 & .742 & .771 & .229 & 358.23 & 35 & 12,538 & 215971 & -.161 & .436 & .436 \\
\hline 323 & 315.53 & 17 & 5,364 & 47686 & .328 & .628 & .372 & 536.40 & 10 & 5,364 & 30409 & .733 & .768 & .232 \\
\hline 324 & 277.21 & 114 & 31,602 & 1994650 & 1.985 & .976 & .024 & 464.74 & 68 & 31,602 & 1241557 & 2.221 & .987 & .013 \\
\hline 325 & 532.38 & 8 & 4,259 & 13758 & -.943 & .173 & .173 & 4259.00 & 1 & 4,259 & 3398 & 1.032 & .849 & .151 \\
\hline 326 & 158.34 & 41 & 6,492 & 137607 & .377 & .647 & .353 & 240.44 & 27 & 6,492 & 93094 & .560 & .712 & .288 \\
\hline 327 & 369.36 & 22 & 8,126 & 71363 & -1.638 & .051 & .051 & 580.43 & 14 & 8,126 & 45100 & -1.342 & .090 & .090 \\
\hline 328 & 975.00 & 1 & 975 & 354 & -.474 & .318 & .318 & -- & -- & -- & -- & -- & -- & -- \\
\hline 329 & 99.71 & 116 & 11,566 & 632474 & -1.067 & .143 & .143 & 156.30 & 74 & 11,566 & 421256 & -.233 & .408 & .408 \\
\hline 330 & 174.77 & 209 & 36,526 & 3814930 & -.013 & .495 & .495 & 341.36 & 107 & 36,526 & 2097928 & 1.318 & .906 & .094 \\
\hline 331 & 418.63 & 16 & 6,698 & 66224 & 1.634 & .949 & .051 & 669.80 & 10 & 6,698 & 41041 & 1.235 & .892 & .108 \\
\hline 332 & 370.71 & 86 & 31,881 & 1388241 & .203 & .581 & .419 & 650.63 & 49 & 31,881 & 855284 & 1.152 & .875 & .125 \\
\hline 333 & 214.36 & 100 & 21,436 & 1060448 & -0.184 & .427 & .427 & 345.74 & 62 & 21,436 & 615031 & -1.016 & .155 & .155 \\
\hline 334 & 1187.85 & 13 & 15,442 & 128214 & 1.732 & .958 & .042 & 1715.78 & 9 & 15,442 & 80253 & .805 & .789 & .211 \\
\hline 335 & 1169.42 & 19 & 22,219 & 190289 & -.744 & .229 & .229 & 4443.80 & 5 & 22,219 & 43507 & -.840 & .201 & .201 \\
\hline 336 & 414.00 & 5 & 2,070 & 4539 & -.476 & .317 & .317 & 1035.00 & 2 & 2,070 & 1902 & -.199 & .421 & .421 \\
\hline
\end{tabular}


Table 7. Summary of interoccurrence intervals for daily precipitation thresholds of 2.5 and 3.0 inches-Continued

\begin{tabular}{|c|c|c|c|c|c|c|c|c|c|c|c|c|c|c|}
\hline \multirow[b]{2}{*}{$\begin{array}{l}\text { Seq. } \\
\text { no. }\end{array}$} & \multicolumn{7}{|c|}{ Daily precipitation threshold of 2.5 inches and greater } & \multicolumn{7}{|c|}{ Daily precipitation threshold of 3.0 inches and greater } \\
\hline & $\begin{array}{l}\text { Mean } \\
\text { inter- } \\
\text { occur- } \\
\text { rence } \\
\text { interval } \\
\text { (days) }\end{array}$ & $\begin{array}{c}\text { Total } \\
\text { no. of } \\
\text { events }\end{array}$ & $\begin{array}{l}\text { Total } \\
\text { no. of } \\
\text { days } \\
\text { avail- } \\
\text { able }\end{array}$ & $\begin{array}{l}\text { Sigma } \\
\mathrm{S}\end{array}$ & $\begin{array}{l}\text { Stan- } \\
\text { dard } \\
\text { normal } \\
\text { variate }\end{array}$ & $\begin{array}{l}\text { Non- } \\
\text { ex- } \\
\text { ceed- } \\
\text { ance } \\
\text { prob- } \\
\text { ability }\end{array}$ & $\begin{array}{c}\text { p- } \\
\text { value }\end{array}$ & $\begin{array}{l}\text { Mean } \\
\text { inter- } \\
\text { occur- } \\
\text { rence } \\
\text { interval } \\
\text { (days) }\end{array}$ & $\begin{array}{c}\text { Total } \\
\text { no. of } \\
\text { events }\end{array}$ & $\begin{array}{l}\text { Total } \\
\text { no. of } \\
\text { days } \\
\text { avail- } \\
\text { able }\end{array}$ & $\begin{array}{c}\text { Sigma } \\
\mathrm{S}\end{array}$ & $\begin{array}{c}\text { Stan- } \\
\text { dard } \\
\text { normal } \\
\text { variate }\end{array}$ & $\begin{array}{l}\text { Non- } \\
\text { ex- } \\
\text { ceed- } \\
\text { ance } \\
\text { prob- } \\
\text { ability }\end{array}$ & $\begin{array}{c}\mathrm{p}- \\
\text { value }\end{array}$ \\
\hline 337 & 379.66 & 29 & 11,010 & 160392 & .044 & .517 & .483 & 734.00 & 15 & 11,010 & 79466 & -.253 & .400 & .400 \\
\hline 338 & 444.39 & 13 & 5,777 & 43331 & .961 & .832 & .168 & 825.29 & 7 & 5,777 & 24847 & 1.049 & .853 & .147 \\
\hline 339 & 161.97 & 30 & 4,859 & 79732 & .891 & .814 & .186 & 303.69 & 16 & 4,859 & 45648 & 1.208 & .886 & .114 \\
\hline 340 & 195.00 & 32 & 6,240 & 99124 & -.070 & .472 & .472 & 367.06 & 17 & 6,240 & 53090 & .007 & .503 & .497 \\
\hline 341 & 608.56 & 16 & 9,737 & 88172 & .914 & .820 & .180 & 973.70 & 10 & 9,737 & 54941 & .704 & .759 & .241 \\
\hline 342 & 683.33 & 6 & 4,100 & 17989 & 1.962 & .975 & .025 & 820.00 & 5 & 4,100 & 13889 & 1.375 & .915 & .085 \\
\hline 343 & 211.70 & 60 & 12,702 & 386193 & .181 & .572 & .428 & 362.91 & 35 & 12,702 & 205412 & -.778 & .218 & .218 \\
\hline 344 & 235.81 & 146 & 34,428 & 2789631 & 2.302 & .989 & .011 & 405.04 & 85 & 34,428 & 1618296 & 1.693 & .955 & .045 \\
\hline 345 & 121.25 & 4 & 485 & 988 & .064 & .526 & .474 & 161.67 & 3 & 485 & 678 & -.204 & .419 & .419 \\
\hline 346 & 828.00 & 26 & 21,528 & 226848 & -1.673 & .047 & .047 & 1794.00 & 12 & 21,528 & 99742 & -1.367 & .086 & .086 \\
\hline 347 & 1192.00 & 1 & 1,192 & 74 & -1.517 & .065 & .065 & 1192.00 & 1 & 1,192 & 74 & -1.517 & .065 & .065 \\
\hline 348 & 587.68 & 19 & 11,166 & 124730 & 1.328 & .908 & .092 & 1395.75 & 8 & 11,166 & 53422 & .961 & .832 & .168 \\
\hline 349 & 429.27 & 26 & 11,161 & 154872 & .595 & .724 & .276 & 744.07 & 15 & 11,161 & 87370 & .294 & .615 & .385 \\
\hline 350 & 512.42 & 55 & 28,183 & 792847 & .295 & .616 & .384 & 805.23 & 35 & 28,183 & 524250 & .645 & .741 & .259 \\
\hline 351 & 386.50 & 10 & 3,865 & 21182 & .526 & .701 & .299 & 966.25 & 4 & 3,865 & 8292 & .252 & .599 & .401 \\
\hline 352 & 1062.00 & 1 & 1,062 & 909 & 1.233 & .891 & .109 & 1062.00 & 1 & 1,062 & 909 & 1.233 & .891 & .109 \\
\hline 353 & 191.59 & 17 & 3,257 & 28089 & .104 & .542 & .458 & 814.25 & 4 & 3,257 & 10068 & 1.890 & .971 & .029 \\
\hline 354 & 286.50 & 24 & 6,876 & 98615 & 1.656 & .951 & .049 & 528.92 & 13 & 6,876 & 46830 & .299 & .617 & .383 \\
\hline 355 & 279.25 & 40 & 11,170 & 213835 & -.469 & .320 & .320 & 507.73 & 22 & 11,170 & 117533 & -.353 & .362 & .362 \\
\hline 356 & 328.38 & 109 & 35,793 & 1864696 & -.797 & .213 & .213 & 586.77 & 61 & 35,793 & 991458 & -1.242 & .107 & .107 \\
\hline 357 & 478.59 & 66 & 31,587 & 1068387 & .351 & .637 & .363 & 957.18 & 33 & 31,587 & 508714 & -.238 & .406 & .406 \\
\hline 358 & 449.62 & 42 & 18,884 & 414950 & .520 & 699 & .301 & 993.90 & 19 & 18,884 & 195339 & .671 & .749 & .251 \\
\hline 359 & 1110.00 & 2 & 2,220 & 3600 & 1.523 & .936 & .064 & 2220.00 & 1 & 2,220 & 2046 & 1.461 & .928 & .072 \\
\hline 360 & 362.00 & 2 & 724 & 828 & .352 & .638 & .362 & 724.00 & 1 & 724 & 110 & -1.206 & .114 & .114 \\
\hline 361 & -- & -- & -- & -- & -- & -- & -- & -- & -- & -- & -- & -- & -- & -- \\
\hline 362 & 175.45 & 121 & 21,229 & 1317190 & .487 & .687 & .313 & 294.85 & 72 & 21,229 & 874019 & 2.111 & .983 & .017 \\
\hline 363 & 136.75 & 16 & 2,188 & 16740 & -.302 & .381 & .381 & 218.80 & 10 & 2,188 & 9646 & -.648 & .259 & .259 \\
\hline 364 & 136.97 & 70 & 9,588 & 324835 & -.464 & .321 & .321 & 228.29 & 42 & 9,588 & 170266 & -1.733 & .042 & .042 \\
\hline 365 & 178.24 & 125 & 22,280 & 1364069 & -.395 & .346 & .346 & 289.35 & 77 & 22,280 & 883044 & .448 & .673 & .327 \\
\hline 366 & 126.25 & 20 & 2,525 & 22675 & -.790 & .215 & .215 & 132.90 & 19 & 2,525 & 21708 & -.718 & .237 & .237 \\
\hline 367 & 164.90 & 70 & 11,543 & 421646 & .633 & .737 & .263 & 295.97 & 39 & 11,543 & 222622 & -.119 & .453 & .453 \\
\hline 368 & 366.35 & 23 & 8,426 & 92696 & -.360 & .359 & .359 & 702.17 & 12 & 8,426 & 38233 & -1.463 & .072 & .072 \\
\hline 369 & -- & -- & -- & -- & -- & -- & -- & -- & -- & -- & -- & -- & -- & -- \\
\hline 370 & 6537.00 & 1 & 6,537 & 2459 & -.429 & .334 & .334 & -- & -- & -- & -- & -- & -- & -- \\
\hline 371 & -- & -- & -- & -- & -- & -- & -- & -- & -- & -- & -- & -- & -- & -- \\
\hline 372 & 543.73 & 15 & 8,156 & 75519 & 1.574 & .942 & .058 & 815.60 & 10 & 8,156 & 46447 & .761 & .777 & .223 \\
\hline 373 & -- & -- & -- & -- & -- & -- & -- & -- & -- & -- & -- & -- & -- & -- \\
\hline 374 & 874.00 & 1 & 874 & 828 & 1.550 & .939 & .061 & -- & -- & -- & -- & -- & -- & -- \\
\hline 375 & 510.52 & 21 & 10,721 & 99856 & -.897 & .185 & .185 & 974.64 & 11 & 10,721 & 37456 & -2.096 & .018 & .018 \\
\hline 376 & 970.00 & 3 & 2,910 & 6164 & 1.236 & .892 & .108 & 970.00 & 3 & 2,910 & 6164 & 1.236 & .892 & .108 \\
\hline 377 & 342.45 & 53 & 18,150 & 501616 & .541 & .706 & .294 & 567.19 & 32 & 18,150 & 292945 & .086 & .534 & .466 \\
\hline 378 & 290.80 & 50 & 14,540 & 367334 & .129 & .551 & .449 & 469.03 & 31 & 14,540 & 236531 & .478 & .684 & .317 \\
\hline 379 & -- & -- & -- & -- & -- & -- & -- & -- & -- & -- & -- & -- & -- & -- \\
\hline 380 & 357.00 & 4 & 1,428 & 3424 & .689 & .754 & .246 & 1428.00 & 1 & 1,428 & 1176 & 1.121 & .869 & .131 \\
\hline 381 & 159.65 & 119 & 18,998 & 1267685 & 2.295 & .989 & .011 & 279.38 & 68 & 18,998 & 685020 & 0.864 & .806 & .194 \\
\hline 382 & 98.67 & 3 & 296 & 305 & -.939 & .174 & .174 & -- & -- & -- & -- & -- & -- & -- \\
\hline 383 & 345.21 & 94 & 32,450 & 1586563 & .676 & .750 & .250 & 550.00 & 59 & 32,450 & 976829 & .272 & .607 & .393 \\
\hline 384 & 388.95 & 21 & 8,168 & 79589 & -.572 & .284 & .284 & 628.31 & 13 & 8,168 & 49253 & -.452 & .326 & .326 \\
\hline
\end{tabular}


Table 7. Summary of interoccurrence intervals for daily precipitation thresholds of 2.5 and 3.0 inches—Continued

\begin{tabular}{|c|c|c|c|c|c|c|c|c|c|c|c|c|c|c|}
\hline \multirow[b]{2}{*}{$\begin{array}{l}\text { Seq. } \\
\text { no. }\end{array}$} & \multicolumn{7}{|c|}{ Daily precipitation threshold of 2.5 inches and greater } & \multicolumn{7}{|c|}{ Daily precipitation threshold of 3.0 inches and greater } \\
\hline & $\begin{array}{c}\text { Mean } \\
\text { inter- } \\
\text { occur- } \\
\text { rence } \\
\text { interval } \\
\text { (days) }\end{array}$ & $\begin{array}{l}\text { Total } \\
\text { no. of } \\
\text { events }\end{array}$ & $\begin{array}{l}\text { Total } \\
\text { no. of } \\
\text { days } \\
\text { avail- } \\
\text { able }\end{array}$ & $\begin{array}{l}\text { Sigma } \\
\mathrm{S}\end{array}$ & $\begin{array}{c}\text { Stan- } \\
\text { dard } \\
\text { normal } \\
\text { variate }\end{array}$ & $\begin{array}{l}\text { Non- } \\
\text { ex- } \\
\text { ceed- } \\
\text { ance } \\
\text { prob- } \\
\text { ability }\end{array}$ & $\begin{array}{c}\mathbf{p}- \\
\text { value }\end{array}$ & $\begin{array}{c}\text { Mean } \\
\text { inter- } \\
\text { occur- } \\
\text { rence } \\
\text { interval } \\
\text { (days) }\end{array}$ & $\begin{array}{l}\text { Total } \\
\text { no. of } \\
\text { events }\end{array}$ & $\begin{array}{l}\text { Total } \\
\text { no. of } \\
\text { days } \\
\text { avail- } \\
\text { able }\end{array}$ & $\begin{array}{l}\text { Sigma } \\
\mathrm{S}\end{array}$ & $\begin{array}{c}\text { Stan- } \\
\text { dard } \\
\text { normal } \\
\text { variate }\end{array}$ & $\begin{array}{l}\text { Non- } \\
\text { ex- } \\
\text { ceed- } \\
\text { ance } \\
\text { prob- } \\
\text { ability }\end{array}$ & $\begin{array}{c}\mathrm{p}- \\
\text { value }\end{array}$ \\
\hline 385 & 197.95 & 87 & 17,222 & 691271 & -1.248 & .106 & .106 & 344.44 & 50 & 17,222 & 379027 & -1.466 & .071 & .071 \\
\hline 386 & -- & -- & -- & -- & -- & -- & -- & -- & -- & -- & -- & -- & -- & -- \\
\hline 387 & 416.20 & 5 & 2,081 & 3648 & -1.157 & .124 & .124 & 693.67 & 3 & 2,081 & 2765 & -.343 & .366 & .366 \\
\hline 388 & 308.00 & 8 & 2,464 & 6914 & -1.462 & .072 & .072 & 1232.00 & 2 & 2,464 & 924 & -1.531 & .063 & .063 \\
\hline 389 & 363.00 & 1 & 363 & 331 & 1.427 & .923 & .077 & 363.00 & 1 & 363 & 331 & 1.427 & .923 & .077 \\
\hline 390 & 133.41 & 131 & 17,476 & 1109857 & -.603 & .273 & .273 & 196.36 & 89 & 17,476 & 714727 & -1.323 & .093 & .093 \\
\hline 391 & 354.93 & 45 & 15,972 & 359033 & -.011 & .496 & .496 & 591.56 & 27 & 15,972 & 223791 & .341 & .633 & .367 \\
\hline 392 & 206.80 & 5 & 1,034 & 2132 & -.679 & .249 & .249 & 258.50 & 4 & 1,034 & 1657 & -.689 & .246 & .246 \\
\hline 393 & 3531.33 & 3 & 10,594 & 11146 & -.896 & .185 & .185 & 5297.00 & 2 & 10,594 & 11045 & .104 & .542 & .458 \\
\hline 394 & -- & -- & -- & -- & -- & -- & -- & -- & -- & -- & -- & -- & -- & -- \\
\hline 395 & 222.32 & 41 & 9,115 & 196236 & .557 & .711 & .289 & 536.18 & 17 & 9,115 & 65118 & -1.139 & .127 & .127 \\
\hline 396 & 205.17 & 96 & 19,696 & 958500 & .235 & .593 & .407 & 364.74 & 54 & 19,696 & 588410 & 1.355 & .912 & .088 \\
\hline 397 & 459.39 & 31 & 14,241 & 226970 & .272 & .607 & .393 & 1017.21 & 14 & 14,241 & 97840 & -.120 & .452 & .452 \\
\hline 398 & 310.27 & 109 & 33,819 & 1819563 & -.231 & .409 & .409 & 490.13 & 69 & 33,819 & 1189219 & .277 & 609 & .391 \\
\hline 399 & 265.11 & 74 & 19,618 & 707964 & -.368 & .357 & .357 & 456.23 & 43 & 19,618 & 422078 & .008 & .503 & .497 \\
\hline 400 & 164.71 & 117 & 19,271 & 1160529 & .551 & .709 & .291 & 296.48 & 65 & 19,271 & 642067 & .351 & .637 & .363 \\
\hline 401 & 1155.80 & 5 & 5,779 & 12958 & -.399 & .345 & .345 & 1444.75 & 4 & 5,779 & 7742 & -1.144 & .126 & .126 \\
\hline 402 & 121.50 & 2 & 243 & 263 & .202 & .580 & .420 & 243.00 & 1 & 243 & 24 & -1.390 & .082 & .082 \\
\hline 403 & 221.89 & 61 & 13,535 & 436144 & .764 & .778 & .222 & 347.05 & 39 & 13,535 & 271205 & .298 & .617 & .383 \\
\hline 404 & 216.95 & 95 & 20,610 & 984084 & .088 & .535 & .465 & 412.20 & 50 & 20,610 & 506025 & -.219 & .413 & .413 \\
\hline 405 & 371.38 & 32 & 11,884 & 213317 & 1.194 & .884 & .116 & 625.47 & 19 & 11,884 & 126044 & .879 & .810 & .190 \\
\hline 406 & 258.16 & 84 & 21,685 & 972961 & 1.084 & .861 & .139 & 409.15 & 53 & 21,685 & 643357 & 1.508 & .934 & .066 \\
\hline 407 & 370.80 & 10 & 3,708 & 20985 & .722 & .765 & .235 & 618.00 & 6 & 3,708 & 12357 & .470 & .681 & .319 \\
\hline 408 & 209.46 & 163 & 34,142 & 2820825 & .304 & .619 & .381 & 371.11 & 92 & 34,142 & 1573816 & .035 & .514 & .486 \\
\hline 409 & 1216.00 & 1 & 1,216 & 423 & -.527 & .299 & .299 & -- & -- & -- & -- & -- & -- & -- \\
\hline 410 & 236.03 & 61 & 14,398 & 449914 & .332 & .630 & .370 & 378.90 & 38 & 14,398 & 272293 & -.050 & .480 & .480 \\
\hline 411 & 562.97 & 34 & 19,141 & 406526 & 2.518 & .994 & .006 & 1472.39 & 13 & 19,141 & 152808 & 1.425 & .923 & .077 \\
\hline 412 & 272.49 & 51 & 13,897 & 428444 & 2.585 & .995 & .005 & 421.12 & 33 & 13,897 & 282503 & 2.309 & .990 & .010 \\
\hline 413 & 310.95 & 97 & 30,162 & 1477100 & .166 & .566 & .434 & 591.41 & 51 & 30,162 & 756639 & -.201 & .420 & .420 \\
\hline 414 & 163.00 & 21 & 3,423 & 36173 & .051 & .520 & .480 & 213.94 & 16 & 3,423 & 28681 & .328 & .629 & .371 \\
\hline 415 & 693.23 & 31 & 21,490 & 320582 & -.362 & .359 & .359 & 1074.50 & 20 & 21,490 & 213551 & -.049 & .481 & .481 \\
\hline 416 & 716.74 & 27 & 19,352 & 234264 & -.930 & .176 & .176 & 1488.62 & 13 & 19,352 & 125425 & -.018 & .493 & .493 \\
\hline 417 & 438.86 & 7 & 3,072 & 16364 & 2.392 & .992 & .008 & 512.00 & 6 & 3,072 & 13789 & 2.105 & .982 & .018 \\
\hline 418 & 958.65 & 26 & 24,925 & 318745 & -.144 & .443 & .443 & 2265.91 & 11 & 24,925 & 127957 & -.383 & .351 & .351 \\
\hline 419 & 248.99 & 91 & 22,658 & 924306 & -1.709 & .044 & .044 & 419.59 & 54 & 22,658 & 530869 & -1.683 & .046 & .046 \\
\hline 420 & 598.06 & 32 & 19,138 & 379722 & 2.352 & .991 & .009 & 1125.77 & 17 & 19,138 & 184154 & .943 & .827 & .173 \\
\hline 421 & 3465.67 & 9 & 31,191 & 147592 & .268 & .606 & .394 & 4455.86 & 7 & 31,191 & 111061 & .079 & .532 & .468 \\
\hline 422 & 1931.50 & 6 & 11,589 & 24648 & -1.235 & .109 & .109 & 2317.80 & 5 & 11,589 & 17234 & -1.569 & .058 & .058 \\
\hline 423 & 527.09 & 22 & 11,596 & 131146 & .229 & .590 & .410 & 892.00 & 13 & 11,596 & 65108 & -.851 & .198 & .198 \\
\hline 424 & -- & -- & -- & -- & -- & -- & -- & -- & -- & -- & -- & -- & -- & -- \\
\hline 425 & 1417.47 & 15 & 21,262 & 157992 & -.062 & .475 & .475 & 3037.43 & 7 & 21,262 & 94005 & 1.206 & .886 & .114 \\
\hline 426 & 992.61 & 18 & 17,867 & 146276 & -.664 & .253 & .253 & 2233.38 & 8 & 17,867 & 63246 & -.564 & .287 & .287 \\
\hline 427 & -- & -- & -- & -- & -- & -- & -- & -- & -- & -- & -- & -- & -- & -- \\
\hline 428 & 282.24 & 17 & 4,798 & 46141 & .938 & .826 & .174 & 436.18 & 11 & 4,798 & 27912 & .332 & .630 & .370 \\
\hline 429 & 541.20 & 10 & 5,412 & 20392 & -1.350 & .089 & .089 & 1353.00 & 4 & 5,412 & 4867 & -1.907 & .028 & .028 \\
\hline 430 & 278.59 & 32 & 8,915 & 160305 & 1.213 & .887 & .113 & 685.77 & 13 & 8,915 & 66888 & .964 & .832 & .168 \\
\hline 431 & 550.86 & 7 & 3,856 & 11573 & -.653 & .257 & .257 & 771.20 & 5 & 3,856 & 7556 & -.837 & .201 & .201 \\
\hline 432 & 131.64 & 66 & 8,688 & 225229 & -3.017 & .001 & .001 & 228.63 & 38 & 8,688 & 127682 & -2.418 & .008 & .008 \\
\hline
\end{tabular}


Table 7. Summary of interoccurrence intervals for daily precipitation thresholds of 2.5 and 3.0 inches-Continued

\begin{tabular}{|c|c|c|c|c|c|c|c|c|c|c|c|c|c|c|}
\hline \multirow[b]{2}{*}{$\begin{array}{l}\text { Seq. } \\
\text { no. }\end{array}$} & \multicolumn{7}{|c|}{ Daily precipitation threshold of 2.5 inches and greater } & \multicolumn{7}{|c|}{ Daily precipitation threshold of 3.0 inches and greater } \\
\hline & $\begin{array}{l}\text { Mean } \\
\text { inter- } \\
\text { occur- } \\
\text { rence } \\
\text { interval } \\
\text { (days) }\end{array}$ & $\begin{array}{l}\text { Total } \\
\text { no. of } \\
\text { events }\end{array}$ & $\begin{array}{l}\text { Total } \\
\text { no. of } \\
\text { days } \\
\text { avail- } \\
\text { able }\end{array}$ & $\begin{array}{l}\text { Sigma } \\
\mathrm{S}\end{array}$ & $\begin{array}{c}\text { Stan- } \\
\text { dard } \\
\text { normal } \\
\text { variate }\end{array}$ & $\begin{array}{l}\text { Non- } \\
\text { ex- } \\
\text { ceed- } \\
\text { ance } \\
\text { prob- } \\
\text { ability }\end{array}$ & $\begin{array}{c}\text { p- } \\
\text { value }\end{array}$ & $\begin{array}{l}\text { Mean } \\
\text { inter- } \\
\text { occur- } \\
\text { rence } \\
\text { interval } \\
\text { (days) }\end{array}$ & $\begin{array}{c}\text { Total } \\
\text { no. of } \\
\text { events }\end{array}$ & $\begin{array}{l}\text { Total } \\
\text { no. of } \\
\text { days } \\
\text { avail- } \\
\text { able }\end{array}$ & $\begin{array}{l}\text { Sigma } \\
\mathbf{S}\end{array}$ & $\begin{array}{c}\text { Stan- } \\
\text { dard } \\
\text { normal } \\
\text { variate }\end{array}$ & $\begin{array}{l}\text { Non- } \\
\text { ex- } \\
\text { ceed- } \\
\text { ance } \\
\text { prob- } \\
\text { ability }\end{array}$ & $\begin{array}{c}p- \\
\text { value }\end{array}$ \\
\hline 433 & 291.00 & 94 & 27,354 & 1243528 & -.550 & .291 & .291 & 463.63 & 59 & 27,354 & 683748 & -2.031 & .021 & .021 \\
\hline 434 & 230.46 & 59 & 13,597 & 395077 & -.200 & .421 & .421 & 543.88 & 25 & 13,597 & 165898 & -.207 & .418 & .418 \\
\hline 435 & 147.57 & 7 & 1,033 & 5323 & 2.164 & .985 & .015 & 258.25 & 4 & 1,033 & 3498 & 2.401 & .992 & .008 \\
\hline 436 & 309.09 & 94 & 29,054 & 1478357 & 1.387 & .917 & .083 & 468.61 & 62 & 29,054 & 968007 & 1.020 & .846 & .154 \\
\hline 437 & 109.62 & 231 & 25,322 & 2960914 & .326 & .628 & .372 & 190.39 & 133 & 25,322 & 1668013 & -.189 & .425 & .425 \\
\hline 438 & 230.51 & 81 & 18,671 & 771761 & .321 & .626 & .374 & 366.10 & 51 & 18,671 & 464415 & -.304 & .381 & .381 \\
\hline 439 & 371.09 & 22 & 8,164 & 82502 & -.661 & .255 & .255 & 680.33 & 12 & 8,164 & 47511 & -.180 & .428 & .428 \\
\hline 440 & 273.59 & 34 & 9,302 & 169138 & .703 & .759 & .241 & 372.08 & 25 & 9,302 & 113189 & -.230 & .409 & .409 \\
\hline 441 & 1483.31 & 16 & 23,733 & 198349 & .310 & .622 & .378 & 3955.50 & 6 & 23,733 & 69689 & -.090 & .464 & .464 \\
\hline 442 & 196.45 & 65 & 12,769 & 481780 & 2.247 & .988 & .012 & 327.41 & 39 & 12,769 & 283853 & 1.514 & .935 & .065 \\
\hline 443 & 295.16 & 44 & 12,987 & 271920 & -.555 & .290 & .290 & 418.94 & 31 & 12,987 & 191941 & -.448 & .327 & .327 \\
\hline 444 & 403.25 & 4 & 1,613 & 4637 & 1.515 & .935 & .065 & 537.67 & 3 & 1,613 & 3070 & .807 & .790 & .210 \\
\hline 445 & 660.00 & 29 & 19,140 & 301219 & .796 & .787 & .213 & 1276.00 & 15 & 19,140 & 148665 & .239 & .594 & .406 \\
\hline 446 & 392.33 & 3 & 1,177 & 2912 & 1.948 & .974 & .026 & 588.50 & 2 & 1,177 & 2147 & 2.019 & .978 & .022 \\
\hline 447 & 573.45 & 40 & 22,938 & 441064 & -.423 & .336 & .336 & 1092.29 & 21 & 22,938 & 227427 & -.442 & .329 & .329 \\
\hline 448 & 244.40 & 129 & 31,527 & 1782422 & -2.429 & .008 & .008 & 404.19 & 78 & 31,527 & 1094310 & -1.683 & .046 & .046 \\
\hline 449 & 166.16 & 32 & 5,317 & 74905 & -1.171 & .121 & .121 & 241.68 & 22 & 5,317 & 46629 & -1.647 & .050 & .050 \\
\hline 450 & 171.11 & 120 & 20,533 & 1244170 & .188 & .574 & .426 & 266.66 & 77 & 20,533 & 813650 & .445 & .672 & .328 \\
\hline 451 & 185.40 & 30 & 5,562 & 82209 & -.139 & .445 & .445 & 231.75 & 24 & 5,562 & 70064 & .422 & .664 & .336 \\
\hline 452 & 304.00 & 1 & 304 & 267 & 1.310 & .905 & .095 & 304.00 & 1 & 304 & 267 & 1.310 & .905 & .095 \\
\hline 453 & 2103.00 & 1 & 2,103 & 1405 & .582 & .720 & .280 & -- & -- & -- & -- & -- & -- & -- \\
\hline 454 & 756.76 & 42 & 31,784 & 650525 & -.285 & .388 & .388 & 1222.46 & 26 & 31,784 & 364338 & -1.044 & .148 & .148 \\
\hline 455 & 887.00 & 1 & 887 & 525 & .318 & .625 & .375 & -- & -- & -- & -- & -- & -- & -- \\
\hline 456 & -- & -- & -- & -- & -- & -- & -- & -- & -- & -- & -- & -- & -- & -- \\
\hline 457 & 113.67 & 3 & 341 & 640 & .754 & .774 & .226 & -- & -- & -- & -- & -- & -- & -- \\
\hline 458 & 151.78 & 41 & 6,223 & 121402 & -.536 & .296 & .296 & 259.29 & 24 & 6,223 & 63769 & -1.239 & .108 & .108 \\
\hline 459 & 160.14 & 7 & 1,121 & 3787 & -.159 & .437 & .437 & 224.20 & 5 & 1,121 & 3024 & .306 & .620 & .380 \\
\hline 460 & 228.90 & 143 & 32,732 & 2245601 & -.838 & .201 & .201 & 404.10 & 81 & 32,732 & 1236357 & -1.050 & .147 & .147 \\
\hline 461 & 282.43 & 109 & 30,785 & 1627288 & -.544 & .293 & .293 & 446.16 & 69 & 30,785 & 997168 & -.879 & .190 & .190 \\
\hline 462 & 249.86 & 71 & 17,740 & 614815 & -.347 & .364 & .364 & 506.86 & 35 & 17,740 & 261964 & -1.600 & .055 & .055 \\
\hline 463 & 207.69 & 36 & 7,477 & 134756 & .013 & .505 & .495 & 373.85 & 20 & 7,477 & 72826 & -.201 & .420 & .420 \\
\hline 464 & 187.50 & 120 & 22,500 & 1331544 & -.259 & .398 & .398 & 330.88 & 68 & 22,500 & 722863 & -.787 & .216 & .216 \\
\hline 465 & 393.20 & 5 & 1,966 & 6395 & 1.166 & .878 & .122 & 983.00 & 2 & 1,966 & 2914 & 1.181 & .881 & .119 \\
\hline 466 & 178.46 & 147 & 26,233 & 1974177 & .502 & .692 & .308 & 305.04 & 86 & 26,233 & 1257357 & 1.842 & .967 & .033 \\
\hline 467 & 2765.75 & 4 & 11,063 & 23562 & .225 & .589 & .411 & 5531.50 & 2 & 11,063 & 13456 & .530 & .702 & .298 \\
\hline 468 & 165.50 & 6 & 993 & 1778 & -1.710 & .044 & .044 & 198.60 & 5 & 993 & 904 & -2.463 & .007 & .007 \\
\hline 469 & 188.87 & 52 & 9,821 & 262553 & .353 & .638 & .362 & 327.37 & 30 & 9,821 & 170154 & 1.471 & .929 & .071 \\
\hline 470 & -- & -- & -- & -- & -- & -- & -- & -- & -- & -- & -- & -- & -- & -- \\
\hline 471 & 252.60 & 53 & 13,388 & 392891 & 1.355 & .912 & .088 & 478.14 & 28 & 13,388 & 208577 & 1.034 & .849 & .151 \\
\hline 472 & 146.61 & 23 & 3,372 & 49348 & 2.264 & .988 & .012 & 210.75 & 16 & 3,372 & 38648 & 2.998 & .999 & .001 \\
\hline 473 & 240.33 & 80 & 19,226 & 811068 & .847 & .801 & .199 & 417.96 & 46 & 19,226 & 453254 & .294 & .616 & .384 \\
\hline 474 & 370.24 & 68 & 25,176 & 855911 & -.001 & .500 & .500 & 645.54 & 39 & 25,176 & 519920 & .639 & .738 & .262 \\
\hline 475 & 194.87 & 164 & 31,959 & 2834839 & 1.813 & .965 & .035 & 301.50 & 106 & 31,959 & 1866767 & 1.821 & .966 & .034 \\
\hline 476 & 175.36 & 104 & 18,237 & 934927 & -.250 & .401 & .401 & 260.53 & 70 & 18,237 & 675038 & .834 & .798 & .202 \\
\hline 477 & 262.56 & 16 & 4,201 & 43014 & 1.939 & .974 & .026 & 381.91 & 11 & 4,201 & 26939 & 0.953 & .830 & .170 \\
\hline 478 & 221.76 & 104 & 23,063 & 1172851 & -.389 & .349 & .349 & 427.09 & 54 & 23,063 & 632650 & .203 & .581 & .419 \\
\hline 479 & 119.47 & 15 & 1,792 & 11157 & -1.140 & .127 & .127 & 224.00 & 8 & 1,792 & 6765 & -.275 & .391 & .391 \\
\hline 480 & 177.63 & 32 & 5,684 & 86185 & -.513 & .304 & .304 & 315.78 & 18 & 5,684 & 58220 & 1.015 & .845 & .155 \\
\hline
\end{tabular}


Table 7. Summary of interoccurrence intervals for daily precipitation thresholds of 2.5 and 3.0 inches—Continued

\begin{tabular}{|c|c|c|c|c|c|c|c|c|c|c|c|c|c|c|}
\hline \multirow[b]{2}{*}{$\begin{array}{l}\text { Seq. } \\
\text { no. }\end{array}$} & \multicolumn{7}{|c|}{ Daily precipitation threshold of 2.5 inches and greater } & \multicolumn{7}{|c|}{ Daily precipitation threshold of 3.0 inches and greater } \\
\hline & $\begin{array}{l}\text { Mean } \\
\text { inter- } \\
\text { occur- } \\
\text { rence } \\
\text { interval } \\
\text { (days) }\end{array}$ & $\begin{array}{l}\text { Total } \\
\text { no. of } \\
\text { events }\end{array}$ & $\begin{array}{l}\text { Total } \\
\text { no. of } \\
\text { days } \\
\text { avail- } \\
\text { able }\end{array}$ & $\begin{array}{l}\text { Sigma } \\
\mathrm{S}\end{array}$ & $\begin{array}{c}\text { Stan- } \\
\text { dard } \\
\text { normal } \\
\text { variate }\end{array}$ & $\begin{array}{l}\text { Non- } \\
\text { ex- } \\
\text { ceed- } \\
\text { ance } \\
\text { prob- } \\
\text { ability }\end{array}$ & $\begin{array}{c}\text { p- } \\
\text { value }\end{array}$ & $\begin{array}{c}\text { Mean } \\
\text { inter- } \\
\text { occur- } \\
\text { rence } \\
\text { interval } \\
\text { (days) }\end{array}$ & $\begin{array}{l}\text { Total } \\
\text { no. of } \\
\text { events }\end{array}$ & $\begin{array}{l}\text { Total } \\
\text { no. of } \\
\text { days } \\
\text { avail- } \\
\text { able }\end{array}$ & $\begin{array}{l}\text { Sigma } \\
\mathbf{S}\end{array}$ & $\begin{array}{c}\text { Stan- } \\
\text { dard } \\
\text { normal } \\
\text { variate }\end{array}$ & $\begin{array}{l}\text { Non- } \\
\text { ex- } \\
\text { ceed- } \\
\text { ance } \\
\text { prob- } \\
\text { ability }\end{array}$ & $\begin{array}{c}\mathrm{p}- \\
\text { value }\end{array}$ \\
\hline 481 & 105.42 & 120 & 12,650 & 905938 & 3.673 & 1.000 & 0 & 164.29 & 77 & 12,650 & 556385 & 2.165 & .985 & .015 \\
\hline 482 & 247.27 & 15 & 3,709 & 22441 & -1.297 & .097 & .097 & 370.90 & 10 & 3,709 & 10148 & -2.480 & .007 & .007 \\
\hline 483 & 453.43 & 7 & 3,174 & 9714 & -.576 & .283 & .283 & 793.50 & 4 & 3,174 & 5020 & -.725 & .234 & .234 \\
\hline 484 & 182.40 & 15 & 2,736 & 19246 & -.417 & .339 & .339 & 248.73 & 11 & 2,736 & 13685 & -.520 & .301 & .301 \\
\hline 485 & 848.00 & 1 & 848 & 200 & -.915 & .180 & .180 & 848.00 & 1 & 848 & 200 & -.915 & .180 & .180 \\
\hline 486 & 409.17 & 85 & 34,779 & 1637199 & 1.719 & .957 & .043 & 772.87 & 45 & 34,779 & 872617 & 1.338 & .909 & .091 \\
\hline 487 & 299.54 & 35 & 10,484 & 163705 & -1.104 & .135 & .135 & 499.24 & 21 & 10,484 & 89190 & -1.506 & .066 & .066 \\
\hline 488 & 1480.95 & 19 & 28,138 & 226901 & -1.141 & .127 & .127 & 3126.44 & 9 & 28,138 & 114129 & -.513 & .304 & .304 \\
\hline 489 & 270.40 & 43 & 11,627 & 266036 & .730 & .767 & .233 & 387.57 & 30 & 11,627 & 193162 & 1.020 & .846 & .154 \\
\hline 490 & 325.08 & 24 & 7,802 & 125017 & 2.845 & .998 & .002 & 780.20 & 10 & 7,802 & 50333 & 1.590 & .944 & .056 \\
\hline 491 & 202.70 & 63 & 12,770 & 413105 & .371 & .645 & .355 & 364.86 & 35 & 12,770 & 221198 & -.104 & .458 & .458 \\
\hline 492 & 272.70 & 92 & 25,088 & 1131296 & -.328 & .372 & .372 & 501.76 & 50 & 25,088 & 568979 & -1.137 & .128 & .128 \\
\hline 493 & 197.02 & 181 & 35,661 & 3386237 & 1.147 & .874 & .126 & 353.08 & 101 & 35,661 & 1875666 & .723 & .765 & .235 \\
\hline 494 & 163.96 & 26 & 4,263 & 61561 & .979 & .836 & .164 & 327.92 & 13 & 4,263 & 31748 & .910 & .819 & .181 \\
\hline 495 & 147.42 & 138 & 20,344 & 1499024 & 1.381 & .916 & .084 & 251.16 & 81 & 20,344 & 852250 & .536 & .704 & .296 \\
\hline 496 & 862.28 & 25 & 21,557 & 192756 & -2.465 & .007 & .007 & 1658.23 & 13 & 21,557 & 96266 & -1.955 & .025 & .025 \\
\hline 497 & -- & -- & -- & -- & -- & -- & -- & -- & -- & -- & -- & -- & -- & -- \\
\hline 498 & 211.88 & 90 & 19,069 & 918608 & 1.159 & .877 & .123 & 353.13 & 54 & 19,069 & 536464 & .534 & .703 & .297 \\
\hline 499 & 493.00 & 36 & 17,748 & 332738 & .432 & .667 & .333 & 806.73 & 22 & 17,748 & 208233 & .541 & .706 & .294 \\
\hline 500 & 231.20 & 5 & 1,156 & 4075 & 1.588 & .944 & .056 & 231.20 & 5 & 1,156 & 4075 & 1.588 & .944 & .056 \\
\hline 501 & 255.60 & 20 & 5,112 & 45139 & -.906 & .182 & .182 & 393.23 & 13 & 5,112 & 34192 & .181 & .572 & .428 \\
\hline 502 & 188.70 & 162 & 30,570 & 2893298 & 3.714 & 1.000 & 0 & 339.67 & 90 & 30,570 & 1641821 & 3.179 & .999 & .001 \\
\hline 503 & 556.36 & 11 & 6,120 & 35060 & .239 & .594 & .406 & 874.29 & 7 & 6,120 & 23750 & .499 & .691 & .309 \\
\hline 504 & 1227.67 & 3 & 3,683 & 7780 & 1.225 & .890 & .110 & 1227.67 & 3 & 3,683 & 7780 & 1.225 & .890 & .110 \\
\hline 505 & -- & -- & -- & -- & -- & -- & -- & -- & -- & -- & -- & -- & -- & -- \\
\hline 506 & 194.80 & 192 & 37,401 & 3962934 & 2.490 & .994 & .006 & 328.08 & 114 & 37,401 & 2292821 & 1.396 & .919 & .081 \\
\hline 507 & 107.39 & 13 & 1,396 & 9955 & .606 & .728 & .272 & 279.20 & 5 & 1,396 & 3742 & .280 & .610 & .390 \\
\hline 508 & 397.05 & 40 & 15,882 & 360670 & 1.484 & .931 & .069 & 661.75 & 24 & 15,882 & 223709 & 1.475 & .930 & .070 \\
\hline 509 & 520.80 & 49 & 25,519 & 618035 & -.139 & .445 & .445 & 850.63 & 30 & 25,519 & 359953 & -.566 & .286 & .286 \\
\hline 510 & -- & -- & -- & -- & -- & -- & -- & -- & -- & -- & -- & -- & -- & -- \\
\hline 511 & 167.49 & 111 & 18,591 & 1165695 & 2.368 & .991 & .009 & 331.98 & 56 & 18,591 & 614811 & 2.347 & .991 & .009 \\
\hline 512 & 264.98 & 119 & 31,532 & 1899486 & .235 & .593 & .407 & 450.46 & 70 & 31,532 & 1108722 & .067 & .527 & .473 \\
\hline 513 & 361.12 & 59 & 21,306 & 707225 & 1.666 & .952 & .048 & 560.68 & 38 & 21,306 & 467074 & 1.642 & .950 & .050 \\
\hline 514 & -- & -- & -- & -- & -- & -- & -- & -- & -- & -- & -- & -- & -- & -- \\
\hline 515 & 1340.33 & 12 & 16,084 & 115729 & 1.195 & .884 & .116 & 2680.67 & 6 & 16,084 & 64498 & 1.429 & .923 & .077 \\
\hline 516 & -- & -- & -- & -- & -- & -- & -- & -- & -- & -- & -- & -- & -- & -- \\
\hline 517 & 1304.00 & 15 & 19,560 & 207871 & 2.797 & .997 & .003 & 3912.00 & 5 & 19,560 & 75935 & 2.141 & .984 & .016 \\
\hline 518 & 582.35 & 63 & 36,688 & 1018146 & -1.636 & .051 & .051 & 940.72 & 39 & 36,688 & 652451 & -.952 & .171 & .171 \\
\hline 519 & 643.00 & 12 & 7,716 & 52143 & .758 & .776 & .224 & 1929.00 & 4 & 7,716 & 16427 & .223 & .588 & .412 \\
\hline 520 & 670.00 & 1 & 670 & 641 & 1.582 & .943 & .057 & -- & -- & -- & -- & -- & -- & -- \\
\hline 521 & 181.86 & 98 & 17,822 & 956856 & 1.641 & .950 & .050 & 371.29 & 48 & 17,822 & 447418 & .552 & .710 & .290 \\
\hline 522 & 389.58 & 19 & 7,402 & 64859 & -.586 & .279 & .279 & 616.83 & 12 & 7,402 & 40073 & -.586 & .279 & .279 \\
\hline 523 & 543.43 & 7 & 3,804 & 14234 & .317 & .624 & .376 & 634.00 & 6 & 3,804 & 12642 & .457 & .676 & .324 \\
\hline 524 & -- & -- & -- & -- & -- & -- & -- & -- & -- & -- & -- & -- & -- & -- \\
\hline 525 & -- & -- & -- & -- & -- & -- & -- & -- & -- & -- & -- & -- & -- & -- \\
\hline 526 & 346.39 & 94 & 32,561 & 1583688 & 0.585 & .721 & .279 & 551.88 & 59 & 32,561 & 983596 & 0.319 & .625 & .375 \\
\hline 527 & 167.15 & 52 & 8,692 & 229133 & .174 & .569 & .431 & 310.43 & 28 & 8,692 & 120395 & -.097 & .461 & .461 \\
\hline 528 & 108.86 & 29 & 3,157 & 47473 & .346 & .635 & .365 & 185.71 & 17 & 3,157 & 25051 & -.475 & .318 & .318 \\
\hline
\end{tabular}


Table 7. Summary of interoccurrence intervals for daily precipitation thresholds of 2.5 and 3.0 inches-Continued

\begin{tabular}{|c|c|c|c|c|c|c|c|c|c|c|c|c|c|c|}
\hline \multirow[b]{2}{*}{$\begin{array}{l}\text { Seq. } \\
\text { no. }\end{array}$} & \multicolumn{7}{|c|}{ Daily precipitation threshold of 2.5 inches and greater } & \multicolumn{7}{|c|}{ Daily precipitation threshold of 3.0 inches and greater } \\
\hline & $\begin{array}{l}\text { Mean } \\
\text { inter- } \\
\text { occur- } \\
\text { rence } \\
\text { interval } \\
\text { (days) }\end{array}$ & $\begin{array}{l}\text { Total } \\
\text { no. of } \\
\text { events }\end{array}$ & $\begin{array}{l}\text { Total } \\
\text { no. of } \\
\text { days } \\
\text { avail- } \\
\text { able }\end{array}$ & $\begin{array}{l}\text { Sigma } \\
\mathrm{S}\end{array}$ & $\begin{array}{c}\text { Stan- } \\
\text { dard } \\
\text { normal } \\
\text { variate }\end{array}$ & $\begin{array}{l}\text { Non- } \\
\text { ex- } \\
\text { ceed- } \\
\text { ance } \\
\text { prob- } \\
\text { ability }\end{array}$ & $\begin{array}{c}\text { p- } \\
\text { value }\end{array}$ & $\begin{array}{l}\text { Mean } \\
\text { inter- } \\
\text { occur- } \\
\text { rence } \\
\text { interval } \\
\text { (days) }\end{array}$ & $\begin{array}{c}\text { Total } \\
\text { no. of } \\
\text { events }\end{array}$ & $\begin{array}{l}\text { Total } \\
\text { no. of } \\
\text { days } \\
\text { avail- } \\
\text { able }\end{array}$ & $\begin{array}{l}\text { Sigma } \\
\mathbf{S}\end{array}$ & $\begin{array}{c}\text { Stan- } \\
\text { dard } \\
\text { normal } \\
\text { variate }\end{array}$ & $\begin{array}{l}\text { Non- } \\
\text { ex- } \\
\text { ceed- } \\
\text { ance } \\
\text { prob- } \\
\text { ability }\end{array}$ & $\begin{array}{c}p- \\
\text { value }\end{array}$ \\
\hline 529 & 186.82 & 62 & 11,583 & 372903 & .525 & .700 & .300 & 297.00 & 39 & 11,583 & 241729 & .760 & .776 & .224 \\
\hline 530 & 180.55 & 189 & 34,124 & 3179008 & -.338 & .368 & .368 & 331.30 & 103 & 34,124 & 1773946 & .166 & .566 & .434 \\
\hline 531 & 212.10 & 30 & 6,363 & 102445 & .696 & .757 & .243 & 353.50 & 18 & 6,363 & 68600 & 1.454 & .927 & .073 \\
\hline 532 & 400.62 & 90 & 36,056 & 1710638 & .892 & .814 & .186 & 680.30 & 53 & 36,056 & 1015890 & .797 & .787 & .213 \\
\hline 533 & 919.89 & 28 & 25,757 & 372170 & .294 & .616 & .384 & 2146.42 & 12 & 25,757 & 170491 & .619 & .732 & .268 \\
\hline 534 & 493.00 & 2 & 986 & 1601 & 1.528 & .937 & .063 & 493.00 & 2 & 986 & 1601 & 1.528 & .937 & .063 \\
\hline 535 & 262.21 & 160 & 41,954 & 3293077 & -.413 & .340 & .340 & 466.16 & 90 & 41,954 & 1876573 & -.099 & .461 & .461 \\
\hline 536 & -- & -- & -- & -- & -- & -- & -- & -- & -- & -- & -- & -- & -- & -- \\
\hline 537 & 354.04 & 90 & 31,864 & 1514423 & .923 & .822 & .178 & 579.35 & 55 & 31,864 & 980385 & 1.526 & .937 & .063 \\
\hline 538 & 572.50 & 4 & 2,290 & 4406 & -.132 & .448 & .448 & 1145.00 & 2 & 2,290 & 2136 & -.165 & .435 & .435 \\
\hline 539 & 704.50 & 28 & 19,726 & 311245 & 1.164 & .878 & .122 & 1232.88 & 16 & 19,726 & 187568 & 1.307 & .904 & .096 \\
\hline 540 & 333.20 & 5 & 1,666 & 5263 & 1.021 & .846 & .154 & 555.33 & 3 & 1,666 & 2853 & .425 & .665 & .335 \\
\hline 541 & 245.00 & 143 & 35,035 & 2529622 & .204 & .581 & .419 & 412.18 & 85 & 35,035 & 1615821 & 1.360 & .913 & .087 \\
\hline 542 & 197.33 & 6 & 1,184 & 2935 & -.737 & .231 & .231 & 394.67 & 3 & 1,184 & 1634 & -.240 & .405 & .405 \\
\hline 543 & 1046.63 & 8 & 8,373 & 31105 & -.349 & .363 & .363 & 1674.60 & 5 & 8,373 & 21600 & .124 & .549 & .451 \\
\hline 544 & 250.68 & 117 & 29,330 & 1636952 & -.861 & .195 & .195 & 437.76 & 67 & 29,330 & 966982 & -.225 & .411 & .411 \\
\hline 545 & 524.81 & 16 & 8,397 & 51548 & -1.612 & .054 & .054 & 645.92 & 13 & 8,397 & 42443 & -1.389 & .082 & .082 \\
\hline 546 & 159.41 & 197 & 31,403 & 3270830 & 1.396 & .919 & .081 & 268.40 & 117 & 31,403 & 1883998 & .479 & .684 & .316 \\
\hline 547 & 339.42 & 52 & 17,650 & 463334 & .121 & .548 & .452 & 767.39 & 23 & 17,650 & 229759 & 1.096 & .863 & .137 \\
\hline 548 & 154.26 & 85 & 13,112 & 549594 & -.220 & .413 & .413 & 312.19 & 42 & 13,112 & 238143 & -1.517 & .065 & .065 \\
\hline 549 & -- & -- & -- & -- & -- & -- & -- & -- & -- & -- & -- & -- & -- & -- \\
\hline 550 & 209.50 & 4 & 838 & 1825 & .308 & .621 & .379 & 279.33 & 3 & 838 & 1342 & .203 & .580 & .420 \\
\hline 551 & 156.74 & 35 & 5,486 & 97418 & .151 & .560 & .440 & 288.74 & 19 & 5,486 & 58361 & .905 & .817 & .183 \\
\hline 552 & 167.24 & 117 & 19,567 & 1249669 & 1.719 & .957 & .043 & 275.59 & 71 & 19,567 & 777566 & 1.743 & .959 & .041 \\
\hline 553 & -- & -- & -- & -- & -- & -- & -- & -- & -- & -- & -- & -- & -- & -- \\
\hline 554 & 167.62 & 71 & 11,901 & 398903 & -.815 & .208 & .208 & 276.77 & 43 & 11,901 & 252178 & -.164 & .435 & .435 \\
\hline 555 & -- & -- & -- & -- & -- & -- & -- & -- & -- & -- & -- & -- & -- & -- \\
\hline 556 & 119.01 & 168 & 19,994 & 1689794 & .138 & .555 & .445 & 197.96 & 101 & 19,994 & 1055271 & .786 & .784 & .216 \\
\hline 557 & -- & -- & -- & -- & -- & -- & -- & -- & -- & -- & -- & -- & -- & -- \\
\hline 558 & 101.38 & 21 & 2,129 & 22349 & -.002 & .499 & .499 & 141.93 & 15 & 2,129 & 16692 & .304 & .620 & .380 \\
\hline 559 & 584.00 & 1 & 584 & 122 & -1.008 & .157 & .157 & 584.00 & 1 & 584 & 122 & -1.008 & .157 & .157 \\
\hline 560 & -- & -- & -- & -- & -- & -- & -- & -- & -- & -- & -- & -- & -- & -- \\
\hline 561 & 144.65 & 123 & 17,792 & 1317844 & 3.926 & 1.000 & 0 & 199.91 & 89 & 17,792 & 950396 & 3.274 & .999 & .001 \\
\hline 562 & 137.94 & 150 & 20,691 & 1651326 & 1.360 & .913 & .087 & 213.31 & 97 & 20,691 & 1045524 & .714 & .762 & .238 \\
\hline 563 & 149.50 & 113 & 16,893 & 1031994 & 1.496 & .933 & .067 & 244.83 & 69 & 16,893 & 621461 & .954 & .830 & .170 \\
\hline 564 & 160.84 & 118 & 18,979 & 1236431 & 1.960 & .975 & .025 & 279.10 & 68 & 18,979 & 731327 & 1.904 & .972 & .028 \\
\hline 565 & 92.40 & 53 & 4,897 & 155307 & 2.481 & .993 & .007 & 139.91 & 35 & 4,897 & 98900 & 1.579 & .943 & .057 \\
\hline 566 & 122.80 & 122 & 14,981 & 935640 & .456 & .676 & .324 & 187.26 & 80 & 14,981 & 629811 & .790 & .785 & .215 \\
\hline 567 & 274.75 & 32 & 8,792 & 176482 & 2.494 & .994 & .006 & 399.64 & 22 & 8,792 & 124070 & 2.298 & .989 & .011 \\
\hline 568 & 163.04 & 97 & 15,815 & 834257 & 1.495 & .933 & .067 & 292.87 & 54 & 15,815 & 491474 & 1.922 & .973 & .027 \\
\hline 569 & 158.93 & 58 & 9,218 & 244577 & -1.122 & .131 & .131 & 263.37 & 35 & 9,218 & 148674 & -.803 & .211 & .211 \\
\hline 570 & 117.32 & 31 & 3,637 & 56310 & -.011 & .496 & .496 & 158.13 & 23 & 3,637 & 45071 & .645 & .740 & .260 \\
\hline 571 & 140.57 & 129 & 18,133 & 1388712 & 3.686 & 1.000 & 0 & 235.49 & 77 & 18,133 & 798074 & 2.176 & .985 & .015 \\
\hline 572 & 266.02 & 48 & 12,769 & 327302 & .816 & .793 & .207 & 425.63 & 30 & 12,769 & 210117 & .920 & .821 & .179 \\
\hline 573 & 420.08 & 12 & 5,041 & 28955 & -0.256 & .399 & .399 & 560.11 & 9 & 5,041 & 22819 & 0.031 & .512 & .488 \\
\hline 574 & 131.33 & 85 & 11,163 & 516347 & 1.411 & .921 & .079 & 195.84 & 57 & 11,163 & 340841 & .933 & .825 & .176 \\
\hline 575 & 372.36 & 47 & 17,501 & 444649 & .964 & .832 & .168 & 760.91 & 23 & 17,501 & 190757 & -.434 & .332 & .332 \\
\hline 576 & 258.36 & 14 & 3,617 & 27321 & .512 & .696 & .304 & 401.89 & 9 & 3,617 & 17807 & .489 & .687 & .313 \\
\hline
\end{tabular}


Table 7. Summary of interoccurrence intervals for daily precipitation thresholds of 2.5 and 3.0 inches—Continued

\begin{tabular}{|c|c|c|c|c|c|c|c|c|c|c|c|c|c|c|}
\hline \multirow[b]{2}{*}{$\begin{array}{l}\text { Seq. } \\
\text { no. }\end{array}$} & \multicolumn{7}{|c|}{ Daily precipitation threshold of 2.5 inches and greater } & \multicolumn{7}{|c|}{ Daily precipitation threshold of 3.0 inches and greater } \\
\hline & $\begin{array}{l}\text { Mean } \\
\text { inter- } \\
\text { occur- } \\
\text { rence } \\
\text { interval } \\
\text { (days) }\end{array}$ & $\begin{array}{l}\text { Total } \\
\text { no. of } \\
\text { events }\end{array}$ & $\begin{array}{l}\text { Total } \\
\text { no. of } \\
\text { days } \\
\text { avail- } \\
\text { able }\end{array}$ & $\begin{array}{l}\text { Sigma } \\
\mathrm{S}\end{array}$ & $\begin{array}{c}\text { Stan- } \\
\text { dard } \\
\text { normal } \\
\text { variate }\end{array}$ & $\begin{array}{l}\text { Non- } \\
\text { ex- } \\
\text { ceed- } \\
\text { ance } \\
\text { prob- } \\
\text { ability }\end{array}$ & $\begin{array}{c}\mathrm{p}- \\
\text { value }\end{array}$ & $\begin{array}{l}\text { Mean } \\
\text { inter- } \\
\text { occur- } \\
\text { rence } \\
\text { interval } \\
\text { (days) }\end{array}$ & $\begin{array}{l}\text { Total } \\
\text { no. of } \\
\text { events }\end{array}$ & $\begin{array}{l}\text { Total } \\
\text { no. of } \\
\text { days } \\
\text { avail- } \\
\text { able }\end{array}$ & $\begin{array}{c}\text { Sigma } \\
\mathrm{S}\end{array}$ & $\begin{array}{c}\text { Stan- } \\
\text { dard } \\
\text { normal } \\
\text { variate }\end{array}$ & $\begin{array}{l}\text { Non- } \\
\text { ex- } \\
\text { ceed- } \\
\text { ance } \\
\text { prob- } \\
\text { ability }\end{array}$ & $\begin{array}{c}p- \\
\text { value }\end{array}$ \\
\hline 577 & 299.48 & 62 & 18,568 & 622871 & 1.120 & .869 & .131 & 488.63 & 38 & 18,568 & 447594 & 2.869 & .998 & .002 \\
\hline 578 & 178.27 & 113 & 20,145 & 1155342 & .277 & .609 & .391 & 305.23 & 66 & 20,145 & 675573 & .228 & .590 & .410 \\
\hline 579 & 281.80 & 50 & 14,090 & 326818 & -.884 & .188 & .188 & 469.67 & 30 & 14,090 & 187776 & -1.058 & .145 & .145 \\
\hline 580 & -- & -- & -- & -- & -- & -- & -- & -- & -- & -- & -- & -- & -- & -- \\
\hline 581 & 139.29 & 17 & 2,368 & 20310 & .065 & .526 & .474 & 197.33 & 12 & 2,368 & 14664 & .193 & .576 & .424 \\
\hline 582 & 240.20 & 77 & 18,495 & 798680 & 1.849 & .968 & .032 & 420.34 & 44 & 18,495 & 458655 & 1.462 & .928 & .072 \\
\hline 583 & 1244.77 & 13 & 16,182 & 120699 & .921 & .821 & .179 & 1798.00 & 9 & 16,182 & 89194 & 1.169 & .879 & .121 \\
\hline 584 & -- & -- & -- & -- & -- & -- & -- & -- & -- & -- & -- & -- & -- & -- \\
\hline 585 & 345.53 & 38 & 13,130 & 252461 & .128 & .551 & .449 & 691.05 & 19 & 13,130 & 105878 & -1.141 & .127 & .127 \\
\hline 586 & -- & -- & -- & -- & -- & -- & -- & -- & -- & -- & -- & -- & -- & -- \\
\hline 587 & 202.83 & 18 & 3,651 & 37112 & .951 & .829 & .171 & 331.91 & 11 & 3,651 & 23203 & .893 & .814 & .186 \\
\hline 588 & 388.64 & 22 & 8,550 & 90207 & -.332 & .370 & .370 & 570.00 & 15 & 8,550 & 54728 & -.983 & .163 & .163 \\
\hline 589 & 168.03 & 39 & 6,553 & 139764 & 1.014 & .845 & .155 & 262.12 & 25 & 6,553 & 91326 & .995 & .840 & .160 \\
\hline 590 & 185.00 & 2 & 370 & 500 & .861 & .805 & .195 & 185.00 & 2 & 370 & 500 & .861 & .805 & .195 \\
\hline 591 & 192.26 & 19 & 3,653 & 36553 & .402 & .656 & .344 & 304.42 & 12 & 3,653 & 22270 & .096 & .538 & .462 \\
\hline 592 & 334.49 & 66 & 22,076 & 766386 & .732 & .768 & .232 & 668.97 & 33 & 22,076 & 371516 & .198 & .579 & .421 \\
\hline 593 & 189.20 & 82 & 15,514 & 674293 & .942 & .827 & .173 & 330.09 & 47 & 15,514 & 417494 & 1.723 & .958 & .042 \\
\hline 594 & 255.39 & 23 & 5,874 & 82807 & 1.876 & .970 & .030 & 419.57 & 14 & 5,874 & 46437 & .838 & .799 & .201 \\
\hline 595 & 341.70 & 27 & 9,226 & 101748 & -1.648 & .050 & .050 & 542.71 & 17 & 9,226 & 60180 & -1.661 & .048 & .048 \\
\hline 596 & 279.46 & 92 & 25,710 & 1317948 & 1.900 & .971 & .029 & 514.20 & 50 & 25,710 & 608068 & -.661 & .254 & .254 \\
\hline 597 & 132.71 & 155 & 20,570 & 1896218 & 4.086 & 1.000 & 0 & 218.83 & 94 & 20,570 & 1179264 & 3.691 & 1.000 & 0 \\
\hline 598 & 149.57 & 7 & 1,047 & 2458 & -1.509 & .066 & .066 & 174.50 & 6 & 1,047 & 2360 & -1.055 & .146 & .146 \\
\hline 599 & 804.50 & 22 & 17,699 & 222645 & 1.167 & .878 & .122 & 1966.56 & 9 & 17,699 & 104431 & 1.617 & .947 & .053 \\
\hline 600 & 217.02 & 90 & 19,532 & 854468 & -.458 & .324 & .324 & 305.19 & 64 & 19,532 & 589014 & -.798 & .212 & .212 \\
\hline 601 & 187.90 & 172 & 32,319 & 2671262 & -.884 & .188 & .188 & 310.76 & 104 & 32,319 & 1634736 & -.482 & .315 & .315 \\
\hline 602 & 183.08 & 84 & 15,379 & 661760 & .389 & .651 & .349 & 341.76 & 45 & 15,379 & 325050 & -.704 & .241 & .241 \\
\hline 603 & 177.89 & 35 & 6,226 & 133699 & 2.327 & .990 & .010 & 270.70 & 23 & 6,226 & 84623 & 1.511 & .935 & .065 \\
\hline 604 & 255.77 & 48 & 12,277 & 306501 & .483 & .685 & .315 & 438.46 & 28 & 12,277 & 199218 & 1.458 & .928 & .072 \\
\hline 605 & 477.69 & 13 & 6,210 & 48581 & 1.271 & .898 & .102 & 1035.00 & 6 & 6,210 & 18812 & .041 & .517 & .483 \\
\hline 606 & 376.14 & 14 & 5,266 & 44437 & 1.332 & .909 & .092 & 478.73 & 11 & 5,266 & 31987 & .600 & .726 & .274 \\
\hline 607 & 973.75 & 4 & 3,895 & 9116 & .590 & .722 & .278 & 1947.50 & 2 & 3,895 & 6513 & 1.646 & .950 & .050 \\
\hline 608 & 246.07 & 86 & 21,162 & 943321 & .589 & .722 & .278 & 450.26 & 47 & 21,162 & 527893 & .730 & .767 & .233 \\
\hline 609 & 2895.00 & 1 & 2,895 & 2440 & 1.188 & .882 & .118 & 2895.00 & 1 & 2,895 & 2440 & 1.188 & .882 & .118 \\
\hline 610 & 423.43 & 72 & 30,487 & 966796 & -1.751 & .040 & .040 & 781.72 & 39 & 30,487 & 522805 & -1.304 & .096 & .096 \\
\hline 611 & 913.22 & 9 & 8,219 & 44097 & .999 & .841 & .159 & 1643.80 & 5 & 8,219 & 28521 & 1.503 & .934 & .066 \\
\hline 612 & 361.00 & 10 & 3,610 & 23968 & 1.796 & .964 & .036 & 601.67 & 6 & 3,610 & 15395 & 1.788 & .963 & .037 \\
\hline 613 & 172.25 & 4 & 689 & 1003 & -.943 & .173 & .173 & 689.00 & 1 & 689 & 200 & -.727 & .234 & .234 \\
\hline 614 & 479.14 & 7 & 3,354 & 7764 & -1.552 & .060 & .060 & 559.00 & 6 & 3,354 & 5240 & -2.033 & .021 & .021 \\
\hline 615 & -- & -- & -- & -- & -- & -- & -- & -- & -- & -- & -- & -- & -- & -- \\
\hline 616 & 156.50 & 12 & 1,878 & 9777 & -.794 & .214 & .214 & 268.29 & 7 & 1,878 & 7060 & .340 & .633 & .367 \\
\hline 617 & 175.30 & 10 & 1,753 & 6434 & -1.457 & .073 & .073 & 350.60 & 5 & 1,753 & 4652 & .238 & .594 & .406 \\
\hline 618 & 191.13 & 108 & 20,642 & 1237155 & 1.978 & .976 & .024 & 322.53 & 64 & 20,642 & 773229 & 2.364 & .991 & .009 \\
\hline 619 & 265.82 & 107 & 28,443 & 1477962 & -.515 & .303 & .303 & 482.09 & 59 & 28,443 & 827281 & -.187 & .426 & .426 \\
\hline 620 & -- & -- & -- & -- & -- & -- & -- & -- & -- & -- & -- & -- & -- & -- \\
\hline 621 & 161.75 & 102 & 16,498 & 849254 & 0.163 & .565 & .435 & 294.61 & 56 & 16,498 & 420805 & -1.154 & .124 & .124 \\
\hline 622 & 206.77 & 175 & 36,185 & 3166373 & .001 & .501 & .499 & 361.85 & 100 & 36,185 & 1790740 & -.177 & .430 & .430 \\
\hline 623 & 460.25 & 16 & 7,364 & 54593 & -.508 & .306 & .306 & 669.46 & 11 & 7,364 & 41666 & .165 & .566 & .434 \\
\hline 624 & 149.54 & 13 & 1,944 & 11432 & -.595 & .276 & .276 & 243.00 & 8 & 1,944 & 8049 & .172 & .568 & .432 \\
\hline
\end{tabular}


Table 7. Summary of interoccurrence intervals for daily precipitation thresholds of 2.5 and 3.0 inches-Continued

\begin{tabular}{|c|c|c|c|c|c|c|c|c|c|c|c|c|c|c|}
\hline \multirow[b]{2}{*}{$\begin{array}{l}\text { Seq. } \\
\text { no. }\end{array}$} & \multicolumn{7}{|c|}{ Daily precipitation threshold of 2.5 inches and greater } & \multicolumn{7}{|c|}{ Daily precipitation threshold of 3.0 inches and greater } \\
\hline & $\begin{array}{l}\text { Mean } \\
\text { inter- } \\
\text { occur- } \\
\text { rence } \\
\text { interval } \\
\text { (days) }\end{array}$ & $\begin{array}{c}\text { Total } \\
\text { no. of } \\
\text { events }\end{array}$ & $\begin{array}{l}\text { Total } \\
\text { no. of } \\
\text { days } \\
\text { avail- } \\
\text { able }\end{array}$ & $\begin{array}{l}\text { Sigma } \\
\mathrm{S}\end{array}$ & $\begin{array}{c}\text { Stan- } \\
\text { dard } \\
\text { normal } \\
\text { variate }\end{array}$ & $\begin{array}{l}\text { Non- } \\
\text { ex- } \\
\text { ceed- } \\
\text { ance } \\
\text { prob- } \\
\text { ability }\end{array}$ & $\begin{array}{c}\text { p- } \\
\text { value }\end{array}$ & $\begin{array}{l}\text { Mean } \\
\text { inter- } \\
\text { occur- } \\
\text { rence } \\
\text { interval } \\
\text { (days) }\end{array}$ & $\begin{array}{c}\text { Total } \\
\text { no. of } \\
\text { events }\end{array}$ & $\begin{array}{l}\text { Total } \\
\text { no. of } \\
\text { days } \\
\text { avail- } \\
\text { able }\end{array}$ & $\begin{array}{l}\text { Sigma } \\
\mathbf{S}\end{array}$ & $\begin{array}{l}\text { Stan- } \\
\text { dard } \\
\text { normal } \\
\text { variate }\end{array}$ & $\begin{array}{l}\text { Non- } \\
\text { ex- } \\
\text { ceed- } \\
\text { ance } \\
\text { prob- } \\
\text { ability }\end{array}$ & $\begin{array}{c}p- \\
\text { value }\end{array}$ \\
\hline 625 & 237.14 & 42 & 9,960 & 227368 & .977 & .836 & .164 & 355.71 & 28 & 9,960 & 148553 & .599 & .725 & .275 \\
\hline 626 & 251.79 & 43 & 10,827 & 225454 & -.358 & .360 & .360 & 386.68 & 28 & 10,827 & 148865 & -.164 & .435 & .435 \\
\hline 627 & 5910.50 & 2 & 11,821 & 18539 & 1.392 & .918 & .082 & -- & -- & -- & -- & -- & -- & -- \\
\hline 628 & 2501.50 & 2 & 5,003 & 1908 & -1.515 & .065 & .065 & 2501.50 & 2 & 5,003 & 1908 & -1.515 & .065 & .065 \\
\hline 629 & 300.59 & 88 & 26,452 & 1139410 & -.342 & .366 & .366 & 448.34 & 59 & 26,452 & 824766 & .758 & .776 & .224 \\
\hline 630 & 242.02 & 41 & 9,923 & 177168 & -1.431 & .076 & .076 & 413.46 & 24 & 9,923 & 101418 & -1.258 & .104 & .104 \\
\hline 631 & 231.79 & 47 & 10,894 & 272181 & .750 & .773 & .227 & 330.12 & 33 & 10,894 & 171132 & -.477 & .317 & .317 \\
\hline 632 & 418.95 & 19 & 7,960 & 90596 & 1.495 & .933 & .067 & 884.44 & 9 & 7,960 & 50410 & 2.117 & .983 & .017 \\
\hline 633 & 189.11 & 9 & 1,702 & 6818 & -.571 & .284 & .284 & 283.67 & 6 & 1,702 & 5037 & -.057 & .477 & .477 \\
\hline 634 & -- & -- & -- & -- & -- & -- & -- & -- & -- & -- & -- & -- & -- & -- \\
\hline 635 & 250.86 & 107 & 26,842 & 1490221 & .676 & .750 & .250 & 400.63 & 67 & 26,842 & 959184 & .946 & .828 & .172 \\
\hline 636 & 112.12 & 185 & 20,742 & 1905762 & -.158 & .437 & .437 & 209.52 & 99 & 20,742 & 1045131 & .309 & .621 & .379 \\
\hline 637 & 542.44 & 45 & 24,410 & 629690 & 1.702 & .956 & .044 & 1109.55 & 22 & 24,410 & 342450 & 2.237 & .987 & .013 \\
\hline 638 & 585.00 & 19 & 11,115 & 113017 & .531 & .702 & .298 & 1010.46 & 11 & 11,115 & 68967 & .736 & .769 & .231 \\
\hline 639 & 326.50 & 16 & 5,224 & 50313 & 1.413 & .921 & .079 & 1044.80 & 5 & 5,224 & 16545 & 1.034 & .849 & .151 \\
\hline 640 & 112.79 & 52 & 5,865 & 161865 & .768 & .779 & .221 & 183.28 & 32 & 5,865 & 105514 & 1.219 & .889 & .111 \\
\hline 641 & 1196.00 & 1 & 1,196 & 1059 & 1.335 & .909 & .091 & 1196.00 & 1 & 1,196 & 1059 & 1.335 & .909 & .091 \\
\hline 642 & -- & -- & -- & -- & -- & -- & -- & -- & -- & -- & -- & -- & -- & -- \\
\hline 643 & 206.62 & 159 & 32,853 & 2580330 & -.263 & .396 & .396 & 309.93 & 106 & 32,853 & 1653434 & -.899 & .184 & .184 \\
\hline 644 & 618.25 & 4 & 2,473 & 7246 & 1.611 & .946 & .054 & 824.33 & 3 & 2,473 & 5774 & 1.670 & .952 & .048 \\
\hline 645 & 365.89 & 75 & 27,442 & 985565 & -.634 & .263 & .263 & 560.04 & 49 & 27,442 & 661816 & -.190 & .425 & .425 \\
\hline 646 & 10357.50 & 2 & 20,715 & 21331 & .073 & .529 & .471 & 20715.00 & 1 & 20,715 & 5666 & -.785 & .216 & .216 \\
\hline 647 & 181.40 & 5 & 907 & 2742 & .811 & .791 & .209 & 907.00 & 1 & 907 & 782 & 1.255 & .895 & .105 \\
\hline 648 & 273.29 & 7 & 1,913 & 6922 & .155 & .562 & .438 & 478.25 & 4 & 1,913 & 3471 & -.321 & .374 & .374 \\
\hline 649 & 4085.00 & 2 & 8,170 & 5783 & -.716 & .237 & .237 & 8170.00 & 1 & 8,170 & 2891 & -.506 & .306 & .306 \\
\hline 650 & 436.09 & 33 & 14,391 & 219972 & -.732 & .232 & .232 & 757.42 & 19 & 14,391 & 140654 & .218 & .586 & .414 \\
\hline 651 & 700.50 & 4 & 2,802 & 8291 & 1.661 & .952 & .048 & 1401.00 & 2 & 2,802 & 4525 & 1.506 & .934 & .066 \\
\hline 652 & 798.00 & 1 & 798 & 490 & .395 & .654 & .346 & -- & -- & -- & -- & -- & -- & -- \\
\hline 653 & 361.00 & 3 & 1,083 & 1254 & -.684 & .247 & .247 & 1083.00 & 1 & 1,083 & 233 & -.987 & .162 & .162 \\
\hline 654 & 796.23 & 13 & 10,351 & 87984 & 1.922 & .973 & .027 & 1725.17 & 6 & 10,351 & 43649 & 1.721 & .957 & .043 \\
\hline 655 & 476.83 & 6 & 2,861 & 4779 & -1.880 & .030 & .030 & 572.20 & 5 & 2,861 & 3932 & -1.744 & .041 & .041 \\
\hline 656 & 128.19 & 37 & 4,743 & 75922 & -1.420 & .078 & .078 & 249.63 & 19 & 4,743 & 41400 & -.613 & .270 & .270 \\
\hline 657 & 552.14 & 21 & 11,595 & 115890 & -.382 & .351 & .351 & 966.25 & 12 & 11,595 & 62197 & -.636 & .262 & .262 \\
\hline 658 & 337.00 & 17 & 5,729 & 43746 & -.726 & .234 & .234 & 572.90 & 10 & 5,729 & 27811 & -.160 & .437 & .437 \\
\hline 659 & 277.60 & 10 & 2,776 & 12238 & -.648 & .259 & .259 & 462.67 & 6 & 2,776 & 5111 & -1.639 & .051 & .051 \\
\hline 660 & 145.61 & 33 & 4,805 & 86145 & .861 & .805 & .195 & 300.31 & 16 & 4,805 & 41411 & .536 & .704 & .296 \\
\hline 661 & 123.13 & 23 & 2,832 & 30195 & -.605 & .273 & .273 & 202.29 & 14 & 2,832 & 23059 & 1.058 & .855 & .145 \\
\hline 662 & 305.00 & 18 & 5,490 & 45952 & -.514 & .304 & .304 & 549.00 & 10 & 5,490 & 24785 & -.532 & .297 & .297 \\
\hline 663 & 1087.40 & 30 & 32,622 & 528270 & .755 & .775 & .225 & 1812.33 & 18 & 32,622 & 294658 & .027 & .511 & .489 \\
\hline 664 & 407.52 & 91 & 37,084 & 1620860 & -.651 & .258 & .258 & 741.68 & 50 & 37,084 & 899510 & -.365 & .358 & .358 \\
\hline 665 & 941.30 & 20 & 18,826 & 188120 & -.006 & .498 & .498 & 1448.15 & 13 & 18,826 & 106314 & -.819 & .206 & .206 \\
\hline 666 & 433.00 & 3 & 1,299 & 2362 & .637 & .738 & .262 & 649.50 & 2 & 1,299 & 2131 & 1.569 & .942 & .058 \\
\hline 667 & 402.62 & 34 & 13,689 & 173564 & -2.567 & .005 & .005 & 622.23 & 22 & 13,689 & 99177 & -2.773 & .003 & .003 \\
\hline 668 & 517.40 & 15 & 7,761 & 46458 & -1.354 & .088 & .088 & 970.13 & 8 & 7,761 & 28748 & -.362 & .359 & .359 \\
\hline 669 & 298.74 & 19 & 5,676 & 59187 & 0.737 & .769 & .231 & 516.00 & 11 & 5,676 & 29607 & -0.296 & .383 & .383 \\
\hline 670 & 424.25 & 20 & 8,485 & 74546 & -.941 & .174 & .174 & 606.07 & 14 & 8,485 & 57706 & -.184 & .427 & .427 \\
\hline 671 & 226.47 & 83 & 18,797 & 894524 & 2.315 & .990 & .010 & 427.21 & 44 & 18,797 & 459953 & 1.290 & .901 & .099 \\
\hline 672 & 429.87 & 45 & 19,344 & 447570 & .329 & .629 & .371 & 690.86 & 28 & 19,344 & 290008 & .650 & .742 & .258 \\
\hline
\end{tabular}


Table 7. Summary of interoccurrence intervals for daily precipitation thresholds of 2.5 and 3.0 inches—Continued

\begin{tabular}{|c|c|c|c|c|c|c|c|c|c|c|c|c|c|c|}
\hline \multirow[b]{2}{*}{$\begin{array}{l}\text { Seq. } \\
\text { no. }\end{array}$} & \multicolumn{7}{|c|}{ Daily precipitation threshold of 2.5 inches and greater } & \multicolumn{7}{|c|}{ Daily precipitation threshold of 3.0 inches and greater } \\
\hline & $\begin{array}{l}\text { Mean } \\
\text { inter- } \\
\text { occur- } \\
\text { rence } \\
\text { interval } \\
\text { (days) }\end{array}$ & $\begin{array}{c}\text { Total } \\
\text { no. of } \\
\text { events }\end{array}$ & $\begin{array}{c}\text { Total } \\
\text { no. of } \\
\text { days } \\
\text { avail- } \\
\text { able }\end{array}$ & $\begin{array}{l}\text { Sigma } \\
\text { S }\end{array}$ & $\begin{array}{c}\text { Stan- } \\
\text { dard } \\
\text { normal } \\
\text { variate }\end{array}$ & $\begin{array}{l}\text { Non- } \\
\text { ex- } \\
\text { ceed- } \\
\text { ance } \\
\text { prob- } \\
\text { ability }\end{array}$ & $\begin{array}{c}\mathrm{p}- \\
\text { value }\end{array}$ & $\begin{array}{c}\text { Mean } \\
\text { inter- } \\
\text { occur- } \\
\text { rence } \\
\text { interval } \\
\text { (days) }\end{array}$ & $\begin{array}{c}\text { Total } \\
\text { no. of } \\
\text { events }\end{array}$ & $\begin{array}{l}\text { Total } \\
\text { no. of } \\
\text { days } \\
\text { avail- } \\
\text { able }\end{array}$ & $\begin{array}{c}\text { Sigma } \\
\mathrm{S}\end{array}$ & $\begin{array}{c}\text { Stan- } \\
\text { dard } \\
\text { normal } \\
\text { variate }\end{array}$ & $\begin{array}{l}\text { Non- } \\
\text { ex- } \\
\text { ceed- } \\
\text { ance } \\
\text { prob- } \\
\text { ability }\end{array}$ & $\begin{array}{c}\mathrm{p}- \\
\text { value }\end{array}$ \\
\hline 673 & 609.00 & 9 & 5,481 & 27999 & .703 & .759 & .241 & 609.00 & 9 & 5,481 & 27999 & .703 & .759 & .241 \\
\hline 674 & 198.67 & 9 & 1,788 & 7302 & -.481 & .315 & .315 & 223.50 & 8 & 1,788 & 6943 & -.143 & .443 & .443 \\
\hline 675 & 240.82 & 11 & 2,649 & 11313 & -1.284 & .100 & .100 & 441.50 & 6 & 2,649 & 7546 & -.214 & .415 & .415 \\
\hline 676 & 277.00 & 12 & 3,324 & 15045 & -1.474 & .070 & .070 & 554.00 & 6 & 3,324 & 8369 & -.682 & .248 & .248 \\
\hline 677 & 856.04 & 24 & 20,545 & 254036 & .258 & .602 & .398 & 1712.08 & 12 & 20,545 & 101314 & -1.069 & .143 & .143 \\
\hline 678 & -- & -- & -- & -- & -- & -- & -- & -- & -- & -- & -- & -- & -- & -- \\
\hline 679 & 838.15 & 27 & 22,630 & 305087 & -.012 & .495 & .495 & 1616.43 & 14 & 22,630 & 150465 & -.325 & .373 & .373 \\
\hline 680 & 436.00 & 10 & 4,360 & 23664 & .468 & .680 & .320 & 726.67 & 6 & 4,360 & 15053 & .640 & .739 & .261 \\
\hline 681 & 419.00 & 2 & 838 & 472 & -1.070 & .142 & .142 & 838.00 & 1 & 838 & 439 & .083 & .533 & .467 \\
\hline 682 & 339.47 & 15 & 5,092 & 39076 & .156 & .562 & .438 & 848.67 & 6 & 5,092 & 19799 & 1.256 & .895 & .105 \\
\hline 683 & 221.80 & 55 & 12,199 & 333214 & -.087 & .465 & .465 & 381.22 & 32 & 12,199 & 176277 & -.949 & .171 & .171 \\
\hline 684 & 228.27 & 68 & 15,522 & 488562 & -1.061 & .145 & .145 & 419.51 & 37 & 15,522 & 298069 & .400 & .656 & .344 \\
\hline 685 & 108.03 & 326 & 35,218 & 6194764 & 2.475 & .993 & .007 & 175.21 & 201 & 35,218 & 3808734 & 1.869 & .969 & .031 \\
\hline 686 & 307.74 & 19 & 5,847 & 70153 & 1.985 & .976 & .024 & 487.25 & 12 & 5,847 & 41304 & 1.064 & .856 & .144 \\
\hline 687 & 774.00 & 2 & 1,548 & 1411 & -.217 & .414 & .414 & 1548.00 & 1 & 1,548 & 1285 & 1.144 & .874 & .126 \\
\hline 688 & 160.25 & 28 & 4,487 & 78934 & 2.351 & .991 & .009 & 213.67 & 21 & 4,487 & 61691 & 2.456 & .993 & .007 \\
\hline 689 & 196.63 & 30 & 5,899 & 93020 & .486 & .687 & .313 & 393.27 & 15 & 5,899 & 45951 & .259 & .602 & .398 \\
\hline 690 & 154.65 & 81 & 12,527 & 497109 & -.315 & .377 & .377 & 313.18 & 40 & 12,527 & 221819 & -1.256 & .105 & .105 \\
\hline 691 & 127.22 & 144 & 18,319 & 1521872 & 3.197 & .999 & .001 & 218.08 & 84 & 18,319 & 893719 & 2.565 & .995 & .005 \\
\hline 692 & 284.73 & 66 & 18,792 & 708766 & 2.011 & .978 & .022 & 447.43 & 42 & 18,792 & 424791 & .858 & .804 & .196 \\
\hline 693 & 1197.69 & 16 & 19,163 & 144315 & -.406 & .342 & .342 & 1742.09 & 11 & 19,163 & 94551 & -.591 & .277 & .277 \\
\hline 694 & 280.81 & 26 & 7,301 & 84137 & -1.003 & .158 & .158 & 456.31 & 16 & 7,301 & 55460 & -.350 & .363 & .363 \\
\hline 695 & 1127.58 & 12 & 13,531 & 98975 & 1.315 & .906 & .094 & 2706.20 & 5 & 13,531 & 29142 & -.537 & .296 & .296 \\
\hline 696 & 854.80 & 15 & 12,822 & 62805 & -2.327 & .010 & .010 & 1424.67 & 9 & 12,822 & 41702 & -1.441 & .075 & .075 \\
\hline 697 & 155.16 & 152 & 23,585 & 1790229 & -.027 & .489 & .489 & 235.85 & 100 & 23,585 & 1163826 & -.227 & .410 & .410 \\
\hline 698 & 469.08 & 80 & 37,526 & 1461466 & -.408 & .342 & .342 & 815.78 & 46 & 37,526 & 854495 & -.117 & .453 & .453 \\
\hline 699 & 395.75 & 4 & 1,583 & 2082 & -1.186 & .118 & .118 & 527.67 & 3 & 1,583 & 1107 & -1.601 & .055 & .055 \\
\hline 700 & 216.07 & 91 & 19,662 & 929879 & .651 & .742 & .258 & 370.98 & 53 & 19,662 & 585556 & 1.561 & .941 & .059 \\
\hline 701 & 199.22 & 9 & 1,793 & 7595 & -.305 & .380 & .380 & 224.13 & 8 & 1,793 & 7008 & -.112 & .455 & .455 \\
\hline 702 & 404.00 & 3 & 1,212 & 1198 & -1.023 & .153 & .153 & 404.00 & 3 & 1,212 & 1198 & -1.023 & .153 & .153 \\
\hline 703 & 224.86 & 21 & 4,722 & 60619 & 1.767 & .961 & .039 & 363.23 & 13 & 4,722 & 35745 & 1.028 & .848 & .152 \\
\hline 704 & 424.00 & 1 & 424 & 24 & -1.536 & .062 & .062 & -- & -- & -- & -- & -- & -- & -- \\
\hline 705 & 217.68 & 59 & 12,843 & 402563 & .832 & .797 & .203 & 428.10 & 30 & 12,843 & 204200 & .569 & .715 & .285 \\
\hline 706 & 213.57 & 168 & 35,879 & 3251254 & 1.769 & .962 & .038 & 348.34 & 103 & 35,879 & 2084175 & 2.249 & .988 & .012 \\
\hline 707 & 157.76 & 59 & 9,308 & 293177 & .901 & .816 & .184 & 310.27 & 30 & 9,308 & 143932 & .293 & .615 & .385 \\
\hline 708 & 850.68 & 19 & 16,163 & 174059 & 1.009 & .843 & .157 & 2309.00 & 7 & 16,163 & 72172 & 1.264 & .897 & .103 \\
\hline 709 & -- & -- & -- & -- & -- & -- & -- & -- & -- & -- & -- & -- & -- & -- \\
\hline 710 & 599.00 & 2 & 1,198 & 1033 & -.337 & .368 & .368 & 1198.00 & 1 & 1,198 & 323 & -.798 & .212 & .212 \\
\hline 711 & 570.00 & 26 & 14,820 & 224391 & 1.455 & .927 & .073 & 1058.57 & 14 & 14,820 & 148464 & 2.794 & .997 & .003 \\
\hline 712 & 447.00 & 3 & 1,341 & 1587 & -.633 & .263 & .263 & 1341.00 & 1 & 1,341 & 714 & .112 & .545 & .455 \\
\hline 713 & 260.33 & 39 & 10,153 & 212531 & .795 & .787 & .213 & 406.12 & 25 & 10,153 & 131913 & .341 & .634 & .366 \\
\hline 714 & -- & -- & -- & -- & -- & -- & -- & -- & -- & -- & -- & -- & -- & -- \\
\hline 715 & 730.00 & 2 & 1,460 & 1412 & -.081 & .468 & .468 & 1460.00 & 1 & 1,460 & 884 & .365 & .643 & .357 \\
\hline 716 & 2105.33 & 3 & 6,316 & 10600 & .357 & .639 & .361 & 3158.00 & 2 & 6,316 & 5985 & -.128 & .449 & .449 \\
\hline 717 & 872.21 & 38 & 33,144 & 591142 & -0.654 & .256 & .256 & 1841.33 & 18 & 33,144 & 296013 & -0.056 & .478 & .478 \\
\hline 718 & 172.13 & 40 & 6,885 & 142808 & .406 & .658 & .342 & 299.35 & 23 & 6,885 & 92168 & 1.363 & .914 & .086 \\
\hline 719 & 114.84 & 38 & 4,364 & 79672 & -.418 & .338 & .338 & 189.74 & 23 & 4,364 & 48332 & -.307 & .379 & .379 \\
\hline 720 & 198.98 & 167 & 33,230 & 2806982 & .260 & .603 & .397 & 307.69 & 108 & 33,230 & 1949479 & 1.555 & .940 & .060 \\
\hline
\end{tabular}


Table 7. Summary of interoccurrence intervals for daily precipitation thresholds of 2.5 and 3.0 inches-Continued

\begin{tabular}{|c|c|c|c|c|c|c|c|c|c|c|c|c|c|c|}
\hline \multirow[b]{2}{*}{$\begin{array}{l}\text { Seq. } \\
\text { no. }\end{array}$} & \multicolumn{7}{|c|}{ Daily precipitation threshold of 2.5 inches and greater } & \multicolumn{7}{|c|}{ Daily precipitation threshold of 3.0 inches and greater } \\
\hline & $\begin{array}{l}\text { Mean } \\
\text { inter- } \\
\text { occur- } \\
\text { rence } \\
\text { interval } \\
\text { (days) }\end{array}$ & $\begin{array}{l}\text { Total } \\
\text { no. of } \\
\text { events }\end{array}$ & $\begin{array}{l}\text { Total } \\
\text { no. of } \\
\text { days } \\
\text { avail- } \\
\text { able }\end{array}$ & $\begin{array}{l}\text { Sigma } \\
\mathrm{S}\end{array}$ & $\begin{array}{c}\text { Stan- } \\
\text { dard } \\
\text { normal } \\
\text { variate }\end{array}$ & $\begin{array}{l}\text { Non- } \\
\text { ex- } \\
\text { ceed- } \\
\text { ance } \\
\text { prob- } \\
\text { ability }\end{array}$ & $\begin{array}{c}\mathrm{p}- \\
\text { value }\end{array}$ & $\begin{array}{l}\text { Mean } \\
\text { inter- } \\
\text { occur- } \\
\text { rence } \\
\text { interval } \\
\text { (days) }\end{array}$ & $\begin{array}{c}\text { Total } \\
\text { no. of } \\
\text { events }\end{array}$ & $\begin{array}{l}\text { Total } \\
\text { no. of } \\
\text { days } \\
\text { avail- } \\
\text { able }\end{array}$ & $\begin{array}{l}\text { Sigma } \\
\mathrm{S}\end{array}$ & $\begin{array}{c}\text { Stan- } \\
\text { dard } \\
\text { normal } \\
\text { variate }\end{array}$ & $\begin{array}{l}\text { Non- } \\
\text { ex- } \\
\text { ceed- } \\
\text { ance } \\
\text { prob- } \\
\text { ability }\end{array}$ & $\begin{array}{c}p- \\
\text { value }\end{array}$ \\
\hline 721 & 223.65 & 163 & 36,455 & 3059467 & 658 & .745 & .255 & 364.55 & 100 & 36,455 & 1958814 & 1.293 & .902 & .098 \\
\hline 722 & 322.80 & 5 & 1,614 & 2731 & -1.252 & .105 & .105 & 322.80 & 5 & 1,614 & 2731 & -1.252 & .105 & .105 \\
\hline 723 & 102.36 & 22 & 2,252 & 24115 & -.216 & .415 & .415 & 281.50 & 8 & 2,252 & 8589 & -.228 & .410 & .410 \\
\hline 724 & 277.30 & 33 & 9,151 & 160242 & .610 & .729 & .271 & 435.76 & 21 & 9,151 & 104742 & .715 & .763 & .237 \\
\hline 725 & 339.33 & 27 & 9,162 & 129868 & .450 & .674 & .326 & 509.00 & 18 & 9,162 & 93070 & .946 & .828 & .172 \\
\hline 726 & 388.33 & 3 & 1,165 & 1120 & -1.077 & .141 & .141 & -- & -- & -- & -- & -- & -- & -- \\
\hline 727 & 173.16 & 146 & 25,281 & 1746115 & -1.127 & .130 & .130 & 297.42 & 85 & 25,281 & 1043129 & -.465 & .321 & .321 \\
\hline 728 & 141.16 & 73 & 10,305 & 429267 & 2.091 & .982 & .018 & 229.00 & 45 & 10,305 & 253264 & 1.073 & .858 & .142 \\
\hline 729 & 208.16 & 31 & 6,453 & 119516 & 1.880 & .970 & .030 & 322.65 & 20 & 6,453 & 75974 & 1.374 & .915 & .085 \\
\hline 730 & -- & -- & -- & -- & -- & -- & -- & -- & -- & -- & -- & -- & -- & -- \\
\hline 731 & 260.50 & 24 & 6,252 & 71974 & -.345 & .365 & .365 & 521.00 & 12 & 6,252 & 38138 & .100 & .540 & .460 \\
\hline 732 & 487.07 & 14 & 6,819 & 61100 & 1.815 & .965 & .035 & 757.67 & 9 & 6,819 & 42702 & 2.035 & .979 & .021 \\
\hline 733 & -- & -- & -- & -- & -- & -- & -- & -- & -- & -- & -- & -- & -- & -- \\
\hline 734 & 2305.70 & 10 & 23,057 & 91896 & -1.111 & .133 & .133 & 11528.50 & 2 & 23,057 & 14440 & -.915 & .180 & .180 \\
\hline 735 & -- & -- & -- & -- & -- & -- & -- & -- & -- & -- & -- & -- & -- & -- \\
\hline 736 & 368.00 & 22 & 8,096 & 122399 & 3.042 & .999 & .001 & 539.73 & 15 & 8,096 & 74929 & 1.570 & .942 & .058 \\
\hline 737 & 2647.00 & 1 & 2,647 & 2018 & .909 & .818 & .182 & 2647.00 & 1 & 2,647 & 2018 & .909 & .818 & .182 \\
\hline 738 & 3583.25 & 4 & 14,333 & 42775 & 1.705 & .956 & .044 & -- & -- & -- & -- & -- & -- & -- \\
\hline 739 & -- & -- & -- & -- & -- & -- & -- & -- & -- & -- & -- & -- & -- & -- \\
\hline 740 & -- & -- & -- & -- & -- & -- & -- & -- & -- & -- & -- & -- & -- & -- \\
\hline 741 & -- & -- & -- & -- & -- & -- & -- & -- & -- & -- & -- & -- & -- & -- \\
\hline 742 & 228.16 & 116 & 26,466 & 1490994 & -.535 & .296 & .296 & 490.11 & 54 & 26,466 & 702465 & -.216 & .415 & .415 \\
\hline 743 & 191.93 & 174 & 33,395 & 2979786 & .585 & .721 & .279 & 407.26 & 82 & 33,395 & 1407923 & .444 & .671 & .329 \\
\hline 744 & 333.32 & 19 & 6,333 & 49062 & -1.393 & .082 & .082 & 575.73 & 11 & 6,333 & 30571 & -.703 & .241 & .241 \\
\hline 745 & 358.16 & 25 & 8,954 & 120010 & .626 & .734 & .266 & 559.63 & 16 & 8,954 & 75049 & .331 & .630 & .371 \\
\hline 746 & 342.53 & 60 & 20,552 & 672589 & 1.219 & .889 & .111 & 622.79 & 33 & 20,552 & 385072 & 1.349 & .911 & .089 \\
\hline 747 & 559.11 & 35 & 19,569 & 379542 & 1.110 & .866 & .134 & 978.45 & 20 & 19,569 & 247345 & 2.045 & .980 & .020 \\
\hline 748 & 129.20 & 255 & 32,946 & 4049576 & -.995 & .160 & .160 & 184.06 & 179 & 32,946 & 2808242 & -1.104 & .135 & .135 \\
\hline 749 & 208.82 & 65 & 13,573 & 373074 & -2.154 & .016 & .016 & 331.05 & 41 & 13,573 & 251342 & -1.072 & .142 & .142 \\
\hline 750 & 137.62 & 135 & 18,579 & 1481557 & 3.650 & 1.000 & 0 & 213.55 & 87 & 18,579 & 983878 & 3.512 & 1.000 & 0 \\
\hline 751 & 152.56 & 50 & 7,628 & 183065 & -.490 & .312 & .312 & 246.07 & 31 & 7,628 & 102111 & -1.315 & .094 & .094 \\
\hline 752 & -- & -- & -- & -- & -- & -- & -- & -- & -- & -- & -- & -- & -- & -- \\
\hline 753 & 508.25 & 4 & 2,033 & 4232 & .141 & .556 & .444 & 2033.00 & 1 & 2,033 & 1004 & -.021 & .491 & .491 \\
\hline 754 & 214.58 & 12 & 2,575 & 14516 & -.363 & .358 & .358 & 429.17 & 6 & 2,575 & 5520 & -1.211 & .113 & .113 \\
\hline 755 & 358.32 & 60 & 21,499 & 631296 & -.284 & .388 & .388 & 551.26 & 39 & 21,499 & 437608 & .474 & .682 & .318 \\
\hline 756 & -- & -- & -- & -- & -- & -- & -- & -- & -- & -- & -- & -- & -- & -- \\
\hline 757 & 649.33 & 9 & 5,844 & 30902 & .910 & .818 & .182 & 1461.00 & 4 & 5,844 & 13935 & .666 & .747 & .253 \\
\hline 758 & 204.71 & 158 & 32,344 & 2660822 & .900 & .816 & .184 & 363.42 & 89 & 32,344 & 1561756 & 1.390 & .918 & .082 \\
\hline 759 & 427.56 & 36 & 15,392 & 317956 & 1.534 & .937 & .063 & 810.11 & 19 & 15,392 & 185183 & 2.012 & .978 & .022 \\
\hline 760 & 374.47 & 57 & 21,345 & 635682 & .588 & .722 & .278 & 576.89 & 37 & 21,345 & 416676 & .582 & .720 & .281 \\
\hline 761 & 322.17 & 42 & 13,531 & 278970 & -.205 & .419 & .419 & 501.15 & 27 & 13,531 & 211433 & 1.417 & .922 & .078 \\
\hline 762 & 1487.71 & 17 & 25,291 & 214916 & -.002 & .499 & .499 & 2529.10 & 10 & 25,291 & 150373 & 1.036 & .850 & .150 \\
\hline 763 & 236.80 & 5 & 1,184 & 3473 & .671 & .749 & .251 & 394.67 & 3 & 1,184 & 2359 & .985 & .838 & .162 \\
\hline 764 & 270.93 & 121 & 32,783 & 1832118 & -1.453 & .073 & .073 & 520.37 & 63 & 32,783 & 865933 & -2.220 & .013 & .013 \\
\hline 765 & 174.21 & 70 & 12,195 & 488083 & 2.080 & .981 & .019 & 290.36 & 42 & 12,195 & 301508 & 1.991 & .977 & .023 \\
\hline 766 & 148.56 & 18 & 2,674 & 19355 & -1.439 & .075 & .075 & 191.00 & 14 & 2,674 & 13976 & -1.642 & .050 & .050 \\
\hline 767 & 721.78 & 46 & 33,202 & 929109 & 2.545 & .995 & .005 & 1844.56 & 18 & 33,202 & 392417 & 2.302 & .989 & .011 \\
\hline 768 & 418.83 & 81 & 33,925 & 1366514 & -.085 & .466 & .466 & 721.81 & 47 & 33,925 & 821551 & .362 & .641 & .359 \\
\hline
\end{tabular}


Table 7. Summary of interoccurrence intervals for daily precipitation thresholds of 2.5 and 3.0 inches—Continued

\begin{tabular}{|c|c|c|c|c|c|c|c|c|c|c|c|c|c|c|}
\hline \multirow[b]{2}{*}{$\begin{array}{l}\text { Seq. } \\
\text { no. }\end{array}$} & \multicolumn{7}{|c|}{ Daily precipitation threshold of 2.5 inches and greater } & \multicolumn{7}{|c|}{ Daily precipitation threshold of 3.0 inches and greater } \\
\hline & $\begin{array}{l}\text { Mean } \\
\text { inter- } \\
\text { occur- } \\
\text { rence } \\
\text { interval } \\
\text { (days) }\end{array}$ & $\begin{array}{l}\text { Total } \\
\text { no. of } \\
\text { events }\end{array}$ & $\begin{array}{l}\text { Total } \\
\text { no. of } \\
\text { days } \\
\text { avail- } \\
\text { able }\end{array}$ & $\begin{array}{l}\text { Sigma } \\
\mathrm{S}\end{array}$ & $\begin{array}{c}\text { Stan- } \\
\text { dard } \\
\text { normal } \\
\text { variate }\end{array}$ & $\begin{array}{l}\text { Non- } \\
\text { ex- } \\
\text { ceed- } \\
\text { ance } \\
\text { prob- } \\
\text { ability }\end{array}$ & $\begin{array}{c}\mathrm{p}- \\
\text { value }\end{array}$ & $\begin{array}{c}\text { Mean } \\
\text { inter- } \\
\text { occur- } \\
\text { rence } \\
\text { interval } \\
\text { (days) }\end{array}$ & $\begin{array}{l}\text { Total } \\
\text { no. of } \\
\text { events }\end{array}$ & $\begin{array}{l}\text { Total } \\
\text { no. of } \\
\text { days } \\
\text { avail- } \\
\text { able }\end{array}$ & $\begin{array}{c}\text { Sigma } \\
\mathrm{S}\end{array}$ & $\begin{array}{c}\text { Stan- } \\
\text { dard } \\
\text { normal } \\
\text { variate }\end{array}$ & $\begin{array}{l}\text { Non- } \\
\text { ex- } \\
\text { ceed- } \\
\text { ance } \\
\text { prob- } \\
\text { ability }\end{array}$ & $\begin{array}{c}\text { p- } \\
\text { value }\end{array}$ \\
\hline 769 & 529.50 & 4 & 2,118 & 6258 & 1.654 & .951 & .049 & 1059.00 & 2 & 2,118 & 2958 & .972 & .834 & .166 \\
\hline 770 & 1356.00 & 6 & 8,136 & 24110 & -.052 & .479 & .479 & 2034.00 & 4 & 8,136 & 12458 & -.812 & .208 & .208 \\
\hline 771 & 1238.00 & 3 & 3,714 & 7276 & .918 & .821 & .179 & 1857.00 & 2 & 3,714 & 3750 & .024 & .509 & .491 \\
\hline 772 & 91.50 & 2 & 183 & 247 & .857 & .804 & .196 & 183.00 & 1 & 183 & 85 & -.123 & .451 & .451 \\
\hline 773 & 129.25 & 4 & 517 & 852 & -.610 & .271 & .271 & 129.25 & 4 & 517 & 852 & -.610 & .271 & .271 \\
\hline 774 & 335.54 & 92 & 30,870 & 1524880 & 1.227 & .890 & .110 & 551.25 & 56 & 30,870 & 938028 & 1.105 & .865 & .135 \\
\hline 775 & -- & -- & -- & -- & -- & -- & -- & -- & -- & -- & -- & -- & -- & -- \\
\hline 776 & 241.90 & 29 & 7,015 & 111218 & .871 & .808 & .192 & 350.75 & 20 & 7,015 & 78379 & .909 & .818 & .182 \\
\hline 777 & 253.78 & 27 & 6,852 & 72038 & -1.991 & .023 & .023 & 428.25 & 16 & 6,852 & 52407 & -.305 & .380 & .380 \\
\hline 778 & 1218.63 & 8 & 9,749 & 31065 & -.996 & .160 & .160 & 1949.80 & 5 & 9,749 & 17318 & -1.121 & .131 & .131 \\
\hline 779 & 640.28 & 25 & 16,007 & 239213 & 1.693 & .955 & .045 & 1333.92 & 12 & 16,007 & 100539 & .281 & .611 & .389 \\
\hline 780 & 215.99 & 160 & 34,558 & 2802517 & .300 & .618 & .382 & 367.64 & 94 & 34,558 & 1691762 & .698 & .757 & .243 \\
\hline 781 & 752.48 & 46 & 34,614 & 778187 & -.265 & .396 & .396 & 1282.00 & 27 & 34,614 & 433811 & -.645 & .260 & .260 \\
\hline 782 & 145.36 & 64 & 9,303 & 308031 & .481 & .685 & .315 & 244.82 & 38 & 9,303 & 186278 & .575 & .717 & .283 \\
\hline 783 & 730.00 & 2 & 1,460 & 1797 & .565 & .714 & .286 & 1460.00 & 1 & 1,460 & 788 & .138 & .555 & .445 \\
\hline 784 & 944.20 & 5 & 4,721 & 9749 & -.674 & .250 & .250 & -- & -- & -- & -- & -- & -- & -- \\
\hline 785 & 1059.94 & 18 & 19,079 & 164141 & -.324 & .373 & .373 & 2119.89 & 9 & 19,079 & 93339 & .453 & .675 & .325 \\
\hline 786 & 1628.42 & 12 & 19,541 & 136926 & 1.007 & .843 & .157 & 3256.83 & 6 & 19,541 & 70619 & .868 & .807 & .193 \\
\hline 787 & 178.09 & 34 & 6,055 & 98781 & -.408 & .342 & .342 & 403.67 & 15 & 6,055 & 42181 & -.477 & .317 & .317 \\
\hline 788 & 161.20 & 10 & 1,612 & 7810 & -.170 & .433 & .433 & 230.29 & 7 & 1,612 & 5262 & -.309 & .379 & .379 \\
\hline 789 & 189.64 & 44 & 8,344 & 199022 & .967 & .833 & .167 & 309.04 & 27 & 8,344 & 121466 & .705 & .760 & .241 \\
\hline 790 & 138.69 & 16 & 2,219 & 15521 & -.871 & .192 & .192 & 221.90 & 10 & 2,219 & 10203 & -.440 & .330 & .330 \\
\hline 791 & 168.36 & 28 & 4,714 & 79423 & 1.865 & .969 & .031 & 392.83 & 12 & 4,714 & 33580 & 1.124 & .869 & .131 \\
\hline 792 & 225.00 & 2 & 450 & 568 & .642 & .740 & .260 & 450.00 & 1 & 450 & 321 & .739 & .770 & .230 \\
\hline 793 & 206.25 & 93 & 19,181 & 869477 & -.420 & .337 & .337 & 355.20 & 54 & 19,181 & 551576 & .828 & .796 & .204 \\
\hline 794 & 220.47 & 53 & 11,685 & 290823 & -.767 & .222 & .222 & 292.13 & 40 & 11,685 & 218690 & -.704 & .241 & .241 \\
\hline 795 & 347.90 & 49 & 17,047 & 463478 & 1.330 & .908 & .092 & 532.72 & 32 & 17,047 & 302816 & 1.080 & .860 & .140 \\
\hline 796 & 580.56 & 9 & 5,225 & 18621 & -1.081 & .140 & .140 & 1045.00 & 5 & 5,225 & 8439 & -1.371 & .085 & .085 \\
\hline 797 & 364.86 & 78 & 28,459 & 1177849 & .937 & .825 & .175 & 547.29 & 52 & 28,459 & 794564 & .922 & .822 & .178 \\
\hline 798 & 694.00 & 1 & 694 & 486 & .694 & .756 & .244 & -- & -- & -- & -- & -- & -- & -- \\
\hline 799 & 653.93 & 14 & 9,155 & 62487 & -.162 & .436 & .436 & 1017.22 & 9 & 9,155 & 46486 & .667 & .748 & .252 \\
\hline 800 & 1148.00 & 1 & 1,148 & 1038 & 1.400 & .919 & .081 & -- & -- & -- & -- & -- & -- & -- \\
\hline 801 & 1345.82 & 11 & 14,804 & 95775 & 1.013 & .844 & .156 & 4934.67 & 3 & 14,804 & 26062 & .521 & .699 & .301 \\
\hline 802 & 1512.00 & 1 & 1,512 & 98 & -1.508 & .066 & .066 & -- & -- & -- & -- & -- & -- & -- \\
\hline 803 & 291.96 & 25 & 7,299 & 97553 & .600 & .726 & .274 & 456.19 & 16 & 7,299 & 58613 & .026 & .510 & .490 \\
\hline 804 & -- & -- & -- & -- & -- & -- & -- & -- & -- & -- & -- & -- & -- & -- \\
\hline 805 & 348.27 & 33 & 11,493 & 189212 & -.022 & .491 & .491 & 522.41 & 22 & 11,493 & 129455 & .195 & .577 & .423 \\
\hline 806 & 178.76 & 96 & 17,161 & 802867 & -.430 & .334 & .334 & 301.07 & 57 & 17,161 & 495754 & .178 & .571 & .429 \\
\hline 807 & 247.76 & 53 & 13,131 & 382246 & 1.242 & .893 & .107 & 410.34 & 32 & 13,131 & 222935 & .599 & .725 & .275 \\
\hline 808 & 295.28 & 61 & 18,012 & 555560 & .153 & .561 & .439 & 514.63 & 35 & 18,012 & 318623 & .111 & .544 & .456 \\
\hline 809 & 737.68 & 25 & 18,442 & 221110 & -.354 & .362 & .362 & 1229.47 & 15 & 18,442 & 134748 & -.173 & .431 & .431 \\
\hline 810 & 894.17 & 23 & 20,566 & 208784 & -.974 & .165 & .165 & 1869.64 & 11 & 20,566 & 96961 & -.820 & .206 & .206 \\
\hline 811 & 189.13 & 39 & 7,376 & 140327 & -.264 & .396 & .396 & 368.80 & 20 & 7,376 & 88572 & 1.556 & .940 & .060 \\
\hline 812 & 393.18 & 17 & 6,684 & 73744 & 2.128 & .983 & .017 & 607.64 & 11 & 6,684 & 41595 & .755 & .775 & .225 \\
\hline 813 & 83.00 & 1 & 83 & 66 & 1.023 & .847 & .153 & -- & -- & -- & -- & -- & -- & -- \\
\hline 814 & 1626.93 & 15 & 24,404 & 186791 & .138 & .555 & .445 & 4067.33 & 6 & 24,404 & 69937 & -0.190 & .425 & .425 \\
\hline 815 & 180.82 & 168 & 30,377 & 2826708 & 2.420 & .992 & .008 & 316.43 & 96 & 30,377 & 1593201 & 1.573 & .942 & .058 \\
\hline 816 & 159.53 & 81 & 12,922 & 597782 & 2.217 & .987 & .013 & 243.81 & 53 & 12,922 & 391796 & 1.818 & .965 & .035 \\
\hline
\end{tabular}


Table 7. Summary of interoccurrence intervals for daily precipitation thresholds of 2.5 and 3.0 inches-Continued

\begin{tabular}{|c|c|c|c|c|c|c|c|c|c|c|c|c|c|c|}
\hline \multirow[b]{2}{*}{$\begin{array}{l}\text { Seq. } \\
\text { no. }\end{array}$} & \multicolumn{7}{|c|}{ Daily precipitation threshold of 2.5 inches and greater } & \multicolumn{7}{|c|}{ Daily precipitation threshold of 3.0 inches and greater } \\
\hline & $\begin{array}{l}\text { Mean } \\
\text { inter- } \\
\text { occur- } \\
\text { rence } \\
\text { interval } \\
\text { (days) }\end{array}$ & $\begin{array}{c}\text { Total } \\
\text { no. of } \\
\text { events }\end{array}$ & $\begin{array}{l}\text { Total } \\
\text { no. of } \\
\text { days } \\
\text { avail- } \\
\text { able }\end{array}$ & $\begin{array}{l}\text { Sigma } \\
\mathrm{S}\end{array}$ & $\begin{array}{c}\text { Stan- } \\
\text { dard } \\
\text { normal } \\
\text { variate }\end{array}$ & $\begin{array}{l}\text { Non- } \\
\text { ex- } \\
\text { ceed- } \\
\text { ance } \\
\text { prob- } \\
\text { ability }\end{array}$ & $\begin{array}{c}\text { p- } \\
\text { value }\end{array}$ & $\begin{array}{c}\text { Mean } \\
\text { inter- } \\
\text { occur- } \\
\text { rence } \\
\text { interval } \\
\text { (days) }\end{array}$ & $\begin{array}{c}\text { Total } \\
\text { no. of } \\
\text { events }\end{array}$ & $\begin{array}{l}\text { Total } \\
\text { no. of } \\
\text { days } \\
\text { avail- } \\
\text { able }\end{array}$ & $\begin{array}{l}\text { Sigma } \\
\mathrm{S}\end{array}$ & $\begin{array}{c}\text { Stan- } \\
\text { dard } \\
\text { normal } \\
\text { variate }\end{array}$ & $\begin{array}{l}\text { Non- } \\
\text { ex- } \\
\text { ceed- } \\
\text { ance } \\
\text { prob- } \\
\text { ability }\end{array}$ & $\begin{array}{c}\mathrm{p}- \\
\text { value }\end{array}$ \\
\hline 817 & 270.44 & 23 & 6,220 & 72251 & .084 & .533 & .467 & 478.46 & 13 & 6,220 & 41900 & .227 & .590 & .410 \\
\hline 818 & 254.27 & 78 & 19,833 & 778322 & .096 & .538 & .462 & 508.54 & 39 & 19,833 & 366345 & -.571 & .284 & .284 \\
\hline 819 & 2061.57 & 14 & 28,862 & 202914 & .028 & .511 & .489 & 2886.20 & 10 & 28,862 & 133961 & -.393 & .347 & .347 \\
\hline 820 & 222.75 & 4 & 891 & 2671 & 1.728 & .958 & .042 & 297.00 & 3 & 891 & 2348 & 2.271 & .988 & .012 \\
\hline 821 & 1137.29 & 7 & 7,961 & 31755 & .640 & .739 & .261 & 1990.25 & 4 & 7,961 & 24768 & 1.925 & .973 & .027 \\
\hline 822 & 352.78 & 54 & 19,050 & 511960 & -.059 & .476 & .476 & 595.31 & 32 & 19,050 & 326911 & .711 & .761 & .239 \\
\hline 823 & 451.94 & 72 & 32,540 & 1279043 & 1.350 & .911 & .089 & 723.11 & 45 & 32,540 & 793635 & .976 & .835 & .165 \\
\hline 824 & 530.50 & 4 & 2,122 & 4140 & -.085 & .466 & .466 & 2122.00 & 1 & 2,122 & 1055 & -.010 & .496 & .496 \\
\hline 825 & 311.15 & 13 & 4,045 & 29859 & .847 & .801 & .199 & 577.86 & 7 & 4,045 & 17981 & 1.238 & .892 & .108 \\
\hline 826 & 187.97 & 141 & 26,503 & 1948909 & .886 & .812 & .188 & 358.15 & 74 & 26,503 & 972314 & -.126 & .450 & .450 \\
\hline 827 & 153.49 & 68 & 10,437 & 402429 & 1.915 & .972 & .028 & 213.00 & 49 & 10,437 & 309125 & 2.533 & .994 & .006 \\
\hline 828 & 198.67 & 130 & 25,827 & 1810486 & 1.550 & .939 & .061 & 379.81 & 68 & 25,827 & 916530 & .625 & .734 & .266 \\
\hline 829 & 120.02 & 45 & 5,401 & 125489 & .379 & .648 & .352 & 192.89 & 28 & 5,401 & 85045 & 1.143 & .873 & .127 \\
\hline 830 & 327.55 & 40 & 13,102 & 243938 & -.757 & .225 & .225 & 623.91 & 21 & 13,102 & 126003 & -.667 & .252 & .252 \\
\hline 831 & 202.51 & 70 & 14,176 & 574007 & 2.274 & .989 & .011 & 329.67 & 43 & 14,176 & 355657 & 1.896 & .971 & .029 \\
\hline 832 & 292.00 & 6 & 1,752 & 3993 & -1.020 & .154 & .154 & 876.00 & 2 & 1,752 & 1221 & -.742 & .229 & .229 \\
\hline 833 & 1641.00 & 1 & 1,641 & 824 & .007 & .503 & .497 & 1641.00 & 1 & 1,641 & 824 & .007 & .503 & .497 \\
\hline 834 & 456.22 & 9 & 4,106 & 23573 & 1.433 & .924 & .076 & 821.20 & 5 & 4,106 & 12485 & .838 & .799 & .201 \\
\hline 835 & 139.47 & 133 & 18,549 & 1279670 & .748 & .773 & .227 & 268.83 & 69 & 18,549 & 721010 & 1.823 & .966 & .034 \\
\hline 836 & 228.73 & 33 & 7,548 & 137692 & 1.051 & .853 & .147 & 377.40 & 20 & 7,548 & 80679 & .534 & .703 & .297 \\
\hline 837 & 145.63 & 82 & 11,942 & 514516 & .797 & .787 & .213 & 265.38 & 45 & 11,942 & 268288 & -.018 & .493 & .493 \\
\hline 838 & 115.42 & 66 & 7,618 & 283463 & 1.795 & .964 & .036 & 173.14 & 44 & 7,618 & 197067 & 2.020 & .978 & .022 \\
\hline 839 & 173.23 & 13 & 2,252 & 11184 & -1.474 & .070 & .070 & 375.33 & 6 & 2,252 & 4502 & -1.416 & .078 & .078 \\
\hline 840 & 248.01 & 147 & 36,458 & 2858109 & 1.398 & .919 & .081 & 434.02 & 84 & 36,458 & 1656314 & 1.297 & .903 & .097 \\
\hline 841 & 130.05 & 139 & 18,077 & 1389983 & 2.172 & .985 & .015 & 203.11 & 89 & 18,077 & 897057 & 1.882 & .970 & .030 \\
\hline 842 & 146.34 & 128 & 18,731 & 1347306 & 2.428 & .992 & .008 & 217.80 & 86 & 18,731 & 921066 & 2.306 & .989 & .011 \\
\hline 843 & 133.11 & 27 & 3,594 & 54436 & 1.098 & .864 & .136 & 211.41 & 17 & 3,594 & 31696 & .268 & .606 & .394 \\
\hline 844 & 307.98 & 57 & 17,555 & 445925 & -1.422 & .078 & .078 & 548.59 & 32 & 17,555 & 239400 & -1.447 & .074 & .074 \\
\hline 845 & 138.52 & 27 & 3,740 & 56645 & 1.097 & .864 & .136 & 340.00 & 11 & 3,740 & 24902 & 1.210 & .887 & .113 \\
\hline 846 & 98.14 & 66 & 6,477 & 224977 & .740 & .770 & .230 & 147.21 & 44 & 6,477 & 152730 & .825 & .795 & .205 \\
\hline 847 & 289.26 & 62 & 17,934 & 520242 & -.876 & .191 & .191 & 427.00 & 42 & 17,934 & 347190 & -.877 & .190 & .190 \\
\hline 848 & 256.81 & 112 & 28,763 & 1654961 & .503 & .693 & .307 & 405.11 & 71 & 28,763 & 1071037 & .714 & .762 & .238 \\
\hline 849 & 169.63 & 40 & 6,785 & 134780 & -.074 & .470 & .470 & 376.94 & 18 & 6,785 & 55013 & -.728 & .233 & .233 \\
\hline 850 & 1431.00 & 1 & 1,431 & 866 & .364 & .642 & .358 & -- & -- & -- & -- & -- & -- & -- \\
\hline 851 & -- & -- & -- & -- & -- & -- & -- & -- & -- & -- & -- & -- & -- & -- \\
\hline 852 & 114.25 & 4 & 457 & 959 & .171 & .568 & .432 & 228.50 & 2 & 457 & 387 & -.375 & .354 & .354 \\
\hline 853 & -- & -- & -- & -- & -- & -- & -- & -- & -- & -- & -- & -- & -- & -- \\
\hline 854 & 609.65 & 31 & 18,899 & 318893 & .855 & .804 & .196 & 1049.94 & 18 & 18,899 & 200671 & 1.321 & .907 & .093 \\
\hline 855 & 192.07 & 29 & 5,570 & 84811 & .467 & .680 & .320 & 278.50 & 20 & 5,570 & 55095 & -.084 & .466 & .466 \\
\hline 856 & 850.41 & 22 & 18,709 & 188180 & -.696 & .243 & .243 & 1870.90 & 10 & 18,709 & 93330 & -.013 & .495 & .495 \\
\hline 857 & 413.64 & 22 & 9,100 & 113494 & 1.087 & .861 & .139 & 827.27 & 11 & 9,100 & 41160 & -1.020 & .154 & .154 \\
\hline 858 & 587.78 & 18 & 10,580 & 117152 & 1.693 & .955 & .045 & 813.85 & 13 & 10,580 & 86762 & 1.634 & .949 & .051 \\
\hline 859 & 720.54 & 13 & 9,367 & 61363 & .049 & .520 & .480 & 1561.17 & 6 & 9,367 & 22398 & -.861 & .195 & .195 \\
\hline 860 & -- & -- & -- & -- & -- & -- & -- & -- & -- & -- & -- & -- & -- & -- \\
\hline 861 & 498.10 & 29 & 14,445 & 179025 & -1.355 & .088 & .088 & 802.50 & 18 & 14,445 & 121394 & -0.487 & .313 & .313 \\
\hline 862 & 331.83 & 23 & 7,632 & 69679 & -1.712 & .043 & .043 & 693.82 & 11 & 7,632 & 39801 & -.298 & .383 & .383 \\
\hline 863 & 426.00 & 1 & 426 & 374 & 1.309 & .905 & .095 & 426.00 & 1 & 426 & 374 & 1.309 & .905 & .095 \\
\hline 864 & 1389.92 & 13 & 18,069 & 106100 & -.603 & .273 & .273 & 2007.67 & 9 & 18,069 & 80780 & -.034 & .486 & .486 \\
\hline
\end{tabular}


Table 7. Summary of interoccurrence intervals for daily precipitation thresholds of 2.5 and 3.0 inches—Continued

\begin{tabular}{|c|c|c|c|c|c|c|c|c|c|c|c|c|c|c|}
\hline \multirow[b]{2}{*}{$\begin{array}{l}\text { Seq. } \\
\text { no. }\end{array}$} & \multicolumn{7}{|c|}{ Daily precipitation threshold of 2.5 inches and greater } & \multicolumn{7}{|c|}{ Daily precipitation threshold of 3.0 inches and greater } \\
\hline & $\begin{array}{l}\text { Mean } \\
\text { inter- } \\
\text { occur- } \\
\text { rence } \\
\text { interval } \\
\text { (days) }\end{array}$ & $\begin{array}{l}\text { Total } \\
\text { no. of } \\
\text { events }\end{array}$ & $\begin{array}{l}\text { Total } \\
\text { no. of } \\
\text { days } \\
\text { avail- } \\
\text { able }\end{array}$ & $\begin{array}{l}\text { Sigma } \\
\mathrm{S}\end{array}$ & $\begin{array}{c}\text { Stan- } \\
\text { dard } \\
\text { normal } \\
\text { variate }\end{array}$ & $\begin{array}{l}\text { Non- } \\
\text { ex- } \\
\text { ceed- } \\
\text { ance } \\
\text { prob- } \\
\text { ability }\end{array}$ & $\begin{array}{c}\mathrm{p}- \\
\text { value }\end{array}$ & $\begin{array}{l}\text { Mean } \\
\text { inter- } \\
\text { occur- } \\
\text { rence } \\
\text { interval } \\
\text { (days) }\end{array}$ & $\begin{array}{c}\text { Total } \\
\text { no. of } \\
\text { events }\end{array}$ & $\begin{array}{l}\text { Total } \\
\text { no. of } \\
\text { days } \\
\text { avail- } \\
\text { able }\end{array}$ & $\begin{array}{l}\text { Sigma } \\
\mathbf{S}\end{array}$ & $\begin{array}{c}\text { Stan- } \\
\text { dard } \\
\text { normal } \\
\text { variate }\end{array}$ & $\begin{array}{l}\text { Non- } \\
\text { ex- } \\
\text { ceed- } \\
\text { ance } \\
\text { prob- } \\
\text { ability }\end{array}$ & $\begin{array}{c}\mathrm{p}- \\
\text { value }\end{array}$ \\
\hline 865 & 585.50 & 4 & 2,342 & 4363 & -.237 & .406 & .406 & 1171.00 & 2 & 2,342 & 2112 & -.241 & .405 & .405 \\
\hline 866 & 680.25 & 4 & 2,721 & 4927 & -.328 & .372 & .372 & 907.00 & 3 & 2,721 & 4710 & .462 & .678 & .322 \\
\hline 867 & 317.98 & 50 & 15,899 & 395631 & -.057 & .477 & .477 & 529.97 & 30 & 15,899 & 239323 & .033 & .513 & .487 \\
\hline 868 & 282.42 & 77 & 21,746 & 897011 & 1.085 & .861 & .139 & 453.04 & 48 & 21,746 & 531550 & .222 & .588 & .412 \\
\hline 869 & 970.53 & 19 & 18,440 & 155601 & -.844 & .199 & .199 & 1676.36 & 11 & 18,440 & 96916 & -.255 & .399 & .399 \\
\hline 870 & 225.00 & 7 & 1,575 & 6773 & 1.048 & .853 & .147 & 393.75 & 4 & 1,575 & 5071 & 2.113 & .983 & .017 \\
\hline 871 & -- & -- & -- & -- & -- & -- & -- & -- & -- & -- & -- & -- & -- & -- \\
\hline 872 & -- & -- & -- & -- & -- & -- & -- & -- & -- & -- & -- & -- & -- & -- \\
\hline 873 & 92.40 & 254 & 23,470 & 2972341 & -.077 & .469 & .469 & 136.45 & 172 & 23,470 & 2023357 & .056 & .522 & .478 \\
\hline 874 & 73.39 & 78 & 5,724 & 214344 & -.609 & .271 & .271 & 110.08 & 52 & 5,724 & 145786 & -.255 & .399 & .399 \\
\hline 875 & 242.50 & 2 & 485 & 545 & .303 & .619 & .381 & 242.50 & 2 & 485 & 545 & .303 & .619 & .381 \\
\hline 876 & -- & -- & -- & -- & -- & -- & -- & -- & -- & -- & -- & -- & -- & -- \\
\hline 877 & -- & -- & -- & -- & -- & -- & -- & -- & -- & -- & -- & -- & -- & -- \\
\hline 878 & 178.02 & 88 & 15,666 & 638615 & -1.195 & .116 & .116 & 326.38 & 48 & 15,666 & 317581 & -1.864 & .031 & .031 \\
\hline 879 & 596.55 & 31 & 18,493 & 253063 & -1.130 & .129 & .129 & 1087.82 & 17 & 18,493 & 122090 & -1.595 & .055 & .055 \\
\hline 880 & -- & -- & -- & -- & -- & -- & -- & -- & -- & -- & -- & -- & -- & -- \\
\hline 881 & 531.29 & 35 & 18,595 & 310327 & -.475 & .317 & .317 & 929.75 & 20 & 18,595 & 200733 & .616 & .731 & .269 \\
\hline 882 & 228.25 & 8 & 1,826 & 7702 & .267 & .605 & .395 & 365.20 & 5 & 1,826 & 3846 & -.610 & .271 & .271 \\
\hline 883 & 473.96 & 23 & 10,901 & 137140 & .781 & .782 & .218 & 726.73 & 15 & 10,901 & 103543 & 1.788 & .963 & .037 \\
\hline 884 & 592.65 & 23 & 13,631 & 173040 & .863 & .806 & .194 & 1239.18 & 11 & 13,631 & 84340 & .718 & .764 & .236 \\
\hline 885 & 479.00 & 62 & 29,698 & 911065 & -.142 & .444 & .444 & 958.00 & 31 & 29,698 & 416972 & -.908 & .182 & .182 \\
\hline 886 & 128.71 & 166 & 21,366 & 1857597 & 1.060 & .855 & .145 & 194.24 & 110 & 21,366 & 1221233 & .713 & .762 & .238 \\
\hline 887 & 191.44 & 131 & 25,079 & 1740833 & 1.185 & .882 & .118 & 338.91 & 74 & 25,079 & 1045190 & 1.883 & .970 & .030 \\
\hline 888 & 295.77 & 64 & 18,929 & 693264 & 2.002 & .977 & .023 & 728.04 & 26 & 18,929 & 273296 & .977 & .836 & .164 \\
\hline 889 & 1372.60 & 10 & 13,726 & 84473 & 1.264 & .897 & .103 & 2745.20 & 5 & 13,726 & 37964 & .412 & .660 & .340 \\
\hline 890 & 1090.42 & 12 & 13,085 & 96387 & 1.366 & .914 & .086 & 1635.63 & 8 & 13,085 & 64057 & 1.097 & .864 & .136 \\
\hline 891 & 490.35 & 37 & 18,143 & 375887 & 1.263 & .897 & .103 & 788.83 & 23 & 18,143 & 208158 & -.019 & .492 & .492 \\
\hline 892 & 425.06 & 17 & 7,226 & 68727 & .850 & .802 & .198 & 602.17 & 12 & 7,226 & 53356 & 1.384 & .917 & .083 \\
\hline 893 & 928.93 & 29 & 26,939 & 405852 & .364 & .642 & .358 & 1584.65 & 17 & 26,939 & 217951 & -.344 & .365 & .365 \\
\hline 894 & 563.10 & 10 & 5,631 & 22636 & -1.074 & .142 & .142 & 1126.20 & 5 & 5,631 & 13153 & -.254 & .400 & .400 \\
\hline 895 & 4267.75 & 4 & 17,071 & 42176 & .815 & .792 & .208 & 5690.33 & 3 & 17,071 & 30356 & .556 & .711 & .289 \\
\hline 896 & 182.42 & 196 & 35,755 & 3918575 & 2.869 & .998 & .002 & 343.80 & 104 & 35,755 & 2197567 & 3.214 & .999 & .001 \\
\hline 897 & -- & -- & -- & -- & -- & -- & -- & -- & -- & -- & -- & -- & -- & -- \\
\hline 898 & 121.10 & 10 & 1,211 & 7465 & 1.276 & .899 & .101 & 134.56 & 9 & 1,211 & 6300 & .811 & .791 & .209 \\
\hline 899 & 58.00 & 1 & 58 & 20 & -.538 & .296 & .296 & -- & -- & -- & -- & -- & -- & -- \\
\hline 900 & 510.33 & 3 & 1,531 & 2951 & .855 & .804 & .196 & 1531.00 & 1 & 1,531 & 987 & .501 & .692 & .308 \\
\hline 901 & 358.42 & 95 & 34,050 & 1559206 & -.607 & .272 & .272 & 577.12 & 59 & 34,050 & 992387 & -.160 & .436 & .436 \\
\hline 902 & 224.80 & 5 & 1,124 & 2072 & -1.017 & .155 & .155 & 224.80 & 5 & 1,124 & 2072 & -1.017 & .155 & .155 \\
\hline 903 & 1944.31 & 13 & 25,276 & 206204 & 1.593 & .944 & .056 & 5055.20 & 5 & 25,276 & 77947 & .905 & .817 & .183 \\
\hline 904 & -- & -- & -- & -- & -- & -- & -- & -- & -- & -- & -- & -- & -- & -- \\
\hline 905 & 1511.46 & 11 & 16,626 & 103808 & .777 & .781 & .219 & 2771.00 & 6 & 16,626 & 50755 & .075 & .530 & .470 \\
\hline 906 & 930.32 & 34 & 31,631 & 607720 & 1.315 & .906 & .094 & 1506.24 & 21 & 31,631 & 391822 & 1.427 & .923 & .077 \\
\hline 907 & -- & -- & -- & -- & -- & -- & -- & -- & -- & -- & -- & -- & -- & -- \\
\hline 908 & 1236.38 & 16 & 19,782 & 162355 & .179 & .571 & .429 & 2472.75 & 8 & 19,782 & 87480 & .517 & .697 & .303 \\
\hline 909 & 762.91 & 11 & 8,392 & 51951 & 0.721 & .765 & .235 & 1049.00 & 8 & 8,392 & 35323 & 0.256 & .601 & .399 \\
\hline 910 & 7085.00 & 1 & 7,085 & 4153 & .299 & .617 & .383 & -- & -- & -- & -- & -- & -- & -- \\
\hline 911 & -- & -- & -- & -- & -- & -- & -- & -- & -- & -- & -- & -- & -- & -- \\
\hline 912 & 302.00 & 1 & 302 & 284 & 1.526 & .936 & .064 & -- & -- & -- & -- & -- & -- & -- \\
\hline
\end{tabular}


Table 7. Summary of interoccurrence intervals for daily precipitation thresholds of 2.5 and 3.0 inches-Continued

\begin{tabular}{|c|c|c|c|c|c|c|c|c|c|c|c|c|c|c|}
\hline \multirow[b]{2}{*}{$\begin{array}{l}\text { Seq. } \\
\text { no. }\end{array}$} & \multicolumn{7}{|c|}{ Daily precipitation threshold of 2.5 inches and greater } & \multicolumn{7}{|c|}{ Daily precipitation threshold of 3.0 inches and greater } \\
\hline & $\begin{array}{l}\text { Mean } \\
\text { inter- } \\
\text { occur- } \\
\text { rence } \\
\text { interval } \\
\text { (days) }\end{array}$ & $\begin{array}{c}\text { Total } \\
\text { no. of } \\
\text { events }\end{array}$ & $\begin{array}{l}\text { Total } \\
\text { no. of } \\
\text { days } \\
\text { avail- } \\
\text { able }\end{array}$ & $\begin{array}{c}\text { Sigma } \\
\mathbf{S}\end{array}$ & $\begin{array}{c}\text { Stan- } \\
\text { dard } \\
\text { normal } \\
\text { variate }\end{array}$ & $\begin{array}{l}\text { Non- } \\
\text { ex- } \\
\text { ceed- } \\
\text { ance } \\
\text { prob- } \\
\text { ability }\end{array}$ & $\begin{array}{c}\text { p- } \\
\text { value }\end{array}$ & $\begin{array}{l}\text { Mean } \\
\text { inter- } \\
\text { occur- } \\
\text { rence } \\
\text { interval } \\
\text { (days) }\end{array}$ & $\begin{array}{c}\text { Total } \\
\text { no. of } \\
\text { events }\end{array}$ & $\begin{array}{l}\text { Total } \\
\text { no. of } \\
\text { days } \\
\text { avail- } \\
\text { able }\end{array}$ & $\begin{array}{c}\text { Sigma } \\
\mathbf{S}\end{array}$ & $\begin{array}{c}\text { Stan- } \\
\text { dard } \\
\text { normal } \\
\text { variate }\end{array}$ & $\begin{array}{l}\text { Non- } \\
\text { ex- } \\
\text { ceed- } \\
\text { ance } \\
\text { prob- } \\
\text { ability }\end{array}$ & $\begin{array}{c}p- \\
\text { value }\end{array}$ \\
\hline 913 & 247.00 & 26 & 6,422 & 106701 & 2.456 & .993 & .007 & 356.78 & 18 & 6,422 & 74430 & 2.115 & .983 & .017 \\
\hline 914 & 517.43 & 7 & 3,622 & 13236 & .202 & .580 & .420 & 724.40 & 5 & 3,622 & 7918 & -.486 & .313 & .313 \\
\hline 915 & 168.25 & 200 & 33,649 & 3644703 & 2.037 & .979 & .021 & 232.06 & 145 & 33,649 & 2783351 & 2.939 & .998 & .002 \\
\hline 916 & 182.20 & 105 & 19,131 & 1110048 & 1.867 & .969 & .031 & 281.34 & 68 & 19,131 & 757441 & 2.349 & .991 & .009 \\
\hline 917 & 1541.80 & 5 & 7,709 & 8792 & -2.106 & .018 & .018 & 2569.67 & 3 & 7,709 & 5508 & -1.571 & .058 & .058 \\
\hline 918 & 121.32 & 73 & 8,856 & 358356 & 1.608 & .946 & .054 & 205.95 & 43 & 8,856 & 226099 & 2.129 & .983 & .017 \\
\hline 919 & 408.38 & 24 & 9,801 & 131994 & 1.038 & .850 & .150 & 700.07 & 14 & 9,801 & 79910 & 1.068 & .857 & .143 \\
\hline 920 & 172.11 & 111 & 19,104 & 1127688 & 1.160 & .877 & .123 & 341.14 & 56 & 19,104 & 600455 & 1.588 & .944 & .056 \\
\hline 921 & 397.09 & 11 & 4,368 & 25749 & .413 & .660 & .340 & 728.00 & 6 & 4,368 & 14540 & .465 & .679 & .321 \\
\hline 922 & 909.76 & 25 & 22,744 & 283131 & -.036 & .486 & .486 & 1624.57 & 14 & 22,744 & 167475 & .337 & .632 & .368 \\
\hline 923 & 955.06 & 35 & 33,427 & 562473 & -.394 & .347 & .347 & 2228.47 & 15 & 33,427 & 279560 & .772 & .780 & .220 \\
\hline 924 & 639.00 & 1 & 639 & 399 & .431 & .667 & .333 & 639.00 & 1 & 639 & 399 & .431 & .667 & .333 \\
\hline 925 & 4205.00 & 1 & 4,205 & 3372 & 1.046 & .852 & .148 & 4205.00 & 1 & 4,205 & 3372 & 1.046 & .852 & .148 \\
\hline 926 & 185.33 & 3 & 556 & 1192 & 1.288 & .901 & .099 & 556.00 & 1 & 556 & 171 & -.667 & .253 & .253 \\
\hline 927 & 184.71 & 24 & 4,433 & 53943 & .119 & .547 & .453 & 277.06 & 16 & 4,433 & 34666 & -.156 & .438 & .438 \\
\hline 928 & 952.88 & 8 & 7,623 & 37698 & 1.158 & .876 & .124 & 2541.00 & 3 & 7,623 & 11135 & -.079 & .469 & .469 \\
\hline 929 & 119.06 & 132 & 15,716 & 1009537 & -.532 & .297 & .297 & 176.58 & 89 & 15,716 & 701700 & .055 & .522 & .478 \\
\hline 930 & 569.25 & 16 & 9,108 & 75679 & .268 & .606 & .394 & 1301.14 & 7 & 9,108 & 32267 & .056 & .522 & .478 \\
\hline 931 & 372.54 & 13 & 4,843 & 46786 & 3.037 & .999 & .001 & 1210.75 & 4 & 4,843 & 13435 & 1.341 & .910 & .090 \\
\hline 932 & 361.67 & 9 & 3,255 & 16349 & .604 & .727 & .273 & 465.00 & 7 & 3,255 & 13407 & .810 & .791 & .209 \\
\hline 933 & -- & -- & -- & -- & -- & -- & -- & -- & -- & -- & -- & -- & -- & -- \\
\hline 934 & 138.68 & 40 & 5,547 & 118182 & .715 & .763 & .237 & 205.44 & 27 & 5,547 & 76593 & .205 & .581 & .419 \\
\hline 935 & 108.21 & 182 & 19,694 & 2007396 & 2.806 & .998 & .003 & 171.25 & 115 & 19,694 & 1209426 & 1.263 & .897 & .103 \\
\hline 936 & 115.98 & 82 & 9,510 & 364108 & -1.038 & .150 & .150 & 161.19 & 59 & 9,510 & 271664 & -.421 & .337 & .337 \\
\hline 937 & 91.96 & 97 & 8,920 & 422174 & -.412 & .340 & .340 & 148.67 & 60 & 8,920 & 261623 & -.300 & .382 & .382 \\
\hline 938 & 269.27 & 94 & 25,311 & 1326043 & 1.926 & .973 & .027 & 414.93 & 61 & 25,311 & 814784 & .750 & .773 & .227 \\
\hline 939 & 133.36 & 162 & 21,605 & 1934249 & 2.321 & .990 & .010 & 198.21 & 109 & 21,605 & 1282467 & 1.613 & .947 & .053 \\
\hline 940 & 299.96 & 53 & 15,898 & 379843 & -1.241 & .107 & .107 & 387.76 & 41 & 15,898 & 288452 & -1.275 & .101 & .101 \\
\hline 941 & 169.61 & 97 & 16,452 & 726174 & -1.534 & .063 & .063 & 228.50 & 72 & 16,452 & 566102 & -.649 & .258 & .258 \\
\hline 942 & 468.96 & 28 & 13,131 & 182218 & -.081 & .468 & .468 & 1193.73 & 11 & 13,131 & 60252 & -.952 & .171 & .171 \\
\hline 943 & 640.14 & 51 & 32,647 & 877312 & .666 & .747 & .253 & 960.21 & 34 & 32,647 & 582120 & .494 & .689 & .311 \\
\hline 944 & 212.59 & 27 & 5,740 & 63041 & -1.678 & .047 & .047 & 318.89 & 18 & 5,740 & 42307 & -1.330 & .092 & .092 \\
\hline 945 & 277.43 & 77 & 21,362 & 876279 & .995 & .840 & .160 & 403.06 & 53 & 21,362 & 574931 & .197 & .578 & .422 \\
\hline 946 & 228.37 & 43 & 9,820 & 264668 & 2.880 & .998 & .002 & 363.70 & 27 & 9,820 & 166682 & 2.316 & .990 & .010 \\
\hline 947 & 275.68 & 50 & 13,784 & 372931 & 1.007 & .843 & .157 & 353.44 & 39 & 13,784 & 293910 & 1.011 & .844 & .156 \\
\hline 948 & 1216.00 & 1 & 1,216 & 472 & -.387 & .349 & .349 & -- & -- & -- & -- & -- & -- & -- \\
\hline 949 & 6627.25 & 4 & 26,509 & 52092 & -.061 & .476 & .476 & 8836.33 & 3 & 26,509 & 33947 & -.439 & .330 & .330 \\
\hline 950 & -- & -- & -- & -- & -- & -- & -- & -- & -- & -- & -- & -- & -- & -- \\
\hline 951 & 206.75 & 60 & 12,405 & 371300 & -.031 & .488 & .488 & 375.91 & 33 & 12,405 & 225523 & 1.013 & .844 & .156 \\
\hline 952 & 273.83 & 18 & 4,929 & 48675 & .715 & .763 & .238 & 492.90 & 10 & 4,929 & 32298 & 1.701 & .956 & .045 \\
\hline 953 & 280.52 & 50 & 14,026 & 391288 & 1.419 & .922 & .078 & 500.93 & 28 & 14,026 & 202292 & .277 & .609 & .391 \\
\hline 954 & 203.59 & 34 & 6,922 & 106987 & -.917 & .180 & .180 & 256.37 & 27 & 6,922 & 85114 & -.803 & .211 & .211 \\
\hline 955 & 472.21 & 70 & 33,055 & 1131683 & -.316 & .376 & .376 & 944.43 & 35 & 33,055 & 554009 & -.433 & .332 & .332 \\
\hline 956 & 496.23 & 70 & 34,736 & 1300085 & 1.005 & .843 & .157 & 914.11 & 38 & 34,736 & 663728 & .061 & .524 & .476 \\
\hline 957 & 194.68 & 22 & 4,283 & 46427 & -0.118 & .453 & .453 & 428.30 & 10 & 4,283 & 17308 & -1.050 & .147 & .147 \\
\hline 958 & 1140.27 & 11 & 12,543 & 73088 & .342 & .634 & .366 & 2090.50 & 6 & 12,543 & 42492 & .548 & .708 & .292 \\
\hline 959 & 191.99 & 72 & 13,823 & 504691 & .209 & .583 & .417 & 337.15 & 41 & 13,823 & 318145 & 1.361 & .913 & .087 \\
\hline 960 & 390.50 & 2 & 781 & 1231 & 1.411 & .921 & .079 & 781.00 & 1 & 781 & 767 & 1.670 & .953 & .047 \\
\hline
\end{tabular}


Table 7. Summary of interoccurrence intervals for daily precipitation thresholds of 2.5 and 3.0 inches—Continued

\begin{tabular}{|c|c|c|c|c|c|c|c|c|c|c|c|c|c|c|}
\hline \multirow[b]{2}{*}{$\begin{array}{l}\text { Seq. } \\
\text { no. }\end{array}$} & \multicolumn{7}{|c|}{ Daily precipitation threshold of 2.5 inches and greater } & \multicolumn{7}{|c|}{ Daily precipitation threshold of $\mathbf{3 . 0}$ inches and greater } \\
\hline & $\begin{array}{c}\text { Mean } \\
\text { inter- } \\
\text { occur- } \\
\text { rence } \\
\text { interval } \\
\text { (days) }\end{array}$ & $\begin{array}{l}\text { Total } \\
\text { no. of } \\
\text { events }\end{array}$ & $\begin{array}{c}\text { Total } \\
\text { no. of } \\
\text { days } \\
\text { avail- } \\
\text { able }\end{array}$ & $\begin{array}{l}\text { Sigma } \\
\mathrm{S}\end{array}$ & $\begin{array}{c}\text { Stan- } \\
\text { dard } \\
\text { normal } \\
\text { variate }\end{array}$ & $\begin{array}{l}\text { Non- } \\
\text { ex- } \\
\text { ceed- } \\
\text { ance } \\
\text { prob- } \\
\text { ability }\end{array}$ & $\begin{array}{c}\text { p- } \\
\text { value }\end{array}$ & $\begin{array}{c}\text { Mean } \\
\text { inter- } \\
\text { occur- } \\
\text { rence } \\
\text { interval } \\
\text { (days) }\end{array}$ & $\begin{array}{c}\text { Total } \\
\text { no. of } \\
\text { events }\end{array}$ & $\begin{array}{l}\text { Total } \\
\text { no. of } \\
\text { days } \\
\text { avail- } \\
\text { able }\end{array}$ & $\begin{array}{l}\text { Sigma } \\
\mathrm{S}\end{array}$ & $\begin{array}{c}\text { Stan- } \\
\text { dard } \\
\text { normal } \\
\text { variate }\end{array}$ & $\begin{array}{l}\text { Non- } \\
\text { ex- } \\
\text { ceed- } \\
\text { ance } \\
\text { prob- } \\
\text { ability }\end{array}$ & $\begin{array}{c}\text { p- } \\
\text { value }\end{array}$ \\
\hline 961 & 340.51 & 59 & 20,090 & 701628 & 2.446 & .993 & .007 & 627.81 & 32 & 20,090 & 380876 & 1.812 & .965 & .035 \\
\hline 962 & -- & -- & -- & -- & -- & -- & -- & -- & -- & -- & -- & -- & -- & -- \\
\hline 963 & 285.25 & 4 & 1,141 & 1776 & -.768 & .221 & .221 & 380.33 & 3 & 1,141 & 1411 & -.527 & .299 & .299 \\
\hline 964 & 335.83 & 6 & 2,015 & 6906 & .604 & .727 & .273 & 671.67 & 3 & 2,015 & 4841 & 1.805 & .964 & .036 \\
\hline 965 & 351.52 & 29 & 10,194 & 111314 & -2.303 & .011 & .011 & 463.36 & 22 & 10,194 & 78699 & -2.422 & .008 & .008 \\
\hline 966 & 987.67 & 9 & 8,889 & 42380 & .309 & .621 & .379 & 1777.80 & 5 & 8,889 & 25183 & .516 & .697 & .303 \\
\hline 967 & 1010.80 & 5 & 5,054 & 12442 & -.059 & .476 & .476 & 1684.67 & 3 & 5,054 & 6372 & -.478 & .316 & .316 \\
\hline 968 & 197.88 & 8 & 1,583 & 5012 & -1.021 & .154 & .154 & 395.75 & 4 & 1,583 & 1934 & -1.348 & .089 & .089 \\
\hline 969 & 202.83 & 6 & 1,217 & 4386 & .854 & .803 & .197 & 243.40 & 5 & 1,217 & 3637 & .757 & .775 & .225 \\
\hline 970 & 297.66 & 110 & 32,743 & 1860896 & .606 & .728 & .272 & 461.17 & 71 & 32,743 & 1145670 & -.210 & .417 & .417 \\
\hline 971 & 191.33 & 9 & 1,722 & 8159 & .275 & .608 & .392 & 215.25 & 8 & 1,722 & 7761 & .621 & .733 & .267 \\
\hline 972 & 411.69 & 45 & 18,526 & 459772 & 1.197 & .884 & .116 & 686.15 & 27 & 18,526 & 291829 & 1.502 & .933 & .067 \\
\hline 973 & 1149.47 & 17 & 19,541 & 217639 & 2.216 & .987 & .013 & 1776.46 & 11 & 19,541 & 142063 & 1.849 & .968 & .032 \\
\hline 974 & 253.66 & 44 & 11,161 & 236539 & -.421 & .337 & .337 & 338.21 & 33 & 11,161 & 181505 & -.143 & .443 & .443 \\
\hline 975 & 193.81 & 64 & 12,404 & 349579 & -1.653 & .049 & .049 & 354.40 & 35 & 12,404 & 199092 & -.849 & .198 & .198 \\
\hline 976 & 444.00 & 2 & 888 & 1649 & 2.099 & .982 & .018 & 888.00 & 1 & 888 & 849 & 1.580 & .943 & .057 \\
\hline 977 & -- & -- & -- & -- & -- & -- & -- & -- & -- & -- & -- & -- & -- & -- \\
\hline 978 & 157.11 & 85 & 13,354 & 626544 & 1.660 & .952 & .048 & 238.46 & 56 & 13,354 & 431514 & 1.997 & .977 & .023 \\
\hline 979 & 158.75 & 36 & 5,715 & 116666 & 1.394 & .918 & .082 & 197.07 & 29 & 5,715 & 94507 & 1.310 & .905 & .095 \\
\hline 980 & 123.21 & 29 & 3,573 & 55034 & .581 & .719 & .281 & 170.14 & 21 & 3,573 & 33589 & -.831 & .203 & .203 \\
\hline 981 & -- & -- & -- & -- & -- & -- & -- & -- & -- & -- & -- & -- & -- & -- \\
\hline 982 & 193.00 & 2 & 386 & 352 & -.216 & .415 & .415 & 193.00 & 2 & 386 & 352 & -.216 & .415 & .415 \\
\hline 983 & 201.06 & 52 & 10,455 & 253090 & -.861 & .195 & .195 & 387.22 & 27 & 10,455 & 132478 & -.553 & .290 & .290 \\
\hline 984 & 392.67 & 9 & 3,534 & 18044 & .700 & .758 & .242 & 589.00 & 6 & 3,534 & 11856 & .502 & .692 & .308 \\
\hline 985 & 242.40 & 5 & 1,212 & 3885 & 1.093 & .863 & .137 & 606.00 & 2 & 1,212 & 853 & -.726 & .234 & .234 \\
\hline 986 & 288.75 & 12 & 3,465 & 20303 & -.141 & .444 & .444 & 866.25 & 4 & 3,465 & 7567 & .318 & .625 & .375 \\
\hline 987 & 244.27 & 92 & 22,473 & 915543 & -1.900 & .029 & .029 & 368.41 & 61 & 22,473 & 613290 & -1.424 & .077 & .077 \\
\hline 988 & 335.14 & 7 & 2,346 & 8173 & -.021 & .492 & .492 & 391.00 & 6 & 2,346 & 6729 & -.186 & .426 & .426 \\
\hline 989 & 156.88 & 103 & 16,159 & 905214 & 1.543 & .939 & .061 & 273.88 & 59 & 16,159 & 484756 & .225 & .589 & .411 \\
\hline 990 & 216.73 & 86 & 18,639 & 833595 & .644 & .740 & .260 & 423.61 & 44 & 18,639 & 449108 & 1.094 & .863 & .137 \\
\hline 991 & 357.19 & 52 & 18,574 & 552897 & 1.810 & .965 & .035 & 663.36 & 28 & 18,574 & 291409 & 1.106 & .866 & .134 \\
\hline 992 & 172.32 & 116 & 19,989 & 1257285 & 1.576 & .942 & .058 & 263.01 & 76 & 19,989 & 805619 & .915 & .820 & .180 \\
\hline 993 & 342.25 & 4 & 1,369 & 3138 & .506 & .694 & .306 & 684.50 & 2 & 1,369 & 1538 & .302 & .619 & .381 \\
\hline 994 & 343.17 & 18 & 6,177 & 61427 & .771 & .780 & .220 & 514.75 & 12 & 6,177 & 45343 & 1.341 & .910 & .090 \\
\hline 995 & 270.00 & 61 & 16,470 & 482977 & -.521 & .301 & .301 & 588.21 & 28 & 16,470 & 233058 & .099 & .539 & .461 \\
\hline 996 & 389.87 & 74 & 28,850 & 1238825 & 2.392 & .992 & .008 & 601.04 & 48 & 28,850 & 792178 & 1.729 & .958 & .042 \\
\hline 997 & 235.51 & 117 & 27,555 & 1851470 & 2.784 & .997 & .003 & 372.37 & 74 & 27,555 & 1149022 & 1.892 & .971 & .029 \\
\hline 998 & 260.05 & 82 & 21,324 & 949908 & 1.357 & .913 & .087 & 380.79 & 56 & 21,324 & 646790 & 1.079 & .860 & .140 \\
\hline 999 & 192.05 & 19 & 3,649 & 27596 & -1.540 & .062 & .062 & 280.69 & 13 & 3,649 & 21818 & -.500 & .308 & .308 \\
\hline 1000 & 180.16 & 50 & 9,008 & 230634 & .296 & .616 & .384 & 360.32 & 25 & 9,008 & 100232 & -.951 & .171 & .171 \\
\hline 1001 & 219.04 & 90 & 19,714 & 992020 & 1.943 & .974 & .026 & 358.44 & 55 & 19,714 & 644241 & 2.419 & .992 & .008 \\
\hline 1002 & -- & -- & -- & -- & -- & -- & -- & -- & -- & -- & -- & -- & -- & -- \\
\hline 1003 & 473.40 & 43 & 20,356 & 475437 & .981 & .837 & .163 & 848.17 & 24 & 20,356 & 238934 & -.185 & .426 & .426 \\
\hline 1004 & 208.00 & 98 & 20,384 & 958238 & -.697 & .243 & .243 & 304.24 & 67 & 20,384 & 721270 & .797 & .787 & .213 \\
\hline 1005 & 547.33 & 39 & 21,346 & 381146 & -0.912 & .181 & .181 & 928.09 & 23 & 21,346 & 233028 & -0.421 & .337 & .337 \\
\hline 1006 & 157.22 & 18 & 2,830 & 20481 & -1.439 & .075 & .075 & 283.00 & 10 & 2,830 & 14930 & .302 & .619 & .381 \\
\hline 1007 & 188.02 & 45 & 8,461 & 170739 & -1.198 & .115 & .115 & 282.03 & 30 & 8,461 & 114386 & -.937 & .175 & .175 \\
\hline 1008 & 210.52 & 66 & 13,894 & 414298 & -1.357 & .087 & .087 & 338.88 & 41 & 13,894 & 277298 & -.293 & .385 & .385 \\
\hline
\end{tabular}


Table 7. Summary of interoccurrence intervals for daily precipitation thresholds of 2.5 and 3.0 inches-Continued

\begin{tabular}{|c|c|c|c|c|c|c|c|c|c|c|c|c|c|c|}
\hline \multirow[b]{2}{*}{$\begin{array}{l}\text { Seq. } \\
\text { no. }\end{array}$} & \multicolumn{7}{|c|}{ Daily precipitation threshold of 2.5 inches and greater } & \multicolumn{7}{|c|}{ Daily precipitation threshold of 3.0 inches and greater } \\
\hline & $\begin{array}{l}\text { Mean } \\
\text { inter- } \\
\text { occur- } \\
\text { rence } \\
\text { interval } \\
\text { (days) }\end{array}$ & $\begin{array}{l}\text { Total } \\
\text { no. of } \\
\text { events }\end{array}$ & $\begin{array}{l}\text { Total } \\
\text { no. of } \\
\text { days } \\
\text { avail- } \\
\text { able }\end{array}$ & $\begin{array}{l}\text { Sigma } \\
\mathrm{S}\end{array}$ & $\begin{array}{c}\text { Stan- } \\
\text { dard } \\
\text { normal } \\
\text { variate }\end{array}$ & $\begin{array}{l}\text { Non- } \\
\text { ex- } \\
\text { ceed- } \\
\text { ance } \\
\text { prob- } \\
\text { ability }\end{array}$ & $\begin{array}{c}\mathrm{p}- \\
\text { value }\end{array}$ & $\begin{array}{c}\text { Mean } \\
\text { inter- } \\
\text { occur- } \\
\text { rence } \\
\text { interval } \\
\text { (days) }\end{array}$ & $\begin{array}{c}\text { Total } \\
\text { no. of } \\
\text { events }\end{array}$ & $\begin{array}{l}\text { Total } \\
\text { no. of } \\
\text { days } \\
\text { avail- } \\
\text { able }\end{array}$ & $\underset{\mathrm{S}}{\text { Sigma }}$ & $\begin{array}{c}\text { Stan- } \\
\text { dard } \\
\text { normal } \\
\text { variate }\end{array}$ & $\begin{array}{l}\text { Non- } \\
\text { ex- } \\
\text { ceed- } \\
\text { ance } \\
\text { prob- } \\
\text { ability }\end{array}$ & $\begin{array}{c}p- \\
\text { value }\end{array}$ \\
\hline 1009 & 176.44 & 71 & 12,527 & 421705 & -.755 & .225 & .225 & 291.33 & 43 & 12,527 & 243795 & -1.077 & .141 & .141 \\
\hline 1010 & 156.55 & 100 & 15,655 & 723135 & -1.319 & .094 & .094 & 233.66 & 67 & 15,655 & 493278 & -.843 & .200 & .200 \\
\hline 1011 & 340.07 & 60 & 20,404 & 636056 & .525 & .700 & .300 & 618.30 & 33 & 20,404 & 323636 & -.385 & .350 & .350 \\
\hline 1012 & 427.57 & 7 & 2,993 & 13692 & 1.407 & .920 & .080 & 997.67 & 3 & 2,993 & 4928 & .293 & .615 & .385 \\
\hline 1013 & 331.06 & 33 & 10,925 & 160991 & -1.064 & .144 & .144 & 496.59 & 22 & 10,925 & 98277 & -1.480 & .069 & .069 \\
\hline 1014 & 195.17 & 12 & 2,342 & 16307 & .963 & .832 & .168 & 292.75 & 8 & 2,342 & 10711 & .702 & .759 & .241 \\
\hline 1015 & 427.00 & 1 & 427 & 208 & -.045 & .482 & .482 & 427.00 & 1 & 427 & 208 & -.045 & .482 & .482 \\
\hline 1016 & 183.83 & 109 & 20,037 & 1114116 & .366 & .643 & .357 & 323.18 & 62 & 20,037 & 644480 & .512 & .696 & .304 \\
\hline 1017 & 373.40 & 5 & 1,867 & 5265 & .496 & .690 & .310 & 373.40 & 5 & 1,867 & 5265 & .496 & .690 & .310 \\
\hline 1018 & 161.40 & 15 & 2,421 & 16296 & -.688 & .246 & .246 & 201.75 & 12 & 2,421 & 13471 & -.436 & .332 & .332 \\
\hline 1019 & 537.47 & 15 & 8,062 & 58019 & -.271 & .393 & .393 & 806.20 & 10 & 8,062 & 37309 & -.408 & .342 & .342 \\
\hline 1020 & 981.33 & 6 & 5,888 & 16295 & -.329 & .371 & .371 & 1472.00 & 4 & 5,888 & 12829 & .310 & .622 & .378 \\
\hline 1021 & 551.21 & 44 & 24,253 & 587622 & 1.164 & .878 & .122 & 1010.54 & 24 & 24,253 & 309259 & .531 & .702 & .298 \\
\hline 1022 & 186.32 & 53 & 9,875 & 270568 & .428 & .666 & .334 & 365.74 & 27 & 9,875 & 135784 & .167 & .566 & .434 \\
\hline 1023 & 232.41 & 29 & 6,740 & 69882 & -2.658 & .004 & .004 & 337.00 & 20 & 6,740 & 46631 & -2.387 & .008 & .008 \\
\hline 1024 & 205.74 & 57 & 11,727 & 349669 & .605 & .727 & .273 & 344.91 & 34 & 11,727 & 186611 & -.646 & .259 & .259 \\
\hline 1025 & 470.67 & 6 & 2,824 & 11607 & 1.570 & .942 & .058 & 706.00 & 4 & 2,824 & 7265 & .992 & .839 & .161 \\
\hline 1026 & 254.85 & 75 & 19,114 & 781552 & 1.356 & .912 & .088 & 406.68 & 47 & 19,114 & 500729 & 1.363 & .914 & .087 \\
\hline 1027 & 307.18 & 22 & 6,758 & 66917 & -.811 & .209 & .209 & 563.17 & 12 & 6,758 & 32366 & -1.211 & .113 & .113 \\
\hline 1028 & 463.93 & 59 & 27,372 & 785252 & -.366 & .357 & .357 & 977.57 & 28 & 27,372 & 397682 & .346 & .635 & .365 \\
\hline 1029 & 242.94 & 64 & 15,548 & 553133 & 1.548 & .939 & .061 & 420.22 & 37 & 15,548 & 344440 & 2.081 & .981 & .019 \\
\hline 1030 & 214.84 & 57 & 12,246 & 378241 & 1.095 & .863 & .137 & 395.03 & 31 & 12,246 & 205875 & .816 & .793 & .207 \\
\hline 1031 & 2538.00 & 1 & 2,538 & 1142 & -.173 & .431 & .431 & -- & -- & -- & -- & -- & -- & -- \\
\hline 1032 & 254.85 & 143 & 36,443 & 2680884 & .598 & .725 & .275 & 418.89 & 87 & 36,443 & 1652113 & .681 & .752 & .248 \\
\hline 1033 & 168.77 & 127 & 21,434 & 1518922 & 2.264 & .988 & .012 & 285.79 & 75 & 21,434 & 902804 & 1.848 & .968 & .032 \\
\hline 1034 & 541.00 & 4 & 2,164 & 3908 & -.336 & .368 & .368 & 1082.00 & 2 & 2,164 & 2548 & .435 & .668 & .332 \\
\hline 1035 & 341.47 & 99 & 33,805 & 1561477 & -1.152 & .125 & .125 & 545.24 & 62 & 33,805 & 925140 & -1.598 & .055 & .055 \\
\hline 1036 & 76.57 & 7 & 536 & 2083 & .506 & .693 & .307 & 134.00 & 4 & 536 & 1186 & .368 & .644 & .356 \\
\hline 1037 & 106.50 & 4 & 426 & 440 & -1.675 & .047 & .047 & 106.50 & 4 & 426 & 440 & -1.675 & .047 & .047 \\
\hline 1038 & 231.40 & 15 & 3,471 & 24601 & -.369 & .356 & .356 & 433.88 & 8 & 3,471 & 14728 & .298 & .617 & .383 \\
\hline 1039 & -- & -- & -- & -- & -- & -- & -- & -- & -- & -- & -- & -- & -- & -- \\
\hline 1040 & 1717.75 & 4 & 6,871 & 19283 & 1.397 & .919 & .081 & 3435.50 & 2 & 6,871 & 8942 & .738 & .770 & .230 \\
\hline 1041 & -- & -- & -- & -- & -- & -- & -- & -- & -- & -- & -- & -- & -- & -- \\
\hline 1042 & 108.90 & 114 & 12,414 & 744369 & .961 & .832 & .168 & 172.42 & 72 & 12,414 & 478346 & 1.034 & .849 & .151 \\
\hline 1043 & -- & -- & -- & -- & -- & -- & -- & -- & -- & -- & -- & -- & -- & -- \\
\hline 1044 & 744.36 & 11 & 8,188 & 39842 & -.662 & .254 & .254 & 1637.60 & 5 & 8,188 & 15234 & -.991 & .161 & .161 \\
\hline 1045 & 952.33 & 21 & 19,999 & 202155 & -.296 & .384 & .384 & 1999.90 & 10 & 19,999 & 97993 & -.110 & .456 & .456 \\
\hline 1046 & 170.60 & 10 & 1,706 & 10357 & 1.173 & .880 & .120 & 213.25 & 8 & 1,706 & 8696 & 1.344 & .910 & .090 \\
\hline 1047 & 252.64 & 80 & 20,211 & 857101 & .933 & .824 & .176 & 404.22 & 50 & 20,211 & 508054 & .067 & .527 & .473 \\
\hline 1048 & 353.24 & 34 & 12,010 & 209462 & .262 & .603 & .397 & 706.47 & 17 & 12,010 & 109060 & .488 & .687 & .313 \\
\hline 1049 & 306.06 & 16 & 4,897 & 37920 & -.222 & .412 & .412 & 349.79 & 14 & 4,897 & 33447 & -.157 & .437 & .437 \\
\hline 1050 & 146.87 & 82 & 12,043 & 533487 & 1.262 & .896 & .104 & 280.07 & 43 & 12,043 & 275323 & .719 & .764 & .236 \\
\hline 1051 & 255.21 & 70 & 17,865 & 629107 & .089 & .535 & .465 & 372.19 & 48 & 17,865 & 414089 & -.411 & .341 & .341 \\
\hline 1052 & 241.33 & 3 & 724 & 483 & -1.666 & .048 & .048 & -- & -- & -- & -- & -- & -- & -- \\
\hline 1053 & 130.50 & 8 & 1,044 & 4268 & 0.108 & .543 & .457 & 348.00 & 3 & 1,044 & 2145 & 1.109 & .866 & .134 \\
\hline 1054 & -- & -- & -- & -- & -- & -- & -- & -- & -- & -- & -- & -- & -- & -- \\
\hline 1055 & 152.00 & 22 & 3,344 & 41461 & 1.033 & .849 & .151 & 222.93 & 15 & 3,344 & 28758 & .984 & .837 & .163 \\
\hline 1056 & 300.29 & 7 & 2,102 & 6830 & -.328 & .371 & .371 & 420.40 & 5 & 2,102 & 3562 & -1.248 & .106 & .106 \\
\hline
\end{tabular}


Table 7. Summary of interoccurrence intervals for daily precipitation thresholds of 2.5 and 3.0 inches—Continued

\begin{tabular}{|c|c|c|c|c|c|c|c|c|c|c|c|c|c|c|}
\hline \multirow[b]{2}{*}{$\begin{array}{l}\text { Seq. } \\
\text { no. }\end{array}$} & \multicolumn{7}{|c|}{ Daily precipitation threshold of 2.5 inches and greater } & \multicolumn{7}{|c|}{ Daily precipitation threshold of 3.0 inches and greater } \\
\hline & $\begin{array}{c}\text { Mean } \\
\text { inter- } \\
\text { occur- } \\
\text { rence } \\
\text { interval } \\
\text { (days) }\end{array}$ & $\begin{array}{c}\text { Total } \\
\text { no. of } \\
\text { events }\end{array}$ & $\begin{array}{l}\text { Total } \\
\text { no. of } \\
\text { days } \\
\text { avail- } \\
\text { able }\end{array}$ & $\begin{array}{l}\text { Sigma } \\
\mathrm{S}\end{array}$ & $\begin{array}{c}\text { Stan- } \\
\text { dard } \\
\text { normal } \\
\text { variate }\end{array}$ & $\begin{array}{l}\text { Non- } \\
\text { ex- } \\
\text { ceed- } \\
\text { ance } \\
\text { prob- } \\
\text { ability }\end{array}$ & $\begin{array}{c}\text { p- } \\
\text { value }\end{array}$ & $\begin{array}{l}\text { Mean } \\
\text { inter- } \\
\text { occur- } \\
\text { rence } \\
\text { interval } \\
\text { (days) }\end{array}$ & $\begin{array}{l}\text { Total } \\
\text { no. of } \\
\text { events }\end{array}$ & $\begin{array}{c}\text { Total } \\
\text { no. of } \\
\text { days } \\
\text { avail- } \\
\text { able }\end{array}$ & $\begin{array}{l}\text { Sigma } \\
\mathrm{S}\end{array}$ & $\begin{array}{c}\text { Stan- } \\
\text { dard } \\
\text { normal } \\
\text { variate }\end{array}$ & $\begin{array}{l}\text { Non- } \\
\text { ex- } \\
\text { ceed- } \\
\text { ance } \\
\text { prob- } \\
\text { ability }\end{array}$ & $\begin{array}{c}\text { p- } \\
\text { value }\end{array}$ \\
\hline 1057 & 508.00 & 1 & 508 & 29 & -1.534 & .063 & .063 & -- & -- & -- & -- & -- & -- & -- \\
\hline 1058 & 225.94 & 156 & 35,247 & 2807754 & .460 & .677 & .323 & 367.16 & 96 & 35,247 & 1724461 & .327 & .628 & .372 \\
\hline 1059 & 178.75 & 4 & 715 & 664 & -1.856 & .032 & .032 & 238.33 & 3 & 715 & 574 & -1.394 & .082 & .082 \\
\hline 1060 & 384.65 & 63 & 24,233 & 791852 & .514 & .696 & .304 & 757.28 & 32 & 24,233 & 335016 & -1.332 & .091 & .091 \\
\hline 1061 & -- & -- & -- & -- & -- & -- & -- & -- & -- & -- & -- & -- & -- & -- \\
\hline 1062 & 2080.00 & 1 & 2,080 & 1088 & .080 & .532 & .468 & -- & -- & -- & -- & -- & -- & -- \\
\hline 1063 & 1084.96 & 22 & 23,869 & 334745 & 2.234 & .987 & .013 & 1491.81 & 16 & 23,869 & 243956 & 1.923 & .973 & .027 \\
\hline 1064 & -- & -- & -- & -- & -- & -- & -- & -- & -- & -- & -- & -- & -- & -- \\
\hline 1065 & 242.80 & 25 & 6,070 & 76278 & .046 & .518 & .482 & 466.92 & 13 & 6,070 & 41914 & .389 & .651 & .349 \\
\hline 1066 & 258.44 & 43 & 11,113 & 262200 & 1.106 & .866 & .134 & 444.52 & 25 & 11,113 & 164366 & 1.587 & .944 & .056 \\
\hline 1067 & 183.40 & 5 & 917 & 2370 & .131 & .552 & .448 & 305.67 & 3 & 917 & 1418 & .093 & .537 & .463 \\
\hline 1068 & 358.00 & 2 & 716 & 365 & -1.201 & .115 & .115 & 716.00 & 1 & 716 & 102 & -1.239 & .108 & .108 \\
\hline 1069 & 301.00 & 18 & 5,418 & 57351 & 1.294 & .902 & .098 & 602.00 & 9 & 5,418 & 21018 & -.717 & .237 & .237 \\
\hline 1070 & 242.93 & 145 & 35,225 & 2573640 & .162 & .564 & .436 & 409.59 & 86 & 35,225 & 1539234 & .260 & .603 & .397 \\
\hline 1071 & 200.32 & 98 & 19,631 & 994956 & .589 & .722 & .278 & 311.60 & 63 & 19,631 & 655151 & .818 & .793 & .207 \\
\hline 1072 & 238.29 & 7 & 1,668 & 3938 & -1.491 & .068 & .068 & 834.00 & 2 & 1,668 & 629 & -1.526 & .064 & .064 \\
\hline 1073 & -- & -- & -- & -- & -- & -- & -- & -- & -- & -- & -- & -- & -- & -- \\
\hline 1074 & 159.29 & 189 & 30,105 & 2784968 & -.502 & .308 & .308 & 244.76 & 123 & 30,105 & 1912424 & .633 & .736 & .264 \\
\hline 1075 & 517.80 & 5 & 2,589 & 7112 & .383 & .649 & .351 & 863.00 & 3 & 2,589 & 4372 & .377 & .647 & .353 \\
\hline 1076 & 273.44 & 66 & 18,047 & 632922 & .883 & .811 & .189 & 410.16 & 44 & 18,047 & 414627 & .509 & .695 & .305 \\
\hline 1077 & 245.57 & 14 & 3,438 & 24114 & .013 & .505 & .495 & 343.80 & 10 & 3,438 & 16247 & -.301 & .382 & .382 \\
\hline 1078 & 809.21 & 34 & 27,513 & 491466 & .513 & .696 & .304 & 2501.18 & 11 & 27,513 & 159792 & .322 & .626 & .374 \\
\hline 1079 & 376.99 & 83 & 31,290 & 1325097 & .323 & .627 & .373 & 782.25 & 40 & 31,290 & 695338 & 1.217 & .888 & .112 \\
\hline 1080 & 161.33 & 3 & 484 & 1144 & 1.727 & .958 & .042 & 161.33 & 3 & 484 & 1144 & 1.727 & .958 & .042 \\
\hline 1081 & 530.80 & 40 & 21,232 & 393100 & -.814 & .208 & .208 & 1179.56 & 18 & 21,232 & 193660 & .099 & .539 & .461 \\
\hline 1082 & 684.75 & 20 & 13,695 & 167113 & 1.706 & .956 & .044 & 1369.50 & 10 & 13,695 & 95724 & 2.180 & .985 & .015 \\
\hline 1083 & 964.38 & 16 & 15,430 & 132777 & .524 & .700 & .300 & 1928.75 & 8 & 15,430 & 79221 & 1.389 & .918 & .082 \\
\hline 1084 & 135.22 & 9 & 1,217 & 5255 & -.210 & .417 & .417 & 152.13 & 8 & 1,217 & 4106 & -.767 & .222 & .222 \\
\hline 1085 & 220.21 & 165 & 36,335 & 3007268 & .072 & .529 & .471 & 399.29 & 91 & 36,335 & 1736087 & .828 & .796 & .204 \\
\hline 1086 & 504.90 & 10 & 5,049 & 24453 & -.172 & .432 & .432 & 1262.25 & 4 & 5,049 & 8646 & -.498 & .309 & .309 \\
\hline 1087 & 3512.50 & 4 & 14,050 & 21441 & -.821 & .206 & .206 & 14050.00 & 1 & 14,050 & 6418 & -.150 & .440 & .440 \\
\hline 1088 & 112.60 & 20 & 2,252 & 24103 & .545 & .707 & .293 & 187.67 & 12 & 2,252 & 13760 & .110 & .544 & .456 \\
\hline 1089 & 593.00 & 2 & 1,186 & 903 & -.585 & .279 & .279 & 1186.00 & 1 & 1,186 & 127 & -1.361 & .087 & .087 \\
\hline 1090 & 424.17 & 24 & 10,180 & 133418 & .782 & .783 & .217 & 598.82 & 17 & 10,180 & 104565 & 1.489 & .932 & .068 \\
\hline 1091 & 895.46 & 22 & 19,700 & 193002 & -.888 & .187 & .187 & 1970.00 & 10 & 19,700 & 76634 & -1.216 & .112 & .112 \\
\hline 1092 & -- & -- & -- & -- & -- & -- & -- & -- & -- & -- & -- & -- & -- & -- \\
\hline 1093 & 158.57 & 30 & 4,757 & 53996 & -2.308 & .011 & .011 & 237.85 & 20 & 4,757 & 37511 & -1.638 & .051 & .051 \\
\hline 1094 & 168.67 & 153 & 25,807 & 2221893 & 2.688 & .996 & .004 & 260.68 & 99 & 25,807 & 1390734 & 1.528 & .937 & .063 \\
\hline 1095 & 152.15 & 13 & 1,978 & 13865 & .490 & .688 & .312 & 247.25 & 8 & 1,978 & 8338 & .264 & .604 & .396 \\
\hline 1096 & 658.76 & 29 & 19,104 & 288596 & .390 & .652 & .348 & 1469.54 & 13 & 19,104 & 142830 & .938 & .826 & .174 \\
\hline 1097 & 250.37 & 76 & 19,028 & 812215 & 1.862 & .969 & .031 & 396.42 & 48 & 19,028 & 489397 & .860 & .805 & .195 \\
\hline 1098 & 289.69 & 51 & 14,774 & 306031 & -2.322 & .010 & .010 & 568.23 & 26 & 14,774 & 171346 & -.953 & .170 & .170 \\
\hline 1099 & -- & -- & -- & -- & -- & -- & -- & -- & -- & -- & -- & -- & -- & -- \\
\hline 1100 & 235.00 & 12 & 2,820 & 20504 & 1.271 & .898 & .102 & 470.00 & 6 & 2,820 & 11325 & 1.437 & .925 & .075 \\
\hline 1101 & 208.56 & 140 & 29,198 & 2013381 & -0.306 & .380 & .380 & 351.78 & 83 & 29,198 & 1213065 & 0.018 & .507 & .493 \\
\hline 1102 & 644.10 & 49 & 31,561 & 818145 & .704 & .759 & .241 & 1502.91 & 21 & 31,561 & 364939 & .804 & .789 & .211 \\
\hline 1103 & 3488.67 & 3 & 10,466 & 17292 & .304 & .620 & .380 & -- & -- & -- & -- & -- & -- & -- \\
\hline 1104 & 217.00 & 36 & 7,812 & 150738 & .748 & .773 & .227 & 289.33 & 27 & 7,812 & 114332 & .757 & .775 & .225 \\
\hline
\end{tabular}


Table 7. Summary of interoccurrence intervals for daily precipitation thresholds of 2.5 and 3.0 inches-Continued

\begin{tabular}{|c|c|c|c|c|c|c|c|c|c|c|c|c|c|c|}
\hline \multirow[b]{2}{*}{$\begin{array}{l}\text { Seq. } \\
\text { no. }\end{array}$} & \multicolumn{7}{|c|}{ Daily precipitation threshold of 2.5 inches and greater } & \multicolumn{7}{|c|}{ Daily precipitation threshold of 3.0 inches and greater } \\
\hline & $\begin{array}{l}\text { Mean } \\
\text { inter- } \\
\text { occur- } \\
\text { rence } \\
\text { interval } \\
\text { (days) }\end{array}$ & $\begin{array}{c}\text { Total } \\
\text { no. of } \\
\text { events }\end{array}$ & $\begin{array}{l}\text { Total } \\
\text { no. of } \\
\text { days } \\
\text { avail- } \\
\text { able }\end{array}$ & $\begin{array}{l}\text { Sigma } \\
\mathbf{S}\end{array}$ & $\begin{array}{c}\text { Stan- } \\
\text { dard } \\
\text { normal } \\
\text { variate }\end{array}$ & $\begin{array}{l}\text { Non- } \\
\text { ex- } \\
\text { ceed- } \\
\text { ance } \\
\text { prob- } \\
\text { ability }\end{array}$ & $\begin{array}{c}\text { p- } \\
\text { value }\end{array}$ & $\begin{array}{c}\text { Mean } \\
\text { inter- } \\
\text { occur- } \\
\text { rence } \\
\text { interval } \\
\text { (days) }\end{array}$ & $\begin{array}{c}\text { Total } \\
\text { no. of } \\
\text { events }\end{array}$ & $\begin{array}{l}\text { Total } \\
\text { no. of } \\
\text { days } \\
\text { avail- } \\
\text { able }\end{array}$ & $\begin{array}{l}\text { Sigma } \\
\mathrm{S}\end{array}$ & $\begin{array}{l}\text { Stan- } \\
\text { dard } \\
\text { normal } \\
\text { variate }\end{array}$ & $\begin{array}{l}\text { Non- } \\
\text { ex- } \\
\text { ceed- } \\
\text { ance } \\
\text { prob- } \\
\text { ability }\end{array}$ & $\begin{array}{c}p- \\
\text { value }\end{array}$ \\
\hline 1105 & 275.90 & 48 & 13,243 & 293559 & -.916 & .180 & .180 & 472.96 & 28 & 13,243 & 153480 & -1.578 & .057 & .057 \\
\hline 1106 & 459.00 & 49 & 22,491 & 518642 & -.713 & .238 & .238 & 775.55 & 29 & 22,491 & 334601 & .243 & .596 & .404 \\
\hline 1107 & 536.67 & 3 & 1,610 & 1656 & -.943 & .173 & .173 & 805.00 & 2 & 1,610 & 1311 & -.455 & .325 & .325 \\
\hline 1108 & -- & -- & -- & -- & -- & -- & -- & -- & -- & -- & -- & -- & -- & -- \\
\hline 1109 & 582.17 & 6 & 3,493 & 10286 & -.078 & .469 & .469 & 1164.33 & 3 & 3,493 & 4270 & -.555 & .289 & .289 \\
\hline 1110 & 319.09 & 11 & 3,510 & 14867 & -1.321 & .093 & .093 & 501.43 & 7 & 3,510 & 9955 & -.869 & .192 & .192 \\
\hline 1111 & 750.67 & 3 & 2,252 & 2172 & -1.071 & .142 & .142 & 2252.00 & 1 & 2,252 & 264 & -1.326 & .092 & .092 \\
\hline 1112 & 141.37 & 85 & 12,016 & 564634 & 1.687 & .954 & .046 & 235.61 & 51 & 12,016 & 331172 & 1.000 & .841 & .159 \\
\hline 1113 & 867.59 & 34 & 29,498 & 510778 & .188 & .574 & .426 & 1404.67 & 21 & 29,498 & 333633 & .613 & .730 & .270 \\
\hline 1114 & 281.95 & 43 & 12,124 & 264763 & .179 & .571 & .429 & 484.96 & 25 & 12,124 & 160900 & .534 & .703 & .297 \\
\hline 1115 & 1162.60 & 5 & 5,813 & 13637 & -.239 & .406 & .406 & 2906.50 & 2 & 5,813 & 5390 & -.178 & .429 & .429 \\
\hline 1116 & 290.60 & 5 & 1,453 & 3404 & -.244 & .404 & .404 & 363.25 & 4 & 1,453 & 2639 & -.318 & .375 & .375 \\
\hline 1117 & 209.69 & 75 & 15,727 & 626017 & .922 & .822 & .178 & 327.65 & 48 & 15,727 & 390827 & .425 & .665 & .335 \\
\hline 1118 & 266.67 & 3 & 800 & 845 & -.888 & .187 & .187 & 266.67 & 3 & 800 & 845 & -.888 & .187 & .187 \\
\hline 1119 & 344.82 & 22 & 7,586 & 84775 & .129 & .552 & .448 & 689.64 & 11 & 7,586 & 44811 & .425 & .665 & .335 \\
\hline 1120 & 698.91 & 35 & 24,462 & 434611 & .156 & .562 & .438 & 1630.80 & 15 & 24,462 & 202501 & .696 & .757 & .243 \\
\hline 1121 & 494.58 & 60 & 29,675 & 834804 & -.836 & .202 & .202 & 847.86 & 35 & 29,675 & 541244 & .433 & .667 & .333 \\
\hline 1122 & 340.33 & 3 & 1,021 & 1194 & -.661 & .254 & .254 & 1021.00 & 1 & 1,021 & 241 & -.914 & .180 & .180 \\
\hline 1123 & 393.17 & 58 & 22,804 & 654926 & -.128 & .449 & .449 & 735.61 & 31 & 22,804 & 339870 & -.371 & .355 & .355 \\
\hline 1124 & 457.00 & 3 & 1,371 & 2994 & 1.368 & .914 & .086 & -- & -- & -- & -- & -- & -- & -- \\
\hline 1125 & 313.33 & 9 & 2,820 & 10877 & -.742 & .229 & .229 & 564.00 & 5 & 2,820 & 7916 & .476 & .683 & .317 \\
\hline 1126 & 429.22 & 9 & 3,863 & 16506 & -.262 & .397 & .397 & 551.86 & 7 & 3,863 & 10129 & -1.150 & .125 & .125 \\
\hline 1127 & 667.40 & 40 & 26,696 & 412280 & -2.496 & .006 & .006 & 1160.70 & 23 & 26,696 & 218401 & -2.397 & .008 & .008 \\
\hline 1128 & 567.12 & 17 & 9,641 & 74203 & -.675 & .250 & .250 & 964.10 & 10 & 9,641 & 46106 & -.239 & .406 & .406 \\
\hline 1129 & 599.67 & 6 & 3,598 & 12931 & .840 & .799 & .201 & 899.50 & 4 & 3,598 & 10187 & 1.440 & .925 & .075 \\
\hline 1130 & -- & -- & -- & -- & -- & -- & -- & -- & -- & -- & -- & -- & -- & -- \\
\hline 1131 & 247.38 & 56 & 13,853 & 430543 & 1.426 & .923 & .077 & 461.77 & 30 & 13,853 & 231654 & 1.089 & .862 & .138 \\
\hline 1132 & -- & -- & -- & -- & -- & -- & -- & -- & -- & -- & -- & -- & -- & -- \\
\hline 1133 & 234.84 & 43 & 10,098 & 191113 & -1.360 & .087 & .087 & 374.00 & 27 & 10,098 & 121751 & -.962 & .168 & .168 \\
\hline 1134 & -- & -- & -- & -- & -- & -- & -- & -- & -- & -- & -- & -- & -- & -- \\
\hline 1135 & 112.71 & 7 & 789 & 2642 & -.198 & .421 & .421 & 197.25 & 4 & 789 & 1536 & -.092 & .463 & .463 \\
\hline 1136 & 1393.75 & 20 & 27,875 & 294456 & .436 & .669 & .331 & 3484.38 & 8 & 27,875 & 136342 & 1.092 & .862 & .138 \\
\hline 1137 & 341.88 & 56 & 19,145 & 591056 & 1.330 & .908 & .092 & 736.35 & 26 & 19,145 & 247767 & -.040 & .484 & .484 \\
\hline 1138 & -- & -- & -- & -- & -- & -- & -- & -- & -- & -- & -- & -- & -- & -- \\
\hline 1139 & -- & -- & -- & -- & -- & -- & -- & -- & -- & -- & -- & -- & -- & -- \\
\hline 1140 & 397.75 & 28 & 11,137 & 142630 & -.781 & .217 & .217 & 655.12 & 17 & 11,137 & 64925 & -2.244 & .012 & .012 \\
\hline 1141 & 150.11 & 133 & 19,965 & 1468634 & 2.121 & .983 & .017 & 249.56 & 80 & 19,965 & 901683 & 2.000 & .977 & .023 \\
\hline 1142 & 162.76 & 201 & 32,715 & 3537710 & 1.866 & .969 & .031 & 268.16 & 122 & 32,715 & 2132713 & 1.314 & .906 & .094 \\
\hline 1143 & 1502.50 & 6 & 9,015 & 20147 & -1.082 & .140 & .140 & 2253.75 & 4 & 9,015 & 10291 & -1.487 & .069 & .069 \\
\hline 1144 & 240.10 & 10 & 2,401 & 13040 & .472 & .682 & .318 & 400.17 & 6 & 2,401 & 8048 & .498 & .691 & .309 \\
\hline 1145 & 714.50 & 2 & 1,429 & 1602 & .297 & .617 & .383 & 1429.00 & 1 & 1,429 & 962 & .600 & .726 & .274 \\
\hline 1146 & 665.58 & 40 & 26,623 & 502463 & -.617 & .269 & .269 & 1023.96 & 26 & 26,623 & 326117 & -.510 & .305 & .305 \\
\hline 1147 & 365.18 & 17 & 6,208 & 52912 & .020 & .508 & .492 & 620.80 & 10 & 6,208 & 33995 & .521 & 699 & .301 \\
\hline 1148 & 764.71 & 17 & 13,000 & 112287 & .116 & .546 & .454 & 2166.67 & 6 & 13,000 & 41468 & .269 & .606 & .394 \\
\hline 1149 & 281.02 & 43 & 12,084 & 285524 & 1.124 & .870 & .131 & 464.77 & 26 & 12,084 & 171988 & 0.838 & .799 & .201 \\
\hline 1150 & 960.21 & 14 & 13,443 & 104158 & .693 & .756 & .244 & 1680.38 & 8 & 13,443 & 65307 & 1.051 & .853 & .147 \\
\hline 1151 & 380.67 & 3 & 1,142 & 1697 & -.028 & .489 & .489 & 571.00 & 2 & 1,142 & 1287 & .311 & .622 & .378 \\
\hline 1152 & 256.95 & 103 & 26,466 & 1416137 & .685 & .753 & .247 & 413.53 & 64 & 26,466 & 898097 & .837 & .799 & .201 \\
\hline
\end{tabular}


Table 7. Summary of interoccurrence intervals for daily precipitation thresholds of 2.5 and 3.0 inches—Continued

\begin{tabular}{|c|c|c|c|c|c|c|c|c|c|c|c|c|c|c|}
\hline \multirow[b]{2}{*}{$\begin{array}{l}\text { Seq. } \\
\text { no. }\end{array}$} & \multicolumn{7}{|c|}{ Daily precipitation threshold of 2.5 inches and greater } & \multicolumn{7}{|c|}{ Daily precipitation threshold of 3.0 inches and greater } \\
\hline & $\begin{array}{l}\text { Mean } \\
\text { inter- } \\
\text { occur- } \\
\text { rence } \\
\text { interval } \\
\text { (days) }\end{array}$ & $\begin{array}{l}\text { Total } \\
\text { no. of } \\
\text { events }\end{array}$ & $\begin{array}{l}\text { Total } \\
\text { no. of } \\
\text { days } \\
\text { avail- } \\
\text { able }\end{array}$ & $\begin{array}{l}\text { Sigma } \\
\mathrm{S}\end{array}$ & $\begin{array}{c}\text { Stan- } \\
\text { dard } \\
\text { normal } \\
\text { variate }\end{array}$ & $\begin{array}{l}\text { Non- } \\
\text { ex- } \\
\text { ceed- } \\
\text { ance } \\
\text { prob- } \\
\text { ability }\end{array}$ & $\begin{array}{c}\mathrm{p}- \\
\text { value }\end{array}$ & $\begin{array}{c}\text { Mean } \\
\text { inter- } \\
\text { occur- } \\
\text { rence } \\
\text { interval } \\
\text { (days) }\end{array}$ & $\begin{array}{c}\text { Total } \\
\text { no. of } \\
\text { events }\end{array}$ & $\begin{array}{c}\text { Total } \\
\text { no. of } \\
\text { days } \\
\text { avail- } \\
\text { able }\end{array}$ & $\begin{array}{l}\text { Sigma } \\
\mathrm{S}\end{array}$ & $\begin{array}{c}\text { Stan- } \\
\text { dard } \\
\text { normal } \\
\text { variate }\end{array}$ & $\begin{array}{l}\text { Non- } \\
\text { ex- } \\
\text { ceed- } \\
\text { ance } \\
\text { prob- } \\
\text { ability }\end{array}$ & $\begin{array}{c}p- \\
\text { value }\end{array}$ \\
\hline 1153 & 81.17 & 6 & 487 & 1493 & .093 & .537 & .463 & 162.33 & 3 & 487 & 867 & .561 & .712 & .288 \\
\hline 1154 & 372.60 & 35 & 13,041 & 229232 & .046 & .518 & .482 & 652.05 & 20 & 13,041 & 101540 & -1.715 & .043 & .043 \\
\hline 1155 & 171.13 & 8 & 1,369 & 4232 & -1.113 & .133 & .133 & 273.80 & 5 & 1,369 & 1806 & -1.829 & .034 & .034 \\
\hline 1156 & 295.75 & 44 & 13,013 & 271996 & -.574 & .283 & .283 & 448.72 & 29 & 13,013 & 157221 & -1.556 & .060 & .060 \\
\hline 1157 & 501.31 & 36 & 18,047 & 350315 & .815 & .792 & .208 & 859.38 & 21 & 18,047 & 211563 & .924 & .822 & .178 \\
\hline 1158 & 209.53 & 17 & 3,562 & 36194 & 1.396 & .919 & .081 & 296.83 & 12 & 3,562 & 27437 & 1.703 & .956 & .044 \\
\hline 1159 & 230.31 & 160 & 36,849 & 2895670 & -.388 & .349 & .349 & 414.03 & 89 & 36,849 & 1571749 & -.678 & .249 & .249 \\
\hline 1160 & 467.00 & 3 & 1,401 & 918 & -1.690 & .046 & .046 & 1401.00 & 1 & 1,401 & 28 & -1.663 & .048 & .048 \\
\hline 1161 & 440.00 & 13 & 5,720 & 37280 & .017 & .507 & .493 & 715.00 & 8 & 5,720 & 16713 & -1.321 & .093 & .093 \\
\hline 1162 & -- & -- & -- & -- & -- & -- & -- & -- & -- & -- & -- & -- & -- & -- \\
\hline 1163 & -- & -- & -- & -- & -- & -- & -- & -- & -- & -- & -- & -- & -- & -- \\
\hline 1164 & 189.70 & 101 & 19,160 & 1027654 & 1.081 & .860 & .140 & 294.77 & 65 & 19,160 & 697767 & 1.683 & .954 & .046 \\
\hline 1165 & 662.27 & 15 & 9,934 & 78612 & .370 & .644 & .356 & 1241.75 & 8 & 9,934 & 37956 & -.220 & .413 & .413 \\
\hline 1166 & 176.31 & 68 & 11,989 & 430619 & .806 & .790 & .210 & 278.81 & 43 & 11,989 & 281174 & 1.032 & .849 & .151 \\
\hline 1167 & 198.56 & 34 & 6,751 & 101691 & -1.151 & .125 & .125 & 375.06 & 18 & 6,751 & 55580 & -.626 & .266 & .266 \\
\hline 1168 & 1249.00 & 2 & 2,498 & 2278 & -.216 & .415 & .415 & 2498.00 & 1 & 2,498 & 2037 & 1.093 & .863 & .137 \\
\hline 1169 & -- & -- & -- & -- & -- & -- & -- & -- & -- & -- & -- & -- & -- & -- \\
\hline 1170 & 163.73 & 101 & 16,537 & 874084 & .812 & .792 & .208 & 246.82 & 67 & 16,537 & 534649 & -.495 & .310 & .310 \\
\hline 1171 & 272.16 & 45 & 12,247 & 299264 & 1.000 & .841 & .159 & 532.48 & 23 & 12,247 & 160007 & 1.130 & .871 & .129 \\
\hline 1172 & 208.23 & 93 & 19,365 & 952875 & .972 & .834 & .166 & 339.74 & 57 & 19,365 & 565318 & .318 & .625 & .375 \\
\hline 1173 & 290.04 & 82 & 23,783 & 955372 & -.317 & .375 & .375 & 495.48 & 48 & 23,783 & 521110 & -1.045 & .148 & .148 \\
\hline 1174 & 282.22 & 18 & 5,080 & 52803 & 1.138 & .872 & .128 & 508.00 & 10 & 5,080 & 29841 & .958 & .831 & .169 \\
\hline 1175 & 467.67 & 58 & 27,125 & 748834 & -.634 & .263 & .263 & 821.97 & 33 & 27,125 & 457414 & .219 & .587 & .413 \\
\hline 1176 & -- & -- & -- & -- & -- & -- & -- & -- & -- & -- & -- & -- & -- & -- \\
\hline 1177 & 203.00 & 7 & 1,421 & 4861 & -.104 & .459 & .459 & 284.20 & 5 & 1,421 & 3703 & .164 & .565 & .435 \\
\hline 1178 & 297.47 & 60 & 17,848 & 579849 & 1.113 & .867 & .133 & 482.38 & 37 & 17,848 & 363249 & 1.055 & .854 & .146 \\
\hline 1179 & 398.48 & 44 & 17,533 & 362864 & -.681 & .248 & .248 & 730.54 & 24 & 17,533 & 216652 & .252 & .600 & .400 \\
\hline 1180 & 558.33 & 3 & 1,675 & 2682 & .202 & .580 & .420 & 1675.00 & 1 & 1,675 & 1559 & 1.492 & .932 & .068 \\
\hline 1181 & 602.50 & 2 & 1,205 & 886 & -.649 & .258 & .258 & 1205.00 & 1 & 1,205 & 826 & .643 & .740 & .260 \\
\hline 1182 & 134.46 & 72 & 9,681 & 343883 & -.195 & .423 & .423 & 268.92 & 36 & 9,681 & 192904 & 1.112 & .867 & .133 \\
\hline 1183 & 164.76 & 119 & 19,606 & 1271715 & 1.703 & .956 & .044 & 272.31 & 72 & 19,606 & 743658 & .788 & .785 & .215 \\
\hline 1184 & 8033.00 & 1 & 8,033 & 3174 & -.363 & .358 & .358 & -- & -- & -- & -- & -- & -- & -- \\
\hline 1185 & 427.80 & 20 & 8,556 & 87421 & .169 & .567 & .433 & 658.15 & 13 & 8,556 & 48501 & -.799 & .212 & .212 \\
\hline 1186 & 172.20 & 35 & 6,027 & 108885 & .332 & .630 & .370 & 287.00 & 21 & 6,027 & 72765 & 1.189 & .883 & .117 \\
\hline 1187 & 111.42 & 103 & 11,476 & 603223 & .363 & .642 & .358 & 225.02 & 51 & 11,476 & 312236 & .828 & .796 & .204 \\
\hline 1188 & 2501.50 & 4 & 10,006 & 15291 & -.817 & .207 & .207 & 3335.33 & 3 & 10,006 & 11083 & -.785 & .216 & .216 \\
\hline 1189 & 445.54 & 43 & 19,158 & 420638 & .241 & .595 & .405 & 736.85 & 26 & 19,158 & 246927 & -.075 & .470 & .470 \\
\hline 1190 & 186.98 & 102 & 19,072 & 1113073 & 2.525 & .994 & .006 & 312.66 & 61 & 19,072 & 648658 & 1.557 & .940 & .060 \\
\hline 1191 & 724.00 & 1 & 724 & 110 & -1.206 & .114 & .114 & 724.00 & 1 & 724 & 110 & -1.206 & .114 & .114 \\
\hline 1192 & 498.77 & 13 & 6,484 & 40448 & -.252 & .401 & .401 & 1296.80 & 5 & 6,484 & 14233 & -.472 & .318 & .318 \\
\hline 1193 & 224.62 & 34 & 7,637 & 131195 & .106 & .542 & .458 & 363.67 & 21 & 7,637 & 80521 & .033 & .513 & .487 \\
\hline 1194 & 225.19 & 37 & 8,332 & 177257 & 1.580 & .943 & .057 & 362.26 & 23 & 8,332 & 102973 & .620 & .732 & .268 \\
\hline 1195 & 202.18 & 11 & 2,224 & 11209 & -.480 & .316 & .316 & 317.71 & 7 & 2,224 & 8584 & .471 & .681 & .319 \\
\hline 1196 & 256.96 & 25 & 6,424 & 85637 & .576 & .718 & .282 & 428.27 & 15 & 6,424 & 53876 & .793 & .786 & .214 \\
\hline 1197 & 347.63 & 57 & 19,815 & 570997 & 0.145 & .558 & .442 & 619.22 & 32 & 19,815 & 335112 & 0.559 & .712 & .288 \\
\hline 1198 & 477.68 & 19 & 9,076 & 94090 & .689 & .754 & .246 & 1008.44 & 9 & 9,076 & 47507 & .848 & .802 & .198 \\
\hline 1199 & -- & -- & -- & -- & -- & -- & -- & -- & -- & -- & -- & -- & -- & -- \\
\hline 1200 & 399.75 & 36 & 14,391 & 255872 & -.127 & .449 & .449 & 625.70 & 23 & 14,391 & 163209 & -.115 & .454 & .454 \\
\hline
\end{tabular}


Table 7. Summary of interoccurrence intervals for daily precipitation thresholds of 2.5 and 3.0 inches-Continued

\begin{tabular}{|c|c|c|c|c|c|c|c|c|c|c|c|c|c|c|}
\hline \multirow[b]{2}{*}{$\begin{array}{l}\text { Seq. } \\
\text { no. }\end{array}$} & \multicolumn{7}{|c|}{ Daily precipitation threshold of 2.5 inches and greater } & \multicolumn{7}{|c|}{ Daily precipitation threshold of 3.0 inches and greater } \\
\hline & $\begin{array}{l}\text { Mean } \\
\text { inter- } \\
\text { occur- } \\
\text { rence } \\
\text { interval } \\
\text { (days) }\end{array}$ & $\begin{array}{l}\text { Total } \\
\text { no. of } \\
\text { events }\end{array}$ & $\begin{array}{l}\text { Total } \\
\text { no. of } \\
\text { days } \\
\text { avail- } \\
\text { able }\end{array}$ & $\begin{array}{l}\text { Sigma } \\
\mathrm{S}\end{array}$ & $\begin{array}{c}\text { Stan- } \\
\text { dard } \\
\text { normal } \\
\text { variate }\end{array}$ & $\begin{array}{l}\text { Non- } \\
\text { ex- } \\
\text { ceed- } \\
\text { ance } \\
\text { prob- } \\
\text { ability }\end{array}$ & $\begin{array}{c}\mathrm{p}- \\
\text { value }\end{array}$ & $\begin{array}{c}\text { Mean } \\
\text { inter- } \\
\text { occur- } \\
\text { rence } \\
\text { interval } \\
\text { (days) }\end{array}$ & $\begin{array}{c}\text { Total } \\
\text { no. of } \\
\text { events }\end{array}$ & $\begin{array}{l}\text { Total } \\
\text { no. of } \\
\text { days } \\
\text { avail- } \\
\text { able }\end{array}$ & $\begin{array}{l}\text { Sigma } \\
\mathbf{S}\end{array}$ & $\begin{array}{c}\text { Stan- } \\
\text { dard } \\
\text { normal } \\
\text { variate }\end{array}$ & $\begin{array}{l}\text { Non- } \\
\text { ex- } \\
\text { ceed- } \\
\text { ance } \\
\text { prob- } \\
\text { ability }\end{array}$ & $\begin{array}{c}\mathrm{p}- \\
\text { value }\end{array}$ \\
\hline 1201 & 958.50 & 2 & 1,917 & 2795 & 1.122 & .869 & .131 & 1917.00 & 1 & 1,917 & 1384 & .769 & .779 & .221 \\
\hline 1202 & 1293.33 & 15 & 19,400 & 189104 & 2.010 & .978 & .022 & 1616.67 & 12 & 19,400 & 153576 & 1.916 & .972 & .028 \\
\hline 1203 & 461.59 & 34 & 15,694 & 237544 & -1.107 & .134 & .134 & 980.88 & 16 & 15,694 & 115801 & -.538 & .295 & .295 \\
\hline 1204 & 673.09 & 23 & 15,481 & 217986 & 1.864 & .969 & .031 & 1290.08 & 12 & 15,481 & 119543 & 1.722 & .957 & .043 \\
\hline 1205 & 135.10 & 48 & 6,485 & 158891 & .251 & .599 & .401 & 240.19 & 27 & 6,485 & 89833 & .235 & .593 & .407 \\
\hline 1206 & 203.69 & 52 & 10,592 & 244908 & -1.383 & .083 & .083 & 392.30 & 27 & 10,592 & 126919 & -1.012 & .156 & .156 \\
\hline 1207 & 210.48 & 29 & 6,104 & 99083 & 1.114 & .867 & .133 & 610.40 & 10 & 6,104 & 31759 & .222 & .588 & .412 \\
\hline 1208 & 928.86 & 21 & 19,506 & 206430 & .063 & .525 & .475 & 1393.29 & 14 & 19,506 & 139794 & .154 & .561 & .439 \\
\hline 1209 & -- & -- & -- & -- & -- & -- & -- & -- & -- & -- & -- & -- & -- & -- \\
\hline 1210 & 137.37 & 27 & 3,709 & 50158 & .016 & .506 & .494 & 309.08 & 12 & 3,709 & 23360 & .298 & .617 & .383 \\
\hline 1211 & 275.11 & 102 & 28,061 & 1359494 & -.875 & .191 & .191 & 425.17 & 66 & 28,061 & 961854 & .545 & .707 & .293 \\
\hline 1212 & 301.06 & 32 & 9,634 & 173148 & 1.208 & .886 & .114 & 401.42 & 24 & 9,634 & 123324 & .566 & .714 & .286 \\
\hline 1213 & 547.40 & 10 & 5,474 & 26954 & -.083 & .467 & .467 & 782.00 & 7 & 5,474 & 18011 & -.275 & .392 & .392 \\
\hline 1214 & 241.33 & 3 & 724 & 1386 & .829 & .796 & .204 & 362.00 & 2 & 724 & 1355 & 2.135 & .984 & .016 \\
\hline 1215 & 2845.67 & 3 & 8,537 & 8667 & -.970 & .166 & .166 & 8537.00 & 1 & 8,537 & 2946 & -.537 & .296 & .296 \\
\hline 1216 & 2523.40 & 10 & 25,234 & 137956 & .512 & .696 & .305 & 6308.50 & 4 & 25,234 & 49749 & -.049 & .480 & .480 \\
\hline 1217 & 226.38 & 58 & 13,130 & 382211 & .050 & .520 & .480 & 423.55 & 31 & 13,130 & 177984 & -1.210 & .113 & .113 \\
\hline 1218 & 204.31 & 16 & 3,269 & 29936 & 1.003 & .842 & .158 & 363.22 & 9 & 3,269 & 21112 & 2.261 & .988 & .012 \\
\hline 1219 & 218.13 & 90 & 19,632 & 979703 & 1.791 & .963 & .037 & 370.42 & 53 & 19,632 & 571886 & 1.252 & .895 & .105 \\
\hline 1220 & 4182.80 & 5 & 20,914 & 57586 & .393 & .653 & .347 & 5228.50 & 4 & 20,914 & 43822 & .165 & .566 & .434 \\
\hline 1221 & 534.27 & 11 & 5,877 & 30095 & -.396 & .346 & .346 & 839.57 & 7 & 5,877 & 21276 & .157 & .563 & .437 \\
\hline 1222 & -- & -- & -- & -- & -- & -- & -- & -- & -- & -- & -- & -- & -- & -- \\
\hline 1223 & 152.11 & 36 & 5,476 & 105466 & .727 & .766 & .234 & 210.62 & 26 & 5,476 & 77638 & .800 & .788 & .212 \\
\hline 1224 & 282.57 & 7 & 1,978 & 8415 & .988 & .838 & .162 & 659.33 & 3 & 1,978 & 2380 & -.594 & .276 & .276 \\
\hline 1225 & 203.77 & 13 & 2,649 & 16711 & -.184 & .427 & .427 & 331.13 & 8 & 2,649 & 10808 & .098 & .539 & .461 \\
\hline 1226 & 1684.69 & 13 & 21,901 & 146388 & .177 & .570 & .430 & 3650.17 & 6 & 21,901 & 69794 & .264 & .604 & .396 \\
\hline 1227 & 347.00 & 18 & 6,246 & 75164 & 2.477 & .993 & .007 & 567.82 & 11 & 6,246 & 45037 & 1.787 & .963 & .037 \\
\hline 1228 & 343.43 & 73 & 25,070 & 972888 & .935 & .825 & .175 & 642.82 & 39 & 25,070 & 496639 & .172 & .568 & .432 \\
\hline 1229 & 155.39 & 95 & 14,762 & 716042 & .358 & .640 & .360 & 268.40 & 55 & 14,762 & 440758 & 1.101 & .865 & .135 \\
\hline 1230 & 163.18 & 88 & 14,360 & 695829 & 1.646 & .950 & .050 & 251.93 & 57 & 14,360 & 451990 & 1.365 & .914 & .086 \\
\hline 1231 & 155.80 & 5 & 779 & 2255 & .612 & .730 & .270 & 779.00 & 1 & 779 & 734 & 1.532 & .937 & .063 \\
\hline 1232 & 243.75 & 4 & 975 & 2936 & 1.752 & .960 & .040 & 487.50 & 2 & 975 & 1604 & 1.580 & .943 & .057 \\
\hline 1233 & 212.92 & 25 & 5,323 & 86872 & 2.647 & .996 & .004 & 443.58 & 12 & 5,323 & 39715 & 1.461 & .928 & .072 \\
\hline 1234 & 413.48 & 27 & 11,164 & 142152 & -.511 & .305 & .305 & 858.77 & 13 & 11,164 & 74305 & .150 & .560 & .440 \\
\hline 1235 & 318.46 & 13 & 4,140 & 35446 & 1.981 & .976 & .024 & 460.00 & 9 & 4,140 & 25483 & 1.911 & .972 & .028 \\
\hline 1236 & 246.22 & 9 & 2,216 & 6053 & -2.042 & .021 & .021 & 554.00 & 4 & 2,216 & 3414 & -.796 & .213 & .213 \\
\hline 1237 & 398.24 & 33 & 13,142 & 142036 & -3.433 & 0 & 0 & 773.06 & 17 & 13,142 & 69043 & -2.728 & .003 & .003 \\
\hline 1238 & 314.49 & 81 & 25,474 & 1057702 & .393 & .653 & .347 & 621.32 & 41 & 25,474 & 514041 & -.174 & .431 & .431 \\
\hline 1239 & 218.10 & 69 & 15,049 & 507125 & -.334 & .369 & .369 & 429.97 & 35 & 15,049 & 253906 & -.368 & .357 & .357 \\
\hline 1240 & 938.50 & 2 & 1,877 & 1963 & .112 & .545 & .455 & 1877.00 & 1 & 1,877 & 114 & -1.522 & .064 & .064 \\
\hline 1241 & 192.26 & 94 & 18,072 & 874499 & .497 & .690 & .310 & 340.98 & 53 & 18,072 & 484280 & .141 & .556 & .444 \\
\hline 1242 & 149.40 & 5 & 747 & 2728 & 1.785 & .963 & .037 & 186.75 & 4 & 747 & 2095 & 1.394 & .918 & .082 \\
\hline 1243 & 133.36 & 132 & 17,604 & 1216998 & .944 & .827 & .173 & 220.05 & 80 & 17,604 & 743075 & .856 & .804 & .196 \\
\hline 1244 & 191.52 & 100 & 19,152 & 919624 & -.687 & .246 & .246 & 336.00 & 57 & 19,152 & 516696 & -.698 & .243 & .243 \\
\hline 1245 & 677.07 & 28 & 18,958 & 338497 & 2.524 & .994 & .006 & 1184.88 & 16 & 18,958 & 169542 & 0.817 & .793 & .207 \\
\hline 1246 & 578.00 & 4 & 2,312 & 5221 & .447 & .673 & .327 & 1156.00 & 2 & 2,312 & 2159 & -.162 & .436 & .436 \\
\hline 1247 & 379.55 & 40 & 15,182 & 285733 & -.646 & .259 & .259 & 1167.85 & 13 & 15,182 & 89613 & -.574 & .283 & .283 \\
\hline 1248 & -- & -- & -- & -- & -- & -- & -- & -- & -- & -- & -- & -- & -- & -- \\
\hline
\end{tabular}


Table 7. Summary of interoccurrence intervals for daily precipitation thresholds of 2.5 and 3.0 inches—Continued

\begin{tabular}{|c|c|c|c|c|c|c|c|c|c|c|c|c|c|c|}
\hline \multirow[b]{2}{*}{$\begin{array}{l}\text { Seq. } \\
\text { no. }\end{array}$} & \multicolumn{7}{|c|}{ Daily precipitation threshold of 2.5 inches and greater } & \multicolumn{7}{|c|}{ Daily precipitation threshold of 3.0 inches and greater } \\
\hline & $\begin{array}{l}\text { Mean } \\
\text { inter- } \\
\text { occur- } \\
\text { rence } \\
\text { interval } \\
\text { (days) }\end{array}$ & $\begin{array}{c}\text { Total } \\
\text { no. of } \\
\text { events }\end{array}$ & $\begin{array}{l}\text { Total } \\
\text { no. of } \\
\text { days } \\
\text { avail- } \\
\text { able }\end{array}$ & $\begin{array}{l}\text { Sigma } \\
\mathrm{S}\end{array}$ & $\begin{array}{c}\text { Stan- } \\
\text { dard } \\
\text { normal } \\
\text { variate }\end{array}$ & $\begin{array}{l}\text { Non- } \\
\text { ex- } \\
\text { ceed- } \\
\text { ance } \\
\text { prob- } \\
\text { ability }\end{array}$ & $\begin{array}{c}\text { p- } \\
\text { value }\end{array}$ & $\begin{array}{l}\text { Mean } \\
\text { inter- } \\
\text { occur- } \\
\text { rence } \\
\text { interval } \\
\text { (days) }\end{array}$ & $\begin{array}{l}\text { Total } \\
\text { no. of } \\
\text { events }\end{array}$ & $\begin{array}{c}\text { Total } \\
\text { no. of } \\
\text { days } \\
\text { avail- } \\
\text { able }\end{array}$ & $\begin{array}{l}\text { Sigma } \\
\mathrm{S}\end{array}$ & $\begin{array}{c}\text { Stan- } \\
\text { dard } \\
\text { normal } \\
\text { variate }\end{array}$ & $\begin{array}{l}\text { Non- } \\
\text { ex- } \\
\text { ceed- } \\
\text { ance } \\
\text { prob- } \\
\text { ability }\end{array}$ & $\begin{array}{c}\text { p- } \\
\text { value }\end{array}$ \\
\hline 1249 & 397.65 & 31 & 12,327 & 215783 & 1.247 & .894 & .106 & 684.83 & 18 & 12,327 & 125561 & .968 & .833 & .167 \\
\hline 1250 & 244.09 & 151 & 36,857 & 2916702 & 1.025 & .847 & .153 & 433.61 & 85 & 36,857 & 1672926 & 1.086 & .861 & .139 \\
\hline 1251 & 554.00 & 2 & 1,108 & 376 & -1.618 & .053 & .053 & -- & -- & -- & -- & -- & -- & -- \\
\hline 1252 & 295.58 & 120 & 35,470 & 2315709 & 1.672 & .953 & .047 & 485.89 & 73 & 35,470 & 1331601 & .422 & .664 & .336 \\
\hline 1253 & 171.73 & 26 & 4,465 & 64847 & 1.035 & .850 & .150 & 235.00 & 19 & 4,465 & 44201 & .317 & .625 & .375 \\
\hline 1254 & 76.00 & 6 & 456 & 1547 & .555 & .711 & .289 & 114.00 & 4 & 456 & 1156 & .927 & .823 & .177 \\
\hline 1255 & 161.78 & 83 & 13,428 & 575057 & .504 & .693 & .307 & 248.67 & 54 & 13,428 & 354738 & -.275 & .392 & .392 \\
\hline 1256 & 511.83 & 40 & 20,473 & 513170 & 2.775 & .997 & .003 & 1023.65 & 20 & 20,473 & 252057 & 1.791 & .963 & .037 \\
\hline 1257 & 300.45 & 65 & 19,529 & 621850 & -.283 & .389 & .389 & 610.28 & 32 & 19,529 & 297521 & -.469 & .320 & .320 \\
\hline 1258 & 213.71 & 17 & 3,633 & 39671 & 2.033 & .979 & .021 & 259.50 & 14 & 3,633 & 31785 & 1.619 & .947 & .053 \\
\hline 1259 & -- & -- & -- & -- & -- & -- & -- & -- & -- & -- & -- & -- & -- & -- \\
\hline 1260 & 170.19 & 128 & 21,784 & 1458245 & .901 & .816 & .184 & 262.46 & 83 & 21,784 & 915818 & .206 & .582 & .418 \\
\hline 1261 & 408.95 & 20 & 8,179 & 96332 & 1.377 & .916 & .084 & 629.15 & 13 & 8,179 & 63950 & 1.267 & .897 & .103 \\
\hline 1262 & 192.33 & 6 & 1,154 & 2720 & -.909 & .182 & .182 & 288.50 & 4 & 1,154 & 1831 & -.716 & .237 & .237 \\
\hline 1263 & -- & -- & -- & -- & -- & -- & -- & -- & -- & -- & -- & -- & -- & -- \\
\hline 1264 & 516.00 & 3 & 1,548 & 3023 & .906 & .817 & .183 & 516.00 & 3 & 1,548 & 3023 & .906 & .817 & .183 \\
\hline 1265 & 164.48 & 21 & 3,454 & 46506 & 2.241 & .987 & .013 & 265.69 & 13 & 3,454 & 24418 & .547 & .708 & .292 \\
\hline 1266 & 288.55 & 66 & 19,044 & 640492 & .270 & .606 & .394 & 577.09 & 33 & 19,044 & 302447 & -.373 & .355 & .355 \\
\hline 1267 & 354.84 & 38 & 13,484 & 245465 & -.447 & .327 & .327 & 612.91 & 22 & 13,484 & 151423 & .170 & .567 & .433 \\
\hline 1268 & 295.88 & 58 & 17,161 & 527220 & .783 & .783 & .217 & 536.28 & 32 & 17,161 & 310459 & 1.280 & .900 & .100 \\
\hline 1269 & -- & -- & -- & -- & -- & -- & -- & -- & -- & -- & -- & -- & -- & -- \\
\hline 1270 & 729.00 & 3 & 2,187 & 3485 & .187 & .574 & .426 & 2187.00 & 1 & 2,187 & 1200 & .169 & .567 & .433 \\
\hline 1271 & 398.56 & 88 & 35,073 & 1388710 & -1.627 & .052 & .052 & 687.71 & 51 & 35,073 & 719695 & -2.416 & .008 & .008 \\
\hline 1272 & 1330.33 & 3 & 3,991 & 6121 & .067 & .527 & .473 & 1330.33 & 3 & 3,991 & 6121 & .067 & .527 & .473 \\
\hline 1273 & 560.53 & 17 & 9,529 & 105729 & 2.181 & .985 & .015 & 1191.13 & 8 & 9,529 & 49648 & 1.482 & .931 & .069 \\
\hline 1274 & 128.19 & 48 & 6,153 & 147944 & .022 & .509 & .491 & 246.12 & 25 & 6,153 & 67189 & -1.095 & .137 & .137 \\
\hline 1275 & 103.03 & 35 & 3,606 & 66278 & .515 & .697 & .303 & 171.71 & 21 & 3,606 & 40439 & .540 & .705 & .295 \\
\hline 1276 & 115.15 & 48 & 5,527 & 137757 & .462 & .678 & .322 & 157.91 & 35 & 5,527 & 94446 & -.241 & .405 & .405 \\
\hline 1277 & 111.30 & 23 & 2,560 & 36897 & 2.104 & .982 & .018 & 134.74 & 19 & 2,560 & 29283 & 1.541 & .938 & .062 \\
\hline 1278 & 194.48 & 149 & 28,977 & 2423132 & 2.589 & .995 & .005 & 329.28 & 88 & 28,977 & 1420842 & 1.859 & .968 & .032 \\
\hline 1279 & 1501.00 & 1 & 1,501 & 58 & -1.598 & .055 & .055 & -- & -- & -- & -- & -- & -- & -- \\
\hline 1280 & 336.19 & 16 & 5,379 & 43418 & .062 & .525 & .475 & 768.43 & 7 & 5,379 & 22756 & .957 & .831 & .169 \\
\hline 1281 & 206.77 & 30 & 6,203 & 95351 & .235 & .593 & .407 & 344.61 & 18 & 6,203 & 64149 & 1.095 & .863 & .137 \\
\hline 1282 & -- & -- & -- & -- & -- & -- & -- & -- & -- & -- & -- & -- & -- & -- \\
\hline 1283 & 581.44 & 9 & 5,233 & 23178 & -.082 & .467 & .467 & 654.13 & 8 & 5,233 & 18016 & -.683 & .248 & .248 \\
\hline 1284 & 137.59 & 71 & 9,769 & 374594 & 1.170 & .879 & .121 & 191.55 & 51 & 9,769 & 265036 & .791 & .785 & .215 \\
\hline 1285 & 1754.67 & 12 & 21,056 & 149096 & 1.081 & .860 & .140 & 3509.33 & 6 & 21,056 & 70656 & .503 & .692 & .308 \\
\hline 1286 & 179.36 & 102 & 18,295 & 980652 & .893 & .814 & .186 & 425.47 & 43 & 18,295 & 426480 & .957 & .831 & .169 \\
\hline 1287 & 398.67 & 15 & 5,980 & 51168 & .945 & .828 & .172 & 664.44 & 9 & 5,980 & 32877 & 1.152 & .875 & .125 \\
\hline 1288 & 401.60 & 30 & 12,048 & 165417 & -.803 & .211 & .211 & 602.40 & 20 & 12,048 & 99184 & -1.369 & .086 & .086 \\
\hline 1289 & 462.27 & 30 & 13,868 & 185918 & -1.008 & .157 & .157 & 1260.73 & 11 & 13,868 & 63165 & -.987 & .162 & .162 \\
\hline 1290 & 476.67 & 3 & 1,430 & 2555 & .573 & .717 & .283 & 715.00 & 2 & 1,430 & 1563 & .228 & .590 & .410 \\
\hline 1291 & 405.33 & 3 & 1,216 & 2619 & 1.308 & .904 & .096 & 608.00 & 2 & 1,216 & 1855 & 1.287 & .901 & .099 \\
\hline 1292 & 182.08 & 108 & 19,665 & 1193196 & 2.225 & .987 & .013 & 289.19 & 68 & 19,665 & 788886 & 2.569 & .995 & .005 \\
\hline 1293 & 274.86 & 62 & 17,041 & 518491 & -0.253 & .400 & .400 & 355.02 & 48 & 17,041 & 411421 & 0.072 & .529 & .471 \\
\hline 1294 & 389.08 & 13 & 5,058 & 28330 & -.864 & .194 & .194 & 843.00 & 6 & 5,058 & 13335 & -.514 & .304 & .304 \\
\hline 1295 & 212.67 & 3 & 638 & 868 & -.279 & .390 & .390 & -- & -- & -- & -- & -- & -- & -- \\
\hline 1296 & 101.36 & 42 & 4,257 & 85557 & -.482 & .315 & .315 & 202.71 & 21 & 4,257 & 48610 & .695 & .756 & .244 \\
\hline
\end{tabular}


Table 7. Summary of interoccurrence intervals for daily precipitation thresholds of 2.5 and 3.0 inches-Continued

\begin{tabular}{|c|c|c|c|c|c|c|c|c|c|c|c|c|c|c|}
\hline \multirow[b]{2}{*}{$\begin{array}{l}\text { Seq. } \\
\text { no. }\end{array}$} & \multicolumn{7}{|c|}{ Daily precipitation threshold of 2.5 inches and greater } & \multicolumn{7}{|c|}{ Daily precipitation threshold of 3.0 inches and greater } \\
\hline & $\begin{array}{c}\text { Mean } \\
\text { inter- } \\
\text { occur- } \\
\text { rence } \\
\text { interval } \\
\text { (days) }\end{array}$ & $\begin{array}{c}\text { Total } \\
\text { no. of } \\
\text { events }\end{array}$ & $\begin{array}{l}\text { Total } \\
\text { no. of } \\
\text { days } \\
\text { avail- } \\
\text { able }\end{array}$ & $\begin{array}{c}\text { Sigma } \\
\mathbf{S}\end{array}$ & $\begin{array}{l}\text { Stan- } \\
\text { dard } \\
\text { normal } \\
\text { variate }\end{array}$ & $\begin{array}{l}\text { Non- } \\
\text { ex- } \\
\text { ceed- } \\
\text { ance } \\
\text { prob- } \\
\text { ability }\end{array}$ & $\begin{array}{c}\mathrm{p}- \\
\text { value }\end{array}$ & $\begin{array}{c}\text { Mean } \\
\text { inter- } \\
\text { occur- } \\
\text { rence } \\
\text { interval } \\
\text { (days) }\end{array}$ & $\begin{array}{c}\text { Total } \\
\text { no. of } \\
\text { events }\end{array}$ & $\begin{array}{l}\text { Total } \\
\text { no. of } \\
\text { days } \\
\text { avail- } \\
\text { able }\end{array}$ & $\begin{array}{c}\text { Sigma } \\
\mathbf{S}\end{array}$ & $\begin{array}{l}\text { Stan- } \\
\text { dard } \\
\text { normal } \\
\text { variate }\end{array}$ & $\begin{array}{l}\text { Non- } \\
\text { ex- } \\
\text { ceed- } \\
\text { ance } \\
\text { prob- } \\
\text { ability }\end{array}$ & $\begin{array}{c}\mathrm{p}- \\
\text { value }\end{array}$ \\
\hline 1297 & 208.39 & 13 & 2,709 & 14316 & -1.168 & .122 & .122 & 270.90 & 10 & 2,709 & 8852 & -1.898 & .029 & .029 \\
\hline 1298 & 185.63 & 8 & 1,485 & 7340 & 1.155 & .876 & .124 & 742.50 & 2 & 1,485 & 1184 & -.497 & .310 & .310 \\
\hline 1299 & 158.95 & 61 & 9,696 & 352693 & 2.606 & .995 & .005 & 269.33 & 36 & 9,696 & 208726 & 2.036 & .979 & .021 \\
\hline 1300 & -- & -- & -- & -- & -- & -- & -- & -- & -- & -- & -- & -- & -- & -- \\
\hline 1301 & 181.61 & 153 & 27,787 & 2128759 & .031 & .512 & .488 & 323.11 & 86 & 27,787 & 1233564 & .521 & 699 & .301 \\
\hline 1302 & 201.02 & 93 & 18,695 & 946786 & 1.489 & .932 & .068 & 327.98 & 57 & 18,695 & 593482 & 1.489 & .932 & .068 \\
\hline 1303 & 291.00 & 1 & 291 & 228 & .982 & .837 & .163 & -- & -- & -- & -- & -- & -- & -- \\
\hline 1304 & 303.33 & 12 & 3,640 & 25576 & 1.026 & .848 & .152 & 606.67 & 6 & 3,640 & 15251 & 1.683 & .954 & .046 \\
\hline 1305 & 11327.50 & 2 & 22,655 & 23673 & .110 & .544 & .456 & 11327.50 & 2 & 22,655 & 23673 & .110 & .544 & .456 \\
\hline 1306 & 383.90 & 39 & 14,972 & 273400 & -.687 & .246 & .246 & 712.95 & 21 & 14,972 & 122454 & -1.755 & .040 & .040 \\
\hline
\end{tabular}


Table 8. Example computation steps of interoccurrence intervals for a Poisson process described in text

[Total time, $T$, is 60 days and mean interoccurrence interval, $\Lambda$, is 10 days. Median number of occurrences to closest integer is 5 (shaded row)]

\begin{tabular}{ccc}
\hline $\begin{array}{c}\text { No. of } \\
\text { occurrences } \\
n\end{array}$ & $\begin{array}{c}\text { Probability } \\
\text { density value, } \\
\text { eq. 5 } \\
f_{n}(T)\end{array}$ & $\begin{array}{c}\text { Cumulative } \\
\text { probability, } \\
\text { eq. } 6\end{array}$ \\
$F_{n}(T)$ \\
\hline 0 & .00248 & .00248 \\
1 & .0149 & .0174 \\
2 & .0466 & .0620 \\
3 & .0892 & .151 \\
4 & .134 & .285 \\
5 & .161 & .446 \\
6 & .161 & .606 \\
7 & .138 & .744 \\
8 & .103 & .847 \\
9 & .0688 & .916 \\
10 & .0413 & .957 \\
\hline
\end{tabular}

Table 9. Summary of statewide interoccurrence interval statistics and diagnostic statistics of interoccurrence interval maps

[RMSE, root-mean-square error; bias computed as observed value for station minus value predicted from map; in., inches]

\begin{tabular}{|c|c|c|c|c|c|c|c|c|c|}
\hline $\begin{array}{l}\text { Daily } \\
\text { precip- } \\
\text { itation } \\
\text { threshold } \\
\text { (in.) }\end{array}$ & $\begin{array}{l}\text { State- } \\
\text { wide } \\
\text { mean } \\
\text { inter- } \\
\text { occur- } \\
\text { rence } \\
\text { interval } \\
\text { (days) }\end{array}$ & $\begin{array}{c}\text { State- } \\
\text { wide } \\
\text { standard } \\
\text { deviation } \\
\text { of } \\
\text { inter- } \\
\text { occur- } \\
\text { rence } \\
\text { interval } \\
\text { (days) }\end{array}$ & $\begin{array}{l}\text { State- } \\
\text { wide } \\
\text { no. of } \\
\text { stations } \\
\text { with } \\
\text { events }\end{array}$ & $\begin{array}{c}\text { State- } \\
\text { wide } \\
\text { no. of } \\
\text { p-values } \\
\text { less } \\
\text { than } \\
\text { or equal } \\
\text { to } .01\end{array}$ & $\begin{array}{l}\text { Percent of } \\
\text { stations } \\
\text { with } \\
\text { p-values } \\
\text { less than } \\
\text { or equal } \\
\text { to } .01\end{array}$ & $\begin{array}{c}\text { Statewide } \\
\text { mean } \\
\text { of inter- } \\
\text { polated } \\
\text { inter- } \\
\text { occur- } \\
\text { rence } \\
\text { interval } \\
\text { map } \\
\text { (days) }\end{array}$ & $\begin{array}{c}\text { Statewide } \\
\text { mean } \\
\text { bias of } \\
\text { inter- } \\
\text { occur- } \\
\text { rence } \\
\text { interval } \\
\text { map } \\
\text { (days) }\end{array}$ & $\begin{array}{c}\text { RMSE } \\
\text { of } \\
\text { inter- } \\
\text { occur- } \\
\text { rence } \\
\text { interval } \\
\text { map } \\
\text { (days) }\end{array}$ & $\begin{array}{c}\text { Percent } \\
\text { change } \\
\text { from } \\
\text { standard } \\
\text { deviation } \\
\text { to } \\
\text { RMSE } \\
\text { (percent) }\end{array}$ \\
\hline 0.05 & 8.04 & 3.68 & 1,299 & 406 & 31.3 & 7.64 & 0.404 & 2.80 & -24 \\
\hline .10 & 9.38 & 4.02 & 1,299 & 309 & 23.8 & 8.95 & .436 & 2.73 & -32 \\
\hline .25 & 14.03 & 7.70 & 1,298 & 184 & 14.2 & 13.4 & 679 & 5.20 & -32 \\
\hline .50 & 23.50 & 18.86 & 1,294 & 171 & 13.2 & 22.3 & 1.22 & 13.7 & -27 \\
\hline .75 & 36.76 & 29.83 & 1,284 & 151 & 11.8 & 35.5 & 1.27 & 17.8 & -40 \\
\hline 1.0 & 55.72 & 52.12 & 1,277 & 140 & 11.0 & 54.5 & 1.23 & 29.5 & -43 \\
\hline 1.5 & 127.7 & 160.1 & 1,258 & 107 & 8.51 & 123 & 5.20 & 86.5 & -46 \\
\hline 2.0 & 273.0 & 458.0 & 1,230 & 87 & 7.07 & 268 & 5.23 & 183 & -60 \\
\hline 2.5 & 533.9 & 882.0 & 1,189 & 65 & 5.47 & 528 & 5.78 & 416 & -53 \\
\hline 3.0 & 876.3 & 1,515 & 1,143 & 37 & 3.24 & 864 & 12.0 & 999 & -34 \\
\hline
\end{tabular}


Table 10. Summary of interoccurrence ratios by month for 0.05 -inch and greater daily precipitation threshold

[A monthly interoccurrence ratio is defined as ratio of mean interoccurrence interval for corresponding month to mean interoccurrence interval for period of record at the station. Mean monthly interoccurrence ratio is arithmetic average of monthly ratios for all stations, and median monthly interoccurrence ratio is median of monthly ratios for all stations. Adjusted ratios have averages of unity and therefore are preferred because statistical bias has been removed.]

\begin{tabular}{lcccc}
\hline Month & $\begin{array}{c}\text { Mean monthly } \\
\text { interoccurrence } \\
\text { ratio }\end{array}$ & $\begin{array}{c}\text { Median monthly } \\
\text { interoccurrence } \\
\text { ratio }\end{array}$ & $\begin{array}{c}\text { Adjusted } \\
\text { mean monthly } \\
\text { interoccurrence } \\
\text { ratio }\end{array}$ & $\begin{array}{c}\text { Adjusted } \\
\text { median monthly } \\
\text { interoccurrence } \\
\text { ratio }\end{array}$ \\
\hline Jan. & 1.21 & 1.08 & 1.12 & 1.07 \\
Feb. & 1.13 & .99 & 1.04 & .98 \\
Mar. & 1.20 & 1.10 & 1.11 & 1.09 \\
Apr. & 1.16 & 1.04 & 1.07 & 1.03 \\
May & .88 & .81 & .79 & .80 \\
June & .84 & .82 & .75 & .81 \\
July & 1.07 & 1.06 & .98 & 1.05 \\
Aug. & 1.15 & 1.14 & 1.06 & 1.13 \\
Sept. & .91 & .88 & .82 & .87 \\
Oct. & 1.09 & 1.05 & 1.00 & 1.04 \\
Nov. & 1.11 & 1.04 & 1.02 & 1.03 \\
Dec. & 1.28 & 1.12 & 1.19 & 1.11 \\
Average & 1.09 & 1.01 & & \\
Incremental adjustment & -.09 & -.01 & & \\
\cline { 2 - 3 } & & & &
\end{tabular}

Table 11. Summary of interoccurrence ratios by month for 0.10 -inch and greater daily precipitation threshold

[A monthly interoccurrence ratio is defined as ratio of mean interoccurrence interval for corresponding month to mean interoccurrence interval for period of record at the station. Mean monthly interoccurrence ratio is arithmetic average of monthly ratios for all stations, and median monthly interoccurrence ratio is median of monthly ratios for all stations. Adjusted ratios have averages of unity and therefore are preferred because statistical bias has been removed.]

\begin{tabular}{lcccc}
\hline Month & $\begin{array}{c}\text { Mean monthly } \\
\text { interoccurrence } \\
\text { ratio }\end{array}$ & $\begin{array}{c}\text { Median monthly } \\
\text { interoccurrence } \\
\text { ratio }\end{array}$ & $\begin{array}{c}\text { Adjusted } \\
\text { mean monthly } \\
\text { interoccurrence } \\
\text { ratio }\end{array}$ & $\begin{array}{c}\text { Adjusted } \\
\text { median monthly } \\
\text { interoccurrence } \\
\text { ratio }\end{array}$ \\
\hline Jan. & 1.25 & 1.13 & 1.15 & 1.11 \\
Feb. & 1.21 & 1.05 & 1.11 & 1.03 \\
Mar. & 1.24 & 1.14 & 1.14 & 1.12 \\
Apr. & 1.19 & 1.06 & 1.09 & 1.04 \\
May & .88 & .80 & .78 & .78 \\
June & .81 & .78 & .71 & .76 \\
July & 1.05 & 1.04 & .95 & 1.02 \\
Aug. & 1.14 & 1.15 & 1.04 & 1.13 \\
Sept. & .91 & .87 & .81 & .85 \\
Oct. & 1.06 & 1.03 & .96 & 1.01 \\
Nov. & 1.10 & 1.04 & 1.00 & 1.02 \\
Dec. & 1.30 & 1.14 & 1.20 & 1.12 \\
Average & 1.10 & 1.02 & & \\
Incremental adjustment & -.10 & -.02 & & \\
\hline
\end{tabular}


Table 12. Summary of interoccurrence ratios by month for 0.25 -inch and greater daily precipitation threshold

[A monthly interoccurrence ratio is defined as ratio of mean interoccurrence interval for corresponding month to mean interoccurrence interval for period of record at the station. Mean monthly interoccurrence ratio is arithmetic average of monthly ratios for all stations, and median monthly interoccurrence ratio is median of monthly ratios for all stations. Adjusted ratios have averages of unity and therefore are preferred because statistical bias has been removed.]

\begin{tabular}{lcccc}
\hline Month & $\begin{array}{c}\text { Mean monthly } \\
\text { interoccurrence } \\
\text { ratio }\end{array}$ & $\begin{array}{c}\text { Median monthly } \\
\text { interoccurrence } \\
\text { ratio }\end{array}$ & $\begin{array}{c}\text { Adjusted } \\
\text { mean monthly } \\
\text { interoccurrence } \\
\text { ratio }\end{array}$ & $\begin{array}{c}\text { Adjusted } \\
\text { median monthly } \\
\text { interoccurrence } \\
\text { ratio }\end{array}$ \\
\hline Jan. & 1.40 & 1.24 & 1.28 & 1.21 \\
Feb. & 1.38 & 1.18 & 1.26 & 1.15 \\
Mar. & 1.42 & 1.23 & 1.30 & 1.20 \\
Apr. & 1.23 & 1.07 & 1.11 & 1.04 \\
May & .88 & .78 & .76 & .75 \\
June & .76 & .73 & .64 & .70 \\
July & 1.01 & 1.00 & .89 & .97 \\
Aug. & 1.13 & 1.12 & 1.00 & 1.09 \\
Sept. & .90 & .86 & .78 & .83 \\
Oct. & 1.00 & .97 & .88 & .94 \\
Nov. & 1.08 & 1.02 & .96 & .99 \\
Dec. & 1.25 & 1.13 & 1.13 & 1.10 \\
Average & 1.12 & 1.03 & & \\
Incremental adjustment & -.12 & -.028 & & \\
\hline
\end{tabular}

Table 13. Summary of interoccurrence ratios by month for 0.50 -inch and greater daily precipitation threshold

[A monthly interoccurrence ratio is defined as ratio of mean interoccurrence interval for corresponding month to mean interoccurrence interval for period of record at the station. Mean monthly interoccurrence ratio is arithmetic average of monthly ratios for all stations, and median monthly interoccurrence ratio is median of monthly ratios for all stations. Adjusted ratios have averages of unity and therefore are preferred because statistical bias has been removed.]

\begin{tabular}{lcccc}
\hline Month & $\begin{array}{c}\text { Mean monthly } \\
\text { interoccurrence } \\
\text { ratio }\end{array}$ & $\begin{array}{c}\text { Median monthly } \\
\text { interoccurrence } \\
\text { ratio }\end{array}$ & $\begin{array}{c}\text { Adjusted } \\
\text { mean monthly } \\
\text { interoccurrence } \\
\text { ratio }\end{array}$ & $\begin{array}{c}\text { Adjusted } \\
\text { median monthly } \\
\text { interoccurrence } \\
\text { ratio }\end{array}$ \\
\hline Jan. & 1.44 & 1.30 & 1.31 & 1.26 \\
Feb. & 1.50 & 1.32 & 1.37 & 1.28 \\
Mar. & 1.52 & 1.33 & 1.39 & 1.29 \\
Apr. & 1.32 & 1.15 & 1.19 & 1.11 \\
May & .90 & .78 & .77 & .74 \\
June & .74 & .69 & .61 & .65 \\
July & .96 & .94 & .83 & .90 \\
Aug. & 1.11 & 1.09 & .98 & 1.05 \\
Sept. & .92 & .88 & .79 & .84 \\
Oct. & .94 & .91 & .81 & .87 \\
Nov. & 1.05 & .98 & .92 & .94 \\
Dec. & 1.19 & 1.11 & 1.06 & 1.07 \\
Average & 1.13 & 1.04 & & \\
Incremental adjustment & -.13 & -.040 & & \\
\hline
\end{tabular}


Table 14. Summary of interoccurrence ratios by month for 0.75 -inch and greater daily precipitation threshold

[A monthly interoccurrence ratio is defined as ratio of mean interoccurrence interval for corresponding month to mean interoccurrence interval for period of record at the station. Mean monthly interoccurrence ratio is arithmetic average of monthly ratios for all stations, and median monthly interoccurrence ratio is median of monthly ratios for all stations. Adjusted ratios have averages of unity and therefore are preferred because statistical bias has been removed.]

\begin{tabular}{lcccc}
\hline Month & $\begin{array}{c}\text { Mean monthly } \\
\text { interoccurrence } \\
\text { ratio }\end{array}$ & $\begin{array}{c}\text { Median monthly } \\
\text { interoccurrence } \\
\text { ratio }\end{array}$ & $\begin{array}{c}\text { Adjusted } \\
\text { mean monthly } \\
\text { interoccurrence } \\
\text { ratio }\end{array}$ & $\begin{array}{c}\text { Adjusted } \\
\text { median monthly } \\
\text { interoccurrence } \\
\text { ratio }\end{array}$ \\
\hline Jan. & 1.41 & 1.31 & 1.29 & 1.27 \\
Feb. & 1.47 & 1.35 & 1.35 & 1.31 \\
Mar. & 1.54 & 1.38 & 1.42 & 1.34 \\
Apr. & 1.36 & 1.24 & 1.24 & 1.20 \\
May & .95 & .84 & .83 & .80 \\
June & .75 & .69 & .63 & .65 \\
July & .93 & .90 & .81 & .86 \\
Aug. & 1.08 & 1.06 & .96 & 1.02 \\
Sept. & .92 & .88 & .80 & .84 \\
Oct. & .89 & .86 & .77 & .82 \\
Nov. & 1.01 & .95 & .89 & .91 \\
Dec. & 1.10 & 1.05 & .98 & 1.01 \\
Average & 1.12 & 1.043 & & \\
Incremental adjustment & -.12 & -.043 & & \\
\cline { 2 - 3 } & & & &
\end{tabular}

Table 15. Summary of interoccurrence ratios by month for 1.0 -inch and greater daily precipitation threshold

[A monthly interoccurrence ratio is defined as ratio of mean interoccurrence interval for corresponding month to mean interoccurrence interval for period of record at the station. Mean monthly interoccurrence ratio is arithmetic average of monthly ratios for all stations, and median monthly interoccurrence ratio is median of monthly ratios for all stations. Adjusted ratios have averages of unity and therefore are preferred because statistical bias has been removed.]

\begin{tabular}{lcccc}
\hline Month & $\begin{array}{c}\text { Mean monthly } \\
\text { interoccurrence } \\
\text { ratio }\end{array}$ & $\begin{array}{c}\text { Median monthly } \\
\text { interoccurrence } \\
\text { ratio }\end{array}$ & $\begin{array}{c}\text { Adjusted } \\
\text { mean monthly } \\
\text { interoccurrence } \\
\text { ratio }\end{array}$ & $\begin{array}{c}\text { Adjusted } \\
\text { median monthly } \\
\text { interoccurrence } \\
\text { ratio }\end{array}$ \\
\hline Jan. & 1.35 & 1.27 & 1.26 & 1.24 \\
Feb. & 1.38 & 1.32 & 1.29 & 1.29 \\
Mar. & 1.47 & 1.36 & 1.38 & 1.33 \\
Apr. & 1.36 & 1.29 & 1.27 & 1.26 \\
May & 1.01 & .92 & .92 & .89 \\
June & .79 & .73 & .70 & .70 \\
July & .90 & .86 & .81 & .83 \\
Aug. & 1.07 & 1.03 & .98 & 1.00 \\
Sept. & .90 & .86 & .81 & .83 \\
Oct. & .84 & .81 & .75 & .78 \\
Nov. & .95 & .91 & .86 & .88 \\
Dec. & 1.02 & .97 & .93 & .94 \\
Average & 1.09 & 1.03 & & \\
Incremental adjustment & -.09 & -.03 & & \\
\hline
\end{tabular}


Table 16. Summary of interoccurrence ratios by month for 1.5 -inch and greater daily precipitation threshold

[A monthly interoccurrence ratio is defined as ratio of mean interoccurrence interval for corresponding month to mean interoccurrence interval for period of record at the station. Mean monthly interoccurrence ratio is arithmetic average of monthly ratios for all stations, and median monthly interoccurrence ratio is median of monthly ratios for all stations. Adjusted ratios have averages of unity and therefore are preferred because statistical bias has been removed.]

\begin{tabular}{lcccc}
\hline Month & $\begin{array}{c}\text { Mean monthly } \\
\text { interoccurrence } \\
\text { ratio }\end{array}$ & $\begin{array}{c}\text { Median monthly } \\
\text { interoccurrence } \\
\text { ratio }\end{array}$ & $\begin{array}{c}\text { Adjusted } \\
\text { mean monthly } \\
\text { interoccurrence } \\
\text { ratio }\end{array}$ & $\begin{array}{c}\text { Adjusted } \\
\text { median monthly } \\
\text { interoccurrence } \\
\text { ratio }\end{array}$ \\
\hline Jan. & 1.17 & 1.06 & 1.13 & 1.06 \\
Feb. & 1.16 & 1.12 & 1.12 & 1.12 \\
Mar. & 1.37 & 1.30 & 1.33 & 1.30 \\
Apr. & 1.30 & 1.25 & 1.26 & 1.25 \\
May & 1.07 & 1.01 & 1.03 & 1.01 \\
June & .89 & .85 & .85 & .85 \\
July & .92 & .88 & .88 & .88 \\
Aug. & 1.02 & .99 & .98 & .99 \\
Sept. & .90 & .87 & .86 & .87 \\
Oct. & .79 & .77 & .75 & .77 \\
Nov. & .92 & .87 & .88 & .87 \\
Dec. & .94 & .86 & .90 & .86 \\
Average & 1.04 & .99 & & \\
Incremental adjustment & -.04 & 0 & & \\
\cline { 1 - 2 } & & & &
\end{tabular}

Table 17. Summary of interoccurrence ratios by month for 2.0 -inch and greater daily precipitation threshold

[A monthly interoccurrence ratio is defined as ratio of mean interoccurrence interval for corresponding month to mean interoccurrence interval for period of record at the station. Mean monthly interoccurrence ratio is arithmetic average of monthly ratios for all stations, and median monthly interoccurrence ratio is median of monthly ratios for all stations. Adjusted ratios have averages of unity and therefore are preferred because statistical bias has been removed.]

\begin{tabular}{lcccc}
\hline Month & $\begin{array}{c}\text { Mean monthly } \\
\text { interoccurrence } \\
\text { ratio }\end{array}$ & $\begin{array}{c}\text { Median monthly } \\
\text { interoccurrence } \\
\text { ratio }\end{array}$ & $\begin{array}{c}\text { Adjusted } \\
\text { mean monthly } \\
\text { interoccurrence } \\
\text { ratio }\end{array}$ & $\begin{array}{c}\text { Adjusted } \\
\text { median monthly } \\
\text { interoccurrence } \\
\text { ratio }\end{array}$ \\
\hline Jan. & 1.06 & .93 & 1.06 & .99 \\
Feb. & 1.00 & .92 & 1.00 & .98 \\
Mar. & 1.30 & 1.20 & 1.30 & 1.26 \\
Apr. & 1.21 & 1.15 & 1.21 & 1.21 \\
May & 1.05 & 1.03 & 1.05 & 1.09 \\
June & .92 & .89 & .92 & .95 \\
July & .96 & .89 & .96 & .95 \\
Aug. & 1.02 & .95 & 1.02 & 1.01 \\
Sept. & .92 & .88 & .92 & .94 \\
Oct. & .80 & .76 & .80 & .82 \\
Nov. & .95 & .86 & .95 & .92 \\
Dec. & .88 & .79 & .88 & .85 \\
Average & 1.01 & .94 & & \\
Incremental adjustment & 0 & .06 & & \\
\hline
\end{tabular}


Table 18. Summary of interoccurrence ratios by month for 2.5 -inch and greater daily precipitation threshold

[A monthly interoccurrence ratio is defined as ratio of mean interoccurrence interval for corresponding month to mean interoccurrence interval for period of record at the station. Mean monthly interoccurrence ratio is arithmetic average of monthly ratios for all stations, and median monthly interoccurrence ratio is median of monthly ratios for all stations. Adjusted ratios have averages of unity and therefore are preferred because statistical bias has been removed.]

\begin{tabular}{lcccc}
\hline Month & $\begin{array}{c}\text { Mean monthly } \\
\text { interoccurrence } \\
\text { ratio }\end{array}$ & $\begin{array}{c}\text { Median monthly } \\
\text { interoccurrence } \\
\text { ratio }\end{array}$ & $\begin{array}{c}\text { Adjusted } \\
\text { mean monthly } \\
\text { interoccurrence } \\
\text { ratio }\end{array}$ & $\begin{array}{c}\text { Adjusted } \\
\text { median monthly } \\
\text { interoccurrence } \\
\text { ratio }\end{array}$ \\
\hline Jan. & 1.04 & .85 & 1.04 & .95 \\
Feb. & .94 & .80 & .94 & .90 \\
Mar. & 1.24 & 1.08 & 1.24 & 1.18 \\
Apr. & 1.19 & 1.09 & 1.19 & 1.19 \\
May & 1.02 & .99 & 1.02 & 1.09 \\
June & .97 & .93 & .97 & 1.03 \\
July & .95 & .88 & .95 & .98 \\
Aug. & 1.00 & .89 & 1.00 & .99 \\
Sept. & .91 & .88 & .91 & .98 \\
Oct. & .80 & .76 & .80 & .86 \\
Nov. & .91 & .83 & .91 & .93 \\
Dec. & .87 & .78 & .87 & .88 \\
Average & .99 & .90 & & \\
Incremental adjustment & .01 & .10 & & \\
\cline { 1 - 3 } & & & &
\end{tabular}

Table 19. Summary of interoccurrence ratios by month for 3.0-inch and greater daily precipitation threshold

[A monthly interoccurrence ratio is defined as ratio of mean interoccurrence interval for corresponding month to mean interoccurrence interval for period of record at the station. Mean monthly interoccurrence ratio is arithmetic average of monthly ratios for all stations, and median monthly interoccurrence ratio is median of monthly ratios for all stations. Adjusted ratios have averages of unity and therefore are preferred because statistical bias has been removed.]

\begin{tabular}{lcccc}
\hline Month & $\begin{array}{c}\text { Mean monthly } \\
\text { interoccurrence } \\
\text { ratio }\end{array}$ & $\begin{array}{c}\text { Median monthly } \\
\text { interoccurrence } \\
\text { ratio }\end{array}$ & $\begin{array}{c}\text { Adjusted } \\
\text { mean monthly } \\
\text { interoccurrence } \\
\text { ratio }\end{array}$ & $\begin{array}{c}\text { Adjusted } \\
\text { median monthly } \\
\text { interoccurrence } \\
\text { ratio }\end{array}$ \\
\hline Jan. & .98 & .74 & 1.01 & .89 \\
Feb. & .90 & .70 & .93 & .85 \\
Mar. & 1.15 & 1.01 & 1.18 & 1.16 \\
Apr. & 1.12 & 1.01 & 1.15 & 1.16 \\
May & .99 & .93 & 1.02 & 1.08 \\
June & .94 & .88 & .97 & 1.03 \\
July & .96 & .82 & .99 & .97 \\
Aug. & 1.01 & .89 & 1.04 & 1.04 \\
Sept. & .91 & .86 & .94 & 1.01 \\
Oct. & .83 & .79 & .86 & .94 \\
Nov. & .91 & .81 & .94 & .96 \\
Dec. & .94 & .77 & .97 & .92 \\
Average & .97 & .85 & & \\
Incremental adjustment & .03 & .15 & & \\
\hline
\end{tabular}


Table 20. Regional estimates of mean interoccurrence interval of precipitation for Austin, Texas

[Regional mean interoccurrence intervals estimated from figures 4-13. For comparison, site-specific mean interoccurrence intervals for 72-year record for Austin Camp Mabry (station no. 428, sequence no. 46) are listed as well as those for 18-year record for Austin Dam (station no. 430, sequence no. 47).]

\begin{tabular}{cccc}
\hline $\begin{array}{c}\text { Daily } \\
\text { precip- } \\
\text { itation } \\
\text { threshold } \\
\text { (inches) }\end{array}$ & $\begin{array}{c}\text { Regional } \\
\text { mean } \\
\text { inter- } \\
\text { occur- } \\
\text { rence } \\
\text { interval } \\
\text { (days) }\end{array}$ & $\begin{array}{c}\text { Station 428 } \\
\text { site- } \\
\text { specific } \\
\text { mean } \\
\text { inter- } \\
\text { occur- } \\
\text { rence } \\
\text { interval } \\
\text { (days) }\end{array}$ & $\begin{array}{c}\text { Station 430 } \\
\text { site- } \\
\text { specific } \\
\text { mean } \\
\text { inter- } \\
\text { occur- } \\
\text { rence } \\
\text { interval } \\
\text { (days) }\end{array}$ \\
\hline 0.05 & 6.6 & 5.96 & 6.55 \\
.10 & 7.8 & 7.37 & 8.03 \\
.25 & 11.3 & 11.06 & 12.60 \\
.50 & 17.5 & 17.66 & 20.20 \\
.75 & 26 & 26.01 & 28.41 \\
1.0 & 36 & 37.41 & 42.42 \\
1.5 & 79 & 76.04 & 79.54 \\
2.0 & 150 & 146.89 & 159.08 \\
2.5 & 265 & 251.00 & 254.52 \\
3.0 & 440 & 403.95 & 454.20 \\
\hline
\end{tabular}

